Supporting Information for:

\title{
Chiral triphenylacetic acid esters - residual stereoisomerism and solid-state variability of molecular architectures
}

Natalia Prusinowska ${ }^{a, b}$, Agnieszka Czapik and Marcin Kwit ${ }^{a, b *}$

a) Faculty of Chemistry, Adam Mickiewicz University, Uniwersytetu Poznańskiego 8, 61614 Poznań, Poland. E-mail: marcin.kwit@amu.edu.pl

b) Centre for Advanced Technologies, Adam Mickiewicz University, Uniwersytetu Poznańskiego 10, 61614 Poznań 


\section{Table of contents:}

Calculations details

Table SI_1. Concentrations $\left(c\right.$, in $\left.\mathrm{mol} \mathrm{L}^{-1}\right)$ of the samples used for UV and ECD measurements.....

Table SI_2. ECD $\left(\Delta \varepsilon\right.$, in $\left.\mathrm{dm}^{3} \cdot \mathrm{mol}^{-1} \cdot \mathrm{cm}^{-1}\right)$ and UV $\left(\varepsilon\right.$, in $\left.\mathrm{dm}^{3} \cdot \mathrm{mol}^{-1} \cdot \mathrm{cm}^{-1}\right)$ data for derivatives 1-22 measured

in acetonitrile solution.

Table SI_3. Total and free energies $(E, \Delta G$, in Hartree), relative energies $(\Delta E, \Delta \Delta G$ in kcal mol-1), percentage populations and number of imaginary frequencies (\#ImFreq) calculated at the B3LYP/6-311++G(d,p) level for individual conformers of $\mathbf{1}$.

Table SI_4. Total and free energies ( $E, \Delta G$, in Hartree), relative energies $\left(\Delta E, \Delta \Delta G\right.$ in $\left.\mathrm{kcal}^{\mathrm{mol}}{ }^{-1}\right)$, percentage populations and number of imaginary frequencies (\#ImFreq) calculated at the M06-2X/6-311++G(d,p) level for individual conformers of $\mathbf{1}$.

Table SI_5. Total and free energies $(E, \Delta G$, in Hartree), relative energies $(\Delta E, \Delta \Delta G$ in kcal mol-1), percentage populations and number of imaginary frequencies (\#ImFreq) calculated at the B3LYP/6-311++G(d,p) level for individual conformers of 4 .

Table SI_6. Total and free energies $\left(E, \Delta G\right.$, in Hartree), relative energies $\left(\Delta E, \Delta \Delta G\right.$ in $\left.\mathrm{kcal}^{\mathrm{mol}}{ }^{-1}\right)$, percentage populations and number of imaginary frequencies (\#ImFreq) calculated at the M06-2X/6-311++G(d,p) level for individual conformers of 4.

Table SI_7. Total and free energies $(E, \Delta G$, in Hartree), relative energies $(\Delta E, \Delta \Delta G$ in kcal mol-1), percentage populations and number of imaginary frequencies (\#ImFreq) calculated at the B3LYP/6-311++G(d,p) level for individual conformers of 6 .

Table SI_8. Total and free energies $\left(E, \Delta G\right.$, in Hartree), relative energies $\left(\Delta E, \Delta \Delta G\right.$ in $\left.\mathrm{kcal}^{\mathrm{mol}}{ }^{-1}\right)$, percentage populations and number of imaginary frequencies (\#ImFreq) calculated at the M06-2X/6-311++G(d,p) level for individual conformers of 6 .

Table SI_9. Total and free energies $(E, \Delta G$, in Hartree), relative energies $(\Delta E, \Delta \Delta G$ in kcal mol-1), percentage populations and number of imaginary frequencies (\#ImFreq) calculated at the B3LYP/6-311++G(d,p) level for individual conformers of $\mathbf{1 0 .}$

Table SI_10. Total and free energies $\left(E, \Delta G\right.$, in Hartree), relative energies $\left(\Delta E, \Delta \Delta G\right.$ in kcal mol$\left.{ }^{-1}\right)$, percentage populations and number of imaginary frequencies (\#ImFreq) calculated at the M06-2X/6$311++G(d, p)$ level for individual conformers of 10 .

Table SI_11. Total and free energies $(E, \Delta G$, in Hartree), relative energies $(\Delta E, \Delta \Delta G$ in kcal mol-1), percentage populations and number of imaginary frequencies (\#ImFreq) calculated at the B3LYP/6$311++G(d, p)$ level for individual conformers of 11.

Table SI_12. Total and free energies $\left(E, \Delta G\right.$, in Hartree), relative energies $\left(\Delta E, \Delta \Delta G\right.$ in kcal mol$\left.{ }^{-1}\right)$, percentage populations and number of imaginary frequencies (\#ImFreq) calculated at the M06-2X/6$311++G(d, p)$ level for individual conformers of 11 .

Table SI_13. Total and free energies $\left(E, \Delta G\right.$, in Hartree), relative energies $\left(\Delta E, \Delta \Delta G\right.$ in $\left.\mathrm{kcal} \mathrm{mol}^{-1}\right)$, percentage populations and number of imaginary frequencies (\#ImFreq) calculated at the B3LYP/6$311++G(d, p)$ level for individual conformers of 14 .

Table SI_14. Total and free energies $\left(E, \Delta G\right.$, in Hartree), relative energies $\left(\Delta E, \Delta \Delta G\right.$ in kcal mol$\left.{ }^{-1}\right)$, percentage populations and number of imaginary frequencies (\#ImFreq) calculated at the M06-2X/6$311++G(d, p)$ level for individual conformers of 14 . 
Table SI_15. Total and free energies ( $E, \Delta G$, in Hartree), relative energies $\left(\Delta E, \Delta \Delta G\right.$ in $\left.\mathrm{kcal} \mathrm{mol}^{-1}\right)$, percentage populations and number of imaginary frequencies (\#ImFreq) calculated at the B3LYP/6$311++G(d, p)$ level for individual conformers of 15 .

Table SI_16. Total and free energies ( $E, \Delta G$, in Hartree), relative energies $\left(\Delta E, \Delta \Delta G\right.$ in $\left.\mathrm{kcal} \mathrm{mol}^{-1}\right)$, percentage populations and number of imaginary frequencies (\#ImFreq) calculated at the M06-2X/6$311++G(d, p)$ level for individual conformers of 15.

Table SI_17. Total and free energies ( $E, \Delta G$, in Hartree), relative energies $\left(\Delta E, \Delta \Delta G\right.$ in $\left.\mathrm{kcal} \mathrm{mol}^{-1}\right)$, percentage populations and number of imaginary frequencies (\#ImFreq) calculated at the B3LYP/6$311 \mathrm{G}(\mathrm{d}, \mathrm{p})$ level for individual conformers of $\mathbf{1 8}$.

Table SI_18. Total and free energies ( $E, \Delta G$, in Hartree), relative energies ( $\Delta E, \Delta \Delta G$ in kcal mol$\left.{ }^{-1}\right)$, percentage populations and number of imaginary frequencies (\#ImFreq) calculated at the B3LYP-GD3BJ/6$311 \mathrm{G}(\mathrm{d}, \mathrm{p})$ level for individual conformers of 18 .

Table SI_19. Total and free energies ( $E, \Delta G$, in Hartree), relative energies $\left(\Delta E, \Delta \Delta G\right.$ in $\left.\mathrm{kcal} \mathrm{mol}^{-1}\right)$, percentage populations and number of imaginary frequencies (\#ImFreq) calculated at the M06-2X/6$311 \mathrm{G}(\mathrm{d}, \mathrm{p})$ level for individual conformers of $\mathbf{1 8}$.

Table SI_20. Total and free energies ( $E, \Delta G$, in Hartree), relative energies $\left(\Delta E, \Delta \Delta G\right.$ in kcal mol$\left.{ }^{-1}\right)$, percentage populations and number of imaginary frequencies (\#ImFreq) calculated at the B3LYP/6$311 \mathrm{G}(\mathrm{d}, \mathrm{p})$ level for individual conformers of $\mathbf{2 0}$.

Table SI_21. Total and free energies ( $E, \Delta G$, in Hartree), relative energies $\left(\Delta E, \Delta \Delta G\right.$ in $\left.\mathrm{kcal} \mathrm{mol}^{-1}\right)$, percentage populations and number of imaginary frequencies (\#ImFreq) calculated at the B3LYP-GD3BJ/6$311 \mathrm{G}(\mathrm{d}, \mathrm{p})$ level for individual conformers of $\mathbf{2 0}$.

Table SI_22. Total and free energies ( $E, \Delta G$, in Hartree), relative energies $\left(\Delta E, \Delta \Delta G\right.$ in $\left.\mathrm{kcal} \mathrm{mol}^{-1}\right)$, percentage populations and number of imaginary frequencies (\#ImFreq) calculated at the M06-2X/6$311 G(d, p)$ level for individual conformers of 20.

Table SI_23. Total and free energies ( $E, \Delta G$, in Hartree), relative energies $\left(\Delta E, \Delta \Delta G\right.$ in $\left.\mathrm{kcal} \mathrm{mol}^{-1}\right)$, percentage populations and number of imaginary frequencies (\#ImFreq) calculated at the B3LYP/6$311 \mathrm{G}(\mathrm{d}, \mathrm{p})$ level for individual conformers of $\mathbf{2 1}$.

Table SI_24. Total and free energies ( $E, \Delta G$, in Hartree), relative energies $\left(\Delta E, \Delta \Delta G\right.$ in $\left.\mathrm{kcal} \mathrm{mol}^{-1}\right)$, percentage populations and number of imaginary frequencies (\#ImFreq) calculated at the B3LYP-GD3BJ/6$311 \mathrm{G}(\mathrm{d}, \mathrm{p})$ level for individual conformers of $\mathbf{2 1}$.

Table SI_25. Total and free energies ( $E, \Delta G$, in Hartree), relative energies $\left(\Delta E, \Delta \Delta G\right.$ in $\left.\mathrm{kcal} \mathrm{mol}^{-1}\right)$, percentage populations and number of imaginary frequencies (\#ImFreq) calculated at the M06-2X/6$311 \mathrm{G}(\mathrm{d}, \mathrm{p})$ level for individual conformers of $\mathbf{2 1}$.

Table SI_26. Total and free energies ( $E, \Delta G$, in Hartree), relative energies $\left(\Delta E, \Delta \Delta G\right.$ in $\left.\mathrm{kcal} \mathrm{mol}^{-1}\right)$, percentage populations and number of imaginary frequencies (\#ImFreq) calculated at the B3LYP/6$311 \mathrm{G}(\mathrm{d}, \mathrm{p})$ level for individual conformers of $\mathbf{2 2}$.

Table SI_27. Total and free energies ( $E, \Delta G$, in Hartree), relative energies $\left(\Delta E, \Delta \Delta G\right.$ in $\left.\mathrm{kcal} \mathrm{mol}^{-1}\right)$, percentage populations and number of imaginary frequencies (\#ImFreq) calculated at the B3LYP-GD3BJ/6$311 \mathrm{G}(\mathrm{d}, \mathrm{p})$ level for individual conformers of $\mathbf{2 2}$.

Table SI_28. Total and free energies ( $E, \Delta G$, in Hartree), relative energies $\left(\Delta E, \Delta \Delta G\right.$ in $\left.\mathrm{kcal} \mathrm{mol}^{-1}\right)$, percentage populations and number of imaginary frequencies (\#ImFreq) calculated at the M06-2X/6$311 \mathrm{G}(\mathrm{d}, \mathrm{p})$ level for individual conformers of $\mathbf{2 2}$ 
Table SI_29. Dihedral angles $\alpha, 6, \gamma, \delta$ and $\zeta$ (in degrees) and selected interatomic distances $I_{1}, I_{2}$ (in $\AA$ ) calculated at the B3LYP/6-311++G(d,p) level for individual low-energy conformers of 1 .

Table SI_30. Dihedral angles $\alpha, b, \gamma, \delta$ and $\zeta$ (in degrees) and selected interatomic distances $I_{1}, I_{2}$ (in $\AA$ ) calculated at the M06-2X/6-311++G(d,p) level for individual low-energy conformers of 1 .

Table SI_31. Dihedral angles $\alpha, b, \gamma, \delta$ and $\zeta$ (in degrees) and selected interatomic distances $I_{1}, I_{2}$ (in $\AA$ ) calculated at the B3LYP/6-311++G(d,p) level for individual low-energy conformers of 4 .

Table SI_32. Dihedral angles $\alpha, b, \gamma, \delta$ and $\zeta$ (in degrees) and selected interatomic distances $I_{1}, I_{2}$ (in $\AA$ ) calculated at the M06-2X/6-311++G(d,p) level for individual low-energy conformers of 4 .

Table SI_33. Dihedral angles $\alpha, 6, \gamma, \delta$ and $\zeta$ (in degrees) and selected interatomic distances $I_{1}, I_{2}$ (in $\AA$ ) calculated at the B3LYP/6-311++G(d,p) level for individual low-energy conformers of 6 . S40

Table SI_34. Dihedral angles $\alpha, 6, y, \delta$ and $\zeta$ (in degrees) and selected interatomic distances $I_{1}$ and $I_{2}$ (in $\AA$ ) calculated at the M06-2X/6-311++G(d,p) level for individual low-energy conformers of 6 .

Table SI_35. Dihedral angles $\alpha, b, y, \delta$ and $\zeta$ (in degrees) and selected interatomic distances $I_{1}, I_{2}$ (in $\AA$ ) calculated at the DFT/6-311++G(d,p) level for individual low-energy conformers of $\mathbf{1 0}$.

Table SI_36. Dihedral angles $\alpha, b, \gamma, \delta$ and $\zeta$ (in degrees) and selected interatomic distances $I_{1}, I_{2}$ (in $\AA$ ) calculated at the B3LYP/6-311++G(d,p) level for individual low-energy conformers of 11 .

Table SI_37. Dihedral angles $\alpha, b, \gamma, \delta$ and $\zeta$ (in degrees) and selected interatomic distances $I_{1}, I_{2}$ (in $\AA$ ) calculated at the M06-2X/6-311++G(d,p) level for individual low-energy conformers of $\mathbf{1 1}$. S43

Table SI_38. Dihedral angles $\alpha, b, \gamma, \delta, \zeta$ and $\varphi$ (in degrees) and selected interatomic distances $I_{1}, I_{2}$ and $I_{3}$ (in $\AA$ ) calculated at the B3LYP/6-311++G(d,p) level for individual low-energy conformers of 14 .

Table SI_39. Dihedral angles $\alpha, b, y, \delta, \zeta$ and $\varphi$ (in degrees) and selected interatomic distances $I_{1}, I_{2}$ and $I_{3}$ (in $\AA$ ) calculated at the M06-2X/6-311++G(d,p) level for individual low-energy conformers of 14 . S44

Table SI_40. Dihedral angles $\alpha, b, \gamma, \delta, \zeta$ and $\varphi$ (in degrees) and selected interatomic distances $I_{1}, I_{2}$ and $I_{3}$ (in $\AA$ ) calculated at the B3LYP/6-311++G(d,p) level for individual low-energy conformers of $\mathbf{1 5}$. S45

Table SI_41. Dihedral angles $\alpha, 6, v, \delta, \zeta$ and $\varphi$ (in degrees) and selected interatomic distances $I_{1}, I_{2}$ and $I_{3}$ (in $\AA$ ) calculated at the M06-2X/6-311++G(d,p) level for individual low-energy conformers of $\mathbf{1 5}$. S46

Table SI_42. Dihedral angles $\alpha, b, \gamma, \delta, \zeta$ and $\varphi$ (in degrees) and selected interatomic distances $I_{1}, I_{2}$ and $I_{3}$ (in $\AA$ ) calculated at the B3LYP/6-311G(d,p) level for individual low-energy conformers of 18 .

Table SI_43. Dihedral angles $\alpha, b, \gamma, \delta, \zeta$ and $\varphi$ (in degrees) and selected interatomic distances $I_{1}, I_{2}$ and $I_{3}$ (in $\AA$ ) calculated at the B3LYP-GD3Bj/6-311G(d,p) level for individual low-energy conformers of 18 ... S49

Table SI_44. Dihedral angles $\alpha, b, y, \delta, \zeta$ and $\varphi$ (in degrees) and selected interatomic distances $I_{1}, I_{2}$ and $I_{3}$ (in $\AA$ ) calculated at the M06-2X/6-311G(d,p) level for individual low-energy conformers of 18 . S50

Table SI_45. Dihedral angles $\alpha, 6, \gamma, \delta, \zeta$ and $\varphi$ (in degrees) and selected interatomic distances $I_{1}, I_{2}$ and $I_{3}$ (in $\AA$ ) calculated at the B3LYP/6-311G $(d, p)$ level for individual low-energy conformers of 20. S51

Table SI_46. Dihedral angles $\alpha, b, \gamma, \delta, \zeta$ and $\varphi$ (in degrees) and selected interatomic distances $I_{1}, I_{2}$ and $I_{3}$ (in $\AA$ ) calculated at the B3LYP-GD3BJ/6-311G $(d, p)$ level for individual low-energy conformers of 20. .....S53

Table SI_47. Dihedral angles $\alpha, b, y, \delta, \zeta$ and $\varphi$ (in degrees) and selected interatomic distances $I_{1}, I_{2}$ and $I_{3}$ (in $\AA$ ) calculated at the M06-2X/6-311G(d,p) level for individual low-energy conformers of 20. S54

Table SI_48. Dihedral angles $\alpha, b, \gamma, \delta, \zeta$ and $\varphi$ (in degrees) and selected interatomic distances $I_{1}, I_{2}$ and $I_{3}$ (in $\AA$ ) calculated at the B3LYP/6-311G(d,p) level for individual low-energy conformers of 21 . .S55 
Table SI_49. Dihedral angles $\alpha, 6, \gamma, \delta, \zeta$ and $\varphi$ (in degrees) and selected interatomic distances $I_{1}, I_{2}$ and $I_{3}$ (in $\AA$ ) calculated at the B3LYP-GD3BJ/6-311G(d,p) level for individual low-energy conformers of 21. .....S56

Table SI_50. Dihedral angles $\alpha, 6, \gamma, \delta, \zeta$ and $\varphi$ (in degrees) and selected interatomic distances $I_{1}, I_{2}$ and $I_{3}$ (in $\AA$ ) calculated at the M06/6-311G(d,p) level for individual low-energy conformers of 21.

Table SI_51. Dihedral angles $\alpha, 8, \gamma, \delta, \zeta$ and $\varphi$ (in degrees) and selected interatomic distances $I_{1}, I_{2}$ and $I_{3}$ (in $\AA$ ) calculated at the B3LYP/6-311G(d,p) level for individual low-energy conformers of 22.

Table SI_52. Dihedral angles $\alpha, 6, \gamma, \delta, \zeta$ and $\varphi$ (in degrees) and selected interatomic distances $I_{1}, I_{2}$ and $I_{3}$ (in $\AA$ ) calculated at the B3LYP-GD3BJ/6-311G(d,p) level for individual low-energy conformers of 22..... S60

Table SI_53. Dihedral angles $\alpha, 6, \gamma, \delta, \zeta$ and $\varphi$ (in degrees) and selected interatomic distances $I_{1}, I_{2}$ and $I_{3}$ (in $\AA$ ) calculated at the M06-2X/6-311G(d,p) level for individual low-energy conformers of 22. S61

Figure SI_1. Structures of individual, low-energy conformers of 1, calculated at the B3LYP/6-311++G(d,p) level of theory.

Figure SI_2. Structures of individual, low-energy conformers of 1, calculated at the M06-2X/6-311++G(d,p) level of theory.

Figure SI_3. Structures of individual, low-energy conformers of 4, calculated at the B3LYP/6-311++G(d,p) level of theory.

Figure SI_4. Structures of individual, low-energy conformers of 4, calculated at the M06-2X/6-311++G(d,p) level of theory. S64

Figure SI_5. Structures of individual, low-energy conformers of 6, calculated at the B3LYP/6-311++G(d,p) level of theory.

Figure SI_6. Structures of individual, low-energy conformers of 6, calculated at the M06-2X/6-311++G(d,p) level of theory.

Figure SI_7. Structure of individual, low-energy conformer of 10, calculated at the B3LYP/6-311++G(d,p) level of theory.

Figure SI_8. Structure of individual, low-energy conformer of 10, calculated at the M06-2X/6-311++G(d,p) level of theory.

Figure SI_9. Structures of individual, low-energy conformers of 11, calculated at the B3LYP/6-311++G(d,p) level of theory. . 568

Figure SI_10. Structures of individual, low-energy conformers of 11, calculated at the M06-2X/6$311++G(d, p)$ level of theory.

Figure SI_11. Structures of individual, low-energy conformers of 14, calculated at the B3LYP/6$311++G(d, p)$ level of theory.

Figure SI_12. Structures of individual, low-energy conformers of 14, calculated at the M06-2X/6$311++G(d, p)$ level of theory.

Figure SI_13. Structures of individual, low-energy conformers of 15, calculated at the B3LYP/6$311++G(d, p)$ level of theory.

Figure SI_14. Structures of individual, low-energy conformers of 15, calculated at the M06-2X/6$311++G(d, p)$ level of theory.

Figure SI_15a. Structures of individual, low-energy conformers of 18, calculated at the B3LYP/6-311G(d,p) level of theory, part I. 
Figure SI_15b. Structures of individual, low-energy conformers of 18, calculated at the B3LYP/6-311G(d,p) level of theory, part II.

Figure SI_16. Structures of individual, low-energy conformers of 11, calculated at the B3LYP-GD3BJ/6$311 G(d, p)$ level of theory.

Figure SI_17. Structures of individual, low-energy conformers of 18, calculated at the M06-2X/6-311G(d,p) level of theory.

Figure SI_18. Structures of individual, low-energy conformers of 20, calculated at the B3LYP/6-311G(d,p) level of theory.

Figure SI_19. Structures of individual, low-energy conformers of 20, calculated at the B3LYP-GD3BJ/6$311 \mathrm{G}(\mathrm{d}, \mathrm{p})$ level of theory.

Figure SI_20. Structures of individual, low-energy conformers of 20, calculated at the M06-2X/6-311G(d,p) level of theory.

Figure SI_21. Structures of individual, low-energy conformers of 21, calculated at the B3LYP/6-311G(d,p) level of theory.

Figure SI_22. Structures of individual, low-energy conformers of 21, calculated at the B3LYP-GD3BJ/6$311 G(d, p)$ level of theory. S80

Figure SI_23. Structures of individual, low-energy conformers of 21, calculated at the M06-2X/6-311G(d,p) level of theory.

Figure SI_24a. Structures of individual, low-energy conformers of 22, calculated at the B3LYP/6-311G(d,p) level of theory, part I.

Figure SI_24b. Structures of individual, low-energy conformers of 22, calculated at the B3LYP/6-311G(d,p) level of theory, part II.

Figure SI_25. Structures of individual, low-energy conformers of 22, calculated at the B3LYP-GD3BJ/6$311 \mathrm{G}(\mathrm{d}, \mathrm{p})$ level of theory.

Figure SI_26. Structures of individual, low-energy conformers of 22, calculated at the M06-2X/6-311G(d,p) level of theory.

Figure SI_27. UV and ECD spectra of the low-energy conformers of compound 1 calculated at TD-CAM$B 3 L Y P / 6-311++G(2 d, 2 p)$ level for structures optimized at B3LYP/6-311++G(d,p) level. Wavelengths were not corrected.

Figure SI_28. UV and ECD spectra of the low-energy conformers of compound 1 calculated at TD-M06$2 X / 6-311++G(2 d, 2 p)$ level for structures optimized at B3LYP/6-311++G(d,p) level. Wavelengths were not corrected.

Figure SI_29. UV and ECD spectra of the low-energy conformers of compound 1 calculated at TD-CAM$B 3 L Y P / 6-311++G(2 d, 2 p)$ level for structures optimized at $M 06-2 X / 6-311++G(d, p)$ level. Wavelengths were not corrected.

Figure SI_30. UV and ECD spectra of the low-energy conformers of compound 1 calculated at TD-M06$2 X / 6-311++G(2 d, 2 p)$ level for structures optimized at M06-2X/6-311++G(d,p) level. Wavelengths were not corrected.

Figure SI_31. UV and ECD spectra of the low-energy conformers of compound 4 calculated at TD-CAM$B 3 L Y P / 6-311++G(2 d, 2 p)$ level for structures optimized at B3LYP/6-311++G(d,p) level. Wavelengths were not corrected. 
Figure SI_32. UV and ECD spectra of the low-energy conformers of compound 4 calculated at TD-M06$2 X / 6-311++G(2 d, 2 p)$ level for structures optimized at $B 3 L Y P / 6-311++G(d, p)$ level. Wavelengths were not corrected.

Figure SI_33. UV and ECD spectra of the low-energy conformers of compound 4 calculated at TD-CAM$B 3 L Y P / 6-311++G(2 d, 2 p)$ level for structures optimized at M06-2X/6-311++G(d,p) level. Wavelengths were not corrected.

Figure SI_34. UV and ECD spectra of the low-energy conformers of compound 4 calculated at TD-M06$2 X / 6-311++G(2 d, 2 p)$ level for structures optimized at M06-2X/6-311++G(d,p) level. Wavelengths were not corrected........

Figure SI_35. UV and ECD spectra of the low-energy conformers of compound 6 calculated at TD-CAM$B 3 L Y P / 6-311++G(2 d, 2 p)$ level for structures optimized at $B 3 L Y P / 6-311++G(d, p)$ level. Wavelengths were not corrected.

Figure SI_36. UV and ECD spectra of the low-energy conformers of compound 6 calculated at TD-M06$2 X / 6-311++G(2 d, 2 p)$ level for structures optimized at B3LYP/6-311++G(d,p) level. Wavelengths were not corrected.......

Figure SI_37. UV and ECD spectra of the low-energy conformers of compound 6 calculated at TD-CAM$B 3 L Y P / 6-311++G(2 d, 2 p)$ level for structures optimized at M06-2X/6-311++G $(d, p)$ level. Wavelengths were not corrected.......

Figure SI_38. UV and ECD spectra of the low-energy conformers of compound 6 calculated at TD-M06$2 X / 6-311++G(2 d, 2 p)$ level for structures optimized at M06-2X/6-311++G(d,p) level. Wavelengths were not corrected.......

Figure SI_39. UV and ECD spectra of the low-energy conformers of compound $\mathbf{1 0}$ calculated at TD-CAM$B 3 L Y P / 6-311++G(2 d, 2 p)$ level for structures optimized at $B 3 L Y P / 6-311++G(d, p)$ level. Wavelengths were not corrected.

Figure SI_40. UV and ECD spectra of the low-energy conformers of compound 10 calculated at TD-M06$2 X / 6-311++G(2 d, 2 p)$ level for structures optimized at B3LYP/6-311++G(d,p) level. Wavelengths were not corrected.......

Figure SI_41. UV and ECD spectra of the low-energy conformers of compound 10 calculated at TD-CAM$B 3 L Y P / 6-311++G(2 d, 2 p)$ level for structures optimized at M06-2X/6-311++G(d,p) level. Wavelengths were not corrected.

Figure SI_42. UV and ECD spectra of the low-energy conformers of compound 10 calculated at TD-M06$2 X / 6-311++G(2 d, 2 p)$ level for structures optimized at M06-2X/6-311++G(d,p) level. Wavelengths were not corrected.......

Figure SI_43. UV and ECD spectra of the low-energy conformers of compound $\mathbf{1 1}$ calculated at TD-CAM$B 3 L Y P / 6-311++G(2 d, 2 p)$ level for structures optimized at $B 3 L Y P / 6-311++G(d, p)$ level. Wavelengths were not corrected.

Figure SI_44. UV and ECD spectra of the low-energy conformers of compound $\mathbf{1 1}$ calculated at TD-M06$2 X / 6-311++G(2 d, 2 p)$ level for structures optimized at B3LYP/6-311++G $(d, p)$ level. Wavelengths were not corrected.......

Figure SI_45. UV and ECD spectra of the low-energy conformers of compound $\mathbf{1 1}$ calculated at TD-CAM$B 3 L Y P / 6-311++G(2 d, 2 p)$ level for structures optimized at M06-2X/6-311++G(d,p) level. Wavelengths were not corrected. 
Figure SI_46. UV and ECD spectra of the low-energy conformers of compound $\mathbf{1 1}$ calculated at TD-M06$2 X / 6-311++G(2 d, 2 p)$ level for structures optimized at M06-2X/6-311++G(d,p) level. Wavelengths were not corrected.

Figure SI_47. UV and ECD spectra of the low-energy conformers of compound $\mathbf{1 4}$ calculated at TD-CAM$B 3 L Y P / 6-311++G(2 d, 2 p)$ level for structures optimized at $B 3 L Y P / 6-311++G(d, p)$ level. Wavelengths were not corrected.

Figure SI_48. UV and ECD spectra of the low-energy conformers of compound 14 calculated at TD-M06$2 X / 6-311++G(2 d, 2 p)$ level for structures optimized at B3LYP/6-311++G $(d, p)$ level. Wavelengths were not corrected........

Figure SI_49. UV and ECD spectra of the low-energy conformers of compound $\mathbf{1 4}$ calculated at TD-CAM$B 3 L Y P / 6-311++G(2 d, 2 p)$ level for structures optimized at $M 06-2 X / 6-311++G(d, p)$ level. Wavelengths were not corrected.

Figure SI_50. UV and ECD spectra of the low-energy conformers of compound 14 calculated at TD-M06$2 X / 6-311++G(2 d, 2 p)$ level for structures optimized at M06-2X/6-311++G $(d, p)$ level. Wavelengths were not corrected.......

Figure SI_51. UV and ECD spectra of the low-energy conformers of compound 15 calculated at TD-CAM$B 3 L Y P / 6-311++G(2 d, 2 p)$ level for structures optimized at $B 3 L Y P / 6-311++G(d, p)$ level. Wavelengths were not corrected....... S100

Figure SI_52. UV and ECD spectra of the low-energy conformers of compound 15 calculated at TD-M06$2 X / 6-311++G(2 d, 2 p)$ level for structures optimized at $B 3 L Y P / 6-311++G(d, p)$ level. Wavelengths were not corrected....... S100

Figure SI_53. UV and ECD spectra of the low-energy conformers of compound 15 calculated at TD-CAM$B 3 L Y P / 6-311++G(2 d, 2 p)$ level for structures optimized at M06-2X/6-311++G(d,p) level. Wavelengths were not corrected... S101

Figure SI_54. UV and ECD spectra of the low-energy conformers of compound 15 calculated at TD-M06$2 X / 6-311++G(2 d, 2 p)$ level for structures optimized at M06-2X/6-311++G(d,p) level. Wavelengths were not corrected....... S101

Figure SI_55. UV and ECD spectra of the low-energy conformers of compound $\mathbf{1 8}$ calculated at TD-CAM$B 3 L Y P / 6-311++G(d, p)$ level for structures optimized at B3LYP/6-311G $(d, p)$ level. Wavelengths were not corrected.

Figure SI_56. UV and ECD spectra of the low-energy conformers of compound 18 calculated at TD-M06$2 X / 6-311++G(d, p)$ level for structures optimized at B3LYP/6-311G $(d, p)$ level. Wavelengths were not corrected.......

Figure SI_57. UV and ECD spectra of the low-energy conformers of compound $\mathbf{1 8}$ calculated at TD-CAM$B 3 L Y P / 6-311++G(d, p)$ level for structures optimized at B3LYP-GD3BJ/6-311G(d,p) level. Wavelengths were not corrected. S106

Figure SI_58. UV and ECD spectra of the low-energy conformers of compound $\mathbf{1 8}$ calculated at TD-M06$2 X / 6-311++G(d, p)$ level for structures optimized at B3LYP-GD3BJ/6-311G $(d, p)$ level. Wavelengths were not corrected.

Figure SI_59. UV and ECD spectra of the low-energy conformers of compound 18 calculated at TD-CAM$B 3 L Y P / 6-311++G(d, p)$ level for structures optimized at M06-2X/6-311G $(d, p)$ level. Wavelengths were not corrected. 
Figure SI_60. UV and ECD spectra of the low-energy conformers of compound $\mathbf{1 8}$ calculated at TD-M06$2 X / 6-311++G(d, p)$ level for structures optimized at M06-2X/6-311G(d,p) level. Wavelengths were not corrected.

Figure SI_61. UV and ECD spectra of the low-energy conformers of compound 20 calculated at TD-CAM$B 3 L Y P / 6-311++G(d, p)$ level for structures optimized at B3LYP/6-311G $(d, p)$ level. Wavelengths were not corrected.

Figure SI_62. UV and ECD spectra of the low-energy conformers of compound 20 calculated at TD-M06$2 X / 6-311++G(d, p)$ level for structures optimized at B3LYP/6-311G(d,p) level. Wavelengths were not corrected........

Figure SI_63. UV and ECD spectra of the low-energy conformers of compound 20 calculated at TD-CAM$B 3 L Y P / 6-311++G(d, p)$ level for structures optimized at B3LYP-GD3BJ/6-311G(d,p) level. Wavelengths were not corrected.

Figure SI_64. UV and ECD spectra of the low-energy conformers of compound 20 calculated at TD-M06$2 X / 6-311++G(d, p)$ level for structures optimized at B3LYP-GD3BJ/6-311G $(d, p)$ level. Wavelengths were not corrected.......

Figure SI_65. UV and ECD spectra of the low-energy conformers of compound 20 calculated at TD-CAM$B 3 L Y P / 6-311++G(d, p)$ level for structures optimized at M06-2X/6-311G(d,p) level. Wavelengths were not corrected.

Figure SI_66. UV and ECD spectra of the low-energy conformers of compound 20 calculated at TD-M06$2 X / 6-311++G(d, p)$ level for structures optimized at M06-2X/6-311G $(d, p)$ level. Wavelengths were not corrected.

Figure SI_67. UV and ECD spectra of the low-energy conformers of compound $\mathbf{2 1}$ calculated at TD-CAM$B 3 L Y P / 6-311++G(d, p)$ level for structures optimized at B3LYP/6-311G $(d, p)$ level. Wavelengths were not corrected.

Figure SI_68. UV and ECD spectra of the low-energy conformers of compound 21 calculated at TD-M06$2 X / 6-311++G(d, p)$ level for structures optimized at B3LYP/6-311G $(d, p)$ level. Wavelengths were not corrected.

Figure SI_69. UV and ECD spectra of the low-energy conformers of compound 21 calculated at TD-CAMB3LYP/6-311++G(d,p) level for structures optimized at B3LYP-GD3BJ/6-311G(d,p) level. Wavelengths were not corrected.

Figure SI_70. UV and ECD spectra of the low-energy conformers of compound 21 calculated at TD-M06$2 X / 6-311++G(d, p)$ level for structures optimized at B3LYP-GD3BJ/6-311G $(d, p)$ level. Wavelengths were not corrected.

Figure SI_71. UV and ECD spectra of the low-energy conformers of compound $\mathbf{2 1}$ calculated at TD-CAM$B 3 L Y P / 6-311++G(d, p)$ level for structures optimized at M06-2X/6-311G $(d, p)$ level. Wavelengths were not corrected.

Figure SI_72. UV and ECD spectra of the low-energy conformers of compound 21 calculated at TD-M06$2 X / 6-311++G(d, p)$ level for structures optimized at M06-2X/6-311G $(d, p)$ level. Wavelengths were not corrected.

Figure SI_73. UV and ECD spectra of the low-energy conformers of compound 22 calculated at TD-CAM$B 3 L Y P / 6-311++G(d, p)$ level for structures optimized at B3LYP/6-311G $(d, p)$ level. Wavelengths were not corrected. 
Figure SI_74. UV and ECD spectra of the low-energy conformers of compound 22 calculated at TD-M06$2 X / 6-311++G(d, p)$ level for structures optimized at B3LYP/6-311G $(d, p)$ level. Wavelengths were not corrected.

Figure SI_75. UV and ECD spectra of the low-energy conformers of compound 22 calculated at TD-CAMB3LYP/6-311++G(d,p) level for structures optimized at B3LYP-GD3BJ/6-311G(d,p) level. Wavelengths were not corrected. S119

Figure SI_76. UV and ECD spectra of the low-energy conformers of compound 22 calculated at TD-M06$2 X / 6-311++G(d, p)$ level for structures optimized at B3LYP-GD3BJ/6-311G $(d, p)$ level. Wavelengths were not corrected. S119

Figure SI_77. UV and ECD spectra of the low-energy conformers of compound $\mathbf{2 2}$ calculated at TD-CAM$B 3 L Y P / 6-311++G(d, p)$ level for structures optimized at M06-2X/6-311G(d,p) level. Wavelengths were not corrected...... S120

Figure SI_78. UV and ECD spectra of the low-energy conformers of compound 22 calculated at TD-M06$2 X / 6-311++G(d, p)$ level for structures optimized at M06-2X/6-311G $(d, p)$ level. Wavelengths were not corrected....... S120

Figure SI_79. UV (upper panel) and ECD (lower panel) spectra of 1, experimental, measured in cyclohexane (solid black lines) and calculated at the TD-CAM-B3LYP/6-311++G $(2 d, 2 p)$ level for structures optimized at the B3LYP/6-311++G(d,p) level, $\Delta \mathrm{E}$-based Boltzmann (red lines) and $\Delta \Delta \mathrm{G}$-based Boltzmann averaged (blue solid lines). Insert shows the comparison between Boltzmann averaged ECD spectrum and that calculated for $\Delta \Delta G$-based on the lowest energy conformer of a given compound (dashed blue line). All calculated spectra were wavelength corrected to match experimental UV maxima. S121

Figure SI_80. UV (upper panel) and ECD (lower panel) spectra of 1, experimental, measured in cyclohexane (solid black lines) and calculated at the TD-M06-2X/6-311++G(2d,2p) level for structures optimized at the B3LYP/6-311++G(d,p) level, $\Delta \mathrm{E}$-based Boltzmann (red lines) and $\Delta \Delta \mathrm{G}$-based Boltzmann averaged (blue solid lines). Insert shows the comparison between Boltzmann averaged ECD spectrum and that calculated for $\Delta \Delta G$-based on the lowest energy conformer of a given compound (dashed blue line). All calculated spectra were wavelength corrected to match experimental UV maxima. S122

Figure SI_81. UV (upper panel) and ECD (lower panel) spectra of 1, experimental, measured in cyclohexane (solid black lines) and calculated at the TD-CAM-B3LYP/6-311++G $(2 d, 2 p)$ level for structures optimized at the M06-2X/6-311++G(d,p) level, $\Delta E$-based Boltzmann (red lines) and $\Delta \Delta G$-based Boltzmann averaged (blue solid lines). Insert shows the comparison between Boltzmann averaged ECD spectrum and that calculated for $\Delta \Delta G$-based on the lowest energy conformer of a given compound (dashed blue line). All calculated spectra were wavelength corrected to match experimental UV maxima. S123

Figure SI_82. UV (upper panel) and ECD (lower panel) spectra of 1, experimental, measured in cyclohexane (solid black lines) and calculated at the TD-M06-2X/6-311++G(2d,2p) level for structures optimized at the M06-2X/6-311++G(d,p) level, $\Delta \mathrm{E}$-based Boltzmann (red lines) and $\Delta \Delta \mathrm{G}$-based Boltzmann averaged (blue solid lines). Insert shows the comparison between Boltzmann averaged ECD spectrum and that calculated for $\Delta \Delta G$-based on the lowest energy conformer of a given compound (dashed blue line). All calculated spectra were wavelength corrected to match experimental UV maxima. S124

Figure SI_83. UV (upper panel) and ECD (lower panel) spectra of 4, experimental, measured in cyclohexane (solid black lines) and calculated at the TD-CAM-B3LYP/6-311++G $(2 d, 2 p)$ level for structures optimized at the B3LYP/6-311++G(d,p) level, $\Delta \mathrm{E}$-based Boltzmann (red lines) and $\Delta \Delta \mathrm{G}$-based Boltzmann averaged (blue solid lines). Insert shows the comparison between Boltzmann averaged ECD spectrum and 
that calculated for $\Delta \Delta G$-based on the lowest energy conformer of a given compound (dashed blue line). All calculated spectra were wavelength corrected to match experimental UV maxima. S125

Figure SI_84. UV (upper panel) and ECD (lower panel) spectra of 4, experimental, measured in cyclohexane (solid black lines) and calculated at the TD-M06-2X/6-311++G(2d,2p) level for structures optimized at the B3LYP/6-311++G(d,p) level, $\Delta E$-based Boltzmann (red lines) and $\Delta \Delta G$-based Boltzmann averaged (blue solid lines). Insert shows the comparison between Boltzmann averaged ECD spectrum and that calculated for $\Delta \Delta G$-based on the lowest energy conformer of a given compound (dashed blue line). All calculated spectra were wavelength corrected to match experimental UV maxima. S126

Figure SI_85. UV (upper panel) and ECD (lower panel) spectra of 4, experimental, measured in cyclohexane (solid black lines) and calculated at the TD-CAM-B3LYP/6-311++G $(2 d, 2 p)$ level for structures optimized at the M06-2X/6-311++G(d,p) level, $\Delta E$-based Boltzmann (red lines) and $\Delta \Delta G$-based Boltzmann averaged (blue solid lines). Insert shows the comparison between Boltzmann averaged ECD spectrum and that calculated for $\Delta \Delta G$-based on the lowest energy conformer of a given compound (dashed blue line). All calculated spectra were wavelength corrected to match experimental UV maxima.

Figure SI_86. UV (upper panel) and ECD (lower panel) spectra of 4, experimental, measured in cyclohexane (solid black lines) and calculated at the TD-M06-2X/6-311++G(2d,2p) level for structures optimized at the M06-2X/6-311++G(d,p) level, $\Delta \mathrm{E}$-based Boltzmann (red lines) and $\Delta \Delta \mathrm{G}$-based Boltzmann averaged (blue solid lines). Insert shows the comparison between Boltzmann averaged ECD spectrum and that calculated for $\Delta \Delta \mathrm{G}$-based on the lowest energy conformer of a given compound (dashed blue line). All calculated spectra were wavelength corrected to match experimental UV maxima. S128

Figure SI_87. UV (upper panel) and ECD (lower panel) spectra of 6, experimental, measured in cyclohexane (solid black lines) and calculated at the TD-CAM-B3LYP/6-311++G $(2 d, 2 p)$ level for structures optimized at the B3LYP/6-311++G(d,p) level, $\Delta \mathrm{E}$-based Boltzmann (red lines) and $\Delta \Delta \mathrm{G}$-based Boltzmann averaged (blue solid lines). Insert shows the comparison between Boltzmann averaged ECD spectrum and that calculated for $\Delta \Delta G$-based on the lowest energy conformer of a given compound (dashed blue line). All calculated spectra were wavelength corrected to match experimental UV maxima.

Figure SI_88. UV (upper panel) and ECD (lower panel) spectra of 6, experimental, measured in cyclohexane (solid black lines) and calculated at the TD-M06-2X/6-311++G $(2 d, 2 p)$ level for structures optimized at the B3LYP/6-311++G(d,p) level, $\Delta \mathrm{E}$-based Boltzmann (red lines) and $\Delta \Delta \mathrm{G}$-based Boltzmann averaged (blue solid lines). Insert shows the comparison between Boltzmann averaged ECD spectrum and that calculated for $\Delta \Delta G$-based on the lowest energy conformer of a given compound (dashed blue line). All calculated spectra were wavelength corrected to match experimental UV maxima. S130

Figure SI_89. UV (upper panel) and ECD (lower panel) spectra of 6, experimental, measured in cyclohexane (solid black lines) and calculated at the TD-CAM-B3LYP/6-311++G $(2 d, 2 p)$ level for structures optimized at the M06-2X/6-311++G(d,p) level, $\Delta \mathrm{E}$-based Boltzmann (red lines) and $\Delta \Delta \mathrm{G}$-based Boltzmann averaged (blue solid lines). Insert shows the comparison between Boltzmann averaged ECD spectrum and that calculated for $\Delta \Delta G$-based on the lowest energy conformer of a given compound (dashed blue line). All calculated spectra were wavelength corrected to match experimental UV maxima. S131

Figure SI_90. UV (upper panel) and ECD (lower panel) spectra of 6, experimental, measured in cyclohexane (solid black lines) and calculated at the TD-M06-2X/6-311++G(2d,2p) level for structures optimized at the M06-2X/6-311++G(d,p) level, $\Delta \mathrm{E}$-based Boltzmann (red lines) and $\Delta \Delta \mathrm{G}$-based Boltzmann averaged (blue solid lines). Insert shows the comparison between Boltzmann averaged ECD spectrum and that calculated for $\Delta \Delta G$-based on the lowest energy conformer of a given compound (dashed blue line). All calculated spectra were wavelength corrected to match experimental UV maxima. 
Figure SI_91. UV (upper panel) and ECD (lower panel) spectra of 10, experimental, measured in cyclohexane (solid black lines) and calculated at the TD-CAM-B3LYP/6-311++G $(2 d, 2 p)$ level for structures optimized at the B3LYP/6-311++G(d,p) level, $\Delta \mathrm{E}$-based Boltzmann (red lines) and $\Delta \Delta \mathrm{G}$-based Boltzmann averaged (blue solid lines). Insert shows the comparison between Boltzmann averaged ECD spectrum and that calculated for $\Delta \Delta G$-based on the lowest energy conformer of a given compound (dashed blue line). All calculated spectra were wavelength corrected to match experimental UV maxima. S133

Figure SI_92. UV (upper panel) and ECD (lower panel) spectra of 10, experimental, measured in cyclohexane (solid black lines) and calculated at the TD-M06-2X/6-311++G(2d,2p) level for structures optimized at the B3LYP/6-311++G(d,p) level, $\Delta \mathrm{E}$-based Boltzmann (red lines) and $\Delta \Delta \mathrm{G}$-based Boltzmann averaged (blue solid lines). Insert shows the comparison between Boltzmann averaged ECD spectrum and that calculated for $\Delta \Delta G$-based on the lowest energy conformer of a given compound (dashed blue line). All calculated spectra were wavelength corrected to match experimental UV maxima. S134

Figure SI_93. UV (upper panel) and ECD (lower panel) spectra of 10, experimental, measured in cyclohexane (solid black lines) and calculated at the TD-CAM-B3LYP/6-311++G $(2 d, 2 p)$ level for structures optimized at the M06-2X/6-311++G(d,p) level, $\Delta E$-based Boltzmann (red lines) and $\Delta \Delta G$-based Boltzmann averaged (blue solid lines). Insert shows the comparison between Boltzmann averaged ECD spectrum and that calculated for $\Delta \Delta G$-based on the lowest energy conformer of a given compound (dashed blue line). All calculated spectra were wavelength corrected to match experimental UV maxima. S135

Figure SI_94. UV (upper panel) and ECD (lower panel) spectra of 10, experimental, measured in cyclohexane (solid black lines) and calculated at the TD-M06-2X/6-311++G(2d,2p) level for structures optimized at the M06-2X/6-311++G(d,p) level, $\Delta \mathrm{E}$-based Boltzmann (red lines) and $\Delta \Delta \mathrm{G}$-based Boltzmann averaged (blue solid lines). Insert shows the comparison between Boltzmann averaged ECD spectrum and that calculated for $\Delta \Delta G$-based on the lowest energy conformer of a given compound (dashed blue line). All calculated spectra were wavelength corrected to match experimental UV maxima. S136

Figure SI_95. UV (upper panel) and ECD (lower panel) spectra of 11, experimental, measured in cyclohexane (solid black lines) and calculated at the TD-CAM-B3LYP/6-311++G $(2 d, 2 p)$ level for structures optimized at the B3LYP/6-311++G(d,p) level, $\Delta \mathrm{E}$-based Boltzmann (red lines) and $\Delta \Delta \mathrm{G}$-based Boltzmann averaged (blue solid lines). Insert shows the comparison between Boltzmann averaged ECD spectrum and that calculated for $\Delta \Delta \mathrm{G}$-based on the lowest energy conformer of a given compound (dashed blue line). All calculated spectra were wavelength corrected to match experimental UV maxima. .. S137

Figure SI_96. UV (upper panel) and ECD (lower panel) spectra of 11, experimental, measured in cyclohexane (solid black lines) and calculated at the TD-M06-2X/6-311++G(2d,2p) level for structures optimized at the B3LYP/6-311++G(d,p) level, $\Delta \mathrm{E}$-based Boltzmann (red lines) and $\Delta \Delta \mathrm{G}$-based Boltzmann averaged (blue solid lines). Insert shows the comparison between Boltzmann averaged ECD spectrum and that calculated for $\Delta \Delta \mathrm{G}$-based on the lowest energy conformer of a given compound (dashed blue line). All calculated spectra were wavelength corrected to match experimental UV maxima. S138

Figure SI_97. UV (upper panel) and ECD (lower panel) spectra of 11, experimental, measured in cyclohexane (solid black lines) and calculated at the TD-CAM-B3LYP/6-311++G $(2 d, 2 p)$ level for structures optimized at the M06-2X/6-311++G(d,p) level, $\Delta E$-based Boltzmann (red lines) and $\Delta \Delta G$-based Boltzmann averaged (blue solid lines). Insert shows the comparison between Boltzmann averaged ECD spectrum and that calculated for $\Delta \Delta G$-based on the lowest energy conformer of a given compound (dashed blue line). All calculated spectra were wavelength corrected to match experimental UV maxima. S139

Figure SI_98. UV (upper panel) and ECD (lower panel) spectra of 11, experimental, measured in cyclohexane (solid black lines) and calculated at the TD-M06-2X/6-311++G(2d,2p) level for structures optimized at the M06-2X/6-311++G(d,p) level, $\Delta \mathrm{E}$-based Boltzmann (red lines) and $\Delta \Delta \mathrm{G}$-based Boltzmann 
averaged (blue solid lines). Insert shows the comparison between Boltzmann averaged ECD spectrum and that calculated for $\Delta \Delta G$-based on the lowest energy conformer of a given compound (dashed blue line). All calculated spectra were wavelength corrected to match experimental UV maxima. S140

Figure SI_99. UV (upper panel) and ECD (lower panel) spectra of 14, experimental, measured in cyclohexane (solid black lines) and calculated at the TD-CAM-B3LYP/6-311++G $(2 d, 2 p)$ level for structures optimized at the B3LYP/6-311++G(d,p) level, $\Delta \mathrm{E}$-based Boltzmann (red lines) and $\Delta \Delta \mathrm{G}$-based Boltzmann averaged (blue solid lines). Insert shows the comparison between Boltzmann averaged ECD spectrum and that calculated for $\Delta \Delta G$-based on the lowest energy conformer of a given compound (dashed blue line). All calculated spectra were wavelength corrected to match experimental UV maxima. S141

Figure SI_100. UV (upper panel) and ECD (lower panel) spectra of 14, experimental, measured in cyclohexane (solid black lines) and calculated at the TD-M06-2X/6-311++G(2d,2p) level for structures optimized at the B3LYP/6-311++G(d,p) level, $\Delta E$-based Boltzmann (red lines) and $\Delta \Delta G$-based Boltzmann averaged (blue solid lines). Insert shows the comparison between Boltzmann averaged ECD spectrum and that calculated for $\Delta \Delta G$-based on the lowest energy conformer of a given compound (dashed blue line). All calculated spectra were wavelength corrected to match experimental UV maxima. S142

Figure SI_101. UV (upper panel) and ECD (lower panel) spectra of 14, experimental, measured in cyclohexane (solid black lines) and calculated at the TD-CAM-B3LYP/6-311++G $(2 d, 2 p)$ level for structures optimized at the M06-2X/6-311++G(d,p) level, $\Delta \mathrm{E}$-based Boltzmann (red lines) and $\Delta \Delta \mathrm{G}$-based Boltzmann averaged (blue solid lines). Insert shows the comparison between Boltzmann averaged ECD spectrum and that calculated for $\Delta \Delta G$-based on the lowest energy conformer of a given compound (dashed blue line). All calculated spectra were wavelength corrected to match experimental UV maxima. S143

Figure SI_102. UV (upper panel) and ECD (lower panel) spectra of 14, experimental, measured in cyclohexane (solid black lines) and calculated at the TD-M06-2X/6-311++G $(2 d, 2 p)$ level for structures optimized at the M06-2X/6-311++G(d,p) level, $\Delta \mathrm{E}$-based Boltzmann (red lines) and $\Delta \Delta \mathrm{G}$-based Boltzmann averaged (blue solid lines). Insert shows the comparison between Boltzmann averaged ECD spectrum and that calculated for $\Delta \Delta G$-based on the lowest energy conformer of a given compound (dashed blue line). All calculated spectra were wavelength corrected to match experimental UV maxima. S144

Figure SI_103. UV (upper panel) and ECD (lower panel) spectra of 15, experimental, measured in cyclohexane (solid black lines) and calculated at the TD-CAM-B3LYP/6-311++G $(2 d, 2 p)$ level for structures optimized at the B3LYP/6-311++G(d,p) level, $\Delta \mathrm{E}$-based Boltzmann (red lines) and $\Delta \Delta \mathrm{G}$-based Boltzmann averaged (blue solid lines). Insert shows the comparison between Boltzmann averaged ECD spectrum and that calculated for $\Delta \Delta G$-based on the lowest energy conformer of a given compound (dashed blue line). All calculated spectra were wavelength corrected to match experimental UV maxima. S145

Figure SI_104. UV (upper panel) and ECD (lower panel) spectra of 15, experimental, measured in cyclohexane (solid black lines) and calculated at the TD-M06-2X/6-311++G $(2 d, 2 p)$ level for structures optimized at the B3LYP/6-311++G(d,p) level, $\Delta \mathrm{E}$-based Boltzmann (red lines) and $\Delta \Delta \mathrm{G}$-based Boltzmann averaged (blue solid lines). Insert shows the comparison between Boltzmann averaged ECD spectrum and that calculated for $\Delta \Delta G$-based on the lowest energy conformer of a given compound (dashed blue line). All calculated spectra were wavelength corrected to match experimental UV maxima. S146

Figure SI_105. UV (upper panel) and ECD (lower panel) spectra of 15, experimental, measured in cyclohexane (solid black lines) and calculated at the TD-CAM-B3LYP/6-311++G $(2 d, 2 p)$ level for structures optimized at the M06-2X/6-311++G(d,p) level, $\Delta E$-based Boltzmann (red lines) and $\Delta \Delta G$-based Boltzmann averaged (blue solid lines). Insert shows the comparison between Boltzmann averaged ECD spectrum and that calculated for $\Delta \Delta G$-based on the lowest energy conformer of a given compound (dashed blue line). All calculated spectra were wavelength corrected to match experimental UV maxima. S147 
Figure SI_106. UV (upper panel) and ECD (lower panel) spectra of 15, experimental, measured in cyclohexane (solid black lines) and calculated at the TD-M06-2X/6-311++G(2d,2p) level for structures optimized at the M06-2X/6-311++G(d,p) level, $\Delta \mathrm{E}$-based Boltzmann (red lines) and $\Delta \Delta \mathrm{G}$-based Boltzmann averaged (blue solid lines). Insert shows the comparison between Boltzmann averaged ECD spectrum and that calculated for $\Delta \Delta G$-based on the lowest energy conformer of a given compound (dashed blue line). All calculated spectra were wavelength corrected to match experimental UV maxima. S148

Figure SI_107. UV (upper panel) and ECD (lower panel) spectra of 18, experimental, measured in cyclohexane (solid black lines) and calculated at the TD-CAM-B3LYP/6-311++G(d,p) level for structures optimized at the B3LYP/6-311G(d,p) level, $\Delta \mathrm{E}$-based Boltzmann (red lines) and $\Delta \Delta \mathrm{G}$-based Boltzmann averaged (blue solid lines). Insert shows the comparison between Boltzmann averaged ECD spectrum and that calculated for $\Delta \Delta G$-based on the lowest energy conformer of a given compound (dashed blue line). All calculated spectra were wavelength corrected to match experimental UV maxima. S149

Figure SI_108. UV (upper panel) and ECD (lower panel) spectra of 18, experimental, measured in cyclohexane (solid black lines) and calculated at the TD-M06-2X/6-311++G(d,p) level for structures optimized at the B3LYP/6-311G(d,p) level, $\Delta \mathrm{E}$-based Boltzmann (red lines) and $\Delta \Delta \mathrm{G}$-based Boltzmann averaged (blue solid lines). Insert shows the comparison between Boltzmann averaged ECD spectrum and that calculated for $\Delta \Delta G$-based on the lowest energy conformer of a given compound (dashed blue line). All calculated spectra were wavelength corrected to match experimental UV maxima. S150

Figure SI_109. UV (upper panel) and ECD (lower panel) spectra of 18, experimental, measured in cyclohexane (solid black lines) and calculated at the TD-CAM-B3LYP/6-311++G(d,p) level for structures optimized at the B3LYP-GD3BJ/6-311G(d,p) level, $\Delta E$-based Boltzmann (red lines) and $\Delta \Delta G$-based Boltzmann averaged (blue solid lines). Insert shows the comparison between Boltzmann averaged ECD spectrum and that calculated for $\Delta \Delta G$-based on the lowest energy conformer of a given compound (dashed blue line). All calculated spectra were wavelength corrected to match experimental UV maxima.

Figure SI_110. UV (upper panel) and ECD (lower panel) spectra of 18, experimental, measured in cyclohexane (solid black lines) and calculated at the TD-M06-2X/6-311++G(d,p) level for structures optimized at the B3LYP-GD3BJ/6-311G(d,p) level, $\Delta \mathrm{E}$-based Boltzmann (red lines) and $\Delta \Delta \mathrm{G}$-based Boltzmann averaged (blue solid lines). Insert shows the comparison between Boltzmann averaged ECD spectrum and that calculated for $\Delta \Delta \mathrm{G}$-based on the lowest energy conformer of a given compound (dashed blue line). All calculated spectra were wavelength corrected to match experimental UV maxima.

Figure SI_111. UV (upper panel) and ECD (lower panel) spectra of 18, experimental, measured in cyclohexane (solid black lines) and calculated the TD-CAM-B3LYP/6-311++G(d,p) level for structures optimized at the M06-2X/6-311G(d,p) level, $\Delta E$-based Boltzmann (red lines) and $\Delta \Delta G$-based Boltzmann averaged (blue solid lines). Insert shows the comparison between Boltzmann averaged ECD spectrum and that calculated for $\Delta \Delta G$-based on the lowest energy conformer of a given compound (dashed blue line). All calculated spectra were wavelength corrected to match experimental UV maxima.

Figure SI_112. UV (upper panel) and ECD (lower panel) spectra of 18, experimental, measured in cyclohexane (solid black lines) and calculated at the TD-M06-2X/6-311++G(d,p) level for structures optimized at the M06-2X/6-311G(d,p) level, $\Delta E$-based Boltzmann (red lines) and $\Delta \Delta G$-based Boltzmann averaged (blue solid lines). Insert shows the comparison between Boltzmann averaged ECD spectrum and that calculated for $\Delta \Delta \mathrm{G}$-based on the lowest energy conformer of a given compound (dashed blue line). All calculated spectra were wavelength corrected to match experimental UV maxima. S154 
Figure SI_113. UV (upper panel) and ECD (lower panel) spectra of 20, experimental, measured in cyclohexane (solid black lines) and calculated at the TD-CAM-B3LYP/6-311++G(d,p) level for structures optimized at the B3LYP/6-311G(d,p) level, $\Delta \mathrm{E}$-based Boltzmann (red lines) and $\Delta \Delta \mathrm{G}$-based Boltzmann averaged (blue solid lines). Insert shows the comparison between Boltzmann averaged ECD spectrum and that calculated for $\Delta \Delta G$-based on the lowest energy conformer of a given compound (dashed blue line). All calculated spectra were wavelength corrected to match experimental UV maxima. S155

Figure SI_114. UV (upper panel) and ECD (lower panel) spectra of 20, experimental, measured in cyclohexane (solid black lines) and calculated at the TD-M06-2X/6-311++G(d,p) level for structures optimized at the B3LYP/6-311G(d,p) level, $\Delta \mathrm{E}$-based Boltzmann (red lines) and $\Delta \Delta \mathrm{G}$-based Boltzmann averaged (blue solid lines). Insert shows the comparison between Boltzmann averaged ECD spectrum and that calculated for $\Delta \Delta G$-based on the lowest energy conformer of a given compound (dashed blue line). All calculated spectra were wavelength corrected to match experimental UV maxima. S156

Figure SI_115. UV (upper panel) and ECD (lower panel) spectra of 20, experimental, measured in cyclohexane (solid black lines) and calculated at the TD-CAM-B3LYP/6-311++G(d,p) level for structures optimized at the B3LYP-GD3BJ/6-311G(d,p) level, $\Delta E$-based Boltzmann (red lines) and $\Delta \Delta G$-based Boltzmann averaged (blue solid lines). Insert shows the comparison between Boltzmann averaged ECD spectrum and that calculated for $\Delta \Delta G$-based on the lowest energy conformer of a given compound (dashed blue line). All calculated spectra were wavelength corrected to match experimental UV maxima.

Figure SI_116. UV (upper panel) and ECD (lower panel) spectra of 20, experimental, measured in cyclohexane (solid black lines) and calculated at the TD-M06-2X/6-311++G(d,p) level for structures optimized at the B3LYP-GD3BJ/6-311G(d,p) level, $\Delta E$-based Boltzmann (red lines) and $\Delta \Delta G$-based Boltzmann averaged (blue solid lines). Insert shows the comparison between Boltzmann averaged ECD spectrum and that calculated for $\Delta \Delta \mathrm{G}$-based on the lowest energy conformer of a given compound (dashed blue line). All calculated spectra were wavelength corrected to match experimental UV maxima.

Figure SI_117. UV (upper panel) and ECD (lower panel) spectra of 20, experimental, measured in cyclohexane (solid black lines) and calculated the TD-CAM-B3LYP/6-311++G(d,p) level for structures optimized at the M06-2X/6-311G(d,p) level, $\Delta \mathrm{E}$-based Boltzmann (red lines) and $\Delta \Delta \mathrm{G}$-based Boltzmann averaged (blue solid lines). Insert shows the comparison between Boltzmann averaged ECD spectrum and that calculated for $\Delta \Delta G$-based on the lowest energy conformer of a given compound (dashed blue line). All calculated spectra were wavelength corrected to match experimental UV maxima. S159

Figure SI_118. UV (upper panel) and ECD (lower panel) spectra of 20, experimental, measured in cyclohexane (solid black lines) and calculated at the TD-M06-2X/6-311++G(d,p) level for structures optimized at the M06-2X/6-311G(d,p) level, $\Delta \mathrm{E}$-based Boltzmann (red lines) and $\Delta \Delta \mathrm{G}$-based Boltzmann averaged (blue solid lines). Insert shows the comparison between Boltzmann averaged ECD spectrum and that calculated for $\Delta \Delta G$-based on the lowest energy conformer of a given compound (dashed blue line). All calculated spectra were wavelength corrected to match experimental UV maxima. S160

Figure SI_119. UV (upper panel) and ECD (lower panel) spectra of 21, experimental, measured in cyclohexane (solid black lines) and calculated at the TD-CAM-B3LYP/6-311++G(d,p) level for structures optimized at the B3LYP/6-311G(d,p) level, $\Delta \mathrm{E}$-based Boltzmann (red lines) and $\Delta \Delta \mathrm{G}$-based Boltzmann averaged (blue solid lines). Insert shows the comparison between Boltzmann averaged ECD spectrum and that calculated for $\Delta \Delta \mathrm{G}$-based on the lowest energy conformer of a given compound (dashed blue line). All calculated spectra were wavelength corrected to match experimental UV maxima. S161 
Figure SI_120. UV (upper panel) and ECD (lower panel) spectra of 21, experimental, measured in cyclohexane (solid black lines) and calculated at the TD-M06-2X/6-311++G(d,p) level for structures optimized at the B3LYP/6-311G(d,p) level, $\Delta \mathrm{E}$-based Boltzmann (red lines) and $\Delta \Delta \mathrm{G}$-based Boltzmann averaged (blue solid lines). Insert shows the comparison between Boltzmann averaged ECD spectrum and that calculated for $\Delta \Delta G$-based on the lowest energy conformer of a given compound (dashed blue line). All calculated spectra were wavelength corrected to match experimental UV maxima. S162

Figure SI_121. UV (upper panel) and ECD (lower panel) spectra of 21, experimental, measured in cyclohexane (solid black lines) and calculated at the TD-CAM-B3LYP/6-311++G(d,p) level for structures optimized at the B3LYP-GD3BJ/6-311G(d,p) level, $\Delta E$-based Boltzmann (red lines) and $\Delta \Delta G$-based Boltzmann averaged (blue solid lines). Insert shows the comparison between Boltzmann averaged ECD spectrum and that calculated for $\Delta \Delta \mathrm{G}$-based on the lowest energy conformer of a given compound (dashed blue line). All calculated spectra were wavelength corrected to match experimental UV maxima.

Figure SI_122. UV (upper panel) and ECD (lower panel) spectra of 21, experimental, measured in cyclohexane (solid black lines) and calculated at the TD-M06-2X/6-311++G(d,p) level for structures optimized at the B3LYP-GD3BJ/6-311G(d,p) level, $\Delta E$-based Boltzmann (red lines) and $\Delta \Delta G$-based Boltzmann averaged (blue solid lines). Insert shows the comparison between Boltzmann averaged ECD spectrum and that calculated for $\Delta \Delta \mathrm{G}$-based on the lowest energy conformer of a given compound (dashed blue line). All calculated spectra were wavelength corrected to match experimental UV maxima.

Figure SI_123. UV (upper panel) and ECD (lower panel) spectra of 21, experimental, measured in cyclohexane (solid black lines) and calculated at the TD-CAM-B3LYP/6-311++G(d,p) level for structures optimized at the M06-2X/6-311G(d,p) level, $\Delta \mathrm{E}$-based Boltzmann (red lines) and $\Delta \Delta \mathrm{G}$-based Boltzmann averaged (blue solid lines). Insert shows the comparison between Boltzmann averaged ECD spectrum and that calculated for $\Delta \Delta G$-based on the lowest energy conformer of a given compound (dashed blue line). All calculated spectra were wavelength corrected to match experimental UV maxima. S165

Figure SI_124. UV (upper panel) and ECD (lower panel) spectra of 21, experimental, measured in cyclohexane (solid black lines) and calculated at the TD-M06-2X/6-311++G(d,p) level for structures optimized at the M06-2X/6-311G(d,p) level, $\Delta \mathrm{E}$-based Boltzmann (red lines) and $\Delta \Delta \mathrm{G}$-based Boltzmann averaged (blue solid lines). Insert shows the comparison between Boltzmann averaged ECD spectrum and that calculated for $\Delta \Delta G$-based on the lowest energy conformer of a given compound (dashed blue line). All calculated spectra were wavelength corrected to match experimental UV maxima. S166

Figure SI_125. UV (upper panel) and ECD (lower panel) spectra of 22, experimental, measured in cyclohexane (solid black lines) and calculated at the TD-CAM-B3LYP/6-311++G(d,p) level for structures optimized at the B3LYP/6-311G(d,p) level, $\Delta \mathrm{E}$-based Boltzmann (red lines) and $\Delta \Delta \mathrm{G}$-based Boltzmann averaged (blue solid lines). Insert shows the comparison between Boltzmann averaged ECD spectrum and that calculated for $\Delta \Delta G$-based on the lowest energy conformer of a given compound (dashed blue line). All calculated spectra were wavelength corrected to match experimental UV maxima.

Figure SI_126. UV (upper panel) and ECD (lower panel) spectra of 22, experimental, measured in cyclohexane (solid black lines) and calculated at the TD-M06-2X/6-311++G(d,p) level for structures optimized at the B3LYP/6-311G(d,p) level, $\Delta \mathrm{E}$-based Boltzmann (red lines) and $\Delta \Delta \mathrm{G}$-based Boltzmann averaged (blue solid lines). Insert shows the comparison between Boltzmann averaged ECD spectrum and that calculated for $\Delta \Delta \mathrm{G}$-based on the lowest energy conformer of a given compound (dashed blue line). All calculated spectra were wavelength corrected to match experimental UV maxima. S168 
Figure SI_127. UV (upper panel) and ECD (lower panel) spectra of 22, experimental, measured in cyclohexane (solid black lines) and calculated at the TD-CAM-B3LYP/6-311++G(d,p) level for structures optimized at the B3LYP-GD3BJ/6-311G(d,p) level, $\Delta \mathrm{E}$-based Boltzmann (red lines) and $\Delta \Delta \mathrm{G}$-based Boltzmann averaged (blue solid lines). Insert shows the comparison between Boltzmann averaged ECD spectrum and that calculated for $\Delta \Delta \mathrm{G}$-based on the lowest energy conformer of a given compound (dashed blue line). All calculated spectra were wavelength corrected to match experimental UV maxima.

Figure SI_128. UV (upper panel) and ECD (lower panel) spectra of 22, experimental, measured in cyclohexane (solid black lines) and calculated at the TD-M06-2X/6-311++G(d,p) level for structures optimized at the B3LYP-GD3BJ/6-311G(d,p) level, $\Delta \mathrm{E}$-based Boltzmann (red lines) and $\Delta \Delta \mathrm{G}$-based Boltzmann averaged (blue solid lines). Insert shows the comparison between Boltzmann averaged ECD spectrum and that calculated for $\Delta \Delta G$-based on the lowest energy conformer of a given compound (dashed blue line). All calculated spectra were wavelength corrected to match experimental UV maxima.

Figure SI_129. UV (upper panel) and ECD (lower panel) spectra of 22, experimental, measured in cyclohexane (solid black lines) and calculated at the TD-CAM-B3LYP/6-311++G(d,p) level for structures optimized at the M06-2X/6-311G (d,p) level, $\Delta \mathrm{E}$-based Boltzmann (red lines) and $\Delta \Delta \mathrm{G}$-based Boltzmann averaged (blue solid lines). Insert shows the comparison between Boltzmann averaged ECD spectrum and that calculated for $\Delta \Delta G$-based on the lowest energy conformer of a given compound (dashed blue line). All calculated spectra were wavelength corrected to match experimental UV maxima. S171

Figure SI_130. UV (upper panel) and ECD (lower panel) spectra of 22, experimental, measured in cyclohexane (solid black lines) and calculated at the TD-M06-2X/6-311++G(d,p) level for structures optimized at the M06-2X/6-311G (d,p) level, $\Delta \mathrm{E}$-based Boltzmann (red lines) and $\Delta \Delta \mathrm{G}$-based Boltzmann averaged (blue solid lines). Insert shows the comparison between Boltzmann averaged ECD spectrum and that calculated for $\Delta \Delta G$-based on the lowest energy conformer of a given compound (dashed blue line). All calculated spectra were wavelength corrected to match experimental UV maxima.....

Single crystal X-ray analysis.

Table SI_54. Dihedral angles $\alpha, 6, \gamma, \delta$ and $\zeta$ (in degrees), selected interatomic distances $I_{1}, I_{2}$ (in $\AA$ ) and helicities of trityl groups observed in the crystal structures of compounds $1,5,10,11,18-21 \ldots \ldots \ldots \ldots . . . S 174$

Table SI_55. Selected crystal data and structure refinement details for 1, 5, 10, 11, 18-21. S176

Figure SI_131. Percentage contributions of various intermolecular interaction to the Hirshfeld surfaces of molecules in crystal structure.

Figure SI_132. Molecular structure of compound a) $\mathbf{1}$ and b) (rac)-1 (2-butyl substituent is disordered and the minor position showed as thinner lines). Atoms numbering scheme presented for one molecule. Displacement ellipsoids are drawn at 50\% probability level. The sixfold phenyl embrace supramolecular synthon in the crystal structure of $\mathbf{1}$ c) top view and d) side view. Comparison of molecular packing in isostructural crystals of compound e) $\mathbf{1}$ (symmetrically independent molecules are indicated with different colors) and f) (rac)-1 (minor position of 2-butyl substituent shown as pink). Hydrogen atoms are omitted for clarity and oxygen atoms are shown as balls. S178

Figure SI_133. Molecular structure of asymmetric unit of a) 5- $\alpha$ and b) 5-6. Atoms numbering scheme presented for one molecule. Displacement ellipsoids are drawn at $50 \%$ probability level. Comparison of molecular packing in the crystals of c) 5- $\alpha$ and d) 5-6. Symmetrically independent molecules are indicated with different colors. Hydrogen atoms are omitted for clarity and oxygen atoms are shown as balls.... S179 
Figure SI_134. a) Molecular structure with atoms numbering scheme and b) molecular packing in crystal of $(\mathrm{rac})$-5. Displacement ellipsoids are drawn at 50\% probability level. Hydrogen atoms are omitted for clarity and oxygen atoms are shown as balls.

Figure SI_135. The recorded X-ray powder diffraction pattern of compound $\mathbf{5}$ (red) and predicted pattern based on single-crystal $X$-ray diffraction indices of polymorphs $5-\alpha$ and $5-6$.

Figure SI_136. Molecular structure and atoms numbering scheme of compound a) $\mathbf{1 0}$ and b) $\mathbf{1 1}$.

Displacement ellipsoids are drawn at $50 \%$ probability level. Comparison of molecular packing in crystal structure of c) $\mathbf{1 0}$ and d) $\mathbf{1 1}$. Hydrogen atoms are omitted for clarity and oxygen atoms are shown as balls.

Figure SI_137. Molecular structure and atoms numbering scheme of compound 18. Only the asymmetric part has been numbered for clarity. Displacement ellipsoids are drawn at 50\% probability level. Molecular packing in crystal structure $b$ ) view along $b$ axis and $c$ ) view along $c$ axis. Hydrogen atoms are omitted for clarity and oxygen atoms are shown as balls. S183

Figure SI_138. Molecular structure and atoms numbering scheme of compound 19. Displacement ellipsoids are drawn at $50 \%$ probability level. b) Scheme of molecular disorder in columns. c) Molecular packing in crystal structure, view along a axis. Hydrogen atoms are omitted for clarity and oxygen atoms are shown as balls. S184

Figure SI_139. Molecular structure of asymmetric unit in crystal structure of compound 20. Displacement ellipsoids are drawn at 50\% probability level. b) Comparison molecular conformation of symmetrically independent molecules. c) Molecular packing in crystal structure -molecules $\mathbf{A}+\mathbf{B}$ and $\mathbf{C}+\mathbf{D}$ forms alternating double layers. Hydrogen atoms are omitted for clarity and oxygen atoms are shown as balls.

Figure SI_140. a) Molecular structure and atoms numbering scheme of compound 21. Only the asymmetric part has been numbered for clarity. Displacement ellipsoids are drawn at $50 \%$ probability level. Molecular packing in crystal structure $b$ ) view along $b$ axis and $c$ ) view along $c$ axis. Hydrogen atoms are omitted for clarity and oxygen atoms are shown as balls. S186

Copies of ${ }^{1} \mathrm{H}$ and ${ }^{13} \mathrm{C}$ NMR spectra S187

Copies of ECD spectra

Cartesian coordinates S260

References S581 


\section{Calculations details}

Starting geometries of esters $1,4,6,10,11,14,15,18,20-22$ were obtained by a conformational search with the use of Scigress[1] software and by pre-optimization of all conformers at the B3LYP/631G(d) level.[2] The conformational searches were done by systematic changes of all rotatable torsion angles with $30^{\circ}$ steps. This allowed to identify the minimum energy structures which were further re-optimized with the independent use of a B3LYP[3] and M06-2X[4] hybrid functionals in conjunction with an enhanced 6-311++G(d,p) basis set. In the case of diesters 18, 20-22 smaller 6$311 \mathrm{G}(\mathrm{d}, \mathrm{p})$ basis set was used for geometry optimization.[2] Expecting the impact of non-covalent (London) interactions on the structure of specific compounds, the diesters 18, 20-22 were additionally optimized with the use of B3LYP hybrid functional with dispersion correction[5] and in conjunction with the 6-311G(d,p) basis set.

The structures thus obtained were the real minimum energy conformers (no imaginary frequencies were found). The total and free energy values were used to obtain the Boltzmann population of conformers at $298.15 \mathrm{~K}$. For density functional theory calculations, only the results for conformers that were different from the most stable one by less than $2 \mathrm{kcal} \mathrm{mol}^{-1}$ were taken into account, following a generally accepted protocol.[6] Relative energies(unit $\mathrm{kcal} \mathrm{mol}^{-1}$ ) discussed in the main text refer to Gibbs free energies $(\Delta \Delta G)$ computed at the respective DFT level of theory. Energetic and structural data have been juxtaposed in Tables SI_1-SI_53, whereas in Figures SI_1-SI_26 have shown structures of individual low-energy conformers of 1, 4, 6, 10, 11, 14, 15, 18, 20-22, calculated at various DFT levels.

ECD spectra for all structures optimized at the DFT level were calculated employing M06-2X[4] and CAM-B3LYP,[7] hybrid functionals, in conjunction with the $6-311++G(2 d, 2 p)$ or $6-311++G(d, p)$ basis sets (see Figures SI_27-SI_78).[2] The calculated ECD spectra were Boltzmann averaged by taking into account thermally accessible conformers of $1,4,6,10,11,14,15,18,20-22$ ranging from 0 to $2.0 \mathrm{kcal} \mathrm{mol}^{-1}$ in relative energies.[6]

Rotatory strengths were calculated using both length and velocity representations. In the present study, the differences between the length and velocity calculated values of the rotator strengths were quite small, and for this reason, only the velocity representations were further used.

The ECD spectra were simulated by overlapping Gaussian functions for each transition, according to the procedure previously described by Harada and Stephens.[8]

The solvent effect on the structure and ECD spectra was not taken into account, since all measurements for esters $1,4,6,10,11,14,15,18,20-22$ were done in non-polar cyclohexane.

Since there are no significant differences between results obtained with the use of M06-2X and CAMB3LYP functionals, we limited our discussion to the results obtained with the use of M06-2X functional only.

The best combination of methods for structure/spectra prediction was chosen by comparison of experimental and Boltzmann averaged CD spectra calculated using all possible combinations of methods (see Figures SI_79-SI_130). The best performed combination for prediction of the structure and the ECD spectra of esters $1,4,6,10,11$ and 14 consists of $B 3 L Y P / 6-311++G(d, p)$ method for 
structure optimization and TD-M06-2X/6-311++G $(2 d, 2 p)$ for calculations of rotatory strengths. The ester 15 is an exception, the much better results were obtained for combination of M06-2X/6$311++G(d, p)$ method for geometry calculations and TD-M06-2X/6-311++G(2d,2p) method for calculations of chiroptical properties. For the particular ester 15 the $\mathrm{CH} \bullet \bullet \mathrm{O}$ interaction between inductor and acceptor may take place.

The comparison of ECD spectra - the experimental ones and those calculated with the use of various methods, provided some general conclusions. Firstly - the use of the B3LYP hybrid functional that have long-range corrections improved the final results only for diesters $\mathbf{1 8}$ and $\mathbf{2 0}$, having the ester groups in the close proximity. In the remaining cases, namely $\mathbf{2 1}$ and $\mathbf{2 2}$, the results obtained with the use of B3LYP-GD3BJ method did not improve the results but elongated the CPU time. Therefore, only for $\mathbf{1 8}$ and 20, the London dispersive interactions control the structure of the given compound. Secondly, the structure of investigated monoesters is controlled by intermolecular interactions satisfactorily reproduced by "classical" B3LYP functional.

Thirdly. In the case of compounds, in which the electrostatic attractive $\mathrm{C}=\mathrm{O} \cdot \cdot \mathrm{H}-\mathrm{C}_{\mathrm{Ar}}$ interactions take place, much better results were obtained with the newer M06-2X hybrid functional than that obtained with "classical" B3LYP hybrid functional. This is especially visible for the derivative 6, whereas for 14, the impact of electrostatic interactions is overestimated. 
Table SI_1. Concentrations $\left(c\right.$, in $\left.\mathrm{mol} \mathrm{L}^{-1}\right)$ of the samples used for UV and ECD measurements.

\begin{tabular}{|c|c|c|}
\hline Compound & c (cyclohexane) & $c$ (acetonitrile) \\
\hline 1 & $2.908 \times 10^{-4}$ & $2.972 \times 10^{-4}$ \\
\hline 2 & $2.839 \times 10^{-4}$ & $2.842 \times 10^{-4}$ \\
\hline 3 & $2.52 \times 10^{-4}$ & $2.496 \times 10^{-4}$ \\
\hline 4 & $2.822 \times 10^{-4}$ & $2.811 \times 10^{-4}$ \\
\hline 5 & $2.596 \times 10^{-4}$ & $2.563 \times 10^{-4}$ \\
\hline 6 & $2.295 \times 10^{-4}$ & $2.293 \times 10^{-4}$ \\
\hline 7 & $2.348 \times 10^{-4}$ & $2.355 \times 10^{-4}$ \\
\hline 8 & $2.833 \times 10^{-4}$ & $2.803 \times 10^{-4}$ \\
\hline 9 & $2.388 \times 10^{-4}$ & $2.393 \times 10^{-4}$ \\
\hline 10 & $2.386 \times 10^{-4}$ & $2.348 \times 10^{-4}$ \\
\hline 11 & $2.376 \times 10^{-4}$ & $2.379 \times 10^{-4}$ \\
\hline 12 & $1.517 \times 10^{-4}$ & $1.532 \times 10^{-4}$ \\
\hline 13 & $1.796 \times 10^{-4}$ & $1.8 \times 10^{-4}$ \\
\hline 14 & $2.546 \times 10^{-4}$ & $2.519 \times 10^{-4}$ \\
\hline 15 & $2.8 \times 10^{-4}$ & $2.809 \times 10^{-4}$ \\
\hline 16 & $2.726 \times 10^{-4}$ & $2.702 \times 10^{-4}$ \\
\hline 17 & $1.607 \times 10^{-4}$ & $1.602 \times 10^{-4}$ \\
\hline 18 & $1.632 \times 10^{-4}$ & $1.647 \times 10^{-4}$ \\
\hline 19 & $1.355 \times 10^{-4}$ & $1.599 \times 10^{-4}$ \\
\hline 20 & $1.588 \times 10^{-4}$ & $1.599 \times 10^{-4}$ \\
\hline 21 & $1.738 \times 10^{-3}$ & $2.989 \times 10^{-3}$ \\
\hline 22 & $1.529 \times 10^{-4}$ & $1.524 \times 10^{-4}$ \\
\hline
\end{tabular}


Table SI_2. ECD $\left(\Delta \varepsilon\right.$, in $\left.\mathrm{dm}^{3} \cdot \mathrm{mol}^{-1} \cdot \mathrm{cm}^{-1}\right)$ and UV $\left(\varepsilon\right.$, in $\left.\mathrm{dm}^{3} \cdot \mathrm{mol}^{-1} \cdot \mathrm{cm}^{-1}\right)$ data for derivatives 1-22 measured in acetonitrile solution.

\begin{tabular}{|c|c|c|}
\hline Compound & $\Delta \varepsilon(\mathrm{nm})$ & $\varepsilon(\mathrm{nm})$ \\
\hline 1 & -2.5 (229); $11.4(200) ;-7.1(185)$ & $73450(196)$ \\
\hline 2 & $3.4(227) ;-13.2(201) ; 10.3(185)$ & $72000(197)$ \\
\hline 3 & $1.1(225) ;-2.5(203) ; 3.8(185)$ & $69550(196)$ \\
\hline 4 & $-3.2(229) ; 15.5(201) ;-8.2(185)$ & $70500(196)$ \\
\hline 5 & $-1.5(232) ; 4.5(203) ; 3.3(192) ;-2.2(185)$ & $120050(189)$ \\
\hline 6 & $6.4(231) ;-33.0(200) ; 21.0(185)$ & $73100(196)$ \\
\hline 7 & $15.6(222) ;-26.9(190)$ & $112600(191)$ \\
\hline 8 & $0.5(222) ;-1.3(202) ;-1.1(197) ; 2.4(185)$ & $71800(196)$ \\
\hline 9 & $0.7(226) ;-2.2(203) ;-2.1(198) ; 1.4(185)$ & $75100(196)$ \\
\hline 10 & $-9.3(229) ; 41.4(201) ;-27.0(185)$ & $71200(196)$ \\
\hline 11 & $-2.9(214) ;-3.0(204) ; 4.5(185)$ & $70100(196)$ \\
\hline 12 & $-0.6(228) ; 2.4(200) ; 1.4(185)$ & $66200(186)$ \\
\hline 13 & $-1.2(328) ; 8.5(227) ; 4.4(185)$ & $73600(196)$ \\
\hline 14 & $10.9(228) ;-51.5(200) ; 31.4(185)$ & $71100(196)$ \\
\hline 15 & -1.5 (231); $5.1(199) ;-0.8(185)$ & $65950(196)$ \\
\hline 16 & $1.2(230) ;-5.3(198)$ & $72750(196)$ \\
\hline 17 & $10.6(231) ;-41.4(204) ; 8.0(185)$ & $142000(193)$ \\
\hline 18 & -0.5 (262); 3.1 (230); -2.9 (209); 0.5 (199); -3.1 (191); 0.3 (185) & $138300(195)$ \\
\hline 19 & $-6.8(223) ; 30.0(196) ;-19.4(185)$ & $141550(196)$ \\
\hline 20 & $-5.6(229) ; 34.1(204) ;-21.8(185)$ & $135250(194)$ \\
\hline 21 & $-3.7(225) ; 14.6(196) ;-27.1(185)$ & $15510(188)$ \\
\hline 22 & $-11.0(230) ; 45.1(202) ;-24.9(186)$ & $131700(196)$ \\
\hline
\end{tabular}


Table SI_3. Total and free energies $\left(E, \Delta G\right.$, in Hartree), relative energies $\left(\Delta E, \Delta \Delta G\right.$ in $\left.\mathrm{kcal}^{\mathrm{mol}}{ }^{-1}\right)$, percentage populations and number of imaginary frequencies (\#ImFreq) calculated at the B3LYP/6$311++G(d, p)$ level for individual conformers of 1 .

\begin{tabular}{cccccccc}
\hline Conformer no $^{[a]}$ & $E$ & $\Delta G$ & $\Delta E$ & Pop. & $\Delta \Delta G$ & Pop & \#ImFreq \\
\hline 1 & -1079.74787 & -1079.38658 & 0.00 & 14.39 & 0.98 & 7.03 & 0 \\
17 & -1079.74654 & -1079.38 & 0.84 & 3.5 & 1.99 & 1.27 & 0 \\
33 & -1079.74678 & -1079.38659 & 0.68 & 4.55 & 0.98 & 7.1 & 0 \\
38 & -1079.7472 & -1079.38815 & 0.42 & 7.08 & 0.00 & 36.97 & 0 \\
39 & -1079.74652 & -1079.38554 & 0.85 & 3.45 & 1.64 & 2.32 & 0 \\
40 & -1079.7472 & -1079.38693 & 0.42 & 7.03 & 0.76 & 10.16 & 0 \\
41 & -1079.74714 & -1079.38684 & 0.46 & 6.63 & 0.82 & 9.2 & 0 \\
42 & -1079.74591 & -1079.38496 & 1.23 & 1.81 & 2.01 & - & 0 \\
43 & -1079.74622 & -1079.38515 & 1.04 & 2.49 & 1.88 & 1.54 & 0 \\
44 & -1079.74747 & -1079.38635 & 0.25 & 9.38 & 1.13 & 5.48 & 0 \\
45 & -1079.74777 & -1079.38634 & 0.06 & 12.98 & 1.14 & 5.43 & 0 \\
49 & -1079.74777 & -1079.38633 & 0.06 & 12.98 & 1.14 & 5.39 & 0 \\
50 & -1079.74667 & -1079.38524 & 0.76 & 4.02 & 1.83 & 1.69 & 0 \\
52 & -1079.74725 & -1079.3865 & 0.39 & 7.48 & 1.04 & 6.42 & 0 \\
53 & -1079.74611 & -1079.38417 & 1.10 & 2.23 & 2.5 & - & 0
\end{tabular}

[a] Conformers are numbered according to their appearance during conformational search. 
Table SI_4. Total and free energies $\left(E, \Delta G\right.$, in Hartree), relative energies $\left(\Delta E, \Delta \Delta G\right.$ in $\left.\mathrm{kcal} \mathrm{mol}^{-1}\right)$, percentage populations and number of imaginary frequencies (\#ImFreq) calculated at the M06-2X/6$311++G(d, p)$ level for individual conformers of 1 .

\begin{tabular}{cccccccc}
\hline Conformer no ${ }^{[a]}$ & $E$ & $\Delta G$ & $\Delta E$ & Pop. & $\Delta \Delta G$ & Pop & $\#$ ImFreq \\
\hline 1 & -1079.30025 & -1078.93348 & 0 & 37.59 & 0.11 & 20.95 & 0 \\
17 & -1079.29975 & -1078.93227 & 0.31 & 22.19 & 0.88 & 5.8 & 0 \\
33 & -1079.29767 & -1078.93 & 1.62 & 2.44 & 0.90 & 5.52 & 0 \\
38 & -1079.29764 & -1078.93165 & 1.64 & 2.37 & 1.26 & 3.01 & 0 \\
39 & -1079.29734 & -1078.93116 & 1.82 & 1.73 & 1.58 & 1.78 & 0 \\
40 & -1079.29722 & -1078.93297 & 1.90 & 1.52 & 0.44 & 12.16 & 0 \\
41 & -1079.29707 & -1078.9309 & 1.99 & 1.29 & 1.74 & 1.36 & 0 \\
44 & -1079.29919 & -1078.93367 & 0.66 & 12.26 & 0.00 & 25.43 & 0 \\
45 & -1079.29898 & -1078.93324 & 0.79 & 9.83 & 0.26 & 16.28 & 0 \\
50 & -1079.29752 & -1078.93175 & 1.71 & 2.1 & 1.20 & 3.32 & 0 \\
52 & -1079.29726 & -1078.93068 & 1.87 & 1.59 & 1.87 & 1.07 & 0 \\
53 & -1079.29836 & -1078.93174 & 1.19 & 5.08 & 1.21 & 3.3 & 0
\end{tabular}

[a] Conformers are numbered according to their appearance during conformational search. 
Table SI_5. Total and free energies $\left(E, \Delta G\right.$, in Hartree), relative energies $\left(\Delta E, \Delta \Delta G\right.$ in $\left.\mathrm{kcal} \mathrm{mol}^{-1}\right)$, percentage populations and number of imaginary frequencies (\#ImFreq) calculated at the B3LYP/6$311++G(d, p)$ level for individual conformers of 4.

\begin{tabular}{cccccccc}
\hline Conformer no $^{[a]}$ & $E$ & $\Delta G$ & $\Delta E$ & Pop. & $\Delta \Delta G$ & Pop & $\#$ ImFreq \\
\hline 1 & -1119.07063 & -1118.68223 & 0 & 21.34 & 0.64 & 9.26 & 0 \\
20 & -1119.07028 & -1118.68245 & 0.22 & 14.7 & 0.5 & 11.68 & 0 \\
22 & -1119.0704 & -1118.68254 & 0.14 & 16.72 & 0.44 & 12.89 & 0 \\
42 & -1119.07051 & -1118.68312 & 0.08 & 18.79 & 0.08 & 23.85 & 0 \\
44 & -1119.06983 & -1118.68269 & 0.5 & 9.11 & 0.35 & 15.09 & 0 \\
46 & -1119.07054 & -1118.68325 & 0.06 & 19.33 & 0 & 27.23 & 0 \\
\hline
\end{tabular}

[a] Conformers are numbered according to their appearance during conformational search.

Table SI_6. Total and free energies $\left(E, \Delta G\right.$, in Hartree), relative energies $\left(\Delta E, \Delta \Delta G\right.$ in $\left.\mathrm{kcal} \mathrm{mol}^{-1}\right)$, percentage populations and number of imaginary frequencies (\#ImFreq) calculated at the M06-2X/6$311++G(d, p)$ level for individual conformers of 4.

\begin{tabular}{cccccccc}
\hline Conformer no $^{[a]}$ & $E$ & $\Delta G$ & $\Delta E$ & Pop. & $\Delta \Delta G$ & Pop & \#ImFreq \\
\hline 1 & -1118.60547 & -1118.213 & 0.00 & 42.42 & 0.00 & 59.64 & 0 \\
20 & -1118.60451 & -1118.21146 & 0.60 & 15.28 & 0.96 & 11.71 & 0 \\
22 & -1118.60511 & -1118.21114 & 0.22 & 29.03 & 1.16 & 8.37 & 0 \\
42 & -1118.60276 & -1118.21138 & 1.70 & 2.39 & 1.01 & 10.78 & 0 \\
44 & -1118.60271 & -1118.21017 & 1.73 & 2.29 & 1.77 & 3 & 0 \\
46 & -1118.60396 & -1118.21091 & 0.95 & 8.59 & 1.31 & 6.5 & 0
\end{tabular}

[a] Conformers are numbered according to their appearance during conformational search. 
Table SI_7. Total and free energies $\left(E, \Delta G\right.$, in Hartree), relative energies $\left(\Delta E, \Delta \Delta G\right.$ in $\left.\mathrm{kcal}^{\mathrm{mol}}{ }^{-1}\right)$, percentage populations and number of imaginary frequencies (\#ImFreq) calculated at the B3LYP/6$311++G(d, p)$ level for individual conformers of 6 .

\begin{tabular}{cccccccc}
\hline Conformer no $^{[a]}$ & $E$ & $\Delta G$ & $\Delta E$ & Pop. & $\Delta \Delta G$ & Pop & \#ImFreq \\
\hline 1 & -1424.44985 & -1423.98764 & 0.00 & 47.62 & 0.00 & 29.07 & 0 \\
4 & -1424.44827 & -1423.99 & 0.99 & 8.97 & 0.90 & 6.37 & 0 \\
7 & -1424.44834 & -1423.99 & 0.95 & 9.63 & 0.70 & 8.97 & 0 \\
8 & -1424.44875 & -1423.99 & 0.69 & 14.81 & 0.59 & 10.81 & 0 \\
10 & -1424.44707 & -1423.99 & 1.74 & 2.5 & 1.21 & 3.75 & 0 \\
14 & -1424.44789 & -1423.99 & 1.23 & 5.95 & 1.12 & 4.39 & 0 \\
17 & -1424.44584 & -1423.98579 & 2.51 & - & 1.16 & 4.09 & 0 \\
18 & -1424.44751 & -1423.98528 & 1.47 & 4 & 1.48 & 2.38 & 0 \\
20 & -1424.44712 & -1423.9869 & 1.71 & 2.64 & 0.47 & 13.16 & 0 \\
21 & -1424.44667 & -1423.98533 & 1.99 & 1.65 & 1.46 & 2.49 & 0 \\
28 & -1424.44696 & -1423.98691 & 1.81 & 2.24 & 0.46 & 13.37 & 0 \\
46 & -1424.44594 & -1423.9846 & 2.45 & - & 1.91 & 1.15 & 0
\end{tabular}

[a] Conformers are numbered according to their appearance during conformational search.

Table SI_8. Total and free energies $\left(E, \Delta G\right.$, in Hartree), relative energies $\left(\Delta E, \Delta \Delta G\right.$ in $\left.\mathrm{kcal} \mathrm{mol}^{-1}\right)$, percentage populations and number of imaginary frequencies (\#ImFreq) calculated at the M06-2X/6$311++G(d, p)$ level for individual conformers of 6 .

\begin{tabular}{cccccccc}
\hline Conformer no ${ }^{[\mathrm{a}]}$ & $E$ & $\Delta G$ & $\Delta E$ & Pop. & $\Delta \Delta G$ & Pop & \#ImFreq \\
\hline 1 & -1423.87727 & -1423.40816 & 0.00 & 67.16 & 0.00 & 62.73 & 0 \\
7 & -1423.87478 & -1423.40654 & 1.56 & 4.82 & 1.02 & 11.2 & 0 \\
11 & -1423.87449 & -1423.40431 & 1.75 & 3.52 & 2.42 & - & 0 \\
14 & -1423.87576 & -1423.40623 & 0.95 & 13.56 & 1.21 & 8.11 & 0 \\
17 & -1423.87435 & -1423.40454 & 1.83 & 3.04 & 2.27 & - & 0 \\
28 & -1423.87391 & -1423.40501 & 2.11 & - & 1.98 & 2.21 & 0 \\
30 & -1423.87492 & -1423.40686 & 1.47 & 5.58 & 0.82 & 15.75 & 0 \\
32 & -1423.8741 & -1423.4032 & 1.99 & 2.33 & 3.11 & - & 0
\end{tabular}

[a] Conformers are numbered according to their appearance during conformational search. 
Table SI_9. Total and free energies $\left(E, \Delta G\right.$, in Hartree), relative energies $\left(\Delta E, \Delta \Delta G\right.$ in $\left.\mathrm{kcal} \mathrm{mol}^{-1}\right)$, percentage populations and number of imaginary frequencies (\#ImFreq) calculated at the B3LYP/6$311++G(d, p)$ level for individual conformers of $\mathbf{1 0}$.

\begin{tabular}{cccccccc}
\hline Conformer no $^{[a]}$ & $E$ & $\Delta G$ & $\Delta E$ & Pop. & $\Delta \Delta G$ & Pop & \#ImFreq \\
\hline 1 & -1314.48305 & -1313.97887 & 0 & 100 & 0 & 100 & 0
\end{tabular}

[a] Conformers are numbered according to their appearance during conformational search.

Table SI_10. Total and free energies $\left(E, \Delta G\right.$, in Hartree), relative energies $\left(\Delta E, \Delta \Delta G\right.$ in $\left.\mathrm{kcal} \mathrm{mol}^{-1}\right)$, percentage populations and number of imaginary frequencies (\#ImFreq) calculated at the M06-2X/6$311++G(d, p)$ level for individual conformers of 10 .

\begin{tabular}{|c|c|c|c|c|c|c|c|}
\hline Conformer no ${ }^{[\mathrm{a}]}$ & $E$ & $\Delta G$ & $\Delta E$ & Pop. & $\Delta \Delta G$ & Pop & \#ImFreq \\
\hline 1 & -1313.9347 & -1313.4237 & 0 & 100 & 0 & 100 & 0 \\
\hline
\end{tabular}

[a] Conformers are numbered according to their appearance during conformational search.

Table SI_11. Total and free energies $\left(E, \Delta G\right.$, in Hartree), relative energies $\left(\Delta E, \Delta \Delta G\right.$ in $\left.\mathrm{kcal} \mathrm{mol}^{-1}\right)$, percentage populations and number of imaginary frequencies (\#ImFreq) calculated at the B3LYP/6$311++G(d, p)$ level for individual conformers of 11.

\begin{tabular}{cccccccc}
\hline Conformer no $^{[a]}$ & $E$ & $\Delta G$ & $\Delta E$ & Pop. & $\Delta \Delta G$ & Pop & \#ImFreq \\
\hline 1 & -1314.4815 & -1313.97671 & 0.30 & 27.31 & 1.34 & 6.18 & 0 \\
22 & -1314.48123 & -1313.97729 & 0.47 & 20.43 & 0.98 & 11.37 & 0 \\
27 & -1314.48199 & -1313.97795 & 0.00 & 45.54 & 0.56 & 23.09 & 0 \\
52 & -1314.47884 & -1313.97413 & 1.97 & 1.62 & 2.96 & - & 0 \\
56 & -1314.4794 & -1313.98 & 1.62 & 2.93 & 0.00 & 59.35 & 0 \\
62 & -1314.47911 & -1313.97387 & 1.80 & 2.16 & 3.12 & - & 0
\end{tabular}

[a] Conformers are numbered according to their appearance during conformational search. 
Table SI_12. Total and free energies $\left(E, \Delta G\right.$, in Hartree), relative energies $\left(\Delta E, \Delta \Delta G\right.$ in $\left.\mathrm{kcal} \mathrm{mol}^{-1}\right)$, percentage populations and number of imaginary frequencies (\#ImFreq) calculated at the M06-2X/6$311++G(d, p)$ level for individual conformers of 11 .

\begin{tabular}{cccccccc}
\hline Conformer no $^{[a]}$ & $E$ & $\Delta G$ & $\Delta E$ & Pop. & $\Delta \Delta G$ & Pop & \#ImFreq \\
\hline 1 & -1313.93677 & -1313.4233 & 0.00 & 82.88 & 0.00 & 65.32 & 0 \\
27 & -1313.93424 & -1313.42235 & 1.58 & 5.73 & 0.60 & 23.69 & 0 \\
31 & -1313.9341 & -1313.42102 & 1.67 & 4.94 & 1.43 & 5.83 & 0 \\
33 & -1313.93381 & -1313.41858 & 1.85 & 3.62 & 2.96 & - & 0 \\
50 & -1313.93358 & -1313.41987 & 1.99 & 2.84 & 2.16 & - & 0 \\
52 & -1313.93306 & -1313.42035 & 2.33 & - & 1.85 & 2.86 & 0 \\
56 & -1313.9327 & -1313.42015 & 2.55 & - & 1.98 & 2.3 & 0 \\
\hline
\end{tabular}

[a] Conformers are numbered according to their appearance during conformational search.

Table SI_13. Total and free energies $\left(E, \Delta G\right.$, in Hartree), relative energies $\left(\Delta E, \Delta \Delta G\right.$ in $\left.\mathrm{kcal}^{\mathrm{mol}}{ }^{-1}\right)$, percentage populations and number of imaginary frequencies (\#ImFreq) calculated at the B3LYP/6$311++G(d, p)$ level for individual conformers of 14 .

\begin{tabular}{cccccccc}
\hline Conformer no ${ }^{[\mathrm{a}]}$ & $E$ & $\Delta G$ & $\Delta E$ & Pop. & $\Delta \Delta G$ & Pop & \#ImFreq \\
\hline 1 & -1306.47214 & -1306.09 & 0.00 & 88.55 & 0.00 & 77.91 & 0 \\
6 & -1306.46911 & -1306.0896 & 1.90 & 3.58 & 1.69 & 4.49 & 0 \\
8 & -1306.46985 & -1306.09067 & 1.43 & 7.86 & 1.02 & 13.97 & 0 \\
39 & -1306.46791 & -1306.0894 & 2.65 & - & 1.81 & 3.64 & 0 \\
\hline
\end{tabular}

[a] Conformers are numbered according to their appearance during conformational search.

Table SI_14. Total and free energies $\left(E, \Delta G\right.$, in Hartree), relative energies $\left(\Delta E, \Delta \Delta G\right.$ in $\left.\mathrm{kcal} \mathrm{mol}^{-1}\right)$, percentage populations and number of imaginary frequencies (\#ImFreq) calculated at the M06-2X/6$311++G(d, p)$ level for individual conformers of 14.

\begin{tabular}{|c|c|c|c|c|c|c|c|}
\hline Conformer $\mathrm{no}^{[\mathrm{a}]}$ & $E$ & $\Delta G$ & $\Delta E$ & Pop. & $\Delta \Delta G$ & Pop & \#ImFreq \\
\hline 1 & -1305.95634 & -1305.56935 & 0.00 & 68.72 & 0.37 & 28.35 & 0 \\
\hline 6 & -1305.95492 & -1305.56849 & 0.89 & 15.22 & 0.91 & 11.36 & 0 \\
\hline 8 & -1305.95337 & -1305.56994 & 1.87 & 2.93 & 0.00 & 53.1 & 0 \\
\hline 27 & -1305.95478 & -1305.56719 & 0.98 & 13.12 & 1.73 & 2.87 & 0 \\
\hline 49 & -1305.95219 & -1305.56757 & 2.61 & & 1.49 & 4.32 & 0 \\
\hline
\end{tabular}

[a] Conformers are numbered according to their appearance during conformational search. 
Table SI_15. Total and free energies $\left(E, \Delta G\right.$, in Hartree), relative energies $\left(\Delta E, \Delta \Delta G\right.$ in $\left.\mathrm{kcal} \mathrm{mol}^{-1}\right)$, percentage populations and number of imaginary frequencies (\#ImFreq) calculated at the B3LYP/6$311++G(d, p)$ level for individual conformers of 15 .

\begin{tabular}{|c|c|c|c|c|c|c|c|}
\hline Conformer no ${ }^{[a]}$ & $E$ & $\Delta G$ & $\Delta E$ & Pop. & $\Delta \Delta G$ & Pop & \#ImFreq \\
\hline 1 & -1153.76091 & -1153.41 & 0.00 & 26.47 & 0.38 & 10.1 & 0 \\
\hline 17 & -1153.75997 & -1153.41 & 0.59 & 9.77 & 0.28 & 11.94 & 0 \\
\hline 18 & -1153.76037 & -1153.42 & 0.34 & 14.89 & 0.00 & 19.11 & 0 \\
\hline 20 & -1153.76014 & -1153.41469 & 0.48 & 11.68 & 0.24 & 12.74 & 0 \\
\hline 34 & -1153.75955 & -1153.41379 & 0.86 & 6.23 & 0.80 & 4.94 & 0 \\
\hline 47 & -1153.75946 & -1153.41486 & 0.91 & 5.69 & 0.13 & 15.22 & 0 \\
\hline 54 & -1153.7601 & -1153.41365 & 0.51 & 11.19 & 0.89 & 4.24 & 0 \\
\hline 65 & -1153.75978 & -1153.41458 & 0.71 & 7.97 & 0.31 & 11.31 & 0 \\
\hline 70 & -1153.75953 & -1153.4145 & 0.87 & 6.10 & 0.36 & 10.39 & 0 \\
\hline
\end{tabular}

[a] Conformers are numbered according to their appearance during conformational search. 
Table SI_16. Total and free energies $\left(E, \Delta G\right.$, in Hartree), relative energies $\left(\Delta E, \Delta \Delta G\right.$ in $\left.\mathrm{kcal}^{\mathrm{mol}}{ }^{-1}\right)$, percentage populations and number of imaginary frequencies (\#ImFreq) calculated at the M06-2X/6$311++G(d, p)$ level for individual conformers of 15.

\begin{tabular}{cccccccc}
\hline Conformer no ${ }^{[\mathrm{a}]}$ & $E$ & $\Delta G$ & $\Delta E$ & Pop. & $\Delta \Delta G$ & Pop & \#ImFreq \\
\hline 1 & -1153.30037 & -1152.95 & 0.02 & 23.95 & 0.33 & 18.11 & 0 \\
17 & -1153.29972 & -1152.94792 & 0.42 & 12.1 & 0.56 & 12.08 & 0 \\
18 & -1153.29966 & -1152.95 & 0.46 & 11.38 & 0.89 & 6.97 & 0 \\
20 & -1153.3004 & -1152.94882 & 0.00 & 24.74 & 0.00 & 31.36 & 0 \\
34 & -1153.29872 & -1152.9457 & 1.06 & 4.16 & 1.96 & 1.15 & 0 \\
47 & -1153.29819 & -1152.94664 & 1.39 & 2.38 & 1.37 & 3.12 & 0 \\
58 & -1153.29923 & -1152.9472 & 0.73 & 7.19 & 1.01 & 5.67 & 0 \\
65 & -1153.29883 & -1152.94747 & 0.98 & 4.69 & 0.84 & 7.56 & 0 \\
70 & -1153.2993 & -1152.94769 & 0.69 & 7.76 & 0.71 & 9.48 & 0 \\
75 & -1153.29785 & -1152.94698 & 1.60 & 1.66 & 1.15 & 4.49 & 0
\end{tabular}

[a] Conformers are numbered according to their appearance during conformational search. 
Table SI_17. Total and free energies $\left(E, \Delta G\right.$, in Hartree), relative energies $\left(\Delta E, \Delta \Delta G\right.$ in $\left.\mathrm{kcal}^{\mathrm{mol}}{ }^{-1}\right)$, percentage populations and number of imaginary frequencies (\#ImFreq) calculated at the B3LYP/6$311 \mathrm{G}(\mathrm{d}, \mathrm{p})$ level for individual conformers of $\mathbf{1 8}$.

\begin{tabular}{|c|c|c|c|c|c|c|c|}
\hline Conformer no ${ }^{[a]}$ & $E$ & $\Delta G$ & $\Delta E$ & Pop. & $\Delta \Delta G$ & Pop & \#ImFreq \\
\hline 1 & -1961.63782 & -1961.0419 & 1.06 & 1.9 & 1.72 & 1.1 & 0 \\
\hline 5 & -1961.63951 & -1961.0439 & 0.00 & 11.3 & 0.47 & 9.13 & 0 \\
\hline 9 & -1961.63811 & -1961.0425 & 0.88 & 2.58 & 1.34 & 2.07 & 0 \\
\hline 13 & -1961.63759 & -1961.04178 & 1.21 & 1.48 & 1.80 & 0.97 & 0 \\
\hline 15 & -1961.63885 & -1961.04311 & 0.41 & 5.67 & 0.96 & 3.96 & 0 \\
\hline 17 & -1961.63843 & -1961.04132 & 0.68 & 3.62 & 2.08 & - & 0 \\
\hline 18 & -1961.63925 & -1961.0429 & 0.16 & 8.66 & 1.09 & 3.18 & 0 \\
\hline 26 & -1961.63797 & -1961.04179 & 0.97 & 2.22 & 1.79 & 0.98 & 0 \\
\hline 28 & -1961.63938 & -1961.04366 & 0.08 & 9.86 & 0.62 & 7.1 & 0 \\
\hline 32 & -1961.63863 & -1961.04151 & 0.55 & 4.47 & 1.96 & 0.73 & 0 \\
\hline 34 & -1961.63951 & -1961.04279 & 0.00 & 11.37 & 1.16 & 2.83 & 0 \\
\hline 35 & -1961.63654 & -1961.04097 & 1.86 & 0.49 & 2.30 & - & 0 \\
\hline 36 & -1961.63874 & -1961.04408 & 0.48 & 5.03 & 0.35 & 11.1 & 0 \\
\hline 42 & -1961.63858 & -1961.04286 & 0.58 & 4.25 & 1.12 & 3.03 & 0 \\
\hline 43 & -1961.63834 & -1961.04275 & 0.73 & 3.3 & 1.19 & 2.7 & 0 \\
\hline 45 & -1961.63715 & -1961.04173 & 1.48 & 0.94 & 1.82 & 0.92 & 0 \\
\hline 46 & -1961.63766 & -1961.0429 & 1.16 & 1.6 & 1.09 & 3.17 & 0 \\
\hline 48 & -1961.6374 & -1961.04175 & 1.33 & 1.21 & 1.81 & 0.94 & 0 \\
\hline 58 & -1961.63862 & -1961.04464 & 0.56 & 4.42 & 0.00 & 20.09 & 0 \\
\hline 59 & -1961.63864 & -1961.04116 & 0.54 & 4.54 & 2.18 & - & 0 \\
\hline 60 & -1961.63672 & -1961.04142 & 1.75 & 0.59 & 2.02 & - & 0 \\
\hline 61 & -1961.6379 & -1961.04149 & 1.01 & 2.06 & 1.98 & 0.71 & 0 \\
\hline 69 & -1961.63704 & -1961.04119 & 1.55 & 0.83 & 2.16 & - & 0 \\
\hline 70 & -1961.63763 & -1961.04265 & 1.18 & 1.55 & 1.25 & 2.43 & 0 \\
\hline 71 & -1961.63746 & -1961.04408 & 1.29 & 1.29 & 0.35 & 11.16 & 0 \\
\hline 73 & -1961.63828 & -1961.04358 & 0.77 & 3.1 & 0.66 & 6.55 & 0 \\
\hline 76 & -1961.63702 & -1961.04266 & 1.57 & 0.81 & 1.24 & 2.46 & 0 \\
\hline 77 & -1961.63708 & -1961.0414 & 1.53 & 0.86 & 2.04 & - & 0 \\
\hline 93 & -1961.63614 & -1961.04274 & 2.12 & - & 1.19 & 2.67 & 0 \\
\hline
\end{tabular}

[a] Conformers are numbered according to their appearance during conformational search. 
Table SI_18. Total and free energies $\left(E, \Delta G\right.$, in Hartree), relative energies $\left(\Delta E, \Delta \Delta G\right.$ in $\left.\mathrm{kcal}^{\mathrm{mol}}{ }^{-1}\right)$, percentage populations and number of imaginary frequencies (\#ImFreq) calculated at the B3LYPGD3BJ/6-311G(d,p) level for individual conformers of 18.

\begin{tabular}{cccccccc}
\hline Conformer no $^{[\mathrm{a}]}$ & $E$ & $\Delta G$ & $\Delta E$ & Pop. & $\Delta \Delta G$ & Pop & \#ImFreq \\
\hline 13 & -1961.8714 & -1961.26924 & 0.08 & 29.26 & 0.54 & 12.19 & 0 \\
15 & -1961.87078 & -1961.26993 & 0.47 & 15.14 & 0.10 & 25.32 & 0 \\
17 & -1961.87087 & -1961.26851 & 0.42 & 16.68 & 1.00 & 5.62 & 0 \\
26 & -1961.86666 & -1961.26711 & 3.06 & - & 1.88 & 1.27 & 0 \\
32 & -1961.87154 & -1961.2701 & 0.00 & 33.68 & 0.00 & 30.23 & 0 \\
35 & -1961.86722 & -1961.26755 & 2.71 & - & 1.60 & 2.02 & 0 \\
36 & -1961.8682 & -1961.26855 & 2.09 & - & 0.97 & 5.87 & 0 \\
46 & -1961.86741 & -1961.26882 & 2.59 & - & 0.80 & 7.77 & 0 \\
60 & -1961.86752 & -1961.26769 & 2.52 & - & 1.51 & 2.36 & 0 \\
63 & -1961.86723 & -1961.26701 & 2.70 & - & 1.94 & 1.14 & 0 \\
77 & -1961.86978 & -1961.26861 & 1.10 & 5.25 & 0.94 & 6.23 & 0
\end{tabular}

[a] Conformers are numbered according to their appearance during conformational search.

Table SI_19. Total and free energies $\left(E, \Delta G\right.$, in Hartree), relative energies $\left(\Delta E, \Delta \Delta G\right.$ in $\left.\mathrm{kcal} \mathrm{mol}^{-1}\right)$, percentage populations and number of imaginary frequencies (\#ImFreq) calculated at the M06-2X/6$311 \mathrm{G}(\mathrm{d}, \mathrm{p})$ level for individual conformers of $\mathbf{1 8}$.

\begin{tabular}{cccccccc}
\hline Conformer no $^{[\mathrm{a}]}$ & $E$ & $\Delta G$ & $\Delta E$ & Pop. & $\Delta \Delta G$ & Pop & \#ImFreq \\
\hline 1 & -1960.87251 & -1960.26243 & 1.82 & 1.46 & 2.16 & - & 0 \\
5 & -1960.87167 & -1960.26353 & 2.35 & - & 1.47 & 3.05 & 0 \\
13 & -1960.87522 & -1960.26485 & 0.12 & 25.65 & 0.64 & 12.29 & 0 \\
15 & -1960.8728 & -1960.26276 & 1.64 & 1.98 & 1.95 & 1.35 & 0 \\
17 & -1960.87385 & -1960.26371 & 0.98 & 5.98 & 1.35 & 3.71 & 0 \\
18 & -1960.87309 & -1960.26465 & 1.46 & 2.69 & 0.77 & 9.98 & 0 \\
28 & -1960.87541 & -1960.26518 & 0.00 & 31.43 & 0.43 & 17.57 & 0 \\
32 & -1960.87347 & -1960.26402 & 1.22 & 4.01 & 1.16 & 5.1 & 0 \\
36 & -1960.87227 & -1960.26456 & 1.97 & 1.13 & 0.83 & 9.05 & 0 \\
42 & -1960.87522 & -1960.26587 & 0.12 & 25.68 & 0.00 & 36.47 & 0 \\
73 & -1960.86827 & -1960.26282 & 4.48 & - & 1.92 & 1.43 & 0 \\
\hline
\end{tabular}

[a] Conformers are numbered according to their appearance during conformational search. 
Table SI_20. Total and free energies $\left(E, \Delta G\right.$, in Hartree), relative energies $\left(\Delta E, \Delta \Delta G\right.$ in $\left.\mathrm{kcal} \mathrm{mol}^{-1}\right)$, percentage populations and number of imaginary frequencies (\#ImFreq) calculated at the B3LYP/6$311 \mathrm{G}(\mathrm{d}, \mathrm{p})$ level for individual conformers of $\mathbf{2 0}$.

\begin{tabular}{cccccccc}
\hline Conformer no $^{[a]}$ & $E$ & $\Delta G$ & $\Delta E$ & Pop. & $\Delta \Delta G$ & Pop & \#ImFreq \\
\hline 1 & -2000.96492 & -2000.3432 & 0.51 & 13.37 & 0.01 & 24.72 & 0 \\
11 & -2000.96394 & -2000.342 & 1.13 & 4.76 & 0.75 & 6.92 & 0 \\
14 & -2000.96381 & -2000.3419 & 1.21 & 4.12 & 0.82 & 6.22 & 0 \\
16 & -2000.96475 & -2000.34164 & 0.62 & 11.2 & 0.98 & 4.74 & 0 \\
17 & -2000.96484 & -2000.34177 & 0.56 & 12.32 & 0.9 & 5.45 & 0 \\
20 & -2000.96356 & -2000.3401 & 1.37 & 3.18 & 1.94 & 0.93 & 0 \\
21 & -2000.96342 & -2000.34138 & 1.46 & 2.73 & 1.14 & 3.6 & 0 \\
22 & -2000.96574 & -2000.3432 & 0.00 & 31.86 & 0.00 & 24.77 & 0 \\
23 & -2000.96431 & -2000.34196 & 0.9 & 6.99 & 0.78 & 6.63 & 0 \\
25 & -2000.96355 & -2000.34063 & 1.37 & 3.13 & 1.61 & 1.63 & 0 \\
43 & -2000.96327 & -2000.34028 & 1.55 & 2.32 & 1.83 & 1.12 & 0 \\
46 & -2000.96172 & -2000.34005 & 2.52 & - & 1.98 & 0.88 & 0 \\
47 & -2000.96378 & -2000.34255 & 1.23 & 4.02 & 0.41 & 12.39 & 0
\end{tabular}

[a] Conformers are numbered according to their appearance during conformational search.

Table SI_21. Total and free energies $\left(E, \Delta G\right.$, in Hartree), relative energies $\left(\Delta E, \Delta \Delta G\right.$ in $\left.\mathrm{kcal} \mathrm{mol}^{-1}\right)$, percentage populations and number of imaginary frequencies (\#ImFreq) calculated at the B3LYPGD3BJ/6-311G(d,p) level for individual conformers of 20.

\begin{tabular}{cccccccc}
\hline Conformer $\mathrm{no}^{[\mathrm{a}]}$ & $E$ & $\Delta G$ & $\Delta E$ & Pop. & $\Delta \Delta G$ & Pop & \#ImFreq \\
\hline 1 & -2001.2047 & -2000.58 & 0.95 & 5.48 & 0.85 & 7.12 & 0 \\
11 & -2001.20602 & -2000.58 & 0.12 & 22.11 & 1.32 & 3.22 & 0 \\
14 & -2001.20508 & -2000.58 & 0.71 & 8.21 & 0.78 & 7.96 & 0 \\
17 & -2001.20621 & -2000.57785 & 0.00 & 27.12 & 0.51 & 12.54 & 0 \\
20 & -2001.20606 & -2000.57805 & 0.10 & 23.07 & 0.39 & 15.51 & 0 \\
21 & -2001.20419 & -2000.57673 & 1.27 & 3.19 & 1.22 & 3.80 & 0 \\
22 & -2001.20378 & -2000.57867 & 1.53 & 2.05 & 0.00 & 29.76 & 0 \\
25 & -2001.20515 & -2000.57813 & 0.67 & 8.77 & 0.34 & 16.79 & 0 \\
43 & -2001.20275 & -2000.57659 & 2.17 & - & 1.30 & 3.30 & 0 \\
\hline
\end{tabular}

[a] Conformers are numbered according to their appearance during conformational search. 
Table SI_22. Total and free energies $\left(E, \Delta G\right.$, in Hartree), relative energies $\left(\Delta E, \Delta \Delta G\right.$ in $\left.\mathrm{kcal} \mathrm{mol}^{-1}\right)$, percentage populations and number of imaginary frequencies (\#ImFreq) calculated at the M06-2X/6$311 \mathrm{G}(\mathrm{d}, \mathrm{p})$ level for individual conformers of $\mathbf{2 0}$.

\begin{tabular}{cccccccc}
\hline Conformer no $^{[a]}$ & $E$ & $\Delta G$ & $\Delta E$ & Pop. & $\Delta \Delta G$ & Pop & \#ImFreq \\
\hline 1 & -2000.18136 & -1999.54587 & 1.52 & 3.4 & 0.95 & 6.89 & 0 \\
11 & -2000.18379 & -1999.54475 & 0.00 & 44.46 & 1.66 & 2.09 & 0 \\
14 & -2000.18141 & -1999.54538 & 1.49 & 3.59 & 1.26 & 4.08 & 0 \\
17 & -2000.18293 & -1999.54673 & 0.54 & 17.95 & 0.41 & 17.03 & 0 \\
20 & -2000.1829 & -1999.54611 & 0.56 & 17.25 & 0.80 & 8.89 & 0 \\
21 & -2000.18175 & -1999.54618 & 1.28 & 5.11 & 0.76 & 9.55 & 0 \\
22 & -2000.1813 & -1999.54621 & 1.56 & 3.19 & 0.74 & 9.79 & 0 \\
23 & -2000.17877 & -1999.54595 & 3.15 & - & 0.90 & 7.48 & 0 \\
25 & -2000.18174 & -1999.54739 & 1.29 & 5.05 & 0.00 & 34.2 & 0
\end{tabular}

[a] Conformers are numbered according to their appearance during conformational search.

Table SI_23. Total and free energies $\left(E, \Delta G\right.$, in Hartree), relative energies $\left(\Delta E, \Delta \Delta G\right.$ in $\left.\mathrm{kcal} \mathrm{mol}^{-1}\right)$, percentage populations and number of imaginary frequencies (\#ImFreq) calculated at the B3LYP/6$311 \mathrm{G}(\mathrm{d}, \mathrm{p})$ level for individual conformers of $\mathbf{2 1}$.

\begin{tabular}{cccccccc}
\hline Conformer no $^{[\mathrm{a}]}$ & $E$ & $\Delta G$ & $\Delta E$ & Pop. & $\Delta \Delta G$ & Pop & \#ImFreq \\
\hline 1 & -2040.29554 & -2039.64705 & 0.00 & 57.34 & 0.00 & 55.91 & 0 \\
6 & -2040.29371 & -2039.64619 & 1.15 & 8.20 & 0.54 & 22.35 & 0 \\
12 & -2040.2943 & -2039.64526 & 0.78 & 15.32 & 1.12 & 8.38 & 0 \\
15 & -2040.29451 & -2039.64511 & 0.65 & 19.14 & 1.22 & 7.12 & 0 \\
31 & -2040.28977 & -2039.64442 & 3.62 & - & 1.65 & 3.45 & 0 \\
54 & -2040.28943 & -2039.64422 & 3.84 & - & 1.77 & 2.79 & 0
\end{tabular}

[a] Conformers are numbered according to their appearance during conformational search.

Table SI_24. Total and free energies $\left(E, \Delta G\right.$, in Hartree), relative energies $\left(\Delta E, \Delta \Delta G\right.$ in $\left.\mathrm{kcal} \mathrm{mol}^{-1}\right)$, percentage populations and number of imaginary frequencies (\#ImFreq) calculated at the B3LYPGD3BJ/6-311G(d,p) level for individual conformers of $\mathbf{2 1 .}$

\begin{tabular}{cccccccc}
\hline Conformer no[a] & $E$ & $\Delta G$ & $\Delta E$ & Pop. & $\Delta \Delta G$ & Pop & \#ImFreq \\
\hline 1 & -2040.54056 & -2039.88688 & 0.00 & 94.76 & 0.00 & 75.15 & 0 \\
6 & -2040.53672 & -2039.88553 & 2.41 & - & 0.85 & 17.94 & 0 \\
16 & -2040.53783 & -2039.88463 & 1.71 & 5.24 & 1.41 & 6.91 & 0 \\
\hline
\end{tabular}

[a] Conformers are numbered according to their appearance during conformational search. 
Table SI_25. Total and free energies $\left(E, \Delta G\right.$, in Hartree), relative energies $\left(\Delta E, \Delta \Delta G\right.$ in $\left.\mathrm{kcal} \mathrm{mol}^{-1}\right)$, percentage populations and number of imaginary frequencies (\#ImFreq) calculated at the M06-2X/6$311 G(d, p)$ level for individual conformers of 21.

\begin{tabular}{|c|c|c|c|c|c|c|c|}
\hline Conformer no ${ }^{[a]}$ & $E$ & $\Delta G$ & $\Delta E$ & Pop. & $\Delta \Delta G$ & Pop & \#ImFreq \\
\hline 1 & -2039.4893 & -2038.82929 & 1.06 & 11.01 & 0.00 & 88.26 & 0 \\
\hline 12 & -2039.48803 & -2038.8257 & 1.85 & 2.89 & 2.25 & - & 0 \\
\hline 15 & -2039.48792 & -2038.82515 & 1.93 & 2.55 & 2.60 & - & 0 \\
\hline 16 & -2039.48878 & -2038.82584 & 1.39 & 6.34 & 2.17 & - & 0 \\
\hline 25 & -2039.48836 & -2038.82512 & 1.65 & 4.06 & 2.62 & - & 0 \\
\hline 35 & -2039.49099 & -2038.82739 & 0.00 & 66.07 & 1.19 & 11.74 & 0 \\
\hline 46 & -2039.48831 & -2038.82377 & 1.68 & 3.89 & 3.46 & - & 0 \\
\hline 61 & -2039.48812 & -2038.82366 & 1.80 & 3.18 & 3.53 & - & 0 \\
\hline
\end{tabular}

[a] Conformers are numbered according to their appearance during conformational search.

Table SI_26. Total and free energies $\left(E, \Delta G\right.$, in Hartree), relative energies $\left(\Delta E, \Delta \Delta G\right.$ in $\left.\mathrm{kcal} \mathrm{mol}^{-1}\right)$, percentage populations and number of imaginary frequencies (\#ImFreq) calculated at the B3LYP/6$311 \mathrm{G}(\mathrm{d}, \mathrm{p})$ level for individual conformers of $\mathbf{2 2}$.

\begin{tabular}{cccccccc}
\hline Conformer no ${ }^{[a]}$ & $E$ & $\Delta G$ & $\Delta E$ & Pop. & $\Delta \Delta G$ & Pop & \#ImFreq \\
\hline 1 & -2079.61752 & -2078.94311 & 0.01 & 16.47 & 0.34 & 15.6 & 0 \\
3 & -2079.61753 & -2078.94365 & 0.00 & 16.52 & 0.00 & 27.73 & 0 \\
5 & -2079.61651 & -2078.94248 & 0.64 & 5.62 & 0.74 & 8.01 & 0 \\
7 & -2079.61649 & -2078.94288 & 0.65 & 5.51 & 0.49 & 12.2 & 0 \\
8 & -2079.61645 & -2078.942 & 0.67 & 5.3 & 1.04 & 4.83 & 0 \\
9 & -2079.61682 & -2078.94166 & 0.44 & 7.8 & 1.25 & 3.36 & 0 \\
10 & -2079.61506 & -2078.93769 & 1.55 & 1.22 & 3.74 & - & 0 \\
11 & -2079.61753 & -2078.94144 & 0.00 & 16.52 & 1.38 & 2.67 & 0 \\
12 & -2079.61684 & -2078.94233 & 0.43 & 7.97 & 0.83 & 6.86 & 0 \\
13 & -2079.61529 & -2078.94021 & 1.40 & 1.54 & 2.16 & - & 0 \\
15 & -2079.61591 & -2078.94248 & 1.02 & 2.97 & 0.74 & 8.01 & 0 \\
17 & -2079.6148 & -2078.93923 & 1.71 & 0.92 & 2.77 & - & 0 \\
26 & -2079.6152 & -2078.93935 & 1.46 & 1.4 & 2.70 & - & 0 \\
31 & -2079.61327 & -2078.94115 & 2.67 & - & 1.57 & 1.96 & 0 \\
36 & -2079.61702 & -2078.94244 & 0.32 & 9.61 & 0.76 & 7.68 & 0 \\
71 & -2079.61446 & -2078.94059 & 1.92 & 0.64 & 1.92 & 1.08 & 0 \\
\hline
\end{tabular}

[a] Conformers are numbered according to their appearance during conformational search. 
Table SI_27. Total and free energies $\left(E, \Delta G\right.$, in Hartree), relative energies $\left(\Delta E, \Delta \Delta G\right.$ in $\left.\mathrm{kcal} \mathrm{mol}^{-1}\right)$, percentage populations and number of imaginary frequencies (\#ImFreq) calculated at the B3LYPGD3BJ/6-311G(d,p) level for individual conformers of 22.

\begin{tabular}{cccccccc}
\hline Conformer no $^{[\mathrm{a}]}$ & $E$ & $\Delta G$ & $\Delta E$ & Pop. & $\Delta \Delta G$ & Pop & \#ImFreq \\
\hline 1 & -2079.87057 & -2079.18934 & 0.01 & 35.17 & 0.40 & 24.96 & 0 \\
3 & -2079.87057 & -2079.18997 & 0.00 & 35.26 & 0.00 & 49.07 & 0 \\
5 & -2079.86797 & -2079.18712 & 1.63 & 2.25 & 1.79 & 2.38 & 0 \\
7 & -2079.86976 & -2079.18928 & 0.51 & 15.0 & 0.43 & 23.59 & 0 \\
17 & -2079.86901 & -2079.18645 & 0.98 & 6.74 & 2.21 & - & 0 \\
26 & -2079.86883 & -2079.18633 & 1.09 & 5.57 & 2.29 & - & 0
\end{tabular}

[a] Conformers are numbered according to their appearance during conformational search.

Table SI_28. Total and free energies $\left(E, \Delta G\right.$, in Hartree), relative energies $\left(\Delta E, \Delta \Delta G\right.$ in kcal mol$\left.{ }^{-1}\right)$, percentage populations and number of imaginary frequencies (\#ImFreq) calculated at the M06-2X/6$311 \mathrm{G}(\mathrm{d}, \mathrm{p})$ level for individual conformers of 22.

\begin{tabular}{|c|c|c|c|c|c|c|c|}
\hline Conformer no ${ }^{[\mathrm{a}]}$ & $E$ & $\Delta G$ & $\Delta E$ & Pop. & $\Delta \Delta G$ & Pop & \#ImFreq \\
\hline 1 & -2078.79565 & -2078.10589 & 0.15 & 26.94 & 0.39 & 29.08 & 0 \\
\hline 3 & -2078.79566 & -2078.10651 & 0.14 & 27.33 & 0.00 & 55.92 & 0 \\
\hline 11 & -2078.79589 & -2078.10509 & 0.00 & 34.79 & 0.89 & 12.39 & 0 \\
\hline 17 & -2078.79342 & -2078.10362 & 1.55 & 2.52 & 1.81 & 2.61 & 0 \\
\hline 26 & -2078.79455 & -2078.1031 & 0.84 & 8.42 & 2.14 & - & 0 \\
\hline
\end{tabular}

[a] Conformers are numbered according to their appearance during conformational search. 
Table SI_29. Dihedral angles $\alpha, 6, \gamma, \delta$ and $\zeta$ (in degrees) and selected interatomic distances $I_{1}, I_{2}$ (in $\AA$ ) calculated at the B3LYP/6-311++G(d,p) level for individual low-energy conformers of 1 .

\begin{tabular}{|c|c|c|c|c|c|c|c|c|c|c|c|}
\hline Conformer no ${ }^{[a]}$ & $\alpha^{[b]}$ & $\mathrm{B}_{1}^{[c]}$ & $B_{2}^{[c]}$ & $B_{3}{ }^{[c]}$ & $\gamma_{1}{ }^{[d]}$ & $V_{2}{ }^{[\mathrm{d}]}$ & $\gamma_{3}{ }^{[d]}$ & $\delta^{[e]}$ & $\zeta^{[f]}$ & $l_{1}^{[\mathrm{g}]}$ & $I_{2}^{[\mathrm{h}]}$ \\
\hline 1 & -174.74 & -110.40 & 127.18 & 9.70 & 66.32 & -11.79 & 49.00 & 33.69 & -97.17 & 2.542 & 2.438 \\
\hline 17 & -176.67 & -113.13 & 124.54 & 7.28 & 67.79 & -13.16 & 47.93 & -32.09 & 62.62 & 2.503 & 2.468 \\
\hline 33 & 178.87 & -110.76 & 127.30 & 6.31 & -1.76 & -60.12 & -54.21 & 34.66 & 63.22 & 2.515 & 2.425 \\
\hline 38 & -175.72 & -113.03 & 124.65 & 7.36 & 67.69 & -13.15 & 47.65 & -34.15 & -174.10 & 2.497 & 2.470 \\
\hline 39 & -171.37 & -111.54 & 126.17 & 8.56 & 65.12 & -9.80 & 50.26 & 33.46 & 63.88 & 2.562 & 2.424 \\
\hline 40 & 171.97 & -125.88 & 111.94 & -8.28 & 10.50 & -66.08 & -49.30 & -28.97 & -175.48 & 2.537 & 2.434 \\
\hline 41 & -172.75 & -117.85 & 120.04 & 2.43 & 61.47 & -2.62 & 52.88 & 32.11 & -173.03 & 2.560 & 2.399 \\
\hline 42 & 168.75 & -132.04 & 105.56 & -14.42 & 21.32 & -71.76 & -44.05 & -25.30 & 65.27 & 2.493 & 2.538 \\
\hline 43 & 169.74 & -156.92 & 81.48 & -38.08 & 49.51 & 75.21 & -12.92 & -0.98 & -68.00 & 2.255 & 2.775 \\
\hline 44 & 171.02 & -145.36 & 92.54 & -27.44 & 43.57 & 3.82 & -22.22 & 39.65 & -64.11 & 2.274 & 2.806 \\
\hline 45 & 178.49 & -132.52 & 106.85 & -12.73 & 48.29 & 44.08 & 54.89 & 39.29 & -64.36 & 2.454 & 2.221 \\
\hline 49 & 178.49 & -132.53 & 106.83 & -12.73 & 48.31 & 44.07 & 54.87 & 39.27 & -64.37 & 2.453 & 2.221 \\
\hline 50 & -177.63 & -134.47 & 104.89 & -14.39 & 47.90 & 40.26 & 56.22 & 35.32 & 63.45 & 2.470 & 2.235 \\
\hline 52 & -178.22 & -135.99 & 103.47 & -15.74 & 48.30 & 40.76 & 56.15 & 34.20 & -172.72 & 2.459 & 2.245 \\
\hline 53 & -177.98 & 153.59 & 31.83 & -85.35 & 65.94 & 42.73 & 38.89 & -31.06 & 61.05 & 2.344 & 2.503 \\
\hline
\end{tabular}

[a] Conformers are numbered according to their appearance during conformational search; $[\mathrm{b}]-\alpha=\mathrm{C}_{\mathrm{Tr}}-\mathrm{C}(=\mathrm{O})-\mathrm{O}-\mathrm{C}^{*} ;[\mathrm{c}]-\mathrm{B}=\mathrm{O}=\mathrm{C}-\mathrm{C}-\mathrm{C}_{\text {ipso }} ;[\mathrm{d}]-\gamma=(\mathrm{O}=) \mathrm{C}-\mathrm{C}_{\mathrm{Tr}}-\mathrm{C}_{\text {ipso }}-\mathrm{C}_{\text {ortho }}$ (of the two possibilities the absolute values $\leq 90^{\circ}$ has been chosen); $[\mathrm{e}]-\delta=\mathrm{C}(=\mathrm{O})-\mathrm{O}-\mathrm{C}^{*}-\mathrm{H} ;[\mathrm{f}]-\zeta=\mathrm{O}-\mathrm{C}^{*}-\mathrm{C}-\mathrm{C}\left({ }^{*}\right) ;[\mathrm{g}]-\mathrm{I}_{1}=\mathrm{C}=\mathrm{O} \cdots \mathrm{HC}$ ortho; $[\mathrm{h}]-\mathrm{I}_{2}=$ $(\mathrm{O}=) \mathrm{CO} \cdots \mathrm{HC}_{\text {ortho }}$. 
Table SI_30. Dihedral angles $\alpha, 6, y, \delta$ and $\zeta$ (in degrees) and selected interatomic distances $I_{1}, I_{2}$ (in $\AA$ ) calculated at the M06-2X/6-311++G(d,p) level for individual low-energy conformers of 1 .

\begin{tabular}{|c|c|c|c|c|c|c|c|c|c|c|c|}
\hline Conformer no ${ }^{[a]}$ & $\alpha^{[\mathrm{b}]}$ & $B_{1}{ }^{[c]}$ & $B_{2}{ }^{[c]}$ & $B_{3}{ }^{[c]}$ & $\gamma_{1}{ }^{[d]}$ & $V_{2}{ }^{[d]}$ & $\mathrm{V}_{3}{ }^{[\mathrm{d}]}$ & $\delta^{[\mathrm{e}]}$ & $\zeta^{[f]}$ & $I_{1}^{[\mathrm{g}]}$ & $I_{2}^{[\mathrm{h}]}$ \\
\hline 1 & -172.82 & -121.12 & 117.14 & -0.55 & 63.62 & -6.68 & 51.45 & 34.22 & -59.23 & 2.496 & 2.442 \\
\hline 17 & -166.73 & -110.93 & 126.91 & 8.79 & 64.12 & -12.04 & 49.92 & -40.17 & 62.48 & 2.535 & 2.415 \\
\hline 33 & 173.77 & -111.60 & 126.83 & 5.78 & 1.05 & -59.82 & -54.92 & 44.43 & 63.91 & 2.510 & 2.419 \\
\hline 38 & -162.70 & -103.44 & 135.00 & 16.51 & 72.18 & -20.93 & 45.29 & -42.51 & -179.59 & 2.517 & 2.465 \\
\hline 39 & -174.62 & -113.48 & 124.78 & 7.07 & 67.99 & -13.93 & 47.91 & 38.67 & 63.48 & 2.491 & 2.449 \\
\hline 40 & 174.90 & -120.96 & 117.14 & -3.60 & 10.27 & -65.68 & -50.73 & -35.47 & -177.67 & 2.502 & 2.446 \\
\hline 41 & -174.68 & -122.45 & 115.81 & -1.92 & 62.18 & -4.12 & 52.23 & 36.53 & -176.63 & 2.497 & 2.430 \\
\hline 44 & 161.98 & -133.56 & 104.96 & -14.90 & 20.71 & -71.49 & -45.03 & 46.18 & -58.84 & 2.506 & 2.477 \\
\hline 45 & -179.60 & -132.50 & 107.21 & -13.14 & 48.67 & 46.84 & 54.08 & 42.40 & -57.46 & 2.422 & 2.205 \\
\hline 50 & 176.92 & -149.11 & 91.57 & -27.83 & 48.72 & 46.93 & 54.90 & 37.52 & 58.71 & 2.365 & 2.294 \\
\hline 52 & 178.58 & -144.86 & 94.91 & -23.72 & 49.23 & 37.51 & 58.02 & 38.72 & -177.09 & 2.422 & 2.291 \\
\hline 53 & -164.57 & -101.04 & 138.30 & 15.74 & 47.64 & 61.86 & 45.29 & -39.73 & 60.40 & 2.485 & 2.302 \\
\hline
\end{tabular}

[a] Conformers are numbered according to their appearance during conformational search; $[\mathrm{b}]-\alpha=\mathrm{C}_{\mathrm{Tr}}-\mathrm{C}(=\mathrm{O})-\mathrm{O}-\mathrm{C}^{*} ;[\mathrm{c}]-\mathrm{B}=\mathrm{O}=\mathrm{C}-\mathrm{C}-\mathrm{C}_{\text {ipso }} ;[\mathrm{d}]-\gamma=(\mathrm{O}=) \mathrm{C}-\mathrm{C}_{\mathrm{Tr}}-\mathrm{C}_{\text {ipso }}-\mathrm{C}_{\text {ortho }}$ (of the two possibilities the absolute values $\leq 90^{\circ}$ has been chosen); $[\mathrm{e}]-\delta=\mathrm{C}(=\mathrm{O})-\mathrm{O}-\mathrm{C}^{*}-\mathrm{H} ;[\mathrm{f}]-\zeta=\mathrm{O}-\mathrm{C}^{*}-\mathrm{C}-\mathrm{C}\left({ }^{*}\right) ;[\mathrm{g}]-\mathrm{I}_{1}=\mathrm{C}=\mathrm{O} \cdots \mathrm{HC}$ ortho; $[\mathrm{h}]-\mathrm{I}_{2}=$ $(\mathrm{O}=) \mathrm{CO} \cdots \mathrm{HC}_{\text {ortho. }}$. 
Table SI_31. Dihedral angles $\alpha, 6, y, \delta$ and $\zeta$ (in degrees) and selected interatomic distances $I_{1}, I_{2}$ (in $\AA$ ) calculated at the B3LYP/6-311++G(d,p) level for individual low-energy conformers of 4 .

\begin{tabular}{|c|c|c|c|c|c|c|c|c|c|c|c|c|}
\hline Conformer no ${ }^{[\mathrm{a}]}$ & $\alpha^{[\mathrm{b}]}$ & $B_{1}{ }^{[c]}$ & $B_{2}{ }^{[c]}$ & $B_{3}[c]$ & $\gamma_{1}{ }^{[\mathrm{d}]}$ & $V_{2}{ }^{[d]}$ & $V_{3}{ }^{[\mathrm{d}]}$ & $\delta^{[\mathrm{e}]}$ & $\zeta^{[f]}$ & $\zeta_{2}^{[f]}$ & $l_{1}^{[\mathrm{g}]}$ & $I_{2}^{[\mathrm{h}]}$ \\
\hline 1 & -175.15 & -114.27 & 123.32 & 5.94 & 63.99 & -7.26 & 50.97 & 33.12 & 59.77 & -66.42 & 2.546 & 2.418 \\
\hline 20 & 171.32 & -126.94 & 110.37 & -9.76 & 19.98 & -70.37 & -44.76 & 40.53 & 60.32 & -66.08 & 2.466 & 2.565 \\
\hline 22 & -176.92 & -116.13 & 121.56 & 4.38 & 66.62 & -10.74 & 48.68 & -31.62 & 65.02 & -168.66 & 2.490 & 2.467 \\
\hline 42 & -171.85 & -114.37 & 123.35 & 5.82 & 64.22 & -8.39 & 50.27 & 30.50 & 65.05 & -168.53 & 2.535 & 2.438 \\
\hline 44 & 170.67 & -149.80 & 88.32 & -31.50 & 47.39 & 77.42 & -15.42 & -22.56 & 65.69 & -168.07 & 2.235 & 2.734 \\
\hline 46 & 178.51 & -44.93 & -166.19 & 72.82 & -41.80 & -66.71 & -34.96 & 28.12 & 61.73 & -64.48 & 2.222 & 2.653 \\
\hline
\end{tabular}

[a] Conformers are numbered according to their appearance during conformational search; $[\mathrm{b}]-\alpha=\mathrm{C}_{\mathrm{Tr}}-\mathrm{C}(=\mathrm{O})-\mathrm{O}-\mathrm{C}^{*} ;[\mathrm{c}]-8=\mathrm{O}=\mathrm{C}-\mathrm{C}-\mathrm{C}_{\text {ipso }} ;[\mathrm{d}]-\gamma=(\mathrm{O}=) \mathrm{C}-\mathrm{C}_{\mathrm{Tr}}-\mathrm{C}_{\text {ipso }}-\mathrm{C}_{\text {ortho }}$ (of the two possibilities the absolute values $\leq 90^{\circ}$ has been chosen); $[\mathrm{e}]-\delta=\mathrm{C}(=\mathrm{O})-\mathrm{O}-\mathrm{C}^{*}-\mathrm{H}$; $[\mathrm{f}]-\zeta=\mathrm{O}-\mathrm{C}^{*}-\mathrm{C}-\mathrm{C}(*)$; $[\mathrm{g}]-\mathrm{I}_{1}=\mathrm{C}=\mathrm{O} \cdots \mathrm{HC}$ ortho; $[\mathrm{h}]-\mathrm{I}_{2}=$ $(\mathrm{O}=) \mathrm{CO} \cdots \mathrm{HC}_{\text {ortho }}$.

Table SI_32. Dihedral angles $\alpha, 6, \gamma, \delta$ and $\zeta$ (in degrees) and selected interatomic distances $I_{1}, I_{2}$ (in $\AA$ ) calculated at the M06-2X/6-311++G(d,p) level for individual low-energy conformers of 4.

\begin{tabular}{|c|c|c|c|c|c|c|c|c|c|c|c|c|}
\hline Conformer no ${ }^{[\mathrm{a}]}$ & $\alpha^{[\mathrm{b}]}$ & $B_{1}^{[c]}$ & $B_{2}^{[c]}$ & $B_{3}^{[c]}$ & $\gamma_{1}{ }^{[\mathrm{d}]}$ & $V_{2}{ }^{[\mathrm{d}]}$ & $\gamma_{3}{ }^{[\mathrm{d}]}$ & $\delta^{[\mathrm{e}]}$ & $\zeta^{[f]}$ & $\zeta_{2}^{[f]}$ & $l_{1}^{[\mathrm{g}]}$ & $I_{2}^{[\mathrm{h}]}$ \\
\hline 1 & -173.79 & -121.37 & 117.11 & -0.43 & 64.62 & -6.12 & 51.44 & 33.75 & 63.06 & -60.80 & 2.489 & 2.432 \\
\hline 20 & 179.24 & -95.53 & 144.10 & 21.76 & -11.40 & -58.22 & -57.44 & 47.25 & 63.86 & -60.02 & 2.454 & 2.445 \\
\hline 22 & -167.71 & -111.86 & 126.21 & 8.28 & 64.19 & -11.02 & 51.02 & -39.06 & 62.88 & -171.88 & 2.540 & 2.410 \\
\hline 42 & -175.36 & -121.15 & 117.30 & -0.23 & 64.70 & -7.08 & 50.69 & 35.92 & 61.02 & -173.58 & 2.478 & 2.444 \\
\hline 44 & 176.45 & -121.25 & 116.57 & -4.28 & 17.59 & -69.19 & -46.61 & -30.48 & 66.04 & -168.74 & 2.438 & 2.531 \\
\hline 46 & 178.04 & -21.49 & -143.45 & 96.39 & -44.03 & -69.83 & -35.84 & 34.84 & 64.62 & -59.70 & 2.455 & 2.474 \\
\hline
\end{tabular}

[a] Conformers are numbered according to their appearance during conformational search; $[\mathrm{b}]-\alpha=\mathrm{C}_{\mathrm{Tr}}-\mathrm{C}(=\mathrm{O})-\mathrm{O}-\mathrm{C}^{*} ;[\mathrm{c}]-B=\mathrm{O}=\mathrm{C}-\mathrm{C}-\mathrm{C}_{i p s o} ;[\mathrm{d}]-\gamma=(\mathrm{O}=) \mathrm{C}-\mathrm{C}_{\mathrm{Tr}}-\mathrm{C}_{i p s o}-\mathrm{C}_{\text {ortho }}$ (of the two possibilities the absolute values $\leq 90^{\circ}$ has been chosen); $[\mathrm{e}]-\delta=\mathrm{C}(=\mathrm{O})-\mathrm{O}-\mathrm{C}^{*}-\mathrm{H}$; $[\mathrm{f}]-\zeta=\mathrm{O}-\mathrm{C}^{*}-\mathrm{C}-\mathrm{C}\left({ }^{*}\right)$; $[\mathrm{g}]-\mathrm{I}_{1}=\mathrm{C}=\mathrm{O} \cdots \mathrm{HC}$ ortho; $[\mathrm{h}]-\mathrm{I}_{2}=$ $(\mathrm{O}=) \mathrm{CO} \cdots \mathrm{HC}_{\text {ortho }}$. 
Table SI_33. Dihedral angles $\alpha, 6, y, \delta$ and $\zeta$ (in degrees) and selected interatomic distances $I_{1}, I_{2}$ (in $\AA$ ) calculated at the B3LYP/6-311++G(d,p) level for individual low-energy conformers of 6 .

\begin{tabular}{|c|c|c|c|c|c|c|c|c|c|c|c|c|}
\hline Conformer no ${ }^{[\mathrm{a}]}$ & $\alpha^{[\mathrm{b}]}$ & $B_{1}^{[c]}$ & $B_{2}{ }^{[c]}$ & $B_{3}^{[c]}$ & $V_{1}{ }^{[\mathrm{d}]}$ & $V_{2}{ }^{[\mathrm{d}]}$ & $\gamma_{3}{ }^{[\mathrm{d}]}$ & $\delta^{[\mathrm{e}]}$ & $\zeta^{[f]}$ & $\zeta_{2}^{[f]}$ & $l_{1}{ }^{[\mathrm{g}]}$ & $I_{2}^{[\mathrm{h}]}$ \\
\hline 1 & 173.94 & -128.20 & 109.38 & -10.46 & 10.62 & -65.13 & -49.76 & -42.80 & 66.26 & -59.39 & 2.569 & 2.431 \\
\hline 4 & 173.00 & -128.90 & 108.95 & -11.14 & 12.24 & -66.98 & -48.84 & -45.37 & 165.41 & -67.19 & 2.560 & 2.441 \\
\hline 7 & -179.35 & -156.54 & 83.19 & -34.74 & 49.73 & 36.46 & 56.62 & -49.20 & 64.53 & -61.35 & 2.383 & 2.449 \\
\hline 8 & 174.89 & -124.42 & 113.36 & -6.82 & 8.39 & -64.58 & -50.68 & -52.51 & 63.89 & -172.07 & 2.556 & 2.430 \\
\hline 10 & 179.20 & -148.97 & 90.74 & -27.59 & 47.29 & 37.80 & 57.73 & -49.95 & 167.58 & -65.12 & 2.439 & 2.347 \\
\hline 14 & 170.48 & -129.40 & 108.34 & -11.38 & 7.54 & -63.83 & -50.73 & -41.80 & 65.12 & -60.68 & 2.594 & 2.373 \\
\hline 17 & -176.61 & -99.11 & 138.23 & 20.91 & 84.28 & -36.78 & 29.00 & -46.86 & 65.15 & -60.80 & 2.313 & 2.721 \\
\hline 18 & -179.81 & -150.96 & 88.52 & -29.39 & 48.91 & 33.62 & 58.40 & -57.23 & 61.21 & -174.74 & 2.443 & 2.402 \\
\hline 20 & 177.90 & -163.74 & 76.22 & -41.75 & 53.54 & 38.00 & 52.40 & -47.29 & 64.68 & -61.20 & 2.294 & 2.544 \\
\hline 21 & 168.48 & -128.94 & 109.03 & -10.86 & 8.49 & -65.12 & -50.08 & -42.75 & 162.90 & -70.13 & 2.580 & 2.385 \\
\hline 28 & 179.03 & -177.30 & 62.39 & -55.98 & 60.44 & 44.83 & 37.33 & -41.87 & 64.99 & -60.85 & 2.160 & 2.699 \\
\hline 46 & 177.82 & -156.21 & 83.71 & -34.41 & 48.97 & 37.89 & 56.17 & -55.96 & 59.95 & -176.39 & 2.375 & 2.444 \\
\hline
\end{tabular}

[a] Conformers are numbered according to their appearance during conformational search; $[\mathrm{b}]-\alpha=\mathrm{C}_{\mathrm{Tr}}-\mathrm{C}(=\mathrm{O})-\mathrm{O}-\mathrm{C}^{*} ;[\mathrm{c}]-B=\mathrm{O}=\mathrm{C}-\mathrm{C}-\mathrm{C}_{i p s o} ;[\mathrm{d}]-\gamma=(\mathrm{O}=) \mathrm{C}-\mathrm{C}_{\mathrm{Tr}}-\mathrm{C}_{i p s o}-\mathrm{C}_{\text {ortho }}$ (of the two possibilities the absolute values $\leq 90^{\circ}$ has been chosen); $[\mathrm{e}]-\delta=\mathrm{C}(=\mathrm{O})-\mathrm{O}-\mathrm{C}^{*}-\mathrm{H} ;[\mathrm{f}]-\zeta=\mathrm{O}-\mathrm{C}^{*}-\mathrm{C}-\mathrm{C}\left({ }^{*}\right)$; $[\mathrm{g}]-\mathrm{I}_{1}=\mathrm{C}=\mathrm{O} \cdots \mathrm{HC}$ ortho; $[\mathrm{h}]-\mathrm{I}_{2}=$ $(\mathrm{O}=) \mathrm{CO} \cdots \mathrm{HC}$ ortho. 
Table SI_34. Dihedral angles $\alpha, b, y, \delta$ and $\zeta$ (in degrees) and selected interatomic distances $I_{1}$ and $I_{2}$ (in $\AA$ ) calculated at the M06-2X/6-311++G(d,p) level for individual low-energy conformers of 6 .

\begin{tabular}{|c|c|c|c|c|c|c|c|c|c|c|c|c|}
\hline Conformer no ${ }^{[a]}$ & $\alpha^{[\mathrm{b}]}$ & $B_{1}{ }^{[c]}$ & $B_{2}{ }^{[c]}$ & $B_{3}{ }^{[c]}$ & $V_{1}{ }^{[d]}$ & $V_{2}{ }^{[d]}$ & $\gamma_{3}{ }^{[\mathrm{d}]}$ & $\delta^{[\mathrm{e}]}$ & $\zeta^{[f]}$ & $\zeta_{2}^{[f]}$ & $l_{1}{ }^{[\mathrm{g}]}$ & $I_{2}^{[\mathrm{h}]}$ \\
\hline 1 & 169.63 & -120.34 & 118.46 & -2.03 & 4.70 & -63.15 & -52.11 & -34.74 & 61.96 & -61.50 & 2.525 & 2.407 \\
\hline 7 & -179.07 & -152.49 & 87.45 & -30.64 & 50.94 & 30.29 & 58.66 & -51.72 & 63.05 & -60.55 & 2.436 & 2.366 \\
\hline 11 & 168.85 & -112.22 & 126.54 & 5.98 & -3.37 & -57.86 & -55.51 & -46.69 & 60.25 & -176.40 & 2.531 & 2.408 \\
\hline 14 & 167.22 & -117.53 & 121.65 & 1.06 & -2.90 & -60.91 & -54.21 & -38.77 & 62.78 & -60.74 & 2.542 & 2.347 \\
\hline 17 & -171.85 & -98.91 & 139.64 & 21.67 & -86.95 & -35.01 & 31.95 & -51.40 & 60.44 & -63.60 & 2.358 & 2.619 \\
\hline 28 & -172.20 & 159.14 & 37.60 & -79.93 & 67.23 & 39.52 & 40.96 & -49.82 & 59.71 & -64.31 & 2.277 & 2.524 \\
\hline 30 & 170.64 & -111.92 & 126.75 & 5.90 & -1.44 & -59.22 & -55.24 & -49.18 & 61.32 & -175.25 & 2.526 & 2.424 \\
\hline 32 & -179.69 & -142.30 & 96.84 & -20.18 & 57.31 & 10.34 & 57.32 & -49.62 & 54.33 & -72.83 & 2.466 & 2.454 \\
\hline
\end{tabular}

[a] Conformers are numbered according to their appearance during conformational search; $[\mathrm{b}]-\alpha=\mathrm{C}_{\mathrm{Tr}}-\mathrm{C}(=\mathrm{O})-\mathrm{O}-\mathrm{C}^{*} ;[\mathrm{c}]-8=\mathrm{O}=\mathrm{C}-\mathrm{C}-\mathrm{C}_{i p s o} ;[\mathrm{d}]-\gamma=(\mathrm{O}=) \mathrm{C}-\mathrm{C}_{\mathrm{Tr}}-\mathrm{C}_{i p s o}-\mathrm{C}_{\text {ortho }}$ (of the two possibilities the absolute values $\leq 90^{\circ}$ has been chosen); $[\mathrm{e}]-\delta=\mathrm{C}(=\mathrm{O})-\mathrm{O}-\mathrm{C}^{*}-\mathrm{H} ;[\mathrm{f}]-\zeta=\mathrm{O}-\mathrm{C}^{*}-\mathrm{C}-\mathrm{C}\left({ }^{*}\right) ;[\mathrm{g}]-\mathrm{I}_{1}=\mathrm{C}=\mathrm{O} \cdots \mathrm{HC}$ ortho; $[\mathrm{h}]-\mathrm{I}_{2}=$

$(\mathrm{O}=) \mathrm{CO} \cdots \mathrm{HC}_{\text {ortho. }}$

Table SI_35. Dihedral angles $\alpha, b, y, \delta$ and $\zeta$ (in degrees) and selected interatomic distances $I_{1}, I_{2}$ (in $\AA$ ) calculated at the DFT/6-311++G(d,p) level for individual low-energy conformers of 10.

\begin{tabular}{|c|c|c|c|c|c|c|c|c|c|c|c|}
\hline Conformer no ${ }^{[\mathrm{a}]}$ & $\alpha^{[\mathrm{b}]}$ & $B_{1}{ }^{[c]}$ & $B_{2}{ }^{[c]}$ & $B_{3}^{[c]}$ & $V_{1}{ }^{[\mathrm{d}]}$ & $V_{2}{ }^{[\mathrm{d}]}$ & $\gamma_{3}{ }^{[d]}$ & $\delta^{[\mathrm{e}]}$ & $\zeta^{[f]}$ & $l_{1}^{[\mathrm{g}]}$ & $I_{2}^{[\mathrm{h}]}$ \\
\hline $1^{[i]}$ & -176.56 & -111.57 & 125.98 & 8.64 & 66.50 & -11.31 & 49.05 & 31.37 & -57.37 & 2.532 & 2.447 \\
\hline $1^{[j]}$ & -172.12 & -119.70 & 118.93 & 1.27 & 64.74 & -6.00 & 52.04 & 26.52 & -55.43 & 2.506 & 2.434 \\
\hline
\end{tabular}

[a] Conformers are numbered according to their appearance during conformational search; $[\mathrm{b}]-\alpha=\mathrm{C}$ Tr $-\mathrm{C}(=\mathrm{O})-\mathrm{O}-\mathrm{C}^{*} ;[\mathrm{c}]-B=\mathrm{O}=\mathrm{C}-\mathrm{C}-\mathrm{C}_{i p s o} ;[\mathrm{d}]-\gamma=(\mathrm{O}=) \mathrm{C}-\mathrm{C}_{\mathrm{Tr}}-\mathrm{C}_{i p s o}-\mathrm{C}_{\text {ortho }}$ (of the two possibilities the absolute values $\leq 90^{\circ}$ has been chosen); [e] $-\delta=\mathrm{C}(=\mathrm{O})-\mathrm{O}-\mathrm{C}^{*}-\mathrm{H}$; $[\mathrm{f}]-\zeta=\mathrm{O}-\mathrm{C}^{*}-\mathrm{C}-\mathrm{C}(*)$; $[\mathrm{g}]-\mathrm{I}_{1}=\mathrm{C}=\mathrm{O} \cdots \mathrm{HC}$ ortho; $[\mathrm{h}]-\mathrm{I}_{2}=$ $(\mathrm{O}=) \mathrm{CO} \cdots \mathrm{HC}_{\text {ortho; }}[\mathrm{i}]$ optimized at the B3LYP/6-311++G(d,p) level; [j] optimized at the M06-2X/6-311++G(d,p) level. 
Table SI_36. Dihedral angles $\alpha, b, y, \delta$ and $\zeta$ (in degrees) and selected interatomic distances $I_{1}, I_{2}$ (in $\AA$ ) calculated at the B3LYP/6-311++G(d,p) level for individual low-energy conformers of $\mathbf{1 1 .}$

\begin{tabular}{|c|c|c|c|c|c|c|c|c|c|c|c|}
\hline Conformer no ${ }^{[a]}$ & $\alpha^{[\mathrm{b}]}$ & $B_{1}^{[c]}$ & $B_{2}{ }^{[c]}$ & $B_{3}{ }^{[c]}$ & $V_{1}{ }^{[\mathrm{d}]}$ & $V_{2}{ }^{[d]}$ & $\gamma_{3}{ }^{[\mathrm{d}]}$ & $\delta^{[\mathrm{e}]}$ & $\zeta^{[f]}$ & $l_{1}^{[\mathrm{g}]}$ & $I_{2}^{[\mathrm{h}]}$ \\
\hline 1 & -174.53 & -108.81 & 128.47 & 11.23 & 70.06 & -18.94 & 45.68 & -37.13 & 60.29 & 2.488 & 2.540 \\
\hline 22 & 176.67 & -123.21 & 114.10 & -6.44 & 16.26 & -69.13 & -46.87 & -18.18 & 61.06 & 2.466 & 2.549 \\
\hline 27 & -176.73 & 153.60 & 31.83 & -85.28 & 66.17 & 42.43 & 39.50 & -31.22 & 58.67 & 2.339 & 2.518 \\
\hline 52 & -174.68 & 160.66 & 39.01 & -78.29 & 66.18 & 42.06 & 37.76 & -30.92 & 66.68 & 2.264 & 2.587 \\
\hline 56 & -175.32 & 161.89 & 40.30 & -77.08 & 66.14 & 42.20 & 37.34 & -30.74 & 65.45 & 2.256 & 2.597 \\
\hline 62 & -178.82 & -21.33 & -142.98 & 95.81 & -44.05 & -66.36 & -39.72 & -33.81 & 66.99 & 2.479 & 2.462 \\
\hline
\end{tabular}

[a] Conformers are numbered according to their appearance during conformational search; $\left.[\mathrm{b}]-\alpha=\mathrm{C}_{\mathrm{Tr}}-\mathrm{C}(=\mathrm{O})-\mathrm{O}-\mathrm{C}^{*} ;[\mathrm{c}]-B=\mathrm{O}=\mathrm{C}-\mathrm{C}-\mathrm{C}_{\text {ipso; }} ; \mathrm{d}\right]-\gamma=(\mathrm{O}=) \mathrm{C}-\mathrm{C}_{\mathrm{Tr}}-\mathrm{C}_{\text {ipso }}-\mathrm{C}_{\text {ortho }}$ (of the two possibilities the absolute values $\leq 90^{\circ}$ has been chosen); $[\mathrm{e}]-\delta=\mathrm{C}(=\mathrm{O})-\mathrm{O}-\mathrm{C}^{*}-\mathrm{H} ;[\mathrm{f}]-\zeta=\mathrm{O}-\mathrm{C}^{*}-\mathrm{C}-\mathrm{C}(*) ;[\mathrm{g}]-\mathrm{I}_{1}=\mathrm{C}=\mathrm{O} \cdots \mathrm{HC} \mathrm{Cortho}$; $[\mathrm{h}]-\mathrm{I}_{2}=$ $(\mathrm{O}=) \mathrm{CO} \cdots \mathrm{HC}_{\text {ortho. }}$ 
Table SI_37. Dihedral angles $\alpha, 6, y, \delta$ and $\zeta$ (in degrees) and selected interatomic distances $I_{1}, I_{2}$ (in $\AA$ ) calculated at the M06-2X/6-311++G(d,p) level for individual low-energy conformers of $\mathbf{1 1 .}$

\begin{tabular}{|c|c|c|c|c|c|c|c|c|c|c|c|}
\hline Conformer no ${ }^{[a]}$ & $\alpha^{[\mathrm{b}]}$ & $B_{1}^{[c]}$ & $B_{2}^{[c]}$ & $B_{3}{ }^{[c]}$ & $\mathrm{V}_{1}{ }^{[\mathrm{d}]}$ & $\mathrm{V}_{2}{ }^{[\mathrm{d}]}$ & $\mathrm{V}_{3}{ }^{[\mathrm{d}]}$ & $\delta^{[\mathrm{e}]}$ & $\zeta^{[f]}$ & $l_{1}^{[\mathrm{g}]}$ & $I_{2}^{[\mathrm{h}]}$ \\
\hline 1 & -167.25 & -113.12 & 125.31 & 7.19 & 67.40 & -14.58 & 48.37 & -42.81 & 57.67 & 2.499 & 2.457 \\
\hline 27 & -166.95 & -102.10 & 137.49 & 15.03 & 45.74 & 64.29 & 44.20 & -38.28 & 56.14 & 2.462 & 2.329 \\
\hline 31 & -168.82 & -116.43 & 121.67 & 4.52 & 72.65 & -20.85 & 44.63 & -49.23 & 61.14 & 2.413 & 2.588 \\
\hline 33 & -168.25 & -115.82 & 122.09 & 5.01 & 75.97 & -27.82 & 39.88 & -51.04 & 61.88 & 2.350 & 2.723 \\
\hline 50 & -179.25 & -132.46 & 107.33 & -10.43 & -67.40 & -42.65 & -44.02 & -41.33 & 63.29 & 2.425 & 2.370 \\
\hline 52 & -168.41 & 151.23 & 29.02 & -89.19 & 70.95 & 42.35 & 33.08 & -38.36 & 65.61 & 2.356 & 2.544 \\
\hline 56 & -165.32 & 149.38 & 27.02 & -90.73 & 71.20 & 41.16 & 36.72 & -39.79 & 67.46 & 2.356 & 2.535 \\
\hline
\end{tabular}

[a] Conformers are numbered according to their appearance during conformational search; $[\mathrm{b}]-\alpha=\mathrm{C}_{\mathrm{Tr}}-\mathrm{C}(=\mathrm{O})-\mathrm{O}-\mathrm{C}^{*} ;[\mathrm{c}]-\beta=\mathrm{O}=\mathrm{C}-\mathrm{C}-\mathrm{C}_{\text {ipso }} ;[\mathrm{d}]-\gamma=(\mathrm{O}=) \mathrm{C}-\mathrm{C}_{\mathrm{Tr}}-\mathrm{C}_{\text {ipso }}-\mathrm{C}_{\text {ortho }}$ (of the two possibilities the absolute values $\leq 90^{\circ}$ has been chosen); $\left.[\mathrm{e}]-\delta=\mathrm{C}(=\mathrm{O})-\mathrm{O}-\mathrm{C}^{*}-\mathrm{H} ;[\mathrm{f}]-\zeta=\mathrm{O}-\mathrm{C}^{*}-\mathrm{C}-\mathrm{C}\left({ }^{*}\right) ;[\mathrm{g}]-\mathrm{I}_{1}=\mathrm{C}=\mathrm{O} \cdots \mathrm{HC} \mathrm{C}_{\text {ortho; }} ; \mathrm{h}\right]-\mathrm{I}_{2}=$ $(\mathrm{O}=) \mathrm{CO} \cdots \mathrm{HC}_{\text {ortho. }}$ 
Table SI_38. Dihedral angles $\alpha, b, y, \delta, \zeta$ and $\varphi$ (in degrees) and selected interatomic distances $I_{1}, I_{2}$ and $I_{3}$ (in $\AA$ ) calculated at the B3LYP/6-311++G(d,p) level for individual low-energy conformers of 14.

\begin{tabular}{|c|c|c|c|c|c|c|c|c|c|c|c|c|c|c|c|c|c|}
\hline $\begin{array}{c}\text { Conf. } \\
\text { no }^{[a]}\end{array}$ & $\alpha^{[\mathrm{b}]}$ & $B_{1}^{[c]}$ & $B_{2}{ }^{[c]}$ & $B_{3}{ }^{[c]}$ & $V_{1}{ }^{[\mathrm{d}]}$ & $V_{2}{ }^{[d]}$ & $\gamma_{3}{ }^{[d]}$ & $\delta^{[\mathrm{e}]}$ & $\zeta^{[f]}$ & $\varphi_{1}^{[\mathrm{g}]}$ & $\varphi_{2}{ }^{[\mathrm{g}]}$ & $\varphi_{3}{ }^{[\mathrm{g}]}$ & $\varphi_{4}^{[\mathrm{g}]}$ & $\varphi_{5}{ }^{[\mathrm{g}]}$ & $I_{1}^{[\mathrm{h}]}$ & $I_{2}^{[i]}$ & $I_{3}^{[j]}$ \\
\hline 1 & 176.4 & -122.0 & -4.5 & 115.7 & 6.5 & -51.8 & -63.6 & -11.9 & -155.3 & -21.2 & 34.0 & -34.1 & 24.4 & -2.1 & 2.560 & 2.443 & 2.441 \\
\hline 6 & -176.4 & -7.8 & -125.0 & 112.8 & -48.1 & 11.7 & -69.0 & 13.1 & -84.6 & 21.6 & -30.9 & 28.6 & -17.9 & -2.2 & 2.510 & 2.458 & 2.721 \\
\hline 8 & -175.8 & -29.4 & -150.9 & 88.5 & 57.8 & 49.4 & 34.6 & -23.5 & -155.3 & -21.2 & 34.3 & -34.4 & 24.8 & -2.4 & 2.430 & 2.392 & 2.661 \\
\hline 39 & -174.2 & 26.1 & -91.5 & 148.0 & 43.8 & 34.6 & 68.7 & 18.9 & -83.9 & 21.5 & -31.1 & 29.1 & -18.5 & -1.7 & 2.431 & 2.509 & \\
\hline
\end{tabular}

[a] Conformers are numbered according to their appearance during conformational search; $[\mathrm{b}]-\alpha=\mathrm{C}_{\mathrm{Tr}}-\mathrm{C}(=\mathrm{O})-\mathrm{O}-\mathrm{C}^{*} ;[\mathrm{c}]-\beta=\mathrm{O}=\mathrm{C}-\mathrm{C}-\mathrm{C}_{\text {ipso }} ;[\mathrm{d}]-\gamma=(\mathrm{O}=) \mathrm{C}-\mathrm{C}_{\mathrm{Tr}}-\mathrm{C}_{\text {ipso }}-\mathrm{C}_{\text {ortho }}$ (of the two possibilities the absolute values $\leq 90^{\circ}$ has been chosen); [e] $-\delta=\mathrm{C}(=\mathrm{O})-\mathrm{O}-\mathrm{C}^{*}-\mathrm{H}$; [f] $-\zeta=\mathrm{O}-\mathrm{C}^{*}-\mathrm{C}-\mathrm{C} ;[\mathrm{g}] \varphi_{1}=(\mathrm{O}=) \mathrm{C}-\mathrm{O}-\mathrm{C}-\mathrm{C} ; \varphi_{2}=\mathrm{O}-\mathrm{C}-\mathrm{C}-\mathrm{C}^{*} ; \varphi_{3}=\mathrm{C}-\mathrm{C}-$ $\mathrm{C}^{*}-\mathrm{C}(=\mathrm{O}) ; \varphi_{4}=\mathrm{C}-\mathrm{C}^{*}-\mathrm{C}(=\mathrm{O})-\mathrm{O} ; \varphi_{5}=\mathrm{C}^{*}-\mathrm{C}(=\mathrm{O})-\mathrm{O}-\mathrm{C} ;[\mathrm{h}]-\mathrm{I}_{1}=\mathrm{C}=\mathrm{O} \cdots \mathrm{HC}$ ortho; $[\mathrm{i}]-\mathrm{I}_{2}=(\mathrm{O}=) \mathrm{CO} \cdots \mathrm{HC}$ ortho; $[\mathrm{j}]-\mathrm{I}_{3}=\mathrm{C}=\mathrm{O}_{\text {lactone }} \cdots \mathrm{HC} \mathrm{C}_{\text {ortho }}$.

Table SI_39. Dihedral angles $\alpha, b, \gamma, \delta, \zeta$ and $\varphi$ (in degrees) and selected interatomic distances $I_{1}, I_{2}$ and $I_{3}$ (in $\AA$ ) calculated at the M06-2X/6-311++G(d,p) level for individual low-energy conformers of 14.

\begin{tabular}{|c|c|c|c|c|c|c|c|c|c|c|c|c|c|c|c|c|c|}
\hline $\begin{array}{l}\text { Conf. } \\
\text { no }^{[a]}\end{array}$ & $\alpha^{[\mathrm{b}]}$ & $B_{1}^{[c]}$ & $B_{2}^{[c]}$ & $B_{3}{ }^{[c]}$ & $V_{1}^{[d]}$ & $V_{2}{ }^{[d]}$ & $\gamma_{3}{ }^{[d]}$ & $\delta^{[\mathrm{e}]}$ & $\zeta^{[f]}$ & $\varphi_{1}^{[\mathrm{g}]}$ & $\varphi_{2}^{[\mathrm{g}]}$ & $\varphi_{3}^{[\mathrm{g}]}$ & $\varphi_{4}^{[\mathrm{g}]}$ & $\varphi_{5}^{[g]}$ & $I_{1}^{[\mathrm{h}]}$ & $I_{2}^{[i]}$ & $I_{3}^{[\mathrm{j}]}$ \\
\hline 1 & 171.5 & -6.7 & -124.9 & 113.9 & -49.9 & 10.8 & -67.4 & -18.0 & -157.1 & -21.7 & 36.2 & -36.9 & 27.0 & -3.4 & 2.521 & 2.433 & 2.522 \\
\hline 6 & 177.7 & -16.9 & -135.5 & 103.2 & -42.6 & 21.7 & -75.4 & 26.5 & -80.5 & 23.4 & -33.5 & 30.9 & -19.3 & -2.4 & 2.484 & 2.450 & \\
\hline 8 & -175.6 & -25.0 & 94.1 & -146.1 & 57.1 & 44.4 & 46.7 & -32.1 & -155.3 & -21.3 & 35.6 & -36.1 & 26.4 & -3.3 & 2.418 & 2.274 & 2.491 \\
\hline 27 & -179.3 & 17.6 & -102.6 & 135.8 & 41.0 & -24.9 & 77.2 & -163.1 & -110.2 & 25.5 & -23.8 & 14.6 & -0.5 & -15.6 & 2.466 & 2.507 & 2.737 \\
\hline 49 & 178.6 & 6.9 & 126.4 & -114.3 & -53.7 & -34.1 & -63.2 & -154.3 & -109.7 & 25.4 & -24.2 & 15.3 & -1.3 & -15.1 & 2.466 & 2.297 & 2.658 \\
\hline
\end{tabular}

[a] Conformers are numbered according to their appearance during conformational search; $[\mathrm{b}]-\alpha=\mathrm{C}_{\mathrm{Tr}}-\mathrm{C}(=\mathrm{O})-\mathrm{O}-\mathrm{C}^{*} ;[\mathrm{c}]-\beta=\mathrm{O}=\mathrm{C}-\mathrm{C}-\mathrm{C}_{\text {ipso; }} ;[\mathrm{d}]-\gamma=(\mathrm{O}=) \mathrm{C}-\mathrm{C}_{\mathrm{Tr}}-\mathrm{C}_{\text {ipso }}-\mathrm{C}_{\text {ortho }}$ (of the two possibilities the absolute values $\leq 90^{\circ}$ has been chosen); [e] $-\delta=\mathrm{C}(=\mathrm{O})-\mathrm{O}-\mathrm{C}^{*}-\mathrm{H} ;[\mathrm{f}]-\zeta=\mathrm{O}-\mathrm{C}^{*}-\mathrm{C}-\mathrm{C} ;[\mathrm{g}] \varphi_{1}=(\mathrm{O}=) \mathrm{C}-\mathrm{O}-\mathrm{C}-\mathrm{C} ; \varphi_{2}=\mathrm{O}-\mathrm{C}-\mathrm{C}-\mathrm{C}^{*} ; \varphi_{3}=\mathrm{C}-\mathrm{C}-$ $\mathrm{C}^{*}-\mathrm{C}(=\mathrm{O}) ; \varphi_{4}=\mathrm{C}-\mathrm{C}^{*}-\mathrm{C}(=\mathrm{O})-\mathrm{O} ; \varphi_{5}=\mathrm{C}^{*}-\mathrm{C}(=\mathrm{O})-\mathrm{O}-\mathrm{C} ;[\mathrm{h}]-\mathrm{I}_{1}=\mathrm{C}=\mathrm{O} \cdots \mathrm{HC} \mathrm{C}_{\text {ortho; }}[\mathrm{i}]-\mathrm{I}_{2}=(\mathrm{O}=) \mathrm{CO} \cdots \mathrm{HC}$ ortho; $[\mathrm{j}]-\mathrm{I}_{3}=\mathrm{C}=\mathrm{O}_{\text {lactone }} \cdots \mathrm{HC}_{\text {ortho. }}$. 
Table SI_40. Dihedral angles $\alpha, 6, \gamma, \delta, \zeta$ and $\varphi$ (in degrees) and selected interatomic distances $I_{1}, I_{2}$ and $I_{3}$ (in $\AA$ ) calculated at the B3LYP/6-311++G(d,p) level for individual low-energy conformers of 15.

\begin{tabular}{|c|c|c|c|c|c|c|c|c|c|c|c|c|c|c|c|c|c|}
\hline $\begin{array}{l}\text { Conf. } \\
\text { no }^{[a]}\end{array}$ & $\alpha^{[\mathrm{b}]}$ & $B_{1}{ }^{[c]}$ & $B_{2}{ }^{[c]}$ & $B_{3}^{[c]}$ & $\nu_{1}{ }^{[\mathrm{d}]}$ & $V_{2}{ }^{[d]}$ & $\gamma_{3}{ }^{[\mathrm{d}]}$ & $\delta^{[\mathrm{e}]}$ & $\zeta^{[f]}$ & $\varphi_{1}^{[\mathrm{g}]}$ & $\varphi_{2}{ }^{[\mathrm{g}]}$ & $\varphi_{3}{ }^{[\mathrm{g}]}$ & $\varphi_{4}{ }^{[\mathrm{g}]}$ & $\varphi_{5}{ }^{[\mathrm{g}]}$ & $I_{1}{ }^{[\mathrm{h}]}$ & $I_{2}^{[\mathrm{i}]}$ & $I_{3}^{[j]}$ \\
\hline 1 & -179.92 & -137.59 & 101.73 & -16.96 & 49.82 & 33.77 & 57.49 & 44.26 & -81.52 & -41.02 & -13.04 & -10.43 & 32.10 & -41.02 & 2.494 & 2.268 & 2.921 \\
\hline 17 & 174.00 & -148.55 & 89.43 & -30.36 & 41.83 & 83.11 & -24.15 & 43.04 & -109.75 & 5.15 & -25.35 & 37.58 & -35.91 & 19.23 & 2.326 & 2.875 & - \\
\hline 18 & -176.56 & -94.59 & 144.83 & 23.35 & -29.25 & -53.35 & -57.02 & 46.29 & -109.39 & 5.45 & -25.77 & 37.99 & -36.12 & 19.18 & 2.446 & 2.342 & - \\
\hline 20 & -173.68 & -109.67 & 128.05 & 10.39 & 67.85 & -14.02 & 47.64 & -36.35 & -110.51 & 6.62 & -26.43 & 37.90 & -35.30 & 17.88 & 2.528 & 2.453 & - \\
\hline 34 & 176.60 & -125.65 & 112.07 & -8.20 & 11.97 & -66.83 & -48.43 & 43.17 & -97.13 & 18.83 & 4.83 & -26.96 & 40.37 & -37.25 & 2.524 & 2.447 & - \\
\hline 47 & 170.32 & -127.30 & 110.43 & -9.56 & 10.95 & -65.74 & -49.57 & -37.75 & -113.50 & 4.83 & -25.30 & 37.83 & -36.36 & 19.70 & 2.561 & 2.437 & - \\
\hline 54 & -179.98 & -105.52 & 134.36 & 14.55 & -50.41 & -46.28 & -52.77 & -45.61 & -91.29 & 27.14 & -4.79 & -18.84 & 37.18 & -40.65 & 2.415 & 2.243 & 3.471 \\
\hline 65 & -177.47 & -102.38 & 137.18 & 16.92 & -42.51 & -48.02 & -55.78 & -38.38 & -115.58 & 2.81 & -23.64 & 37.07 & -36.92 & 21.31 & 2.452 & 2.255 & - \\
\hline 70 & -177.12 & 152.23 & 30.39 & -86.76 & 66.39 & 42.27 & 39.11 & -35.15 & -106.85 & 10.21 & -28.31 & 37.47 & -32.50 & 13.87 & 2.361 & 2.501 & - \\
\hline
\end{tabular}

[a] Conformers are numbered according to their appearance during conformational search; $[\mathrm{b}]-\alpha=\mathrm{C}_{\mathrm{Tr}}-\mathrm{C}(=\mathrm{O})-\mathrm{O}-\mathrm{C}^{*} ;[\mathrm{c}]-\mathrm{B}=\mathrm{O}=\mathrm{C}-\mathrm{C}-\mathrm{C}_{i p s o} ;[\mathrm{d}]-\gamma=(\mathrm{O}=) \mathrm{C}-\mathrm{C}_{\mathrm{Tr}}-\mathrm{C}_{i p s o}-\mathrm{C}_{\text {ortho }}$ (of the two possibilities the absolute values $\leq 90^{\circ}$ has been chosen); [e] $-\delta=\mathrm{C}(=\mathrm{O})-\mathrm{O}-\mathrm{C}^{*}-\mathrm{H} ;[\mathrm{f}]-\zeta=\mathrm{O}-\mathrm{C} *-\mathrm{C}-\mathrm{X} ;[\mathrm{g}] \varphi_{1}=\mathrm{X}-\mathrm{C} 2-\mathrm{C} 3^{*}-\mathrm{C} 4 ; \varphi_{2}=\mathrm{C} 2-\mathrm{C} 3^{*}-\mathrm{C} 4-\mathrm{C} 5 ; \varphi_{3}=$ $\mathrm{C}^{*}-\mathrm{C} 4-\mathrm{C} 5-\mathrm{X} ; \varphi_{4}=\mathrm{C} 4-\mathrm{C} 5-\mathrm{X}-\mathrm{C} 2 ; \varphi_{5}=\mathrm{C} 5-\mathrm{X}-\mathrm{C} 2-\mathrm{C} 3^{*} ;[\mathrm{h}]-\mathrm{I}_{1}=\mathrm{C}=\mathrm{O} \cdots \mathrm{HC}_{\text {ortho }}[\mathrm{i}]-\mathrm{I}_{2}=(\mathrm{O}=) \mathrm{CO} \cdots \mathrm{HC}_{\text {ortho; }}[\mathrm{j}]-\mathrm{I}_{3}=\mathrm{X} \cdots \mathrm{HC}$ ortho. 
Table SI_41. Dihedral angles $\alpha, b, \gamma, \delta, \zeta$ and $\varphi$ (in degrees) and selected interatomic distances $I_{1}, I_{2}$ and $I_{3}$ (in $\AA$ ) calculated at the M06-2X/6-311++G(d,p) level for individual low-energy conformers of 15.

\begin{tabular}{|c|c|c|c|c|c|c|c|c|c|c|c|c|c|c|c|c|c|}
\hline $\begin{array}{l}\text { Conf. } \\
\text { no }^{[\mathrm{a}]}\end{array}$ & $\alpha^{[\mathrm{b}]}$ & $B_{1}{ }^{[c]}$ & $B_{2}^{[c]}$ & $B_{3}{ }^{[c]}$ & $V_{1}{ }^{[\mathrm{d}]}$ & $V_{2}{ }^{[\mathrm{d}]}$ & $\gamma_{3}{ }^{[\mathrm{d}]}$ & $\delta^{[e]}$ & $\zeta^{[\mathrm{f}]}$ & $\varphi_{1}^{[\mathrm{g}]}$ & $\varphi_{2}{ }^{[g]}$ & $\varphi_{3}{ }^{[\mathrm{g}]}$ & $\varphi_{4}{ }^{[\mathrm{g}]}$ & $\varphi_{5}{ }^{[\mathrm{g}]}$ & $I_{1}^{[\mathrm{h}]}$ & $I_{2}^{[i]}$ & $I_{3}^{[j]}$ \\
\hline 1 & 172.52 & -147.69 & 92.43 & -26.06 & 50.29 & 36.49 & 58.28 & 49.39 & -76.08 & 35.51 & -37.39 & 27.36 & -5.47 & -18.99 & 2.435 & 2.306 & 2.498 \\
\hline 17 & 166.12 & -136.77 & 101.97 & -18.23 & 24.20 & -75.45 & -42.50 & 55.98 & -113.31 & 0.46 & -24.25 & 40.74 & -42.41 & 26.17 & 2.482 & 2.507 & - \\
\hline 18 & 172.57 & 170.77 & 146.93 & 26.04 & -38.92 & -46.04 & -59.44 & 54.00 & -76.80 & 34.33 & -38.59 & 30.67 & -9.70 & -15.63 & 2.464 & 2.308 & 2.906 \\
\hline 20 & -163.94 & -111.62 & 126.49 & 8.10 & 64.95 & -12.48 & 49.42 & -51.74 & -92.27 & 22.37 & -36.08 & 38.38 & -25.72 & 1.93 & 2.528 & 2.440 & - \\
\hline 34 & 163.50 & -157.86 & 81.72 & -38.17 & 51.41 & 69.42 & -10.79 & 47.45 & -78.80 & 34.35 & -14.21 & -10.12 & 32.99 & -42.54 & 2.253 & 2.548 & 2.837 \\
\hline 47 & 175.73 & -117.11 & 121.11 & 0.34 & 8.05 & -64.53 & -50.88 & -41.34 & -110.26 & 15.71 & -33.06 & 40.09 & -31.82 & 9.98 & 2.484 & 2.457 & - \\
\hline 58 & -165.64 & -110.14 & 140.96 & 20.14 & -56.09 & -44.94 & -52.00 & -55.70 & -78.02 & 37.67 & -20.83 & -2.19 & 26.76 & -40.84 & 2.364 & 2.236 & 2.754 \\
\hline 65 & -176.74 & -118.03 & 122.42 & 3.52 & -62.94 & -40.30 & -50.08 & -48.37 & -83.69 & 31.44 & -37.24 & 31.20 & -12.27 & -12.25 & 2.424 & 2.279 & 3.317 \\
\hline 70 & -166.35 & -100.56 & 138.94 & 16.33 & 46.87 & 62.46 & 44.92 & -48.91 & -83.26 & 30.69 & -38.37 & 33.82 & -15.55 & -9.69 & $\begin{array}{l}2.487 \\
2.511\end{array}$ & 2.306 & 3.440 \\
\hline 75 & -170.88 & 148.07 & 25.74 & -91.22 & 64.12 & 43.64 & 43.98 & -49.44 & -84.83 & 31.49 & -9.30 & -15.49 & 36.61 & -43.04 & $\begin{array}{l}2.384 \\
2.555\end{array}$ & 2.368 & 3.499 \\
\hline
\end{tabular}


Table SI_42. Dihedral angles $\alpha, b, y, \delta, \zeta$ and $\varphi$ (in degrees) and selected interatomic distances $I_{1}, I_{2}$ and $I_{3}$ (in $\AA$ ) calculated at the B3LYP/6-311G(d,p) level for individual low-energy conformers of $\mathbf{1 8}$.

\begin{tabular}{|c|c|c|c|c|c|c|c|c|c|c|c|c|c|}
\hline Conf. no ${ }^{[\mathrm{a}]}$ & $\alpha^{[\mathrm{b}]}$ & $B_{1}^{[c]}$ & $B_{2}^{[c]}$ & $b_{3}^{[c]}$ & $\gamma_{1}{ }^{[\mathrm{d}]}$ & $V_{2}{ }^{[d]}$ & $\gamma_{3}{ }^{[\mathrm{d}]}$ & $\delta^{[\mathrm{e}]}$ & $\zeta^{[f]}$ & $\varphi^{[\mathrm{g}]}$ & $l_{1}^{[\mathrm{h}]}$ & $I_{2}^{[i]}$ & $I_{3}^{[j]}$ \\
\hline 1 & 168.80 & -139.44 & 98.45 & -21.34 & 28.06 & -78.30 & -37.89 & -33.80 & 63.63 & -56.93 & 2.447 & 2.548 & 6.926 \\
\hline 5 & 177.31 & -141.83 & 98.31 & -21.27 & 45.64 & 51.92 & 52.01 & -8.33 & -74.71 & 167.19 & 2.353 & 2.291 & 6.430 \\
\hline 9 & 172.46 & -139.87 & 98.02 & -21.94 & 31.45 & -81.61 & -34.05 & 35.88 & 69.86 & -52.39 & 2.383 & 2.603 & 7.000 \\
\hline 13 & 174.98 & -138.11 & 101.80 & -17.94 & 45.79 & 50.58 & 52.94 & -32.27 & 65.73 & -55.09 & 2.383 & 2.264 & 6.924 \\
\hline 15 & 177.01 & -144.47 & 95.84 & -23.67 & 46.36 & 51.82 & 52.10 & -9.88 & -70.34 & 171.54 & 2.344 & 2.306 & 6.130 \\
\hline 17 & 178.24 & -94.12 & 145.47 & 24.31 & -35.84 & -50.29 & -56.24 & -31.66 & 64.74 & -55.92 & 2.411 & 2.324 & 6.841 \\
\hline 18 & 165.50 & -148.01 & 89.82 & -29.58 & 42.22 & 84.16 & -25.96 & -30.59 & 72.88 & -48.17 & 2.338 & $\begin{array}{l}2.753 \\
2.880\end{array}$ & 6.454 \\
\hline 26 & -169.47 & -96.21 & 141.89 & 23.63 & 79.98 & -28.96 & 36.44 & 33.46 & 70.98 & -51.57 & 2.438 & 2.540 & 6.930 \\
\hline 28 & 177.06 & -143.76 & 96.44 & -23.09 & 46.31 & 52.69 & 50.97 & 0.46 & -77.24 & 164.38 & 2.328 & 2.309 & 6.515 \\
\hline 32 & 173.77 & -42.78 & -164.58 & 74.50 & -42.78 & -67.69 & -37.10 & -34.84 & 68.75 & -51.76 & 2.233 & 2.639 & 6.147 \\
\hline 34 & 168.38 & -138.54 & 99.34 & -20.31 & 26.15 & -75.32 & -40.85 & -9.34 & -68.13 & 174.00 & 2.505 & 2.543 & 6.193 \\
\hline 35 & -175.71 & -83.61 & 154.60 & 35.99 & -75.94 & -48.00 & 13.91 & -29.92 & 66.45 & -54.37 & 2.248 & $\begin{array}{l}2.746 \\
2.769\end{array}$ & 6.948 \\
\hline 36 & 169.79 & -137.33 & 100.59 & -19.13 & 20.86 & -72.95 & -43.65 & 34.01 & -70.95 & 169.33 & 2.527 & 2.469 & 6.848 \\
\hline 42 & 171.32 & -140.43 & 99.59 & -19.95 & 46.38 & 53.02 & 51.59 & -29.84 & 75.13 & -45.87 & 2.348 & 2.285 & 6.553 \\
\hline 43 & 166.99 & -141.00 & 97.03 & -22.60 & 26.24 & -75.51 & -41.42 & -2.17 & -71.84 & 170.00 & 2.539 & 2.518 & 6.512 \\
\hline 45 & 177.77 & -96.92 & 142.89 & 22.26 & -43.83 & -49.54 & -53.40 & -32.83 & 66.41 & -54.64 & 2.370 & 2.291 & 7.053 \\
\hline 46 & 167.73 & -138.12 & 99.67 & -19.99 & 24.73 & -75.37 & -40.50 & -31.77 & 63.90 & -57.25 & 2.478 & 2.518 & 7.050 \\
\hline 48 & -178.34 & -104.11 & 136.02 & 16.10 & -55.77 & -42.67 & -52.17 & 32.13 & 69.91 & -52.46 & 2.380 & 2.289 & 6.970 \\
\hline 58 & -169.15 & -101.51 & 136.13 & 18.18 & 73.13 & -21.45 & 42.64 & 31.21 & 72.62 & -50.14 & 2.495 & 2.488 & 7.038 \\
\hline
\end{tabular}




\begin{tabular}{|c|c|c|c|c|c|c|c|c|c|c|c|c|c|}
\hline 59 & 165.70 & -145.43 & 92.75 & -26.82 & 30.83 & -79.08 & -38.62 & -17.25 & -93.32 & 149.16 & 2.528 & 2.561 & 6.080 \\
\hline 60 & 178.72 & 151.46 & 29.72 & -87.07 & 63.09 & 43.88 & 41.92 & 39.01 & -79.16 & -161.27 & $\begin{array}{l}2.379 \\
2.630\end{array}$ & 2.419 & 6.366 \\
\hline 61 & 174.32 & -149.93 & 90.35 & -28.36 & 48.46 & 46.75 & 53.98 & -24.25 & -94.94 & 147.68 & 2.356 & 2.339 & 6.082 \\
\hline 69 & 174.69 & -31.83 & -153.83 & 84.92 & -41.20 & -65.14 & -42.28 & 31.60 & -70.72 & 169.68 & $\begin{array}{l}2.334 \\
2.600\end{array}$ & 2.477 & 6.539 \\
\hline 70 & -179.12 & -133.86 & 165.63 & -14.00 & 44.94 & 44.78 & 55.48 & 38.20 & 71.54 & -51.70 & 2.455 & 2.224 & 7.088 \\
\hline 71 & 175.80 & -136.59 & 101.13 & -18.86 & 29.60 & -78.87 & -35.95 & 37.03 & -176.64 & 63.35 & 2.390 & 2.602 & 8.047 \\
\hline 73 & 169.68 & -142.03 & 96.02 & -23.50 & 28.27 & -77.83 & -38.19 & 28.69 & -74.36 & 166.25 & 2.475 & 2.538 & 6.750 \\
\hline 76 & 175.80 & -131.67 & 106.04 & -14.04 & 22.35 & -73.32 & -41.95 & 37.50 & -173.58 & 66.38 & 2.450 & 2.537 & 8.094 \\
\hline 77 & 173.20 & -30.87 & -152.98 & 85.72 & -41.35 & -62.98 & -43.48 & 37.37 & -72.40 & 167.88 & $\begin{array}{l}2.346 \\
2.583\end{array}$ & 2.433 & 6.801 \\
\hline 93 & -171.47 & -110.74 & 127.12 & 9.40 & 67.41 & -12.86 & 47.64 & 30.43 & -177.33 & 62.56 & 2.513 & 2.455 & 8.044 \\
\hline
\end{tabular}

[a] Conformers are numbered according to their appearance during conformational search; $[\mathrm{b}]-\alpha=\mathrm{C}_{\mathrm{Tr}}-\mathrm{C}(=\mathrm{O})-\mathrm{O}-\mathrm{C}^{*} ;[\mathrm{c}]-B=\mathrm{O}=\mathrm{C}-\mathrm{C}-\mathrm{C}_{i p s o} ;[\mathrm{d}]-\gamma=(\mathrm{O}=) \mathrm{C}-\mathrm{C}_{\mathrm{Tr}}-\mathrm{C}_{i p s o}-\mathrm{C}_{\text {ortho }}$ (of the two possibilities the absolute values $\leq 90^{\circ}$ has been chosen); $[\mathrm{e}]-\delta=\mathrm{C}(=\mathrm{O})-\mathrm{O}-\mathrm{C}^{*}-\mathrm{H} ;[\mathrm{f}]-\zeta=\mathrm{O}-\mathrm{C}^{*}-\mathrm{C}-\mathrm{O}[\mathrm{g}] \varphi=\mathrm{C} 3-\mathrm{C} 2-\mathrm{C} 1-\mathrm{O}$; $[\mathrm{h}]-\mathrm{I}_{1}=\mathrm{C}=\mathrm{O} \cdots \mathrm{HC}$ ortho; $[\mathrm{i}]-$ $\mathrm{I}_{2}=(\mathrm{O}=) \mathrm{CO} \cdots \mathrm{HC}_{\text {ortho }}[\mathrm{j}]-\mathrm{I}_{3}=\mathrm{C}_{\mathrm{Tr}} \cdots \mathrm{C}_{\mathrm{Tr}}$ 
Table SI_43. Dihedral angles $\alpha, b, y, \delta, \zeta$ and $\varphi$ (in degrees) and selected interatomic distances $I_{1}, I_{2}$ and $I_{3}$ (in $\AA$ ) calculated at the B3LYP-GD3Bj/6-311G(d,p) level for individual low-energy conformers of 18.

\begin{tabular}{|c|c|c|c|c|c|c|c|c|c|c|c|c|c|}
\hline Conf. no ${ }^{[a]}$ & $\alpha^{[\mathrm{b}]}$ & $B_{1}^{[c]}$ & $B_{2}{ }^{[c]}$ & $B_{3}{ }^{[c]}$ & $\gamma_{1}{ }^{[\mathrm{d}]}$ & $V_{2}{ }^{[\mathrm{d}]}$ & $V_{3}{ }^{[\mathrm{d}]}$ & $\delta^{[\mathrm{e}]}$ & $\zeta^{[f]}$ & $\varphi^{[\mathrm{g}]}$ & $l_{1}{ }^{[\mathrm{h}]}$ & $\mathrm{I}^{[\mathrm{i}]}$ & $I_{3}^{[j]}$ \\
\hline 13 & 178.87 & -124.44 & 115.79 & -5.43 & 41.24 & 55.96 & 52.18 & -31.03 & 58.27 & -60.96 & 2.449 & 2.193 & 6.373 \\
\hline 15 & -178.91 & -160.98 & 79.26 & -40.03 & 55.75 & 54.79 & 31.86 & -8.65 & -60.05 & -177.91 & $\begin{array}{l}2.108 \\
2.184\end{array}$ & $\begin{array}{l}2.459 \\
2.795\end{array}$ & 5.530 \\
\hline 17 & -176.49 & -88.55 & 152.19 & 30.43 & -42.43 & -43.43 & -57.50 & -33.15 & 60.12 & -59.23 & 2.405 & 2.313 & 6.402 \\
\hline 26 & -176.82 & -100.93 & 138.50 & 20.13 & -89.55 & -25.65 & 36.71 & 42.10 & 58.71 & -61.76 & 2.415 & $\begin{array}{l}2.469 \\
2.870\end{array}$ & 6.356 \\
\hline 32 & 173.34 & -45.27 & -166.80 & 71.79 & -32.61 & -69.44 & -41.80 & -35.66 & 59.20 & -59.40 & $\begin{array}{l}2.215 \\
2.597\end{array}$ & 2.666 & 5.484 \\
\hline 35 & -162.83 & -93.51 & 144.47 & 25.68 & -98.53 & -26.44 & 39.92 & -18.76 & 58.32 & -61.08 & 2.523 & 2.443 & 6.367 \\
\hline 36 & 164.14 & -134.70 & 103.66 & -16.27 & 24.78 & -74.78 & -42.03 & 39.67 & -64.04 & 177.03 & 2.478 & 3.080 & 6.309 \\
\hline 46 & 177.96 & -140.51 & 97.87 & -22.92 & 44.00 & 77.44 & -15.95 & -23.16 & 59.13 & -60.19 & 2.193 & $\begin{array}{l}2.597 \\
2.801\end{array}$ & 6.520 \\
\hline 60 & 177.51 & 169.98 & 49.43 & -67.90 & 61.82 & 43.77 & 42.90 & -1.30 & -73.62 & 168.68 & $\begin{array}{l}2.220 \\
2.634\end{array}$ & 2.546 & 5.473 \\
\hline 63 & -173.50 & 159.71 & 37.93 & -79.15 & 70.38 & 34.87 & 40.17 & 176.18 & -62.93 & 168.90 & $\begin{array}{l}2.266 \\
2.541\end{array}$ & 2.605 & 5.430 \\
\hline 77 & 173.13 & -115.82 & 123.65 & 4.58 & -47.16 & -40.86 & -55.62 & -21.92 & -58.47 & -176.21 & $\begin{array}{l}2.509 \\
2.389\end{array}$ & 2.160 & 5.482 \\
\hline
\end{tabular}


Table SI_44. Dihedral angles $\alpha, b, y, \delta, \zeta$ and $\varphi$ (in degrees) and selected interatomic distances $I_{1}, I_{2}$ and $I_{3}$ (in $\AA$ ) calculated at the M06-2X/6-311G(d,p) level for individual low-energy conformers of 18.

\begin{tabular}{|c|c|c|c|c|c|c|c|c|c|c|c|c|c|}
\hline Conf. no ${ }^{[a]}$ & $\alpha^{[\mathrm{b}]}$ & $B_{1}^{[c]}$ & $B_{2}^{[c]}$ & $B_{3}^{[c]}$ & $\gamma_{1}{ }^{[\mathrm{d}]}$ & $V_{2}{ }^{[\mathrm{d}]}$ & $V_{3}{ }^{[\mathrm{d}]}$ & $\delta^{[\mathrm{e}]}$ & $\zeta^{[f]}$ & $\varphi^{[\mathrm{g}]}$ & $l_{1}^{[\mathrm{h}]}$ & $I_{2}^{[i]}$ & $I_{3}^{[j]}$ \\
\hline 1 & 176.05 & -138.44 & 100.23 & -21.27 & 43.39 & 77.80 & -22.21 & -28.71 & 58.98 & -59.40 & 2.228 & $\begin{array}{l}2.556 \\
2.791\end{array}$ & 6.356 \\
\hline 5 & 179.11 & -130.46 & 109.77 & -11.03 & 40.51 & 61.20 & 51.34 & -7.25 & -66.20 & 176.51 & $\begin{array}{l}2.393 \\
2.528\end{array}$ & 2.270 & 6.002 \\
\hline 13 & 179.61 & -122.66 & 117.50 & -3.43 & 41.80 & 55.63 & 52.95 & -34.83 & 58.68 & -59.97 & $\begin{array}{l}2.459 \\
2.891\end{array}$ & 2.183 & 6.308 \\
\hline 15 & 175.98 & -137.06 & 103.19 & -17.42 & 45.54 & 63.40 & 41.42 & -24.92 & -62.12 & -179.13 & $\begin{array}{l}2.213 \\
2.265\end{array}$ & 2.330 & 5.475 \\
\hline 17 & -174.94 & -86.10 & 154.65 & 32.70 & -41.98 & -41.45 & -59.48 & -35.72 & 60.95 & -58.11 & 2.421 & 2.316 & 6.341 \\
\hline 18 & 163.44 & -143.15 & 95.41 & -24.96 & 49.94 & 74.94 & -17.08 & -20.87 & 69.11 & -49.71 & 2.212 & 2.512 & 6.001 \\
\hline 28 & 173.77 & -157.57 & 83.27 & -36.09 & 50.44 & 55.01 & 45.82 & 34.82 & -65.21 & 175.38 & 2.220 & $\begin{array}{l}2.380 \\
2.669\end{array}$ & 6.059 \\
\hline 32 & 174.99 & -34.56 & -156.64 & 82.10 & -33.22 & -75.95 & -39.40 & -42.85 & 62.67 & -56.12 & $\begin{array}{l}2.277 \\
2.479\end{array}$ & 2.712 & 5.493 \\
\hline 36 & 158.52 & -134.89 & 103.70 & -15.81 & 21.77 & -72.34 & -44.33 & 45.92 & -61.71 & 179.88 & $\begin{array}{l}2.507 \\
2.979\end{array}$ & 2.497 & 6.230 \\
\hline 42 & -175.12 & -113.95 & 125.80 & 3.81 & 42.42 & 55.80 & 51.09 & -33.01 & 69.16 & -48.44 & $\begin{array}{l}2.483 \\
2.709\end{array}$ & 2.179 & 6.077 \\
\hline 73 & 161.11 & -140.19 & 98.39 & -21.15 & 29.04 & -77.96 & -38.50 & 28.76 & -67.40 & 174.53 & 2.443 & 2.543 & 6.281 \\
\hline
\end{tabular}

[a] Conformers are numbered according to their appearance during conformational search; $[\mathrm{b}]-\alpha=\mathrm{C}_{\mathrm{Tr}}-\mathrm{C}(=\mathrm{O})-\mathrm{O}-\mathrm{C}^{*} ;[\mathrm{c}]-8=\mathrm{O}=\mathrm{C}-\mathrm{C}-\mathrm{C}_{i p s o} ;[\mathrm{d}]-\gamma=(\mathrm{O}=) \mathrm{C}-\mathrm{C}_{\mathrm{Tr}}-\mathrm{C}_{i p s o}-\mathrm{C}_{\text {ortho }}$ (of the two possibilities the absolute values $\leq 90^{\circ}$ has been chosen); [e] $-\delta=\mathrm{C}(=\mathrm{O})-\mathrm{O}-\mathrm{C}^{*}-\mathrm{H} ;[\mathrm{f}]-\zeta=\mathrm{O}-\mathrm{C}^{*}-\mathrm{C}-\mathrm{O}[\mathrm{g}] \varphi=\mathrm{C} 3-\mathrm{C} 2-\mathrm{C} 1-\mathrm{O} ;[\mathrm{h}]-\mathrm{I}_{1}=\mathrm{C}=\mathrm{O} \cdots \mathrm{HC}$ ortho; $[\mathrm{i}]-$ $\mathrm{I}_{2}=(\mathrm{O}=) \mathrm{CO} \cdots \mathrm{HC}_{\text {ortho }}[\mathrm{j}]-\mathrm{I}_{3}=\mathrm{C}_{\mathrm{Tr}} \cdots \mathrm{C}_{\mathrm{Tr}}$ 
Table SI_45. Dihedral angles $\alpha, 6, \gamma, \delta, \zeta$ and $\varphi$ (in degrees) and selected interatomic distances $I_{1}, I_{2}$ and $I_{3}$ (in $\AA$ ) calculated at the B3LYP/6-311G(d,p) level for individual low-energy conformers of 20.

\begin{tabular}{|c|c|c|c|c|c|c|c|c|c|c|c|c|c|c|}
\hline & & $\alpha^{[\mathrm{b}]}$ & $B_{1}^{[c]}$ & $B_{2}{ }^{[c]}$ & $B_{3}[c]$ & $V_{1}{ }^{[d]}$ & $\mathrm{V}_{2}{ }^{[\mathrm{d}]}$ & $\gamma_{3}{ }^{[d]}$ & $\delta^{[\mathrm{e}]}$ & $\zeta^{[f]}$ & $\varphi^{[\mathrm{g}]}$ & $l_{1}{ }^{[\mathrm{h}]}$ & $l_{2}^{[i]}$ & $l_{3}^{[j]}$ \\
\hline \multirow[t]{2}{*}{1} & $\mathrm{C} 2$ & 172.66 & -139.32 & 98.37 & -21.69 & 39.12 & 86.32 & -24.84 & 41.43 & \multirow[t]{2}{*}{67.18} & \multirow[t]{2}{*}{-178.46} & 2.284 & $\begin{array}{l}2.772 \\
2.872\end{array}$ & \multirow[t]{2}{*}{6.934} \\
\hline & C3 & -172.52 & -100.32 & 137.36 & 19.14 & 69.89 & -17.33 & 45.32 & 37.64 & & & 2.557 & 2.407 & \\
\hline \multirow{2}{*}{11} & $\mathrm{C} 2$ & -178.80 & -97.27 & 140.37 & 22.65 & 82.94 & -34.29 & 31.76 & -1.66 & \multirow{2}{*}{65.15} & \multirow{2}{*}{-177.47} & 2.350 & 2.644 & \multirow{2}{*}{6.955} \\
\hline & C3 & -178.80 & -97.27 & 140.37 & 22.65 & 82.94 & -34.29 & 31.76 & -1.66 & & & 2.350 & 2.644 & \\
\hline \multirow[t]{2}{*}{14} & $\mathrm{C} 2$ & 166.25 & -153.58 & 64.94 & -34.55 & 44.27 & 79.40 & -19.31 & -9.89 & \multirow[t]{2}{*}{61.72} & \multirow[t]{2}{*}{179.80} & 2.293 & $\begin{array}{l}2.698 \\
2.814\end{array}$ & \multirow[t]{2}{*}{6.948} \\
\hline & $\mathrm{C} 3$ & -178.24 & -99.81 & 138.21 & 20.47 & 81.23 & -31.33 & 33.94 & 0.74 & & & 2.366 & 2.615 & \\
\hline \multirow{3}{*}{16} & $\mathrm{C} 2$ & 169.98 & -148.55 & 89.33 & -30.34 & 46.63 & 79.11 & -19.09 & 36.03 & \multirow{3}{*}{70.16} & \multirow{3}{*}{-175.73} & 2.255 & $\begin{array}{l}2.760 \\
2.824\end{array}$ & \multirow{3}{*}{7.108} \\
\hline & & & & & & & & & & & & & & \\
\hline & C3 & 169.98 & -148.55 & 89.33 & -30.34 & 46.63 & 79.11 & -19.09 & 36.03 & & & 2.255 & $\begin{array}{l}2.760 \\
2.824\end{array}$ & \\
\hline \multirow{2}{*}{17} & $\mathrm{C} 2$ & 174.76 & -107.48 & 132.16 & 12.53 & -46.90 & -45.80 & -54.42 & -7.85 & \multirow{2}{*}{61.60} & \multirow{2}{*}{179.23} & 2.441 & 2.220 & \multirow{2}{*}{6.881} \\
\hline & $\mathrm{C} 3$ & -177.44 & -95.71 & 141.76 & 24.14 & 85.47 & -37.76 & 28.46 & -4.04 & & & 2.317 & 2.698 & \\
\hline \multirow{2}{*}{20} & $\mathrm{C} 2$ & 170.41 & -152.00 & 88.83 & -30.40 & 47.71 & 53.31 & 50.70 & -11.41 & \multirow{2}{*}{62.24} & \multirow{2}{*}{179.73} & 2.287 & 2.372 & \multirow{2}{*}{6.957} \\
\hline & C3 & -178.35 & -97.41 & 140.49 & 22.69 & 84.39 & -34.62 & 30.69 & -2.53 & & & 2.337 & 2.651 & \\
\hline \multirow{2}{*}{21} & $\mathrm{C} 2$ & 177.06 & -116.52 & 123.38 & 4.53 & -62.24 & -39.51 & -50.26 & 35.99 & \multirow{2}{*}{66.81} & \multirow{2}{*}{-179.59} & 2.417 & 2.339 & \multirow{2}{*}{7.012} \\
\hline & C3 & 174.52 & -133.34 & 104.15 & -16.00 & 32.11 & -80.78 & -34.10 & 40.20 & & & 2.333 & 2.707 & \\
\hline \multirow[b]{2}{*}{22} & $\mathrm{C} 2$ & -173.38 & -105.17 & 132.07 & 14.44 & 68.78 & -17.59 & 45.56 & 32.82 & \multirow[b]{2}{*}{66.43} & \multirow[b]{2}{*}{-178.84} & 2.515 & 2.464 & \\
\hline & C3 & -178.67 & -112.85 & 126.24 & 4.71 & 45.70 & 58.75 & 47.28 & 36.45 & & & $\begin{array}{l}2.437 \\
2.762\end{array}$ & 2.264 & 7.012 \\
\hline 23 & $\mathrm{C} 2$ & 172.20 & -27.29 & -149.55 & 89.12 & -38.67 & -64.05 & -44.78 & 39.37 & -71.03 & 50.24 & $\begin{array}{l}2.395 \\
2.489\end{array}$ & 2.424 & 6.535 \\
\hline & C3 & 165.09 & -142.78 & 95.18 & -24.27 & 29.53 & -78.32 & -37.90 & 34.16 & & & 2.472 & 2.569 & \\
\hline 25 & $\mathrm{C} 2$ & 171.23 & -153.21 & 87.49 & -31.57 & 49.79 & 55.71 & 47.32 & 25.88 & -71.67 & 49.17 & 2.240 & $\begin{array}{l}2.403 \\
2.806\end{array}$ & 6.344 \\
\hline
\end{tabular}




\begin{tabular}{|c|c|c|c|c|c|c|c|c|c|c|c|c|c|c|}
\hline & C3 & 173.37 & -140.53 & 98.19 & -18.32 & -62.93 & -46.15 & -39.89 & 41.08 & & & $\begin{array}{l}2.420 \\
2.526\end{array}$ & 2.358 & \\
\hline \multirow[b]{2}{*}{43} & $\mathrm{C} 2$ & 161.60 & -142.83 & 95.61 & -23.76 & 23.89 & -74.70 & -42.24 & -0.27 & \multirow[b]{2}{*}{-75.86} & \multirow[b]{2}{*}{47.05} & 2.571 & 2.466 & \multirow[b]{2}{*}{6.263} \\
\hline & C3 & 175.78 & 152.54 & 30.94 & -85.74 & 62.97 & 43.10 & 43.37 & 39.20 & & & $\begin{array}{l}2.369 \\
2.629\end{array}$ & 2.426 & \\
\hline \multirow[b]{2}{*}{46} & $\mathrm{C} 2$ & 166.27 & -140.24 & 97.74 & -21.89 & 26.81 & -76.09 & -40.65 & -2.69 & \multirow[b]{2}{*}{-68.30} & \multirow[b]{2}{*}{46.26} & 2.513 & 2.539 & \multirow[b]{2}{*}{6.087} \\
\hline & C3 & -171.41 & 161.65 & 39.71 & -77.16 & 64.83 & 39.77 & 42.54 & 175.19 & & & $\begin{array}{l}2.259 \\
2.674\end{array}$ & 2.534 & \\
\hline \multirow{2}{*}{47} & $\mathrm{C} 2$ & 171.77 & -28.88 & -152.14 & 86.80 & -43.39 & -63.61 & -42.08 & 29.21 & \multirow{2}{*}{-71.18} & \multirow{2}{*}{50.58} & $\begin{array}{l}2.351 \\
2.629\end{array}$ & 2.429 & \multirow{2}{*}{6.241} \\
\hline & C3 & 171.77 & -28.89 & -152.15 & 86.79 & -43.39 & -63.61 & -42.09 & 29.21 & & & $\begin{array}{l}2.351 \\
2.629\end{array}$ & 2.429 & \\
\hline
\end{tabular}

[a] Conformers are numbered according to their appearance during conformational search; $[\mathrm{b}]-\alpha=\mathrm{C}_{\mathrm{Tr}}-\mathrm{C}(=\mathrm{O})-\mathrm{O}-\mathrm{C}^{*} ;[\mathrm{c}]-8=\mathrm{O}=\mathrm{C}-\mathrm{C}-\mathrm{C}_{i p s o} ;[\mathrm{d}]-\gamma=(\mathrm{O}=) \mathrm{C}-\mathrm{C}_{\mathrm{Tr}}-\mathrm{C}_{i p s o}-\mathrm{C}_{\text {ortho }}$ (of the two possibilities the absolute values $\leq 90^{\circ}$ has been chosen); [e] $-\delta=\mathrm{C}(=\mathrm{O})-\mathrm{O}-\mathrm{C}^{*}-\mathrm{H}$; $[\mathrm{f}]-\zeta=\mathrm{O}-\mathrm{C}^{*}-\mathrm{C}^{*}-\mathrm{O}$; [g] $\varphi=\mathrm{C} 1-\mathrm{C} 2-\mathrm{C} 3-\mathrm{C} 4 ;[\mathrm{h}]-\mathrm{I}_{1}=\mathrm{C}=\mathrm{O} \cdots \mathrm{HC}$ ortho; [i] $-\mathrm{I}_{2}=(\mathrm{O}=) \mathrm{CO} \cdots \mathrm{HC}$ ortho; $[\mathrm{j}]-\mathrm{I}_{3}=\mathrm{C}_{\mathrm{Tr}} \cdots \mathrm{C}_{\mathrm{Tr}}$ 
Table SI_46. Dihedral angles $\alpha, 6, \gamma, \delta, \zeta$ and $\varphi$ (in degrees) and selected interatomic distances $I_{1}, I_{2}$ and $I_{3}$ (in $\AA$ ) calculated at the B3LYP-GD3BJ/6-311G(d,p) level for individual low-energy conformers of 20.

\begin{tabular}{|c|c|c|c|c|c|c|c|c|c|c|c|c|c|c|}
\hline \multicolumn{2}{|c|}{ Conf. no ${ }^{[a]}$} & \multirow{2}{*}{$\begin{array}{c}\alpha^{[b]} \\
167.77\end{array}$} & \multirow{2}{*}{$\begin{array}{c}B_{1}{ }^{[c]} \\
-131.03\end{array}$} & \multirow{2}{*}{$\begin{array}{c}b_{2}^{[c]} \\
106.50\end{array}$} & \multirow{2}{*}{$\begin{array}{c}B_{3}{ }^{[c]} \\
-13.82\end{array}$} & \multirow{2}{*}{$\frac{v_{1}{ }^{[d]}}{20.51}$} & \multirow{2}{*}{$\begin{array}{c}\gamma_{2}{ }^{[\mathrm{d}]} \\
-69.73\end{array}$} & \multirow{2}{*}{$\begin{array}{c}\gamma_{3}{ }^{[\mathrm{d}]} \\
-44.34\end{array}$} & \multirow{2}{*}{$\begin{array}{c}\delta^{[\mathrm{e}]} \\
41.72\end{array}$} & \multirow{3}{*}{$\begin{array}{c}\zeta^{[f]} \\
58.94\end{array}$} & \multirow{3}{*}{$\begin{array}{c}\varphi^{[\mathrm{g}]} \\
178.09\end{array}$} & \multirow{3}{*}{$\begin{array}{c}l_{1}^{[\mathrm{h}]} \\
2.484 \\
2.462\end{array}$} & \multirow{2}{*}{$\begin{array}{c}I_{2}^{[i]} \\
2.504\end{array}$} & \multirow{3}{*}{$\begin{array}{c}l_{3}^{[j]} \\
6.365\end{array}$} \\
\hline 1 & $\mathrm{C} 2$ & & & & & & & & & & & & & \\
\hline & C3 & -177.17 & -102.28 & 136.67 & 18.30 & 76.83 & -22.97 & 40.18 & 42.18 & & & & 2.447 & \\
\hline \multirow{2}{*}{11} & C2 & -174.72 & -101.67 & 136.82 & 18.80 & 80.19 & -30.21 & 35.49 & -12.38 & \multirow{2}{*}{61.63} & \multirow{2}{*}{-176.83} & 2.383 & 2.580 & \multirow{2}{*}{6.503} \\
\hline & C3 & -174.72 & -101.67 & 136.82 & 18.80 & 80.19 & -30.21 & 35.49 & -12.38 & & & 2.383 & 2.580 & \\
\hline \multirow{2}{*}{14} & $\mathrm{C} 2$ & 168.41 & -151.17 & 88.12 & -32.22 & 45.92 & 74.84 & -16.34 & -6.42 & \multirow{2}{*}{55.83} & \multirow{2}{*}{177.72} & 2.243 & $2.595 ; 2.731$ & \multirow{2}{*}{6.439} \\
\hline & C3 & -171.61 & -98.17 & 139.92 & 21.91 & 78.12 & -27.78 & 38.24 & -8.60 & & & 2.451 & 2.507 & \\
\hline \multirow{2}{*}{17} & C2 & -172.80 & -101.13 & 136.73 & 18.88 & 78.66 & -31.11 & 35.97 & -10.47 & \multirow{2}{*}{58.79} & \multirow{2}{*}{179.74} & 2.394 & 2.604 & \multirow{2}{*}{6.489} \\
\hline & C3 & -179.49 & -103.68 & 136.98 & 16.61 & -49.48 & -38.71 & -56.81 & -16.16 & & & 2.467 & $2.185 ; 2.697$ & \\
\hline \multirow{2}{*}{20} & $\mathrm{C} 2$ & 170.98 & -143.30 & 97.91 & -22.22 & 43.58 & 55.81 & 52.07 & -9.17 & \multirow{2}{*}{54.83} & \multirow{2}{*}{175.89} & 2.349 & $2.261 ; 2.750$ & \multirow{2}{*}{6.422} \\
\hline & C3 & -171.05 & -98.52 & 139.65 & 21.58 & 78.94 & -28.17 & 37.85 & -10.24 & & & 2.441 & 2.520 & \\
\hline \multirow{2}{*}{21} & $\mathrm{C} 2$ & 174.84 & -106.96 & 134.38 & 14.18 & -63.58 & -36.50 & -51.28 & 43.03 & ז0 & גרס & 2.378 & $2.335 ; 2.681$ & $6 \cos ^{\circ}$ \\
\hline & C3 & 169.53 & -130.21 & 107.63 & -12.68 & 23.45 & -73.59 & -42.14 & 42.53 & נ5.50 & 171.00 & 2.441 & 2.562 & 0.444 \\
\hline 2 & $\mathrm{C} 2$ & -171.10 & -109.69 & 128.49 & 10.09 & 63.31 & -8.50 & 50.52 & 37.46 & & & 2.582 & 2.356 & \\
\hline & C3 & -177.19 & -125.47 & 114.77 & -6.57 & 49.08 & 52.55 & 51.03 & 42.72 & 03.12 & $-1 / 0.02$ & 2.424 & 2.190 & 0.301 \\
\hline & $\mathrm{C} 2$ & 172.25 & -153.09 & 87.55 & -31.83 & 52.23 & 56.65 & 43.49 & 18.21 & & & 2.203 & $2.365 ; 2.812$ & \\
\hline 25 & C3 & 174.59 & -135.69 & 103.46 & -13.53 & -63.76 & -45.19 & -39.77 & 38.51 & -67.77 & 54.25 & $\begin{array}{l}2.360 \\
2.580\end{array}$ & 2.321 & 5.962 \\
\hline & & & & & & & & & & & & 2.661 & & \\
\hline & C2 & 163.47 & -125.42 & 113.81 & -4.84 & -28.36 & -51.38 & -58.12 & -5.82 & & & 2.784 & 2.149 & \\
\hline 43 & & & & & & & & & & -68.94 & 56.19 & 2.256 & & 5.427 \\
\hline & C3 & 177.06 & 170.74 & 50.25 & -67.14 & 62.12 & 43.48 & 42.32 & -2.97 & & & $\begin{array}{l}2.213 \\
2.571\end{array}$ & 2.565 & \\
\hline
\end{tabular}

[a] Conformers are numbered according to their appearance during conformational search; $[\mathrm{b}]-\alpha=\mathrm{C}_{\mathrm{Tr}}-\mathrm{C}(=\mathrm{O})-\mathrm{O}-\mathrm{C}^{*} ;[\mathrm{c}]-\mathrm{B}=\mathrm{O}=\mathrm{C}-\mathrm{C}-\mathrm{C}_{i p s o} ;[\mathrm{d}]-\gamma=(\mathrm{O}=) \mathrm{C}-\mathrm{C}_{\mathrm{Tr}}-\mathrm{C}_{i p s o}-\mathrm{C}_{\text {ortho }}$ (of the two possibilities the absolute values $\leq 90^{\circ}$ has been chosen); [e] $-\delta=\mathrm{C}(=\mathrm{O})-\mathrm{O}-\mathrm{C}^{*}-\mathrm{H} ;[\mathrm{f}]-\zeta=\mathrm{O}-\mathrm{C}^{*}-\mathrm{C}-\mathrm{C}(*)$; $[\mathrm{g}] \varphi=\mathrm{C} 1-\mathrm{C} 2-\mathrm{C} 3-\mathrm{C} 4$; $[\mathrm{h}]-\mathrm{I}_{1}=\mathrm{C}=\mathrm{O} \cdots \mathrm{HC}$ ortho; $[\mathrm{i}]-\mathrm{I}_{2}=(\mathrm{O}=) \mathrm{CO} \cdots \mathrm{HC}_{\text {ortho }} ;[\mathrm{j}]-\mathrm{I}_{3}=\mathrm{C}_{\mathrm{Tr}} \cdots \mathrm{C}_{\mathrm{Tr}}$ 
Table SI_47. Dihedral angles $\alpha, b, y, \delta, \zeta$ and $\varphi$ (in degrees) and selected interatomic distances $I_{1}, I_{2}$ and $I_{3}$ (in $\AA$ ) calculated at the M06-2X/6-311G(d,p) level for individual low-energy conformers of 20.

\begin{tabular}{|c|c|c|c|c|c|c|c|c|c|c|c|c|c|c|}
\hline & & $\alpha^{[\mathrm{b}]}$ & $B_{1}[c]$ & $B_{2}^{[c]}$ & $B_{3}[c]$ & $\gamma_{1}{ }^{[d]}$ & $\mathrm{V}_{2}{ }^{[\mathrm{d}]}$ & $\gamma_{3}{ }^{[d]}$ & $\delta^{[\mathrm{e}]}$ & $\zeta^{[f]}$ & $\varphi^{[\mathrm{g}]}$ & $l_{1}[\mathrm{~h}]$ & $I_{2}^{[i]}$ & $l_{3}^{[j]}$ \\
\hline \multirow{2}{*}{1} & $\mathrm{C} 2$ & 164.40 & -134.61 & 102.81 & -16.60 & 23.57 & -72.01 & -42.50 & 45.86 & \multirow{2}{*}{61.12} & \multirow{2}{*}{-178.64} & 2.469 & 2.488 & \multirow{2}{*}{6.342} \\
\hline & $\mathrm{C} 3$ & 179.84 & -102.44 & 136.56 & 18.45 & 82.10 & -28.29 & 35.98 & 45.92 & & & 2.396 & 2.508 & \\
\hline \multirow{2}{*}{11} & $\mathrm{C} 2$ & -174.82 & -101.63 & 137.59 & 19.06 & 80.13 & -28.05 & 38.08 & -15.21 & \multirow{2}{*}{63.24} & \multirow{2}{*}{173.78} & 2.413 & 2.525 & \multirow{2}{*}{6.499} \\
\hline & $\mathrm{C} 3$ & -174.82 & -101.63 & 137.59 & 19.06 & 80.13 & -28.05 & 38.08 & -15.21 & & & 2.413 & 2.525 & \\
\hline \multirow{2}{*}{14} & $\mathrm{C} 2$ & 167.32 & -148.32 & 90.93 & -29.56 & 45.71 & 75.15 & -18.66 & -11.98 & \multirow{2}{*}{55.61} & \multirow{2}{*}{178.88} & 2.263 & $2.547 ; 2.745$ & \multirow{2}{*}{6.364} \\
\hline & C3 & -171.39 & -93.72 & 144.20 & 25.88 & 79.26 & -30.31 & 36.51 & -13.37 & & & 2.446 & 2.479 & \\
\hline \multirow{2}{*}{17} & $\mathrm{C} 2$ & -173.09 & -98.59 & 139.39 & 21.14 & 78.50 & -31.72 & 36.28 & -15.23 & \multirow{2}{*}{58.98} & \multirow{2}{*}{-178.80} & 2.407 & 2.564 & \multirow{2}{*}{6.426} \\
\hline & $\mathrm{C} 3$ & -178.91 & -101.16 & 139.66 & 19.08 & -49.32 & -35.59 & -58.40 & -19.89 & & & 2.474 & $2.185 ; 2.569$ & \\
\hline \multirow{2}{*}{20} & $\mathrm{C} 2$ & 169.39 & -140.34 & 100.69 & -19.96 & 44.62 & 57.84 & 51.21 & -14.86 & \multirow{2}{*}{54.26} & \multirow{2}{*}{176.46} & 2.349 & 2.249 & \multirow{2}{*}{6.341} \\
\hline & $\mathrm{C} 3$ & -170.42 & -94.09 & 143.96 & 25.64 & 80.54 & -31.07 & 36.13 & -14.05 & & & 2.437 & 2.506 & \\
\hline \multirow{2}{*}{21} & $\mathrm{C} 2$ & 173.57 & -109.88 & 131.50 & 11.69 & -65.50 & -35.40 & -51.55 & 48.16 & \multirow{2}{*}{62.25} & \multirow{2}{*}{-178.77} & 2.419 & 2.546 & \multirow{2}{*}{6.421} \\
\hline & $\mathrm{C} 3$ & 167.00 & -134.16 & 103.95 & -16.31 & 26.32 & -77.11 & -39.86 & 46.44 & & & 2.419 & 2.546 & \\
\hline \multirow{2}{*}{22} & $\mathrm{C} 2$ & -173.90 & -120.41 & 119.99 & -2.12 & 48.65 & 54.45 & 50.55 & 42.87 & \multirow{2}{*}{63.95} & \multirow{2}{*}{-176.54} & 2.436 & 2.181 & \multirow{2}{*}{6.570} \\
\hline & $\mathrm{C} 3$ & -171.48 & -116.81 & 121.33 & 3.18 & 60.69 & -4.82 & 52.17 & 36.97 & & & 2.533 & 2.369 & \\
\hline \multirow{2}{*}{23} & $\mathrm{C} 2$ & 156.10 & -138.32 & 100.54 & -19.08 & 24.16 & -74.25 & -44.02 & 35.86 & \multirow{2}{*}{-66.33} & \multirow{2}{*}{56.45} & 2.526 & 2.513 & \multirow{2}{*}{6.208} \\
\hline & $\mathrm{C} 3$ & 178.43 & -21.22 & -142.85 & 96.13 & -43.86 & -60.90 & -44.94 & 34.60 & & & $2.452 ; 2.476$ & 2.294 & \\
\hline 25 & $\mathrm{C} 2$ & 171.62 & -155.62 & 85.11 & -34.32 & 52.33 & 56.83 & 43.77 & 21.84 & 1 & 5007 & 2.202 & $2.376 ; 2.746$ & \\
\hline & $\mathrm{C} 3$ & 174.96 & -138.36 & 101.05 & -16.15 & -64.82 & -44.40 & -39.43 & 42.08 & & & $2.363 ; 2.522$ & 2.334 & \\
\hline
\end{tabular}

[a] Conformers are numbered according to their appearance during conformational search; $[\mathrm{b}]-\alpha=\mathrm{C}_{\mathrm{Tr}}-\mathrm{C}(=\mathrm{O})-\mathrm{O}-\mathrm{C}^{*} ;[\mathrm{c}]-B=\mathrm{O}=\mathrm{C}-\mathrm{C}-\mathrm{C}_{i p s o} ;[\mathrm{d}]-\gamma=(\mathrm{O}=) \mathrm{C}-\mathrm{C}_{\mathrm{Tr}}-\mathrm{C}_{i p s o}-\mathrm{C}_{\text {ortho }}$ (of the two possibilities the absolute values $\leq 90^{\circ}$ has been chosen); [e] $-\delta=\mathrm{C}(=\mathrm{O})-\mathrm{O}-\mathrm{C}^{*}-\mathrm{H}$; [f] $-\zeta=\mathrm{O}-\mathrm{C}^{*}-\mathrm{C}-\mathrm{C}\left({ }^{*}\right)$; [g] $\varphi=\mathrm{C} 1-\mathrm{C} 2-\mathrm{C} 3-\mathrm{C} 4 ;[\mathrm{h}]-\mathrm{I}_{1}=\mathrm{C}=\mathrm{O} \cdots \mathrm{HC}$ ortho; $\left.[\mathrm{i}]-\mathrm{I}_{2}=(\mathrm{O}=) \mathrm{CO} \cdots \mathrm{HC}_{\text {ortho }} ; \mathrm{j}\right]-\mathrm{I}_{3}=\mathrm{C}_{\mathrm{Tr}} \cdots \mathrm{C}_{\mathrm{Tr}}$ 
Table SI_48. Dihedral angles $\alpha, 6, \gamma, \delta, \zeta$ and $\varphi$ (in degrees) and selected interatomic distances $I_{1}, I_{2}$ and $I_{3}$ (in $\AA$ ) calculated at the B3LYP/6-311G(d,p) level for individual low-energy conformers of $\mathbf{2 1 .}$

\begin{tabular}{|c|c|c|c|c|c|c|c|c|c|c|c|c|c|c|c|c|}
\hline \multicolumn{2}{|c|}{ Conf. no ${ }^{[a]}$} & $\alpha^{[\mathrm{b}]}$ & $B_{1}{ }^{[c]}$ & $B_{2}{ }^{[c]}$ & $B_{3}{ }^{[c]}$ & $V_{1}{ }^{[d]}$ & $V_{2}{ }^{[\mathrm{d}]}$ & $\gamma_{3}{ }^{[\mathrm{d}]}$ & $\delta^{[\mathrm{e}]}$ & $\zeta^{[f]}$ & $\varphi_{1}{ }^{[\mathrm{g}]}$ & $\varphi_{2}{ }^{[\mathrm{g}]}$ & $l_{1}^{[\mathrm{h}]}$ & $I_{2}^{[i]}$ & $I_{3}^{[j]}$ & $I_{4}^{[k]}$ \\
\hline \multirow{2}{*}{1} & $\mathrm{C} 2$ & 178.46 & -43.61 & -165.07 & 73.95 & -45.46 & -65.44 & -36.42 & 25.79 & -63.28 & \multirow{2}{*}{177.29} & \multirow{2}{*}{173.74} & 2.229 & 2.599 & \multirow{2}{*}{6.582} & 2.675 \\
\hline & C4 & 175.68 & -137.54 & 102.24 & -17.23 & 47.75 & 49.52 & 53.20 & 29.92 & -66.28 & & & 2.386 & 2.264 & & 2.681 \\
\hline \multirow{2}{*}{6} & $\mathrm{C} 2$ & 173.60 & -133.68 & 103.87 & -16.12 & 26.50 & -75.40 & -40.91 & 43.22 & -63.16 & \multirow{2}{*}{176.63} & \multirow{2}{*}{172.26} & 2.458 & 2.603 & \multirow{2}{*}{6.971} & \\
\hline & $\mathrm{C} 4$ & -177.87 & -80.84 & 157.12 & 38.60 & -75.03 & -49.47 & 11.53 & 36.67 & -68.01 & & & 2.242 & $2.740 ; 2.814$ & & \\
\hline \multirow{2}{*}{12} & $\mathrm{C} 2$ & -178.30 & -118.33 & 119.54 & 2.54 & 67.73 & -11.15 & 48.17 & -7.43 & -66.36 & \multirow{2}{*}{175.50} & \multirow{2}{*}{175.07} & 2.457 & 2.502 & \multirow{2}{*}{6.521} & 2.427 \\
\hline & $\mathrm{C} 4$ & 170.11 & -148.08 & 89.83 & -29.92 & 39.90 & -86.10 & -30.92 & 33.59 & -64.93 & & & 2.418 & 2.706 & & 2.993 \\
\hline \multirow{2}{*}{15} & $\mathrm{C} 2$ & 168.41 & -150.25 & 88.00 & -31.68 & 36.96 & -84.64 & -32.85 & -6.32 & -65.41 & \multirow{2}{*}{176.37} & \multirow{2}{*}{175.68} & 2.469 & 2.605 & \multirow{2}{*}{6.536} & 2.416 \\
\hline & $\mathrm{C} 4$ & 168.88 & -147.14 & 90.70 & -29.02 & 38.32 & -83.93 & -34.13 & 30.16 & -64.40 & & & 2.466 & 2.696 & & 2.744 \\
\hline \multirow{2}{*}{31} & $\mathrm{C} 2$ & 170.96 & -137.00 & 100.91 & -18.95 & 25.20 & -75.93 & -39.77 & -30.37 & -178.60 & \multirow{2}{*}{63.80} & \multirow{2}{*}{63.94} & 2.453 & 2.535 & \multirow{2}{*}{8.400} & \\
\hline & $\mathrm{C} 4$ & -172.88 & -97.75 & 140.23 & 22.13 & 82.33 & -32.88 & 32.94 & -33.98 & -178.61 & & & 2.371 & 2.621 & & \\
\hline \multirow{2}{*}{54} & $\mathrm{C} 2$ & -175.12 & -109.45 & 128.41 & 10.85 & 71.76 & -18.89 & 43.87 & -36.64 & -179.27 & \multirow{2}{*}{63.32} & \multirow{2}{*}{63.14} & 2.453 & 2.518 & \multirow{2}{*}{8.336} & \\
\hline & C4 & 176.59 & -108.23 & 131.44 & 11.90 & -48.17 & -46.49 & -53.52 & -34.16 & -179.18 & & & 2.432 & 2.226 & & \\
\hline
\end{tabular}

[a] Conformers are numbered according to their appearance during conformational search; $[\mathrm{b}]-\alpha=\mathrm{C}_{\mathrm{Tr}}-\mathrm{C}(=\mathrm{O})-\mathrm{O}-\mathrm{C}^{*} ;[\mathrm{c}]-B=\mathrm{O}=\mathrm{C}-\mathrm{C}-\mathrm{C}_{i p s o} ;[\mathrm{d}]-\gamma=(\mathrm{O}=) \mathrm{C}-\mathrm{C}_{\mathrm{Tr}}-\mathrm{C}_{i p s o}-\mathrm{C}_{\text {ortho }}$ (of the two possibilities the absolute values $\leq 90^{\circ}$ has been chosen); [e] $-\delta=\mathrm{C}(=\mathrm{O})-\mathrm{O}-\mathrm{C}^{*}-\mathrm{H}$; [f] $-\zeta=\mathrm{O}-\mathrm{C}^{*}-\mathrm{C}-\mathrm{C}\left({ }^{*}\right)$; [g] $\varphi_{1}=\mathrm{C} 1-\mathrm{C} 2-\mathrm{C} 3-\mathrm{C} 4 ; \varphi_{2}=\mathrm{C} 2-\mathrm{C} 3-\mathrm{C} 4-\mathrm{C} 5$; [h] $\mathrm{I}_{1}=\mathrm{C}=\mathrm{O} \cdots \mathrm{HC} \mathrm{C}_{\text {ortho }}[\mathrm{i}]-\mathrm{I}_{2}=(\mathrm{O}=) \mathrm{CO} \cdots \mathrm{HC}_{\text {ortho }}[\mathrm{j}]-\mathrm{I}_{3}=\mathrm{C}_{\mathrm{Tr}} \cdots \mathrm{C}_{\mathrm{Tr}} ;[\mathrm{k}]-\mathrm{I}_{4}=\mathrm{C}=\mathrm{O} \mathrm{HC}_{\text {ortho }}$ of the second trityl group. 
Table SI_49. Dihedral angles $\alpha, 6, \gamma, \delta, \zeta$ and $\varphi$ (in degrees) and selected interatomic distances $I_{1}, I_{2}$ and $I_{3}$ (in $\AA$ ) calculated at the B3LYP-GD3BJ/6-311G(d,p) level for individual low-energy conformers of 21.

\begin{tabular}{|c|c|c|c|c|c|c|c|c|c|c|c|c|c|c|c|}
\hline Con & $10^{[\mathrm{a}]}$ & $\alpha^{[\mathrm{b}]}$ & $B_{1}{ }^{[c]}$ & $B_{2}{ }^{[c]}$ & $B_{3}{ }^{[c]}$ & $\gamma_{1}{ }^{[\mathrm{d}]}$ & $V_{2}{ }^{[\mathrm{d}]}$ & $\gamma_{3}{ }^{[\mathrm{d}]}$ & $\delta^{[\mathrm{e}]}$ & $\zeta^{[f]}$ & $\varphi_{1}^{[\mathrm{g}]}$ & $\varphi_{2}^{[g]}$ & $l_{1}^{[h]}$ & $I_{2}{ }^{[\mathrm{i}]}$ & $I_{3}^{[j]}$ \\
\hline \multirow{2}{*}{1} & $\mathrm{C} 2$ & 179.12 & -145.64 & 94.60 & -25.02 & 50.87 & 51.46 & 47.58 & 45.60 & -57.55 & \multirow{2}{*}{178.20} & \multirow{2}{*}{-177.81} & 2.272 & 2.279 & \multirow{2}{*}{6.248} \\
\hline & $\mathrm{C} 4$ & -172.62 & -84.91 & 152.76 & 34.56 & -86.04 & -39.98 & 26.47 & 49.00 & -57.42 & & & 2.377 & 2.571 & \\
\hline \multirow{2}{*}{6} & $\mathrm{C} 2$ & 178.53 & -83.94 & 156.31 & 34.98 & -44.62 & -53.09 & -52.45 & 43.78 & -60.44 & \multirow{2}{*}{179.67} & \multirow{2}{*}{-177.49} & 2.301 & 2.425 & \multirow{2}{*}{6.336} \\
\hline & $\mathrm{C} 4$ & -176.98 & -72.10 & 166.32 & 47.23 & -67.36 & -56.53 & -3.63 & 41.16 & -57.60 & & & 2.181 & 2.744 & \\
\hline \multirow{2}{*}{16} & $\mathrm{C} 2$ & -173.26 & -133.92 & 105.29 & -11.82 & 67.68 & 4.56 & 51.04 & 30.44 & -52.69 & \multirow{2}{*}{-173.23} & \multirow{2}{*}{-173.58} & 2.395 & 2.430 & \multirow{2}{*}{6.123} \\
\hline & $\mathrm{C} 4$ & -164.30 & -85.58 & 153.09 & 34.00 & -71.13 & -54.87 & 9.05 & -179.56 & -46.73 & & & 2.197 & 2.553 & \\
\hline
\end{tabular}

[a] Conformers are numbered according to their appearance during conformational search; $[\mathrm{b}]-\alpha=\mathrm{C}_{\mathrm{Tr}}-\mathrm{C}(=\mathrm{O})-\mathrm{O}-\mathrm{C}^{*} ;[\mathrm{c}]-\mathrm{B}=\mathrm{O}=\mathrm{C}-\mathrm{C}-\mathrm{C}_{\text {ipso }} ;[\mathrm{d}]-\gamma=(\mathrm{O}=) \mathrm{C}-\mathrm{C}_{\mathrm{Tr}}-\mathrm{C}_{\text {ipso }}-\mathrm{C}_{\text {ortho }}$ (of the two possibilities the absolute values $\leq 90^{\circ}$ has been chosen); [e] $-\delta=\mathrm{C}(=\mathrm{O})-\mathrm{O}-\mathrm{C}^{*}-\mathrm{H} ;[\mathrm{f}]-\zeta=\mathrm{O}-\mathrm{C}^{*}-\mathrm{C}-\mathrm{C}\left({ }^{*}\right) ;[\mathrm{g}] \varphi_{1}=\mathrm{C} 1-\mathrm{C} 2-\mathrm{C} 3-\mathrm{C} 4 ; \varphi_{2}=\mathrm{C} 2-\mathrm{C} 3-\mathrm{C} 4-\mathrm{C} 5$; [h] $\mathrm{I}_{1}=\mathrm{C}=\mathrm{O} \cdots \mathrm{HC}_{\text {ortho; }}[\mathrm{i}]-\mathrm{I}_{2}=(\mathrm{O}=) \mathrm{CO} \cdots \mathrm{HC}_{\text {ortho; }}[\mathrm{j}]-\mathrm{I}_{3}=\mathrm{C}_{\mathrm{Tr}} \cdots \mathrm{C}_{\mathrm{Tr}}$ 
Table SI_50. Dihedral angles $\alpha, b, y, \delta, \zeta$ and $\varphi$ (in degrees) and selected interatomic distances $I_{1}, I_{2}$ and $I_{3}$ (in $\AA$ ) calculated at the M06/6-311G(d,p) level for individual low-energy conformers of 21.

\begin{tabular}{|c|c|c|c|c|c|c|c|c|c|c|c|c|c|c|c|c|}
\hline Con & $10^{[a]}$ & $\alpha^{[\mathrm{b}]}$ & $B_{1}{ }^{[c]}$ & $B_{2}{ }^{[c]}$ & $B_{3}^{[c]}$ & $\nu_{1}{ }^{[\mathrm{d}]}$ & $V_{2}{ }^{[\mathrm{d}]}$ & $\gamma_{3}{ }^{[d]}$ & $\delta^{[\mathrm{e}]}$ & $\zeta^{[f]}$ & $\varphi_{1}^{[\mathrm{g}]}$ & $\varphi_{2}^{[\mathrm{g}]}$ & $l_{1}^{[\mathrm{h}]}$ & $I_{2}^{[i]}$ & $I_{3}^{[j]}$ & $I_{4}^{[\mathrm{k}]}$ \\
\hline \multirow[t]{2}{*}{1} & $\mathrm{C} 2$ & -178.71 & -68.65 & 171.59 & 50.16 & 28.66 & -59.24 & -38.68 & 42.07 & -58.53 & \multirow[t]{2}{*}{-177.90} & \multirow[t]{2}{*}{-178.79} & 2.151 & $\begin{array}{l}2.642 \\
2.716\end{array}$ & \multirow[t]{2}{*}{6.237} & 2.753 \\
\hline & $\mathrm{C} 4$ & 177.33 & -146.40 & 93.39 & -24.97 & 48.26 & 37.28 & 61.41 & 42.28 & -58.70 & & & 2.500 & 2.299 & & 2.551 \\
\hline \multirow[b]{2}{*}{12} & $\mathrm{C} 2$ & -169.25 & -98.71 & 140.16 & 21.15 & 79.52 & -29.32 & 38.02 & -20.45 & -64.67 & \multirow[b]{2}{*}{178.08} & \multirow[b]{2}{*}{179.01} & 2.431 & 2.538 & \multirow[b]{2}{*}{6.037} & 2.477 \\
\hline & $\mathrm{C} 4$ & 170.20 & -156.17 & 82.72 & -37.45 & 43.91 & 80.16 & -28.85 & 34.35 & -61.50 & & & 2.431 & $\begin{array}{c}2.658 \\
2.808\end{array}$ & & 2.731 \\
\hline \multirow{3}{*}{15} & $\mathrm{C} 2$ & 158.27 & -143.33 & 95.40 & -25.00 & 34.43 & -79.03 & -43.10 & 15.13 & -56.44 & \multirow{3}{*}{-175.04} & \multirow{3}{*}{-175.04} & 2.584 & 2.650 & \multirow{3}{*}{5.972} & $\begin{array}{l}2.358 \\
2.412\end{array}$ \\
\hline & & & & & & & & & & & & & & & & \\
\hline & $\mathrm{C} 4$ & 158.29 & -143.33 & 95.39 & -25.00 & 34.43 & -79.02 & -43.10 & 15.10 & -56.45 & & & 2.584 & 2.650 & & $\begin{array}{l}2.357 \\
2.413\end{array}$ \\
\hline \multirow[b]{2}{*}{16} & $\mathrm{C} 2$ & -159.19 & -91.76 & 146.28 & 27.42 & -83.23 & -45.21 & 25.35 & -179.63 & -41.16 & \multirow[b]{2}{*}{-167.19} & \multirow[b]{2}{*}{-172.22} & 2.323 & $\begin{array}{l}2.785 \\
2.795\end{array}$ & \multirow[b]{2}{*}{6.184} & \\
\hline & $\mathrm{C} 4$ & 177.25 & -140.53 & 99.72 & -20.10 & 44.92 & 51.34 & 55.45 & 38.95 & -51.93 & & & 2.410 & $\begin{array}{l}2.245 \\
2.782\end{array}$ & & 2.586 \\
\hline \multirow{2}{*}{25} & $\mathrm{C} 2$ & -162.13 & -118.77 & 119.59 & 0.99 & 57.75 & -1.17 & 54.41 & 38.92 & -59.63 & \multirow{2}{*}{179.94} & \multirow{2}{*}{171.15} & 2.555 & 2.364 & \multirow{2}{*}{6.614} & \\
\hline & $\mathrm{C4}$ & 171.23 & -133.38 & 104.93 & -14.97 & 26.05 & -72.61 & -41.38 & -179.90 & -61.56 & & & 2.438 & 2.544 & & \\
\hline \multirow{2}{*}{35} & $\mathrm{C} 2$ & -169.22 & -97.80 & 142.12 & 20.94 & -39.78 & -53.92 & -55.54 & -179.08 & -47.16 & \multirow{2}{*}{-174.22} & \multirow{2}{*}{-170.51} & 2.387 & 2.239 & \multirow{2}{*}{6063} & \\
\hline & $\mathrm{C} 4$ & -173.28 & -142.14 & 97.33 & -20.57 & 64.20 & 11.59 & 50.42 & 38.34 & -49.86 & & & 2.340 & 2.420 & & \\
\hline \multirow{2}{*}{46} & $\mathrm{C} 2$ & 173.24 & -137.30 & 101.63 & -18.86 & 30.31 & -79.14 & -37.10 & -176.18 & -47.38 & \multirow{2}{*}{-174.36} & \multirow{2}{*}{-174.36} & 2.399 & 2.569 & \multirow{2}{*}{6.426} & \\
\hline & $\mathrm{C} 4$ & 173.24 & -137.30 & 101.63 & -18.86 & 30.31 & -79.14 & -37.10 & -176.18 & -47.38 & & & 2.399 & 2.569 & & \\
\hline \multirow{2}{*}{61} & $\mathrm{C} 2$ & -175.82 & -138.30 & 101.98 & -18.18 & 43.07 & 43.59 & 58.66 & -175.88 & -42.43 & \multirow{2}{*}{-169.01} & \multirow{2}{*}{-172.09} & 2.476 & 2.204 & \multirow{2}{*}{6.306} & \\
\hline & $\mathrm{C} 4$ & 172.34 & -141.32 & 96.01 & -23.34 & 35.77 & -77.86 & -34.22 & -177.80 & -44.71 & & & 2.399 & 2.615 & & \\
\hline
\end{tabular}

[a] Conformers are numbered according to their appearance during conformational search; $[\mathrm{b}]-\alpha=\mathrm{C}_{\mathrm{Tr}} \mathrm{C}(=\mathrm{O})-\mathrm{O}-\mathrm{C}^{*} ;[\mathrm{c}]-\theta=\mathrm{O}=\mathrm{C}-\mathrm{C}-\mathrm{C}_{\text {ipso }} ;[\mathrm{d}]-\gamma=(\mathrm{O}=) \mathrm{C}-\mathrm{C}_{\mathrm{Tr}}-\mathrm{C}_{\text {ipso }}-\mathrm{C}_{\text {ortho }}$ (of the two possibilities the absolute values $\leq 90^{\circ}$ has been chosen); [e] $-\delta=\mathrm{C}(=\mathrm{O})-\mathrm{O}-\mathrm{C}^{*}-\mathrm{H} ;[\mathrm{f}]-\zeta=\mathrm{O}-\mathrm{C}^{*}-\mathrm{C}-\mathrm{C}\left({ }^{*}\right) ;[\mathrm{g}] \varphi_{1}=\mathrm{C} 1-\mathrm{C} 2-\mathrm{C} 3-\mathrm{C} 4 ; \varphi_{2}=\mathrm{C} 2-\mathrm{C} 3-\mathrm{C} 4-\mathrm{C} 5$; [h] $\left.\mathrm{I}_{1}=\mathrm{C}=\mathrm{O} \cdots \mathrm{H} \mathrm{C}_{\text {ortho }}[\mathrm{i}]-\mathrm{I}_{2}=(\mathrm{O}=) \mathrm{CO} \cdots \mathrm{HC} \mathrm{C}_{\text {ortho }} ; \mathrm{j}\right]-\mathrm{I}_{3}=\mathrm{C}_{\mathrm{Tr}} \cdots \mathrm{C}_{\mathrm{Tr}} ;[\mathrm{k}]-\mathrm{I}_{4}=\mathrm{C}=\mathrm{O} \cdots \mathrm{H} \mathrm{C}_{\text {ortho }}$ of the second trityl group. 
Table SI_51. Dihedral angles $\alpha, 6, \gamma, \delta, \zeta$ and $\varphi$ (in degrees) and selected interatomic distances $I_{1}, I_{2}$ and $I_{3}$ (in $\AA$ ) calculated at the B3LYP/6-311G(d,p) level for individual low-energy conformers of 22.

\begin{tabular}{|c|c|c|c|c|c|c|c|c|c|c|c|c|c|c|c|c|}
\hline & & $\alpha^{[\mathrm{b}]}$ & $B_{1}[\mathrm{c}]$ & $B_{2}[c]$ & $B_{3}{ }^{[c]}$ & $V_{1}{ }^{[d]}$ & $V_{2}{ }^{[d]}$ & $\gamma_{3}{ }^{[d]}$ & $\delta^{[\mathrm{e}]}$ & $\zeta^{[f]}$ & $\varphi_{1}[\mathrm{~g}]$ & $\varphi_{2}{ }^{[\mathrm{g}]}$ & $\varphi_{3}[\mathrm{~g}]$ & $l_{1}{ }^{[\mathrm{h}]}$ & $I_{2}^{[i]}$ & $l_{3}^{[\mathrm{j}]}$ \\
\hline \multirow[b]{2}{*}{1} & $\mathrm{C} 2$ & -173.95 & -108.33 & 129.42 & 11.78 & 69.88 & -16.18 & 45.32 & 31.81 & -67.14 & \multirow[b]{2}{*}{172.52} & \multirow[b]{2}{*}{-177.78} & \multirow[b]{2}{*}{172.52} & 2.483 & 2.472 & \multirow[b]{2}{*}{7.717} \\
\hline & C5 & -173.95 & -108.33 & 129.42 & 11.78 & 69.88 & -16.18 & 45.32 & 31.81 & -67.14 & & & & 2.483 & 2.472 & \\
\hline \multirow{2}{*}{3} & $\mathrm{C} 2$ & -173.93 & -108.37 & 129.38 & 11.74 & 69.89 & -16.19 & 45.30 & 31.67 & -67.09 & \multirow{2}{*}{172.57} & \multirow{2}{*}{-177.77} & \multirow{2}{*}{172.57} & 2.482 & 2.473 & \multirow{2}{*}{7.716} \\
\hline & $\mathrm{C} 5$ & -173.93 & -108.37 & 129.38 & 11.74 & 69.90 & -16.19 & 45.29 & 31.67 & -67.09 & & & & 2.482 & 2.473 & \\
\hline \multirow[b]{2}{*}{5} & $\mathrm{C} 2$ & 178.20 & -109.03 & 128.83 & 7.38 & 8.51 & -66.29 & -49.07 & 42.56 & -62.38 & \multirow[b]{2}{*}{177.31} & \multirow[b]{2}{*}{179.45} & \multirow[b]{2}{*}{175.59} & 2.400 & 2.585 & \multirow{2}{*}{7.744} \\
\hline & C5 & -172.65 & -101.74 & 136.06 & 17.99 & 71.31 & -19.01 & 44.07 & 36.15 & -64.04 & & & & 2.520 & 2.445 & \\
\hline \multirow[t]{2}{*}{7} & $\mathrm{C} 2$ & 169.31 & -156.92 & 81.50 & -37.94 & 49.63 & 75.11 & -13.33 & 19.01 & -69.45 & \multirow[t]{2}{*}{171.21} & \multirow[t]{2}{*}{-176.95} & \multirow[t]{2}{*}{172.27} & 2.250 & $\begin{array}{c}2.769 \\
2.770\end{array}$ & \multirow[t]{2}{*}{7.872} \\
\hline & $\mathrm{C} 5$ & -174.61 & -107.13 & 130.52 & 12.96 & 71.11 & -18.85 & 43.85 & 32.52 & -67.41 & & & & 2.470 & 2.498 & \\
\hline \multirow[b]{2}{*}{8} & $\mathrm{C} 2$ & 171.73 & -139.53 & 98.47 & -21.32 & 28.00 & -78.23 & -37.39 & -31.32 & -173.60 & \multirow[b]{2}{*}{68.32} & \multirow[b]{2}{*}{170.03} & \multirow[b]{2}{*}{172.12} & 2.444 & 2.548 & \multirow[b]{2}{*}{7.208} \\
\hline & C5 & 169.69 & -148.94 & 89.03 & -30.47 & 44.08 & 81.46 & -21.57 & -4.26 & -69.68 & & & & 2.283 & $\begin{array}{l}2.758 \\
2.822\end{array}$ & \\
\hline \multirow{2}{*}{9} & $\mathrm{C} 2$ & -174.83 & -104.66 & 132.98 & 15.21 & 72.11 & -20.20 & 42.91 & 33.72 & -66.87 & \multirow{2}{*}{173.03} & \multirow{2}{*}{-73.79} & \multirow{2}{*}{173.03} & 2.467 & 2.495 & \multirow{2}{*}{9.051} \\
\hline & $\mathrm{C} 5$ & -174.83 & -104.66 & 132.98 & 15.21 & 72.11 & -20.20 & 42.91 & 33.72 & -66.87 & & & & 2.467 & 2.495 & \\
\hline 10 & $\mathrm{C} 2$ & 164.76 & -137.37 & 100.60 & -19.23 & 28.29 & -77.50 & -38.58 & -28.11 & 56.82 & 6280 & 17157 & 9200 & 2.456 & 2.589 & 6717 \\
\hline 10 & $\mathrm{C5}$ & 164.76 & -137.37 & 100.60 & -19.23 & 28.29 & -77.50 & -38.58 & -28.11 & 56.82 & -02.00 & $-1 / 4.3 /$ & -02.00 & 2.456 & 2.589 & 0.111 \\
\hline 11 & $\mathrm{C} 2$ & 177.57 & -122.43 & 117.00 & -1.05 & -60.65 & -43.41 & -47.51 & 35.50 & -68.28 & 171.69 & 177.85 & 173.73 & $\begin{array}{c}2.409 \\
2.837\end{array}$ & 2.297 & 7.575 \\
\hline & $\mathrm{C} 5$ & -174.36 & -109.20 & 128.24 & 10.89 & 70.29 & -18.71 & 44.40 & 30.25 & -66.13 & & & & 2.461 & 2.522 & \\
\hline 12 & $\mathrm{C} 2$ & 177.07 & -178.56 & 61.37 & -56.59 & 60.22 & 46.67 & 38.87 & 33.49 & -68.96 & 171.15 & 174.86 & 65.94 & 2.153 & $\begin{array}{l}2.701 \\
2.803\end{array}$ & 7.401 \\
\hline & C5 & -171.24 & -102.57 & 135.31 & 17.33 & 73.37 & -22.76 & 42.36 & -30.89 & -176.36 & & & & 2.490 & 2.503 & \\
\hline & $\mathrm{C} 2$ & 178.53 & -128.29 & 109.75 & -6.75 & 64.50 & -6.28 & 48.97 & -11.67 & -74.50 & & & & 2.408 & 2.539 & \\
\hline 13 & C5 & -171.64 & -83.19 & 155.45 & 36.57 & -73.15 & -49.26 & 13.59 & -12.28 & 179.78 & 167.71 & 176.10 & 62.14 & 2.251 & $\begin{array}{l}2.692 ; \\
2.771\end{array}$ & 7.280 \\
\hline
\end{tabular}




\begin{tabular}{|c|c|c|c|c|c|c|c|c|c|c|c|c|c|c|c|c|}
\hline \multirow[b]{2}{*}{15} & $\mathrm{C} 2$ & -174.56 & -105.84 & 131.82 & 14.05 & 70.40 & -17.55 & 44.76 & 34.93 & -66.40 & \multirow[b]{2}{*}{173.52} & \multirow[b]{2}{*}{-71.84} & \multirow[b]{2}{*}{176.48} & 2.495 & 2.464 & \multirow[b]{2}{*}{9.106} \\
\hline & C5 & 170.61 & -147.18 & 90.72 & -28.99 & 46.86 & 78.96 & -17.88 & 40.21 & -63.49 & & & & 2.234 & $\begin{array}{l}2.750 \\
2.842\end{array}$ & \\
\hline \multirow{2}{*}{17} & $\mathrm{C} 2$ & 164.27 & -133.97 & 103.81 & -15.93 & 23.65 & -73.23 & -43.34 & -22.10 & 57.05 & \multirow{2}{*}{-62.71} & \multirow{2}{*}{-178.69} & \multirow{2}{*}{-62.01} & 2.512 & 2.555 & \multirow{2}{*}{6.790} \\
\hline & C5 & 173.24 & -139.69 & 100.28 & -19.15 & 47.65 & 48.27 & 53.82 & -32.21 & 57.74 & & & & 2.399 & 2.262 & \\
\hline \multirow[t]{2}{*}{26} & C2 & 170.80 & -139.08 & 98.80 & -21.08 & 28.93 & -78.81 & -36.82 & -32.20 & -176.12 & \multirow[t]{2}{*}{67.28} & \multirow[t]{2}{*}{81.67} & \multirow[t]{2}{*}{168.77} & $\begin{array}{c}2.421 \\
2.389\end{array}$ & 2.566 & \multirow[t]{2}{*}{6.953} \\
\hline & C5 & 174.79 & -45.64 & -167.29 & 71.64 & -42.34 & -65.92 & -37.83 & 36.48 & -71.09 & & & & 2.209 & 2.638 & \\
\hline \multirow{3}{*}{31} & $\mathrm{C} 2$ & 165.57 & -143.89 & 94.26 & -25.33 & 34.29 & -82.86 & -32.37 & -23.24 & 61.56 & \multirow{2}{*}{-58.64} & \multirow{2}{*}{174.57} & \multirow{2}{*}{-61.59} & 2.396 & 2.630 & \multirow{2}{*}{7.768} \\
\hline & C5 & 171.09 & -129.30 & 108.35 & -11.77 & 18.10 & -70.89 & -44.96 & 40.15 & 60.20 & & & & 2.474 & 2.509 & \\
\hline & $\mathrm{C} 2$ & 179.87 & -115.91 & 123.43 & 2.05 & 43.44 & 60.67 & 47.14 & 37.12 & -61.95 & \multirow[b]{2}{*}{177.86} & \multirow[b]{2}{*}{-175.17} & \multirow[b]{2}{*}{177.77} & 2.412 & 2.293 & \multirow[b]{2}{*}{8.142} \\
\hline 36 & C5 & 171.22 & -139.46 & 98.17 & -21.80 & 38.63 & -86.07 & -27.72 & 41.99 & -61.87 & & & & 2.294 & $\begin{array}{l}2.758 \\
2.878\end{array}$ & \\
\hline \multirow{2}{*}{71} & $\mathrm{C} 2$ & -177.67 & -134.88 & 104.57 & -14.80 & 49.14 & 40.59 & 55.14 & 29.51 & -172.03 & \multirow{2}{*}{67.96} & \multirow{2}{*}{65.22} & \multirow{2}{*}{156.66} & 2.450 & 2.231 & \multirow{2}{*}{7.446} \\
\hline & C5 & 171.45 & -42.40 & -164.38 & 74.88 & -45.93 & -64.38 & -38.31 & 32.64 & -84.09 & & & & 2.231 & 2.563 & \\
\hline
\end{tabular}

[a] Conformers are numbered according to their appearance during conformational search; $[\mathrm{b}]-\alpha=\mathrm{C}_{\mathrm{Tr}}-\mathrm{C}(=\mathrm{O})-\mathrm{O}-\mathrm{C}^{*} ;[\mathrm{c}]-B=\mathrm{O}=\mathrm{C}-\mathrm{C}-\mathrm{C}_{i p s o} ;[\mathrm{d}]-\gamma=(\mathrm{O}=) \mathrm{C}-\mathrm{C}_{\mathrm{Tr}}-\mathrm{C}_{i p s o}-\mathrm{C}_{\text {ortho }}$ (of the two possibilities the absolute values $\leq 90^{\circ}$ has been chosen); $[\mathrm{e}]-\delta=\mathrm{C}(=\mathrm{O})-\mathrm{O}-\mathrm{C}^{*}-\mathrm{H} ;[\mathrm{f}]-\zeta=\mathrm{O}-\mathrm{C} *-\mathrm{C}-\mathrm{C}\left({ }^{*}\right) ;[\mathrm{g}] \varphi_{1}=\mathrm{C} 1-\mathrm{C} 2-\mathrm{C} 3-\mathrm{C} 4 ; \varphi_{2}=\mathrm{C} 2-\mathrm{C} 3-\mathrm{C} 4-\mathrm{C} 5 ; \varphi_{3}=$ $\mathrm{C} 3-\mathrm{C} 4-\mathrm{C} 5-\mathrm{C} 6 ; \mathrm{h}]-\mathrm{I}_{1}=\mathrm{C}=\mathrm{O} \cdots \mathrm{HC}_{\text {ortho; }}[\mathrm{i}]-\mathrm{I}_{2}=(\mathrm{O}=) \mathrm{CO} \cdots \mathrm{HC}_{\text {ortho; }}[\mathrm{j}]-\mathrm{I}_{3}=\mathrm{C}=\mathrm{O} \cdots \mathrm{HC}_{\text {ortho; }}$; distance between $\mathrm{C}=\mathrm{O}$ and $\mathrm{HC}$ ortho of the second trityl group. 
Table SI_52. Dihedral angles $\alpha, 6, \gamma, \delta, \zeta$ and $\varphi$ (in degrees) and selected interatomic distances $I_{1}, I_{2}$ and $I_{3}$ (in $\AA$ ) calculated at the B3LYP-GD3BJ/6-311G(d,p) level for individual low-energy conformers of 22.

\begin{tabular}{|c|c|c|c|c|c|c|c|c|c|c|c|c|c|c|c|c|}
\hline \multicolumn{2}{|c|}{ Conf. no ${ }^{[a]}$} & $\alpha^{[\mathrm{b}]}$ & $B_{1}[c]$ & $B_{2}{ }^{[c]}$ & $B_{3}[c]$ & $V_{1}{ }^{[\mathrm{d}]}$ & $V_{2}{ }^{[\mathrm{d}]}$ & $V_{3}{ }^{[d]}$ & $\delta^{[\mathrm{e}]}$ & $\zeta^{[f]}$ & $\varphi_{1}{ }^{[\mathrm{g}]}$ & $\varphi_{2}{ }^{[\mathrm{g}]}$ & $\varphi_{3}{ }^{[\mathrm{g}]}$ & $l_{1}^{[\mathrm{h}]}$ & $I_{2}^{[i]}$ & $\left.\right|_{3} ^{[j]}$ \\
\hline \multirow{2}{*}{1} & $\mathrm{C} 2$ & -173.84 & -109.63 & 128.33 & 10.75 & 70.07 & -19.91 & 43.73 & 18.64 & -66.52 & \multirow{2}{*}{174.64} & \multirow{2}{*}{175.81} & \multirow{2}{*}{174.64} & 2.452 & 2.499 & \multirow{2}{*}{6.983} \\
\hline & $\mathrm{C} 5$ & -173.84 & -109.63 & 128.33 & 10.75 & 70.07 & -19.91 & 43.73 & 18.64 & -66.52 & & & & 2.452 & 2.499 & \\
\hline \multirow[b]{2}{*}{3} & $\mathrm{C} 2$ & -173.83 & -109.59 & 128.37 & 10.79 & 70.10 & -19.96 & 43.69 & 18.63 & -66.54 & \multirow{2}{*}{174.63} & \multirow{2}{*}{175.84} & \multirow{2}{*}{174.64} & 2.452 & 2.500 & \multirow{2}{*}{6.983} \\
\hline & C5 & -173.83 & -109.60 & 128.37 & 10.79 & 70.09 & -19.96 & 43.70 & 18.62 & -66.53 & & & & 2.452 & 2.500 & \\
\hline \multirow{2}{*}{5} & $\mathrm{C} 2$ & 173.58 & -110.80 & 126.42 & 6.00 & -1.18 & -58.00 & -54.82 & 39.21 & -67.74 & \multirow{2}{*}{173.16} & \multirow{2}{*}{160.53} & \multirow{2}{*}{-179.30} & 2.501 & 2.434 & \multirow{2}{*}{6.580} \\
\hline & $\mathrm{C} 5$ & -164.91 & -108.63 & 130.64 & 10.73 & 59.72 & 6.89 & 54.78 & 39.63 & -59.46 & & & & 2.671 & 2.199 & \\
\hline \multirow{2}{*}{7} & $\mathrm{C} 2$ & 177.35 & -146.76 & 93.10 & -25.20 & 49.83 & 33.05 & 57.25 & 31.25 & -68.52 & \multirow{2}{*}{171.75} & \multirow{2}{*}{174.25} & \multirow{2}{*}{174.54} & 2.417 & 2.309 & \multirow{2}{*}{7.098} \\
\hline & $\mathrm{C} 5$ & -171.89 & -107.59 & 130.34 & 12.90 & 73.16 & -20.71 & 43.24 & 16.82 & -65.78 & & & & 2.456 & 2.500 & \\
\hline \multirow{2}{*}{17} & $\mathrm{C} 2$ & 167.14 & -154.42 & 85.20 & -33.69 & 50.43 & 71.04 & 1.58 & -24.44 & 59.96 & \multirow{2}{*}{-58.77} & \multirow{2}{*}{-169.88} & \multirow{2}{*}{-54.53} & 2.141 & 2.575 & \multirow{2}{*}{5.819} \\
\hline & $\mathrm{C} 5$ & 179.07 & -154.31 & 86.31 & -32.52 & 46.30 & 41.28 & 62.73 & -35.08 & 64.30 & & & & 2.512 & 2.371 & \\
\hline \multirow[b]{2}{*}{26} & $\mathrm{C} 2$ & 175.15 & -138.31 & 99.75 & -20.60 & 30.18 & -84.63 & -34.45 & -43.06 & -154.13 & \multirow[b]{2}{*}{88.05} & \multirow[b]{2}{*}{83.79} & \multirow[b]{2}{*}{170.24} & 2.346 & 2.567 & \multirow[b]{2}{*}{5.502} \\
\hline & $\mathrm{C} 5$ & 177.67 & -42.65 & -165.09 & 73.41 & -10.43 & -70.90 & -43.44 & 32.80 & -70.07 & & & & $\begin{array}{l}2.235 \\
2.271\end{array}$ & 2.676 & \\
\hline
\end{tabular}

[a] Conformers are numbered according to their appearance during conformational search; $[\mathrm{b}]-\alpha=\mathrm{C}_{\mathrm{Tr}}-\mathrm{C}(=\mathrm{O})-\mathrm{O}-\mathrm{C}^{*} ;[\mathrm{c}]-B=\mathrm{O}=\mathrm{C}-\mathrm{C}-\mathrm{C}_{i p s o} ;[\mathrm{d}]-\gamma=(\mathrm{O}=) \mathrm{C}-\mathrm{C}_{\mathrm{Tr}}-\mathrm{C}_{i p s o}-\mathrm{C}_{\text {ortho }}$ (of the two possibilities the absolute values $\leq 90^{\circ}$ has been chosen); $[\mathrm{e}]-\delta=\mathrm{C}(=\mathrm{O})-\mathrm{O}-\mathrm{C}^{*}-\mathrm{H} ;[\mathrm{f}]-\zeta=\mathrm{O}-\mathrm{C}^{*}-\mathrm{C}-\mathrm{C}\left({ }^{*}\right)$; $[\mathrm{g}] \varphi_{1}=\mathrm{C} 1-\mathrm{C} 2-\mathrm{C} 3-\mathrm{C} 4 ; \varphi_{2}=\mathrm{C} 2-\mathrm{C} 3-\mathrm{C} 4-\mathrm{C} 5 ; \varphi_{3}=$ C3-C4-C5-C6; [h] $-\mathrm{I}_{1}=\mathrm{C}=\mathrm{O} \cdots \mathrm{HC}$ ortho; $\left.[\mathrm{i}]-\mathrm{I}_{2}=(\mathrm{O}=) \mathrm{CO} \cdots \mathrm{HC}_{\text {ortho }} ; \mathrm{j}\right]-\mathrm{I}_{3}=\mathrm{C}_{\mathrm{Tr}} \cdots \mathrm{C}_{\mathrm{Tr}}$ 
Table SI_53. Dihedral angles $\alpha, b, y, \delta, \zeta$ and $\varphi$ (in degrees) and selected interatomic distances $I_{1}, I_{2}$ and $I_{3}$ (in $\AA$ ) calculated at the M06-2X/6-311G(d,p) level for individual low-energy conformers of 22.

\begin{tabular}{|c|c|c|c|c|c|c|c|c|c|c|c|c|c|c|c|c|c|}
\hline \multicolumn{2}{|c|}{ Conf. no ${ }^{[a]}$} & $\alpha^{[\mathrm{b}]}$ & $B_{1}[c]$ & $B_{2}{ }^{[c]}$ & $B_{3}[c]$ & $V_{1}{ }^{[d]}$ & $V_{2}{ }^{[d]}$ & $\gamma_{3}{ }^{[d]}$ & $\delta^{[\mathrm{e}]}$ & $\zeta^{[f]}$ & $\varphi_{1}^{[\mathrm{g}]}$ & $\varphi_{2}{ }^{[\mathrm{g}]}$ & $\varphi_{3}^{[\mathrm{g}]}$ & $l_{1}^{[\mathrm{h}]}$ & $I_{2}^{[i]}$ & $I_{3}^{[j]}$ & $I_{4}^{[k]}$ \\
\hline \multirow{2}{*}{1} & $\mathrm{C} 2$ & -174.18 & -116.68 & 121.87 & 4.35 & 69.39 & -17.08 & 45.99 & 25.93 & -67.18 & \multirow{2}{*}{173.93} & \multirow{2}{*}{176.69} & \multirow{2}{*}{173.93} & 2.433 & 2.514 & \multirow{2}{*}{7.011} & \\
\hline & C5 & -174.18 & -116.68 & 121.87 & 4.35 & 69.39 & -17.08 & 45.99 & 25.93 & -67.18 & & & & 2.433 & 2.514 & & \\
\hline 3 & C5 & -174.19 & -116.66 & 121.90 & 4.36 & 69.38 & -17.06 & 45.97 & 25.87 & -67.16 & 173.95 & 176.72 & 173.95 & 2.433 & 2.513 & 7.011 & \\
\hline \multirow[t]{2}{*}{11} & $\mathrm{C} 2$ & 178.99 & -125.18 & 115.70 & -2.99 & -61.41 & -36.59 & -50.49 & 35.37 & -67.06 & \multirow[t]{2}{*}{174.04} & \multirow[t]{2}{*}{171.84} & \multirow[t]{2}{*}{177.15} & $\begin{array}{l}2.470 \\
2.761\end{array}$ & 2.213 & \multirow[t]{2}{*}{7.016} & \\
\hline & C5 & -174.60 & -115.08 & 122.80 & 5.49 & 69.95 & -22.53 & 43.62 & 27.79 & -64.03 & & & & 2.407 & 2.599 & & \\
\hline \multirow[b]{2}{*}{17} & $\mathrm{C} 2$ & 163.40 & -136.67 & 102.34 & -17.40 & 20.65 & -75.22 & -41.18 & -29.51 & 62.24 & \multirow[b]{2}{*}{-56.27} & \multirow[b]{2}{*}{-169.93} & \multirow[b]{2}{*}{-54.20} & 2.444 & 2.468 & \multirow[b]{2}{*}{5.904} & 2.691 \\
\hline & C5 & 176.45 & -152.39 & 87.95 & -30.86 & 45.59 & 38.51 & 65.19 & -37.77 & 64.17 & & & & 2.565 & $\begin{array}{c}2.347 \\
2.648\end{array}$ & & 2.815 \\
\hline \multirow{2}{*}{26} & $\mathrm{C} 2$ & 178.31 & -135.58 & 102.71 & -18.20 & 30.22 & -85.98 & -34.18 & -45.32 & -151.73 & \multirow{2}{*}{90.98} & \multirow{2}{*}{85.21} & \multirow{2}{*}{-167.92} & 2.320 & 2.569 & \multirow{2}{*}{5.417} & \multirow[t]{2}{*}{2.339} \\
\hline & C5 & 177.68 & -159.46 & 79.10 & -37.75 & -70.73 & -43.80 & -17.96 & 32.79 & -71.90 & & & & 2.298 & 2.613 & & \\
\hline
\end{tabular}

[a] Conformers are numbered according to their appearance during conformational search; $[\mathrm{b}]-\alpha=\mathrm{C}_{\mathrm{Tr}}-\mathrm{C}(=\mathrm{O})-\mathrm{O}-\mathrm{C}^{*} ;[\mathrm{c}]-B=\mathrm{O}=\mathrm{C}-\mathrm{C}-\mathrm{C}_{i p s o} ;[\mathrm{d}]-\gamma=(\mathrm{O}=) \mathrm{C}-\mathrm{C}_{\mathrm{Tr}}-\mathrm{C}_{i p s o}-\mathrm{C}_{\text {ortho }}$ (of the two possibilities the absolute values $\leq 90^{\circ}$ has been chosen); $[\mathrm{e}]-\delta=\mathrm{C}(=\mathrm{O})-\mathrm{O}-\mathrm{C}^{*}-\mathrm{H}$; $[\mathrm{f}]-\zeta=\mathrm{O}-\mathrm{C}^{*}-\mathrm{C}-\mathrm{C}\left({ }^{*}\right)$; $[\mathrm{g}] \varphi_{1}=\mathrm{C} 1-\mathrm{C} 2-\mathrm{C} 3-\mathrm{C} 4 ; \varphi_{2}=\mathrm{C} 2-\mathrm{C} 3-\mathrm{C} 4-\mathrm{C} 5 ; \varphi_{3}=$ $\mathrm{C} 3-\mathrm{C} 4-\mathrm{C} 5-\mathrm{C} 6 ;[\mathrm{h}]-\mathrm{I}_{1}=\mathrm{C}=\mathrm{O} \cdots \mathrm{HC}_{\text {ortho; }}[\mathrm{i}]-\mathrm{I}_{2}=(\mathrm{O}=) \mathrm{CO} \cdots \mathrm{HC}_{\text {ortho; }}[\mathrm{j}]-\mathrm{I}_{3}=\mathrm{C}_{\mathrm{Tr}} \cdots \mathrm{C}_{\mathrm{Tr}} ;[\mathrm{k}]-\mathrm{I}_{4}=\mathrm{C}=\mathrm{O} \cdots \mathrm{HC}$ ortho; distance between $\mathrm{C}=\mathrm{O}$ and $\mathrm{HC}$ ortho of the second trityl group. 


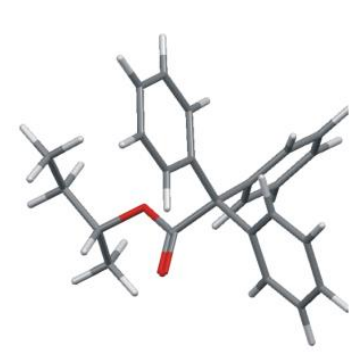

$1(\operatorname{conf} 1)$

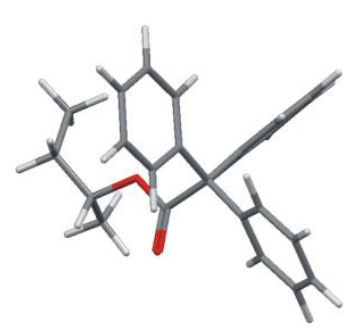

1 (conf 39)

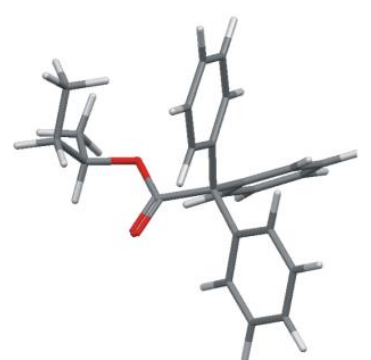

1 (conf 17)

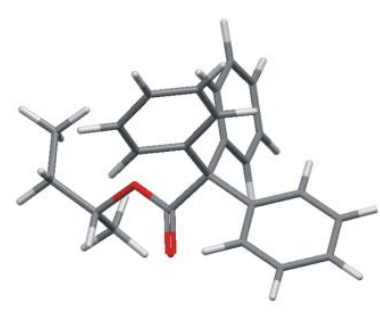

1 (conf 33)

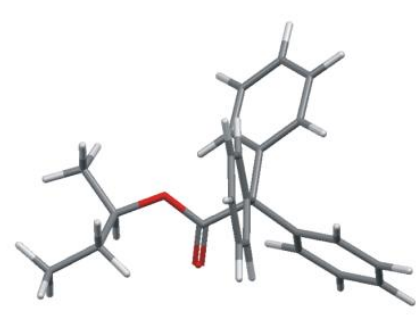

$1(\operatorname{conf} 38)$

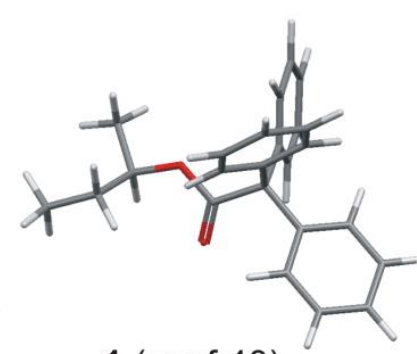

1 (conf 40)

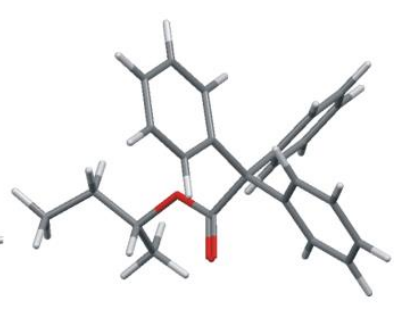

$1(\operatorname{conf} 41)$

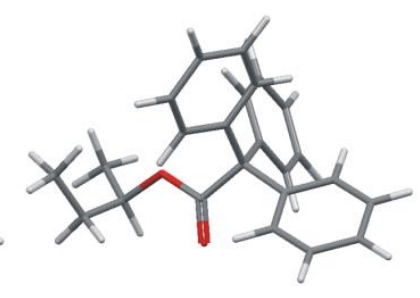

$1(\operatorname{conf} 42)$

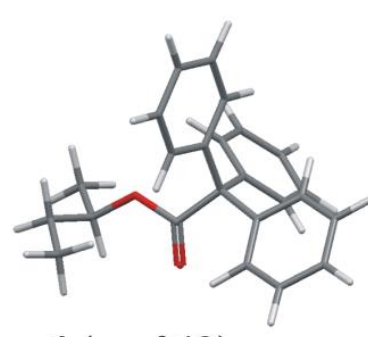

$1(\operatorname{conf} 43)$
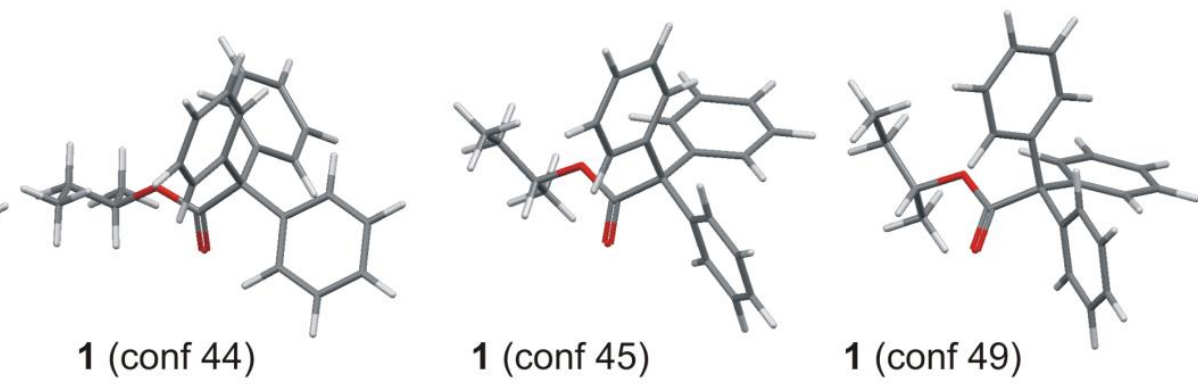

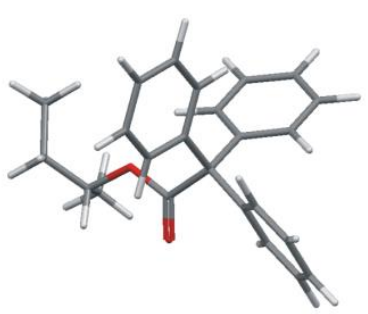

1 (conf 50)
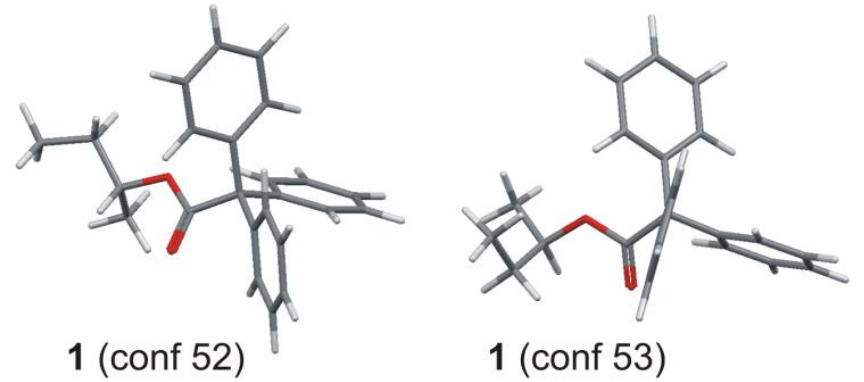

Figure SI_1. Structures of individual, low-energy conformers of 1, calculated at the B3LYP/6$311++G(d, p)$ level of theory. 


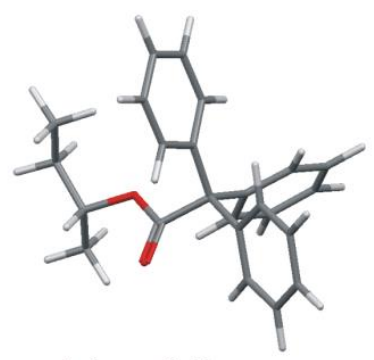

1 (conf 1)

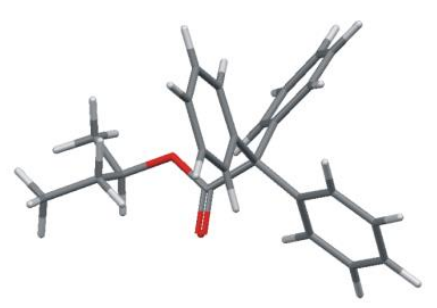

1 (conf 38)

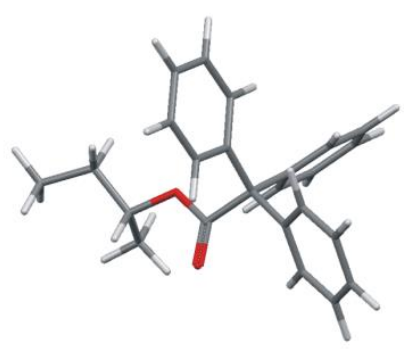

$1(\operatorname{conf} 41)$

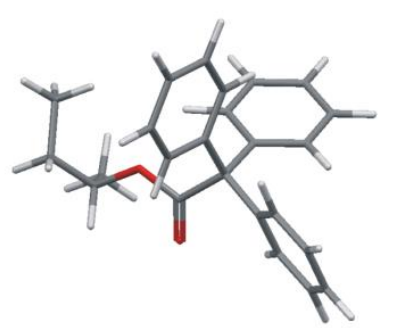

$1(\operatorname{conf} 50)$

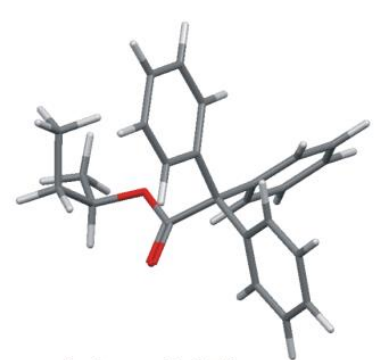

1 (conf 17)

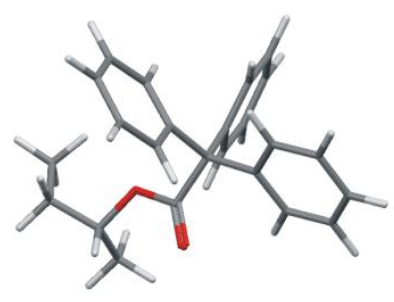

1 (conf 39)

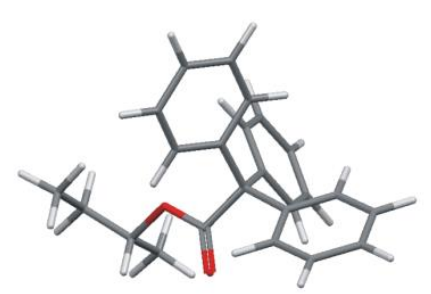

1 (conf 44)

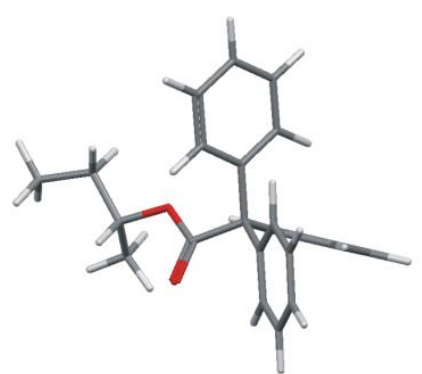

1 (conf 52)

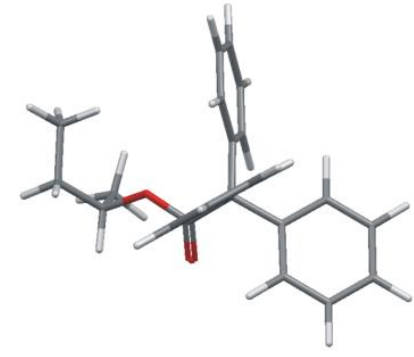

1 (conf 33)

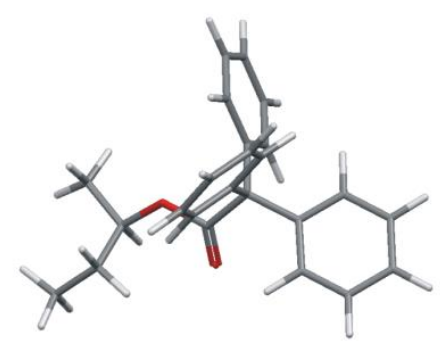

$1(\operatorname{conf} 40)$
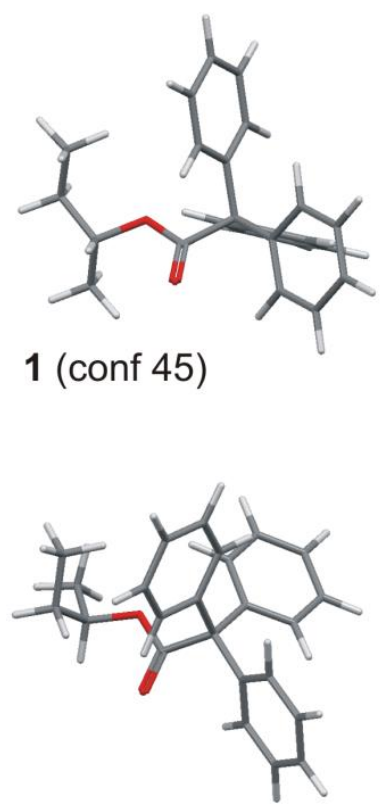

$1(\operatorname{conf} 53)$

Figure SI_2. Structures of individual, low-energy conformers of 1, calculated at the M06-2X/6$311++G(d, p)$ level of theory. 


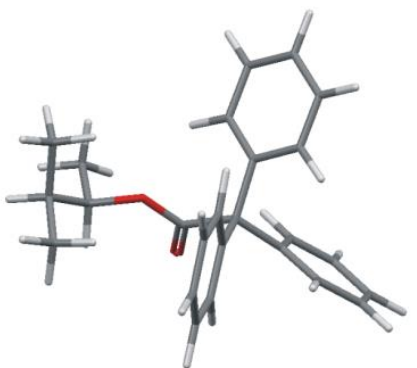

$4(\operatorname{conf} 1)$

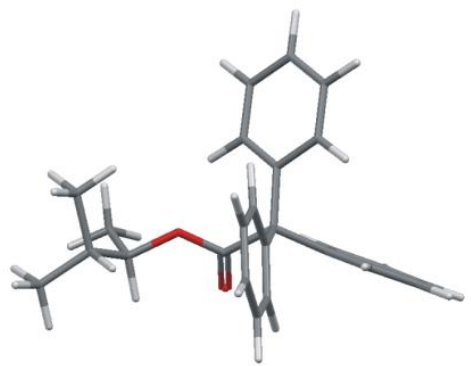

$4(\operatorname{conf} 42)$

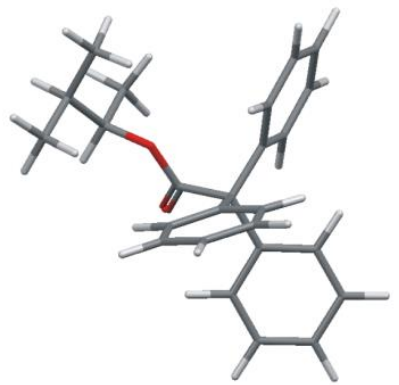

$4(\operatorname{conf} 20)$

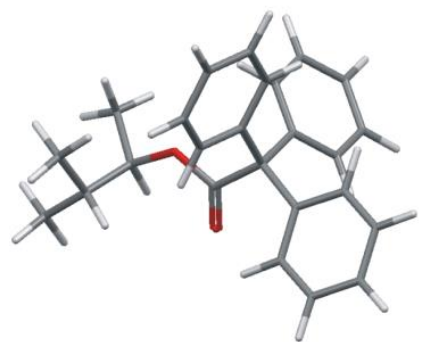

$4(\operatorname{conf} 44)$

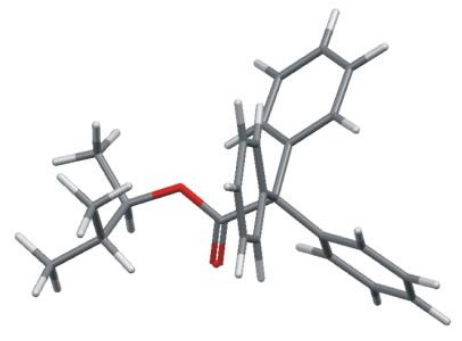

$4(\operatorname{conf} 22)$

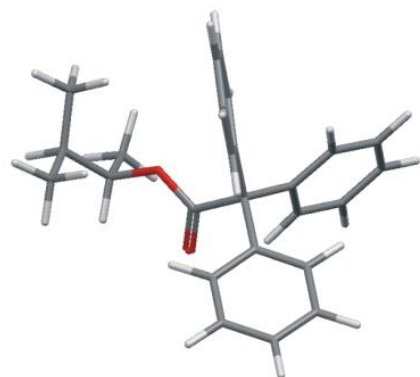

$4(\operatorname{conf} 46)$

Figure SI_3. Structures of individual, low-energy conformers of 4, calculated at the B3LYP/6$311++G(d, p)$ level of theory.

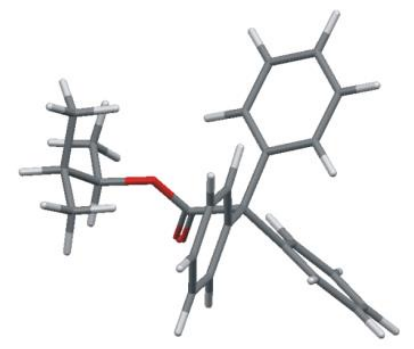

$4(\operatorname{conf} 1)$

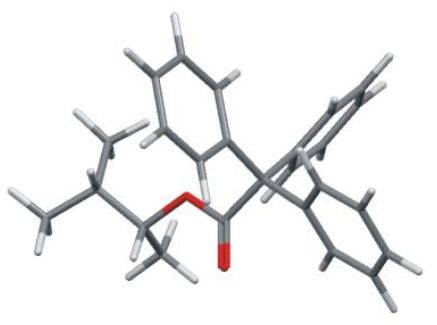

4 (conf 42)

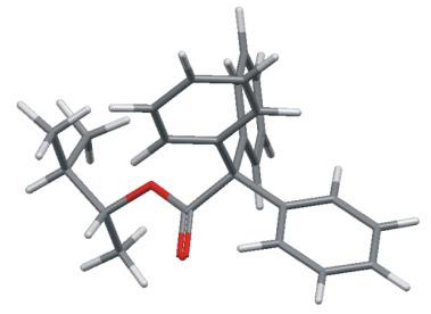

$4(\operatorname{conf} 20)$

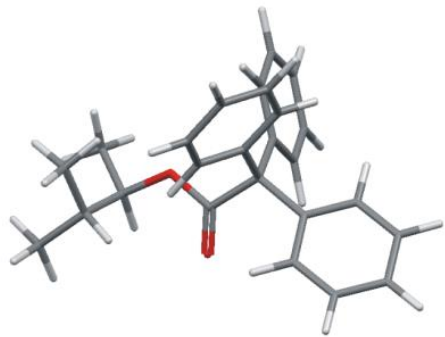

$4(\operatorname{conf} 44)$

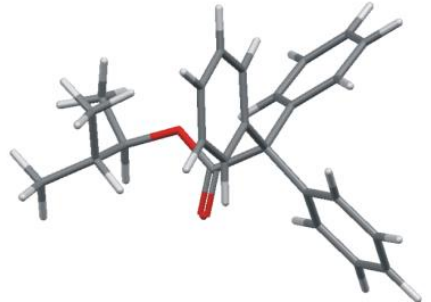

$4(\operatorname{conf} 22)$

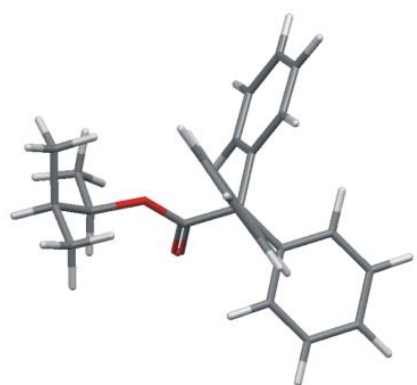

$4(\operatorname{conf} 46)$

Figure SI_4. Structures of individual, low-energy conformers of 4, calculated at the M06-2X/6$311++G(d, p)$ level of theory. 


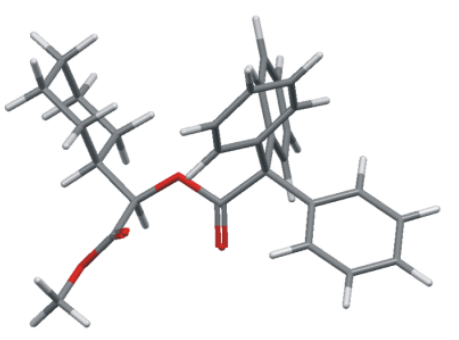

$6(\operatorname{conf} 1)$

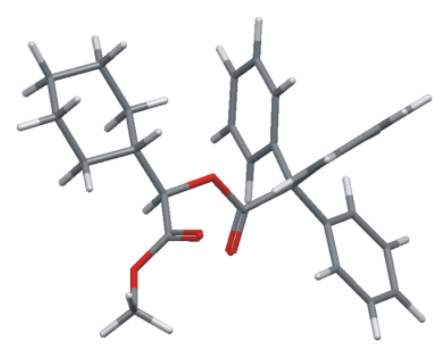

$6(\operatorname{conf} 8)$

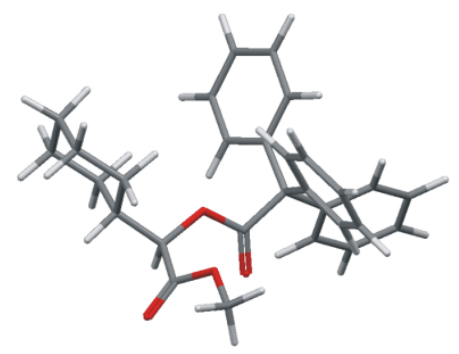

$6(\operatorname{conf} 17)$

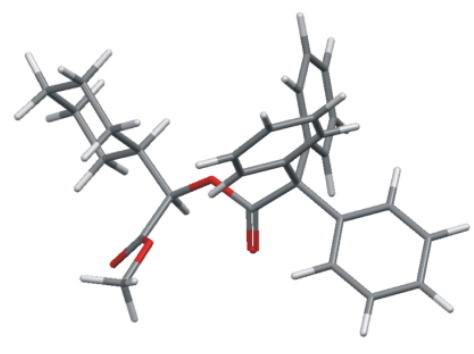

$6(\operatorname{conf} 21)$
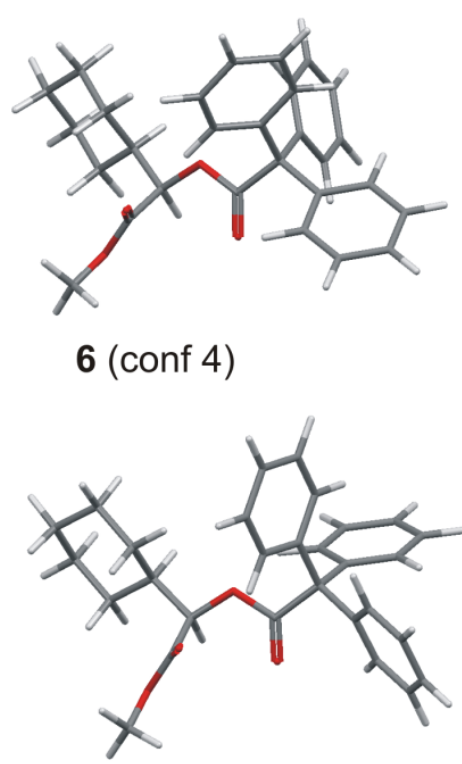

$6(\operatorname{conf} 10)$

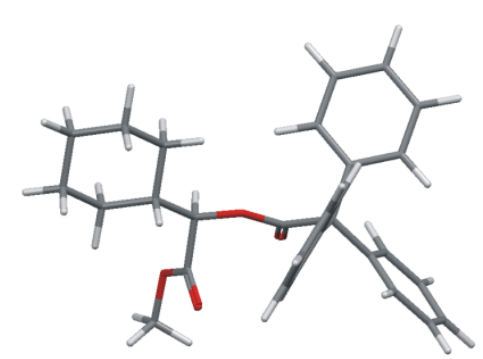

6 (conf 18)

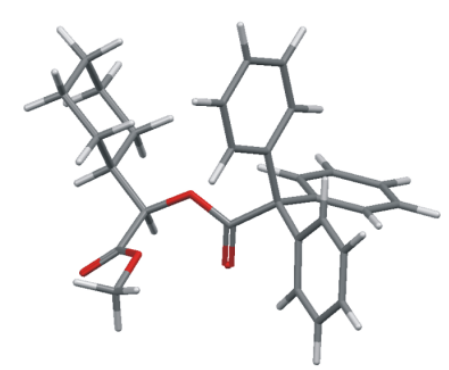

$6(\operatorname{conf} 28)$
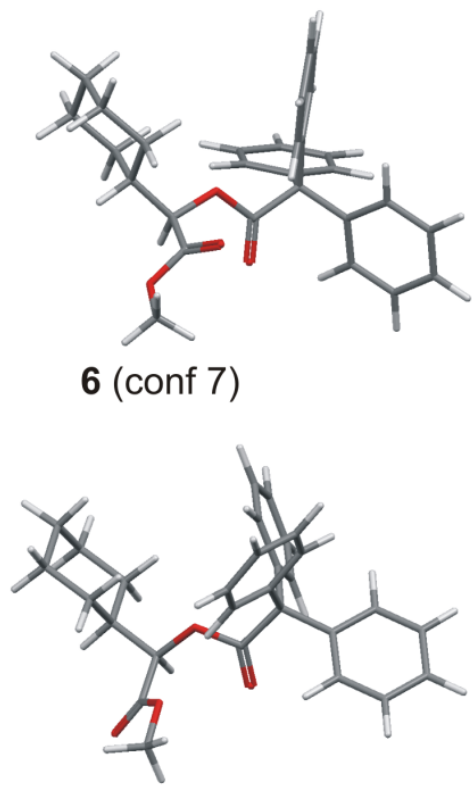

$6(\operatorname{conf} 14)$

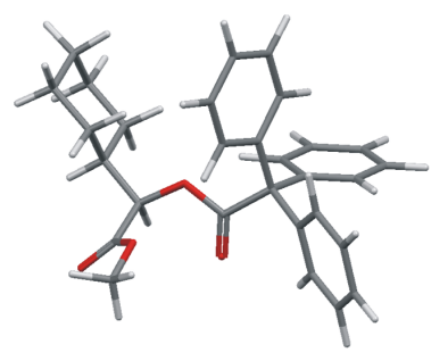

6 (conf 20)

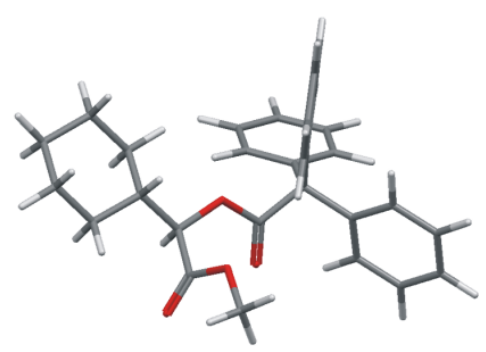

$6(\operatorname{conf} 46)$

Figure SI_5. Structures of individual, low-energy conformers of 6, calculated at the B3LYP/6$311++G(d, p)$ level of theory. 


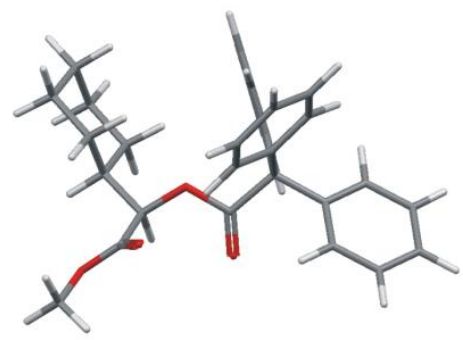

$6(\operatorname{conf} 1)$

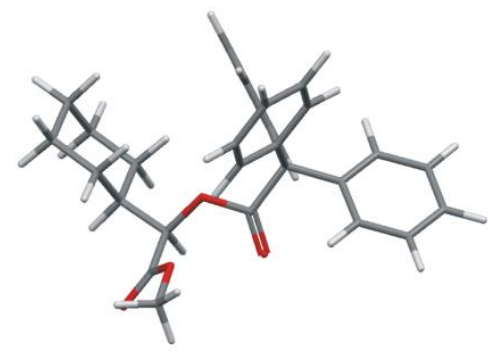

$6(\operatorname{conf} 14)$

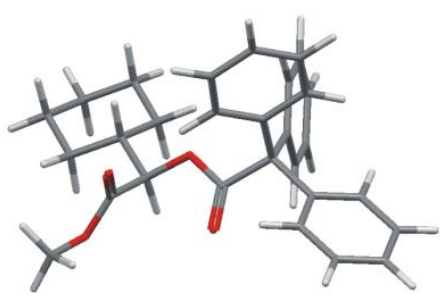

$6(\operatorname{conf} 30)$

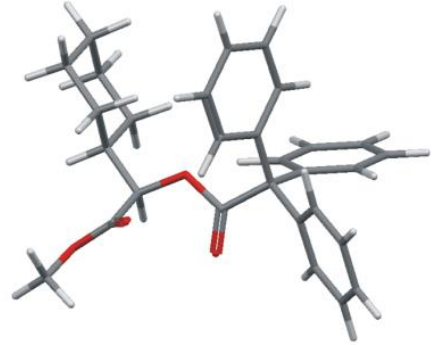

$6(\operatorname{conf} 7)$

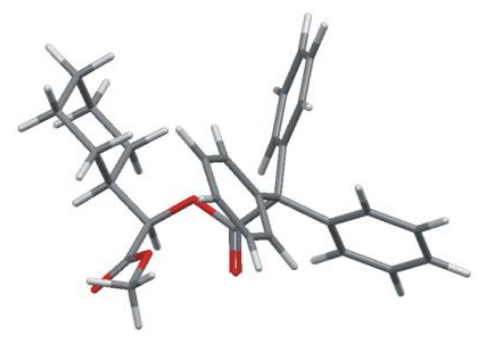

$6(\operatorname{conf} 17)$

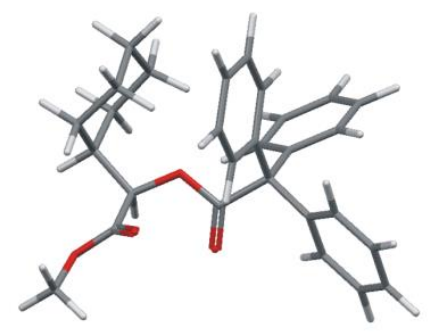

$6(\operatorname{conf} 32)$

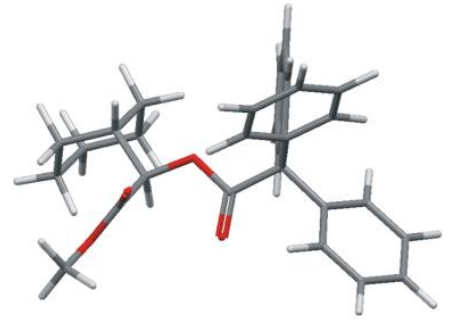

$6(\operatorname{conf} 11)$

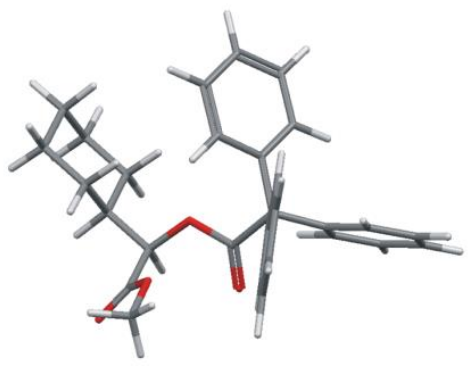

6 (conf 28)

Figure SI_6. Structures of individual, low-energy conformers of 6, calculated at the M06-2X/6$311++G(d, p)$ level of theory. 


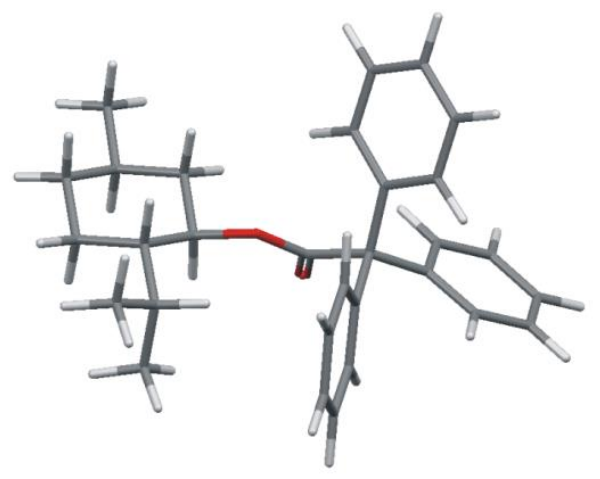

$10($ conf 1)

Figure SI_7. Structure of individual, low-energy conformer of 10, calculated at the B3LYP/6$311++G(d, p)$ level of theory.

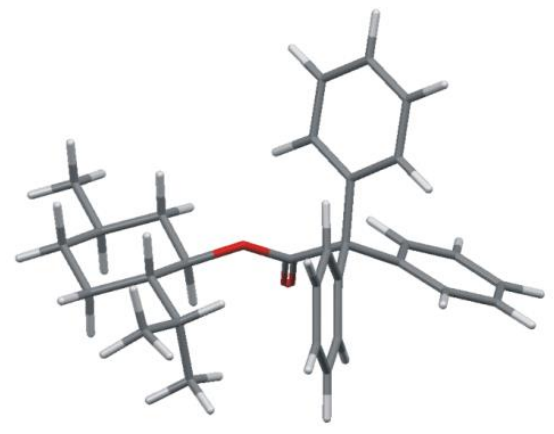

$10(\operatorname{conf} 1)$

Figure SI_8. Structure of individual, low-energy conformer of 10, calculated at the M06-2X/6$311++G(d, p)$ level of theory. 


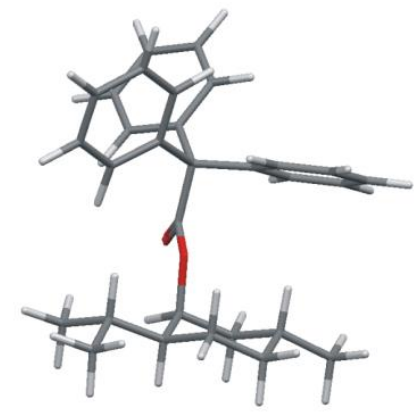

$11(\operatorname{conf} 1)$

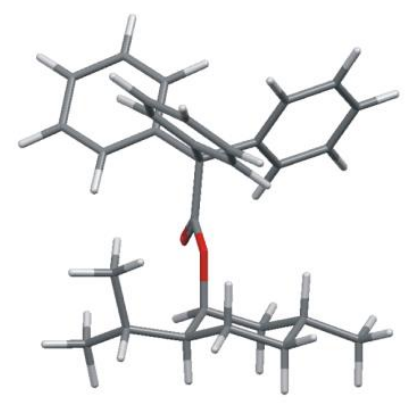

$11(\operatorname{conf} 52)$

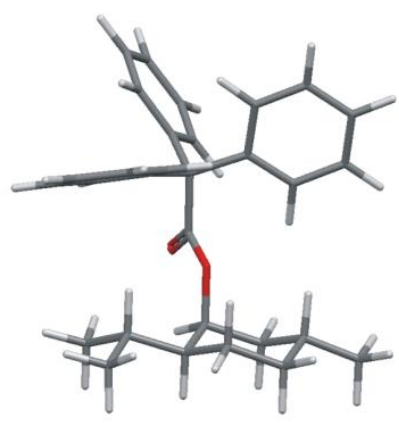

$11(\operatorname{conf} 22)$

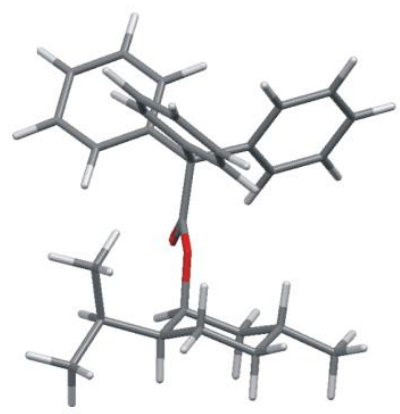

$11(\operatorname{conf} 56)$

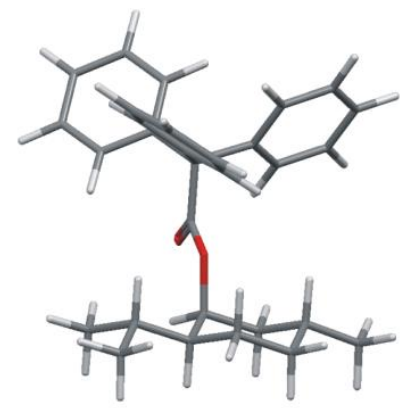

$11(\operatorname{conf} 27)$

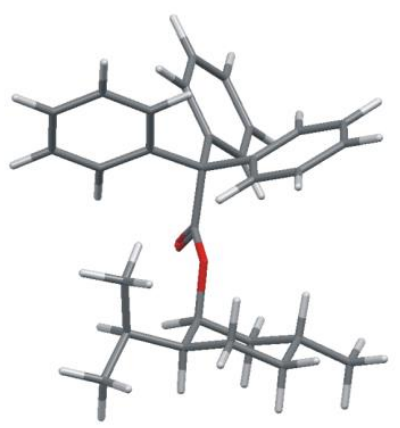

$11(\operatorname{conf} 62)$

Figure SI_9. Structures of individual, low-energy conformers of 11, calculated at the B3LYP/6$311++G(d, p)$ level of theory. 


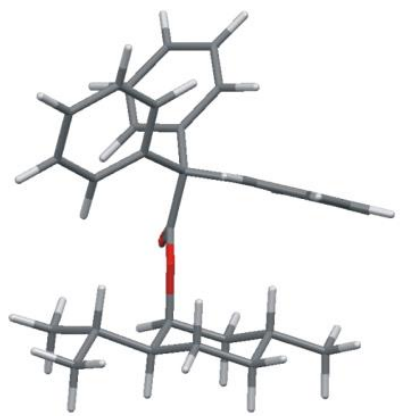

$11(\operatorname{conf} 1)$

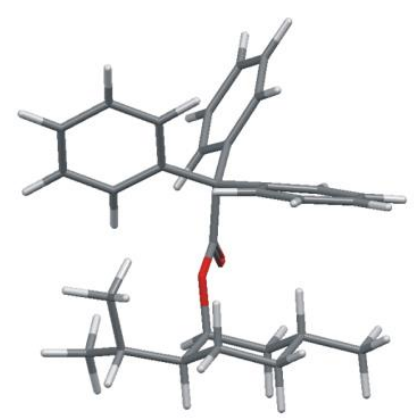

$11(\operatorname{conf} 33)$

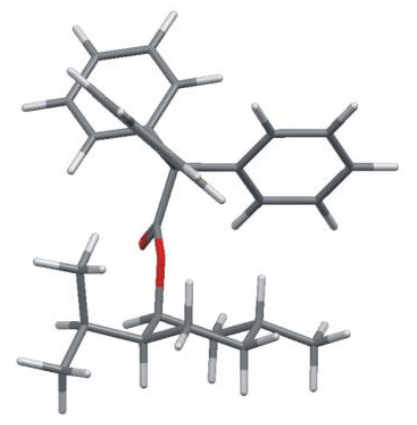

11 (conf 56)

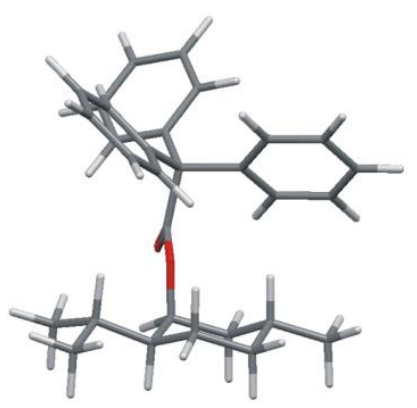

11 (conf 27)

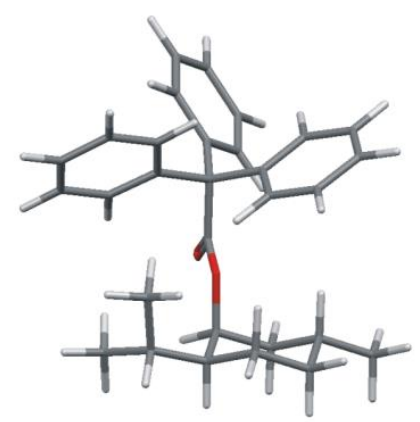

11 (conf 50)

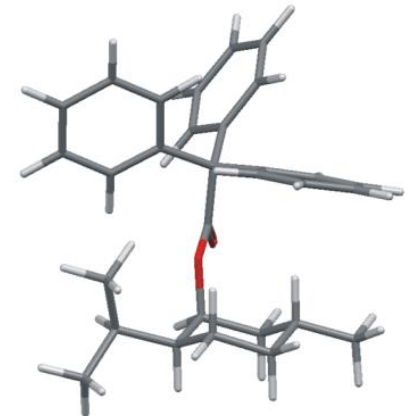

$11($ conf 31)

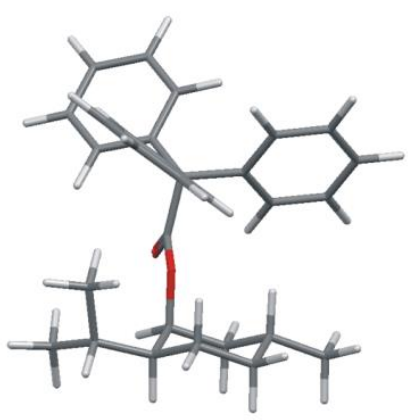

$11(\operatorname{conf} 52)$

Figure SI_10. Structures of individual, low-energy conformers of 11, calculated at the M06-2X/6$311++G(d, p)$ level of theory. 


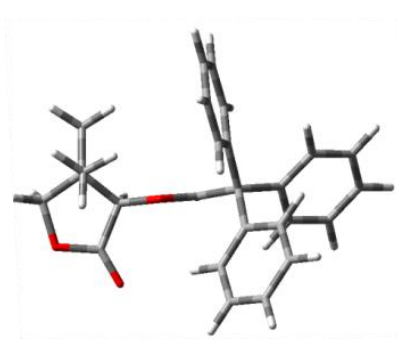

14 (conf. 1)

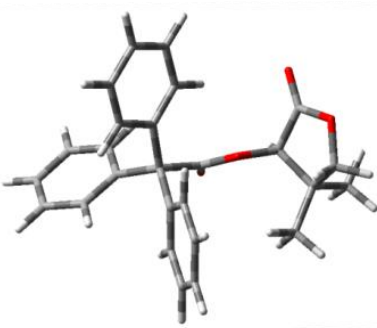

14 (conf. 6)

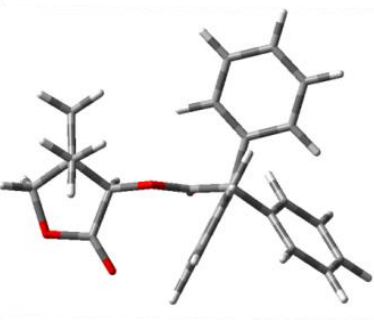

14 (conf. 8)

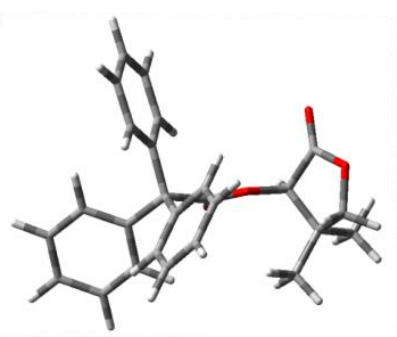

14 (conf. 39)

Figure SI_11. Structures of individual, low-energy conformers of 14, calculated at the B3LYP/6$311++G(d, p)$ level of theory.

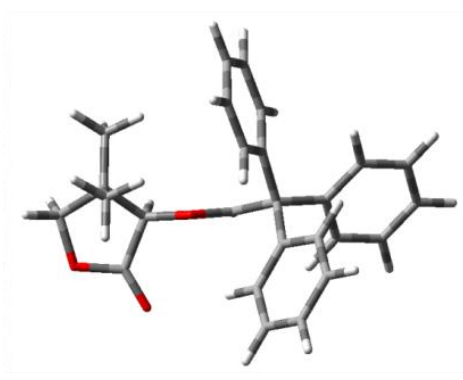

14 (conf. 1)

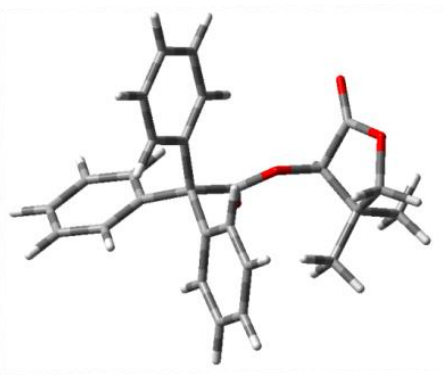

14 (conf. 6)

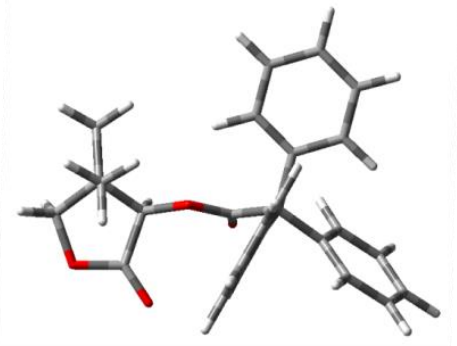

14 (conf. 8)

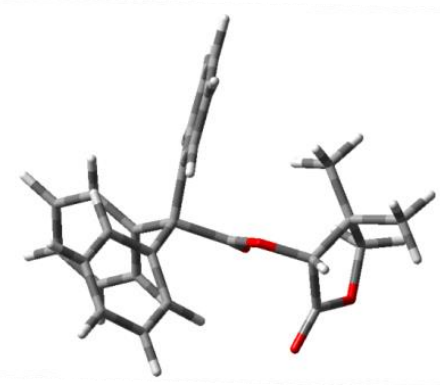

14 (conf. 27)

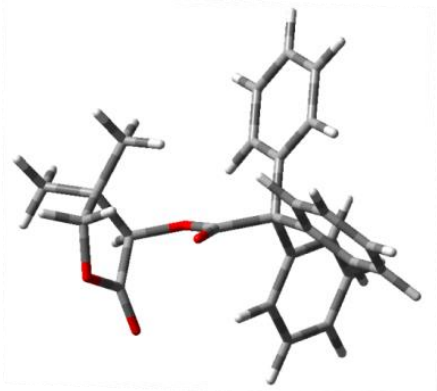

14 (conf. 49)

Figure SI_12. Structures of individual, low-energy conformers of 14, calculated at the M06-2X/6$311++G(d, p)$ level of theory. 


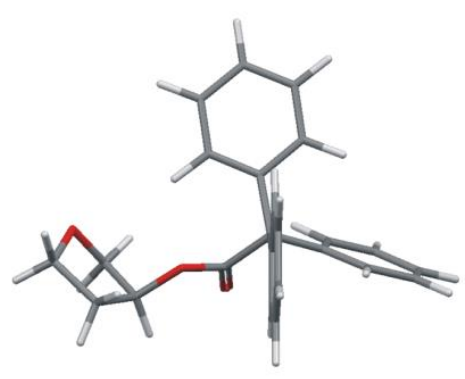

$15(\operatorname{conf} 1)$

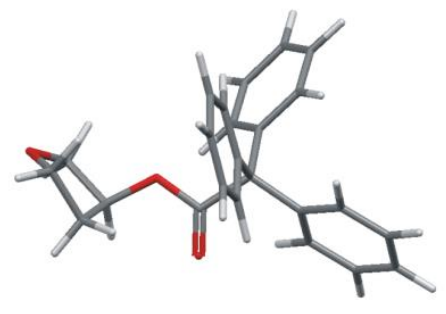

$15(\operatorname{conf} 20)$

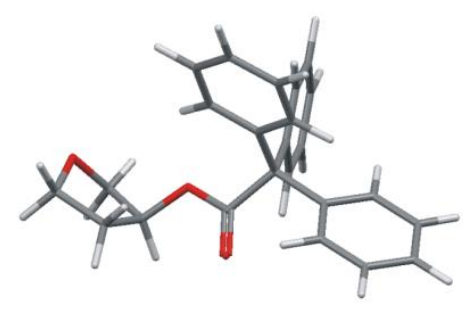

$15(\operatorname{conf} 54)$

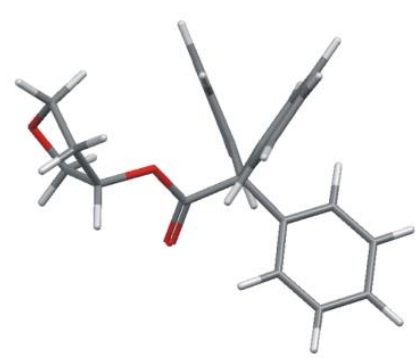

$15(\operatorname{conf} 17)$

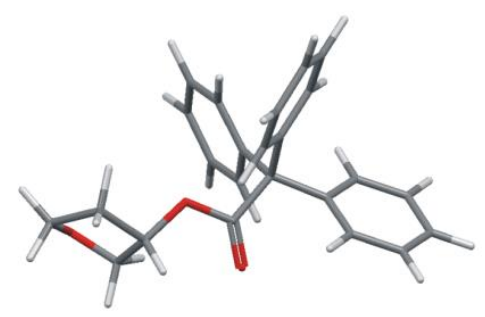

$15(\operatorname{conf} 34)$

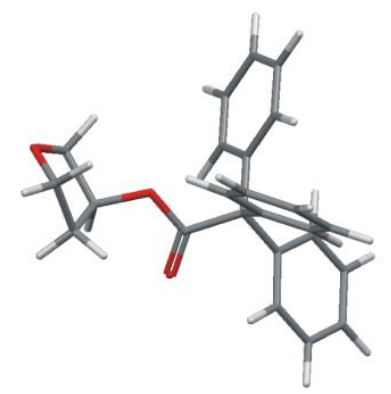

$15(\operatorname{conf} 65)$

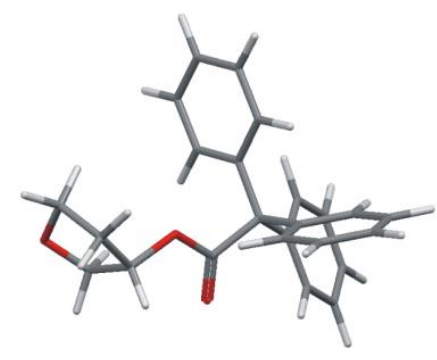

$15(\operatorname{conf} 18$

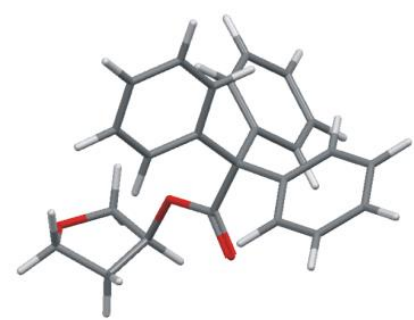

$15(\operatorname{conf} 47)$

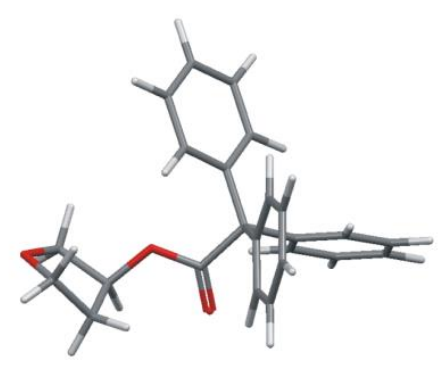

$15(\operatorname{conf} 70)$

Figure SI_13. Structures of individual, low-energy conformers of 15, calculated at the B3LYP/6$311++G(d, p)$ level of theory. 


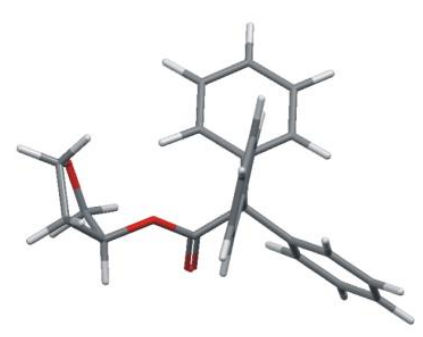

$15(\operatorname{conf} 1)$

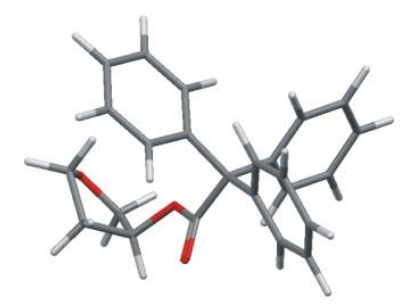

15 (conf 20)

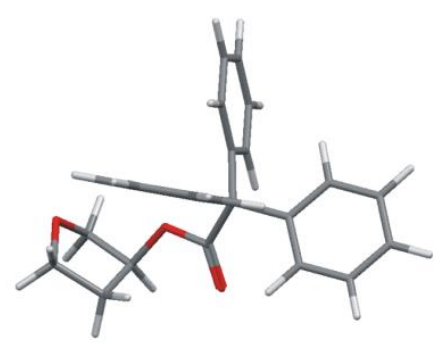

15 (conf 58)

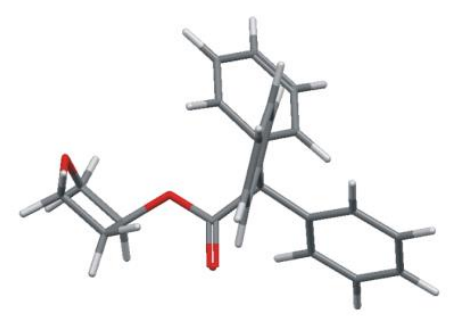

$15(\operatorname{conf} 75)$

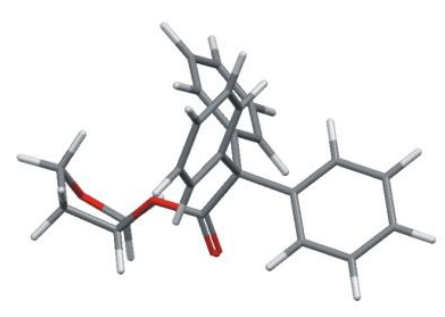

$15(\operatorname{conf} 17)$

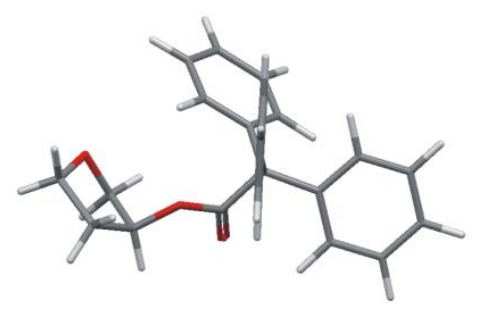

15 (conf 34)

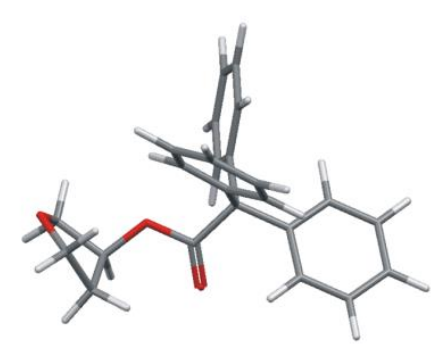

15 (conf 65)

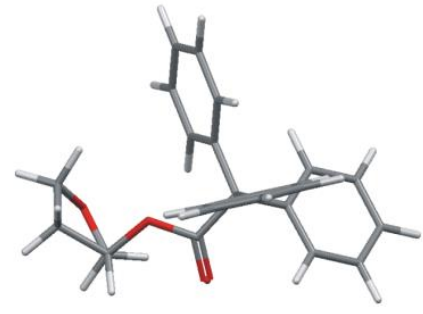

$15(\operatorname{conf} 18$

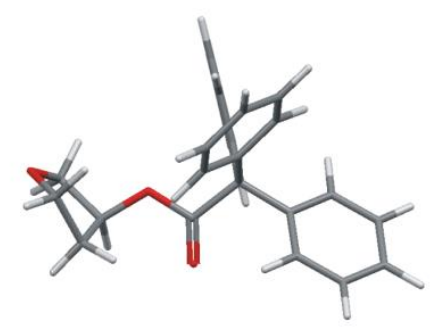

15 (conf 47)

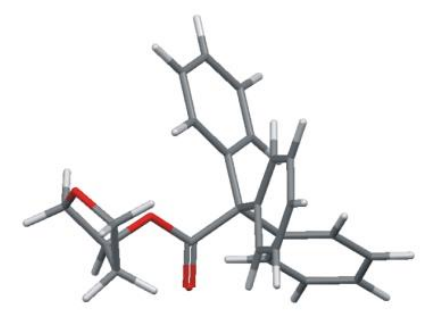

$15(\operatorname{conf} 70)$

Figure SI_14. Structures of individual, low-energy conformers of 15, calculated at the M06-2X/6$311++G(d, p)$ level of theory. 


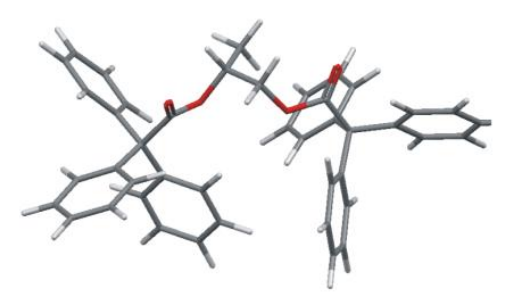

$18(\operatorname{conf} 1)$

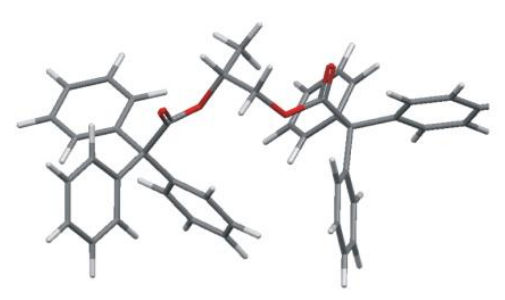

18 (conf 13)

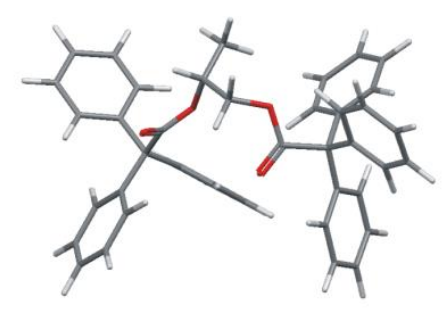

18 (conf 18)

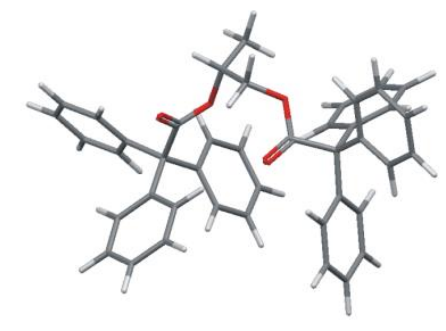

18 (conf 32)

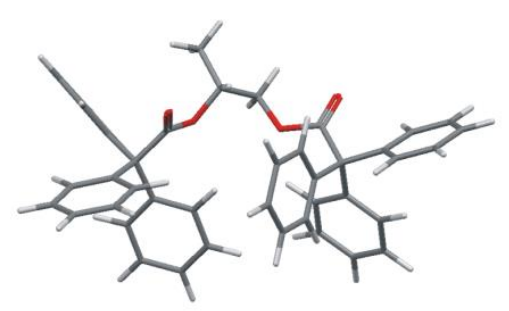

18 (conf 36)

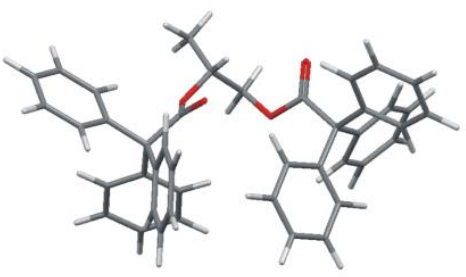

$18(\operatorname{conf} 5)$

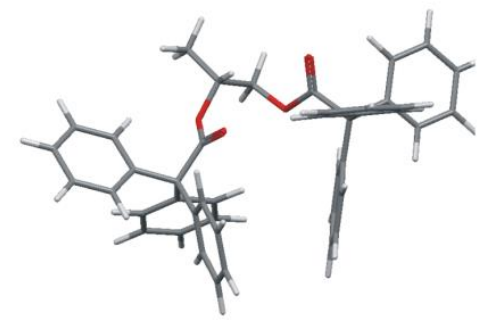

18 (conf 15)

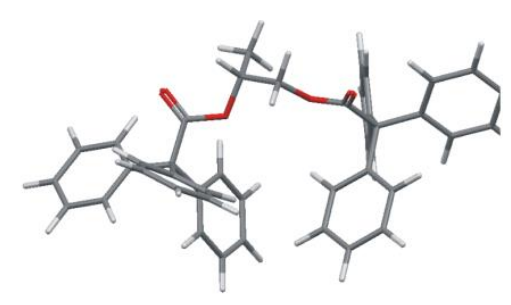

18 (conf 26)

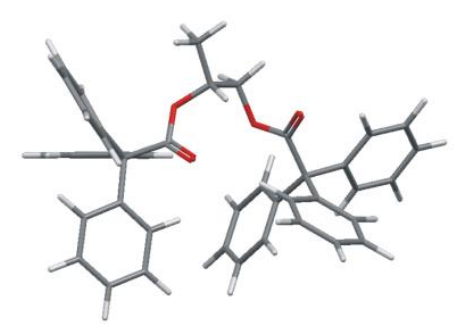

18 (conf 34)

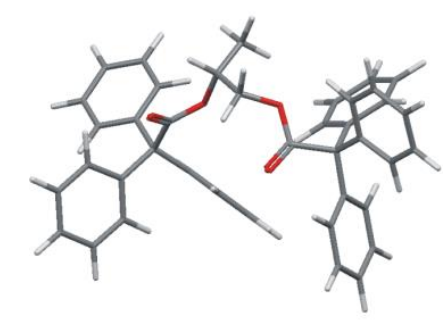

18 (conf 42)

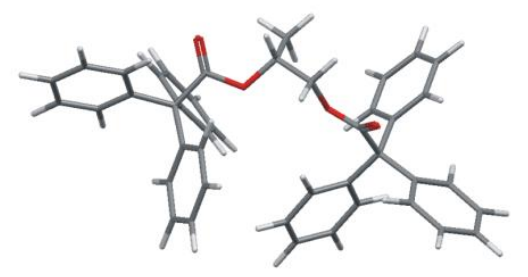

18 (conf 9)

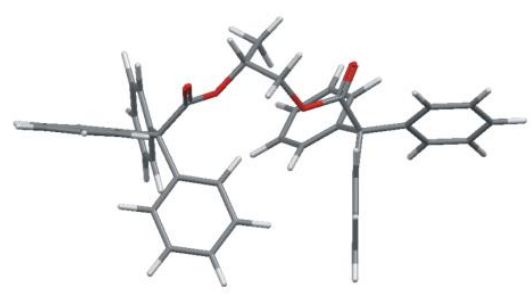

18 (conf 17)

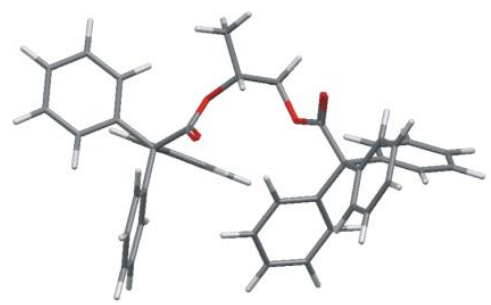

18 (conf 28)

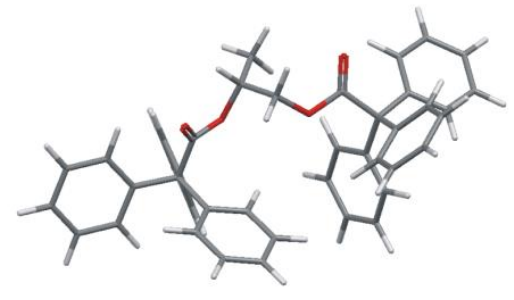

18 (conf 35)

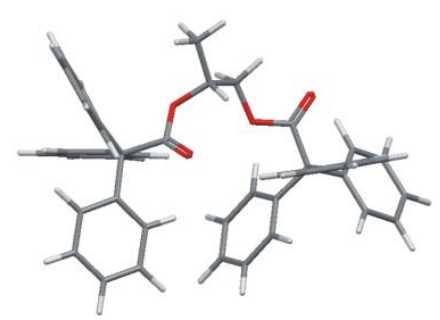

$18(\operatorname{conf} 43)$

Figure SI_15a. Structures of individual, low-energy conformers of 18, calculated at the B3LYP/6$311 G(d, p)$ level of theory, part I. 


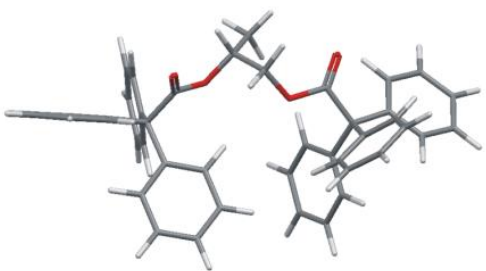

18 (conf 45)

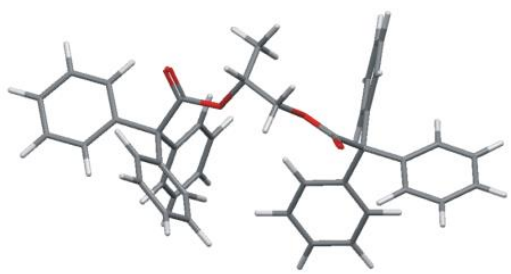

$18(\operatorname{conf} 58)$

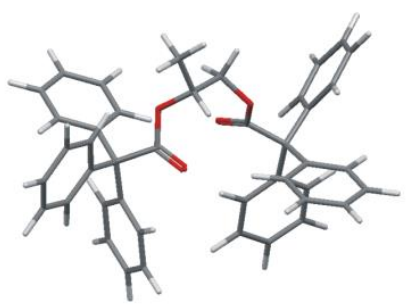

$18(\operatorname{conf} 61)$

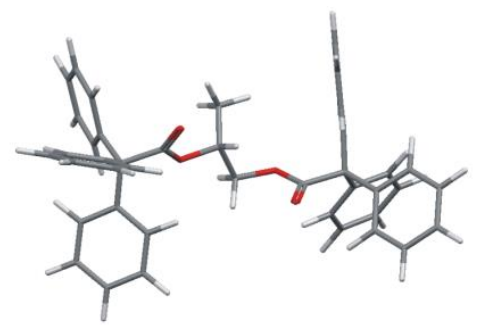

$18(\operatorname{conf} 71)$

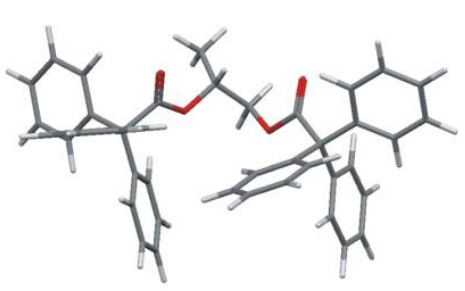

18 (conf 77)

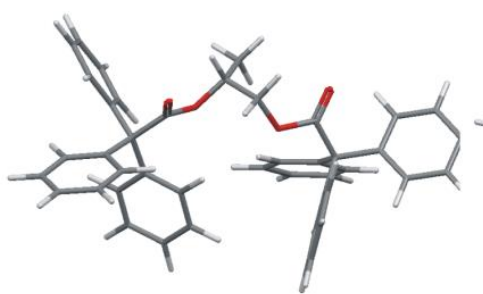

18 (conf 46)

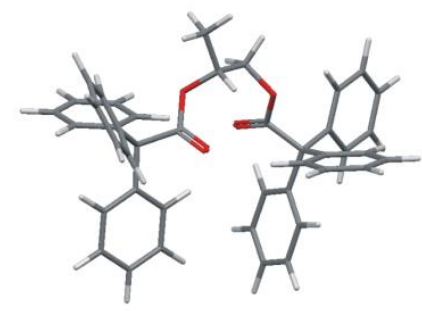

18 (conf 59)

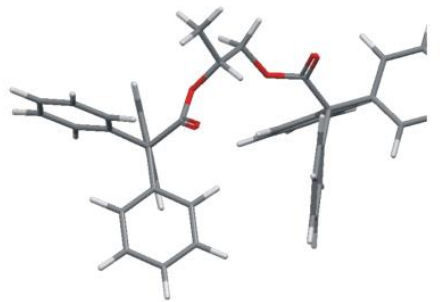

18 (conf 69)

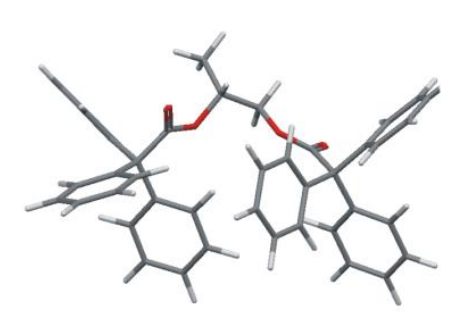

18 (conf 73)

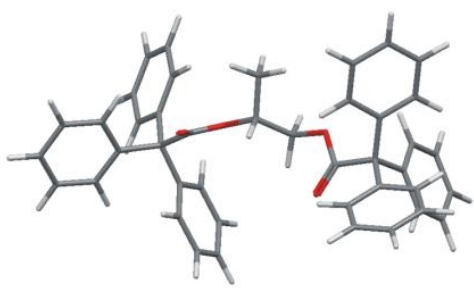

18 (conf 93)

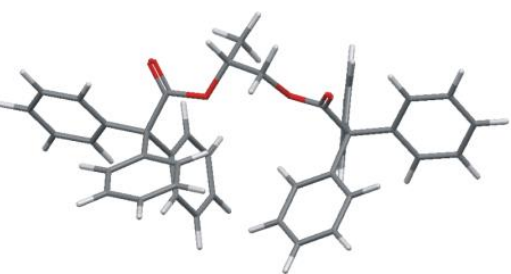

18 (conf 48)

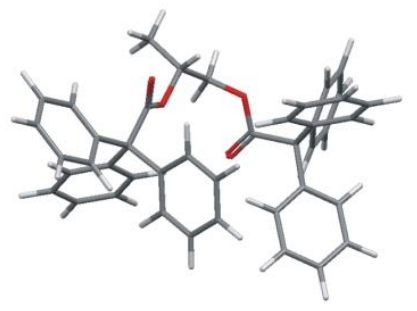

18 (conf 60)

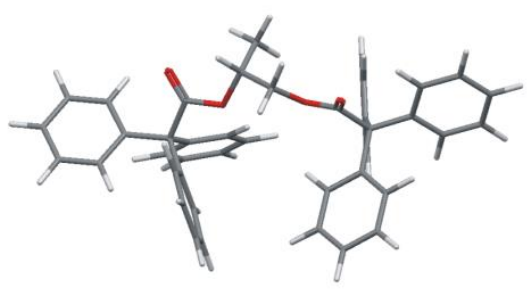

$18(\operatorname{conf} 70)$

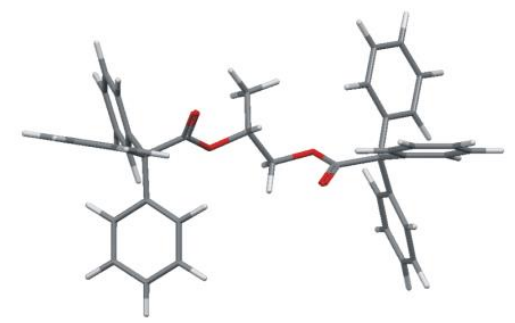

18 (conf 76)

Figure SI_15b. Structures of individual, low-energy conformers of 18, calculated at the B3LYP/6$311 G(d, p)$ level of theory, part II. 


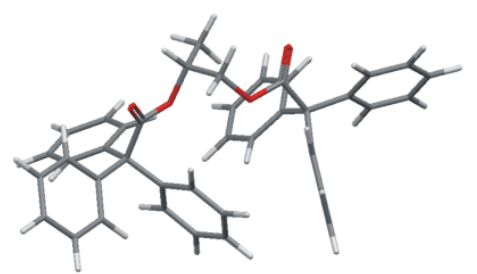

18 (conf 13)

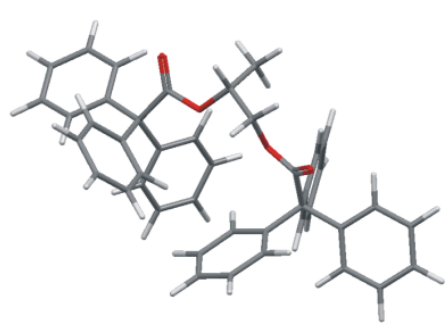

18 (conf 26)

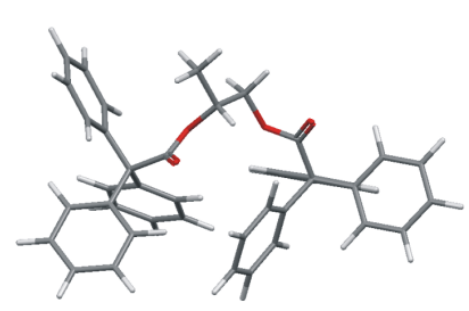

18 (conf 36)

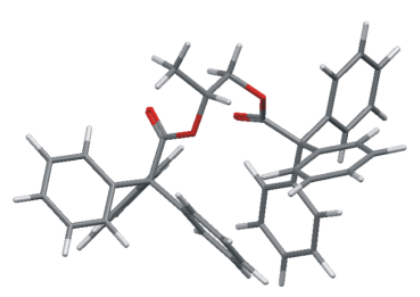

18 (conf 63)

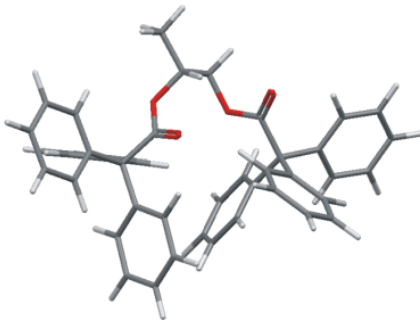

18 (conf 15)

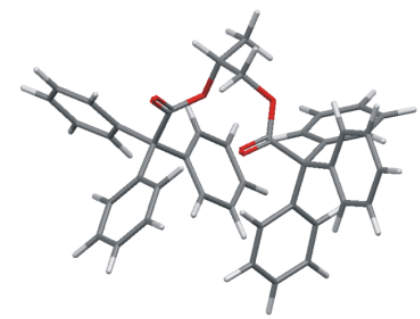

$18(\operatorname{conf} 32)$

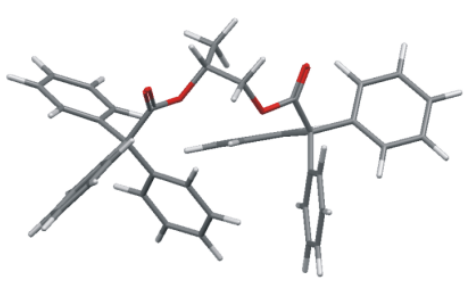

18 (conf 46)

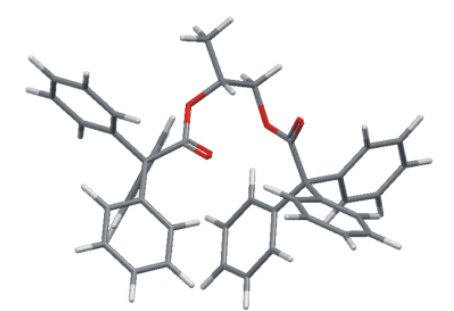

18 (conf 77)

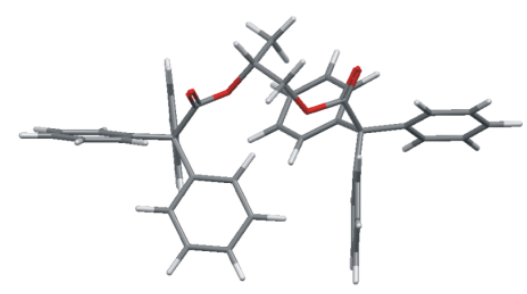

18 (conf 17)

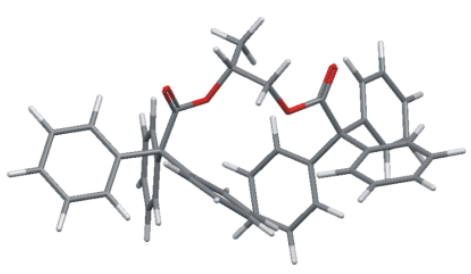

18 (conf 35)

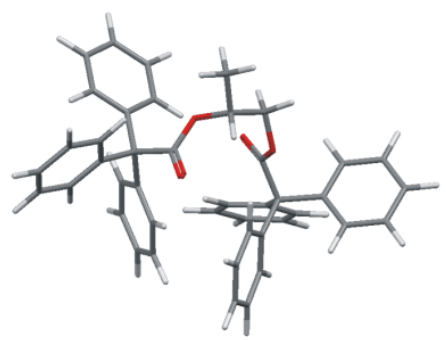

18 (conf 60$)$

Figure SI_16. Structures of individual, low-energy conformers of 11, calculated at the B3LYP-GD3BJ/6$311 G(d, p)$ level of theory. 


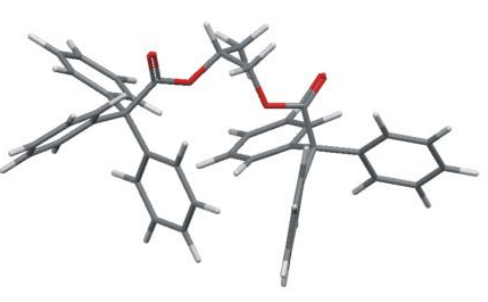

$18(\operatorname{conf} 1)$

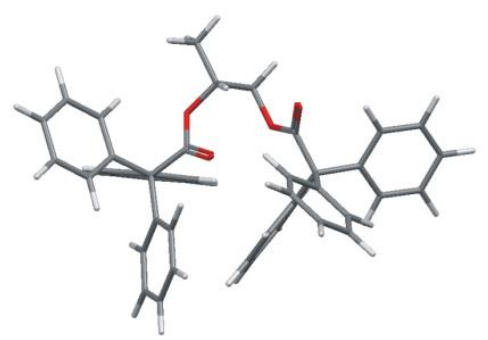

18 (conf 15)

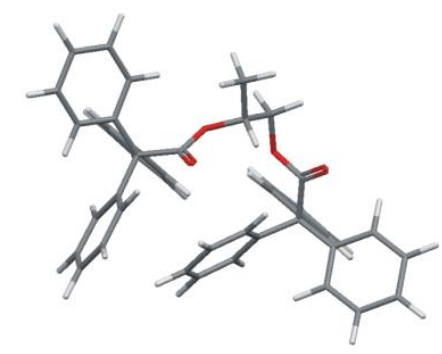

18 (conf 28)

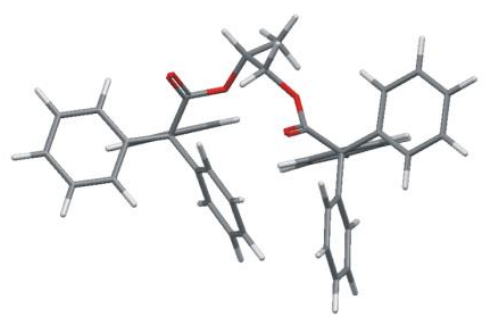

18 (conf 42)

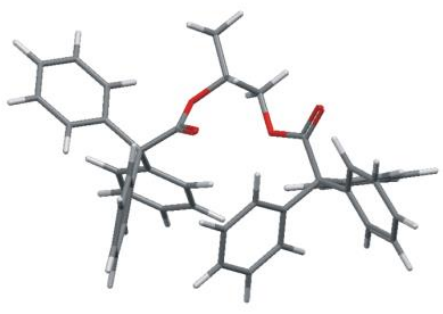

$18(\operatorname{conf} 5)$

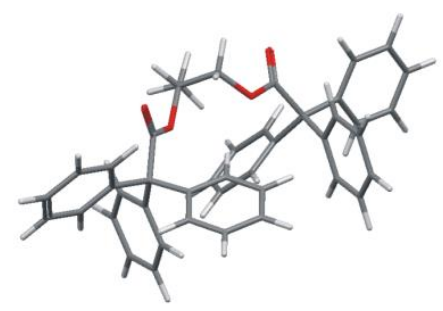

18 (conf 17)

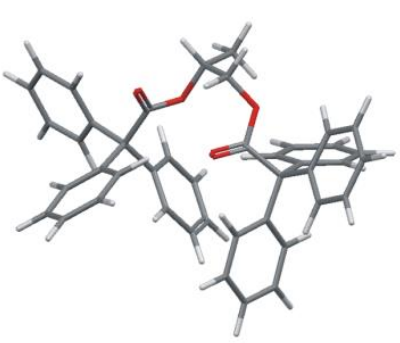

18 (conf 32)

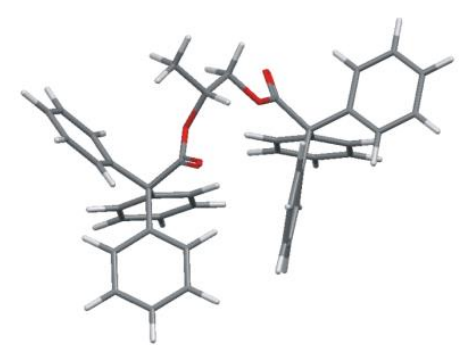

$18($ conf 73)

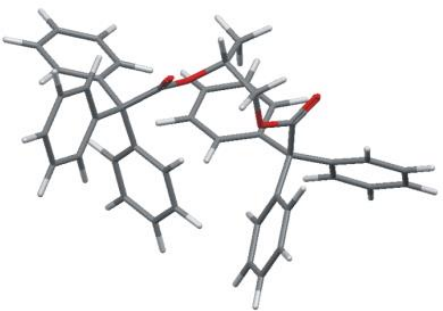

18 (conf 13)

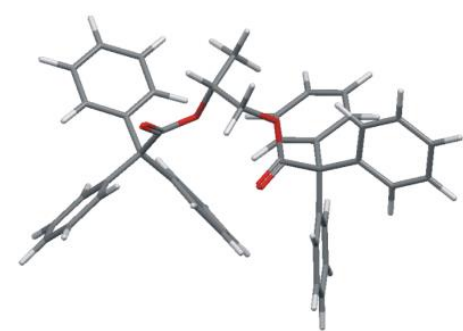

18 (conf 18)

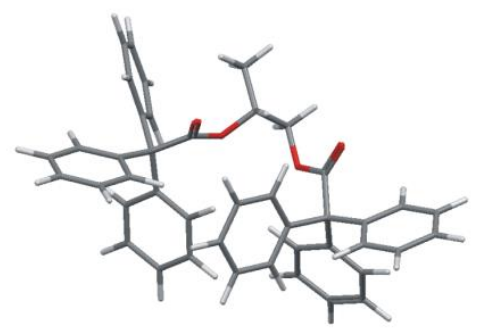

18 (conf 36)

Figure SI_17. Structures of individual, low-energy conformers of 18, calculated at the M06-2X/6$311 \mathrm{G}(\mathrm{d}, \mathrm{p})$ level of theory. 


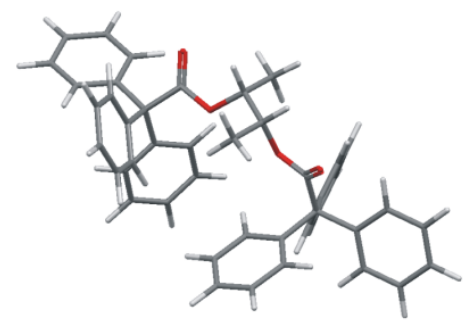

$20(\operatorname{conf} 1)$

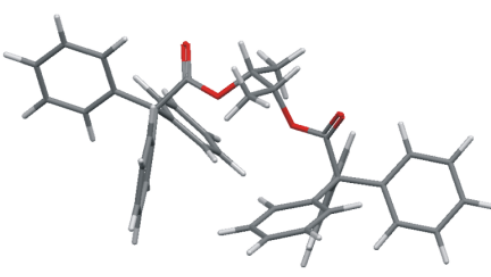

20 (conf 16)

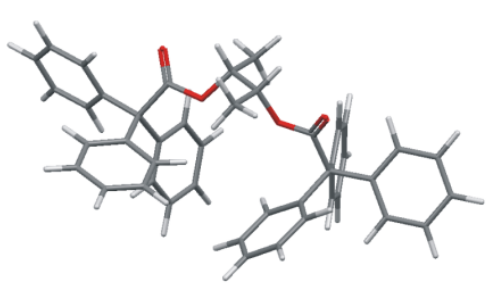

$20($ conf 21$)$

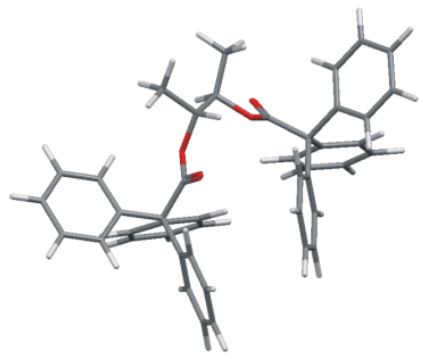

20 (conf 25)

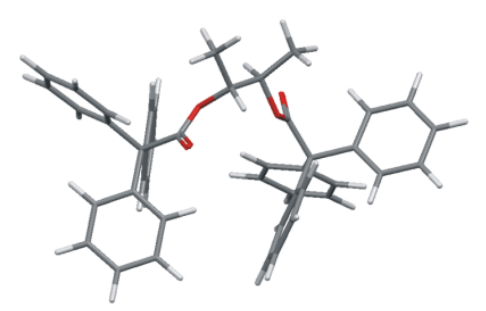

$20($ conf 47)

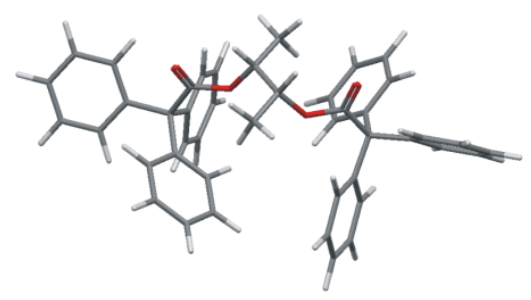

$20(\operatorname{conf} 11)$

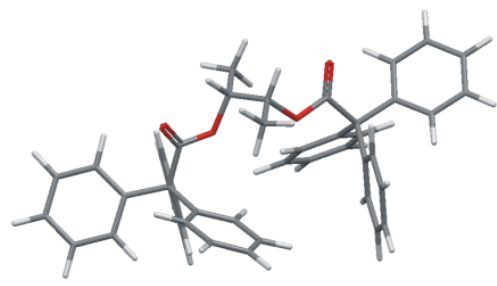

20 (conf 17)

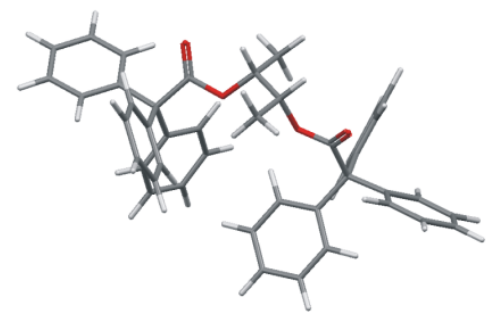

20 (conf 22)

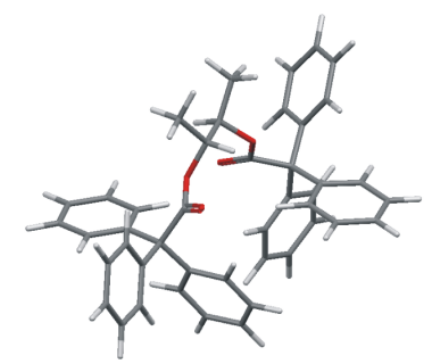

$20(\operatorname{conf} 43)$

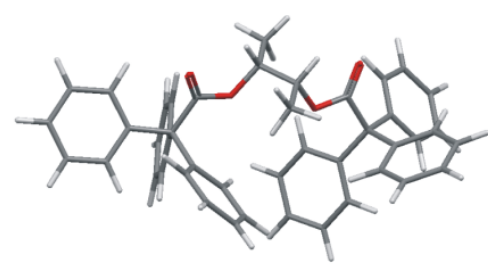

$20($ conf 14)

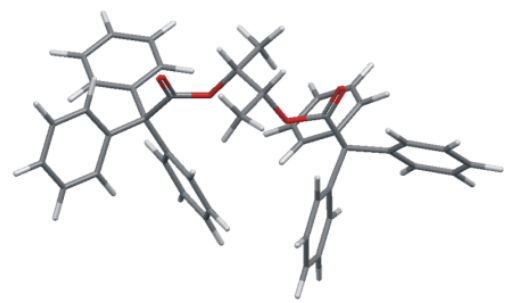

20 (conf 20)

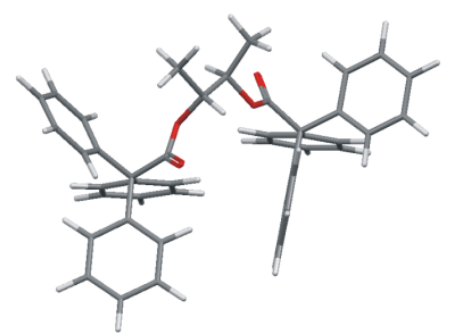

20 (conf 23)

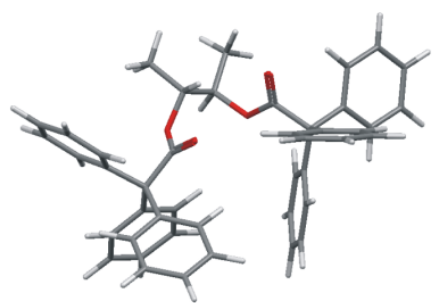

20 (conf 46)

Figure SI_18. Structures of individual, low-energy conformers of 20, calculated at the B3LYP/6$311 G(d, p)$ level of theory. 


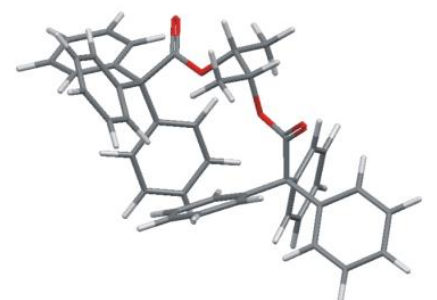

$20(\operatorname{conf} 1)$

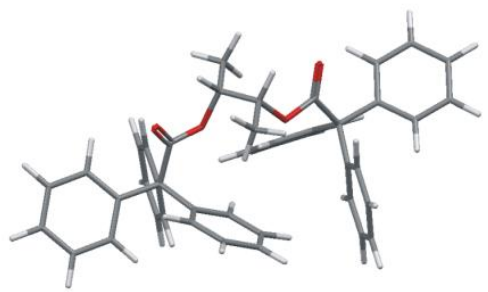

$20(\operatorname{conf} 17)$

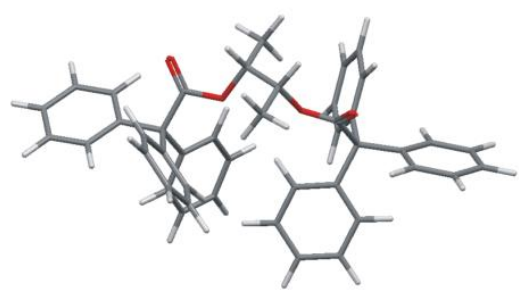

$20(\operatorname{conf} 22)$

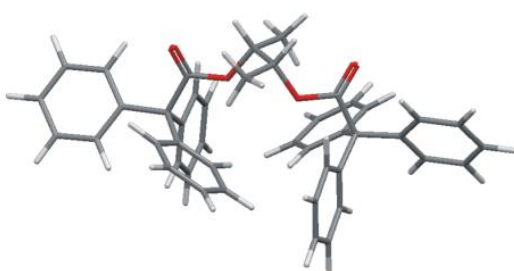

$20(\operatorname{conf} 11)$

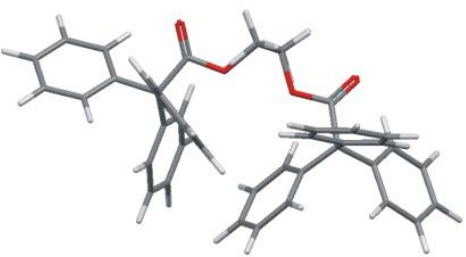

$20($ conf 14)

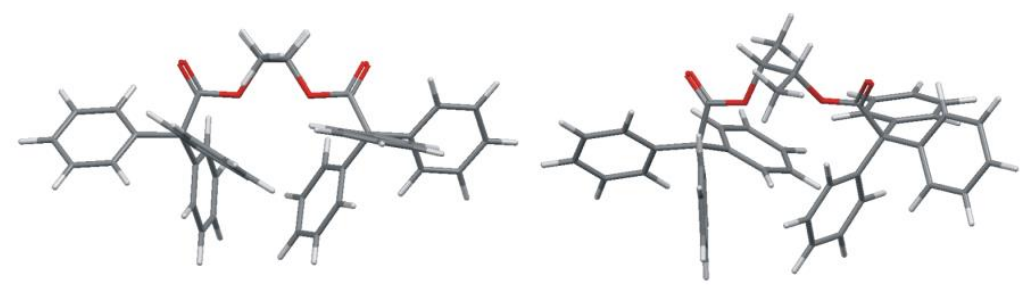

$20(\operatorname{conf} 20)$

$20($ conf 21)

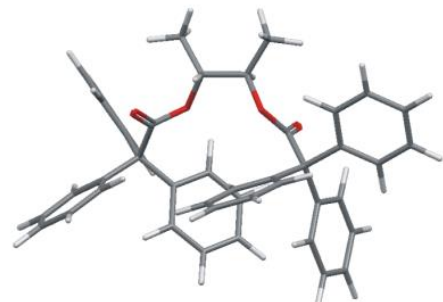

$20($ conf 25)

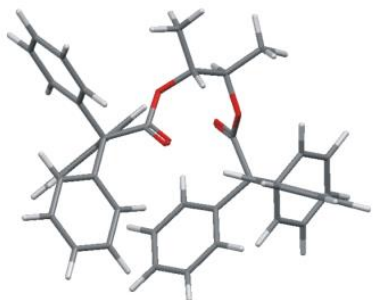

$20($ conf 43)

Figure SI_19. Structures of individual, low-energy conformers of 20, calculated at the B3LYP-GD3BJ/6$311 G(d, p)$ level of theory. 


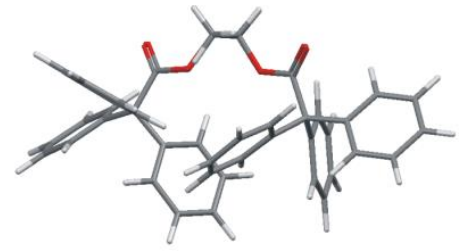

$20(\operatorname{conf} 1)$

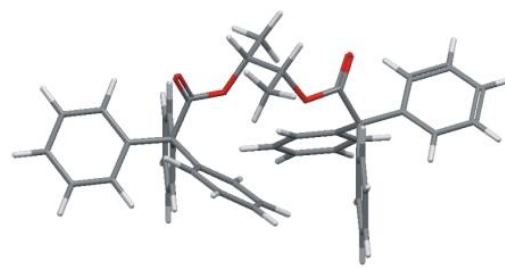

$20($ conf 17)

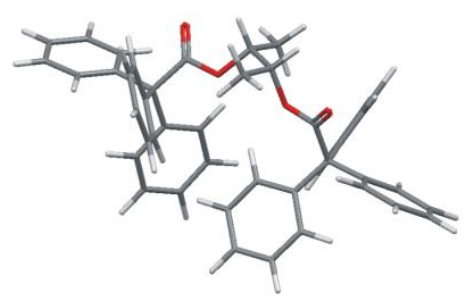

$20($ conf 22)

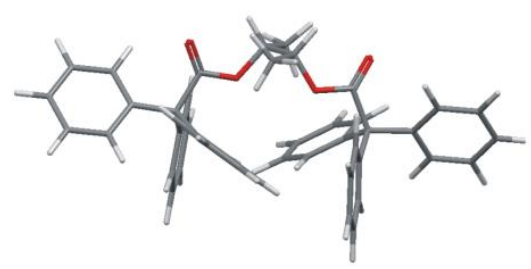

$20(\operatorname{conf} 11)$

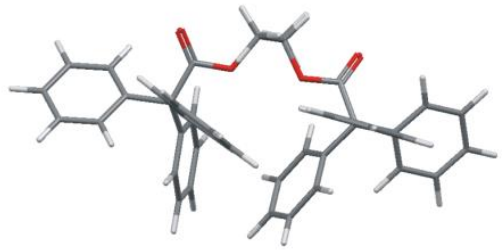

$20(\operatorname{conf} 20)$

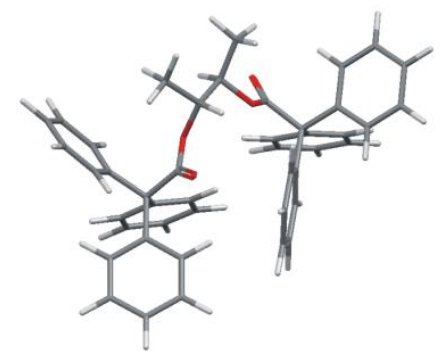

$20($ conf 23)

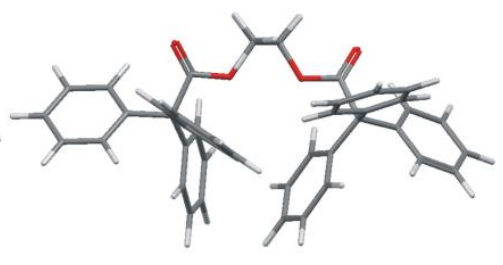

$20($ conf 14)

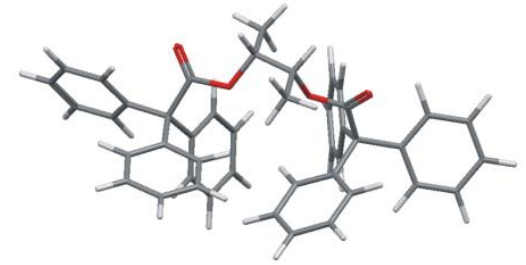

$20($ conf 21)

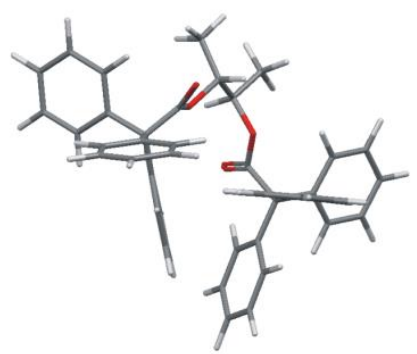

$20($ conf 25)

Figure SI_20. Structures of individual, low-energy conformers of 20, calculated at the M06-2X/6$311 G(d, p)$ level of theory. 


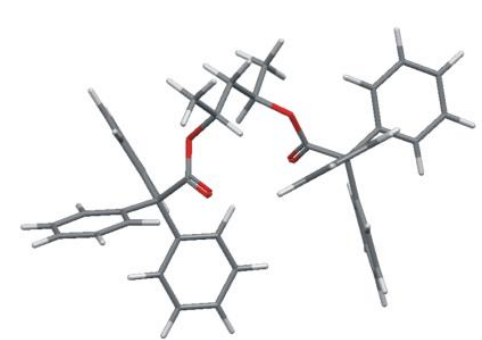

21 (conf 1)

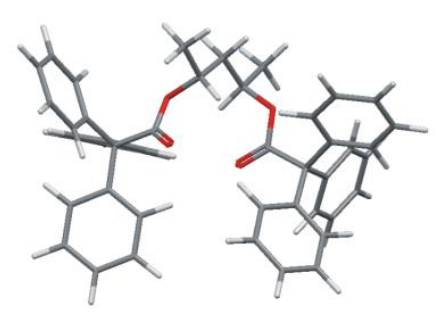

21 (conf 15)

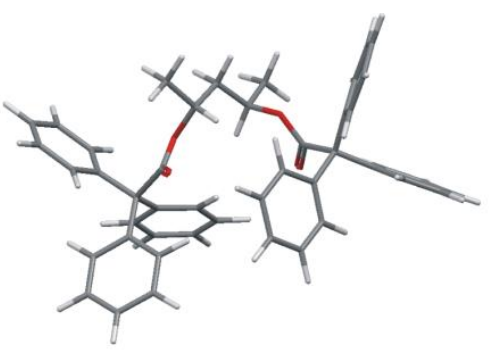

$21(\operatorname{conf} 6)$

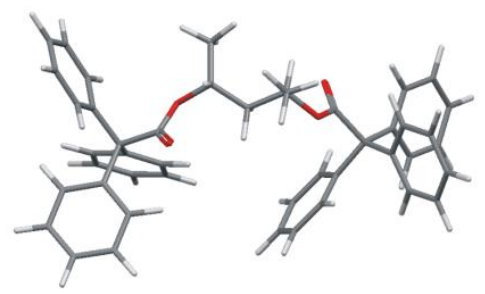

21 (conf 31)

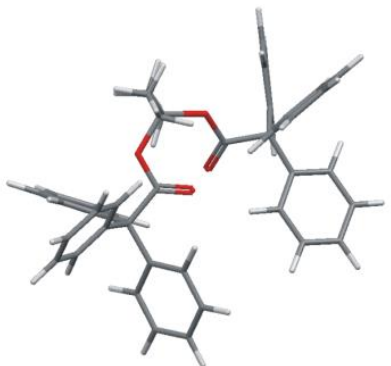

21 (conf 12)

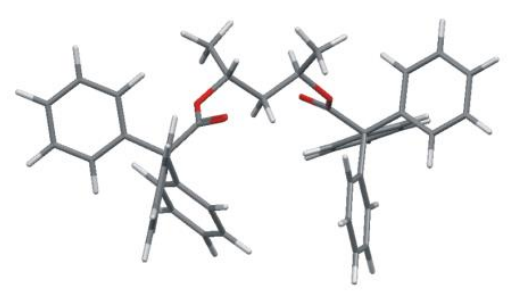

21 (conf 54)

Figure SI_21. Structures of individual, low-energy conformers of 21, calculated at the B3LYP/6$311 G(d, p)$ level of theory.

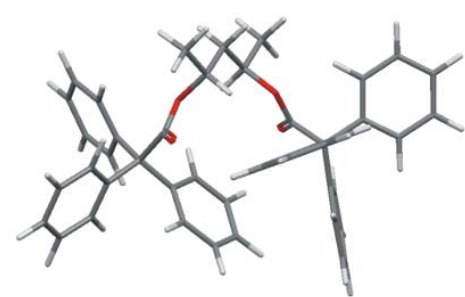

$21(\operatorname{conf} 1)$

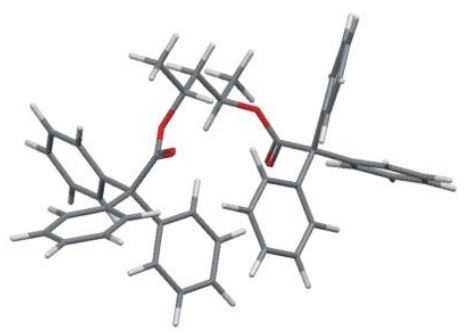

$21(\operatorname{conf} 6)$

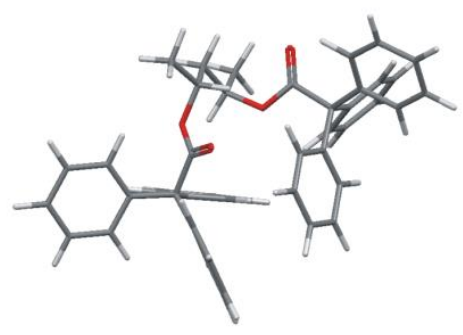

21 (conf 16)

Figure SI_22. Structures of individual, low-energy conformers of 21, calculated at the B3LYP-GD3BJ/6$311 G(d, p)$ level of theory. 


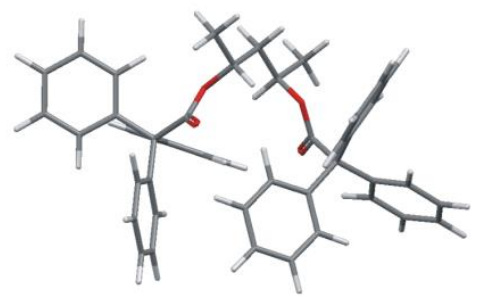

$21(\operatorname{conf} 1)$

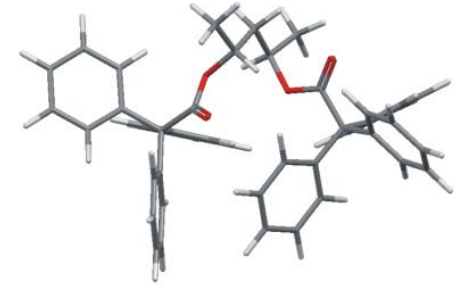

$21(\operatorname{conf} 16)$

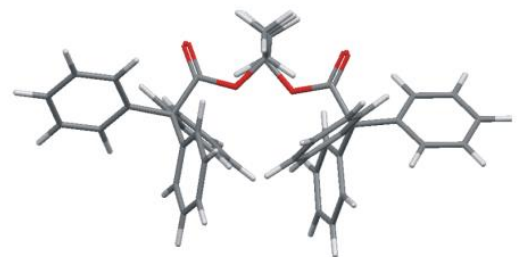

$21(\operatorname{conf} 46)$

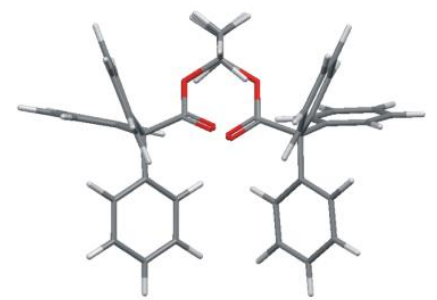

$21($ conf 12)

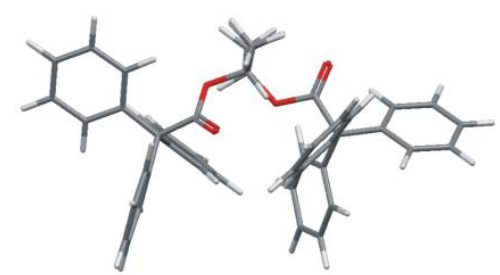

21 (conf 25)

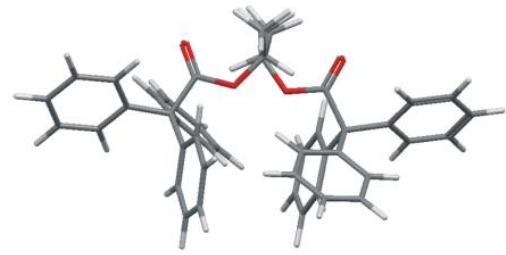

$21(\operatorname{conf} 61)$

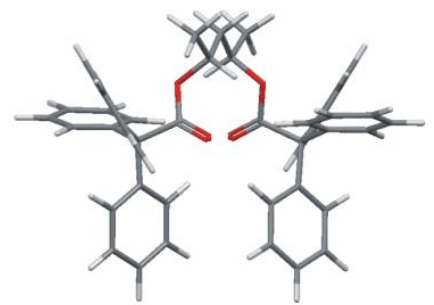

$21($ conf 15)

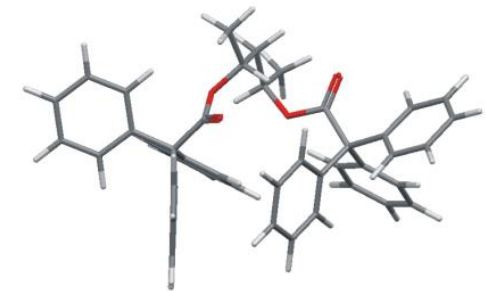

21 (conf 35)

Figure SI_23. Structures of individual, low-energy conformers of 21, calculated at the M06-2X/6$311 \mathrm{G}(\mathrm{d}, \mathrm{p})$ level of theory. 


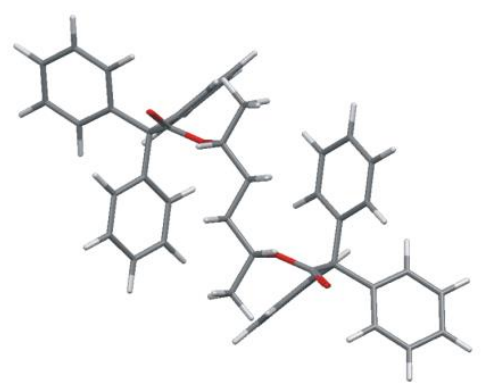

22 (conf 1)

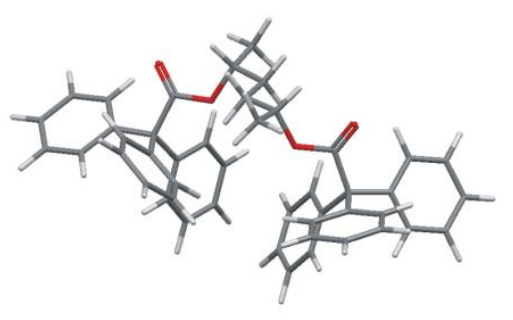

22 (conf 7)

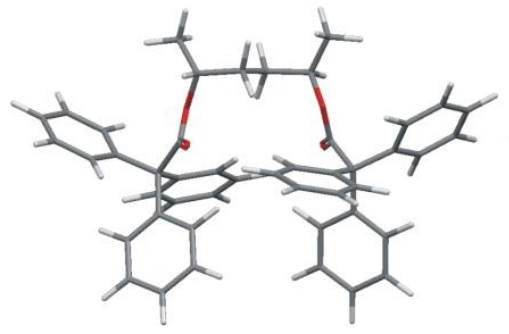

22 (conf 10)

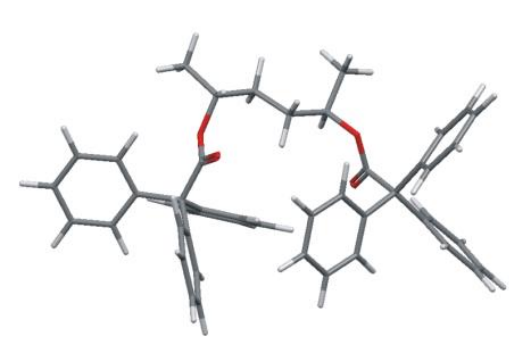

22 (conf 13)

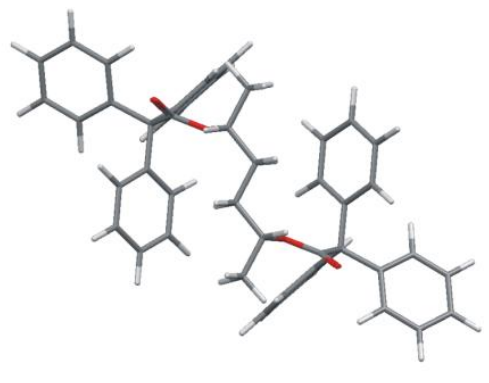

22 (conf 3)

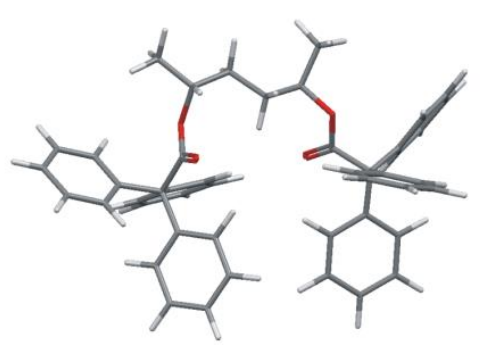

22 (conf 8)

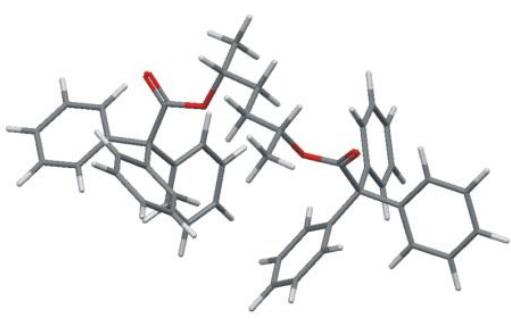

22 (conf 5)

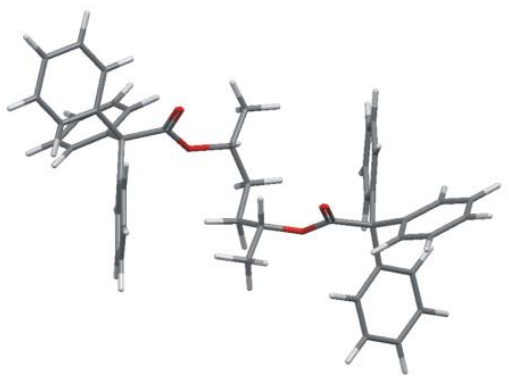

22 (conf 9)

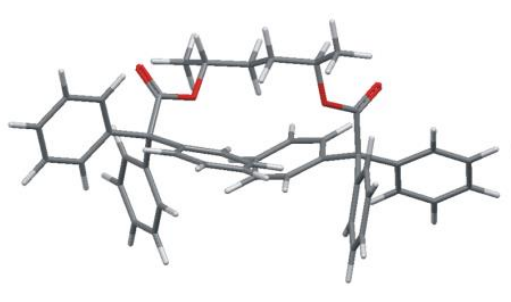

22 (conf 11)

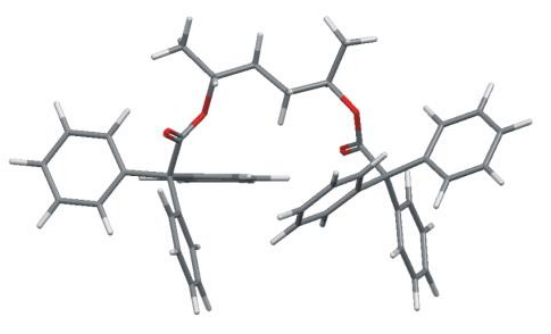

22 (conf 12)

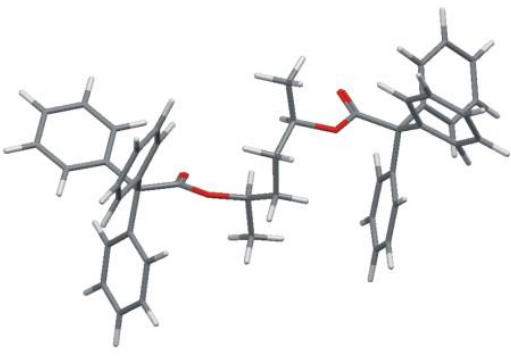

22 (conf 15)

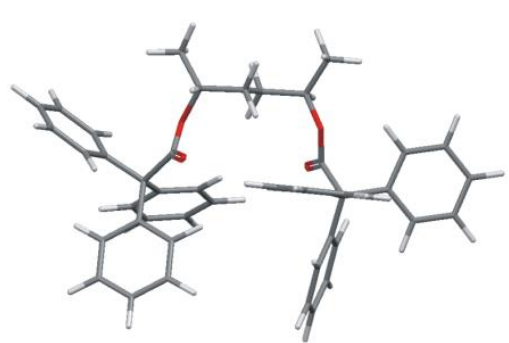

22 (conf 17)

Figure SI_24a. Structures of individual, low-energy conformers of 22, calculated at the B3LYP/6$311 G(d, p)$ level of theory, part I. 


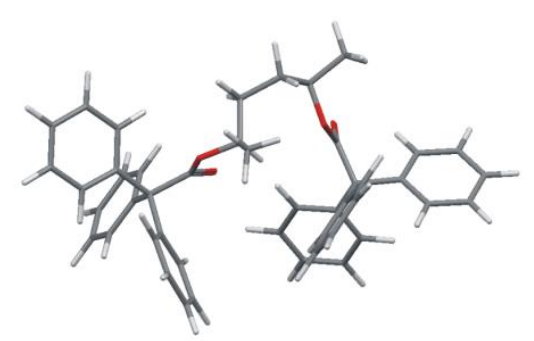

22 (conf 26)

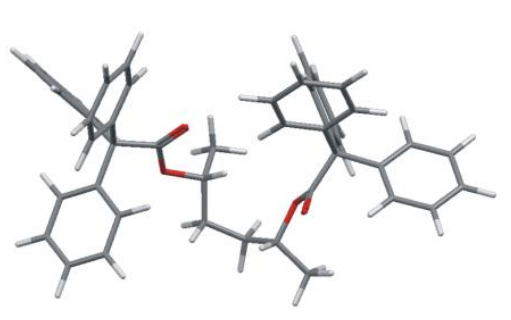

22 (conf 71)

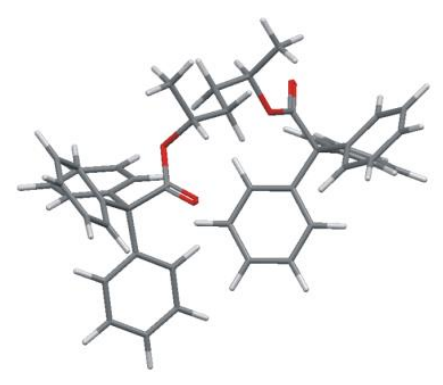

$22($ conf 31)

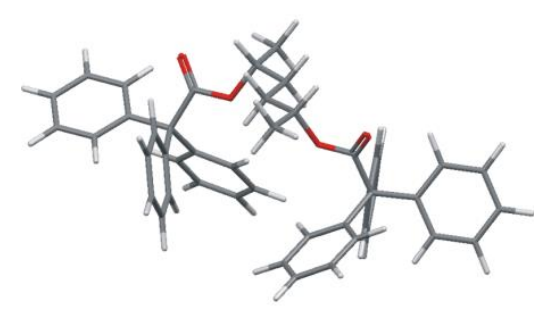

22 (conf 36)

Figure SI_24b. Structures of individual, low-energy conformers of 22, calculated at the B3LYP/6$311 \mathrm{G}(\mathrm{d}, \mathrm{p})$ level of theory, part II.

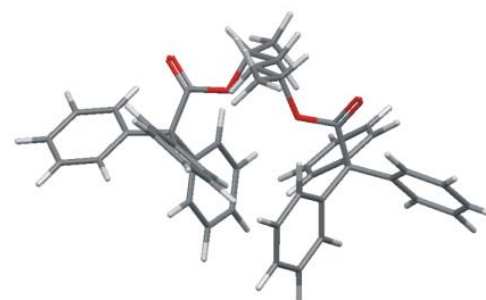

$22(\operatorname{conf} 1)$

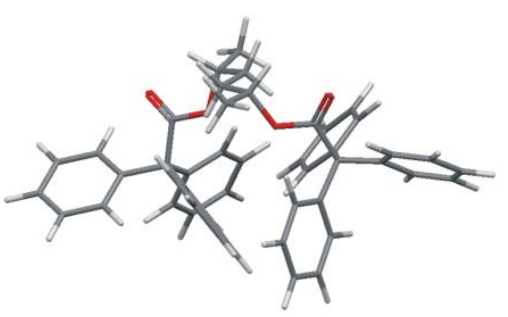

$22(\operatorname{conf} 7)$

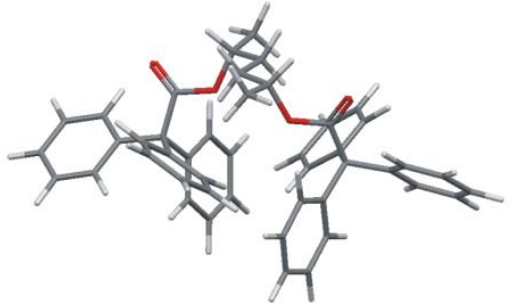

$22(\operatorname{conf} 3)$

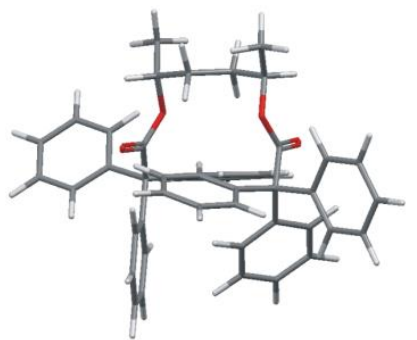

$22($ conf 17)

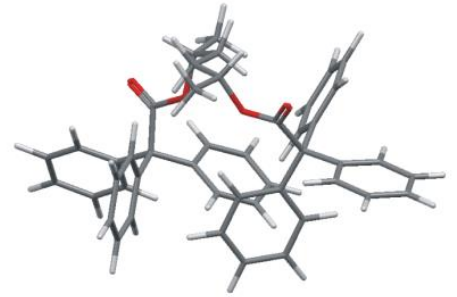

$22(\operatorname{conf} 5)$

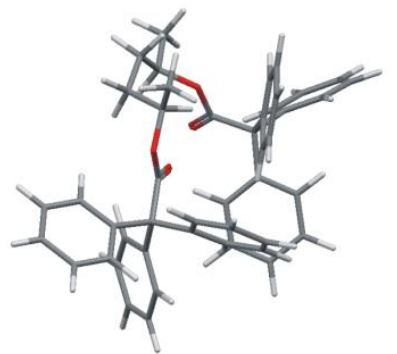

$22(\operatorname{conf} 26)$

Figure SI_25. Structures of individual, low-energy conformers of 22, calculated at the B3LYP-GD3BJ/6$311 G(d, p)$ level of theory. 


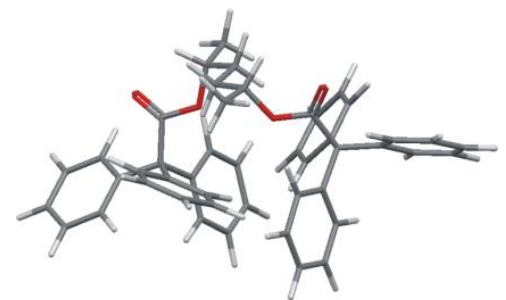

$22(\operatorname{conf} 1)$

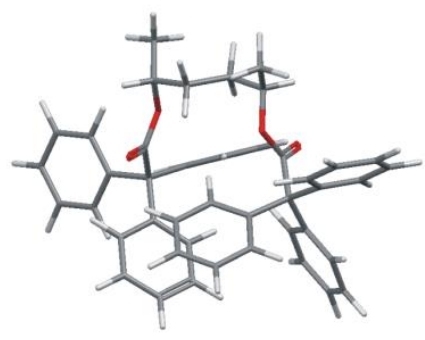

$22(\operatorname{conf} 17)$

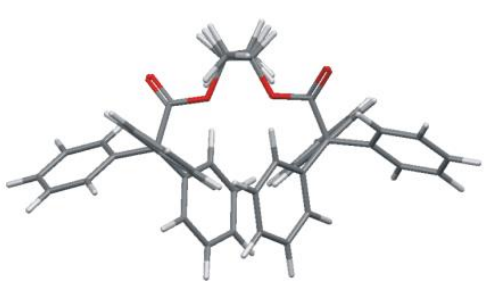

$22(\operatorname{conf} 3)$

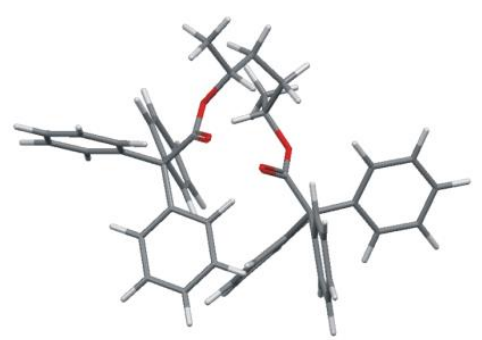

$22($ conf 26)

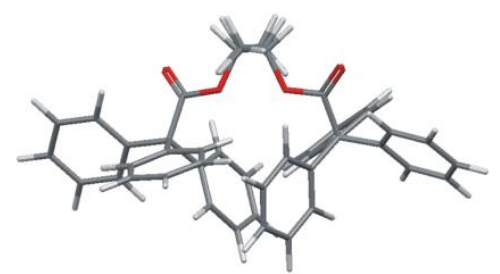

$22(\operatorname{conf} 11)$

Figure SI_26. Structures of individual, low-energy conformers of 22, calculated at the M06-2X/6$311 G(d, p)$ level of theory. 

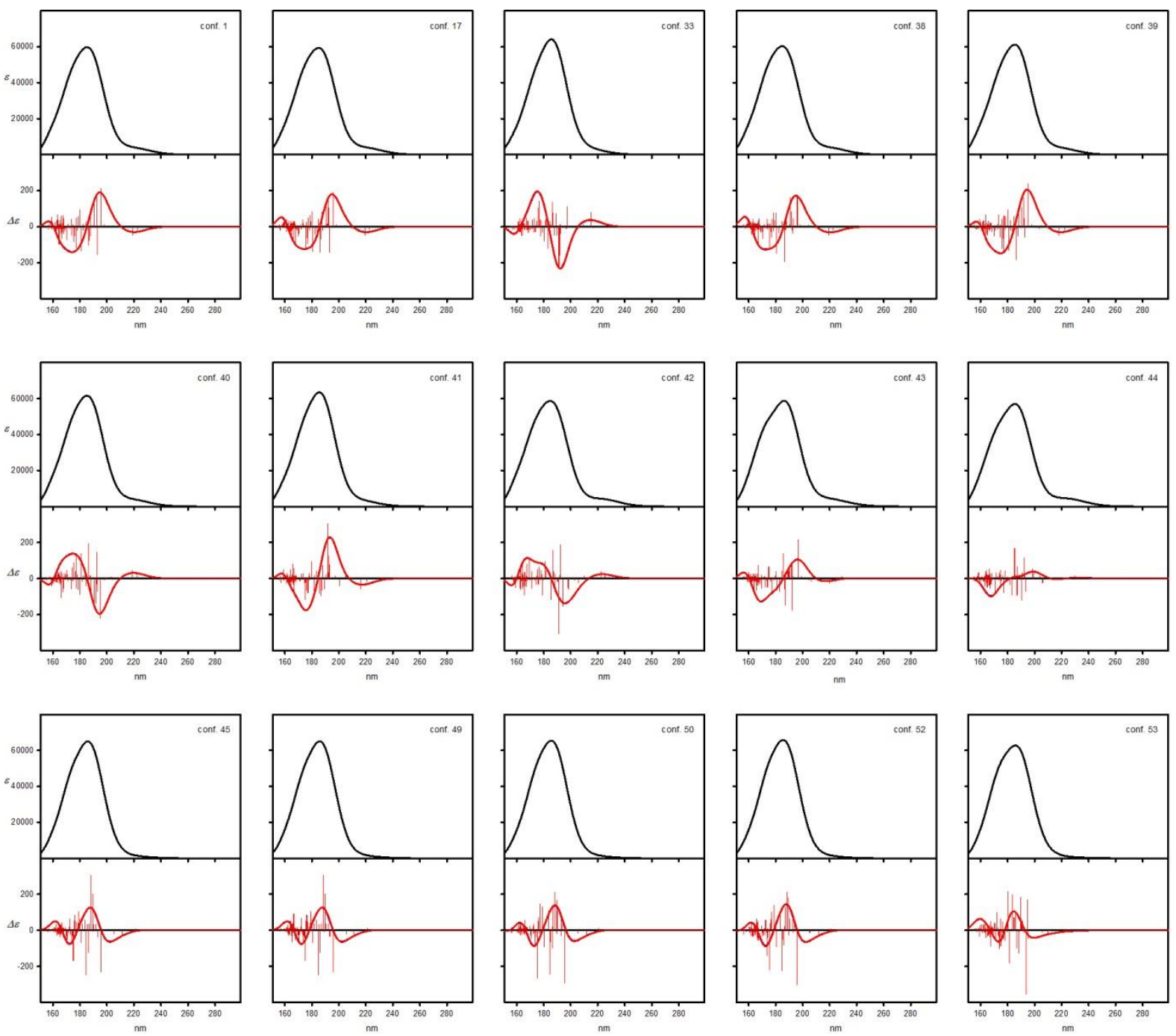

Figure SI_27. UV and ECD spectra of the low-energy conformers of compound $\mathbf{1}$ calculated at TDCAM-B3LYP/6-311++G(2d,2p) level for structures optimized at B3LYP/6-311++G(d,p) level. Wavelengths were not corrected. 

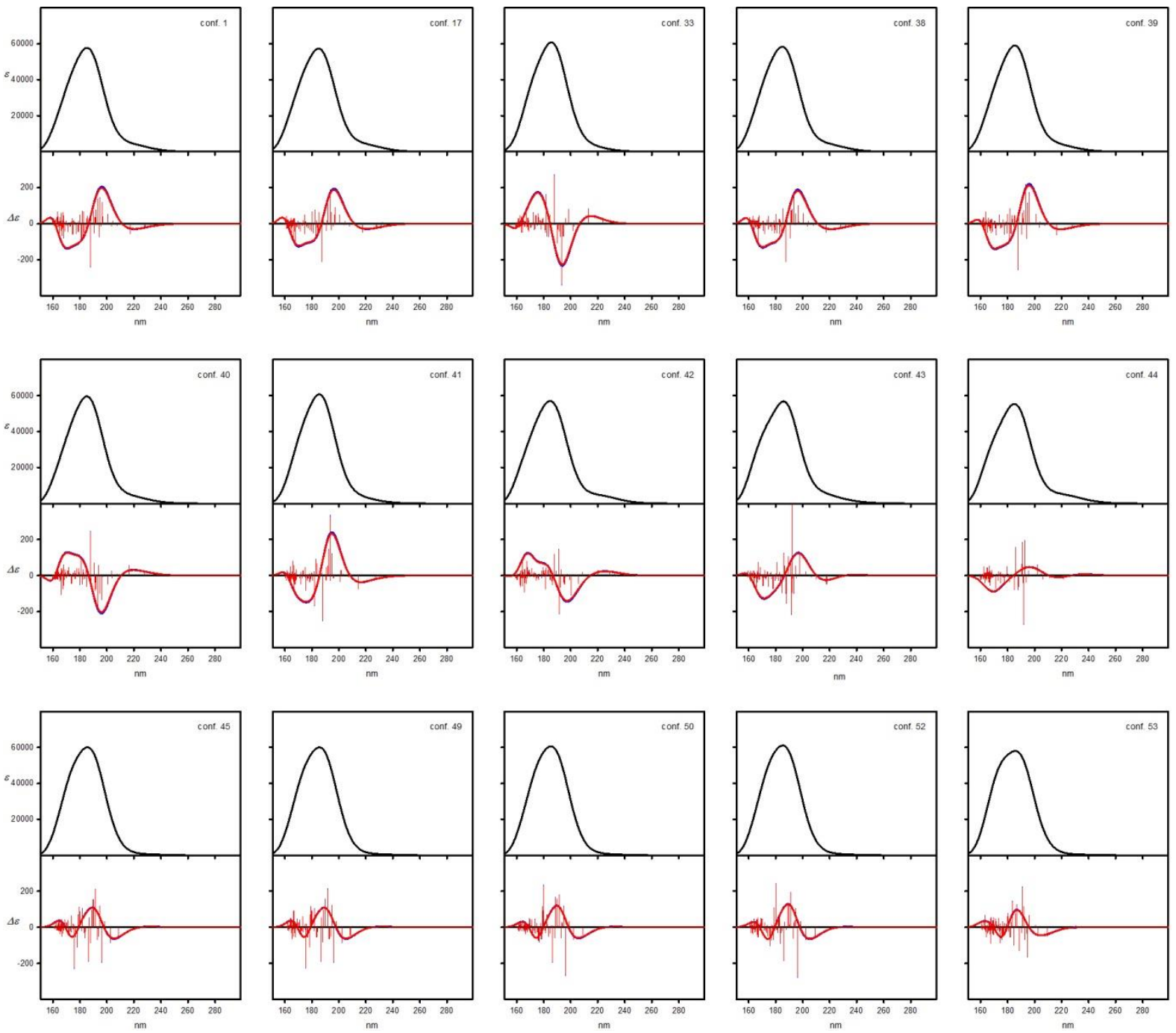

Figure SI_28. UV and ECD spectra of the low-energy conformers of compound 1 calculated at TDM06-2X/6-311++G $(2 d, 2 p)$ level for structures optimized at $B 3 L Y P / 6-311++G(d, p)$ level. Wavelengths were not corrected. 

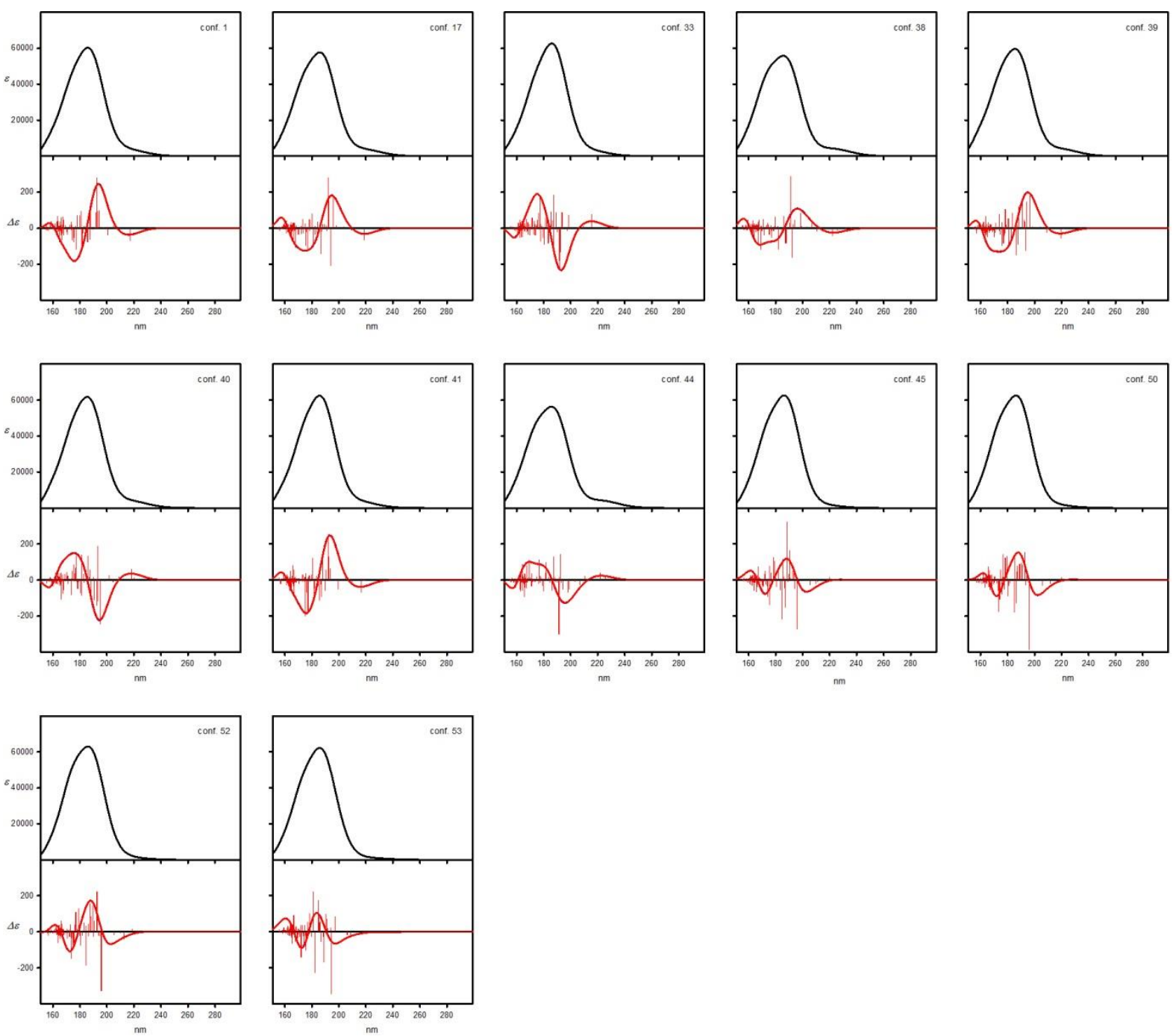

Figure SI_29. UV and ECD spectra of the low-energy conformers of compound 1 calculated at TDCAM-B3LYP/6-311++G(2d,2p) level for structures optimized at M06-2X/6-311++G(d,p) level. Wavelengths were not corrected. 

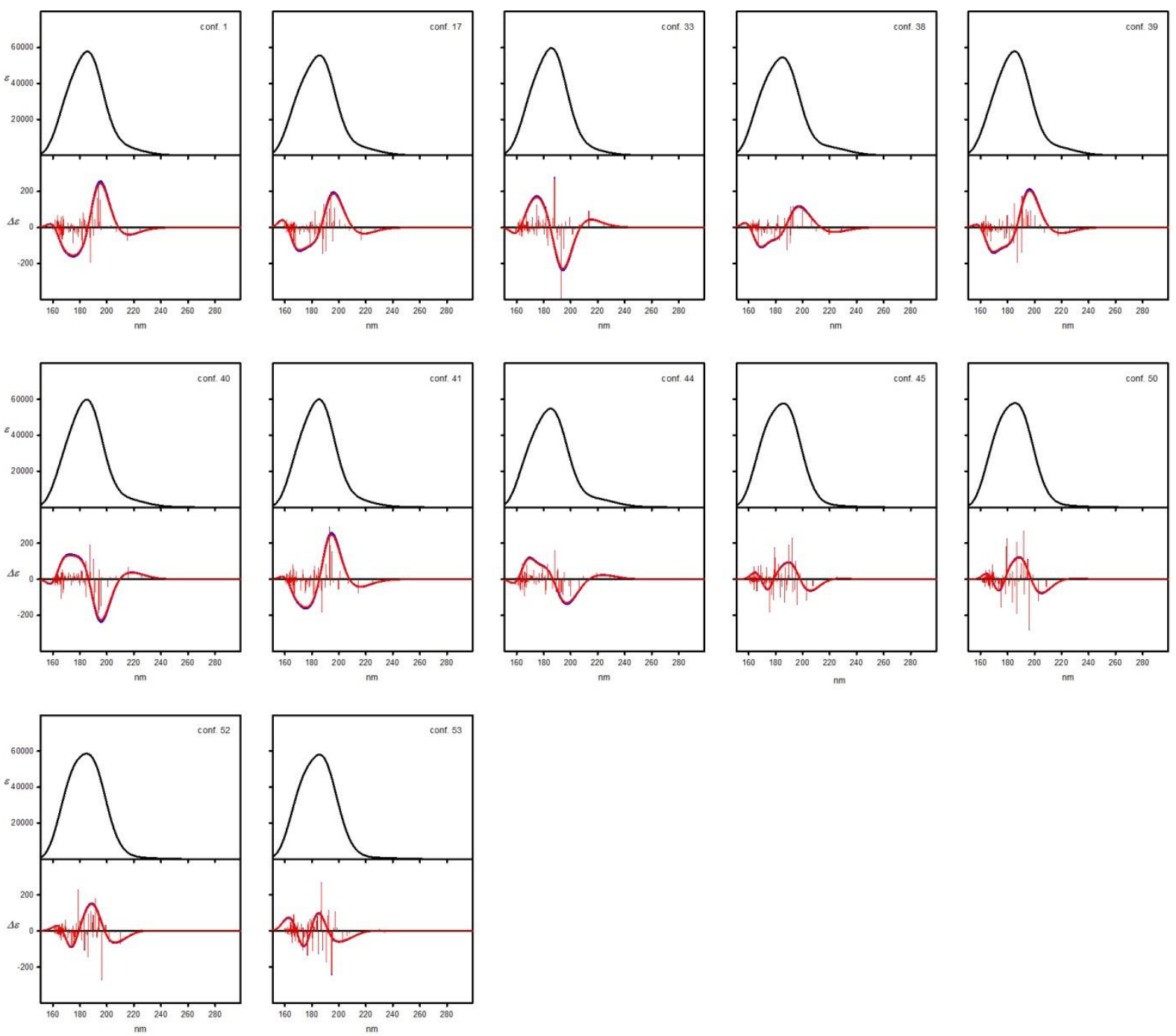

Figure SI_30. UV and ECD spectra of the low-energy conformers of compound 1 calculated at TDM06-2X/6-311++G(2d,2p) level for structures optimized at M06-2X/6-311++G(d,p) level. Wavelengths were not corrected. 

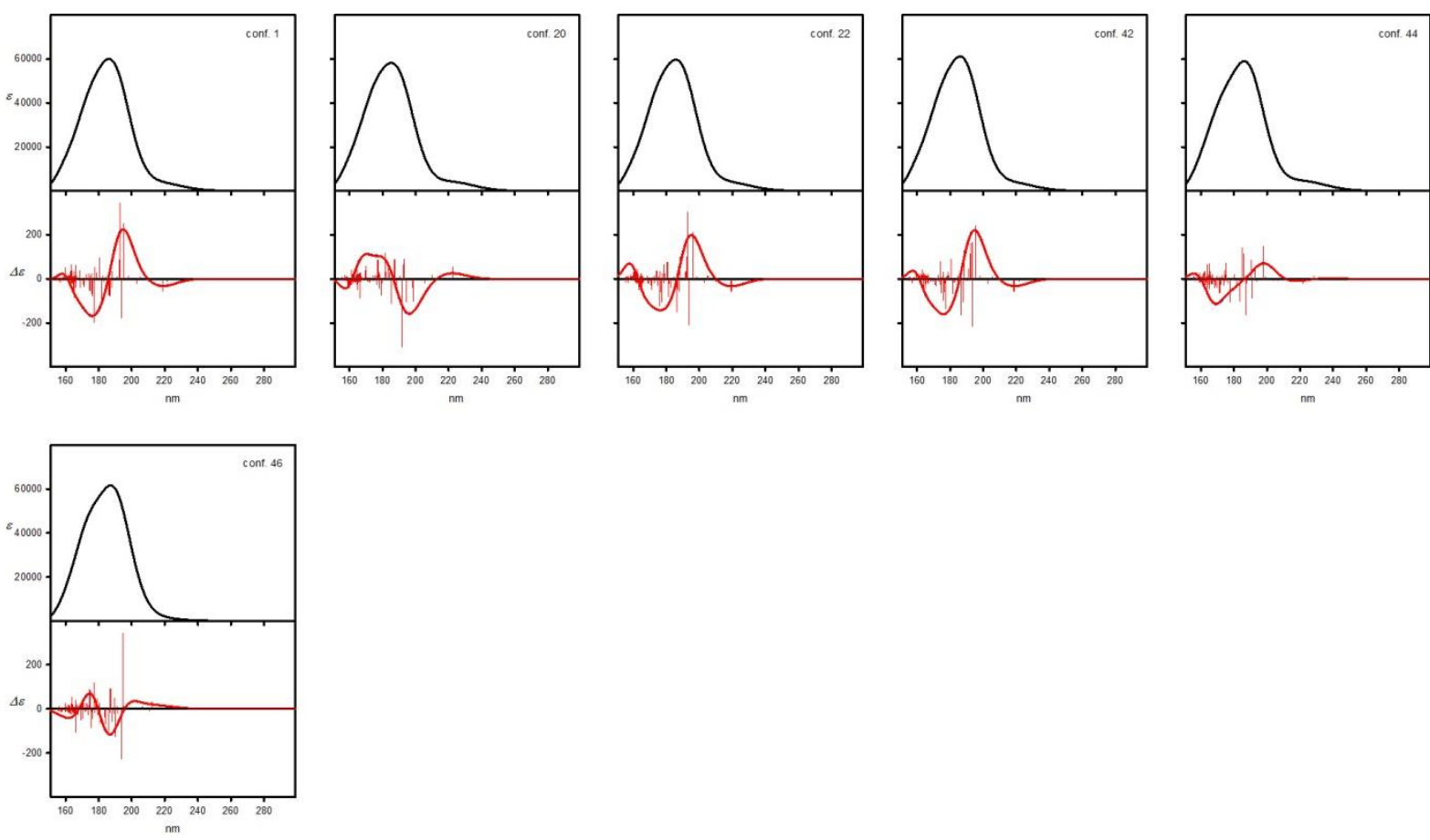

Figure SI_31. UV and ECD spectra of the low-energy conformers of compound 4 calculated at TDCAM-B3LYP/6-311++G(2d,2p) level for structures optimized at B3LYP/6-311++G(d,p) level. Wavelengths were not corrected.
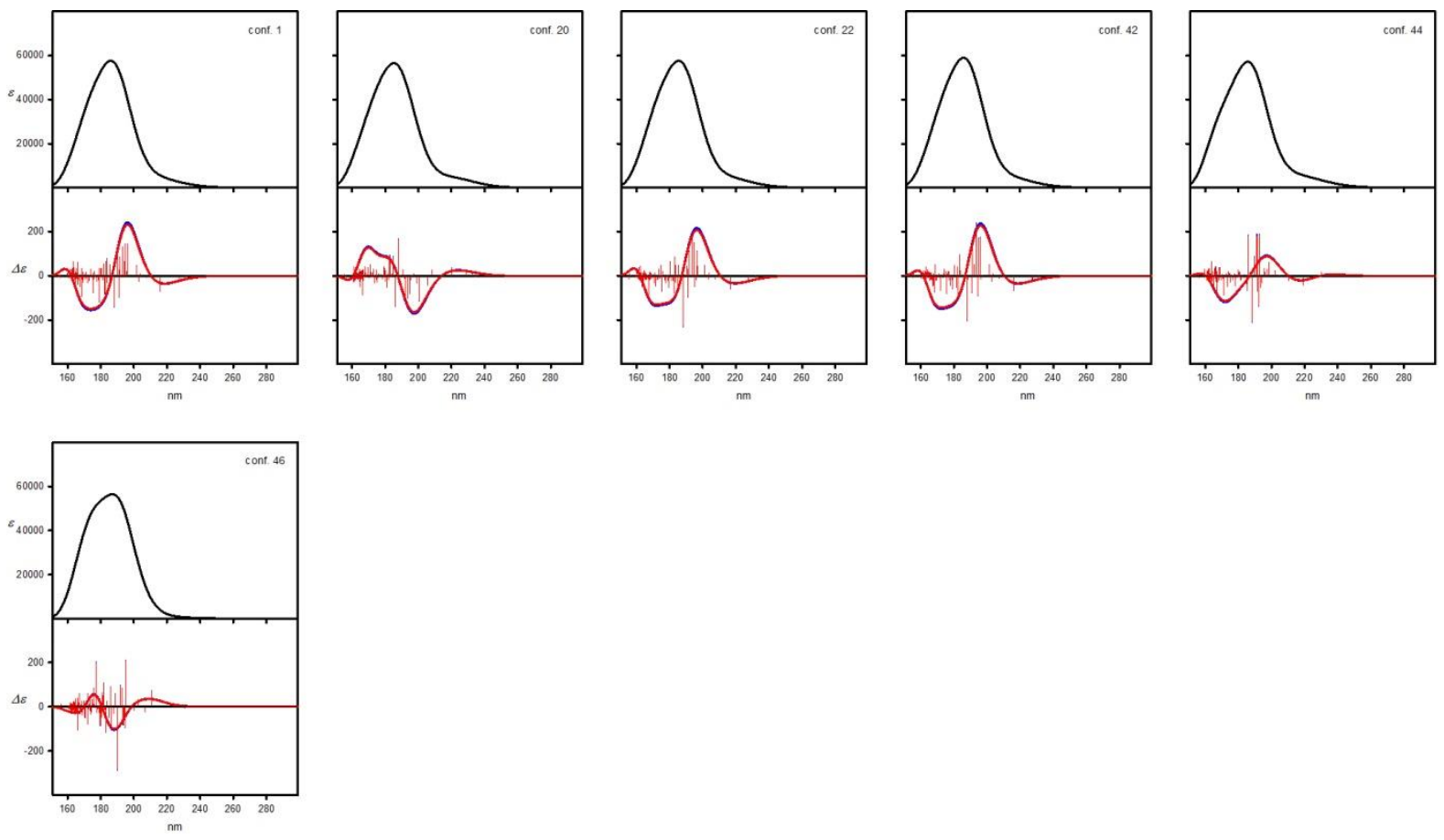

Figure SI_32. UV and ECD spectra of the low-energy conformers of compound 4 calculated at TDM06-2X/6-311++G $(2 d, 2 p)$ level for structures optimized at $B 3 L Y P / 6-311++G(d, p)$ level. Wavelengths were not corrected. 

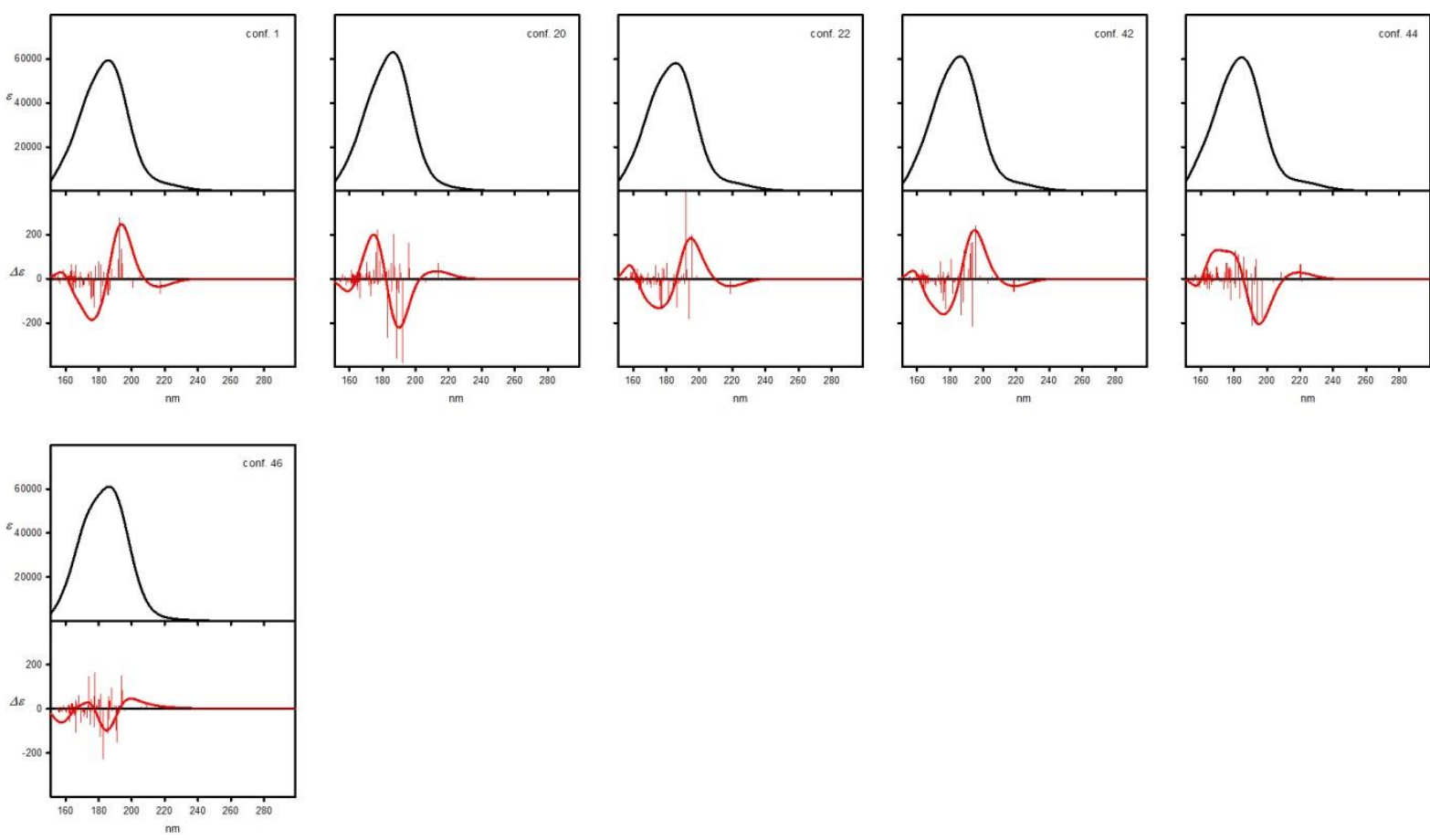

Figure SI_33. UV and ECD spectra of the low-energy conformers of compound 4 calculated at TDCAM-B3LYP/6-311++G(2d,2p) level for structures optimized at $M 06-2 X / 6-311++G(d, p)$ level. Wavelengths were not corrected.
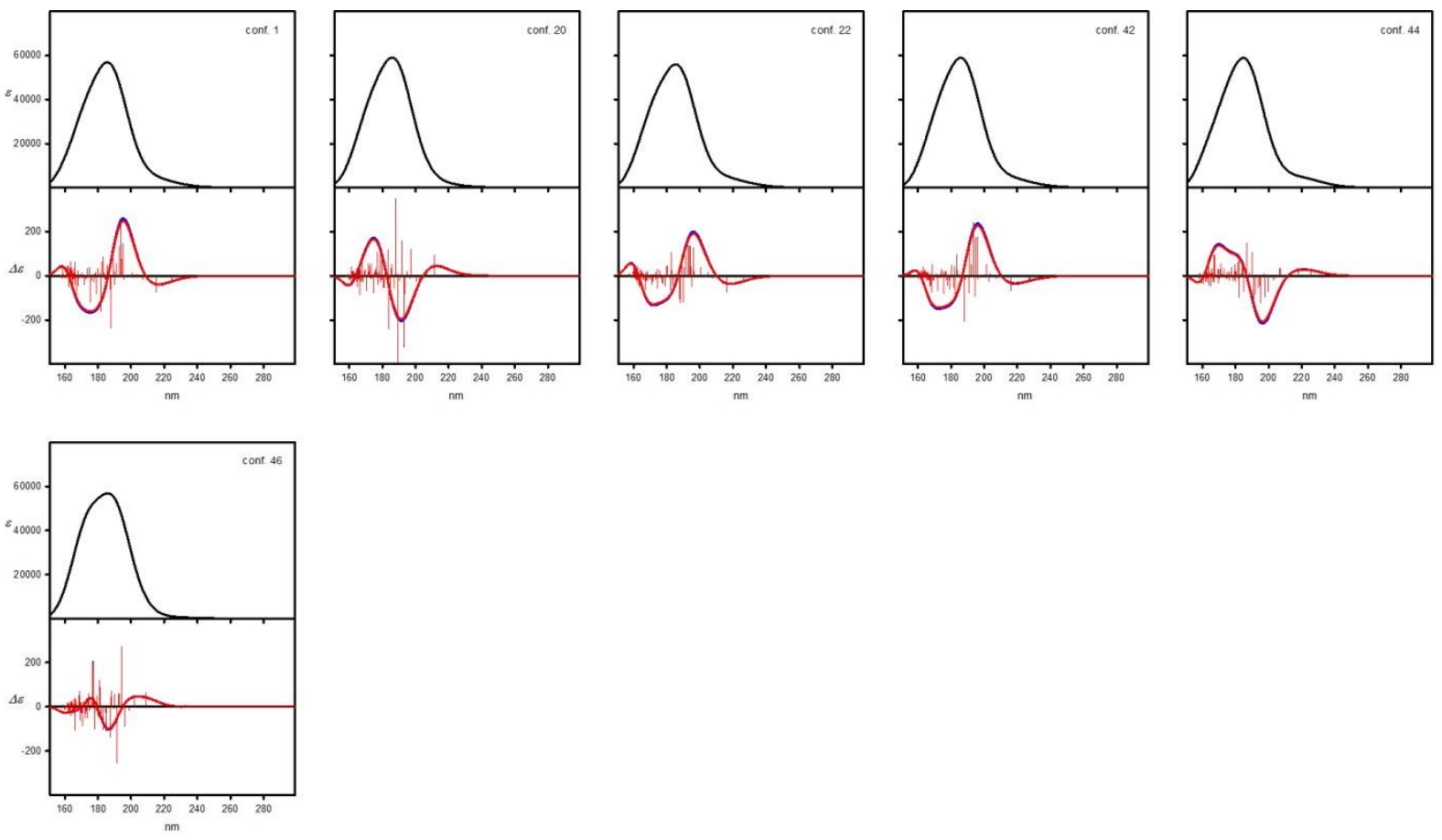

Figure SI_34. UV and ECD spectra of the low-energy conformers of compound 4 calculated at TDM06-2X/6-311++G $(2 d, 2 p)$ level for structures optimized at M06-2X/6-311++G(d,p) level. Wavelengths were not corrected. 

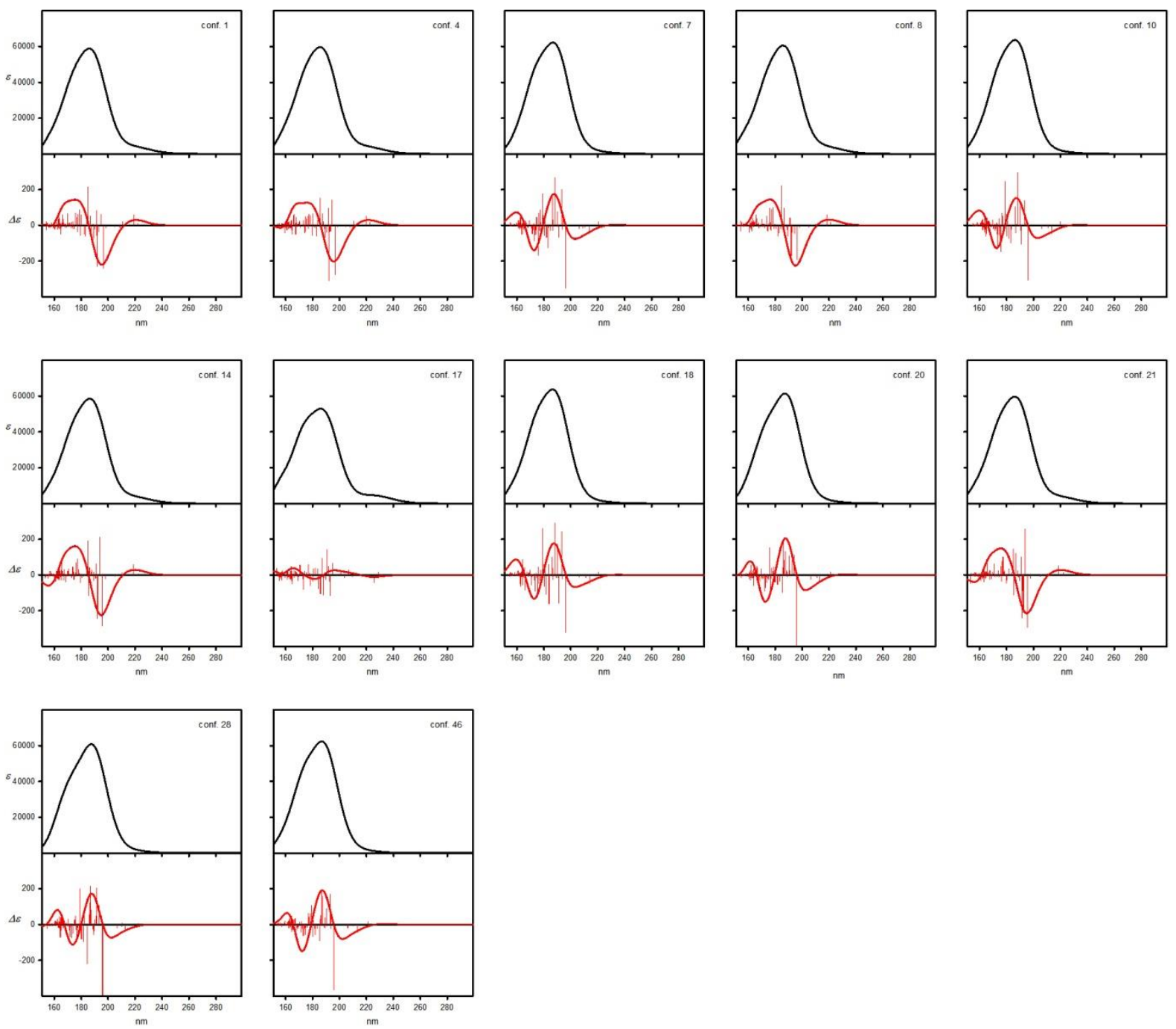

Figure SI_35. UV and ECD spectra of the low-energy conformers of compound 6 calculated at TDCAM-B3LYP/6-311++G(2d,2p) level for structures optimized at B3LYP/6-311++G(d,p) level. Wavelengths were not corrected. 

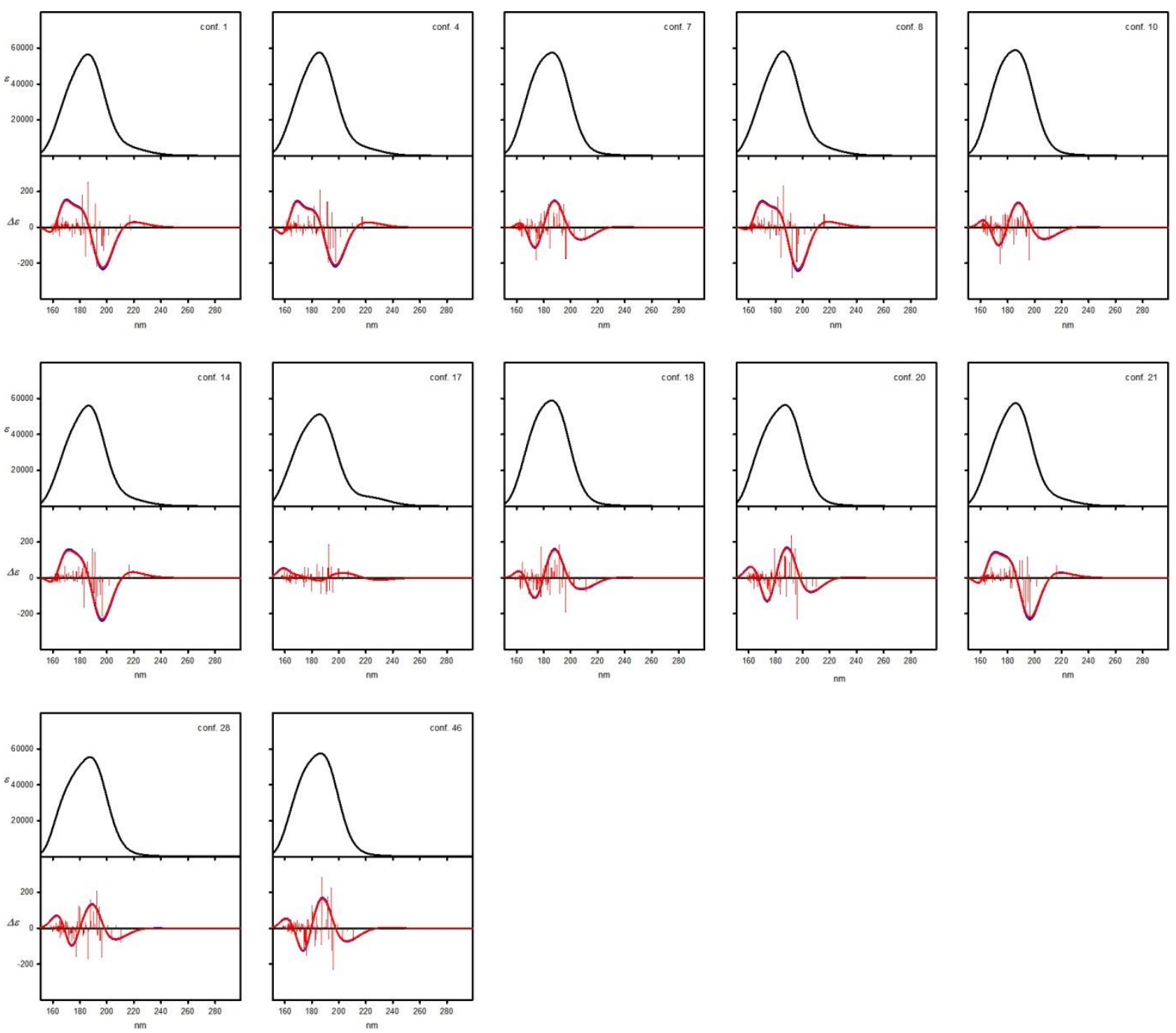

Figure SI_36. UV and ECD spectra of the low-energy conformers of compound 6 calculated at TDM06-2X/6-311++G $(2 d, 2 p)$ level for structures optimized at $B 3 L Y P / 6-311++G(d, p)$ level. Wavelengths were not corrected. 

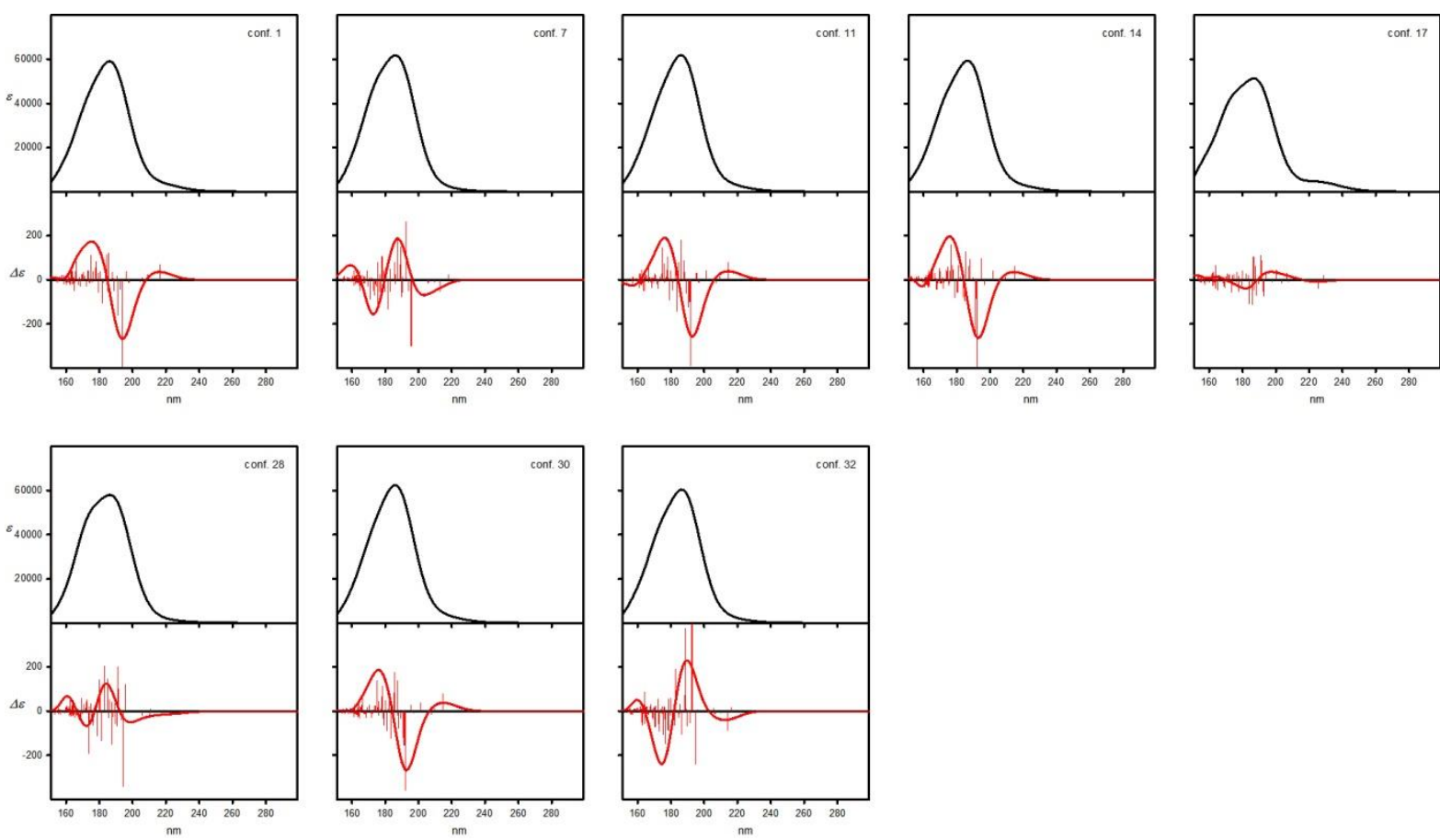

Figure SI_37. UV and ECD spectra of the low-energy conformers of compound 6 calculated at TDCAM-B3LYP/6-311++G(2d,2p) level for structures optimized at M06-2X/6-311++G(d,p) level. Wavelengths were not corrected.
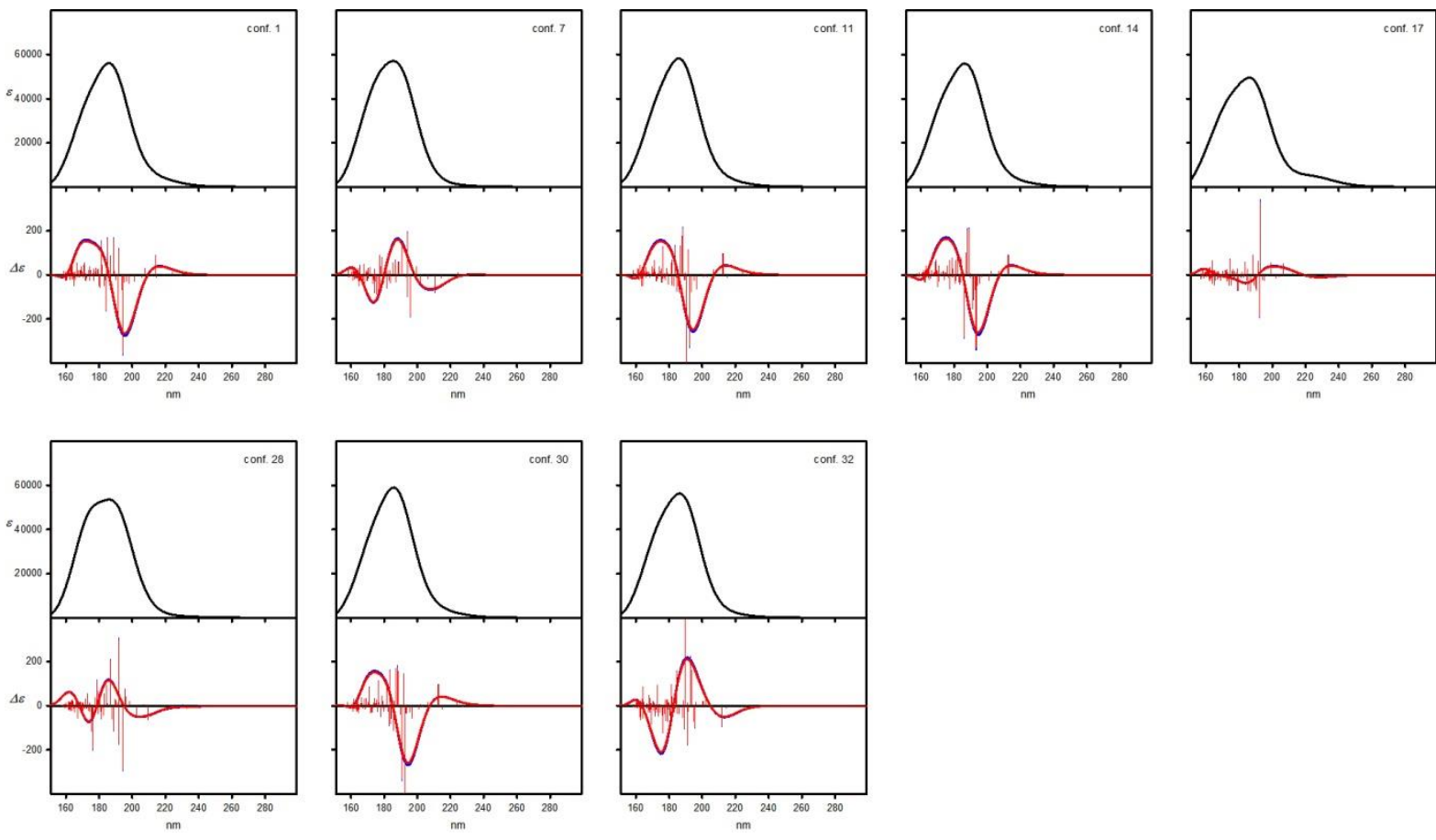

Figure SI_38. UV and ECD spectra of the low-energy conformers of compound 6 calculated at TDM06-2X/6-311++G(2d,2p) level for structures optimized at $M 06-2 X / 6-311++G(d, p)$ level. Wavelengths were not corrected. 


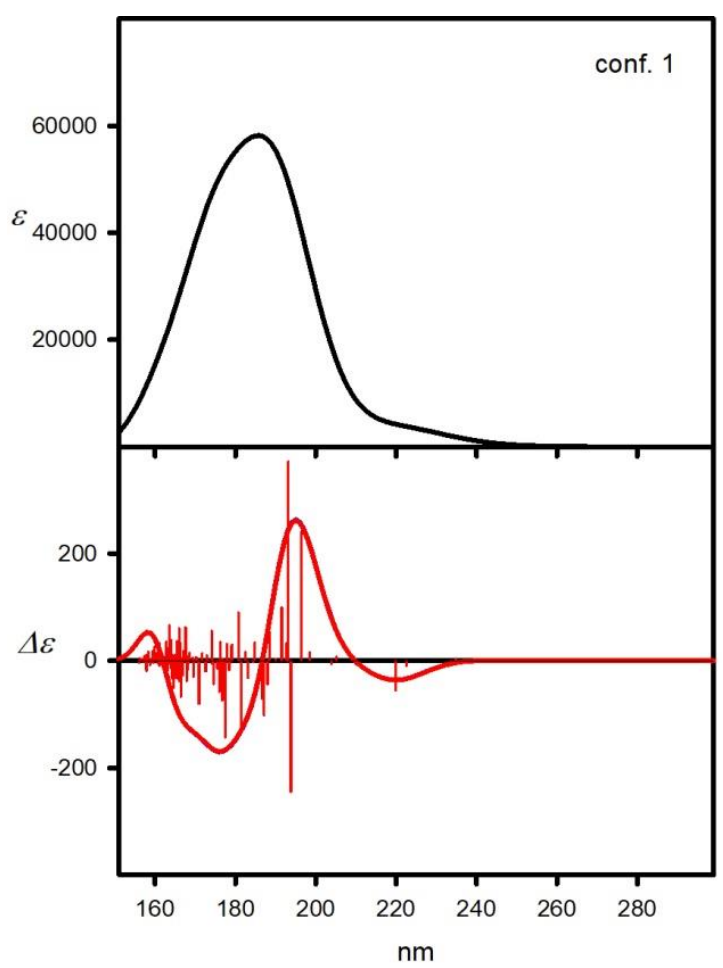

Figure SI_39. UV and ECD spectra of the low-energy conformers of compound $\mathbf{1 0}$ calculated at TDCAM-B3LYP/6-311++G(2d,2p) level for structures optimized at B3LYP/6-311++G(d,p) level. Wavelengths were not corrected.

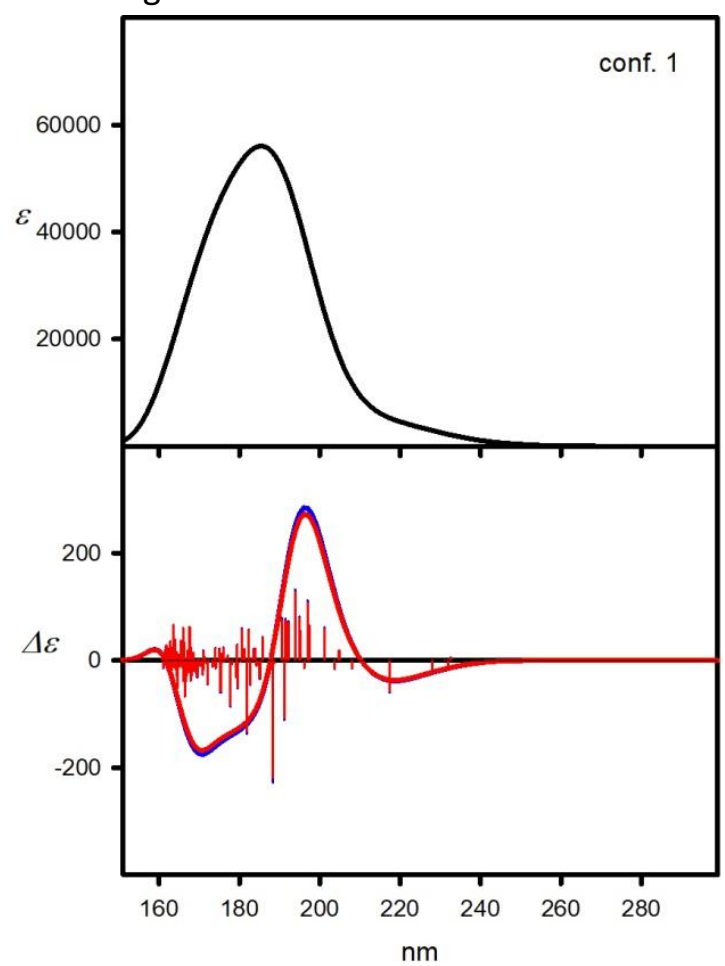

Figure SI_40. UV and ECD spectra of the low-energy conformers of compound $\mathbf{1 0}$ calculated at TDM06-2X/6-311++G $(2 d, 2 p)$ level for structures optimized at B3LYP/6-311++G(d,p) level. Wavelengths were not corrected. 


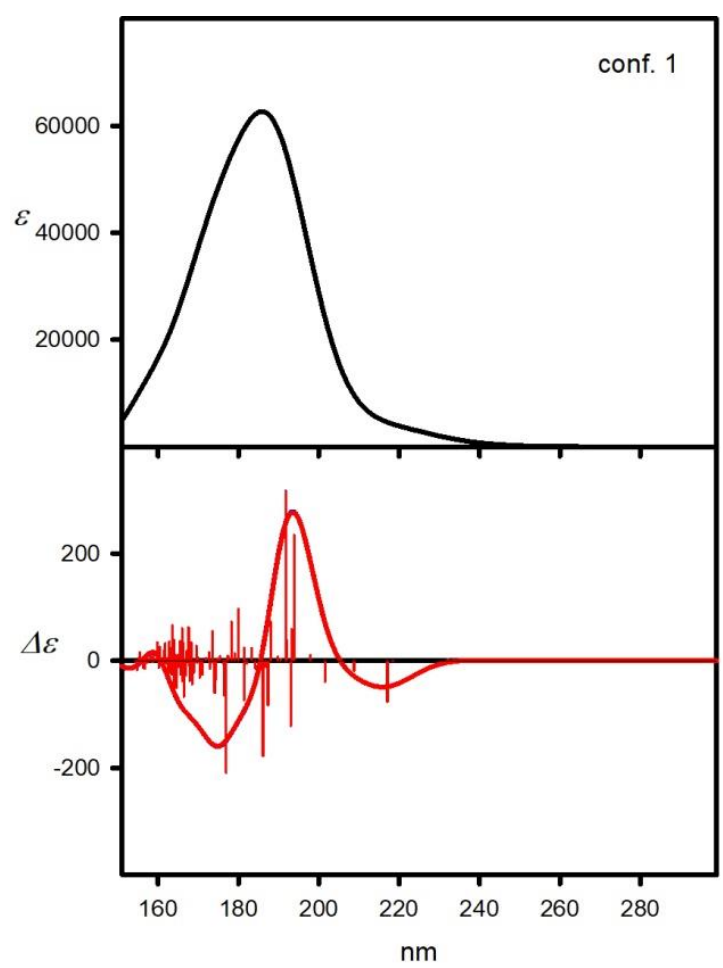

Figure SI_41. UV and ECD spectra of the low-energy conformers of compound $\mathbf{1 0}$ calculated at TDCAM-B3LYP/6-311++G(2d,2p) level for structures optimized at M06-2X/6-311++G(d,p) level. Wavelengths were not corrected.

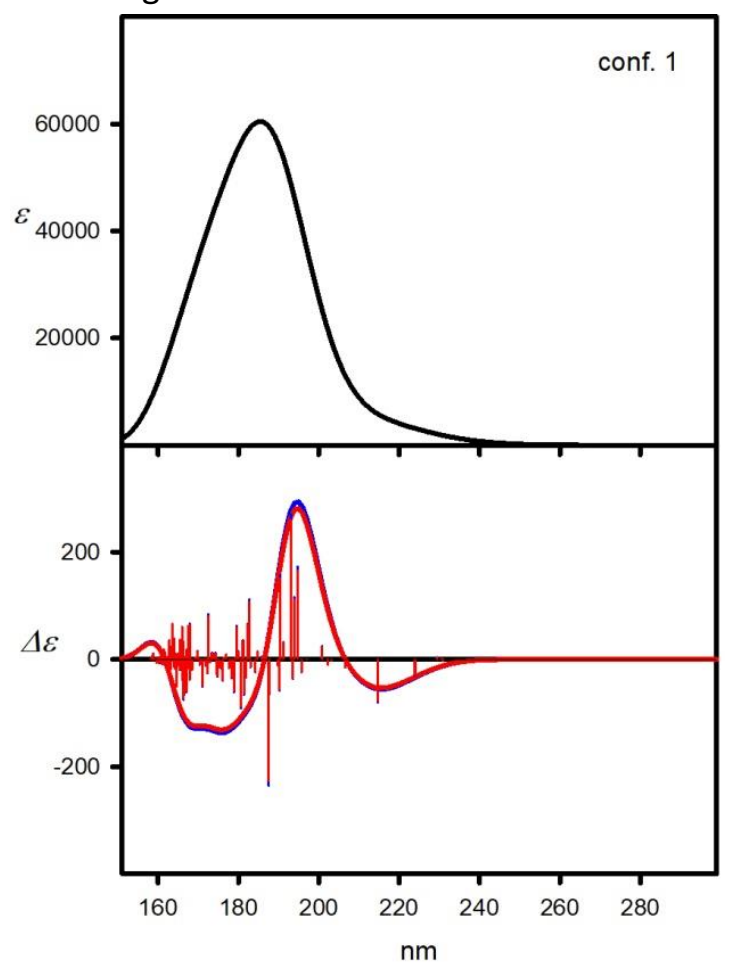

Figure SI_42. UV and ECD spectra of the low-energy conformers of compound $\mathbf{1 0}$ calculated at TDM06-2X/6-311++G(2d,2p) level for structures optimized at M06-2X/6-311++G(d,p) level. Wavelengths were not corrected. 

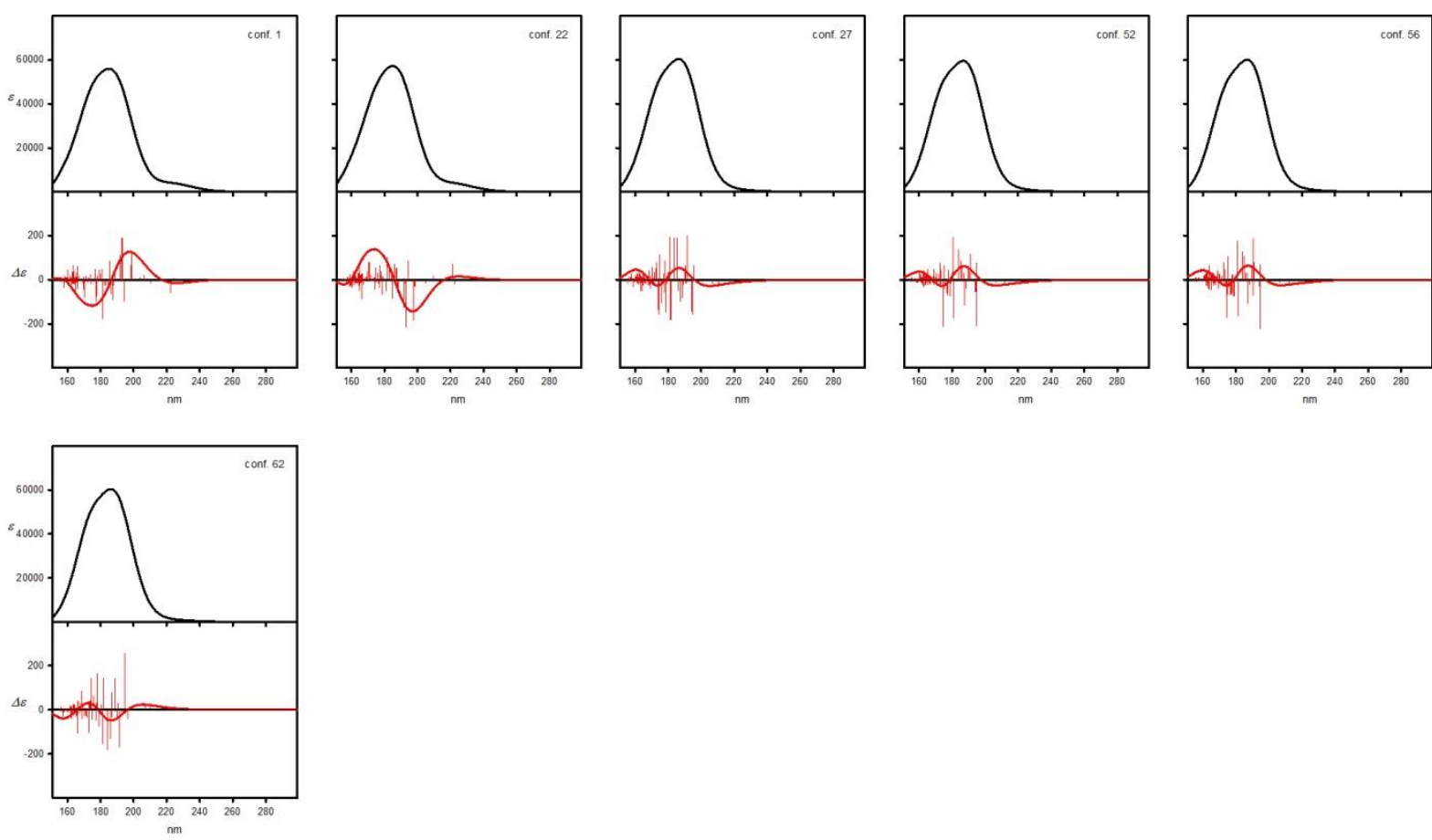

Figure SI_43. UV and ECD spectra of the low-energy conformers of compound $\mathbf{1 1}$ calculated at TDCAM-B3LYP/6-311++G(2d,2p) level for structures optimized at B3LYP/6-311++G(d,p) level. Wavelengths were not corrected.
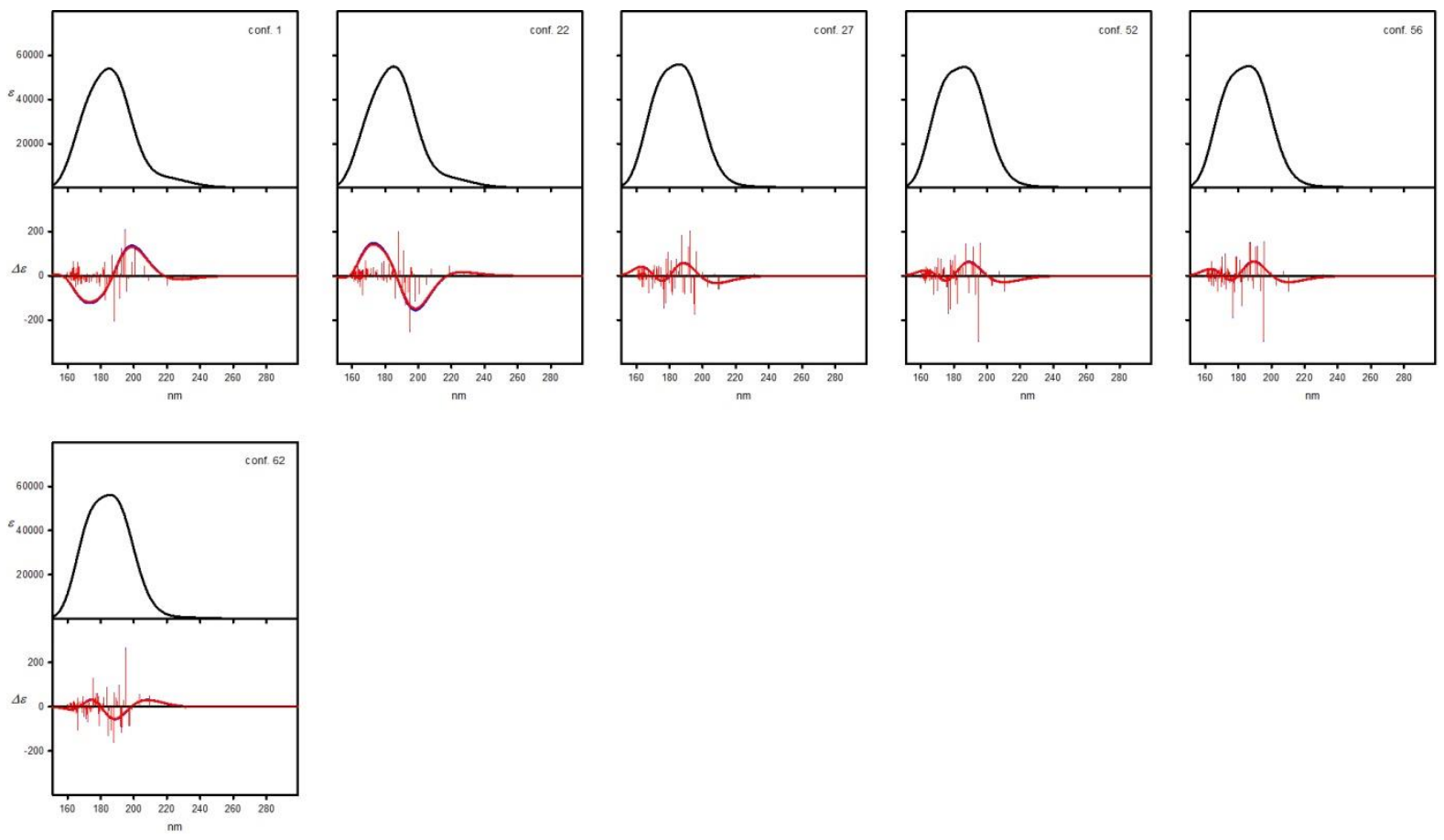

Figure SI_44. UV and ECD spectra of the low-energy conformers of compound $\mathbf{1 1}$ calculated at TDM06-2X/6-311++G $(2 d, 2 p)$ level for structures optimized at $B 3 L Y P / 6-311++G(d, p)$ level. Wavelengths were not corrected. 

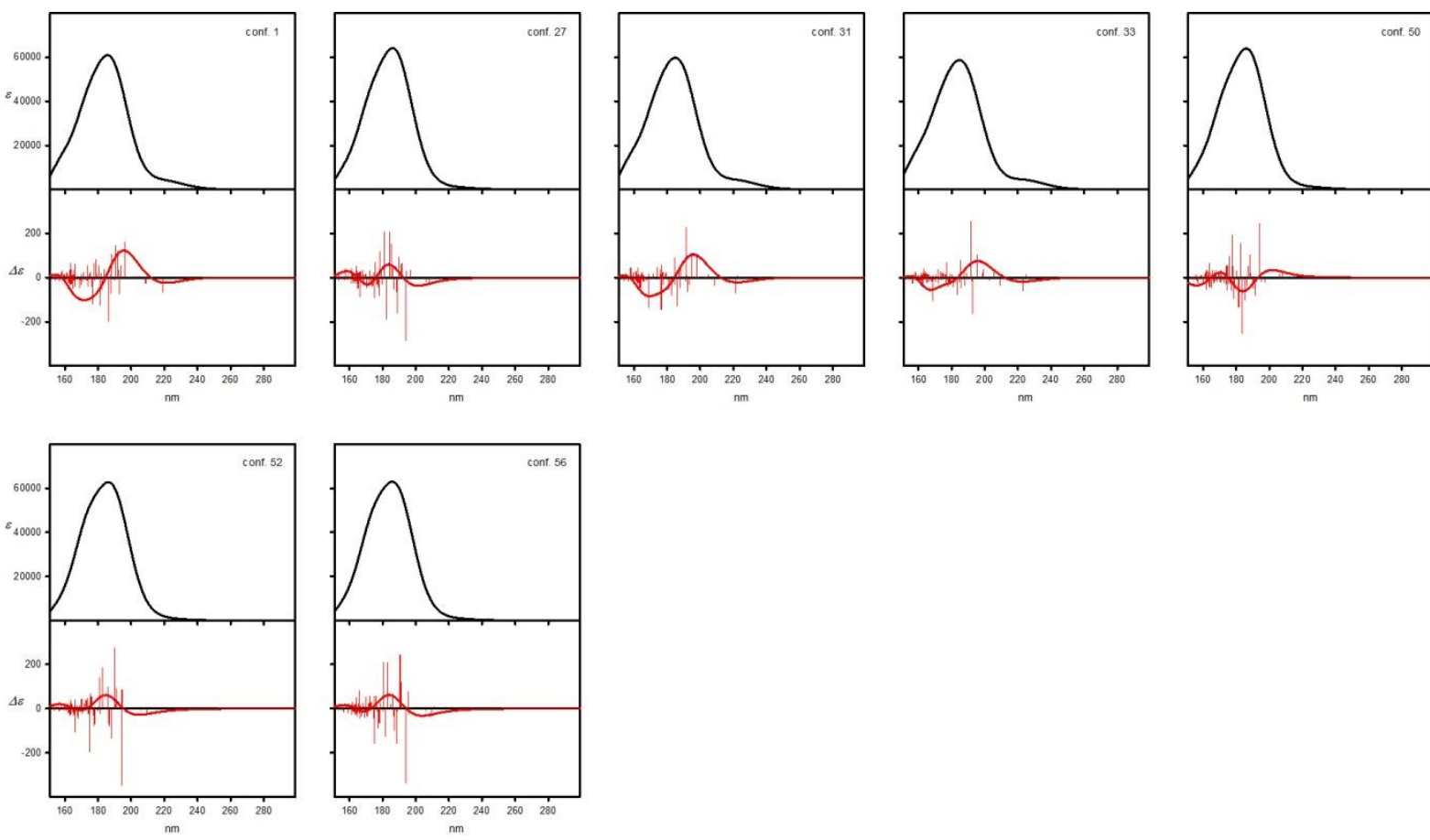

Figure SI_45. UV and ECD spectra of the low-energy conformers of compound $\mathbf{1 1}$ calculated at TDCAM-B3LYP/6-311++G(2d,2p) level for structures optimized at M06-2X/6-311++G(d,p) level. Wavelengths were not corrected.
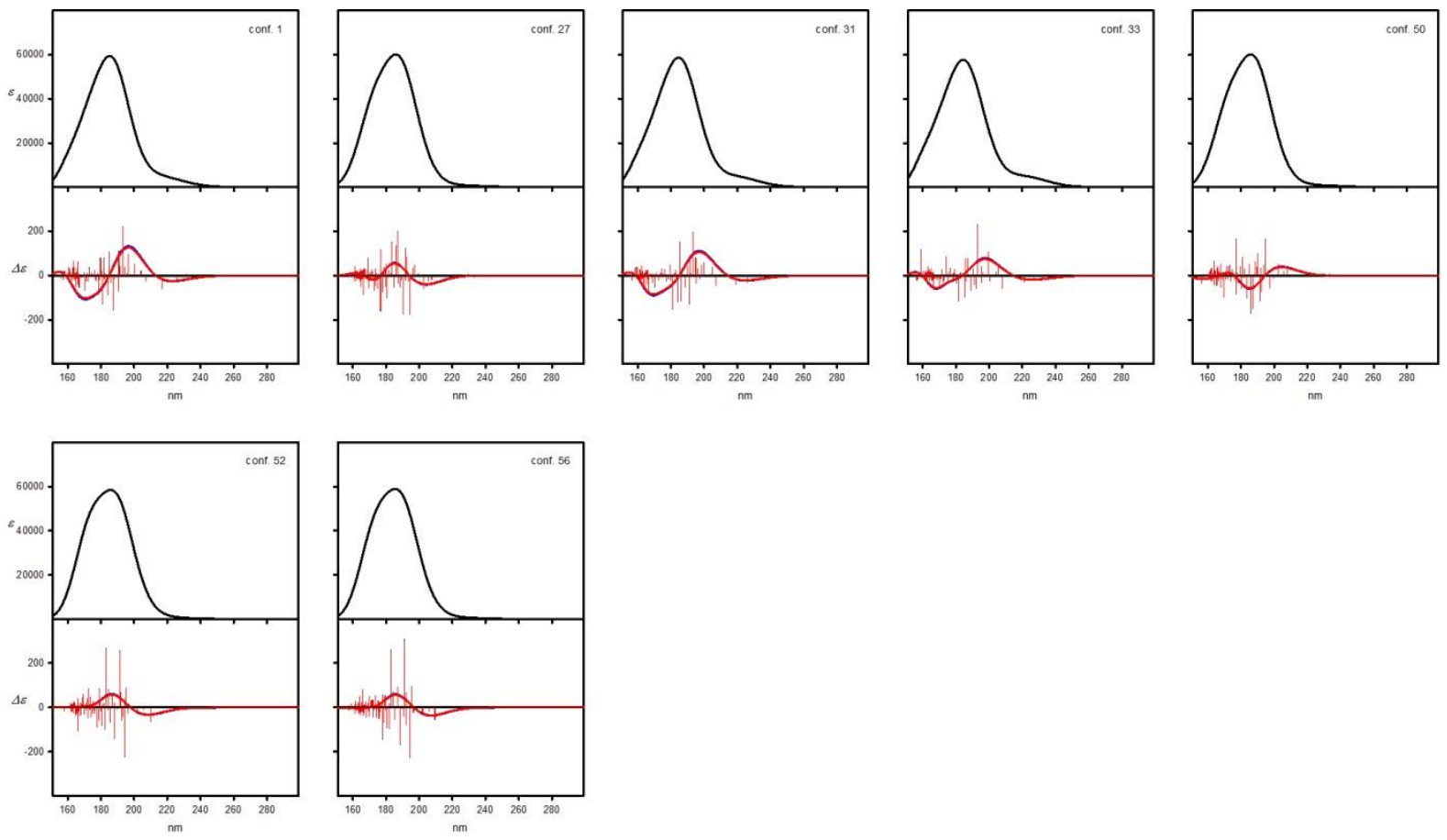

Figure SI_46. UV and ECD spectra of the low-energy conformers of compound $\mathbf{1 1}$ calculated at TDM06-2X/6-311++G(2d,2p) level for structures optimized at M06-2X/6-311++G(d,p) level. Wavelengths were not corrected. 

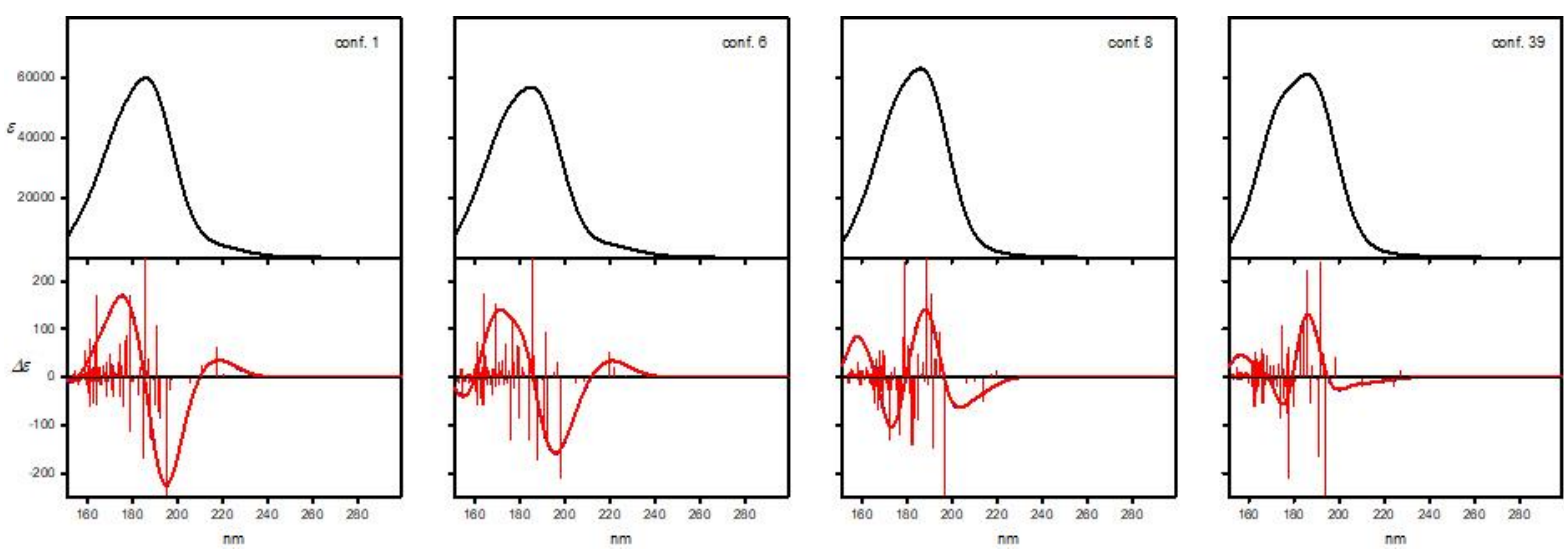

Figure SI_47. UV and ECD spectra of the low-energy conformers of compound $\mathbf{1 4}$ calculated at TDCAM-B3LYP/6-311++G(2d,2p) level for structures optimized at B3LYP/6-311++G(d,p) level. Wavelengths were not corrected.
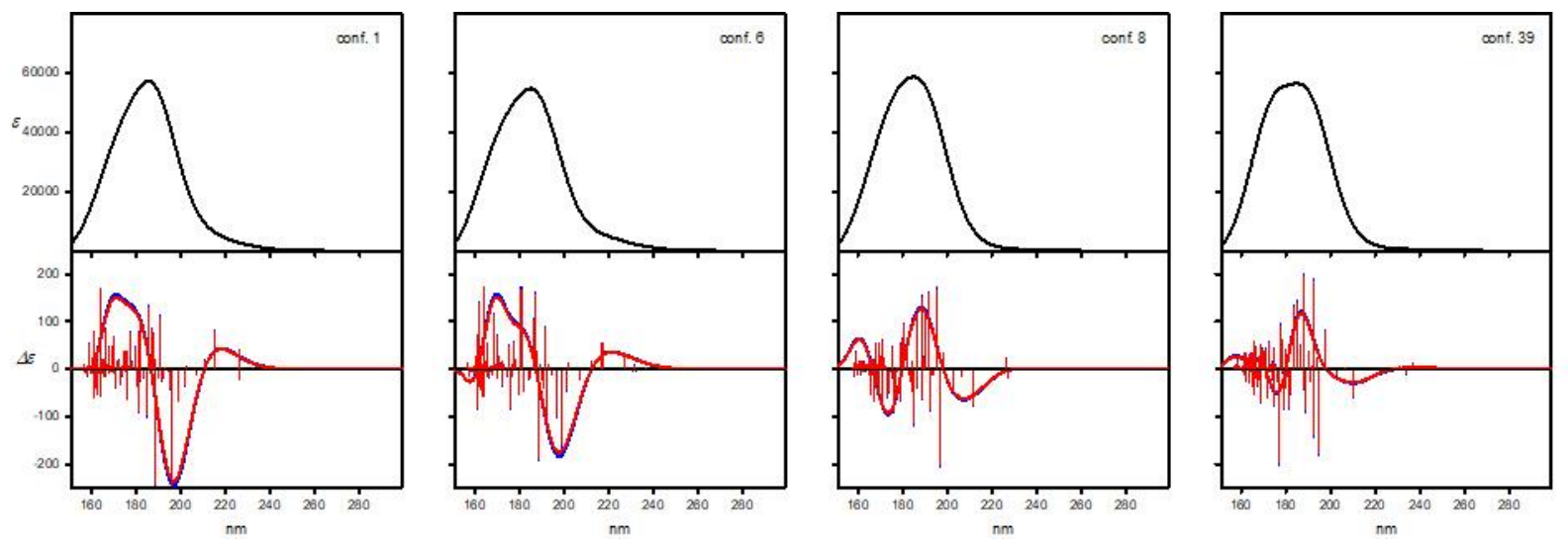

Figure SI_48. UV and ECD spectra of the low-energy conformers of compound $\mathbf{1 4}$ calculated at TDM06-2X/6-311++G $(2 d, 2 p)$ level for structures optimized at B3LYP/6-311++G(d,p) level. Wavelengths were not corrected. 

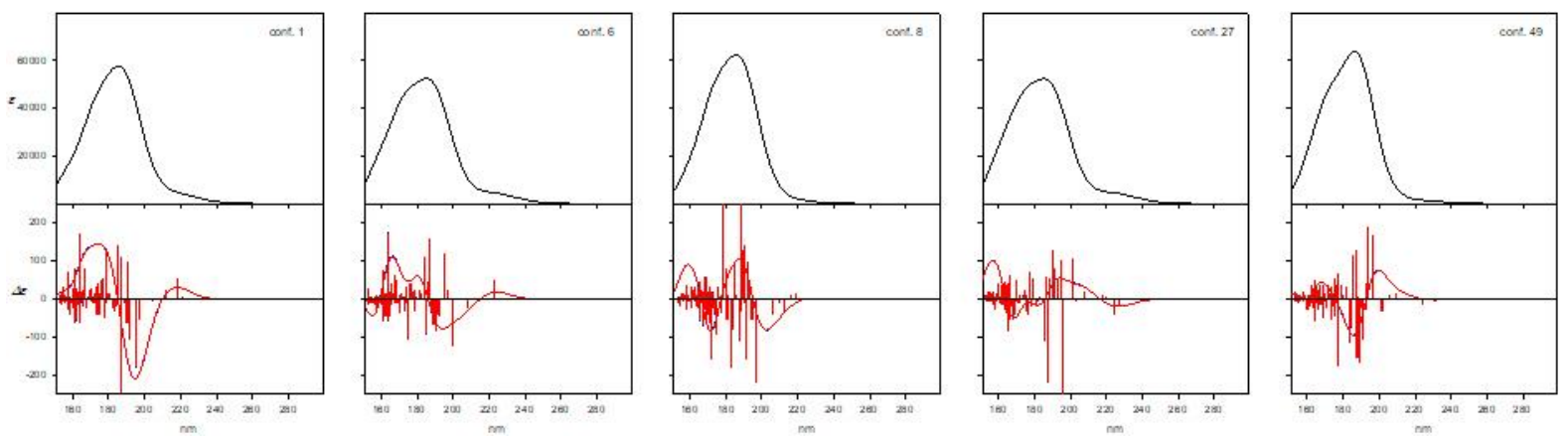

Figure SI_49. UV and ECD spectra of the low-energy conformers of compound $\mathbf{1 4}$ calculated at TDCAM-B3LYP/6-311++G(2d,2p) level for structures optimized at M06-2X/6-311++G(d,p) level. Wavelengths were not corrected.
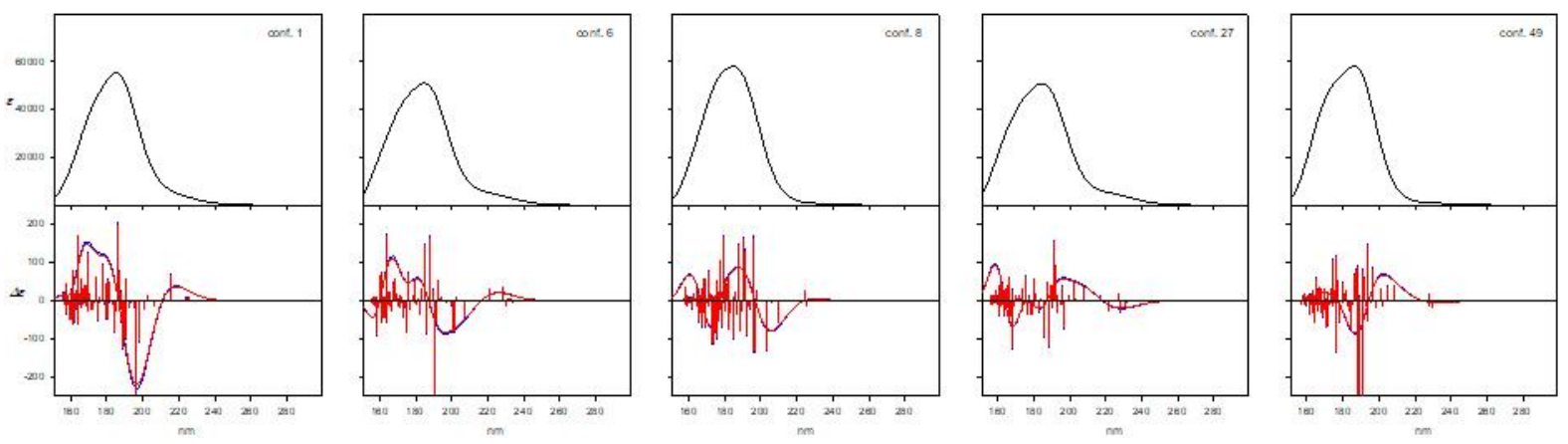

Figure SI_50. UV and ECD spectra of the low-energy conformers of compound $\mathbf{1 4}$ calculated at TDM06-2X/6-311++G(2d,2p) level for structures optimized at M06-2X/6-311++G(d,p) level. Wavelengths were not corrected. 

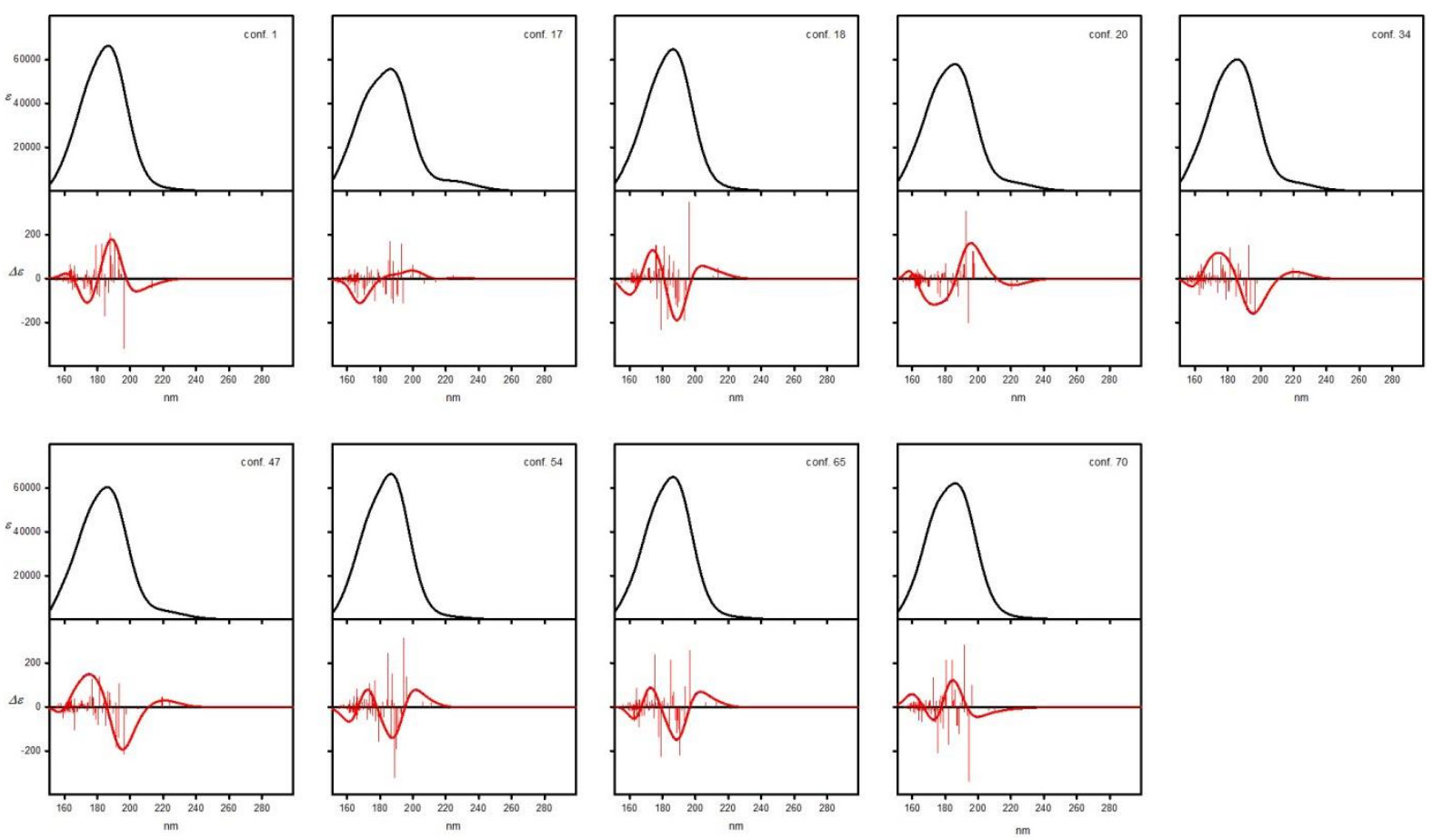

Figure SI_51. UV and ECD spectra of the low-energy conformers of compound $\mathbf{1 5}$ calculated at TDCAM-B3LYP/6-311++G(2d,2p) level for structures optimized at B3LYP/6-311++G(d,p) level. Wavelengths were not corrected.
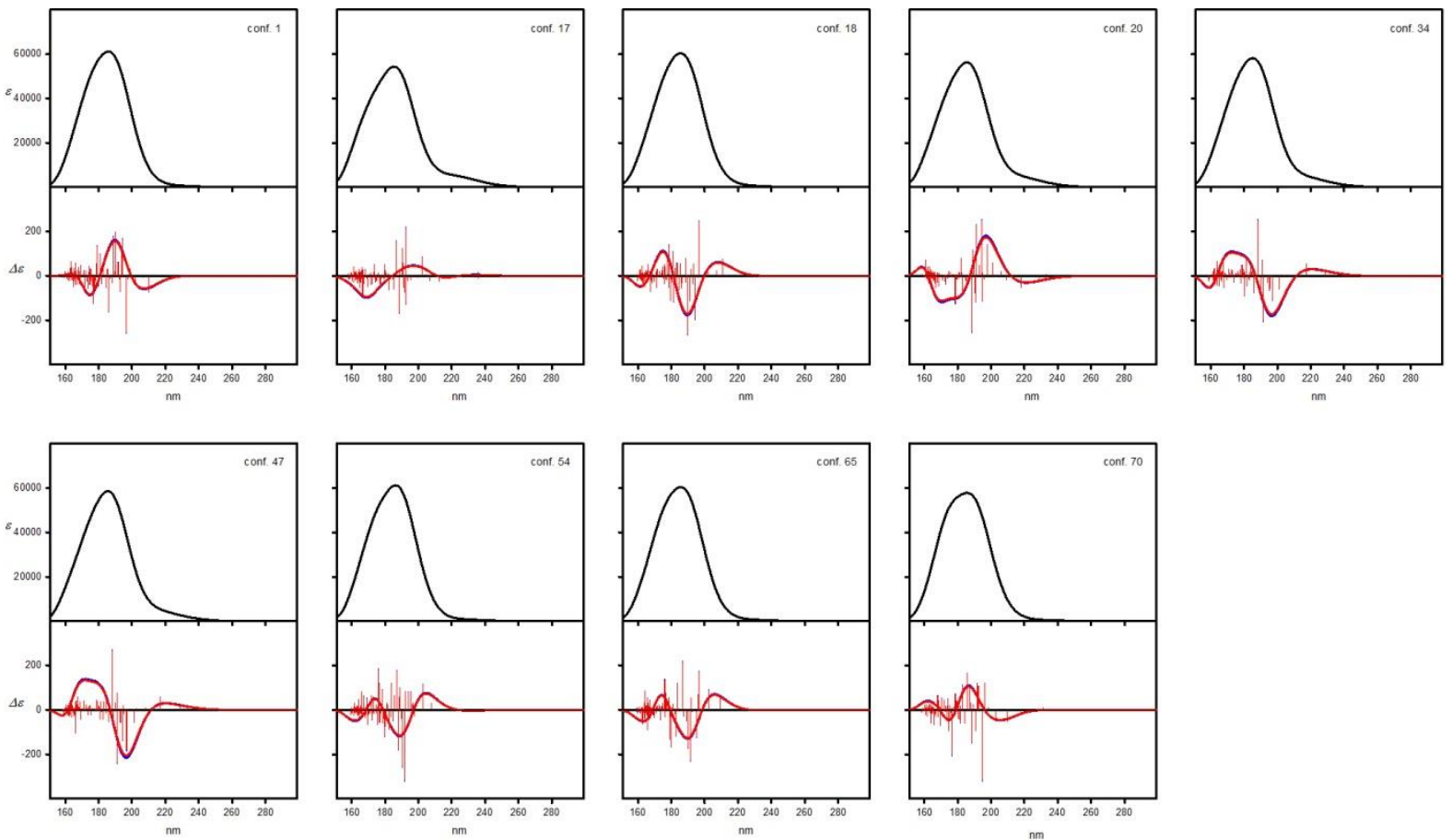

Figure SI_52. UV and ECD spectra of the low-energy conformers of compound $\mathbf{1 5}$ calculated at TDM06-2X/6-311++G $(2 d, 2 p)$ level for structures optimized at B3LYP/6-311++G(d,p) level. Wavelengths were not corrected. 

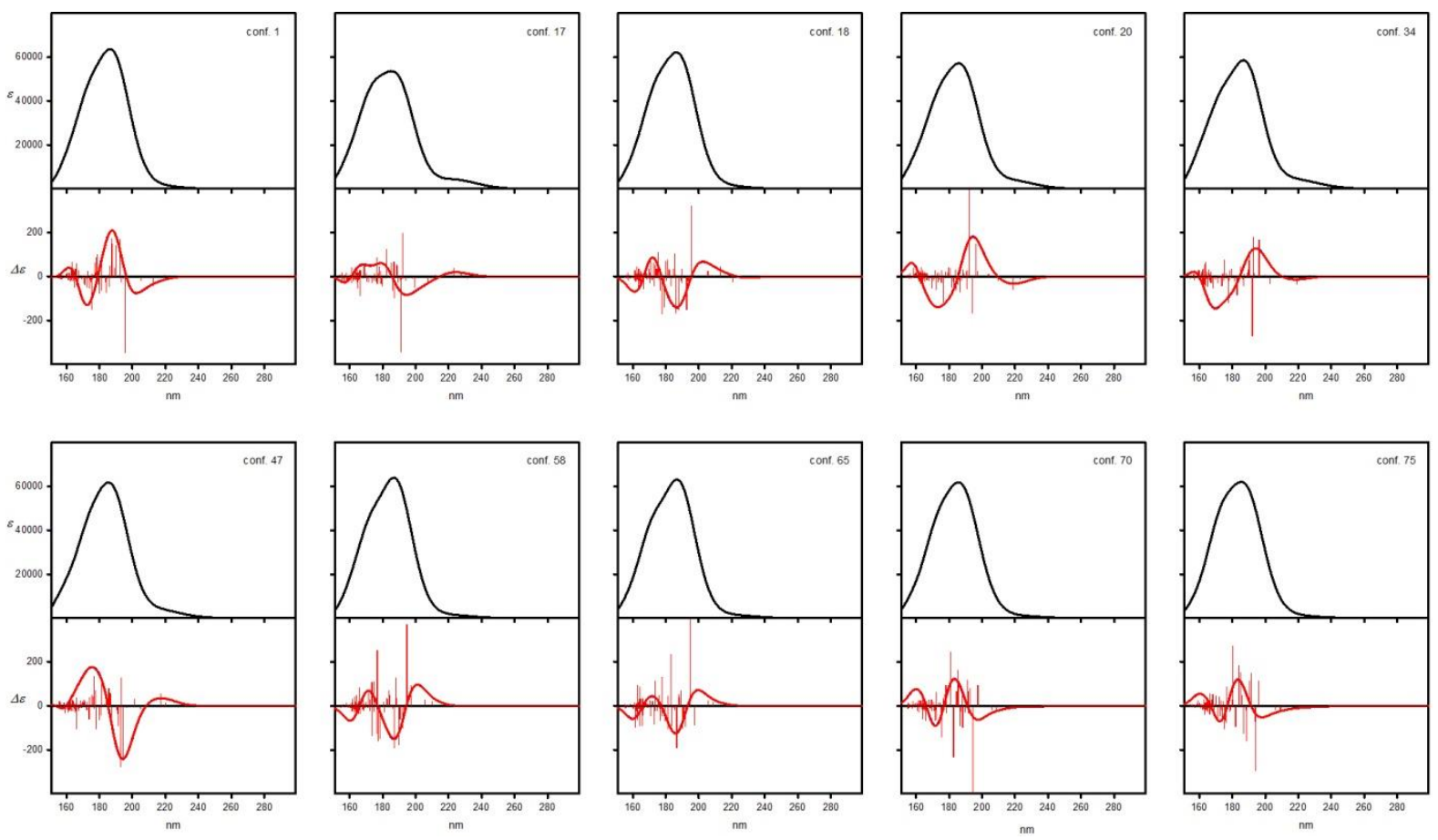

Figure SI_53. UV and ECD spectra of the low-energy conformers of compound $\mathbf{1 5}$ calculated at TDCAM-B3LYP/6-311++G(2d,2p) level for structures optimized at M06-2X/6-311++G(d,p) level. Wavelengths were not corrected.
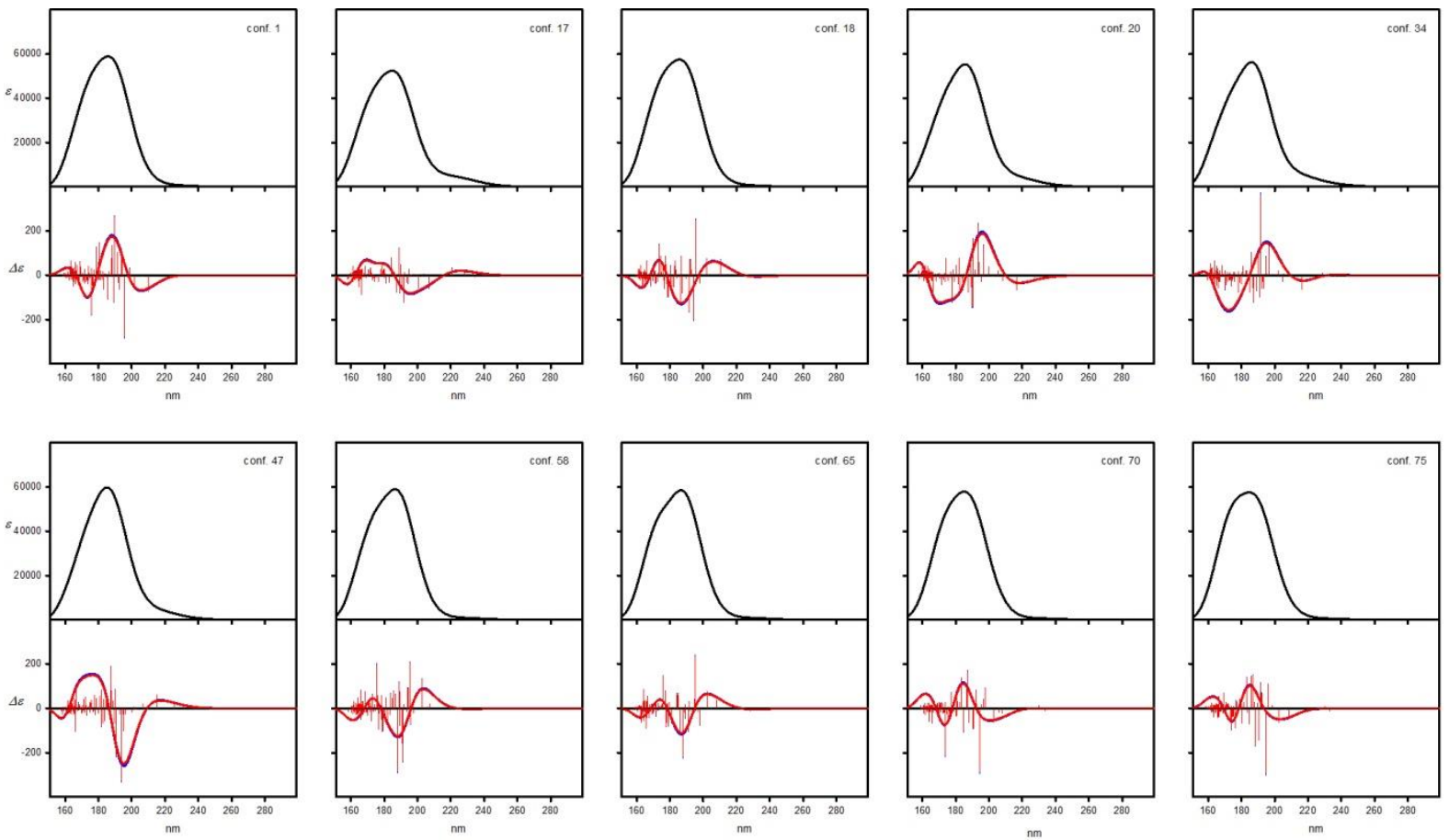

Figure SI_54. UV and ECD spectra of the low-energy conformers of compound $\mathbf{1 5}$ calculated at TDM06-2X/6-311++G(2d,2p) level for structures optimized at $M 06-2 X / 6-311++G(d, p)$ level. Wavelengths were not corrected. 

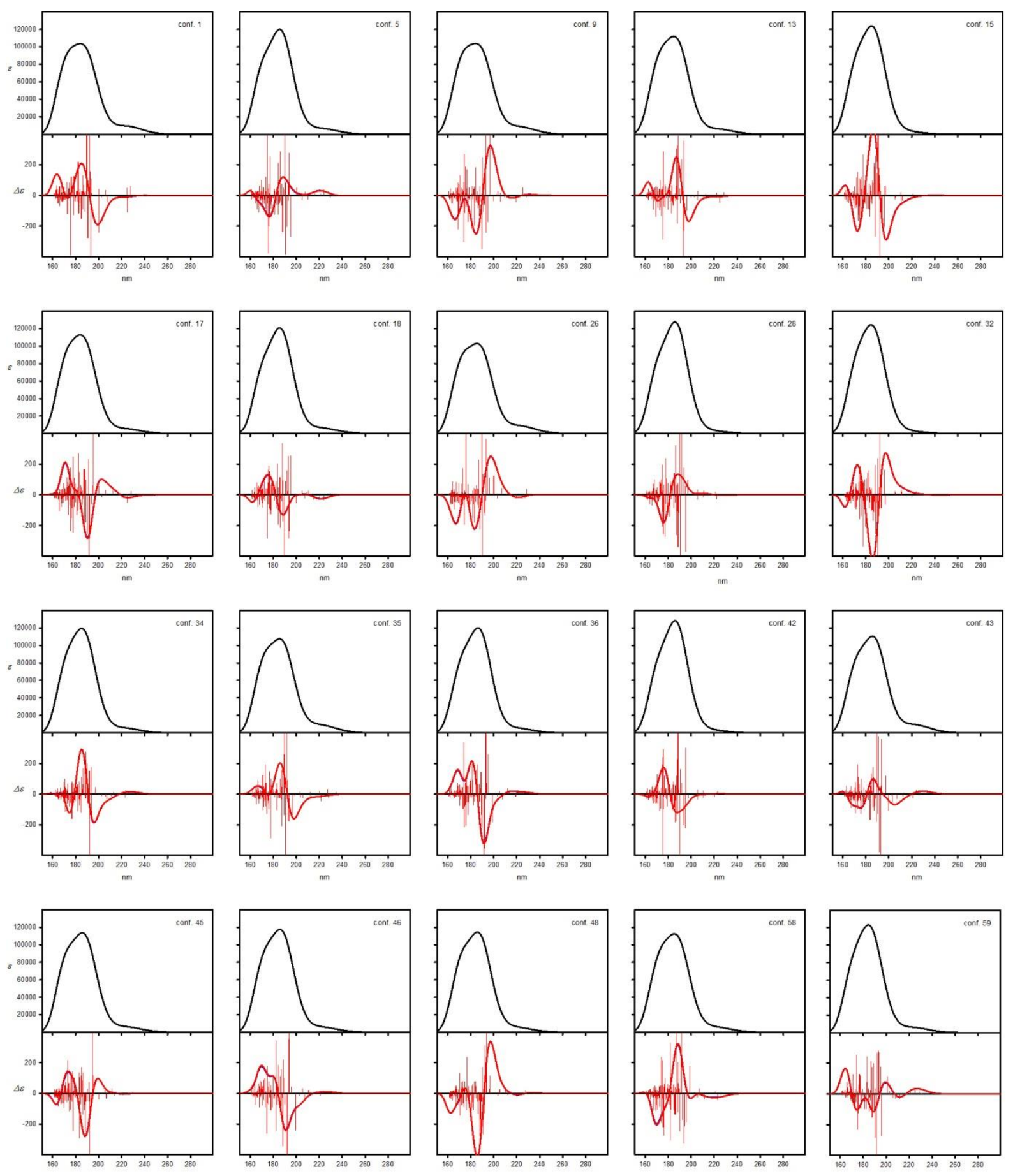

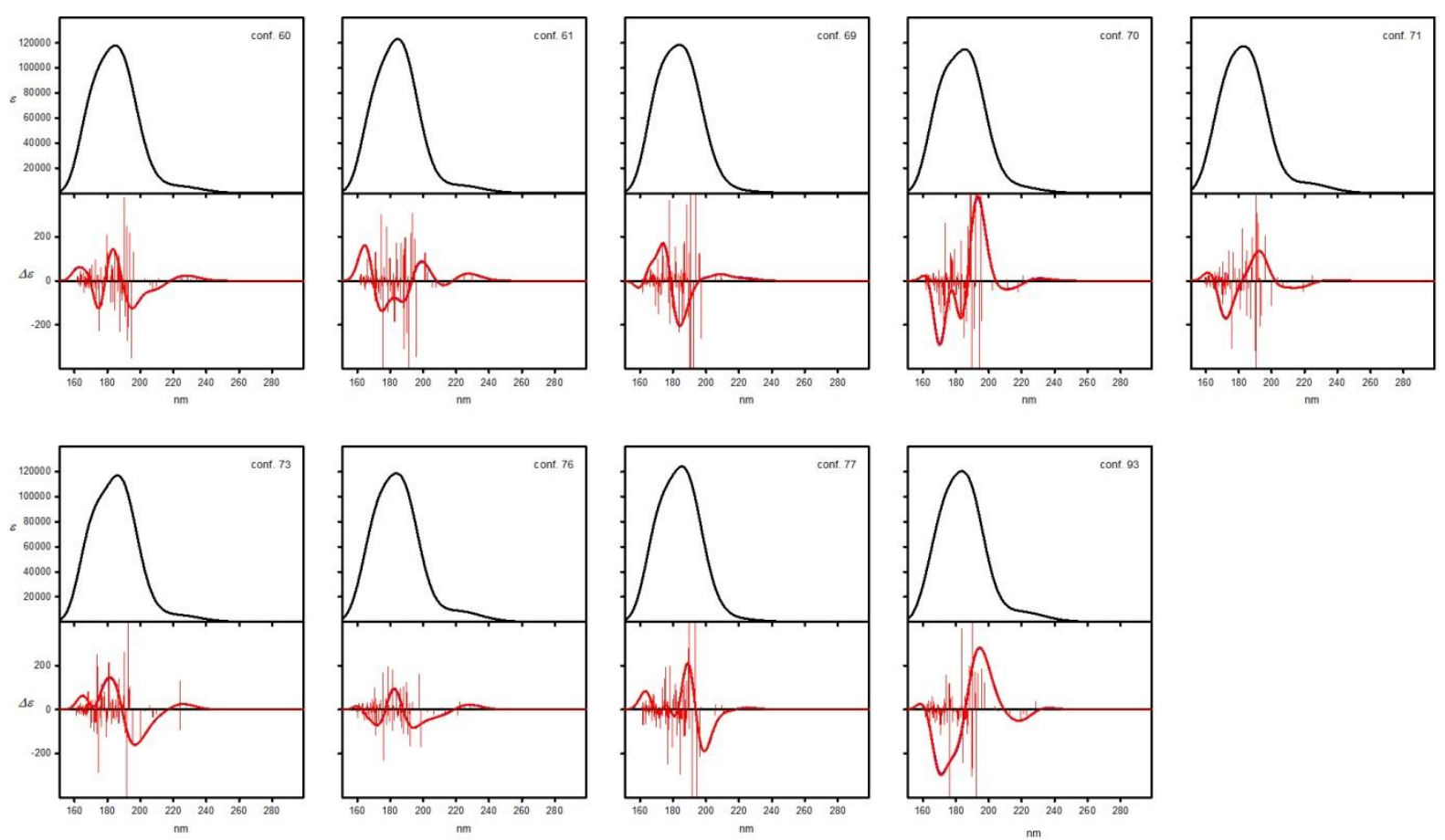

Figure SI_55. UV and ECD spectra of the low-energy conformers of compound $\mathbf{1 8}$ calculated at TDCAM-B3LYP/6-311++G $(d, p)$ level for structures optimized at B3LYP/6-311G(d,p) level. Wavelengths were not corrected. 

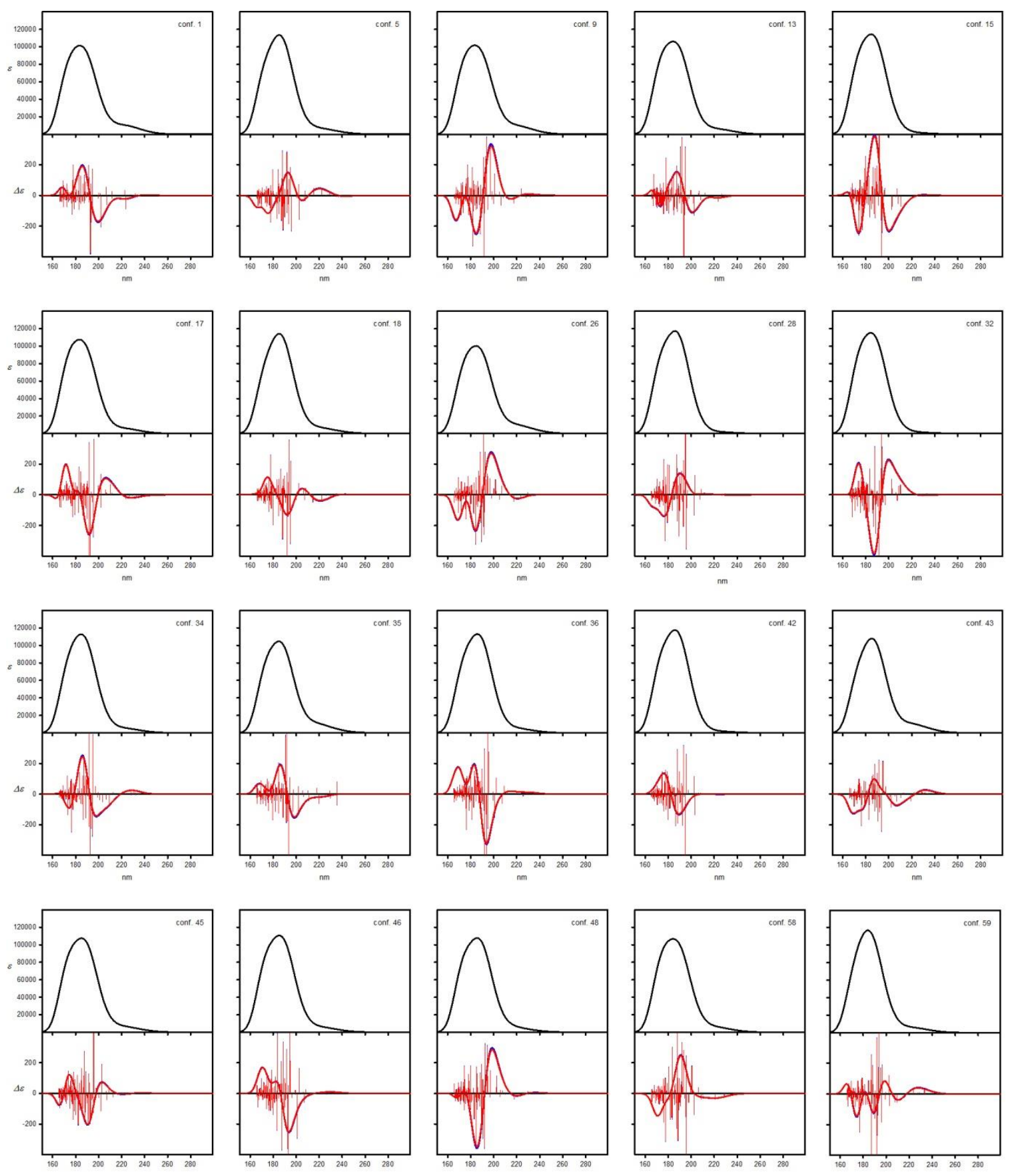

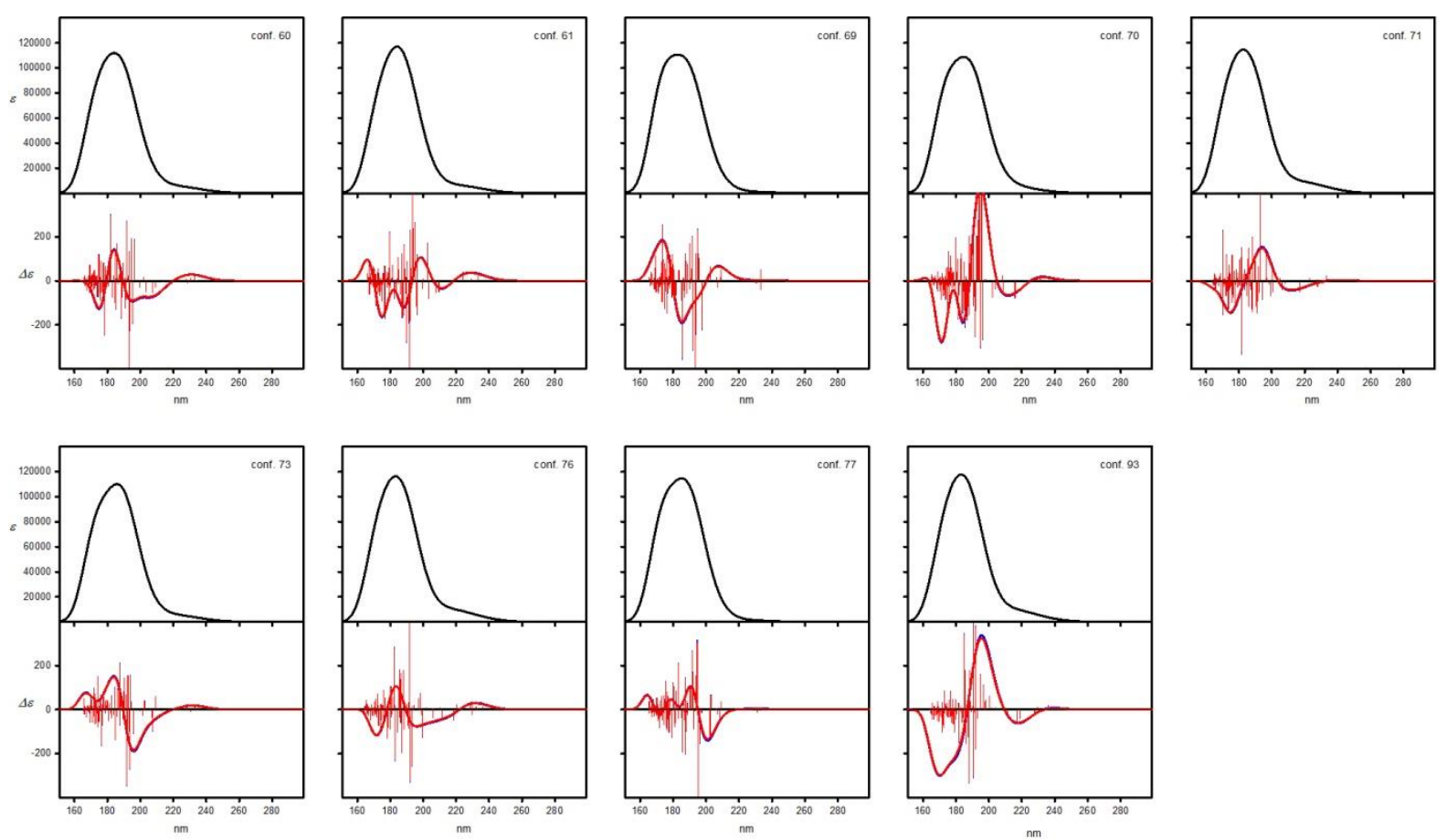

Figure SI_56. UV and ECD spectra of the low-energy conformers of compound $\mathbf{1 8}$ calculated at TDM06-2X/6-311++G(d,p) level for structures optimized at B3LYP/6-311G(d,p) level. Wavelengths were not corrected. 

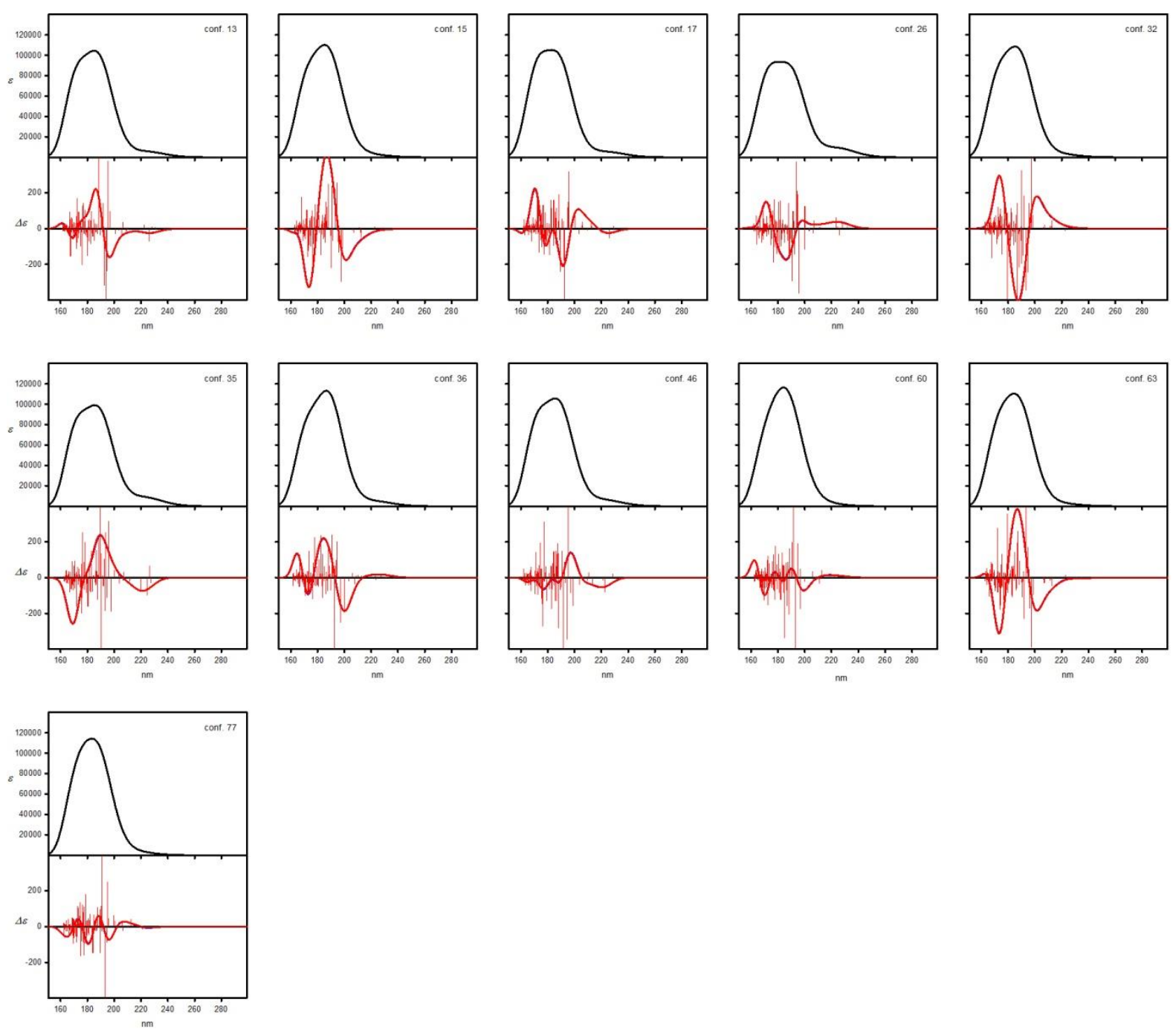

Figure SI_57. UV and ECD spectra of the low-energy conformers of compound $\mathbf{1 8}$ calculated at TDCAM-B3LYP/6-311++G(d,p) level for structures optimized at B3LYP-GD3BJ/6-311G(d,p) level. Wavelengths were not corrected. 

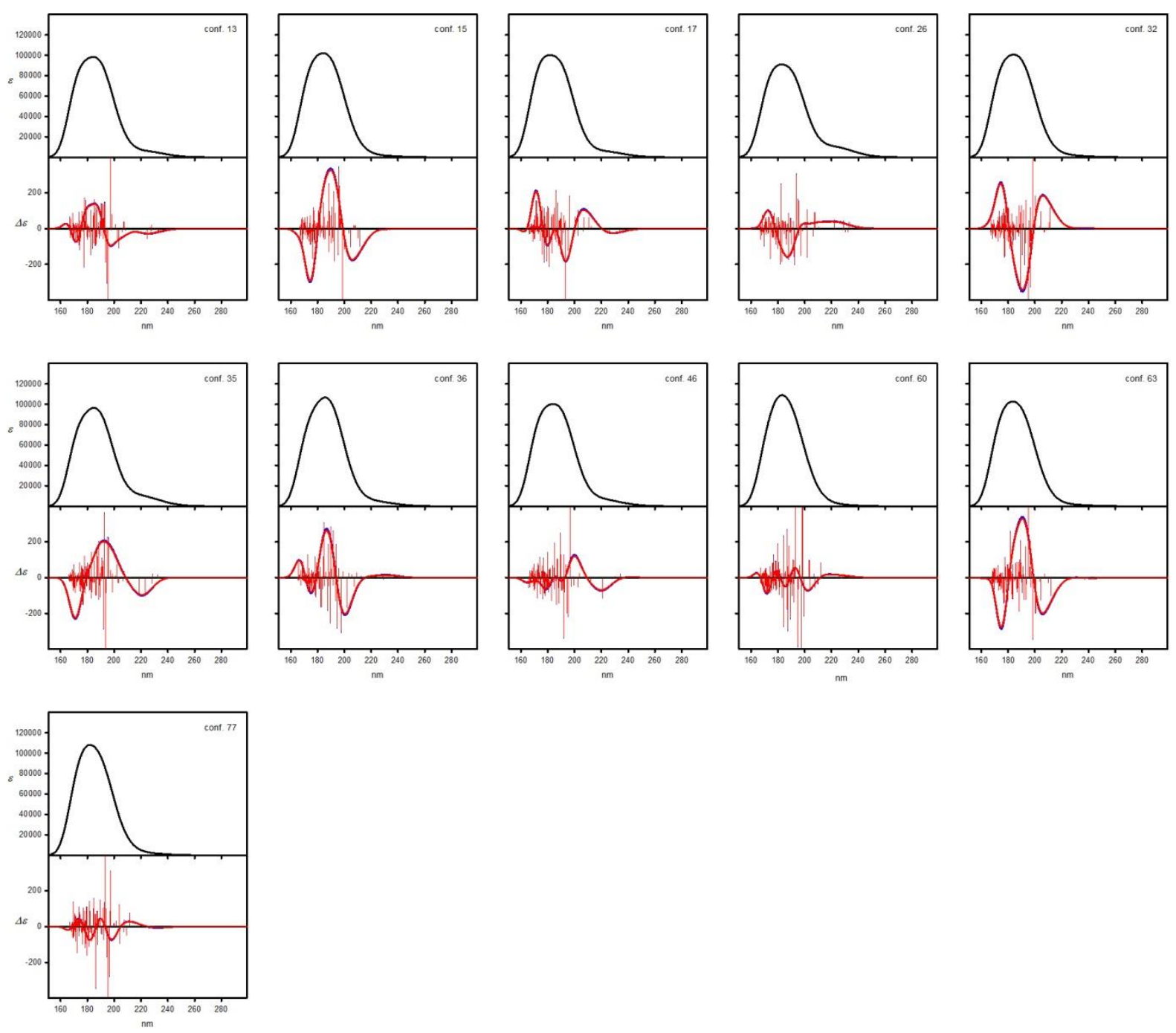

Figure SI_58. UV and ECD spectra of the low-energy conformers of compound 18 calculated at TDM06-2X/6-311++G(d,p) level for structures optimized at B3LYP-GD3BJ/6-311G(d,p) level. Wavelengths were not corrected. 

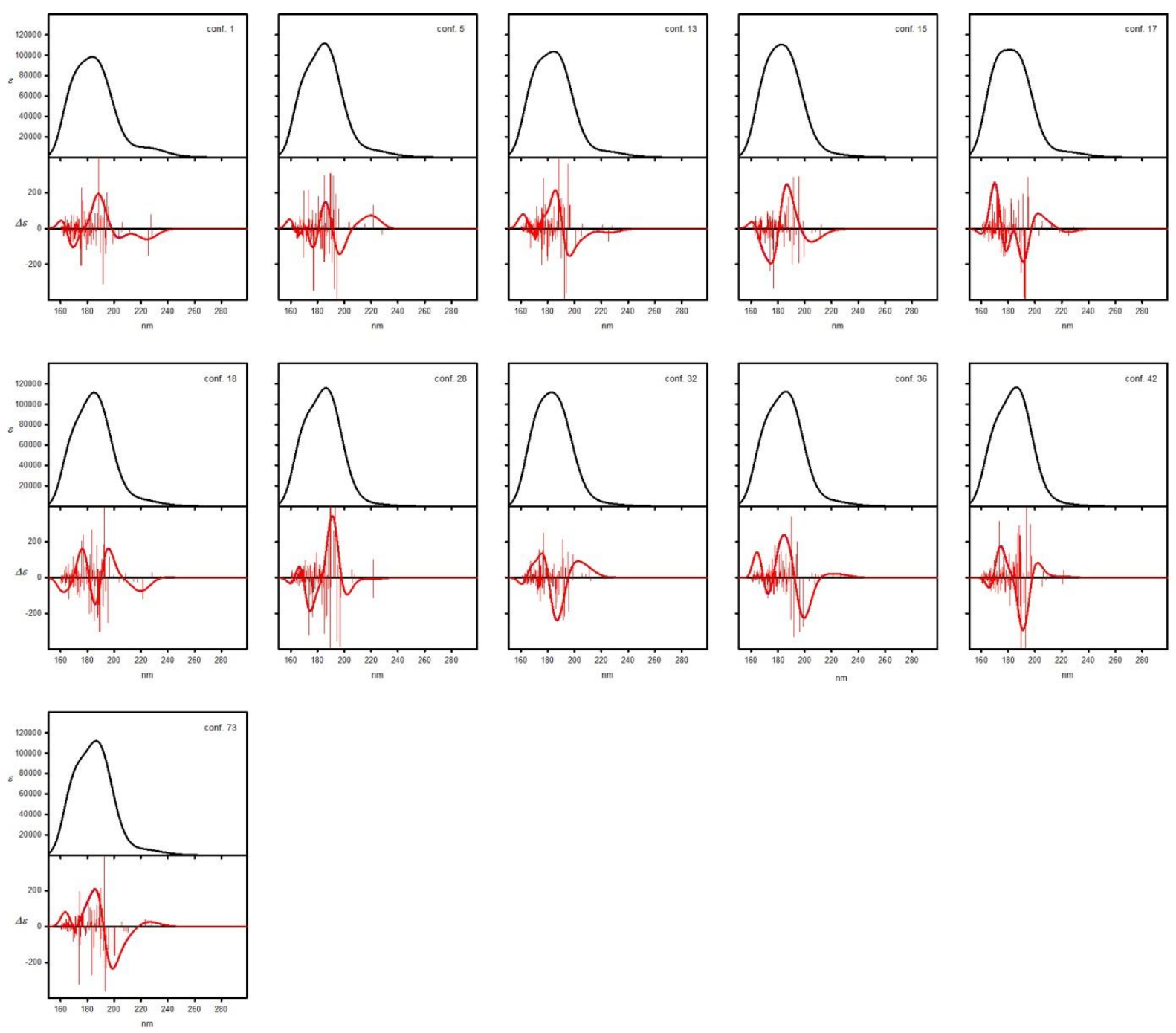

Figure SI_59. UV and ECD spectra of the low-energy conformers of compound $\mathbf{1 8}$ calculated at TDCAM-B3LYP/6-311++G $(d, p)$ level for structures optimized at M06-2X/6-311G $(d, p)$ level. Wavelengths were not corrected. 

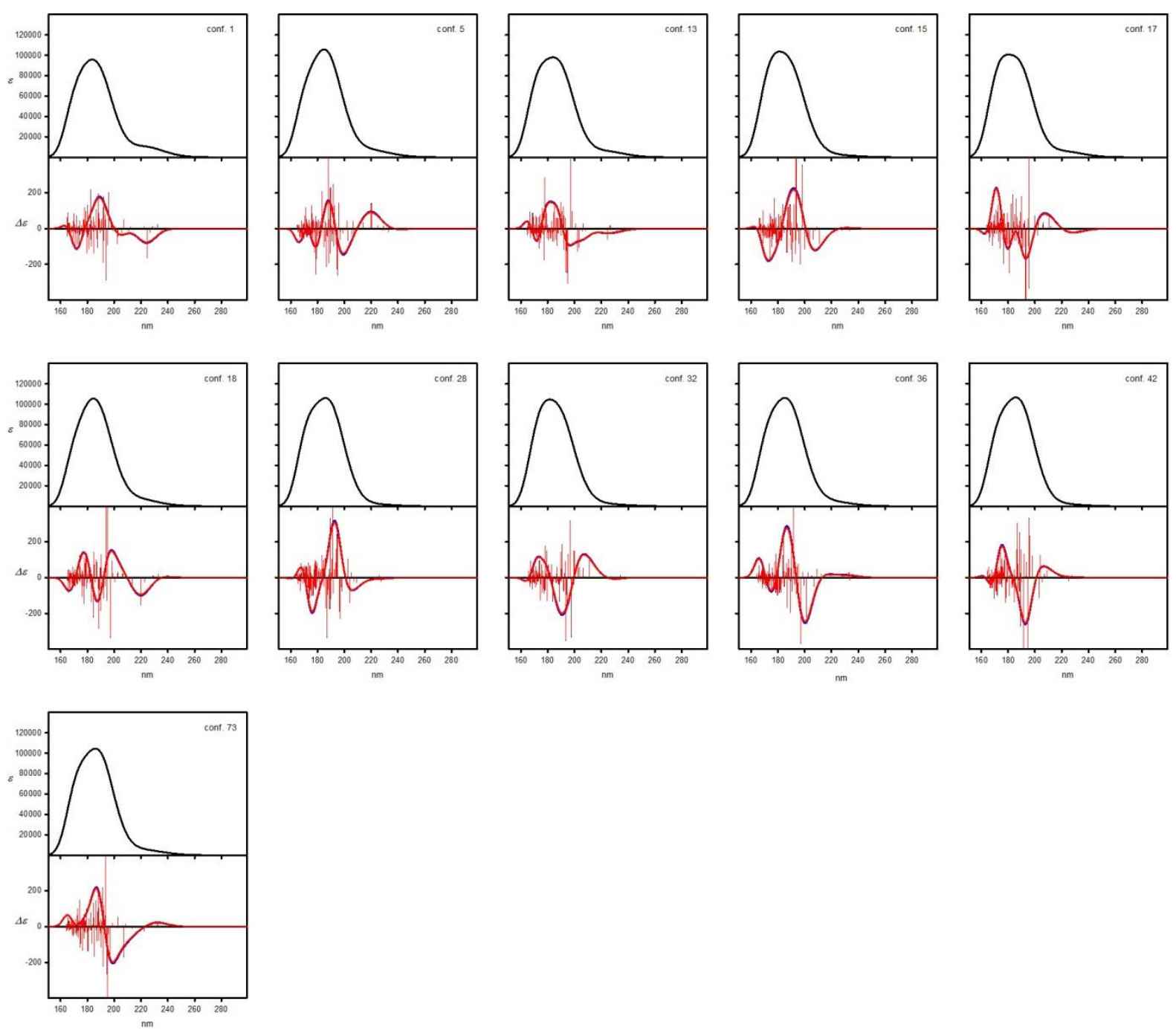

Figure SI_60. UV and ECD spectra of the low-energy conformers of compound 18 calculated at TDM06-2X/6-311++G(d,p) level for structures optimized at M06-2X/6-311G(d,p) level. Wavelengths were not corrected. 

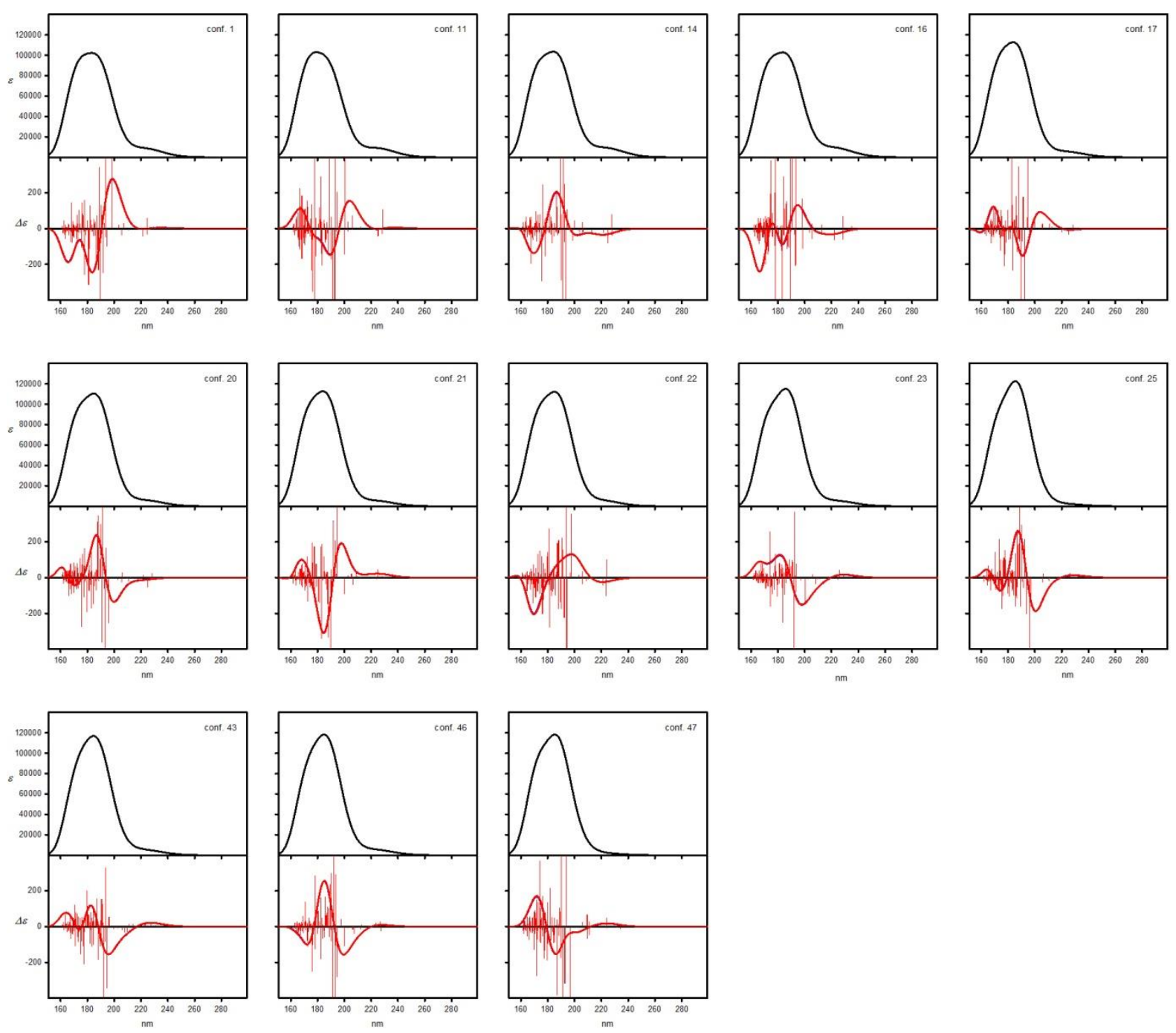

Figure SI_61. UV and ECD spectra of the low-energy conformers of compound $\mathbf{2 0}$ calculated at TDCAM-B3LYP/6-311++G $(d, p)$ level for structures optimized at B3LYP/6-311G $(d, p)$ level. Wavelengths were not corrected. 

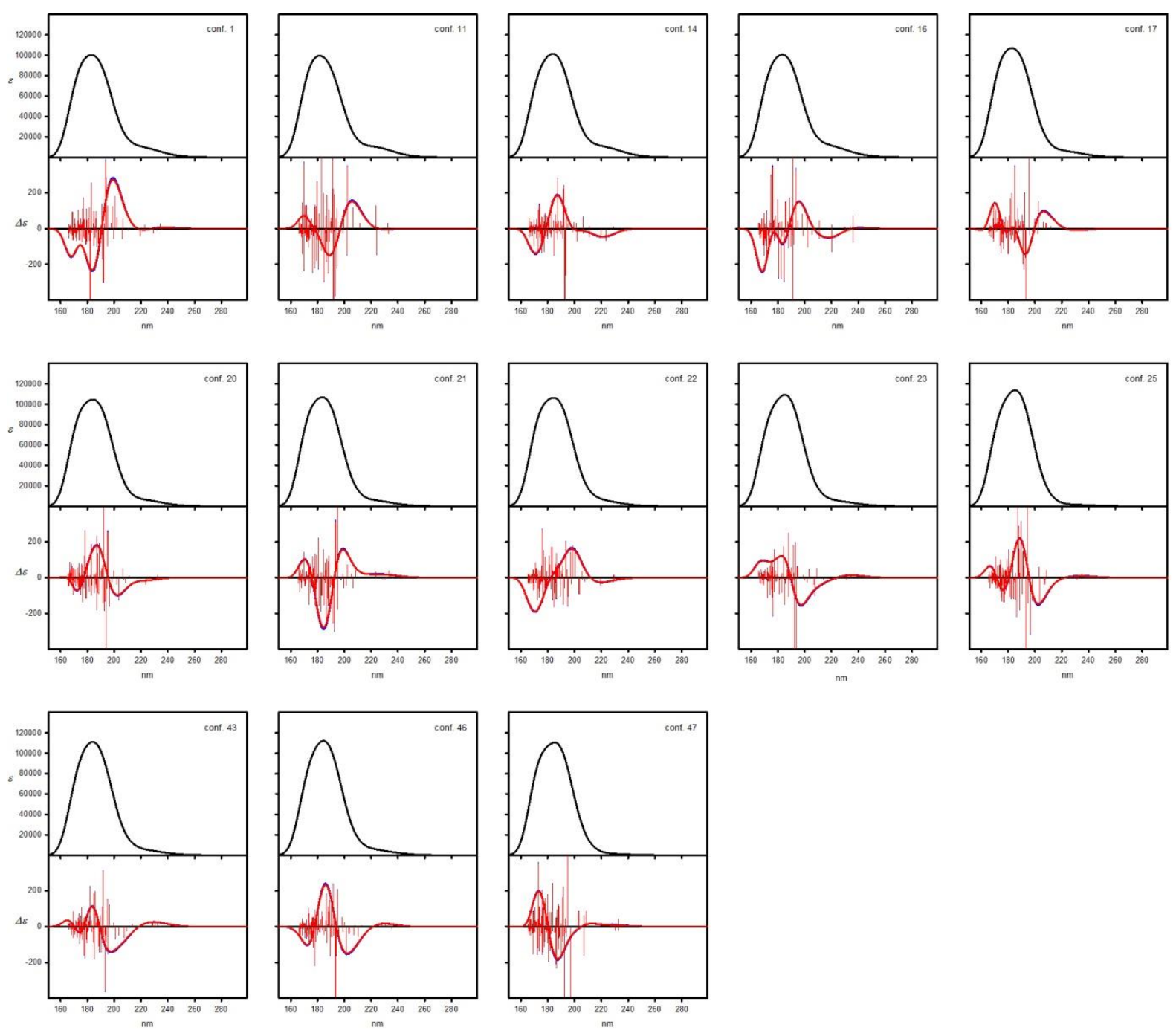

Figure SI_62. UV and ECD spectra of the low-energy conformers of compound 20 calculated at TDM06-2X/6-311++G(d,p) level for structures optimized at B3LYP/6-311G(d,p) level. Wavelengths were not corrected. 

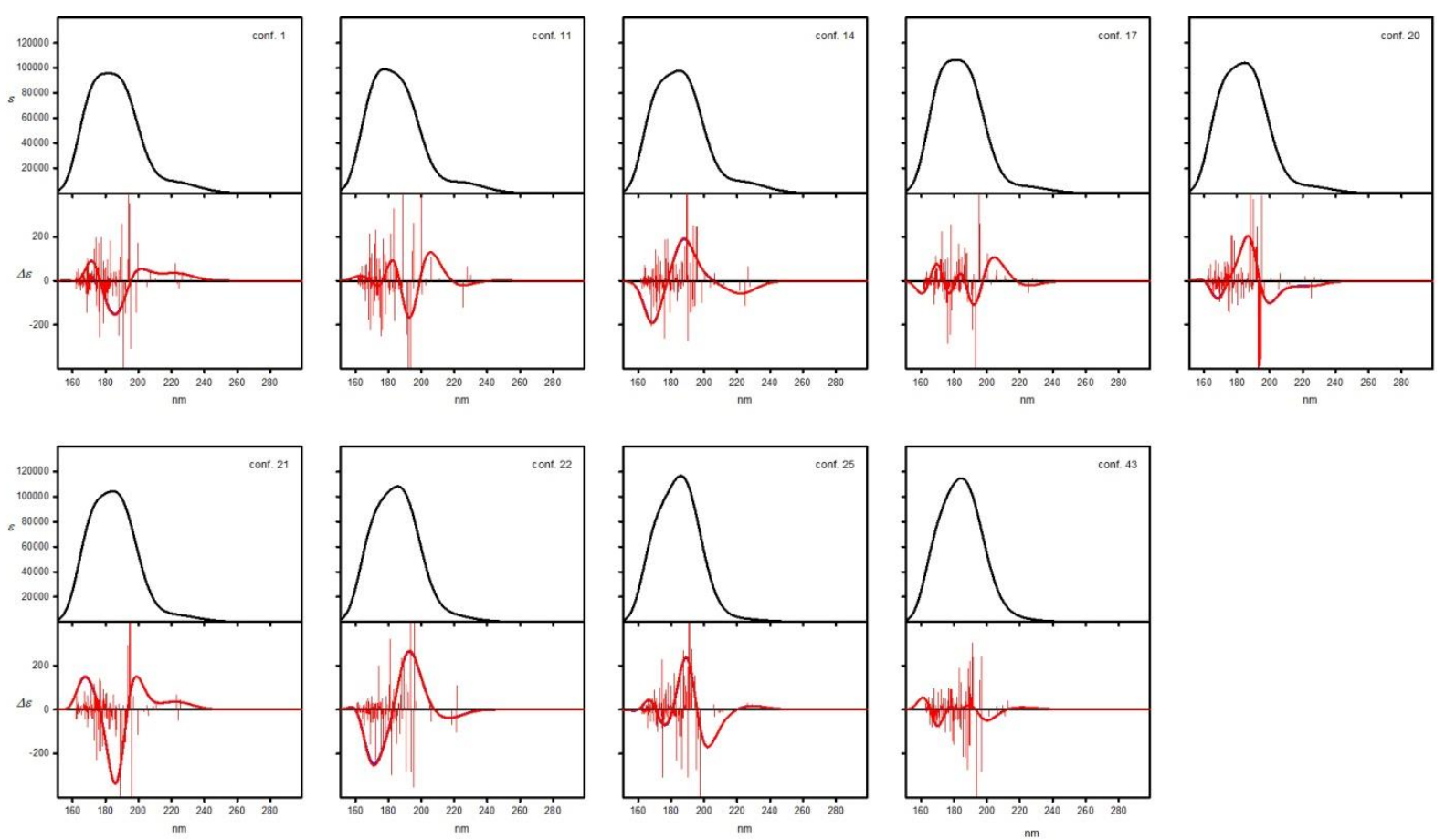

Figure SI_63. UV and ECD spectra of the low-energy conformers of compound $\mathbf{2 0}$ calculated at TDCAM-B3LYP/6-311++G(d,p) level for structures optimized at B3LYP-GD3BJ/6-311G(d,p) level. Wavelengths were not corrected.
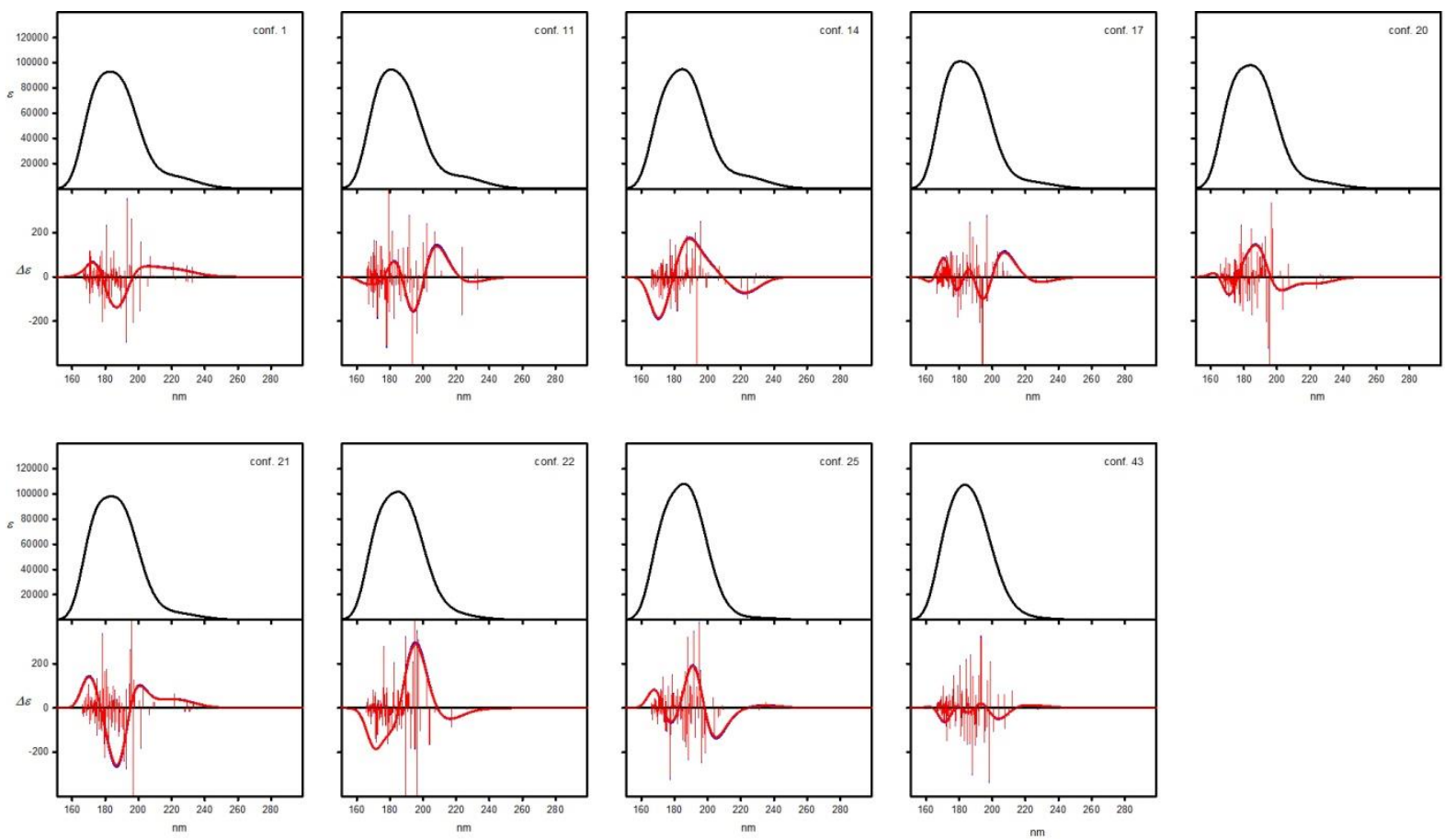

Figure SI_64. UV and ECD spectra of the low-energy conformers of compound $\mathbf{2 0}$ calculated at TDM06-2X/6-311++G(d,p) level for structures optimized at B3LYP-GD3BJ/6-311G(d,p) level. Wavelengths were not corrected. 

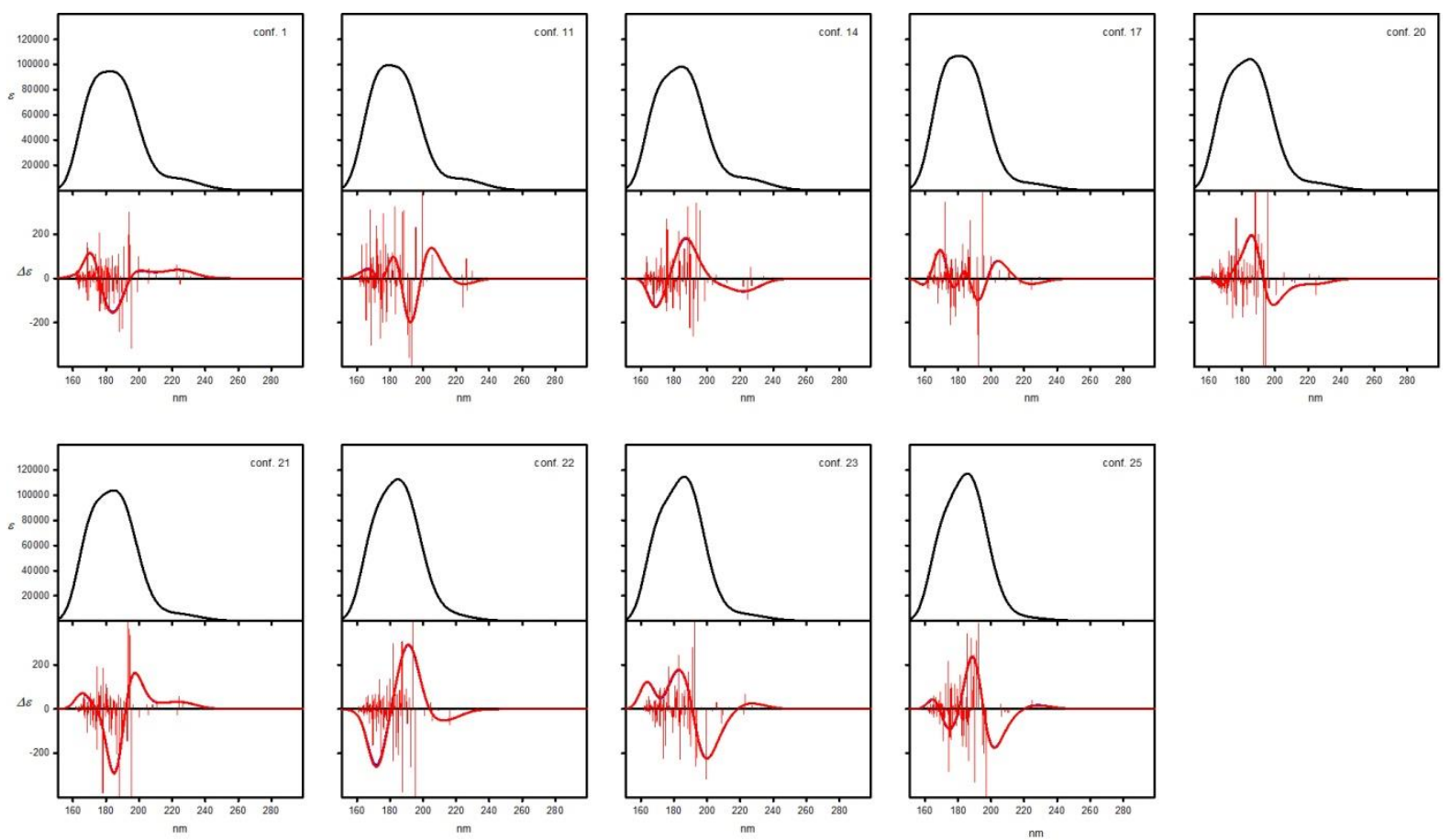

Figure SI_65. UV and ECD spectra of the low-energy conformers of compound $\mathbf{2 0}$ calculated at TDCAM-B3LYP/6-311++G(d,p) level for structures optimized at M06-2X/6-311G(d,p) level. Wavelengths were not corrected.
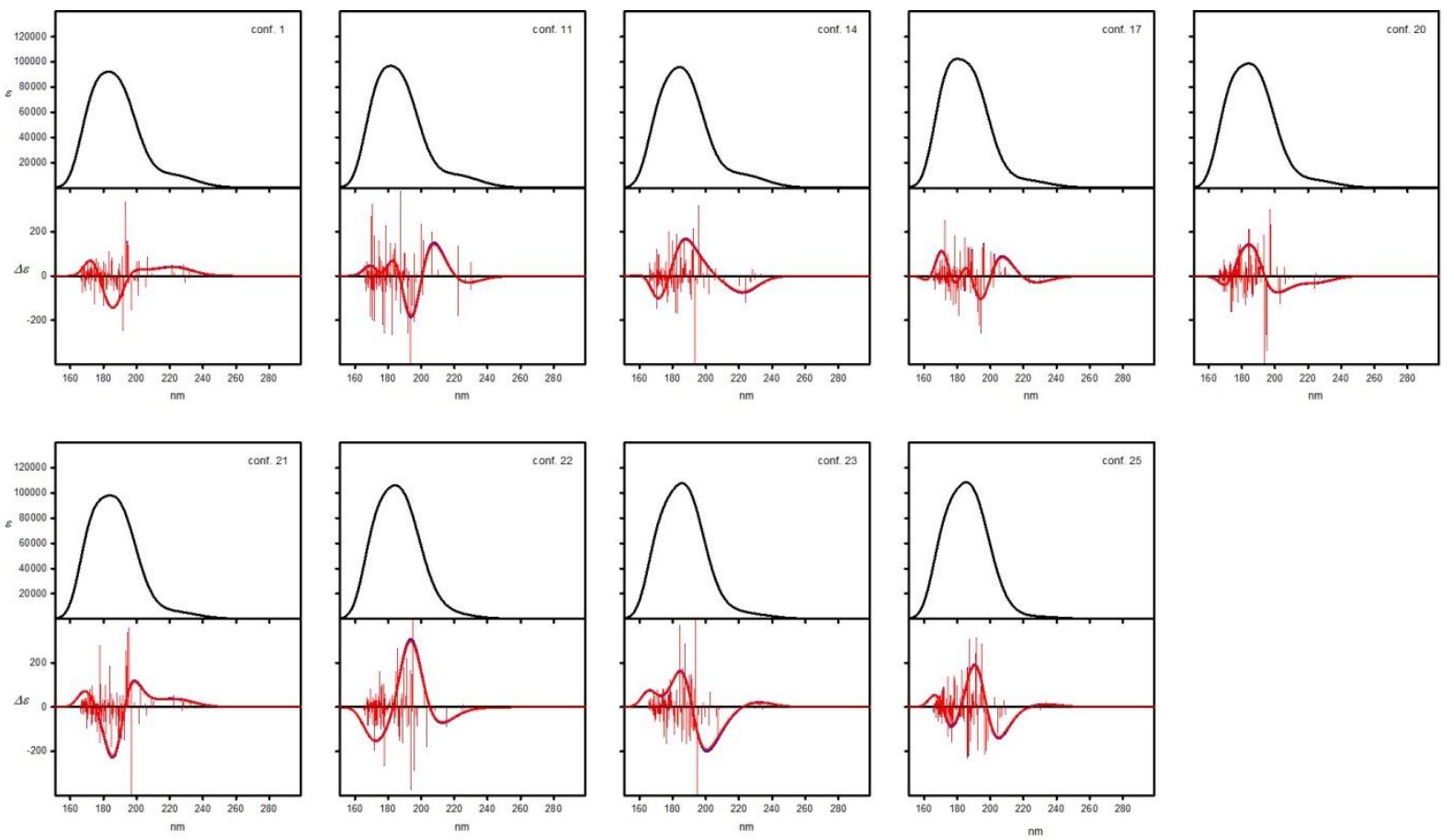

Figure SI_66. UV and ECD spectra of the low-energy conformers of compound $\mathbf{2 0}$ calculated at TDM06-2X/6-311++G(d,p) level for structures optimized at M06-2X/6-311G(d,p) level. Wavelengths were not corrected. 

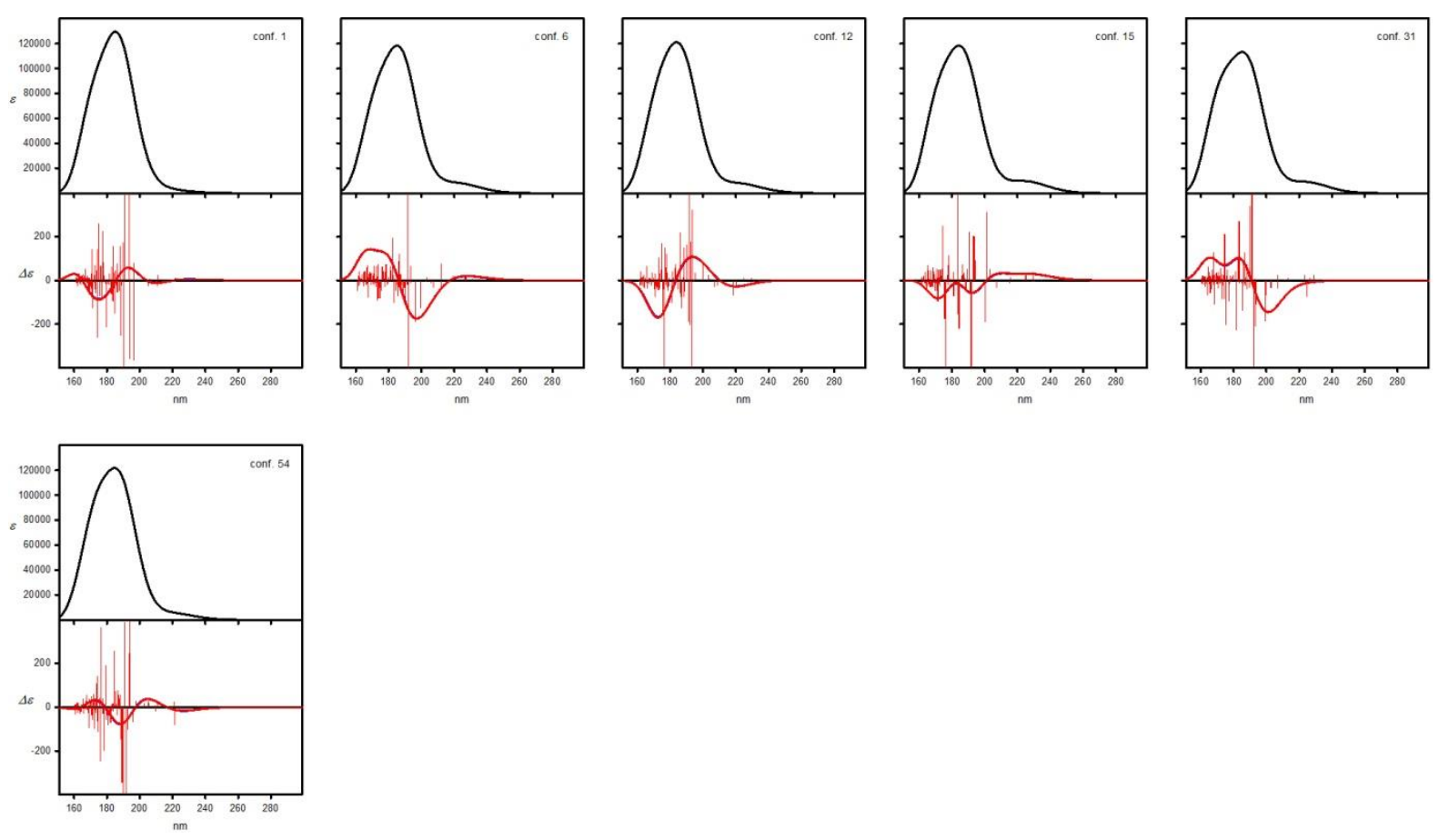

Figure SI_67. UV and ECD spectra of the low-energy conformers of compound $\mathbf{2 1}$ calculated at TDCAM-B3LYP/6-311++G(d,p) level for structures optimized at B3LYP/6-311G $(d, p)$ level. Wavelengths were not corrected.
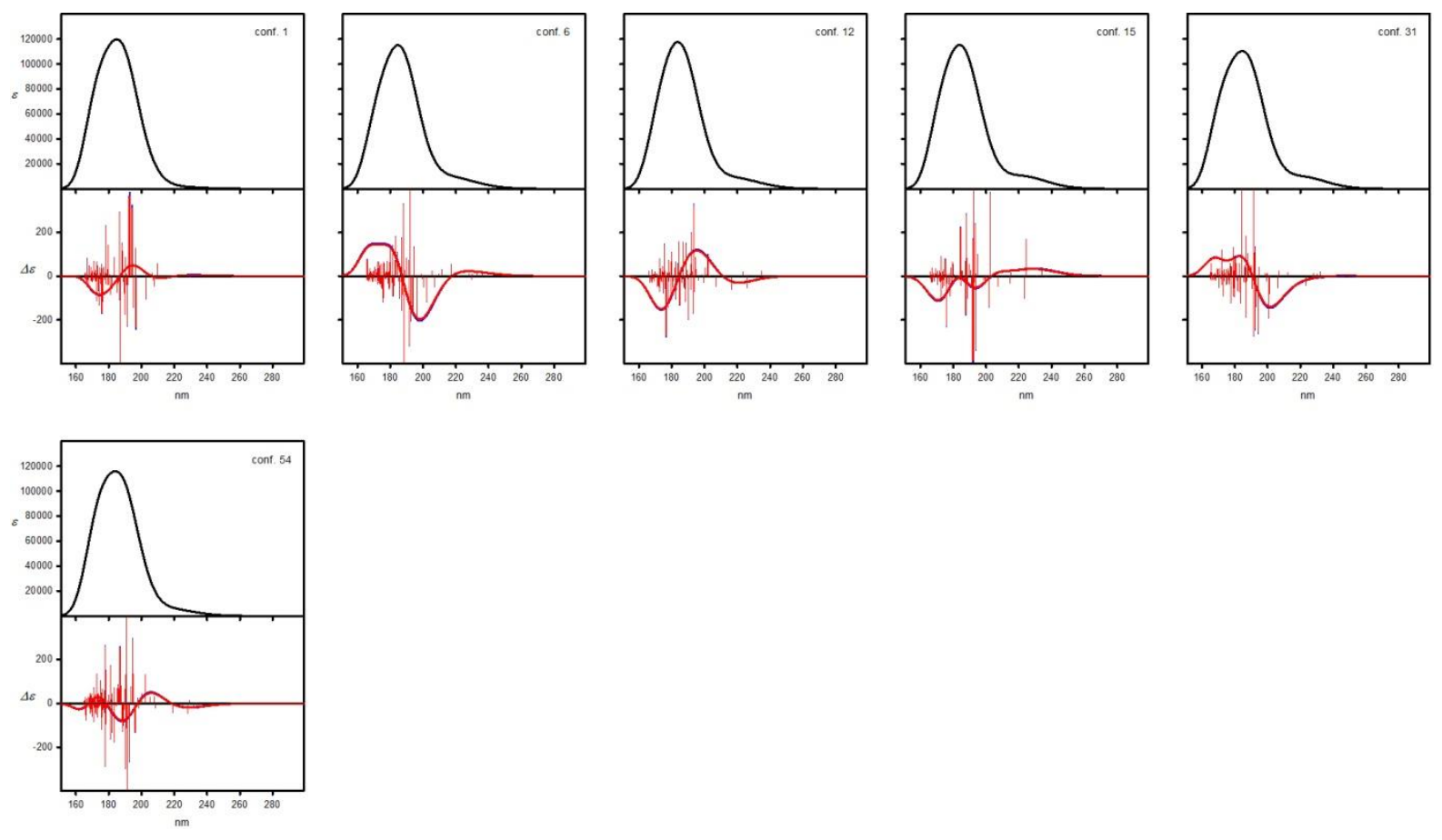

Figure SI_68. UV and ECD spectra of the low-energy conformers of compound 21 calculated at TDM06-2X/6-311++G $(d, p)$ level for structures optimized at B3LYP/6-311G(d,p) level. Wavelengths were not corrected. 

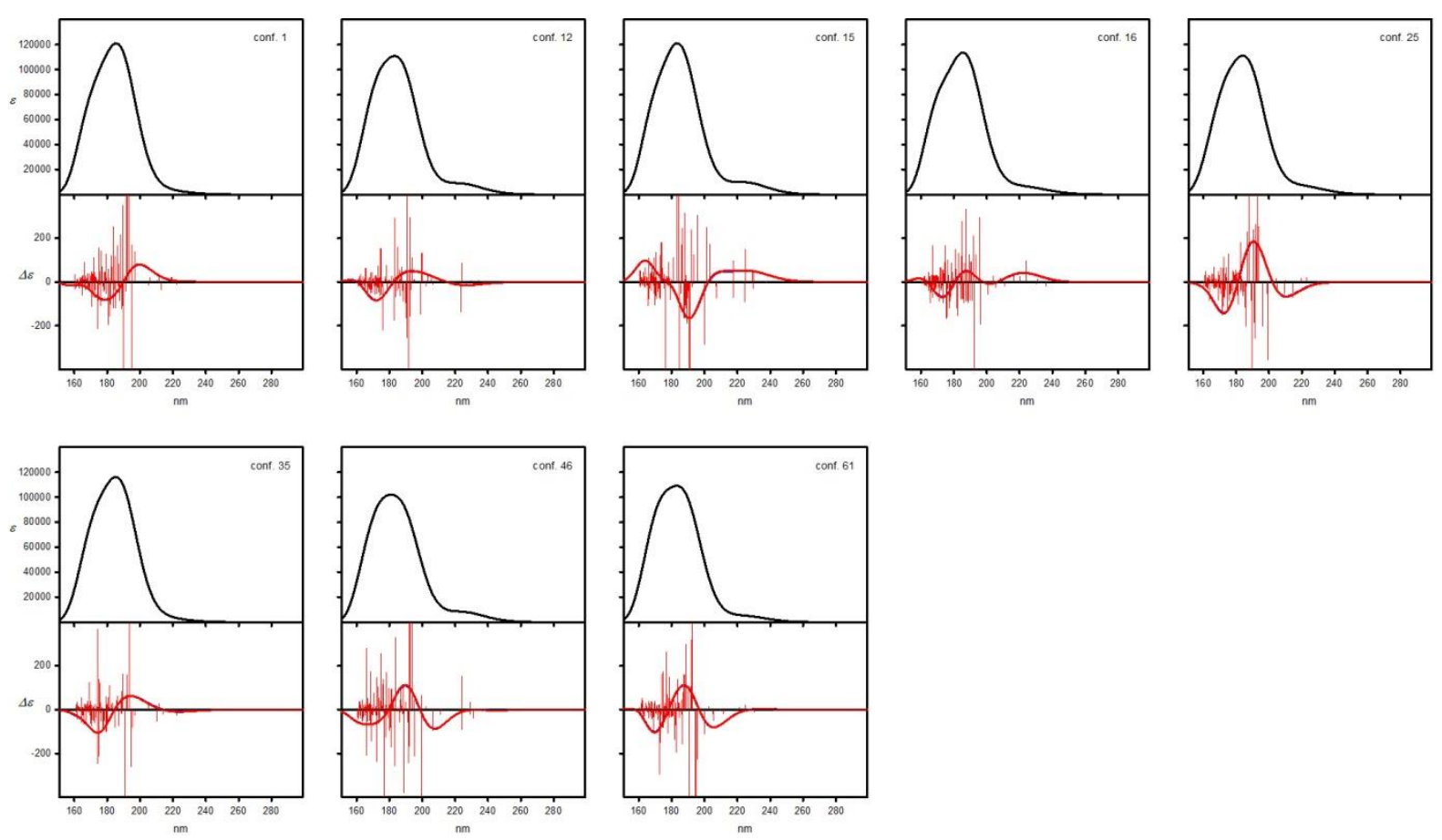

Figure SI_69. UV and ECD spectra of the low-energy conformers of compound 21 calculated at TDCAM-B3LYP/6-311++G(d,p) level for structures optimized at B3LYP-GD3BJ/6-311G(d,p) level. Wavelengths were not corrected.
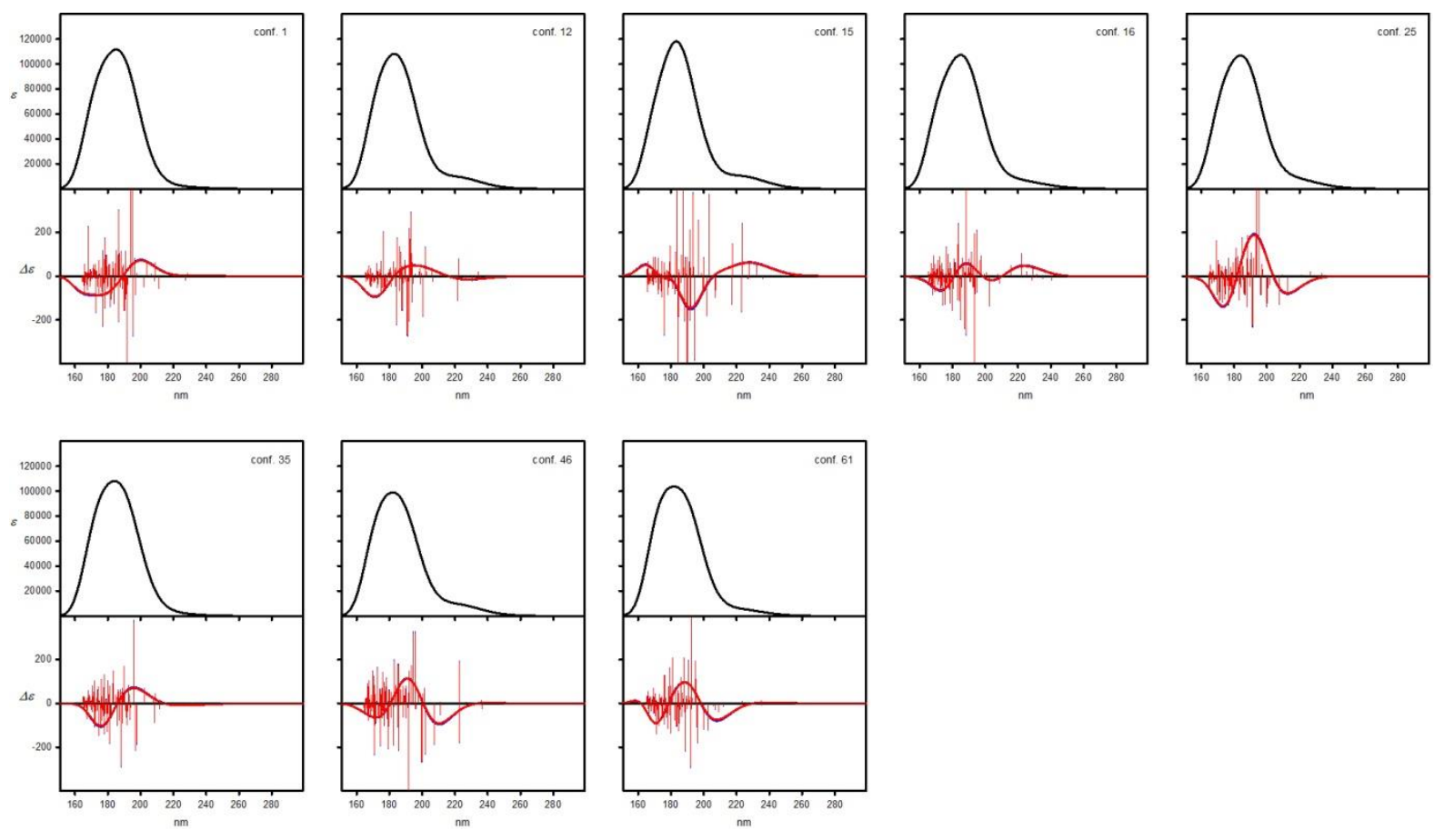

Figure SI_70. UV and ECD spectra of the low-energy conformers of compound $\mathbf{2 1}$ calculated at TDM06-2X/6-311++G(d,p) level for structures optimized at B3LYP-GD3BJ/6-311G(d,p) level. Wavelengths were not corrected. 

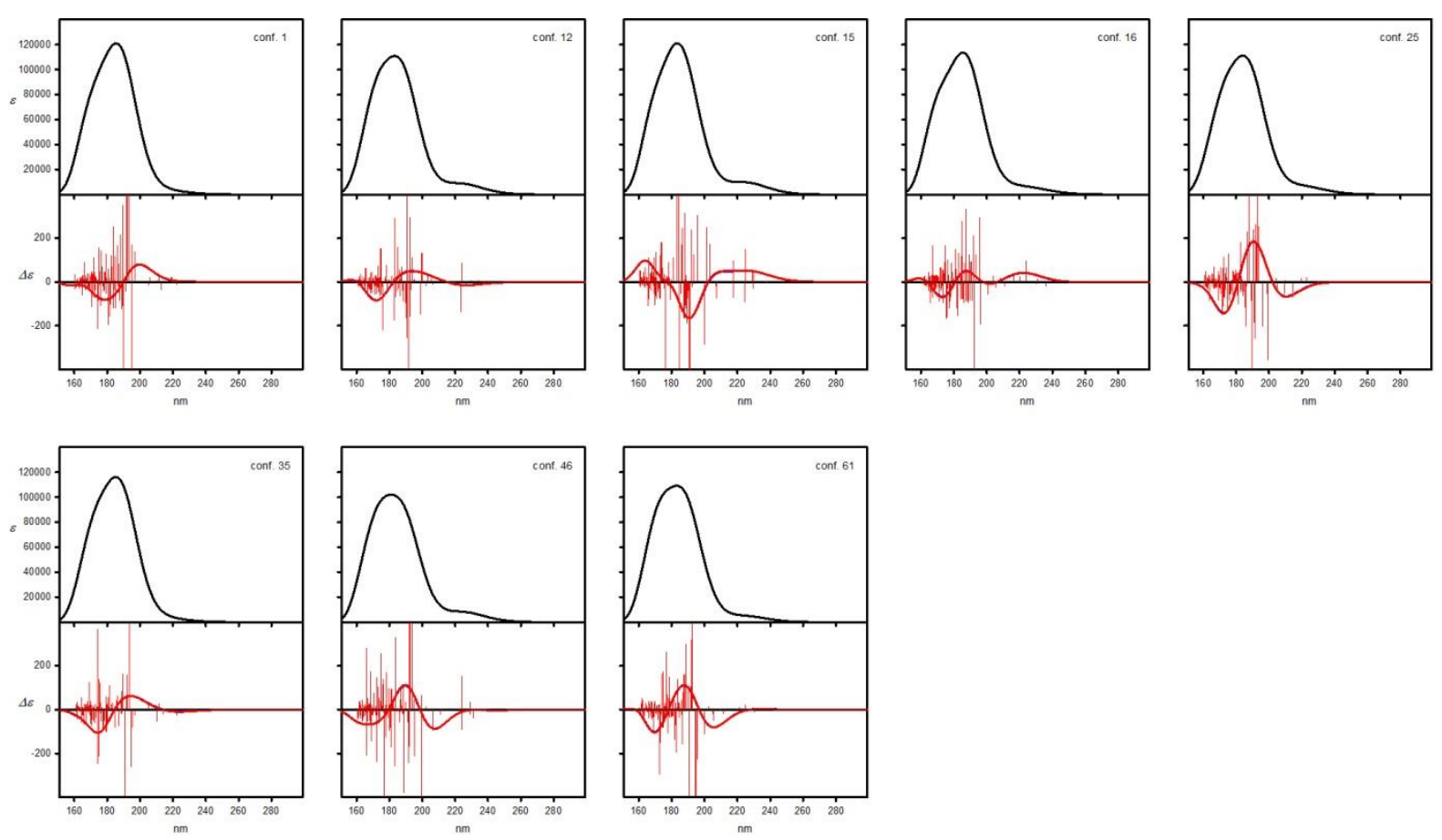

Figure SI_71. UV and ECD spectra of the low-energy conformers of compound 21 calculated at TDCAM-B3LYP/6-311++G $(d, p)$ level for structures optimized at M06-2X/6-311G(d,p) level. Wavelengths were not corrected.
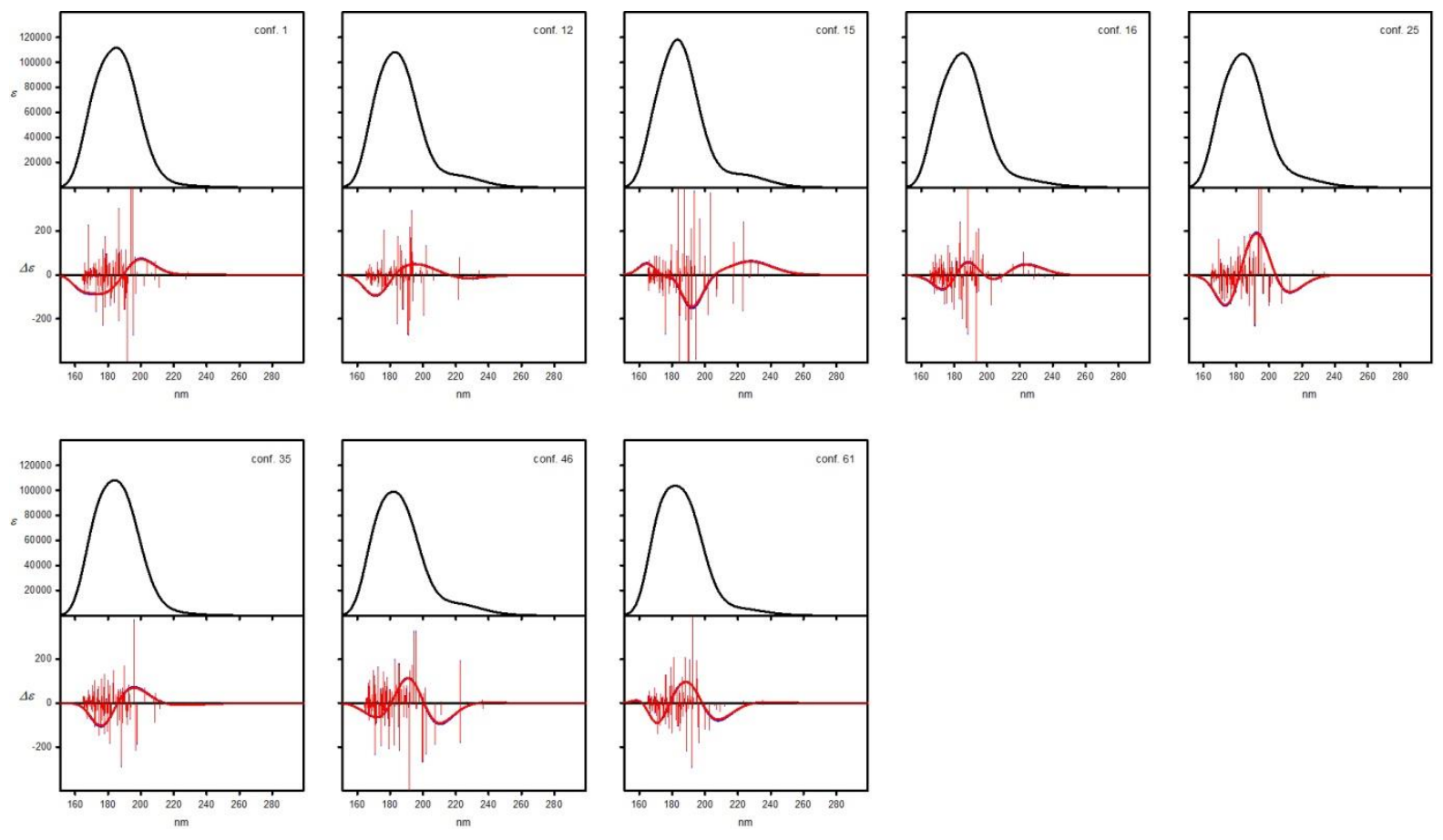

Figure SI_72. UV and ECD spectra of the low-energy conformers of compound 21 calculated at TDM06-2X/6-311++G(d,p) level for structures optimized at M06-2X/6-311G(d,p) level. Wavelengths were not corrected. 

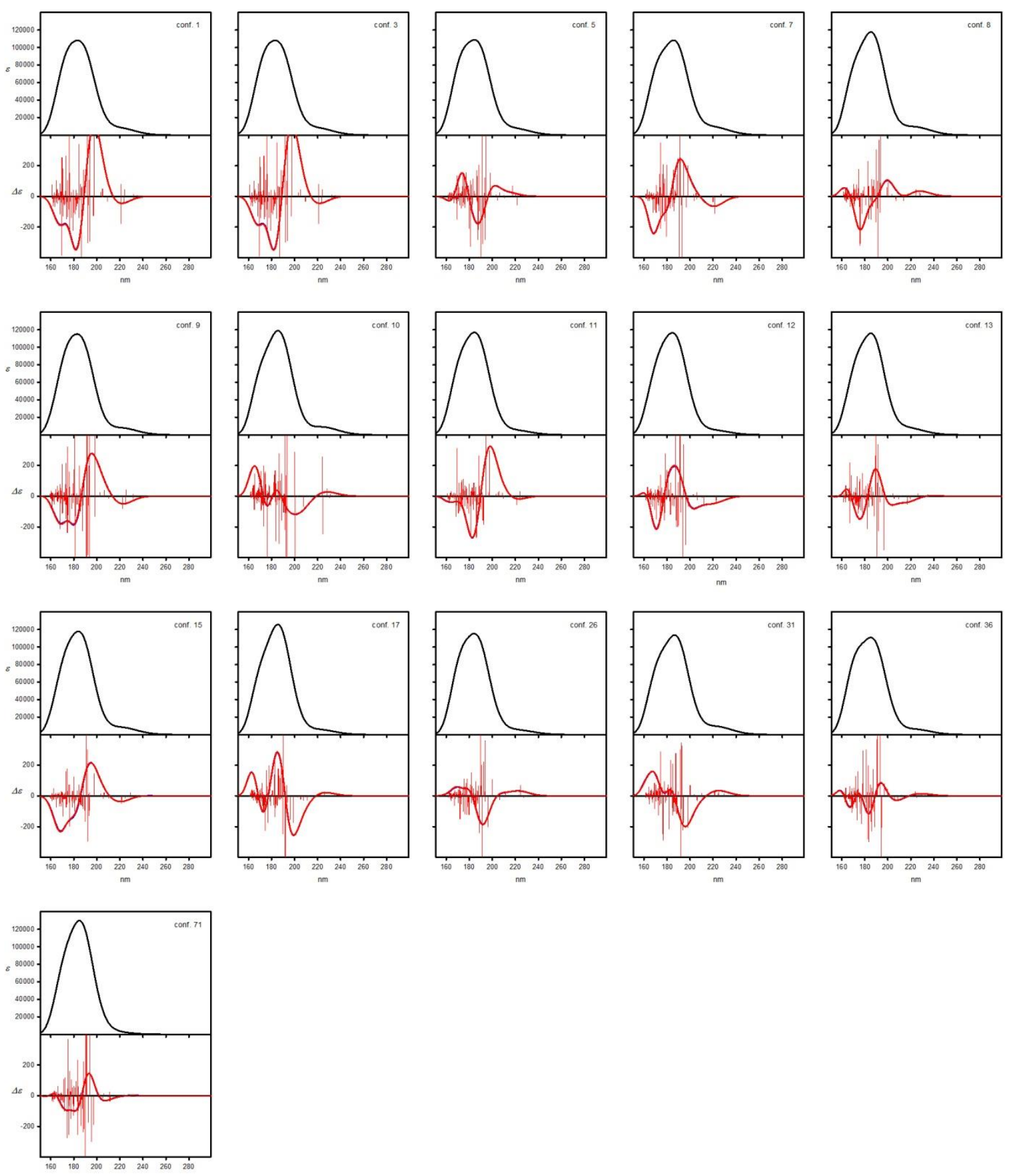

Figure SI_73. UV and ECD spectra of the low-energy conformers of compound $\mathbf{2 2}$ calculated at TDCAM-B3LYP/6-311++G $(d, p)$ level for structures optimized at B3LYP/6-311G $(d, p)$ level. Wavelengths were not corrected. 

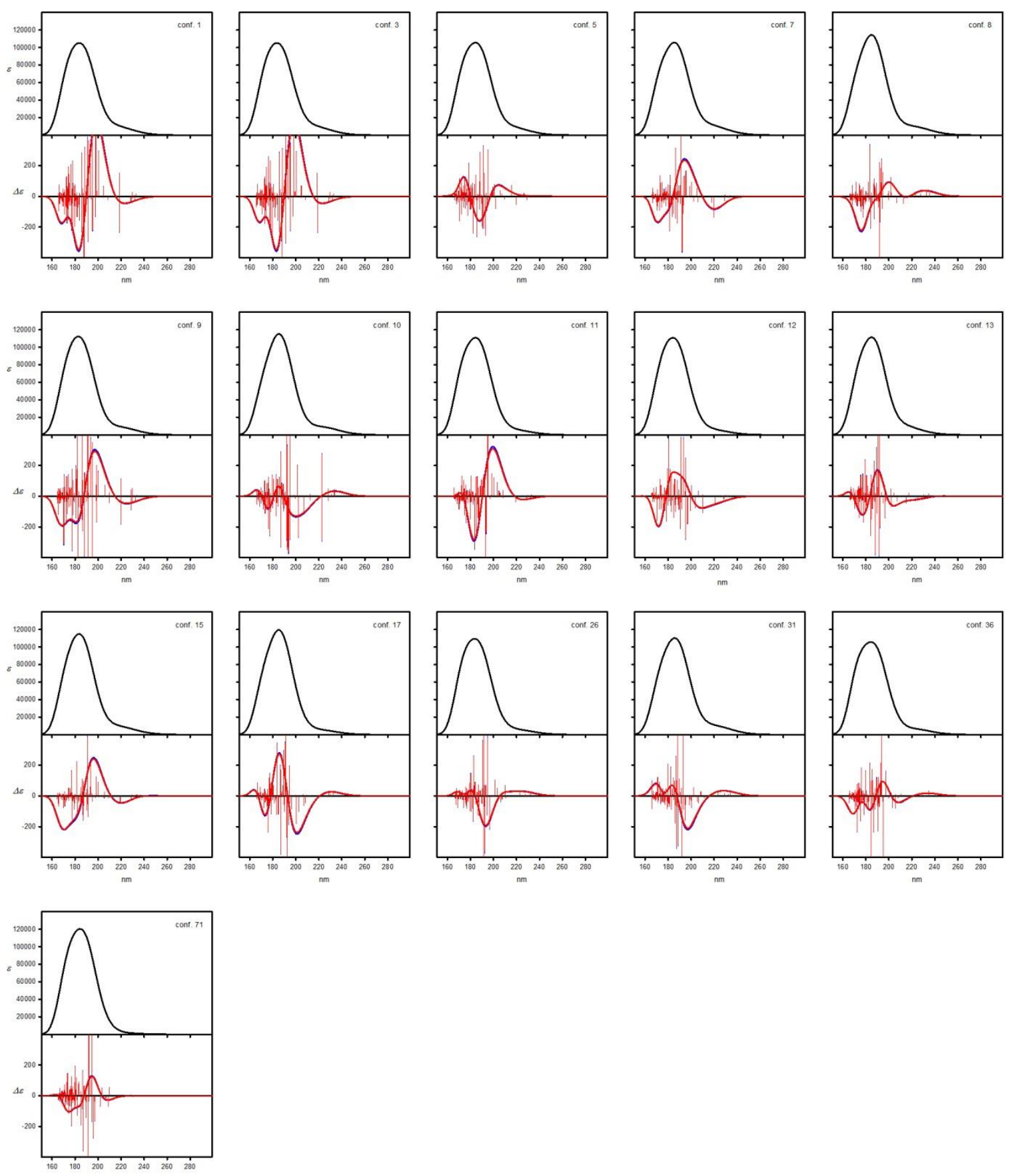

Figure SI_74. UV and ECD spectra of the low-energy conformers of compound 22 calculated at TDM06-2X/6-311++G(d,p) level for structures optimized at B3LYP/6-311G(d,p) level. Wavelengths were not corrected. 

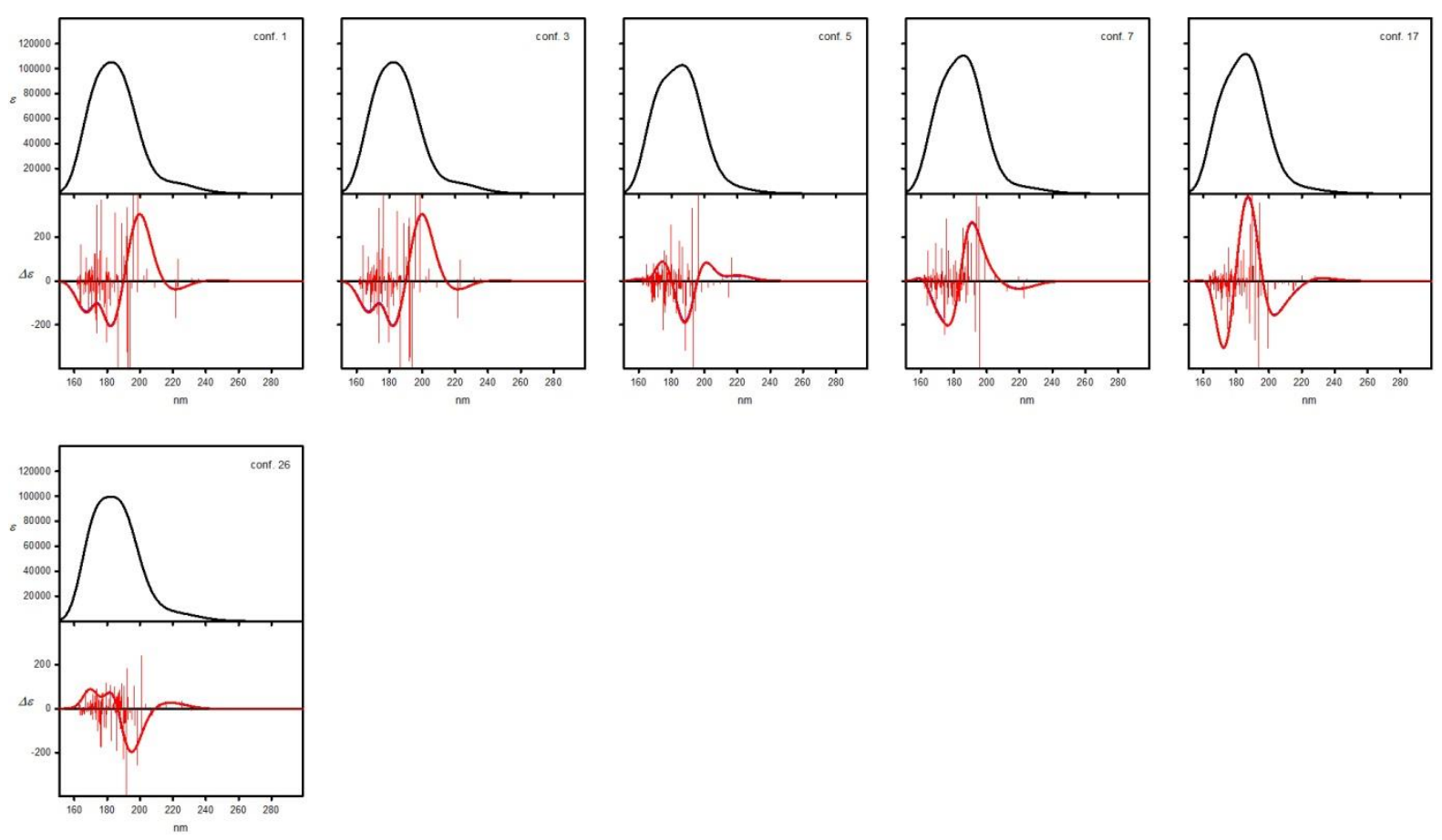

Figure SI_75. UV and ECD spectra of the low-energy conformers of compound $\mathbf{2 2}$ calculated at TDCAM-B3LYP/6-311++G(d,p) level for structures optimized at B3LYP-GD3BJ/6-311G(d,p) level. Wavelengths were not corrected.
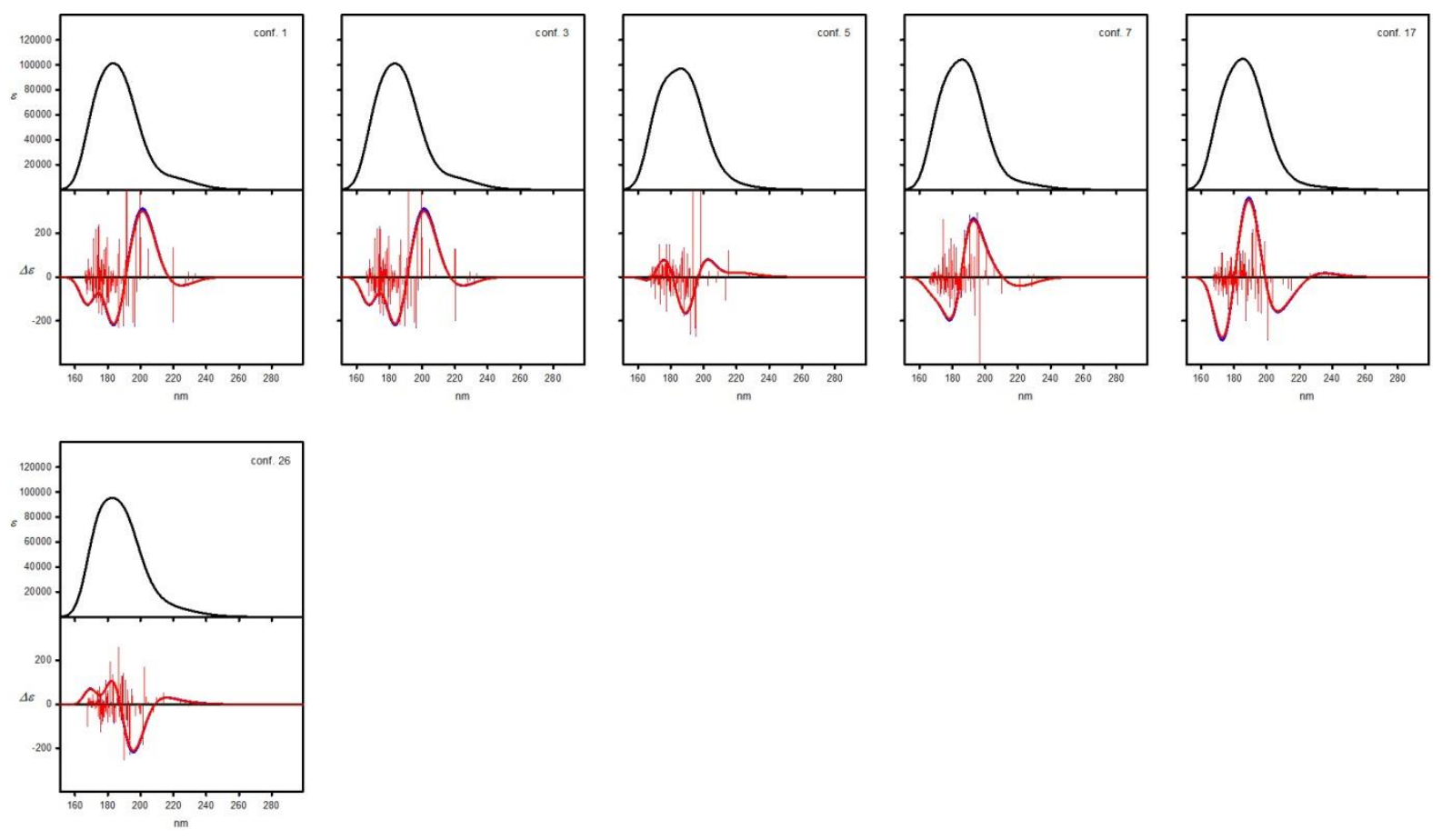

Figure SI_76. UV and ECD spectra of the low-energy conformers of compound $\mathbf{2 2}$ calculated at TDM06-2X/6-311++G(d,p) level for structures optimized at B3LYP-GD3BJ/6-311G(d,p) level. Wavelengths were not corrected. 

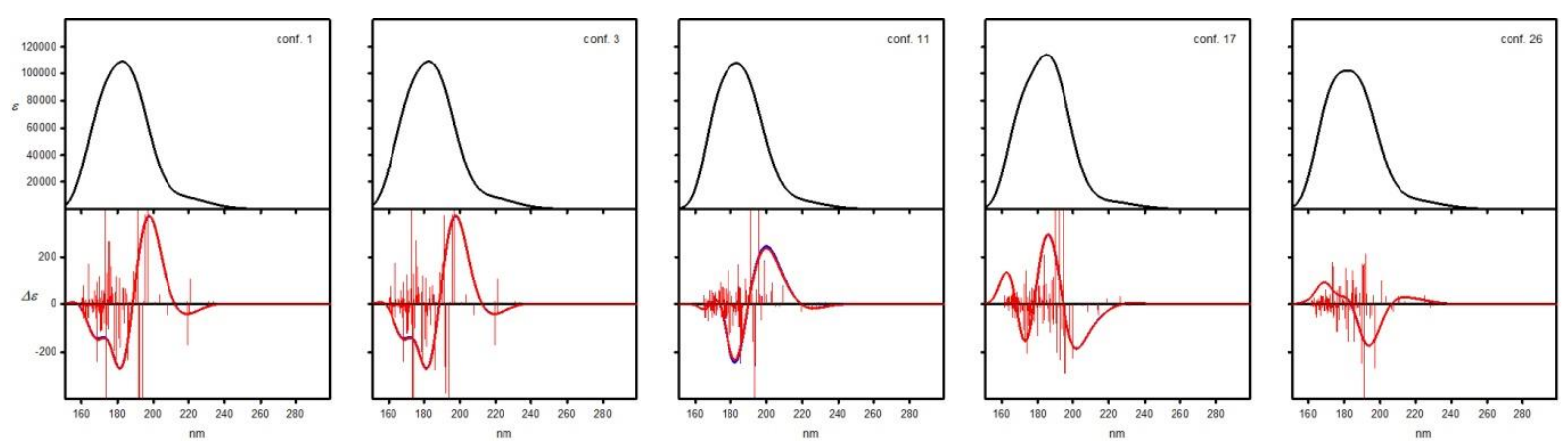

Figure SI_77. UV and ECD spectra of the low-energy conformers of compound 22 calculated at TDCAM-B3LYP/6-311++G(d,p) level for structures optimized at M06-2X/6-311G(d,p) level. Wavelengths were not corrected.
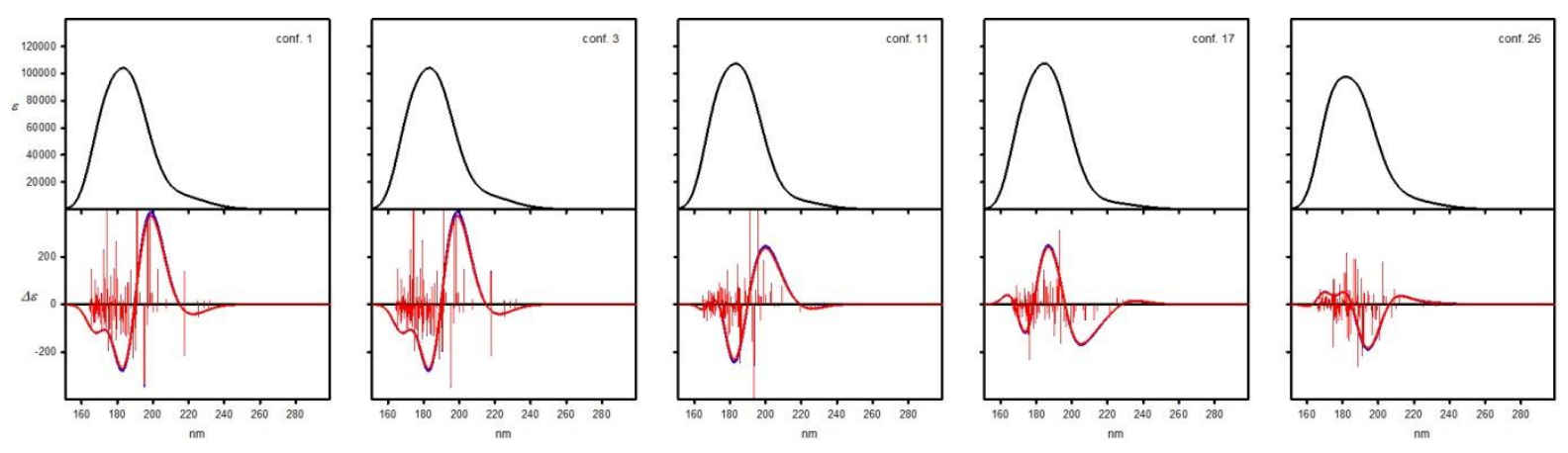

Figure SI_78. UV and ECD spectra of the low-energy conformers of compound 22 calculated at TDM06-2X/6-311++G(d,p) level for structures optimized at M06-2X/6-311G(d,p) level. Wavelengths were not corrected. 


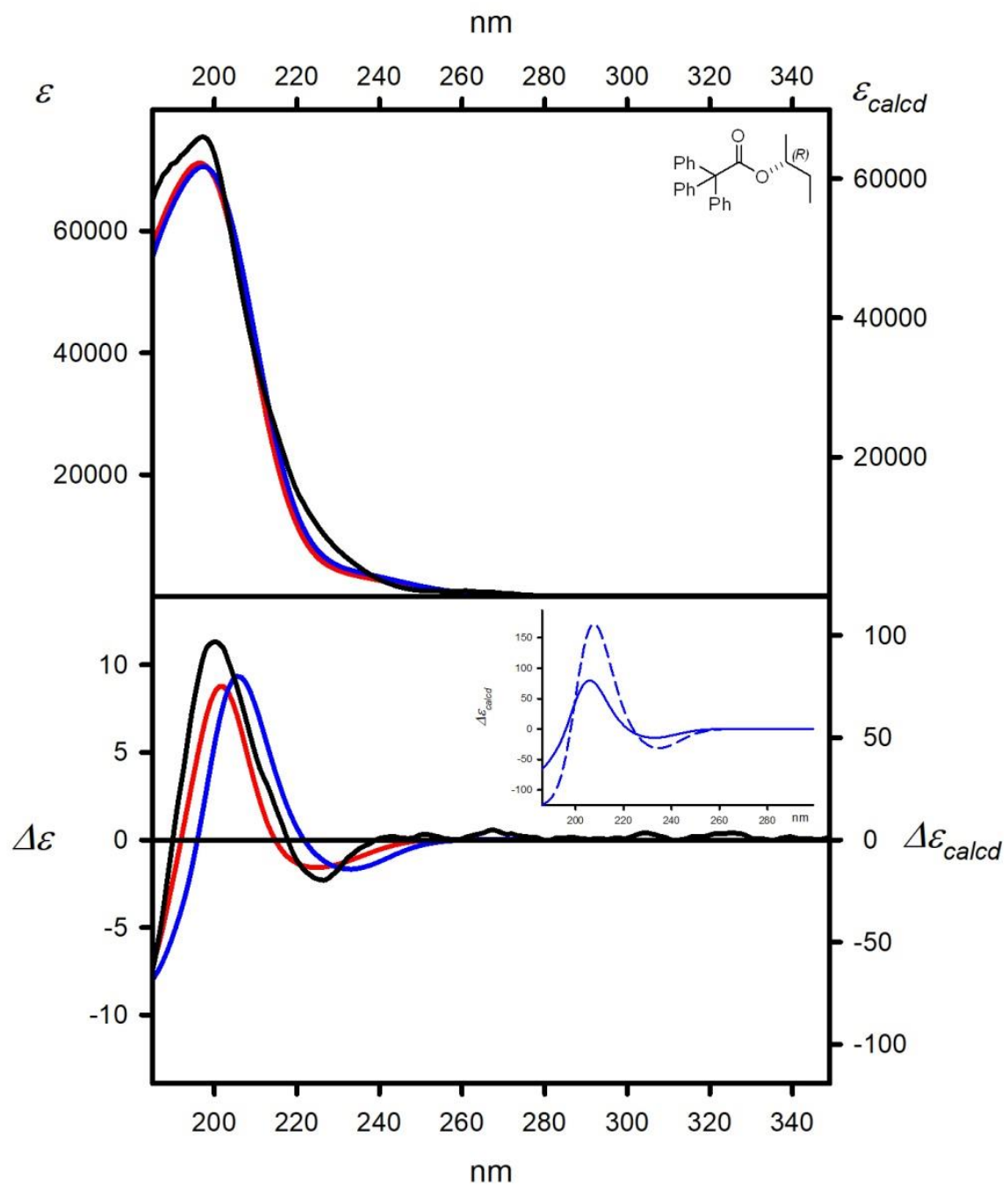

Experimental (cyclohexane, black lines)

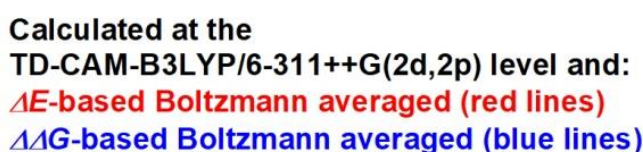

$\triangle \triangle G$-based Boltzmann averaged (blue lines)

Figure SI_79. UV (upper panel) and ECD (lower panel) spectra of 1, experimental, measured in cyclohexane (solid black lines) and calculated at the TD-CAM-B3LYP/6-311++G(2d,2p) level for structures optimized at the B3LYP/6-311++G(d,p) level, $\Delta E$-based Boltzmann (red lines) and $\Delta \Delta G$ based Boltzmann averaged (blue solid lines). Insert shows the comparison between Boltzmann averaged ECD spectrum and that calculated for $\Delta \Delta G$-based on the lowest energy conformer of a given compound (dashed blue line). All calculated spectra were wavelength corrected to match experimental UV maxima. 


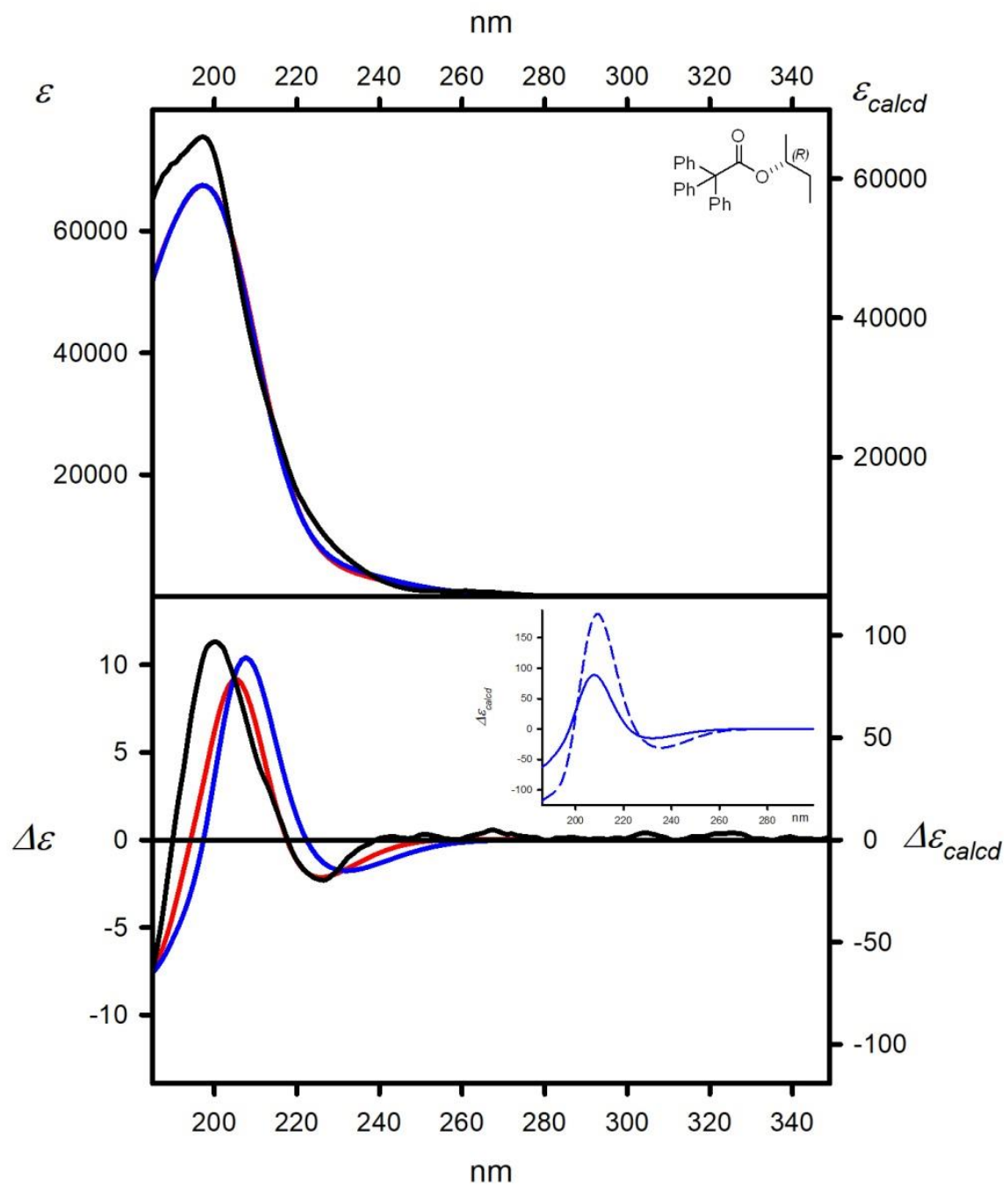

Experimental (cyclohexane, black lines)

\section{Calculated at the}

TD-M06-2X/6-311++G(2d,2p) level and:

$\triangle E$-based Boltzmann averaged (red lines)

$\triangle \triangle G$-based Boltzmann averaged (blue lines)

Figure SI_80. UV (upper panel) and ECD (lower panel) spectra of 1, experimental, measured in cyclohexane (solid black lines) and calculated at the TD-M06-2X/6-311++G(2d,2p) level for structures optimized at the B3LYP/6-311++G(d,p) level, $\Delta \mathrm{E}$-based Boltzmann (red lines) and $\Delta \Delta \mathrm{G}$-based Boltzmann averaged (blue solid lines). Insert shows the comparison between Boltzmann averaged ECD spectrum and that calculated for $\Delta \Delta G$-based on the lowest energy conformer of a given compound (dashed blue line). All calculated spectra were wavelength corrected to match experimental UV maxima. 


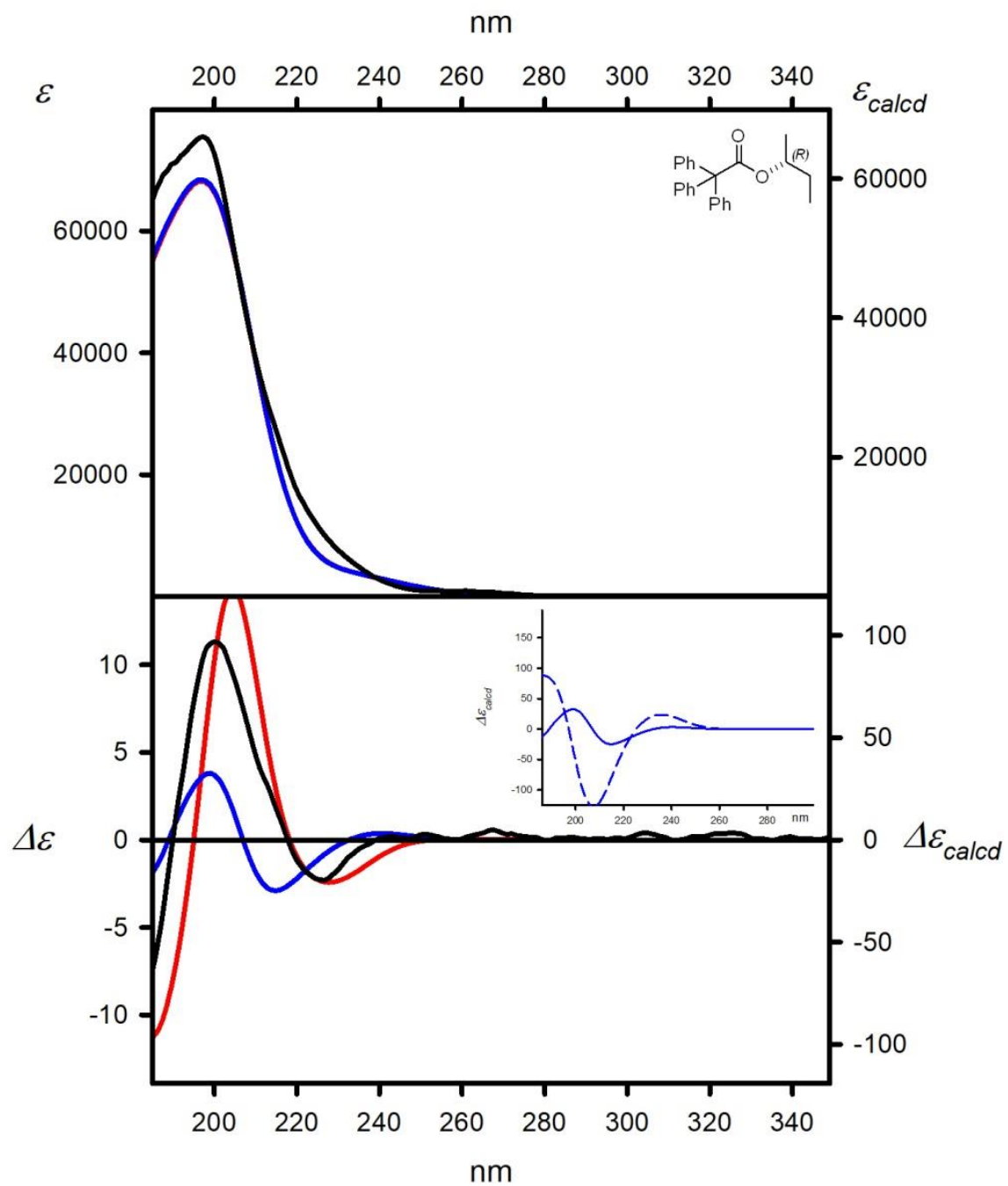

Experimental (cyclohexane, black lines)

\section{Calculated at the}

TD-CAM-B3LYP/6-311++G(2d,2p) level and:

$\triangle E$-based Boltzmann averaged (red lines)

$\triangle \triangle G$-based Boltzmann averaged (blue lines)

Figure SI_81. UV (upper panel) and ECD (lower panel) spectra of 1, experimental, measured in cyclohexane (solid black lines) and calculated at the TD-CAM-B3LYP/6-311++G(2d,2p) level for structures optimized at the M06-2X/6-311++G(d,p) level, $\Delta \mathrm{E}$-based Boltzmann (red lines) and $\Delta \Delta \mathrm{G}-$ based Boltzmann averaged (blue solid lines). Insert shows the comparison between Boltzmann averaged ECD spectrum and that calculated for $\Delta \Delta G$-based on the lowest energy conformer of a given compound (dashed blue line). All calculated spectra were wavelength corrected to match experimental UV maxima. 


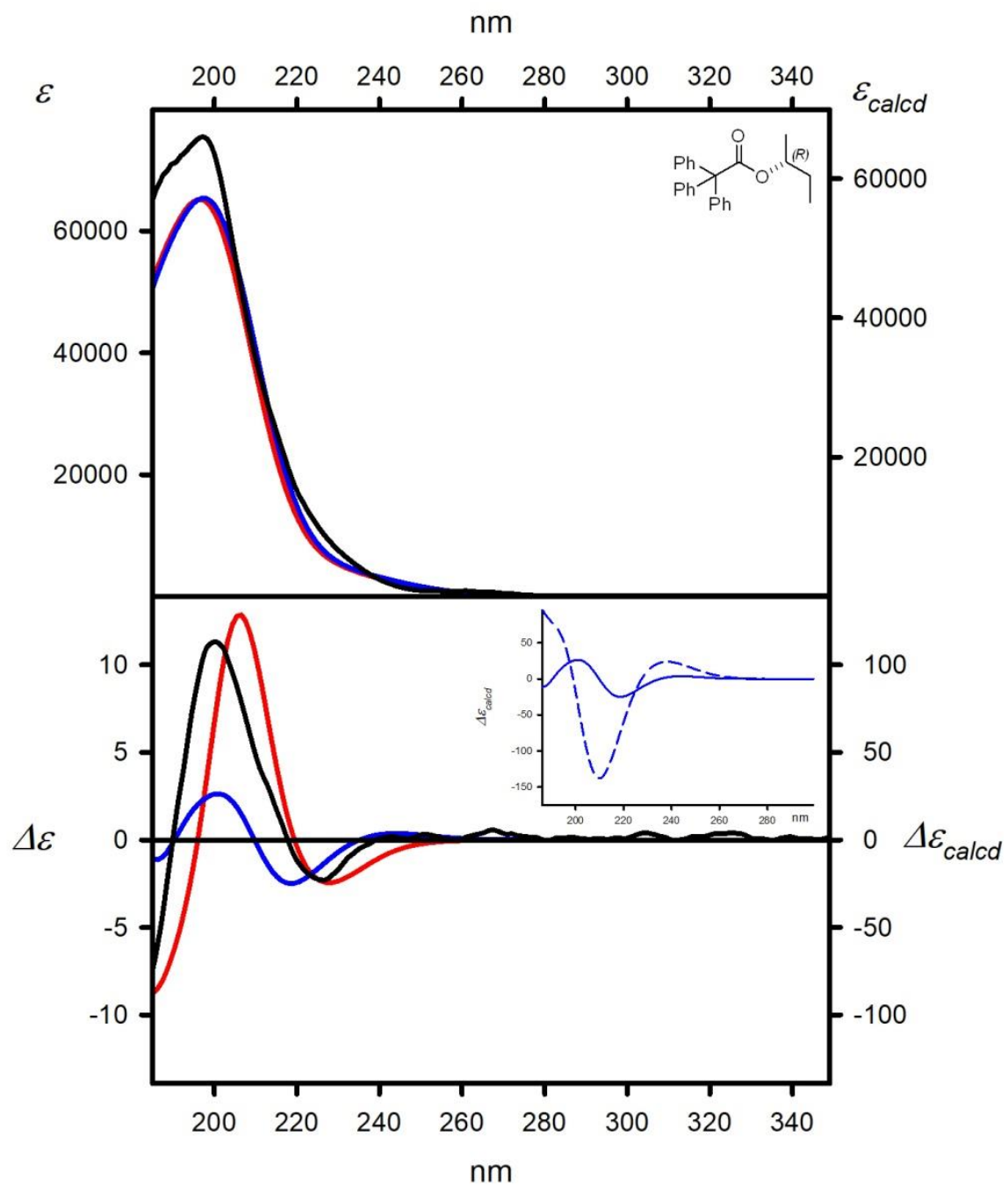

Experimental (cyclohexane, black lines)

\section{Calculated at the}

TD-M06-2X/6-311++G(2d,2p) level and:

$\triangle E$-based Boltzmann averaged (red lines)

$\triangle \triangle G$-based Boltzmann averaged (blue lines)

Figure SI_82. UV (upper panel) and ECD (lower panel) spectra of 1, experimental, measured in cyclohexane (solid black lines) and calculated at the TD-M06-2X/6-311++G(2d,2p) level for structures optimized at the M06-2X/6-311++G(d,p) level, $\Delta E$-based Boltzmann (red lines) and $\Delta \Delta G$-based Boltzmann averaged (blue solid lines). Insert shows the comparison between Boltzmann averaged $E C D$ spectrum and that calculated for $\Delta \Delta G$-based on the lowest energy conformer of a given compound (dashed blue line). All calculated spectra were wavelength corrected to match experimental UV maxima. 


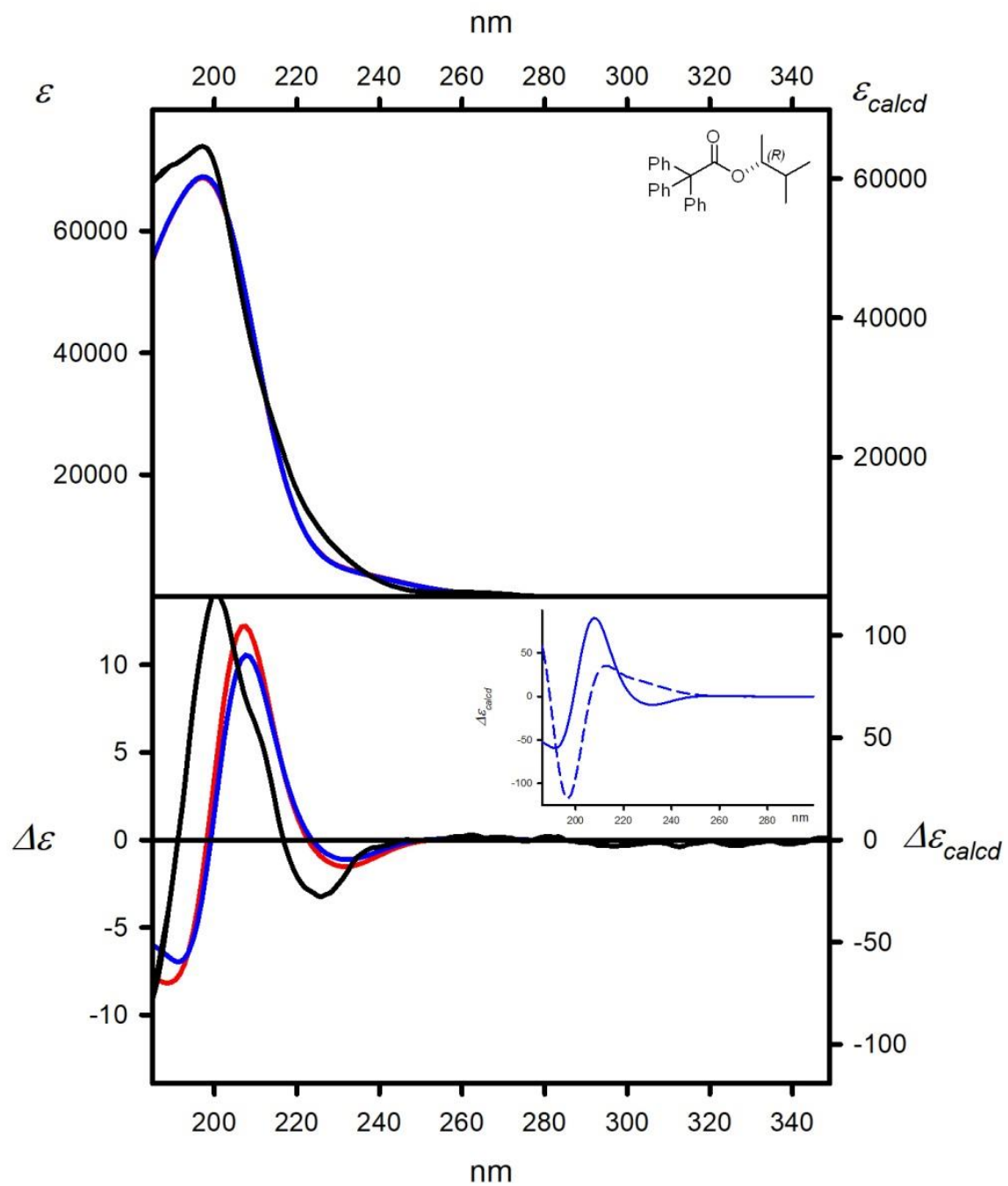

Experimental (cyclohexane, black lines)

\section{Calculated at the}

TD-CAM-B3LYP/6-311++G(2d,2p) level and:

$\triangle E$-based Boltzmann averaged (red lines)

$\triangle \triangle G$-based Boltzmann averaged (blue lines)

Figure SI_83. UV (upper panel) and ECD (lower panel) spectra of 4, experimental, measured in cyclohexane (solid black lines) and calculated at the TD-CAM-B3LYP/6-311++G(2d,2p) level for structures optimized at the B3LYP/6-311++G(d,p) level, $\Delta E$-based Boltzmann (red lines) and $\Delta \Delta G$ based Boltzmann averaged (blue solid lines). Insert shows the comparison between Boltzmann averaged ECD spectrum and that calculated for $\Delta \Delta G$-based on the lowest energy conformer of a given compound (dashed blue line). All calculated spectra were wavelength corrected to match experimental UV maxima. 


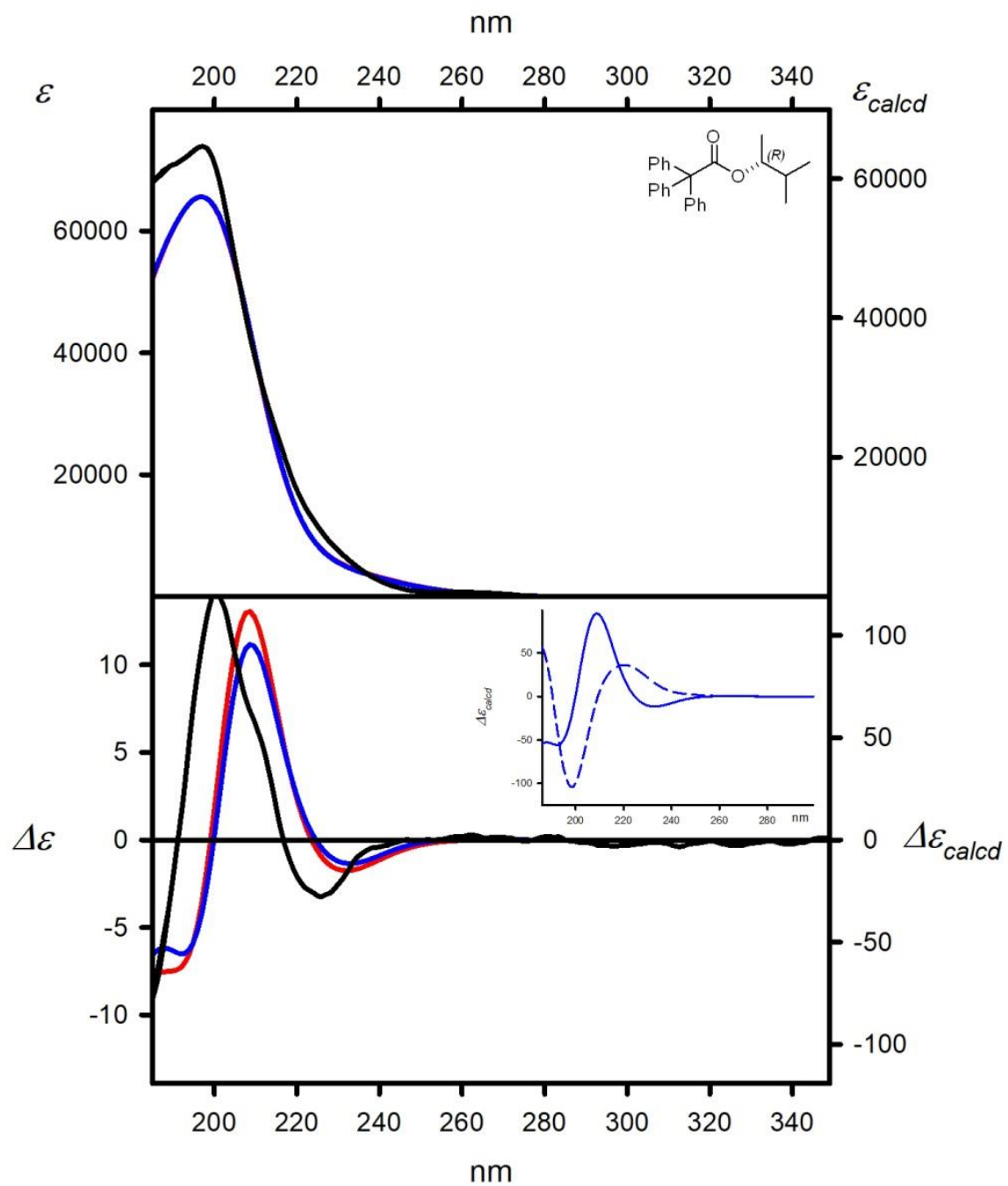

Experimental (cyclohexane, black lines)

\section{Calculated at the}

TD-M06-2X/6-311++G(2d,2p) level and:

$\triangle E$-based Boltzmann averaged (red lines)

$\triangle \triangle G$-based Boltzmann averaged (blue lines)

Figure SI_84. UV (upper panel) and ECD (lower panel) spectra of 4, experimental, measured in cyclohexane (solid black lines) and calculated at the TD-M06-2X/6-311++G(2d,2p) level for structures optimized at the B3LYP/6-311++G(d,p) level, $\Delta \mathrm{E}$-based Boltzmann (red lines) and $\Delta \Delta \mathrm{G}$-based Boltzmann averaged (blue solid lines). Insert shows the comparison between Boltzmann averaged ECD spectrum and that calculated for $\Delta \Delta G$-based on the lowest energy conformer of a given compound (dashed blue line). All calculated spectra were wavelength corrected to match experimental UV maxima. 


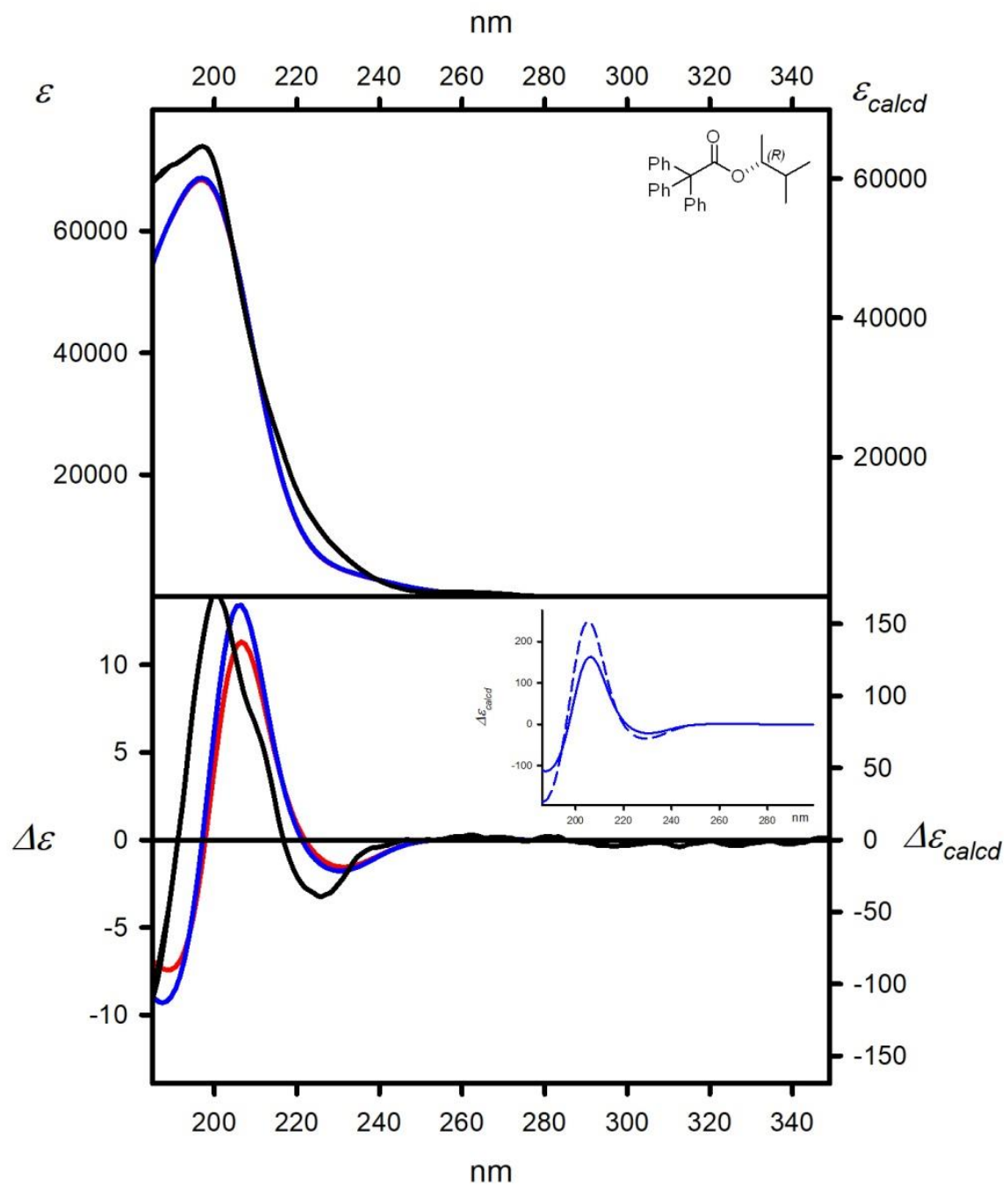

Experimental (cyclohexane, black lines)

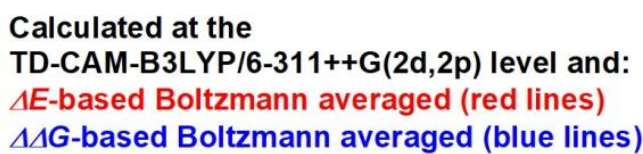

Figure SI_85. UV (upper panel) and ECD (lower panel) spectra of 4, experimental, measured in cyclohexane (solid black lines) and calculated at the TD-CAM-B3LYP/6-311++G(2d,2p) level for structures optimized at the M06-2X/6-311++G(d,p) level, $\Delta \mathrm{E}$-based Boltzmann (red lines) and $\Delta \Delta \mathrm{G}-$ based Boltzmann averaged (blue solid lines). Insert shows the comparison between Boltzmann averaged ECD spectrum and that calculated for $\Delta \Delta G$-based on the lowest energy conformer of a given compound (dashed blue line). All calculated spectra were wavelength corrected to match experimental UV maxima. 


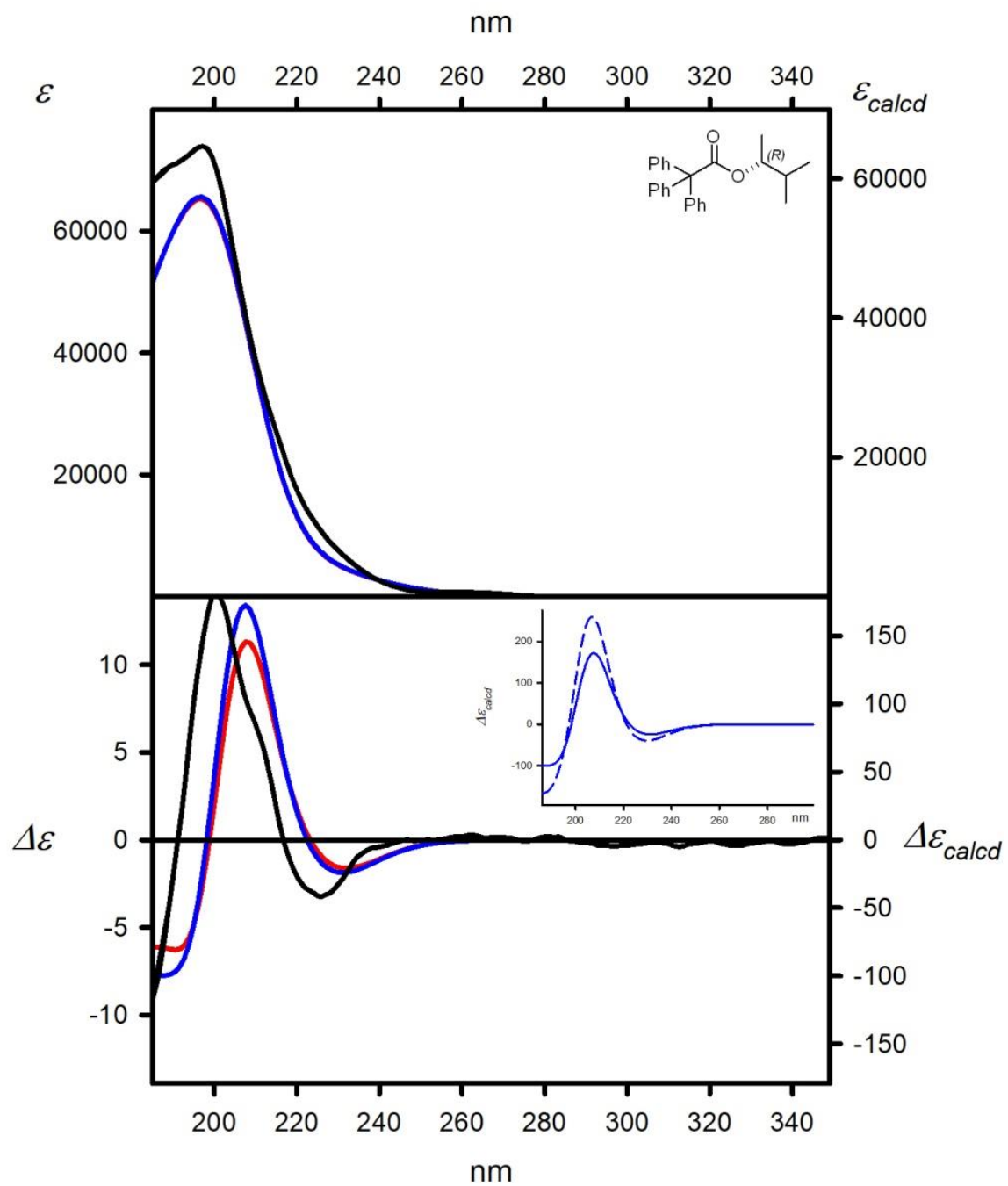

Experimental (cyclohexane, black lines)

\section{Calculated at the}

TD-M06-2X/6-311++G(2d,2p) level and:

$\triangle E$-based Boltzmann averaged (red lines)

$\triangle \triangle G$-based Boltzmann averaged (blue lines)

Figure SI_86. UV (upper panel) and ECD (lower panel) spectra of 4, experimental, measured in cyclohexane (solid black lines) and calculated at the TD-M06-2X/6-311++G(2d,2p) level for structures optimized at the M06-2X/6-311++G(d,p) level, $\Delta \mathrm{E}$-based Boltzmann (red lines) and $\Delta \Delta \mathrm{G}$-based Boltzmann averaged (blue solid lines). Insert shows the comparison between Boltzmann averaged $E C D$ spectrum and that calculated for $\Delta \Delta G$-based on the lowest energy conformer of a given compound (dashed blue line). All calculated spectra were wavelength corrected to match experimental UV maxima. 


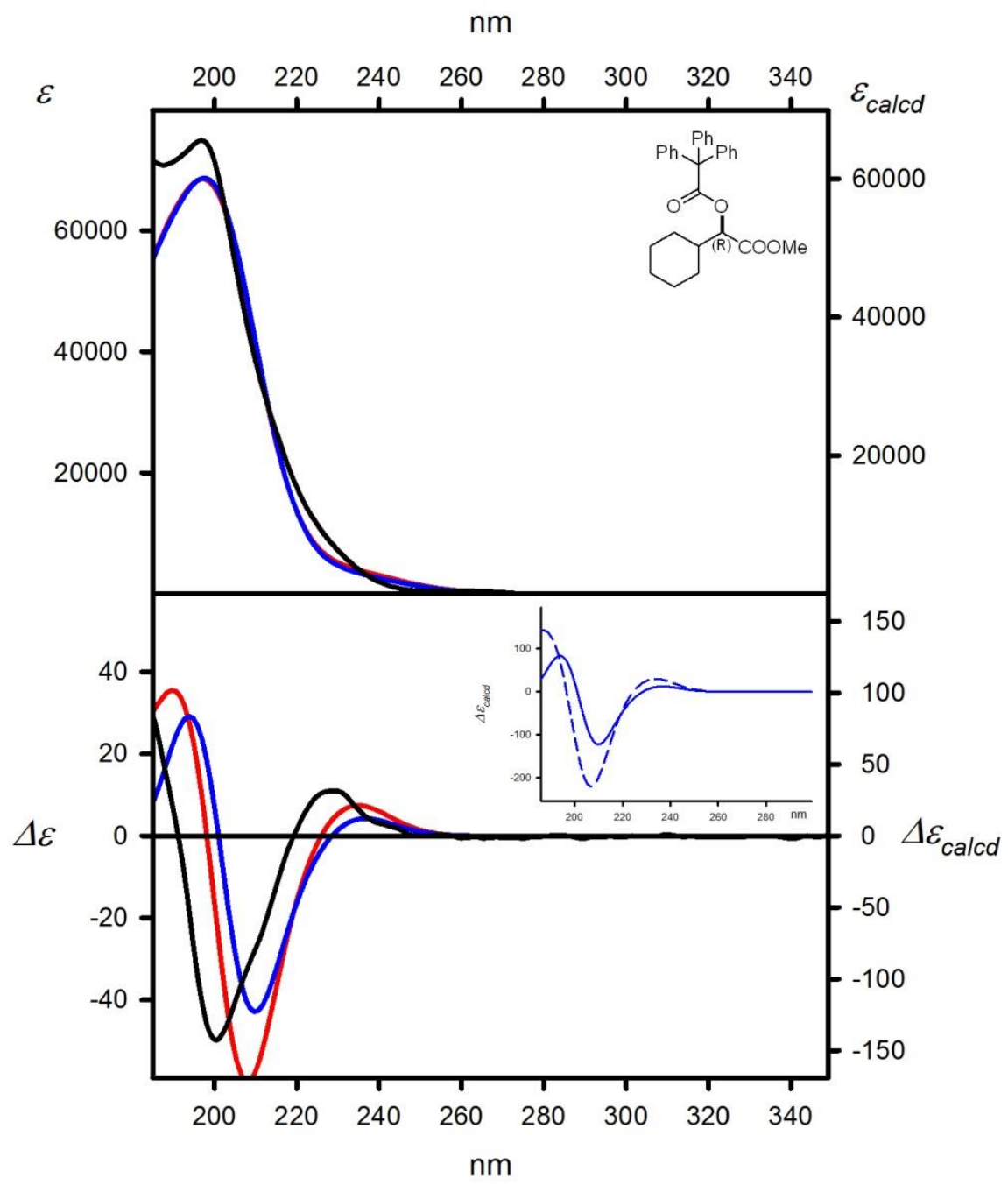

Experimental (cyclohexane, black lines)

Calculated at the

TD-CAM-B3LYP/6-311++G(2d,2p) level and:

$\triangle E$-based Boltzmann averaged (red lines)

$\triangle \Lambda G$-based Boltzmann averaged (blue lines)

Figure SI_87. UV (upper panel) and ECD (lower panel) spectra of 6, experimental, measured in cyclohexane (solid black lines) and calculated at the TD-CAM-B3LYP/6-311++G(2d,2p) level for structures optimized at the B3LYP/6-311++G(d,p) level, $\Delta E$-based Boltzmann (red lines) and $\Delta \Delta G$ based Boltzmann averaged (blue solid lines). Insert shows the comparison between Boltzmann averaged ECD spectrum and that calculated for $\Delta \Delta G$-based on the lowest energy conformer of a given compound (dashed blue line). All calculated spectra were wavelength corrected to match experimental UV maxima. 


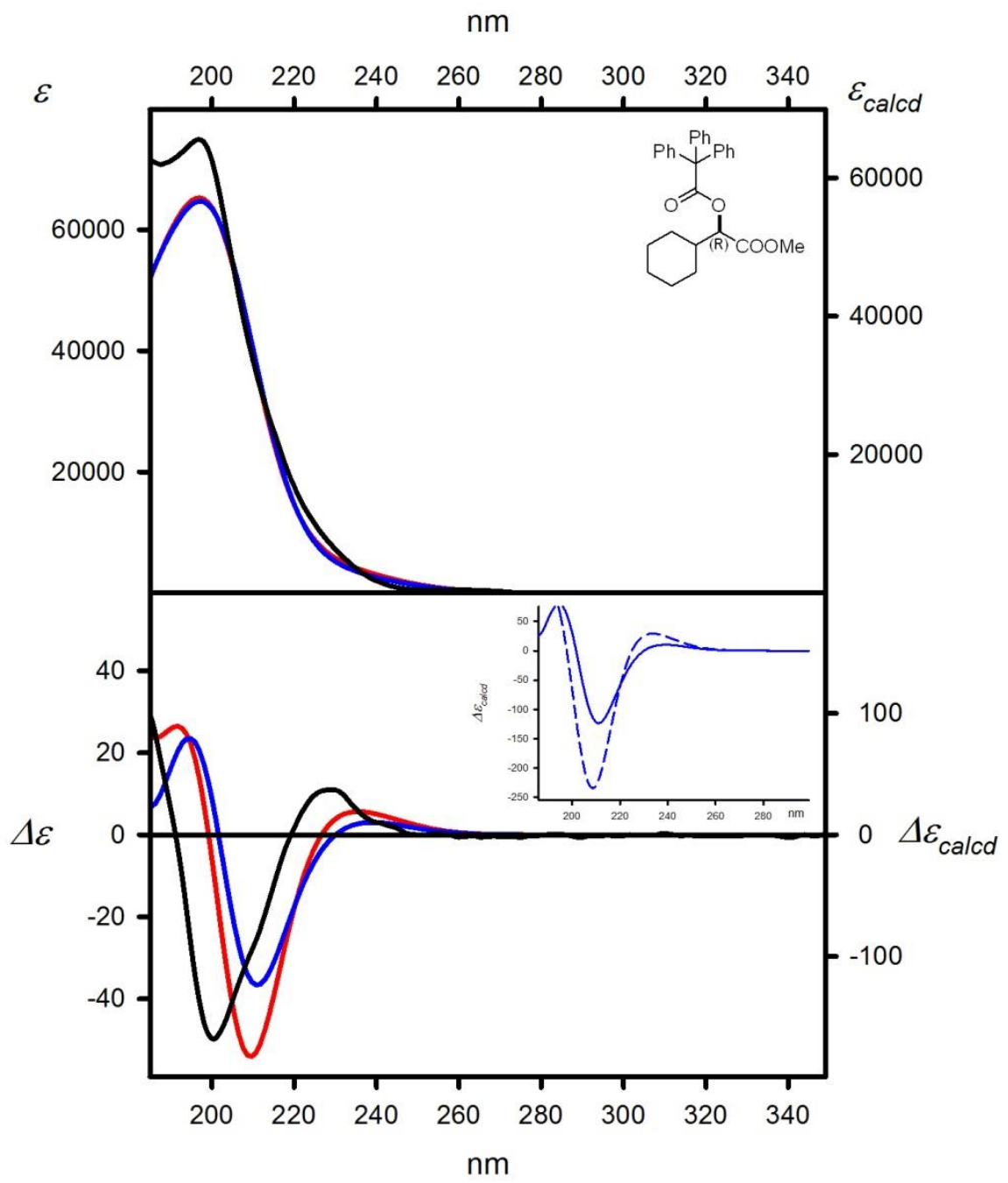

Experimental (cyclohexane, black lines)

Calculated at the

TD-M06-2X/6-311++G(2d,2p) level and:

$\triangle E$-based Boltzmann averaged (red lines)

$\triangle \Lambda G$-based Boltzmann averaged (blue lines)

Figure SI_88. UV (upper panel) and ECD (lower panel) spectra of 6, experimental, measured in cyclohexane (solid black lines) and calculated at the TD-M06-2X/6-311++G $(2 d, 2 p)$ level for structures optimized at the B3LYP/6-311++G(d,p) level, $\Delta \mathrm{E}$-based Boltzmann (red lines) and $\Delta \Delta \mathrm{G}$-based Boltzmann averaged (blue solid lines). Insert shows the comparison between Boltzmann averaged ECD spectrum and that calculated for $\Delta \Delta G$-based on the lowest energy conformer of a given compound (dashed blue line). All calculated spectra were wavelength corrected to match experimental UV maxima. 


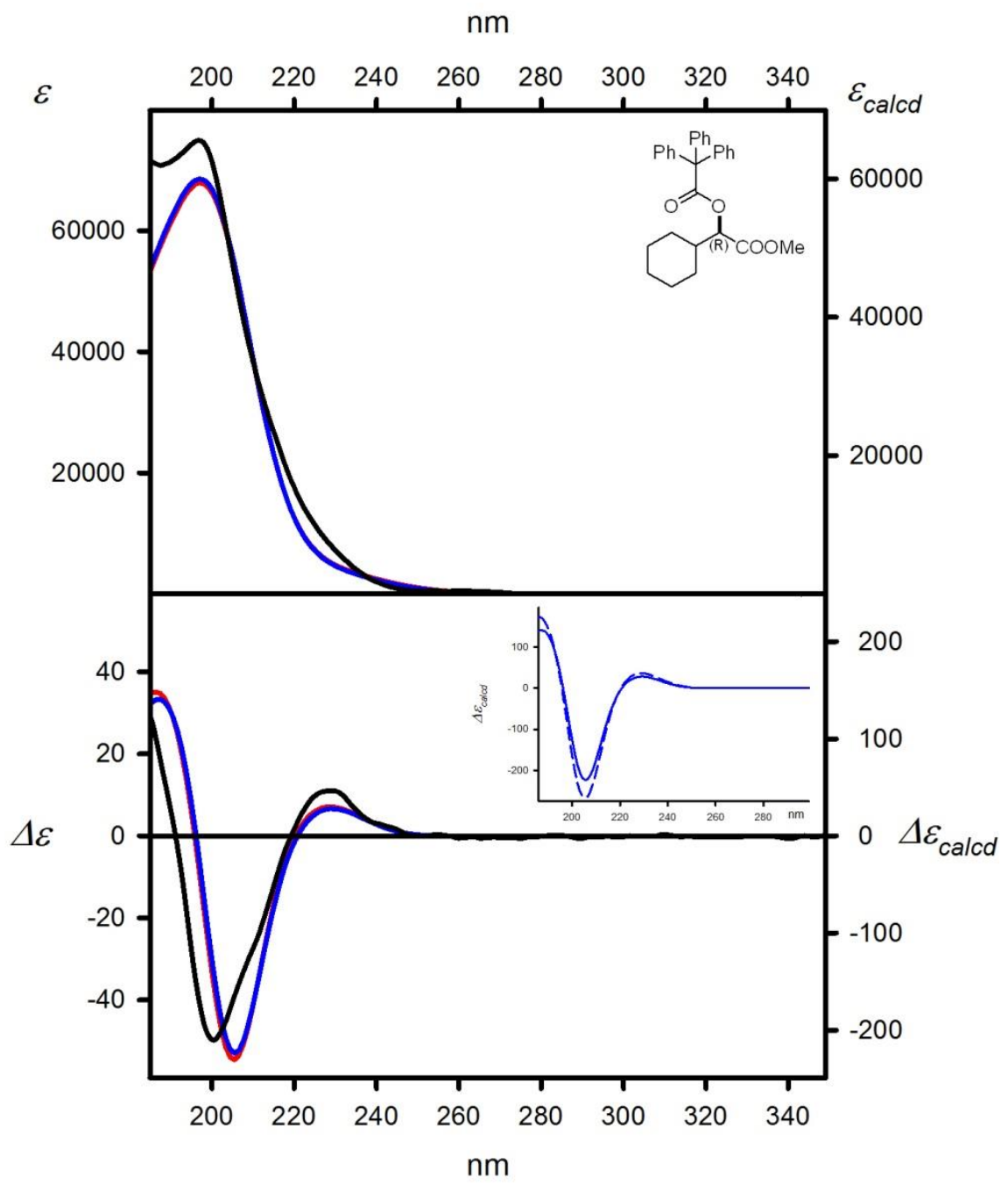

Experimental (cyclohexane, black lines)

Calculated at the

TD-CAM-B3LYP/6-311++G(2d,2p) level and:

$\triangle E$-based Boltzmann averaged (red lines)

$\triangle \Lambda G$-based Boltzmann averaged (blue lines)

Figure SI_89. UV (upper panel) and ECD (lower panel) spectra of 6, experimental, measured in cyclohexane (solid black lines) and calculated at the TD-CAM-B3LYP/6-311++G(2d,2p) level for structures optimized at the M06-2X/6-311++G(d,p) level, $\Delta \mathrm{E}$-based Boltzmann (red lines) and $\Delta \Delta \mathrm{G}-$ based Boltzmann averaged (blue solid lines). Insert shows the comparison between Boltzmann averaged ECD spectrum and that calculated for $\Delta \Delta G$-based on the lowest energy conformer of a given compound (dashed blue line). All calculated spectra were wavelength corrected to match experimental UV maxima. 


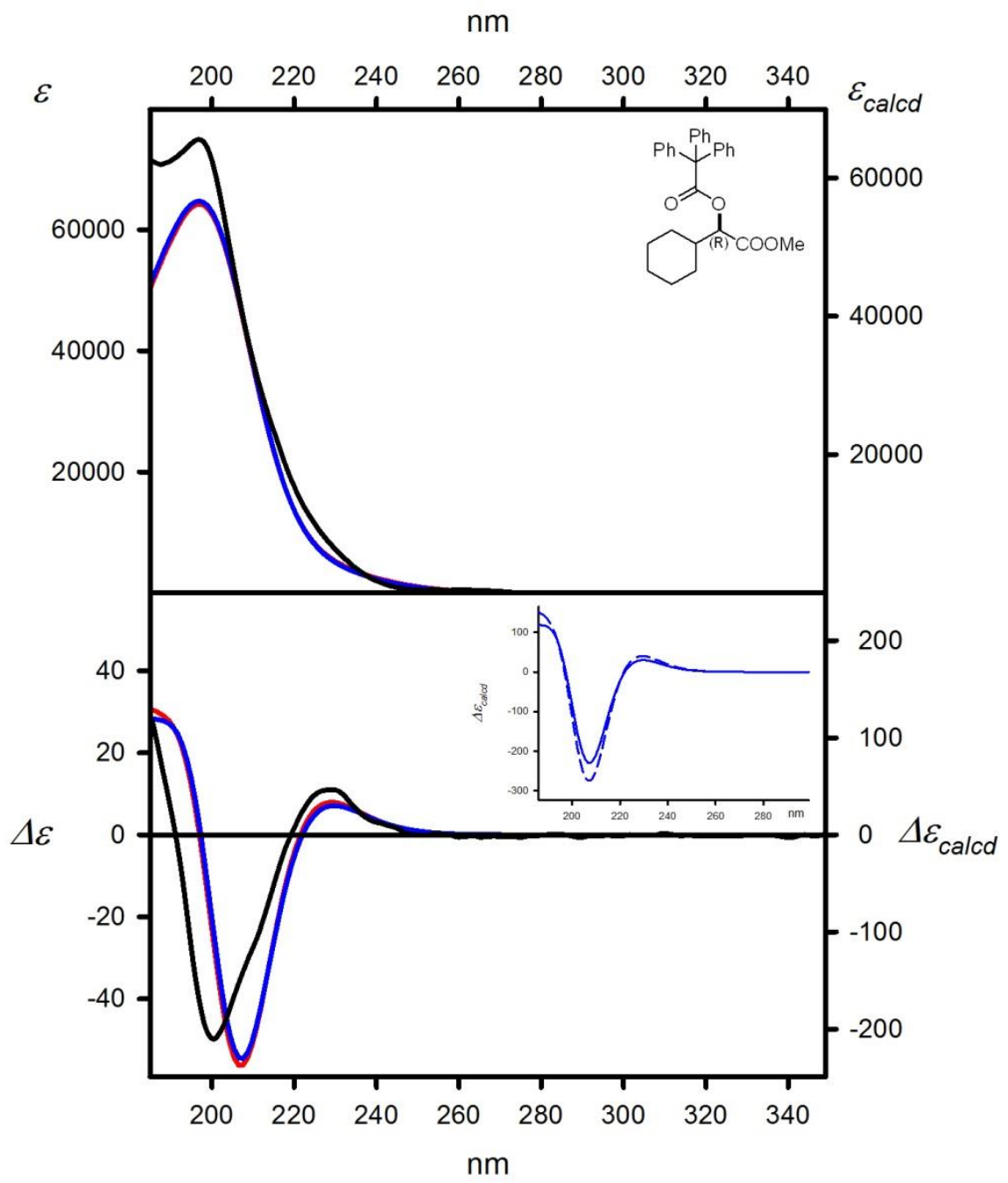

Experimental (cyclohexane, black lines)

Calculated at the

TD-M06-2X/6-311++G(2d,2p) level and:

$\triangle E$-based Boltzmann averaged (red lines)

$\triangle \Lambda G$-based Boltzmann averaged (blue lines)

Figure SI_90. UV (upper panel) and ECD (lower panel) spectra of 6, experimental, measured in cyclohexane (solid black lines) and calculated at the TD-M06-2X/6-311++G(2d,2p) level for structures optimized at the M06-2X/6-311++G(d,p) level, $\Delta \mathrm{E}$-based Boltzmann (red lines) and $\Delta \Delta \mathrm{G}$-based Boltzmann averaged (blue solid lines). Insert shows the comparison between Boltzmann averaged ECD spectrum and that calculated for $\Delta \Delta G$-based on the lowest energy conformer of a given compound (dashed blue line). All calculated spectra were wavelength corrected to match experimental UV maxima. 


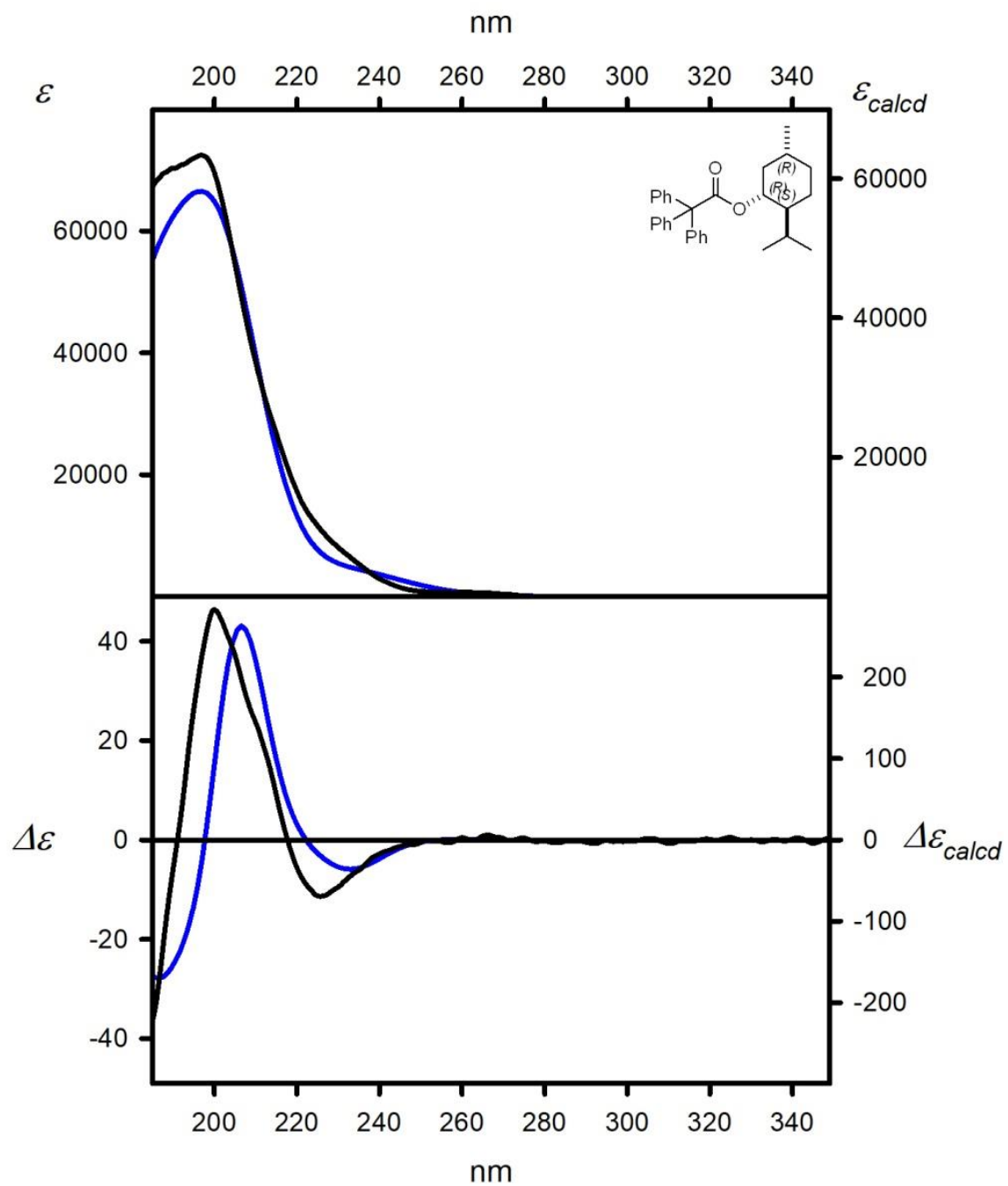

Experimental (cyclohexane, black lines)

\section{Calculated at the}

TD-CAM-B3LYP/6-311++G(2d,2p) level and:

$\triangle E$-based Boltzmann averaged (red lines)

$\triangle \triangle G$-based Boltzmann averaged (blue lines)

Figure SI_91. UV (upper panel) and ECD (lower panel) spectra of 10, experimental, measured in cyclohexane (solid black lines) and calculated at the TD-CAM-B3LYP/6-311++G(2d,2p) level for structures optimized at the B3LYP/6-311++G(d,p) level, $\Delta E$-based Boltzmann (red lines) and $\Delta \Delta G$ based Boltzmann averaged (blue solid lines). Insert shows the comparison between Boltzmann averaged ECD spectrum and that calculated for $\Delta \Delta G$-based on the lowest energy conformer of a given compound (dashed blue line). All calculated spectra were wavelength corrected to match experimental UV maxima. 


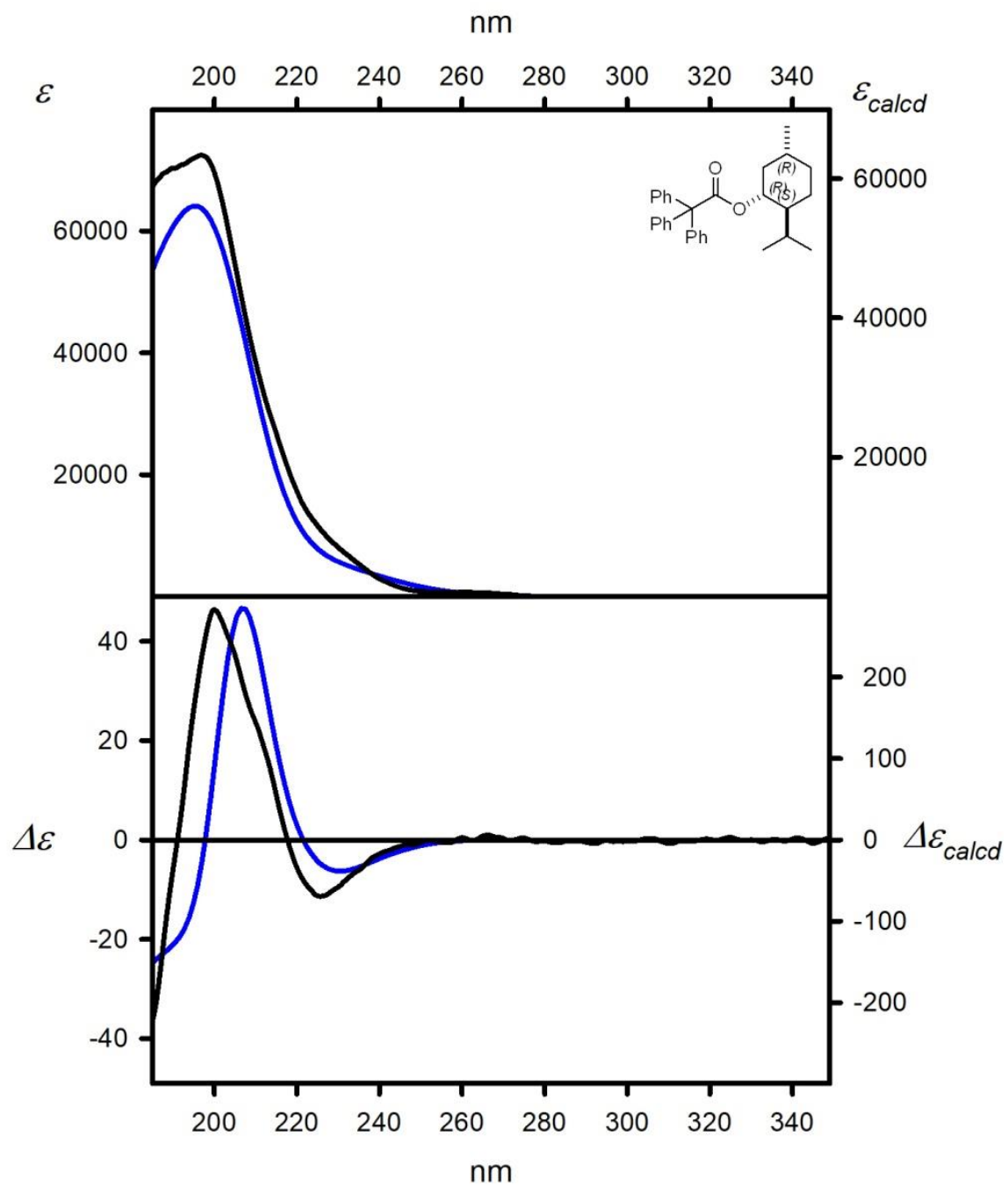

Experimental (cyclohexane, black lines)

\section{Calculated at the}

TD-M06-2X/6-311++G(2d,2p) level and:

$\triangle E$-based Boltzmann averaged (red lines)

$\triangle \triangle G$-based Boltzmann averaged (blue lines)

Figure SI_92. UV (upper panel) and ECD (lower panel) spectra of 10, experimental, measured in cyclohexane (solid black lines) and calculated at the TD-M06-2X/6-311++G(2d,2p) level for structures optimized at the B3LYP/6-311++G(d,p) level, $\Delta \mathrm{E}$-based Boltzmann (red lines) and $\Delta \Delta \mathrm{G}$-based Boltzmann averaged (blue solid lines). Insert shows the comparison between Boltzmann averaged ECD spectrum and that calculated for $\Delta \Delta G$-based on the lowest energy conformer of a given compound (dashed blue line). All calculated spectra were wavelength corrected to match experimental UV maxima. 


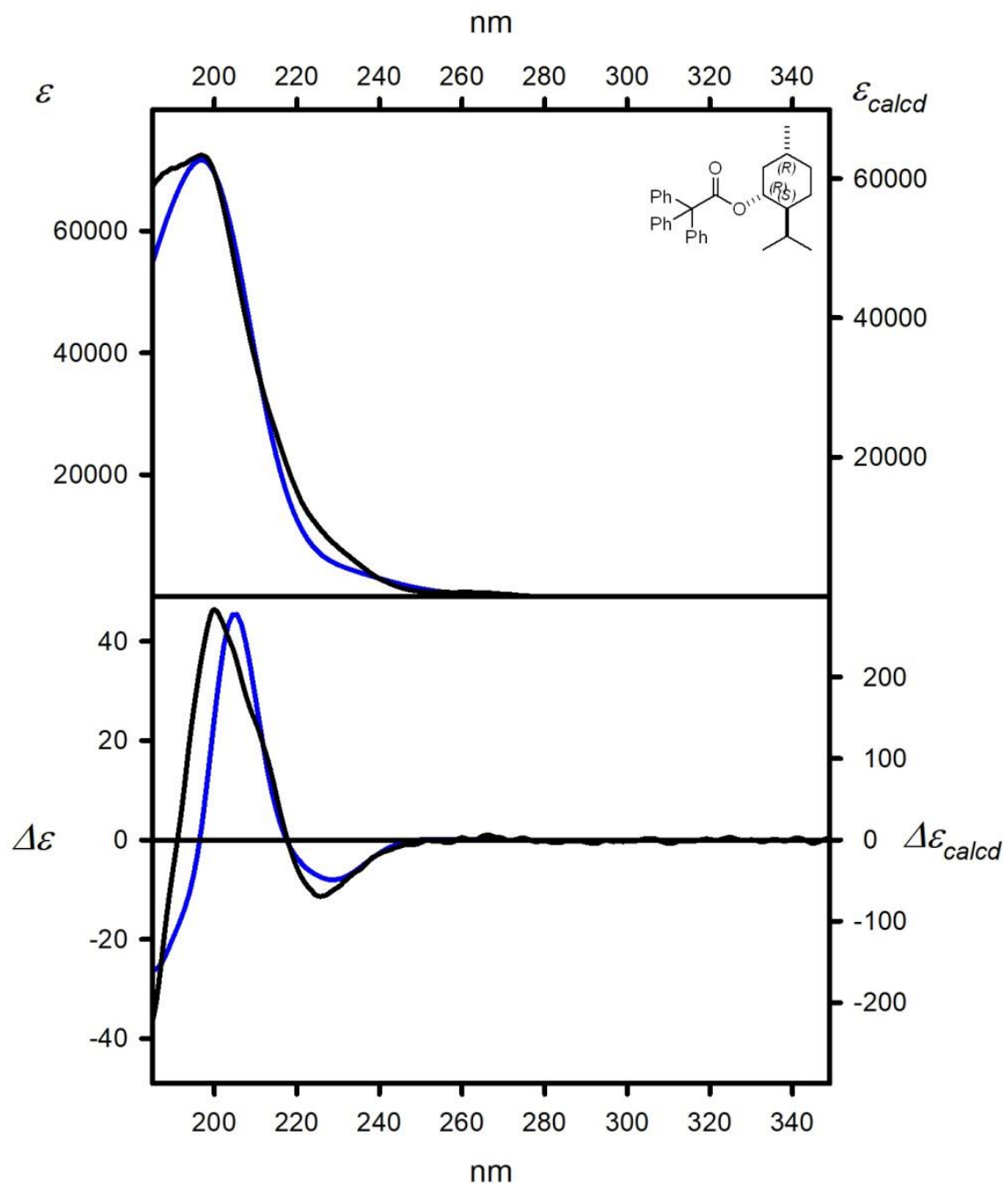

Experimental (cyclohexane, black lines)

\section{Calculated at the}

TD-CAM-B3LYP/6-311++G(2d,2p) level and:

$\triangle E$-based Boltzmann averaged (red lines)

$\triangle \triangle G$-based Boltzmann averaged (blue lines)

Figure SI_93. UV (upper panel) and ECD (lower panel) spectra of 10, experimental, measured in cyclohexane (solid black lines) and calculated at the TD-CAM-B3LYP/6-311++G(2d,2p) level for structures optimized at the M06-2X/6-311++G(d,p) level, $\Delta \mathrm{E}$-based Boltzmann (red lines) and $\Delta \Delta \mathrm{G}-$ based Boltzmann averaged (blue solid lines). Insert shows the comparison between Boltzmann averaged ECD spectrum and that calculated for $\Delta \Delta G$-based on the lowest energy conformer of a given compound (dashed blue line). All calculated spectra were wavelength corrected to match experimental UV maxima. 


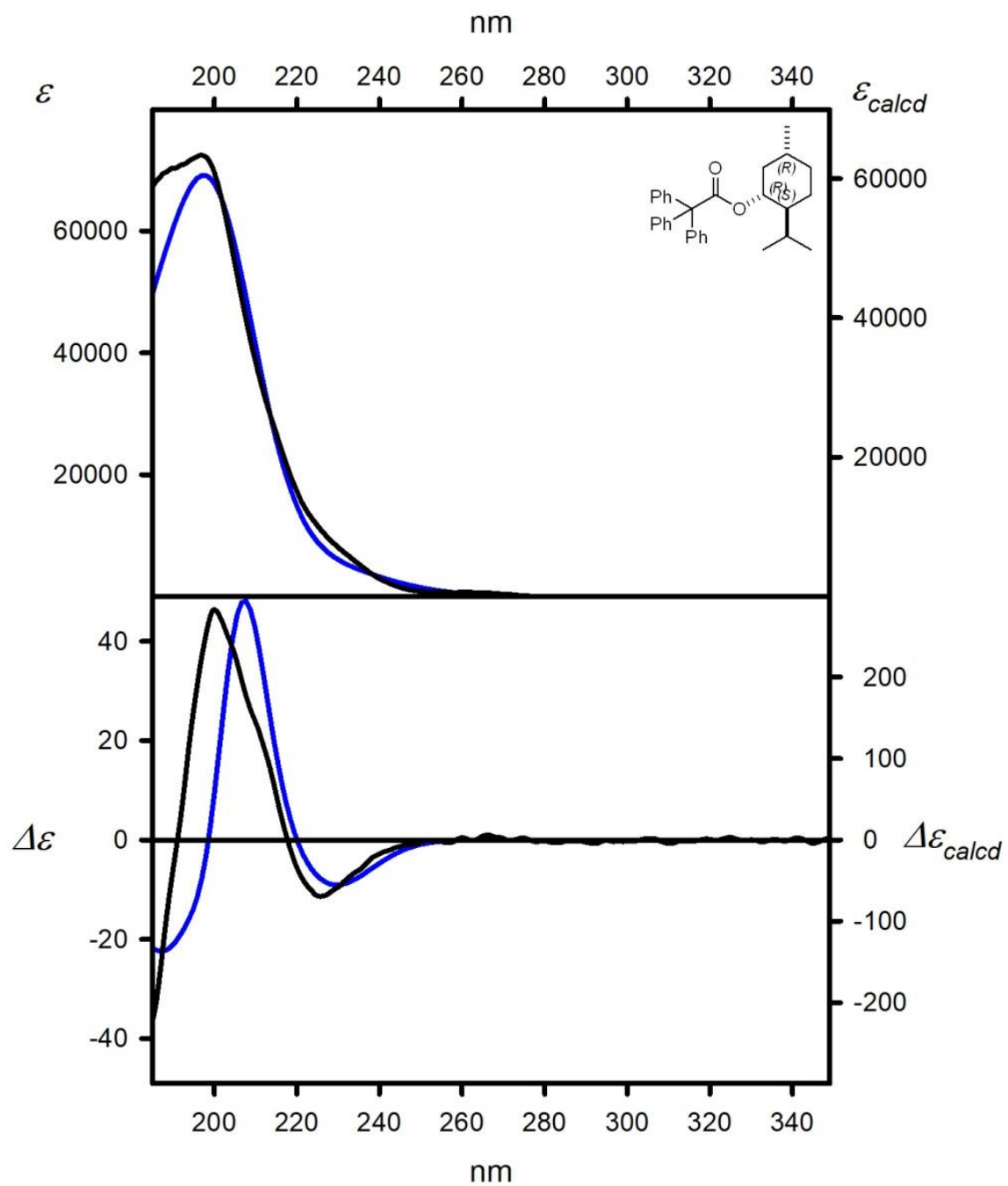

Experimental (cyclohexane, black lines)

\section{Calculated at the}

TD-M06-2X/6-311++G(2d,2p) level and:

$\triangle E$-based Boltzmann averaged (red lines)

$\triangle \triangle G$-based Boltzmann averaged (blue lines)

Figure SI_94. UV (upper panel) and ECD (lower panel) spectra of 10, experimental, measured in cyclohexane (solid black lines) and calculated at the TD-M06-2X/6-311++G(2d,2p) level for structures optimized at the M06-2X/6-311++G(d,p) level, $\Delta \mathrm{E}$-based Boltzmann (red lines) and $\Delta \Delta \mathrm{G}$-based Boltzmann averaged (blue solid lines). Insert shows the comparison between Boltzmann averaged ECD spectrum and that calculated for $\Delta \Delta G$-based on the lowest energy conformer of a given compound (dashed blue line). All calculated spectra were wavelength corrected to match experimental UV maxima. 


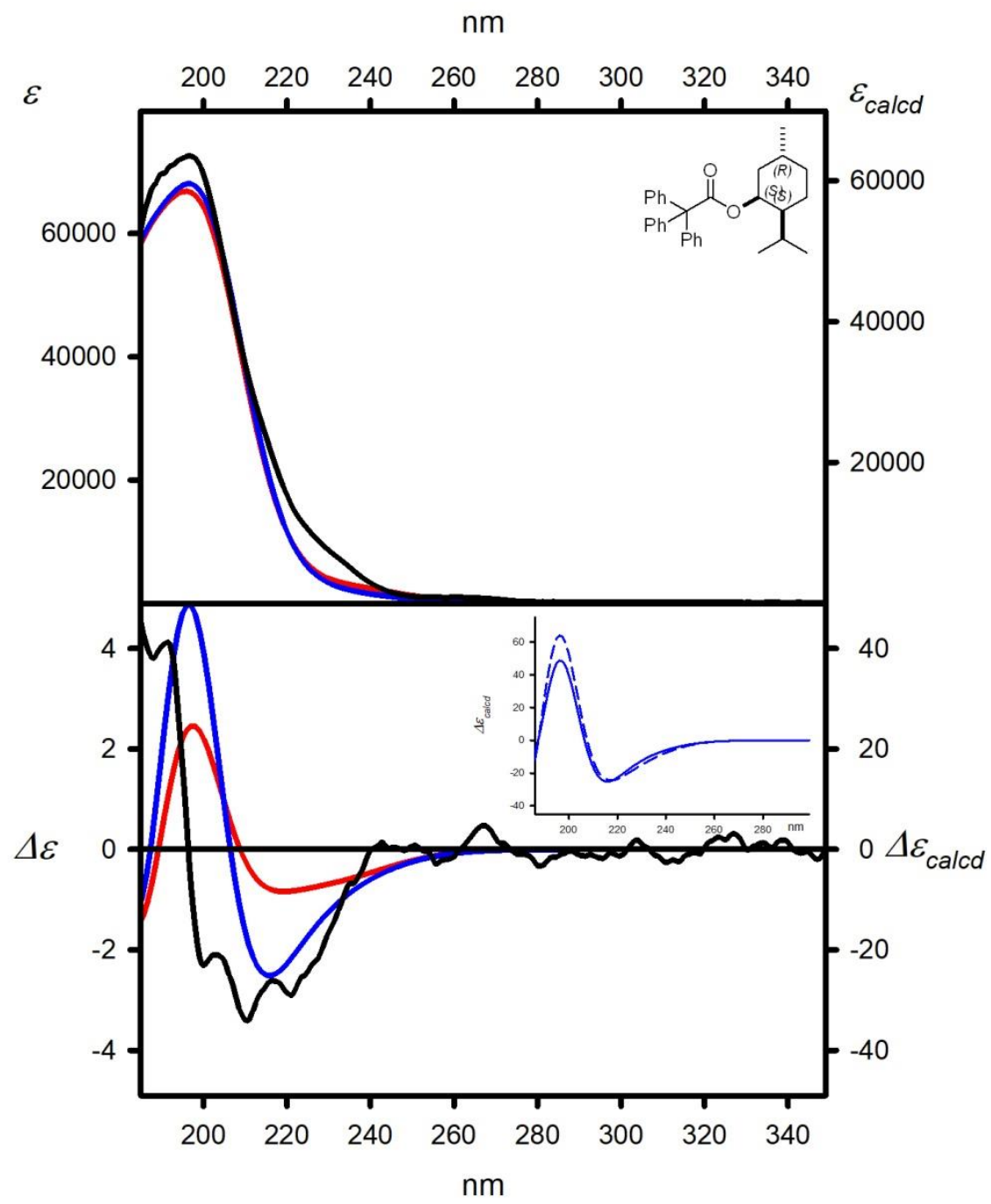

Experimental (cyclohexane, black lines)

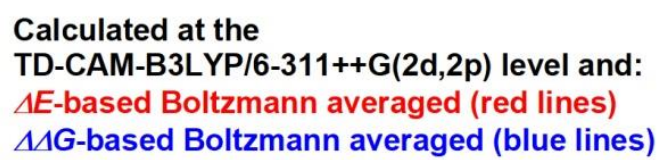

Figure SI_95. UV (upper panel) and ECD (lower panel) spectra of 11, experimental, measured in cyclohexane (solid black lines) and calculated at the TD-CAM-B3LYP/6-311++G(2d,2p) level for structures optimized at the B3LYP/6-311++G(d,p) level, $\Delta E$-based Boltzmann (red lines) and $\Delta \Delta G$ based Boltzmann averaged (blue solid lines). Insert shows the comparison between Boltzmann averaged ECD spectrum and that calculated for $\Delta \Delta G$-based on the lowest energy conformer of a given compound (dashed blue line). All calculated spectra were wavelength corrected to match experimental UV maxima. 


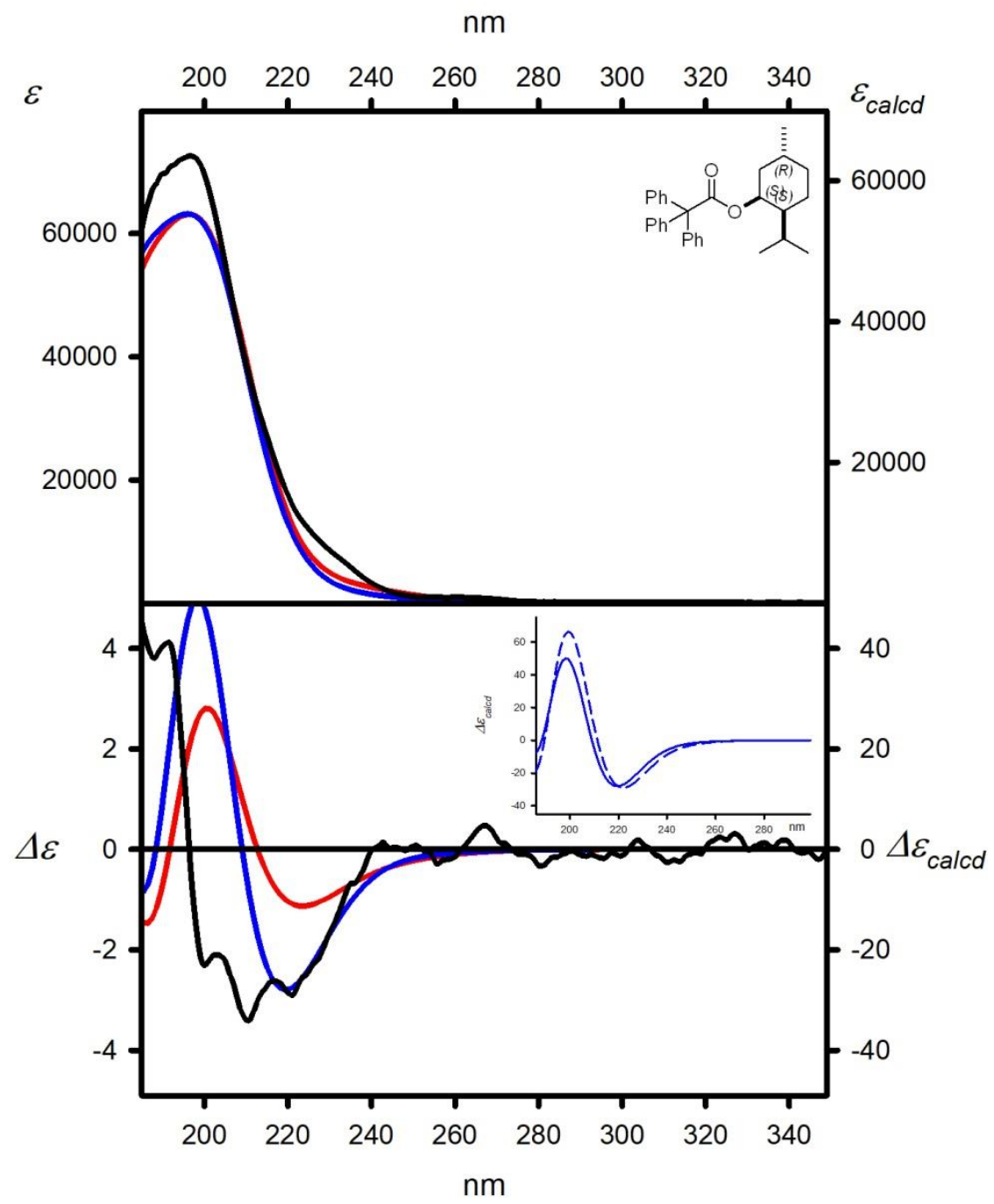

Experimental (cyclohexane, black lines)

\section{Calculated at the}

TD-M06-2X/6-311++G(2d,2p) level and:

$\triangle E$-based Boltzmann averaged (red lines)

$\triangle \Lambda G$-based Boltzmann averaged (blue lines)

Figure SI_96. UV (upper panel) and ECD (lower panel) spectra of 11, experimental, measured in cyclohexane (solid black lines) and calculated the TD-M06-2X/6-311++G $(2 d, 2 p)$ level for structures optimized at the B3LYP/6-311++G(d,p) level, $\Delta \mathrm{E}$-based Boltzmann (red lines) and $\Delta \Delta \mathrm{G}$-based Boltzmann averaged (blue solid lines). Insert shows the comparison between Boltzmann averaged ECD spectrum and that calculated for $\Delta \Delta G$-based on the lowest energy conformer of a given compound (dashed blue line). All calculated spectra were wavelength corrected to match experimental UV maxima. 


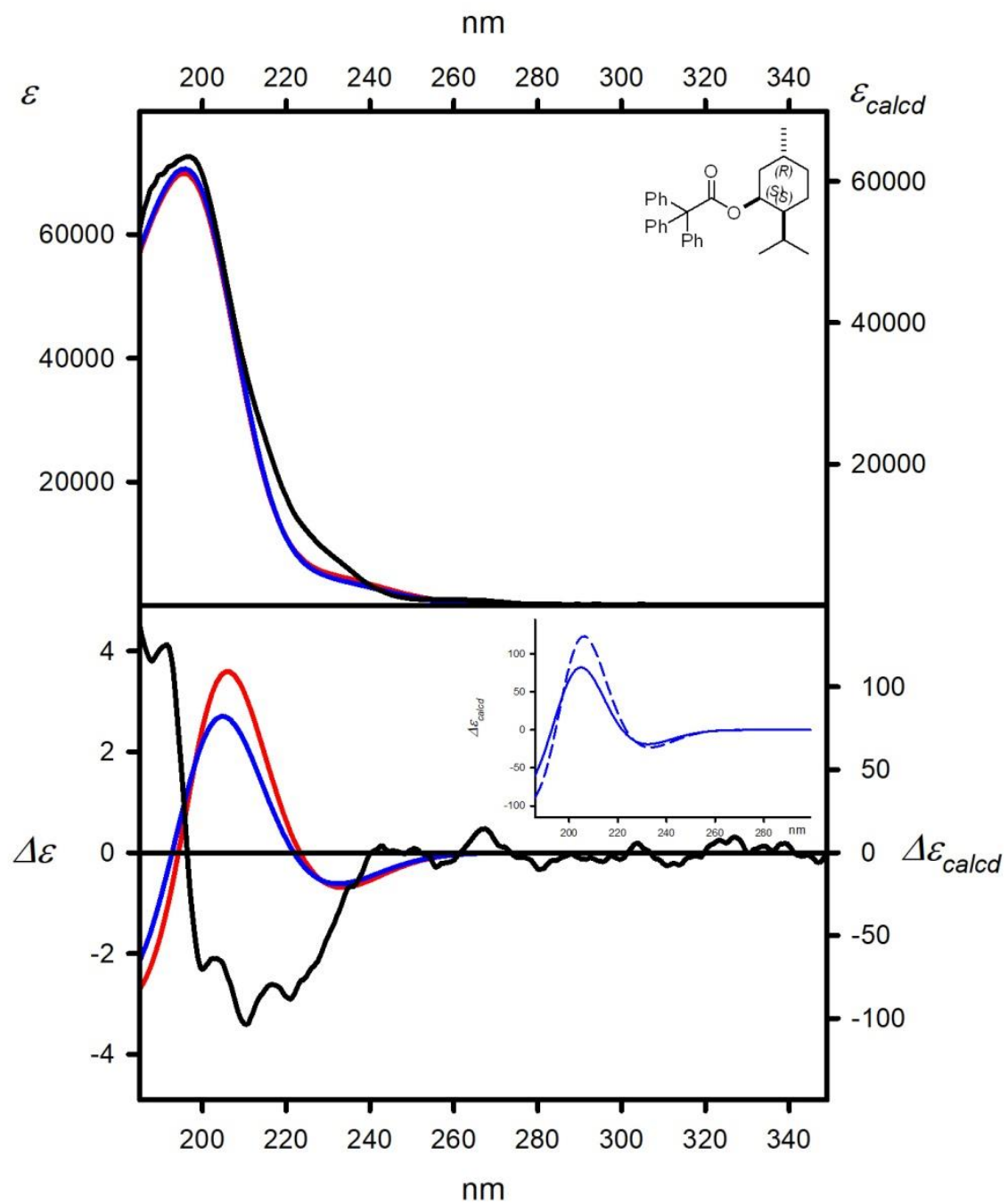

Experimental (cyclohexane, black lines)

Calculated at the

TD-CAM-B3LYP/6-311++G(2d,2p) level and:

$\triangle E$-based Boltzmann averaged (red lines)

$\triangle \triangle G$-based Boltzmann averaged (blue lines)

Figure SI_97. UV (upper panel) and ECD (lower panel) spectra of 11, experimental, measured in cyclohexane (solid black lines) and calculated at the TD-CAM-B3LYP/6-311++G(2d,2p) level for structures optimized at the M06-2X/6-311++G(d,p) level, $\Delta \mathrm{E}$-based Boltzmann (red lines) and $\Delta \Delta \mathrm{G}-$ based Boltzmann averaged (blue solid lines). Insert shows the comparison between Boltzmann averaged ECD spectrum and that calculated for $\Delta \Delta G$-based on the lowest energy conformer of a given compound (dashed blue line). All calculated spectra were wavelength corrected to match experimental UV maxima. 


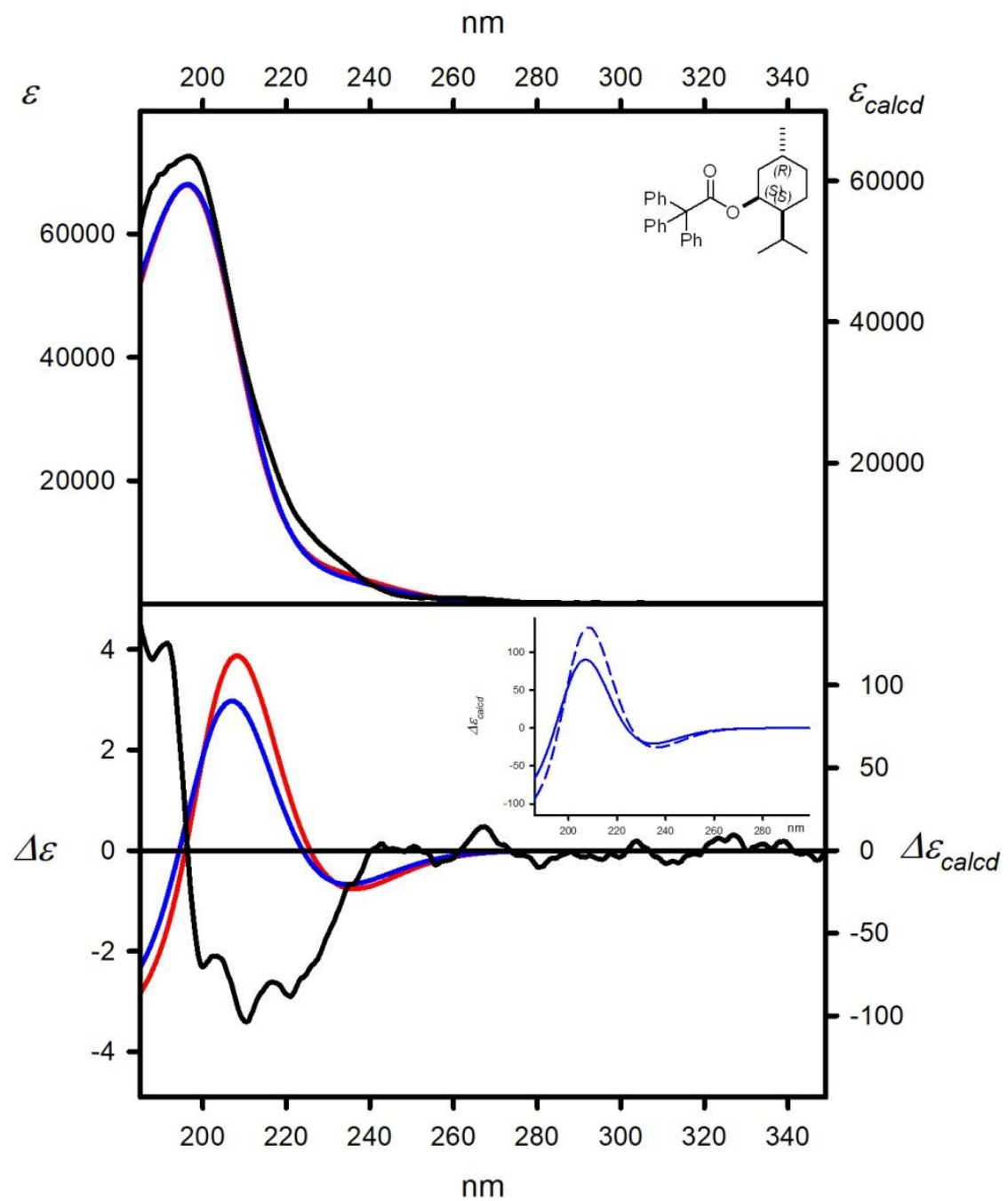

Experimental (cyclohexane, black lines)

\section{Calculated at the}

TD-M06-2X/6-311++G(2d,2p) level and:

$\triangle E$-based Boltzmann averaged (red lines)

$\triangle \triangle G$-based Boltzmann averaged (blue lines)

Figure SI_98. UV (upper panel) and ECD (lower panel) spectra of 11, experimental, measured in cyclohexane (solid black lines) and calculated at the TD-M06-2X/6-311++G(2d,2p) level for structures optimized at the M06-2X/6-311++G(d,p) level, $\Delta \mathrm{E}$-based Boltzmann (red lines) and $\Delta \Delta \mathrm{G}$-based Boltzmann averaged (blue solid lines). Insert shows the comparison between Boltzmann averaged ECD spectrum and that calculated for $\Delta \Delta G$-based on the lowest energy conformer of a given compound (dashed blue line). All calculated spectra were wavelength corrected to match experimental UV maxima. 


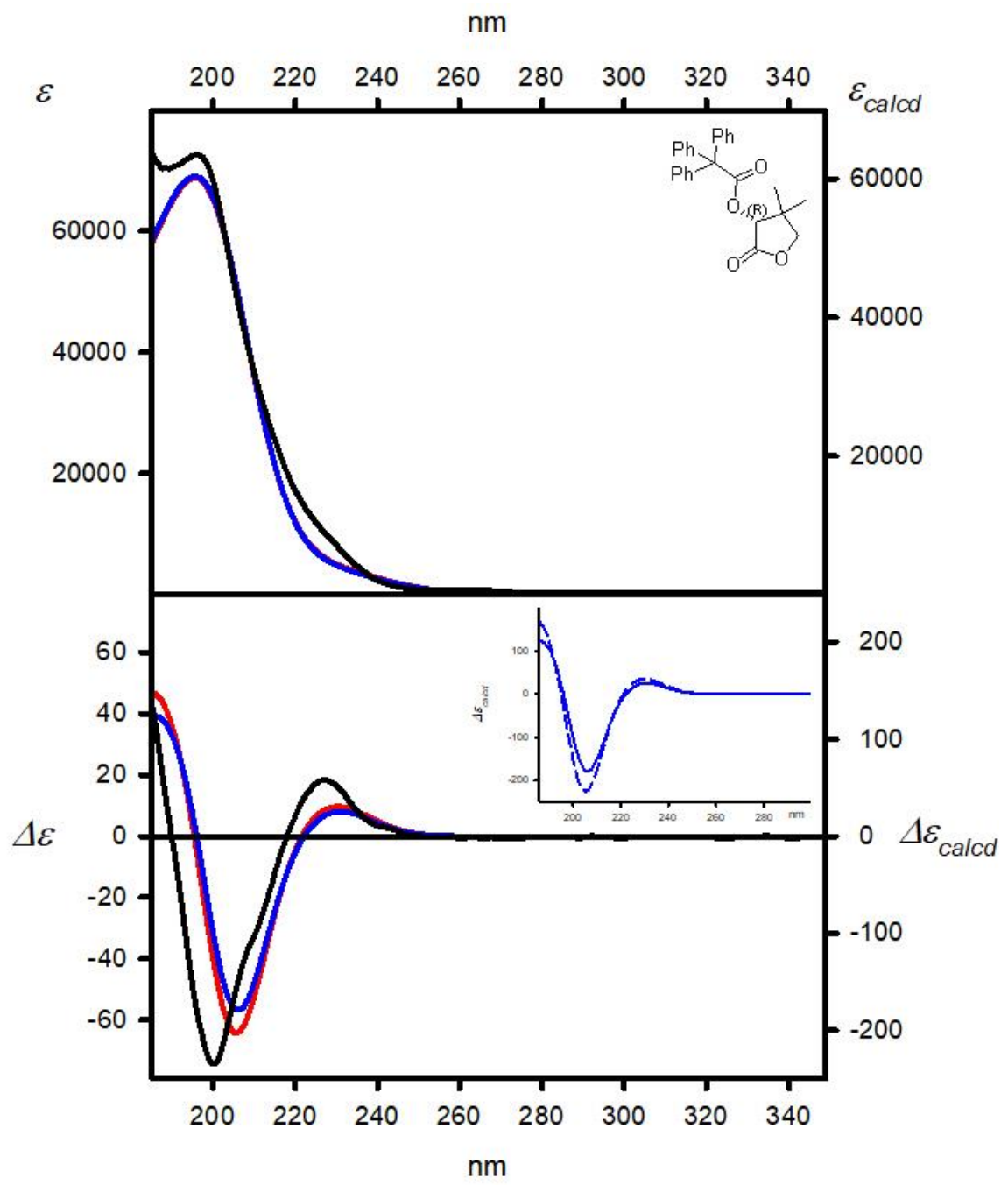

Experimental (cyclohexane, black lines)

Calculated at the

TD-CAM-B3LYP/6-311++G(2d,2p) level and:

$\triangle E$-based Boltzmann averaged (red lines)

$\Delta \triangle G$-based Boltzmann averaged (blue lines)

Figure SI_99. UV (upper panel) and ECD (lower panel) spectra of 14, experimental, measured in cyclohexane (solid black lines) and calculated at the TD-CAM-B3LYP/6-311++G(2d,2p) level for structures optimized at the B3LYP/6-311++G(d,p) level, $\Delta E$-based Boltzmann (red lines) and $\Delta \Delta G$ based Boltzmann averaged (blue solid lines). Insert shows the comparison between Boltzmann averaged ECD spectrum and that calculated for $\Delta \Delta G$-based on the lowest energy conformer of a given compound (dashed blue line). All calculated spectra were wavelength corrected to match experimental UV maxima. 


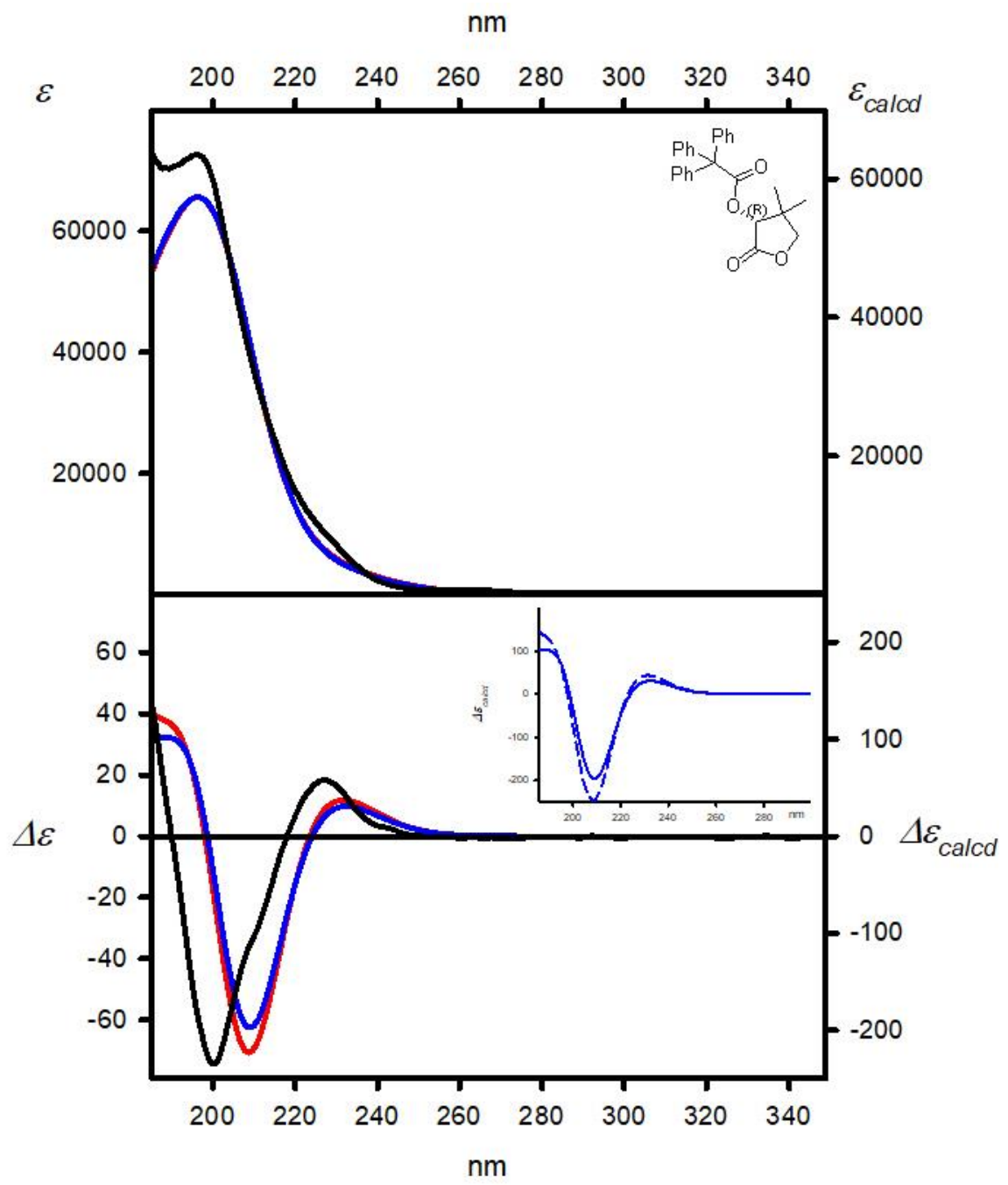

Experimental (cyclohexane, black lines)

Calculated at the TD-M06-2X/6-311++G(2d,2p) level and:

$\triangle E$-based Boltzmann averaged (red lines) $\Delta \Delta G$-based Boltzmann averaged (blue lines)

Figure SI_100. UV (upper panel) and ECD (lower panel) spectra of 14, experimental, measured in cyclohexane (solid black lines) and calculated at the TD-M06-2X/6-311++G $(2 d, 2 p)$ level for structures optimized at the B3LYP/6-311++G(d,p) level, $\Delta \mathrm{E}$-based Boltzmann (red lines) and $\Delta \Delta \mathrm{G}$-based Boltzmann averaged (blue solid lines). Insert shows the comparison between Boltzmann averaged ECD spectrum and that calculated for $\Delta \Delta G$-based on the lowest energy conformer of a given compound (dashed blue line). All calculated spectra were wavelength corrected to match experimental UV maxima. 


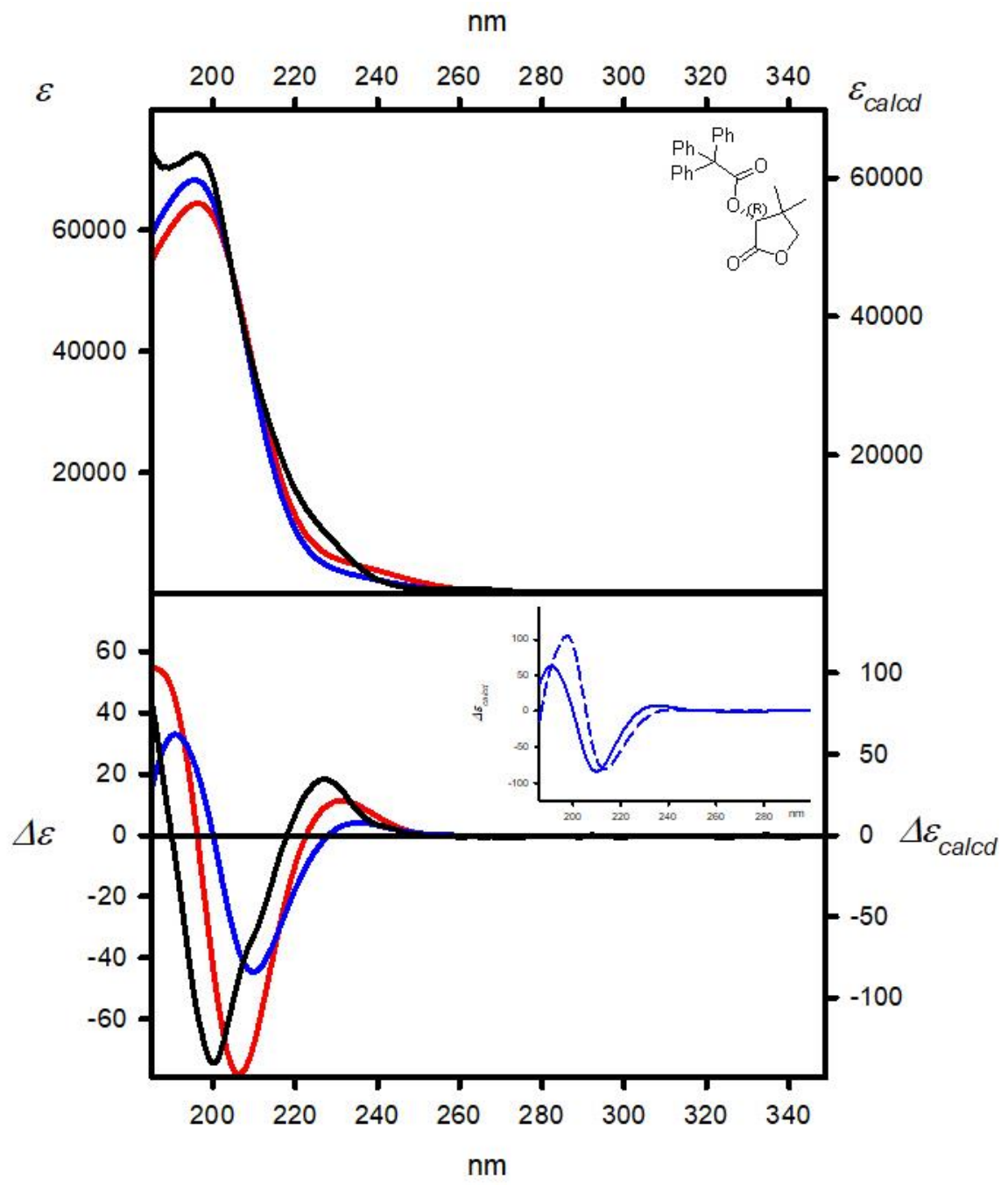

Experimental (cyclohexane, black lines)

Calculated at the

TD-CAM-B3LYP/6-311++G(2d,2p) level and:

$\triangle E$-based Boltzmann averaged (red lines)

$\Delta \triangle G$-based Boltzmann averaged (blue lines)

Figure SI_101. UV (upper panel) and ECD (lower panel) spectra of 14, experimental, measured in cyclohexane (solid black lines) and calculated at the TD-CAM-B3LYP/6-311++G(2d,2p) level for structures optimized at the M06-2X/6-311++G(d,p) level, $\Delta \mathrm{E}$-based Boltzmann (red lines) and $\Delta \Delta \mathrm{G}-$ based Boltzmann averaged (blue solid lines). Insert shows the comparison between Boltzmann averaged ECD spectrum and that calculated for $\Delta \Delta G$-based on the lowest energy conformer of a given compound (dashed blue line). All calculated spectra were wavelength corrected to match experimental UV maxima. 


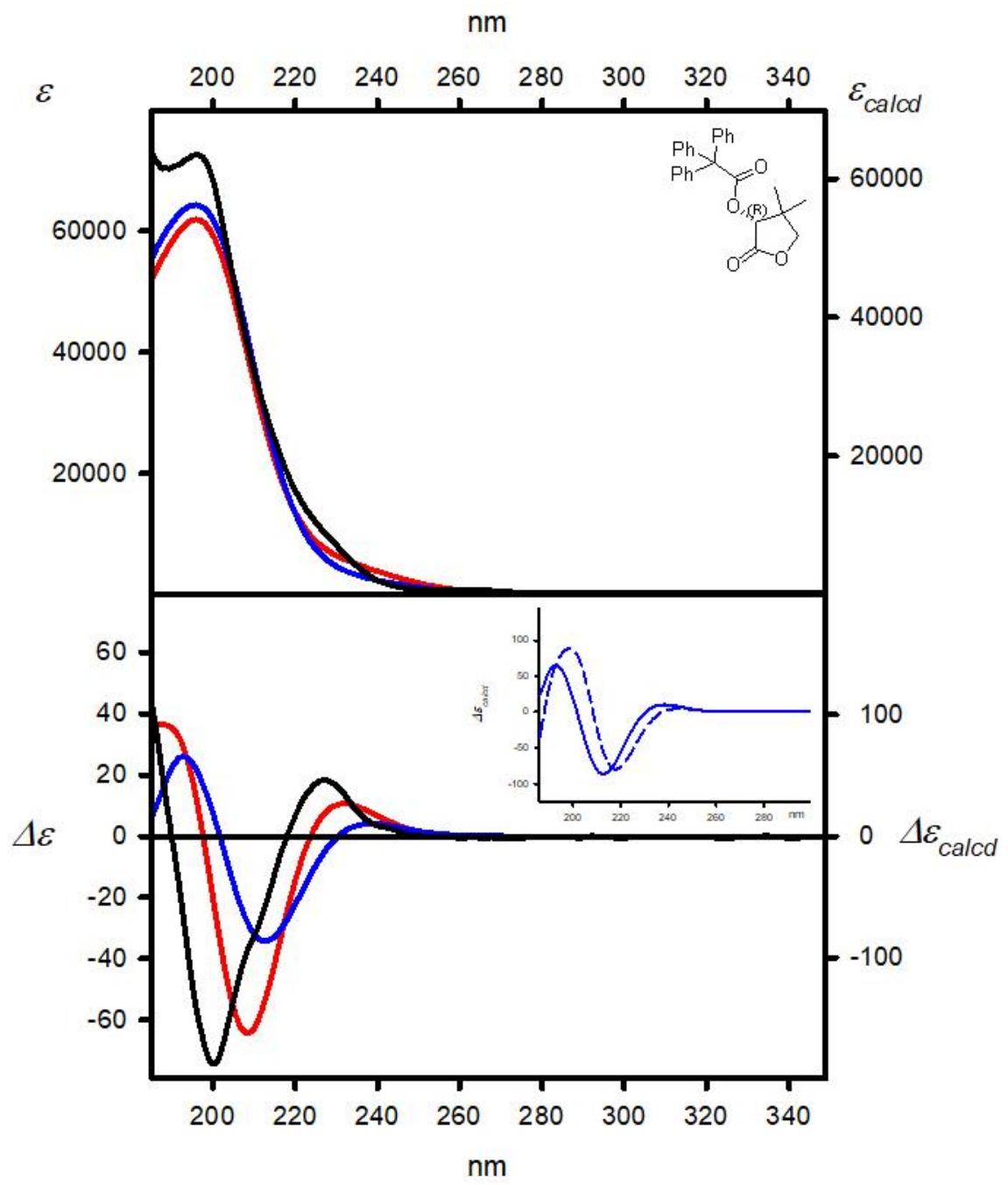

Experimental (cyclohexane, black lines)

Calculated at the

TD-M06-2X/6-311++G(2d,2p) level and:

$\triangle E$-based Boltzmann averaged (red lines)

$\Delta \Delta G$-based Boltzmann averaged (blue lines)

Figure SI_102. UV (upper panel) and ECD (lower panel) spectra of 14, experimental, measured in cyclohexane (solid black lines) and calculated at the TD-M06-2X/6-311++G(2d,2p) level for structures optimized at the M06-2X/6-311++G(d,p) level, $\Delta E$-based Boltzmann (red lines) and $\Delta \Delta G$-based Boltzmann averaged (blue solid lines). Insert shows the comparison between Boltzmann averaged ECD spectrum and that calculated for $\Delta \Delta G$-based on the lowest energy conformer of a given compound (dashed blue line). All calculated spectra were wavelength corrected to match experimental UV maxima. 


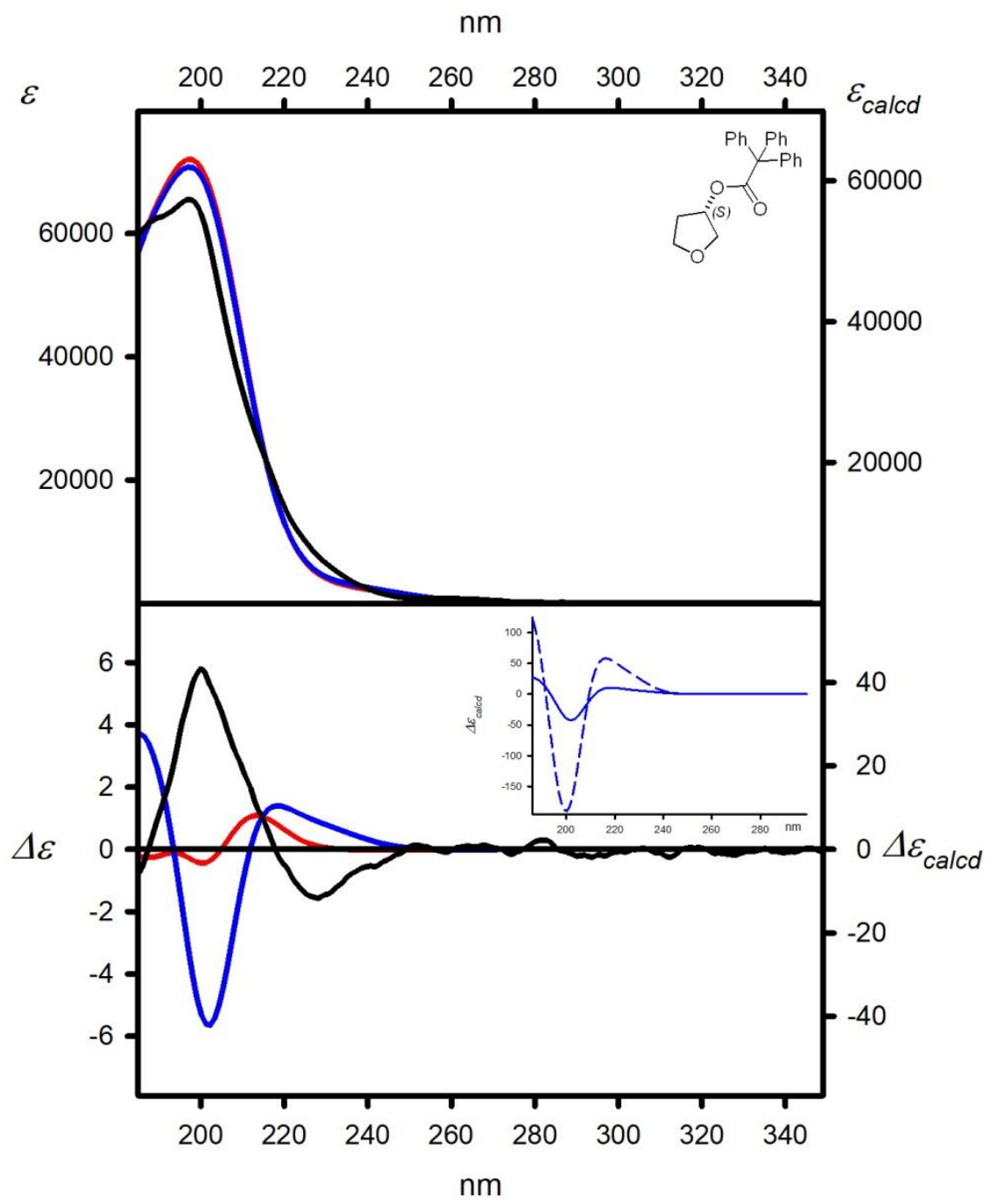

Experimental (cyclohexane, black lines)

Calculated at the

TD-CAM-B3LYP/6-311++G(2d,2p) level and:

$\triangle E$-based Boltzmann averaged (red lines)

$\triangle 4 G$-based Boltzmann averaged (blue lines)

Figure SI_103. UV (upper panel) and ECD (lower panel) spectra of 15, experimental, measured in cyclohexane (solid black lines) and calculated at the TD-CAM-B3LYP/6-311++G(2d,2p) level for structures optimized at the B3LYP/6-311++G(d,p) level, $\Delta E$-based Boltzmann (red lines) and $\Delta \Delta G$ based Boltzmann averaged (blue solid lines). Insert shows the comparison between Boltzmann averaged ECD spectrum and that calculated for $\Delta \Delta G$-based on the lowest energy conformer of a given compound (dashed blue line). All calculated spectra were wavelength corrected to match experimental UV maxima. 


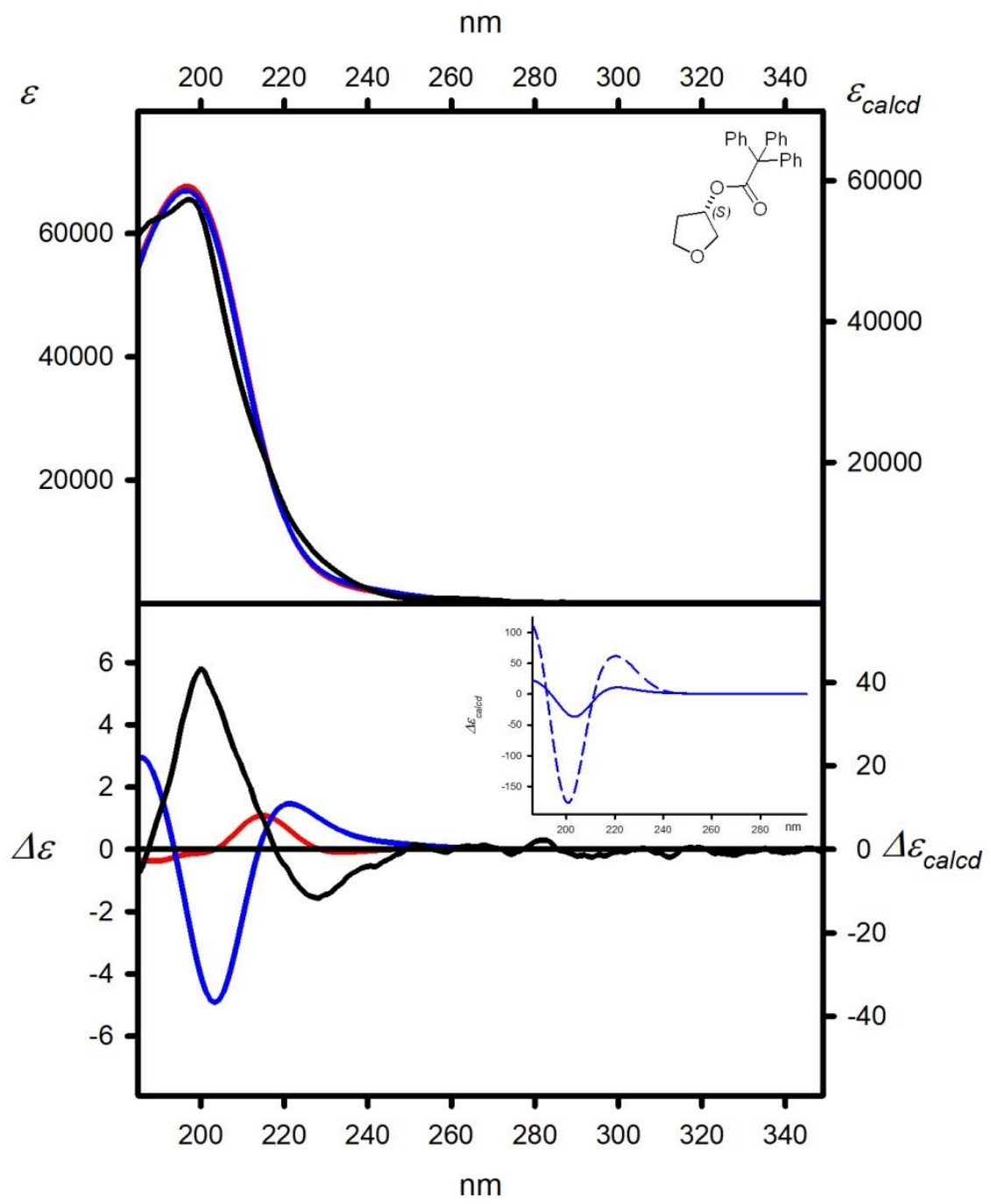

Experimental (cyclohexane, black lines)

Calculated at the

TD-M06-2X/6-311++G(2d,2p) level and:

$\triangle E$-based Boltzmann averaged (red lines)

$\triangle 4 G$-based Boltzmann averaged (blue lines)

Figure SI_104. UV (upper panel) and ECD (lower panel) spectra of 15, experimental, measured in cyclohexane (solid black lines) and calculated at the TD-M06-2X/6-311++G $(2 d, 2 p)$ level for structures optimized at the B3LYP/6-311++G(d,p) level, $\Delta \mathrm{E}$-based Boltzmann (red lines) and $\Delta \Delta \mathrm{G}$-based Boltzmann averaged (blue solid lines). Insert shows the comparison between Boltzmann averaged ECD spectrum and that calculated for $\Delta \Delta G$-based on the lowest energy conformer of a given compound (dashed blue line). All calculated spectra were wavelength corrected to match experimental UV maxima. 


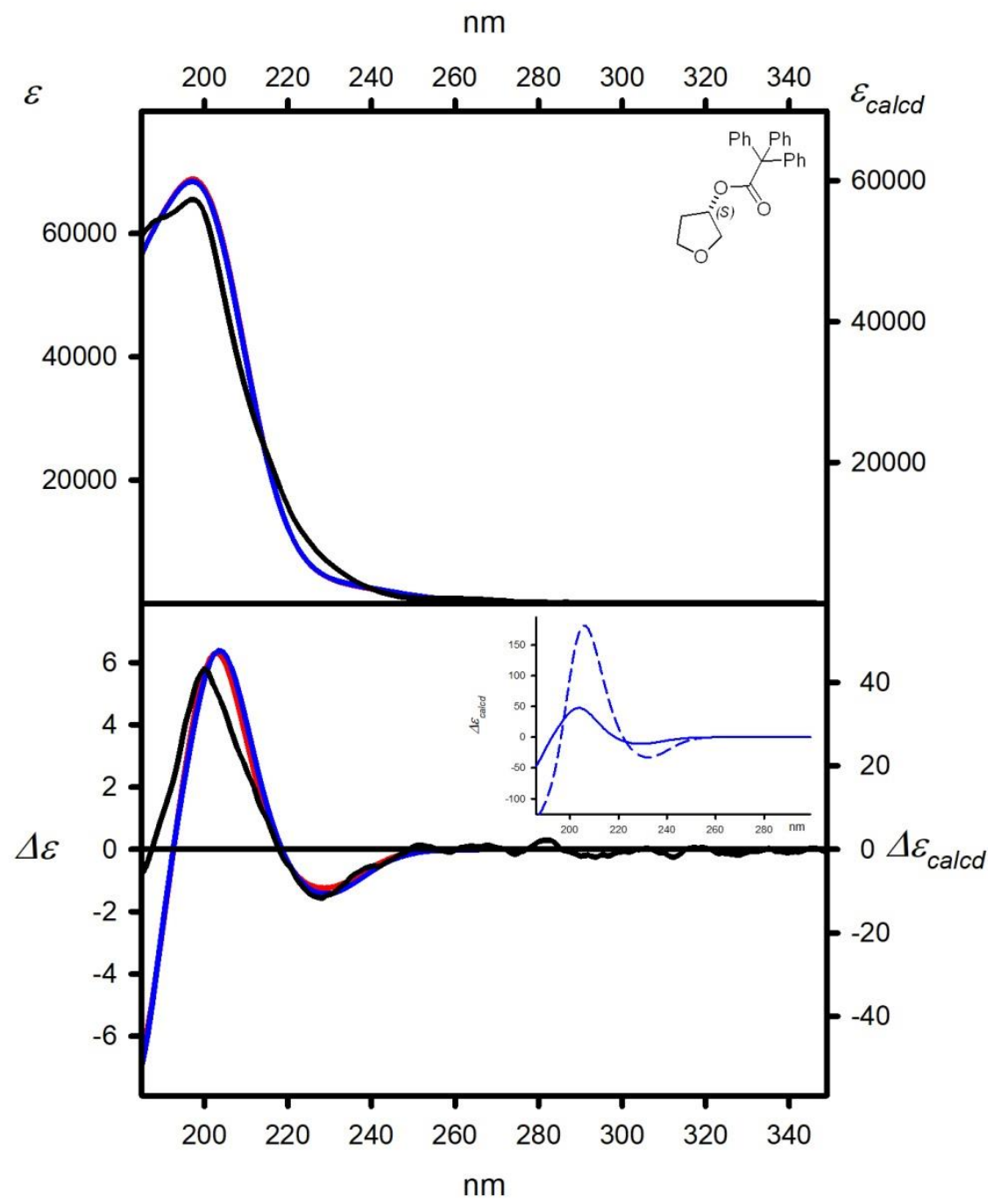

Experimental (cyclohexane, black lines)

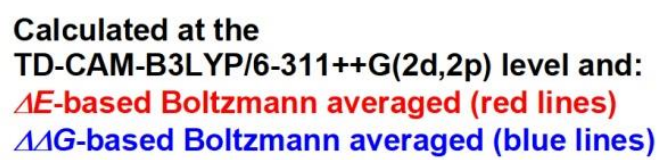

Figure SI_105. UV (upper panel) and ECD (lower panel) spectra of 15, experimental, measured in cyclohexane (solid black lines) and calculated at the TD-CAM-B3LYP/6-311++G(2d,2p) level for structures optimized at the M06-2X/6-311++G(d,p) level, $\Delta \mathrm{E}$-based Boltzmann (red lines) and $\Delta \Delta \mathrm{G}-$ based Boltzmann averaged (blue solid lines). Insert shows the comparison between Boltzmann averaged ECD spectrum and that calculated for $\Delta \Delta G$-based on the lowest energy conformer of a given compound (dashed blue line). All calculated spectra were wavelength corrected to match experimental UV maxima. 


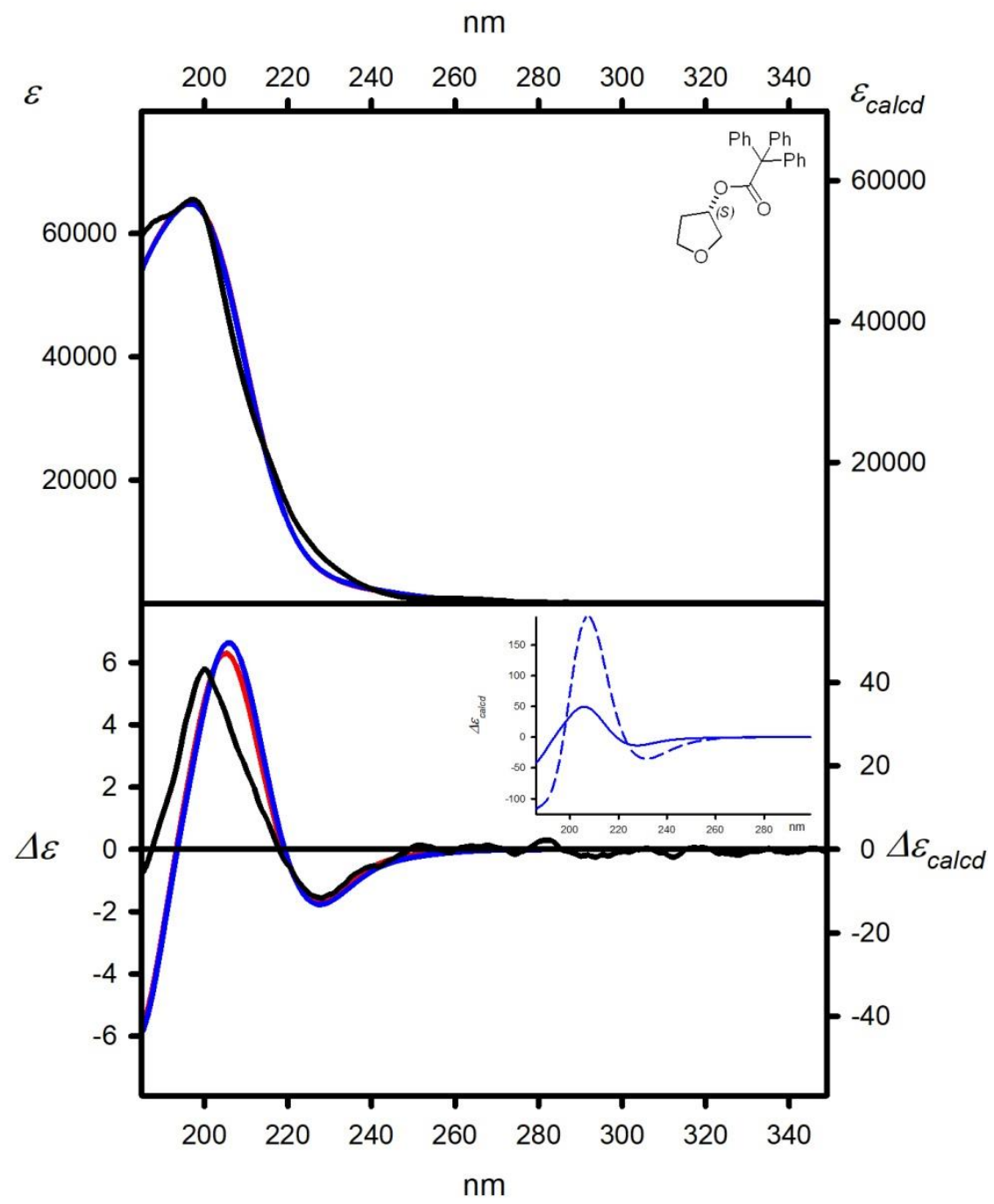

Experimental (cyclohexane, black lines)

Calculated at the

TD-M06-2X/6-311++G(2d,2p) level and:

$\triangle E$-based Boltzmann averaged (red lines)

$\triangle M G$-based Boltzmann averaged (blue lines)

Figure SI_106. UV (upper panel) and ECD (lower panel) spectra of 15, experimental, measured in cyclohexane (solid black lines) and calculated at the TD-M06-2X/6-311++G $(2 d, 2 p)$ level for structures optimized at the M06-2X/6-311++G(d,p) level, $\Delta E$-based Boltzmann (red lines) and $\Delta \Delta G$-based Boltzmann averaged (blue solid lines). Insert shows the comparison between Boltzmann averaged ECD spectrum and that calculated for $\Delta \Delta G$-based on the lowest energy conformer of a given compound (dashed blue line). All calculated spectra were wavelength corrected to match experimental UV maxima. 


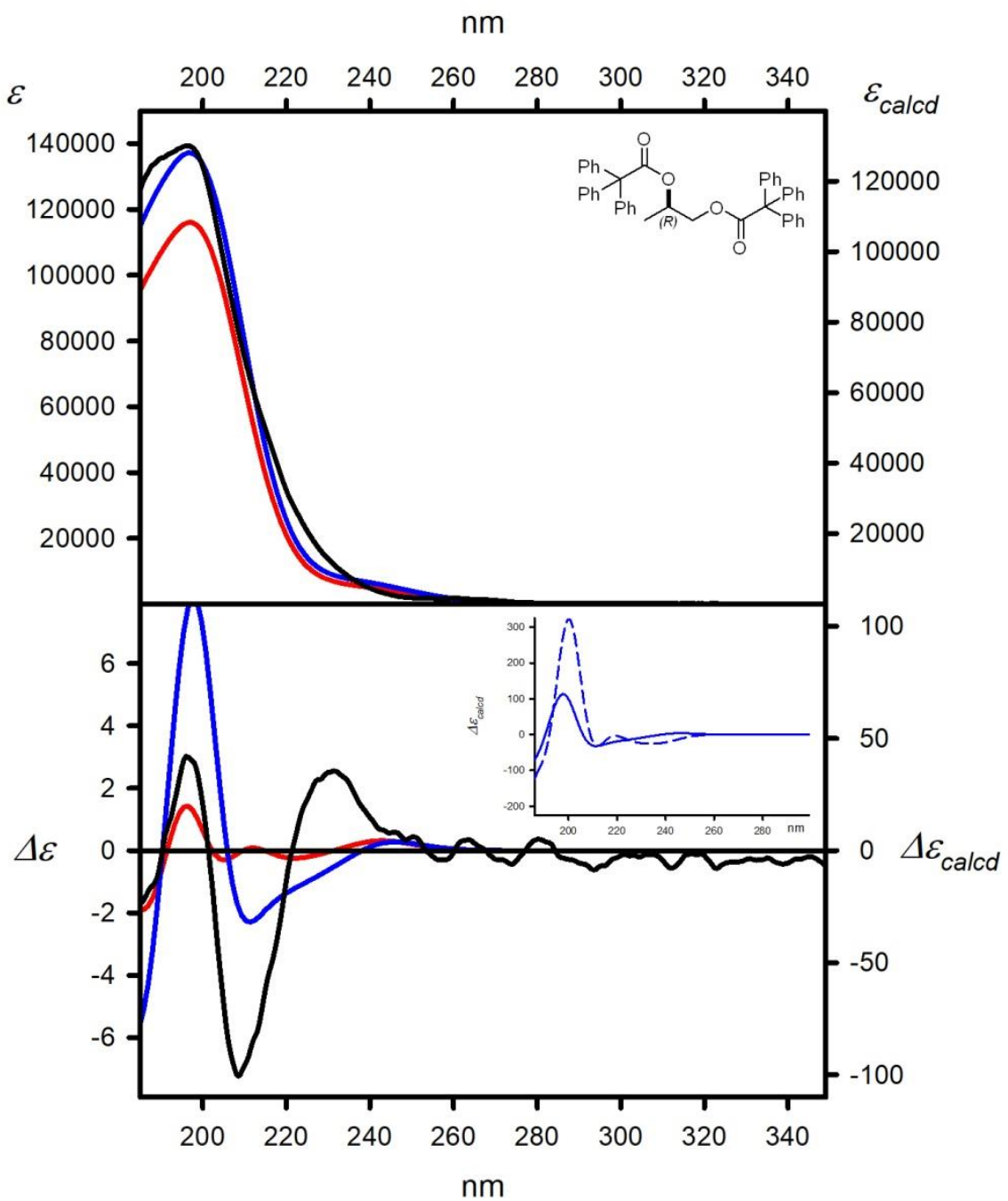

Experimental (cyclohexane, black lines)

\section{Calculated at the}

TD-CAM-B3LYP/6-311++G(d,p) level and:

$\triangle E$-based Boltzmann averaged (red lines)

$\triangle \triangle G$-based Boltzmann averaged (blue lines)

Figure SI_107. UV (upper panel) and ECD (lower panel) spectra of 18, experimental, measured in cyclohexane (solid black lines) and calculated at the TD-CAM-B3LYP/6-311++G(d,p) level for structures optimized at the B3LYP/6-311G(d,p) level, $\Delta \mathrm{E}$-based Boltzmann (red lines) and $\Delta \Delta \mathrm{G}$-based Boltzmann averaged (blue solid lines). Insert shows the comparison between Boltzmann averaged ECD spectrum and that calculated for $\Delta \Delta G$-based on the lowest energy conformer of a given compound (dashed blue line). All calculated spectra were wavelength corrected to match experimental UV maxima. 


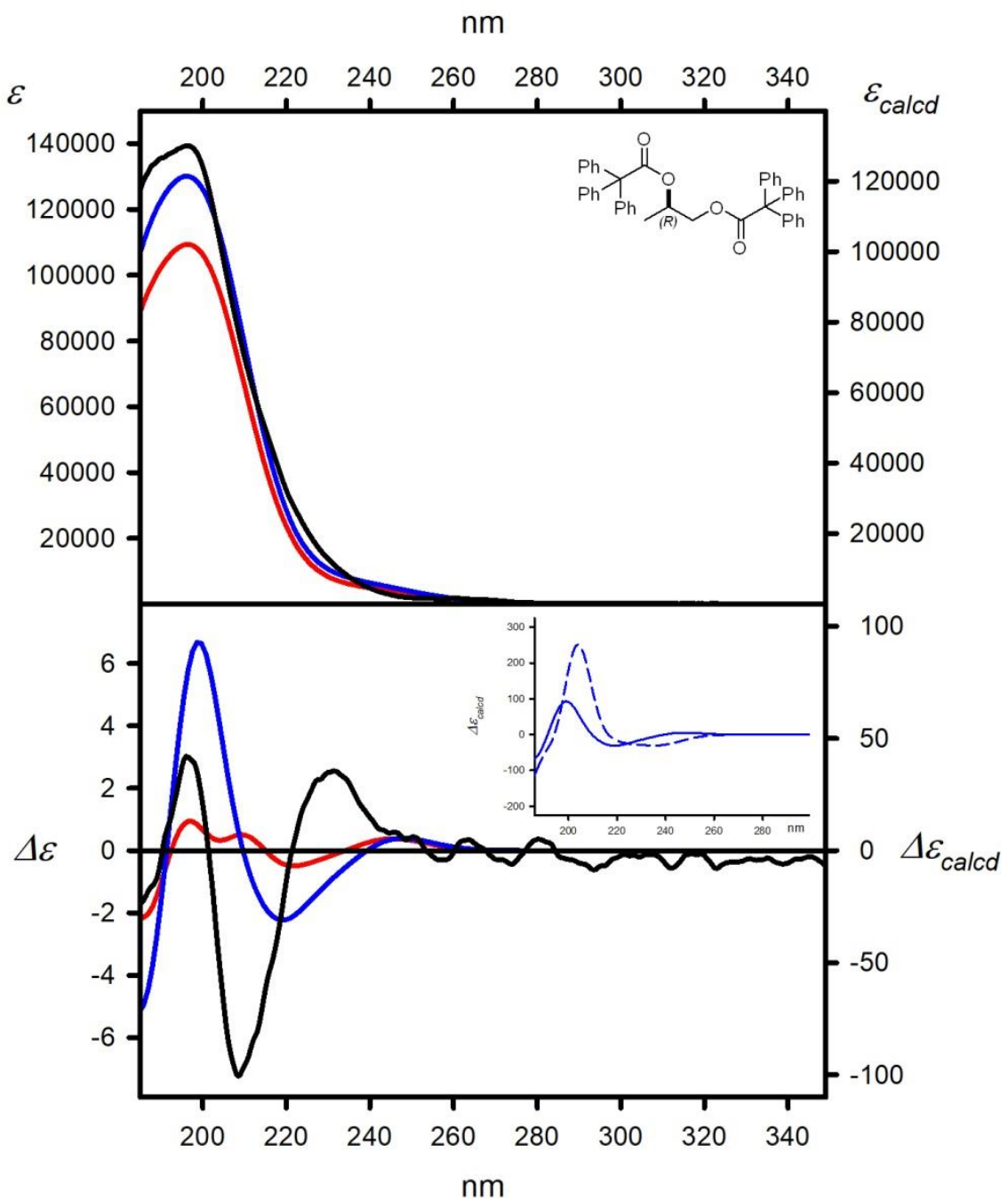

Experimental (cyclohexane, black lines)

\section{Calculated at the}

TD-M06-2X/6-311++G(d,p) level and:

$\triangle E$-based Boltzmann averaged (red lines)

$\triangle \triangle G$-based Boltzmann averaged (blue lines)

Figure SI_108. UV (upper panel) and ECD (lower panel) spectra of 18, experimental, measured in cyclohexane (solid black lines) and calculated at the TD-M06-2X/6-311++G(d,p) level for structures optimized at the B3LYP/6-311G(d,p) level, $\Delta \mathrm{E}$-based Boltzmann (red lines) and $\Delta \Delta \mathrm{G}$-based Boltzmann averaged (blue solid lines). Insert shows the comparison between Boltzmann averaged ECD spectrum and that calculated for $\Delta \Delta G$-based on the lowest energy conformer of a given compound (dashed blue line). All calculated spectra were wavelength corrected to match experimental UV maxima. 


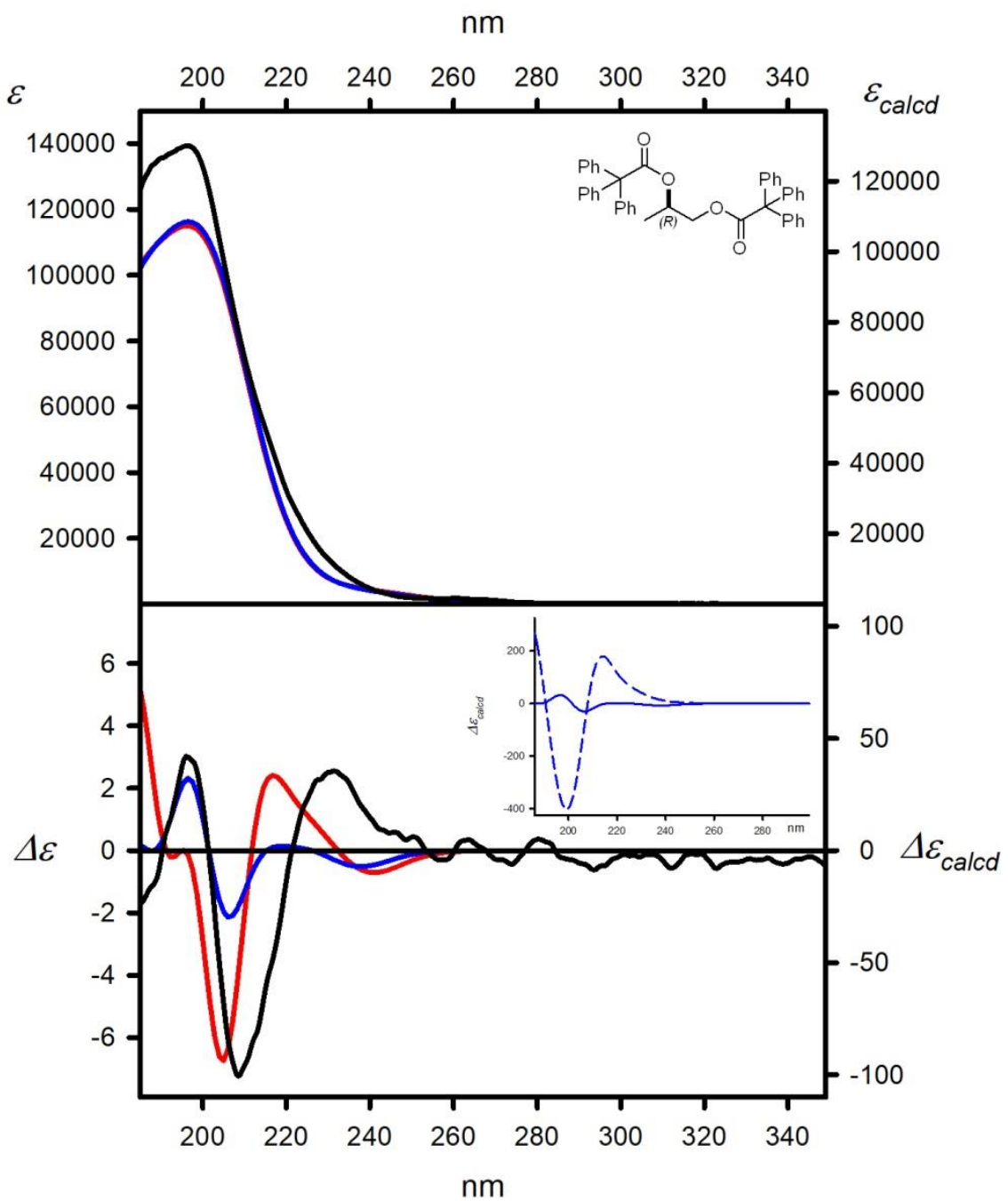

Experimental (cyclohexane, black lines)

\section{Calculated at the}

TD-CAM-B3LYP/6-311++G(d,p) level and:

$\triangle E$-based Boltzmann averaged (red lines)

$\triangle \triangle G$-based Boltzmann averaged (blue lines)

Figure SI_109. UV (upper panel) and ECD (lower panel) spectra of 18, experimental, measured in cyclohexane (solid black lines) and calculated at the TD-CAM-B3LYP/6-311++G(d,p) level for structures optimized at the B3LYP-GD3BJ/6-311G(d,p) level, $\Delta \mathrm{E}$-based Boltzmann (red lines) and $\Delta \Delta G$-based Boltzmann averaged (blue solid lines). Insert shows the comparison between Boltzmann averaged ECD spectrum and that calculated for $\Delta \Delta G$-based on the lowest energy conformer of a given compound (dashed blue line). All calculated spectra were wavelength corrected to match experimental UV maxima. 


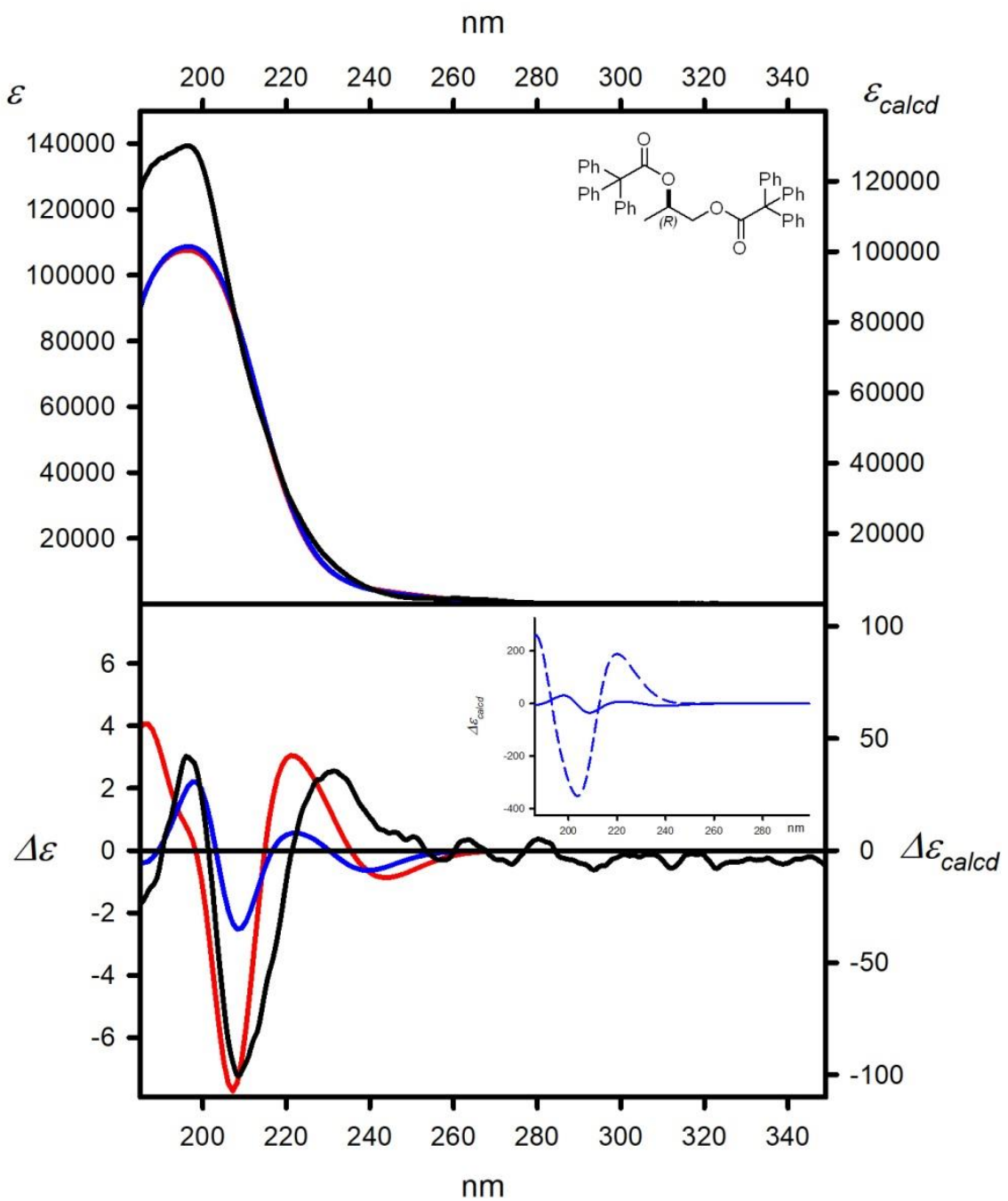

Experimental (cyclohexane, black lines)

\section{Calculated at the}

TD-M06-2X/6-311++G(d,p) level and:

$\triangle E$-based Boltzmann averaged (red lines)

$\triangle \triangle G$-based Boltzmann averaged (blue lines)

Figure SI_110. UV (upper panel) and ECD (lower panel) spectra of 18, experimental, measured in cyclohexane (solid black lines) and calculated at the TD-M06-2X/6-311++G(d,p) level for structures optimized at the B3LYP-GD3BJ/6-311G(d,p) level, $\Delta E$-based Boltzmann (red lines) and $\Delta \Delta G$-based Boltzmann averaged (blue solid lines). Insert shows the comparison between Boltzmann averaged $E C D$ spectrum and that calculated for $\Delta \Delta G$-based on the lowest energy conformer of a given compound (dashed blue line). All calculated spectra were wavelength corrected to match experimental UV maxima. 


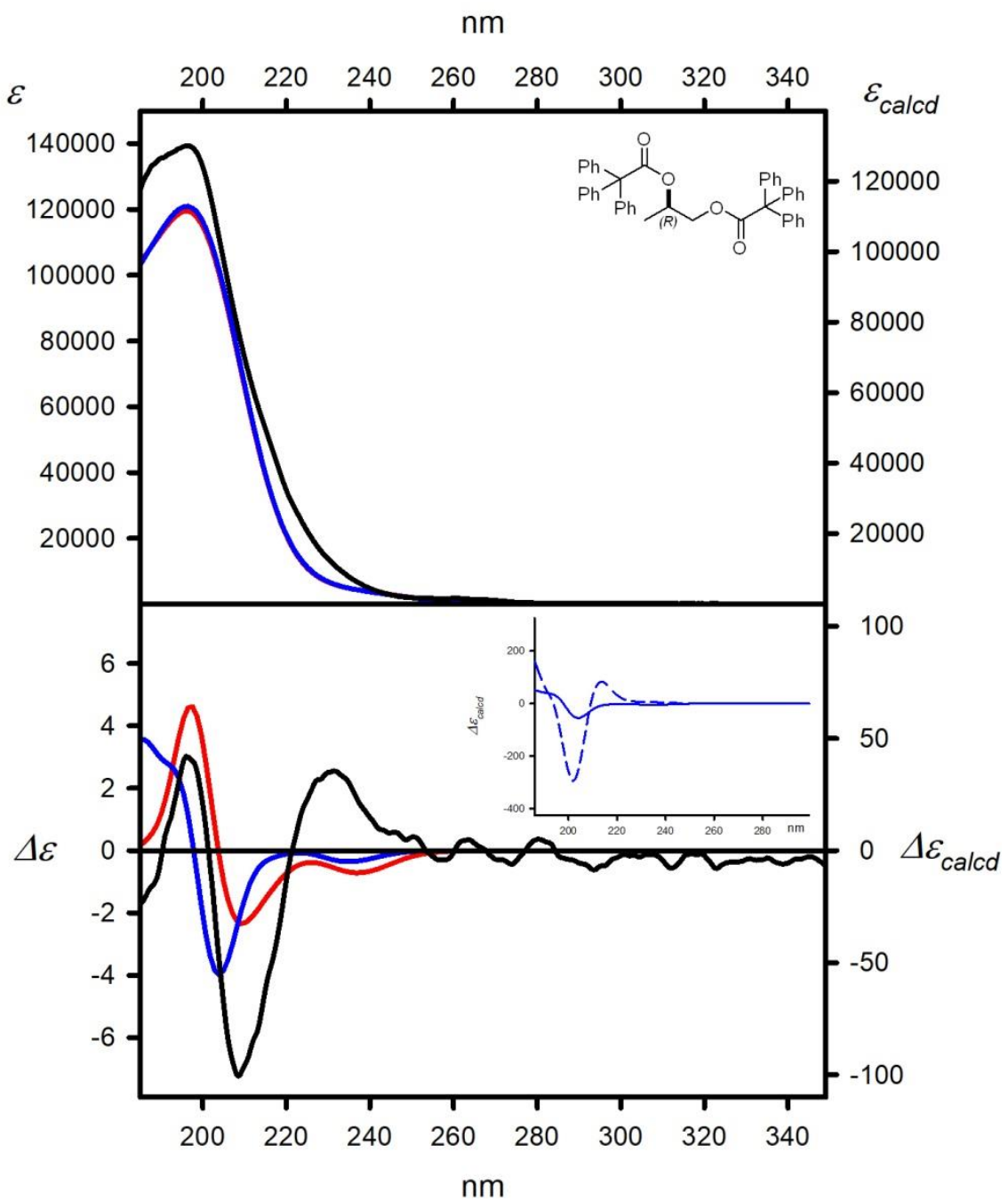

Experimental (cyclohexane, black lines)

\section{Calculated at the}

TD-CAM-B3LYP/6-311++G(d,p) level and:

$\triangle E$-based Boltzmann averaged (red lines)

$\triangle \triangle G$-based Boltzmann averaged (blue lines)

Figure SI_111. UV (upper panel) and ECD (lower panel) spectra of 18, experimental, measured in cyclohexane (solid black lines) and calculated at the TD-CAM-B3LYP/6-311++G(d,p) level for structures optimized at the M06-2X/6-311G(d,p) level, $\Delta \mathrm{E}$-based Boltzmann (red lines) and $\Delta \Delta \mathrm{G}$ based Boltzmann averaged (blue solid lines). Insert shows the comparison between Boltzmann averaged ECD spectrum and that calculated for $\Delta \Delta G$-based on the lowest energy conformer of a given compound (dashed blue line). All calculated spectra were wavelength corrected to match experimental UV maxima. 


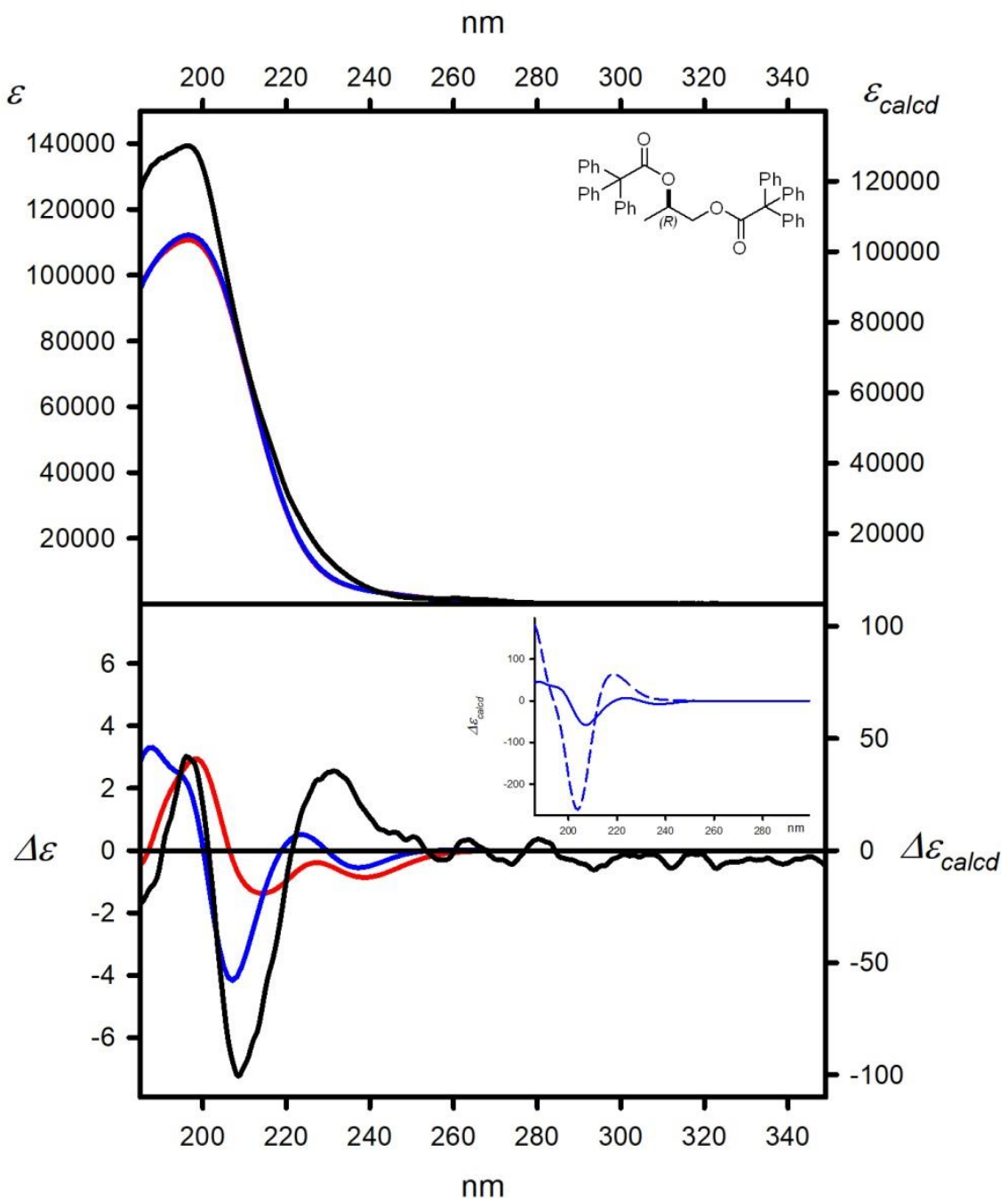

Experimental (cyclohexane, black lines)

\section{Calculated at the}

TD-M06-2X/6-311++G(d,p) level and:

$\triangle E$-based Boltzmann averaged (red lines)

$\triangle \triangle G$-based Boltzmann averaged (blue lines)

Figure SI_112. UV (upper panel) and ECD (lower panel) spectra of 18, experimental, measured in cyclohexane (solid black lines) and calculated at the TD-M06-2X/6-311++G(d,p) level for structures optimized at the M06-2X/6-311G(d,p) level, $\Delta E$-based Boltzmann (red lines) and $\Delta \Delta G$-based Boltzmann averaged (blue solid lines). Insert shows the comparison between Boltzmann averaged ECD spectrum and that calculated for $\Delta \Delta G$-based on the lowest energy conformer of a given compound (dashed blue line). All calculated spectra were wavelength corrected to match experimental UV maxima. 


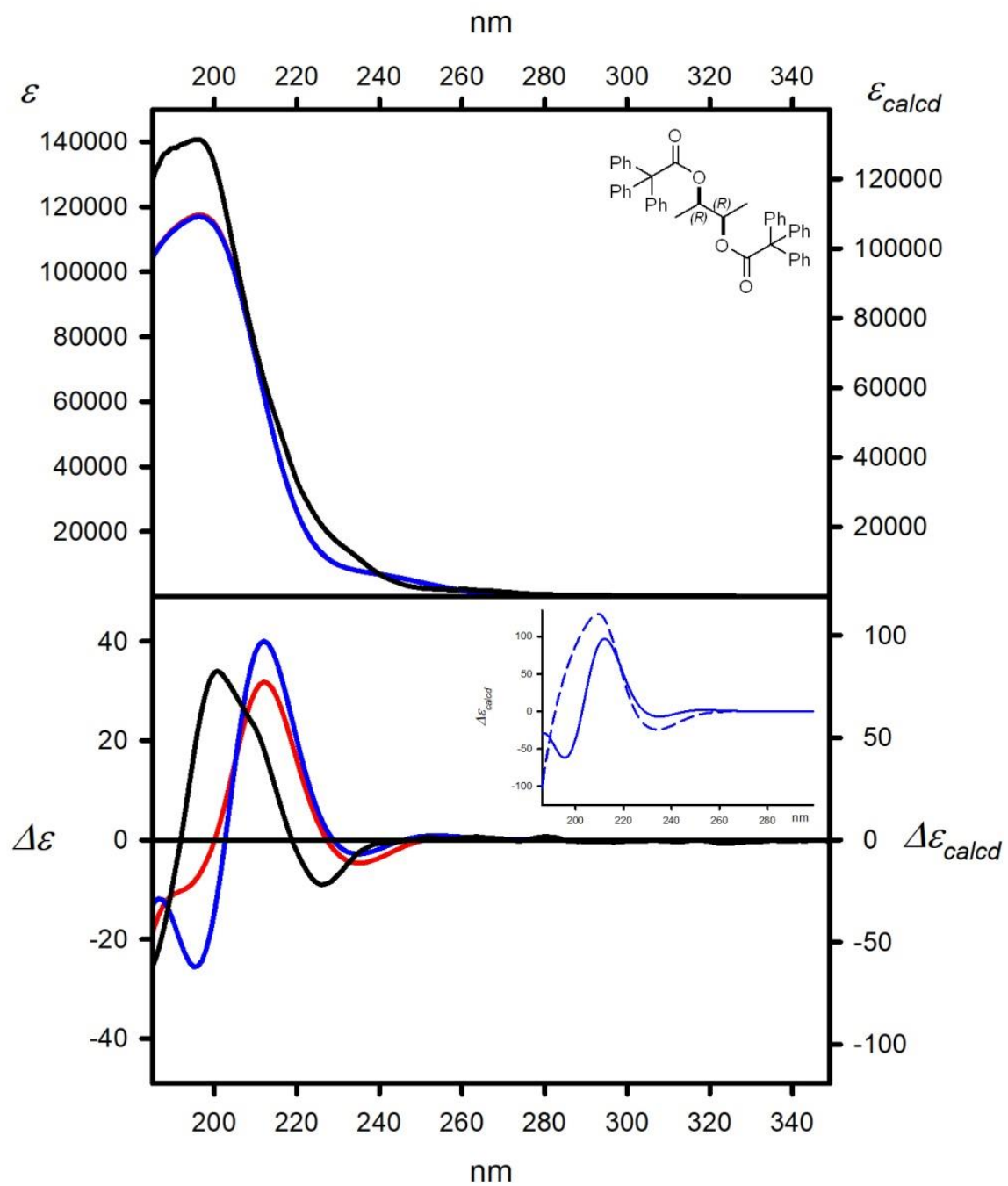

Experimental (cyclohexane, black lines)

\section{Calculated at the}

TD-CAM-B3LYP/6-311++G(d,p) level and:

$\triangle E$-based Boltzmann averaged (red lines)

$\triangle \triangle G$-based Boltzmann averaged (blue lines)

Figure SI_113. UV (upper panel) and ECD (lower panel) spectra of 20, experimental, measured in cyclohexane (solid black lines) and calculated at the TD-CAM-B3LYP/6-311++G(d,p) level for structures optimized at the B3LYP/6-311G(d,p) level, $\Delta \mathrm{E}$-based Boltzmann (red lines) and $\Delta \Delta \mathrm{G}$-based Boltzmann averaged (blue solid lines). Insert shows the comparison between Boltzmann averaged ECD spectrum and that calculated for $\Delta \Delta G$-based on the lowest energy conformer of a given compound (dashed blue line). All calculated spectra were wavelength corrected to match experimental UV maxima. 


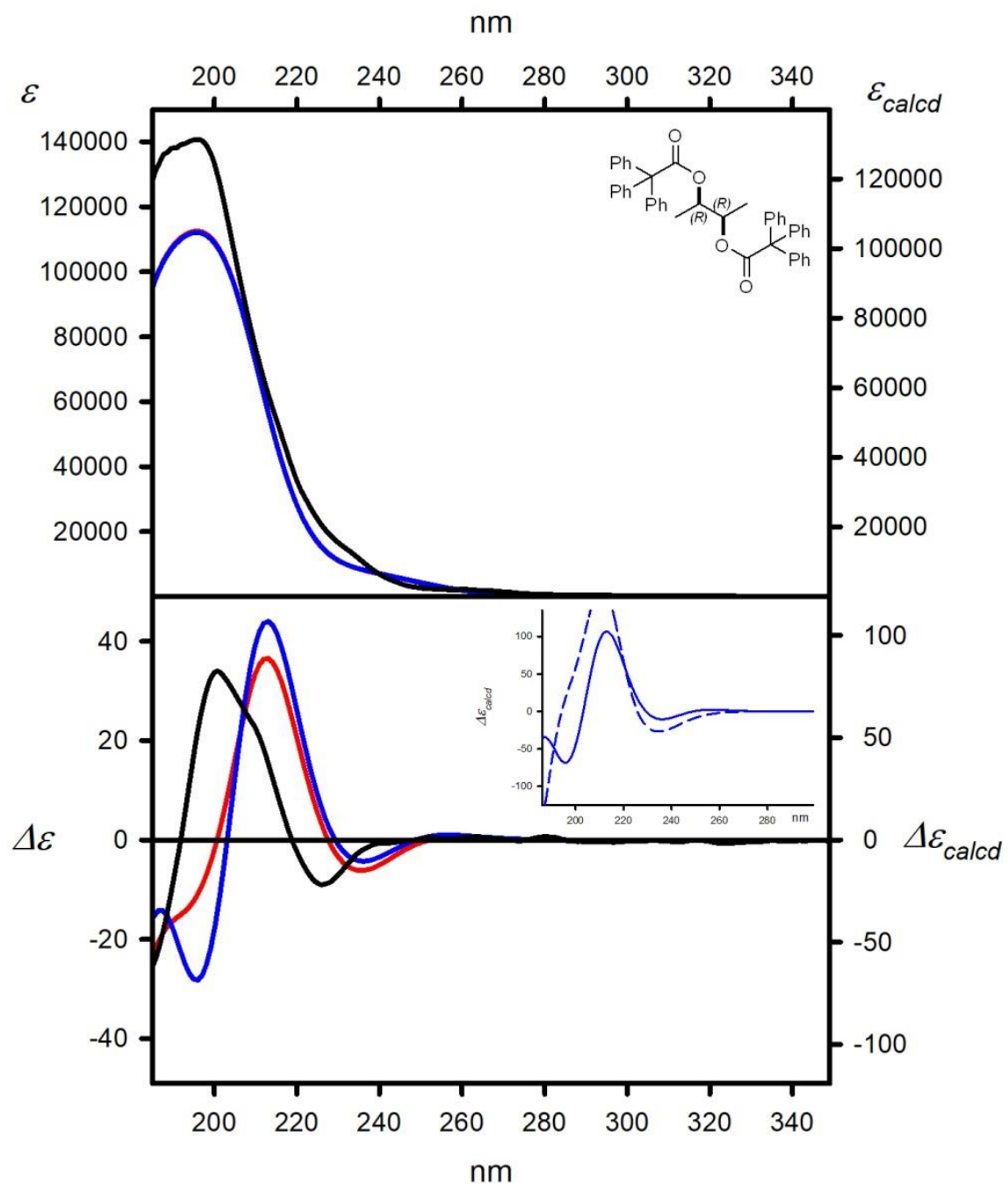

Experimental (cyclohexane, black lines)

\section{Calculated at the}

TD-M06-2X/6-311++G(d,p) level and:

$\triangle E$-based Boltzmann averaged (red lines)

$\triangle \triangle G$-based Boltzmann averaged (blue lines)

Figure SI_114. UV (upper panel) and ECD (lower panel) spectra of 20, experimental, measured in cyclohexane (solid black lines) and calculated at the TD-M06-2X/6-311++G(d,p) level for structures optimized at the B3LYP/6-311G(d,p) level, $\Delta \mathrm{E}$-based Boltzmann (red lines) and $\Delta \Delta \mathrm{G}$-based Boltzmann averaged (blue solid lines). Insert shows the comparison between Boltzmann averaged ECD spectrum and that calculated for $\Delta \Delta G$-based on the lowest energy conformer of a given compound (dashed blue line). All calculated spectra were wavelength corrected to match experimental UV maxima. 


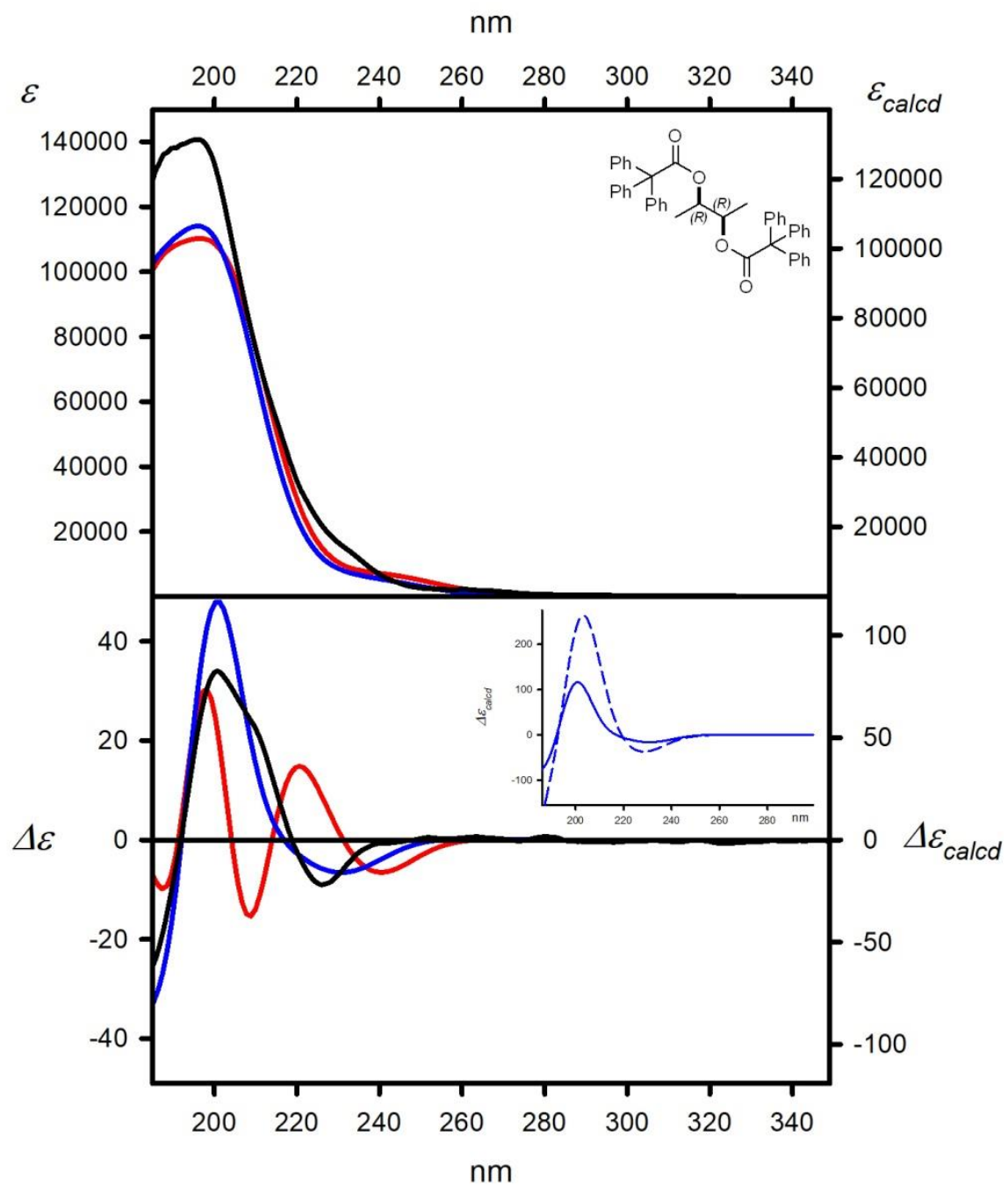

Experimental (cyclohexane, black lines)

\section{Calculated at the}

TD-CAM-B3LYP/6-311++G(d,p) level and:

$\triangle E$-based Boltzmann averaged (red lines)

$\triangle \triangle G$-based Boltzmann averaged (blue lines)

Figure SI_115. UV (upper panel) and ECD (lower panel) spectra of 20, experimental, measured in cyclohexane (solid black lines) and calculated at the TD-CAM-B3LYP/6-311++G(d,p) level for structures optimized at the B3LYP-GD3BJ/6-311G(d,p) level, $\Delta \mathrm{E}$-based Boltzmann (red lines) and $\Delta \Delta G$-based Boltzmann averaged (blue solid lines). Insert shows the comparison between Boltzmann averaged ECD spectrum and that calculated for $\Delta \Delta G$-based on the lowest energy conformer of a given compound (dashed blue line). All calculated spectra were wavelength corrected to match experimental UV maxima. 


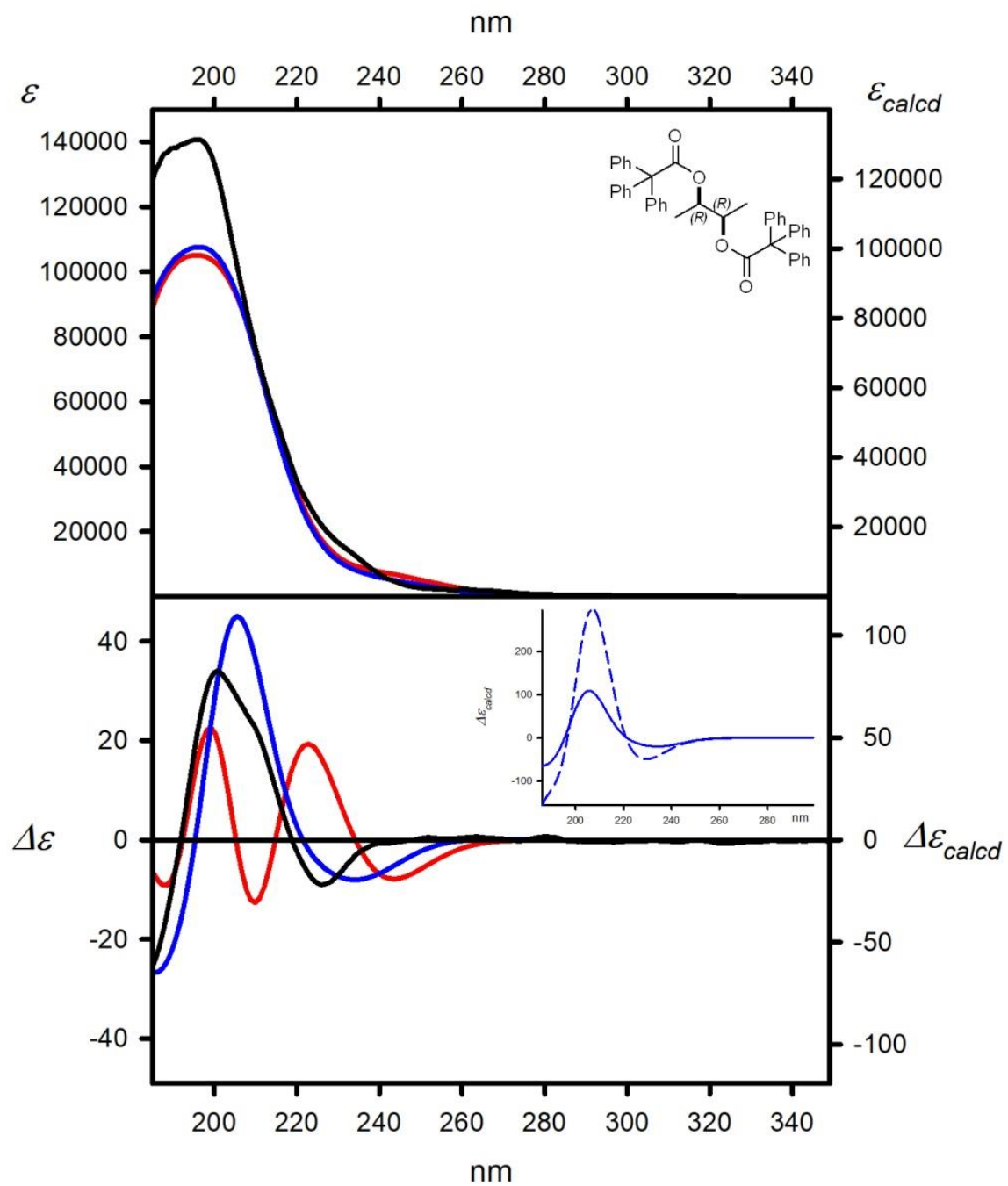

Experimental (cyclohexane, black lines)

\section{Calculated at the}

TD-M06-2X/6-311++G(d,p) level and:

$\triangle E$-based Boltzmann averaged (red lines)

$\triangle \triangle G$-based Boltzmann averaged (blue lines)

Figure SI_116. UV (upper panel) and ECD (lower panel) spectra of 20, experimental, measured in cyclohexane (solid black lines) and calculated at the TD-M06-2X/6-311++G(d,p) level for structures optimized at the B3LYP-GD3BJ/6-311G(d,p) level, $\Delta \mathrm{E}$-based Boltzmann (red lines) and $\Delta \Delta \mathrm{G}$-based Boltzmann averaged (blue solid lines). Insert shows the comparison between Boltzmann averaged ECD spectrum and that calculated for $\Delta \Delta G$-based on the lowest energy conformer of a given compound (dashed blue line). All calculated spectra were wavelength corrected to match experimental UV maxima. 


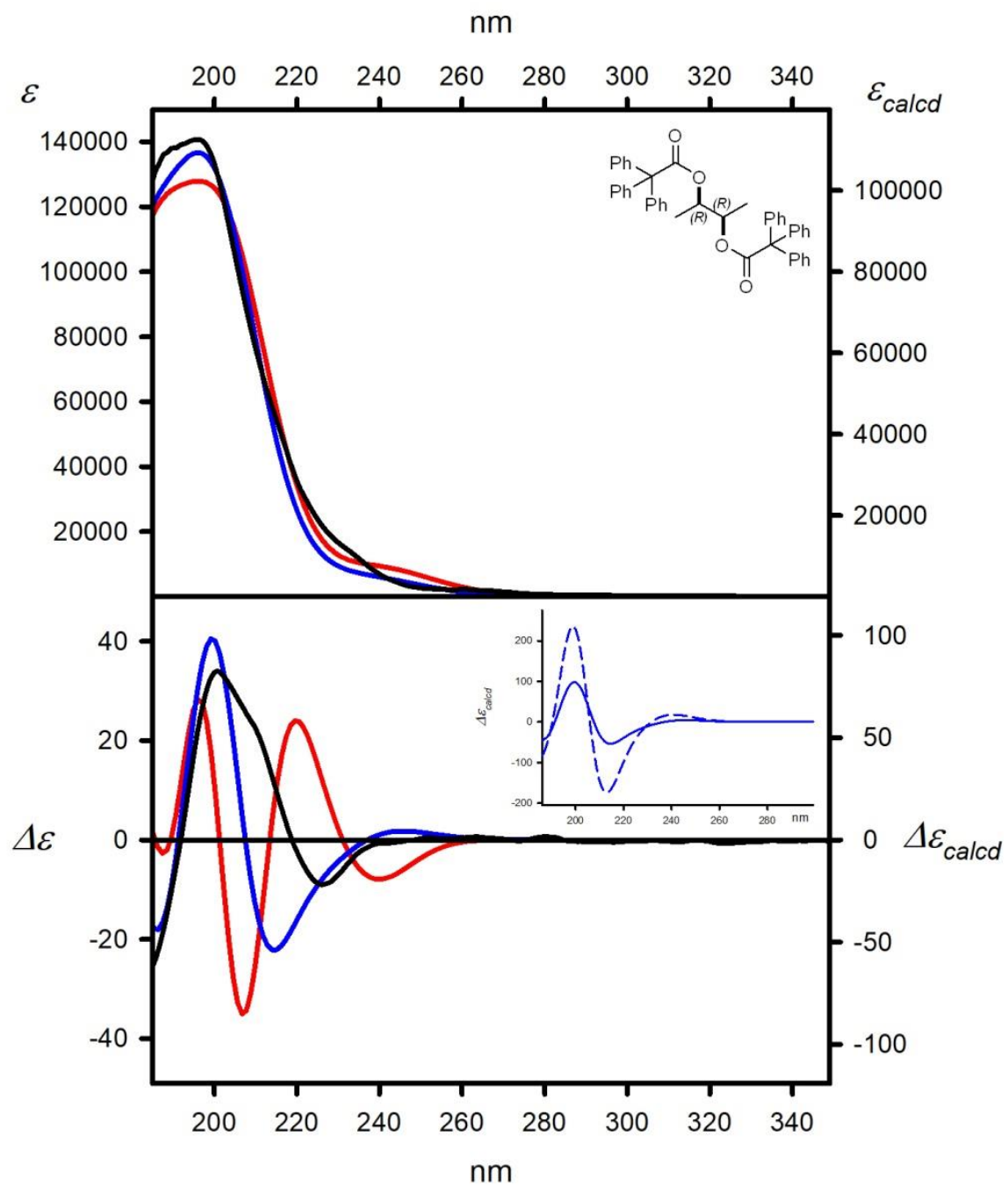

Experimental (cyclohexane, black lines)

\section{Calculated at the}

TD-CAM-B3LYP/6-311++G(d,p) level and:

$\triangle E$-based Boltzmann averaged (red lines)

$\triangle \triangle G$-based Boltzmann averaged (blue lines)

Figure SI_117. UV (upper panel) and ECD (lower panel) spectra of 20, experimental, measured in cyclohexane (solid black lines) and calculated at the TD-CAM-B3LYP/6-311++G(d,p) level for structures optimized at the M06-2X/6-311G(d,p) level, $\Delta \mathrm{E}$-based Boltzmann (red lines) and $\Delta \Delta \mathrm{G}$ based Boltzmann averaged (blue solid lines). Insert shows the comparison between Boltzmann averaged ECD spectrum and that calculated for $\Delta \Delta G$-based on the lowest energy conformer of a given compound (dashed blue line). All calculated spectra were wavelength corrected to match experimental UV maxima. 


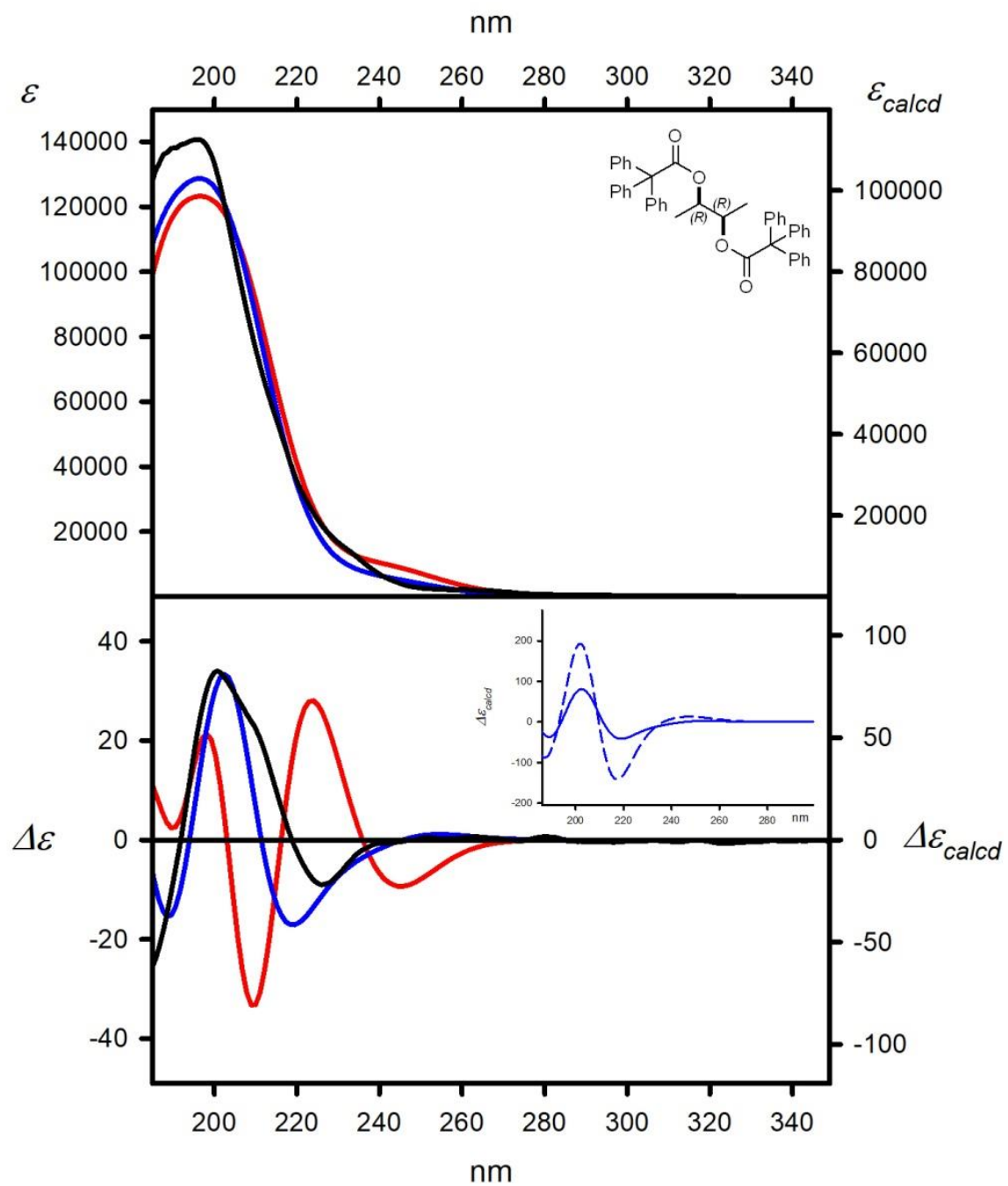

Experimental (cyclohexane, black lines)

\section{Calculated at the}

TD-M06-2X/6-311++G(d,p) level and:

$\triangle E$-based Boltzmann averaged (red lines)

$\triangle \triangle G$-based Boltzmann averaged (blue lines)

Figure SI_118. UV (upper panel) and ECD (lower panel) spectra of 20, experimental, measured in cyclohexane (solid black lines) and calculated at the TD-M06-2X/6-311++G(d,p) level for structures optimized at the M06-2X/6-311G(d,p) level, $\Delta E$-based Boltzmann (red lines) and $\Delta \Delta G$-based Boltzmann averaged (blue solid lines). Insert shows the comparison between Boltzmann averaged ECD spectrum and that calculated for $\Delta \Delta G$-based on the lowest energy conformer of a given compound (dashed blue line). All calculated spectra were wavelength corrected to match experimental UV maxima. 


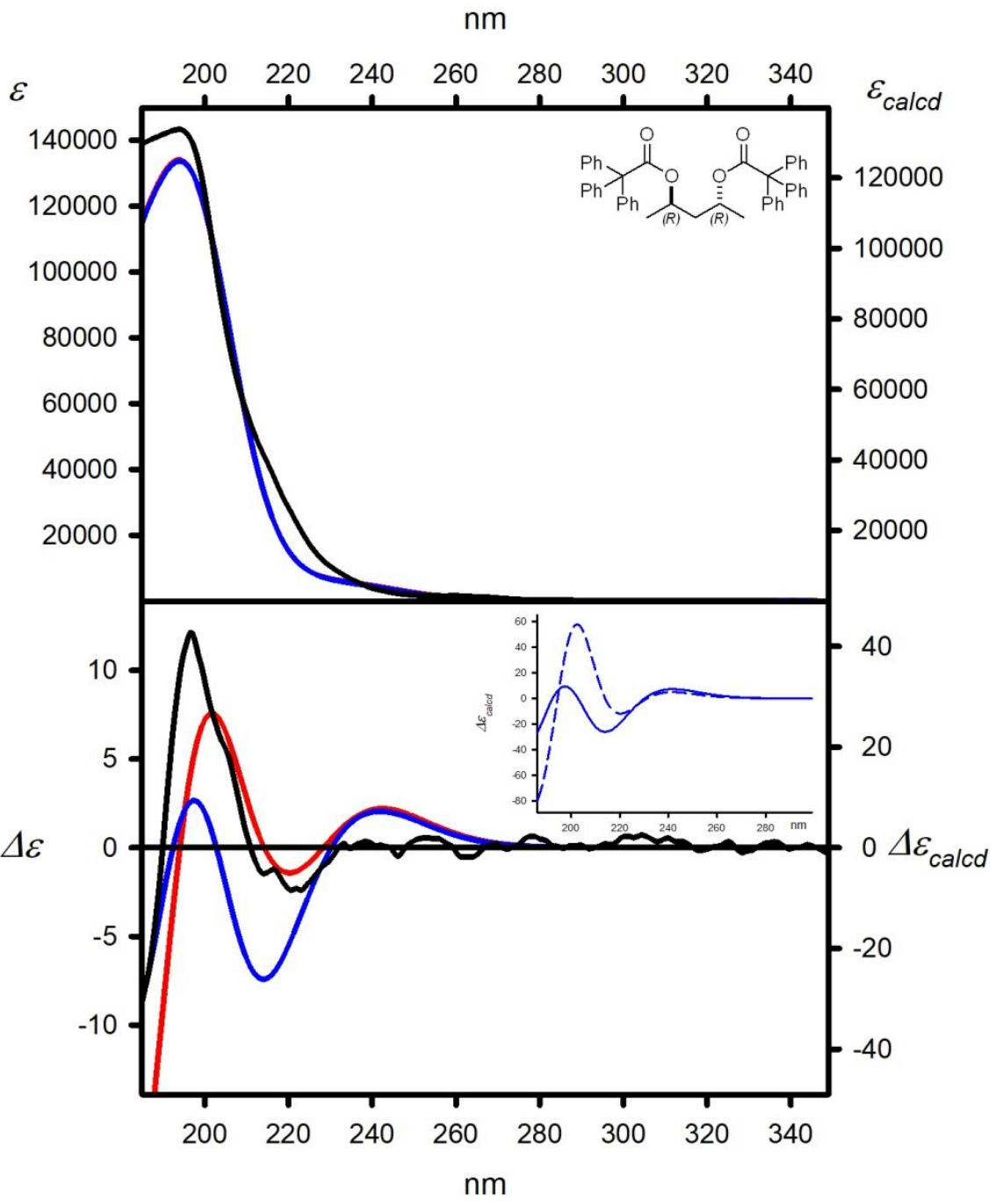

Experimental (cyclohexane, black lines)

Calculated at the

TD-CAM-B3LYP/6-311++G(d,p) level and:

$\triangle E$-based Boltzmann averaged (red lines)

$\triangle \triangle G$-based Boltzmann averaged (blue lines)

Figure SI_119. UV (upper panel) and ECD (lower panel) spectra of 21, experimental, measured in cyclohexane (solid black lines) and calculated at the TD-CAM-B3LYP/6-311++G(d,p) level for structures optimized at the B3LYP/6-311G(d,p) level, $\Delta \mathrm{E}$-based Boltzmann (red lines) and $\Delta \Delta \mathrm{G}$-based Boltzmann averaged (blue solid lines). Insert shows the comparison between Boltzmann averaged ECD spectrum and that calculated for $\Delta \Delta G$-based on the lowest energy conformer of a given compound (dashed blue line). All calculated spectra were wavelength corrected to match experimental UV maxima. 


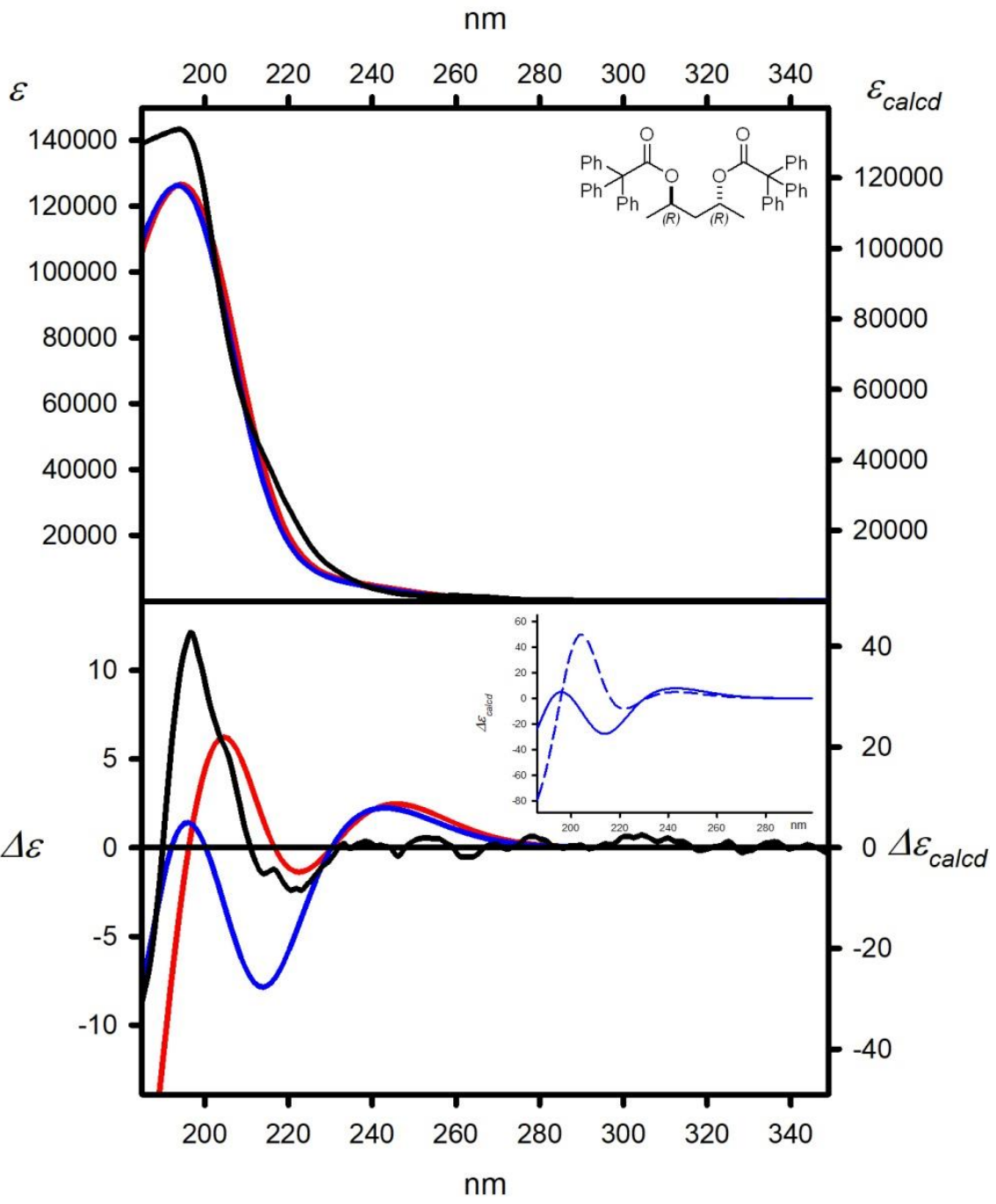

Experimental (cyclohexane, black lines)

Calculated at the

TD-M06-2X/6-311++G(d,p) level and:

$\triangle E$-based Boltzmann averaged (red lines)

$\triangle \Lambda$ G-based Boltzmann averaged (blue lines)

Figure SI_120. UV (upper panel) and ECD (lower panel) spectra of 21, experimental, measured in cyclohexane (solid black lines) and calculated at the TD-M06-2X/6-311++G(d,p) level for structures optimized at the B3LYP/6-311G(d,p) level, $\Delta \mathrm{E}$-based Boltzmann (red lines) and $\Delta \Delta \mathrm{G}$-based Boltzmann averaged (blue solid lines). Insert shows the comparison between Boltzmann averaged ECD spectrum and that calculated for $\Delta \Delta G$-based on the lowest energy conformer of a given compound (dashed blue line). All calculated spectra were wavelength corrected to match experimental UV maxima. 


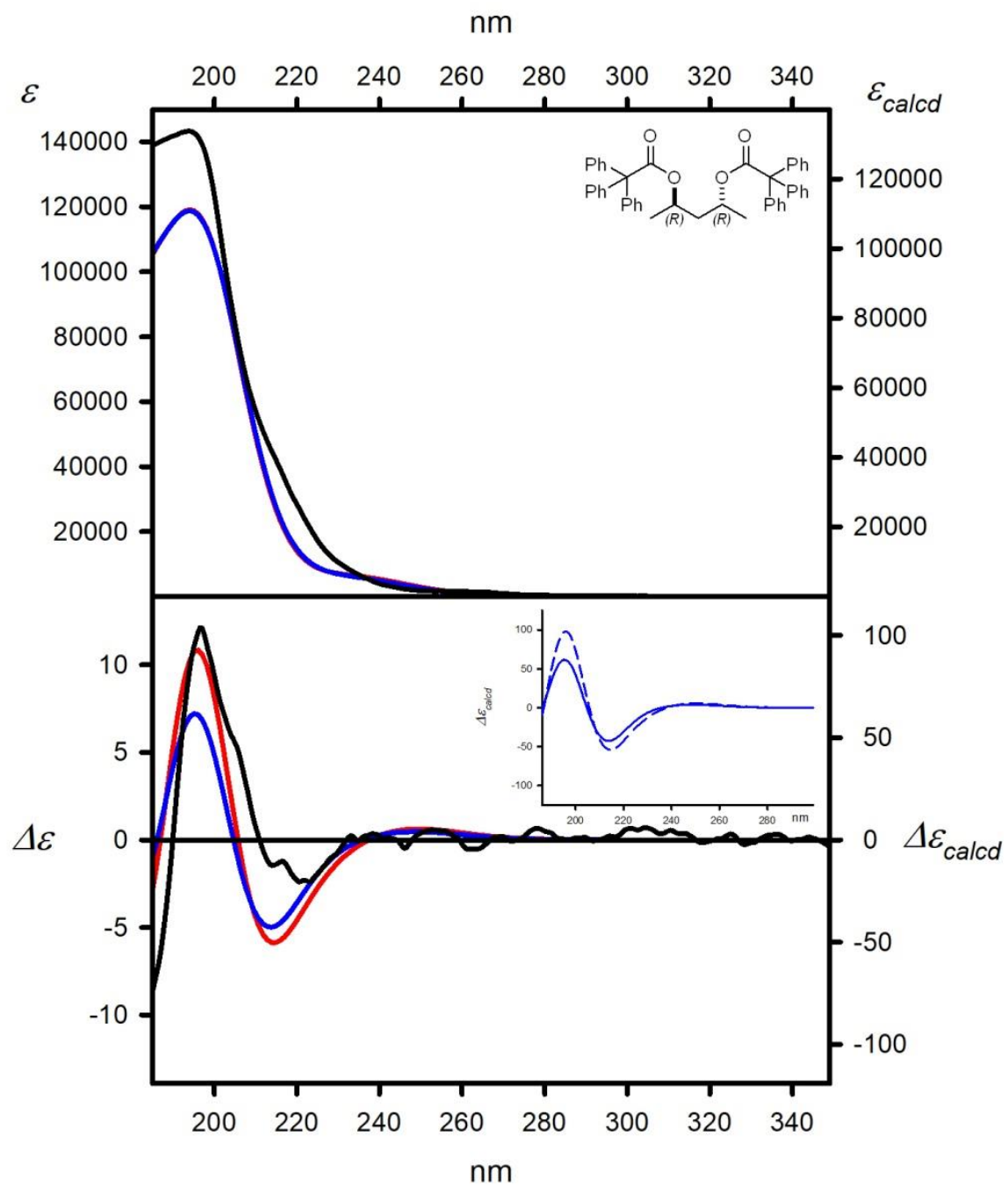

Experimental (cyclohexane, black lines)

\section{Calculated at the}

TD-CAM-B3LYP/6-311++G(d,p) level and:

$\triangle E$-based Boltzmann averaged (red lines)

$\triangle \triangle G$-based Boltzmann averaged (blue lines)

Figure SI_121. UV (upper panel) and ECD (lower panel) spectra of 21, experimental, measured in cyclohexane (solid black lines) and calculated at the TD-CAM-B3LYP/6-311++G(d,p) level for structures optimized at the B3LYP-GD3BJ/6-311G(d,p) level, $\Delta \mathrm{E}$-based Boltzmann (red lines) and $\Delta \Delta G$-based Boltzmann averaged (blue solid lines). Insert shows the comparison between Boltzmann averaged ECD spectrum and that calculated for $\Delta \Delta G$-based on the lowest energy conformer of a given compound (dashed blue line). All calculated spectra were wavelength corrected to match experimental UV maxima. 


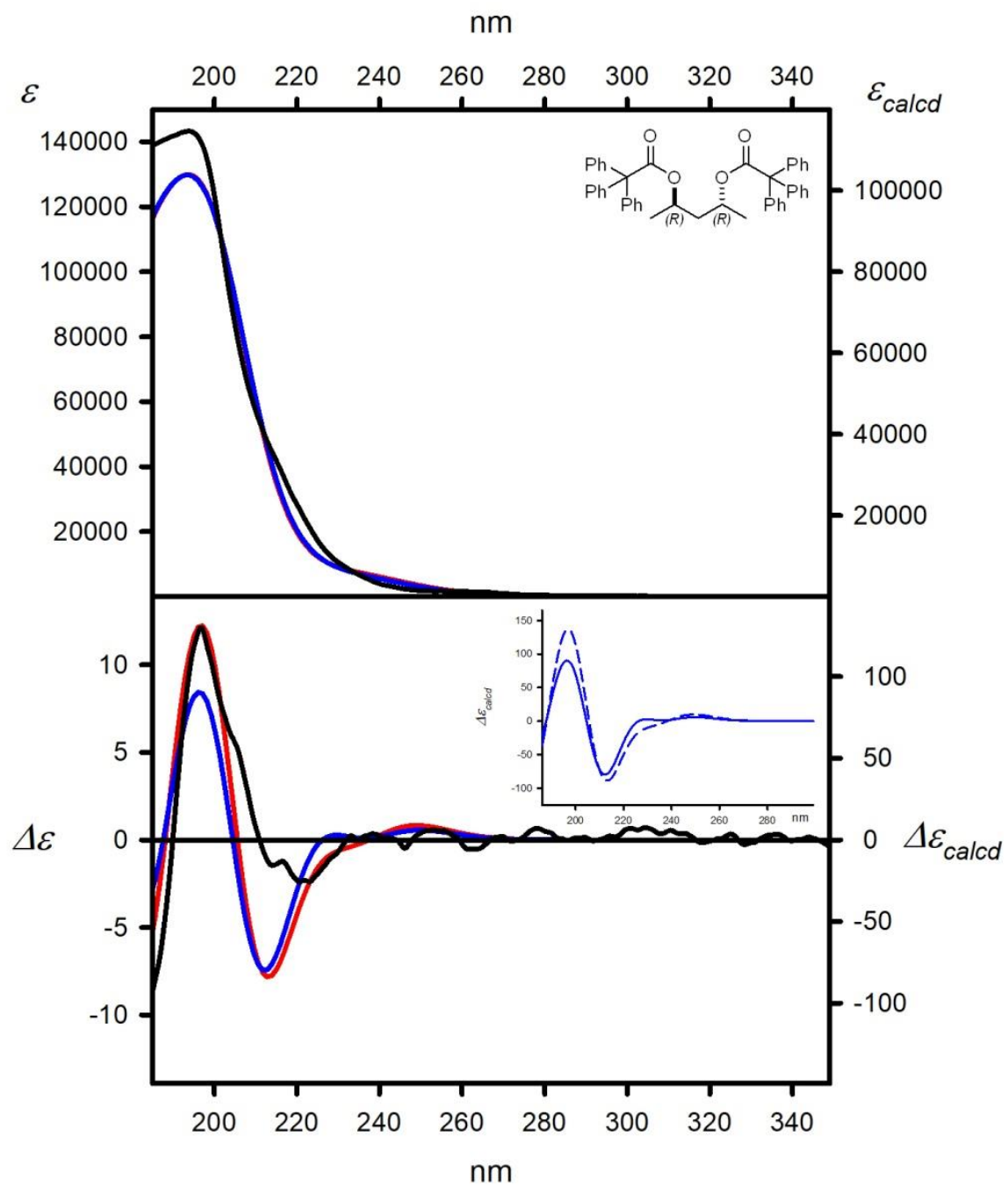

Experimental (cyclohexane, black lines)

\section{Calculated at the}

TD-M06-2X/6-311++G(d,p) level and:

$\triangle E$-based Boltzmann averaged (red lines)

$\triangle \triangle G$-based Boltzmann averaged (blue lines)

Figure SI_122. UV (upper panel) and ECD (lower panel) spectra of 21, experimental, measured in cyclohexane (solid black lines) and calculated at the TD-M06-2X/6-311++G(d,p) level for structures optimized at the B3LYP-GD3BJ/6-311G(d,p) level, $\Delta \mathrm{E}$-based Boltzmann (red lines) and $\Delta \Delta \mathrm{G}$-based Boltzmann averaged (blue solid lines). Insert shows the comparison between Boltzmann averaged ECD spectrum and that calculated for $\Delta \Delta G$-based on the lowest energy conformer of a given compound (dashed blue line). All calculated spectra were wavelength corrected to match experimental UV maxima. 


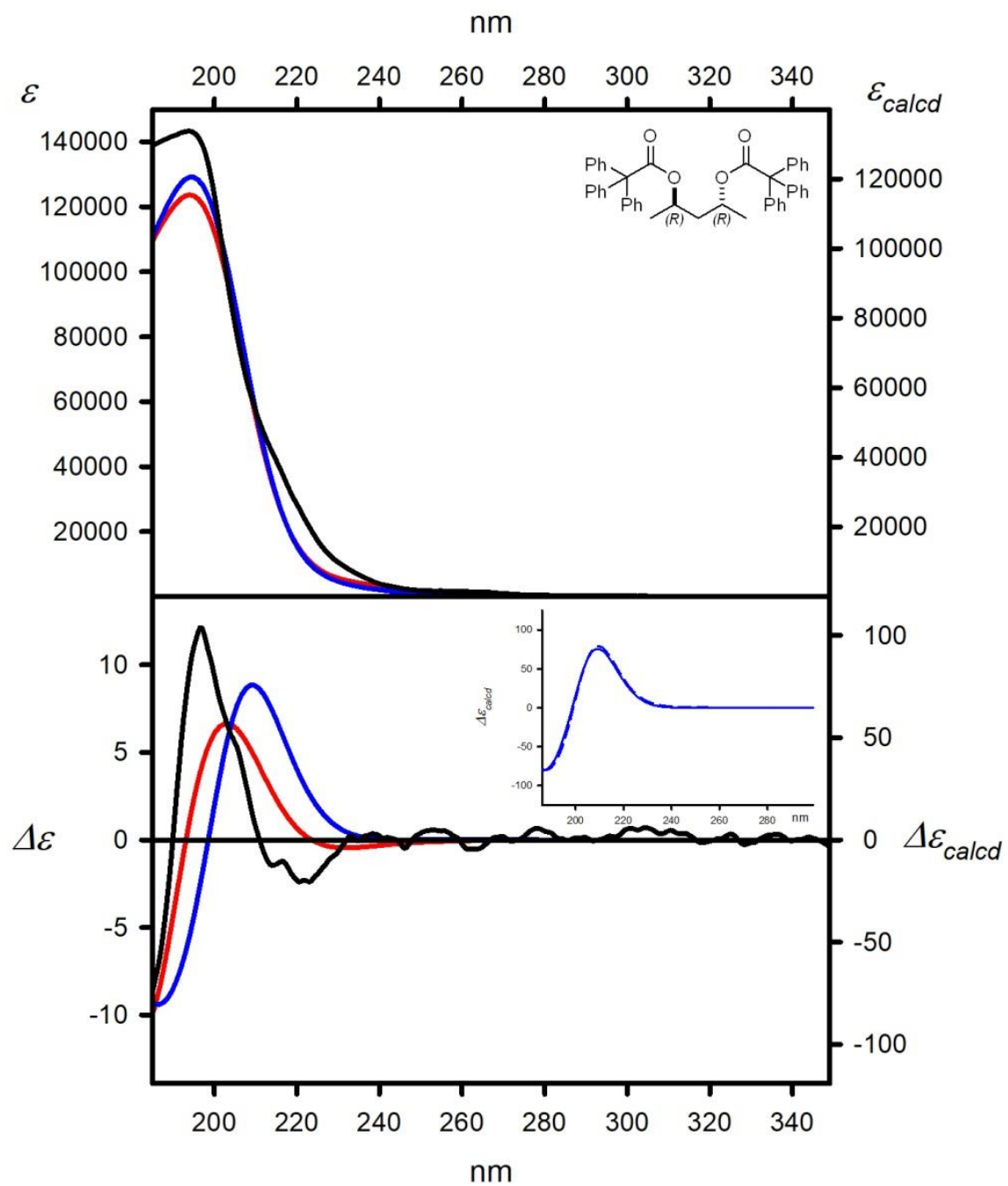

Experimental (cyclohexane, black lines)

\section{Calculated at the}

TD-CAM-B3LYP/6-311++G(d,p) level and:

$\triangle E$-based Boltzmann averaged (red lines)

$\triangle \triangle G$-based Boltzmann averaged (blue lines)

Figure SI_123. UV (upper panel) and ECD (lower panel) spectra of 21, experimental, measured in cyclohexane (solid black lines) and calculated at the TD-CAM-B3LYP/6-311++G(d,p) level for structures optimized at the M06-2X/6-311G(d,p) level, $\Delta \mathrm{E}$-based Boltzmann (red lines) and $\Delta \Delta \mathrm{G}$ based Boltzmann averaged (blue solid lines). Insert shows the comparison between Boltzmann averaged ECD spectrum and that calculated for $\Delta \Delta G$-based on the lowest energy conformer of a given compound (dashed blue line). All calculated spectra were wavelength corrected to match experimental UV maxima. 


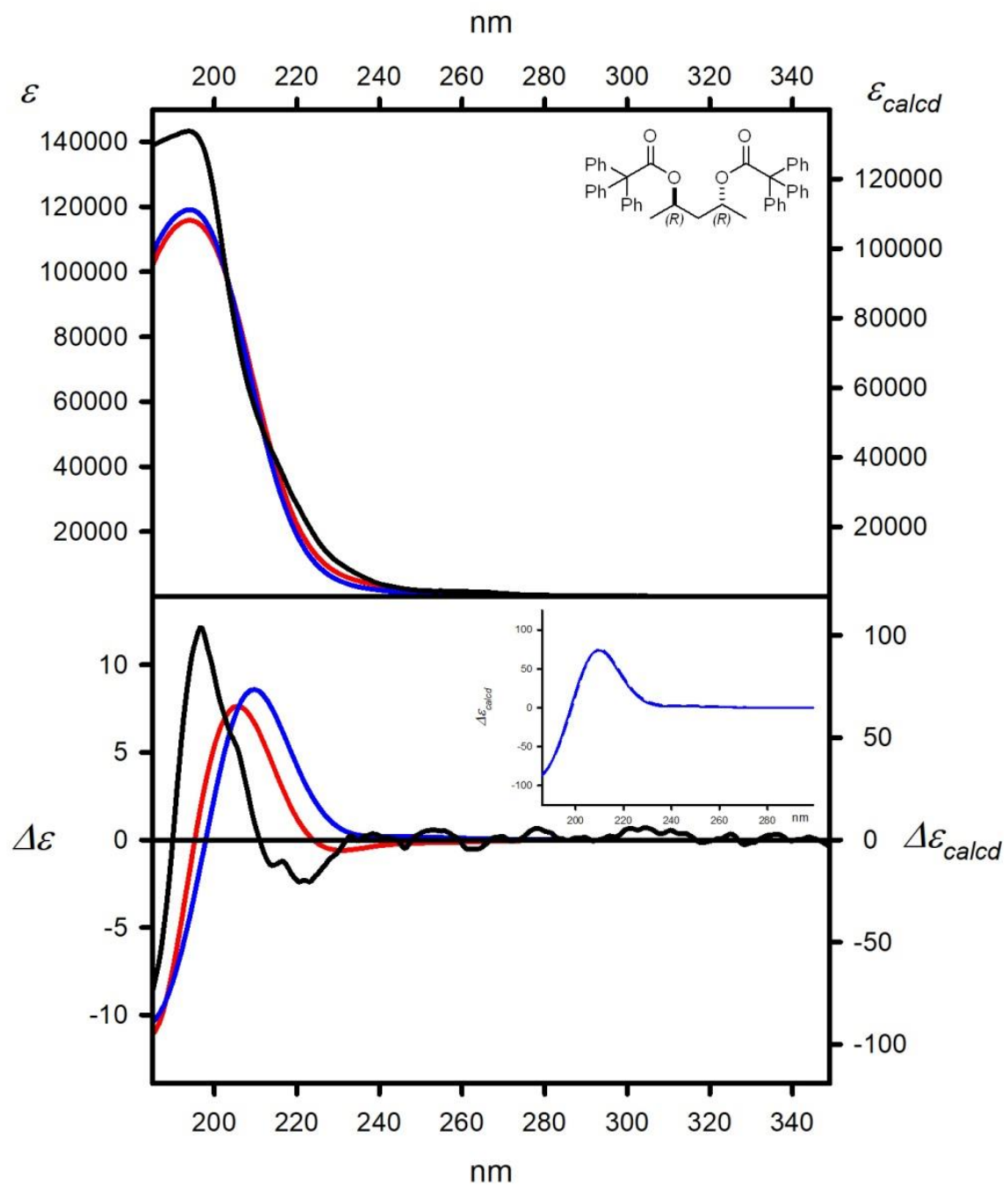

Experimental (cyclohexane, black lines)

\section{Calculated at the}

TD-M06-2X/6-311++G(d,p) level and:

$\triangle E$-based Boltzmann averaged (red lines)

$\triangle \triangle G$-based Boltzmann averaged (blue lines)

Figure SI_124. UV (upper panel) and ECD (lower panel) spectra of 21, experimental, measured in cyclohexane (solid black lines) and calculated at the TD-M06-2X/6-311++G(d,p) level for structures optimized at the M06-2X/6-311G(d,p) level, $\Delta E$-based Boltzmann (red lines) and $\Delta \Delta G$-based Boltzmann averaged (blue solid lines). Insert shows the comparison between Boltzmann averaged ECD spectrum and that calculated for $\Delta \Delta G$-based on the lowest energy conformer of a given compound (dashed blue line). All calculated spectra were wavelength corrected to match experimental UV maxima. 


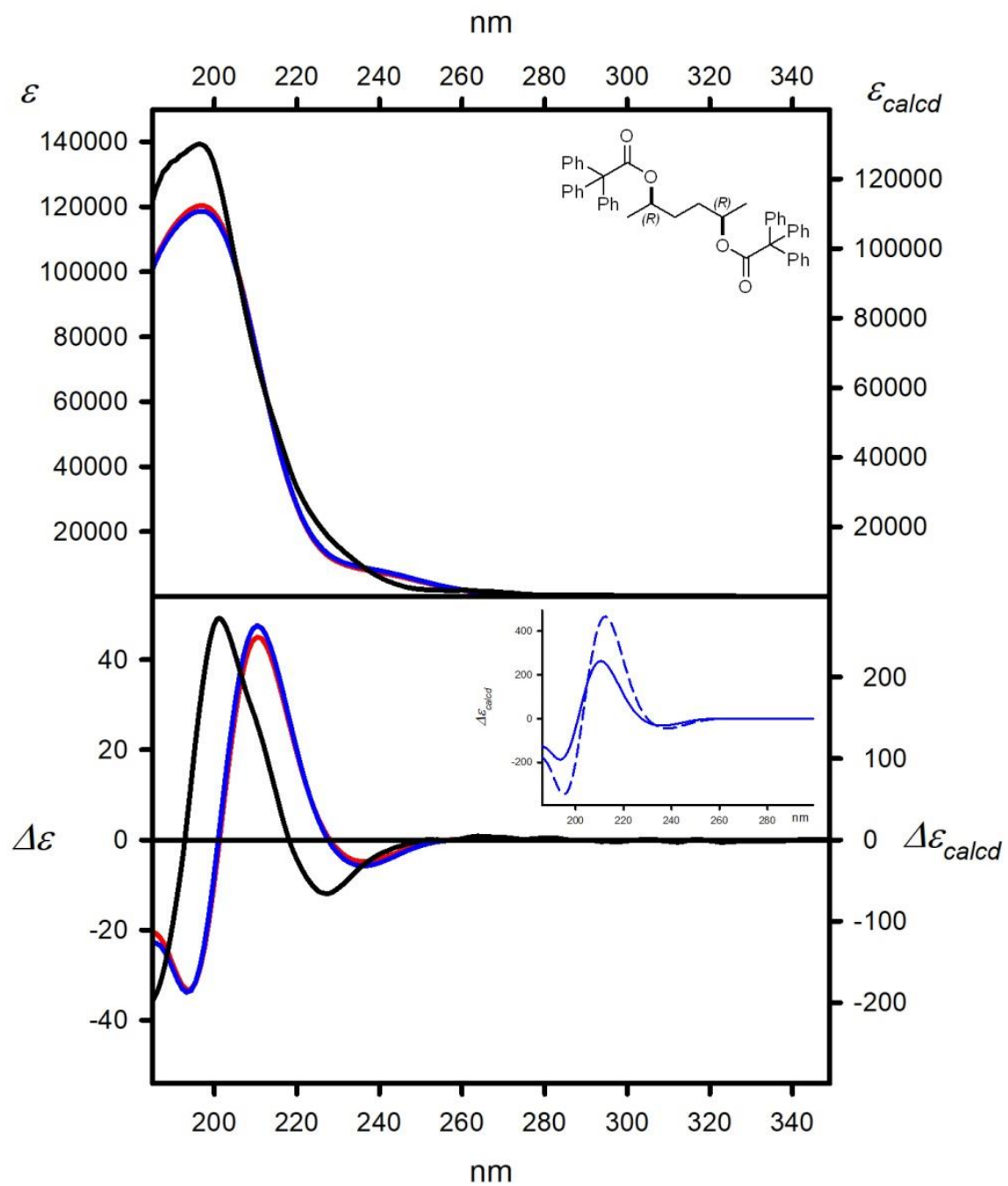

Experimental (cyclohexane, black lines)

\section{Calculated at the}

TD-CAM-B3LYP/6-311++G(d,p) level and:

$\triangle E$-based Boltzmann averaged (red lines)

$\triangle \triangle G$-based Boltzmann averaged (blue lines)

Figure SI_125. UV (upper panel) and ECD (lower panel) spectra of 22, experimental, measured in cyclohexane (solid black lines) and calculated at the TD-CAM-B3LYP/6-311++G(d,p) level for structures optimized at the B3LYP/6-311G(d,p) level, $\Delta \mathrm{E}$-based Boltzmann (red lines) and $\Delta \Delta \mathrm{G}$-based Boltzmann averaged (blue solid lines). Insert shows the comparison between Boltzmann averaged ECD spectrum and that calculated for $\Delta \Delta G$-based on the lowest energy conformer of a given compound (dashed blue line). All calculated spectra were wavelength corrected to match experimental UV maxima. 


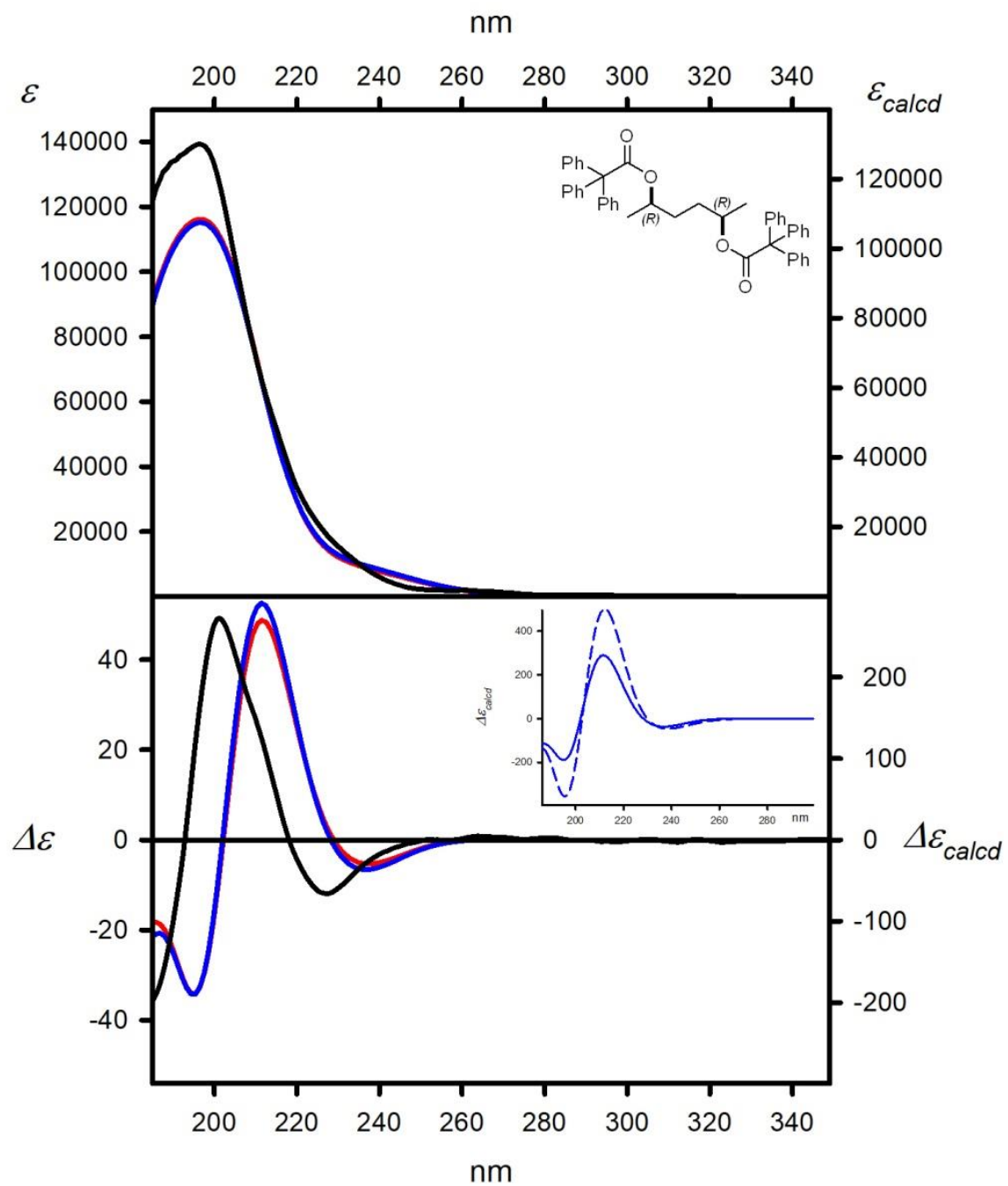

Experimental (cyclohexane, black lines)

\section{Calculated at the}

TD-M06-2X/6-311++G(d,p) level and:

$\triangle E$-based Boltzmann averaged (red lines)

$\triangle \triangle G$-based Boltzmann averaged (blue lines)

Figure SI_126. UV (upper panel) and ECD (lower panel) spectra of 22, experimental, measured in cyclohexane (solid black lines) and calculated at the TD-M06-2X/6-311++G(d,p) level for structures optimized at the B3LYP/6-311G(d,p) level, $\Delta \mathrm{E}$-based Boltzmann (red lines) and $\Delta \Delta \mathrm{G}$-based Boltzmann averaged (blue solid lines). Insert shows the comparison between Boltzmann averaged ECD spectrum and that calculated for $\Delta \Delta G$-based on the lowest energy conformer of a given compound (dashed blue line). All calculated spectra were wavelength corrected to match experimental UV maxima. 


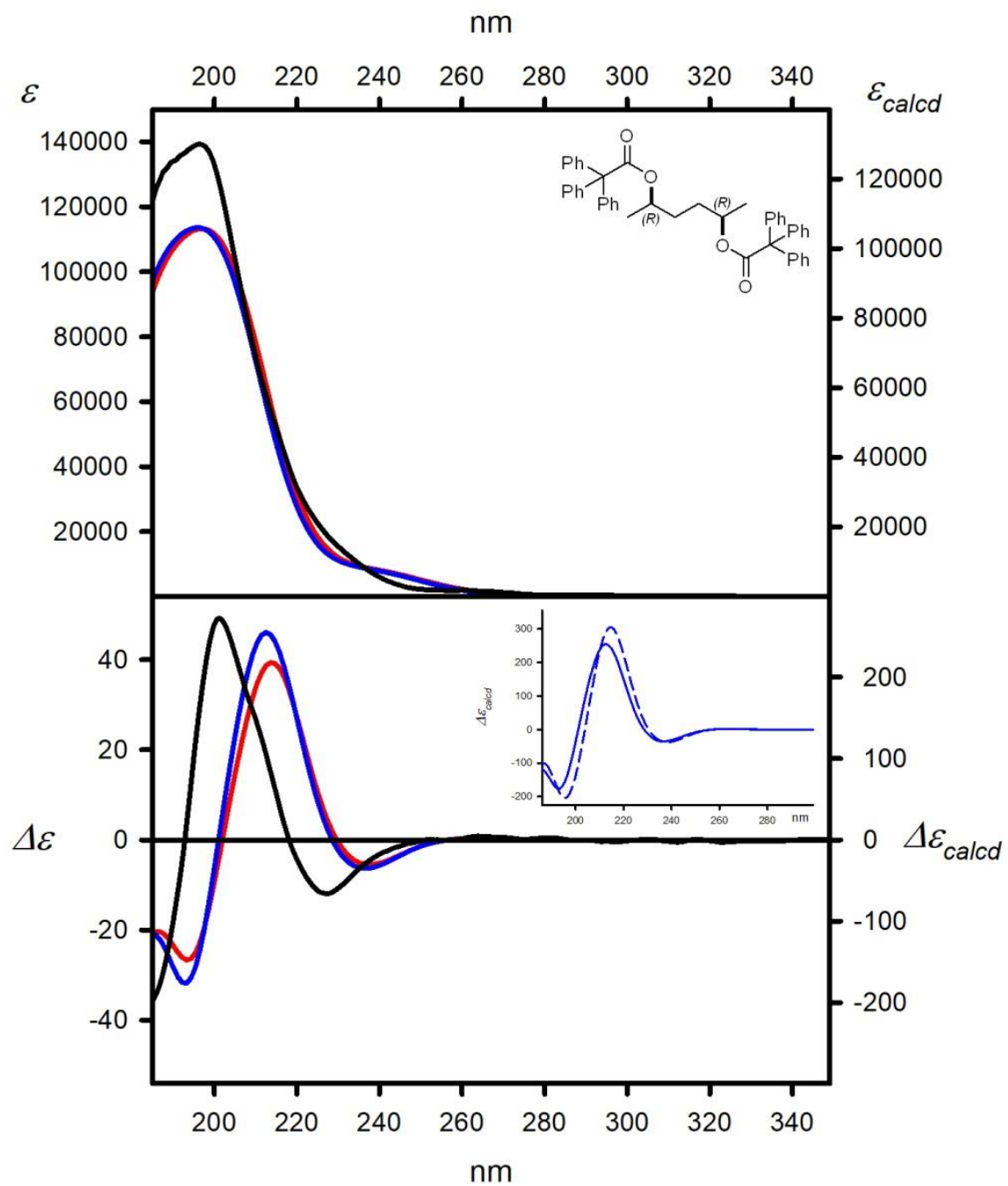

Experimental (cyclohexane, black lines)

\section{Calculated at the}

TD-CAM-B3LYP/6-311++G(d,p) level and:

$\triangle E$-based Boltzmann averaged (red lines)

$\triangle \triangle G$-based Boltzmann averaged (blue lines)

Figure SI_127. UV (upper panel) and ECD (lower panel) spectra of 22, experimental, measured in cyclohexane (solid black lines) and calculated at the TD-CAM-B3LYP/6-311++G(d,p) level for structures optimized at the B3LYP-GD3BJ/6-311G(d,p) level, $\Delta \mathrm{E}$-based Boltzmann (red lines) and $\Delta \Delta G$-based Boltzmann averaged (blue solid lines). Insert shows the comparison between Boltzmann averaged ECD spectrum and that calculated for $\Delta \Delta G$-based on the lowest energy conformer of a given compound (dashed blue line). All calculated spectra were wavelength corrected to match experimental UV maxima. 


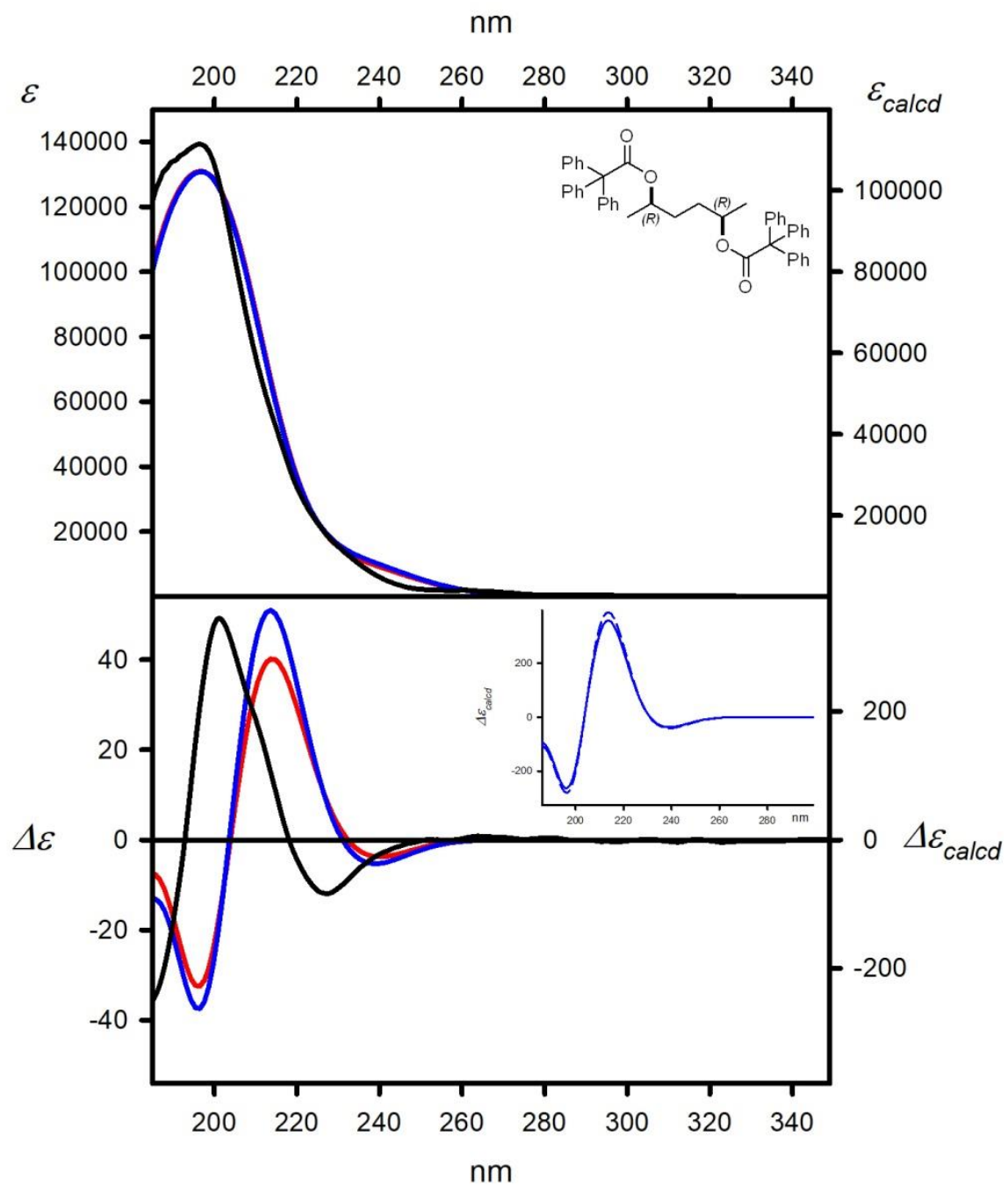

Experimental (cyclohexane, black lines)

\section{Calculated at the}

TD-M06-2X/6-311++G(d,p) level and:

$\triangle E$-based Boltzmann averaged (red lines)

$\triangle \triangle G$-based Boltzmann averaged (blue lines)

Figure SI_128. UV (upper panel) and ECD (lower panel) spectra of 22, experimental, measured in cyclohexane (solid black lines) and calculated at the TD-M06-2X/6-311++G(d,p) level for structures optimized at the B3LYP-GD3BJ/6-311G(d,p) level, $\Delta \mathrm{E}$-based Boltzmann (red lines) and $\Delta \Delta \mathrm{G}$-based Boltzmann averaged (blue solid lines). Insert shows the comparison between Boltzmann averaged ECD spectrum and that calculated for $\Delta \Delta G$-based on the lowest energy conformer of a given compound (dashed blue line). All calculated spectra were wavelength corrected to match experimental UV maxima. 


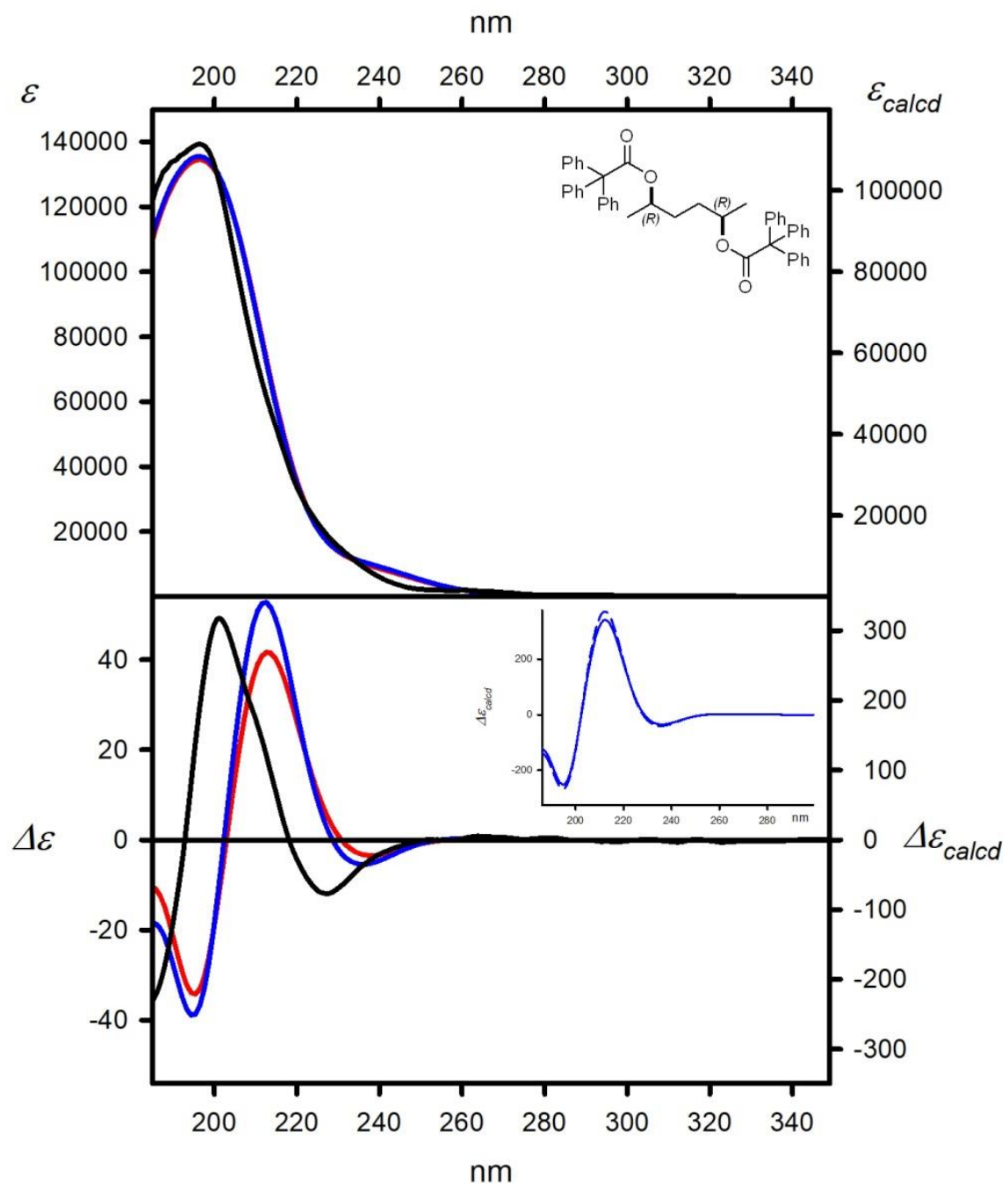

Experimental (cyclohexane, black lines)

\section{Calculated at the}

TD-CAM-B3LYP/6-311++G(d,p) level and:

$\triangle E$-based Boltzmann averaged (red lines)

$\triangle \triangle G$-based Boltzmann averaged (blue lines)

Figure SI_129. UV (upper panel) and ECD (lower panel) spectra of 22, experimental, measured in cyclohexane (solid black lines) and calculated at the TD-CAM-B3LYP/6-311++G(d,p) level for structures optimized at the M06-2X/6-311G(d,p) level, $\Delta \mathrm{E}$-based Boltzmann (red lines) and $\Delta \Delta \mathrm{G}$ based Boltzmann averaged (blue solid lines). Insert shows the comparison between Boltzmann averaged ECD spectrum and that calculated for $\Delta \Delta G$-based on the lowest energy conformer of a given compound (dashed blue line). All calculated spectra were wavelength corrected to match experimental UV maxima. 


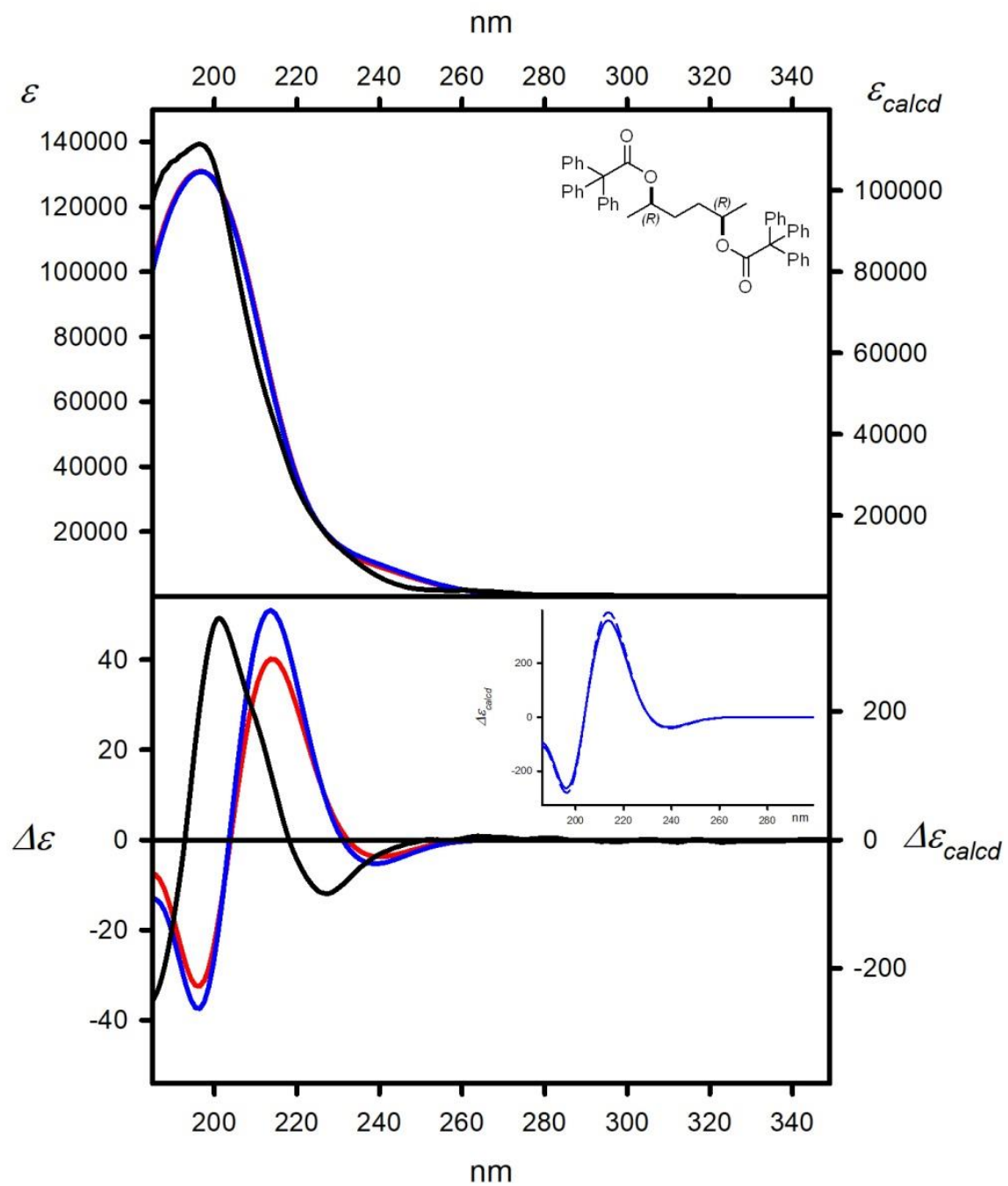

Experimental (cyclohexane, black lines)

\section{Calculated at the}

TD-M06-2X/6-311++G(d,p) level and:

$\triangle E$-based Boltzmann averaged (red lines)

$\triangle \triangle G$-based Boltzmann averaged (blue lines)

Figure SI_130. UV (upper panel) and ECD (lower panel) spectra of 22, experimental, measured in cyclohexane (solid black lines) and calculated at the TD-M06-2X/6-311++G(d,p) level for structures optimized at the M06-2X/6-311G(d,p) level, $\Delta E$-based Boltzmann (red lines) and $\Delta \Delta G$-based Boltzmann averaged (blue solid lines). Insert shows the comparison between Boltzmann averaged ECD spectrum and that calculated for $\Delta \Delta G$-based on the lowest energy conformer of a given compound (dashed blue line). All calculated spectra were wavelength corrected to match experimental UV maxima. 


\section{Single crystal X-ray analysis}

The colourless single crystals of compounds $1,5,10,11,18-21$ suitable for $X$-ray structural analysis were obtained by slow evaporation of solvent (dichloromethane, $n$-hexane or mixture thereof). The diffraction data were collected at $130 \mathrm{~K}$ with an Oxford Diffraction SuperNova diffractometer (Oxford Diffraction), using Cu K $\alpha$ radiation $(\lambda=1.54184 \AA$ ) equipped with a mirror monochromator and CCD detector (Atlas). The intensity data were collected and processed using the CrysAlisPro software.[9] The structures were solved by direct methods with the program SHELXT 2018/2 [10] and refined by full-matrix least-squares method on $\mathrm{F}^{2}$ with SHELXL 2018/3.[11] The carbon-bound hydrogen atoms were refined as riding on their carriers and their displacement parameters were set equal to $1.5 \mathrm{Ueq}(\mathrm{C})$ for the methyl groups and $1.2 \mathrm{Ueq}(\mathrm{C})$ for the remaining $\mathrm{H}$ atoms. Absolute structures of the compounds were specified by the synthetic procedure and confirmed using Flack parameter.[12]

A powder pattern of compound $\mathbf{5}$ was registered with a four-circle SuperNova diffractometer (Oxford Diffraction) using $\mathrm{Cu} K \alpha$ radiation ( $\lambda=1.54184 \AA$ ) , equipped with a mirror monochromator and CCD detector (Atlas). A $0.3 \mathrm{~mm}$ pinhole collimator was used and the detector was set at $70 \mathrm{~mm}$ from the sample. The exposure time was fixed to 300 second per scan and images were collected with a 360 degrees phi rotation. The powder patterns of polymorph 5- $\alpha$ and 5-6 for comparison were calculated with Mercury 4.3.0 software.[13]

Selected geometrical data are given in Table SI_54. A summary of the crystallographic data is given in Table SI_55. Molecular graphics were generated with Olex2[14] and Mercury 4.3.0 software. The analysis of intermolecular interactions was carried out using the Crystal Explorer.[15] Figure SI_131 presents the contribution to the Hirshfeld surface area for various intermolecular interactions for molecules in the studied crystal structures.

In crystal structure of 1-rac molecule is slightly disordered in 2-butyl part. The site occupancy factors for the alkyl chains are 0.78 and 0.22 . (Figure SI_132b). For disordered part the following restrains was used: RIGU 01 C2A C3A C4A C5A and RIGU C2 C3 C4 C5.

In refinement process of crystal structure of $\mathbf{1 1}$ four reflection have been omitted: $7-4$ 4, 8 -2 3, $7-34,7-24$.

The molecule in crystal structure of $\mathbf{1 8}$ lies on the two-fold axis passing through the $\mathrm{C}_{\mathrm{sp2} 2}-\mathrm{C}_{\mathrm{sp2}}$ bond. it is impossible to locate the methyl group unambiguously and in the adopted model it is equally likely attached to the C2 or C2' (Figure SI_137). It is similar in the crystal of 19. The methyl group is equally likely attached to the $\mathrm{C} 2$ and $\mathrm{C} 4$ atom. The molecules in crystal structure are arranged in columns and the additional disorder is the result of the molecule shifting in the column by half its length (Figure SI_138). The site occupancy factors for molecules are 0.85 and 0.15 . For disordered part the following restrains was used: DFIX 1.54 0.005 C2B C6B, SIMU 0.04 0.08 1.7 C11B C12B C13B C14B C15B C16B, SIMU 0.040 .081 .7 C41B C42B C43B C44B C45B C46B, SIMU 0.04 0.08 1.7 C51B C52B C53B C54B C55B C56B, SIMU 0.04 0.08 1.7 C61B C62B C63B C64B C65B C66B, SIMU 0.040 .081 .7 C31B С32B С33B С34B C35B C36B, SIMU 0.040 .081 .7 C21B C22B C23B C24B C25B C26B. 
Table SI_54. Dihedral angles $\alpha, 6, y, \delta$ and $\zeta$ (in degrees), selected interatomic distances $I_{1}, I_{2}$ (in $\AA$ ) and helicities of trityl groups observed in the crystal structures of compounds $1,5,10,11,18-21$.

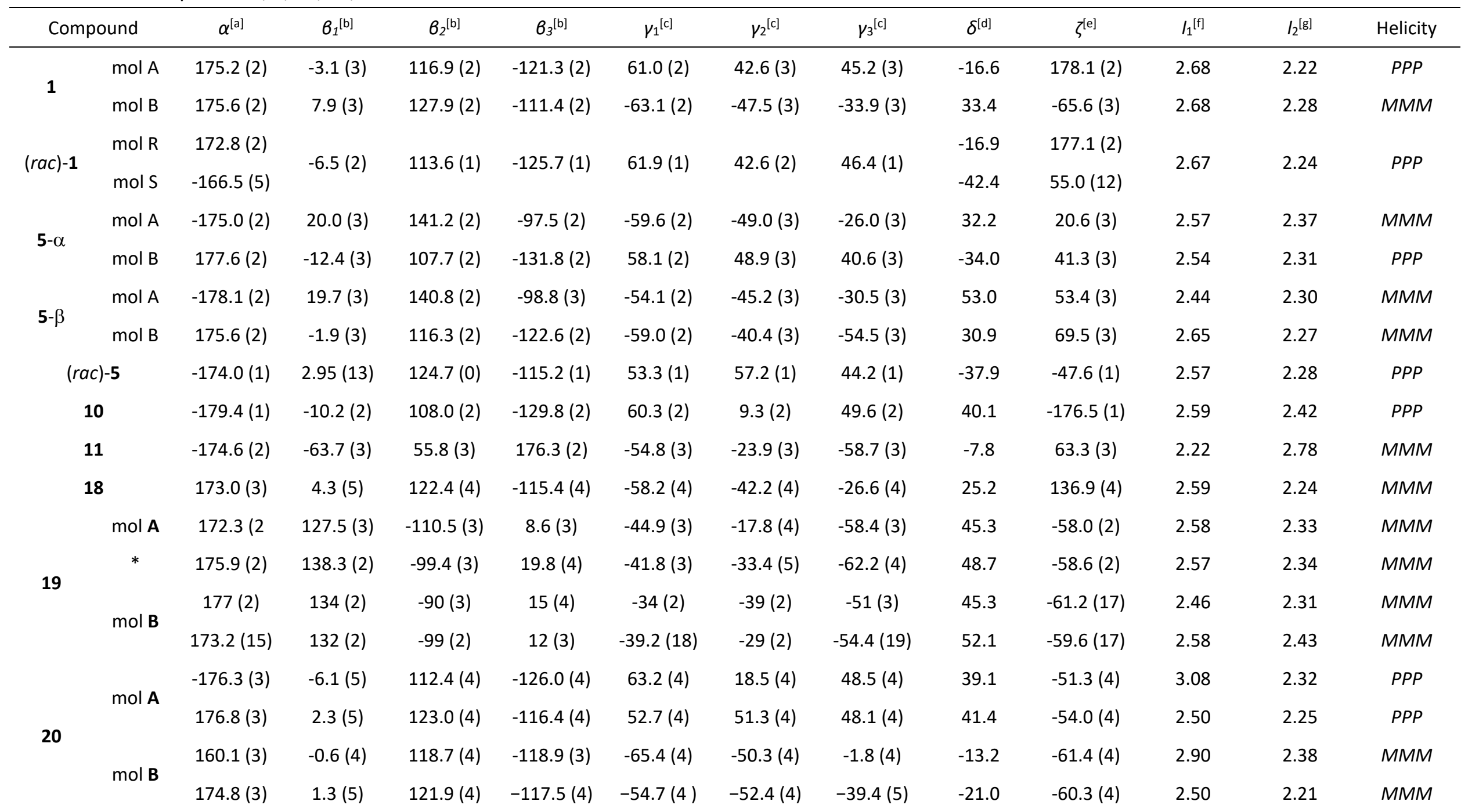




\begin{tabular}{|c|c|c|c|c|c|c|c|c|c|c|c|c|}
\hline \multirow{2}{*}{$\mathrm{mol} \mathrm{C}$} & $173.3(3)$ & $4.6(5)$ & $123.9(4)$ & $-115.5(4)$ & $52.7(4)$ & $53.1(4)$ & $46.8(4)$ & 38.8 & $-53.3(4)$ & 2.51 & 2.23 & $P P P$ \\
\hline & $-174.9(3)$ & $-2.1(4)$ & $115.8(4)$ & $-122.2(3)$ & $64.7(4)$ & $12.2(4)$ & $49.0(4)$ & 41.4 & $-51.3(4)$ & 2.78 & 2.34 & $P P P$ \\
\hline \multirow{2}{*}{ mol D } & $172.4(3)$ & $1.2(5)$ & 120.7 (4) & $-118.8(4)$ & $-53.8(4)$ & $-53.1(4)$ & $-38.9(4)$ & -19.8 & $-61.0(4)$ & 2.52 & 2.22 & $M M M$ \\
\hline & $159.4(3)$ & $0.8(5)$ & $120.0(4)$ & $-118.1(4)$ & $-64.7(4)$ & $-49.6(4)$ & $-7.6(4)$ & -9.2 & $-61.2(4)$ & 2.83 & 2.35 & $M M M$ \\
\hline 21 & $174.8(2)$ & $19.0(3)$ & $139.3(2)$ & $-99.7(2)$ & $-59.9(2)$ & $-47.1(2)$ & $-23.1(3)$ & 46.7 & $-61.2(1)$ & 2.56 & 2.38 & $M M M$ \\
\hline
\end{tabular}

$[\mathrm{a}]-\alpha=\mathrm{C}_{\mathrm{Tr}} \mathrm{C}(=\mathrm{O})-\mathrm{O}-\mathrm{C}^{*} ;[\mathrm{b}]-\mathrm{B}=\mathrm{O}=\mathrm{C}-\mathrm{C}-\mathrm{C}_{\text {ipso }} ;[\mathrm{c}]-\gamma=(\mathrm{O}=) \mathrm{C}-\mathrm{C}_{\mathrm{Tr}}-\mathrm{C}_{\text {ipso }}-\mathrm{C}_{\text {ortho }}$ (of the two possibilities the absolute values $\leq 90^{\circ}$ has been chosen); [d] $-\delta=\mathrm{C}(=\mathrm{O})-$ $\mathrm{O}-\mathrm{C}^{*}-\mathrm{H} ;[\mathrm{e}]-\zeta=\mathrm{O}-\mathrm{C}^{*}-\mathrm{C}-\mathrm{C}\left({ }^{*}\right) ;[\mathrm{f}]-\mathrm{I}_{1}=\mathrm{C}=\mathrm{O} \cdots \mathrm{HC}$ ortho; $[\mathrm{g}]-\mathrm{I}_{2}=(\mathrm{O}=) \mathrm{CO} \cdots \mathrm{HC}_{\text {ortho }}$. 
Table SI_55. Selected crystal data and structure refinement details for 1, 5, 10, 11, 18-21.

\begin{tabular}{|c|c|c|c|c|c|c|c|c|c|c|c|}
\hline & 1 & $(r a c)-1$ & $5-\alpha$ & $5-\beta$ & $(r a c)-5$ & 10 & 11 & 18 & 19 & 20 & 21 \\
\hline Chemical formula & $\mathrm{C}_{24} \mathrm{H}_{24} \mathrm{O}_{2}$ & $\mathrm{C}_{24} \mathrm{H}_{24} \mathrm{O}_{2}$ & $\mathrm{C}_{28} \mathrm{H}_{24} \mathrm{O}_{2}$ & $\mathrm{C}_{28} \mathrm{H}_{24} \mathrm{O}_{2}$ & $\mathrm{C}_{28} \mathrm{H}_{24} \mathrm{O}_{2}$ & $\mathrm{C}_{30} \mathrm{H}_{34} \mathrm{O}_{2}$ & $\mathrm{C}_{30} \mathrm{H}_{34} \mathrm{O}_{2}$ & $\mathrm{C}_{43} \mathrm{H}_{36} \mathrm{O}_{4}$ & $\mathrm{C}_{44} \mathrm{H}_{38} \mathrm{O}_{4}$ & $\mathrm{C}_{44} \mathrm{H}_{38} \mathrm{O}_{4}$ & $\mathrm{C}_{45} \mathrm{H}_{40} \mathrm{O}_{4}$ \\
\hline Mr & 344.43 & 344.43 & 392.47 & 392.47 & 392.47 & 426.57 & 426.57 & 616.72 & 630.74 & 630.74 & 644.77 \\
\hline $\begin{array}{l}\text { Crystal system, } \\
\text { space group }\end{array}$ & $\begin{array}{l}\text { Monoclinic, } \\
P 2_{1}\end{array}$ & $\begin{array}{l}\text { Monoclinic, } \\
P 2_{1} / c\end{array}$ & $\begin{array}{l}\text { Monoclinic, } \\
P 2_{1}\end{array}$ & $\begin{array}{l}\text { Monoclinic, } \\
P 2_{1}\end{array}$ & $\begin{array}{l}\text { Monoclinic, } \\
P 2_{1} / c\end{array}$ & $\begin{array}{l}\text { Monoclinic, } \\
P 2_{1}\end{array}$ & $\begin{array}{l}\text { Monoclinic, } \\
P 2_{1}\end{array}$ & $\begin{array}{l}\text { Monoclinic, } \\
C 2\end{array}$ & $\begin{array}{l}\text { Monoclinic, } \\
P 2_{1}\end{array}$ & $\begin{array}{l}\text { Monoclinic, } \\
\text { P2 }\end{array}$ & $\begin{array}{l}\text { Monoclinic, } \\
\text { C2 }\end{array}$ \\
\hline Temperature (K) & 130 & 130 & 130 & 130 & 130 & 130 & 130 & 130 & 130 & 130 & 130 \\
\hline$a, b, c(\AA)$ & $\begin{array}{l}8.6848(1), \\
13.1859(1), \\
16.7979(1)\end{array}$ & $\begin{array}{l}8.7143(2), \\
13.1260(3), \\
16.7238(3)\end{array}$ & $\begin{array}{l}8.56847(8), \\
27.5418(3), \\
9.4209(1)\end{array}$ & $\begin{array}{l}8.76286(12) \\
17.3973(3) \\
14.2707(2)\end{array}$ & $\begin{array}{l}15.90336(5), \\
8.05841(3), \\
16.20465(6)\end{array}$ & $\begin{array}{l}8.81491(6), \\
15.42044(11), \\
9.11196(8)\end{array}$ & $\begin{array}{l}8.5973(2), \\
16.5110(4), \\
9.2226(3)\end{array}$ & $\begin{array}{l}15.8708(8), \\
7.9327(2) \\
14.1241(7)\end{array}$ & $\begin{array}{l}14.2775(1), \\
8.0436(1), \\
14.4025(2)\end{array}$ & $\begin{array}{l}20.0936(2), \\
9.9377(1) \\
34.7742(3)\end{array}$ & $\begin{array}{l}16.3482(4), \\
8.2085(1) \\
14.2443(4)\end{array}$ \\
\hline$\beta\left(\left(^{\circ}\right)\right.$ & $99.848(1)$ & $99.740(2)$ & $100.4459(10)$ & $101.8322(12)$ & $90.0241(3)$ & $93.3367(7)$ & $113.412(3)$ & $114.416(6)$ & $91.020(1)$ & $103.090(1)$ & $117.606(3)$ \\
\hline$V\left(\AA^{3}\right)$ & $1895.30(3)$ & $1885.36(7)$ & $2186.40(4)$ & $2129.34(5)$ & $2076.72(1)$ & $1236.49(2)$ & $1201.37(6)$ & $1619.17(14)$ & $1653.76(3)$ & $6763.42(12)$ & $1693.89(8)$ \\
\hline$z$ & 4 & 4 & 4 & 4 & 4 & 2 & 2 & 2 & 2 & 8 & 2 \\
\hline$D_{x}\left(\mathrm{Mg} \mathrm{m}^{-3}\right)$ & 1.207 & 1.213 & 1.192 & 1.243 & 1.255 & 1.146 & 1.179 & 1.265 & 1.267 & 1.239 & 1.264 \\
\hline Radiation type & $\mathrm{Cu} K \alpha$ & $\mathrm{Cu} K \alpha$ & $\mathrm{Cu} K \alpha$ & $\mathrm{Cu} K \alpha$ & $\mathrm{Cu} K \alpha$ & $\mathrm{Cu} K \alpha$ & $\mathrm{Cu} K \alpha$ & $\mathrm{Cu} K \alpha$ & $\mathrm{Cu} K \alpha$ & $\mathrm{Cu} K \alpha$ & $\mathrm{Cu} K \alpha$ \\
\hline$\mu\left(\mathrm{mm}^{-1}\right)$ & 0.59 & 0.59 & 0.57 & 0.59 & 0.61 & 0.54 & 0.55 & 0.63 & 0.63 & 0.62 & 0.62 \\
\hline Crystal size (mm) & $\begin{array}{l}0.24 \times 0.10 \times \\
0.05\end{array}$ & $\begin{array}{l}0.50 \times 0.07 \times \\
0.06\end{array}$ & $\begin{array}{l}0.20 \times 0.07 \times \\
0.02\end{array}$ & $\begin{array}{l}0.16 \times 0.10 \times \\
0.03\end{array}$ & $0.4 \times 0.3 \times 0.07$ & $\begin{array}{l}0.45 \times 0.34 \times \\
0.05\end{array}$ & $\begin{array}{l}0.23 \times 0.20 \times \\
0.02\end{array}$ & $\begin{array}{l}0.45 \times 0.10 \times \\
0.02\end{array}$ & $\begin{array}{l}0.30 \times 0.20 \times \\
0.03\end{array}$ & $\begin{array}{l}0.55 \times 0.35 \times \\
0.15\end{array}$ & $\begin{array}{l}0.40 \times 0.16 \times \\
0.02\end{array}$ \\
\hline $\begin{array}{l}\text { No. of measured, } \\
\text { independent and } \\
\text { observed }[I>2 \sigma(l)] \\
\text { reflections }\end{array}$ & $\begin{array}{l}28779 \\
7191 \\
6962\end{array}$ & $\begin{array}{l}13757 \\
3711 \\
3358\end{array}$ & $\begin{array}{l}23089 \\
7327 \\
6906\end{array}$ & $\begin{array}{l}21638 \\
7691 \\
7164\end{array}$ & $\begin{array}{l}61948, \\
4368 \\
4197\end{array}$ & $\begin{array}{l}13582 \\
4521 \\
4463\end{array}$ & $\begin{array}{l}17587 \\
4553 \\
4296\end{array}$ & $\begin{array}{l}11856 \\
3082 \\
2881\end{array}$ & $\begin{array}{l}47791 \\
6267 \\
6126\end{array}$ & $\begin{array}{l}112063 \\
25552 \\
24266\end{array}$ & $\begin{array}{l}11151 \\
3485 \\
3374\end{array}$ \\
\hline$R_{\text {int }}$ & 0.027 & 0.036 & 0.030 & 0.036 & 0.023 & 0.023 & 0.052 & 0.046 & 0.036 & 0.057 & 0.029 \\
\hline $\begin{array}{l}R\left[F^{2}>2 \sigma\left(F^{2}\right)\right], \\
w R\left(F^{2}\right), S\end{array}$ & $\begin{array}{l}0.036,0.095 \\
1.04\end{array}$ & $\begin{array}{l}0.043,0.108, \\
1.07\end{array}$ & $\begin{array}{l}0.031,0.075, \\
1.05\end{array}$ & $\begin{array}{l}0.034,0.080 \\
1.04\end{array}$ & $\begin{array}{l}0.034,0.085, \\
1.05\end{array}$ & $\begin{array}{l}0.029,0.076 \\
1.07\end{array}$ & $\begin{array}{l}0.040,0.110 \\
1.08\end{array}$ & $\begin{array}{l}0.047,0.128 \\
1.10\end{array}$ & $\begin{array}{l}0.039,0.110 \\
1.05\end{array}$ & $\begin{array}{l}0.082,0.217, \\
1.05\end{array}$ & $\begin{array}{l}0.033,0.092, \\
1.09\end{array}$ \\
\hline No. of parameters & 473 & 277 & 543 & 543 & 272 & 292 & 292 & 218 & 577 & 1737 & 223 \\
\hline$\Delta>_{\max }, \Delta>_{\min }\left(\mathrm{e} \AA^{-3}\right)$ & $0.24,-0.27$ & $0.29,-0.18$ & $0.14,-0.14$ & $0.16,-0.13$ & $0.27,-0.20$ & $0.18,-0.16$ & $0.18,-0.14$ & $0.16,-0.21$ & $0.24,-0.22$ & $0.87,-0.37$ & $0.20,-0.17$ \\
\hline $\begin{array}{l}\text { Absolute structure } \\
\text { parameter }\end{array}$ & $0.03(6)$ & - & $-0.05(9)$ & $-0.06(11)$ & - & $0.01(8)$ & 0.24 (19) & $0.0(2)$ & $0.00(6)$ & $-0.04(8)$ & $-0.06(11)$ \\
\hline
\end{tabular}




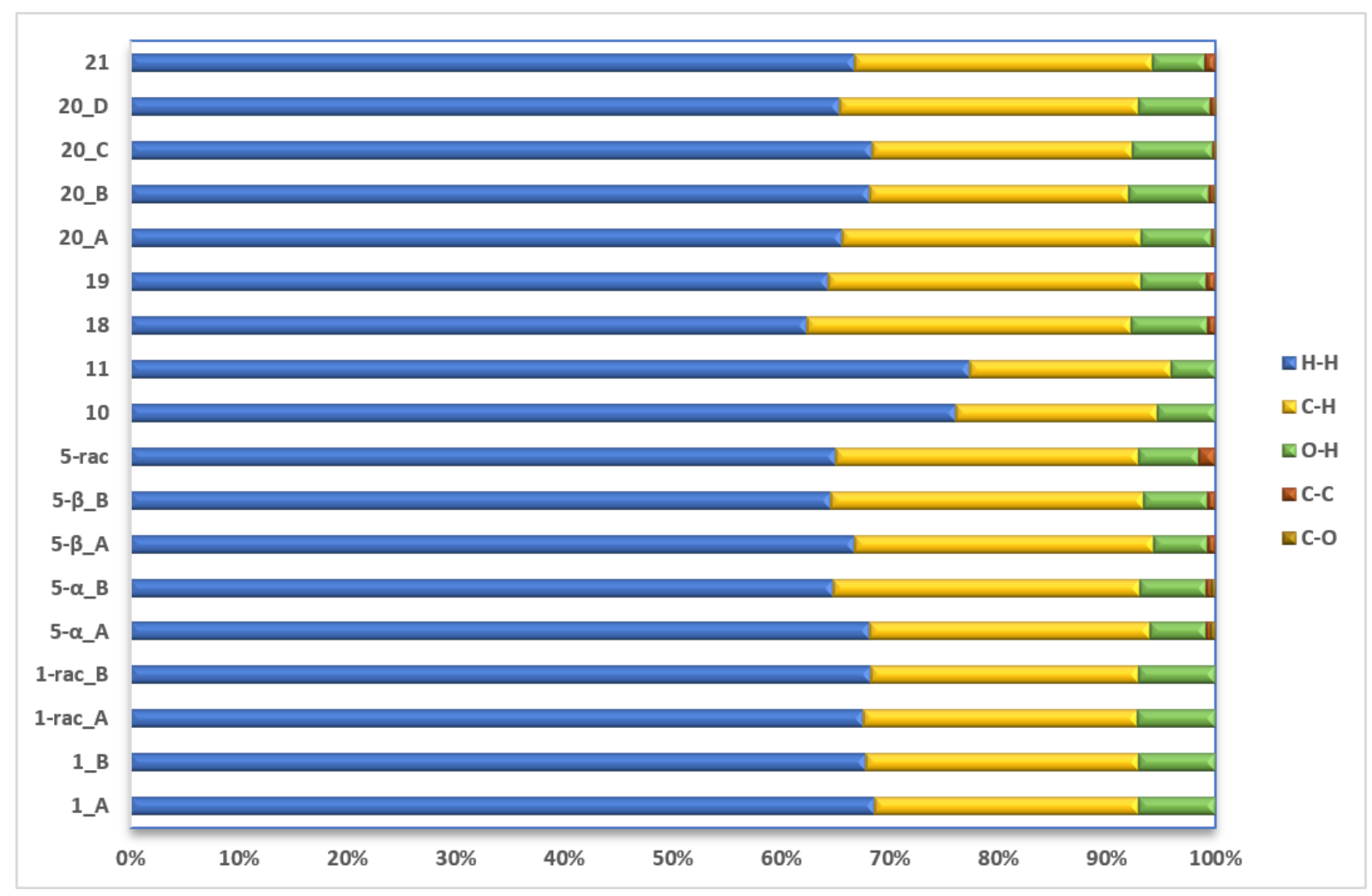

Figure SI_131. Percentage contributions of various intermolecular interaction to the Hirshfeld surfaces of molecules in crystal structure. 
a)

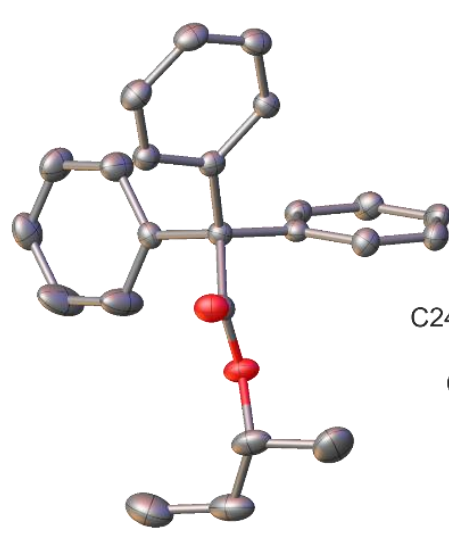

c)

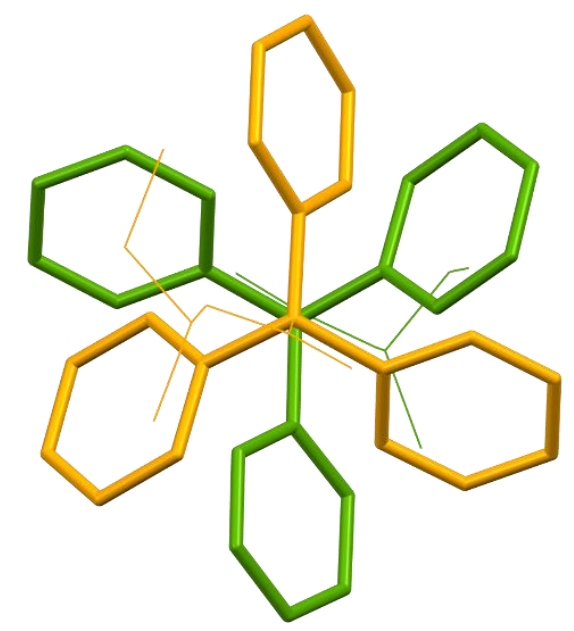

e)

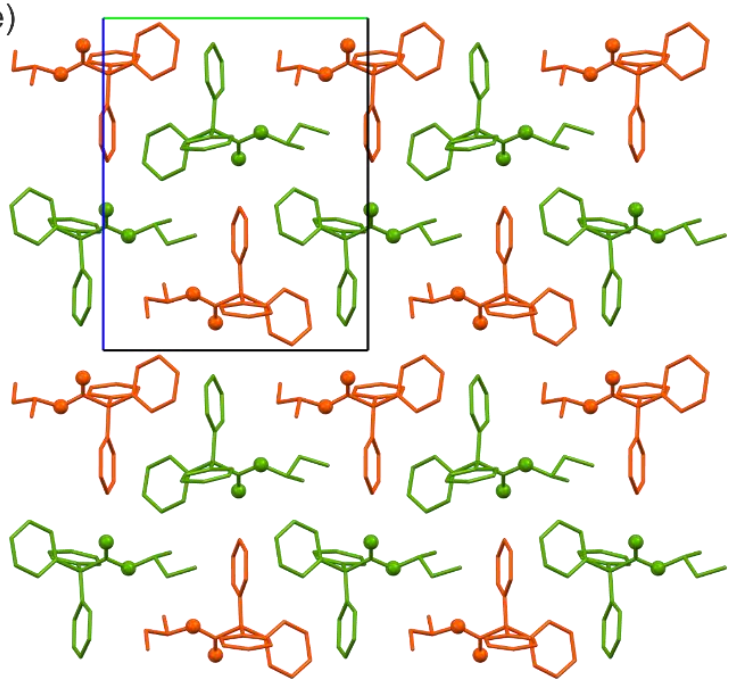

b)

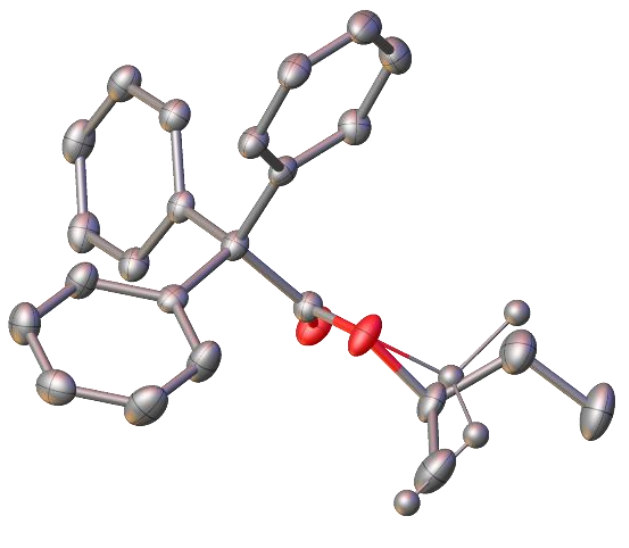

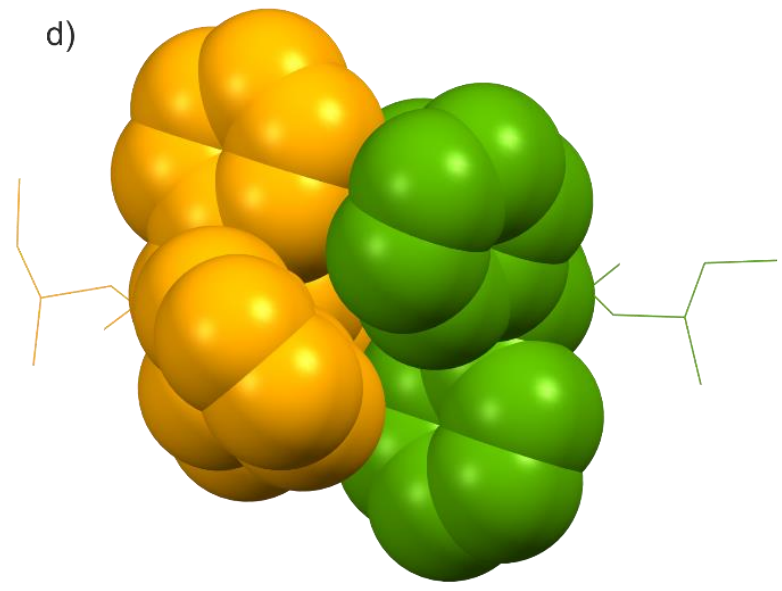

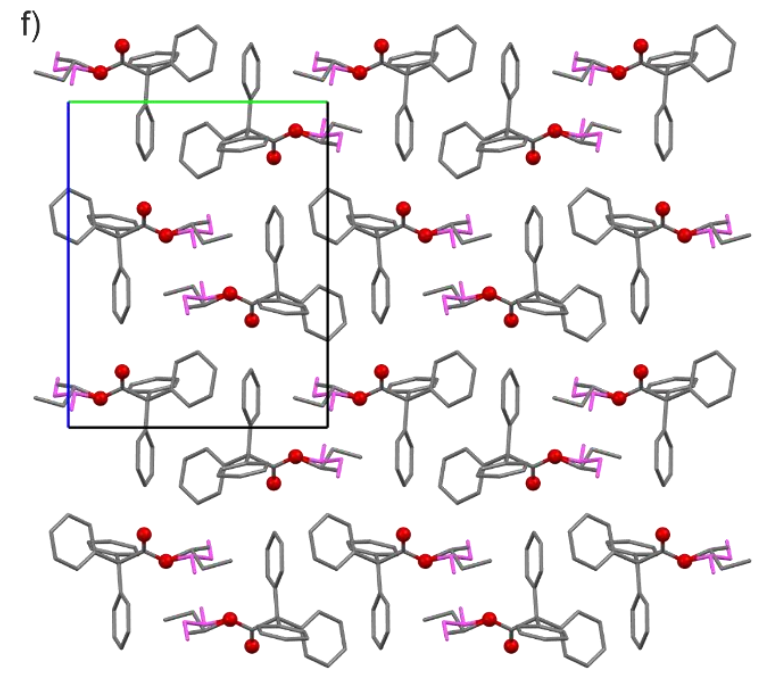

Figure SI_132. Molecular structure of compound a) $\mathbf{1}$ and b) (rac)-1 (2-butyl substituent is disordered and the minor position showed as thinner lines). Atoms numbering scheme presented for one molecule. Displacement ellipsoids are drawn at 50\% probability level. The sixfold phenyl embrace supramolecular synthon in the crystal structure of $\mathbf{1}$ c) top view and d) side view. Comparison of molecular packing in isostructural crystals of compound e) $\mathbf{1}$ (symmetrically independent molecules are indicated with different colors) and f) ( $r a c)-1$ (minor position of 2-butyl substituent shown as pink). Hydrogen atoms are omitted for clarity and oxygen atoms are shown as balls. 

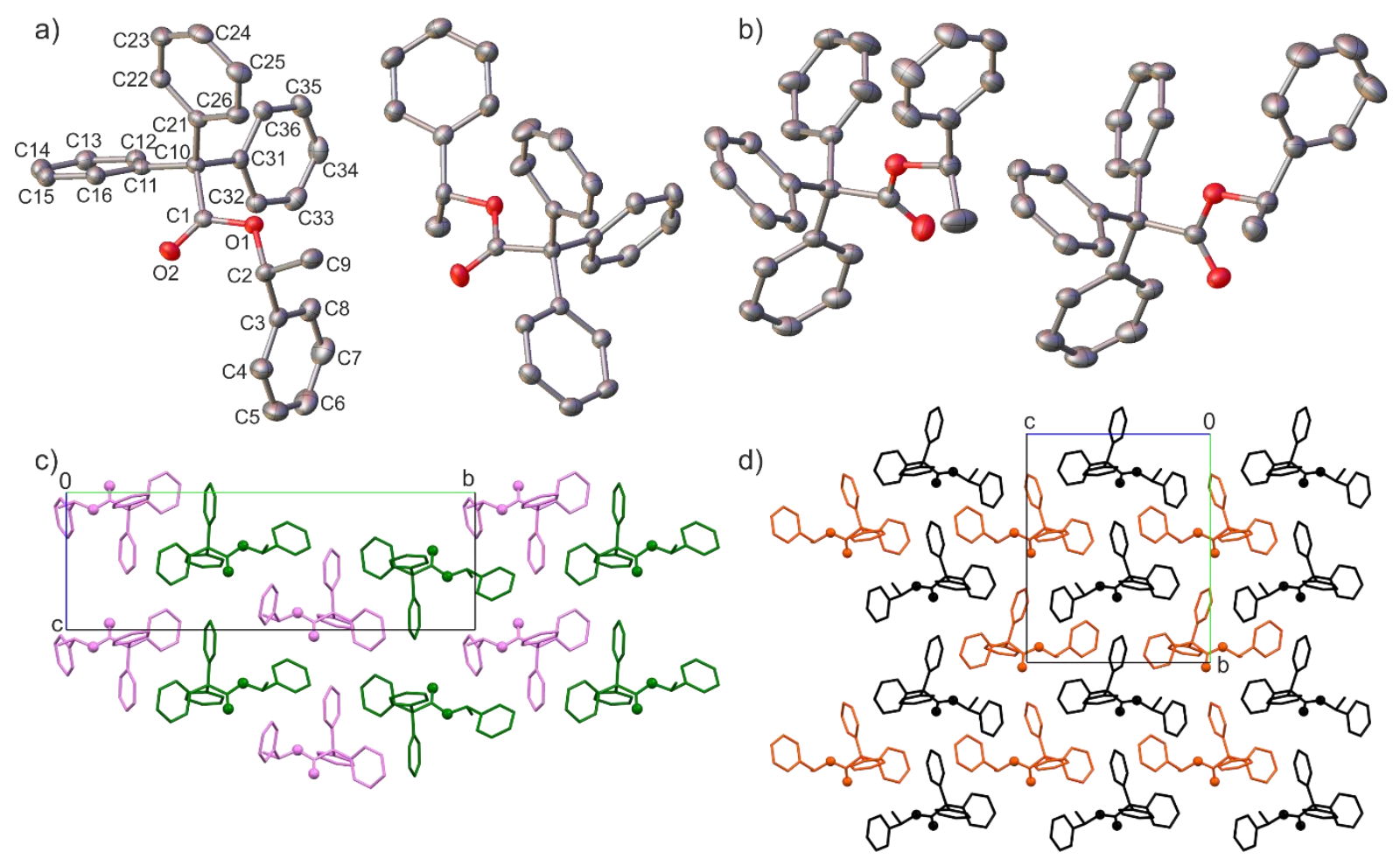

Figure SI_133. Molecular structure of asymmetric unit of a) 5- $\alpha$ and b) 5-6. Atoms numbering scheme presented for one molecule. Displacement ellipsoids are drawn at $50 \%$ probability level. Comparison of molecular packing in the crystals of c) 5- $\alpha$ and d) 5-6. Symmetrically independent molecules are indicated with different colors. Hydrogen atoms are omitted for clarity and oxygen atoms are shown as balls. 

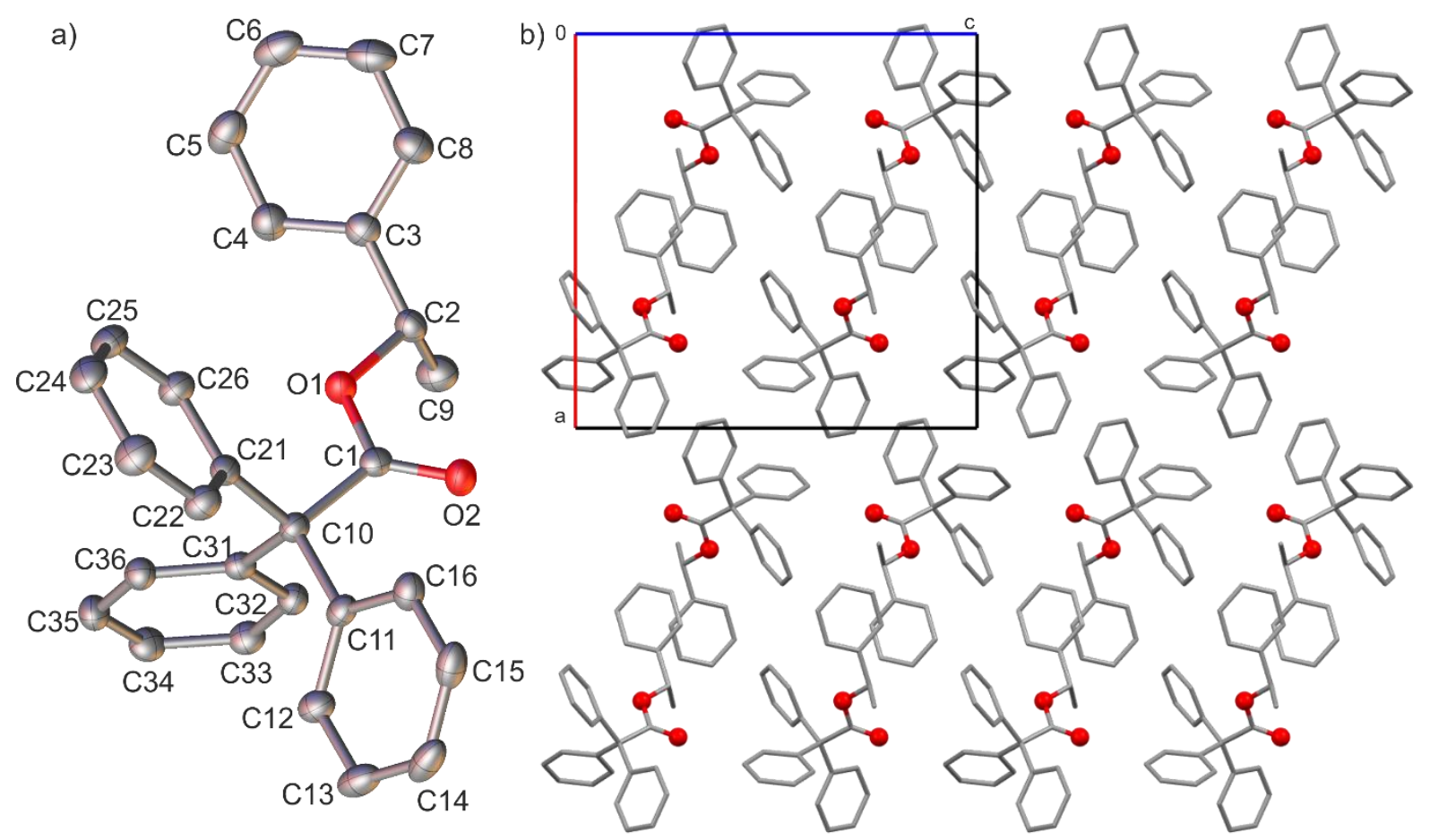

Figure SI_134. a) Molecular structure with atoms numbering scheme and b) molecular packing in crystal of (rac)-5. Displacement ellipsoids are drawn at 50\% probability level. Hydrogen atoms are omitted for clarity and oxygen atoms are shown as balls. 


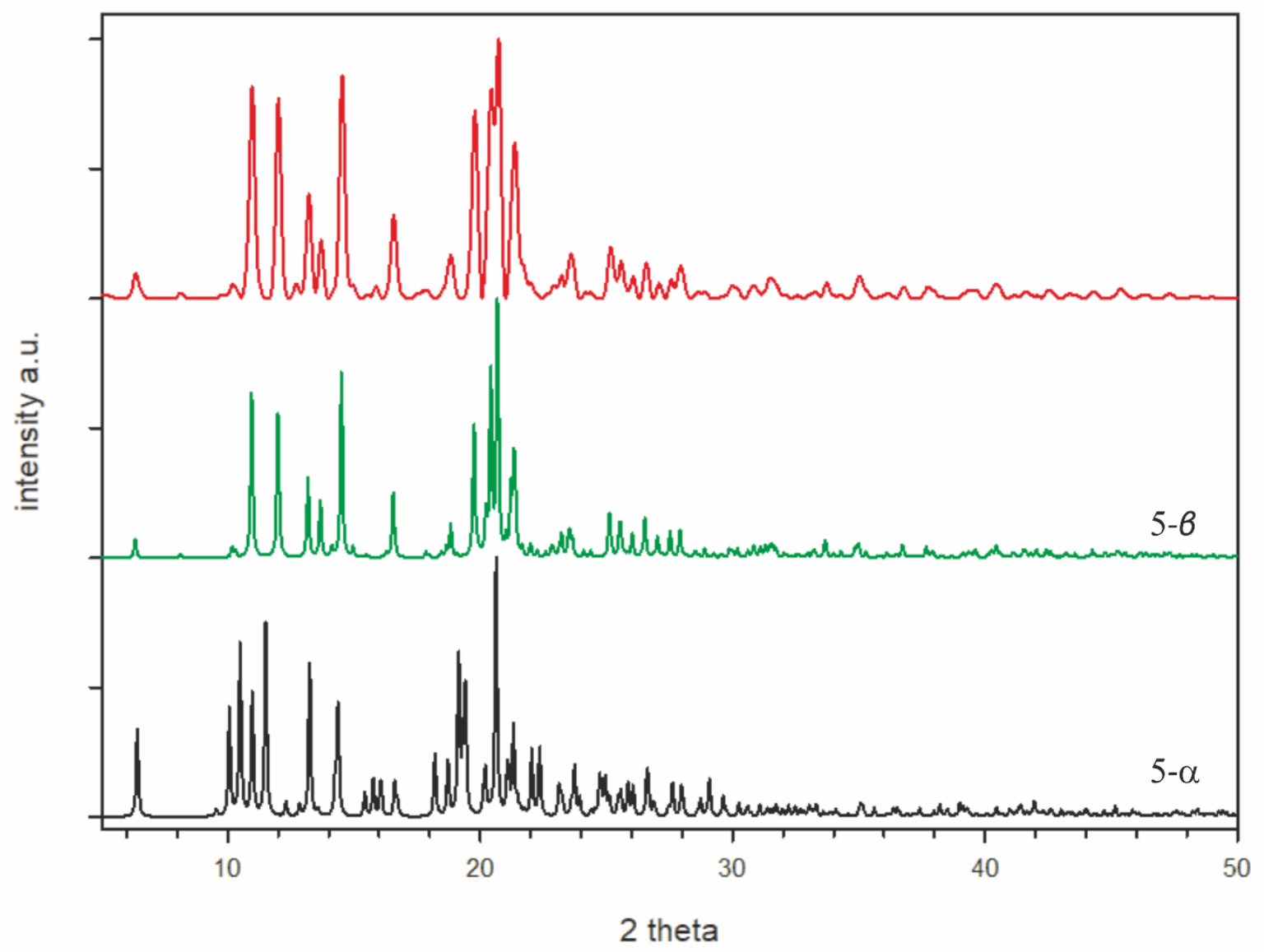

Figure SI_135. The recorded X-ray powder diffraction pattern of compound $\mathbf{5}$ (red) and predicted pattern based on single-crystal X-ray diffraction indices of polymorphs 5- $\alpha$ and 5- 6 . 

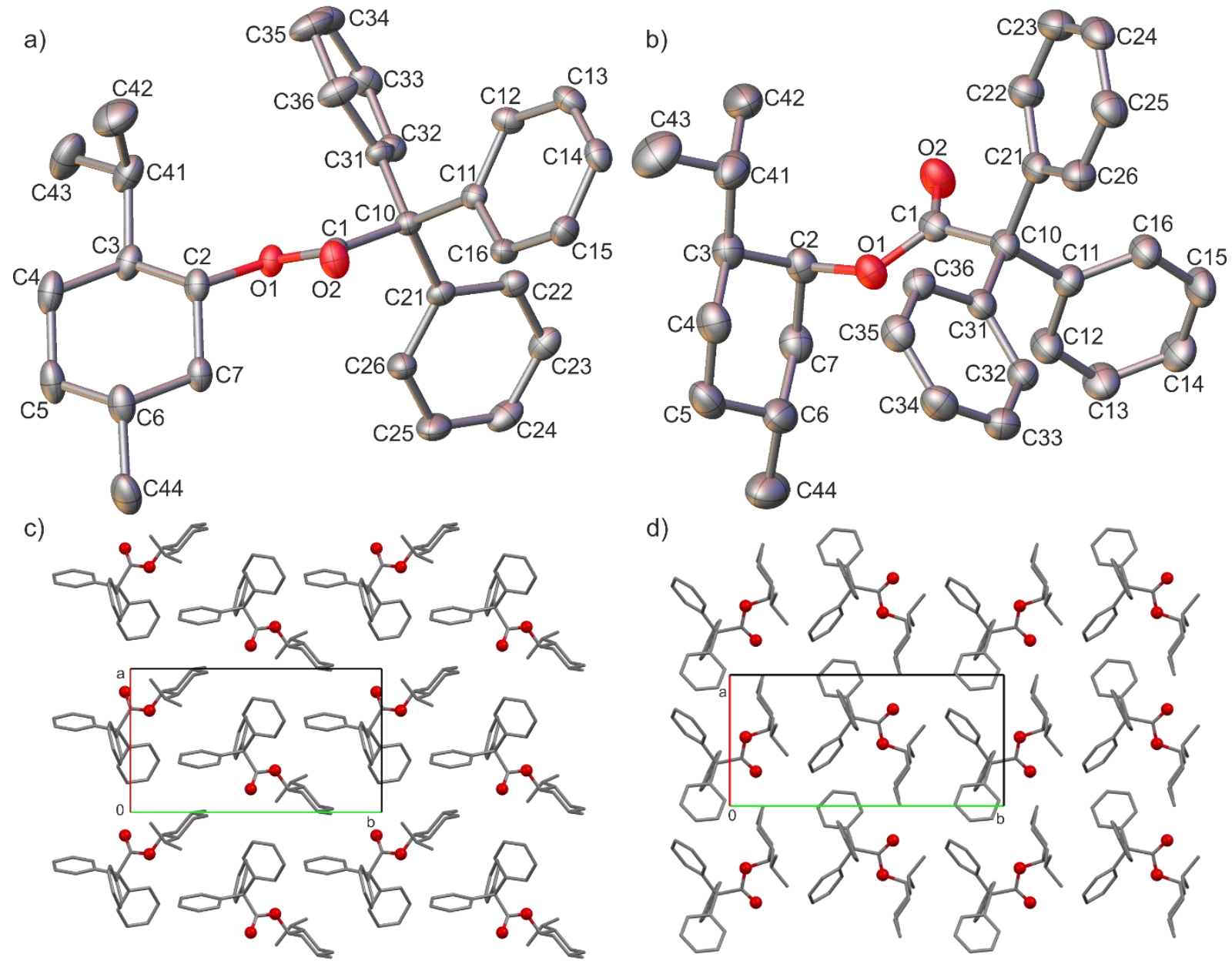

d)

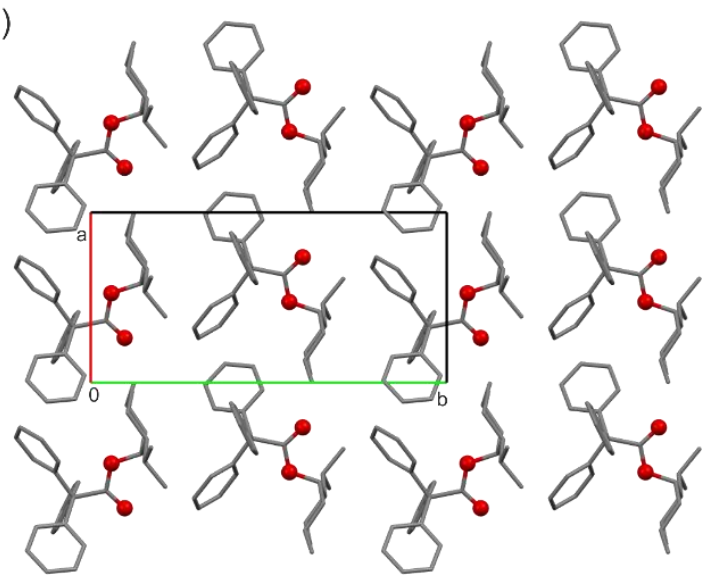

Figure SI_136. Molecular structure and atoms numbering scheme of compound a) $\mathbf{1 0}$ and b) $\mathbf{1 1}$. Displacement ellipsoids are drawn at $50 \%$ probability level. Comparison of molecular packing in crystal structure of c) $\mathbf{1 0}$ and d) $\mathbf{1 1}$. Hydrogen atoms are omitted for clarity and oxygen atoms are shown as balls. 
a)
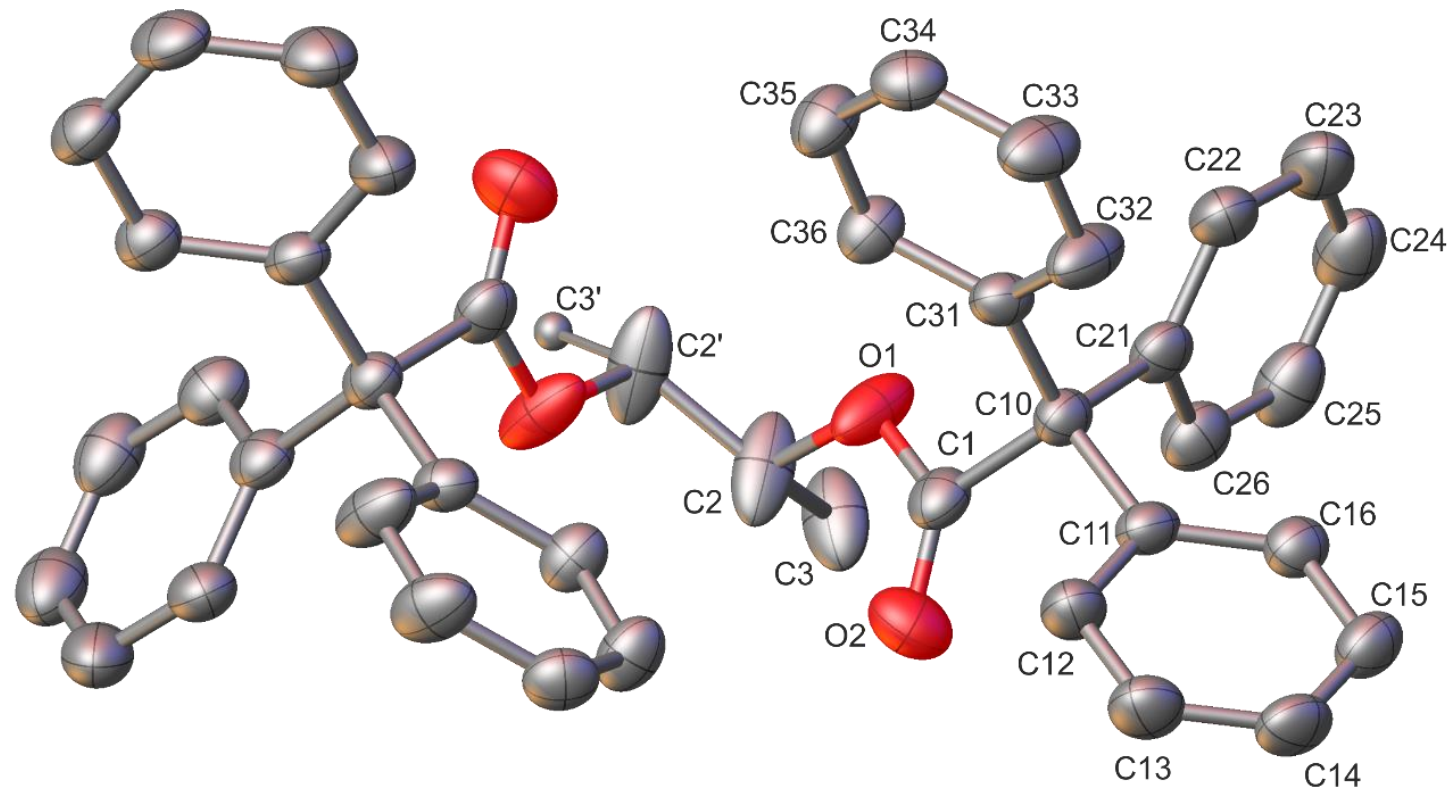

b)
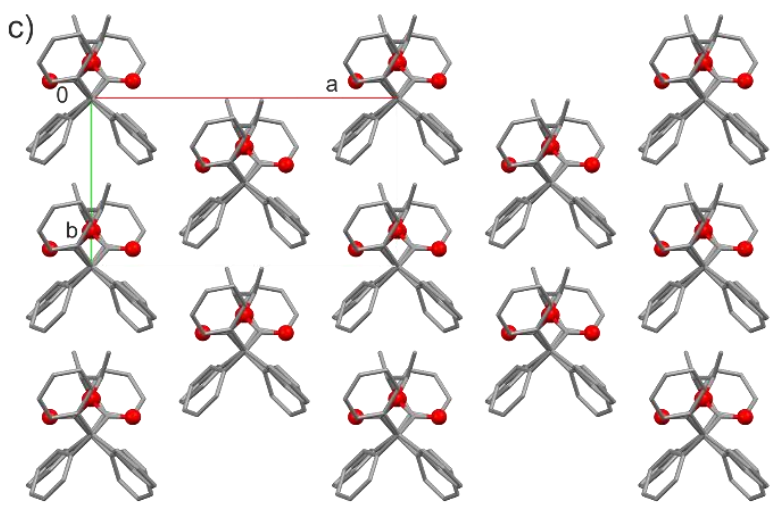

Figure SI_137. Molecular structure and atoms numbering scheme of compound 18. Only the asymmetric part has been numbered for clarity. Displacement ellipsoids are drawn at 50\% probability level. Molecular packing in crystal structure b) view along b axis and c) view along $c$ axis. Hydrogen atoms are omitted for clarity and oxygen atoms are shown as balls. 
a)

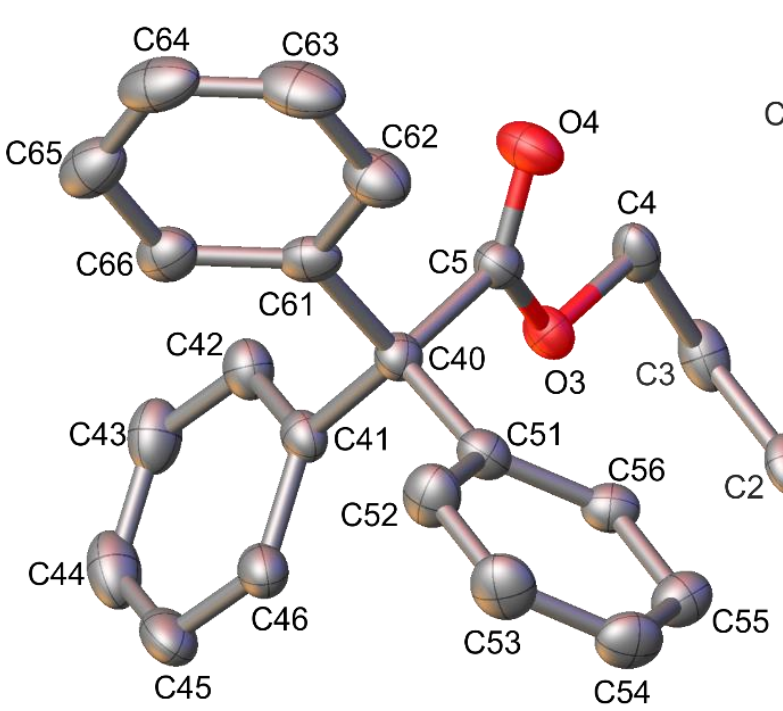

$\mathrm{C} 24$

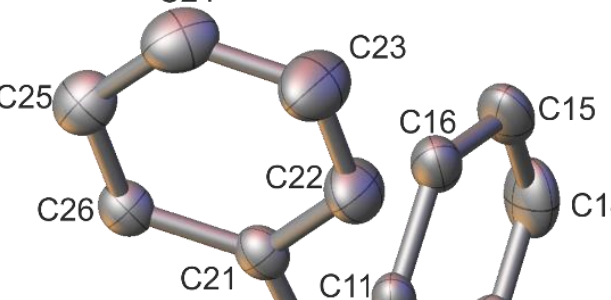

C14
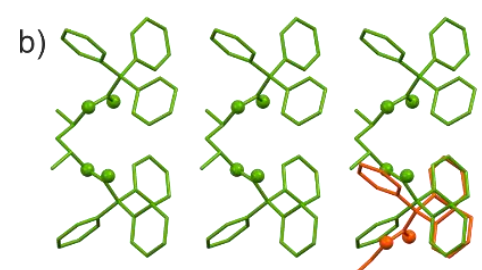

80

3o0
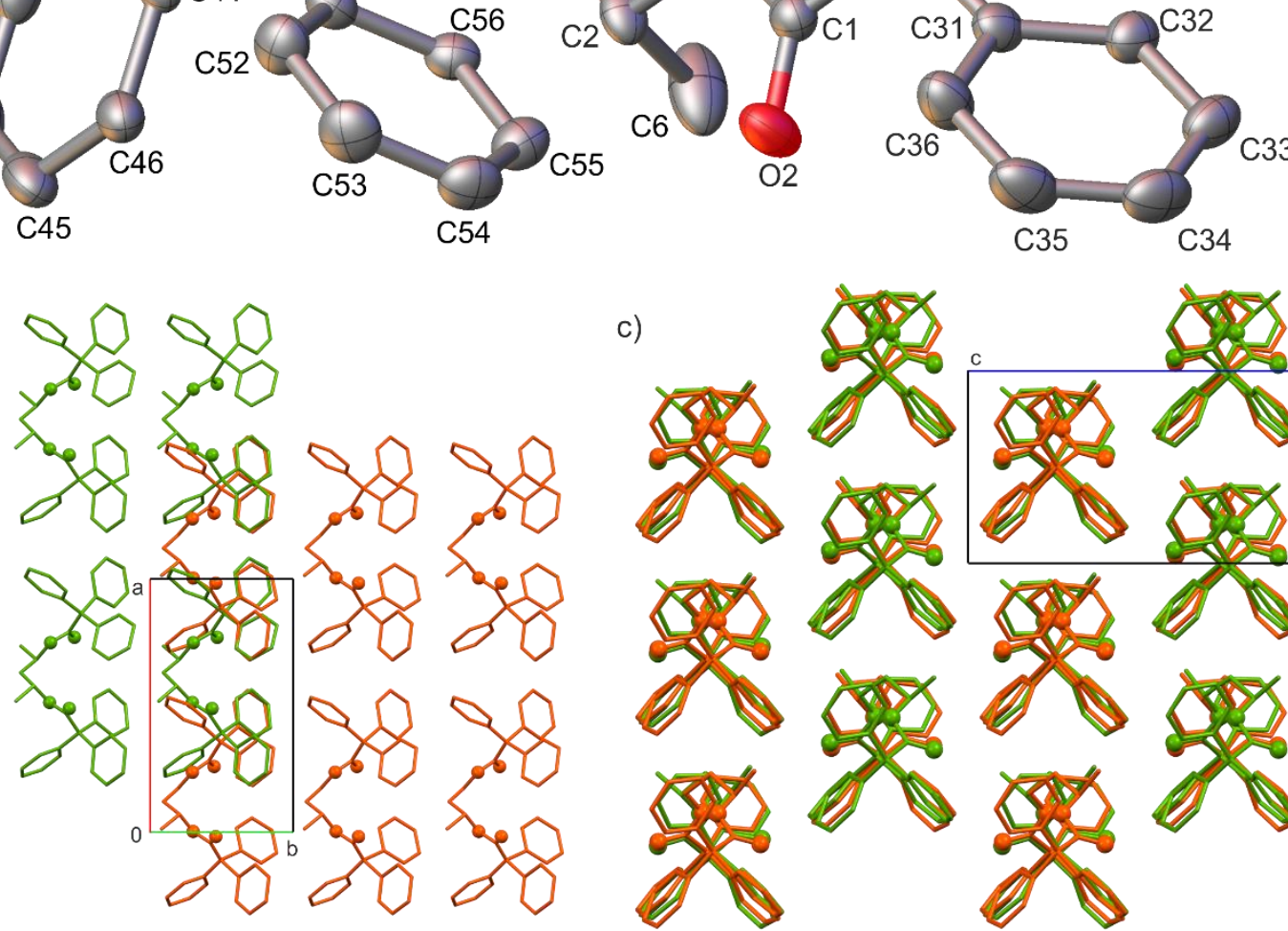

Figure SI_138. Molecular structure and atoms numbering scheme of compound 19. Displacement ellipsoids are drawn at $50 \%$ probability level. b) Scheme of molecular disorder in columns. c) Molecular packing in crystal structure, view along a axis. Hydrogen atoms are omitted for clarity and oxygen atoms are shown as balls. 
a)

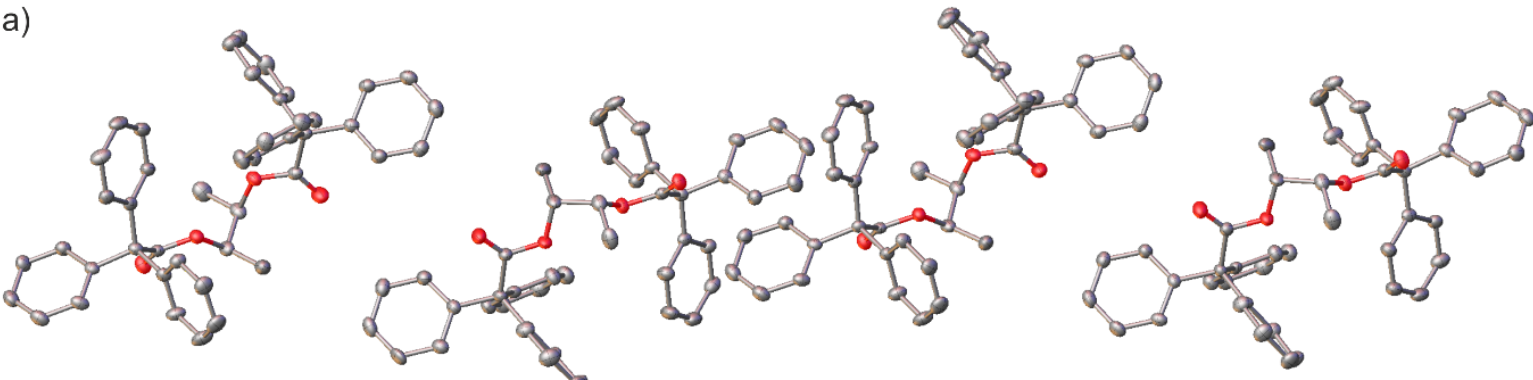

b)

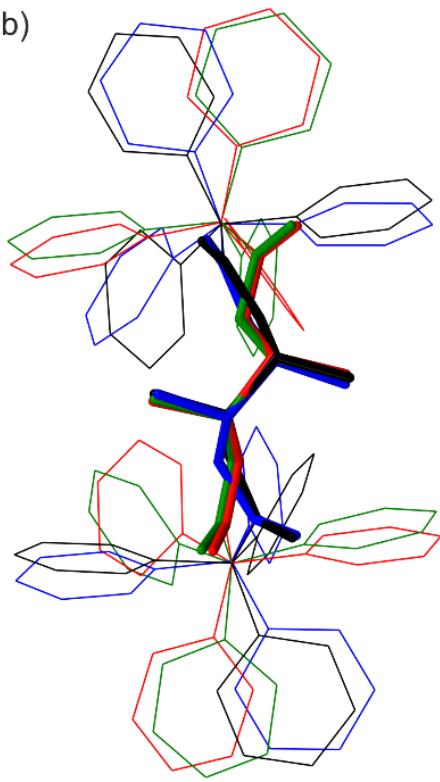

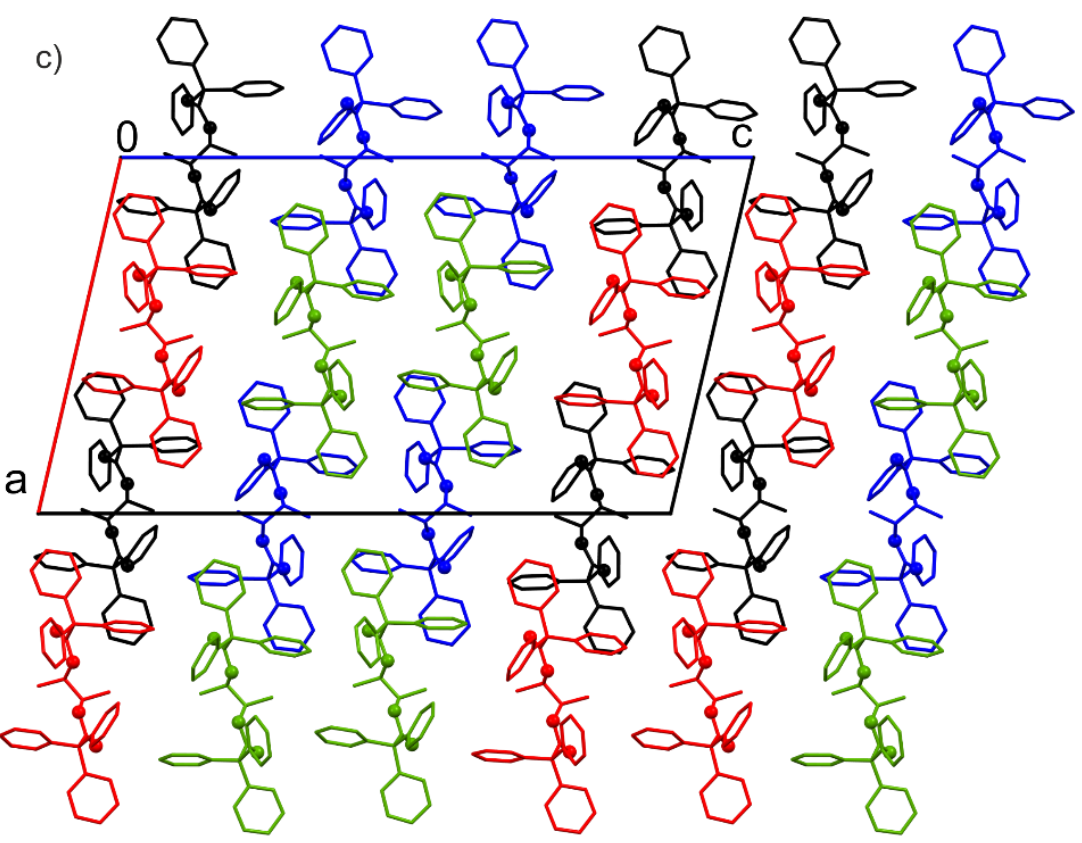

Figure SI_139. Molecular structure of asymmetric unit in crystal structure of compound 20. Displacement ellipsoids are drawn at $50 \%$ probability level. b) Comparison molecular conformation of symmetrically independent molecules. c) Molecular packing in crystal structure -molecules A + B and C + D forms alternating double layers. Hydrogen atoms are omitted for clarity and oxygen atoms are shown as balls. 
a)

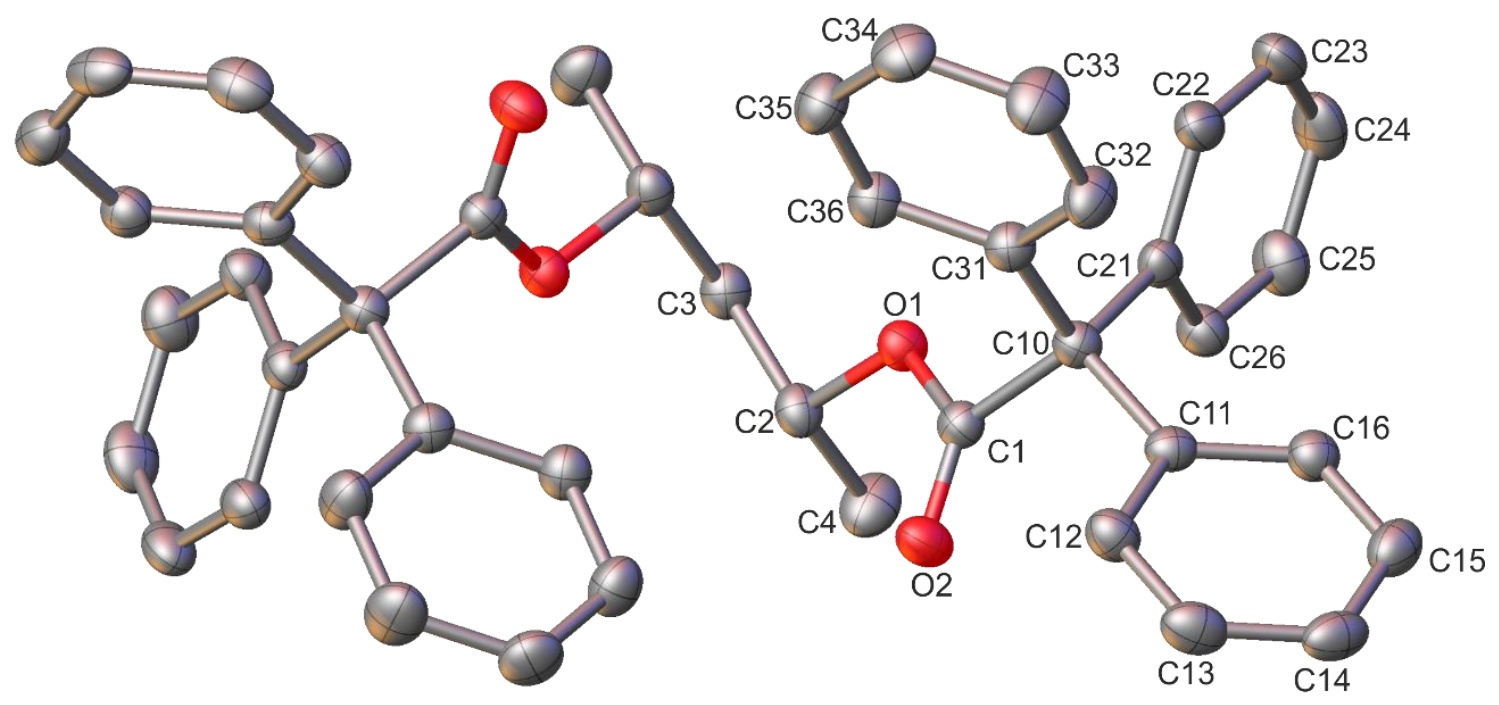

b)

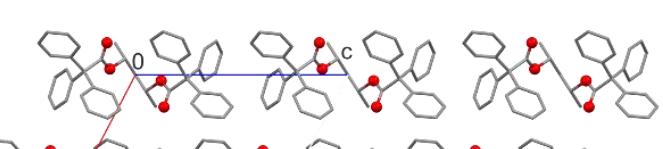
828984898980

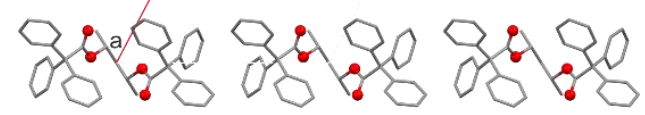

c)
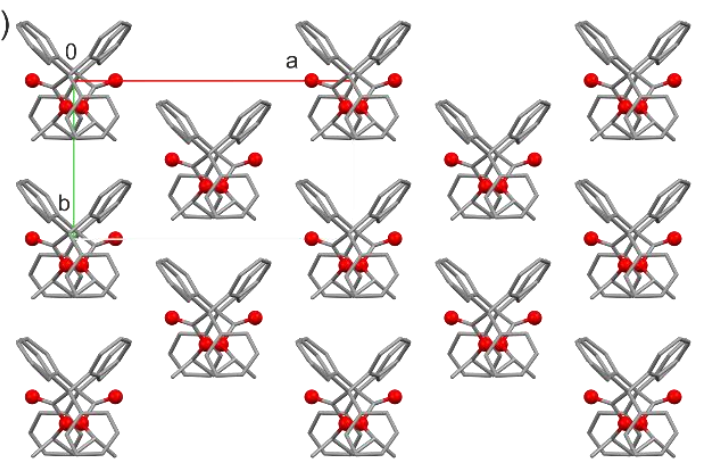

Figure SI_140. a) Molecular structure and atoms numbering scheme of compound 21. Only the asymmetric part has been numbered for clarity. Displacement ellipsoids are drawn at $50 \%$ probability level. Molecular packing in crystal structure b) view along b axis and c) view along $c$ axis. Hydrogen atoms are omitted for clarity and oxygen atoms are shown as balls. 
Copies of ${ }^{1} \mathrm{H}$ and ${ }^{13} \mathrm{C}$ NMR spectra 
NPTrO7 19.10.fid

PROTON CDCl3 \{C:|IconNMR|prusin\} prusin 1

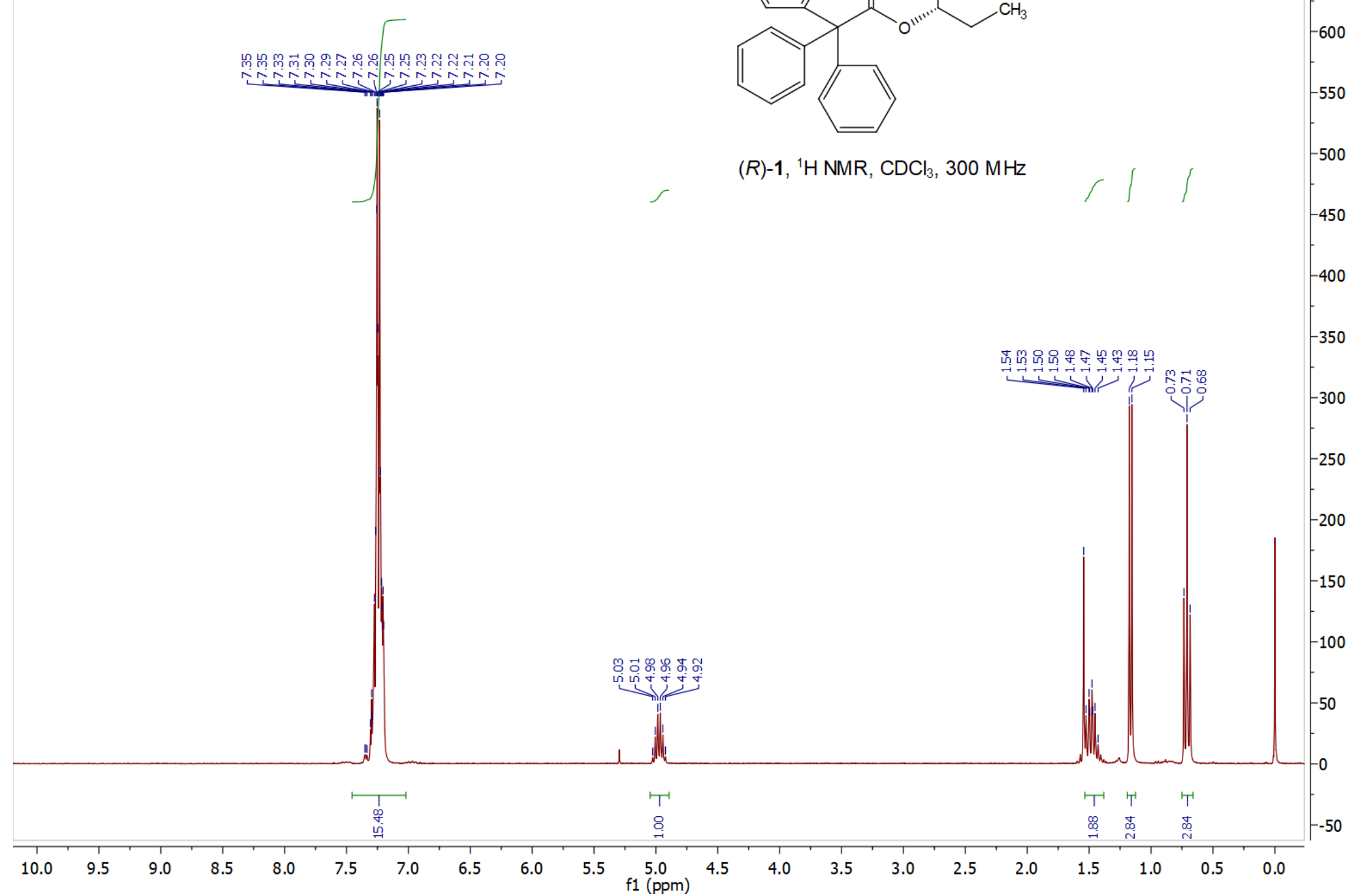

$-650$ 
NP07_19_C.10.fid

C13CPD_512 CDCl3 \{C:|IconNMR $\mid$ Stereochemia_org $\}$ Stereochemia_org 2

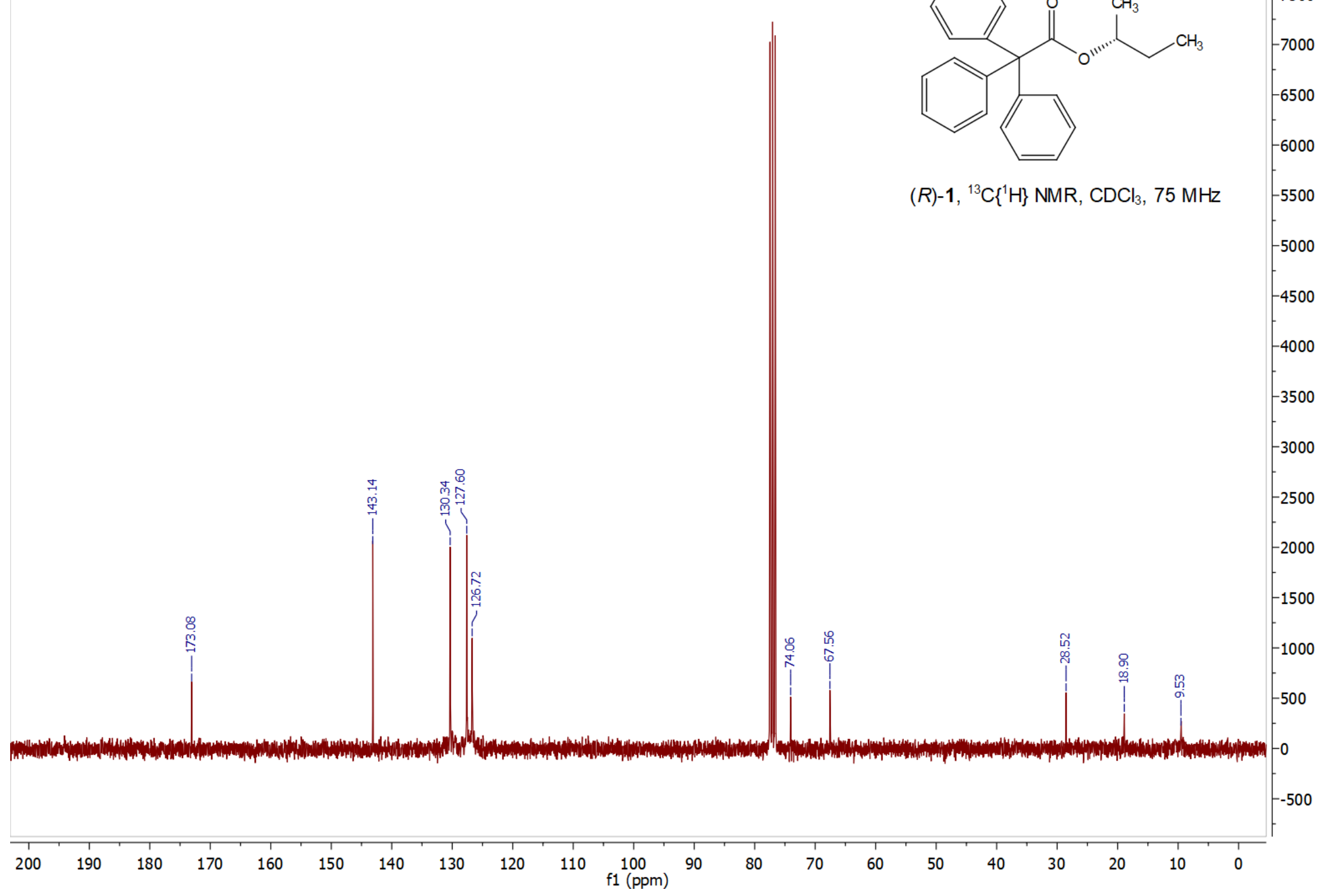




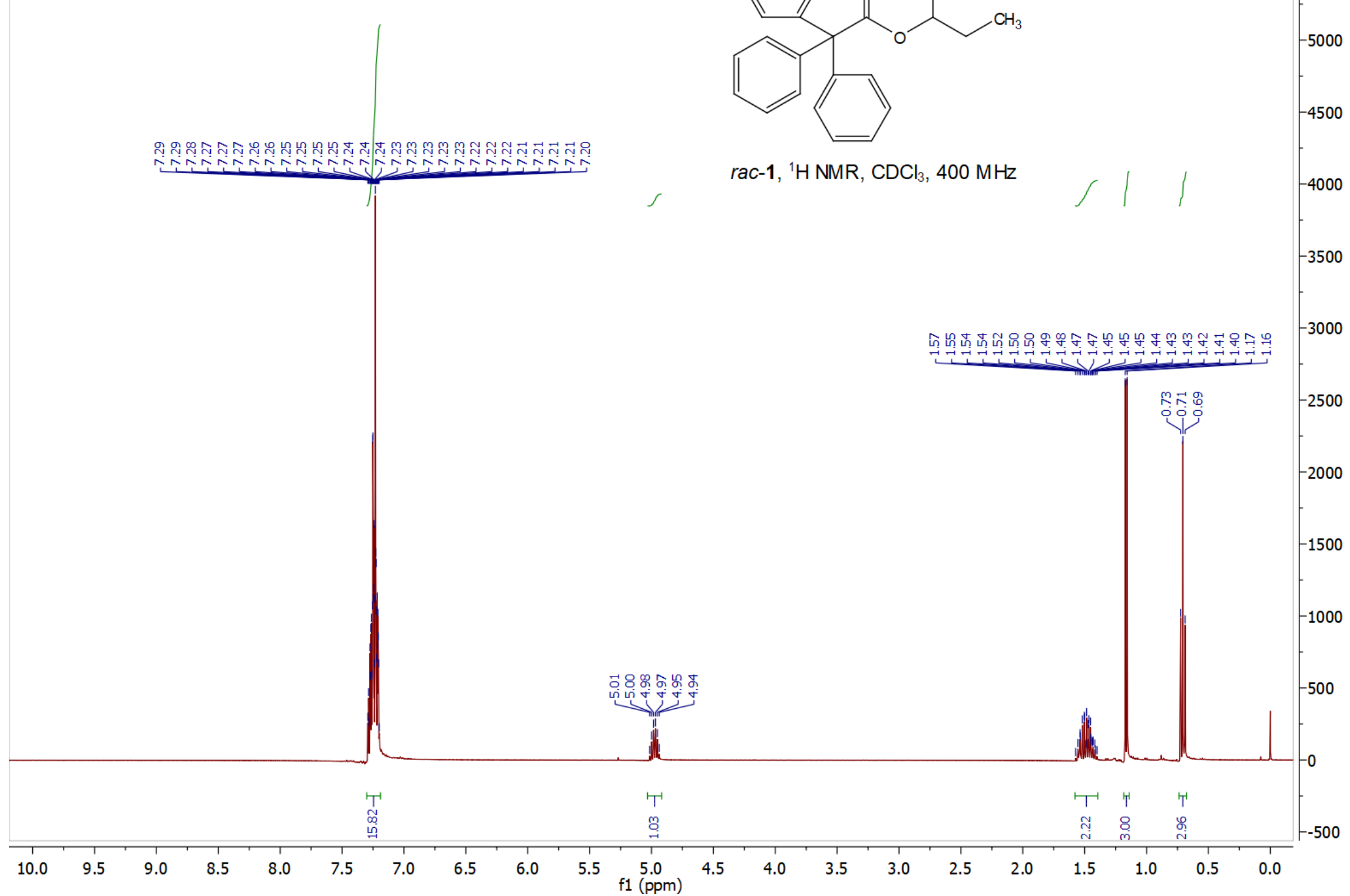




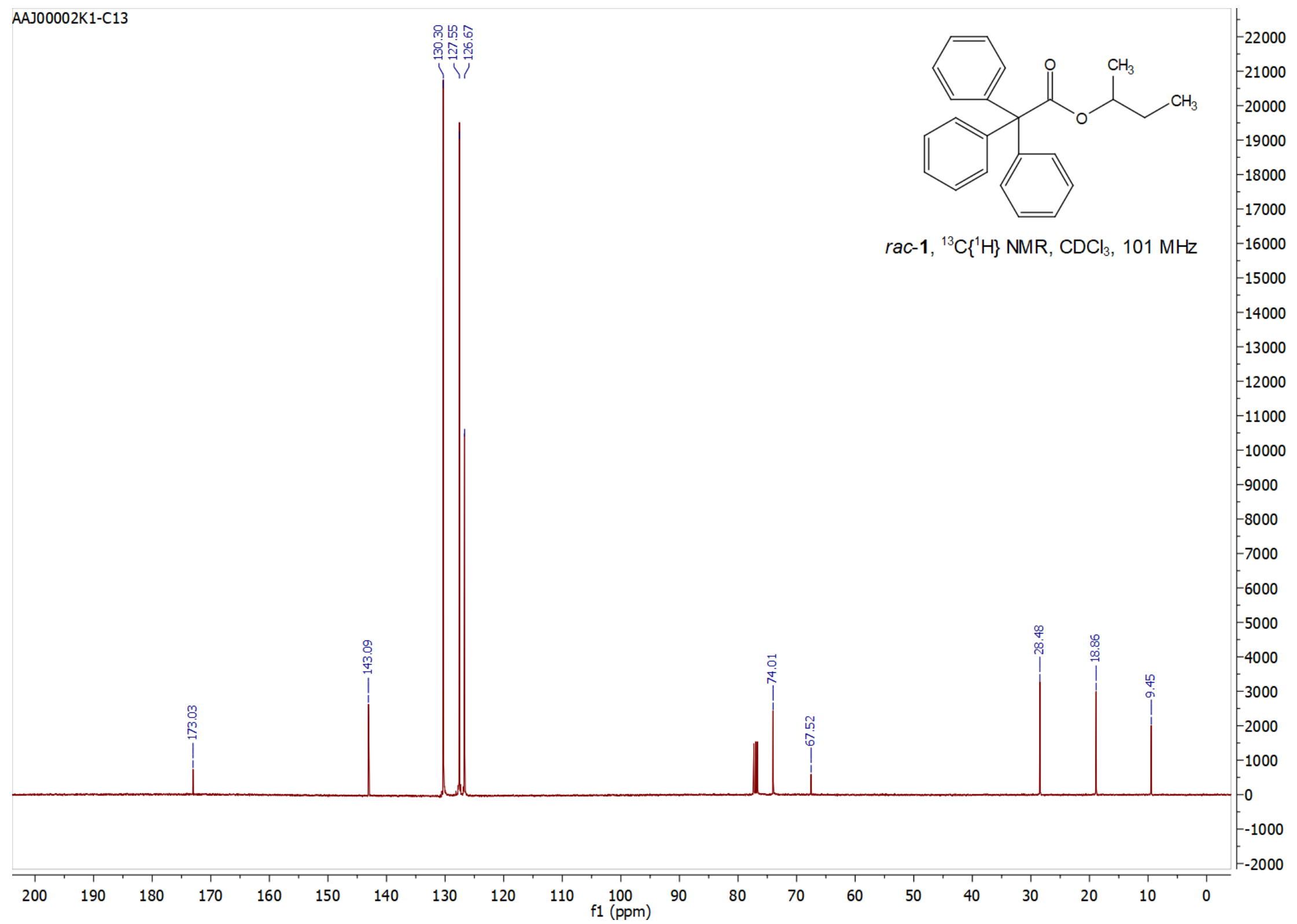


NPTr15_19.10.fid

PROTON CDCI3 \{C:|IconNMR|Stereochemia_org\} Stereochemia_org 11

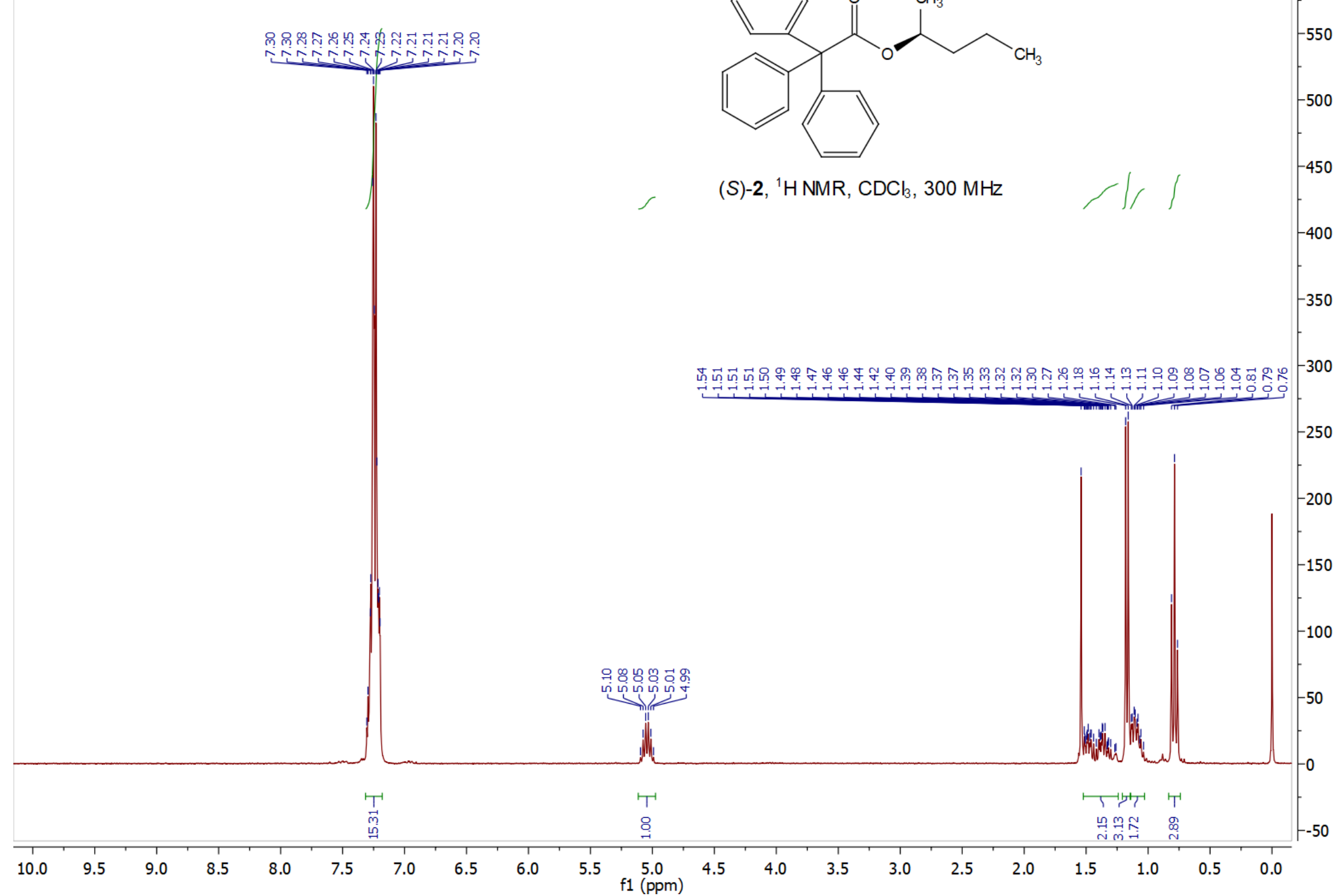


NPTr15_19_C.10.fid

C13CPD_512 CDCl3 \{C:|IconNMR $\mid$ Stereochemia_org $\}$ Stereochemia_org 7

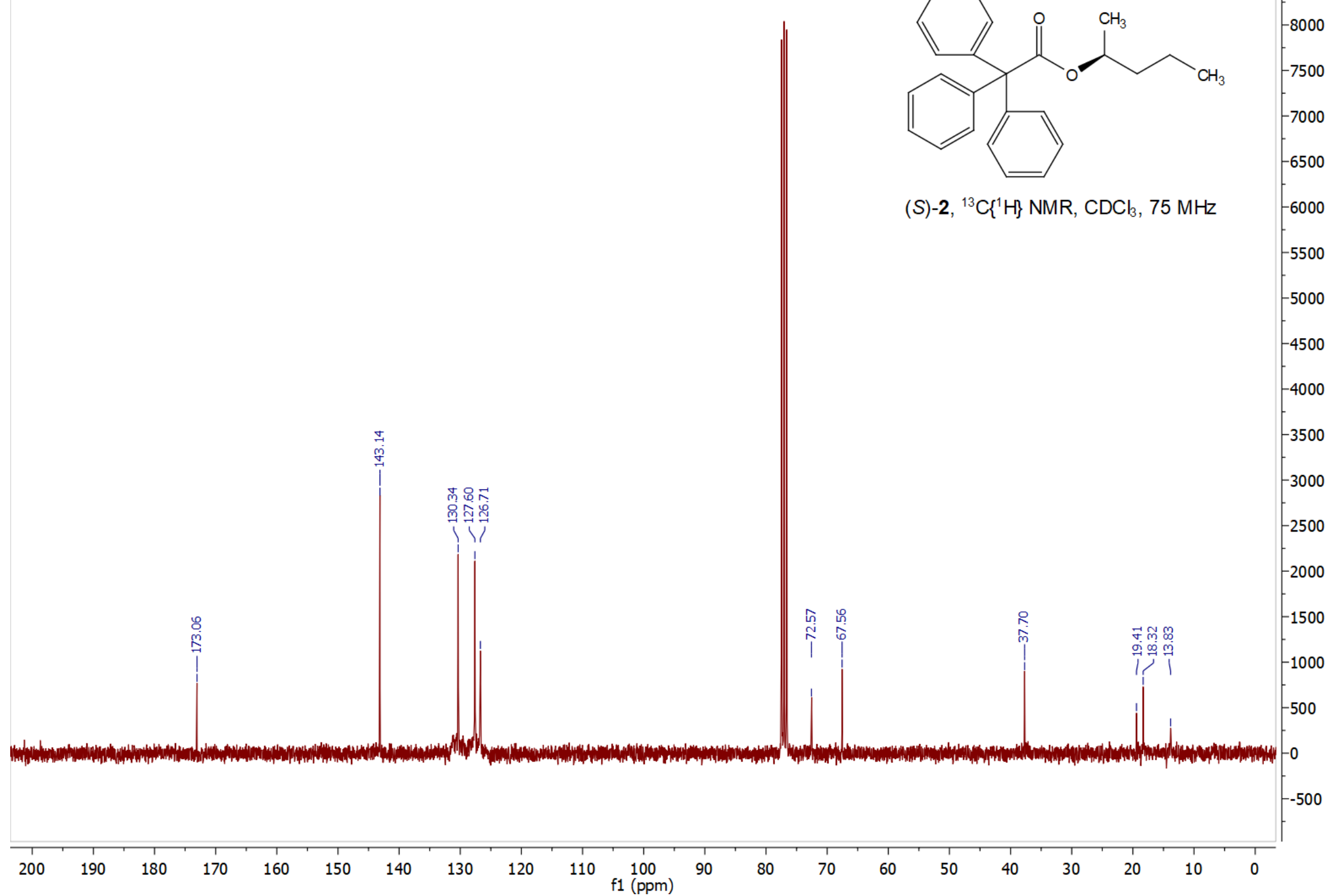


NPTr10_19.10.fid

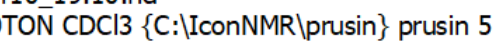

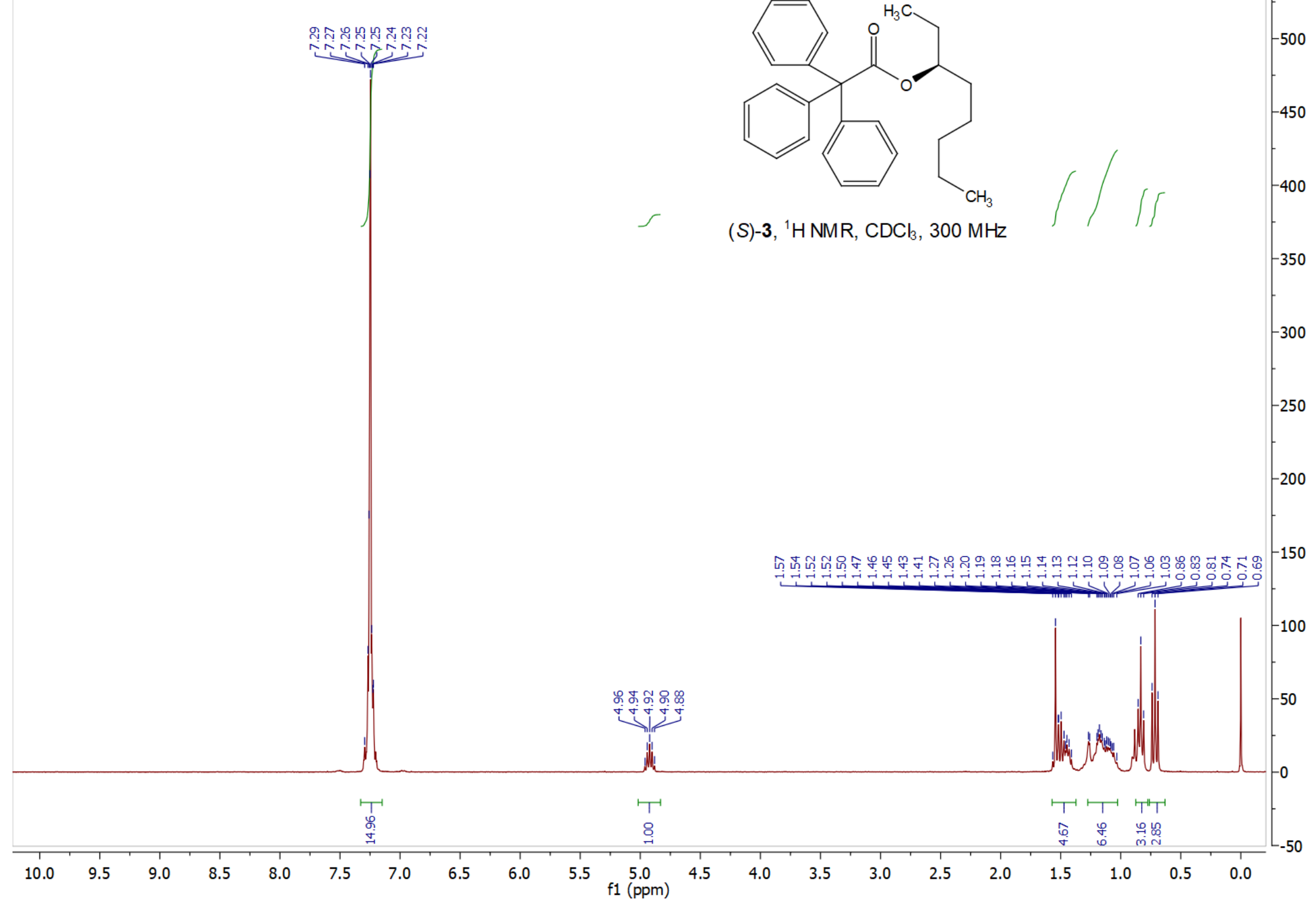




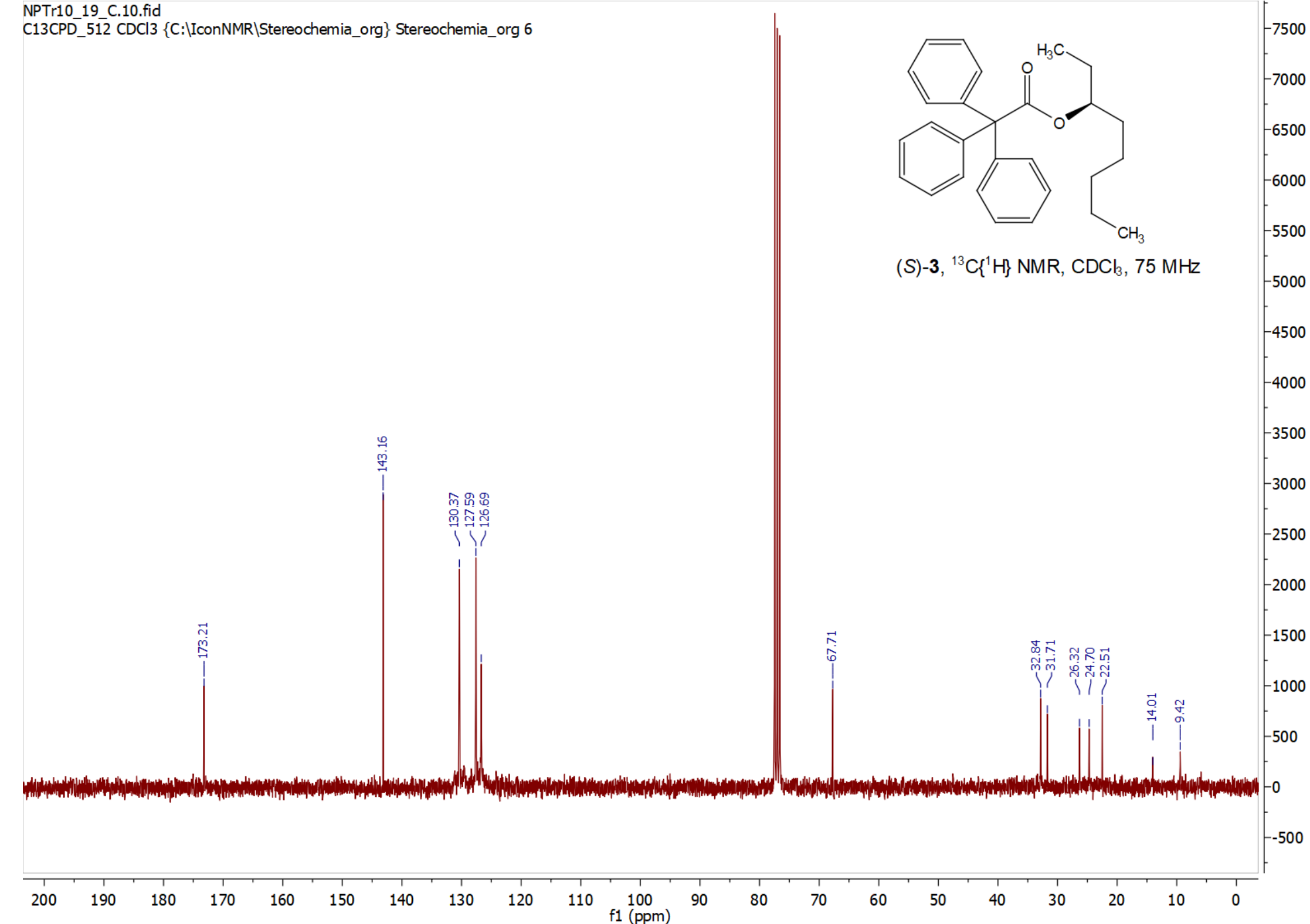

$-7500$ 


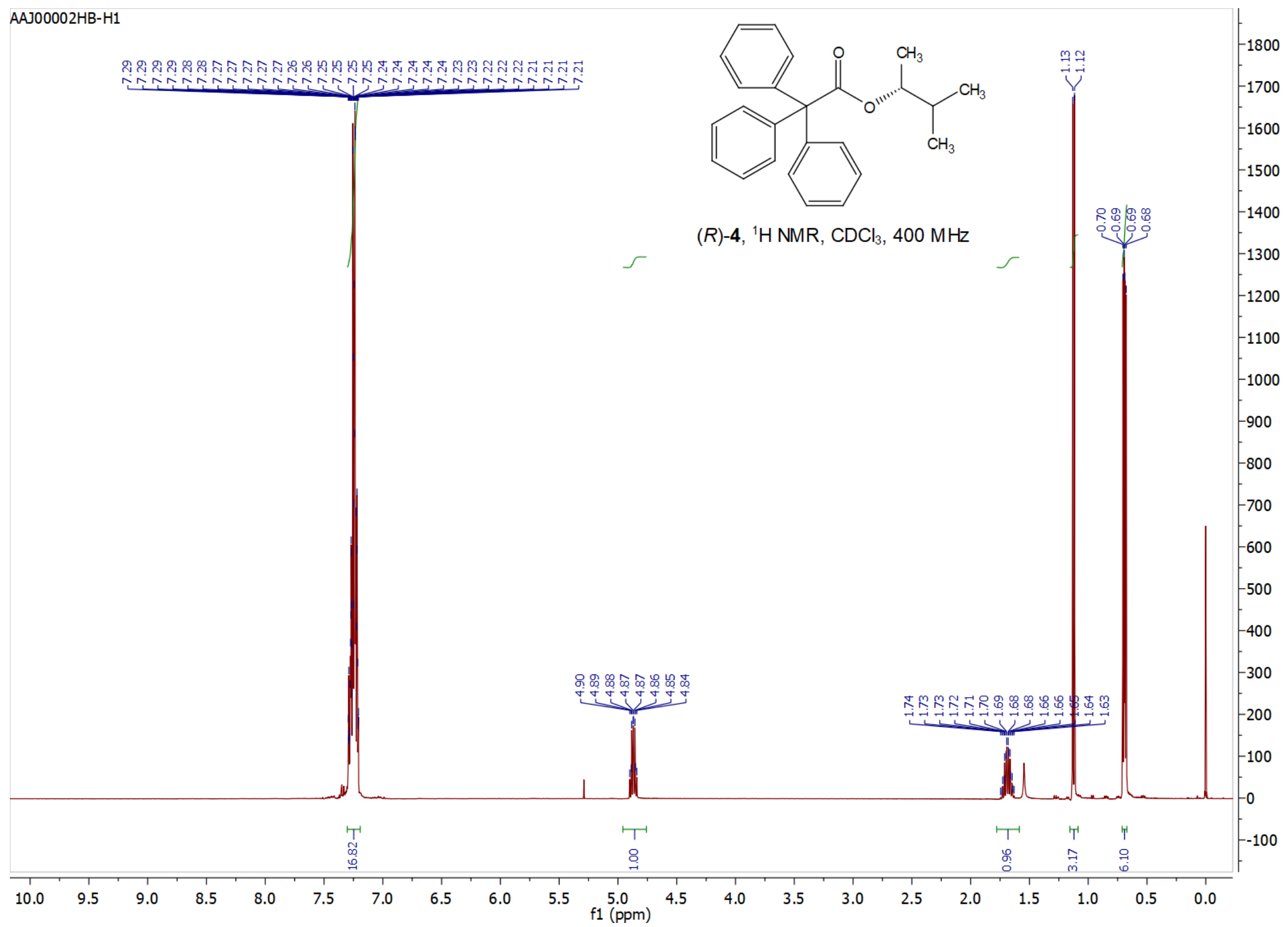




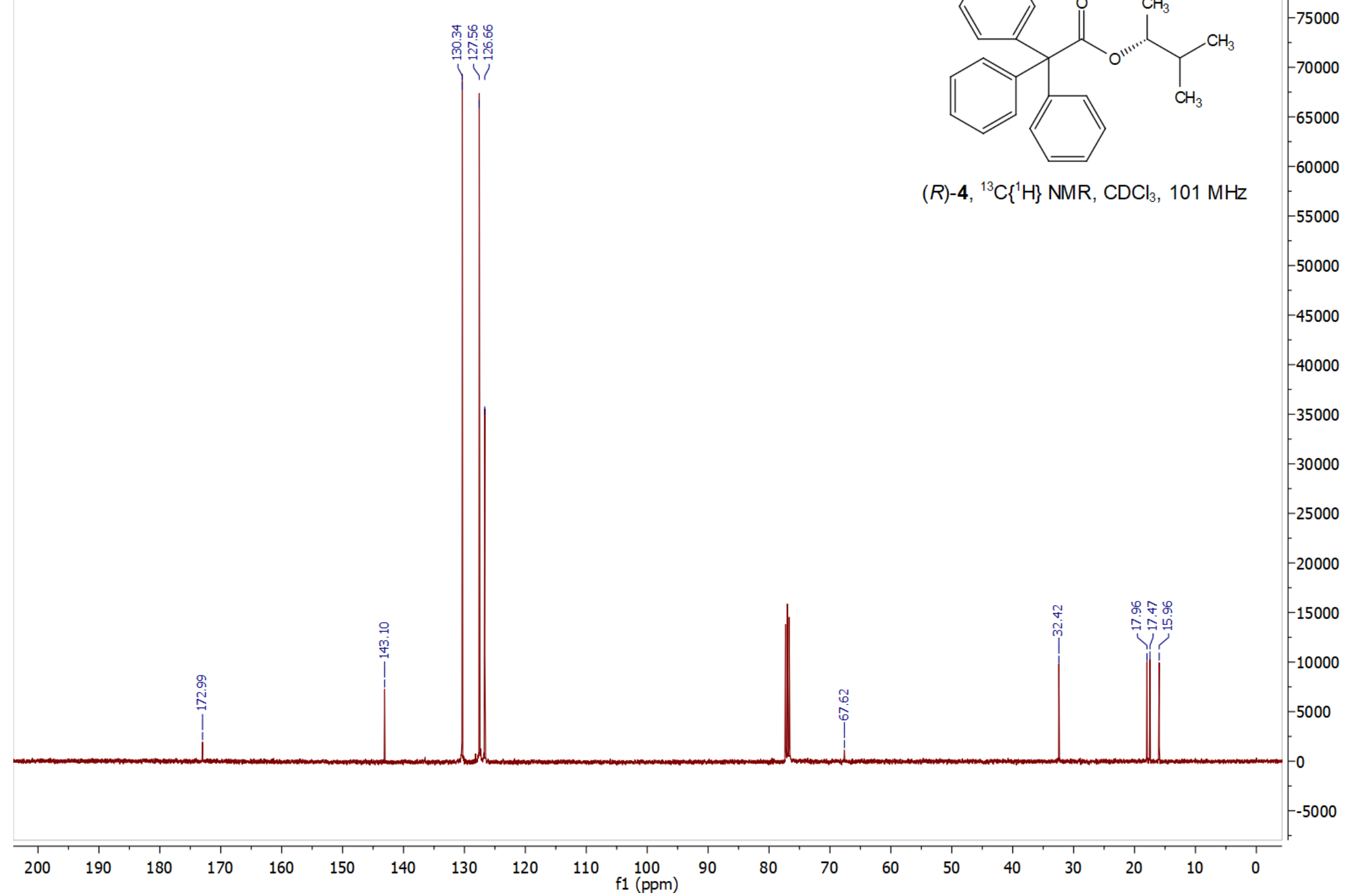




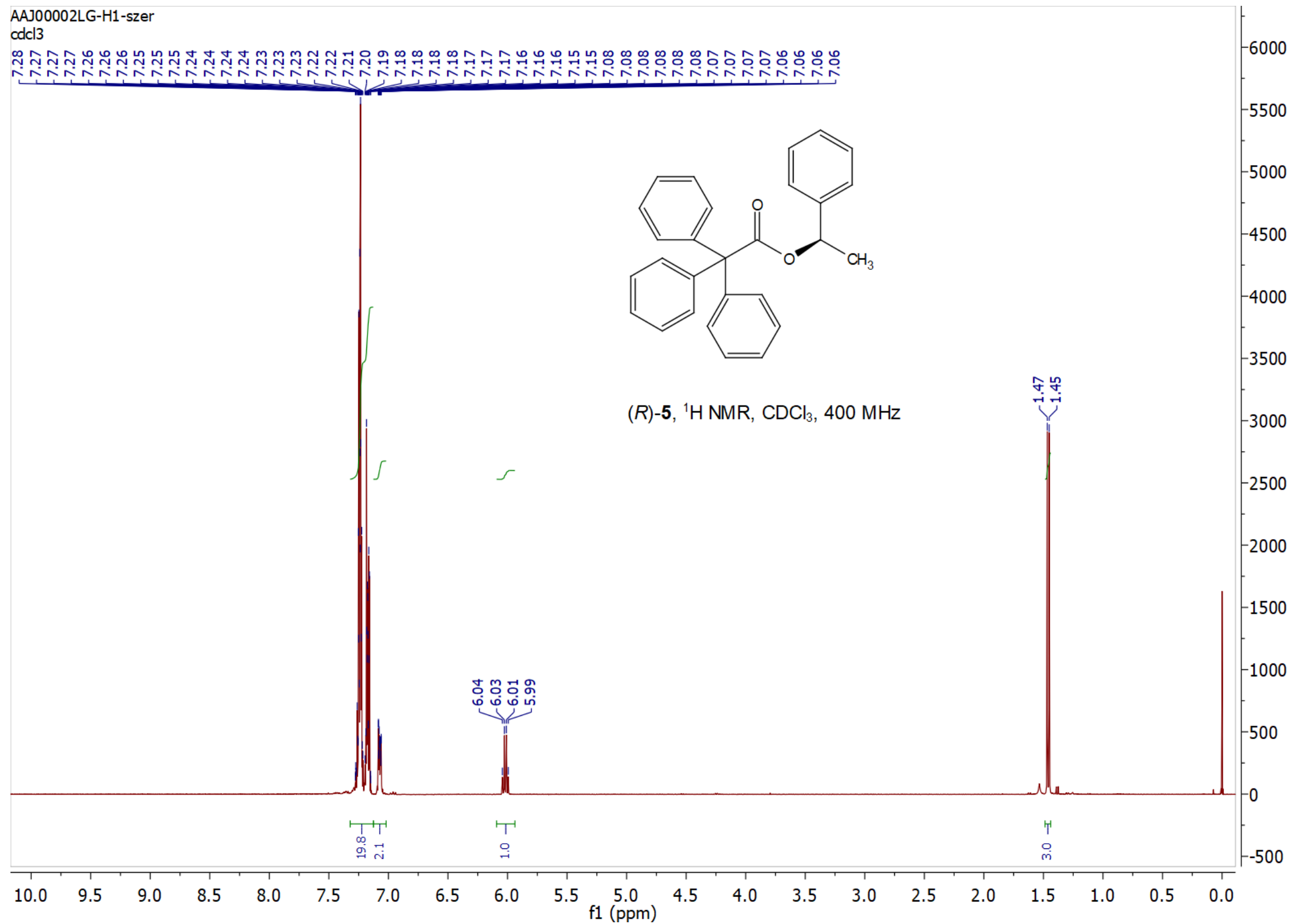




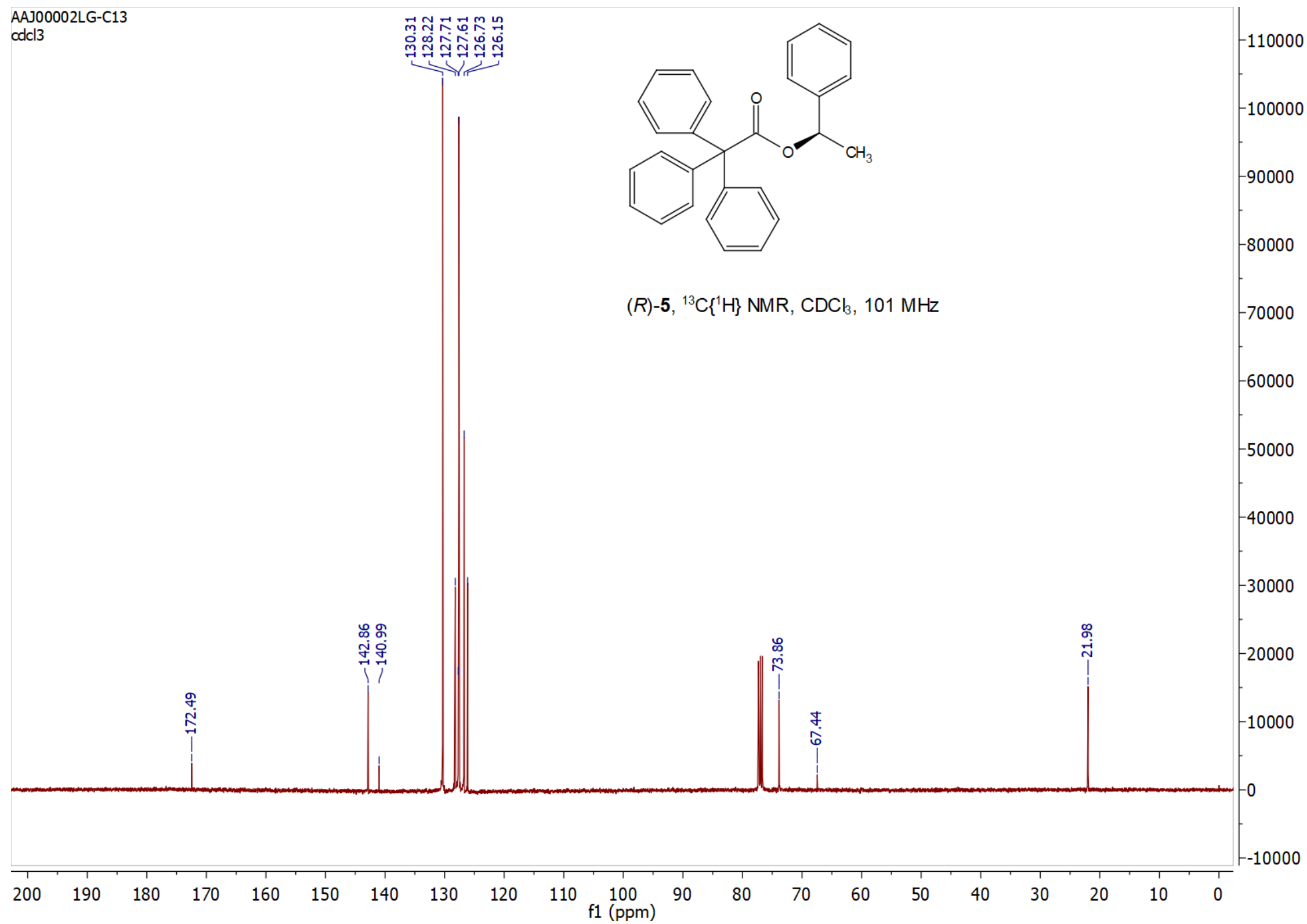


AAJ00002JY-szer

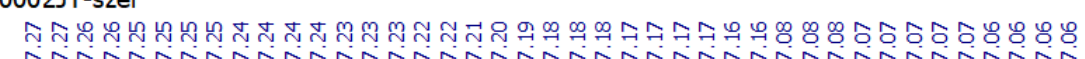

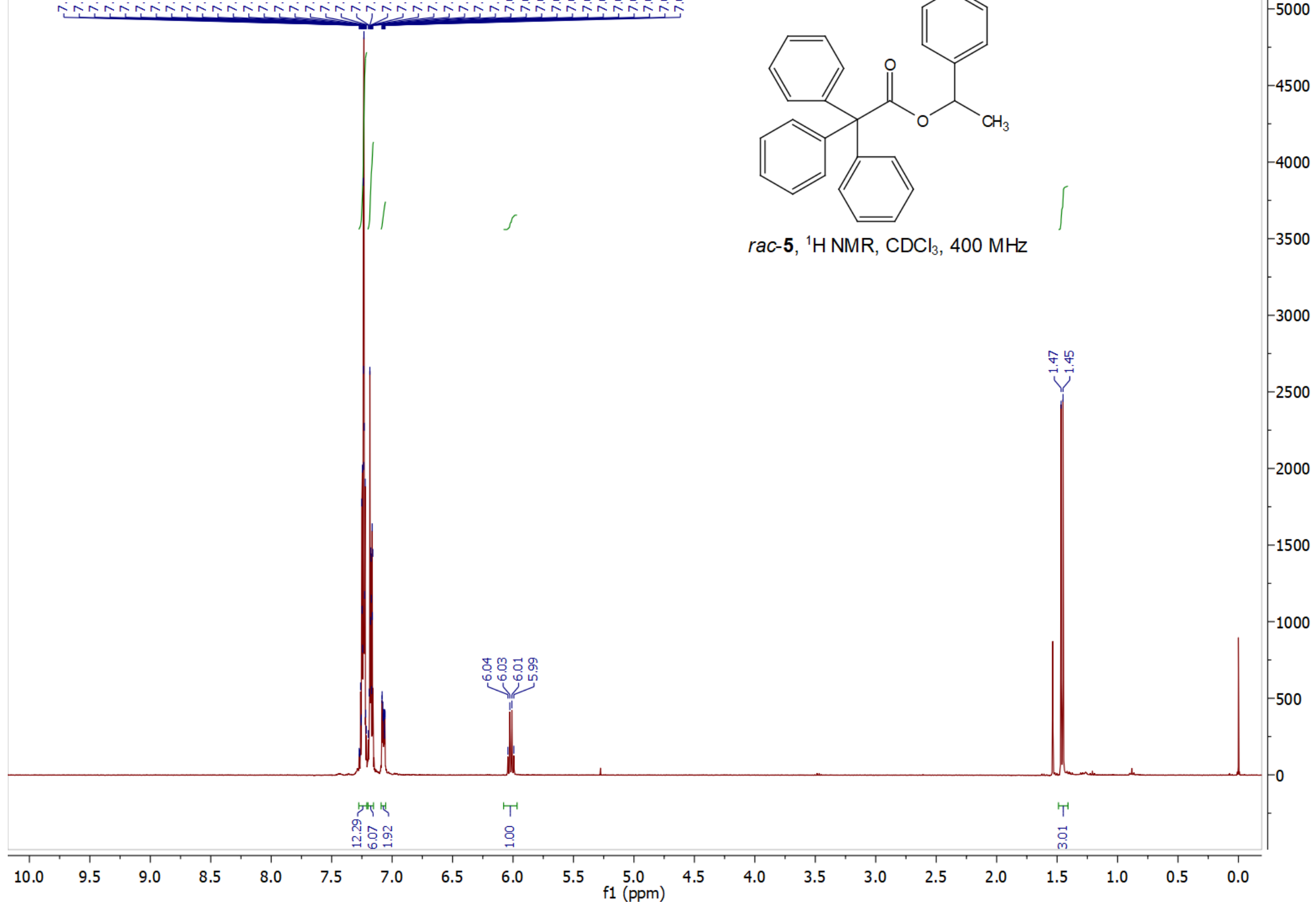




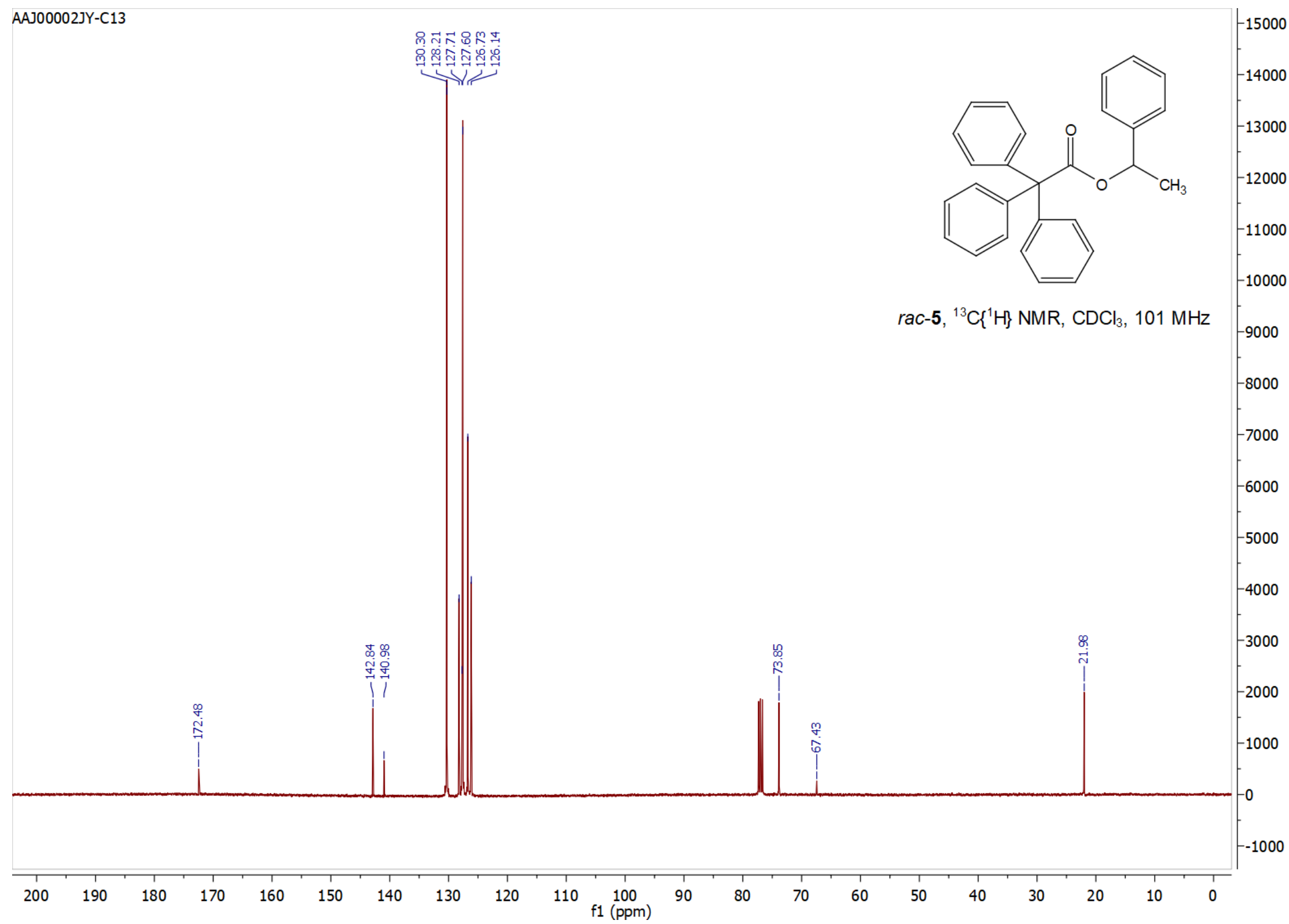




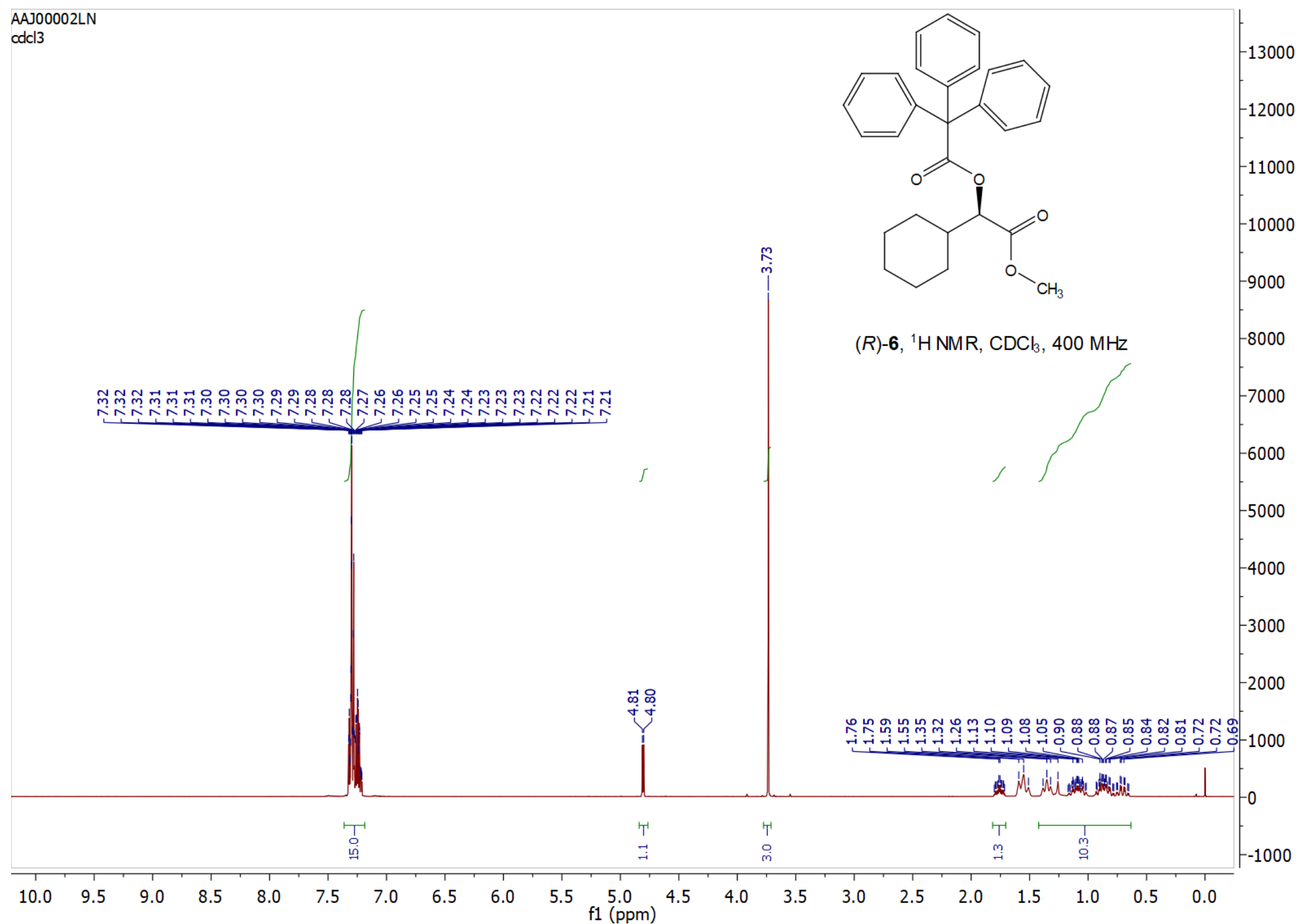




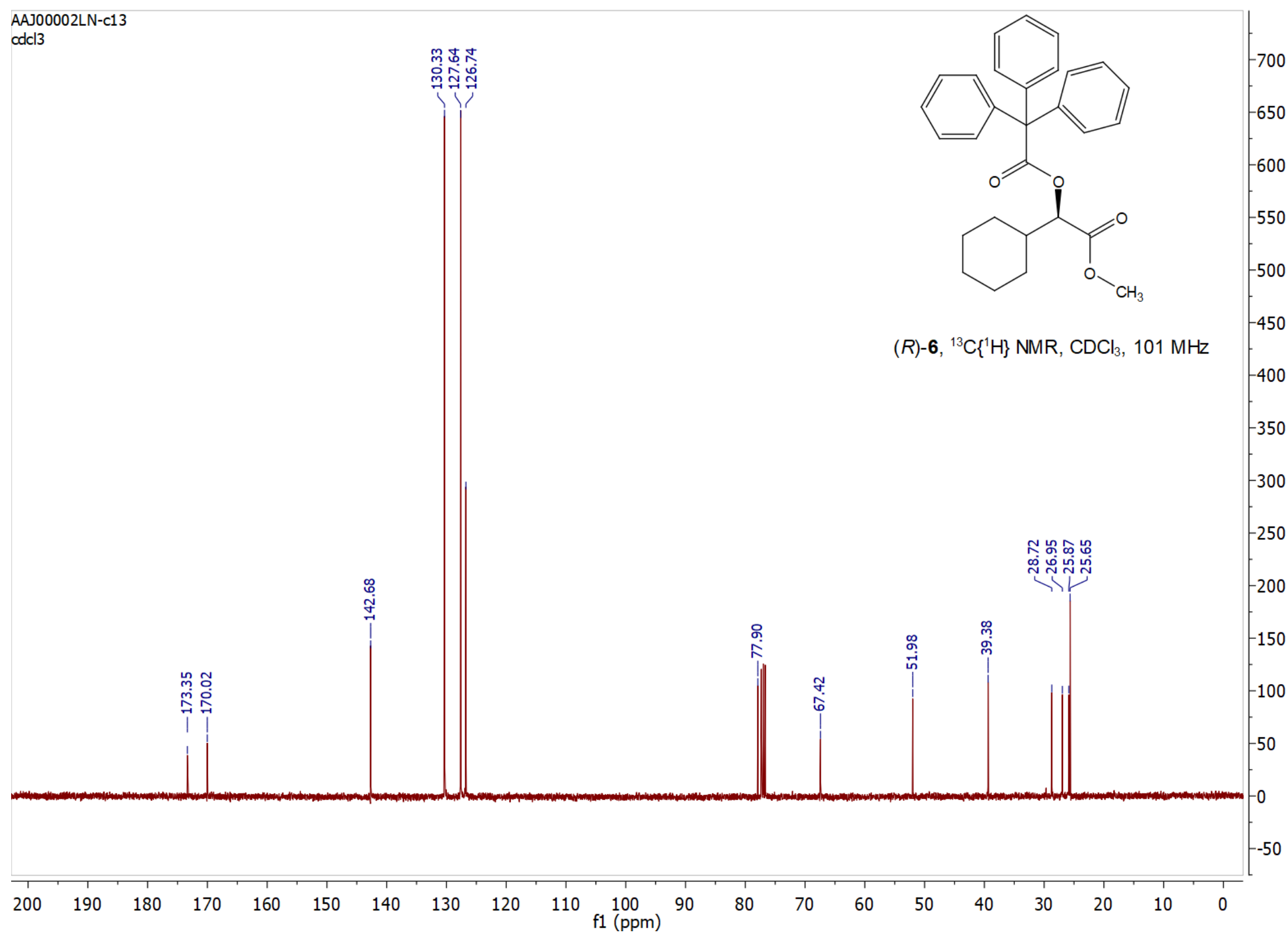




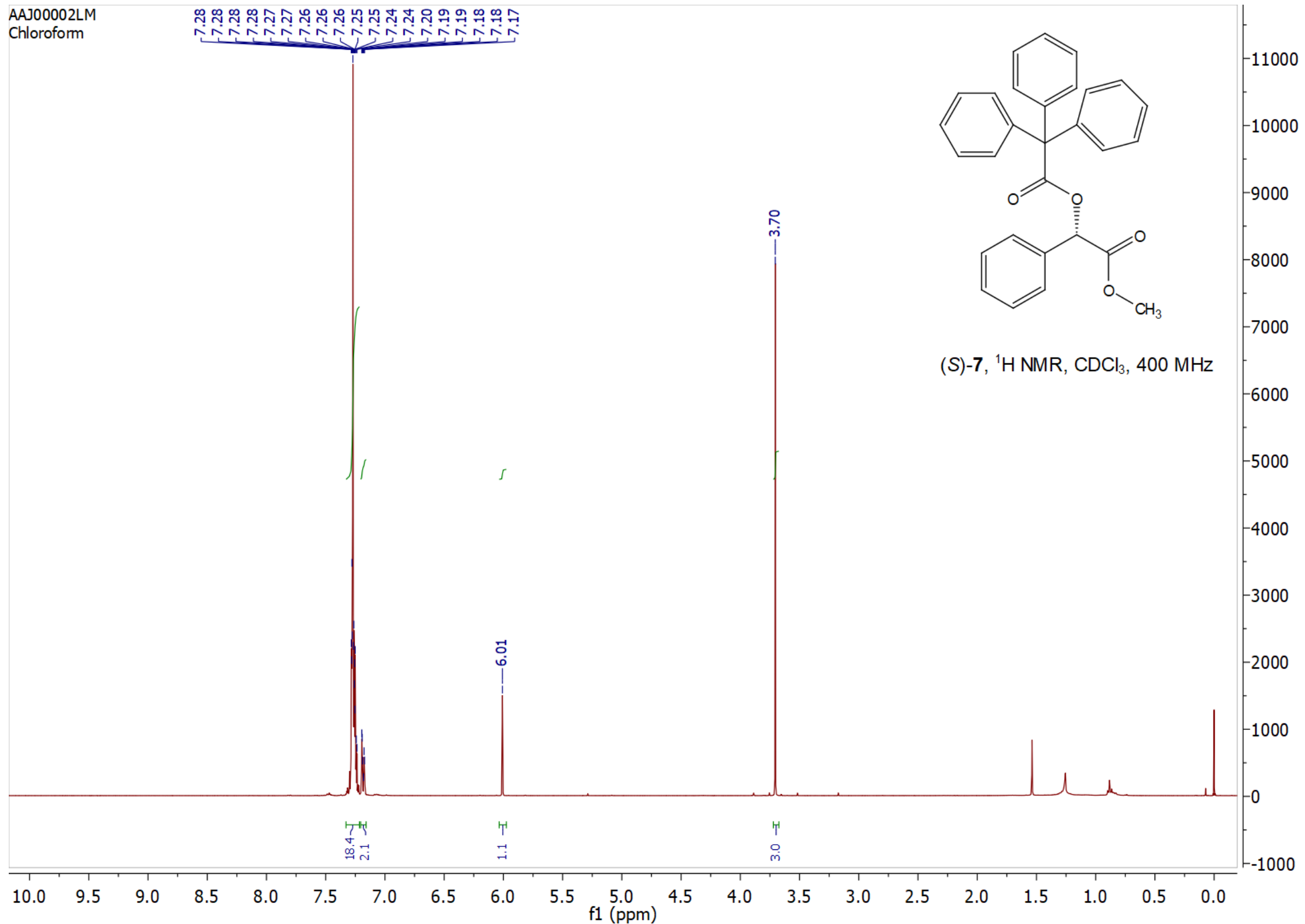




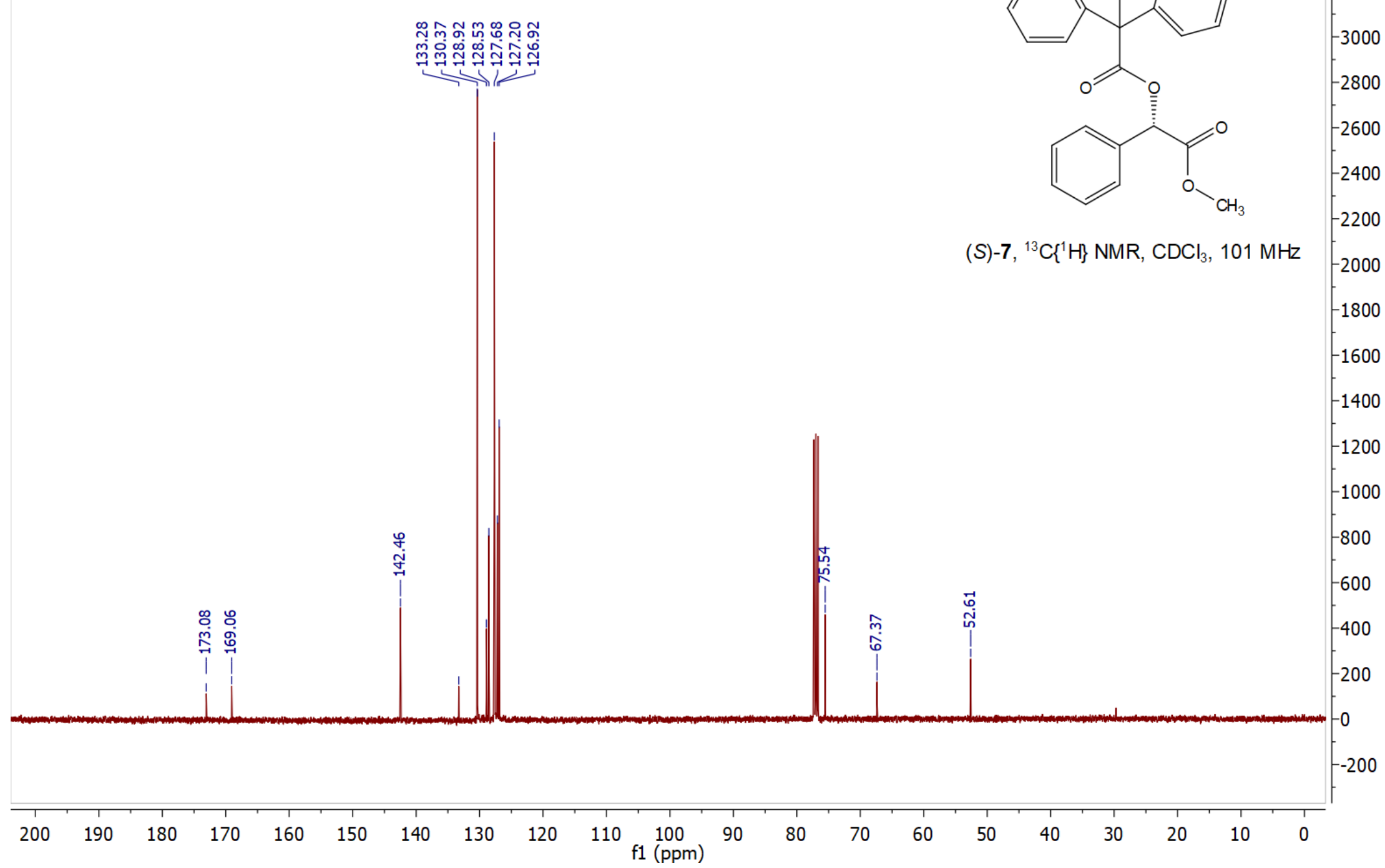


NPTr12_19.10.fid

PROTON CDCl3 \{C:|IconNMR|prusin $\}$ prusin 16

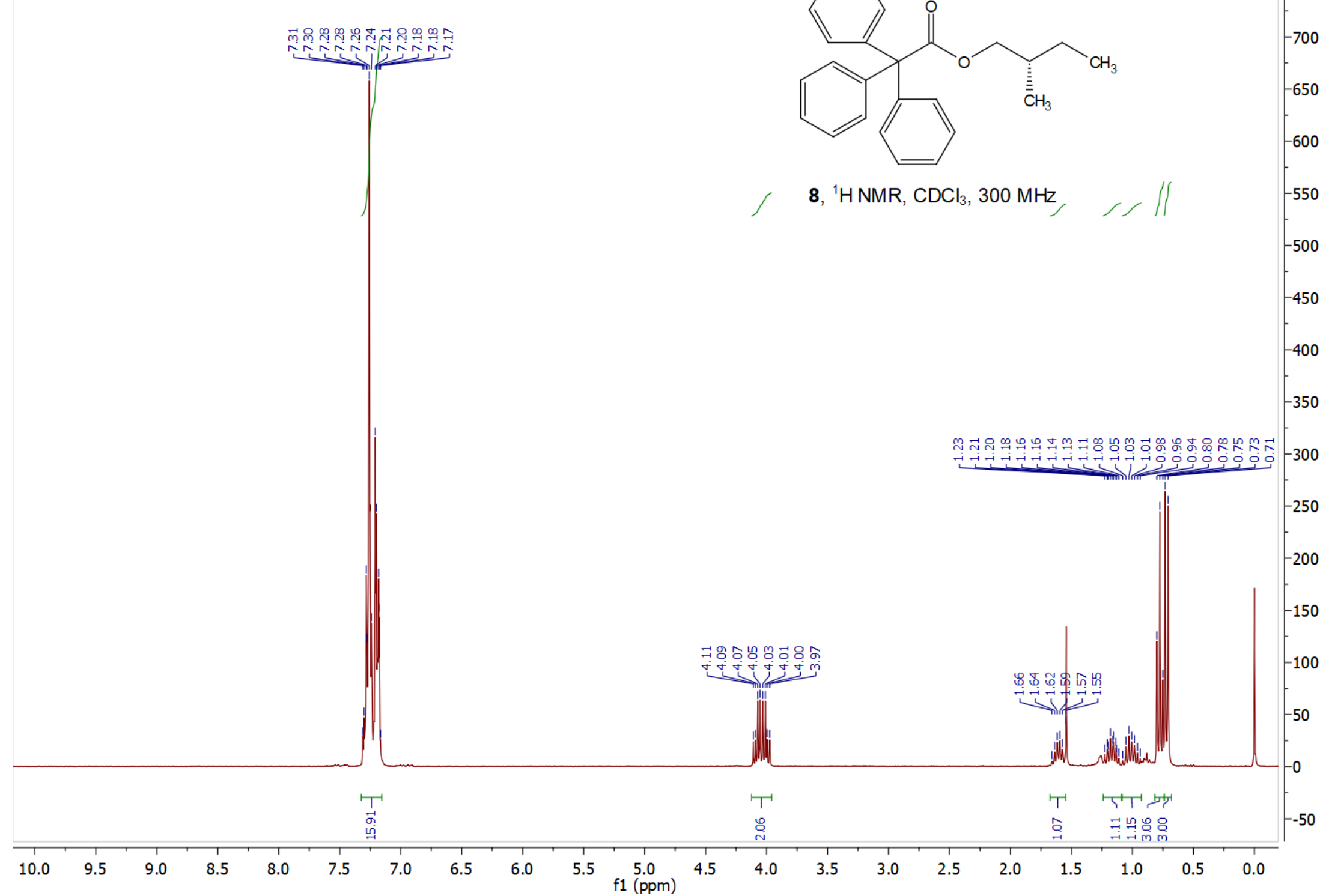




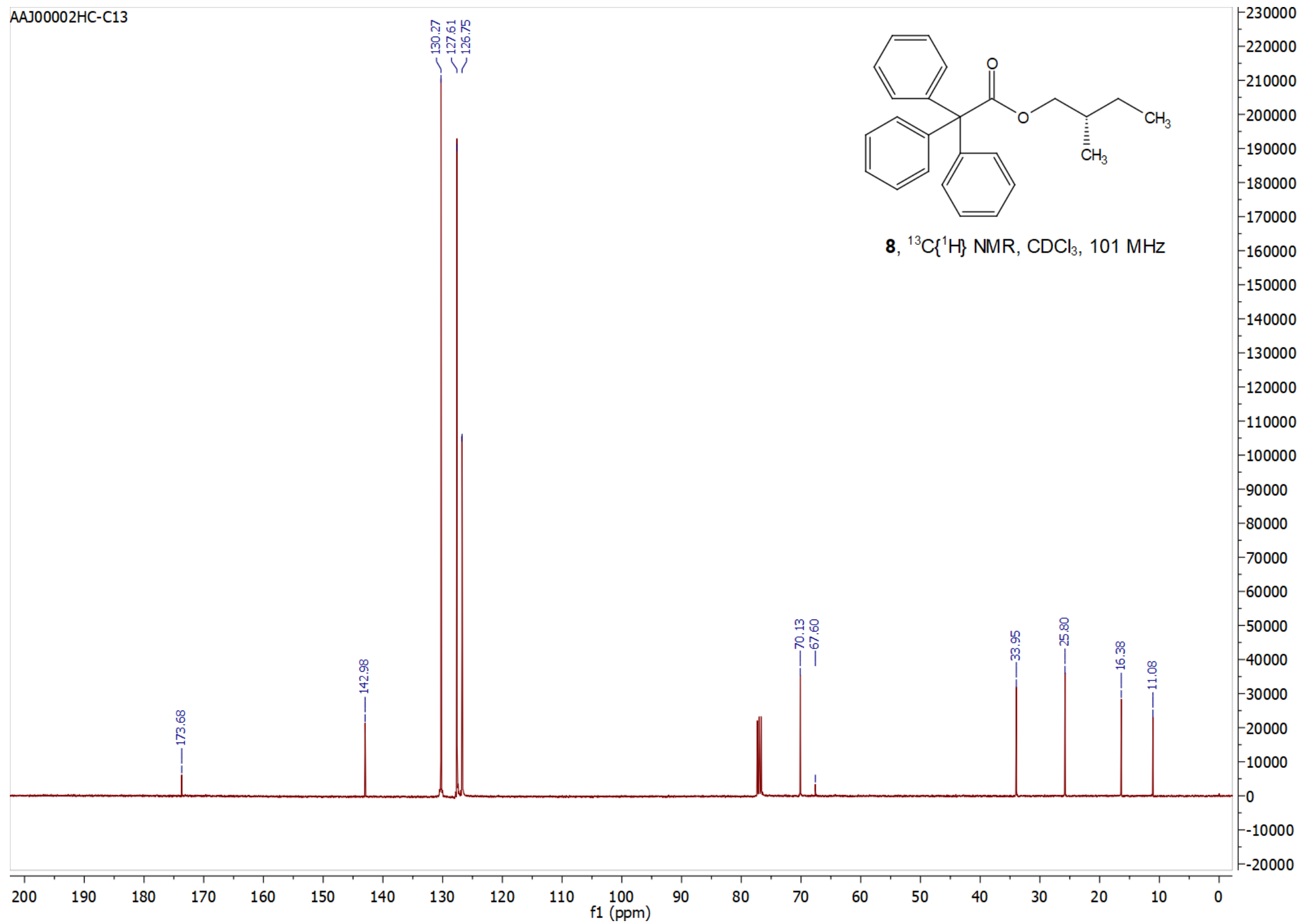


AAJ00002LH-H1

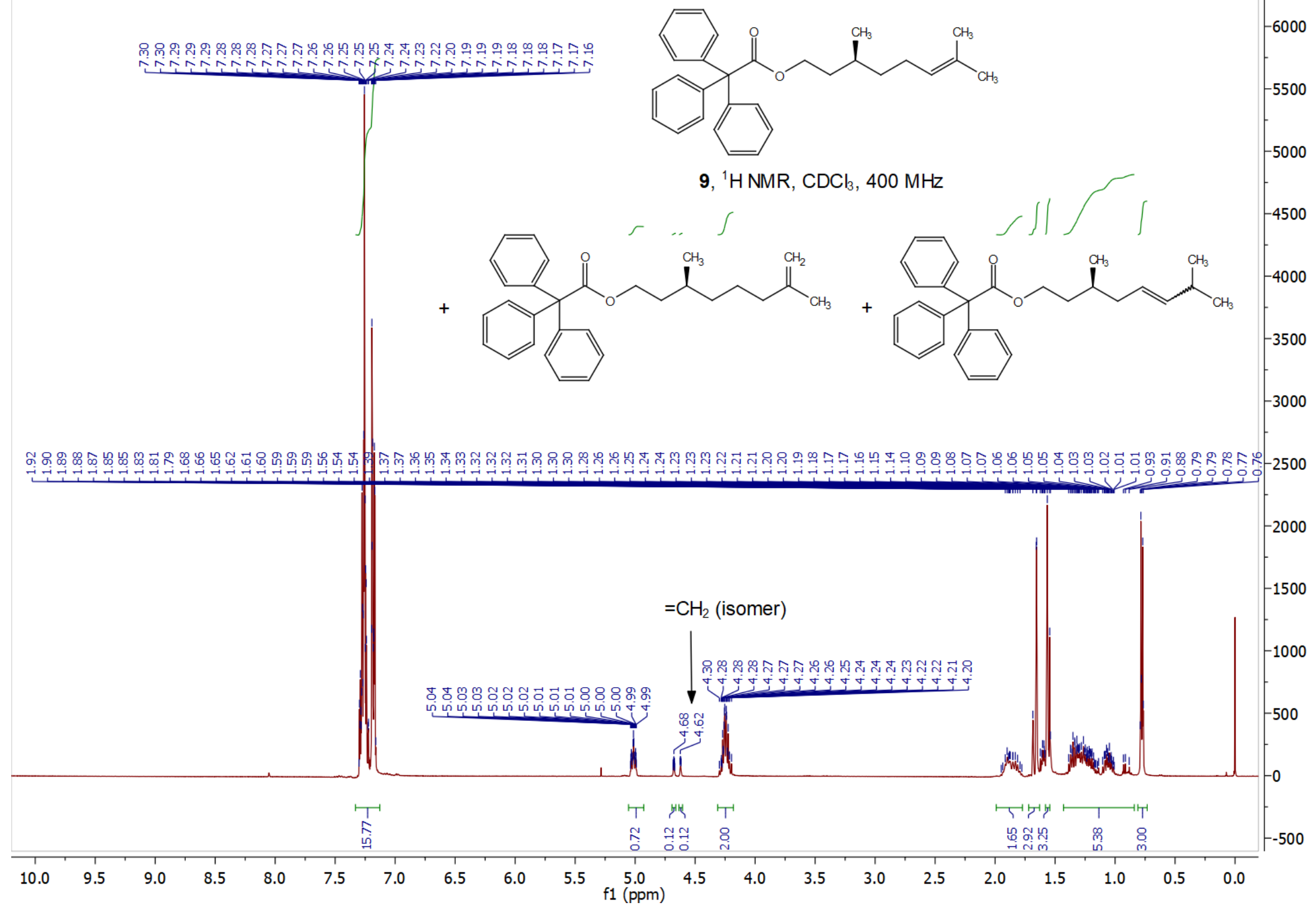




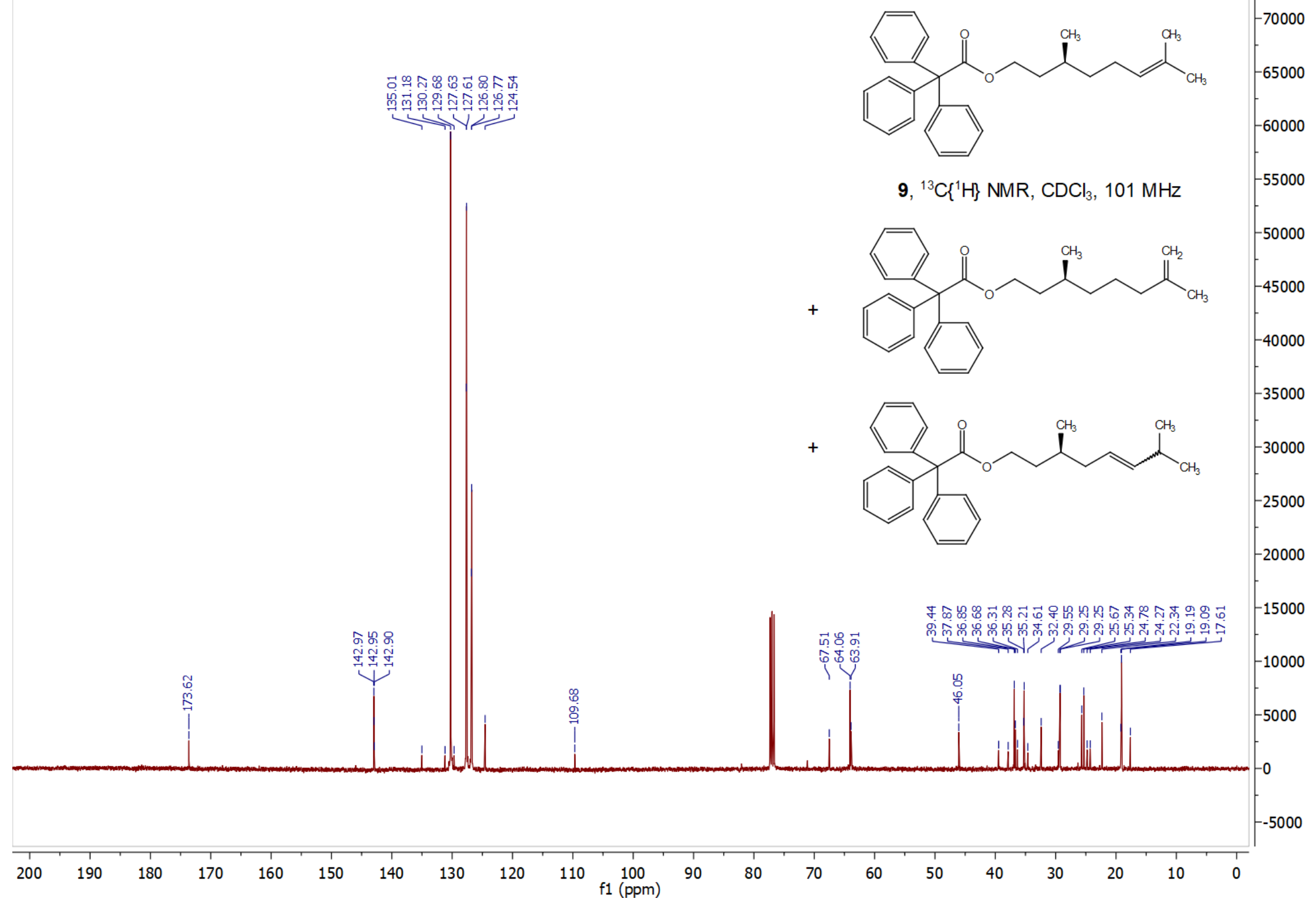


NPTr05_19.10.fid

PROTON CDCl3 \{C:|IconNMR|prusin\} prusin 14

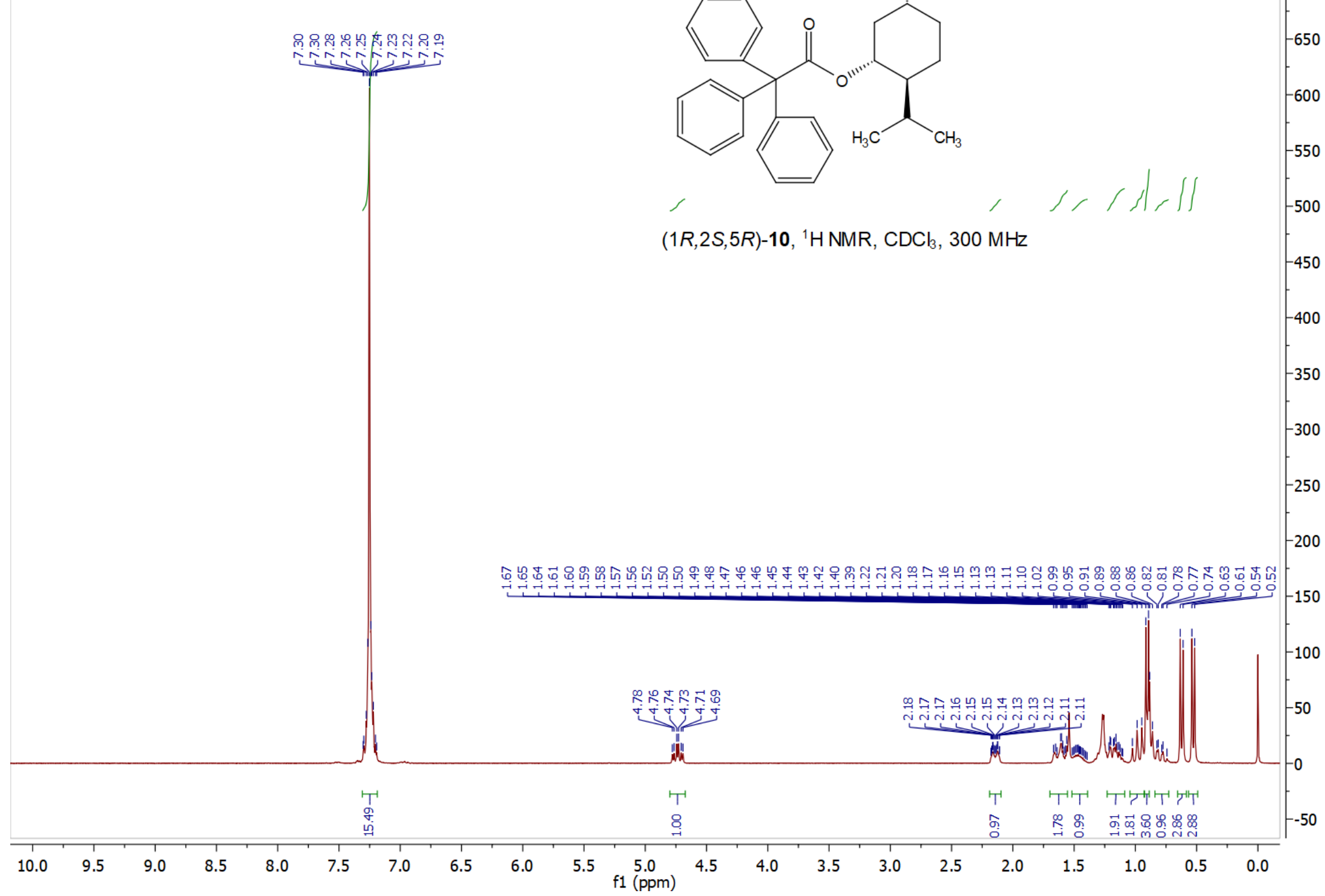


NPTr05_19_C.10.fid

C13CPD_512 CDCl3 $\{\mathrm{C}: \mid$ IconNMR $\mid$ Stereochemia_org $\}$ Stereochemia_org 10

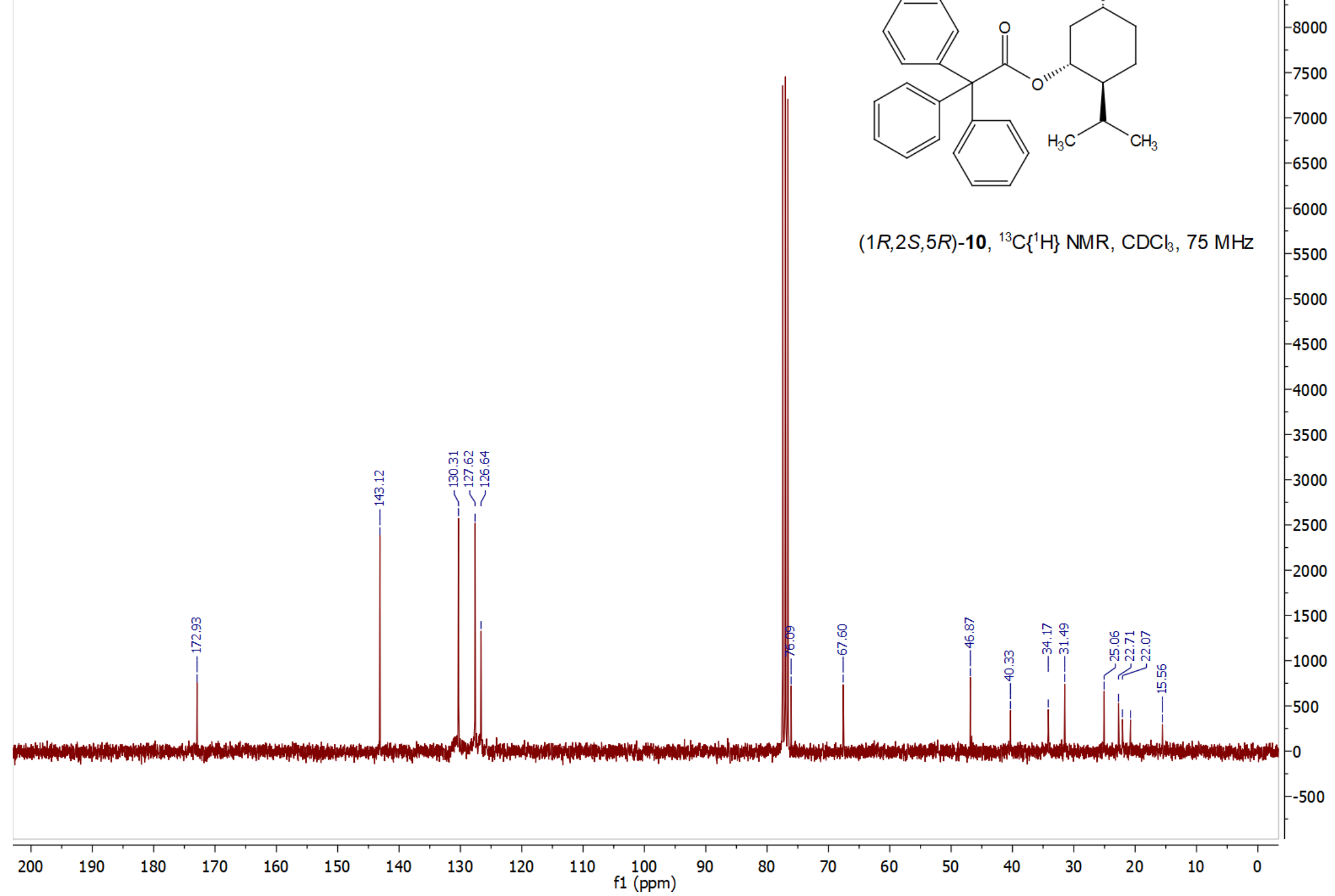


NPTr09_19.10.fid

PROTON CDCl3 \{C:|IconNMR|prusin\} prusin 15

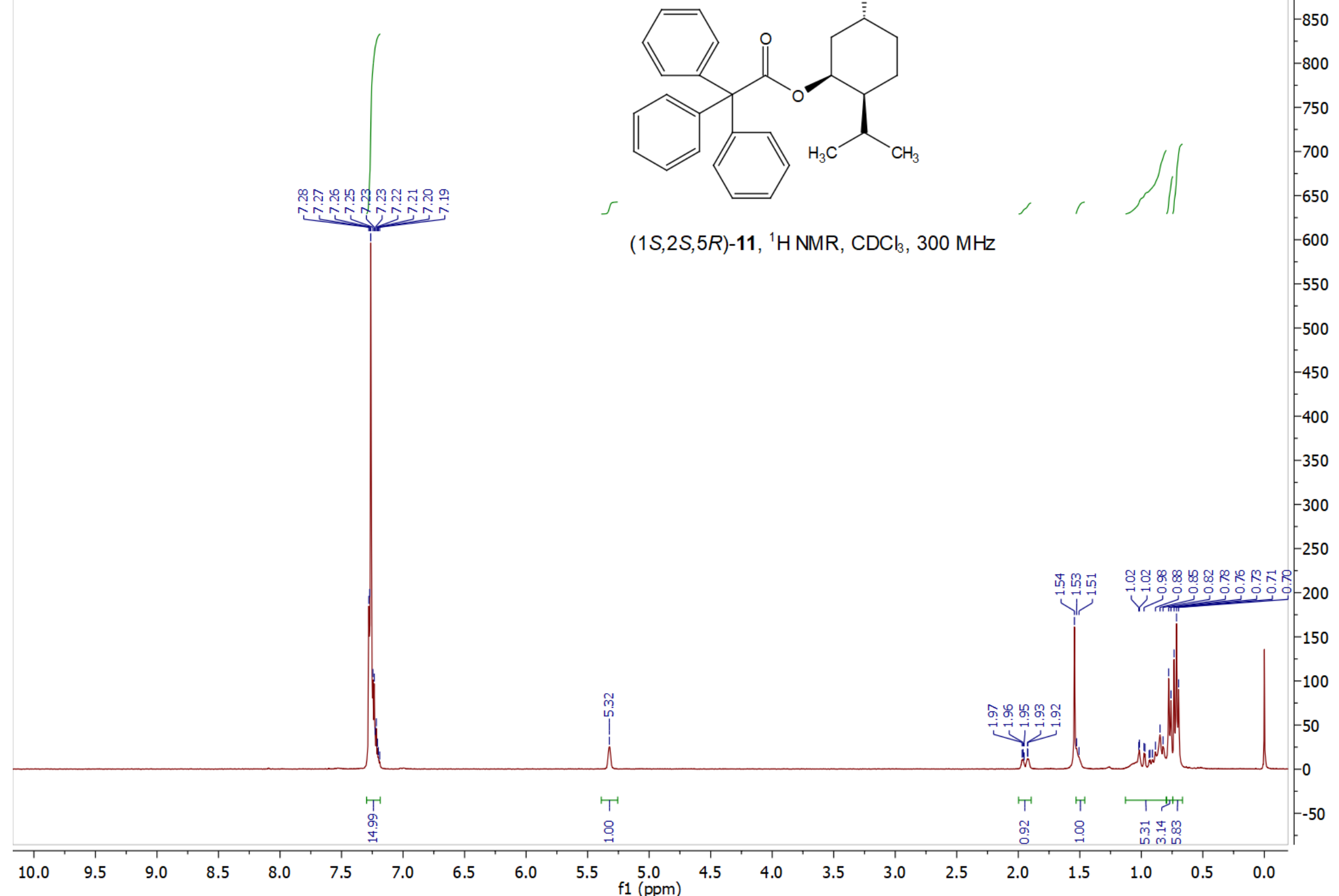


NPTr09_19_C.10.fid

C13CPD_512 CDCl3 \{C:|IconNMR $\mid$ Stereochemia_org $\}$ Stereochemia_org 3

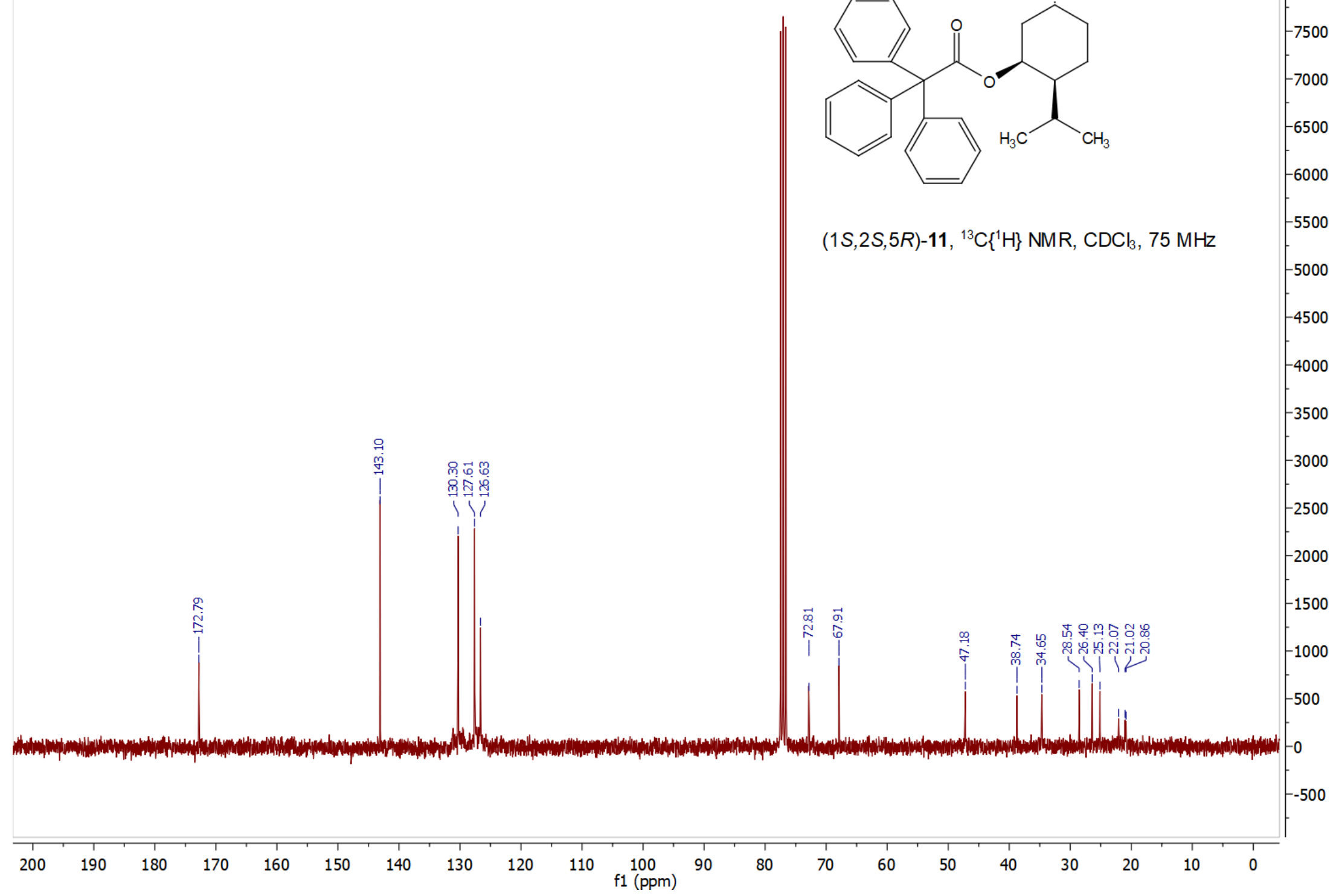


NPTr14_19.10.fid

PROTON CDCI3 \{C:|IconNMR|Stereochemia_org\} Stereochemia_org 10

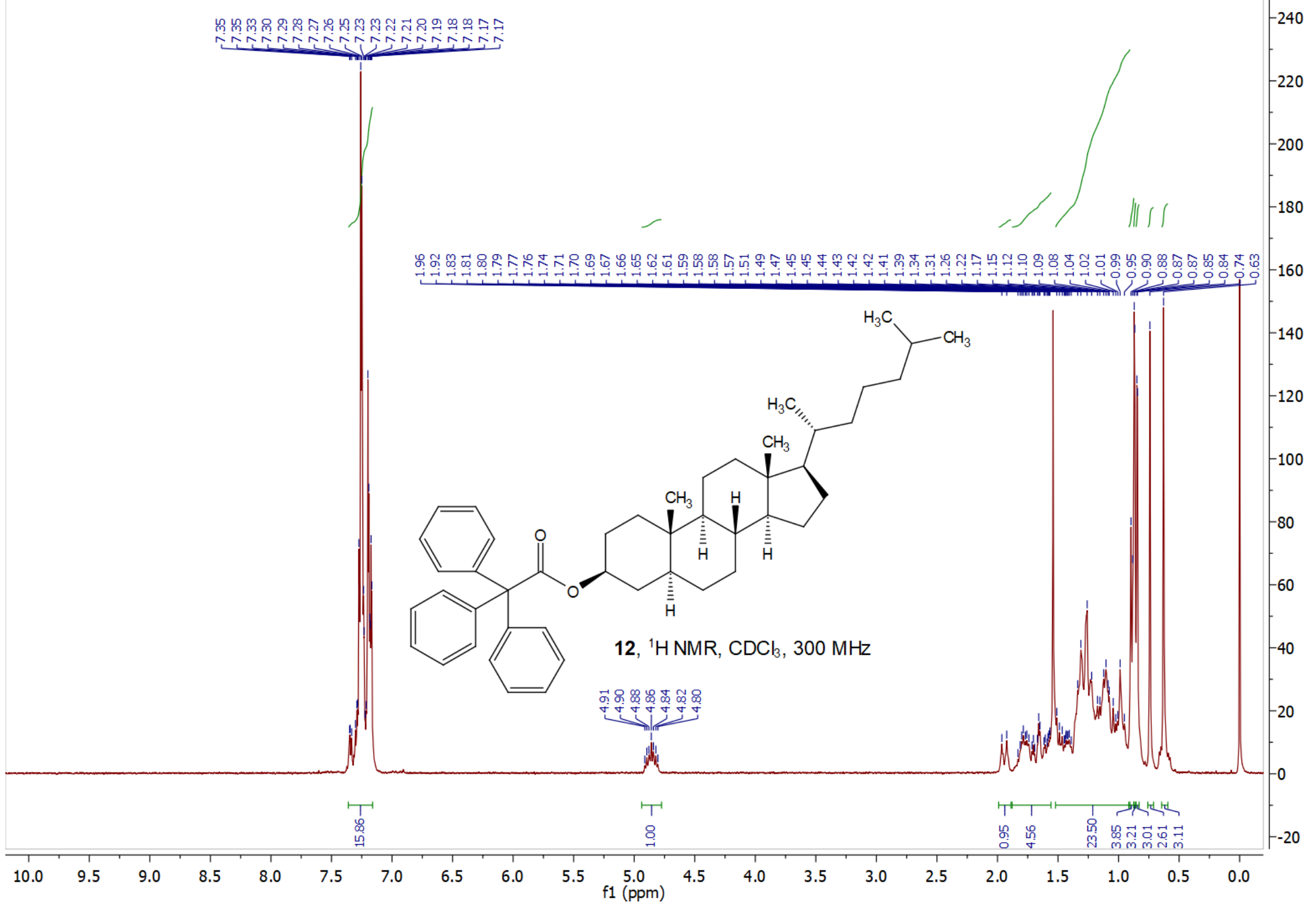



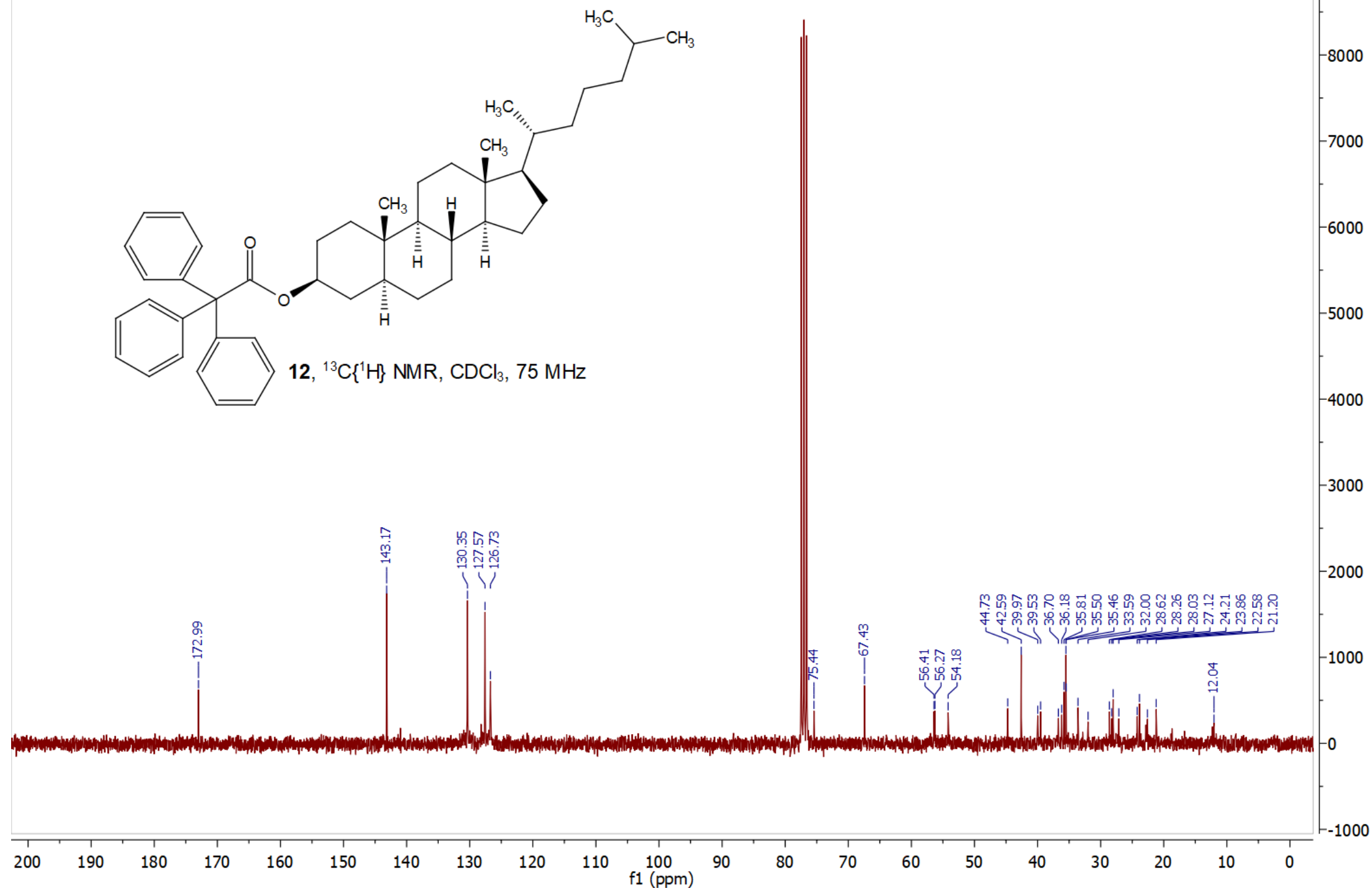
NPTr22 19.10.fid

PROTON CDCI3 \{C:|IconNMR|Stereochemia_org\} Stereochemia_org 16

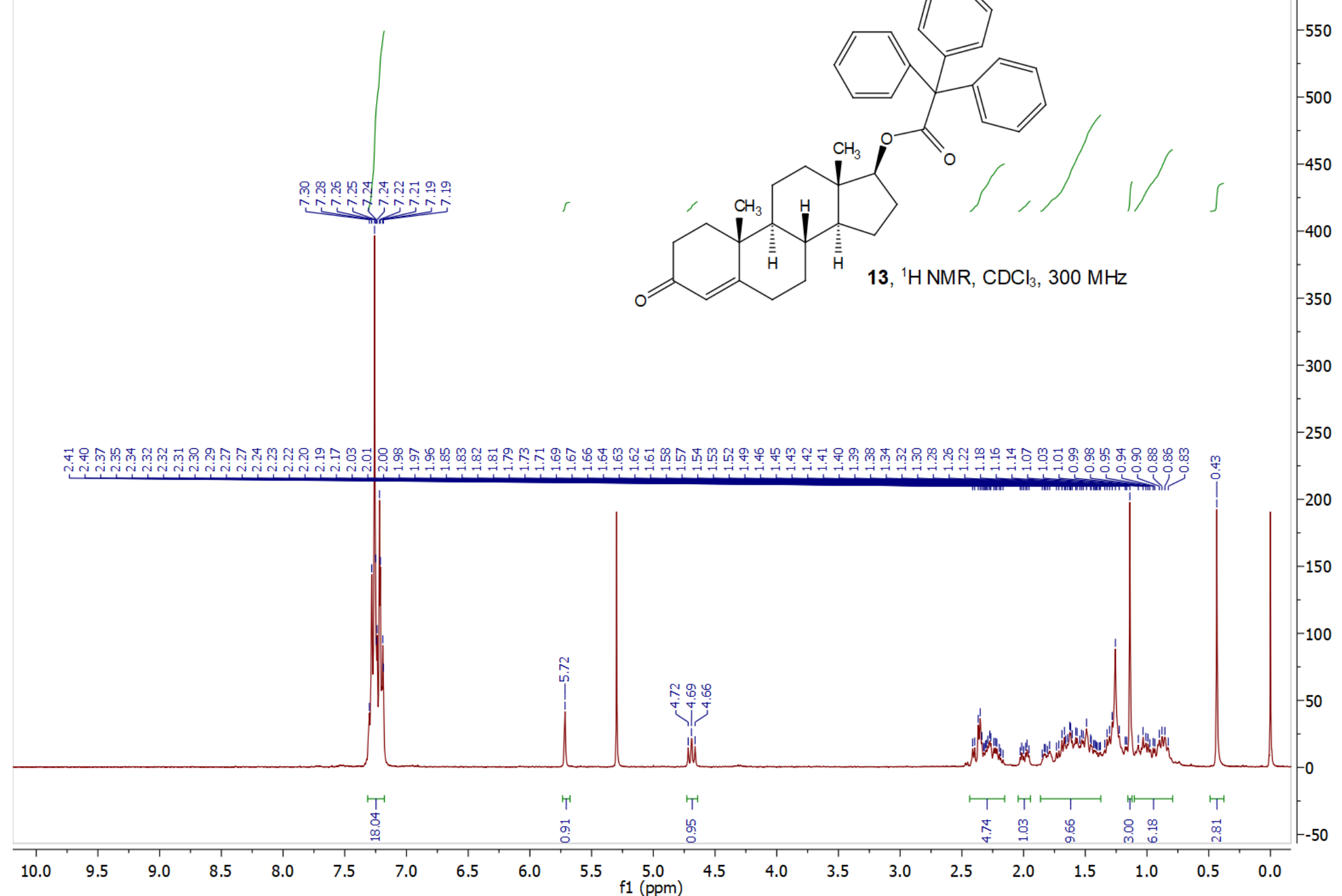

$-600$ 


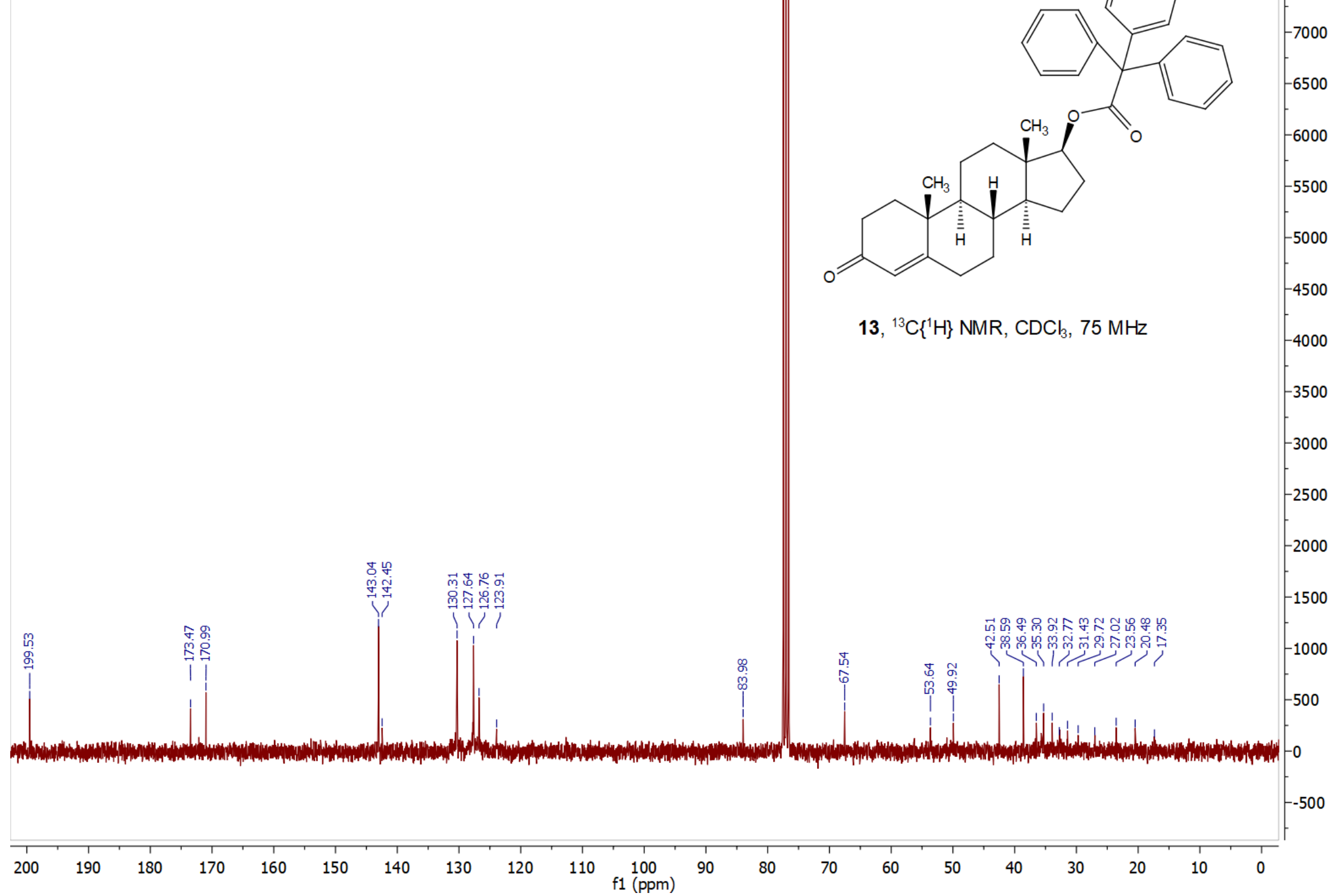




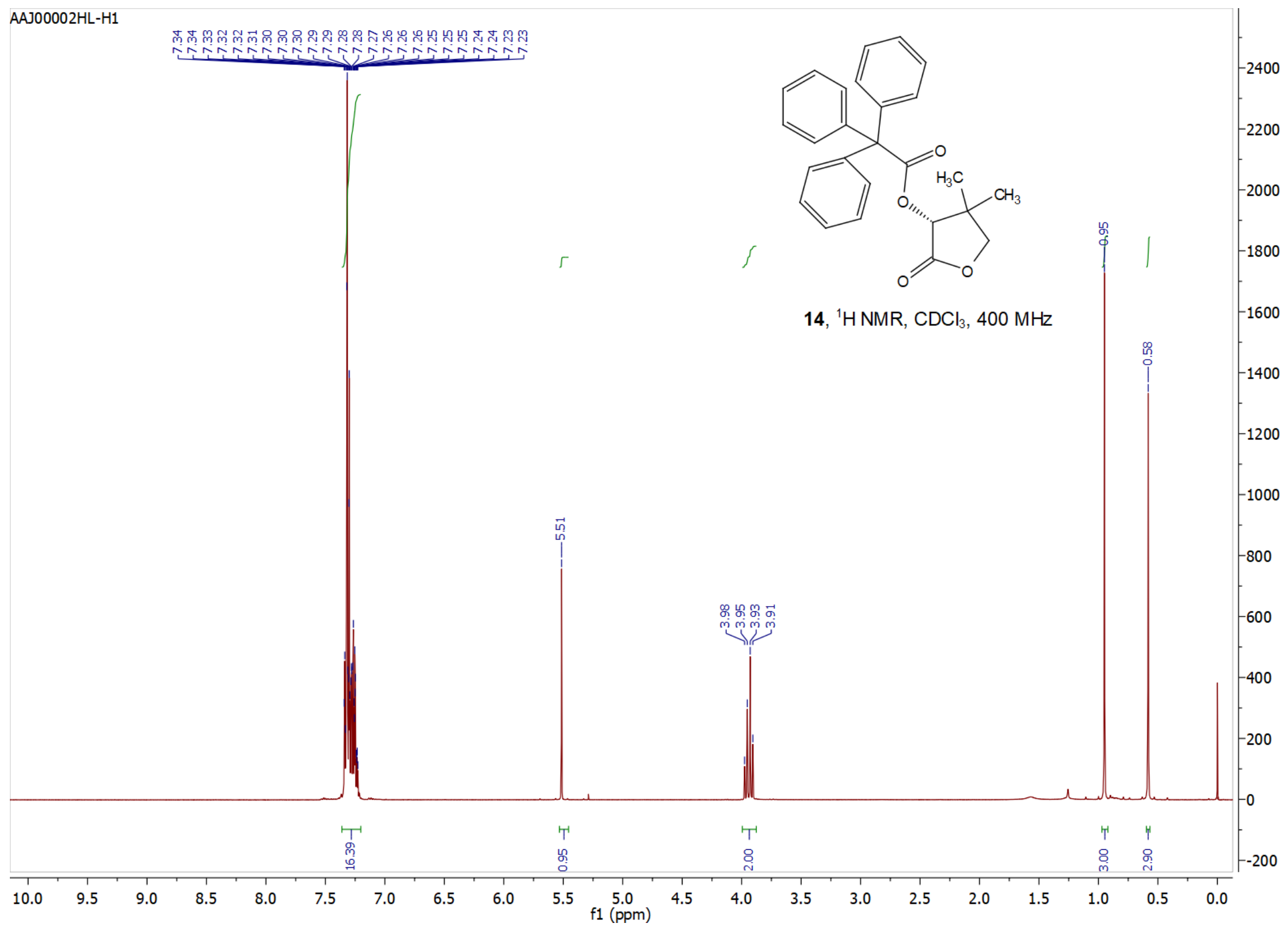




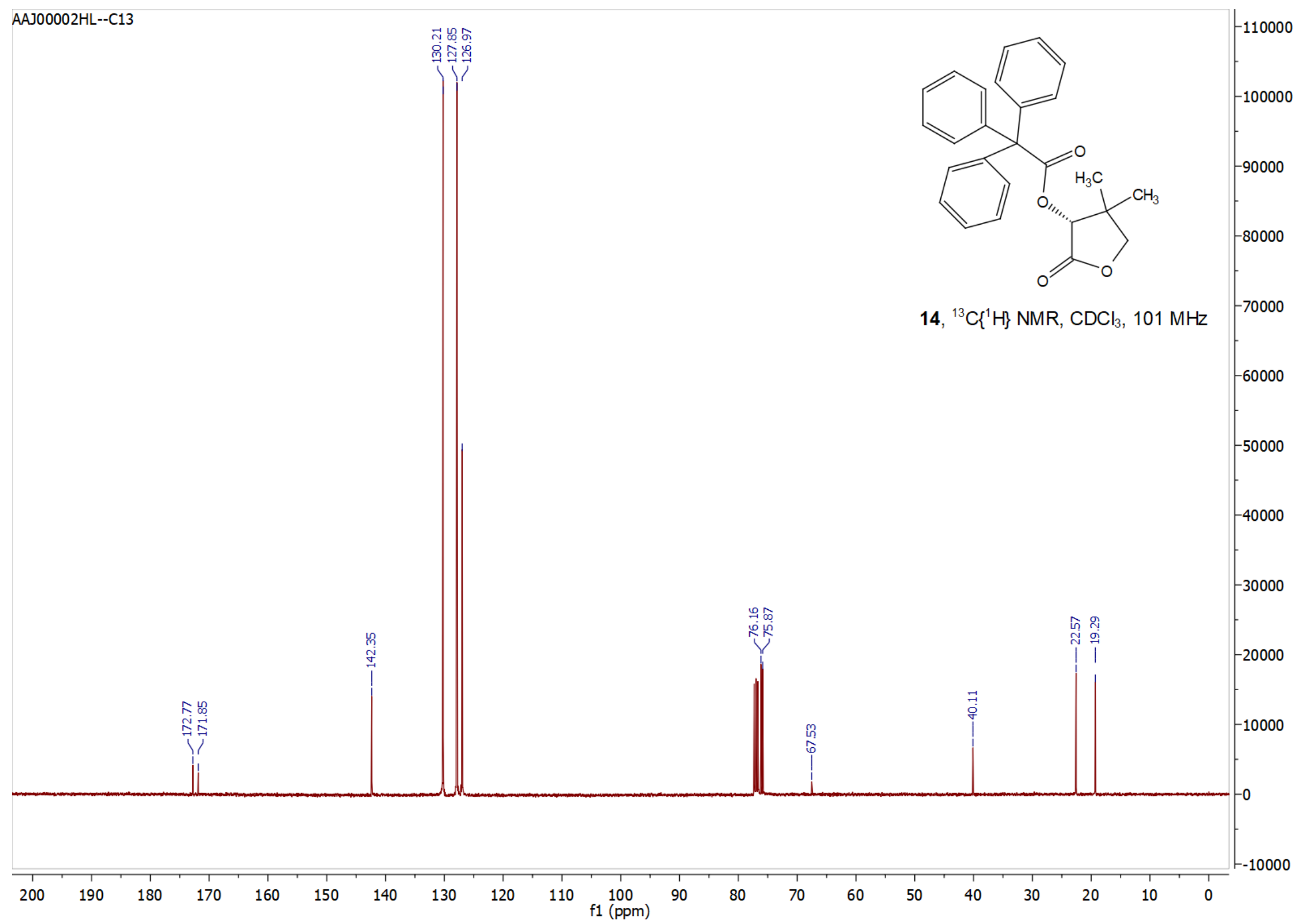


NPTr23 19.10.fid

PROTON CDCl3 \{C:|IconNMR|Stereochemia_org $\}$ Stereochemia_org 1

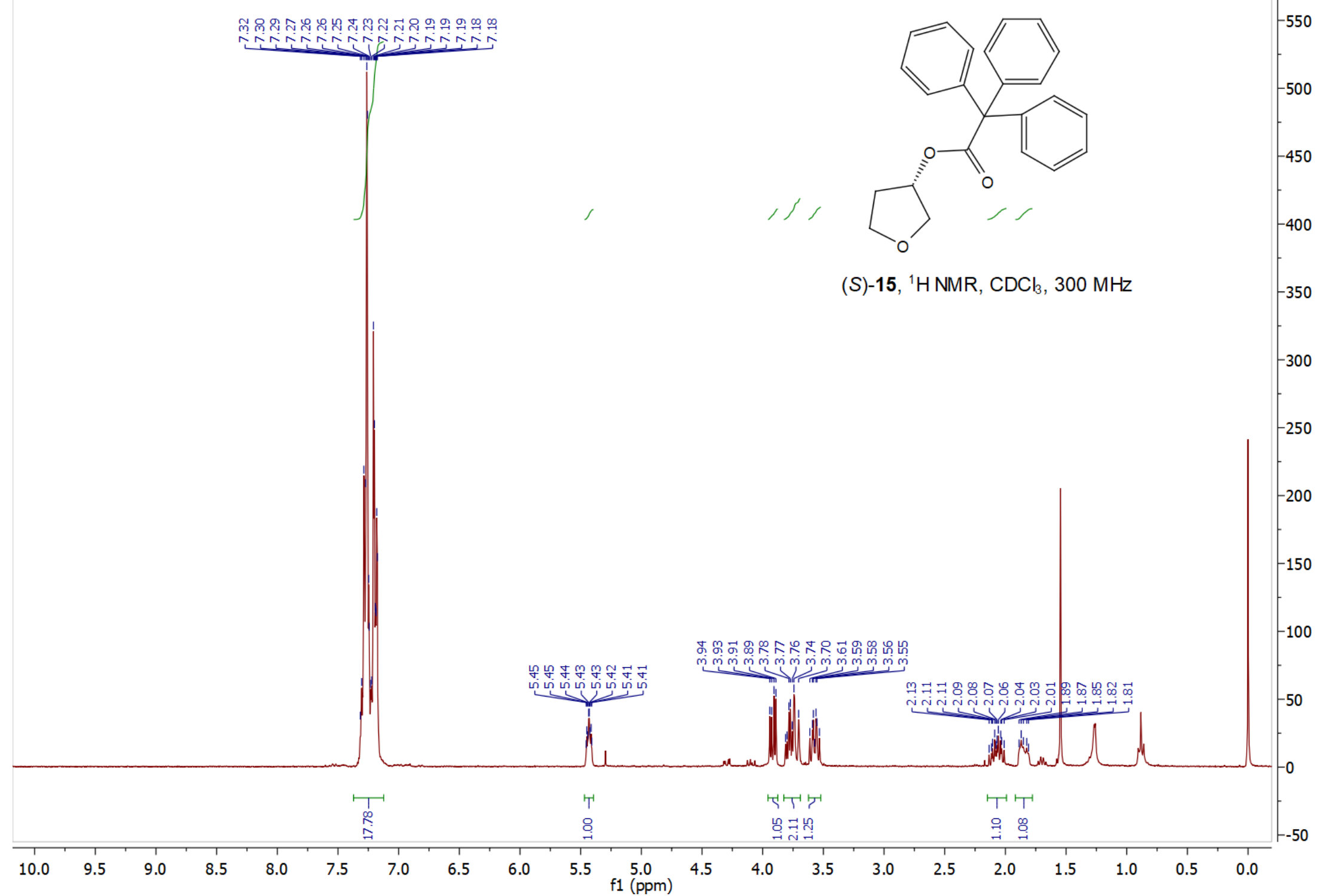




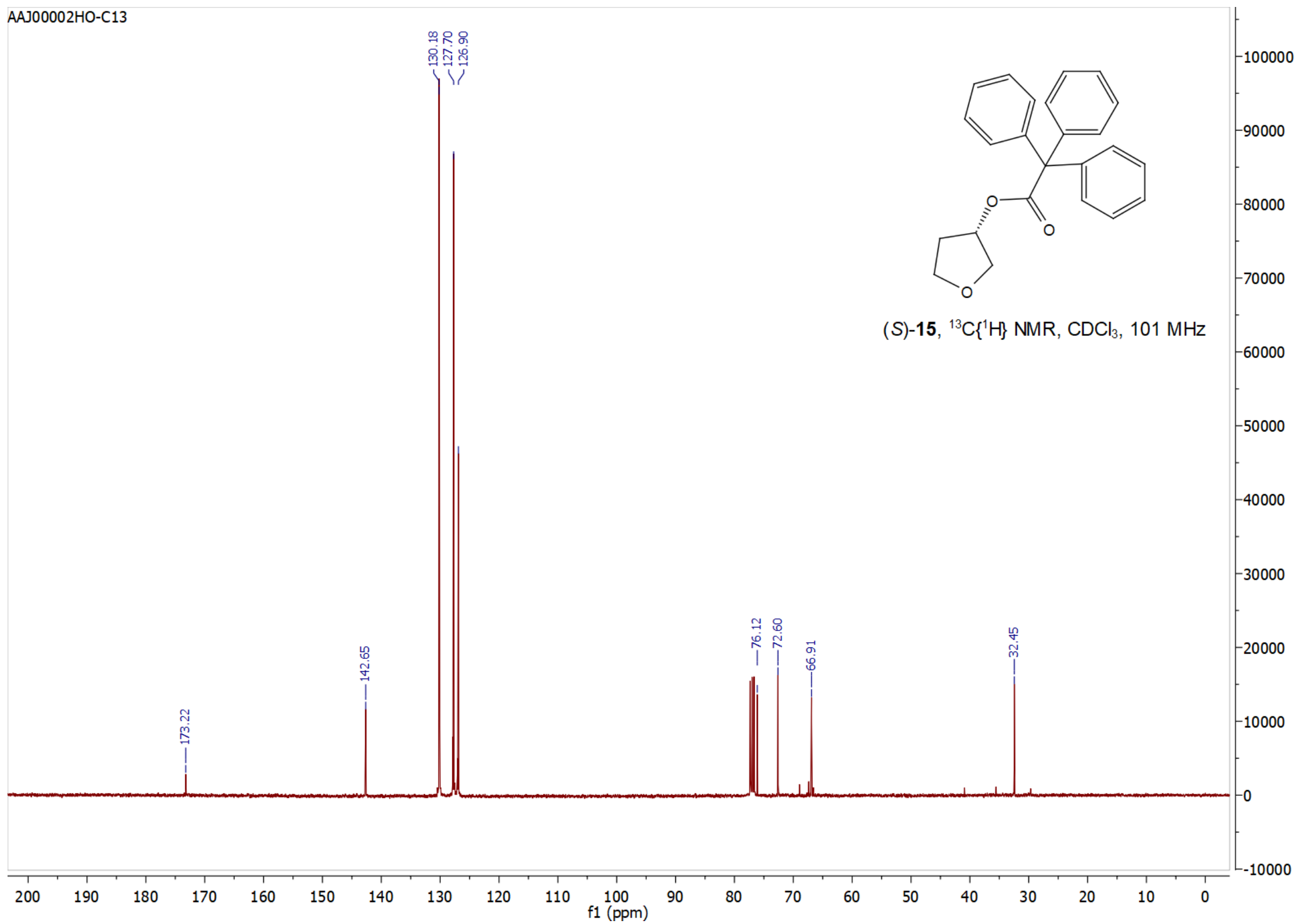


NPTr35 19.10.fid

PROTON CDCI3 \{C:|IconNMR|Stereochemia_org\} Stereochemia_org 8

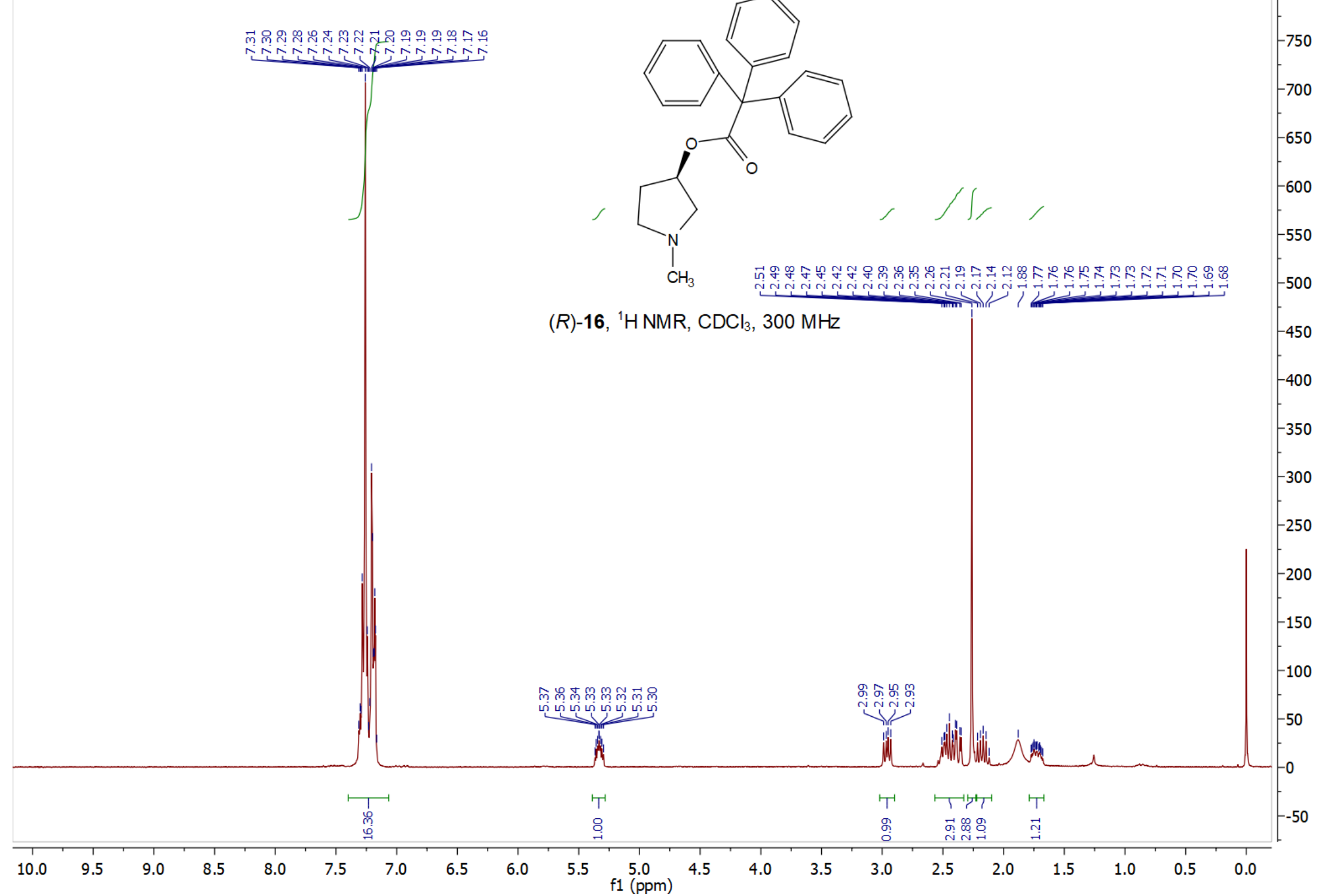

800 


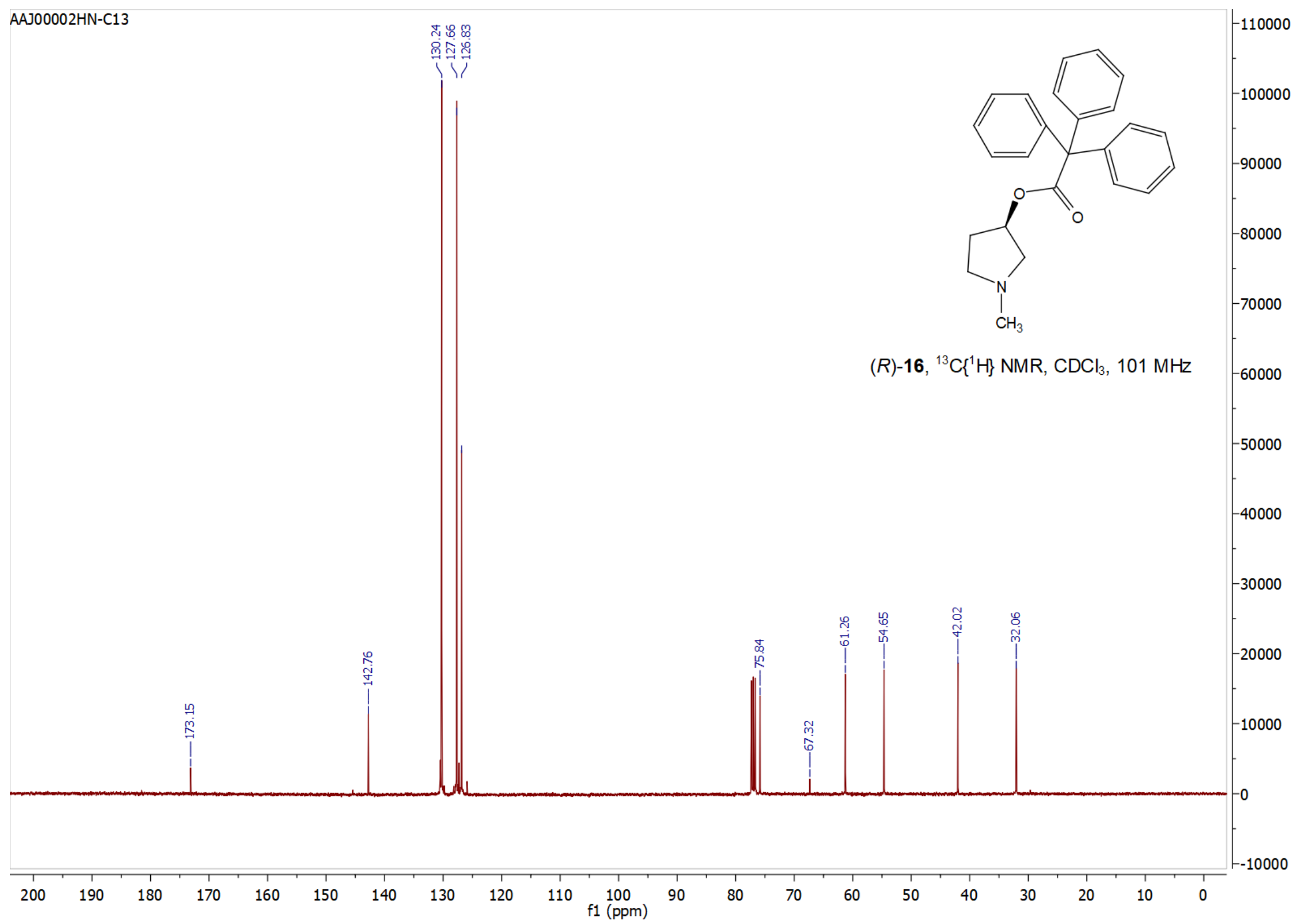




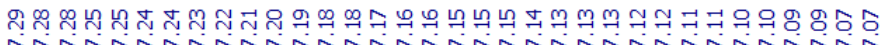

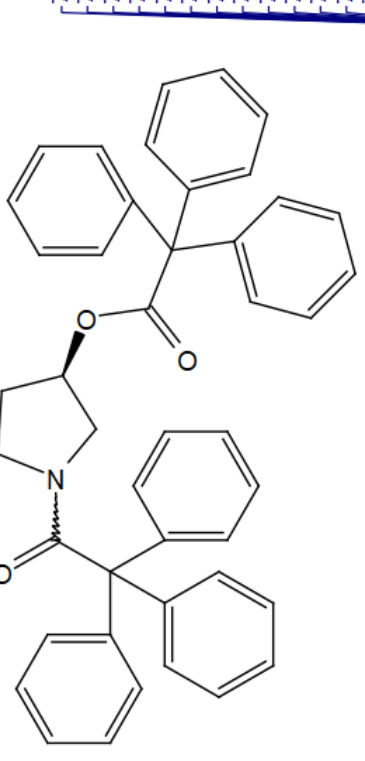

(R)-17, ${ }^{1} \mathrm{H} \mathrm{NMR}, \mathrm{CDCl}_{3}, 400 \mathrm{MHz}$

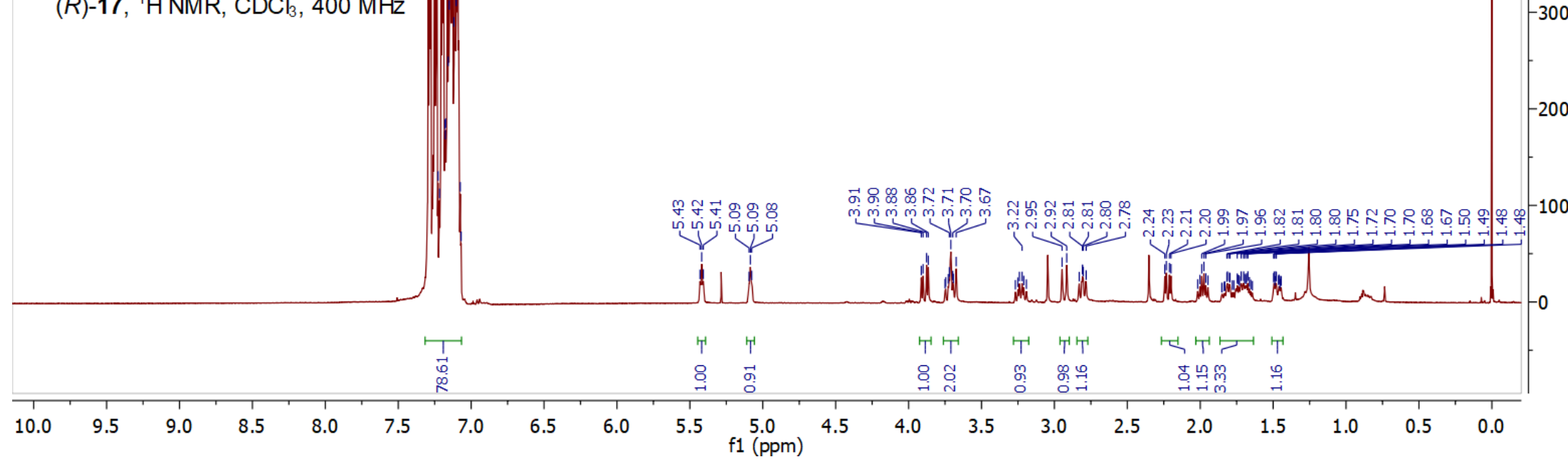




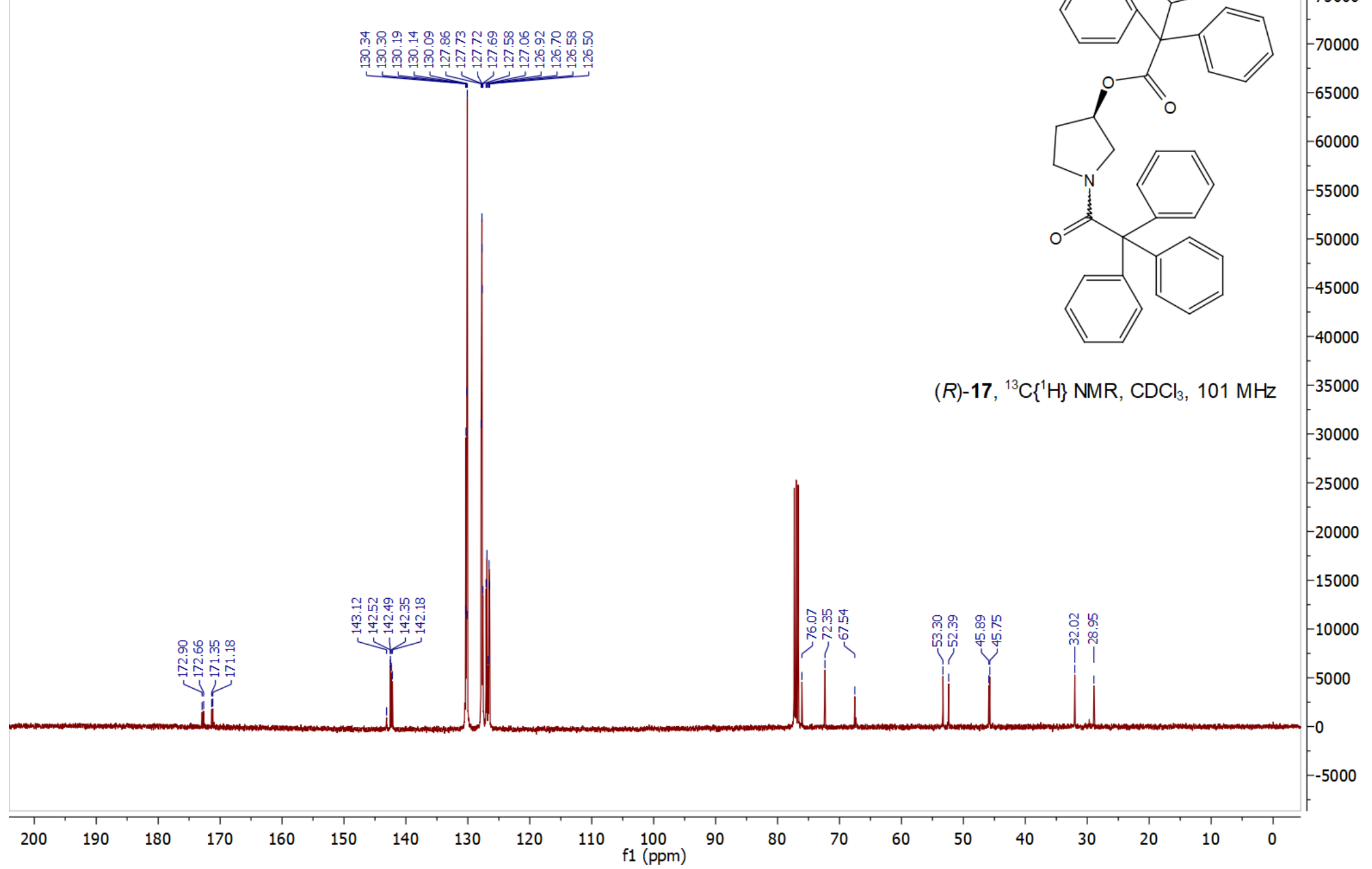




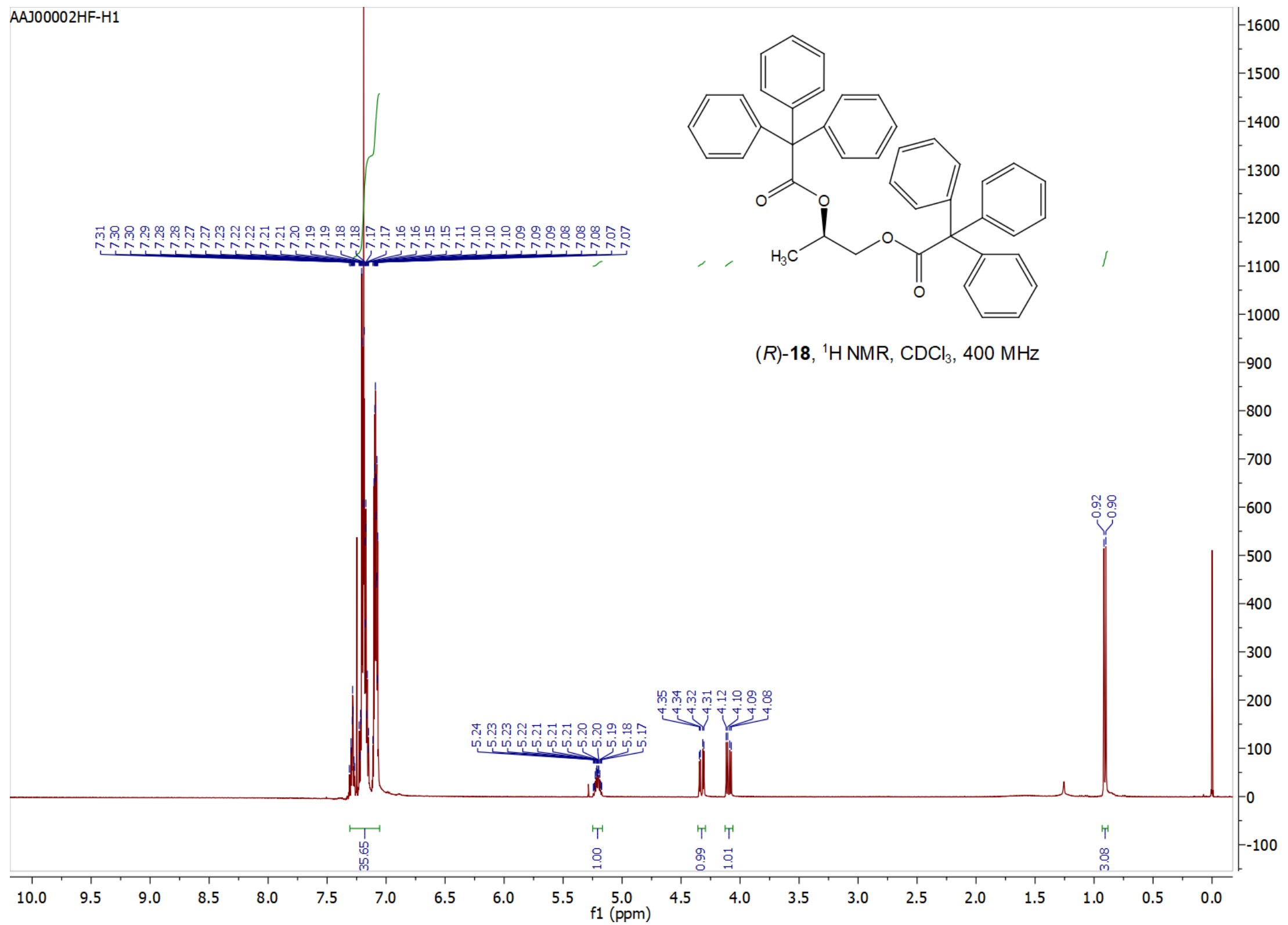




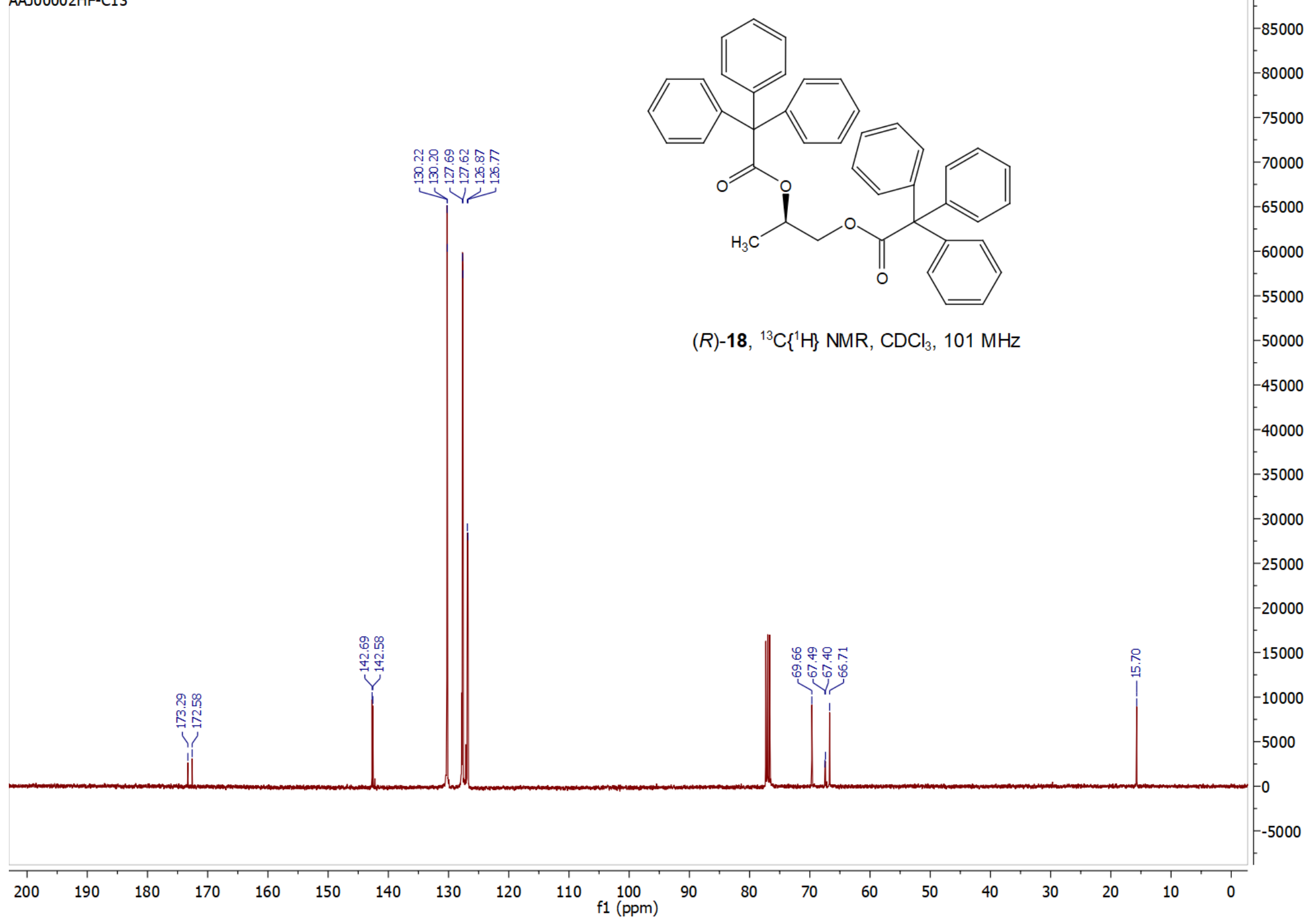


NPTr26_19.10.fid

PROTON CDCI3 \{C:|IconNMR|Stereochemia_org\} Stereochemia_org 15

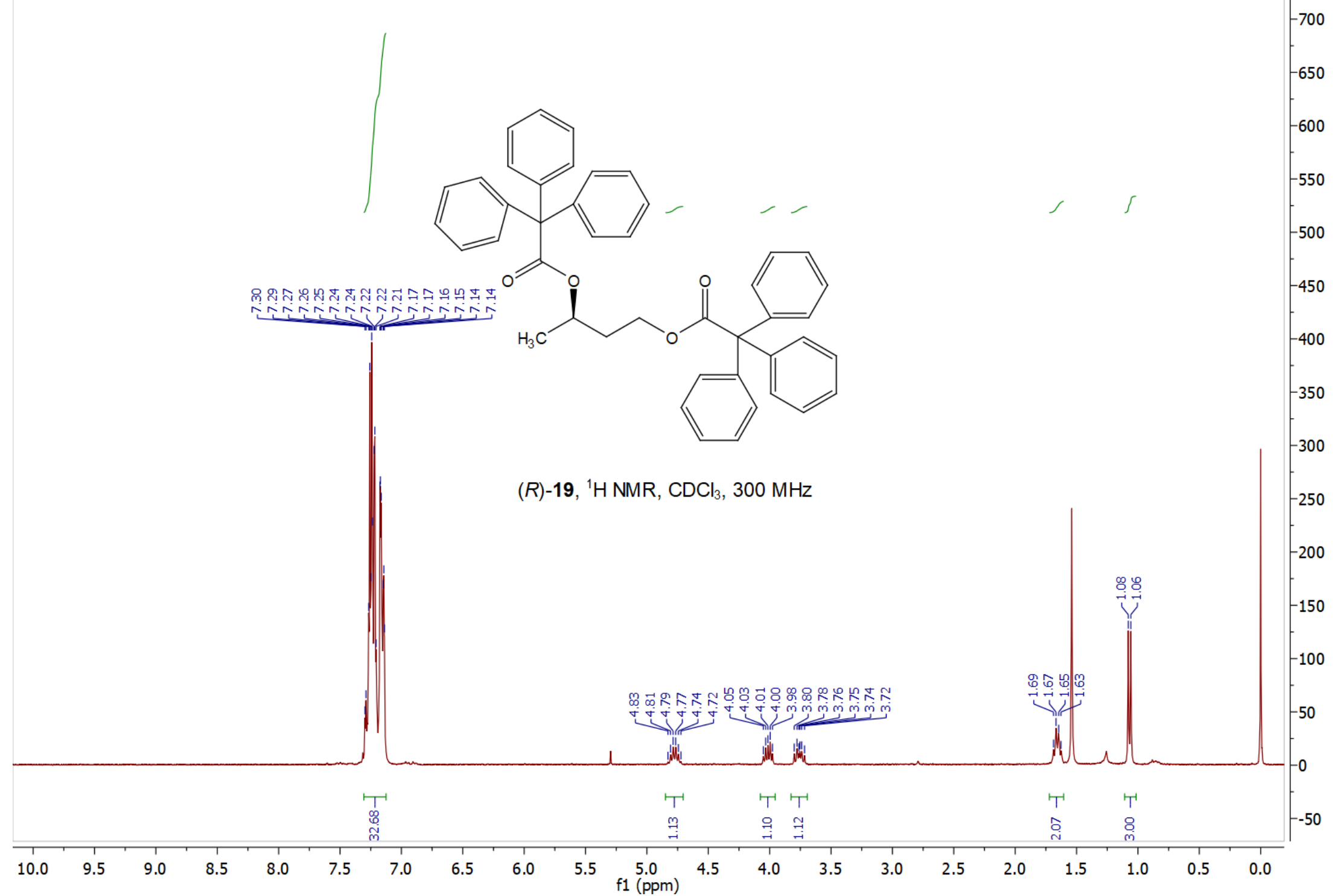




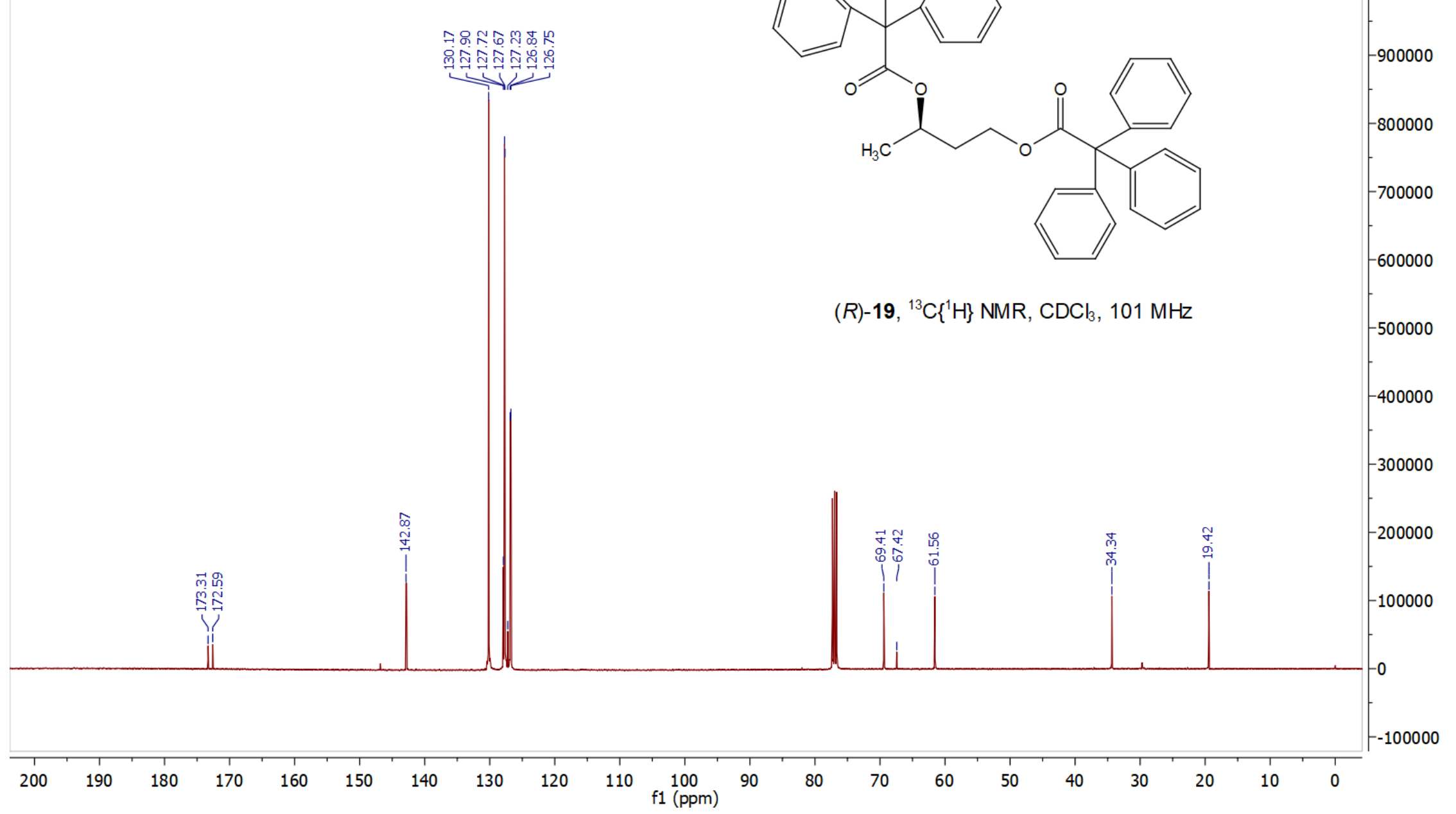




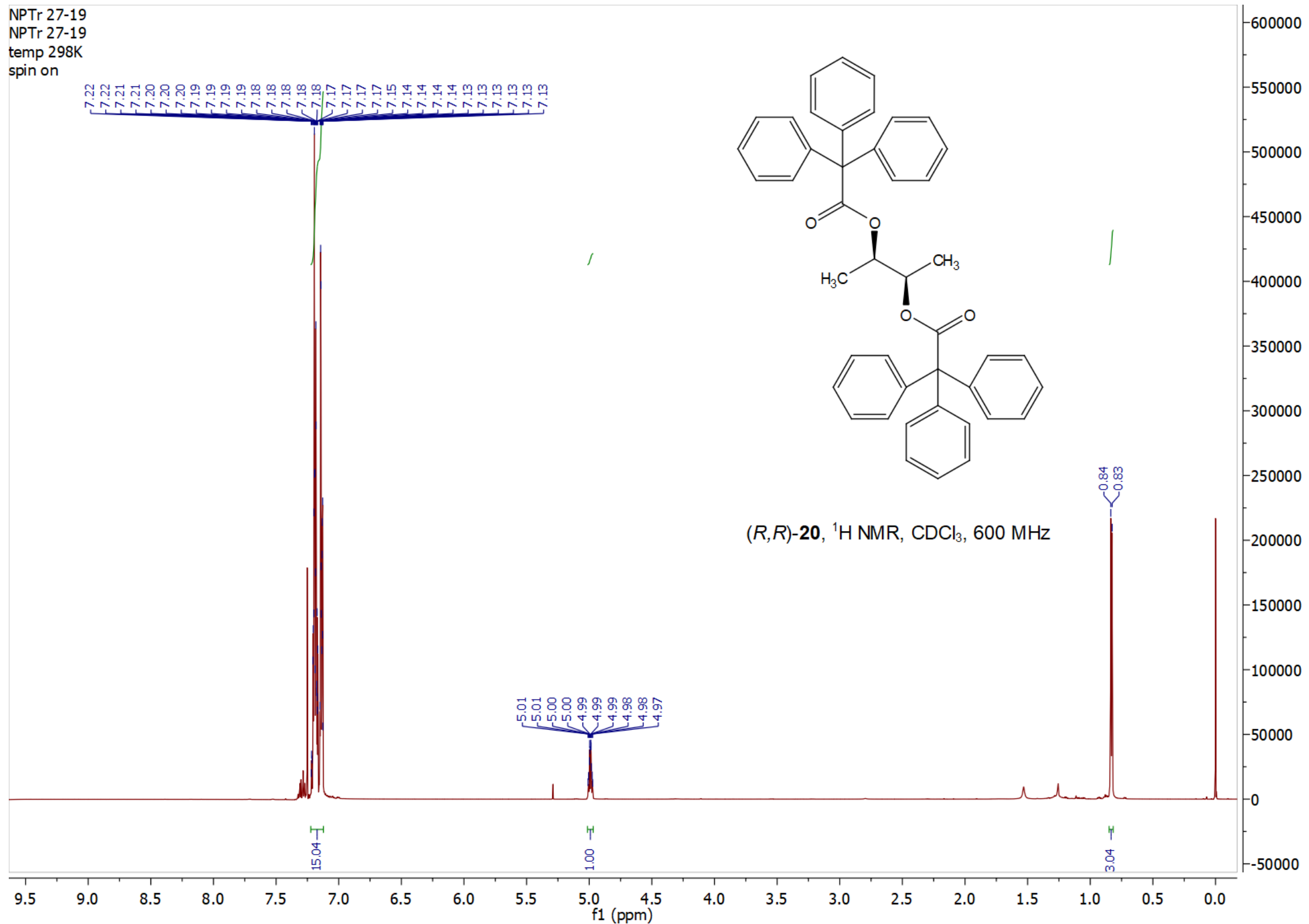




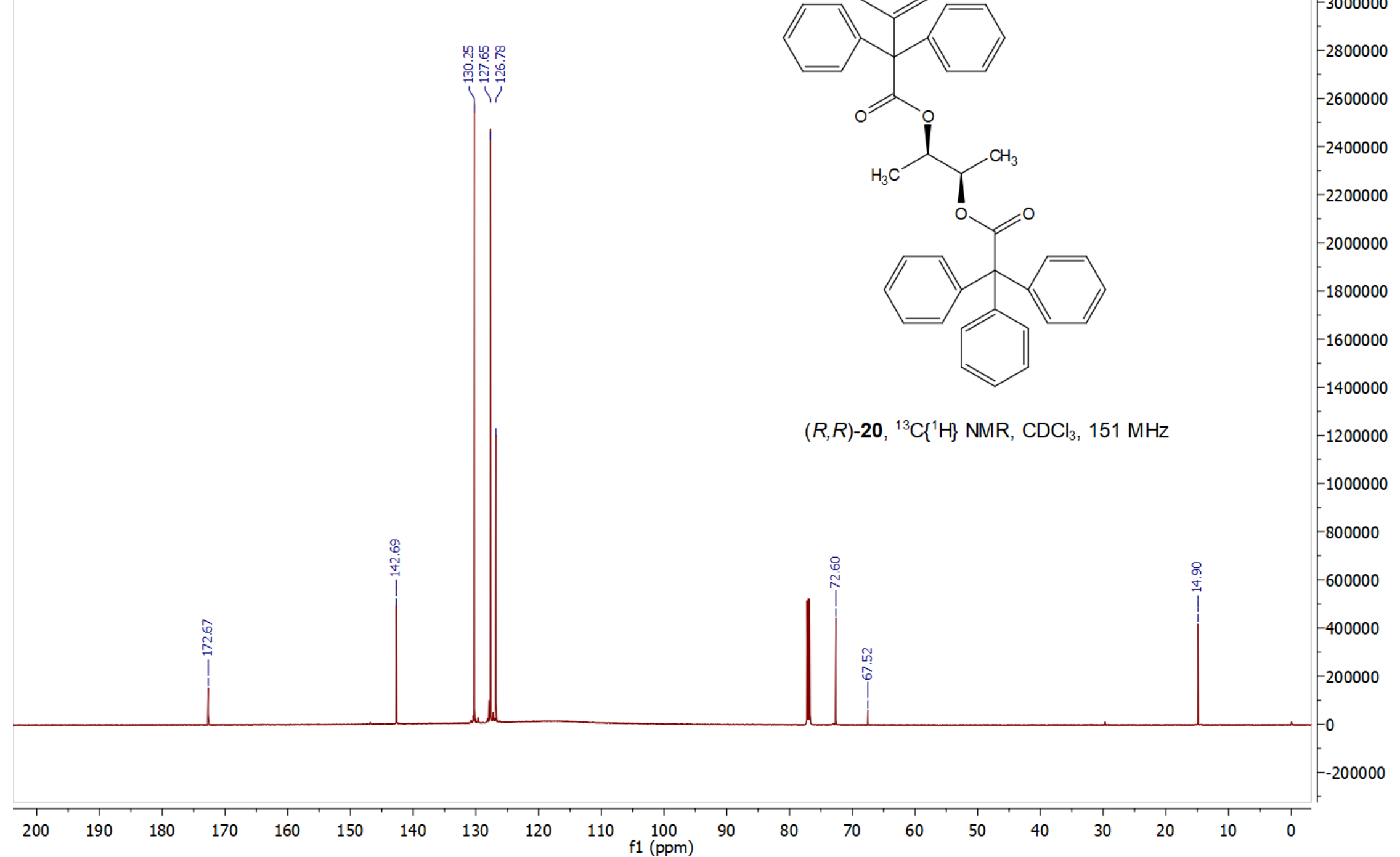




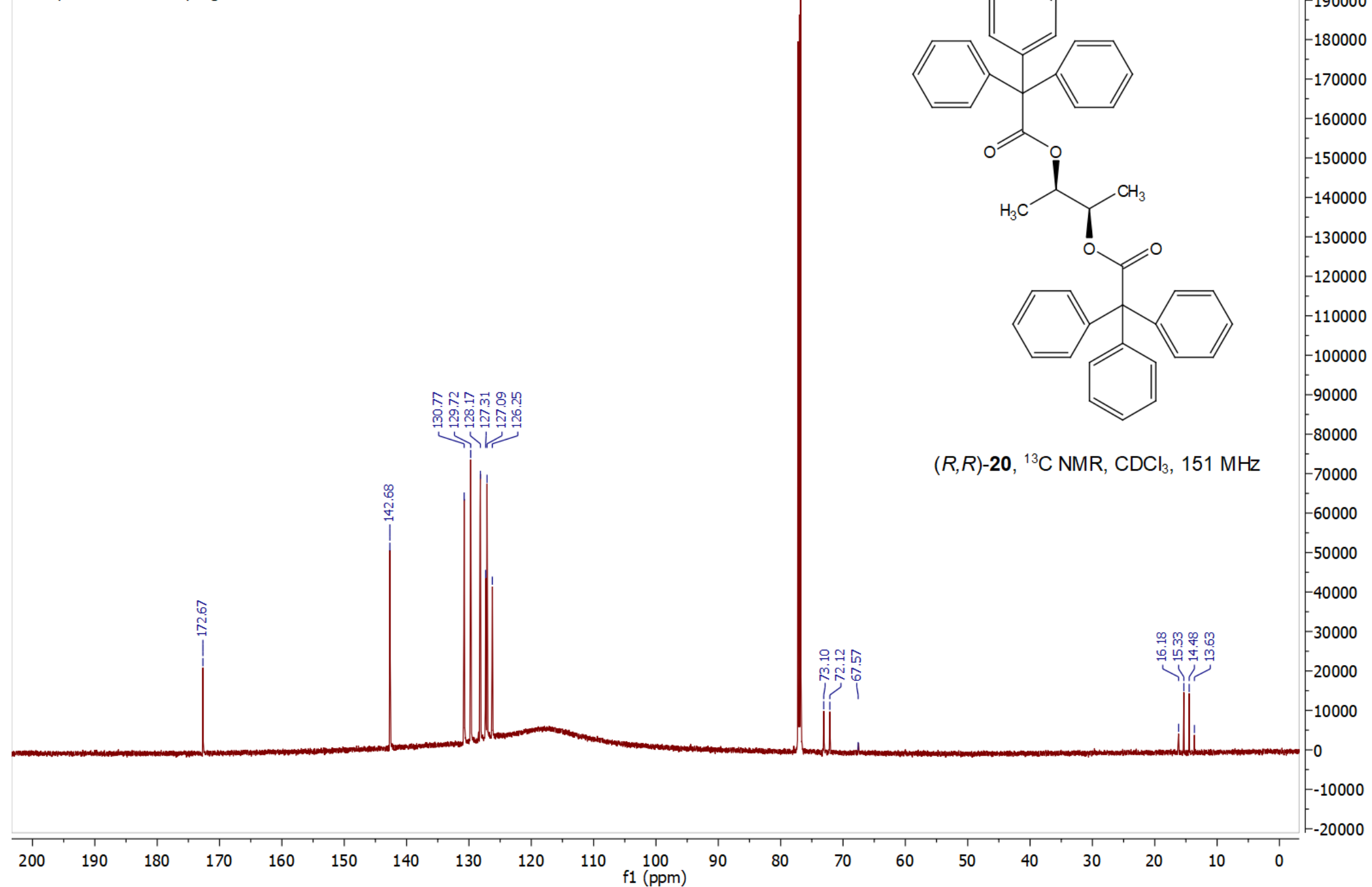




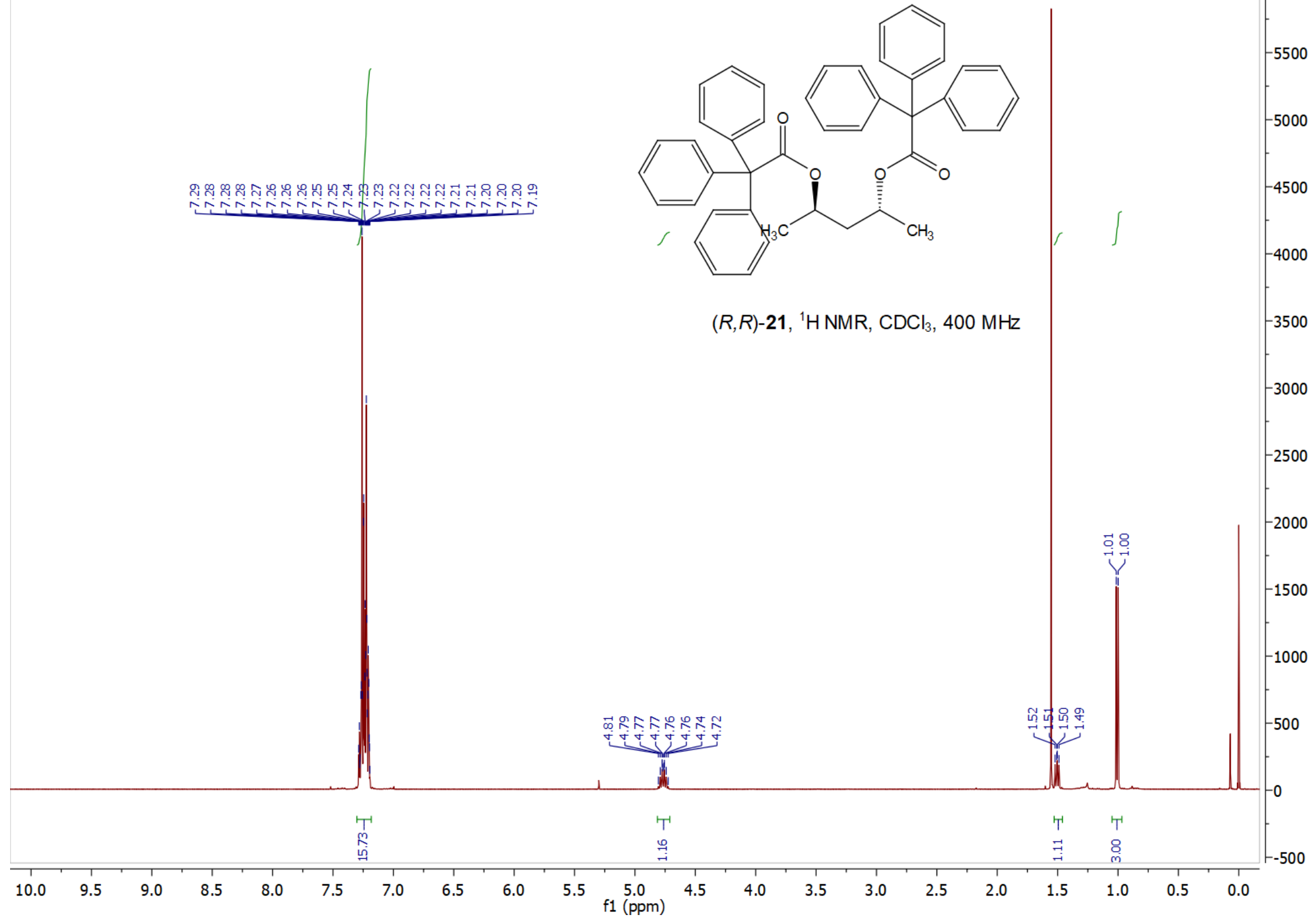




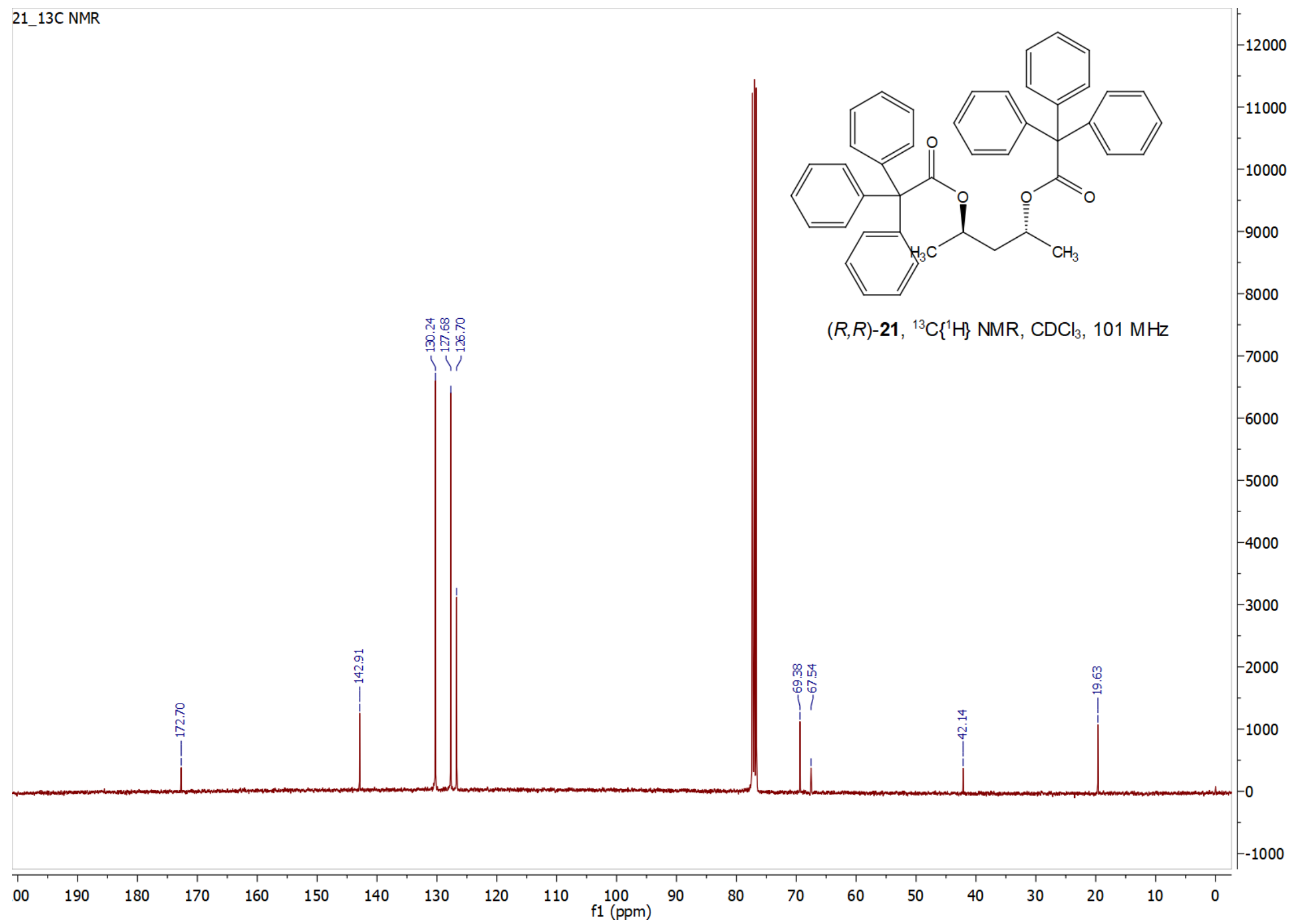




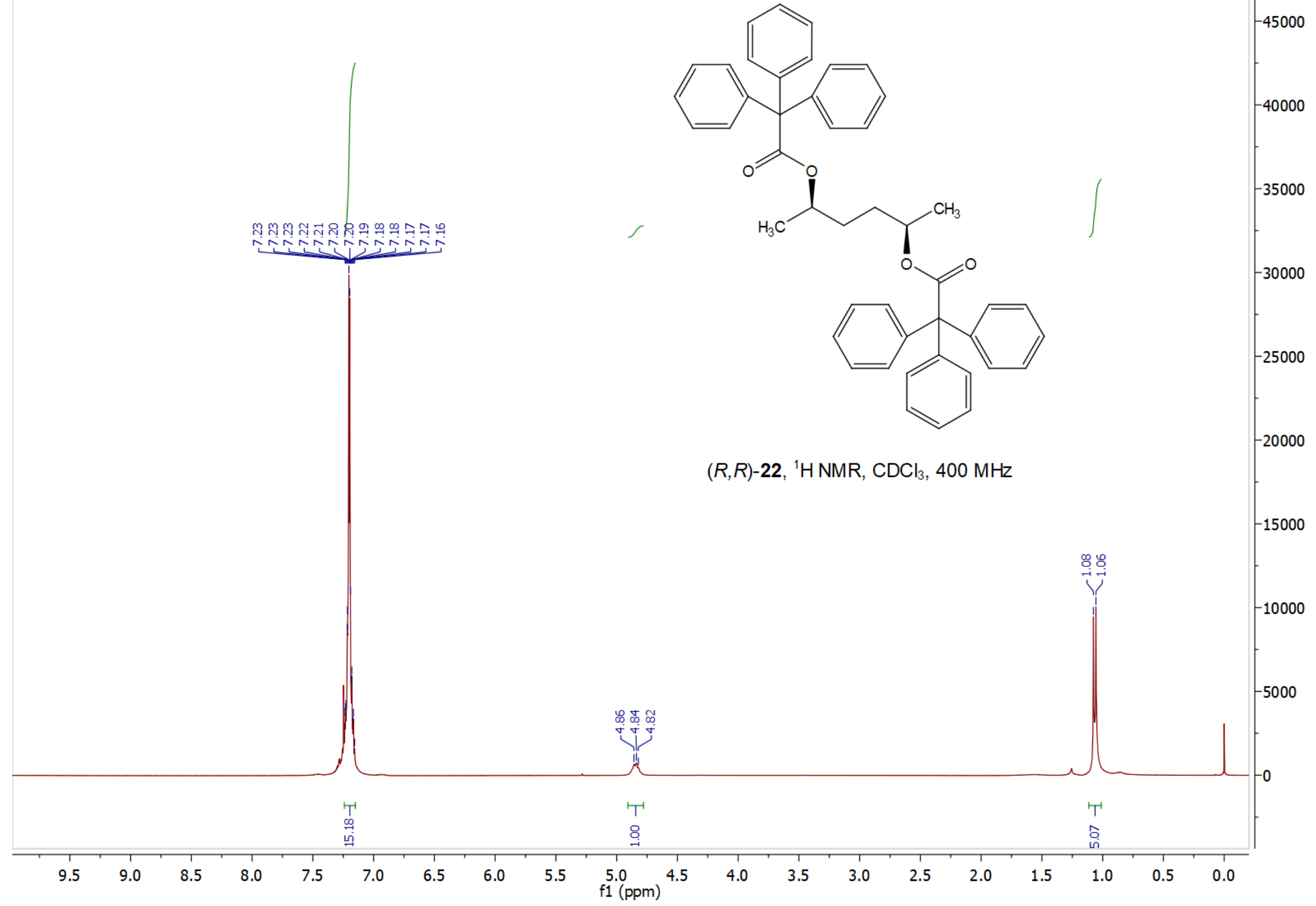




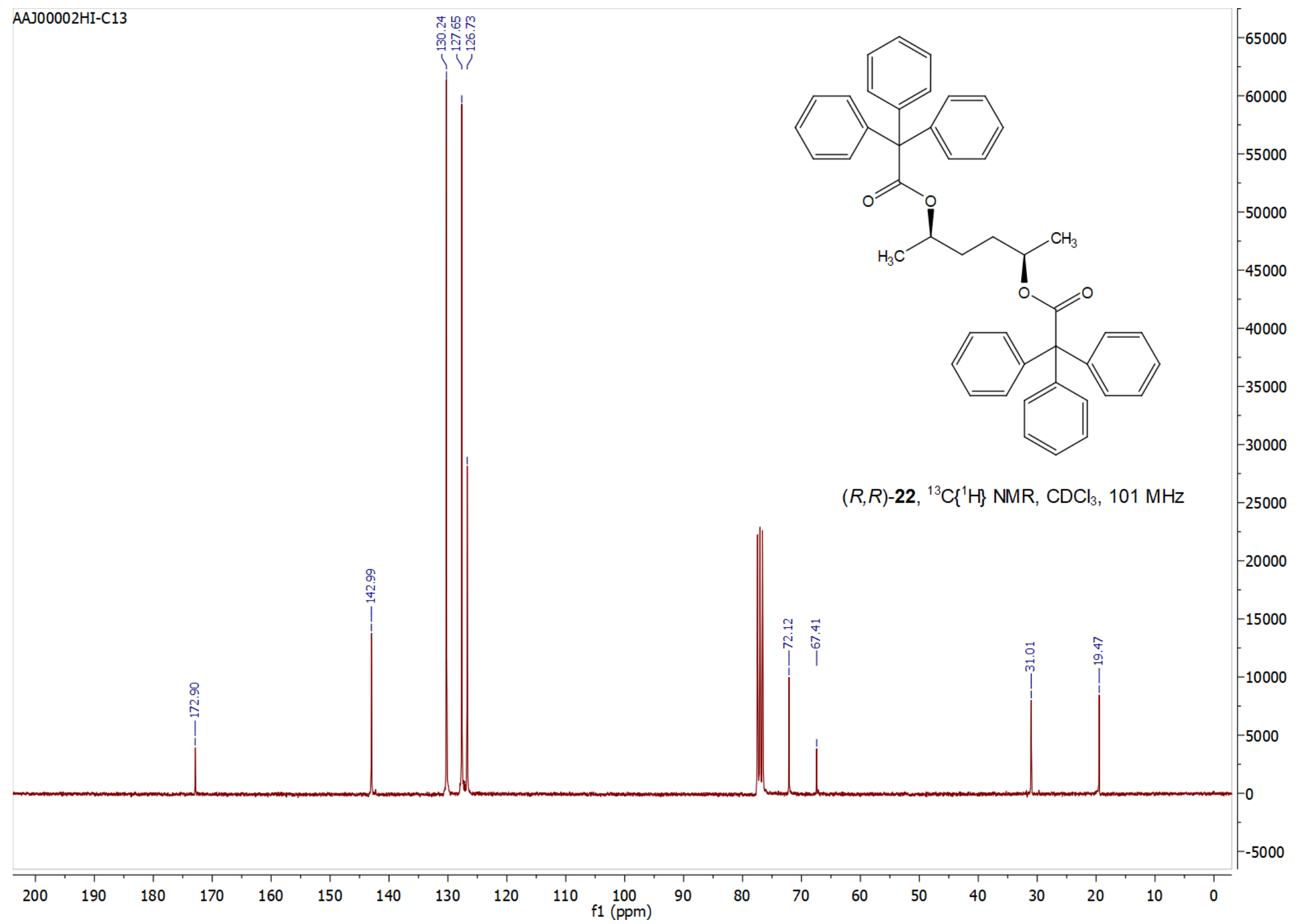


Copies of ECD spectra 
$\mathrm{nm}$

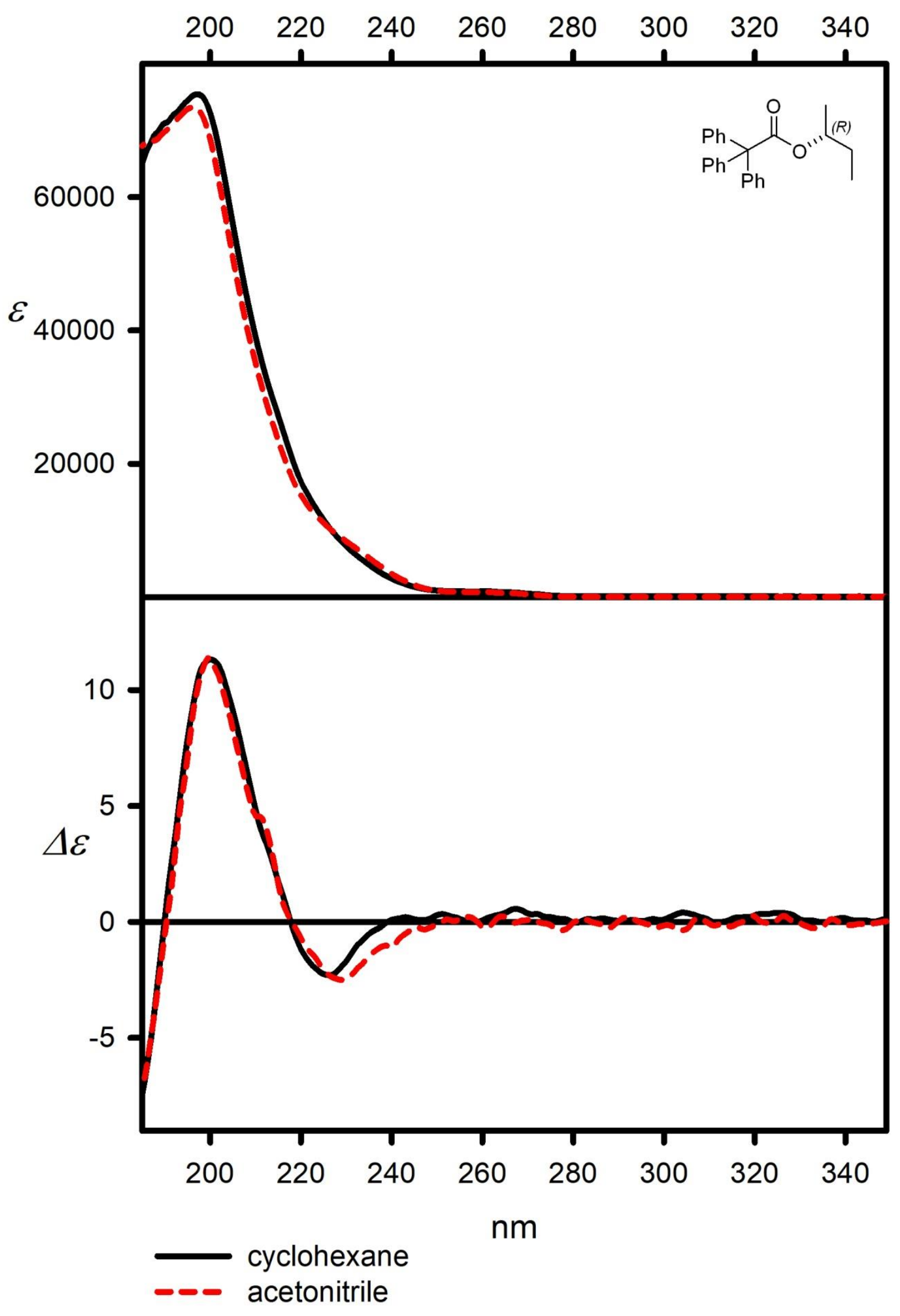


$\mathrm{nm}$

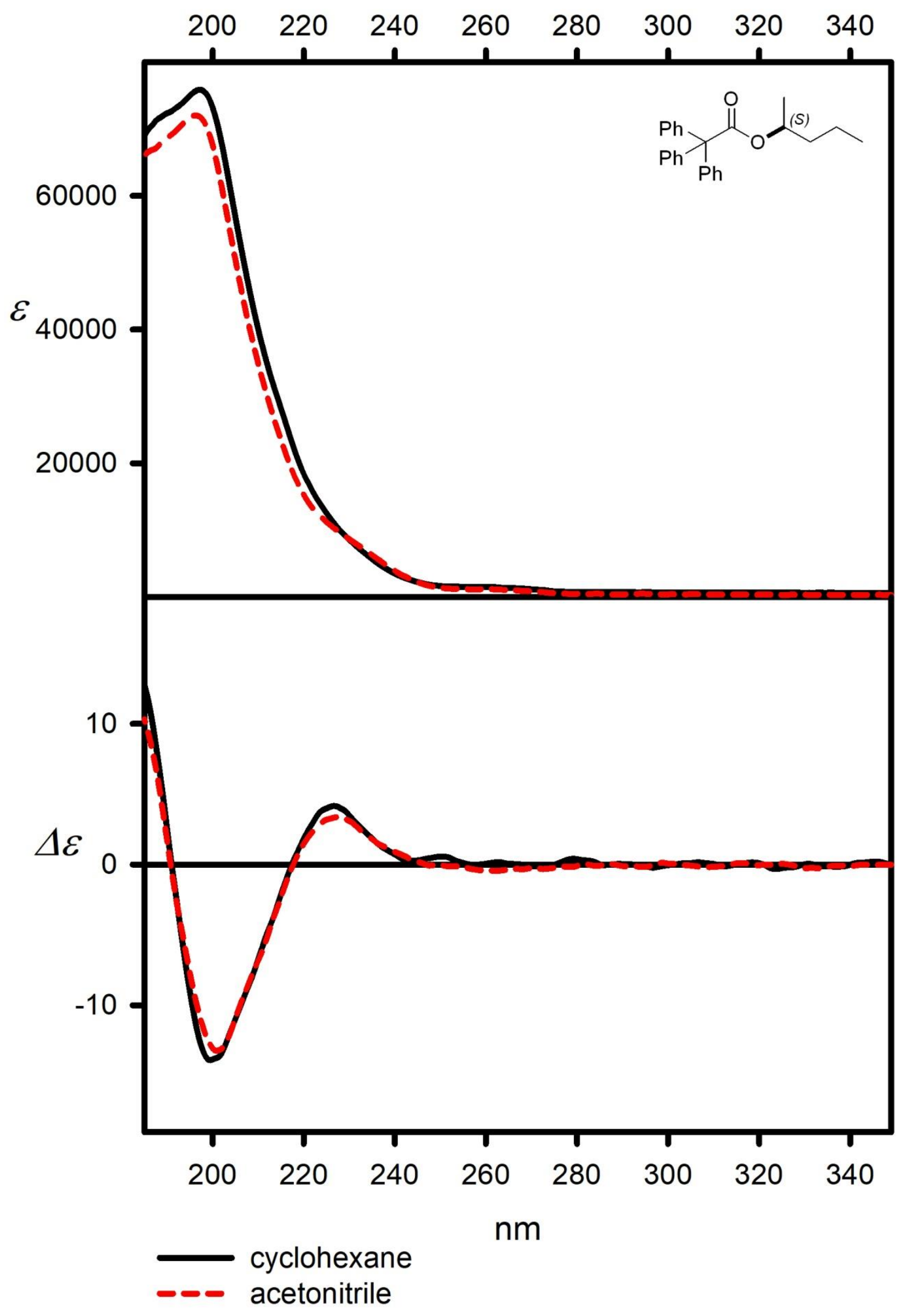


$\mathrm{nm}$

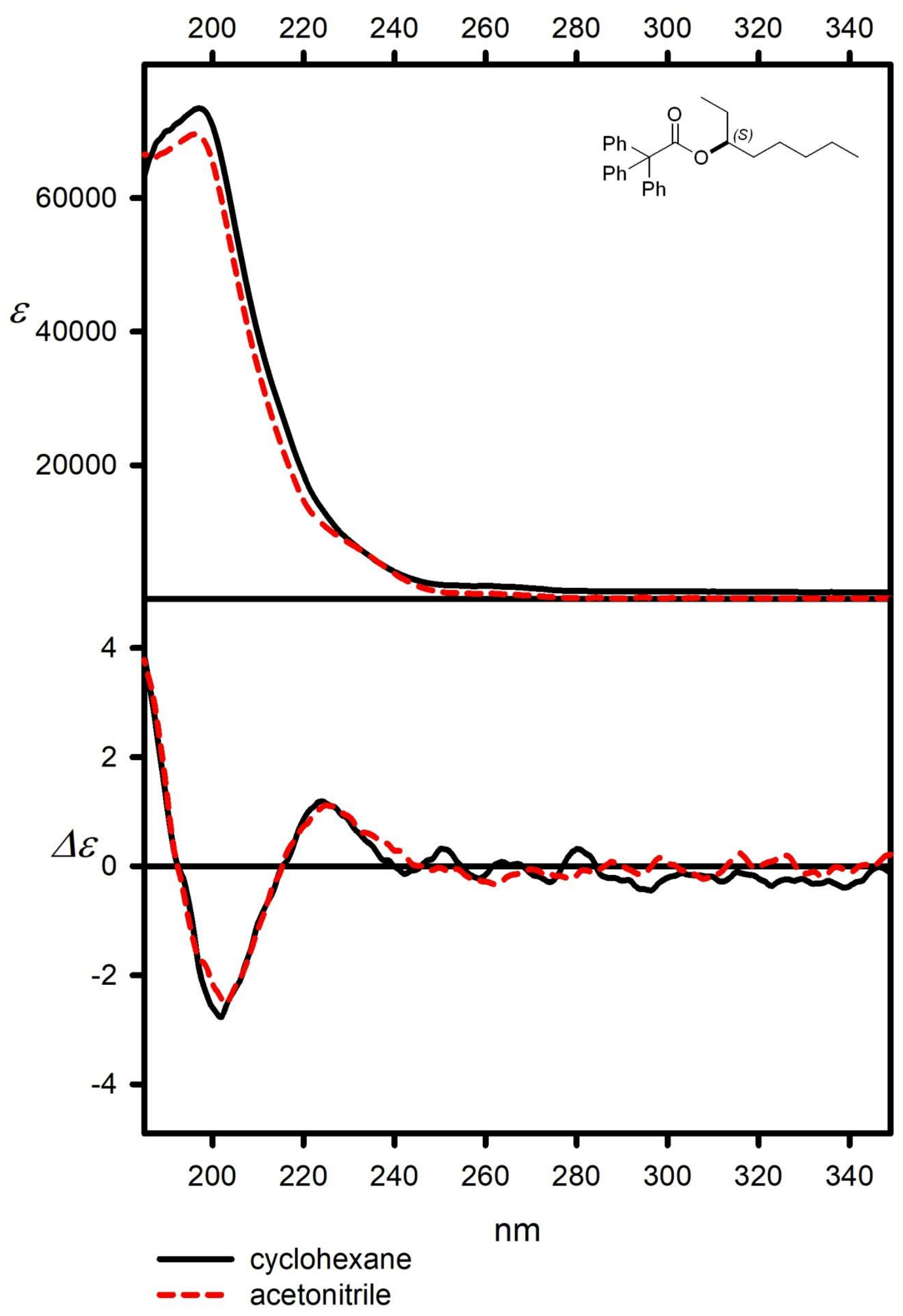


$\mathrm{nm}$

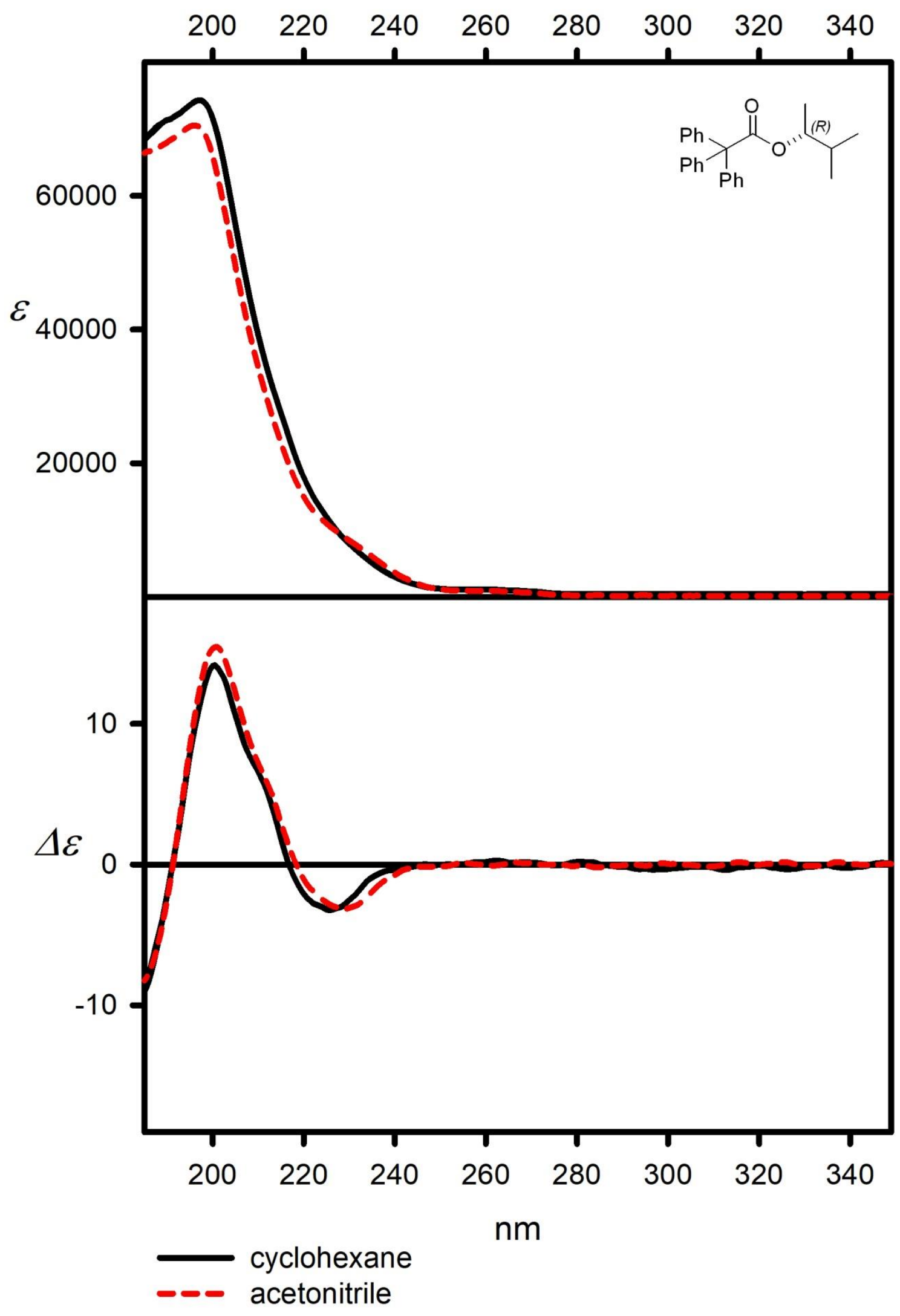


$\mathrm{nm}$

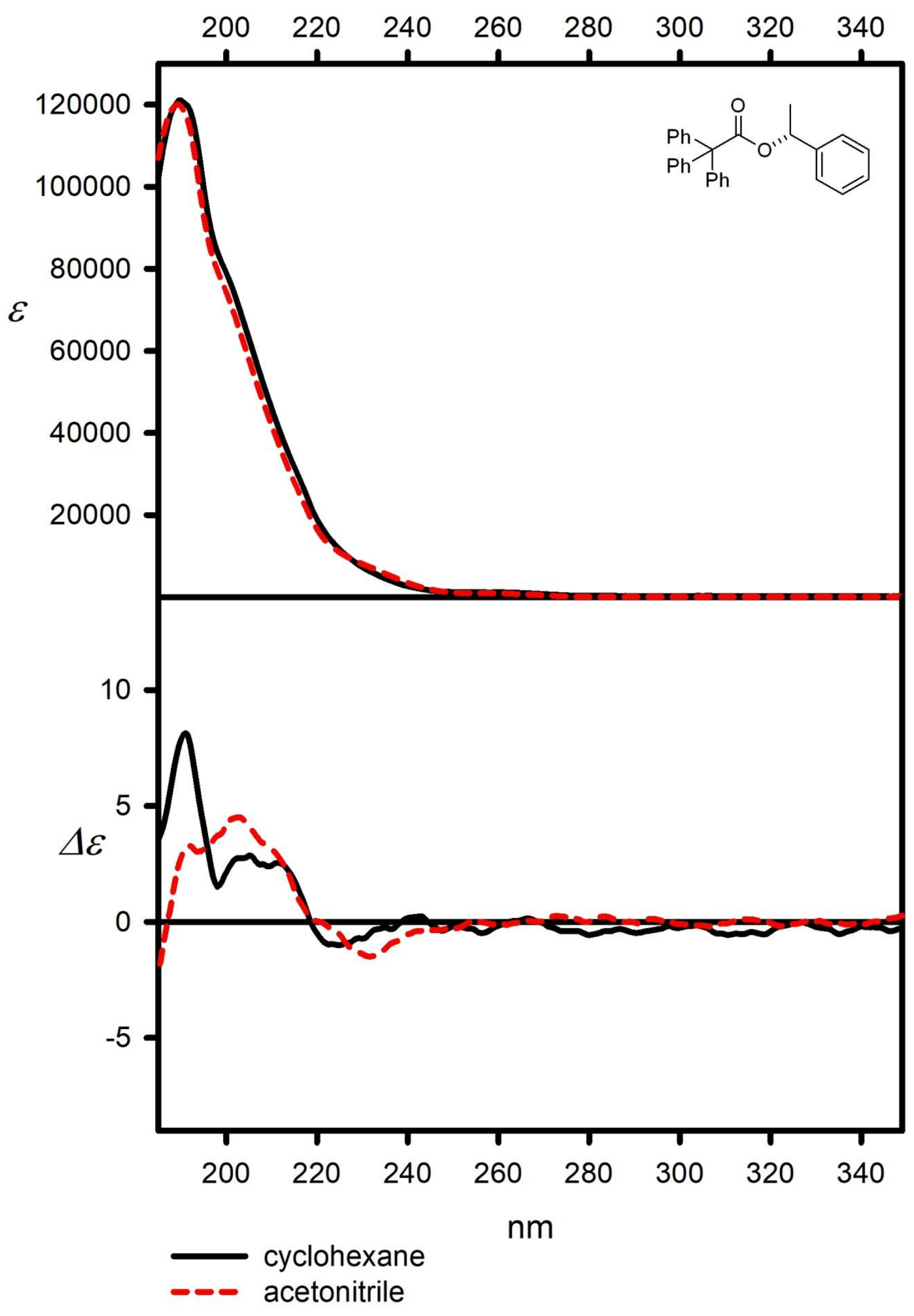


$\mathrm{nm}$

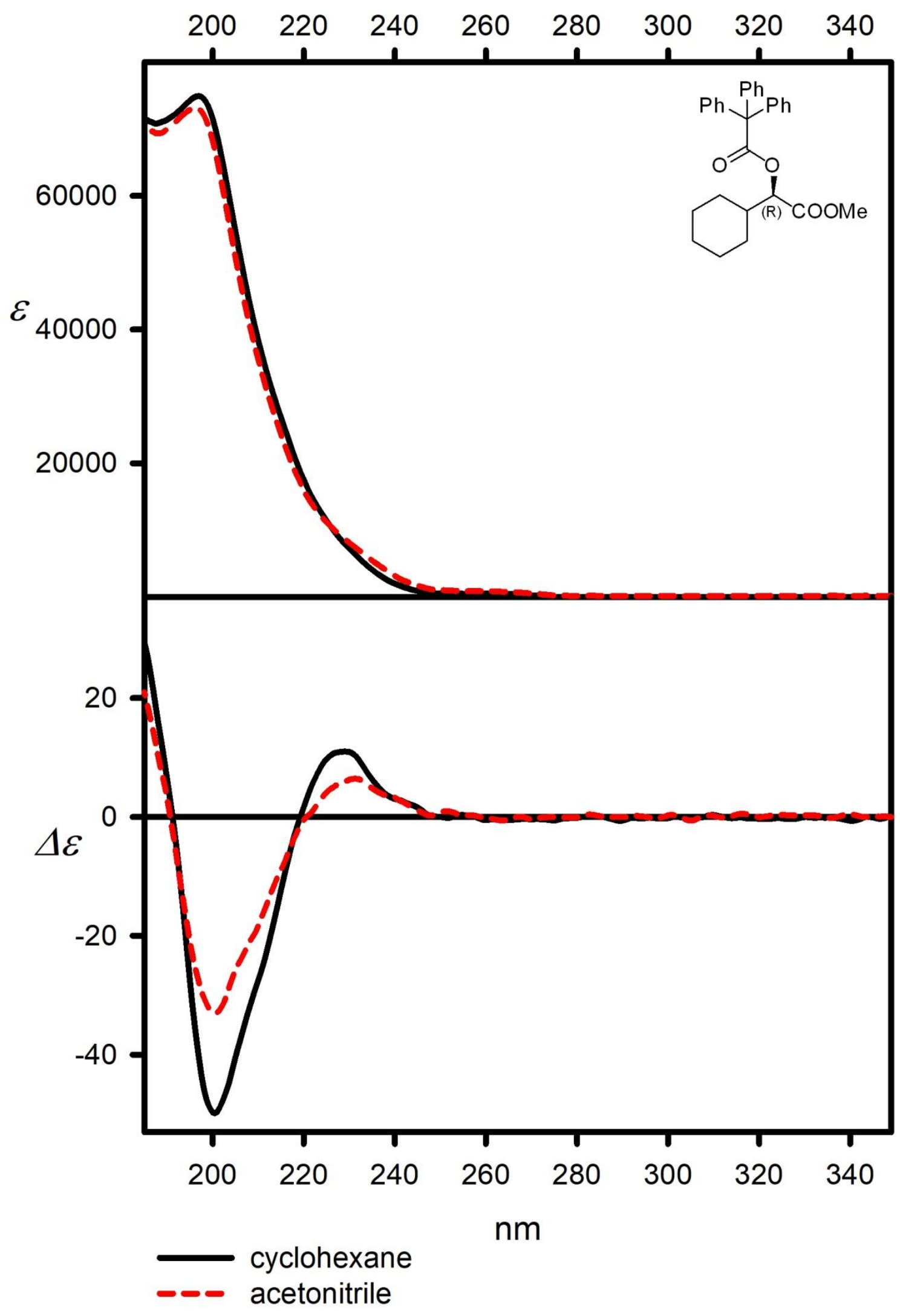


$\mathrm{nm}$

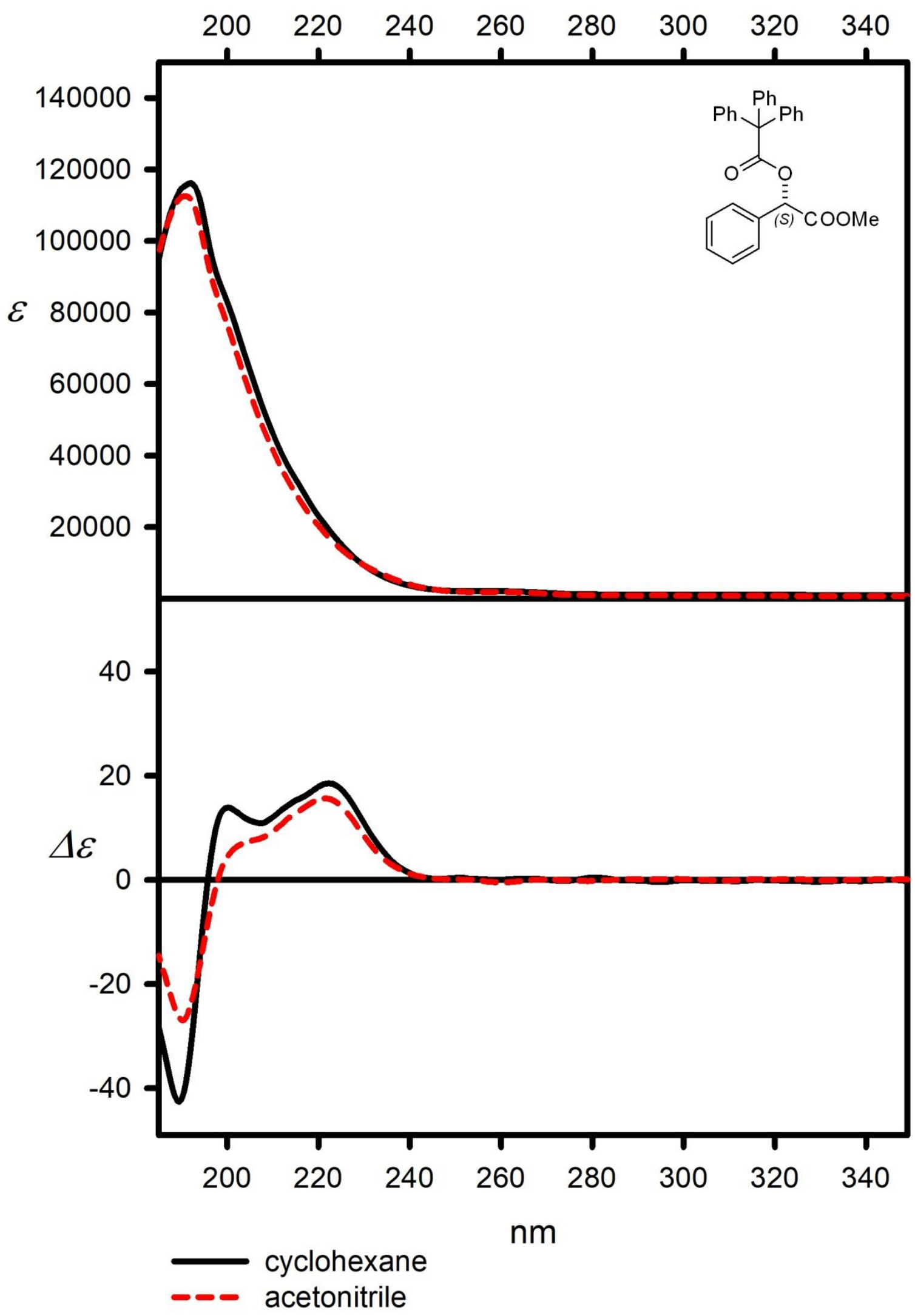


$\mathrm{nm}$

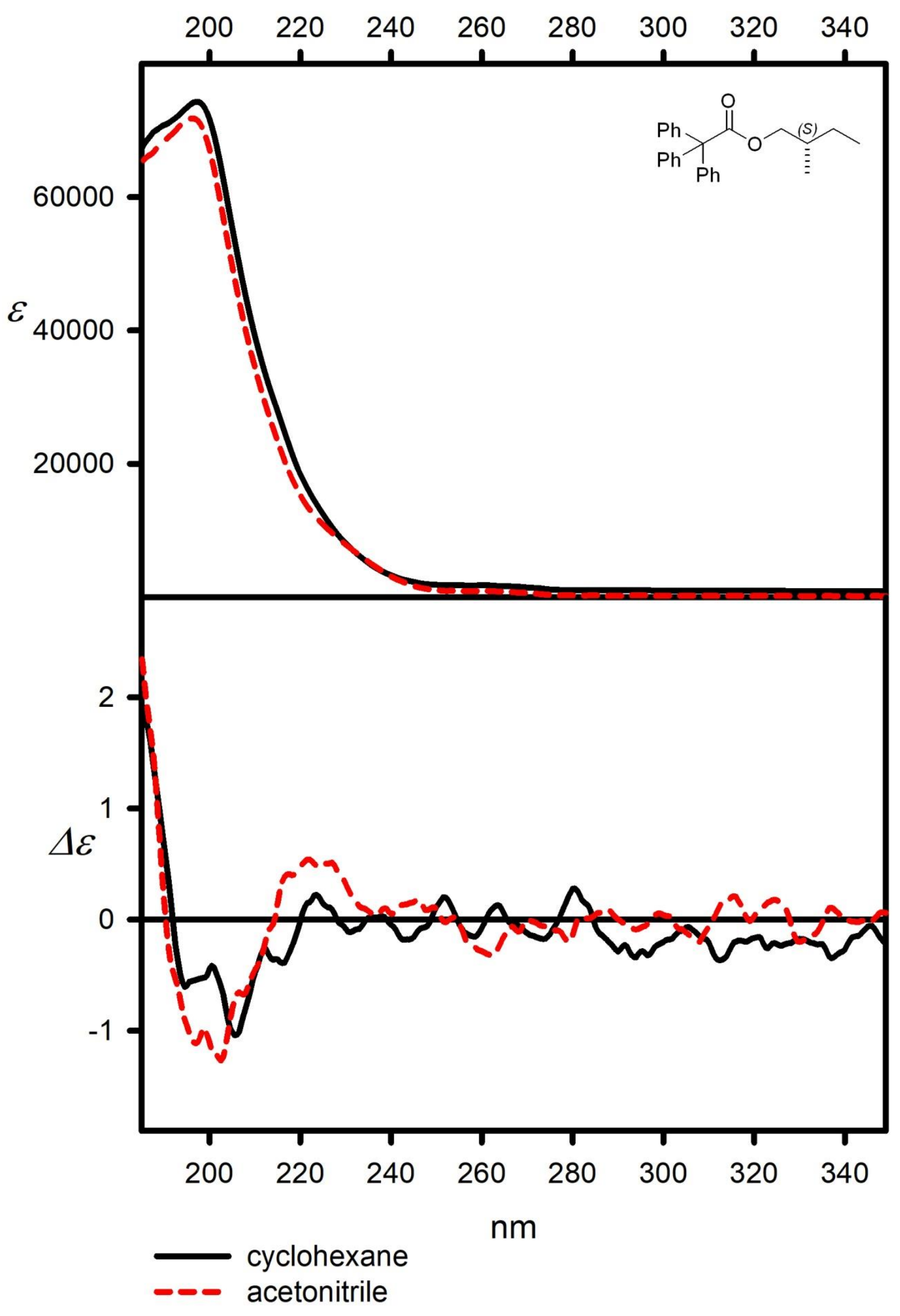


$\mathrm{nm}$

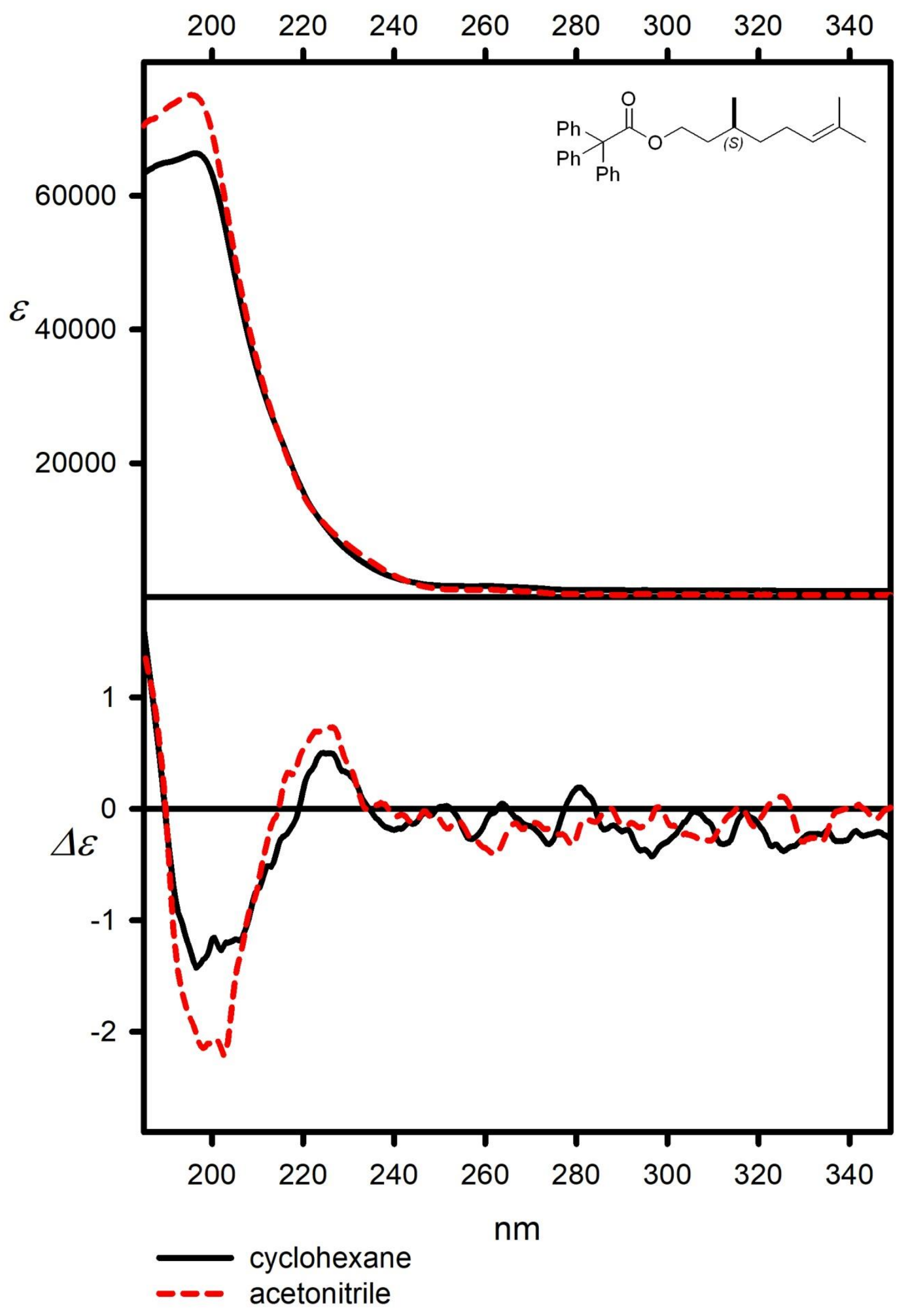


$\mathrm{nm}$

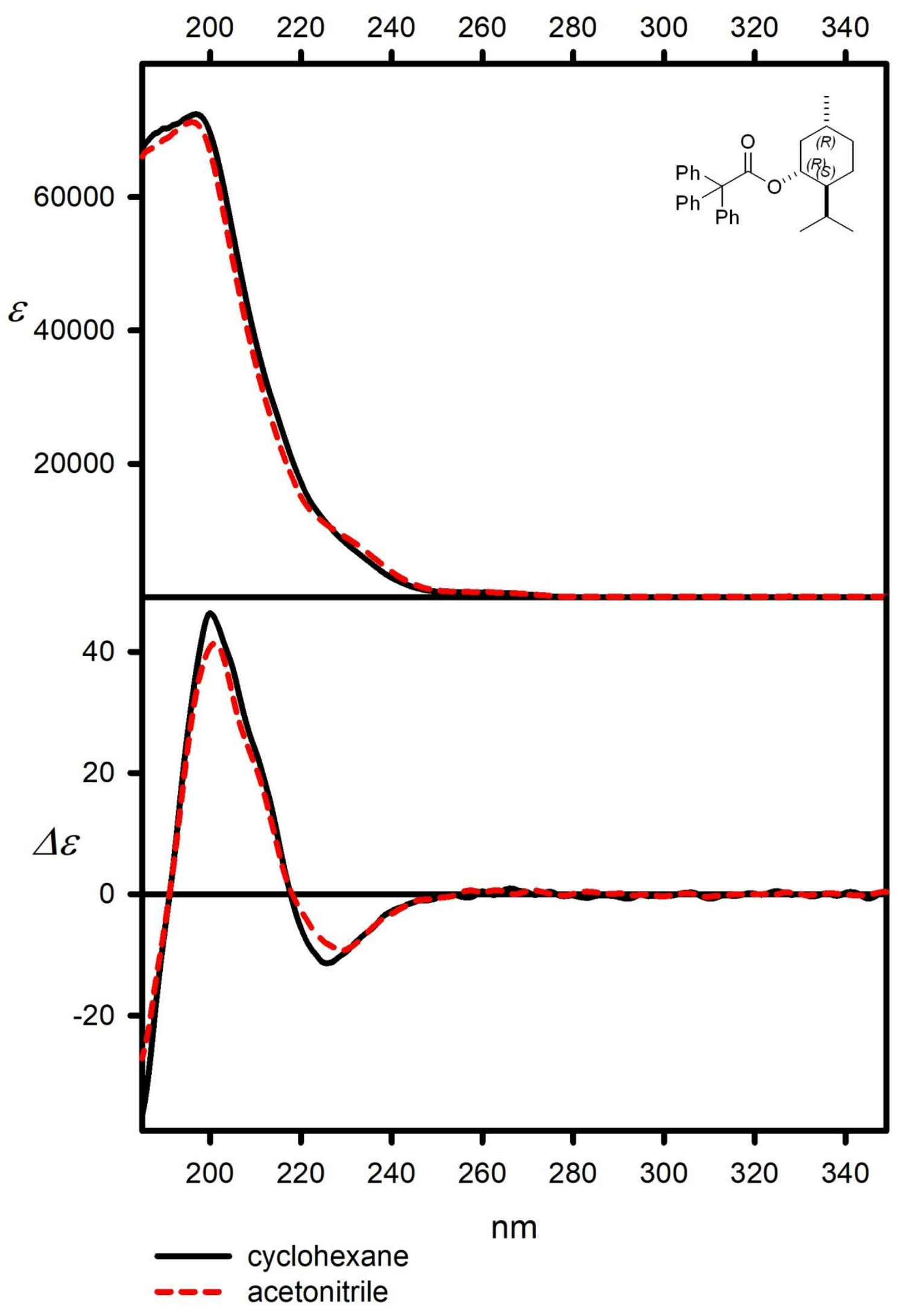


$\mathrm{nm}$

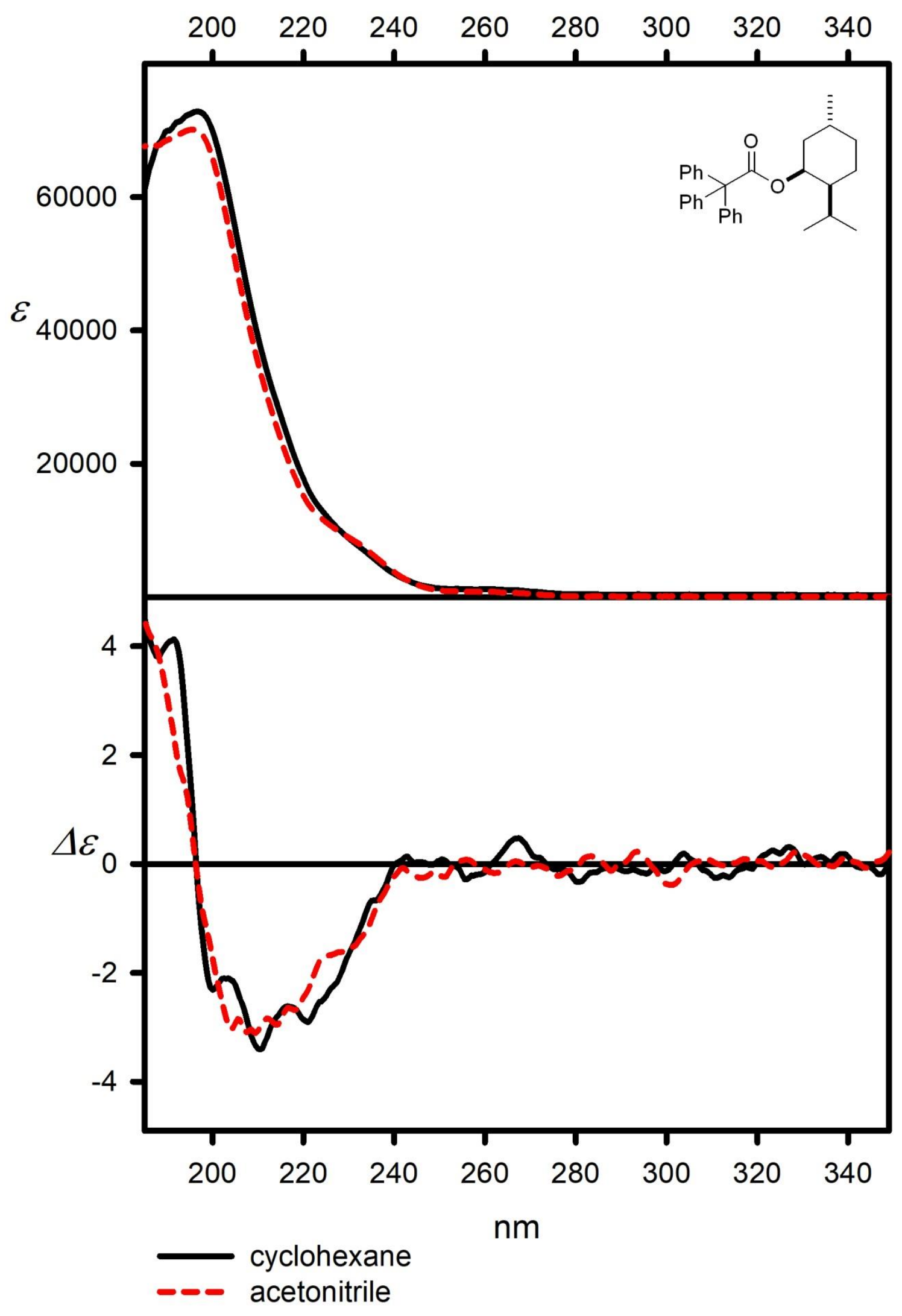


$\mathrm{nm}$

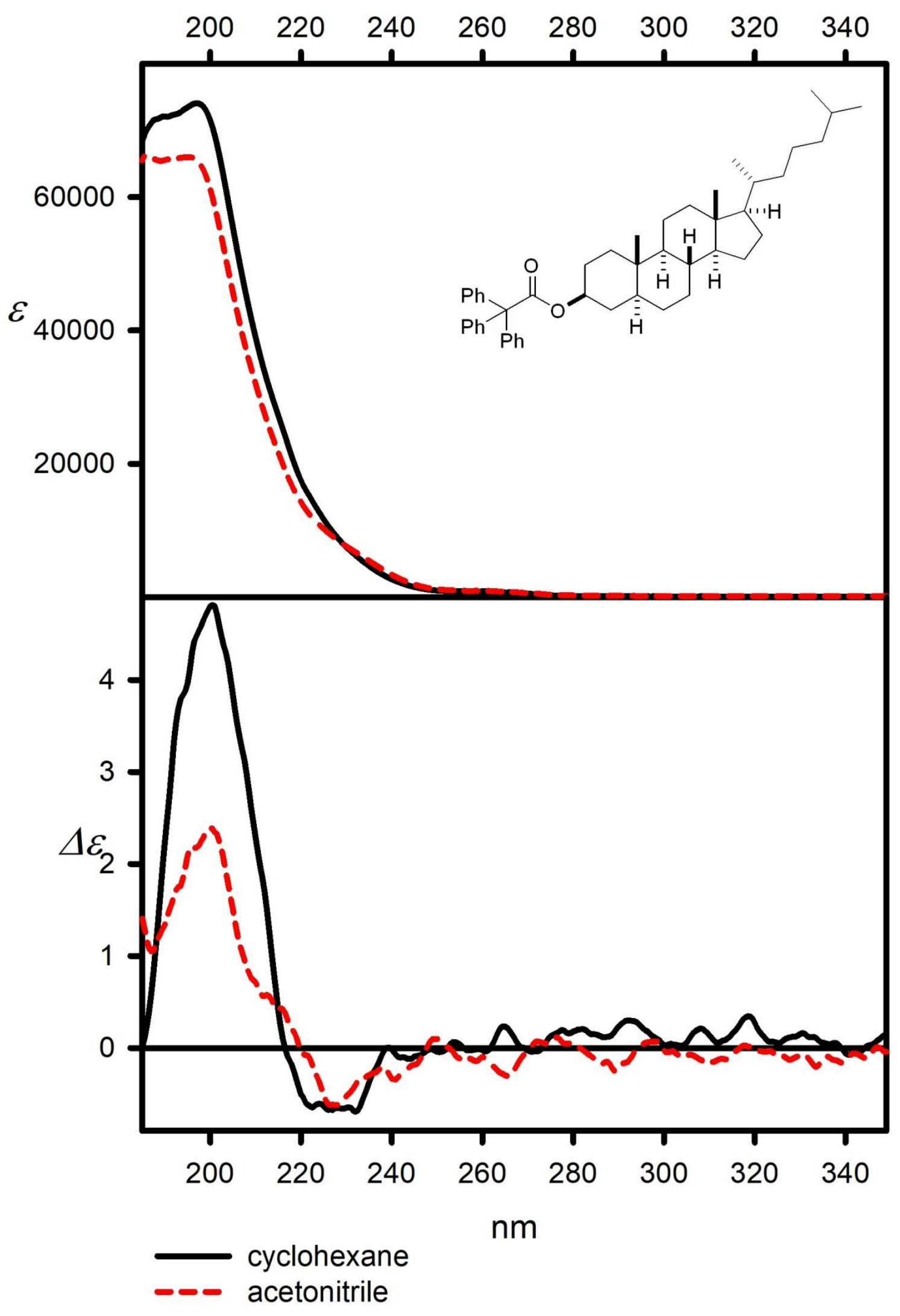


$\mathrm{nm}$

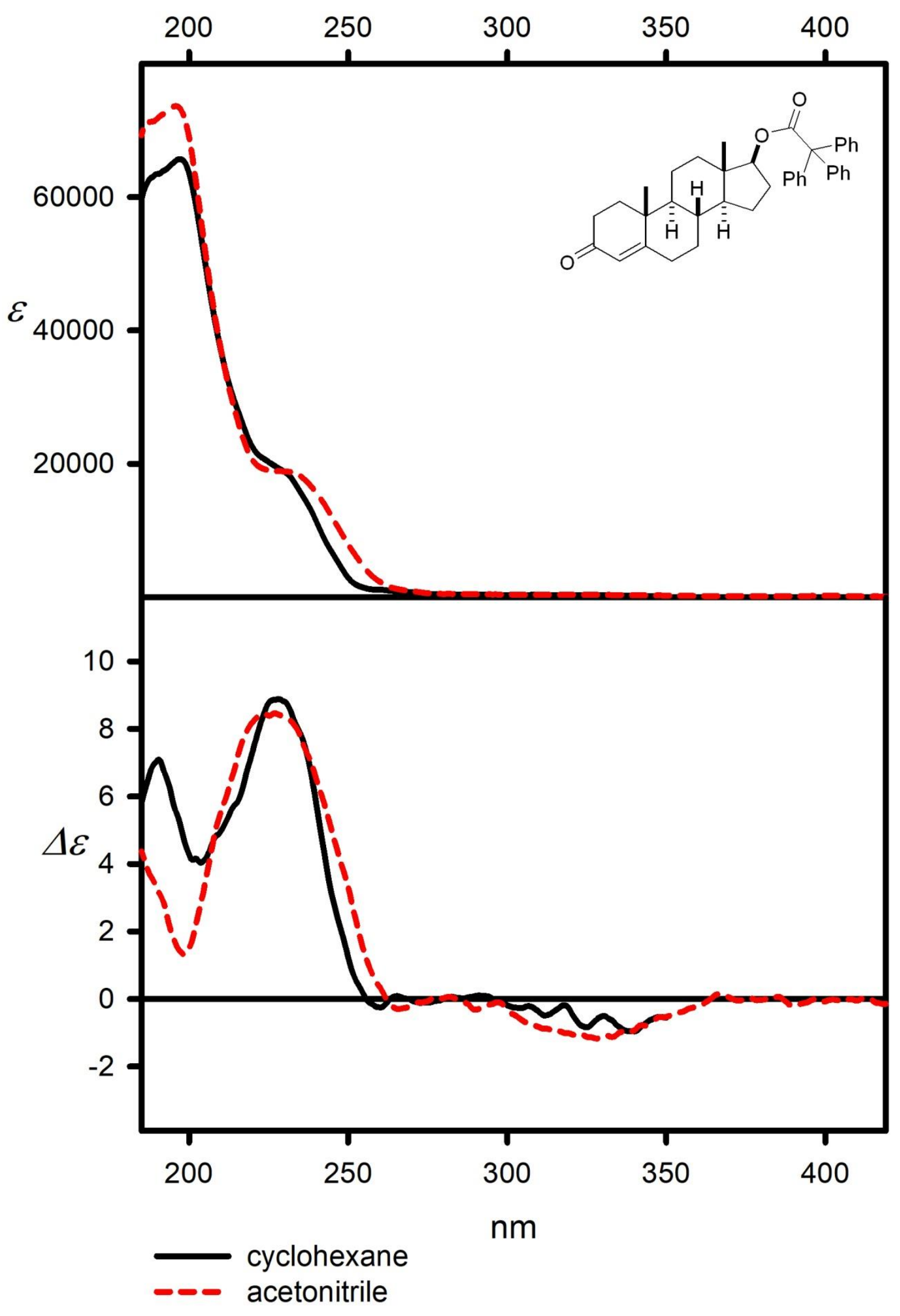


$\mathrm{nm}$

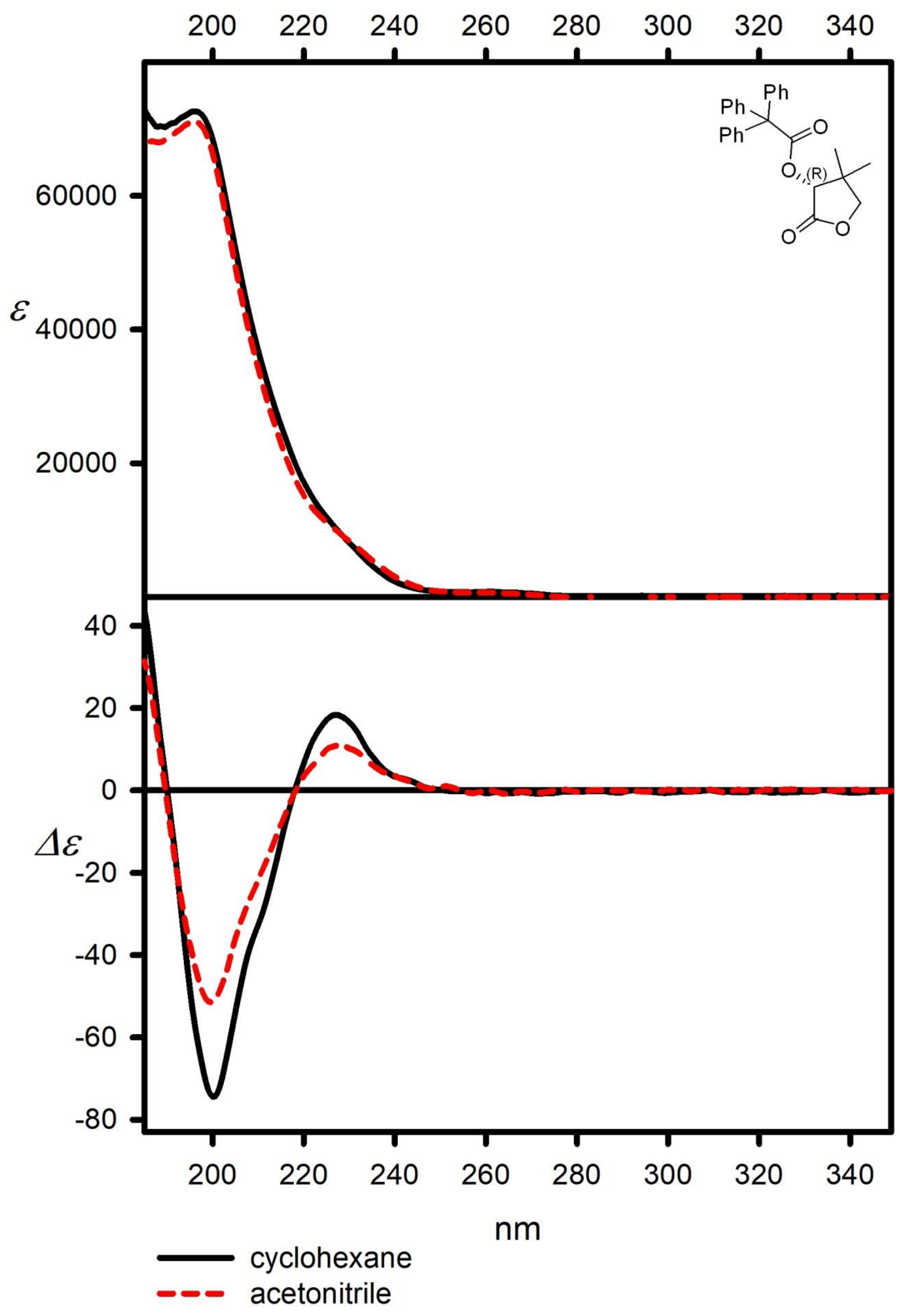


$\mathrm{nm}$

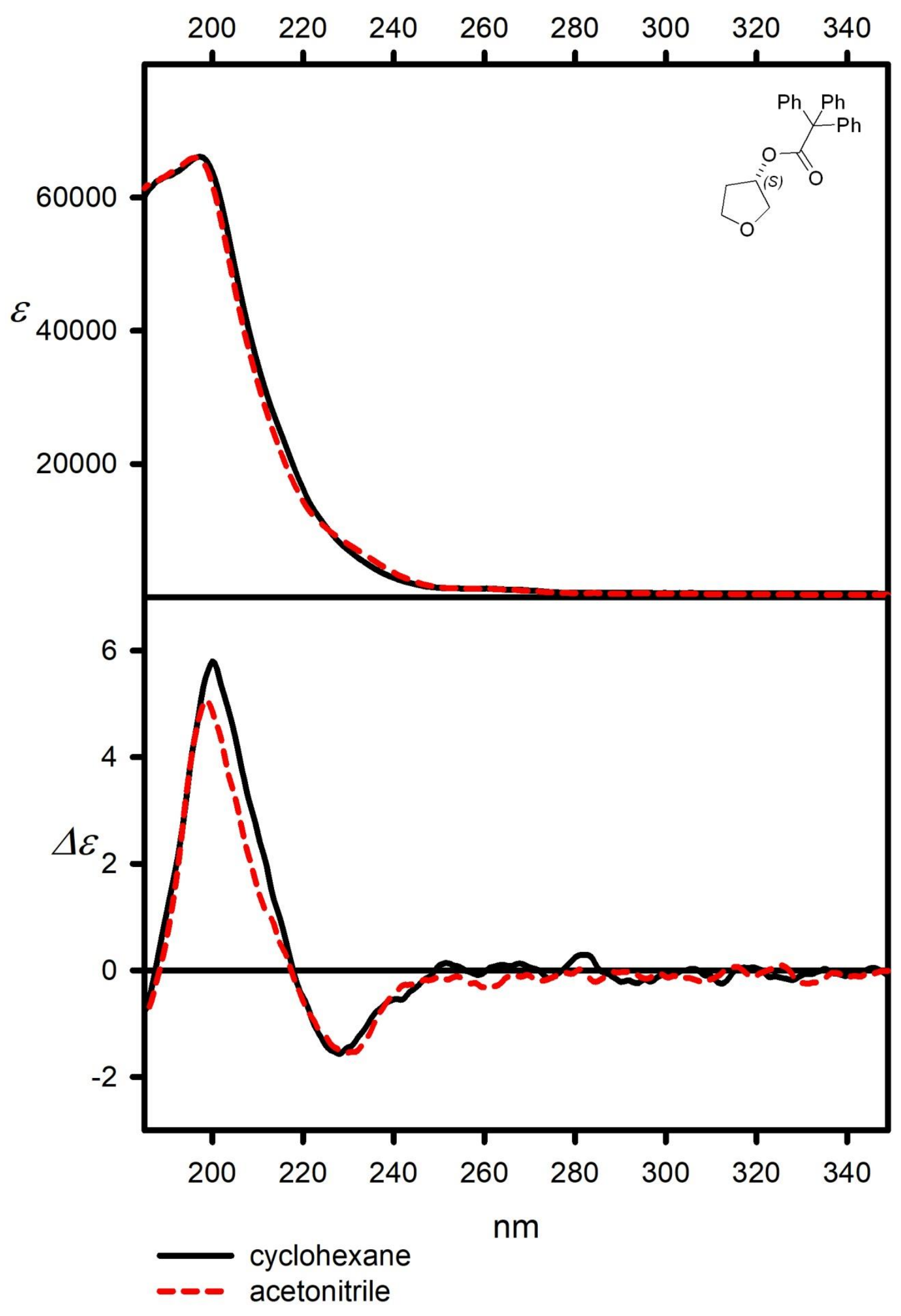


$\mathrm{nm}$

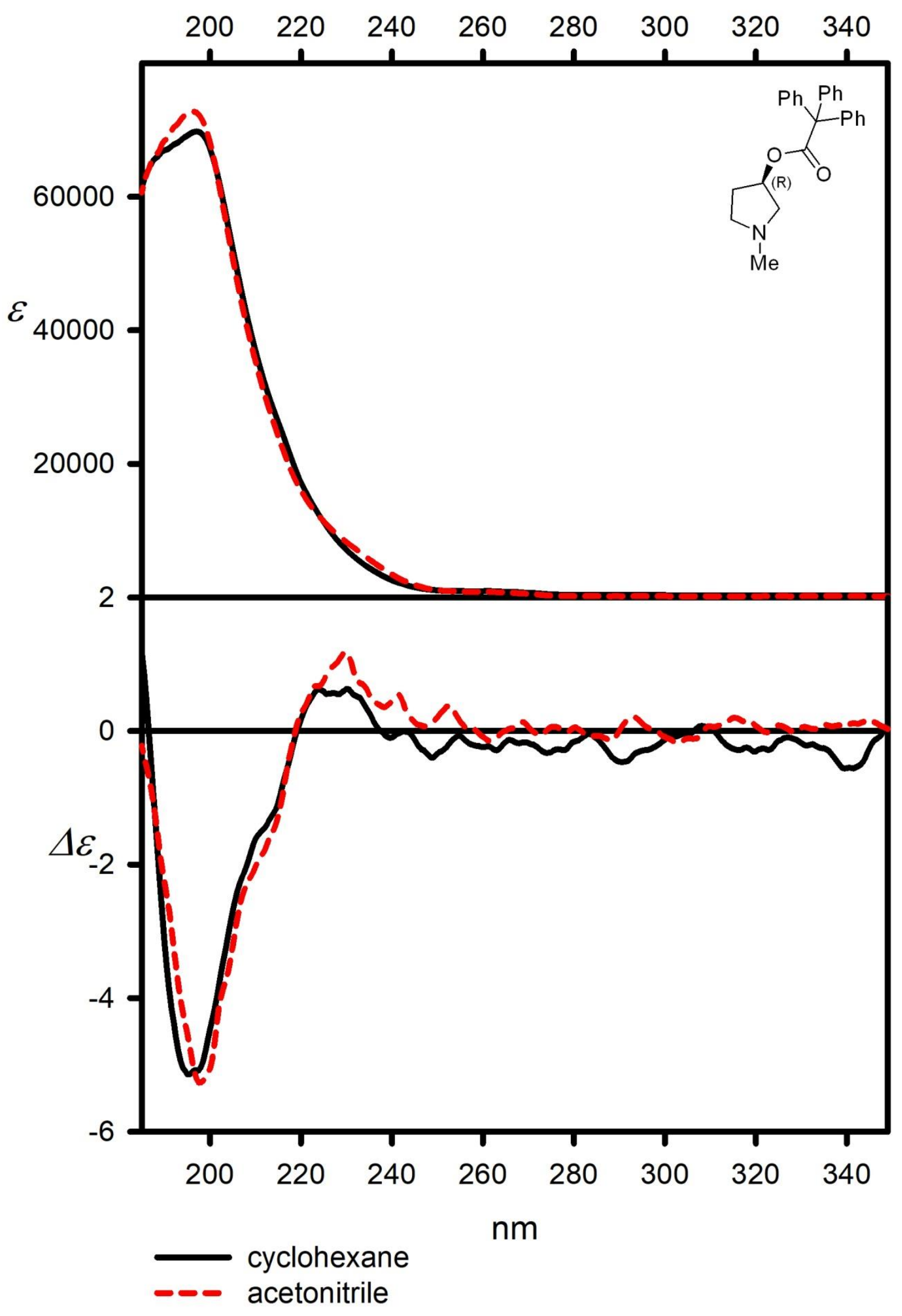


$\mathrm{nm}$

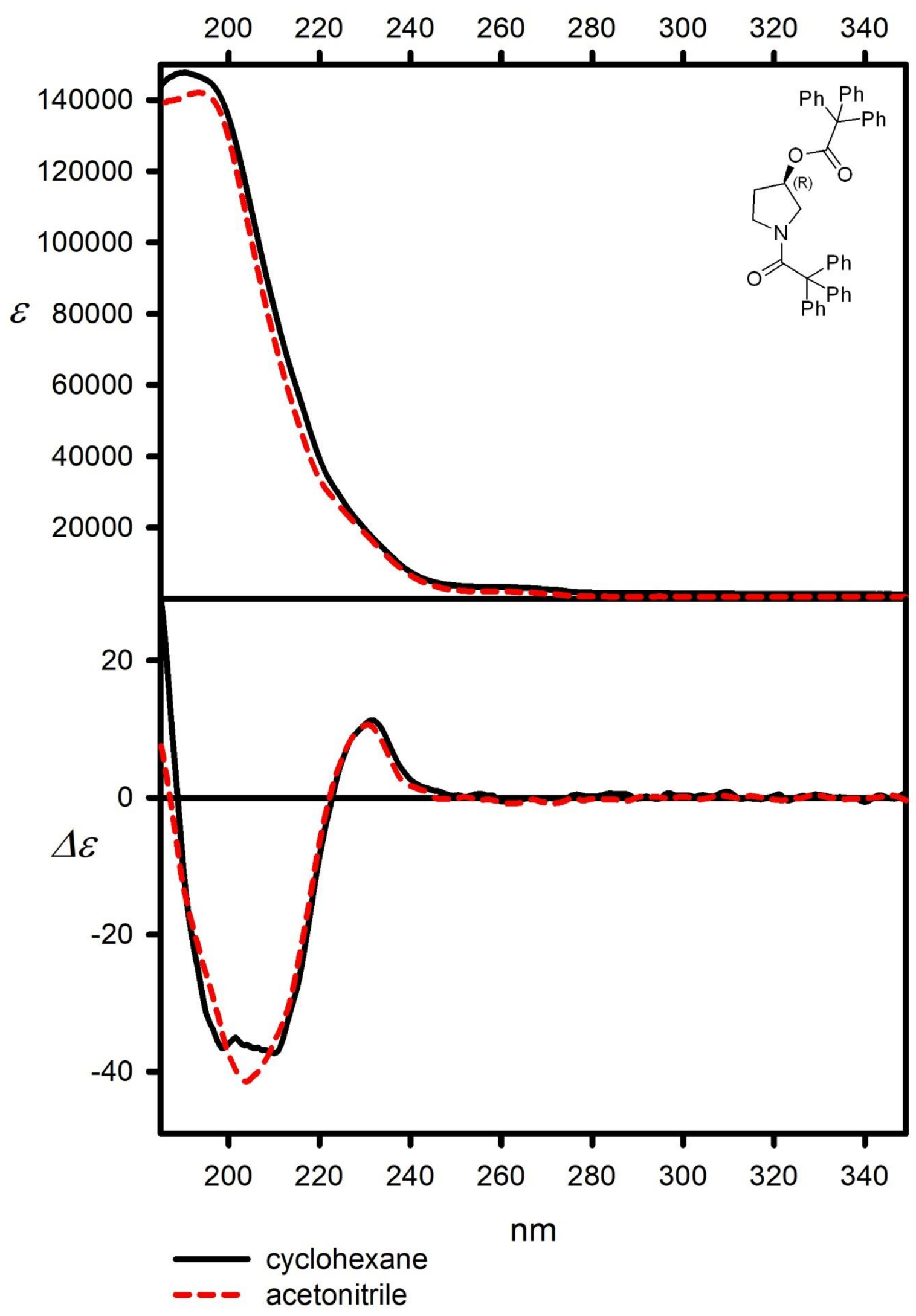


$\mathrm{nm}$

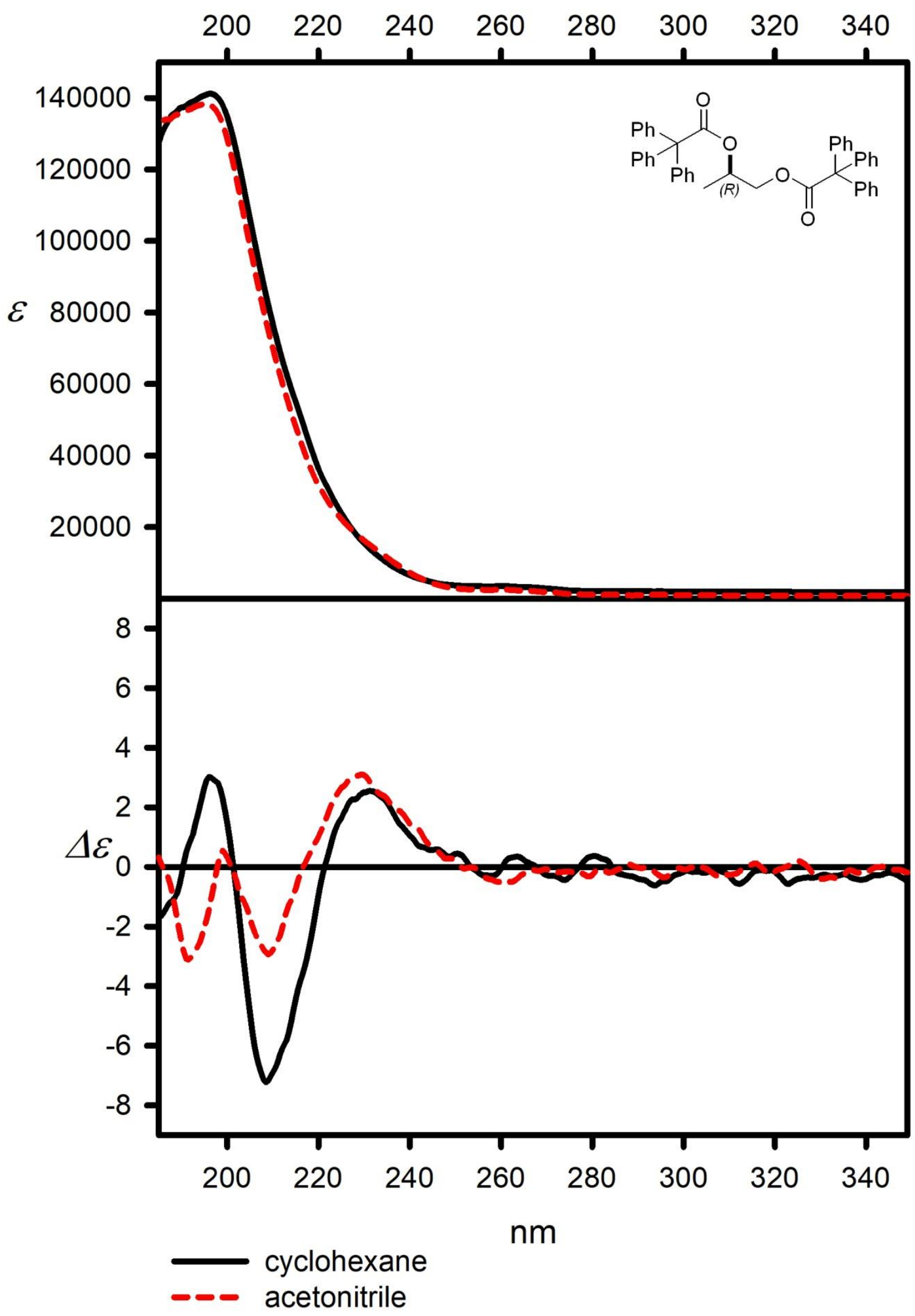


$\mathrm{nm}$

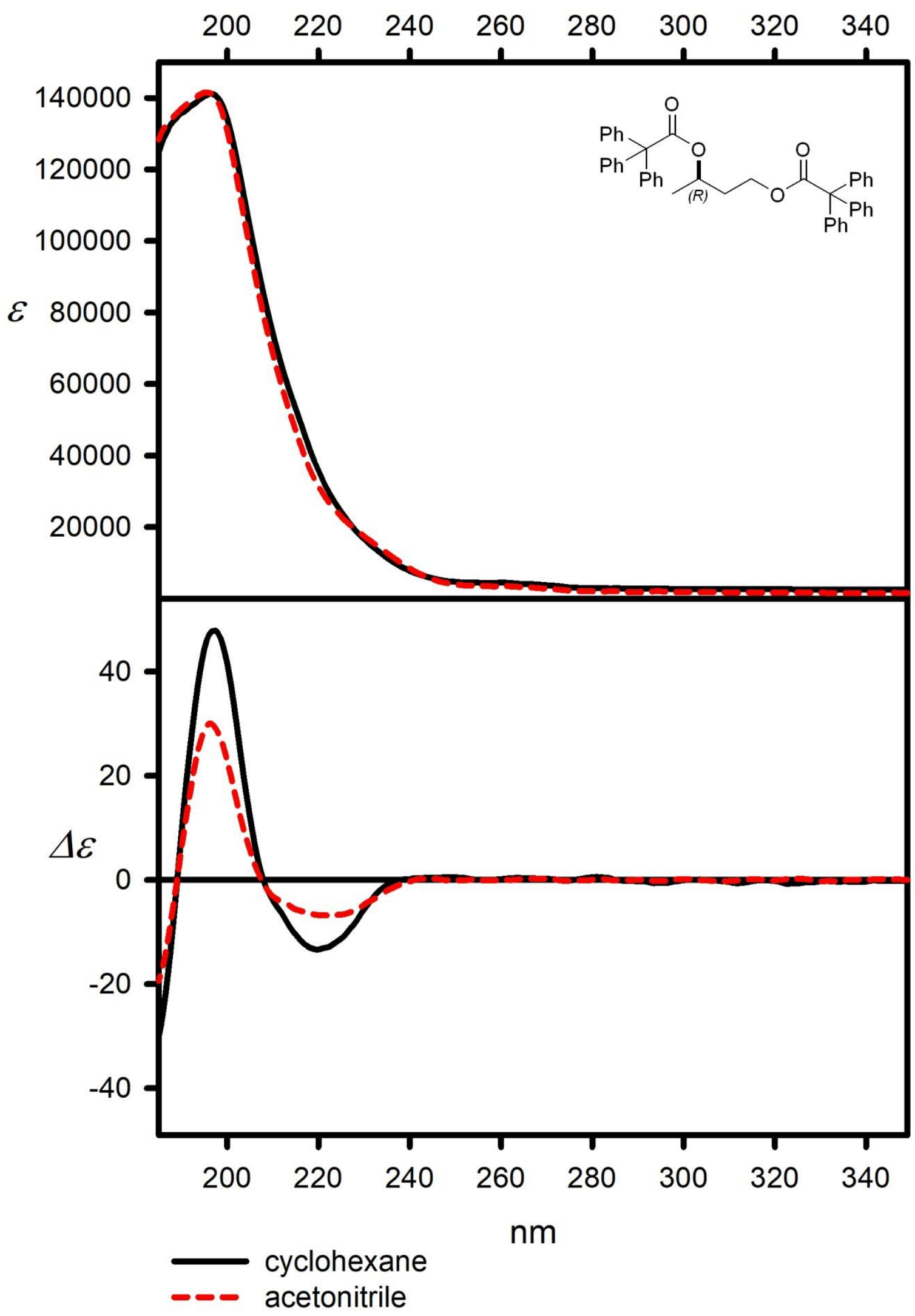


$\mathrm{nm}$

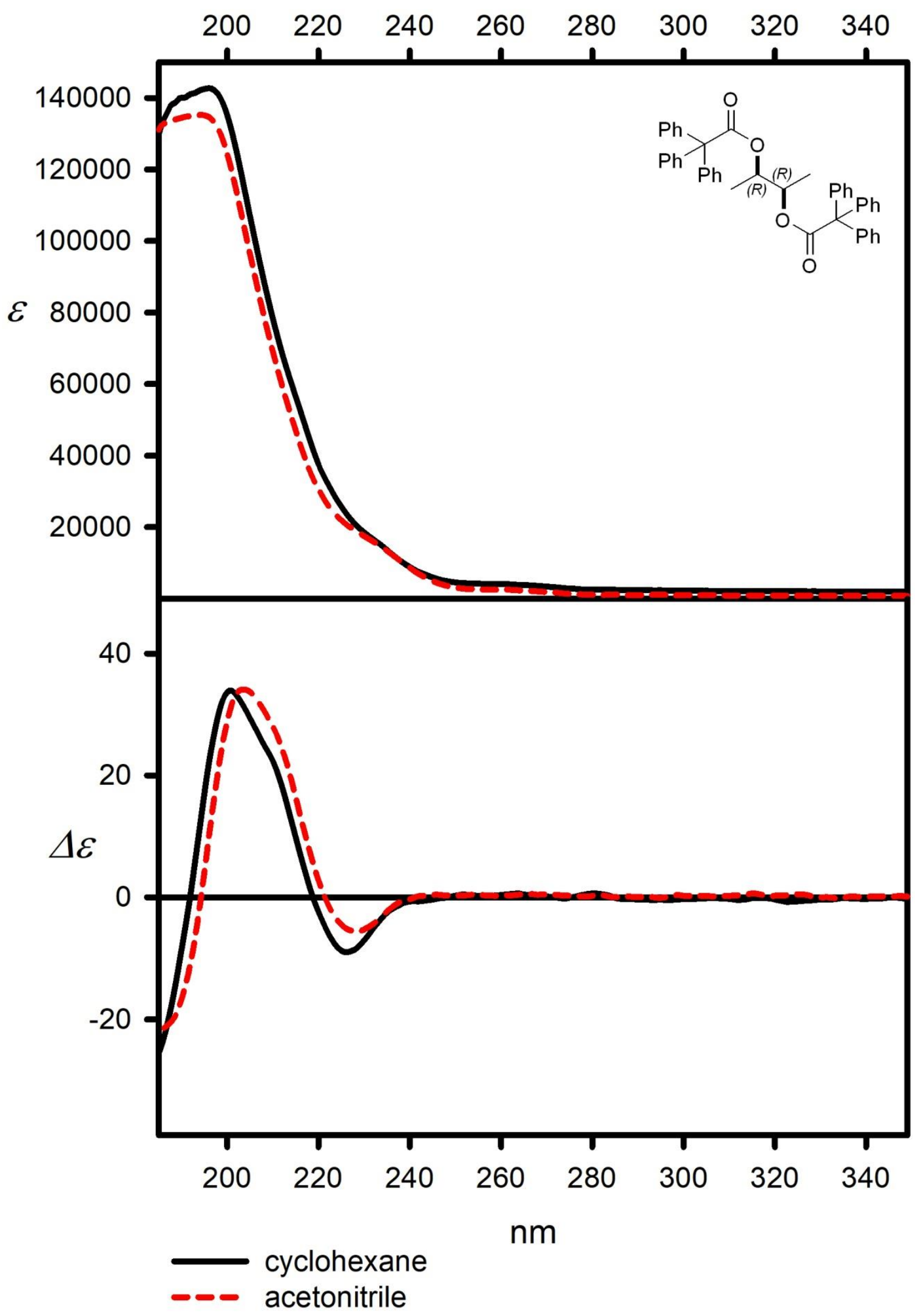


$\mathrm{nm}$

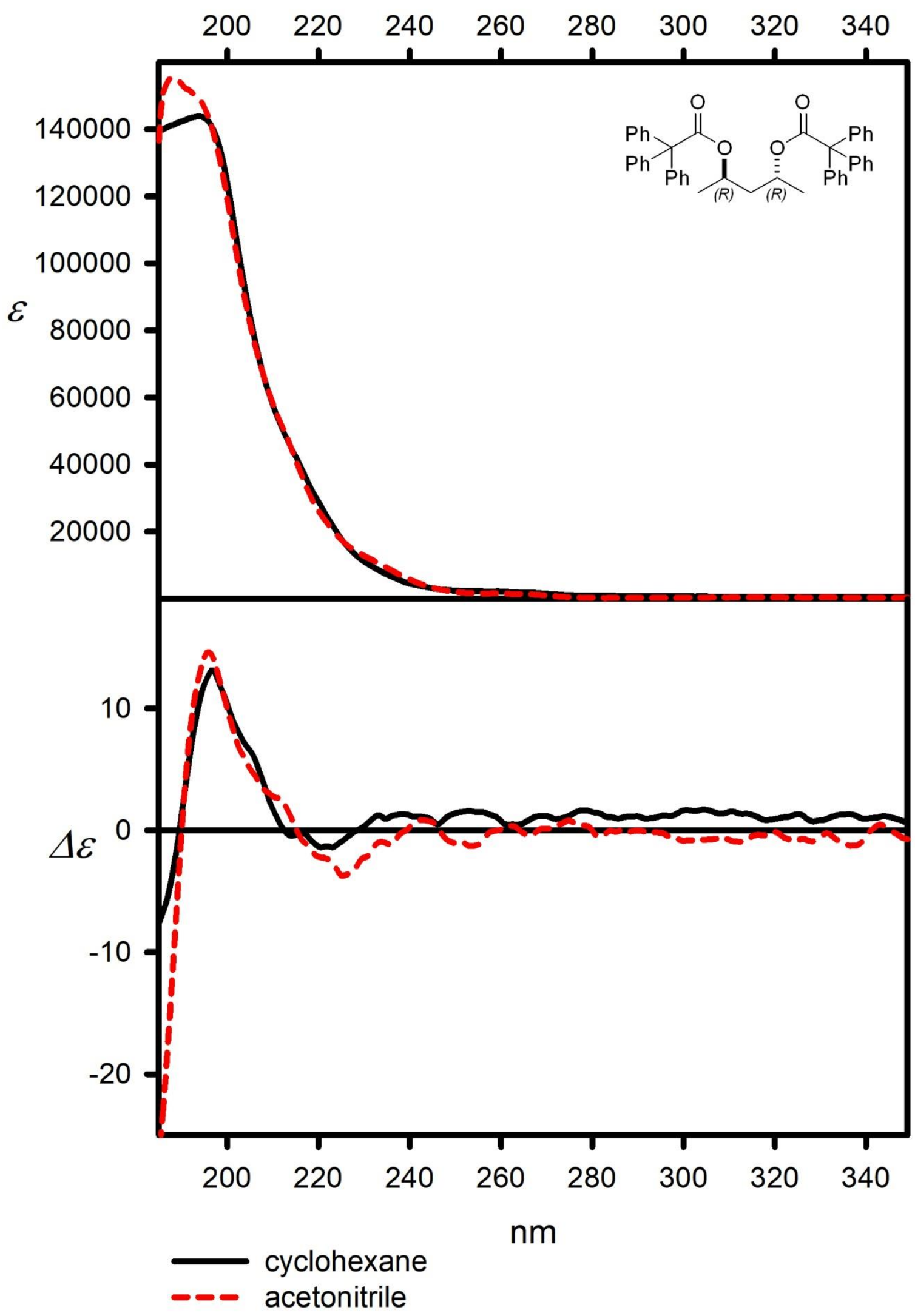


$\mathrm{nm}$

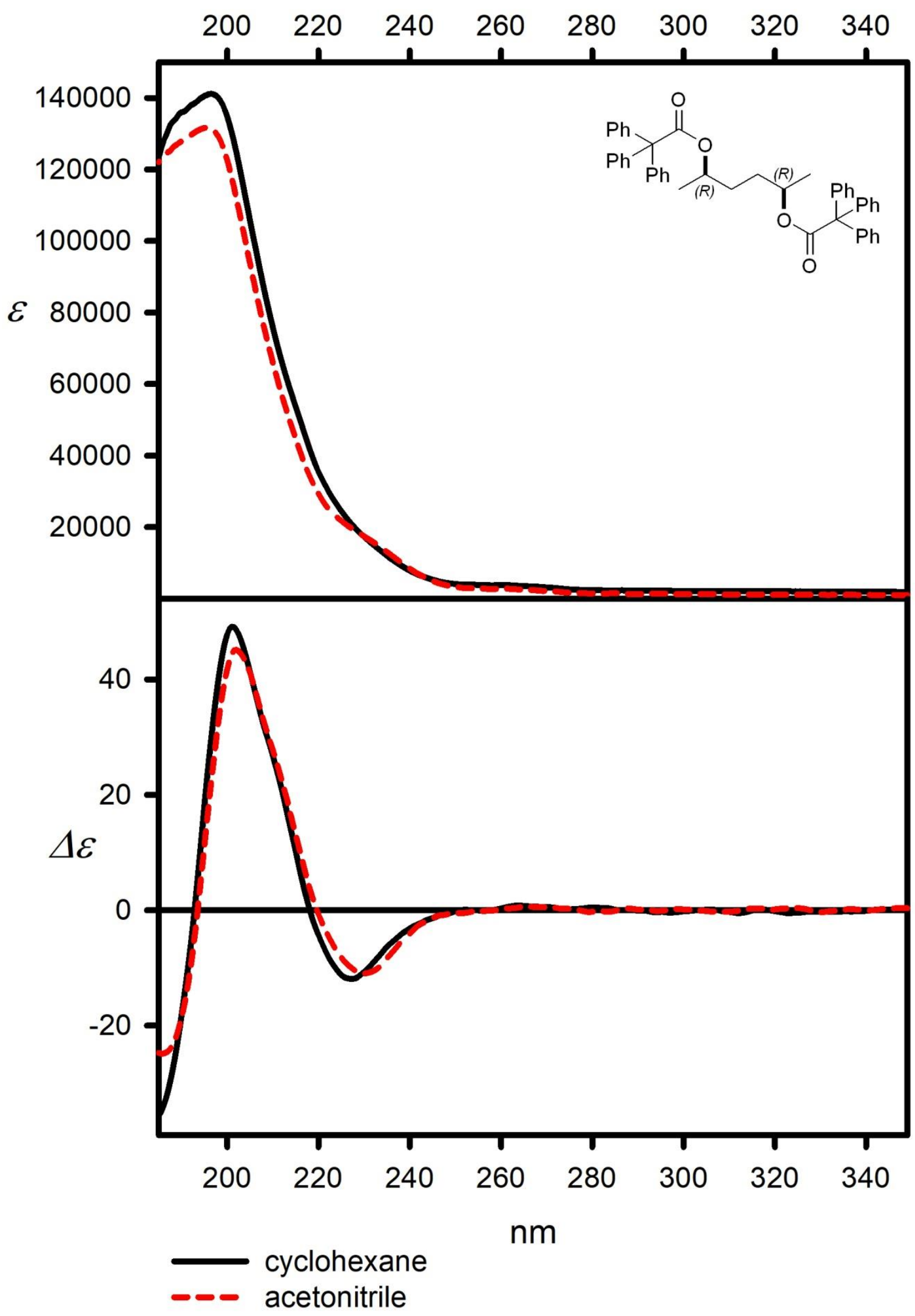




\section{Cartesian coordinates}

1 (optimized at the B3LYP/6-311++G(d,p) level)

\begin{tabular}{|c|c|c|c|c|}
\hline Conformer no & & & & \\
\hline 1 & 0 & 1.74300100 & -0.78681200 & -0.59855500 \\
\hline & C & 0.61085500 & -0.33759900 & -1.16640700 \\
\hline & C & 2.87194500 & -1.11236900 & -1.47491800 \\
\hline & $\mathrm{H}$ & 2.83814900 & -0.41258000 & -2.31319700 \\
\hline & C & 4.13583100 & -0.89758000 & -0.64855200 \\
\hline & $\mathrm{H}$ & 4.08648900 & -1.54025900 & 0.23761500 \\
\hline & C & 2.72349500 & -2.53916300 & -1.98922000 \\
\hline & $\mathrm{H}$ & 3.55630200 & -2.78049900 & -2.65522900 \\
\hline & $\mathrm{H}$ & 1.79767400 & -2.65650000 & -2.55478800 \\
\hline & C & 4.37633800 & 0.55614000 & -0.23573500 \\
\hline & $\mathrm{H}$ & 3.55812500 & 0.93415500 & 0.37964900 \\
\hline & 0 & 0.46559100 & -0.20848900 & -2.35685400 \\
\hline & C & -0.43253000 & 0.08220600 & -0.08528300 \\
\hline & C & -1.77723800 & 0.46253100 & -0.75299200 \\
\hline & C & -2.35126600 & -0.39298800 & -1.70289900 \\
\hline & C & -3.60334200 & -0.12585100 & -2.24580100 \\
\hline & C & -4.32170500 & 0.99931500 & -1.84260300 \\
\hline & C & -3.77202500 & 1.84774100 & -0.88751100 \\
\hline & C & -2.51262600 & 1.58046400 & -0.34835900 \\
\hline & C & 0.26170900 & 1.29288800 & 0.59189300 \\
\hline & C & 0.46134000 & 2.46114100 & -0.15850600 \\
\hline & C & 1.13561800 & 3.55331100 & 0.37956400 \\
\hline & C & 1.63322000 & 3.49937000 & 1.68108600 \\
\hline & C & 1.46088900 & 2.33725000 & 2.42724000 \\
\hline & C & 0.78824600 & 1.24133000 & 1.88488200 \\
\hline & C & -0.77041100 & -1.06803700 & 0.89352800 \\
\hline & C & -0.38941400 & -2.39471100 & 0.67530100 \\
\hline & C & -0.79869100 & -3.41339300 & 1.53758600 \\
\hline & C & -1.59757700 & -3.12637600 & 2.63881500 \\
\hline & C & -1.99577100 & -1.80872700 & 2.86290300 \\
\hline & C & -1.59411000 & -0.79785600 & 1.99647800 \\
\hline & $\mathrm{H}$ & -1.81758700 & -1.27609900 & -2.02774000 \\
\hline & $\mathrm{H}$ & -4.01898700 & -0.80028500 & -2.98636400 \\
\hline & $\mathrm{H}$ & -5.29791400 & 1.20771200 & -2.26593700 \\
\hline & $\mathrm{H}$ & -4.31881000 & 2.72366500 & -0.55580600 \\
\hline & $\mathrm{H}$ & -2.10816100 & 2.25775200 & 0.39268600 \\
\hline & $\mathrm{H}$ & 0.08473500 & 2.51563300 & -1.17341900 \\
\hline & $\mathrm{H}$ & 1.27223100 & 4.44652900 & -0.22000300 \\
\hline & $\mathrm{H}$ & 2.15505700 & 4.35054400 & 2.10387000 \\
\hline & $\mathrm{H}$ & 1.85464100 & 2.27446000 & 3.43580300 \\
\hline & $\mathrm{H}$ & 0.68448200 & 0.34148800 & 2.47566500 \\
\hline & $\mathrm{H}$ & 0.23539100 & -2.65419200 & -0.16769300 \\
\hline & $\mathrm{H}$ & -0.48729700 & -4.43350300 & 1.34127400 \\
\hline & $\mathrm{H}$ & -1.91339000 & -3.91695800 & 3.31006800 \\
\hline & $\mathrm{H}$ & -2.62945200 & -1.56725400 & 3.70918000 \\
\hline & $\mathrm{H}$ & -1.93627500 & 0.21422400 & 2.17547700 \\
\hline
\end{tabular}




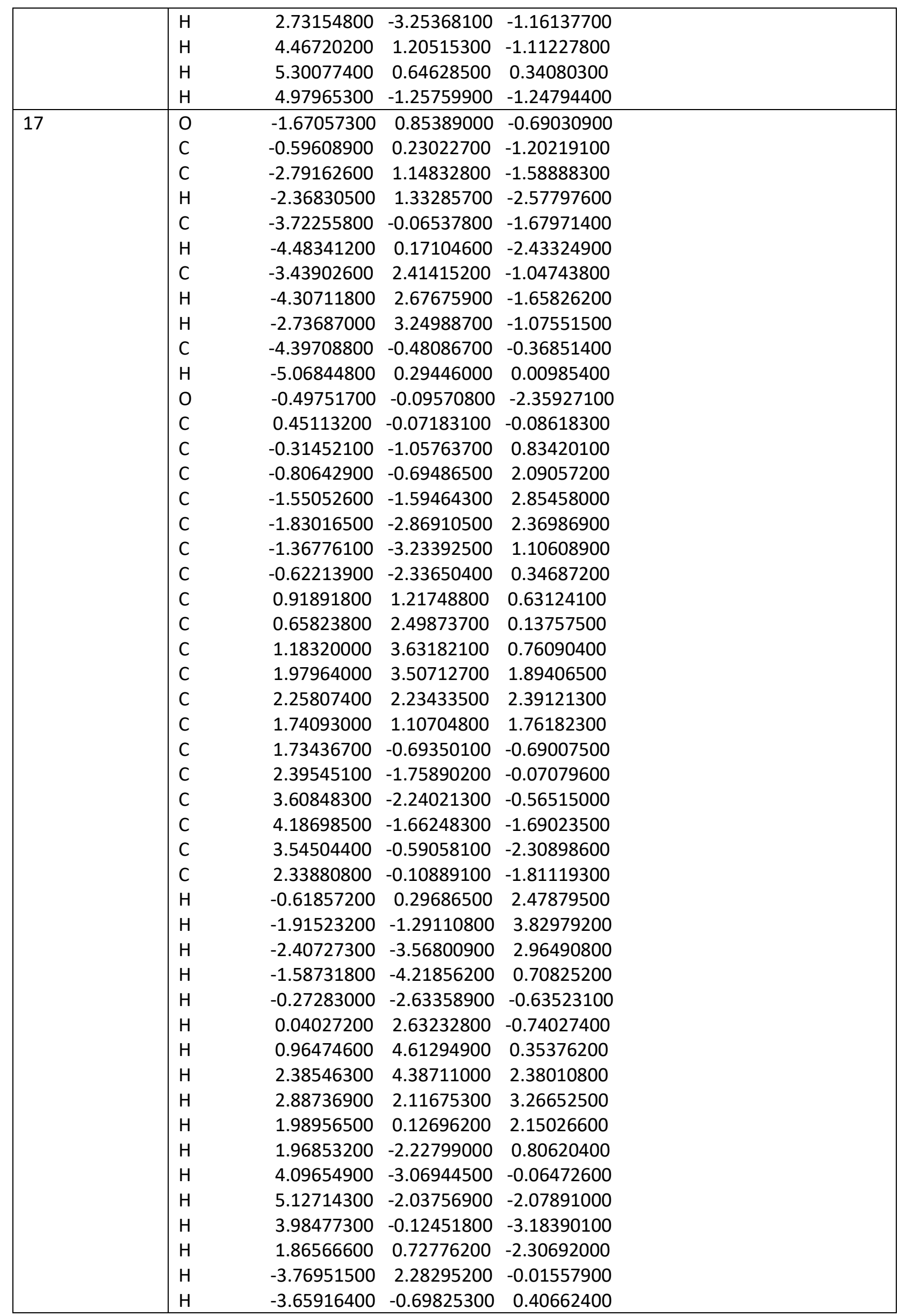




\begin{tabular}{|c|c|c|c|c|}
\hline & $\begin{array}{l}\mathrm{H} \\
\mathrm{H}\end{array}$ & $\begin{array}{l}-4.99425500 \\
-3.14510200\end{array}$ & $\begin{array}{l}-1.38343000 \\
-0.90129300\end{array}$ & $\begin{array}{l}-0.52161300 \\
-2.08416500\end{array}$ \\
\hline \multirow[t]{50}{*}{33} & 0 & 1.78253100 & 0.23065700 & -0.82087300 \\
\hline & C & 0.54257000 & -0.01534200 & -1.27062000 \\
\hline & C & 2.87357700 & 0.27696900 & -1.79938600 \\
\hline & $\mathrm{H}$ & 2.46340500 & 0.70564100 & -2.71610800 \\
\hline & C & 3.92964100 & 1.22225500 & -1.22481900 \\
\hline & $\mathrm{H}$ & 4.69804900 & 1.35448300 & -1.99528800 \\
\hline & C & 3.36175100 & -1.13870000 & -2.08186700 \\
\hline & $\mathrm{H}$ & 4.21460500 & -1.10471100 & -2.76604500 \\
\hline & $\mathrm{H}$ & 2.57331600 & -1.72709200 & -2.55320200 \\
\hline & C & 4.57721600 & 0.77376900 & 0.08929200 \\
\hline & $\mathrm{H}$ & 3.82360100 & 0.60217100 & 0.86062900 \\
\hline & 0 & 0.28378400 & -0.19718200 & -2.42364100 \\
\hline & C & -0.47940000 & -0.05532000 & -0.09002800 \\
\hline & C & -0.68929500 & 1.36501000 & 0.49495700 \\
\hline & C & -0.01369200 & 2.49835200 & 0.03535400 \\
\hline & C & -0.27834900 & 3.76099200 & 0.57043300 \\
\hline & C & -1.22031100 & 3.91481000 & 1.58113100 \\
\hline & C & -1.90826000 & 2.79324700 & 2.04458700 \\
\hline & C & -1.65211600 & 1.53959800 & 1.50065800 \\
\hline & C & 0.13495300 & -1.04416200 & 0.93160900 \\
\hline & C & 0.52866900 & -0.67137400 & 2.21749500 \\
\hline & C & 1.10912100 & -1.59844700 & 3.08572600 \\
\hline & C & 1.31795100 & -2.91201500 & 2.67901100 \\
\hline & C & 0.94707800 & -3.29234800 & 1.38889600 \\
\hline & C & 0.36595500 & -2.36783300 & 0.52772800 \\
\hline & C & -1.86445300 & -0.52359600 & -0.60596900 \\
\hline & C & -2.46629800 & 0.14856900 & -1.67958800 \\
\hline & C & -3.73624700 & -0.20163300 & -2.12371700 \\
\hline & C & -4.44668300 & -1.22526800 & -1.49624600 \\
\hline & C & -3.87100100 & -1.88611500 & -0.41743600 \\
\hline & C & -2.59230700 & -1.53745100 & 0.02301400 \\
\hline & $\mathrm{H}$ & 0.73156000 & 2.41509800 & -0.74378300 \\
\hline & $\mathrm{H}$ & 0.25807000 & 4.62315600 & 0.18964500 \\
\hline & $\mathrm{H}$ & -1.42413700 & 4.89466000 & 1.99789400 \\
\hline & $\mathrm{H}$ & -2.65356300 & 2.89525500 & 2.82568500 \\
\hline & $\mathrm{H}$ & -2.21520200 & 0.68489900 & 1.85601000 \\
\hline & $\mathrm{H}$ & 0.39575200 & 0.34888900 & 2.55060200 \\
\hline & $\mathrm{H}$ & 1.40359400 & -1.28360900 & 4.08091600 \\
\hline & $\mathrm{H}$ & 1.76873100 & -3.63088800 & 3.35397800 \\
\hline & $\mathrm{H}$ & 1.11050600 & -4.31022100 & 1.05221000 \\
\hline & $\mathrm{H}$ & 0.08194500 & -2.68096700 & -0.47132300 \\
\hline & $\mathrm{H}$ & -1.93908400 & 0.95185100 & -2.17595000 \\
\hline & $\mathrm{H}$ & -4.17348100 & 0.32962000 & -2.96193900 \\
\hline & $\mathrm{H}$ & -5.43803100 & -1.49656500 & -1.84174500 \\
\hline & $\mathrm{H}$ & -4.41226100 & -2.67626400 & 0.09147300 \\
\hline & $\mathrm{H}$ & -2.17266600 & -2.06402100 & 0.86958900 \\
\hline & $\mathrm{H}$ & 3.67449600 & -1.64116300 & -1.16471100 \\
\hline & $\mathrm{H}$ & 5.26359200 & 1.54227000 & 0.45428600 \\
\hline & $\mathrm{H}$ & 5.15295900 & -0.14709200 & -0.03259800 \\
\hline & $\mathrm{H}$ & 3.46332000 & 2.20299600 & -1.08538700 \\
\hline
\end{tabular}




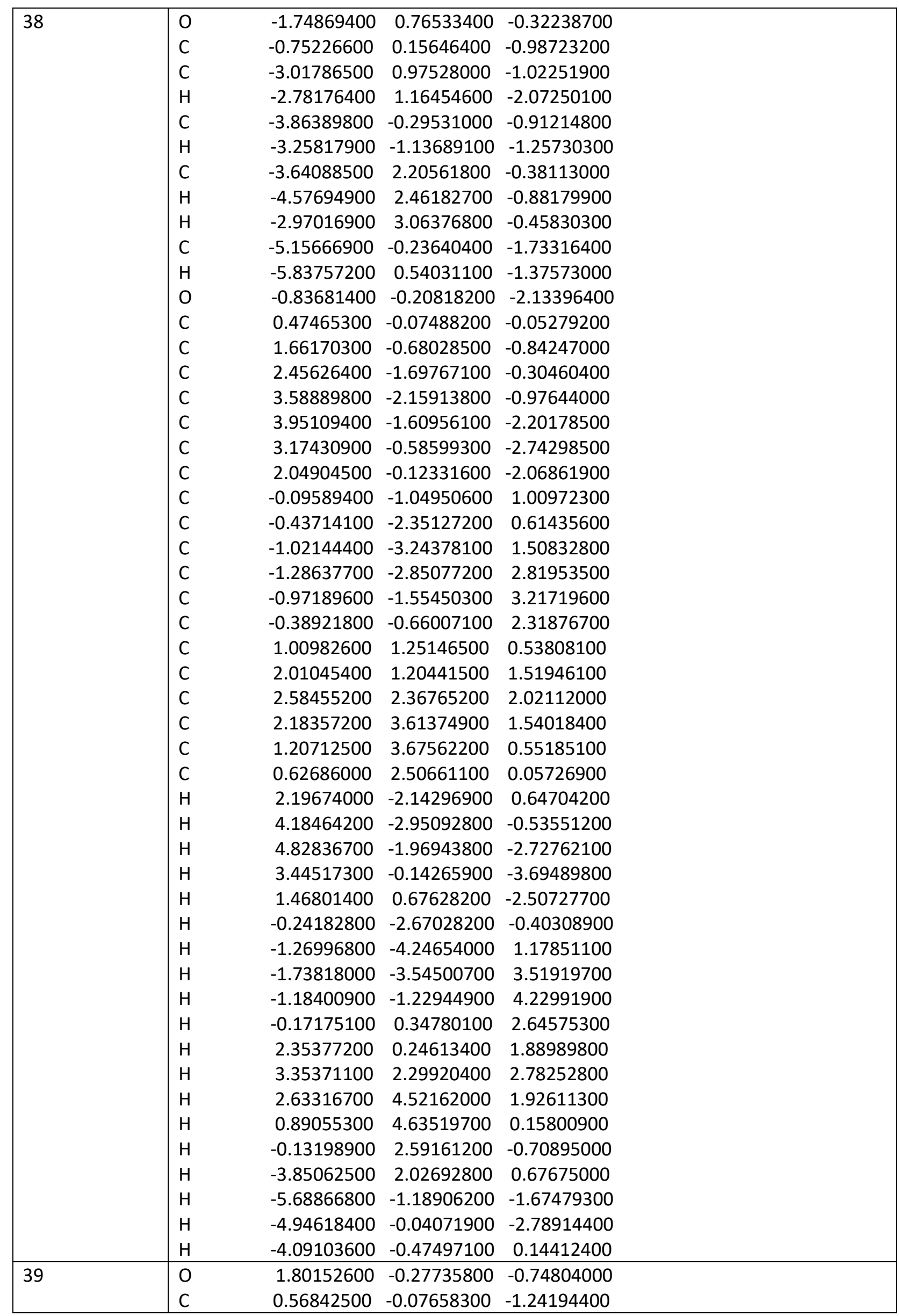




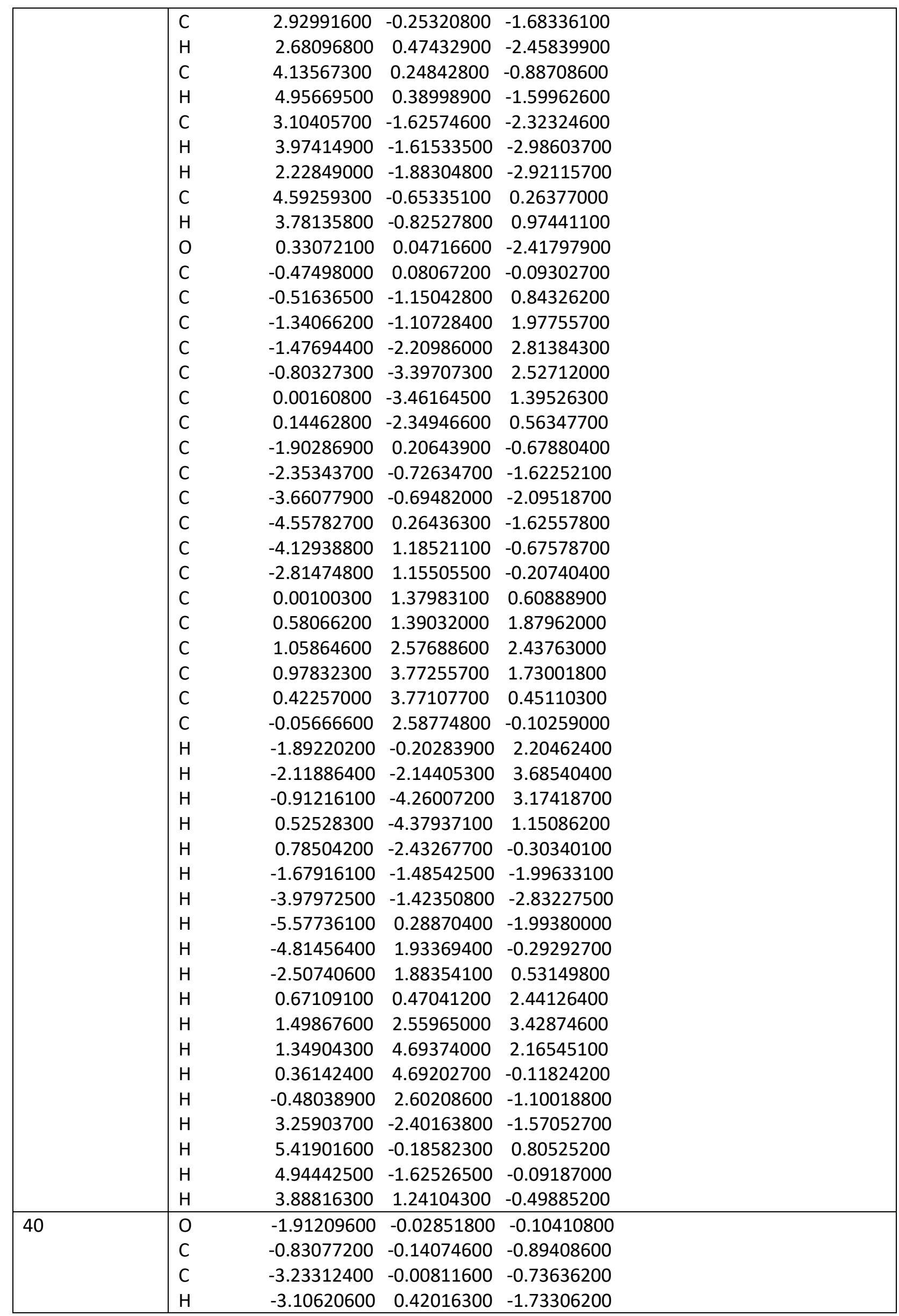




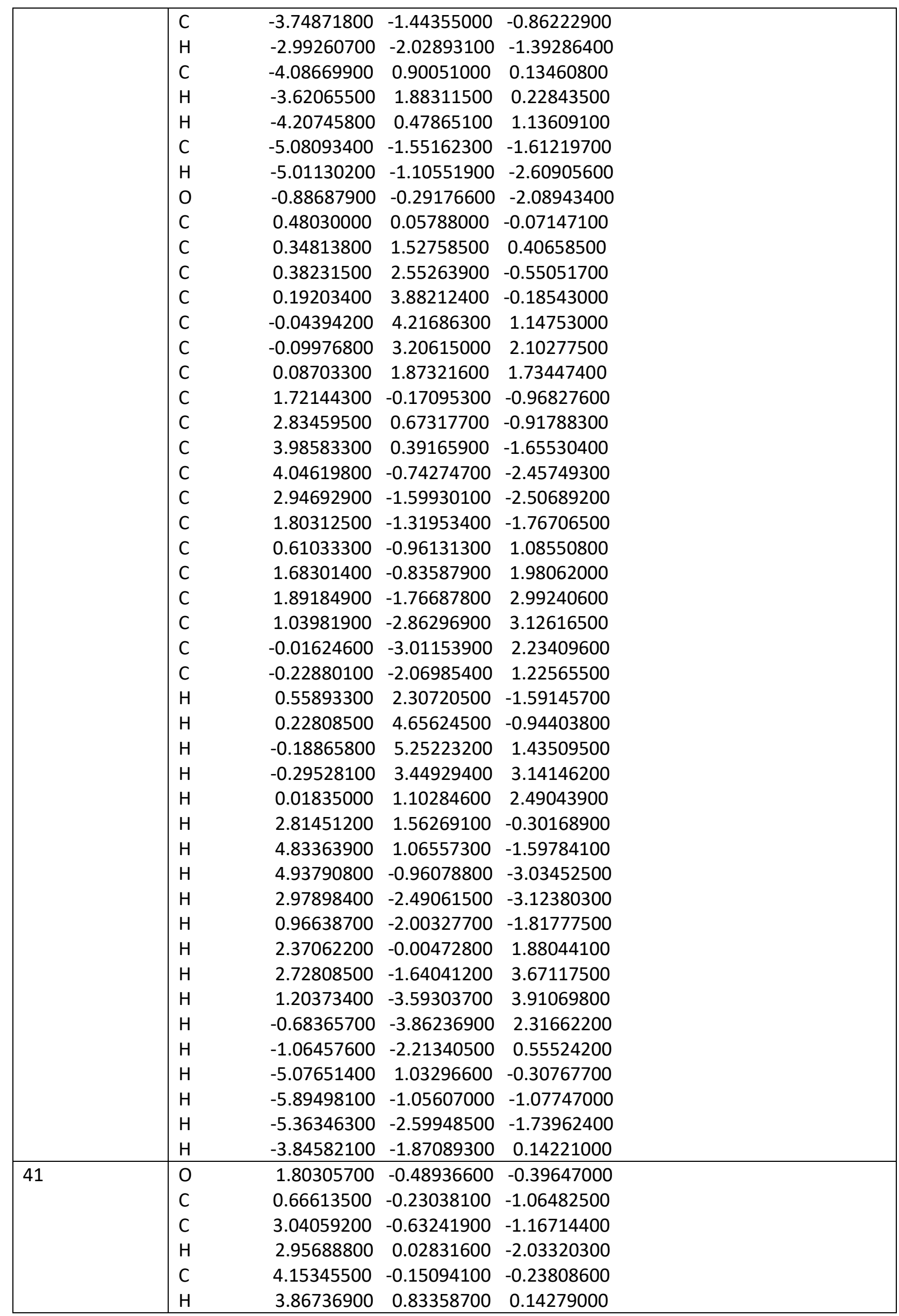




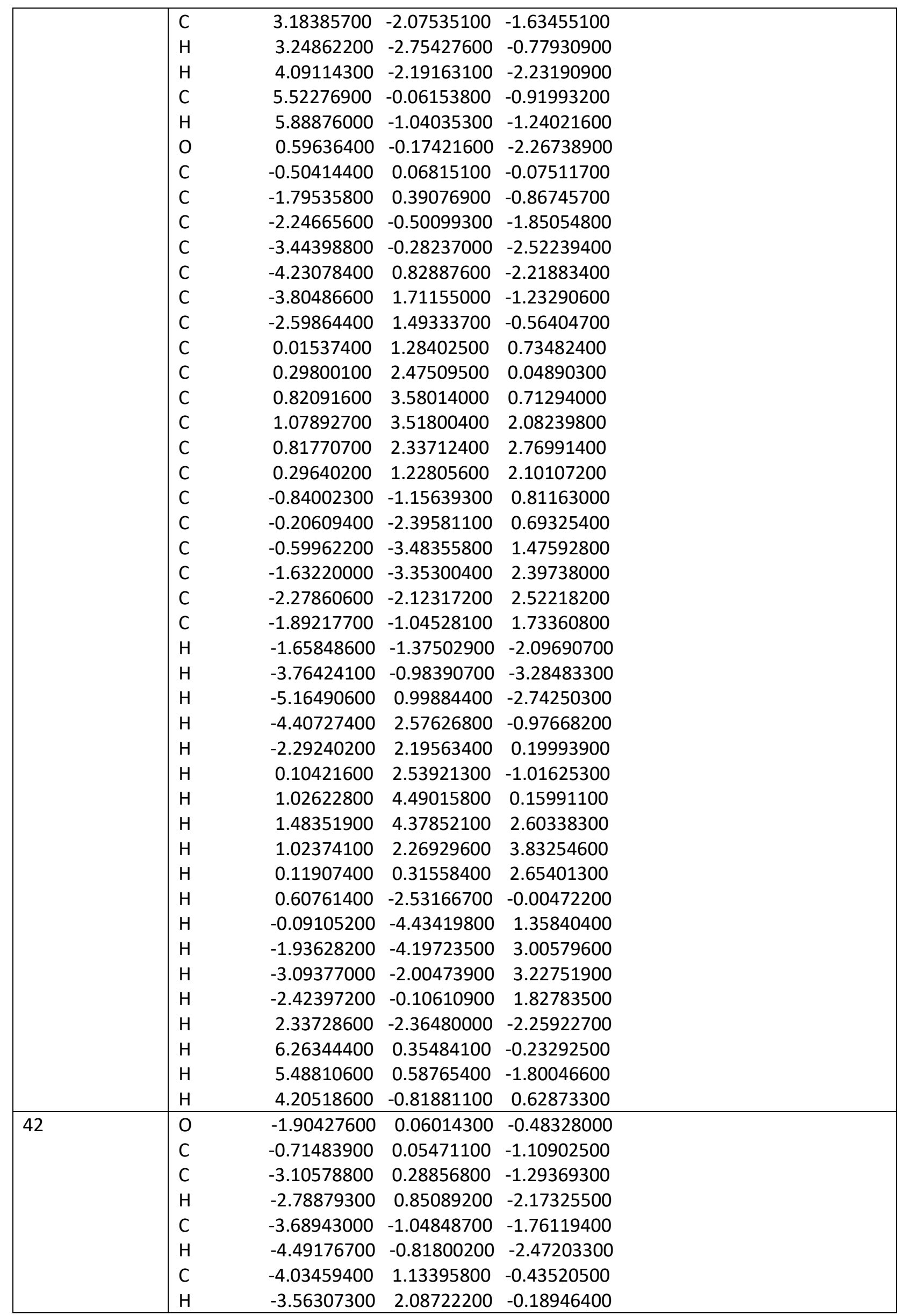




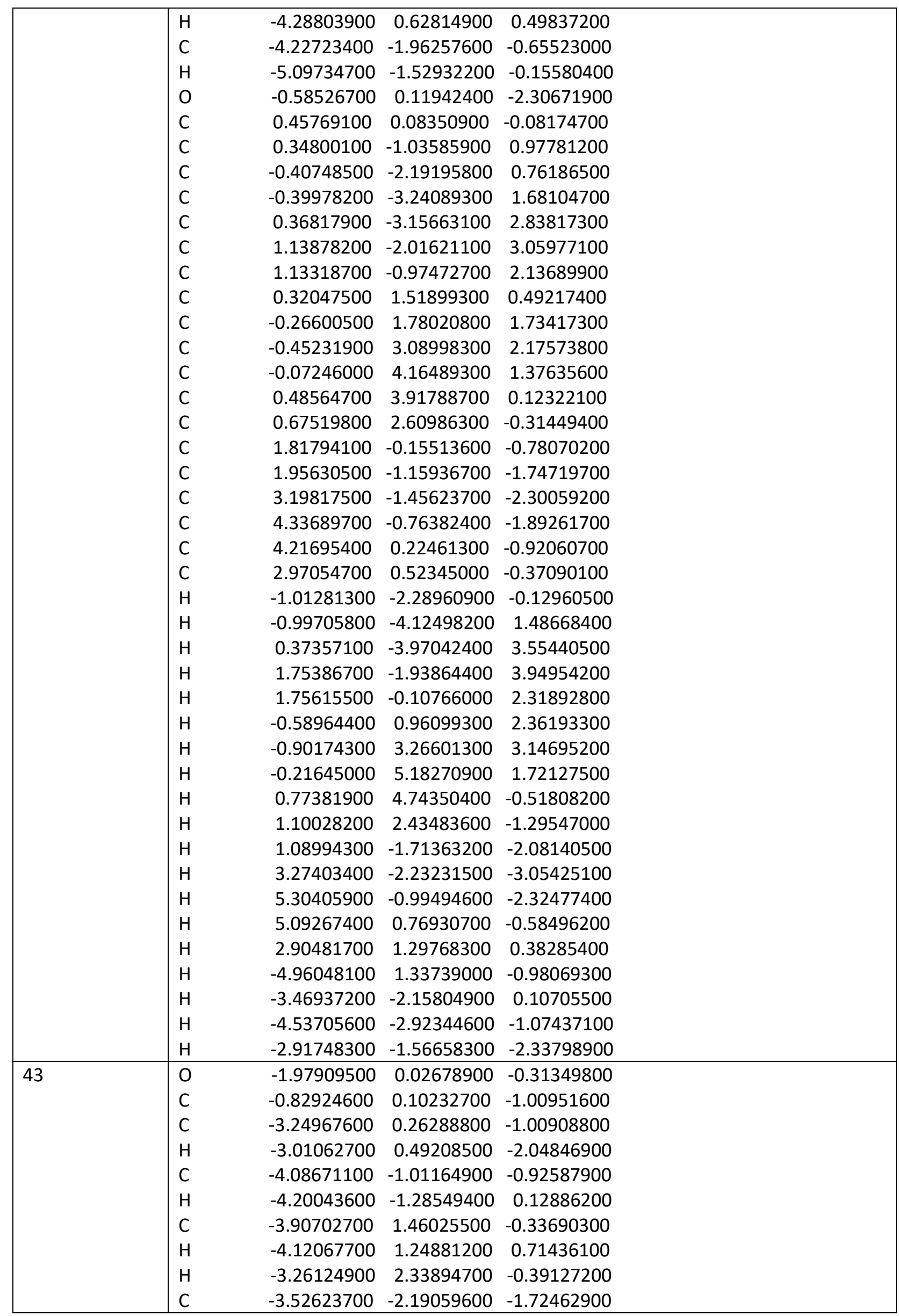




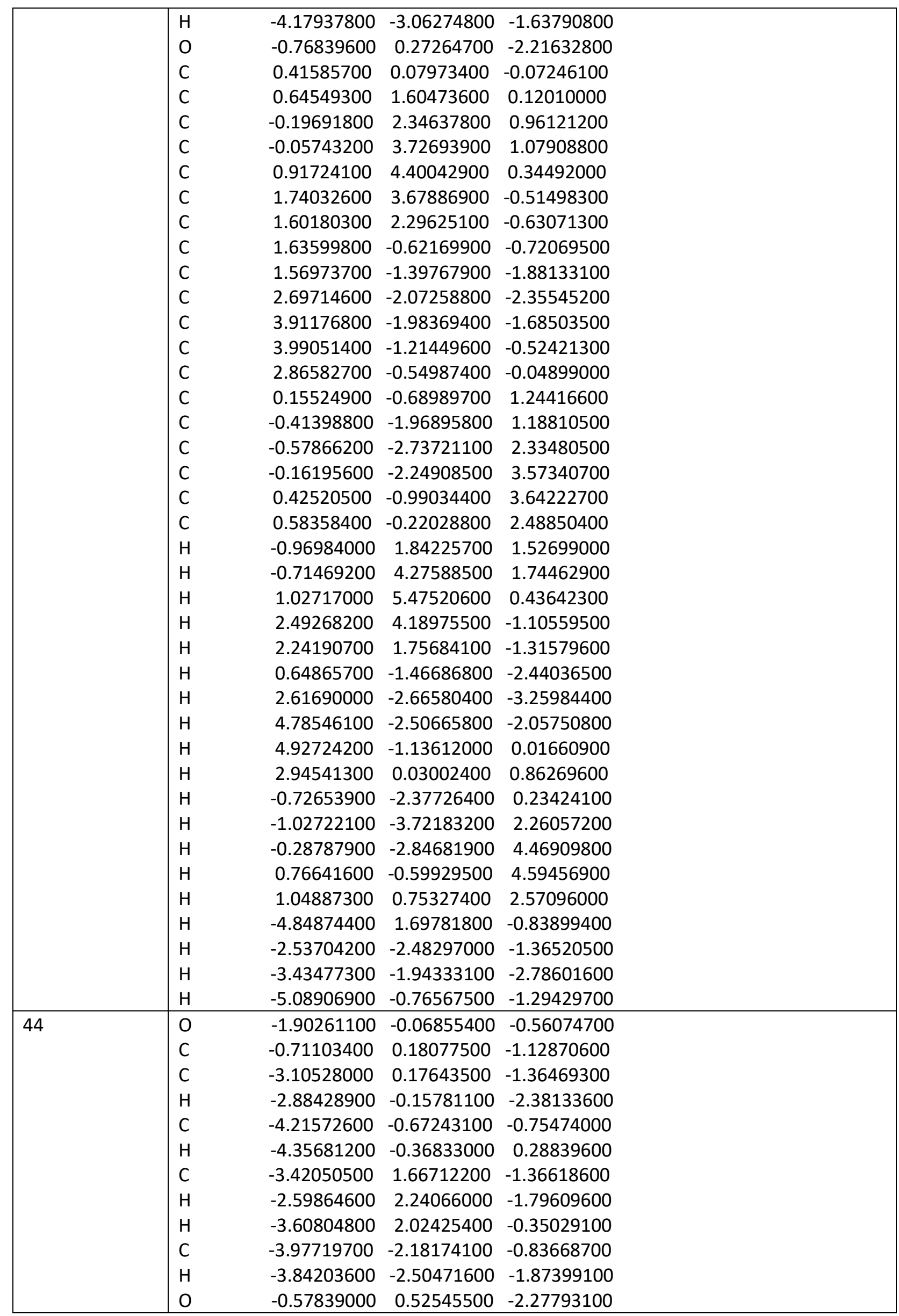




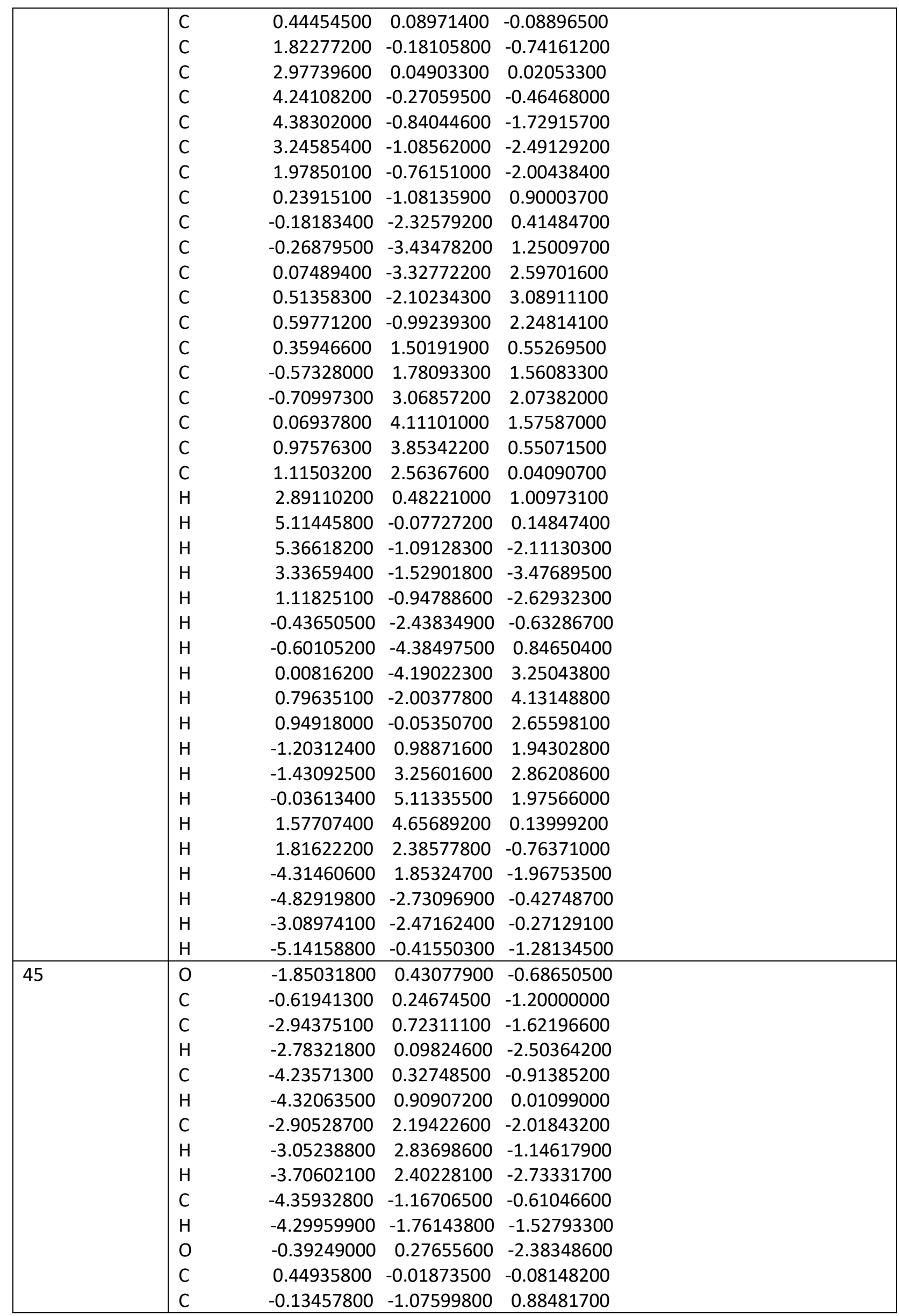




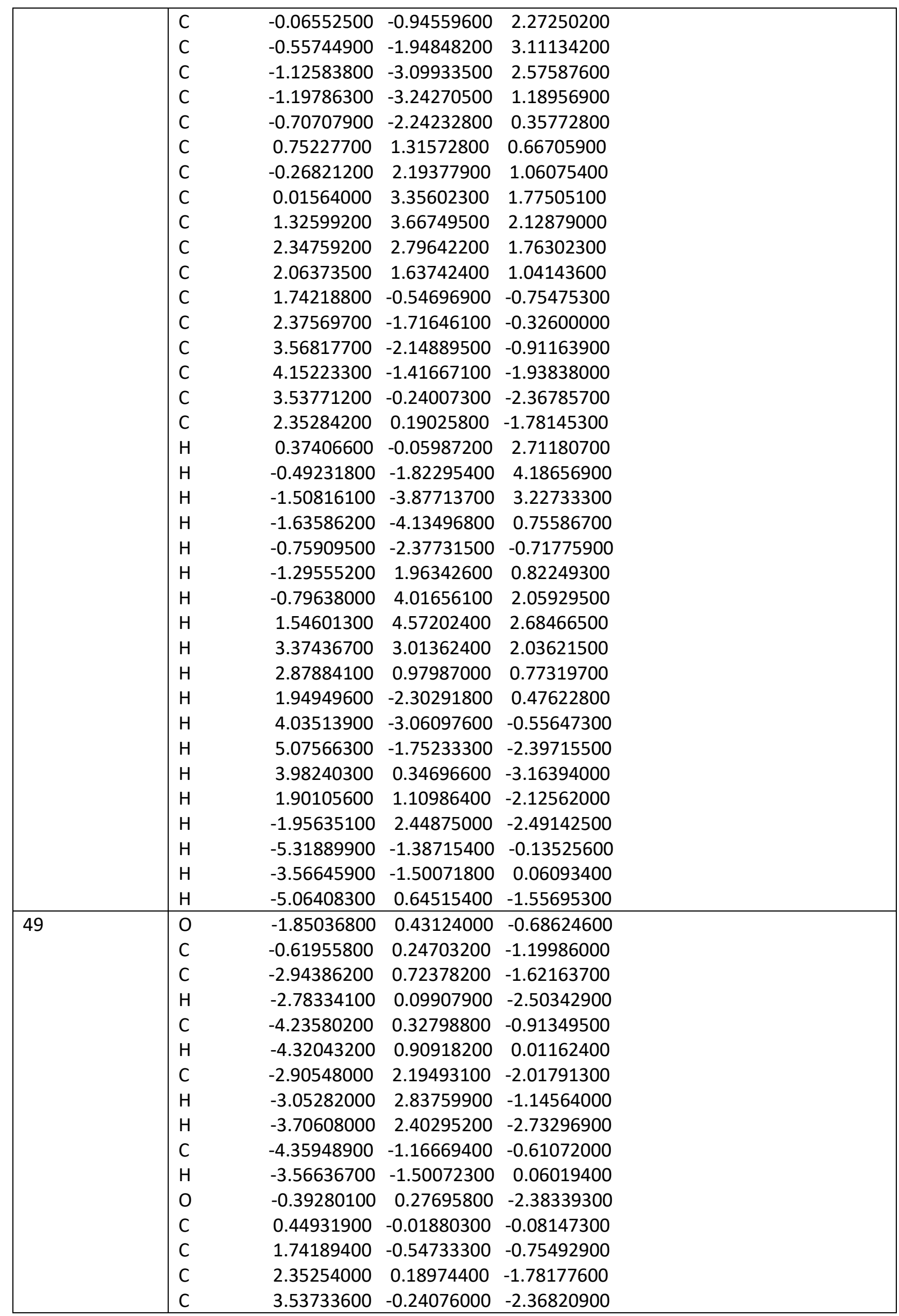




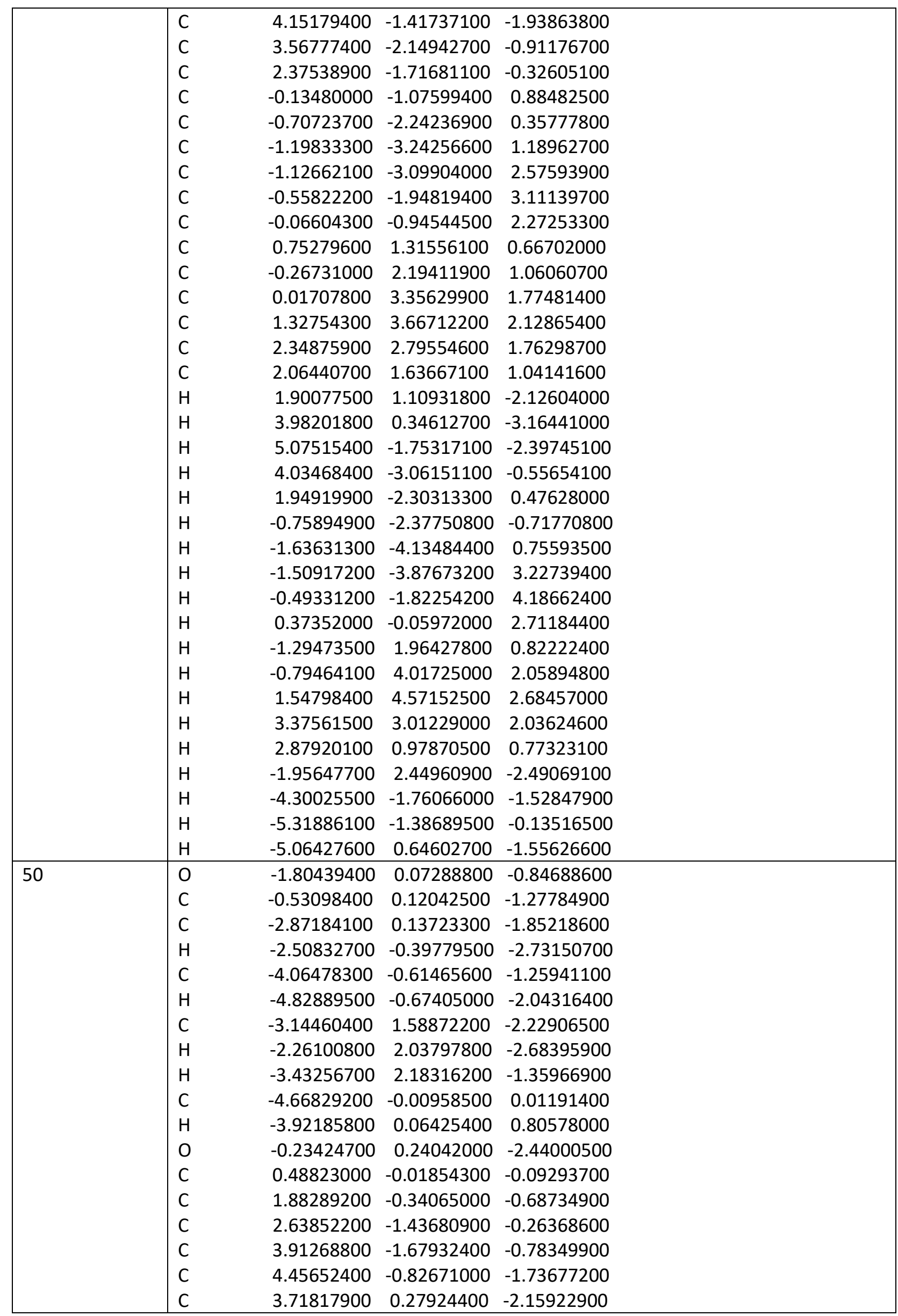




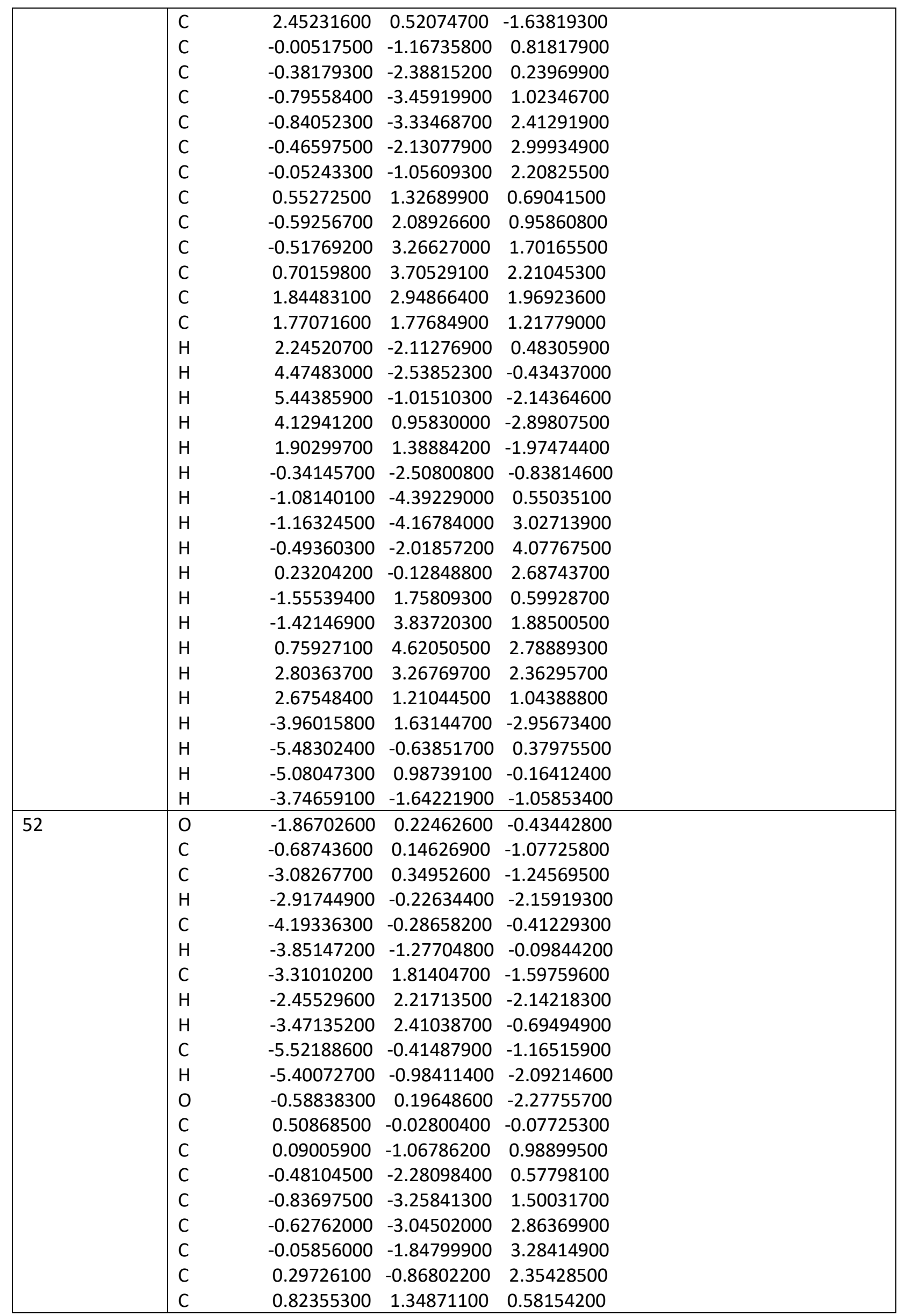




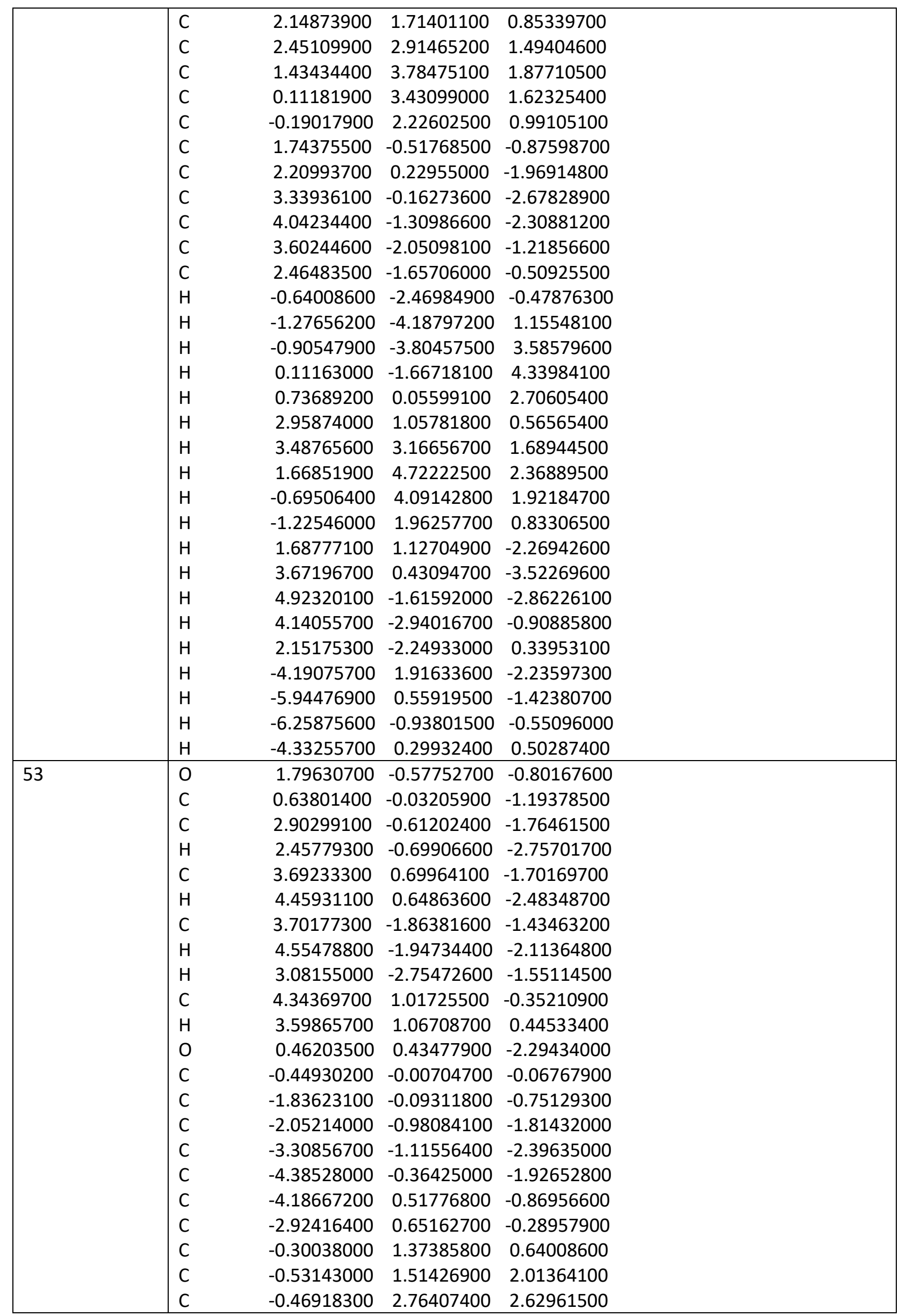




\begin{tabular}{|l|lrrr|}
\hline & C & -0.17238700 & 3.90250800 & 1.88559400 \\
C & 0.04653400 & 3.77851200 & 0.51548100 \\
C & -0.02449700 & 2.53112000 & -0.10118100 \\
C & -0.25397500 & -1.17594700 & 0.93551700 \\
C & -1.19960700 & -2.19630100 & 1.07544200 \\
C & -1.01323000 & -3.23787000 & 1.98808400 \\
C & 0.12214300 & -3.27908400 & 2.78749200 \\
C & 1.07027500 & -2.26215600 & 2.66901300 \\
C & 0.88279100 & -1.22845500 & 1.75916900 \\
H & -1.23281100 & -1.57514400 & -2.19967100 \\
H & -3.44439400 & -1.80544900 & -3.22194900 \\
H & -5.36403300 & -0.46455800 & -2.38218400 \\
H & -5.01201300 & 1.11148700 & -0.49226900 \\
H & -2.79486300 & 1.34960700 & 0.52700600 \\
H & -0.76777200 & 0.64577000 & 2.61382100 \\
H & -0.65504200 & 2.84201000 & 3.69526400 \\
H & -0.11828200 & 4.87344800 & 2.36508500 \\
H & 0.26707700 & 4.65593100 & -0.08255000 \\
H & 0.11961400 & 2.46390200 & -1.17172900 \\
H & -2.10020600 & -2.18792600 & 0.47778600 \\
H & -1.76769800 & -4.01259900 & 2.06993200 \\
H & 0.26713500 & -4.08562000 & 3.49760000 \\
H & 1.95951300 & -2.27192900 & 3.29001400 \\
H & 1.62952300 & -0.44966700 & 1.69203500 \\
H & 4.07667900 & -1.84350300 & -0.40974800 \\
H & 4.84734600 & 1.98628600 & -0.39398900 \\
H & 5.09152400 & 0.27135600 & -0.07102100 \\
H & 3.01884600 & 1.51139600 & -1.99013200 \\
\hline
\end{tabular}

1 (optimized at the M06-2X/6-311++G(d,p) level)

\begin{tabular}{|l|llll|}
\hline Conformer no & & & & \\
\hline 1 & O & -1.69643000 & 0.76038800 & -0.65441700 \\
& C & -0.54324600 & 0.36375200 & -1.19990600 \\
& C & -2.80097800 & 1.05438300 & -1.53942300 \\
& H & -2.73399900 & 0.36516200 & -2.38591200 \\
& C & -4.05982700 & 0.77907900 & -0.73388900 \\
& H & -4.08826600 & 1.47111600 & 0.11436100 \\
& C & -2.68941600 & 2.48944900 & -2.02269700 \\
& H & -3.52678000 & 2.72835700 & -2.68160200 \\
& H & -1.76212600 & 2.63671300 & -2.57870800 \\
& C & -4.13993300 & -0.66370500 & -0.24174400 \\
& H & -3.31255400 & -0.88965900 & 0.43418700 \\
& O & -0.34602100 & 0.31128800 & -2.38263600 \\
& C & 0.44322000 & -0.07007500 & -0.09103300 \\
& C & 1.74816400 & -0.60847600 & -0.69935200 \\
& C & 2.42965100 & 0.15334200 & -1.65298400 \\
& C & 3.65610000 & -0.26387600 & -2.15080200 \\
& C & 4.23643600 & -1.44416200 & -1.69361700 \\
& C & 3.57924200 & -2.19602500 & -0.73032400 \\
& C & 2.34428200 & -1.78033800 & -0.23664100 \\
& C & -0.34149500 & -1.15409200 & 0.67099300 \\
\hline
\end{tabular}




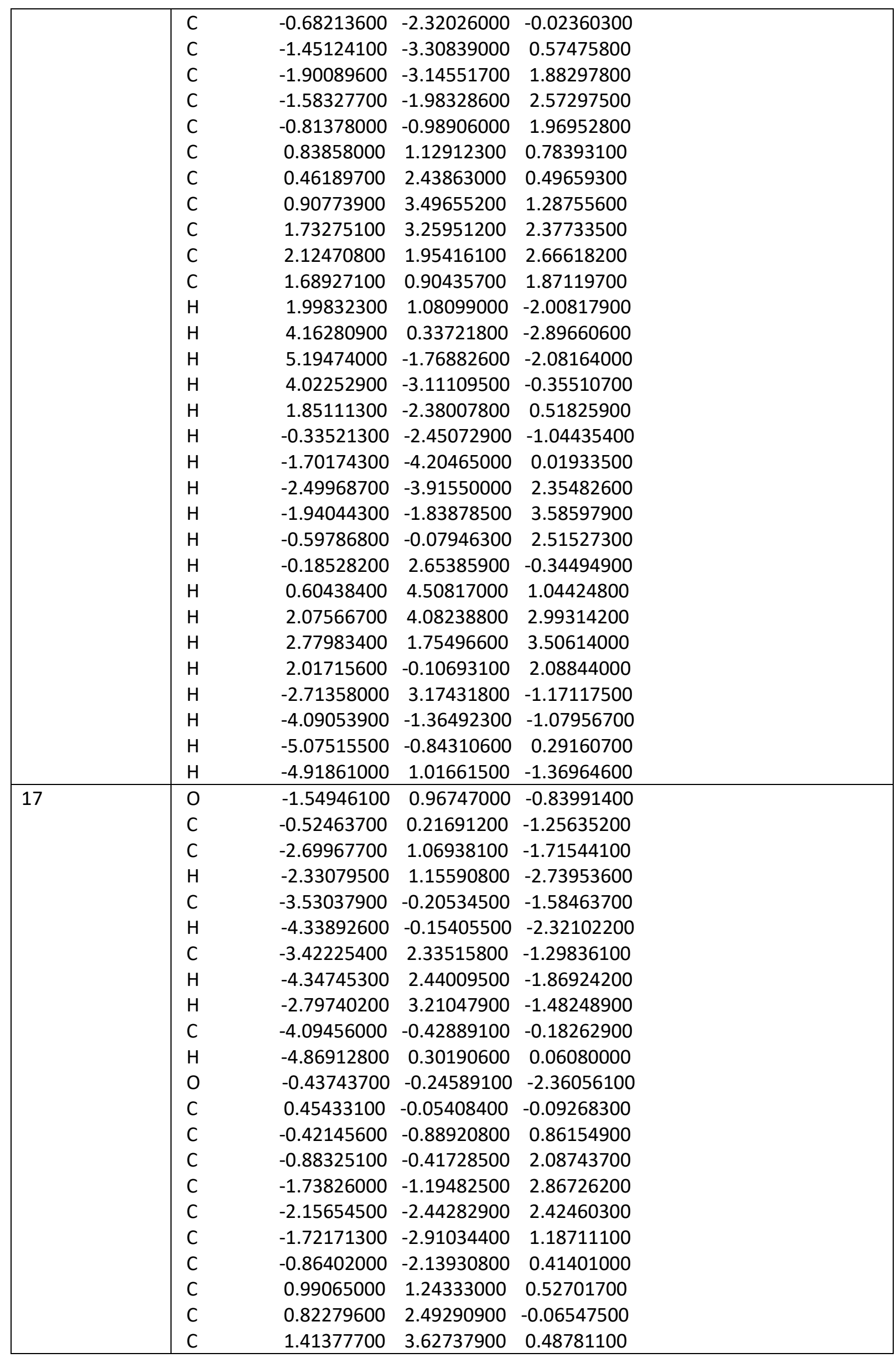




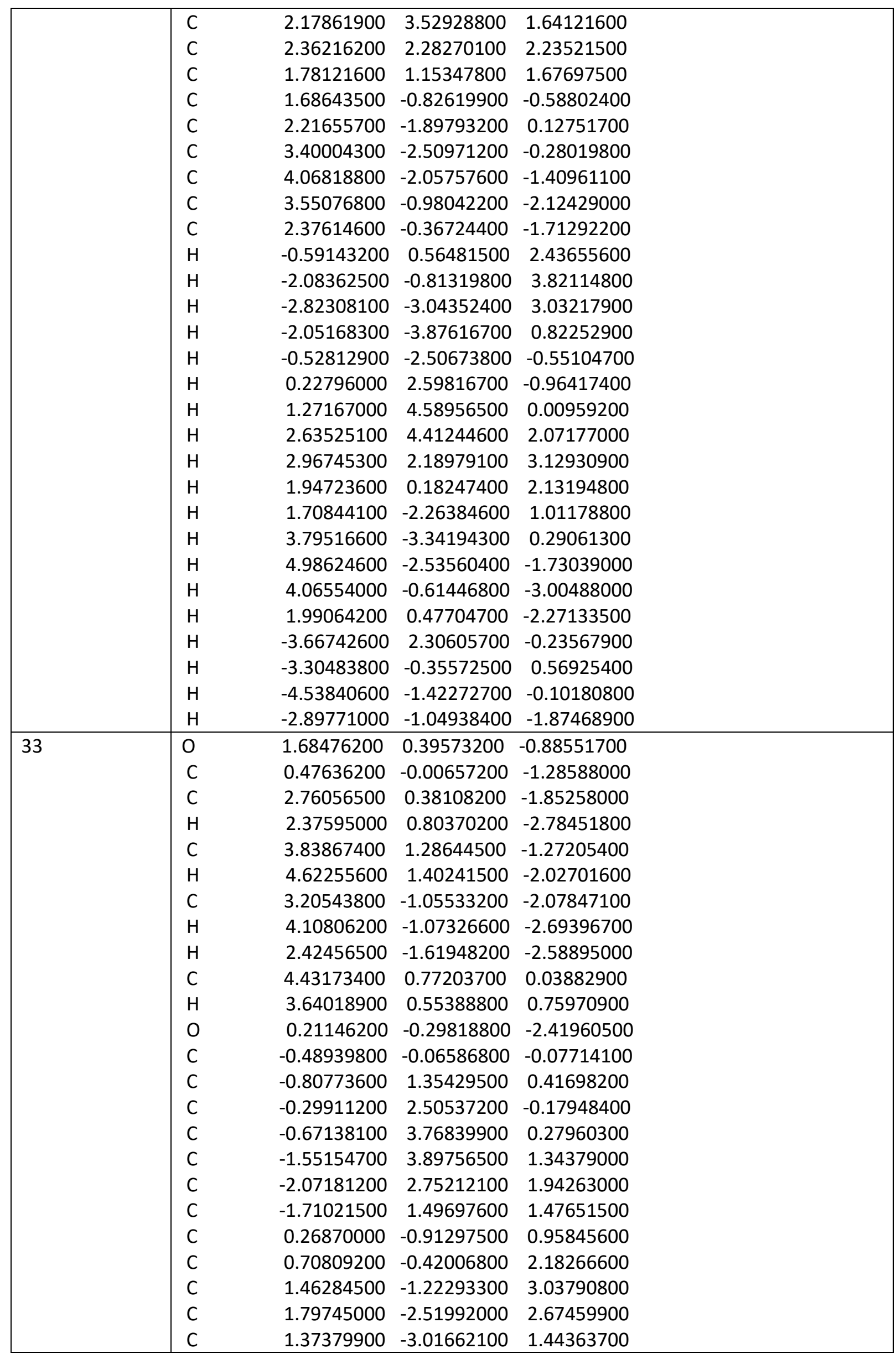




\begin{tabular}{|c|c|c|c|c|}
\hline & $\mathrm{C}$ & 0.61805400 & -2.21917500 & 0.59680500 \\
\hline & C & -1.82696600 & -0.69564000 & -0.49735300 \\
\hline & C & -2.52121100 & -0.13868700 & -1.57598600 \\
\hline & C & -3.76763700 & -0.62093900 & -1.94483500 \\
\hline & C & -4.35590700 & -1.66296900 & -1.23191400 \\
\hline & $\mathrm{C}$ & -3.68593700 & -2.20792700 & -0.14624100 \\
\hline & C & -2.42907000 & -1.72712300 & 0.21989200 \\
\hline & $\mathrm{H}$ & 0.39268100 & 2.43665500 & -1.00997400 \\
\hline & $\mathrm{H}$ & -0.26765600 & 4.65070200 & -0.20299100 \\
\hline & $\mathrm{H}$ & -1.83952200 & 4.87936000 & 1.70008700 \\
\hline & $\mathrm{H}$ & -2.77054400 & 2.83787100 & 2.76649500 \\
\hline & $\mathrm{H}$ & -2.13888300 & 0.60987600 & 1.93138200 \\
\hline & $\mathrm{H}$ & 0.48094200 & 0.59784600 & 2.47290200 \\
\hline & $\mathrm{H}$ & 1.79649500 & -0.82245600 & 3.98827600 \\
\hline & $\mathrm{H}$ & 2.38817000 & -3.13936200 & 3.33915700 \\
\hline & $\mathrm{H}$ & 1.63425600 & -4.02507500 & 1.14388800 \\
\hline & $\mathrm{H}$ & 0.29117500 & -2.60983800 & -0.36302500 \\
\hline & $\mathrm{H}$ & -2.08053300 & 0.68259400 & -2.12742500 \\
\hline & $\mathrm{H}$ & -4.28398400 & -0.17884400 & -2.78877100 \\
\hline & $\mathrm{H}$ & -5.33100200 & -2.03870500 & -1.51862800 \\
\hline & $\mathrm{H}$ & -4.13694300 & -3.01064100 & 0.42557000 \\
\hline & $\mathrm{H}$ & -1.92705400 & -2.16206800 & 1.07531100 \\
\hline & $\mathrm{H}$ & 3.42128300 & -1.53690600 & -1.12254800 \\
\hline & $\mathrm{H}$ & 5.09847400 & 1.51690000 & 0.47642500 \\
\hline & $\mathrm{H}$ & 5.01162400 & -0.14020400 & -0.11534500 \\
\hline & $\mathrm{H}$ & 3.39720900 & 2.27543100 & -1.11729500 \\
\hline \multirow[t]{26}{*}{38} & 0 & -1.55040200 & 0.97240600 & -0.63489500 \\
\hline & C & -0.62585400 & 0.12720300 & -1.10031500 \\
\hline & C & -2.83761800 & 0.98164200 & -1.29565800 \\
\hline & $\mathrm{H}$ & -2.66488100 & 0.93539200 & -2.37451500 \\
\hline & C & -3.61147900 & -0.25085600 & -0.84075400 \\
\hline & $\mathrm{H}$ & -3.02145300 & -1.13606000 & -1.09323500 \\
\hline & C & -3.48528000 & 2.29649500 & -0.91029200 \\
\hline & $\mathrm{H}$ & -4.43419400 & 2.42183000 & -1.43351200 \\
\hline & $\mathrm{H}$ & -2.83460500 & 3.13177000 & -1.17345200 \\
\hline & C & -4.99165100 & -0.35176600 & -1.48642500 \\
\hline & $\mathrm{H}$ & -5.65291000 & 0.45259500 & -1.15789000 \\
\hline & 0 & -0.72184600 & -0.46890900 & -2.13794700 \\
\hline & C & 0.49636200 & -0.07595900 & -0.05935900 \\
\hline & C & 1.68953700 & -0.82306700 & -0.66898100 \\
\hline & C & 2.39846400 & -1.77835100 & 0.05834700 \\
\hline & C & 3.54987800 & -2.36091800 & -0.46465700 \\
\hline & C & 4.01082600 & -1.99382500 & -1.72220300 \\
\hline & C & 3.31676900 & -1.03162200 & -2.44949000 \\
\hline & $\mathrm{C}$ & 2.17122300 & -0.44887100 & -1.92485000 \\
\hline & C & -0.26151000 & -0.90140800 & 0.99946000 \\
\hline & C & -0.57946700 & -2.23155700 & 0.70853000 \\
\hline & C & -1.36056700 & -2.98234500 & 1.57841600 \\
\hline & C & -1.84697000 & -2.41101500 & 2.75036000 \\
\hline & C & -1.55709500 & -1.08160100 & 3.03443200 \\
\hline & C & -0.77764700 & -0.32841300 & 2.16093100 \\
\hline & $\mathrm{C}$ & 1.06188900 & 1.24789700 & 0.46863300 \\
\hline
\end{tabular}




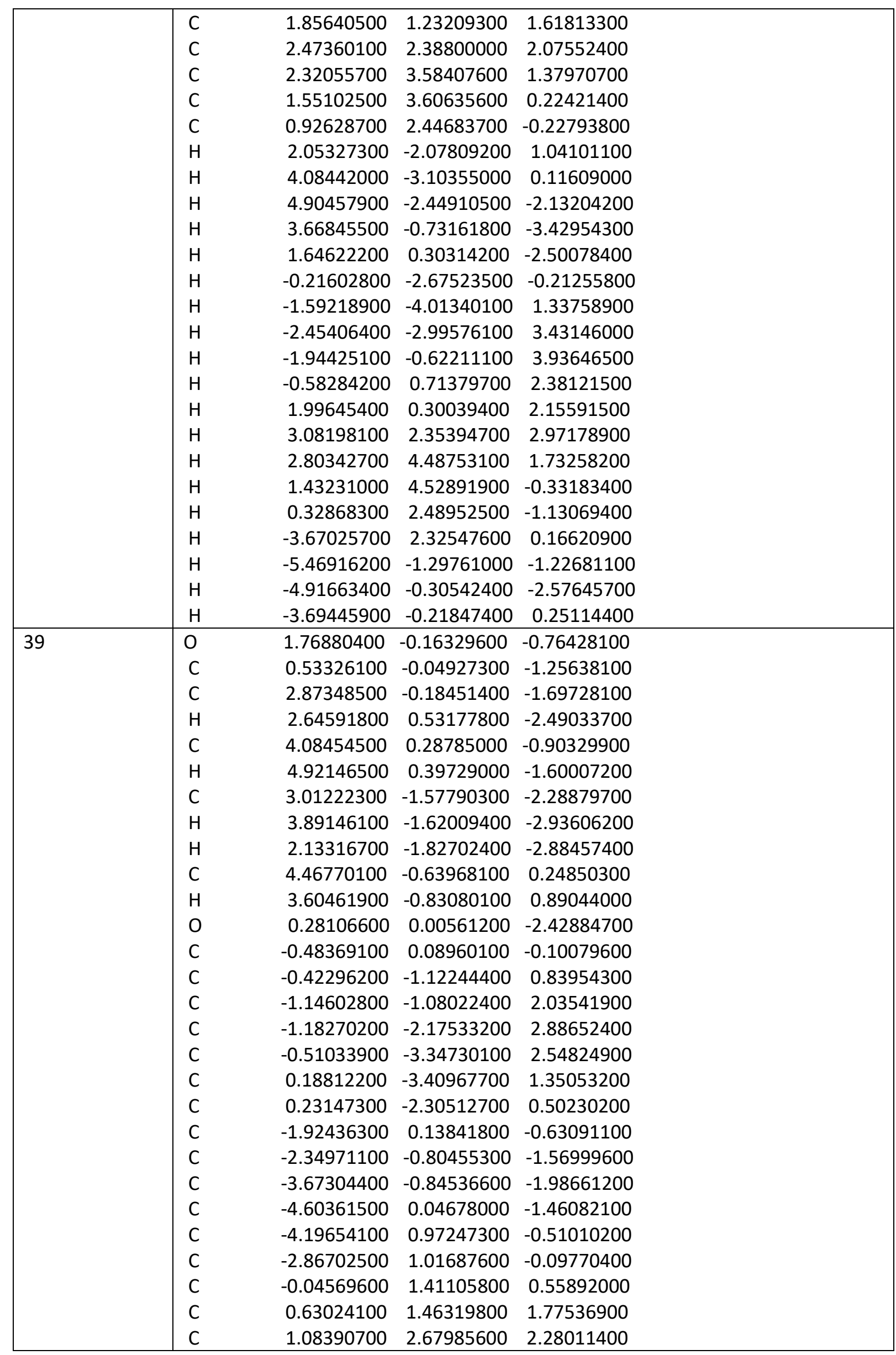




\begin{tabular}{|c|c|c|c|c|}
\hline & $\mathrm{C}$ & 0.88259600 & 3.85601000 & 1.56949000 \\
\hline & $\mathrm{C}$ & 0.23109800 & 3.80926500 & 0.34005800 \\
\hline & $\mathrm{C}$ & -0.22453900 & 2.59685300 & -0.16059500 \\
\hline & $\mathrm{H}$ & -1.68864500 & -0.17776400 & 2.29693700 \\
\hline & $\mathrm{H}$ & -1.74478600 & -2.11797000 & 3.81129900 \\
\hline & $\mathrm{H}$ & -0.53970500 & -4.20505300 & 3.20957000 \\
\hline & $\mathrm{H}$ & 0.70509400 & -4.31943500 & 1.06848300 \\
\hline & $\mathrm{H}$ & 0.78504500 & -2.38031300 & -0.42599700 \\
\hline & $\mathrm{H}$ & -1.64108100 & -1.51337100 & -1.98018500 \\
\hline & $\mathrm{H}$ & -3.97986600 & -1.57889400 & -2.72296900 \\
\hline & $\mathrm{H}$ & -5.63655900 & 0.01443600 & -1.78682000 \\
\hline & $\mathrm{H}$ & -4.91127500 & 1.66708500 & -0.08409000 \\
\hline & $\mathrm{H}$ & -2.56938800 & 1.74623400 & 0.64617200 \\
\hline & $\mathrm{H}$ & 0.82039400 & 0.55325700 & 2.33023800 \\
\hline & $\mathrm{H}$ & 1.60413400 & 2.70191800 & 3.23063800 \\
\hline & $\mathrm{H}$ & 1.23715800 & 4.80100900 & 1.96409300 \\
\hline & $\mathrm{H}$ & 0.07796200 & 4.71764100 & -0.23070900 \\
\hline & $\mathrm{H}$ & -0.72711300 & 2.56417900 & -1.12211500 \\
\hline & $\mathrm{H}$ & 3.13059700 & -2.32141800 & -1.49798300 \\
\hline & $\mathrm{H}$ & 5.25180300 & -0.18839400 & 0.85860800 \\
\hline & $\mathrm{H}$ & 4.84368200 & -1.59881900 & -0.11390300 \\
\hline & $\mathrm{H}$ & 3.85658600 & 1.28493200 & -0.51602000 \\
\hline \multirow[t]{30}{*}{40} & 0 & -1.88251000 & 0.07261900 & -0.14328000 \\
\hline & $\mathrm{C}$ & -0.80257200 & -0.13477700 & -0.90277200 \\
\hline & $\mathrm{C}$ & -3.17565900 & 0.03836400 & -0.78886300 \\
\hline & $\mathrm{H}$ & -3.05805400 & 0.46489600 & -1.78875700 \\
\hline & $\mathrm{C}$ & -3.63500100 & -1.41034700 & -0.90398300 \\
\hline & $\mathrm{H}$ & -2.86614400 & -1.96664700 & -1.44713000 \\
\hline & $\mathrm{C}$ & -4.07568700 & 0.91056400 & 0.06441500 \\
\hline & $\mathrm{H}$ & -3.62510300 & 1.89607200 & 0.19238800 \\
\hline & $\mathrm{H}$ & -4.21549000 & 0.46149400 & 1.05085300 \\
\hline & $\mathrm{C}$ & -4.97268700 & -1.54832500 & -1.62775600 \\
\hline & $\mathrm{H}$ & -4.93624900 & -1.06278600 & -2.60669300 \\
\hline & $\mathrm{O}$ & -0.84807300 & -0.37906400 & -2.07727200 \\
\hline & $\mathrm{C}$ & 0.48445700 & 0.06549800 & -0.06825500 \\
\hline & $\mathrm{C}$ & 0.36010600 & 1.52528300 & 0.40398000 \\
\hline & $\mathrm{C}$ & 0.40835400 & 2.53396000 & -0.56413800 \\
\hline & $\mathrm{C}$ & 0.21903200 & 3.86442800 & -0.21660600 \\
\hline & $\mathrm{C}$ & -0.02957500 & 4.21097000 & 1.10904400 \\
\hline & $\mathrm{C}$ & -0.09925000 & 3.21374700 & 2.07285000 \\
\hline & $\mathrm{C}$ & 0.08686100 & 1.87799000 & 1.72246400 \\
\hline & $\mathrm{C}$ & 1.73356700 & -0.17858900 & -0.92981700 \\
\hline & $\mathrm{C}$ & 2.83832000 & 0.67012300 & -0.89082900 \\
\hline & $\mathrm{C}$ & 3.99576100 & 0.36551100 & -1.60524900 \\
\hline & $\mathrm{C}$ & 4.06547900 & -0.79351000 & -2.36532800 \\
\hline & $\mathrm{C}$ & 2.97277400 & -1.65584900 & -2.39618200 \\
\hline & $\mathrm{C}$ & 1.82332100 & -1.35433900 & -1.68056300 \\
\hline & $\mathrm{C}$ & 0.55911900 & -0.95041000 & 1.08045800 \\
\hline & $\mathrm{C}$ & 1.60670100 & -0.84069900 & 2.00028900 \\
\hline & $\mathrm{C}$ & 1.76346400 & -1.77031000 & 3.01805200 \\
\hline & $\mathrm{C}$ & 0.88378700 & -2.84507100 & 3.12552400 \\
\hline & $\mathrm{C}$ & -0.14446500 & -2.97743100 & 2.20352400 \\
\hline
\end{tabular}




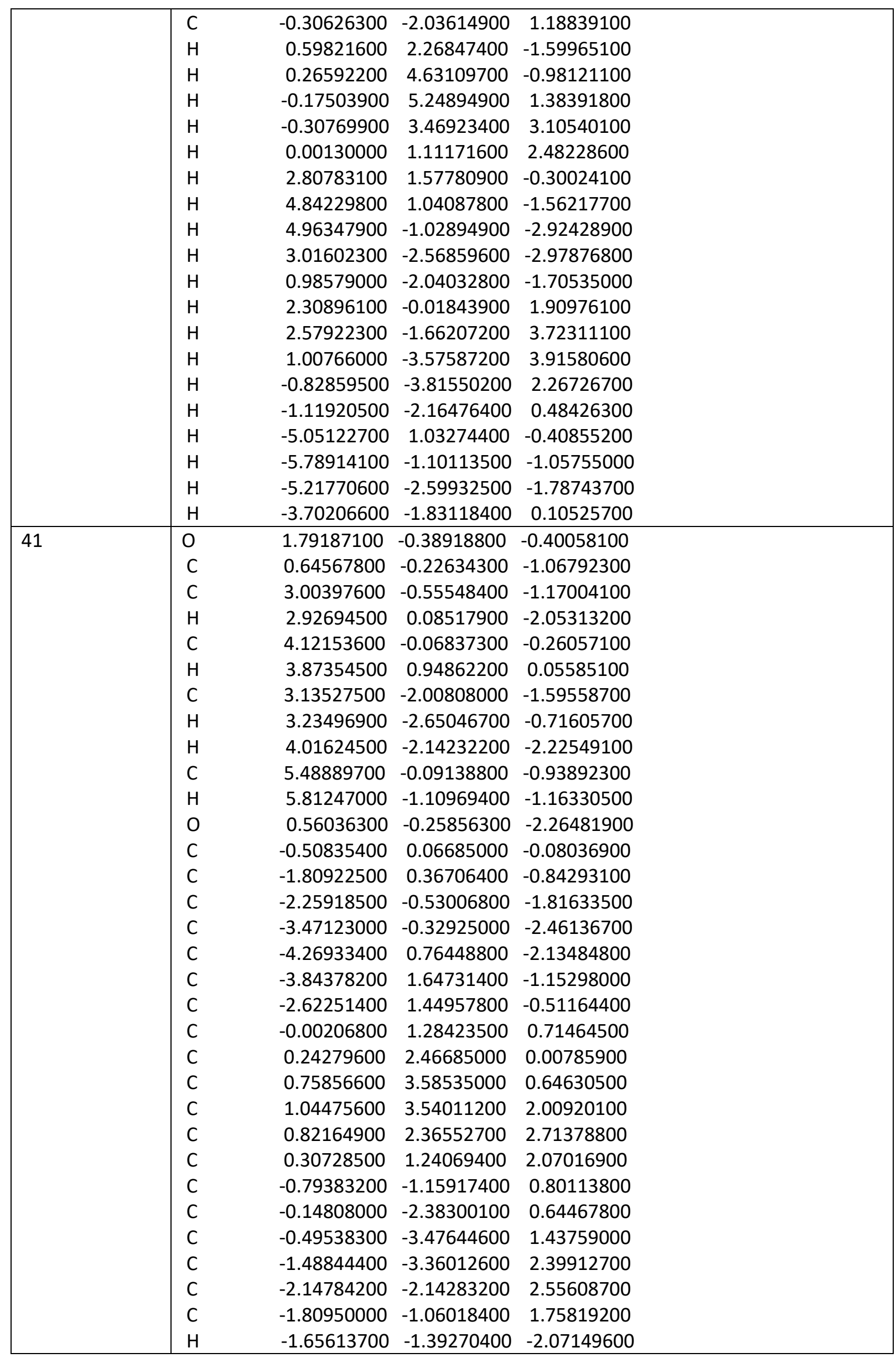




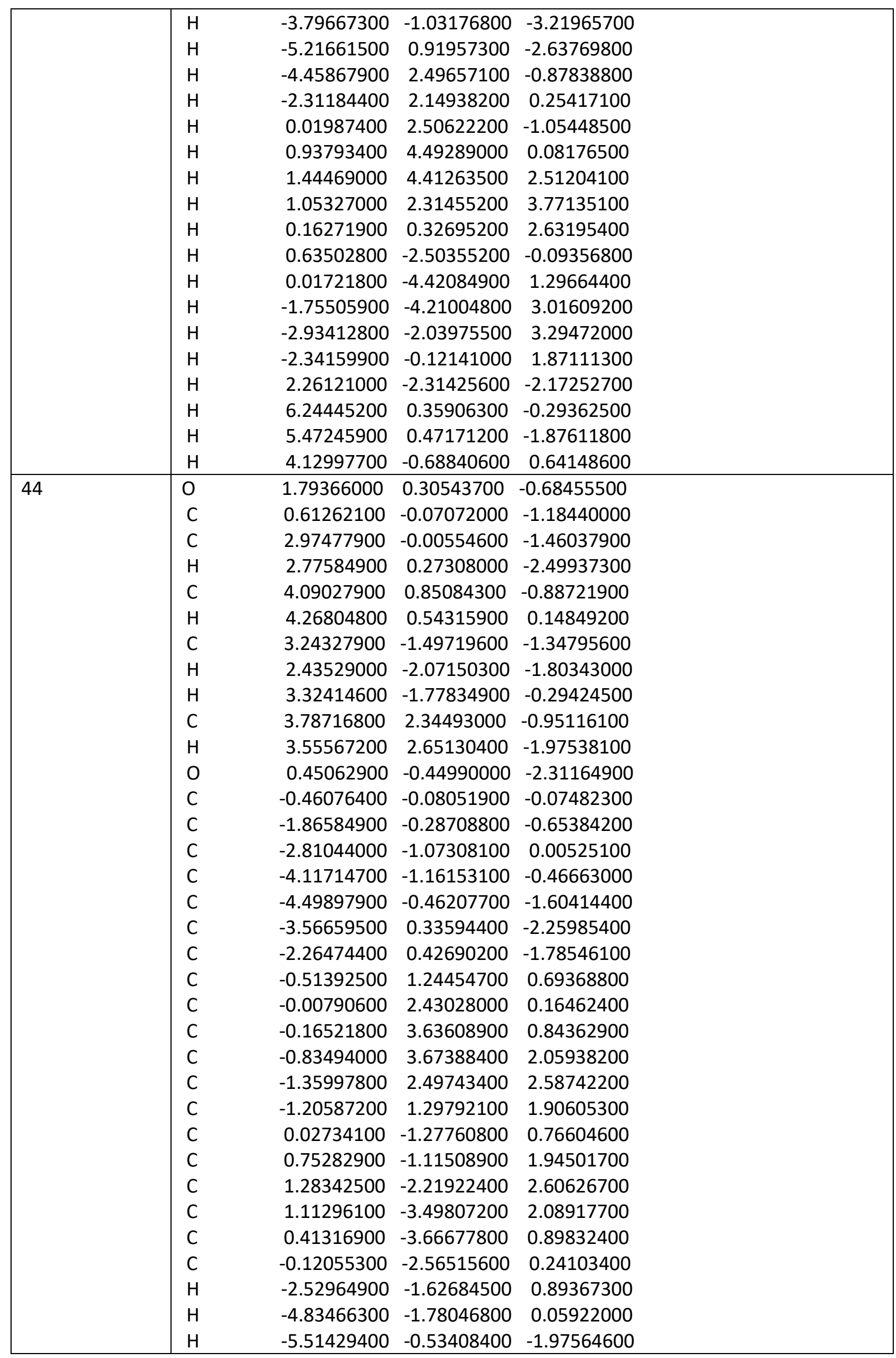




\begin{tabular}{|c|c|c|c|c|}
\hline & $\mathrm{H}$ & -3.85276700 & 0.89196600 & -3.14496300 \\
\hline & $\mathrm{H}$ & -1.55255200 & 1.05648300 & -2.30411600 \\
\hline & $\mathrm{H}$ & 0.51743600 & 2.42556600 & -0.78341500 \\
\hline & $\mathrm{H}$ & 0.23827800 & 4.54664700 & 0.41591900 \\
\hline & $\mathrm{H}$ & -0.95526900 & 4.61198200 & 2.58790700 \\
\hline & $\mathrm{H}$ & -1.89781300 & 2.51512700 & 3.52802200 \\
\hline & $\mathrm{H}$ & -1.63194700 & 0.38877400 & 2.31697100 \\
\hline & $\mathrm{H}$ & 0.91850200 & -0.12256500 & 2.34476600 \\
\hline & $\mathrm{H}$ & 1.83809100 & -2.07410200 & 3.52599600 \\
\hline & $\mathrm{H}$ & 1.52877700 & -4.35584400 & 2.60447600 \\
\hline & $\mathrm{H}$ & 0.28541200 & -4.65675900 & 0.47612800 \\
\hline & $\mathrm{H}$ & -0.65043500 & -2.69942300 & -0.69624600 \\
\hline & $\mathrm{H}$ & 4.17645000 & -1.74983900 & -1.85563000 \\
\hline & $\mathrm{H}$ & 4.64103600 & 2.93299400 & -0.61040000 \\
\hline & $\mathrm{H}$ & 2.93172300 & 2.59111300 & -0.31956700 \\
\hline & $\mathrm{H}$ & 5.00072000 & 0.62277400 & -1.44979700 \\
\hline \multirow[t]{36}{*}{45} & 0 & -1.81050800 & 0.42395100 & -0.74661500 \\
\hline & C & -0.57895200 & 0.25018000 & -1.23939400 \\
\hline & C & -2.88495000 & 0.66570900 & -1.68606200 \\
\hline & $\mathrm{H}$ & -2.74073500 & -0.01885800 & -2.52707800 \\
\hline & $\mathrm{C}$ & -4.16788800 & 0.33014400 & -0.94312200 \\
\hline & $\mathrm{H}$ & -4.28879100 & 1.04192000 & -0.11936200 \\
\hline & C & -2.83342200 & 2.10686700 & -2.16321300 \\
\hline & $\mathrm{H}$ & -2.93604700 & 2.78684900 & -1.31369200 \\
\hline & $\mathrm{H}$ & -3.65521400 & 2.29584200 & -2.85715000 \\
\hline & C & -4.19644200 & -1.09958800 & -0.41127400 \\
\hline & $\mathrm{H}$ & -4.03969000 & -1.81990900 & -1.21950900 \\
\hline & $\mathrm{O}$ & -0.33409800 & 0.27152300 & -2.41386700 \\
\hline & C & 0.44805600 & -0.00690200 & -0.10204800 \\
\hline & C & -0.17986000 & -1.04816200 & 0.83780200 \\
\hline & C & -0.16348300 & -0.92227200 & 2.22242600 \\
\hline & C & -0.69169800 & -1.93079000 & 3.02927300 \\
\hline & C & -1.23883200 & -3.07251100 & 2.46113200 \\
\hline & C & -1.25620800 & -3.20685100 & 1.07369500 \\
\hline & C & -0.73161700 & -2.20296600 & 0.27405900 \\
\hline & C & 0.72818200 & 1.32021400 & 0.64462400 \\
\hline & C & -0.31367300 & 2.16226600 & 1.05155200 \\
\hline & C & -0.05634100 & 3.31737900 & 1.78095500 \\
\hline & C & 1.24551400 & 3.65350900 & 2.13537300 \\
\hline & C & 2.28541000 & 2.81549700 & 1.75604700 \\
\hline & C & 2.03017500 & 1.66244400 & 1.01866500 \\
\hline & C & 1.74961800 & -0.55202800 & -0.71299900 \\
\hline & C & 2.37309000 & -1.69054400 & -0.20618100 \\
\hline & C & 3.59478200 & -2.12580600 & -0.71871100 \\
\hline & C & 4.21209400 & -1.42710600 & -1.74521900 \\
\hline & C & 3.60371100 & -0.28062800 & -2.25139000 \\
\hline & C & 2.39033600 & 0.15458800 & -1.73802300 \\
\hline & $\mathrm{H}$ & 0.26409600 & -0.03999300 & 2.68299100 \\
\hline & $\mathrm{H}$ & -0.67044700 & -1.81670500 & 4.10680900 \\
\hline & $\mathrm{H}$ & -1.64802600 & -3.85391000 & 3.09037700 \\
\hline & $\mathrm{H}$ & -1.68079200 & -4.09323500 & 0.61692900 \\
\hline & $\mathrm{H}$ & -0.73783600 & -2.31954200 & -0.80664300 \\
\hline
\end{tabular}




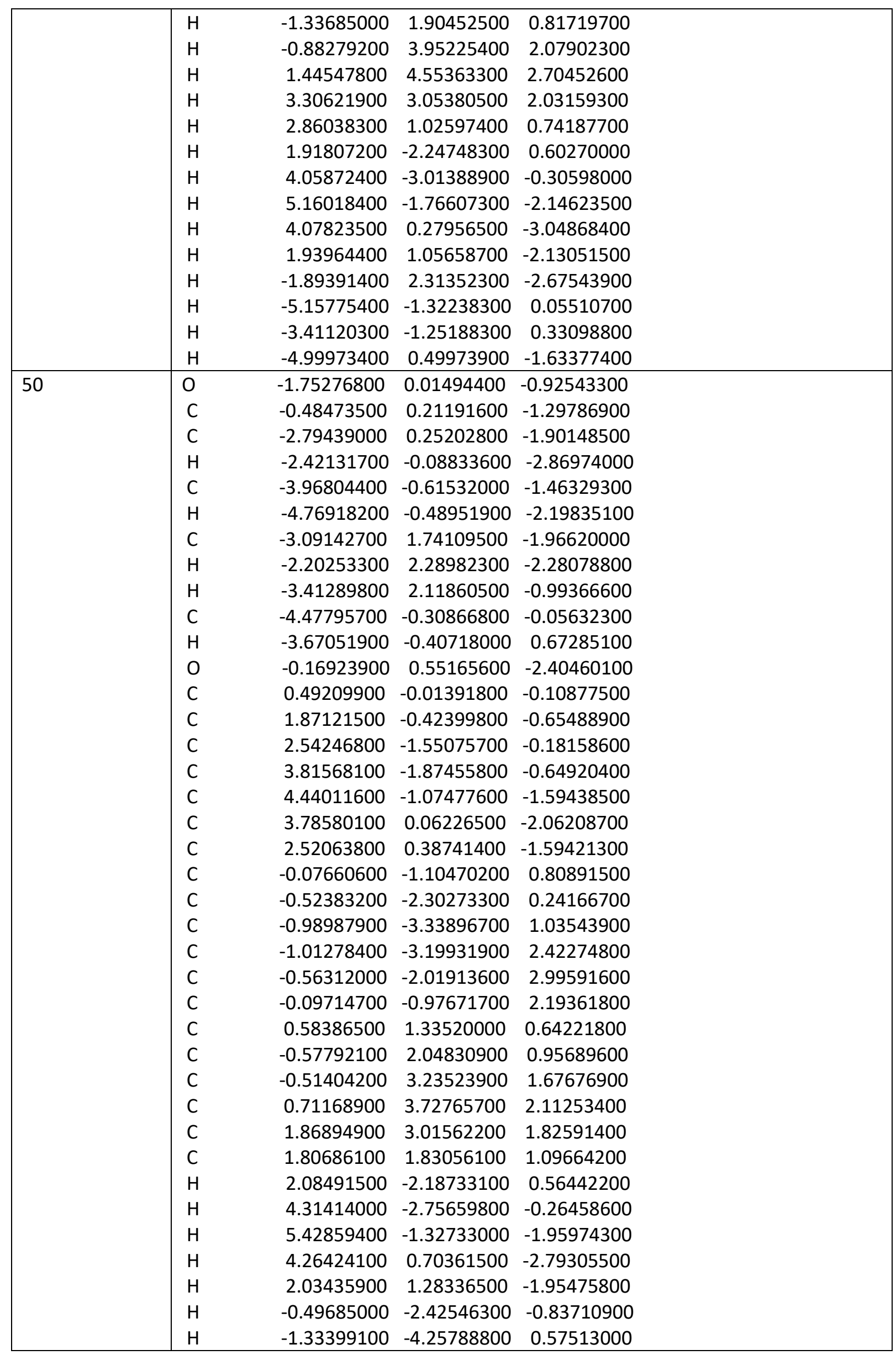




\begin{tabular}{|c|c|c|c|c|}
\hline & $\mathrm{H}$ & -1.37625700 & -4.00713900 & 3.04673500 \\
\hline & $\mathrm{H}$ & -0.57109000 & -1.89952700 & 4.07300600 \\
\hline & $\mathrm{H}$ & 0.25187700 & -0.06316700 & 2.65963800 \\
\hline & $\mathrm{H}$ & -1.54371600 & 1.66169800 & 0.65753200 \\
\hline & $\mathrm{H}$ & -1.42788300 & 3.77205200 & 1.90361000 \\
\hline & $\mathrm{H}$ & 0.76177500 & 4.65238200 & 2.67509500 \\
\hline & $\mathrm{H}$ & 2.83079600 & 3.37878000 & 2.16887400 \\
\hline & $\mathrm{H}$ & 2.72185200 & 1.29120600 & 0.88762500 \\
\hline & $\mathrm{H}$ & -3.88791600 & 1.92784100 & -2.69007000 \\
\hline & $\mathrm{H}$ & -5.27021700 & -1.00546000 & 0.22228000 \\
\hline & $\mathrm{H}$ & -4.88860600 & 0.70064400 & 0.01568000 \\
\hline & $\mathrm{H}$ & -3.64849800 & -1.66029400 & -1.51413800 \\
\hline \multirow[t]{40}{*}{52} & 0 & -1.86596600 & 0.10987300 & -0.42720500 \\
\hline & $\mathrm{C}$ & -0.68901300 & 0.18401700 & -1.05925800 \\
\hline & $\mathrm{C}$ & -3.05872500 & 0.32643800 & -1.21863400 \\
\hline & $\mathrm{H}$ & -2.90152200 & -0.14881600 & -2.19062700 \\
\hline & $\mathrm{C}$ & -4.17578200 & -0.37383400 & -0.46134900 \\
\hline & $\mathrm{H}$ & -3.87795900 & -1.41486700 & -0.30894900 \\
\hline & $\mathrm{C}$ & -3.27536500 & 1.81914400 & -1.40450500 \\
\hline & $\mathrm{H}$ & -2.38943700 & 2.28047900 & -1.84267800 \\
\hline & $\mathrm{H}$ & -3.49240500 & 2.29316000 & -0.44305700 \\
\hline & $\mathrm{C}$ & -5.51312300 & -0.30856500 & -1.19504300 \\
\hline & $\mathrm{H}$ & -5.42139400 & -0.69361600 & -2.21437800 \\
\hline & 0 & -0.58876400 & 0.41032500 & -2.23348500 \\
\hline & $\mathrm{C}$ & 0.50029400 & -0.03341400 & -0.08378500 \\
\hline & $\mathrm{C}$ & 0.09456400 & -1.08009300 & 0.96254100 \\
\hline & $\mathrm{C}$ & -0.47602700 & -2.28080300 & 0.52655800 \\
\hline & $\mathrm{C}$ & -0.81466800 & -3.27929400 & 1.42608100 \\
\hline & $\mathrm{C}$ & -0.58765800 & -3.09580500 & 2.78962400 \\
\hline & $\mathrm{C}$ & -0.02156700 & -1.90957400 & 3.23193100 \\
\hline & $\mathrm{C}$ & 0.31757300 & -0.90571600 & 2.32395800 \\
\hline & $\mathrm{C}$ & 0.81535500 & 1.32187700 & 0.58865300 \\
\hline & $\mathrm{C}$ & 2.13198200 & 1.64252700 & 0.92996600 \\
\hline & $\mathrm{C}$ & 2.42826600 & 2.82233900 & 1.60401100 \\
\hline & $\mathrm{C}$ & 1.41493300 & 3.71071300 & 1.94489600 \\
\hline & $\mathrm{C}$ & 0.10160500 & 3.39988500 & 1.61440200 \\
\hline & $\mathrm{C}$ & -0.19675600 & 2.21406200 & 0.95013800 \\
\hline & $\mathrm{C}$ & 1.72242800 & -0.50168800 & -0.89469900 \\
\hline & $\mathrm{C}$ & 2.20209900 & 0.30870100 & -1.93099000 \\
\hline & $\mathrm{C}$ & 3.33105700 & -0.04991800 & -2.65159000 \\
\hline & $\mathrm{C}$ & 4.01686800 & -1.22360100 & -2.34591400 \\
\hline & $\mathrm{C}$ & 3.56238100 & -2.02450500 & -1.30942700 \\
\hline & $\mathrm{C}$ & 2.42362500 & -1.66584800 & -0.58697500 \\
\hline & $\mathrm{H}$ & -0.64620200 & -2.43597800 & -0.53523400 \\
\hline & $\mathrm{H}$ & -1.25419200 & -4.20231700 & 1.06605200 \\
\hline & $\mathrm{H}$ & -0.85244300 & -3.87341700 & 3.49622900 \\
\hline & $\mathrm{H}$ & 0.15852600 & -1.75390000 & 4.28924600 \\
\hline & $\mathrm{H}$ & 0.75431000 & 0.01519100 & 2.69060600 \\
\hline & $\mathrm{H}$ & 2.93614500 & 0.96454700 & 0.67179700 \\
\hline & $\mathrm{H}$ & 3.45776000 & 3.04560600 & 1.85923000 \\
\hline & $\mathrm{H}$ & 1.64620400 & 4.63319100 & 2.46401400 \\
\hline & $\mathrm{H}$ & -0.70113000 & 4.07891600 & 1.87733200 \\
\hline
\end{tabular}




\begin{tabular}{|c|c|c|c|c|}
\hline & $\mathrm{H}$ & -1.23042300 & 1.98032600 & 0.73278200 \\
\hline & $\mathrm{H}$ & 1.68919400 & 1.23131900 & -2.16788600 \\
\hline & $\mathrm{H}$ & 3.67972100 & 0.59138600 & -3.45250000 \\
\hline & $\mathrm{H}$ & 4.90031700 & -1.50315600 & -2.90800500 \\
\hline & $\mathrm{H}$ & 4.09169700 & -2.93402900 & -1.05047000 \\
\hline & $\mathrm{H}$ & 2.09895500 & -2.30178500 & 0.22649500 \\
\hline & $\mathrm{H}$ & -4.11641000 & 1.99897000 & -2.07617100 \\
\hline & $\mathrm{H}$ & -5.89147900 & 0.71378600 & -1.25446900 \\
\hline & $\mathrm{H}$ & -6.26440800 & -0.90782600 & -0.67894700 \\
\hline & $\mathrm{H}$ & -4.26166000 & 0.07896600 & 0.53157200 \\
\hline \multirow[t]{42}{*}{53} & 0 & -1.56878600 & 0.73592800 & -1.06952000 \\
\hline & $\mathrm{C}$ & -0.47430100 & 0.01663400 & -1.31920700 \\
\hline & $\mathrm{C}$ & -2.70279400 & 0.57448600 & -1.95507700 \\
\hline & $\mathrm{H}$ & -2.32160000 & 0.48971600 & -2.97438400 \\
\hline & $\mathrm{C}$ & -3.43405800 & -0.71195400 & -1.57683300 \\
\hline & $\mathrm{H}$ & -4.25160900 & -0.85331700 & -2.29092300 \\
\hline & $\mathrm{C}$ & -3.52820400 & 1.83608000 & -1.79582900 \\
\hline & $\mathrm{H}$ & -4.43681500 & 1.76574700 & -2.39776800 \\
\hline & $\mathrm{H}$ & -2.95814000 & 2.70711300 & -2.12166500 \\
\hline & $\mathrm{C}$ & -3.96704700 & -0.72455700 & -0.14446900 \\
\hline & $\mathrm{H}$ & -3.17160000 & -0.49900100 & 0.57132600 \\
\hline & 0 & -0.31375900 & -0.61761100 & -2.32634300 \\
\hline & $\mathrm{C}$ & 0.48907200 & -0.00004800 & -0.09987900 \\
\hline & $\mathrm{C}$ & 1.82931500 & -0.61192400 & -0.52183900 \\
\hline & $\mathrm{C}$ & 2.43639300 & -0.22449200 & -1.71979000 \\
\hline & $\mathrm{C}$ & 3.69437600 & -0.69797800 & -2.06626300 \\
\hline & $\mathrm{C}$ & 4.37490200 & -1.56760100 & -1.21815200 \\
\hline & $\mathrm{C}$ & 3.78387200 & -1.95404400 & -0.02316200 \\
\hline & $\mathrm{C}$ & 2.51950100 & -1.48024000 & 0.32128200 \\
\hline & $\mathrm{C}$ & -0.22696600 & -0.94000200 & 0.89771500 \\
\hline & $\mathrm{C}$ & -0.36285000 & -0.64791600 & 2.25274700 \\
\hline & $\mathrm{C}$ & -1.00224700 & -1.54293000 & 3.10952900 \\
\hline & $\mathrm{C}$ & -1.51090800 & -2.74086500 & 2.62574500 \\
\hline & $\mathrm{C}$ & -1.35545600 & -3.05299900 & 1.27791500 \\
\hline & $\mathrm{C}$ & -0.71314600 & -2.16495400 & 0.42605700 \\
\hline & $\mathrm{C}$ & 0.69415000 & 1.42079700 & 0.47363300 \\
\hline & $\mathrm{C}$ & 1.95914400 & 2.00839800 & 0.51896300 \\
\hline & $\mathrm{C}$ & 2.14102100 & 3.28937900 & 1.03923900 \\
\hline & $\mathrm{C}$ & 1.06337400 & 4.00682100 & 1.53420600 \\
\hline & $\mathrm{C}$ & -0.20177200 & 3.42583400 & 1.51506700 \\
\hline & $\mathrm{C}$ & -0.38309700 & 2.15169000 & 0.99640300 \\
\hline & $\mathrm{H}$ & 1.92482500 & 0.45854400 & -2.38626400 \\
\hline & $\mathrm{H}$ & 4.14365900 & -0.38725500 & -3.00225700 \\
\hline & $\mathrm{H}$ & 5.35620300 & -1.93863000 & -1.48940600 \\
\hline & $\mathrm{H}$ & 4.30256400 & -2.62815100 & 0.64840000 \\
\hline & $\mathrm{H}$ & 2.07517700 & -1.79218000 & 1.25898500 \\
\hline & $\mathrm{H}$ & 0.03328200 & 0.27703300 & 2.65203900 \\
\hline & $\mathrm{H}$ & -1.09827000 & -1.29658900 & 4.16059900 \\
\hline & $\mathrm{H}$ & -2.01382000 & -3.43075500 & 3.29298400 \\
\hline & $\mathrm{H}$ & -1.73088000 & -3.99257100 & 0.88895900 \\
\hline & $\mathrm{H}$ & -0.57495000 & -2.42921300 & -0.61731100 \\
\hline & $\mathrm{H}$ & 2.82451100 & 1.47269700 & 0.15315200 \\
\hline
\end{tabular}




\begin{tabular}{|l|lrrr|}
\hline & $H$ & 3.13653400 & 3.71715700 & 1.05667200 \\
H & 1.20370900 & 5.00231800 & 1.93885600 \\
H & -1.05473600 & 3.96531400 & 1.91037600 \\
H & -1.37171200 & 1.71406000 & 1.00537500 \\
H & -3.81242900 & 1.98314200 & -0.75239900 \\
H & -4.36194500 & -1.71100000 & 0.10539000 \\
H & -4.77259900 & -0.00011700 & -0.00569700 \\
& $H$ & -2.74263800 & -1.54628900 & -1.72613900 \\
\hline
\end{tabular}

4 (optimized at the B3LYP/6-311++G(d,p) level)

\begin{tabular}{|c|c|c|c|c|}
\hline \multicolumn{5}{|c|}{ Conformer no } \\
\hline \multirow[t]{40}{*}{1} & 0 & 1.62566600 & -0.50851900 & -0.68420100 \\
\hline & C & 0.42884000 & -0.19034600 & -1.20761900 \\
\hline & $\mathrm{C}$ & 2.75305800 & -0.68522100 & -1.60483800 \\
\hline & $\mathrm{H}$ & 2.59688900 & 0.01034300 & -2.43193600 \\
\hline & $\mathrm{C}$ & 4.02769800 & -0.28267000 & -0.84573500 \\
\hline & $\mathrm{H}$ & 4.84636300 & -0.47386300 & -1.55250200 \\
\hline & C & 4.02873600 & 1.21786800 & -0.52210100 \\
\hline & $\mathrm{H}$ & 3.22455100 & 1.47125400 & 0.17248300 \\
\hline & C & 2.75151000 & -2.10985400 & -2.14794700 \\
\hline & $\mathrm{H}$ & 3.60993400 & -2.25283300 & -2.81019700 \\
\hline & $\mathrm{H}$ & 1.84635000 & -2.29615600 & -2.72835200 \\
\hline & C & 4.28278900 & -1.12077500 & 0.41573800 \\
\hline & $\mathrm{H}$ & 4.37295300 & -2.18689000 & 0.19374500 \\
\hline & $\mathrm{O}$ & 0.22936000 & -0.06629700 & -2.39056900 \\
\hline & C & -0.62386700 & 0.07845100 & -0.08692700 \\
\hline & C & -2.00651200 & 0.38361200 & -0.71622600 \\
\hline & C & -2.80725300 & 1.43980700 & -0.27259800 \\
\hline & C & -4.09082900 & 1.63545500 & -0.78588000 \\
\hline & C & -4.59926100 & 0.77563000 & -1.75307800 \\
\hline & C & -3.81505600 & -0.28988200 & -2.19469200 \\
\hline & $\mathrm{C}$ & -2.53935200 & -0.48603500 & -1.67774400 \\
\hline & C & -0.03064500 & 1.29879200 & 0.66358000 \\
\hline & C & 0.45970800 & 1.22573000 & 1.96912700 \\
\hline & C & 1.03400700 & 2.34115500 & 2.58123400 \\
\hline & C & 1.14117100 & 3.54625200 & 1.89385300 \\
\hline & $\mathrm{C}$ & 0.67659000 & 3.62524400 & 0.58120700 \\
\hline & C & 0.10101500 & 2.51342000 & -0.02604300 \\
\hline & C & -0.84304400 & -1.15632900 & 0.82144500 \\
\hline & C & -0.28374600 & -2.41094600 & 0.56513900 \\
\hline & C & -0.58430500 & -3.51153300 & 1.37050600 \\
\hline & $\mathrm{C}$ & -1.44819900 & -3.37963600 & 2.45161700 \\
\hline & $\mathrm{C}$ & -2.02100400 & -2.13528600 & 2.71446300 \\
\hline & $\mathrm{C}$ & -1.72779200 & -1.04387400 & 1.90457400 \\
\hline & $\mathrm{H}$ & -2.43798700 & 2.12360400 & 0.48047600 \\
\hline & $\mathrm{H}$ & -4.68879200 & 2.46451700 & -0.42342600 \\
\hline & $\mathrm{H}$ & -5.59424300 & 0.92849400 & -2.15592900 \\
\hline & $\mathrm{H}$ & -4.19796700 & -0.97312400 & -2.94475500 \\
\hline & $\mathrm{H}$ & -1.95413800 & -1.32448400 & -2.03117000 \\
\hline & $\mathrm{H}$ & 0.40292800 & 0.29520500 & 2.51730600 \\
\hline & $\mathrm{H}$ & 1.40206600 & 2.25986200 & 3.59814900 \\
\hline
\end{tabular}




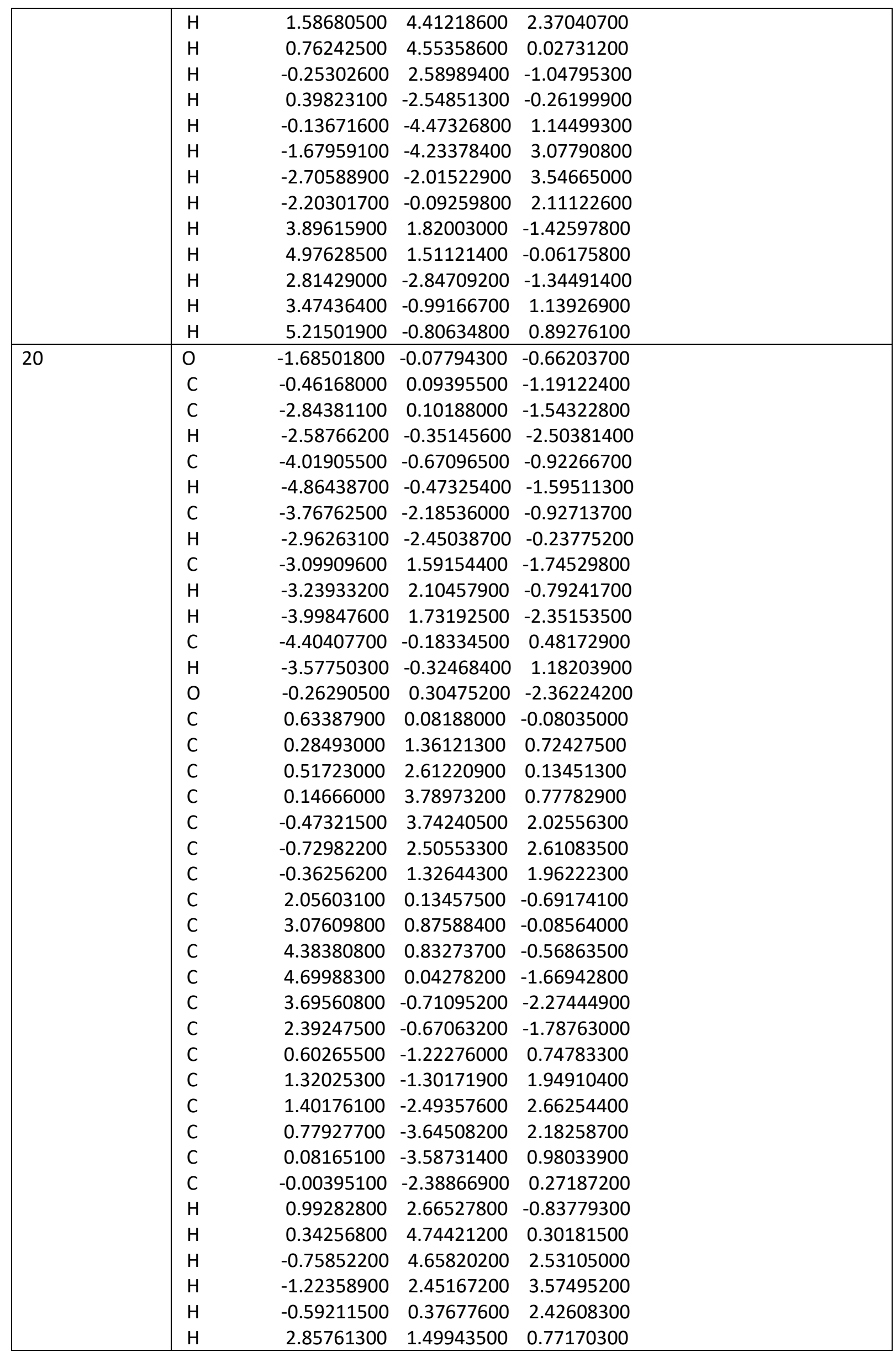




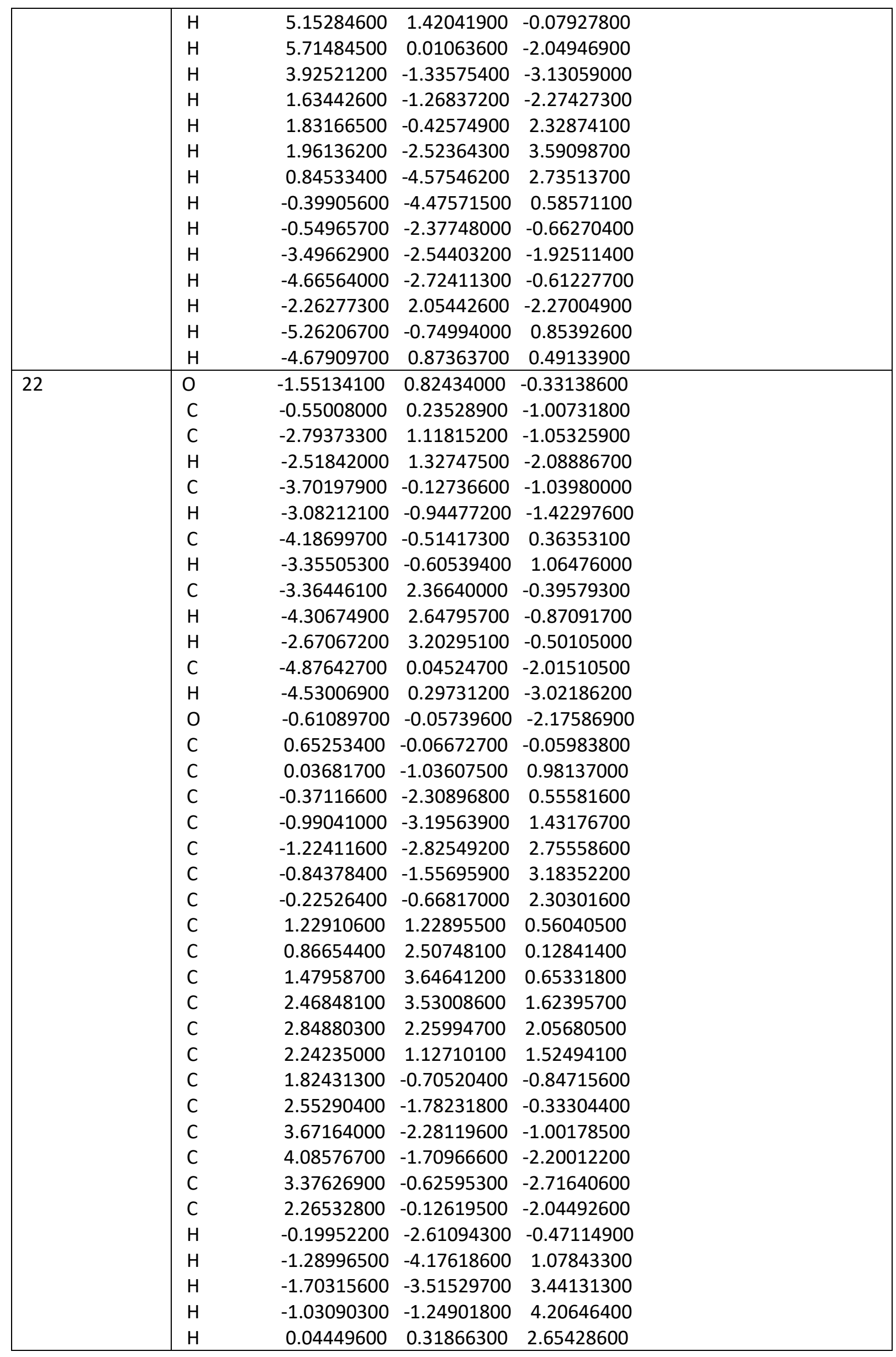




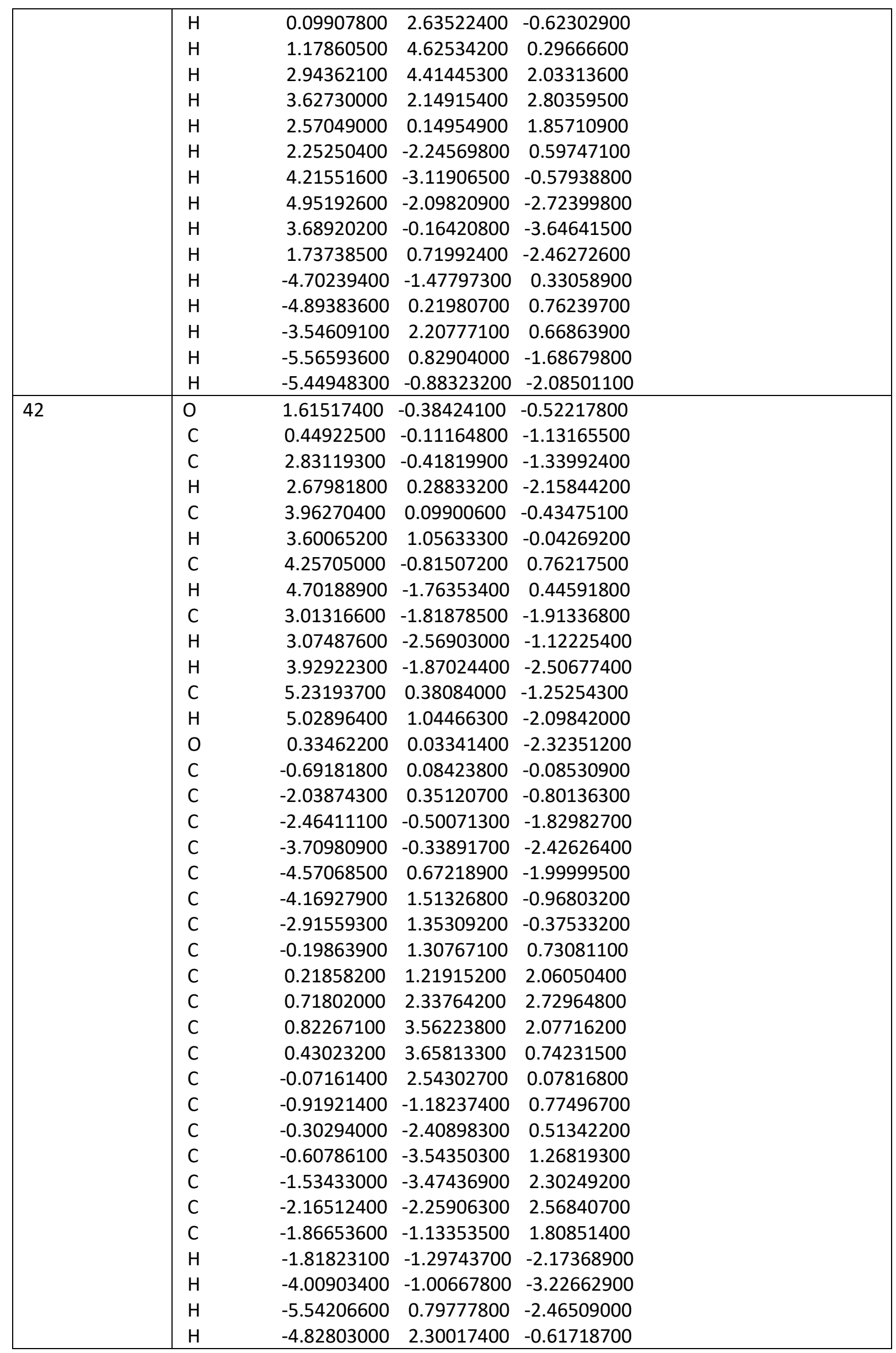




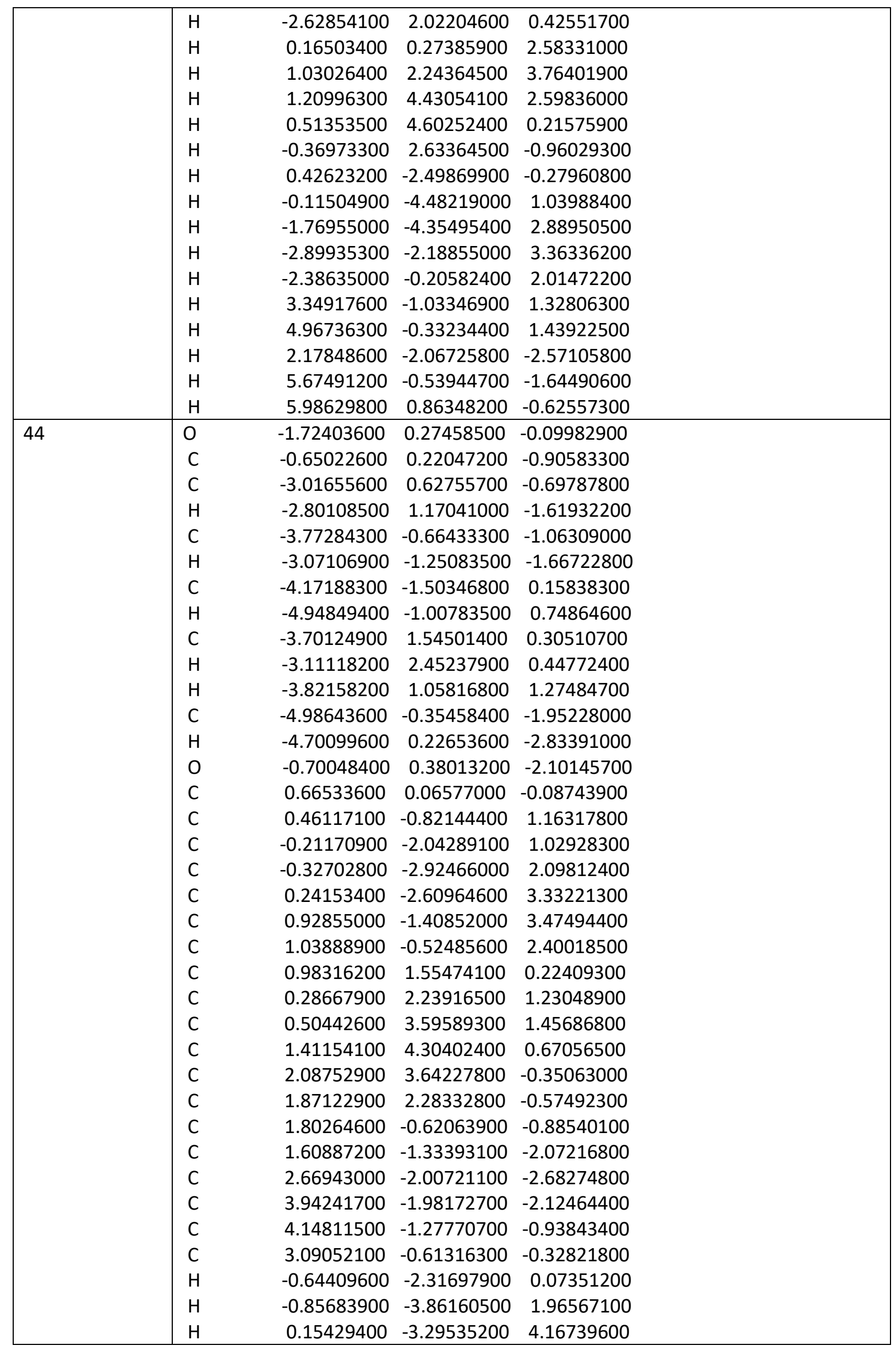




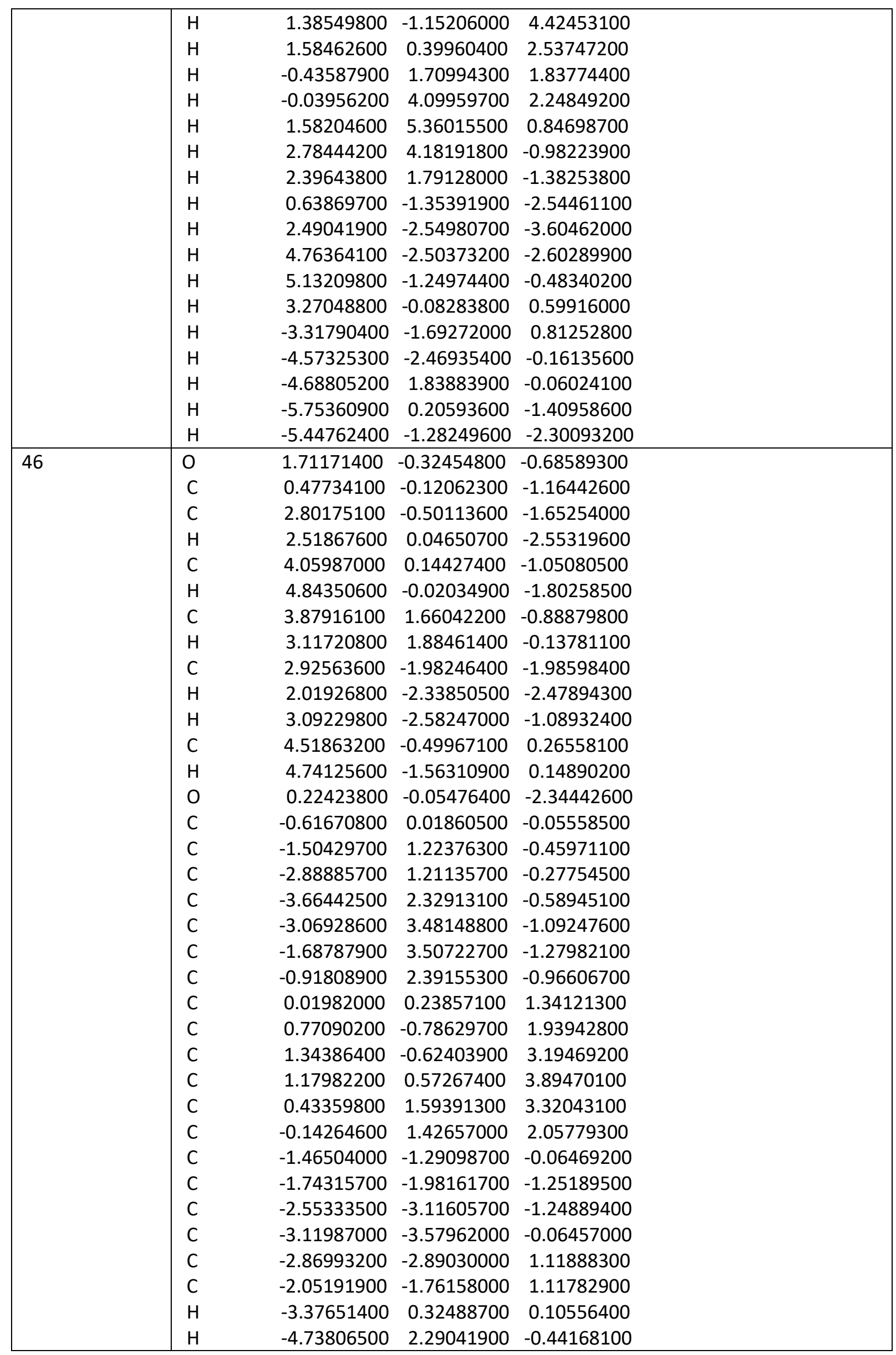




\begin{tabular}{|l|lrrr|}
\hline & $H$ & -3.67214300 & 4.34790500 & -1.34035000 \\
H & -1.20742800 & 4.39557900 & -1.67472700 \\
$\mathrm{H}$ & 0.15391200 & 2.43764000 & -1.11995400 \\
$\mathrm{H}$ & 0.90489800 & -1.72523100 & 1.41791300 \\
$\mathrm{H}$ & 1.91766100 & -1.43504000 & 3.62997400 \\
$\mathrm{H}$ & 1.62412100 & 0.69995800 & 4.87560600 \\
$\mathrm{H}$ & 0.28733500 & 2.52818600 & 3.85135800 \\
$\mathrm{H}$ & -0.72756500 & 2.23592500 & 1.64325400 \\
$\mathrm{H}$ & -1.33817500 & -1.62702400 & -2.18938100 \\
$\mathrm{H}$ & -2.74452400 & -3.63325200 & -2.18281400 \\
$\mathrm{H}$ & -3.75067000 & -4.46151600 & -0.06446300 \\
$\mathrm{H}$ & -3.30976300 & -3.22785000 & 2.05100500 \\
$\mathrm{H}$ & -1.87454100 & -1.24481900 & 2.05162500 \\
$\mathrm{H}$ & 3.57955800 & 2.13026700 & -1.83063700 \\
$\mathrm{H}$ & 4.81327200 & 2.12756000 & -0.56541300 \\
$\mathrm{H}$ & 3.76549500 & -2.14046400 & -2.66846800 \\
$\mathrm{H}$ & 3.75401900 & -0.39353300 & 1.03890700 \\
$\mathrm{H}$ & 5.42955200 & -0.01272100 & 0.62444600 \\
\hline
\end{tabular}

4 (optimized at the M06-2X/6-311++G(d,p) level)

\begin{tabular}{|c|c|c|c|c|}
\hline Conformer no & & & & \\
\hline 1 & 0 & -1.56743500 & 0.49260400 & -0.73644500 \\
\hline & C & -0.36478800 & 0.20821400 & -1.24186100 \\
\hline & C & -2.67234100 & 0.65940400 & -1.65371700 \\
\hline & $\mathrm{H}$ & -2.50747200 & -0.02562300 & -2.48969900 \\
\hline & C & -3.92413500 & 0.22785400 & -0.88868800 \\
\hline & $\mathrm{H}$ & -4.75488100 & 0.32416600 & -1.59853700 \\
\hline & C & -3.80505200 & -1.23750100 & -0.46631700 \\
\hline & $\mathrm{H}$ & -3.01729000 & -1.35630300 & 0.28250400 \\
\hline & C & -2.68862600 & 2.09104800 & -2.16124900 \\
\hline & $\mathrm{H}$ & -3.56530100 & 2.25459500 & -2.79230200 \\
\hline & $\mathrm{H}$ & -1.79513600 & 2.29059600 & -2.75532700 \\
\hline & C & -4.20288200 & 1.11635800 & 0.32494700 \\
\hline & $\mathrm{H}$ & -4.41947200 & 2.14847200 & 0.04224400 \\
\hline & 0 & -0.13068300 & 0.14460000 & -2.41726400 \\
\hline & C & 0.63222900 & -0.08151600 & -0.09560600 \\
\hline & C & 2.00560100 & -0.48528000 & -0.65447300 \\
\hline & C & 2.72223900 & -1.56600600 & -0.14341600 \\
\hline & C & 4.01035000 & -1.84518900 & -0.59585100 \\
\hline & C & 4.60088600 & -1.04600300 & -1.56426800 \\
\hline & C & 3.89868800 & 0.04530100 & -2.06903200 \\
\hline & C & 2.61893600 & 0.32655700 & -1.61313700 \\
\hline & C & -0.05055900 & -1.21960000 & 0.68521900 \\
\hline & C & -0.59538400 & -1.05050600 & 1.95508800 \\
\hline & C & -1.27403400 & -2.09686500 & 2.57850200 \\
\hline & C & -1.42893400 & -3.31792400 & 1.93572300 \\
\hline & C & -0.90765100 & -3.48765800 & 0.65528400 \\
\hline & C & -0.22855800 & -2.44651500 & 0.03700800 \\
\hline & C & 0.86911500 & 1.17908700 & 0.75118600 \\
\hline & C & 0.35113800 & 2.42919500 & 0.42267700 \\
\hline & C & 0.66140400 & 3.55138200 & 1.19053000 \\
\hline
\end{tabular}




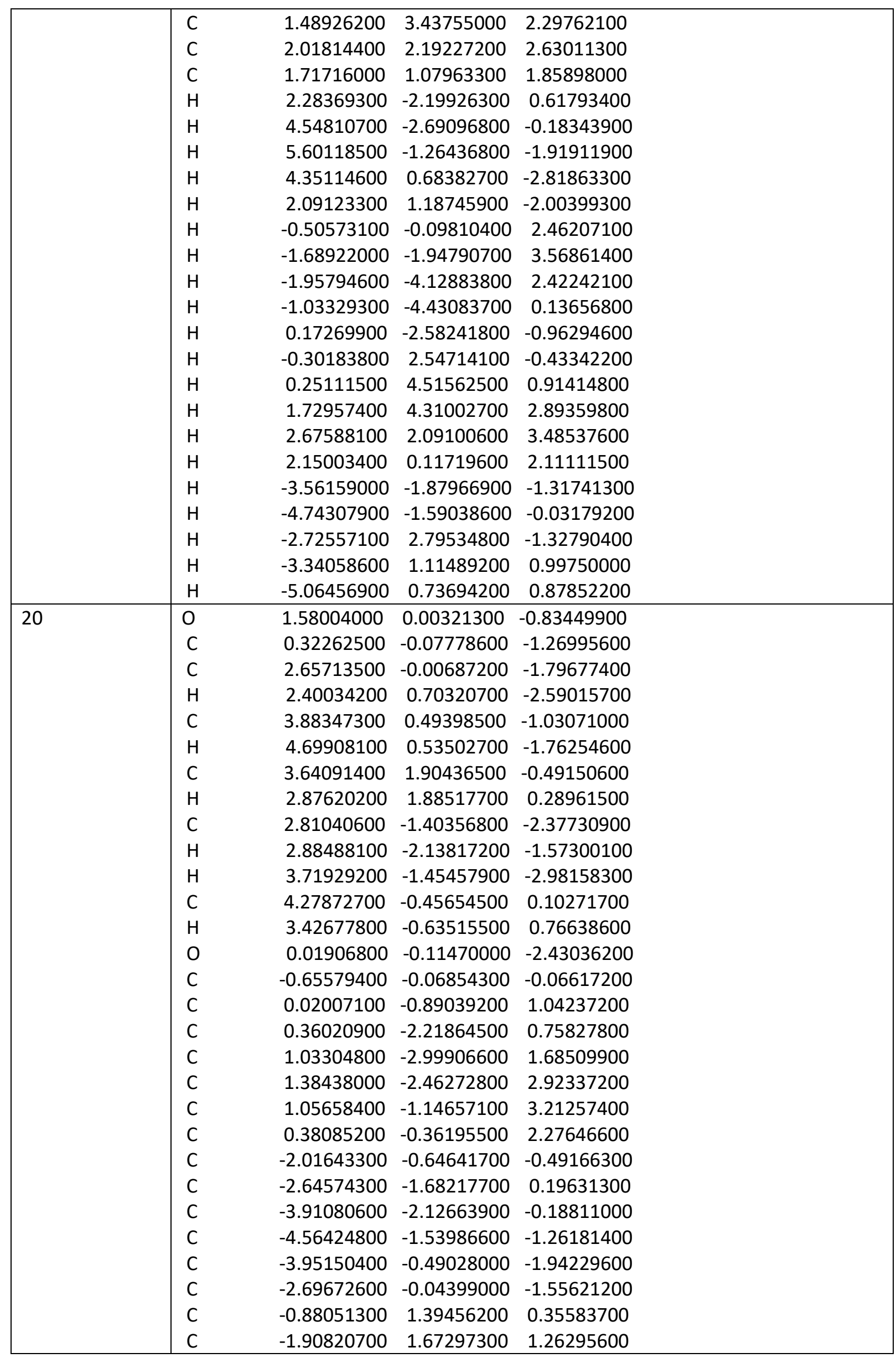




\begin{tabular}{|c|c|c|c|c|}
\hline & $\mathrm{C}$ & -2.14482400 & 2.96948400 & 1.69613100 \\
\hline & C & -1.36510900 & 4.02061000 & 1.21884700 \\
\hline & C & -0.35272500 & 3.75750900 & 0.30730500 \\
\hline & C & -0.11016400 & 2.45279200 & -0.12024900 \\
\hline & $\mathrm{H}$ & 0.09395600 & -2.63944500 & -0.20773400 \\
\hline & $\mathrm{H}$ & 1.28733000 & -4.02465500 & 1.44323600 \\
\hline & $\mathrm{H}$ & 1.91256400 & -3.06787200 & 3.65056700 \\
\hline & $\mathrm{H}$ & 1.33118100 & -0.71572600 & 4.16841100 \\
\hline & $\mathrm{H}$ & 0.15271400 & 0.66898700 & 2.51648800 \\
\hline & $\mathrm{H}$ & -2.16246200 & -2.14743400 & 1.04593100 \\
\hline & $\mathrm{H}$ & -4.38166400 & -2.93327400 & 0.36183100 \\
\hline & $\mathrm{H}$ & -5.54570900 & -1.88724300 & -1.56225400 \\
\hline & $\mathrm{H}$ & -4.45580500 & -0.01283600 & -2.77427600 \\
\hline & $\mathrm{H}$ & -2.23775400 & 0.78371200 & -2.08131900 \\
\hline & $\mathrm{H}$ & -2.52964300 & 0.86125600 & 1.62617000 \\
\hline & $\mathrm{H}$ & -2.94495600 & 3.16122100 & 2.40141800 \\
\hline & $\mathrm{H}$ & -1.55283200 & 5.03489200 & 1.55045000 \\
\hline & $\mathrm{H}$ & 0.25575100 & 4.56624100 & -0.08029500 \\
\hline & $\mathrm{H}$ & 0.68825400 & 2.27993400 & -0.83173400 \\
\hline & $\mathrm{H}$ & 3.30728900 & 2.58260300 & -1.28276100 \\
\hline & $\mathrm{H}$ & 4.55708100 & 2.31373700 & -0.06069500 \\
\hline & $\mathrm{H}$ & 1.95748200 & -1.65382400 & -3.00749400 \\
\hline & $\mathrm{H}$ & 5.08587100 & -0.01938600 & 0.69465500 \\
\hline & $\mathrm{H}$ & 4.62813200 & -1.41992900 & -0.27340200 \\
\hline \multirow[t]{28}{*}{22} & 0 & -1.42402400 & 0.96372500 & -0.56072000 \\
\hline & $\mathrm{C}$ & -0.47334800 & 0.18531000 & -1.08593800 \\
\hline & C & -2.67192200 & 1.07534900 & -1.28643300 \\
\hline & $\mathrm{H}$ & -2.43469900 & 1.11675600 & -2.35259800 \\
\hline & C & -3.50877000 & -0.18157000 & -1.01572900 \\
\hline & $\mathrm{H}$ & -2.87014000 & -1.02854800 & -1.29129200 \\
\hline & C & -3.88625800 & -0.32503500 & 0.45851800 \\
\hline & $\mathrm{H}$ & -3.01185700 & -0.22401800 & 1.10626300 \\
\hline & C & -3.29078500 & 2.38156400 & -0.82773300 \\
\hline & $\mathrm{H}$ & -4.25751600 & 2.53702100 & -1.30967100 \\
\hline & $\mathrm{H}$ & -2.63918700 & 3.21678400 & -1.08919500 \\
\hline & C & -4.74302300 & -0.21476700 & -1.91693700 \\
\hline & $\mathrm{H}$ & -4.47329000 & -0.10409400 & -2.97043100 \\
\hline & 0 & -0.53800500 & -0.30817300 & -2.17831400 \\
\hline & C & 0.65080500 & -0.06609300 & -0.05510500 \\
\hline & C & -0.09800600 & -0.84351200 & 1.04342000 \\
\hline & C & -0.62044000 & -2.10000800 & 0.71727400 \\
\hline & C & -1.38301600 & -2.81368800 & 1.63246700 \\
\hline & $\mathrm{C}$ & -1.64117200 & -2.28045600 & 2.89260700 \\
\hline & C & -1.14148800 & -1.02617700 & 3.21776300 \\
\hline & $\mathrm{C}$ & -0.38163900 & -0.30711600 & 2.29684900 \\
\hline & C & 1.28809200 & 1.24033000 & 0.43820800 \\
\hline & C & 1.04898700 & 2.47720100 & -0.15585700 \\
\hline & C & 1.73045500 & 3.61504700 & 0.27281200 \\
\hline & C & 2.65697900 & 3.53337300 & 1.30238900 \\
\hline & C & 2.91131800 & 2.29903200 & 1.89580200 \\
\hline & C & 2.24055300 & 1.16606200 & 1.45963800 \\
\hline & $\mathrm{C}$ & 1.78824600 & -0.87685000 & -0.69314100 \\
\hline
\end{tabular}




\begin{tabular}{|c|c|c|c|c|}
\hline & $\mathrm{C}$ & 2.38739900 & -1.95642000 & -0.04728600 \\
\hline & C & 3.48943500 & -2.59819100 & -0.61001700 \\
\hline & C & 4.00584300 & -2.16882300 & -1.82443900 \\
\hline & C & 3.41987400 & -1.08319400 & -2.47063400 \\
\hline & C & 2.32744300 & -0.44184000 & -1.90682700 \\
\hline & $\mathrm{H}$ & -0.42486600 & -2.51714100 & -0.26607000 \\
\hline & $\mathrm{H}$ & -1.77691200 & -3.78594500 & 1.36021400 \\
\hline & $\mathrm{H}$ & -2.23447000 & -2.83541500 & 3.60962000 \\
\hline & $\mathrm{H}$ & -1.34802000 & -0.59352300 & 4.18986100 \\
\hline & $\mathrm{H}$ & -0.02431500 & 0.68065700 & 2.55780100 \\
\hline & $\mathrm{H}$ & 0.32737900 & 2.57140300 & -0.95763200 \\
\hline & $\mathrm{H}$ & 1.53157400 & 4.56704200 & -0.20546000 \\
\hline & $\mathrm{H}$ & 3.18417400 & 4.41945600 & 1.63540900 \\
\hline & $\mathrm{H}$ & 3.64215800 & 2.21671100 & 2.69172800 \\
\hline & $\mathrm{H}$ & 2.46120600 & 0.20378500 & 1.90996900 \\
\hline & $\mathrm{H}$ & 2.00119800 & -2.30541000 & 0.90321400 \\
\hline & $\mathrm{H}$ & 3.94162700 & -3.43588700 & -0.09185300 \\
\hline & $\mathrm{H}$ & 4.86028000 & -2.66978500 & -2.26384800 \\
\hline & $\mathrm{H}$ & 3.81695300 & -0.73344600 & -3.41639100 \\
\hline & $\mathrm{H}$ & 1.88734800 & 0.40798500 & -2.41435900 \\
\hline & $\mathrm{H}$ & -4.32480500 & -1.30927300 & 0.63932200 \\
\hline & $\mathrm{H}$ & -4.62606200 & 0.42553300 & 0.75229800 \\
\hline & $\mathrm{H}$ & -3.43081100 & 2.38273100 & 0.25418100 \\
\hline & $\mathrm{H}$ & -5.44661500 & 0.58076600 & -1.65652500 \\
\hline & $\mathrm{H}$ & -5.26747700 & -1.16571200 & -1.80230800 \\
\hline 42 & 0 & 1.59316400 & -0.27006900 & -0.54421900 \\
\hline & C & 0.41365600 & -0.12026700 & -1.15147400 \\
\hline & C & 2.77809600 & -0.37670200 & -1.36468700 \\
\hline & $\mathrm{H}$ & 2.64059700 & 0.28091900 & -2.22731600 \\
\hline & C & 3.91996000 & 0.15796300 & -0.49752500 \\
\hline & $\mathrm{H}$ & 3.61604900 & 1.17115200 & -0.21049900 \\
\hline & C & 4.11610400 & -0.65724500 & 0.78093300 \\
\hline & $\mathrm{H}$ & 4.51289500 & -1.65196700 & 0.55951900 \\
\hline & C & 2.92892900 & -1.81305900 & -1.83889700 \\
\hline & $\mathrm{H}$ & 3.00484300 & -2.49574600 & -0.98994200 \\
\hline & $\mathrm{H}$ & 3.82450800 & -1.91908500 & -2.45379200 \\
\hline & C & 5.21662200 & 0.25076100 & -1.30128300 \\
\hline & $\mathrm{H}$ & 5.07311500 & 0.79565900 & -2.23821100 \\
\hline & 0 & 0.27111200 & -0.10564100 & -2.34314500 \\
\hline & C & -0.69716500 & 0.08940400 & -0.09563400 \\
\hline & C & -2.05594400 & 0.33373100 & -0.77079300 \\
\hline & C & -2.50082200 & -0.54830700 & -1.75984900 \\
\hline & $\mathrm{C}$ & -3.76171500 & -0.40789300 & -2.32194500 \\
\hline & C & -4.61272300 & 0.60828700 & -1.89531600 \\
\hline & $\mathrm{C}$ & -4.19050300 & 1.47438500 & -0.89684100 \\
\hline & C & -2.92111200 & 1.33773300 & -0.33863400 \\
\hline & C & -0.20102800 & 1.31093200 & 0.69964600 \\
\hline & C & 0.25323600 & 1.23133800 & 2.01276000 \\
\hline & C & 0.76808600 & 2.35749200 & 2.65249700 \\
\hline & C & 0.84696900 & 3.57205800 & 1.98487600 \\
\hline & C & 0.41446300 & 3.65536700 & 0.66336200 \\
\hline & $\mathrm{C}$ & -0.10107700 & 2.53411800 & 0.02830200 \\
\hline
\end{tabular}




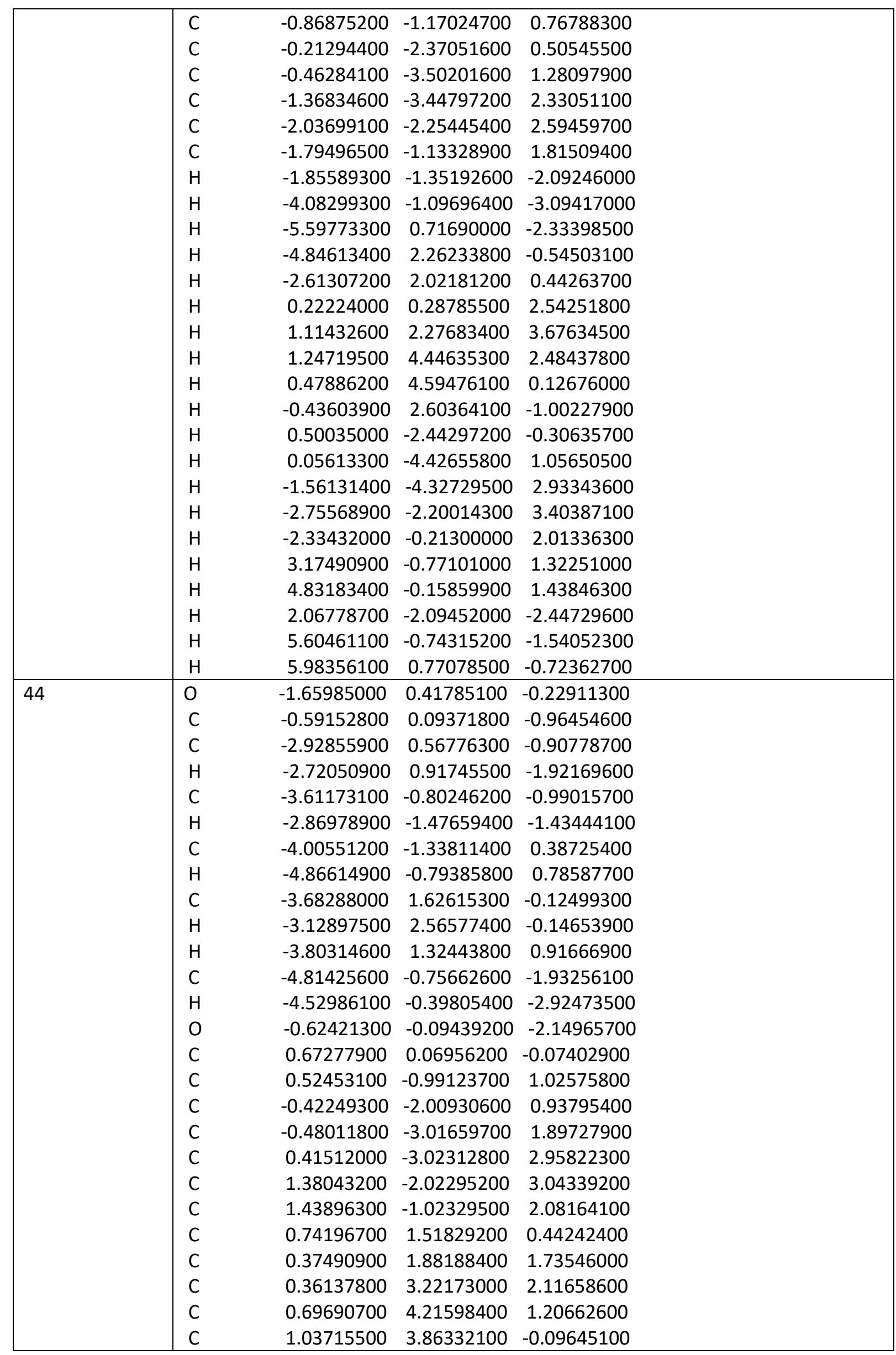




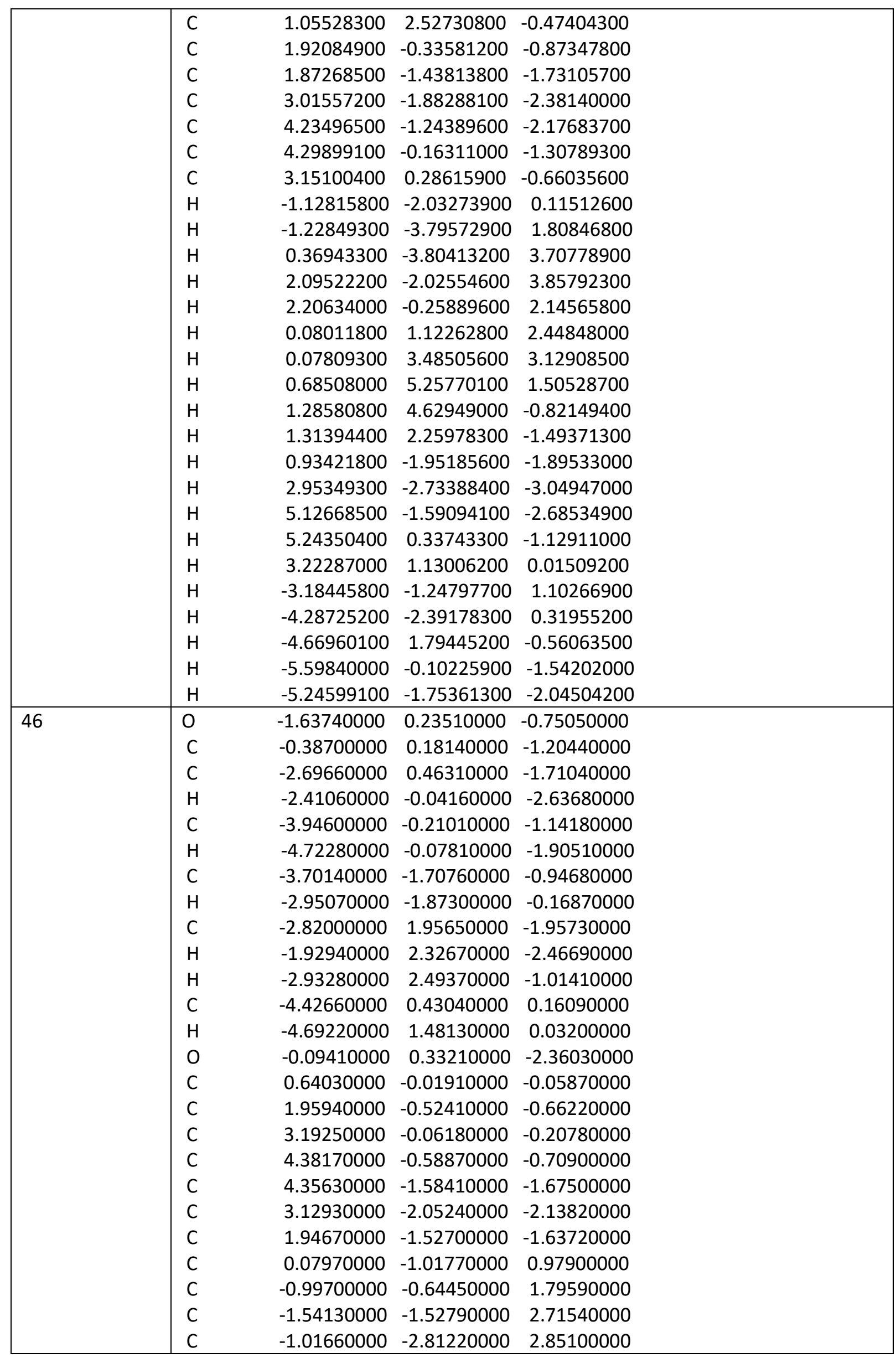




\begin{tabular}{|l|lrrr|}
\hline & C & 0.06140000 & -3.18770000 & 2.06480000 \\
C & 0.60780000 & -2.29710000 & 1.13950000 \\
C & 0.84860000 & 1.37860000 & 0.56260000 \\
C & 0.83670000 & 2.51700000 & -0.24630000 \\
C & 1.09360000 & 3.77400000 & 0.29070000 \\
C & 1.38160000 & 3.91380000 & 1.64340000 \\
C & 1.41640000 & 2.78370000 & 2.45270000 \\
C & 1.15050000 & 1.52770000 & 1.91720000 \\
H & 3.23970000 & 0.72010000 & 0.53970000 \\
H & 5.32830000 & -0.21040000 & -0.34080000 \\
H & 5.28080000 & -1.99020000 & -2.06850000 \\
H & 3.09160000 & -2.82650000 & -2.89580000 \\
H & 1.00170000 & -1.90400000 & -2.00990000 \\
H & -1.40660000 & 0.35390000 & 1.70950000 \\
H & -2.37660000 & -1.21260000 & 3.33050000 \\
H & -1.43970000 & -3.50440000 & 3.56950000 \\
H & 0.49280000 & -4.17660000 & 2.16760000 \\
H & 1.45810000 & -2.61520000 & 0.55140000 \\
H & 0.64490000 & 2.42420000 & -1.30950000 \\
H & 1.07470000 & 4.64460000 & -0.35450000 \\
H & 1.58160000 & 4.89290000 & 2.06230000 \\
H & 1.65120000 & 2.87580000 & 3.50670000 \\
H & 1.18620000 & 0.65590000 & 2.55990000 \\
H & -3.34780000 & -2.17630000 & -1.86920000 \\
H & -4.62170000 & -2.21100000 & -0.64300000 \\
H & -3.68970000 & 2.16250000 & -2.58580000 \\
H & -3.64850000 & 0.35970000 & 0.92530000 \\
H & -5.30980000 & -0.09370000 & 0.53270000 \\
\hline
\end{tabular}

6 (optimized at the B3LYP/6-311++G(d,p) level)

\begin{tabular}{|l|llll|}
\hline Conformer no & \multicolumn{4}{|c|}{} \\
\hline 1 & C & -4.68569900 & 2.13362200 & 0.99457900 \\
& C & -4.43744900 & 2.22194600 & -0.51687600 \\
& C & -3.18608400 & 1.43472900 & -0.93018600 \\
& C & -3.26356200 & -0.03216800 & -0.46781800 \\
& C & -3.50757200 & -0.11879400 & 1.04993000 \\
& C & -4.75779500 & 0.67338900 & 1.46130200 \\
& C & -2.02738500 & -0.82141200 & -0.93604500 \\
& C & -2.15638400 & -2.32136800 & -0.66887600 \\
& O & -0.86978300 & -0.30383200 & -0.26068300 \\
& C & 0.33297000 & -0.60597400 & -0.80352000 \\
& O & 0.44889900 & -1.29032900 & -1.78719500 \\
& C & 1.48596900 & 0.13475800 & -0.07068400 \\
& C & 1.52232300 & -0.16490700 & 1.44776200 \\
& C & 2.44686900 & 0.52757500 & 2.24398100 \\
& C & 2.58185800 & 0.24887000 & 3.59949300 \\
& C & 1.80439400 & -0.74657100 & 4.19135000 \\
& C & 0.89928500 & -1.45438900 & 3.40824700 \\
& C & 0.75689700 & -1.16871800 & 2.04825600 \\
& C & 2.85326200 & -0.33625100 & -0.62400600 \\
& C & 3.12514500 & -1.70690700 & -0.72960500 \\
\hline
\end{tabular}




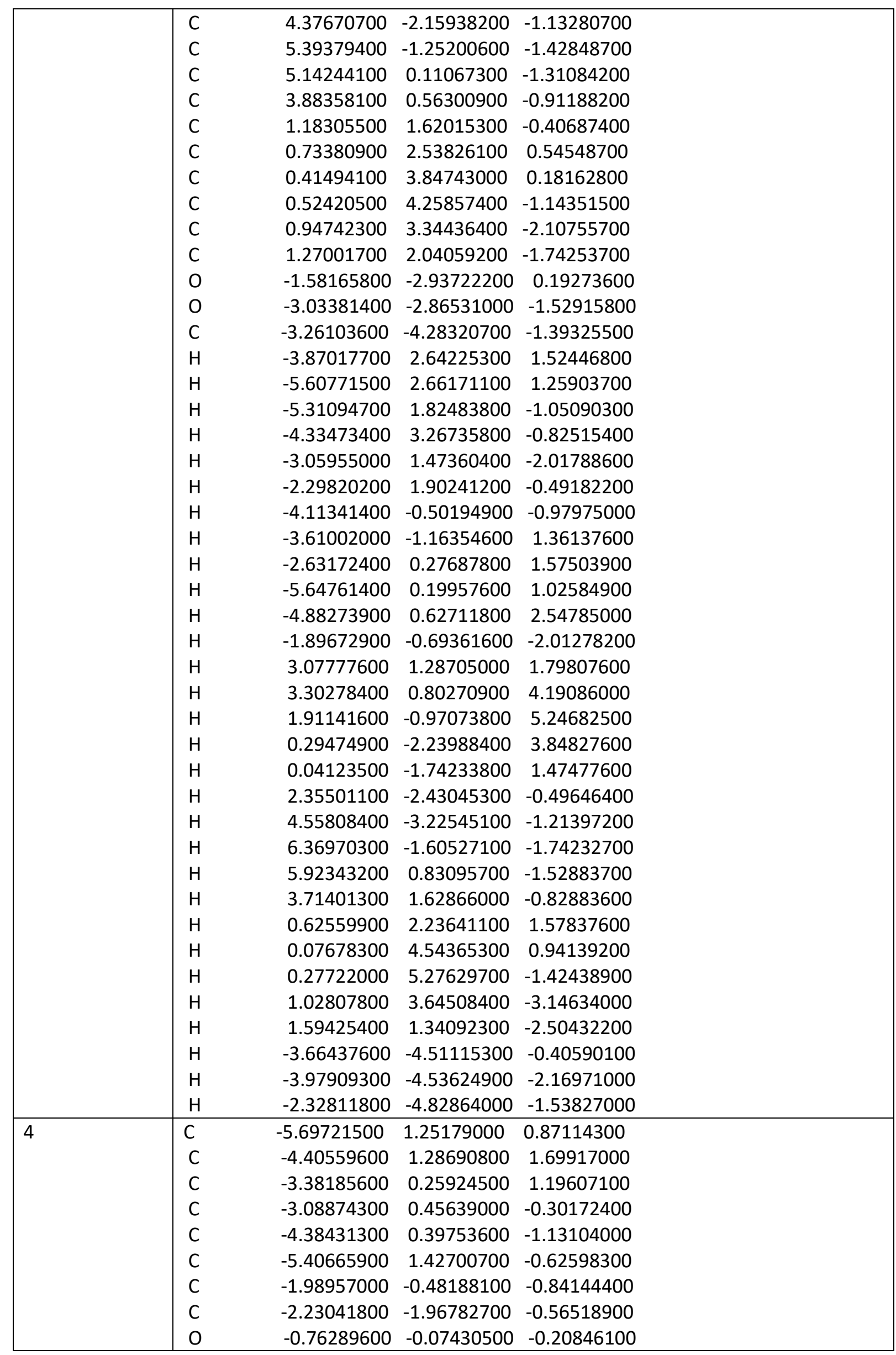




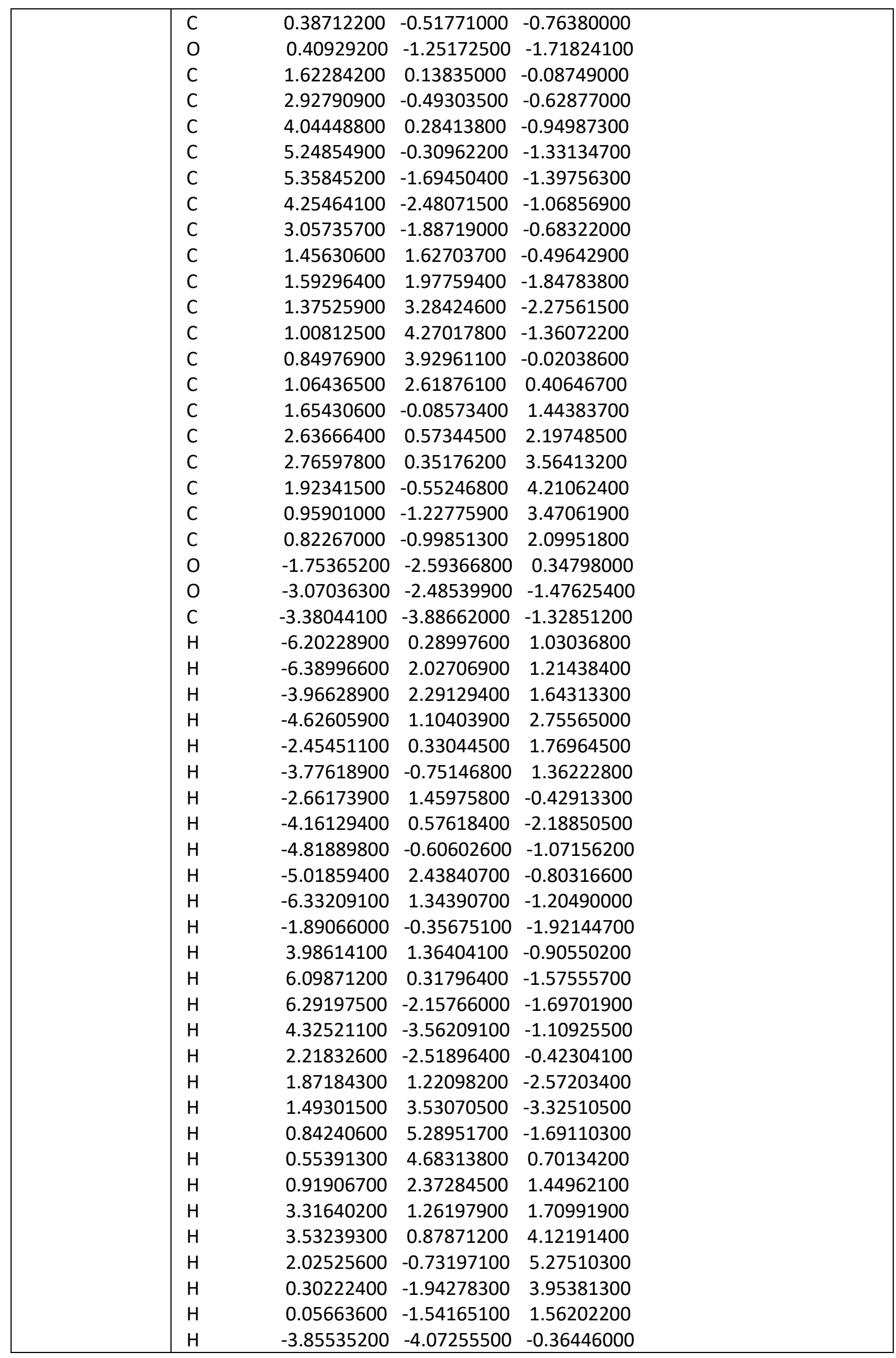




\begin{tabular}{|c|c|c|c|c|}
\hline & $\begin{array}{l}\mathrm{H} \\
\mathrm{H}\end{array}$ & $\begin{array}{l}-4.06084500 \\
-2.47007100\end{array}$ & $\begin{array}{l}-4.11900200 \\
-4.48178600\end{array}$ & $\begin{array}{l}-2.14434400 \\
-1.40451700\end{array}$ \\
\hline \multirow[t]{50}{*}{7} & $C$ & 4.83304100 & -2.21544700 & 0.57159800 \\
\hline & $\mathrm{C}$ & 4.52761300 & -2.19225000 & -0.93237100 \\
\hline & $\mathrm{C}$ & 3.23782100 & -1.41514000 & -1.23431000 \\
\hline & $\mathrm{C}$ & 3.29125100 & 0.01275000 & -0.66005100 \\
\hline & $\mathrm{C}$ & 3.59107100 & -0.01603400 & 0.85063700 \\
\hline & $\mathrm{C}$ & 4.87964000 & -0.79549900 & 1.15023100 \\
\hline & $\mathrm{C}$ & 2.02405300 & 0.81109800 & -1.01720900 \\
\hline & C & 2.12343700 & 2.27175300 & -0.57043000 \\
\hline & $\mathrm{O}$ & 0.89394800 & 0.19025000 & -0.37974600 \\
\hline & C & -0.32738000 & 0.62606100 & -0.76993500 \\
\hline & $\mathrm{O}$ & -0.46584500 & 1.44069000 & -1.64497500 \\
\hline & C & -1.48942800 & -0.09359200 & -0.01213400 \\
\hline & C & -2.67203200 & 0.90563200 & 0.13553000 \\
\hline & C & -3.25185700 & 1.18737500 & 1.37598900 \\
\hline & $\mathrm{C}$ & -4.34055200 & 2.05573500 & 1.48345300 \\
\hline & $\mathrm{C}$ & -4.87397900 & 2.65832900 & 0.35038800 \\
\hline & $\mathrm{C}$ & -4.31413900 & 2.37541000 & -0.89529300 \\
\hline & $\mathrm{C}$ & -3.23320300 & 1.50686000 & -1.00257900 \\
\hline & C & -1.92267600 & -1.29028300 & -0.90576100 \\
\hline & $\mathrm{C}$ & -0.98686700 & -2.02385100 & -1.64479800 \\
\hline & $\mathrm{C}$ & -1.37516000 & -3.12187700 & -2.41033600 \\
\hline & $\mathrm{C}$ & -2.70893300 & -3.52071400 & -2.44205500 \\
\hline & $\mathrm{C}$ & -3.64779500 & -2.80931700 & -1.69909100 \\
\hline & $\mathrm{C}$ & -3.25911100 & -1.70496800 & -0.94272200 \\
\hline & $\mathrm{C}$ & -1.00305000 & -0.58753700 & 1.37001400 \\
\hline & $\mathrm{C}$ & -0.32283300 & 0.29481700 & 2.22347900 \\
\hline & $\mathrm{C}$ & 0.07981700 & -0.10511500 & 3.49288200 \\
\hline & $\mathrm{C}$ & -0.18902300 & -1.39872600 & 3.94304900 \\
\hline & $\mathrm{C}$ & -0.86128600 & -2.28232100 & 3.10629900 \\
\hline & $\mathrm{C}$ & -1.26403600 & -1.87935900 & 1.83056800 \\
\hline & $\mathrm{O}$ & 1.59684300 & 2.74735600 & 0.40206800 \\
\hline & $\mathrm{O}$ & 2.92377100 & 2.95184700 & -1.40948000 \\
\hline & C & 3.12114500 & 4.34746600 & -1.10300600 \\
\hline & $\mathrm{H}$ & 4.05503000 & -2.79076100 & 1.08937600 \\
\hline & $\mathrm{H}$ & 5.78012100 & -2.73272800 & 0.75675100 \\
\hline & $\mathrm{H}$ & 5.36593600 & -1.72478800 & -1.46534200 \\
\hline & $\mathrm{H}$ & 4.44404300 & -3.21272300 & -1.31989800 \\
\hline & $\mathrm{H}$ & 3.06720900 & -1.37489500 & -2.31627100 \\
\hline & $\mathrm{H}$ & 2.38577100 & -1.94512900 & -0.79454500 \\
\hline & $\mathrm{H}$ & 4.11020000 & 0.54389200 & -1.16283300 \\
\hline & $\mathrm{H}$ & 3.67409000 & 1.00243000 & 1.24268000 \\
\hline & $\mathrm{H}$ & 2.74856100 & -0.48070300 & 1.37338000 \\
\hline & $\mathrm{H}$ & 5.73736000 & -0.25929600 & 0.72274900 \\
\hline & $\mathrm{H}$ & 5.04513900 & -0.83201500 & 2.23164300 \\
\hline & $\mathrm{H}$ & 1.87332600 & 0.80602900 & -2.09894100 \\
\hline & $\mathrm{H}$ & -2.86391300 & 0.72978900 & 2.27499100 \\
\hline & $\mathrm{H}$ & -4.76677000 & 2.25451300 & 2.46067100 \\
\hline & $\mathrm{H}$ & -5.71762700 & 3.33467900 & 0.43250400 \\
\hline & $\mathrm{H}$ & -4.72204200 & 2.83040700 & -1.79130900 \\
\hline & $\mathrm{H}$ & -2.82128100 & 1.29730400 & -1.97887700 \\
\hline
\end{tabular}




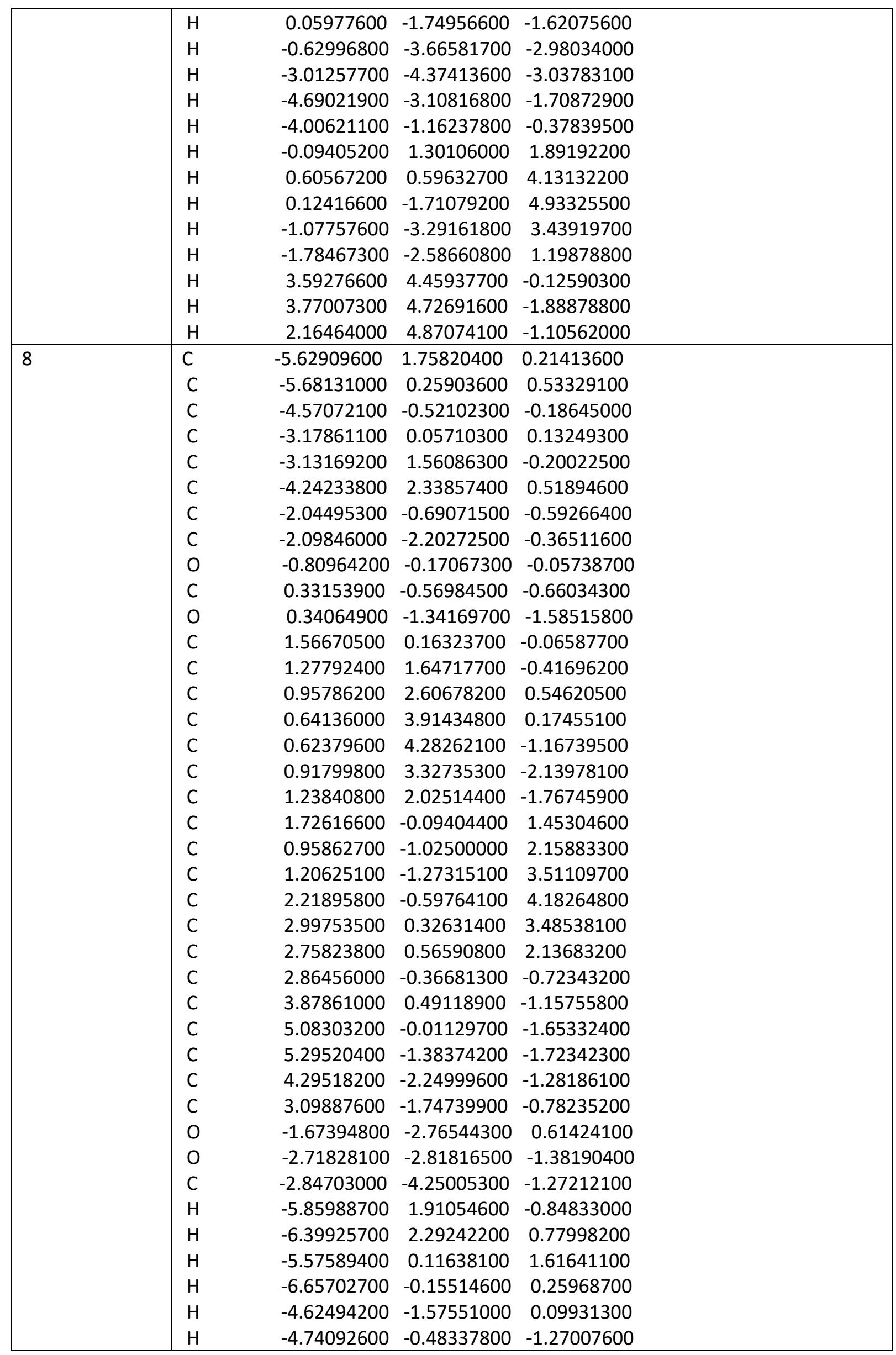




\begin{tabular}{|c|c|c|c|c|}
\hline & $\mathrm{H}$ & -2.99298400 & -0.06963400 & 1.20682800 \\
\hline & $\mathrm{H}$ & -2.15336400 & 1.97004200 & 0.05889500 \\
\hline & $\mathrm{H}$ & -3.24951500 & 1.68373500 & -1.28564800 \\
\hline & $\mathrm{H}$ & -4.06445800 & 2.30436300 & 1.60156400 \\
\hline & $\mathrm{H}$ & -4.19830700 & 3.39395100 & 0.23147200 \\
\hline & $\mathrm{H}$ & -2.07458900 & -0.50367800 & -1.66798600 \\
\hline & $\mathrm{H}$ & 0.94750100 & 2.33879900 & 1.59391300 \\
\hline & $\mathrm{H}$ & 0.40397600 & 4.64301900 & 0.94198500 \\
\hline & $\mathrm{H}$ & 0.37841000 & 5.29901300 & -1.45434000 \\
\hline & $\mathrm{H}$ & 0.89917200 & 3.59470700 & -3.19059100 \\
\hline & $\mathrm{H}$ & 1.46251300 & 1.29366200 & -2.53562000 \\
\hline & $\mathrm{H}$ & 0.15726000 & -1.57011600 & 1.67802300 \\
\hline & $\mathrm{H}$ & 0.59718400 & -2.00296000 & 4.03300900 \\
\hline & $\mathrm{H}$ & 2.40723300 & -0.79166700 & 5.23268200 \\
\hline & $\mathrm{H}$ & 3.80003800 & 0.85440900 & 3.98866600 \\
\hline & $\mathrm{H}$ & 3.39096100 & 1.26820800 & 1.60722200 \\
\hline & $\mathrm{H}$ & 3.73895200 & 1.56338000 & -1.11341600 \\
\hline & $\mathrm{H}$ & 5.85252900 & 0.67789400 & -1.98367400 \\
\hline & $\mathrm{H}$ & 6.22827800 & -1.77620900 & -2.11205300 \\
\hline & $\mathrm{H}$ & 4.44739100 & -3.32290200 & -1.32338400 \\
\hline & $\mathrm{H}$ & 2.34267300 & -2.43897200 & -0.43522300 \\
\hline & $\mathrm{H}$ & -3.41855900 & -4.51508300 & -0.38157400 \\
\hline & $\mathrm{H}$ & -3.36995500 & -4.56283700 & -2.17275800 \\
\hline & $\mathrm{H}$ & -1.86034000 & -4.71107700 & -1.22000500 \\
\hline \multirow[t]{28}{*}{10} & $C$ & 5.75599300 & -1.42697600 & 0.41707500 \\
\hline & $\mathrm{C}$ & 4.46654700 & -1.68552500 & 1.20806200 \\
\hline & $\mathrm{C}$ & 3.41081200 & -0.60235400 & 0.94332500 \\
\hline & $\mathrm{C}$ & 3.12134400 & -0.46730500 & -0.56198800 \\
\hline & $\mathrm{C}$ & 4.41397100 & -0.18721700 & -1.35044200 \\
\hline & $\mathrm{C}$ & 5.46840400 & -1.27187600 & -1.08282600 \\
\hline & $\mathrm{C}$ & 1.99912200 & 0.53945100 & -0.88731500 \\
\hline & $\mathrm{C}$ & 2.22836500 & 1.94507400 & -0.32296900 \\
\hline & 0 & 0.78770500 & -0.00391900 & -0.32534800 \\
\hline & $\mathrm{C}$ & -0.36935800 & 0.59468200 & -0.69210200 \\
\hline & 0 & -0.39522300 & 1.51656600 & -1.46550000 \\
\hline & $\mathrm{C}$ & -1.61701300 & -0.08569600 & -0.04168000 \\
\hline & $\mathrm{C}$ & -2.75496200 & 0.96805900 & 0.03795800 \\
\hline & $\mathrm{C}$ & -3.39212600 & 1.28299400 & 1.24125200 \\
\hline & $\mathrm{C}$ & -4.43560300 & 2.21086600 & 1.28366100 \\
\hline & $\mathrm{C}$ & -4.86406700 & 2.84074800 & 0.12129700 \\
\hline & $\mathrm{C}$ & -4.24496600 & 2.52677000 & -1.08871100 \\
\hline & $\mathrm{C}$ & -3.20911400 & 1.60014900 & -1.13055400 \\
\hline & $\mathrm{C}$ & -2.04232200 & -1.25326000 & -0.97915100 \\
\hline & $\mathrm{C}$ & -1.09698900 & -2.04783300 & -1.64004700 \\
\hline & $\mathrm{C}$ & -1.49033500 & -3.11818100 & -2.44161000 \\
\hline & $\mathrm{C}$ & -2.83934800 & -3.42964700 & -2.58938800 \\
\hline & $\mathrm{C}$ & -3.78917700 & -2.65904500 & -1.92382300 \\
\hline & $\mathrm{C}$ & -3.39486400 & -1.58248000 & -1.13129500 \\
\hline & $\mathrm{C}$ & -1.24889300 & -0.61705800 & 1.36352200 \\
\hline & $\mathrm{C}$ & -0.55214700 & 0.20991200 & 2.25707500 \\
\hline & $\mathrm{C}$ & -0.25108500 & -0.22074400 & 3.54462300 \\
\hline & $\mathrm{C}$ & -0.64228200 & -1.49023500 & 3.97212700 \\
\hline
\end{tabular}




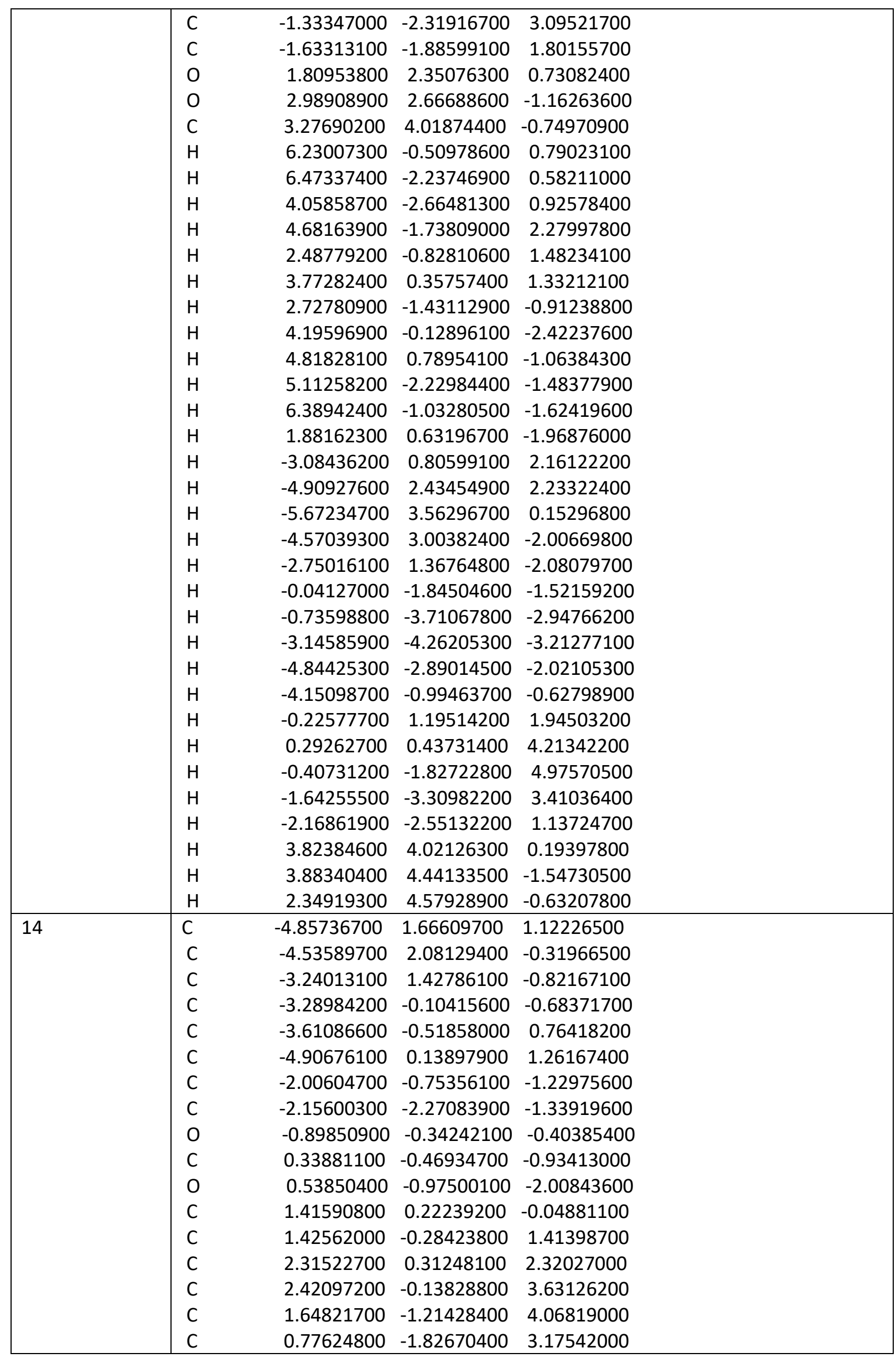




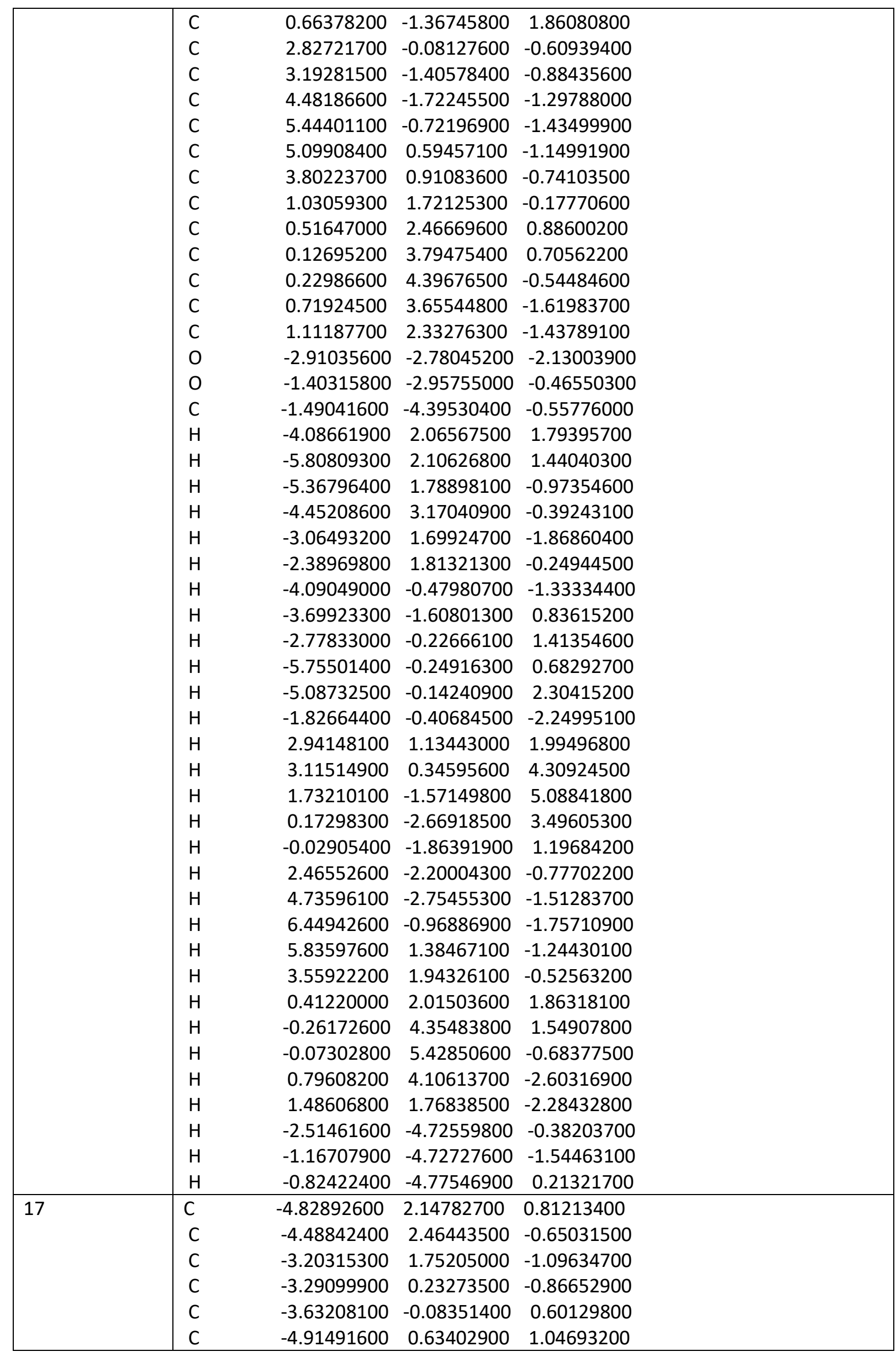




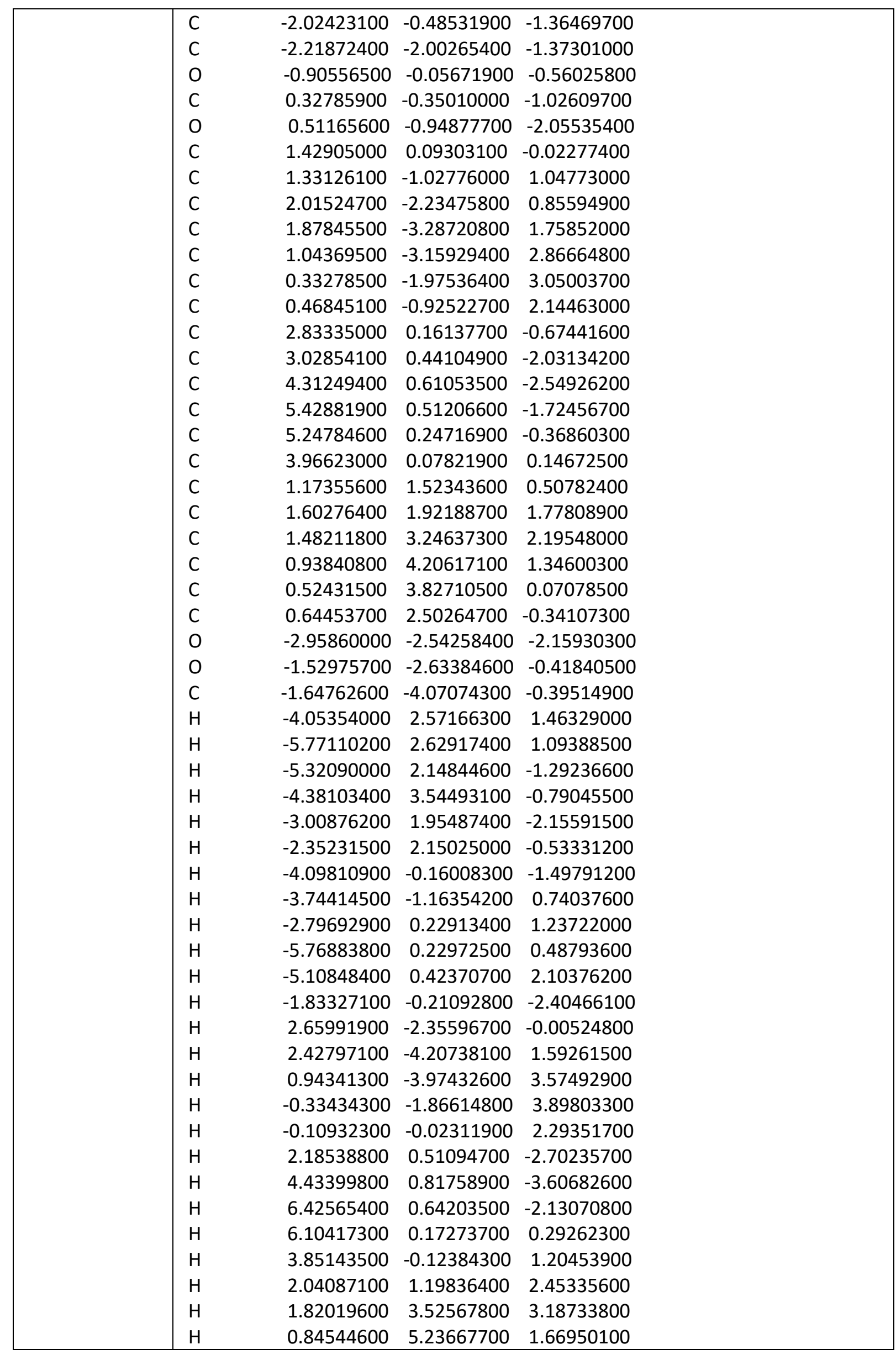




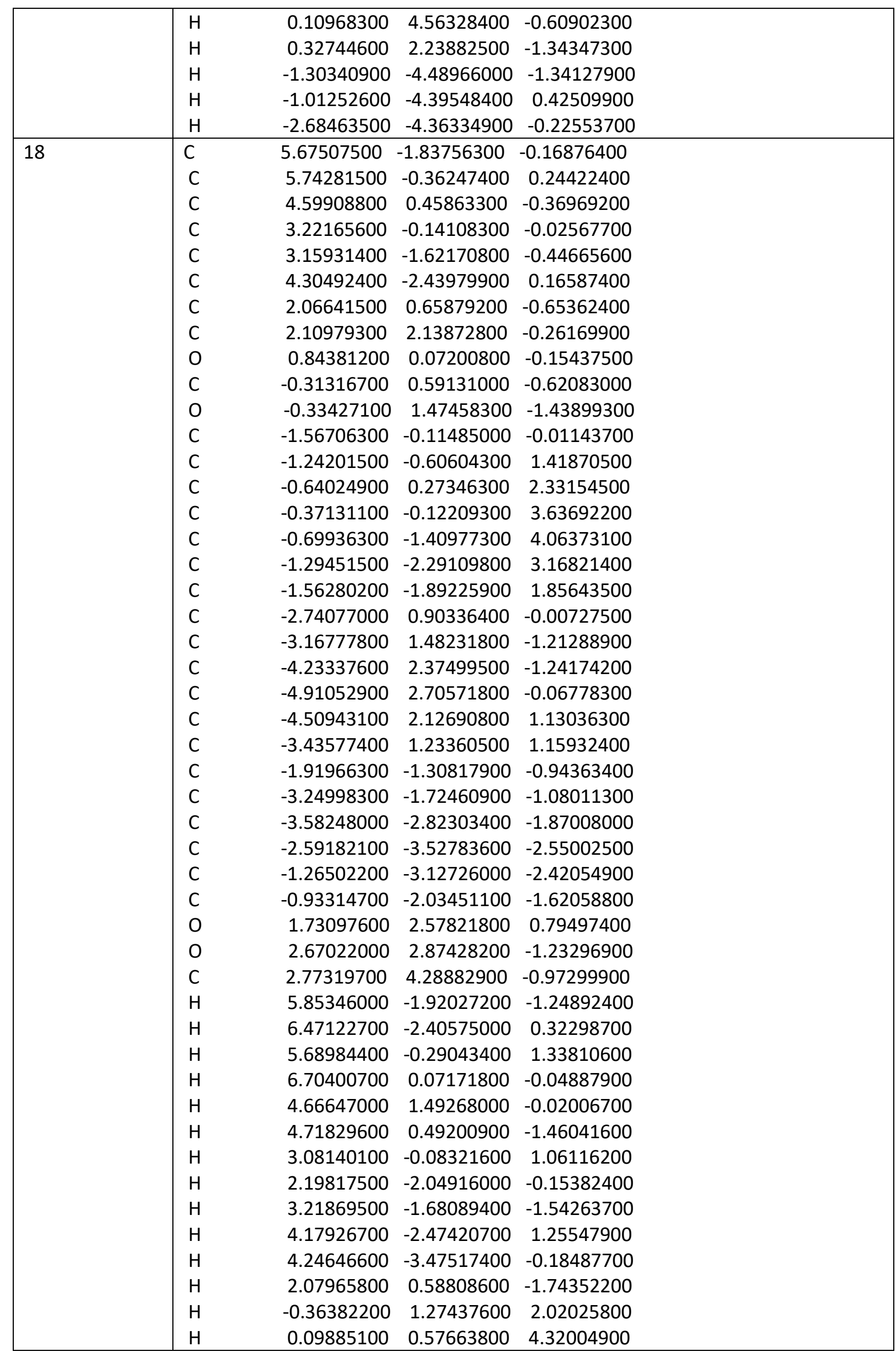




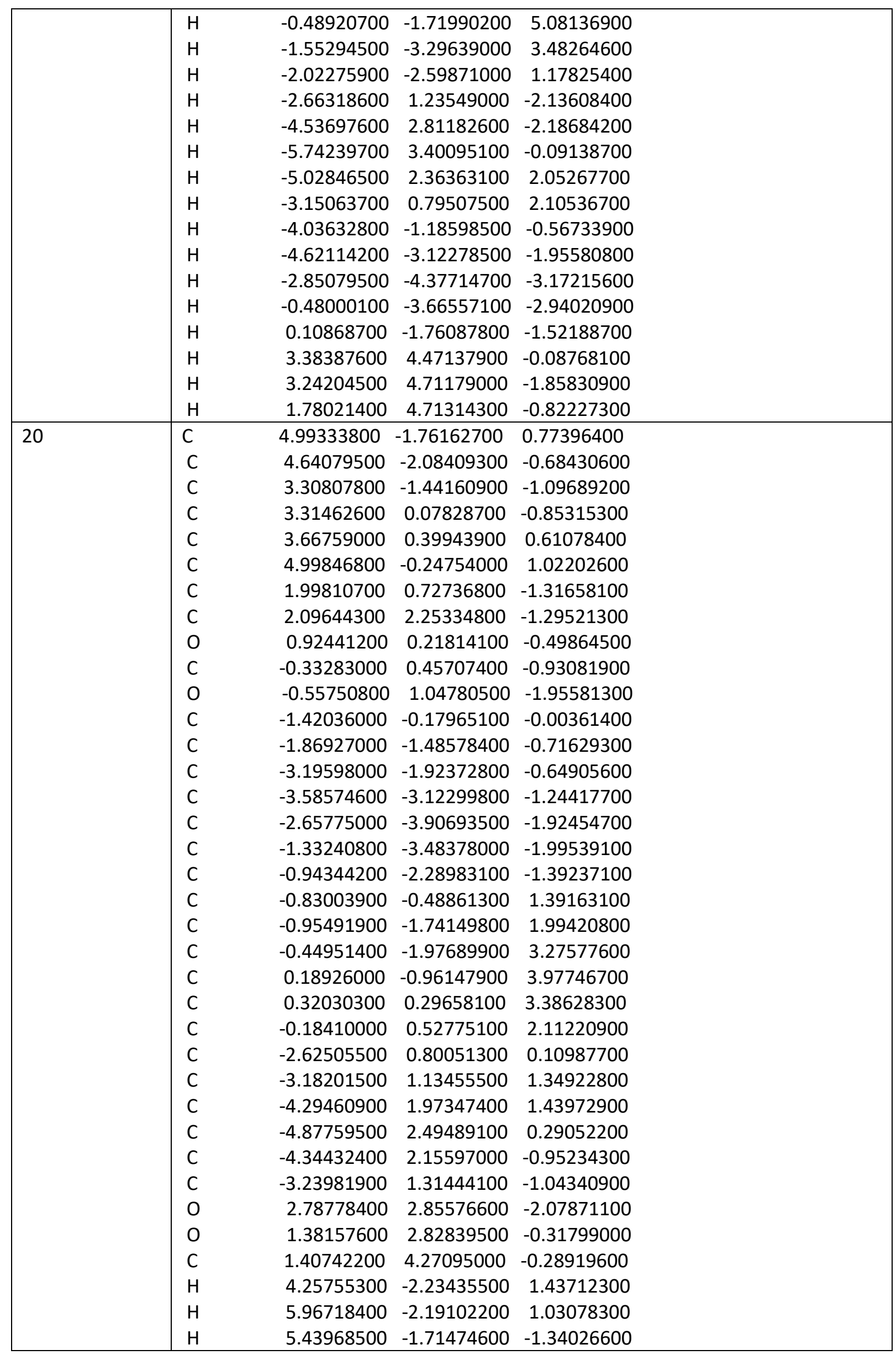




\begin{tabular}{|c|c|c|c|c|}
\hline & $\mathrm{H}$ & 4.59167100 & -3.16749200 & -0.83374900 \\
\hline & $\mathrm{H}$ & 3.10226800 & -1.64635500 & -2.15383800 \\
\hline & $\mathrm{H}$ & 2.49515500 & -1.89332400 & -0.51745500 \\
\hline & $\mathrm{H}$ & 4.08421400 & 0.52123100 & -1.49811600 \\
\hline & $\mathrm{H}$ & 3.72364000 & 1.48315500 & 0.75723600 \\
\hline & $\mathrm{H}$ & 2.86533600 & 0.03485700 & 1.26086700 \\
\hline & $\mathrm{H}$ & 5.81666100 & 0.20961800 & 0.45032900 \\
\hline & $\mathrm{H}$ & 5.20122900 & -0.03656200 & 2.07691000 \\
\hline & $\mathrm{H}$ & 1.80247700 & 0.46379200 & -2.35864700 \\
\hline & $\mathrm{H}$ & -3.93510000 & -1.32668400 & -0.13111000 \\
\hline & $\mathrm{H}$ & -4.62102100 & -3.43884400 & -1.17756600 \\
\hline & $\mathrm{H}$ & -2.96296900 & -4.83492300 & -2.39468900 \\
\hline & $\mathrm{H}$ & -0.59615800 & -4.08258900 & -2.52002400 \\
\hline & $\mathrm{H}$ & 0.09698400 & -1.99381200 & -1.44578500 \\
\hline & $\mathrm{H}$ & -1.44868100 & -2.54915500 & 1.47047100 \\
\hline & $\mathrm{H}$ & -0.56106500 & -2.96004800 & 3.71973400 \\
\hline & $\mathrm{H}$ & 0.58144900 & -1.14334200 & 4.97189900 \\
\hline & $\mathrm{H}$ & 0.81769400 & 1.09939300 & 3.91961100 \\
\hline & $\mathrm{H}$ & -0.06758000 & 1.50929100 & 1.66850500 \\
\hline & $\mathrm{H}$ & -2.75725500 & 0.73958700 & 2.26122700 \\
\hline & $\mathrm{H}$ & -4.70131400 & 2.21265500 & 2.41624400 \\
\hline & $\mathrm{H}$ & -5.73969900 & 3.14910000 & 0.35891000 \\
\hline & $\mathrm{H}$ & -4.79250700 & 2.54369700 & -1.86067200 \\
\hline & $\mathrm{H}$ & -2.85039000 & 1.05942300 & -2.01751800 \\
\hline & $\mathrm{H}$ & 0.77718300 & 4.55506100 & 0.55019400 \\
\hline & $\mathrm{H}$ & 2.42732800 & 4.62994400 & -0.14758600 \\
\hline & $\mathrm{H}$ & 1.00798600 & 4.67013500 & -1.22180400 \\
\hline 21 & $C$ & -5.83770200 & 0.86021500 & 0.82372700 \\
\hline & C & -4.61836800 & 0.61777000 & 1.72379200 \\
\hline & C & -3.54339700 & -0.21388800 & 1.00880800 \\
\hline & C & -3.13045700 & 0.43484500 & -0.32428200 \\
\hline & C & -4.35389600 & 0.66023200 & -1.23128100 \\
\hline & C & -5.42654200 & 1.49264400 & -0.51297100 \\
\hline & C & -1.99582200 & -0.31626800 & -1.05013200 \\
\hline & C & -2.30290500 & -1.79342900 & -1.29182900 \\
\hline & 0 & -0.79886200 & -0.10804600 & -0.27097200 \\
\hline & C & 0.38027700 & -0.32119400 & -0.89550100 \\
\hline & 0 & 0.45646800 & -0.77509900 & -2.00824900 \\
\hline & C & 1.57259000 & 0.20970700 & -0.04879700 \\
\hline & C & 2.91185300 & -0.17362500 & -0.72348400 \\
\hline & C & 3.96629100 & 0.73567900 & -0.84464900 \\
\hline & C & 5.20108800 & 0.33995800 & -1.36156900 \\
\hline & C & 5.40419900 & -0.97471600 & -1.76674200 \\
\hline & C & 4.36278700 & -1.89384300 & -1.64106700 \\
\hline & $\mathrm{C}$ & 3.13551500 & -1.49865500 & -1.12038400 \\
\hline & C & 1.31223900 & 1.74040000 & -0.05339400 \\
\hline & C & 1.38804600 & 2.43544700 & -1.26959800 \\
\hline & C & 1.09196400 & 3.79370600 & -1.33903400 \\
\hline & C & 0.70658500 & 4.48727300 & -0.19242300 \\
\hline & C & 0.60973000 & 3.80365200 & 1.01602500 \\
\hline & C & 0.90247700 & 2.44094400 & 1.08360200 \\
\hline & $\mathrm{C}$ & 1.62489500 & -0.40017200 & 1.37258900 \\
\hline
\end{tabular}




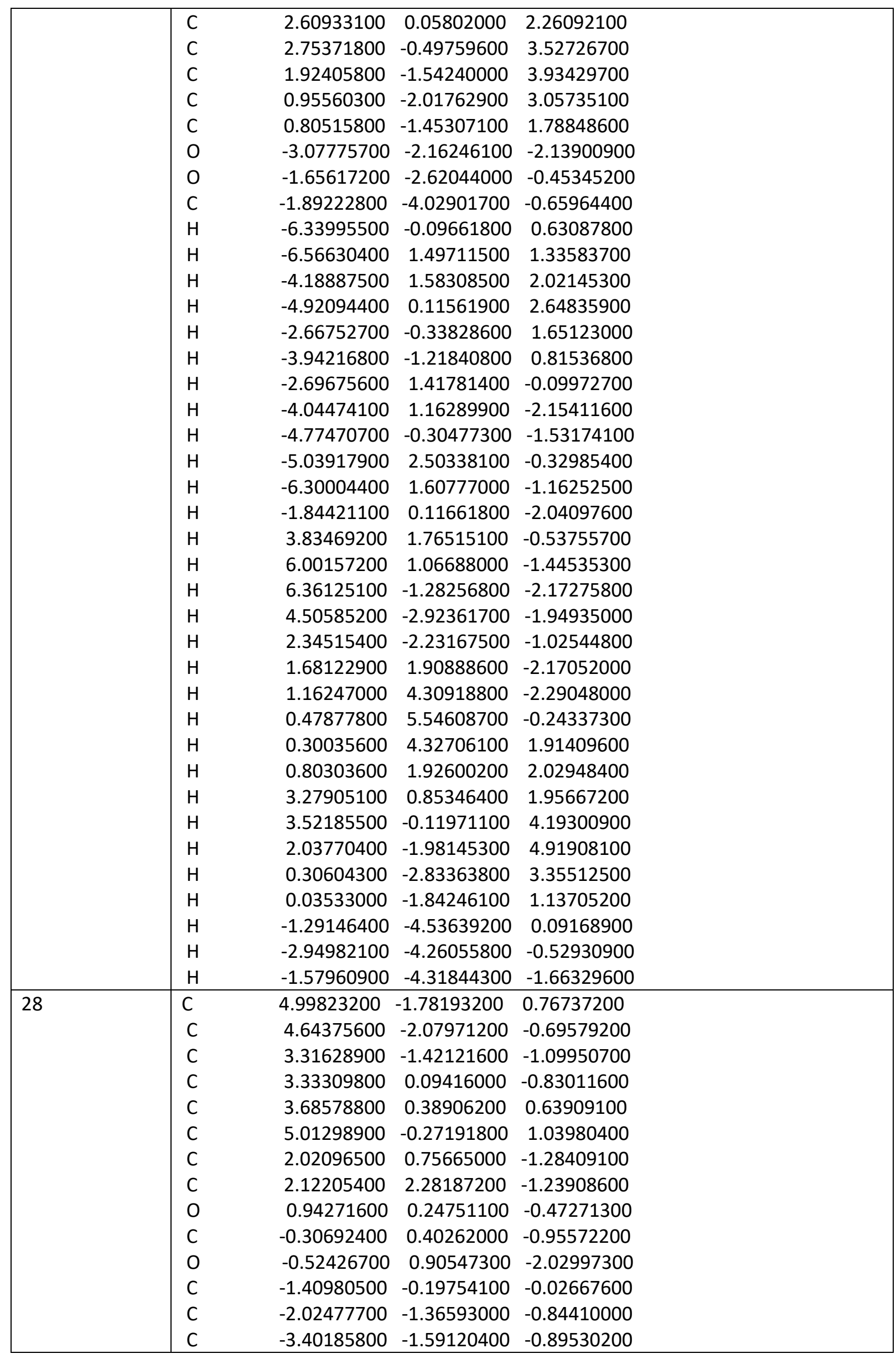




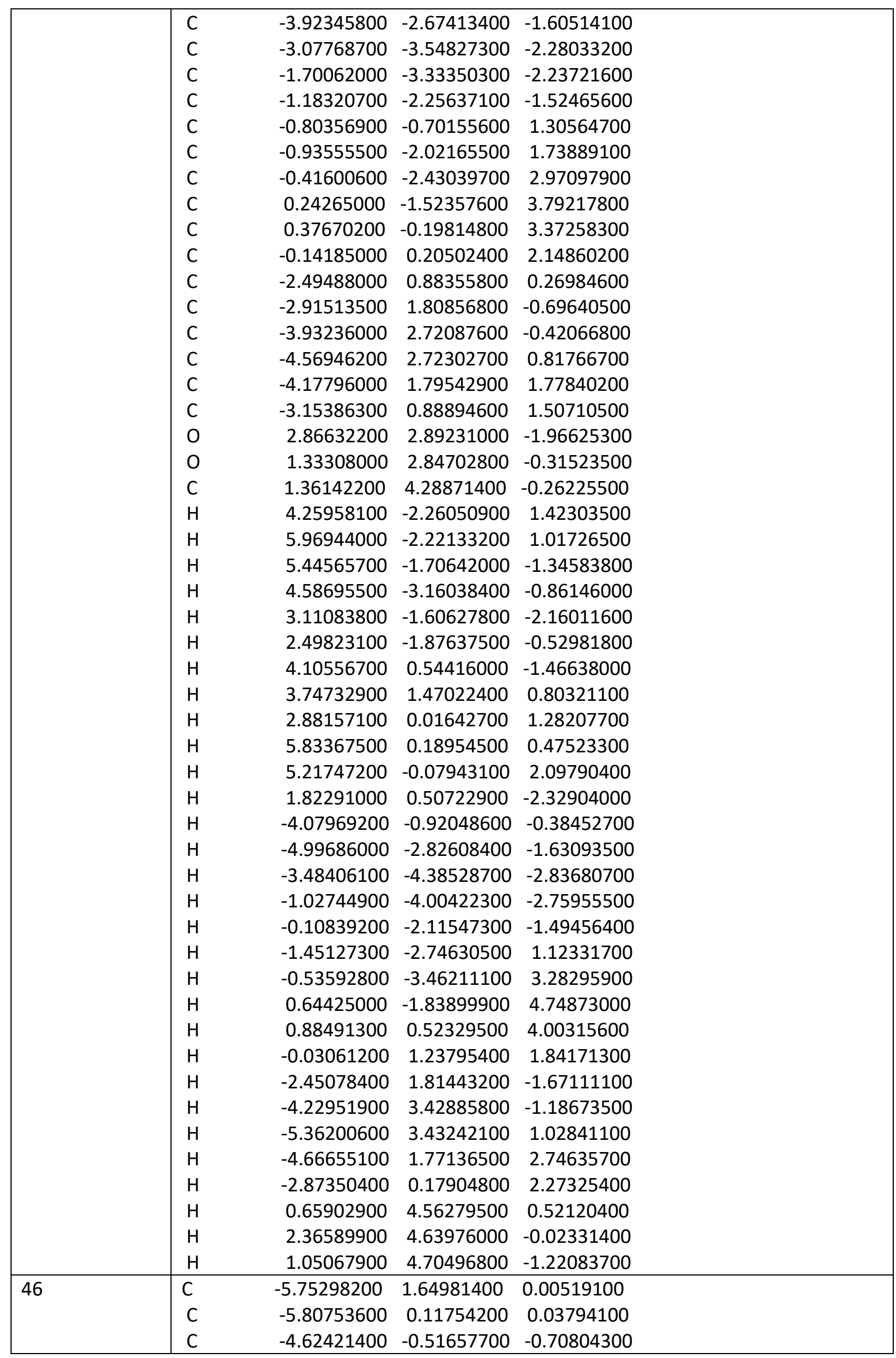




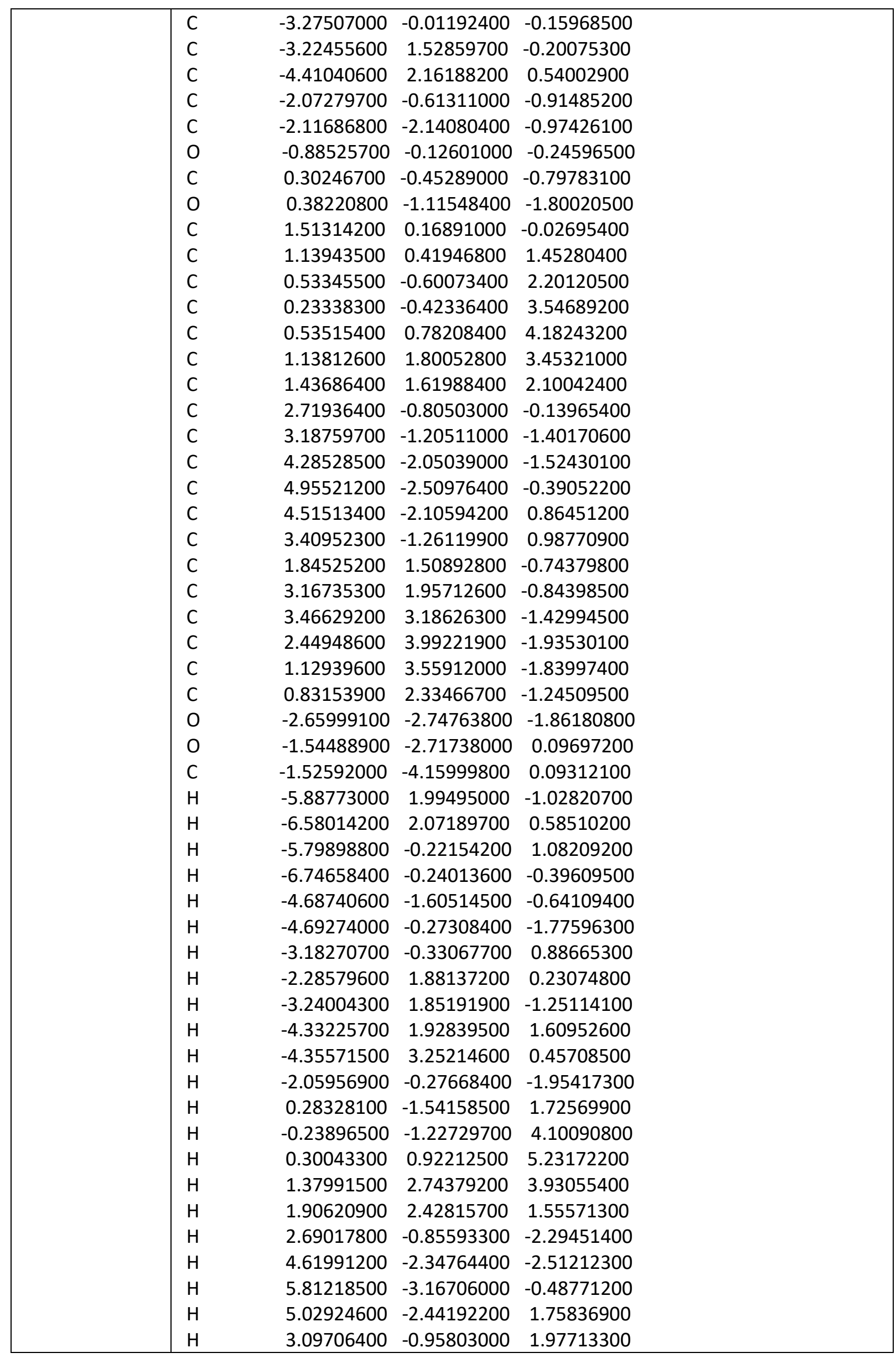




\begin{tabular}{|l|lrrr|}
\hline & $H$ & 3.97393000 & 1.34485800 & -0.46261200 \\
& $H$ & 4.49969700 & 3.50892800 & -1.49276700 \\
$H$ & 2.68247900 & 4.94466800 & -2.39781900 \\
$H$ & 0.32462000 & 4.17446800 & -2.22679900 \\
$H$ & -0.20483200 & 2.03392900 & -1.16421200 \\
H & -1.01889600 & -4.44589600 & 1.01185200 \\
& $H$ & -2.54259000 & -4.55393100 & 0.07451600 \\
& $H$ & -0.97942200 & -4.52411600 & -0.77725900 \\
\hline
\end{tabular}

6 (optimized at the M06-2X/6-311++G(d,p) level)

\begin{tabular}{|c|c|c|c|c|}
\hline Conformer no & & & & \\
\hline \multirow[t]{40}{*}{1} & $C$ & -4.22105000 & 2.26854900 & 1.02424900 \\
\hline & C & -3.96308000 & 2.42340200 & -0.47511500 \\
\hline & C & -2.83427500 & 1.49833800 & -0.92996400 \\
\hline & $\mathrm{C}$ & -3.12885300 & 0.03582600 & -0.57577800 \\
\hline & C & -3.40876400 & -0.11912800 & 0.92350000 \\
\hline & C & -4.53556800 & 0.81270500 & 1.37396000 \\
\hline & C & -1.96576000 & -0.85120000 & -1.02753800 \\
\hline & C & -2.21257200 & -2.32599900 & -0.75272000 \\
\hline & $\mathrm{O}$ & -0.81471700 & -0.43004700 & -0.31239800 \\
\hline & C & 0.38659400 & -0.66205600 & -0.86825600 \\
\hline & $\mathrm{O}$ & 0.53165200 & -1.29123200 & -1.87641200 \\
\hline & C & 1.47967000 & 0.09009200 & -0.07772700 \\
\hline & C & 1.59928900 & -0.44371900 & 1.35814500 \\
\hline & C & 2.53237000 & 0.16027600 & 2.20763300 \\
\hline & C & 2.71725600 & -0.29929500 & 3.50310400 \\
\hline & C & 1.98353300 & -1.38876800 & 3.96890300 \\
\hline & C & 1.07178700 & -2.00612400 & 3.12544100 \\
\hline & C & 0.87540700 & -1.53765300 & 1.82603800 \\
\hline & $\mathrm{C}$ & 2.84855300 & -0.11353300 & -0.74212700 \\
\hline & C & 3.27916400 & -1.41375900 & -1.02115600 \\
\hline & C & 4.54356400 & -1.64589000 & -1.54215800 \\
\hline & C & 5.41052900 & -0.58254100 & -1.78175600 \\
\hline & $\mathrm{C}$ & 5.00063300 & 0.70999900 & -1.48720600 \\
\hline & C & 3.72742700 & 0.94317400 & -0.97022100 \\
\hline & C & 0.99208400 & 1.55155700 & -0.14026800 \\
\hline & C & 0.51711900 & 2.24283400 & 0.97104000 \\
\hline & $\mathrm{C}$ & -0.00272800 & 3.52984700 & 0.83454900 \\
\hline & C & -0.06728700 & 4.13530300 & -0.41362200 \\
\hline & C & 0.38893900 & 3.44339300 & -1.53300700 \\
\hline & C & 0.91068000 & 2.16387300 & -1.39560000 \\
\hline & $\mathrm{O}$ & -1.57546400 & -3.00932000 & -0.00026500 \\
\hline & $\mathrm{O}$ & -3.25963900 & -2.76308900 & -1.45805200 \\
\hline & C & -3.58744000 & -4.14442100 & -1.27756900 \\
\hline & $\mathrm{H}$ & -3.32592100 & 2.58484300 & 1.57424400 \\
\hline & $\mathrm{H}$ & -5.04000600 & 2.92027400 & 1.34053500 \\
\hline & $\mathrm{H}$ & -4.88075900 & 2.18442100 & -1.02686300 \\
\hline & $\mathrm{H}$ & -3.70799900 & 3.45976200 & -0.71348600 \\
\hline & $\mathrm{H}$ & -2.67007500 & 1.59416200 & -2.00859800 \\
\hline & $\mathrm{H}$ & -1.90468800 & 1.79997100 & -0.43477500 \\
\hline & $\mathrm{H}$ & -4.01058400 & -0.29640100 & -1.13746100 \\
\hline
\end{tabular}




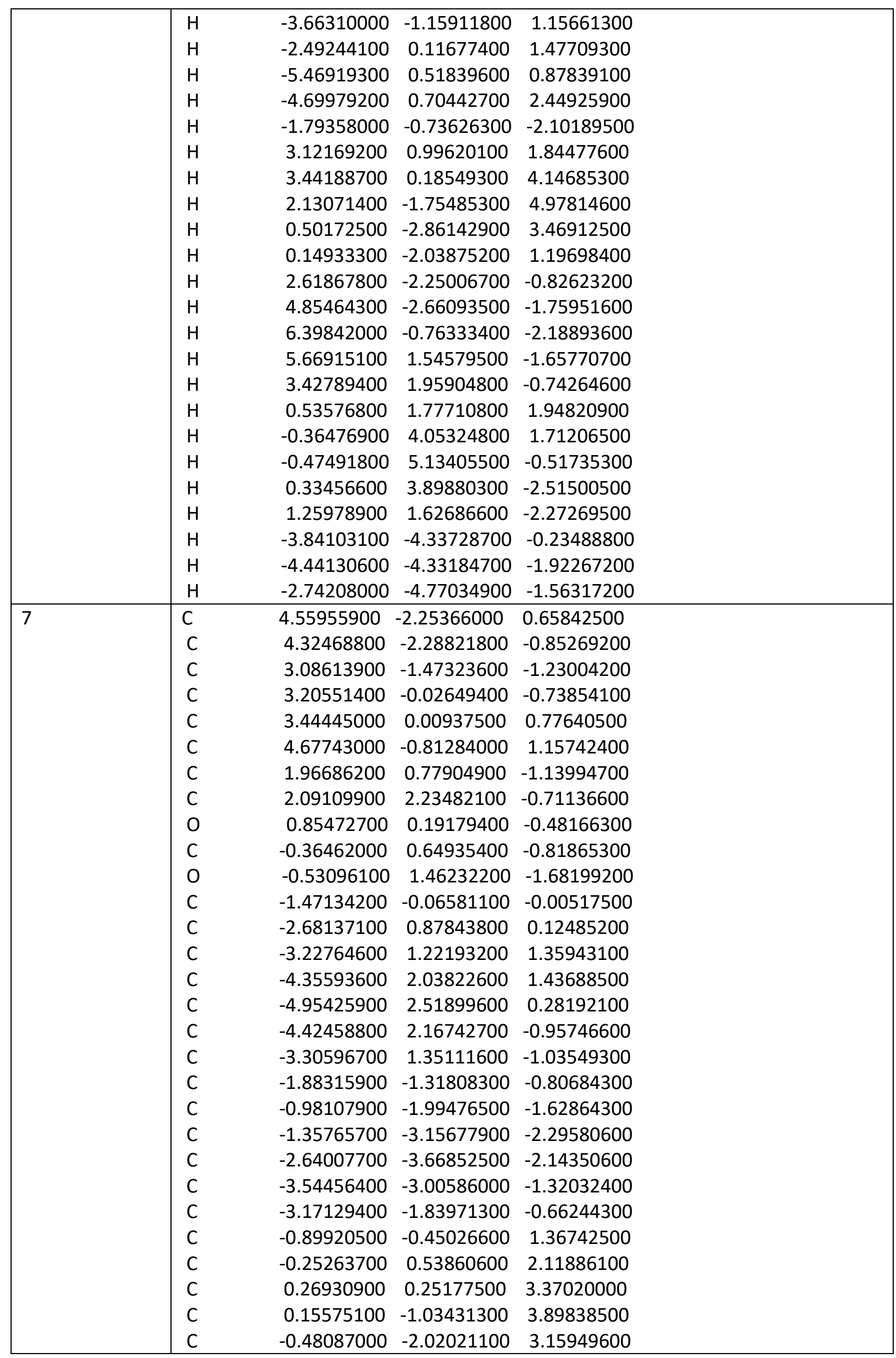




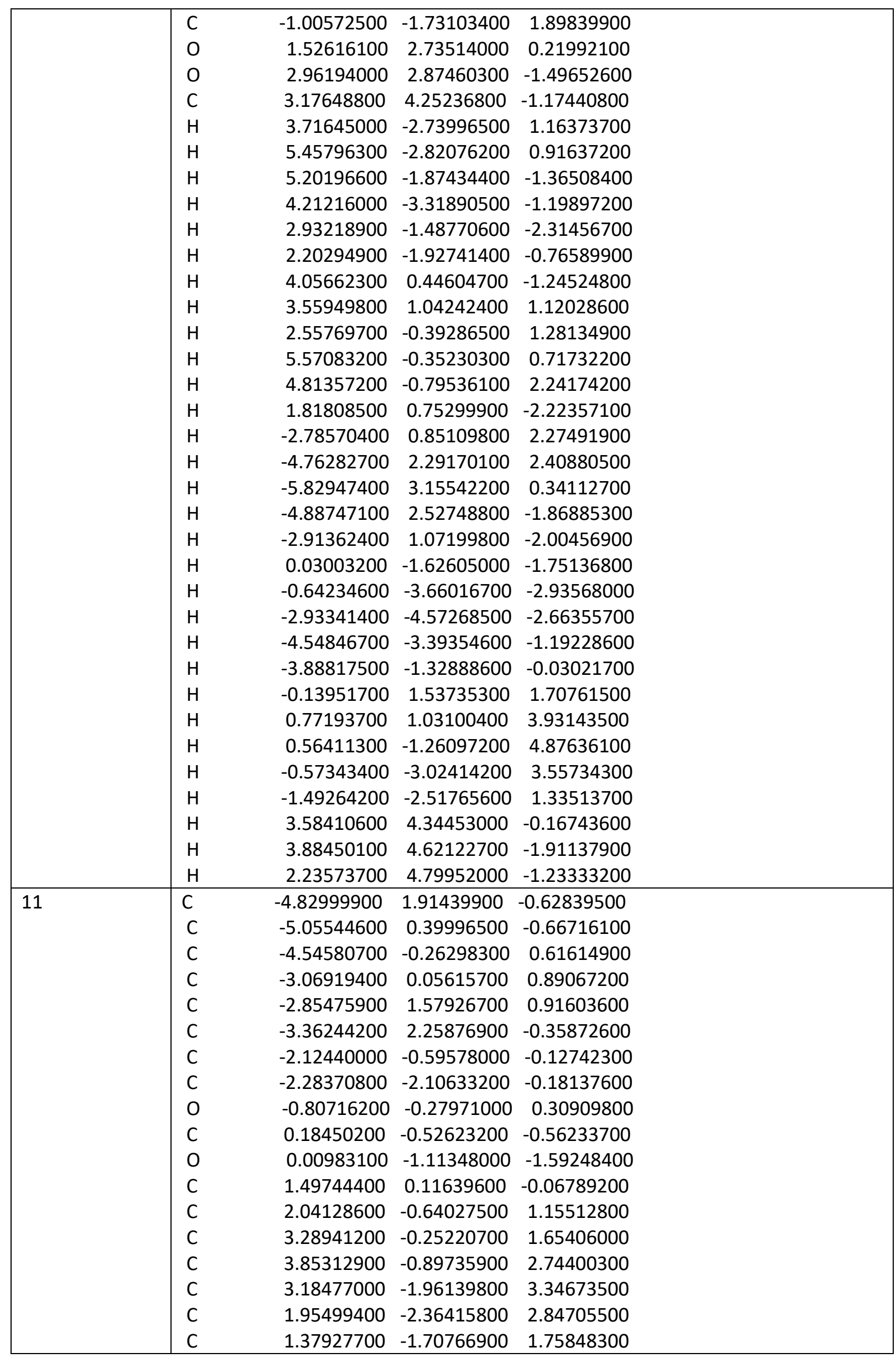




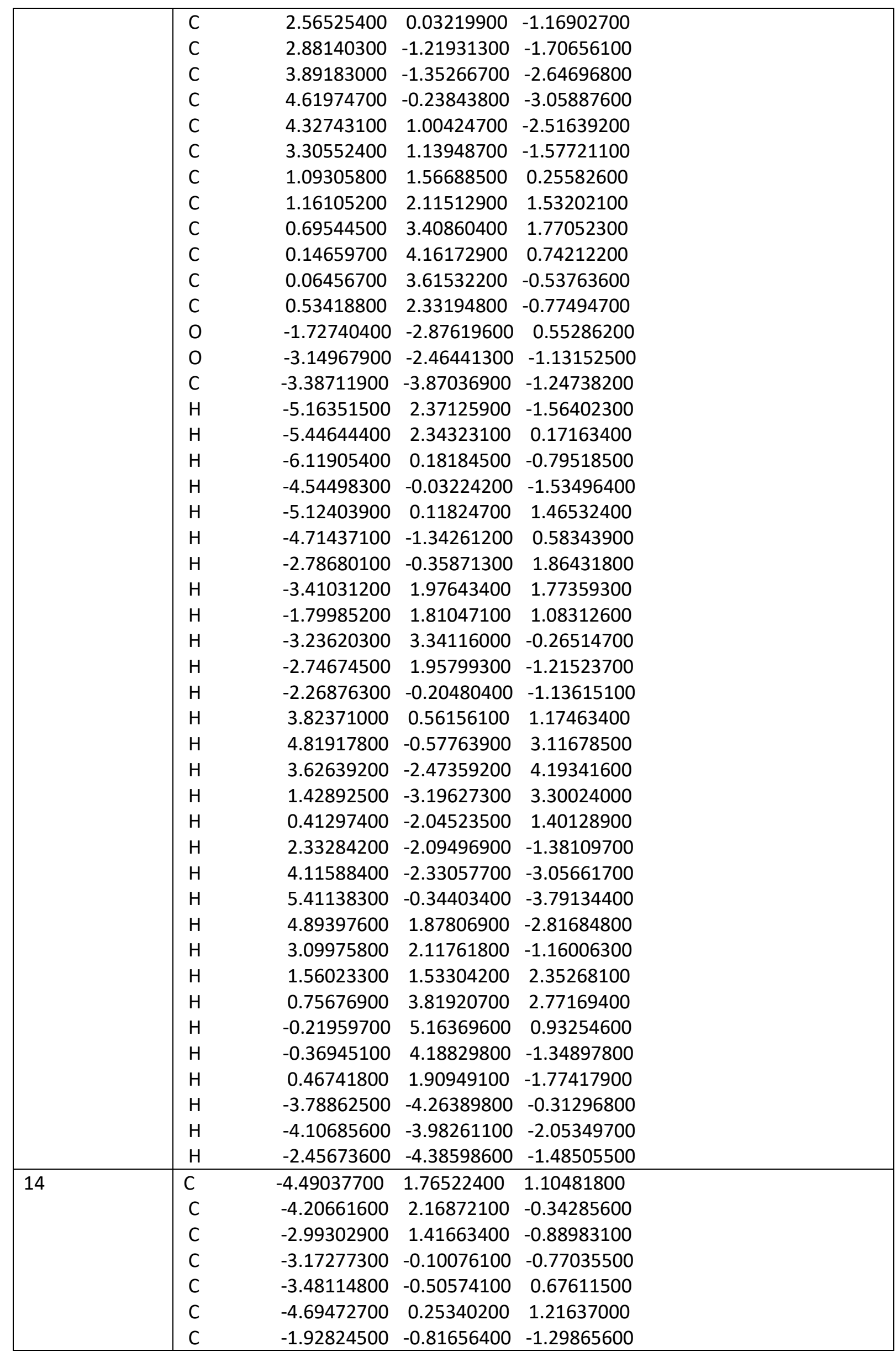




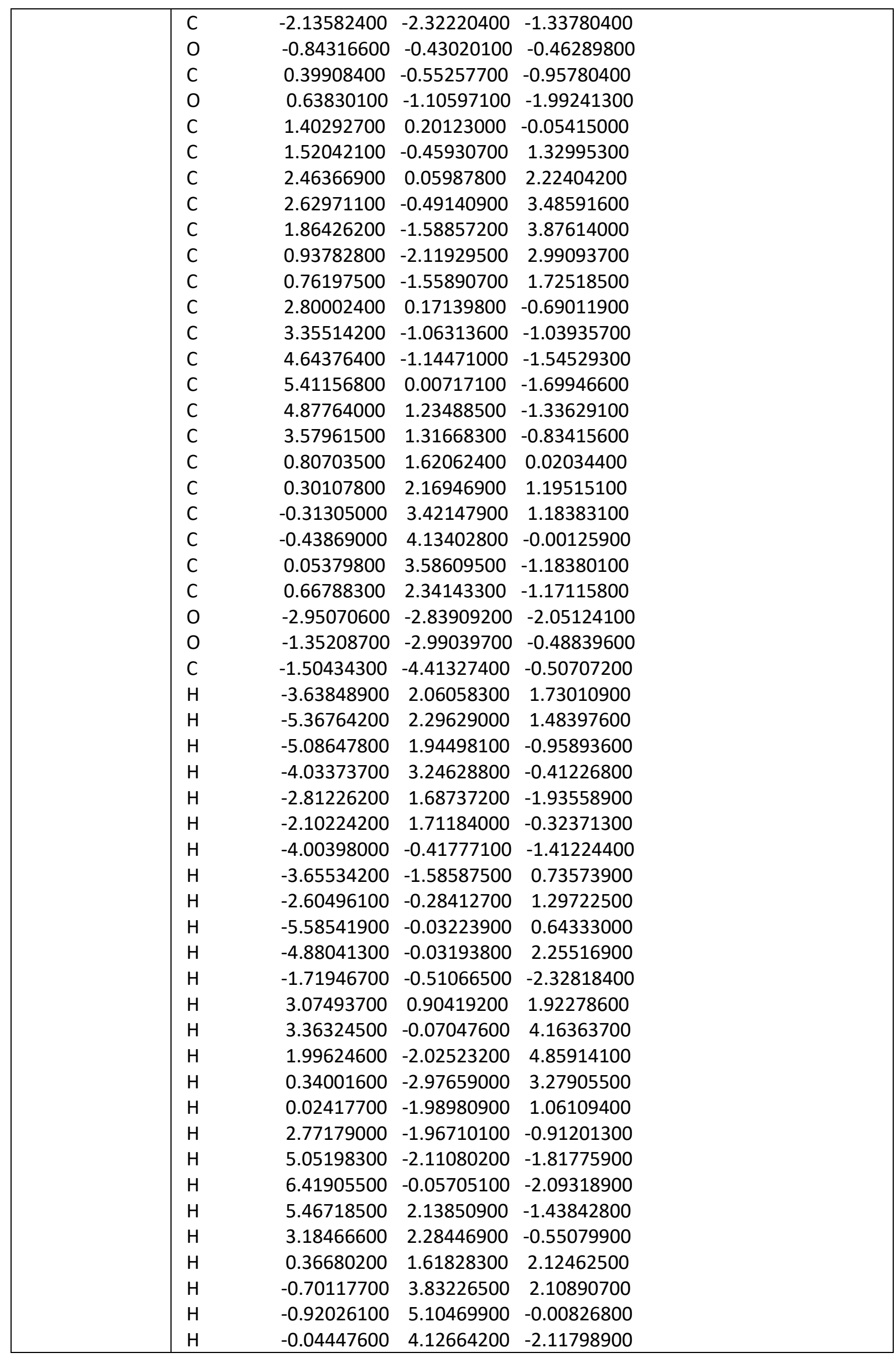




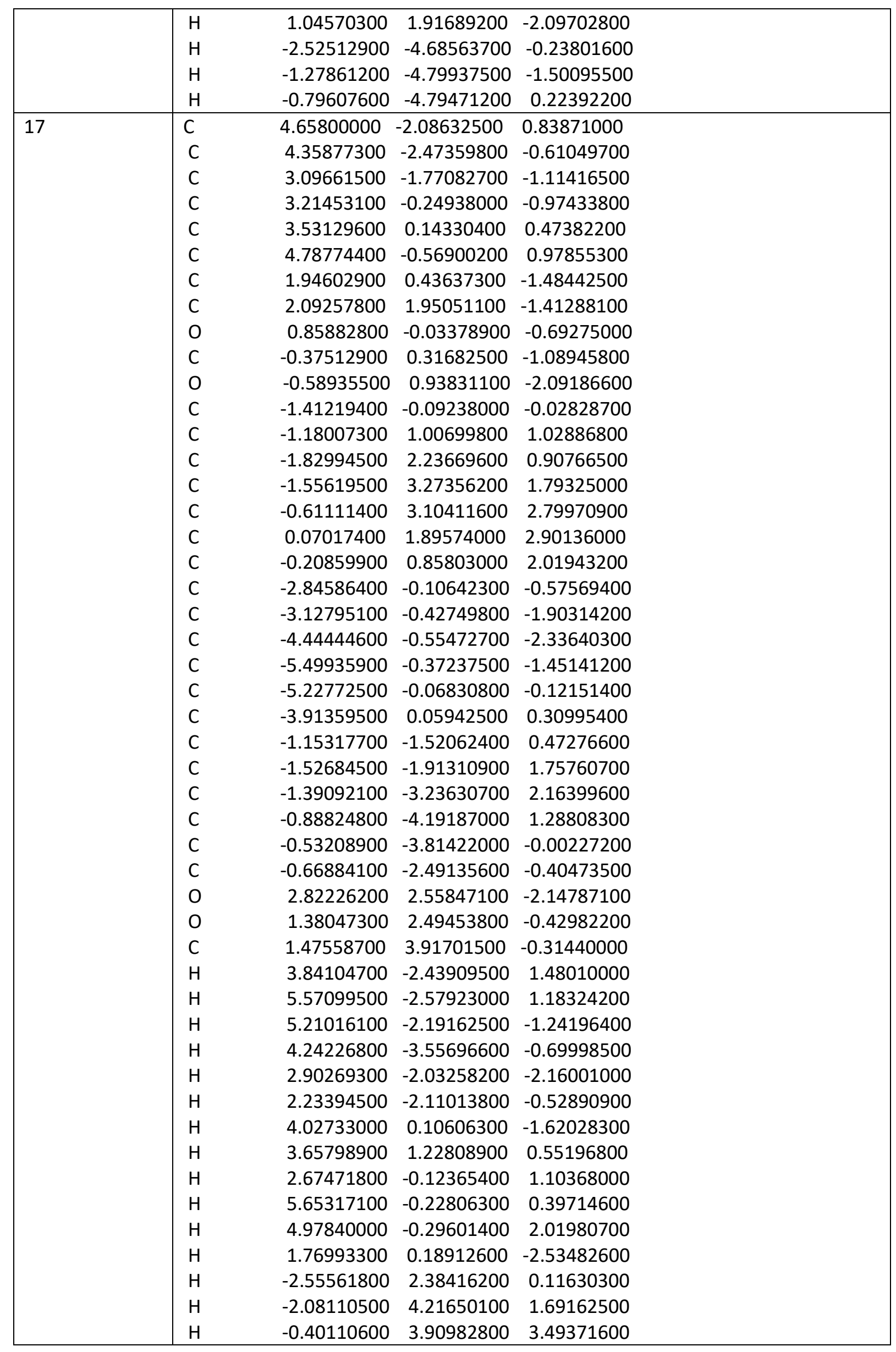




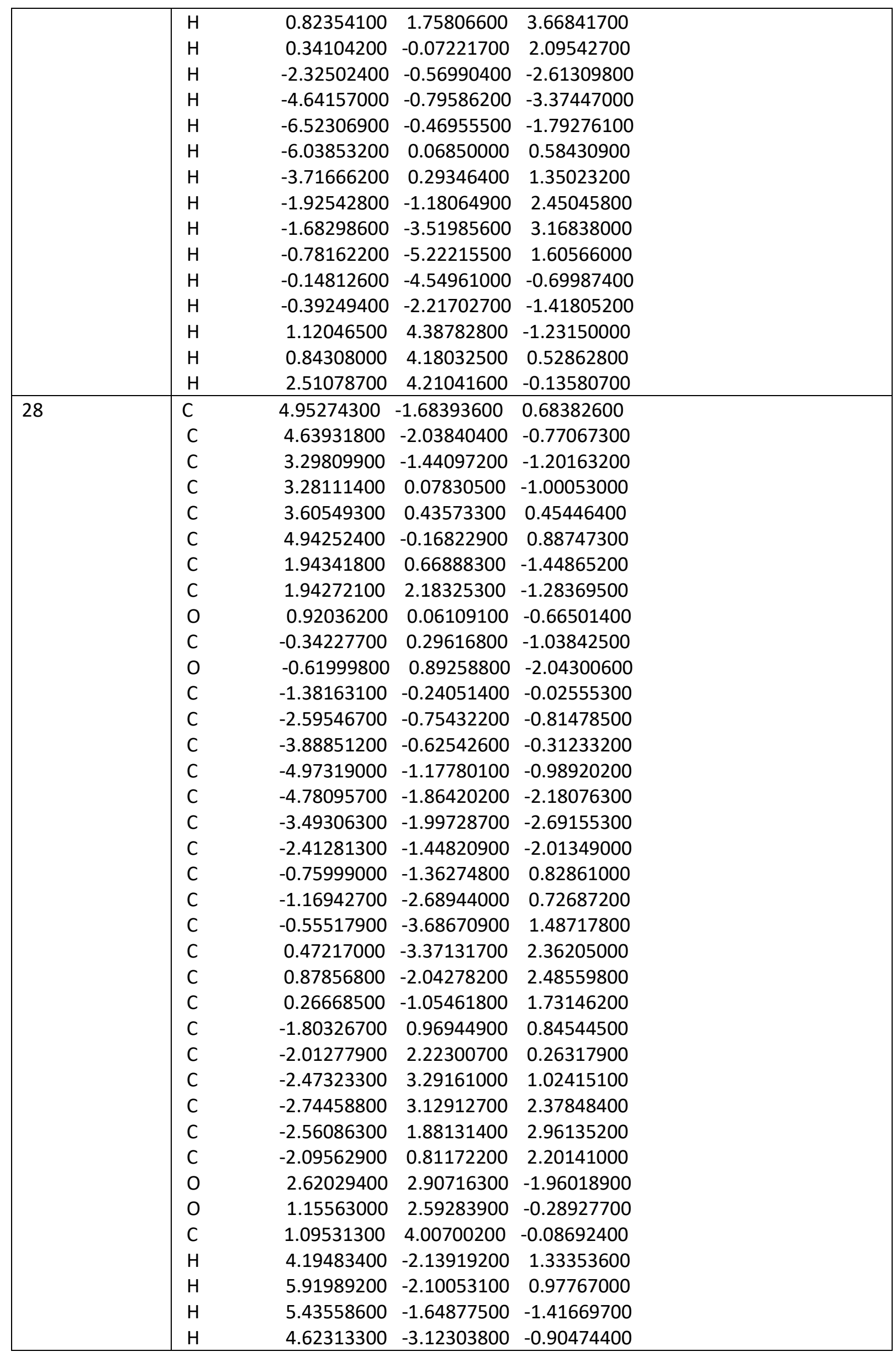




\begin{tabular}{|c|c|c|c|c|}
\hline & $\mathrm{H}$ & 3.09116000 & -1.67914500 & -2.25020500 \\
\hline & $\mathrm{H}$ & 2.49396900 & -1.88412300 & -0.60160700 \\
\hline & $\mathrm{H}$ & 4.03931900 & 0.53293900 & -1.65085700 \\
\hline & $\mathrm{H}$ & 3.62503900 & 1.52296700 & 0.58354100 \\
\hline & $\mathrm{H}$ & 2.80545900 & 0.04596800 & 1.09385500 \\
\hline & $\mathrm{H}$ & 5.74983200 & 0.28105200 & 0.29623900 \\
\hline & $\mathrm{H}$ & 5.14145100 & 0.07833700 & 1.93384100 \\
\hline & $\mathrm{H}$ & 1.76580000 & 0.46574000 & -2.50777400 \\
\hline & $\mathrm{H}$ & -4.05961200 & -0.08961700 & 0.61321600 \\
\hline & $\mathrm{H}$ & -5.97060000 & -1.06358000 & -0.58096200 \\
\hline & $\mathrm{H}$ & -5.62540200 & -2.28915700 & -2.71028300 \\
\hline & $\mathrm{H}$ & -3.32807000 & -2.52578900 & -3.62302600 \\
\hline & $\mathrm{H}$ & -1.41687800 & -1.56448900 & -2.42605700 \\
\hline & $\mathrm{H}$ & -1.97403200 & -2.96317800 & 0.05676900 \\
\hline & $\mathrm{H}$ & -0.89177200 & -4.71200300 & 1.38817000 \\
\hline & $\mathrm{H}$ & 0.94917600 & -4.14664300 & 2.95004300 \\
\hline & $\mathrm{H}$ & 1.67431000 & -1.77732800 & 3.17266800 \\
\hline & $\mathrm{H}$ & 0.58683600 & -0.02440000 & 1.83560400 \\
\hline & $\mathrm{H}$ & -1.82722100 & 2.36554100 & -0.79399200 \\
\hline & $\mathrm{H}$ & -2.62775500 & 4.25457000 & 0.55016700 \\
\hline & $\mathrm{H}$ & -3.10335600 & 3.96291900 & 2.97040500 \\
\hline & $\mathrm{H}$ & -2.78192700 & 1.73147000 & 4.01171600 \\
\hline & $\mathrm{H}$ & -1.97012700 & -0.15507000 & 2.67305800 \\
\hline & $\mathrm{H}$ & 0.37858300 & 4.15432600 & 0.71695200 \\
\hline & $\mathrm{H}$ & 2.07911600 & 4.38856200 & 0.18886000 \\
\hline & $\mathrm{H}$ & 0.76111900 & 4.50027600 & -1.00029300 \\
\hline \multirow[t]{26}{*}{30} & $C$ & -5.46389600 & 1.80433400 & 0.06818700 \\
\hline & $\mathrm{C}$ & -5.60549800 & 0.31350400 & 0.37742800 \\
\hline & C & -4.49271000 & -0.50469500 & -0.28172000 \\
\hline & C & -3.11009200 & 0.01021600 & 0.13228300 \\
\hline & C & -2.97041500 & 1.50753300 & -0.17331400 \\
\hline & C & -4.08574200 & 2.31645500 & 0.48937600 \\
\hline & C & -1.98297100 & -0.75960600 & -0.55597100 \\
\hline & C & -2.06360200 & -2.25869000 & -0.31049100 \\
\hline & 0 & -0.76919700 & -0.26194400 & -0.00356500 \\
\hline & C & 0.36295600 & -0.63288800 & -0.62338700 \\
\hline & 0 & 0.37833900 & -1.43337500 & -1.51538400 \\
\hline & C & 1.56136600 & 0.16192100 & -0.06390300 \\
\hline & C & 2.80923400 & -0.10444700 & -0.92007200 \\
\hline & C & 3.60519100 & 0.92306700 & -1.42079900 \\
\hline & C & 4.77866200 & 0.63770200 & -2.11799400 \\
\hline & C & 5.16900300 & -0.67742400 & -2.32333500 \\
\hline & C & 4.38565800 & -1.71046900 & -1.81393900 \\
\hline & C & 3.22340900 & -1.42613800 & -1.11300100 \\
\hline & C & 1.09242600 & 1.62724400 & -0.13117200 \\
\hline & C & 0.74165600 & 2.14581400 & -1.38291200 \\
\hline & C & 0.22845100 & 3.42915500 & -1.50591900 \\
\hline & C & 0.05304300 & 4.22116600 & -0.37307000 \\
\hline & C & 0.39046900 & 3.71175800 & 0.87306500 \\
\hline & C & 0.90253500 & 2.41977200 & 0.99582300 \\
\hline & C & 1.88870700 & -0.29908400 & 1.36601800 \\
\hline & $\mathrm{C}$ & 3.00746500 & 0.26321700 & 1.99036300 \\
\hline
\end{tabular}




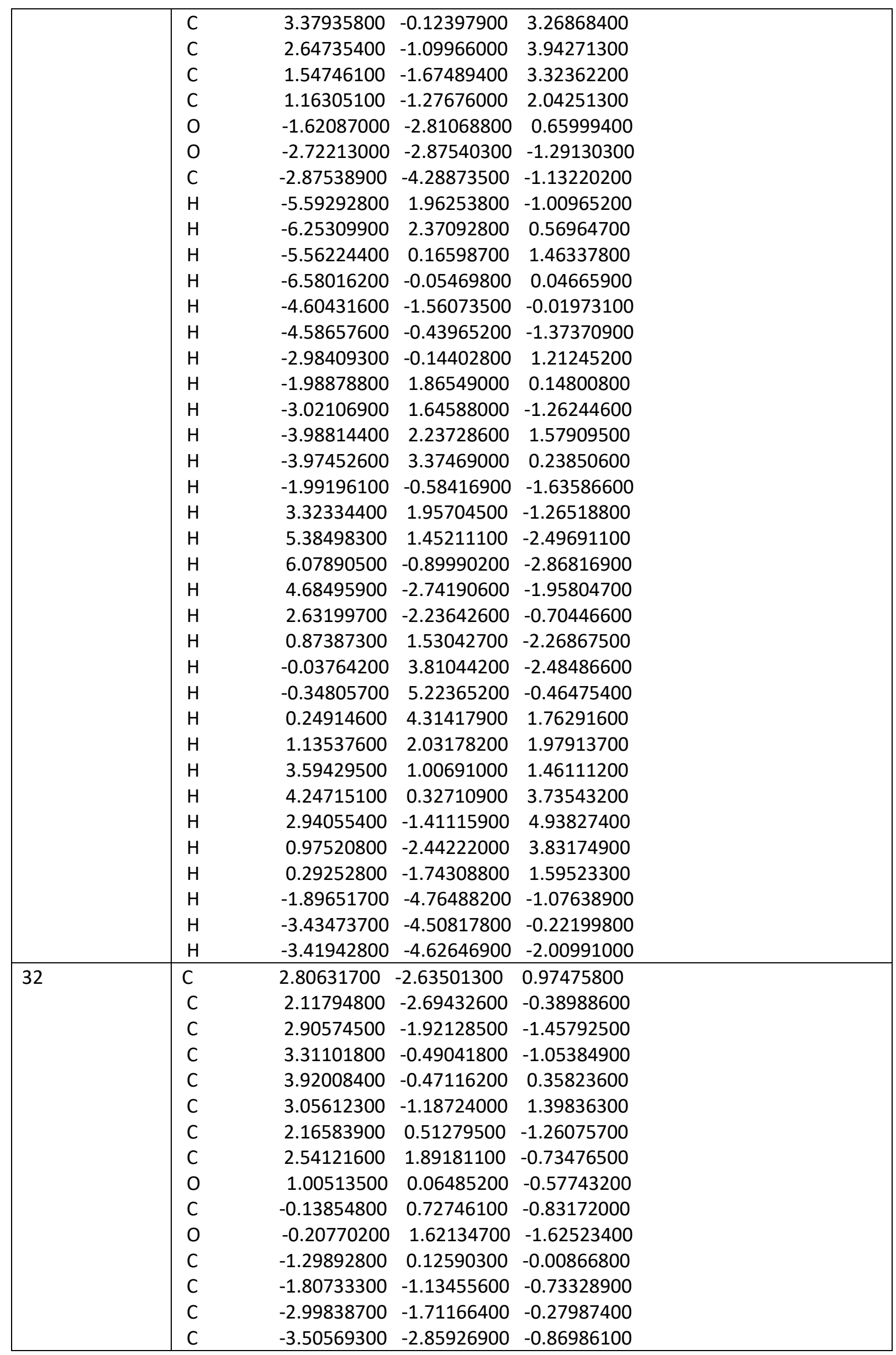




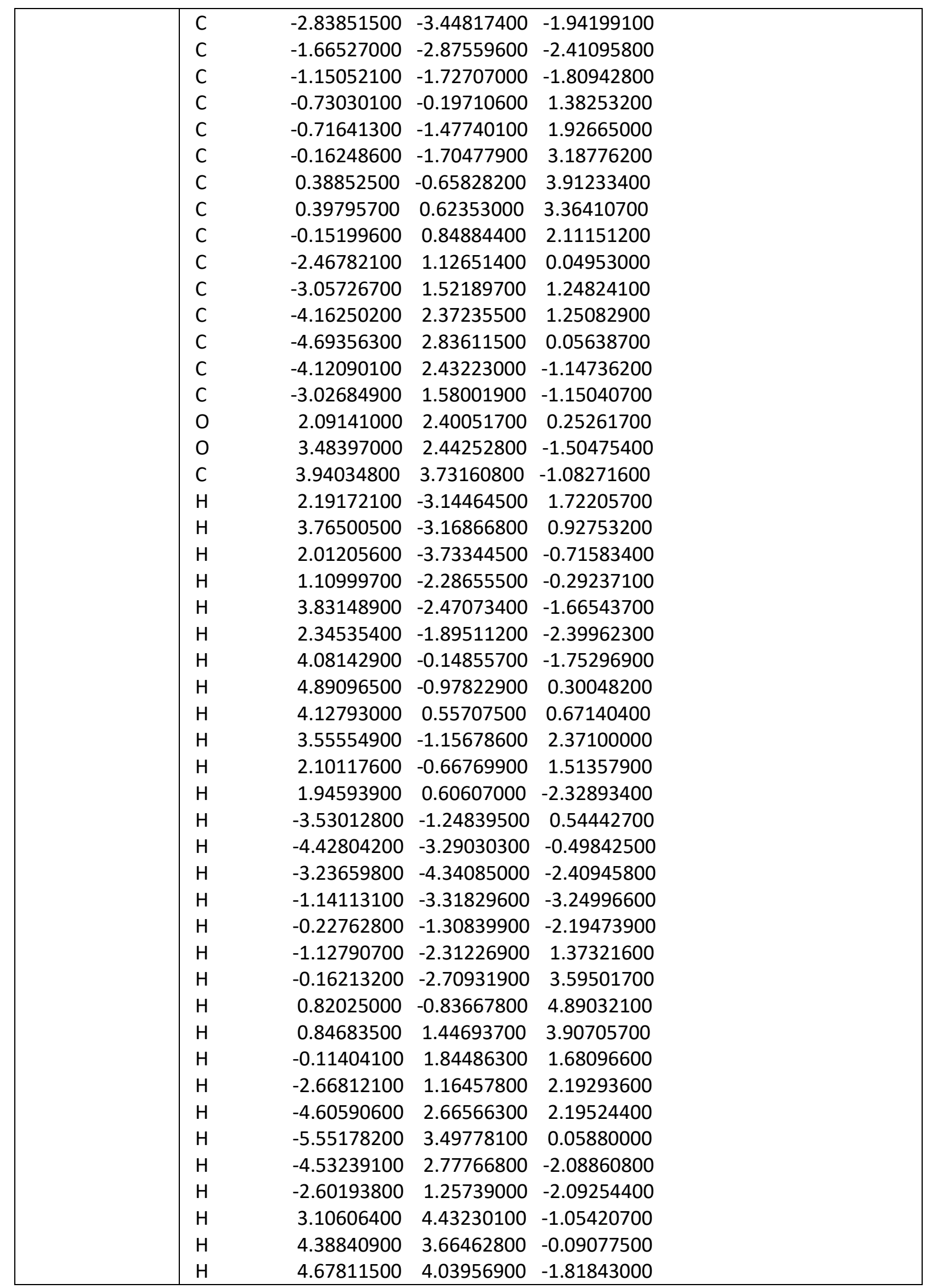

10 (optimized at the B3LYP/6-311++G(d,p) level)

Conformer no 


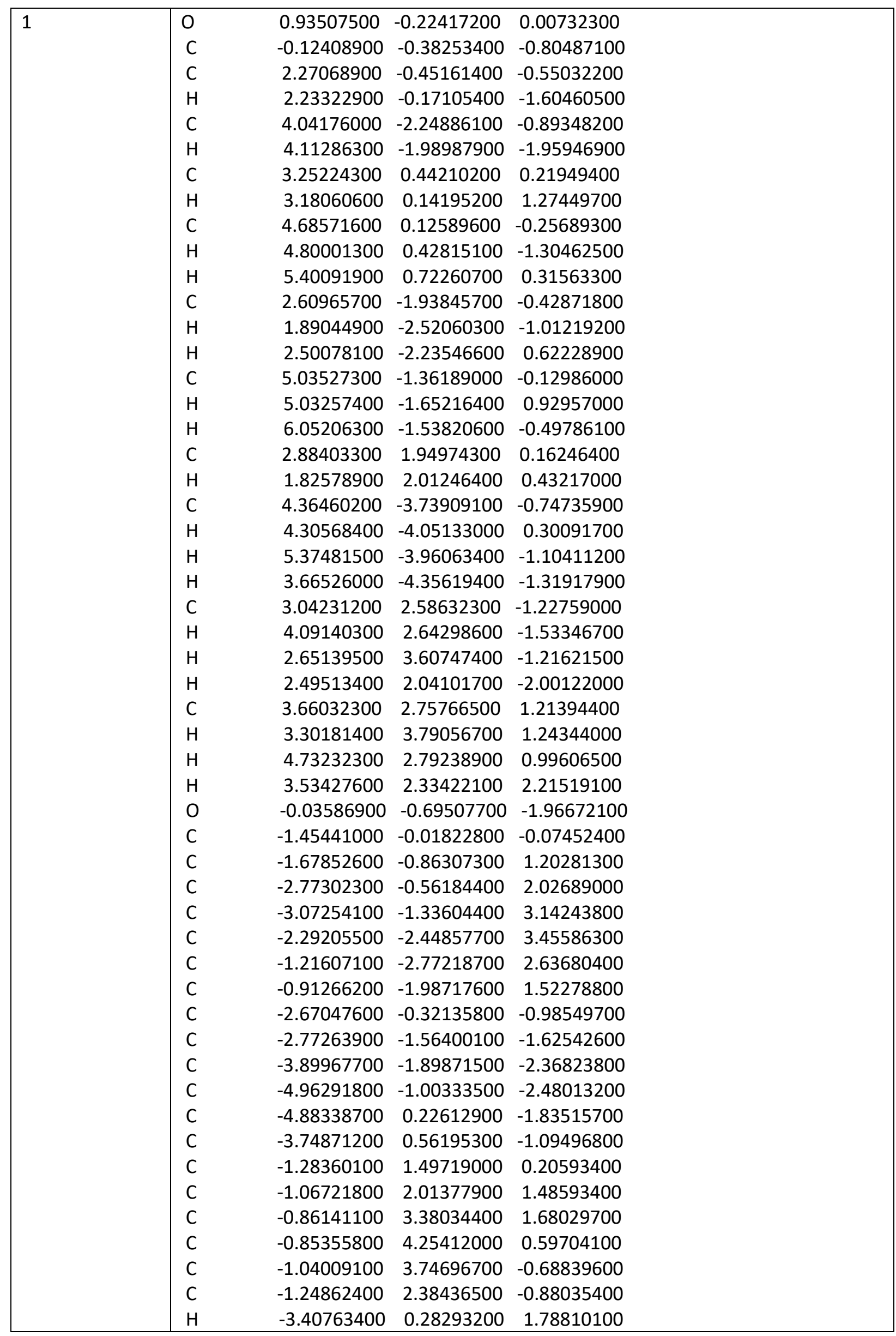




\begin{tabular}{|l|lrrr|}
\hline & $H$ & -3.92333300 & -1.07489200 & 3.76212700 \\
$H$ & -2.52595100 & -3.05700300 & 4.32216100 \\
$H$ & -0.60376600 & -3.63936700 & 2.85878500 \\
$H$ & -0.06531800 & -2.26555000 & 0.91190400 \\
$H$ & -1.96534200 & -2.27980900 & -1.54860500 \\
H & -3.94702700 & -2.86393600 & -2.86032100 \\
$H$ & -5.84161900 & -1.26443900 & -3.05921700 \\
$H$ & -5.70339500 & 0.93267100 & -1.90338700 \\
$H$ & -3.71499300 & 1.52551000 & -0.60338100 \\
H & -1.05050200 & 1.35189000 & 2.34114200 \\
H & -0.70243400 & 3.75723600 & 2.68473400 \\
H & -0.69696800 & 5.31610500 & 0.74990100 \\
H & -1.02400000 & 4.41182500 & -1.54496400 \\
H & -1.38826200 & 2.00496100 & -1.88610800 \\
\hline
\end{tabular}

10 (optimized at the M06-2X/6-311++G(d,p) level)

\begin{tabular}{|c|c|c|c|c|}
\hline Conformer no & & & & \\
\hline 1 & 0 & 0.90263700 & -0.34292700 & 0.01234900 \\
\hline & C & -0.14528400 & -0.45409400 & -0.80855500 \\
\hline & C & 2.22381600 & -0.52055500 & -0.53963900 \\
\hline & $\mathrm{H}$ & 2.18477600 & -0.25678900 & -1.59990500 \\
\hline & C & 4.06749000 & -2.19078800 & -0.90994200 \\
\hline & $\mathrm{H}$ & 4.08878100 & -1.92788200 & -1.97658600 \\
\hline & C & 3.15182000 & 0.43001500 & 0.21099900 \\
\hline & $\mathrm{H}$ & 3.12520800 & 0.12744700 & 1.26824400 \\
\hline & C & 4.58306200 & 0.21632600 & -0.29990500 \\
\hline & $\mathrm{H}$ & 4.64736900 & 0.51582400 & -1.35284000 \\
\hline & $\mathrm{H}$ & 5.27086700 & 0.85925800 & 0.25469500 \\
\hline & C & 2.63860200 & -1.97791300 & -0.40391300 \\
\hline & $\mathrm{H}$ & 1.93712600 & -2.60601700 & -0.96277100 \\
\hline & $\mathrm{H}$ & 2.57909500 & -2.26434600 & 0.65459000 \\
\hline & C & 5.01947900 & -1.24508400 & -0.17366400 \\
\hline & $\mathrm{H}$ & 5.04586000 & -1.52879400 & 0.88691500 \\
\hline & $\mathrm{H}$ & 6.03597500 & -1.36921700 & -0.55982700 \\
\hline & C & 2.66121100 & 1.89255800 & 0.14944500 \\
\hline & $\mathrm{H}$ & 1.65265000 & 1.89671500 & 0.57451300 \\
\hline & C & 4.49436000 & -3.64791800 & -0.76192400 \\
\hline & $\mathrm{H}$ & 4.48734200 & -3.94290800 & 0.29204700 \\
\hline & $\mathrm{H}$ & 5.50536300 & -3.80212400 & -1.14689300 \\
\hline & $\mathrm{H}$ & 3.81968700 & -4.31534900 & -1.30359600 \\
\hline & C & 2.56569600 & 2.44869900 & -1.27346700 \\
\hline & $\mathrm{H}$ & 3.54035500 & 2.45088500 & -1.77042800 \\
\hline & $\mathrm{H}$ & 2.20901600 & 3.48118200 & -1.24113300 \\
\hline & $\mathrm{H}$ & 1.86412700 & 1.88459700 & -1.89423900 \\
\hline & C & 3.52396800 & 2.80947400 & 1.01813500 \\
\hline & $\mathrm{H}$ & 3.04542700 & 3.78656500 & 1.12377000 \\
\hline & $\mathrm{H}$ & 4.51188000 & 2.97201500 & 0.57731300 \\
\hline & $\mathrm{H}$ & 3.66310300 & 2.39312600 & 2.02005200 \\
\hline & 0 & -0.06239700 & -0.77322800 & -1.96269700 \\
\hline & C & -1.44074300 & -0.03737500 & -0.07313200 \\
\hline & C & -1.75158300 & -0.99035200 & 1.09149700 \\
\hline
\end{tabular}




\begin{tabular}{|l|lrrr|}
\hline & C & -2.87535200 & -0.71343600 & 1.87709000 \\
C & -3.25035500 & -1.55667200 & 2.91150300 \\
C & -2.51566800 & -2.71142800 & 3.17230100 \\
C & -1.41027000 & -3.00612800 & 2.38749800 \\
C & -1.02789900 & -2.15061600 & 1.35425600 \\
C & -2.64853800 & -0.11349700 & -1.02002300 \\
C & -2.87901100 & -1.29314100 & -1.73400800 \\
C & -4.01396200 & -1.43965600 & -2.51768200 \\
C & -4.95234900 & -0.41338700 & -2.59416300 \\
C & -4.74283400 & 0.75439000 & -1.87482200 \\
C & -3.59916000 & 0.90281600 & -1.09238500 \\
C & -1.12935300 & 1.39827700 & 0.38976500 \\
C & -0.91426400 & 1.73503400 & 1.72388700 \\
C & -0.53447100 & 3.02915400 & 2.07503400 \\
C & -0.35754400 & 4.00094400 & 1.09790800 \\
C & -0.55721900 & 3.66899200 & -0.23945900 \\
C & -0.93394200 & 2.37881900 & -0.58875900 \\
H & -3.46348300 & 0.17359800 & 1.66603800 \\
H & -4.12288600 & -1.31866700 & 3.50863000 \\
H & -2.80939100 & -3.37625100 & 3.97576800 \\
H & -0.83584500 & -3.90600600 & 2.57420600 \\
H & -0.15529400 & -2.40231800 & 0.76340800 \\
H & -2.16497600 & -2.10510400 & -1.67186700 \\
H & -4.16946100 & -2.36078100 & -3.06688100 \\
H & -5.84029000 & -0.52833600 & -3.20454100 \\
H & -5.46878900 & 1.55807700 & -1.91602300 \\
H & -3.45926400 & 1.82008900 & -0.53388500 \\
H & -1.02279800 & 0.98491700 & 2.49671800 \\
H & -0.36903500 & 3.27073600 & 3.11859100 \\
H & -0.06168000 & 5.00638500 & 1.37371000 \\
H & -0.41364900 & 4.41407800 & -1.01362600 \\
H & -1.07731500 & 2.12387000 & -1.63464400 \\
\hline & & & \\
\hline
\end{tabular}

11 (optimized at the B3LYP/6-311++G(d,p) level)

\begin{tabular}{|l|lrrr|}
\hline Conformer no & & & & \\
\hline 1 & O & 1.02377600 & -0.22135000 & -0.46333300 \\
& C & -0.15565400 & -0.03160000 & -1.07899500 \\
& C & 2.23841600 & -0.16240600 & -1.29341500 \\
& H & 1.99081900 & -0.61841300 & -2.25050600 \\
& C & 3.07848300 & 2.03031000 & -0.25291300 \\
& H & 2.21558800 & 2.08408700 & 0.42068800 \\
& C & 3.33674600 & -0.96026000 & -0.57088100 \\
& H & 4.19331700 & -0.93733000 & -1.26272600 \\
& C & 3.76298500 & -0.22924400 & 0.71823300 \\
& H & 2.93209200 & -0.25345300 & 1.43265300 \\
& H & 4.59643100 & -0.75596200 & 1.18788600 \\
& C & 2.63439500 & 1.29809400 & -1.52796100 \\
& H & 1.80509900 & 1.82259200 & -2.01014100 \\
& H & 3.46030000 & 1.29586800 & -2.25206700 \\
& C & 4.17696100 & 1.22501600 & 0.45442300 \\
& H & 5.08503500 & 1.23734800 & -0.16534000 \\
\hline
\end{tabular}




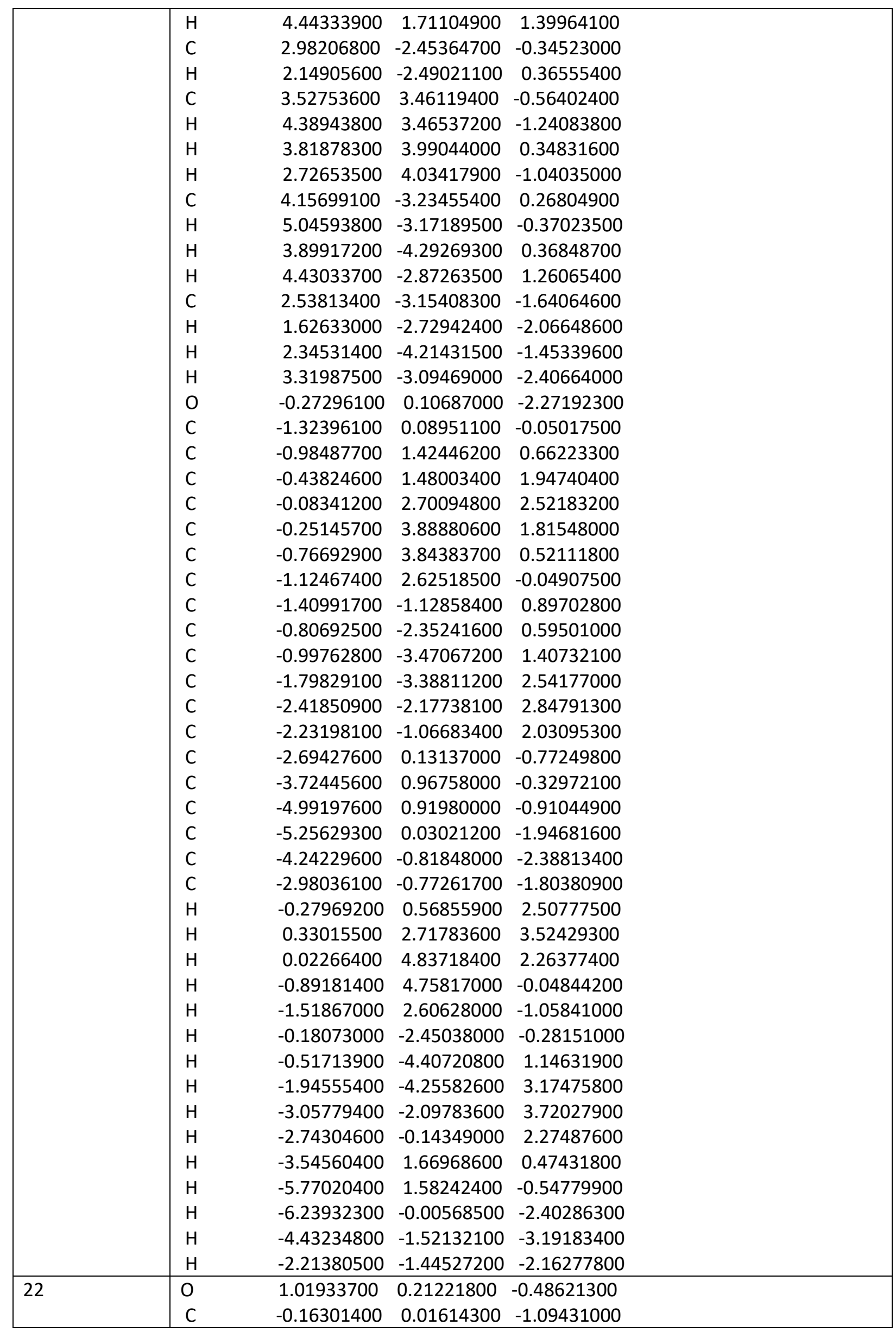




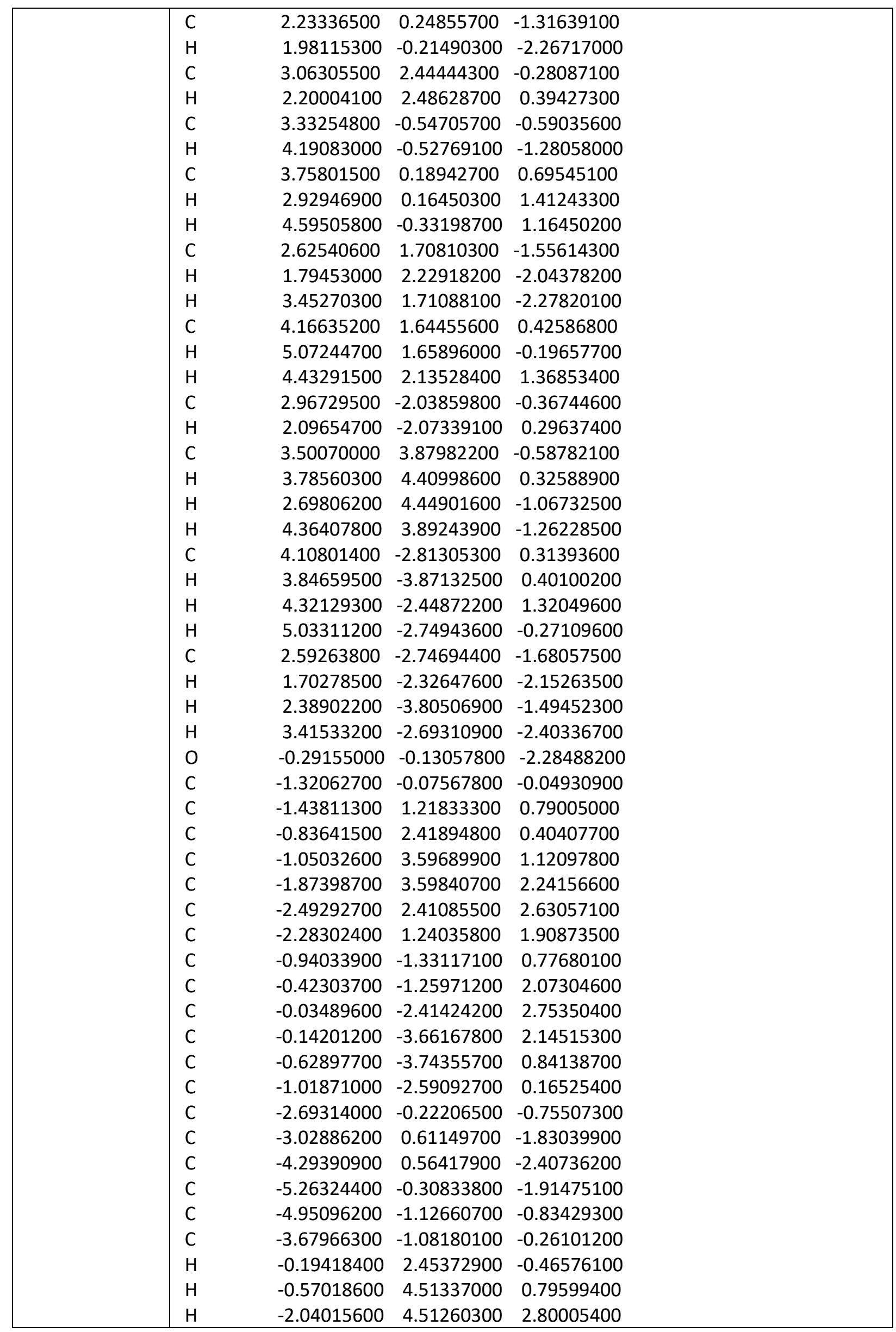




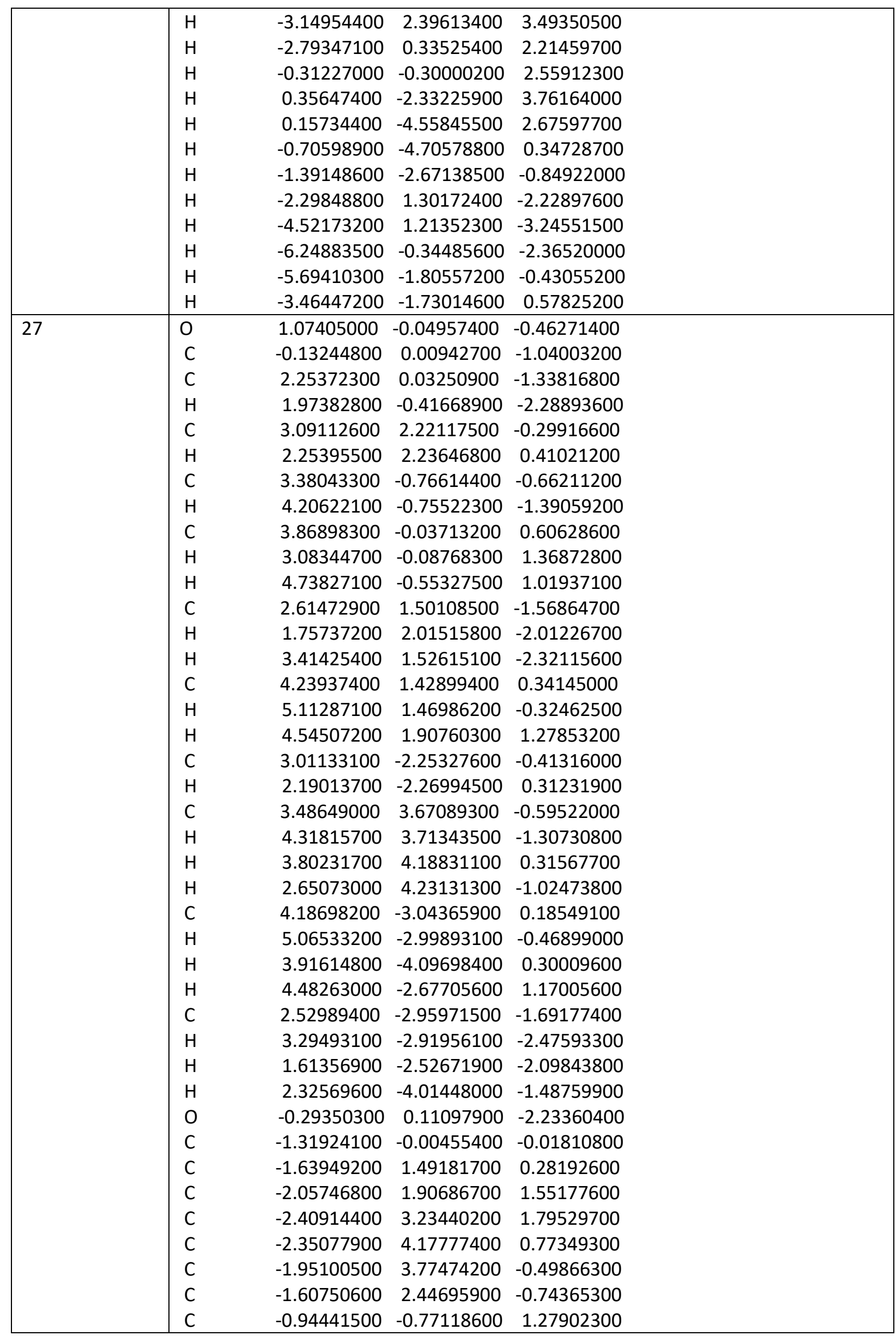




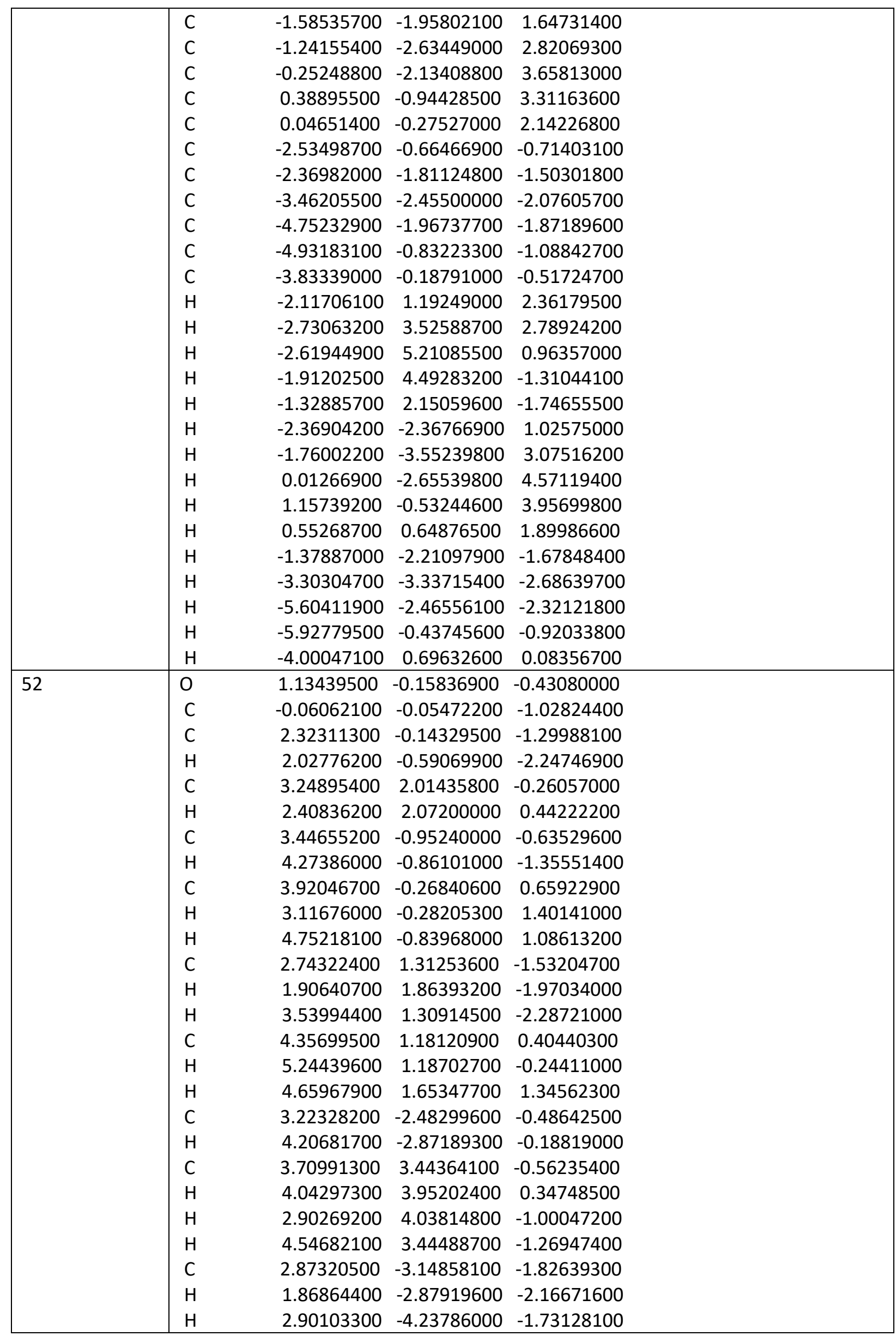




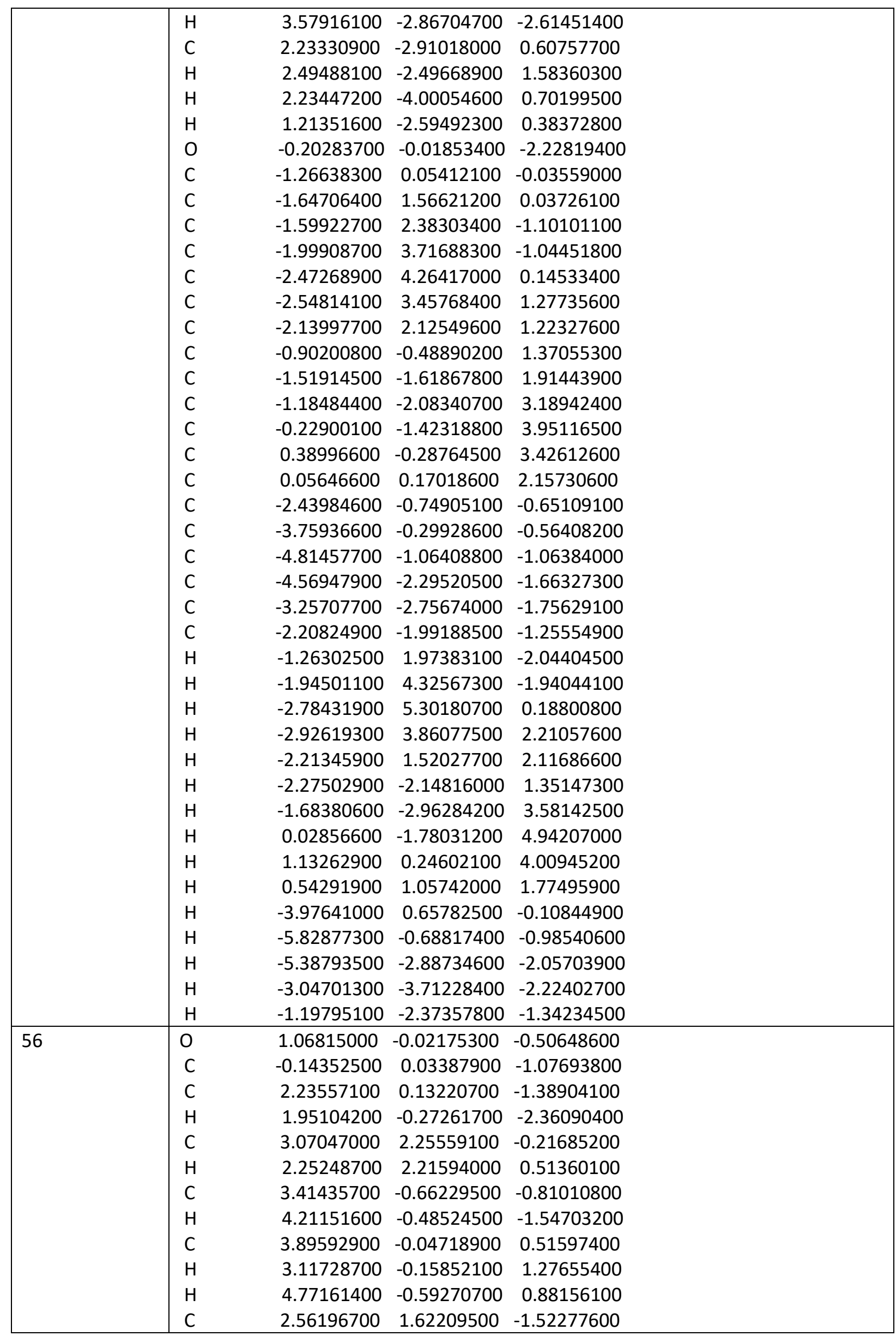




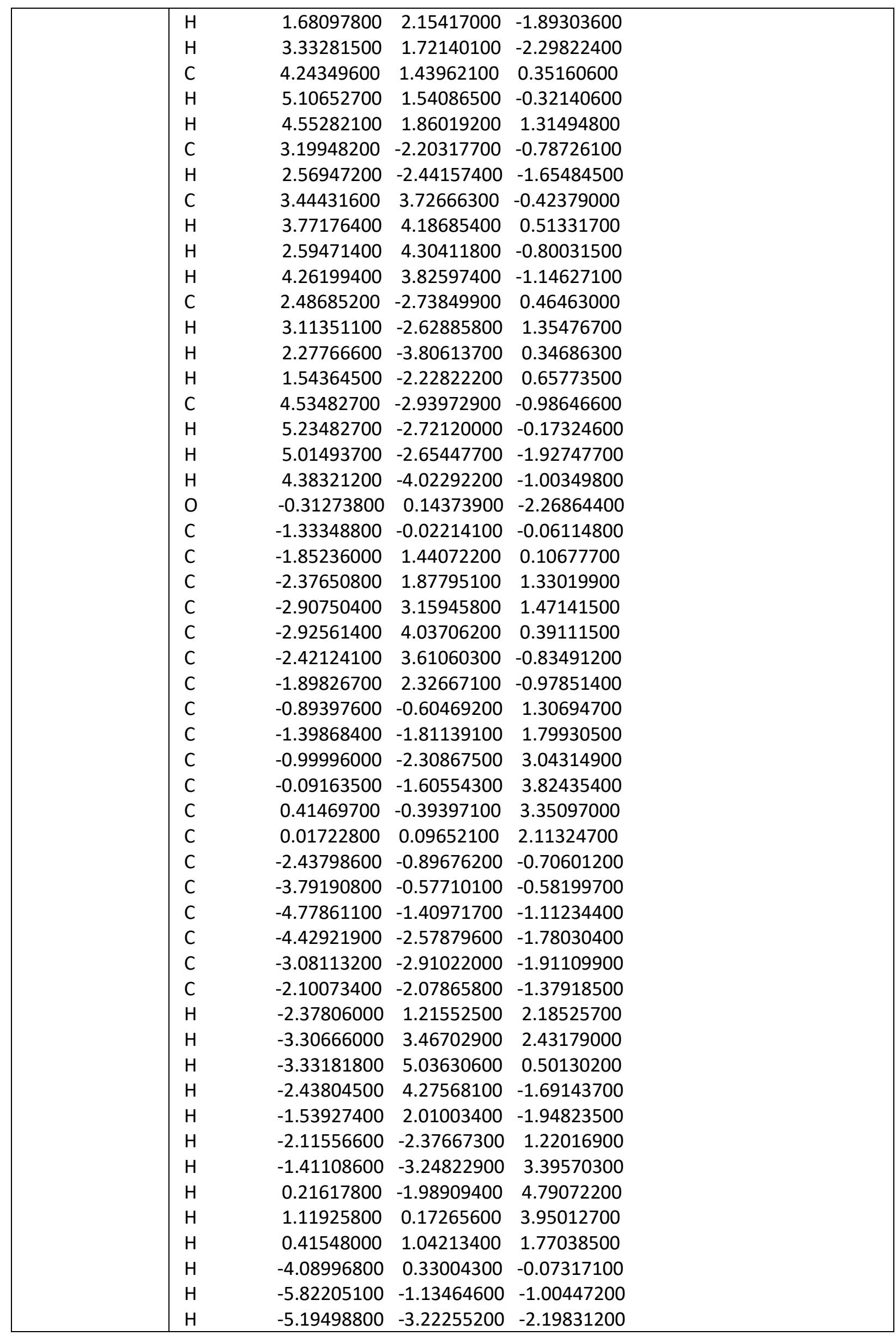




\begin{tabular}{|c|c|c|c|c|}
\hline & $\begin{array}{l}\mathrm{H} \\
\mathrm{H}\end{array}$ & $\begin{array}{l}-2.79023300 \\
-1.06032000\end{array}$ & $\begin{array}{l}-3.81535100 \\
-2.35872100\end{array}$ & $\begin{array}{l}-2.43270500 \\
-1.49483100\end{array}$ \\
\hline 62 & 0 & -1.04659400 & -0.02902600 & -0.51966700 \\
\hline & C & 0.16710400 & -0.09249500 & -1.08780100 \\
\hline & C & -2.21180700 & -0.13685100 & -1.41166000 \\
\hline & $\mathrm{H}$ & -1.93480300 & 0.33794900 & -2.35374800 \\
\hline & C & -2.99181100 & -2.34430400 & -0.37196600 \\
\hline & $\mathrm{H}$ & -2.16713200 & -2.32191800 & 0.35084200 \\
\hline & C & -3.40703400 & 0.59317600 & -0.78291800 \\
\hline & $\mathrm{H}$ & -4.20281700 & 0.43381000 & -1.52534200 \\
\hline & C & -3.86538900 & -0.11259700 & 0.50645300 \\
\hline & $\mathrm{H}$ & -3.08377200 & -0.03651200 & 1.26811200 \\
\hline & $\mathrm{H}$ & -4.74977300 & 0.38833300 & 0.91217700 \\
\hline & C & -2.51644300 & -1.62043700 & -1.64328200 \\
\hline & $\mathrm{H}$ & -1.63280000 & -2.11000700 & -2.06251900 \\
\hline & $\mathrm{H}$ & -3.29748100 & -1.68157700 & -2.41246100 \\
\hline & C & -4.17949700 & -1.59441600 & 0.25320300 \\
\hline & $\mathrm{H}$ & -5.04644700 & -1.67473400 & -0.41766500 \\
\hline & $\mathrm{H}$ & -4.46853400 & -2.08019400 & 1.19164000 \\
\hline & C & -3.24753500 & 2.13698700 & -0.67863200 \\
\hline & $\mathrm{H}$ & -2.66888200 & 2.44858000 & -1.55850900 \\
\hline & C & -3.33099200 & -3.80969500 & -0.66170600 \\
\hline & $\mathrm{H}$ & -4.15251100 & -3.88884300 & -1.38251100 \\
\hline & $\mathrm{H}$ & -3.63750500 & -4.33132100 & 0.24991700 \\
\hline & $\mathrm{H}$ & -2.47091000 & -4.34314000 & -1.07770600 \\
\hline & C & -2.49568600 & 2.63318600 & 0.56536000 \\
\hline & $\mathrm{H}$ & -3.03840200 & 2.38785400 & 1.48333400 \\
\hline & $\mathrm{H}$ & -2.39286200 & 3.72167000 & 0.53058600 \\
\hline & $\mathrm{H}$ & -1.49369200 & 2.21090500 & 0.63534800 \\
\hline & C & -4.61752600 & 2.83038700 & -0.77446000 \\
\hline & $\mathrm{H}$ & -5.26446500 & 2.55831400 & 0.06592800 \\
\hline & $\mathrm{H}$ & -5.13941500 & 2.56366900 & -1.69850700 \\
\hline & $\mathrm{H}$ & -4.50347900 & 3.91811900 & -0.75791700 \\
\hline & $\mathrm{O}$ & 0.33667300 & -0.15691700 & -2.28183700 \\
\hline & C & 1.34106500 & 0.00227600 & -0.05299900 \\
\hline & C & 1.51109600 & 1.51822100 & 0.25096900 \\
\hline & C & 1.86984000 & 1.97859000 & 1.52221500 \\
\hline & C & 2.09095800 & 3.33575800 & 1.75788200 \\
\hline & C & 1.95765700 & 4.26106300 & 0.72663900 \\
\hline & C & 1.61294800 & 3.81348500 & -0.54718000 \\
\hline & C & 1.39895300 & 2.45777400 & -0.78309400 \\
\hline & C & 2.62824900 & -0.53508000 & -0.72755500 \\
\hline & C & 2.60230200 & -1.70952000 & -1.49340200 \\
\hline & C & 3.76517100 & -2.23427400 & -2.04813100 \\
\hline & C & 4.98988100 & -1.59772900 & -1.84972100 \\
\hline & C & 5.03252500 & -0.43520900 & -1.08797200 \\
\hline & C & 3.86325700 & 0.08919400 & -0.53430700 \\
\hline & C & 1.01732500 & -0.80832800 & 1.23393800 \\
\hline & C & 1.75806900 & -1.93731200 & 1.60043400 \\
\hline & C & 1.45945300 & -2.65745700 & 2.75965600 \\
\hline & C & 0.41630700 & -2.26060800 & 3.58700800 \\
\hline & C & -0.32338200 & -1.12861500 & 3.24479200 \\
\hline
\end{tabular}




\begin{tabular}{|l|lrrr|}
\hline & C & -0.02586300 & -0.41536500 & 2.08868200 \\
$H$ & 1.98607700 & 1.27778300 & 2.33831100 \\
H & 2.36975700 & 3.66478600 & 2.75297100 \\
$H$ & 2.12526500 & 5.31627100 & 0.91100600 \\
H & 1.51482900 & 4.51923100 & -1.36470200 \\
H & 1.15752700 & 2.12956300 & -1.78682900 \\
H & 1.66627000 & -2.22398200 & -1.66677800 \\
H & 3.71180400 & -3.14065200 & -2.64118300 \\
H & 5.89596800 & -2.00311000 & -2.28601700 \\
H & 5.97521100 & 0.07473800 & -0.92257600 \\
$\mathrm{H}$ & 3.92560800 & 0.99766500 & 0.04995500 \\
$\mathrm{H}$ & 2.58511700 & -2.26725900 & 0.98809800 \\
$\mathrm{H}$ & 2.05534500 & -3.52805300 & 3.01084400 \\
$\mathrm{H}$ & 0.18568100 & -2.81651200 & 4.48898200 \\
$\mathrm{H}$ & -1.13421400 & -0.79450800 & 3.88315300 \\
$\mathrm{H}$ & -0.61036800 & 0.46180900 & 1.85315200 \\
\hline
\end{tabular}

11 (optimized at the M06-2X/6-311++G(d,p) level)

\begin{tabular}{|l|lrrr|}
\hline Conformer no & \multicolumn{4}{|c|}{} \\
\hline 1 & O & 0.97753200 & -0.29277400 & -0.57265200 \\
& C & -0.20746700 & -0.05419400 & -1.13902700 \\
& C & 2.16475600 & -0.16155400 & -1.39322800 \\
& H & 1.95888500 & -0.61924700 & -2.36146900 \\
& C & 2.80555500 & 2.02388600 & -0.27297700 \\
& H & 1.92282200 & 1.96180800 & 0.37318700 \\
& C & 3.26510900 & -0.91928100 & -0.64763500 \\
& H & 4.14490100 & -0.89819100 & -1.30883500 \\
& C & 3.61686000 & -0.18535900 & 0.65530100 \\
& H & 2.75976800 & -0.26102900 & 1.33615400 \\
& H & 4.46097500 & -0.67635600 & 1.14319500 \\
& C & 2.47750300 & 1.31816100 & -1.58848000 \\
& H & 1.63232400 & 1.79991800 & -2.09011100 \\
& H & 3.33650000 & 1.39101800 & -2.26840500 \\
& C & 3.94916800 & 1.28976500 & 0.42586200 \\
& H & 4.85650600 & 1.37167500 & -0.18932200 \\
& H & 4.17096800 & 1.77421700 & 1.38225700 \\
& C & 2.89116300 & -2.39566400 & -0.40101200 \\
& H & 2.08268500 & -2.40566500 & 0.33998500 \\
& C & 3.12800500 & 3.49562900 & -0.50340300 \\
& H & 4.02191700 & 3.60418600 & -1.12694100 \\
& H & 3.31226300 & 4.00768500 & 0.44496800 \\
& H & 2.30081500 & 4.00433600 & -1.00594600 \\
& C & 4.07457100 & -3.18416000 & 0.16704400 \\
& H & 4.92823300 & -3.14099400 & -0.51787000 \\
& H & 3.80630100 & -4.23536200 & 0.29630000 \\
& H & 4.39998900 & -2.80873700 & 1.13775000 \\
& C & 2.38724200 & -3.08863600 & -1.66997200 \\
& H & 1.45837700 & -2.65590300 & -2.04908100 \\
& H & 2.20137100 & -4.14803100 & -1.47631700 \\
& H & 3.13408900 & -3.02339100 & -2.46899800 \\
O & -0.36794300 & 0.11280000 & -2.31684600 \\
\hline
\end{tabular}




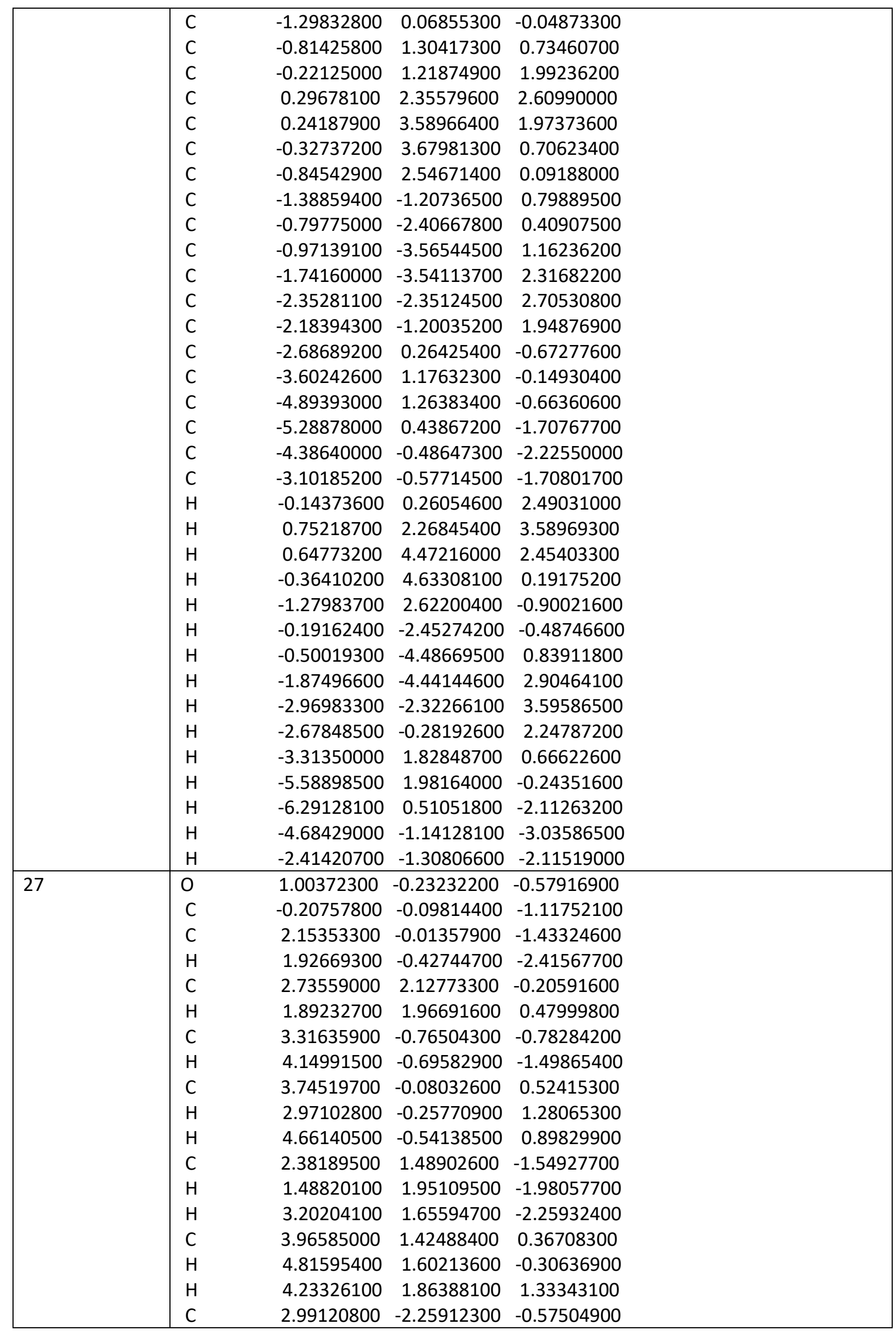




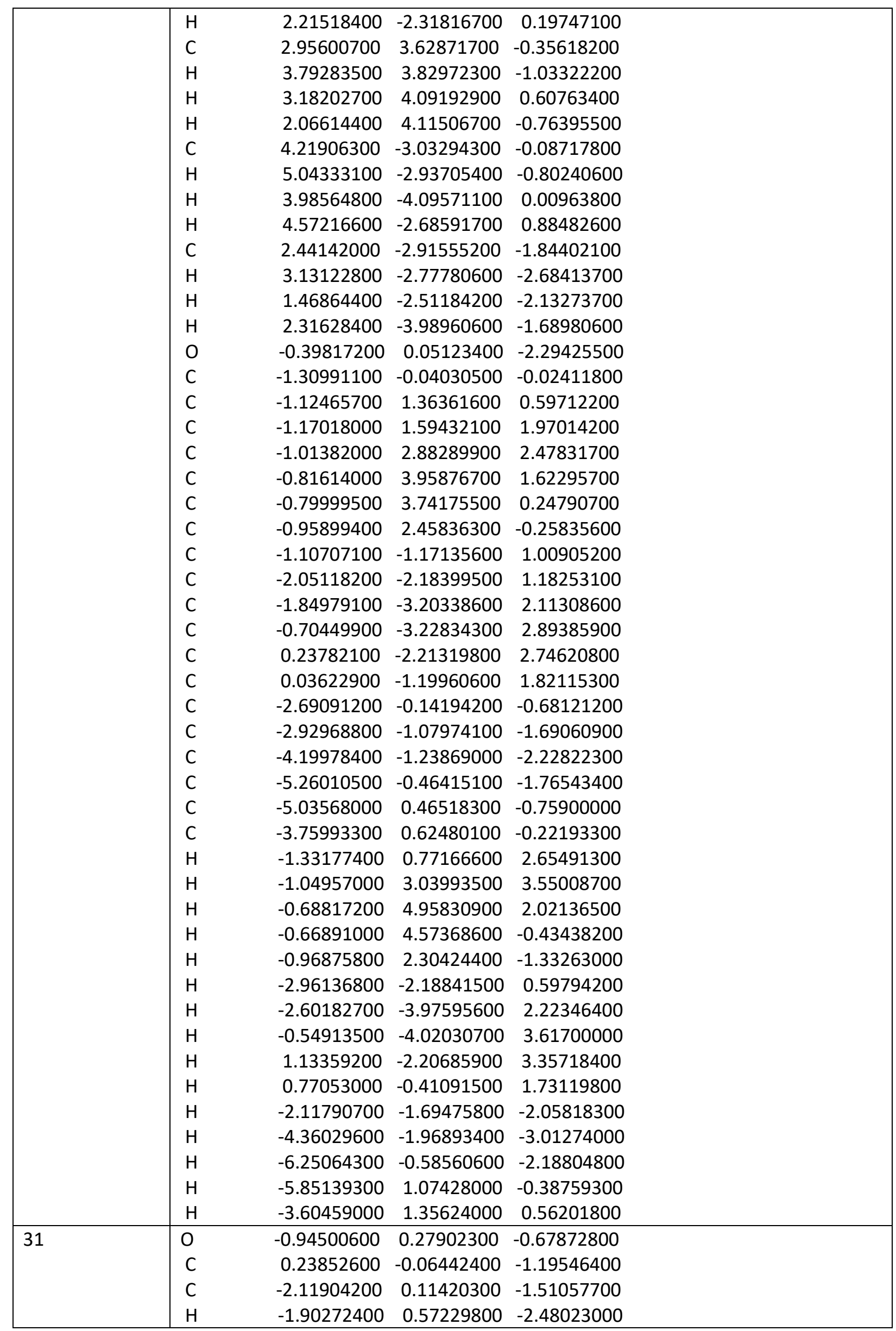




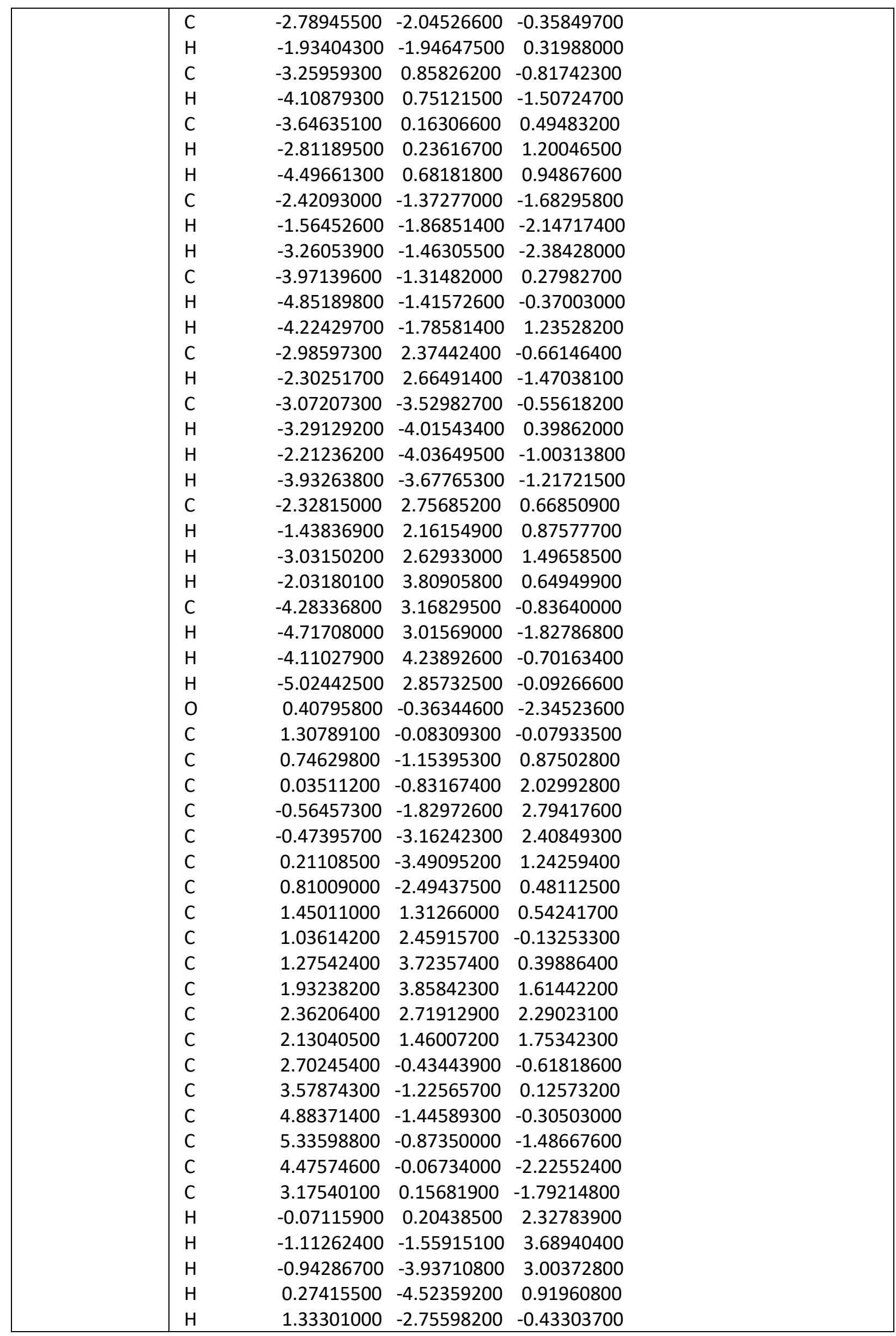




\begin{tabular}{|c|c|c|c|c|}
\hline & $\mathrm{H}$ & 0.51346300 & 2.37838900 & -1.08019000 \\
\hline & $\mathrm{H}$ & 0.94222200 & 4.60212500 & -0.14142900 \\
\hline & $\mathrm{H}$ & 2.11468900 & 4.84181500 & 2.03130100 \\
\hline & $\mathrm{H}$ & 2.88644100 & 2.81198000 & 3.23405500 \\
\hline & $\mathrm{H}$ & 2.48625300 & 0.57829600 & 2.27573600 \\
\hline & $\mathrm{H}$ & 3.24657000 & -1.67779100 & 1.05267500 \\
\hline & $\mathrm{H}$ & 5.54426100 & -2.06656500 & 0.28926800 \\
\hline & $\mathrm{H}$ & 6.35004000 & -1.04716000 & -1.82676400 \\
\hline & $\mathrm{H}$ & 4.81832600 & 0.39287200 & -3.14494500 \\
\hline & $\mathrm{H}$ & 2.52490100 & 0.79492900 & -2.37568200 \\
\hline \multirow[t]{42}{*}{33} & 0 & -0.98589000 & 0.42870000 & -0.62277400 \\
\hline & $\mathrm{C}$ & 0.15556300 & -0.00539300 & -1.16545400 \\
\hline & $\mathrm{C}$ & -2.18358900 & 0.40237100 & -1.44082400 \\
\hline & $\mathrm{H}$ & -1.94865500 & 0.89873600 & -2.38488200 \\
\hline & $\mathrm{C}$ & -2.97408100 & -1.79289200 & -0.42944600 \\
\hline & $\mathrm{H}$ & -2.09572600 & -1.80116300 & 0.22448100 \\
\hline & $\mathrm{C}$ & -3.26860900 & 1.16116400 & -0.67240500 \\
\hline & $\mathrm{H}$ & -4.13400200 & 1.14199000 & -1.35077300 \\
\hline & $\mathrm{C}$ & -3.65919200 & 0.39767000 & 0.59942300 \\
\hline & $\mathrm{H}$ & -2.79798200 & 0.37265300 & 1.27596500 \\
\hline & $\mathrm{H}$ & -4.45818200 & 0.94103000 & 1.11490500 \\
\hline & $\mathrm{C}$ & -2.59849400 & -1.04492100 & -1.70966800 \\
\hline & $\mathrm{H}$ & -1.79549500 & -1.56176600 & -2.24039100 \\
\hline & $\mathrm{H}$ & -3.46350800 & -1.02084100 & -2.38490900 \\
\hline & $\mathrm{C}$ & -4.08431500 & -1.03960500 & 0.30302900 \\
\hline & $\mathrm{H}$ & -4.99334700 & -1.04437800 & -0.31445300 \\
\hline & $\mathrm{H}$ & -4.32920500 & -1.55865400 & 1.23554400 \\
\hline & $\mathrm{C}$ & -2.96895300 & 2.65423100 & -0.39092500 \\
\hline & $\mathrm{H}$ & -3.94919800 & 3.11836500 & -0.22309000 \\
\hline & $\mathrm{C}$ & -3.35952600 & -3.23717400 & -0.72667100 \\
\hline & $\mathrm{H}$ & -4.24462000 & -3.28006500 & -1.37026900 \\
\hline & $\mathrm{H}$ & -3.58647900 & -3.77693300 & 0.19674700 \\
\hline & $\mathrm{H}$ & -2.54633900 & -3.76346000 & -1.23399600 \\
\hline & $\mathrm{C}$ & -2.33662200 & 3.35084500 & -1.59678700 \\
\hline & $\mathrm{H}$ & -1.30695600 & 3.01288400 & -1.74696800 \\
\hline & $\mathrm{H}$ & -2.30929000 & 4.43179900 & -1.44212700 \\
\hline & $\mathrm{H}$ & -2.89459800 & 3.15523100 & -2.51722700 \\
\hline & $\mathrm{C}$ & -2.13597500 & 2.90467800 & 0.87194100 \\
\hline & $\mathrm{H}$ & -2.67922700 & 2.61644900 & 1.77410500 \\
\hline & $\mathrm{H}$ & -1.89933500 & 3.96889900 & 0.95657600 \\
\hline & $\mathrm{H}$ & -1.19584200 & 2.35185600 & 0.84735000 \\
\hline & $\mathrm{O}$ & 0.28628800 & -0.27094500 & -2.32878000 \\
\hline & $\mathrm{C}$ & 1.22658200 & -0.17289300 & -0.06025200 \\
\hline & $\mathrm{C}$ & 0.57522400 & -1.23575500 & 0.84589600 \\
\hline & $\mathrm{C}$ & -0.16238600 & -0.90239800 & 1.98204200 \\
\hline & $\mathrm{C}$ & -0.84138000 & -1.88334400 & 2.70001200 \\
\hline & $\mathrm{C}$ & -0.80917600 & -3.20981500 & 2.28357000 \\
\hline & $\mathrm{C}$ & -0.10253700 & -3.54707600 & 1.13356900 \\
\hline & $\mathrm{C}$ & 0.57907100 & -2.56748300 & 0.41991700 \\
\hline & $\mathrm{C}$ & 1.48960700 & 1.17958800 & 0.61472100 \\
\hline & $\mathrm{C}$ & 1.29894500 & 2.36999100 & -0.08529700 \\
\hline & $\mathrm{C}$ & 1.62868400 & 3.59505200 & 0.48454400 \\
\hline
\end{tabular}




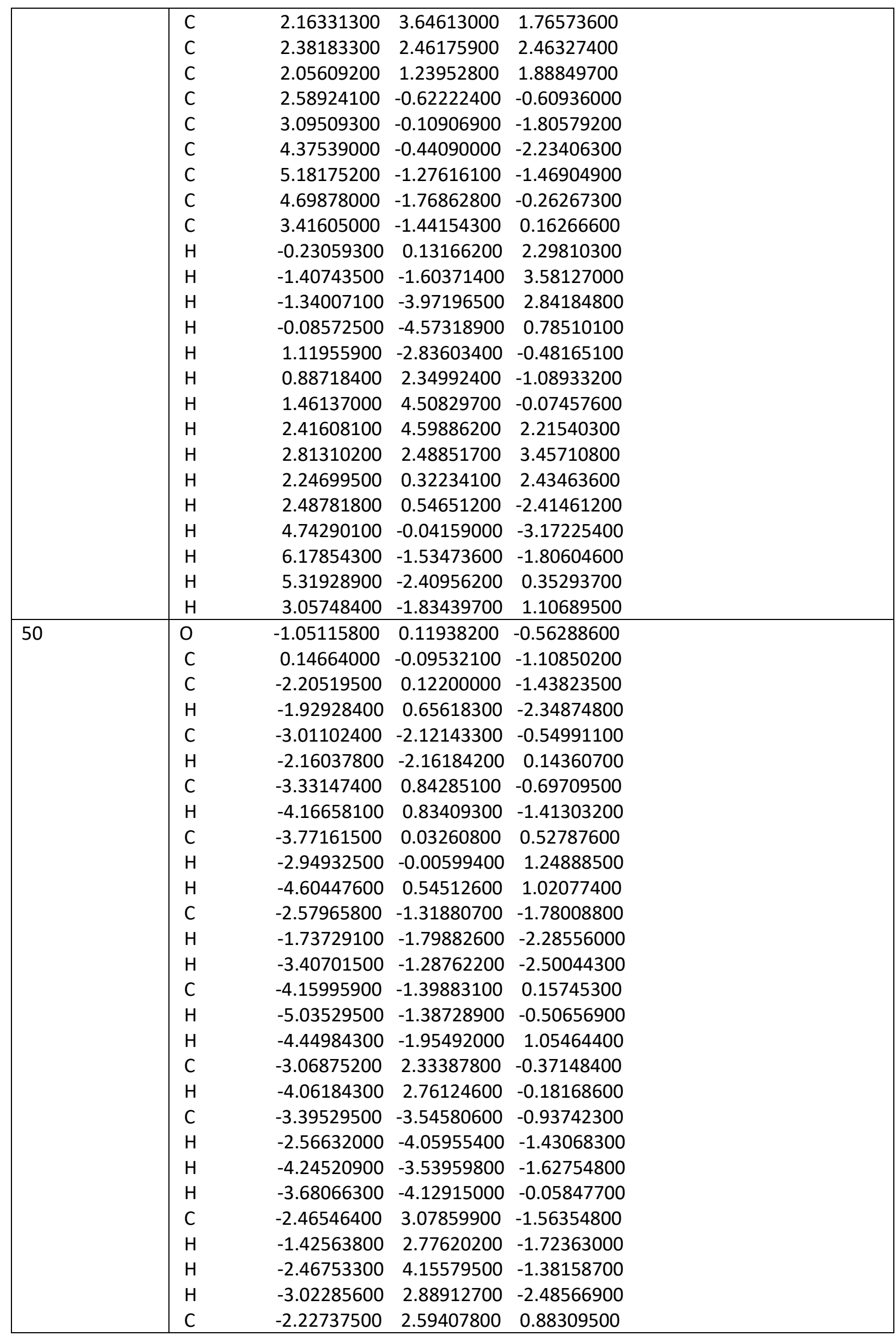




\begin{tabular}{|c|c|c|c|c|}
\hline & $\mathrm{H}$ & -1.21000000 & 2.21626400 & 0.76907100 \\
\hline & $\mathrm{H}$ & -2.66963200 & 2.14344200 & 1.77444400 \\
\hline & $\mathrm{H}$ & -2.15873800 & 3.67093100 & 1.06032700 \\
\hline & $\mathrm{O}$ & 0.32549600 & -0.21327400 & -2.29036800 \\
\hline & C & 1.26449800 & -0.08318200 & -0.02718700 \\
\hline & $\mathrm{C}$ & 2.60041600 & -0.50164200 & -0.65501300 \\
\hline & C & 2.67024100 & -1.58752900 & -1.53428000 \\
\hline & $\mathrm{C}$ & 3.88864300 & -2.01345900 & -2.04572500 \\
\hline & C & 5.06728200 & -1.36579800 & -1.68607400 \\
\hline & C & 5.01171300 & -0.29303200 & -0.80750500 \\
\hline & C & 3.78784100 & 0.13537600 & -0.29790000 \\
\hline & C & 0.86115800 & -1.03832700 & 1.12042800 \\
\hline & C & 1.54996400 & -2.22397300 & 1.37451200 \\
\hline & $\mathrm{C}$ & 1.14249800 & -3.08723100 & 2.39232700 \\
\hline & C & 0.04410100 & -2.77905500 & 3.18012800 \\
\hline & C & -0.63936500 & -1.58657100 & 2.95275700 \\
\hline & $\mathrm{C}$ & -0.23327100 & -0.72924500 & 1.94144800 \\
\hline & C & 1.34273600 & 1.38610300 & 0.43523700 \\
\hline & C & 1.28542200 & 2.39724800 & -0.52793200 \\
\hline & $\mathrm{C}$ & 1.35792100 & 3.73517500 & -0.16267500 \\
\hline & C & 1.50840900 & 4.08680000 & 1.17540000 \\
\hline & C & 1.60282800 & 3.08754200 & 2.13584900 \\
\hline & $\mathrm{C}$ & 1.52351000 & 1.74594600 & 1.76872700 \\
\hline & $\mathrm{H}$ & 1.76602000 & -2.10779200 & -1.82281300 \\
\hline & $\mathrm{H}$ & 3.91602700 & -2.85353600 & -2.72975700 \\
\hline & $\mathrm{H}$ & 6.01759400 & -1.69648300 & -2.08855600 \\
\hline & $\mathrm{H}$ & 5.91987900 & 0.22174200 & -0.51663900 \\
\hline & $\mathrm{H}$ & 3.76723400 & 0.97921000 & 0.38066700 \\
\hline & $\mathrm{H}$ & 2.41792900 & -2.48879700 & 0.78552100 \\
\hline & $\mathrm{H}$ & 1.69599600 & -4.00302500 & 2.56370600 \\
\hline & $\mathrm{H}$ & -0.27268200 & -3.45120400 & 3.96882600 \\
\hline & $\mathrm{H}$ & -1.49186000 & -1.31956200 & 3.56745000 \\
\hline & $\mathrm{H}$ & -0.76813500 & 0.19847700 & 1.78866100 \\
\hline & $\mathrm{H}$ & 1.18661600 & 2.13480300 & -1.57691500 \\
\hline & $\mathrm{H}$ & 1.29426600 & 4.50311300 & -0.92494500 \\
\hline & $\mathrm{H}$ & 1.55863600 & 5.12985400 & 1.46481400 \\
\hline & $\mathrm{H}$ & 1.73816200 & 3.34665400 & 3.17943500 \\
\hline & $\mathrm{H}$ & 1.60328800 & 0.98030700 & 2.53050800 \\
\hline 52 & 0 & 1.06595200 & -0.29598100 & -0.55921200 \\
\hline & C & -0.13294800 & -0.10640400 & -1.10617300 \\
\hline & C & 2.22309800 & -0.19019400 & -1.42477200 \\
\hline & $\mathrm{H}$ & 1.96522500 & -0.63285300 & -2.38732400 \\
\hline & C & 2.91904000 & 1.96567200 & -0.28174900 \\
\hline & $\mathrm{H}$ & 2.04214000 & 1.91201900 & 0.37787400 \\
\hline & C & 3.36257900 & -0.95112600 & -0.75012800 \\
\hline & $\mathrm{H}$ & 4.21337200 & -0.82070500 & -1.43512700 \\
\hline & C & 3.73112400 & -0.27705400 & 0.57636400 \\
\hline & $\mathrm{H}$ & 2.88624500 & -0.36648900 & 1.26736300 \\
\hline & $\mathrm{H}$ & 4.57342900 & -0.80760300 & 1.03254500 \\
\hline & $\mathrm{C}$ & 2.55036600 & 1.28974700 & -1.60443100 \\
\hline & $\mathrm{H}$ & 1.69957000 & 1.79237700 & -2.07464200 \\
\hline & $\mathrm{H}$ & 3.39308800 & 1.36959300 & -2.30257400 \\
\hline
\end{tabular}




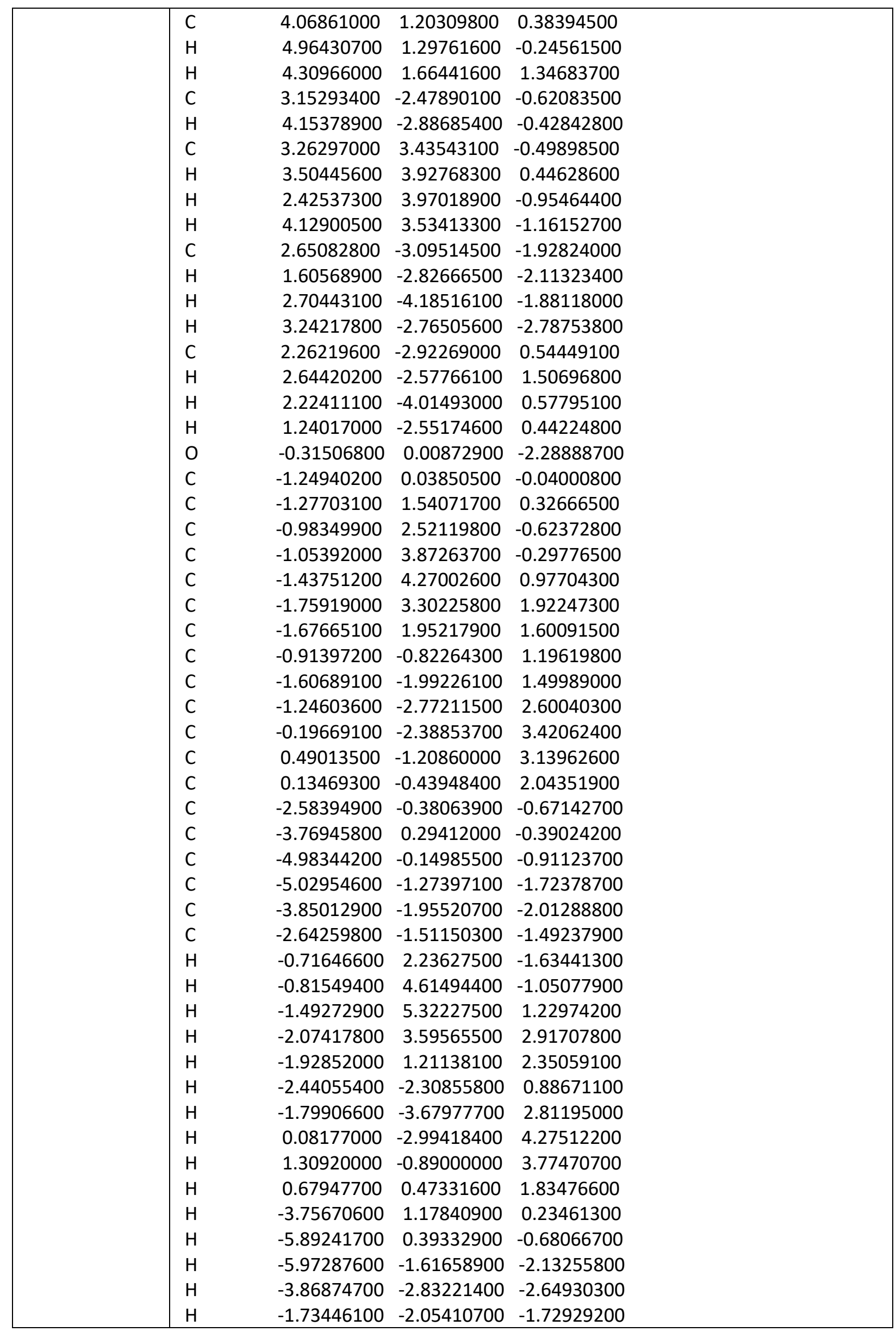




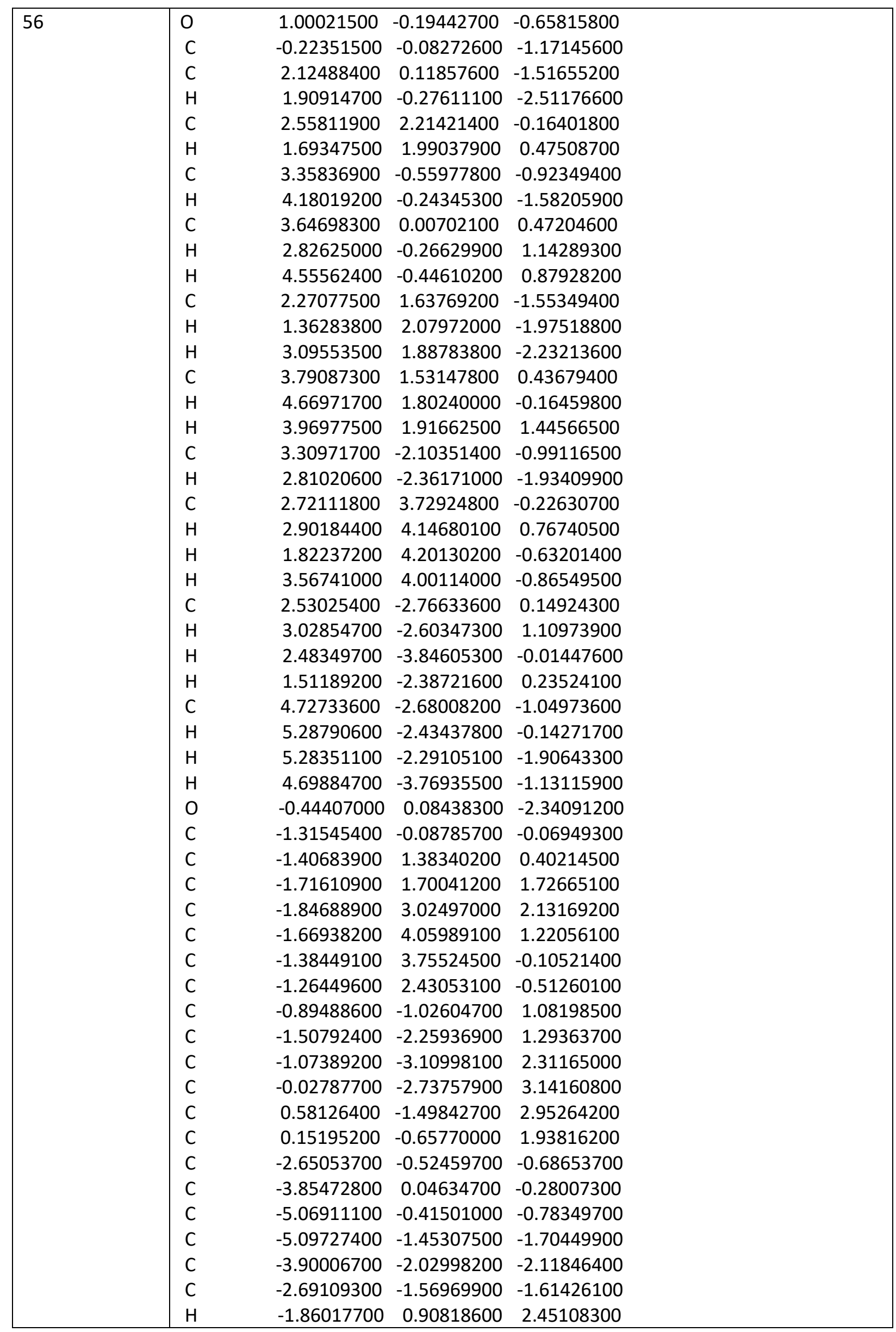




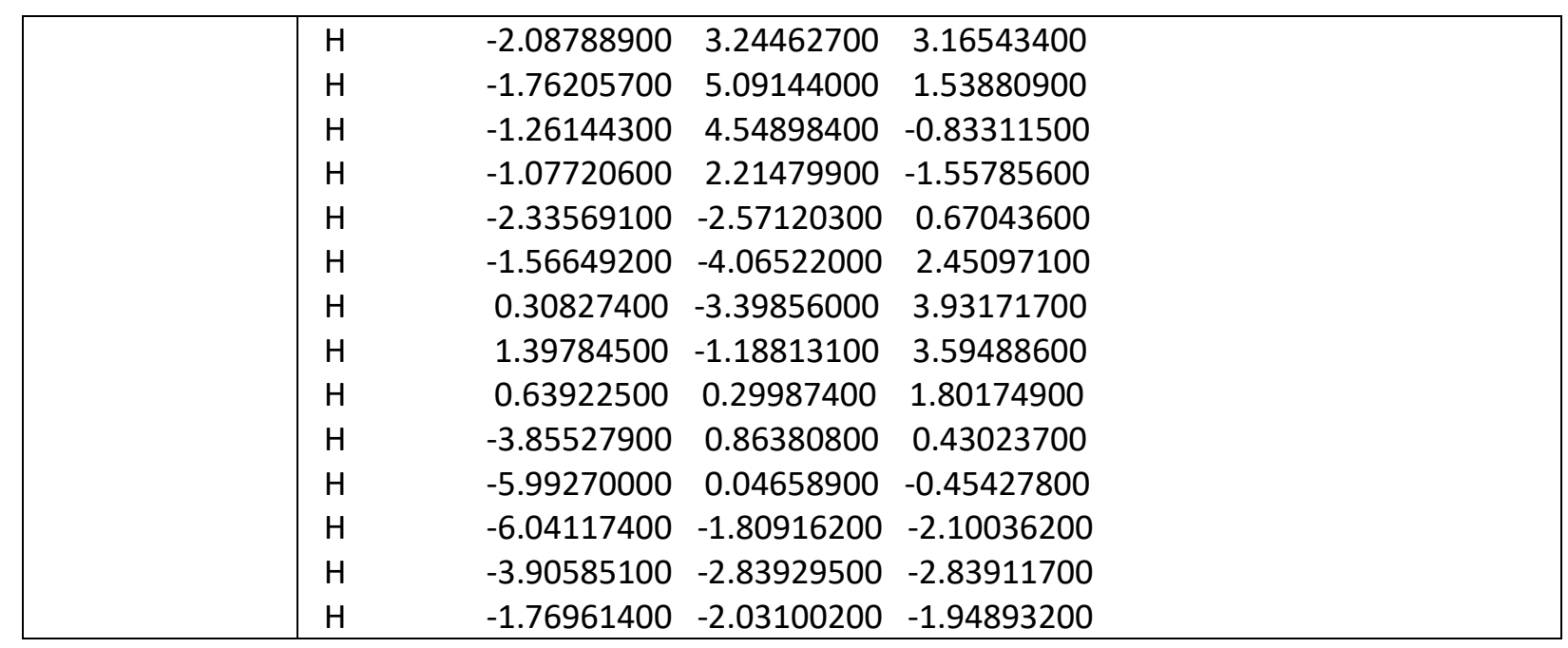

14 (optimized at the B3LYP/6-311++G(d,p) level)

\begin{tabular}{|c|c|c|c|c|}
\hline Conformer no & & & & \\
\hline \multirow[t]{35}{*}{1} & 0 & -1.31685100 & -0.21812100 & -0.22959000 \\
\hline & $\mathrm{C}$ & -0.18101200 & -0.13744300 & -0.97783000 \\
\hline & C & -2.55672200 & -0.34359900 & -0.91588000 \\
\hline & $\mathrm{H}$ & -2.39951300 & -0.14181100 & -1.98050100 \\
\hline & C & -3.67884900 & 0.54601500 & -0.34801200 \\
\hline & C & -4.88834200 & -0.28503300 & -0.83072900 \\
\hline & $\mathrm{C}$ & -3.12392000 & -1.76061300 & -0.79246400 \\
\hline & $\mathrm{O}$ & -0.18730100 & -0.16174300 & -2.17884400 \\
\hline & C & 1.05943200 & 0.08510100 & -0.06810600 \\
\hline & $\mathrm{C}$ & 0.73318700 & 1.42727700 & 0.63967600 \\
\hline & C & 0.46163000 & 1.51918900 & 2.00643100 \\
\hline & C & 0.11114600 & 2.74041400 & 2.58496800 \\
\hline & C & 0.01283400 & 3.88899500 & 1.80525800 \\
\hline & C & 0.26152900 & 3.80586000 & 0.43533400 \\
\hline & $\mathrm{C}$ & 0.61502700 & 2.58839300 & -0.13908500 \\
\hline & C & 2.34022000 & 0.16286700 & -0.93615000 \\
\hline & C & 2.60884900 & -0.85298700 & -1.86402900 \\
\hline & C & 3.79178200 & -0.86175900 & -2.59420300 \\
\hline & C & 4.74526000 & 0.13858300 & -2.40516700 \\
\hline & C & 4.50013600 & 1.14143500 & -1.47393100 \\
\hline & $\mathrm{C}$ & 3.30836700 & 1.15260100 & -0.74649400 \\
\hline & C & 1.27861500 & -1.09372600 & 0.91225900 \\
\hline & C & 2.34184300 & -1.01193300 & 1.82424200 \\
\hline & C & 2.63224800 & -2.06288400 & 2.68673200 \\
\hline & $\mathrm{C}$ & 1.87414200 & -3.23346500 & 2.64701100 \\
\hline & C & 0.83037700 & -3.33404600 & 1.73444600 \\
\hline & $\mathrm{C}$ & 0.53260600 & -2.27452900 & 0.87390400 \\
\hline & $\mathrm{H}$ & 0.51615000 & 0.63619800 & 2.62842200 \\
\hline & $\mathrm{H}$ & -0.08769500 & 2.78705700 & 3.65008100 \\
\hline & $\mathrm{H}$ & -0.25718800 & 4.83715800 & 2.25649100 \\
\hline & $\mathrm{H}$ & 0.18429800 & 4.69015100 & -0.18774100 \\
\hline & $\mathrm{H}$ & 0.80608600 & 2.54036200 & -1.20532300 \\
\hline & $\mathrm{H}$ & 1.89094100 & -1.64738900 & -2.02008800 \\
\hline & $\mathrm{H}$ & 3.97025600 & -1.65556200 & -3.31102800 \\
\hline & $\mathrm{H}$ & 5.66851400 & 0.13056900 & -2.97359800 \\
\hline
\end{tabular}




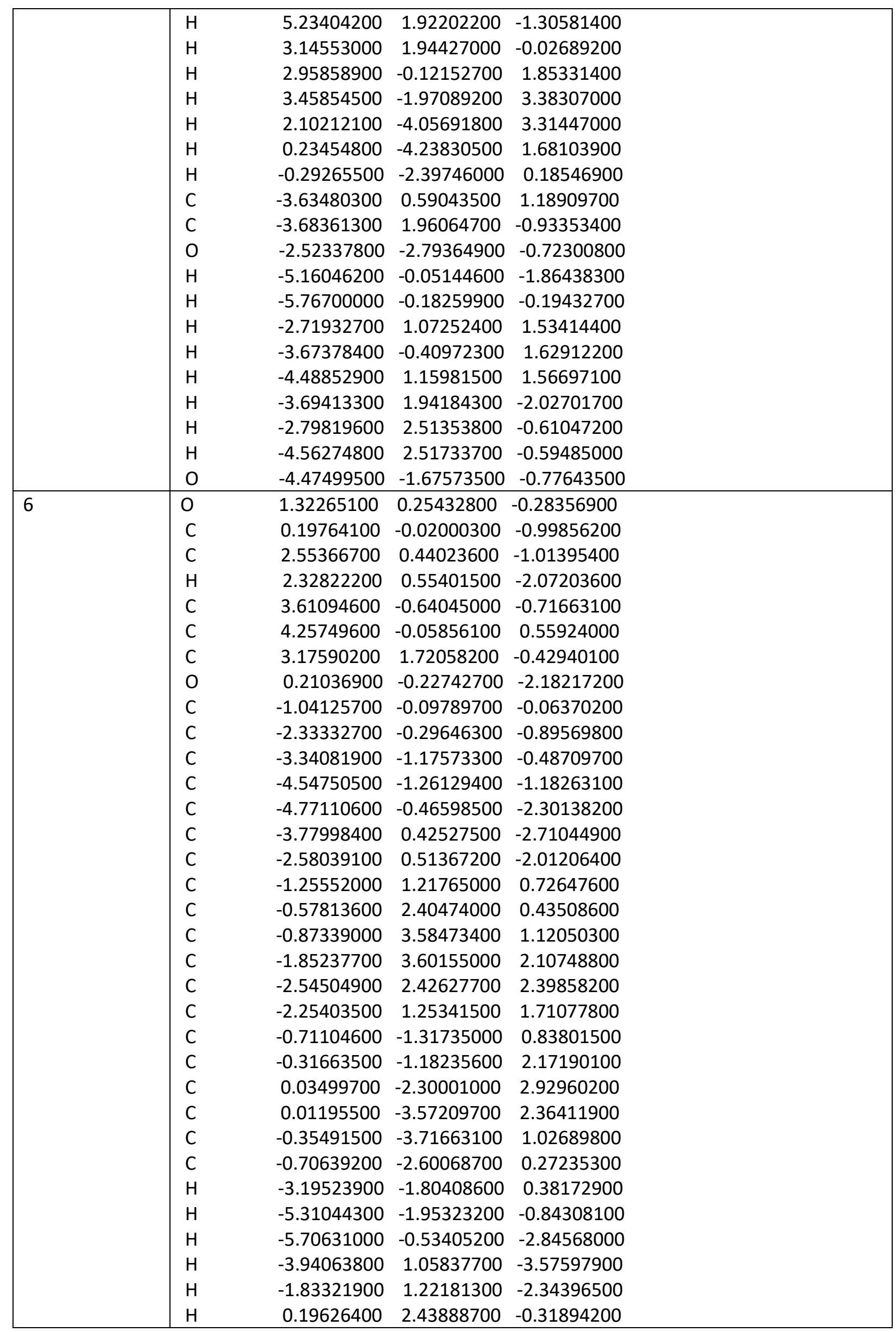




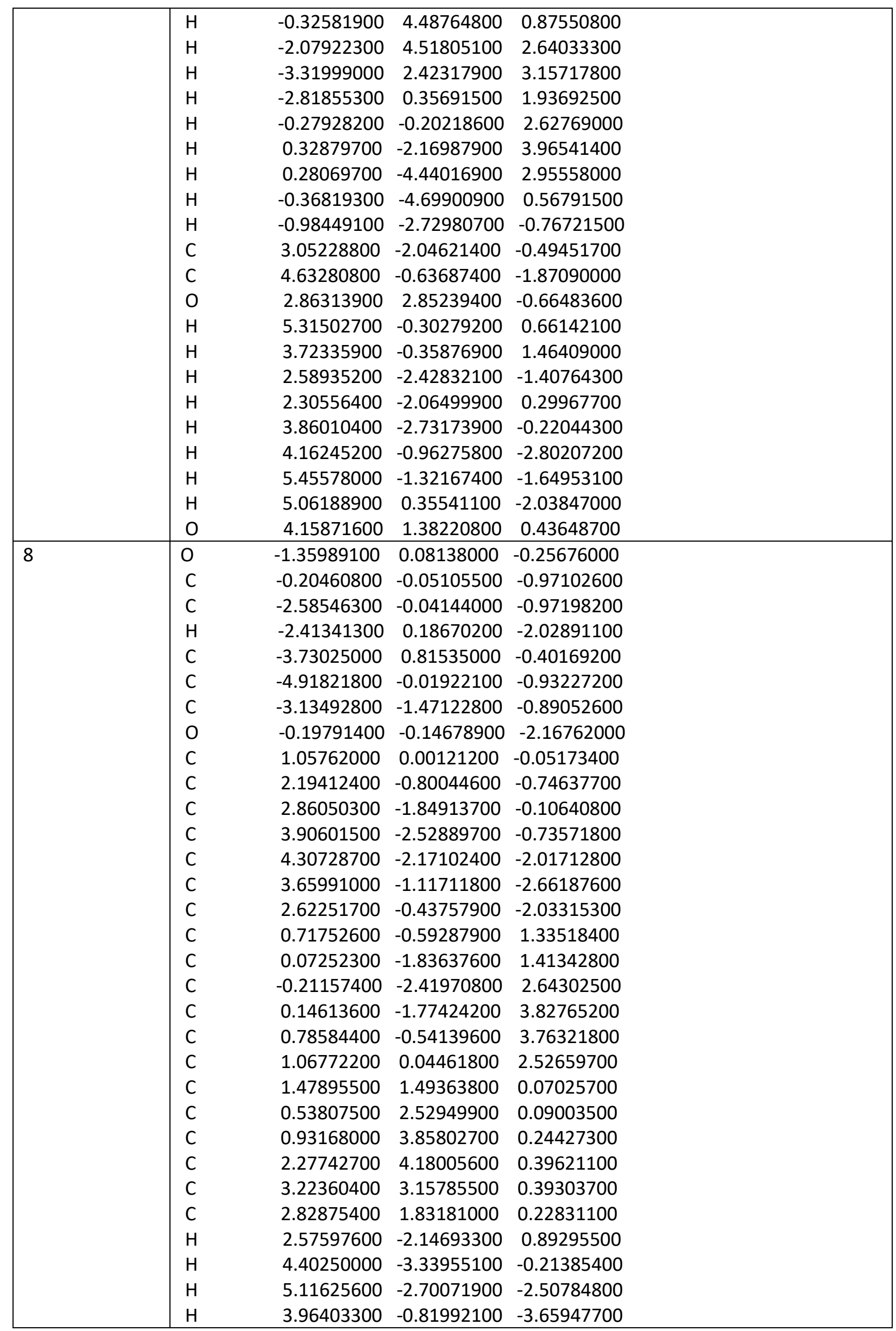




\begin{tabular}{|c|c|c|c|c|}
\hline & $\begin{array}{l}\mathrm{H} \\
\mathrm{H} \\
\mathrm{H} \\
\mathrm{H} \\
\mathrm{H} \\
\mathrm{H} \\
\mathrm{H} \\
\mathrm{H} \\
\mathrm{H} \\
\mathrm{H} \\
\mathrm{H} \\
\mathrm{C} \\
\mathrm{C} \\
\mathrm{O} \\
\mathrm{H} \\
\mathrm{H} \\
\mathrm{H} \\
\mathrm{H} \\
\mathrm{H} \\
\mathrm{H} \\
\mathrm{H} \\
\mathrm{H} \\
\mathrm{O}\end{array}$ & $\begin{array}{r}2.14262400 \\
-0.22391500 \\
-0.71573000 \\
-0.07459200 \\
1.06911600 \\
1.56344700 \\
-0.51595800 \\
0.18108400 \\
2.58486000 \\
4.27612600 \\
3.58110100 \\
-3.72285300 \\
-3.75443300 \\
-2.52278700 \\
-5.17077200 \\
-5.81117200 \\
-2.82295500 \\
-3.75731100 \\
-4.59284600 \\
-3.73505200 \\
-2.89407000 \\
-4.65545900 \\
-4.48906500\end{array}$ & $\begin{array}{c}0.38126100 \\
-2.35398400 \\
-3.37918200 \\
-2.22863600 \\
-0.02527400 \\
1.00597700 \\
2.30559900 \\
4.64084300 \\
5.21282100 \\
3.38899700 \\
1.05399600 \\
0.81686700 \\
2.24485300 \\
-2.49578400 \\
0.23869100 \\
0.05832900 \\
1.29782700 \\
-0.19557400 \\
1.36404900 \\
2.25649800 \\
2.81475800 \\
2.77100300 \\
-1.40378500\end{array}$ & $\begin{array}{r}-2.54974200 \\
0.50835000 \\
2.67392000 \\
4.78715800 \\
4.67401800 \\
2.50417500 \\
-0.00316200 \\
0.24711000 \\
0.51528300 \\
0.51411400 \\
0.22146900 \\
1.13685300 \\
-0.95006400 \\
-0.82381400 \\
-1.96519100 \\
-0.31235500 \\
1.52215400 \\
1.54697200 \\
1.51022900 \\
-2.04338400 \\
-0.59057900 \\
-0.62038000 \\
-0.90327800\end{array}$ \\
\hline 39 & $\begin{array}{l}\text { O } \\
\mathrm{C} \\
\mathrm{C} \\
\mathrm{H} \\
\mathrm{C} \\
\mathrm{C} \\
\mathrm{C} \\
\mathrm{O} \\
\mathrm{C} \\
\mathrm{C} \\
\mathrm{C} \\
\mathrm{C} \\
\mathrm{C} \\
\mathrm{C} \\
\mathrm{C} \\
\mathrm{C} \\
\mathrm{C} \\
\mathrm{C} \\
\mathrm{C} \\
\mathrm{C} \\
\mathrm{C} \\
\mathrm{C} \\
\mathrm{C} \\
\mathrm{C} \\
\mathrm{C} \\
\mathrm{C} \\
\mathrm{C} \\
\mathrm{H} \\
\mathrm{H} \\
\end{array}$ & $\begin{array}{r}1.36960100 \\
0.22530300 \\
2.60018000 \\
2.37364800 \\
3.52219000 \\
4.23671800 \\
3.37914100 \\
0.20759600 \\
-1.04275200 \\
-1.44175800 \\
-2.07182400 \\
-2.49359200 \\
-2.28957200 \\
-1.67234900 \\
-1.26067100 \\
-0.73259000 \\
0.12253500 \\
0.41517900 \\
-0.14414900 \\
-1.00216100 \\
-1.29504200 \\
-2.16554900 \\
-1.89376500 \\
-2.90515200 \\
-4.22006500 \\
-4.50511000 \\
-3.48745300 \\
-2.24010800 \\
-2.97984000 \\
\end{array}$ & $\begin{array}{c}0.12676700 \\
-0.11956500 \\
0.18904700 \\
0.35294100 \\
-1.01869600 \\
-0.54878100 \\
1.37201400 \\
-0.33836200 \\
-0.01842600 \\
1.48672300 \\
2.05621600 \\
3.38456600 \\
4.17398200 \\
3.61615900 \\
2.28501300 \\
-0.52206700 \\
0.20971900 \\
-0.22682200 \\
-1.40798800 \\
-2.13651300 \\
-1.69591700 \\
-0.85846300 \\
-2.12961400 \\
-2.91895500 \\
-2.45581200 \\
-1.19754000 \\
-0.40766700 \\
1.46252400 \\
3.80006900 \\
\end{array}$ & $\begin{array}{c}-0.26369200 \\
-0.94500200 \\
-1.01499300 \\
-2.06603200 \\
-0.75136900 \\
0.53460900 \\
-0.41301000 \\
-2.12840400 \\
-0.04157200 \\
-0.03421700 \\
1.07874700 \\
1.06212100 \\
-0.06672300 \\
-1.18298900 \\
-1.16993200 \\
1.39749100 \\
2.23870000 \\
3.52570400 \\
4.01547600 \\
3.20102800 \\
1.90758400 \\
-0.69937200 \\
-1.22489700 \\
-1.76296800 \\
-1.78882600 \\
-1.26993100 \\
-0.73270400 \\
1.96767900 \\
1.93786900 \\
\end{array}$ \\
\hline
\end{tabular}




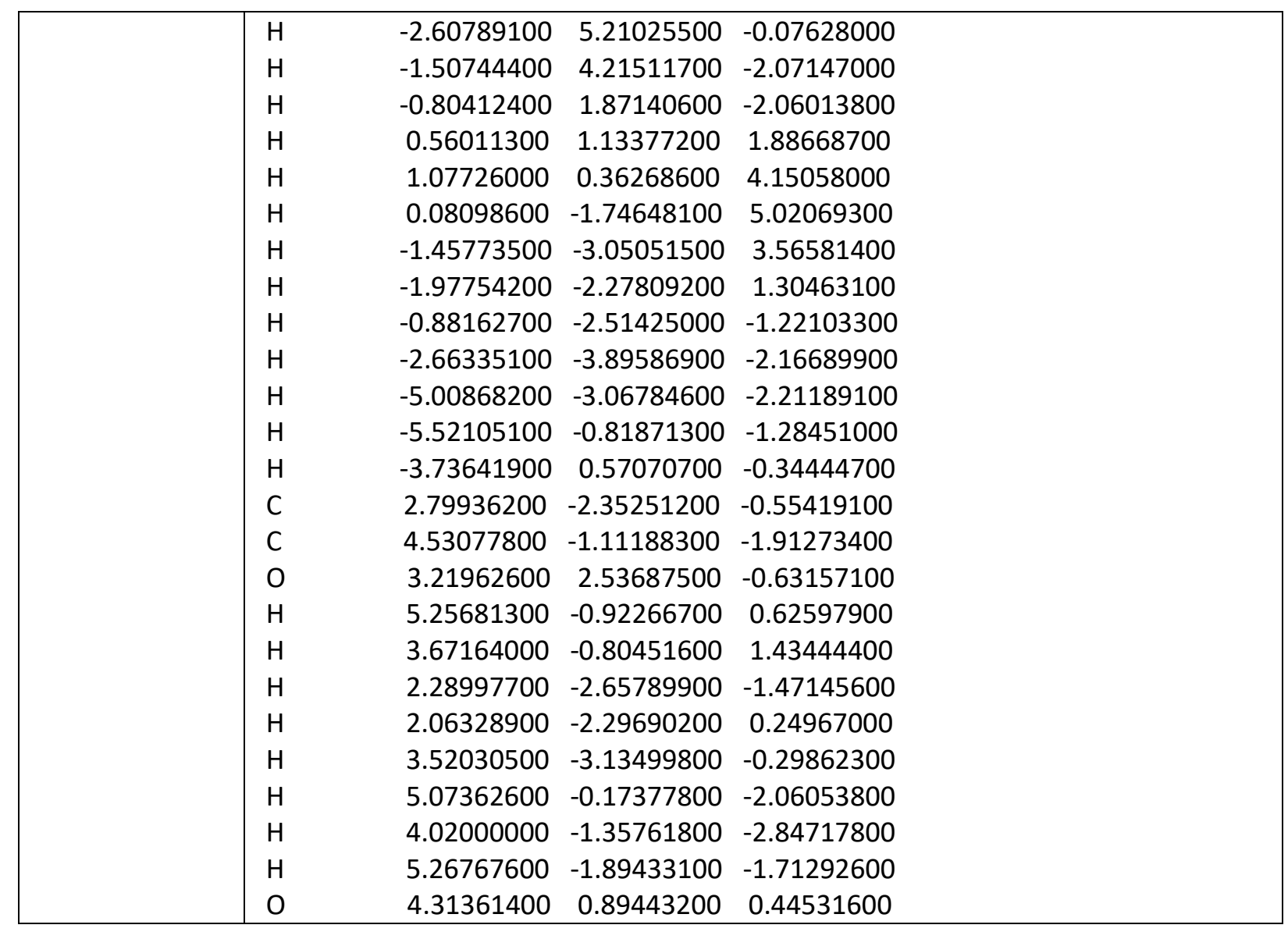

14 (optimized at the M06-2X/6-311++G(d,p) level)

\begin{tabular}{|l|llll|}
\hline Conformer no & & & & \\
\hline 1 & O & -1.25239700 & -0.25287900 & -0.28574500 \\
& C & -0.11629900 & -0.16355100 & -1.01287000 \\
C & -2.47728300 & -0.30731100 & -0.97280000 \\
H & -2.35172100 & 0.04746700 & -2.00202400 \\
& C & -3.58501300 & 0.46218400 & -0.25513300 \\
C & -4.78724200 & -0.27032100 & -0.87124000 \\
& C & -3.03212100 & -1.72571300 & -1.02469600 \\
O & -0.09547500 & -0.19462700 & -2.20730000 \\
C & 1.07303500 & 0.09634800 & -0.06957700 \\
C & 0.67676700 & 1.44538500 & 0.56347900 \\
& C & 0.20137600 & 1.54957000 & 1.86870100 \\
C & -0.26433100 & 2.76879600 & 2.35666400 \\
& C & -0.27149800 & 3.89600200 & 1.54388700 \\
C & 0.18508800 & 3.79658400 & 0.23238900 \\
& C & 0.64951900 & 2.58065600 & -0.25364100 \\
& C & 2.38517300 & 0.15816700 & -0.86254100 \\
C & 2.67921100 & -0.86557800 & -1.76711900 \\
& C & 3.89614000 & -0.89675500 & -2.43223800 \\
& C & 4.85076100 & 0.08874500 & -2.19561800 \\
& C & 4.57684500 & 1.09774900 & -1.28263400 \\
& C & 3.35210400 & 1.13162400 & -0.61894500 \\
& C & 1.23400500 & -1.03823700 & 0.95299100 \\
& C & 2.19056000 & -0.88104000 & 1.96057400 \\
C & 2.43128400 & -1.89346200 & 2.87734500 \\
\hline
\end{tabular}




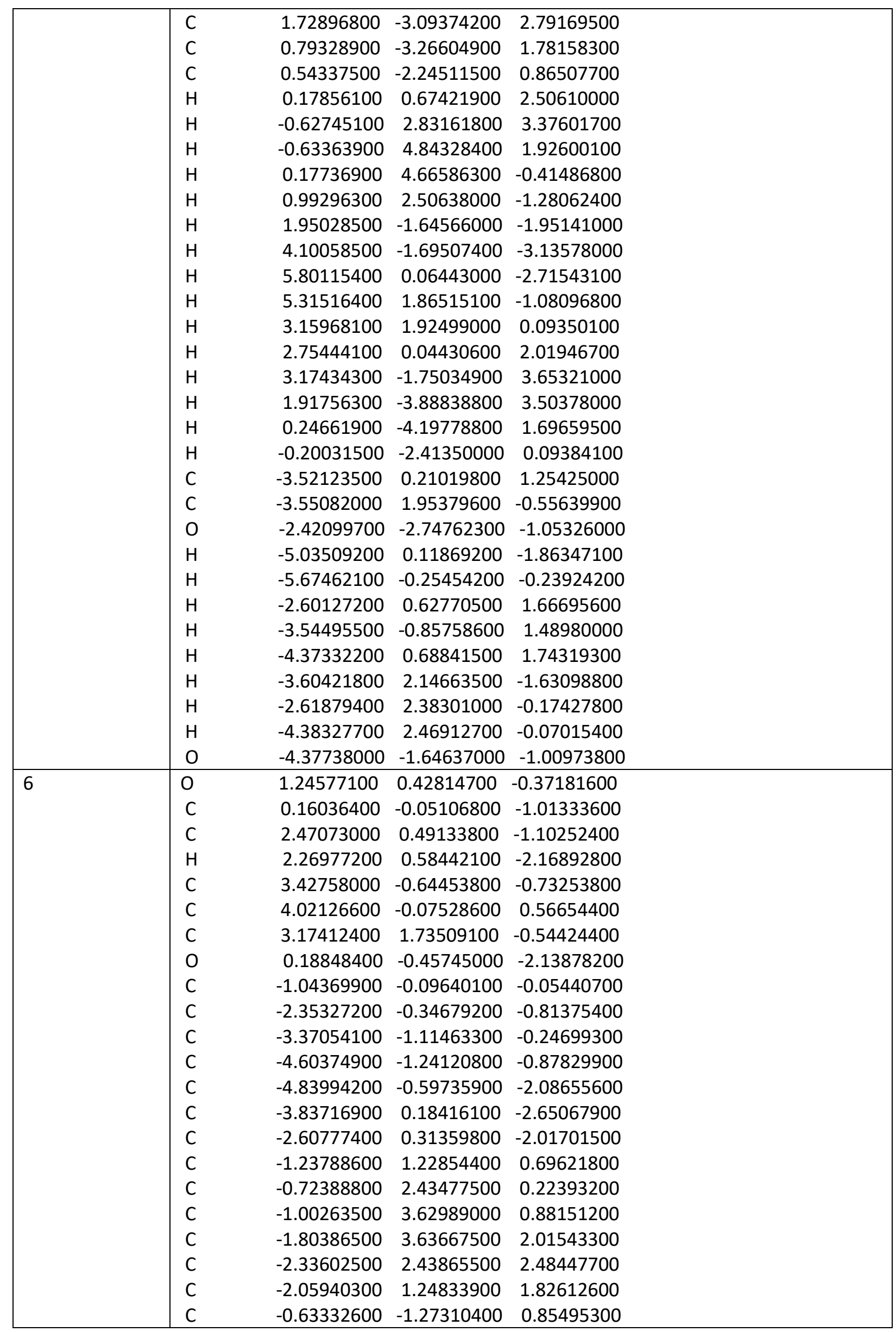




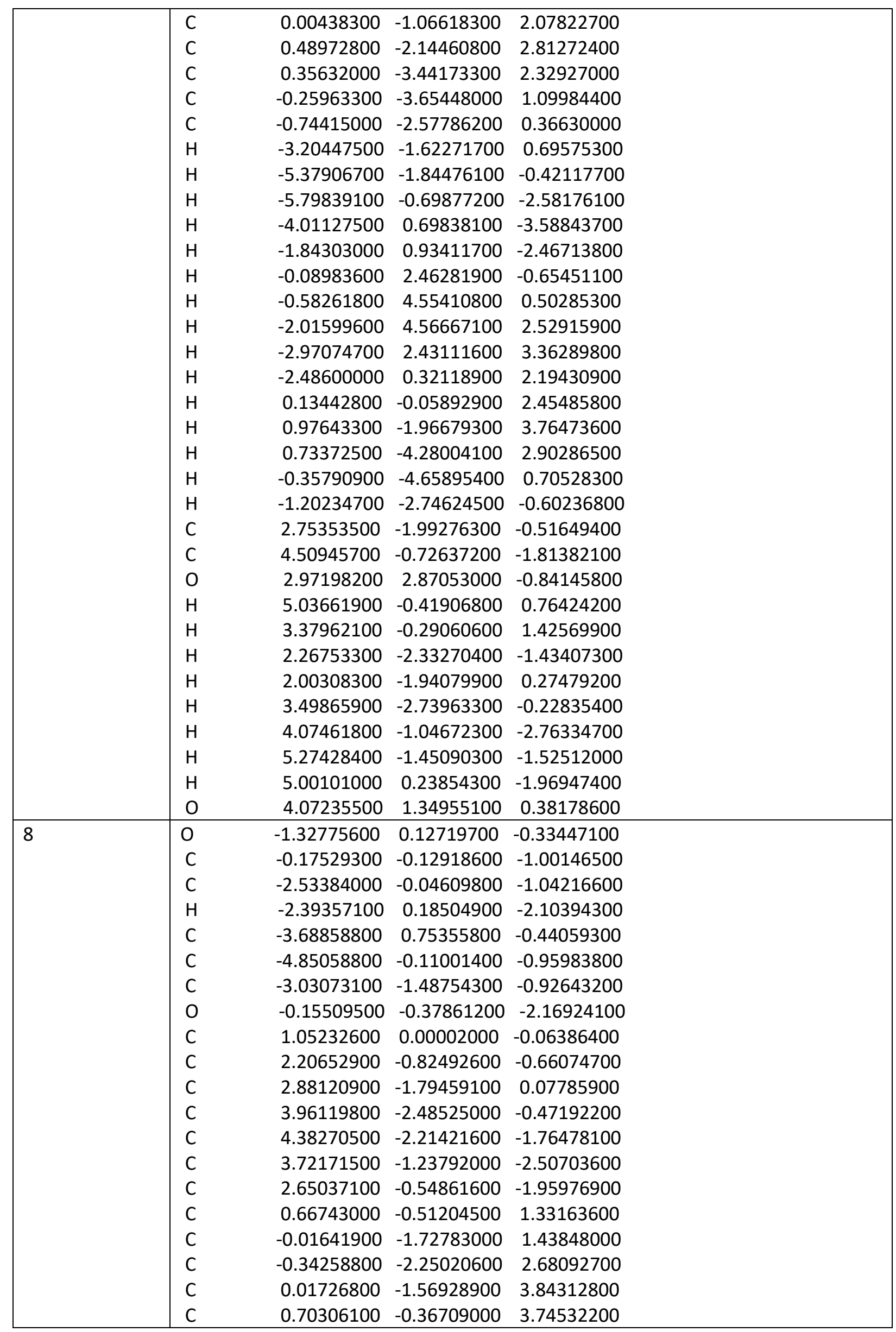




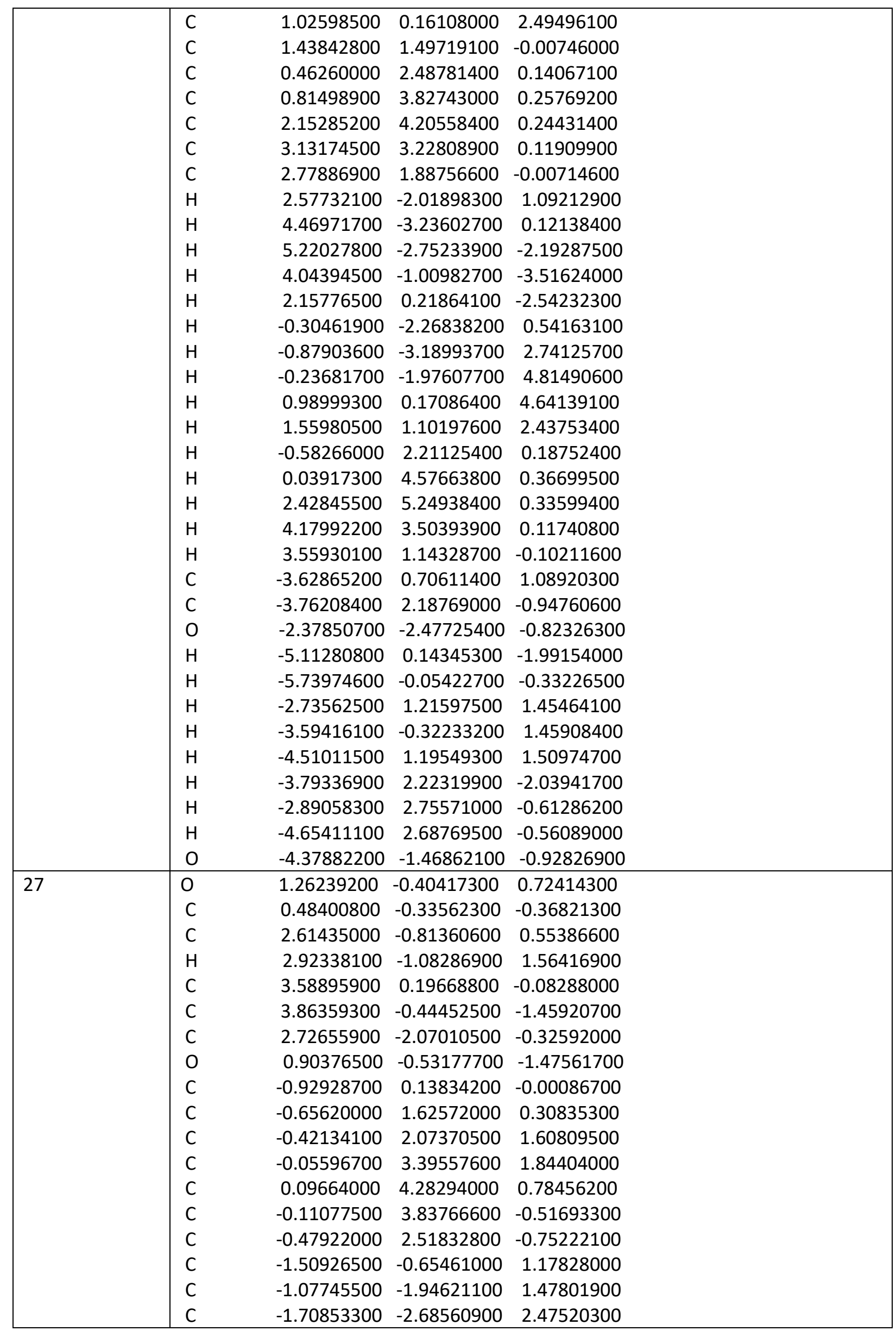




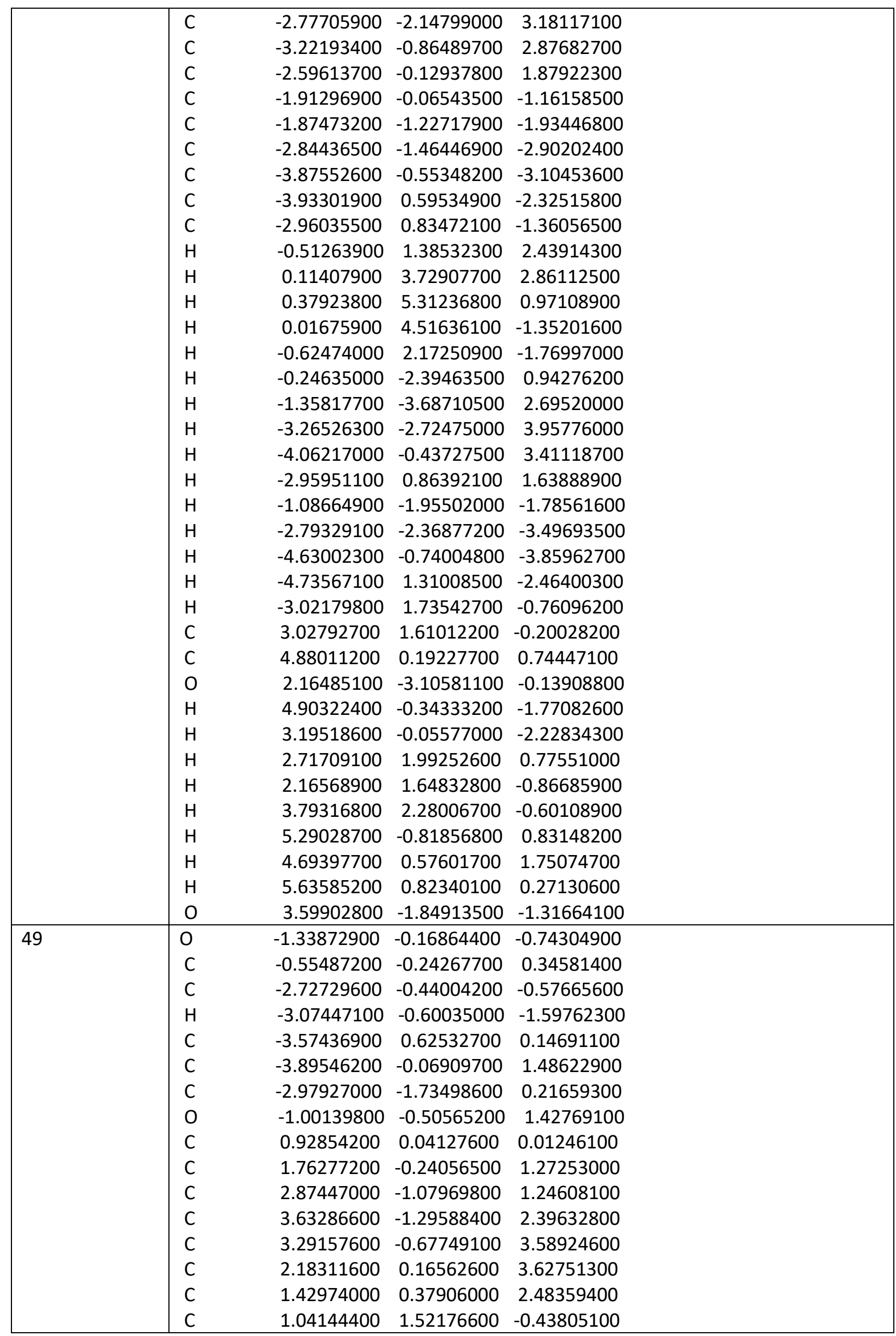




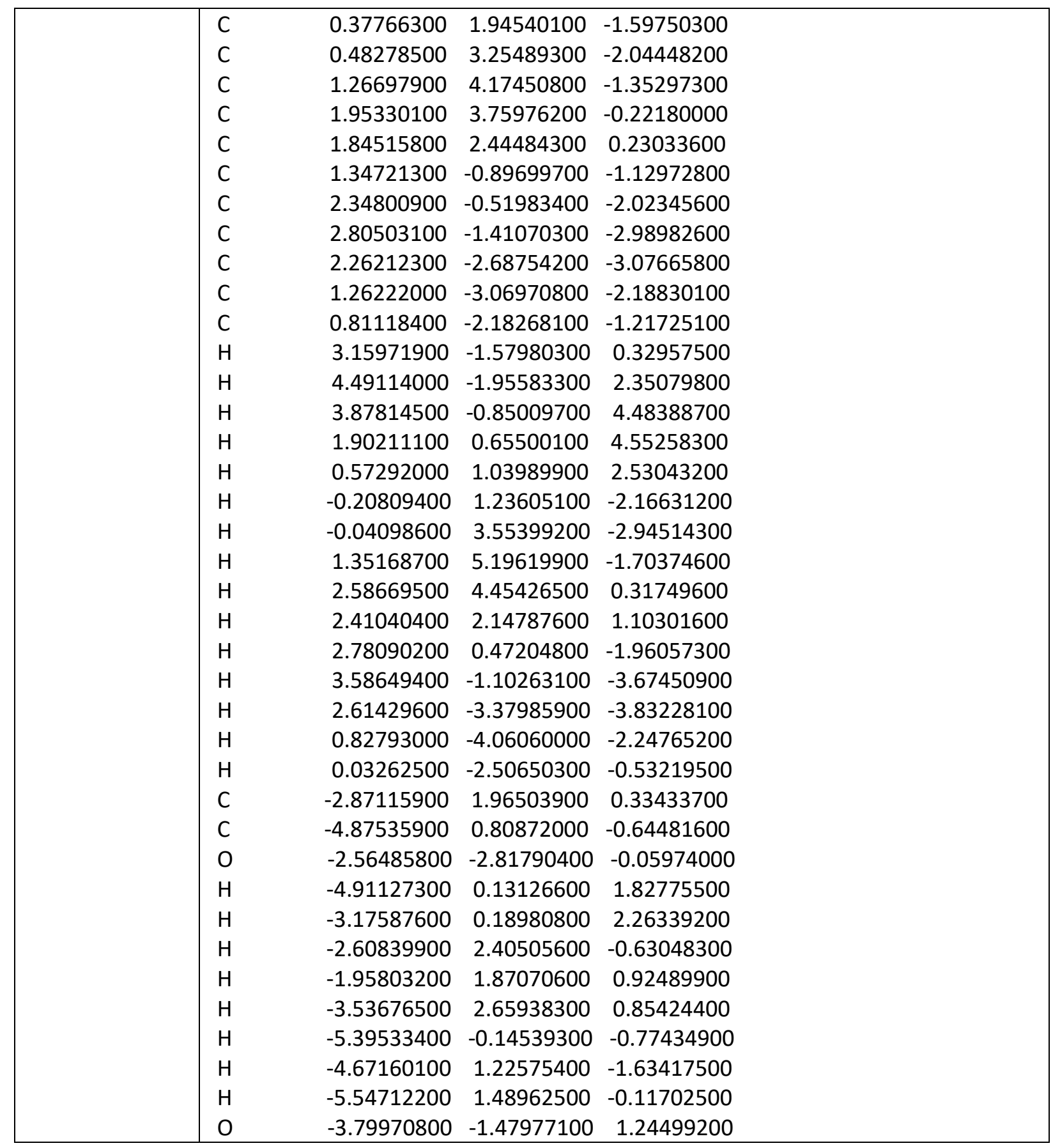

15 (optimized at the B3LYP/6-311++G(d,p) level)

\begin{tabular}{|l|llll|}
\hline Conformer no & & & \\
\hline 1 & O & 1.67283900 & 0.38268200 & -0.64553200 \\
& C & 0.49155800 & -0.01470800 & -1.15977200 \\
& C & 2.81734000 & 0.39610100 & -1.54376500 \\
& H & 2.50496100 & 0.83939200 & -2.48793900 \\
& C & 3.40412500 & -1.00603800 & -1.71332200 \\
& O & 4.23009300 & -1.19593200 & -0.57121700 \\
& C & 3.94332400 & 1.15725000 & -0.82407800 \\
& C & 4.91260900 & 0.04245800 & -0.35658000 \\
& H & 3.52832700 & 1.72510100 & 0.00830400 \\
& O & 0.36262900 & -0.35072500 & -2.30999400 \\
\hline
\end{tabular}




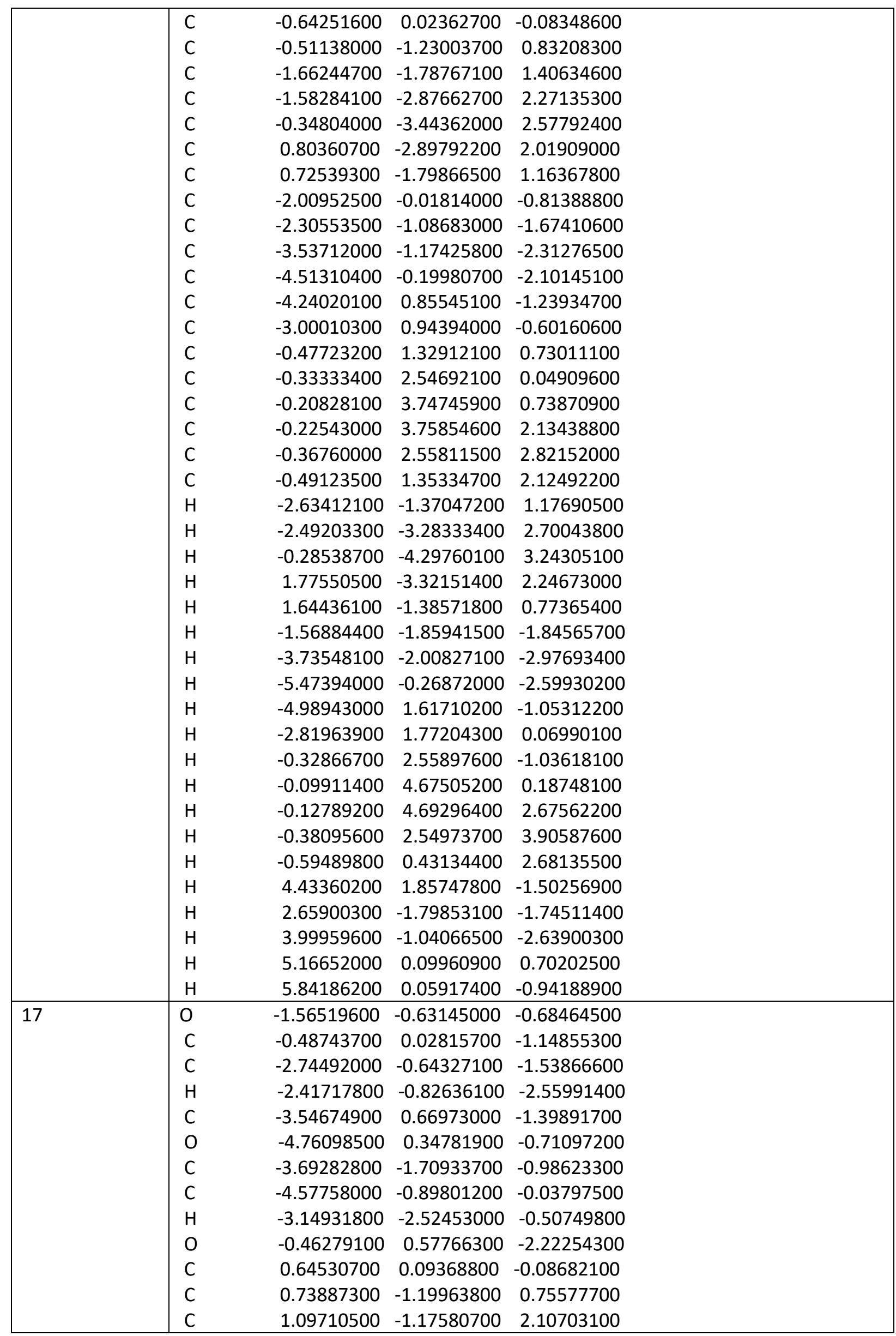




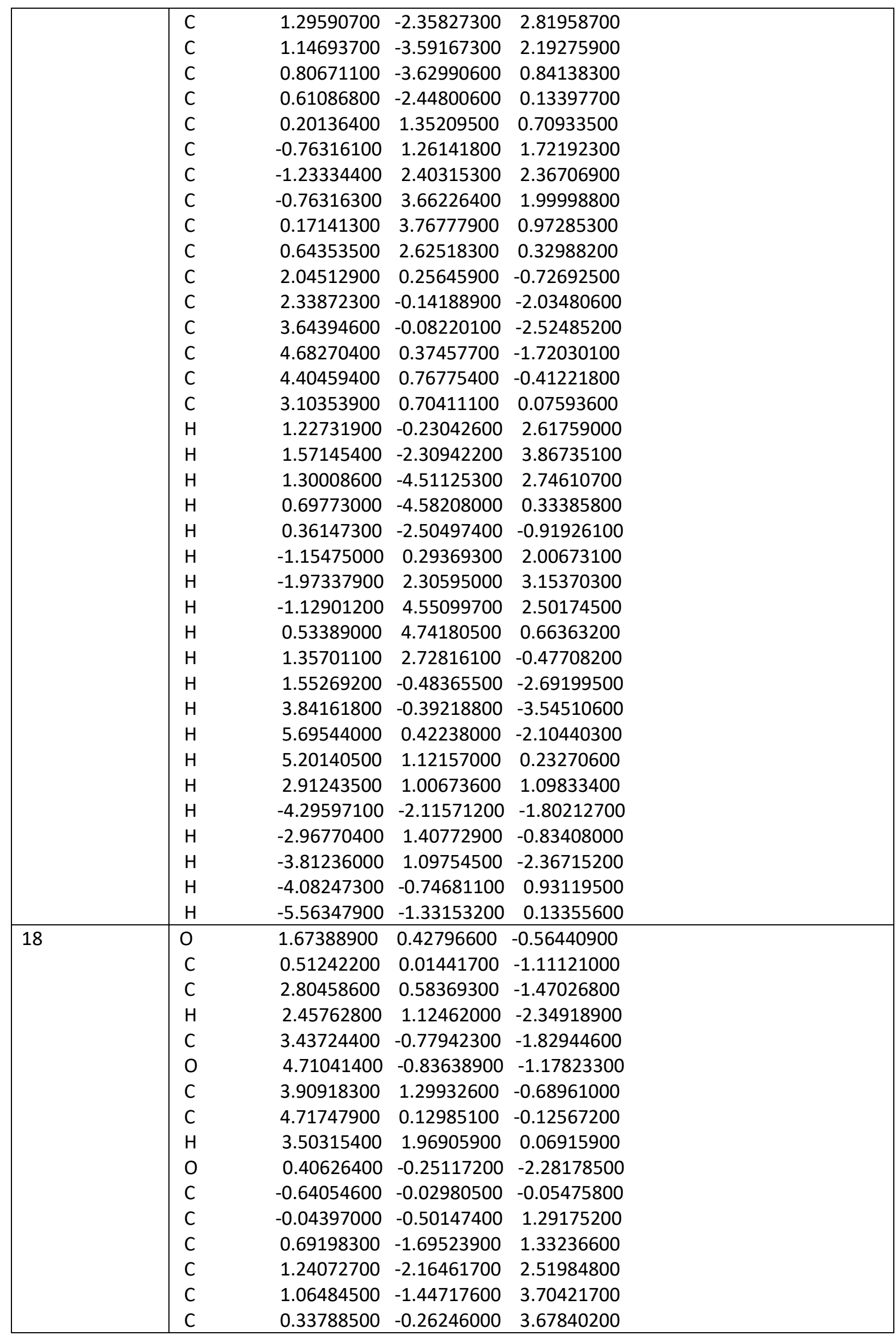




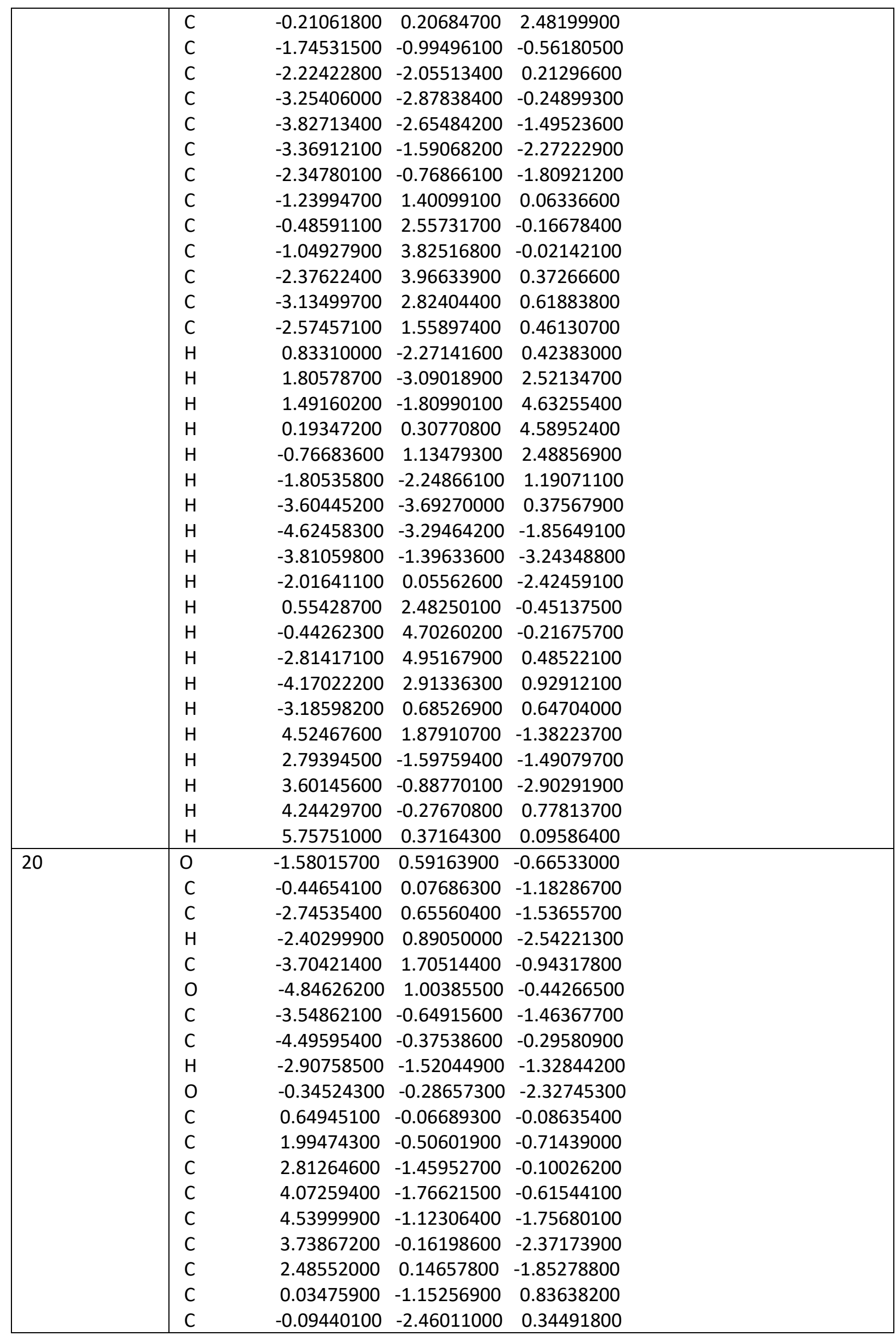




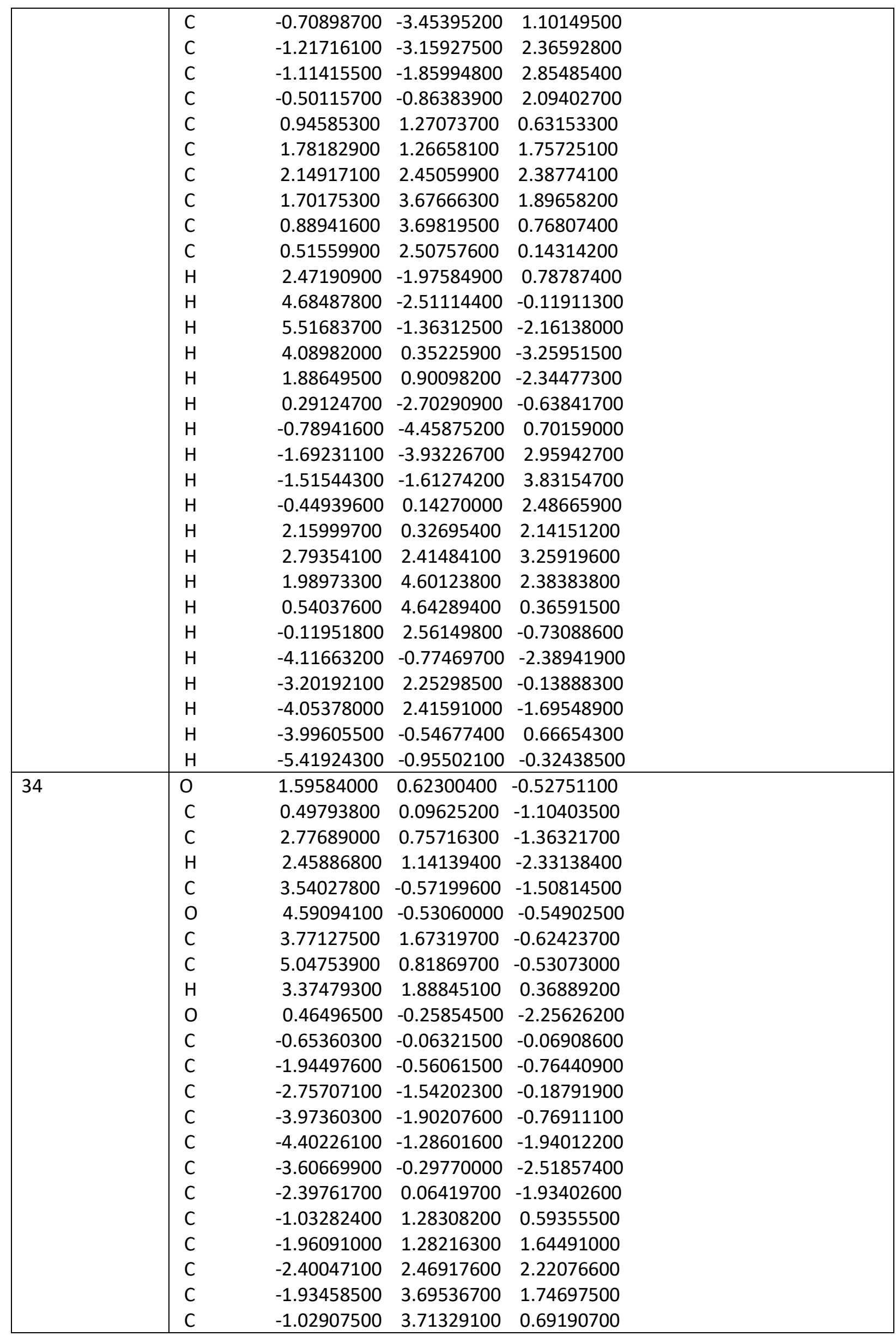




\begin{tabular}{|c|c|c|c|c|}
\hline & $C$ & -0.58239300 & 2.51945700 & 0.12278300 \\
\hline & C & -0.06677600 & -1.10384800 & 0.92050000 \\
\hline & $\mathrm{C}$ & 0.35324000 & -0.77074500 & 2.21034100 \\
\hline & C & 0.94356500 & -1.72624300 & 3.03789900 \\
\hline & $\mathrm{C}$ & 1.13890200 & -3.02776200 & 2.58594900 \\
\hline & $\mathrm{C}$ & 0.74641600 & -3.36619000 & 1.29170400 \\
\hline & $\mathrm{C}$ & 0.15382100 & -2.41324500 & 0.46837600 \\
\hline & $\mathrm{H}$ & -2.44595200 & -2.03786700 & 0.72235700 \\
\hline & $\mathrm{H}$ & -4.58235300 & -2.66759600 & -0.30050100 \\
\hline & $\mathrm{H}$ & -5.34496600 & -1.56770500 & -2.39566900 \\
\hline & $\mathrm{H}$ & -3.92835600 & 0.19620700 & -3.42882400 \\
\hline & $\mathrm{H}$ & -1.80278700 & 0.83933300 & -2.39802500 \\
\hline & $\mathrm{H}$ & -2.35397000 & 0.34196300 & 2.01207300 \\
\hline & $\mathrm{H}$ & -3.11546600 & 2.43574200 & 3.03538400 \\
\hline & $\mathrm{H}$ & -2.27979600 & 4.62226800 & 2.19075800 \\
\hline & $\mathrm{H}$ & -0.66322800 & 4.65750900 & 0.30370700 \\
\hline & $\mathrm{H}$ & 0.12512600 & 2.57180400 & -0.69369200 \\
\hline & $\mathrm{H}$ & 0.23048000 & 0.23968300 & 2.57597600 \\
\hline & $\mathrm{H}$ & 1.25738700 & -1.44461600 & 4.03702100 \\
\hline & $\mathrm{H}$ & 1.60004700 & -3.76826600 & 3.22965100 \\
\hline & $\mathrm{H}$ & 0.90288400 & -4.37275600 & 0.91994000 \\
\hline & $\mathrm{H}$ & -0.14326400 & -2.69015700 & -0.53673500 \\
\hline & $\mathrm{H}$ & 3.93271400 & 2.61871900 & -1.14452900 \\
\hline & $\mathrm{H}$ & 2.92326700 & -1.44717400 & -1.31223800 \\
\hline & $\mathrm{H}$ & 3.95377100 & -0.64523800 & -2.52479500 \\
\hline & $\mathrm{H}$ & 5.61269600 & 0.97079500 & 0.38878500 \\
\hline & $\mathrm{H}$ & 5.71212400 & 1.00445100 & -1.38808600 \\
\hline \multirow[t]{25}{*}{47} & 0 & -1.72450400 & 0.10735100 & -0.43909300 \\
\hline & $\mathrm{C}$ & -0.54228200 & 0.03871800 & -1.08428300 \\
\hline & $\mathrm{C}$ & -2.91570900 & 0.30849800 & -1.25152000 \\
\hline & $\mathrm{H}$ & -2.65557900 & 0.99397100 & -2.05560200 \\
\hline & $\mathrm{C}$ & -4.01775800 & 0.83976500 & -0.31364100 \\
\hline & 0 & -5.03073900 & -0.16867100 & -0.23505200 \\
\hline & $\mathrm{C}$ & -3.48841600 & -1.01960500 & -1.76430200 \\
\hline & $\mathrm{C}$ & -4.45417800 & -1.40925500 & -0.64494400 \\
\hline & $\mathrm{H}$ & -2.71074800 & -1.75377400 & -1.97469500 \\
\hline & 0 & -0.44407200 & 0.04673600 & -2.28541600 \\
\hline & $\mathrm{C}$ & 0.64671500 & 0.06715100 & -0.07849400 \\
\hline & $\mathrm{C}$ & 0.57642000 & -1.07483800 & 0.96283900 \\
\hline & $\mathrm{C}$ & -0.31215400 & -2.14857800 & 0.86340300 \\
\hline & $\mathrm{C}$ & -0.27429500 & -3.20263200 & 1.77792700 \\
\hline & $\mathrm{C}$ & 0.65345800 & -3.20380200 & 2.81351000 \\
\hline & $\mathrm{C}$ & 1.55385600 & -2.14390300 & 2.91930000 \\
\hline & $\mathrm{C}$ & 1.51929100 & -1.10021300 & 2.00104600 \\
\hline & $\mathrm{C}$ & 0.51150800 & 1.48399500 & 0.54081300 \\
\hline & $\mathrm{C}$ & 0.09182000 & 1.70066600 & 1.85549200 \\
\hline & $\mathrm{C}$ & -0.08420600 & 2.99553900 & 2.34492000 \\
\hline & $\mathrm{C}$ & 0.14139100 & 4.09697100 & 1.52465200 \\
\hline & $\mathrm{C}$ & 0.53746300 & 3.89302200 & 0.20334900 \\
\hline & $\mathrm{C}$ & 0.71668600 & 2.60116100 & -0.28248300 \\
\hline & $\mathrm{C}$ & 1.98698800 & -0.13380500 & -0.82737800 \\
\hline & $\mathrm{C}$ & 3.11595100 & 0.63770500 & -0.53736800 \\
\hline
\end{tabular}




\begin{tabular}{|c|c|c|c|c|}
\hline & $C$ & 4.34330800 & 0.37489100 & -1.14766900 \\
\hline & C & 4.46481900 & -0.66751100 & -2.06013900 \\
\hline & $\mathrm{C}$ & 3.34866300 & -1.45223700 & -2.34863600 \\
\hline & C & 2.12830600 & -1.19211500 & -1.73459200 \\
\hline & $\mathrm{H}$ & -1.05060700 & -2.17720100 & 0.07433100 \\
\hline & $\mathrm{H}$ & -0.97617800 & -4.02282300 & 1.67457000 \\
\hline & $\mathrm{H}$ & 0.68148700 & -4.02097500 & 3.52528300 \\
\hline & $\mathrm{H}$ & 2.29198900 & -2.13305500 & 3.71361400 \\
\hline & $\mathrm{H}$ & 2.24292300 & -0.29869200 & 2.08721600 \\
\hline & $\mathrm{H}$ & -0.10771700 & 0.86005900 & 2.50607200 \\
\hline & $\mathrm{H}$ & -0.40472100 & 3.13777500 & 3.37113300 \\
\hline & $\mathrm{H}$ & 0.00456800 & 5.10242000 & 1.90655600 \\
\hline & $\mathrm{H}$ & 0.70787400 & 4.73999100 & -0.45204900 \\
\hline & $\mathrm{H}$ & 1.02072400 & 2.45862700 & -1.31308900 \\
\hline & $\mathrm{H}$ & 3.04823100 & 1.45553500 & 0.16814400 \\
\hline & $\mathrm{H}$ & 5.20220100 & 0.99094100 & -0.90483000 \\
\hline & $\mathrm{H}$ & 5.41635200 & -0.87066600 & -2.53852200 \\
\hline & $\mathrm{H}$ & 3.42757400 & -2.27235200 & -3.05368900 \\
\hline & $\mathrm{H}$ & 1.27935300 & -1.82065000 & -1.96891400 \\
\hline & $\mathrm{H}$ & -4.04279500 & -0.83642200 & -2.68856800 \\
\hline & $\mathrm{H}$ & -3.59175800 & 1.04342400 & 0.67411400 \\
\hline & $\mathrm{H}$ & -4.48478600 & 1.74982200 & -0.69528700 \\
\hline & $\mathrm{H}$ & -3.92338000 & -1.88302700 & 0.19282900 \\
\hline & $\mathrm{H}$ & -5.26636200 & -2.06378000 & -0.96294200 \\
\hline \multirow[t]{28}{*}{54} & 0 & -1.66055400 & 0.37054700 & -0.62840400 \\
\hline & $\mathrm{C}$ & -0.48930100 & -0.04695900 & -1.14691400 \\
\hline & $\mathrm{C}$ & -2.81138800 & 0.38106000 & -1.51619100 \\
\hline & $\mathrm{H}$ & -2.50206800 & 0.82915400 & -2.45941100 \\
\hline & $\mathrm{C}$ & -3.91607900 & 1.16282400 & -0.80528000 \\
\hline & 0 & -4.66319700 & 0.19465400 & -0.07949200 \\
\hline & $\mathrm{C}$ & -3.42318700 & -1.02777400 & -1.68951900 \\
\hline & $\mathrm{C}$ & -4.77328500 & -0.93604900 & -0.94513000 \\
\hline & $\mathrm{H}$ & -2.76628000 & -1.77280000 & -1.24068100 \\
\hline & 0 & -0.37768800 & -0.39749700 & -2.29458900 \\
\hline & $\mathrm{C}$ & 0.65838300 & -0.00639800 & -0.08114100 \\
\hline & $\mathrm{C}$ & 2.01976000 & -0.13084700 & -0.81043700 \\
\hline & $\mathrm{C}$ & 3.07110000 & 0.75502600 & -0.55670400 \\
\hline & $\mathrm{C}$ & 4.30963600 & 0.60701400 & -1.18497100 \\
\hline & $\mathrm{C}$ & 4.52282100 & -0.43378200 & -2.08112400 \\
\hline & $\mathrm{C}$ & 3.48720100 & -1.33295300 & -2.33497500 \\
\hline & $\mathrm{C}$ & 2.25566700 & -1.18637000 & -1.70555100 \\
\hline & $\mathrm{C}$ & 0.45138400 & -1.20187200 & 0.90031700 \\
\hline & $\mathrm{C}$ & 1.51827900 & -2.03456600 & 1.25944700 \\
\hline & $\mathrm{C}$ & 1.34775100 & -3.07347400 & 2.17504600 \\
\hline & $\mathrm{C}$ & 0.10537400 & -3.30541900 & 2.75586300 \\
\hline & $\mathrm{C}$ & -0.96258500 & -2.47600900 & 2.42018400 \\
\hline & $\mathrm{C}$ & -0.79153600 & -1.43532200 & 1.51039200 \\
\hline & $\mathrm{C}$ & 0.56608000 & 1.34604700 & 0.66477400 \\
\hline & $\mathrm{C}$ & 0.70792400 & 1.44742300 & 2.04921100 \\
\hline & $\mathrm{C}$ & 0.67520500 & 2.69193100 & 2.68265400 \\
\hline & $\mathrm{C}$ & 0.49914600 & 3.85549400 & 1.94139800 \\
\hline & $\mathrm{C}$ & 0.36209500 & 3.76733800 & 0.55542000 \\
\hline
\end{tabular}




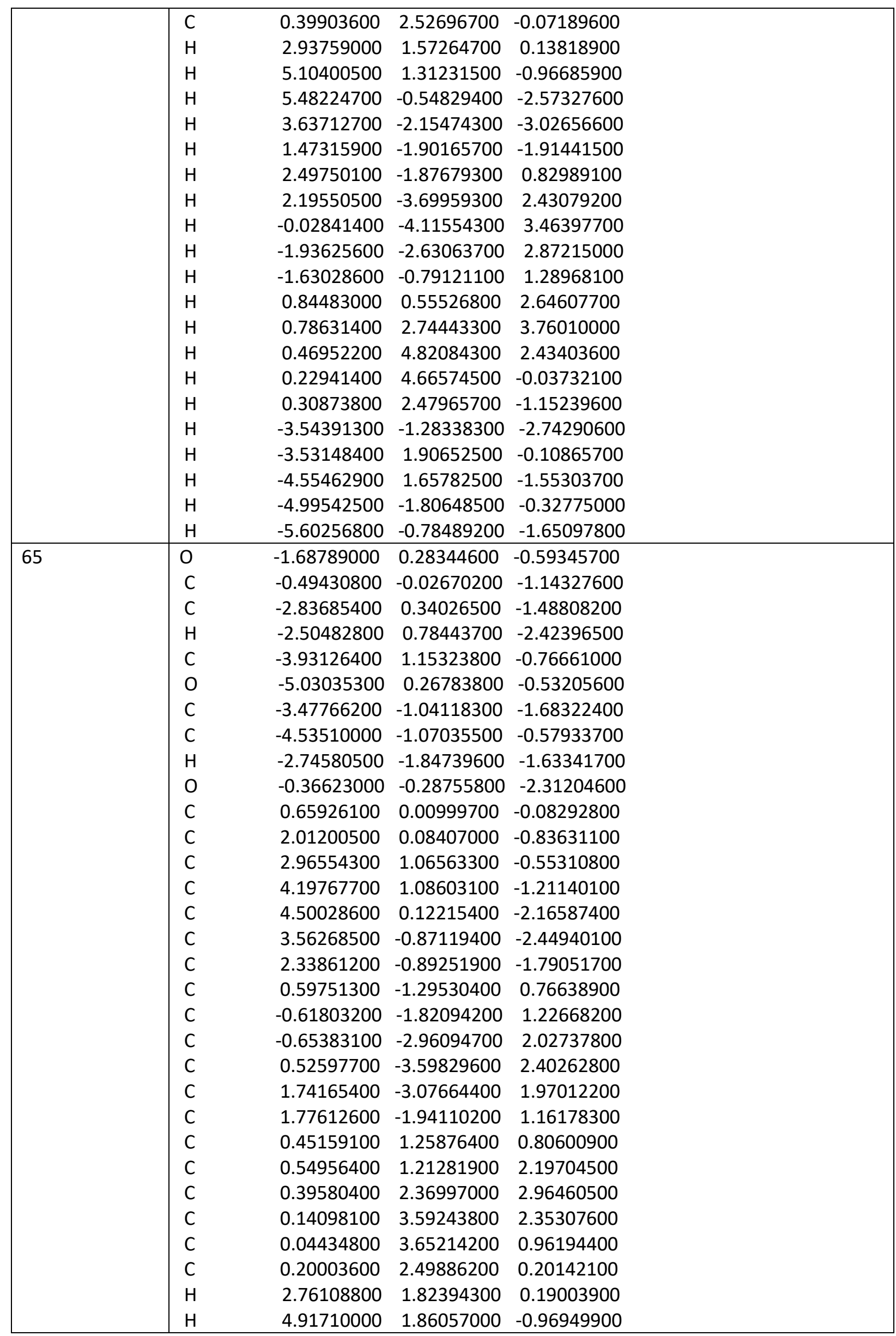




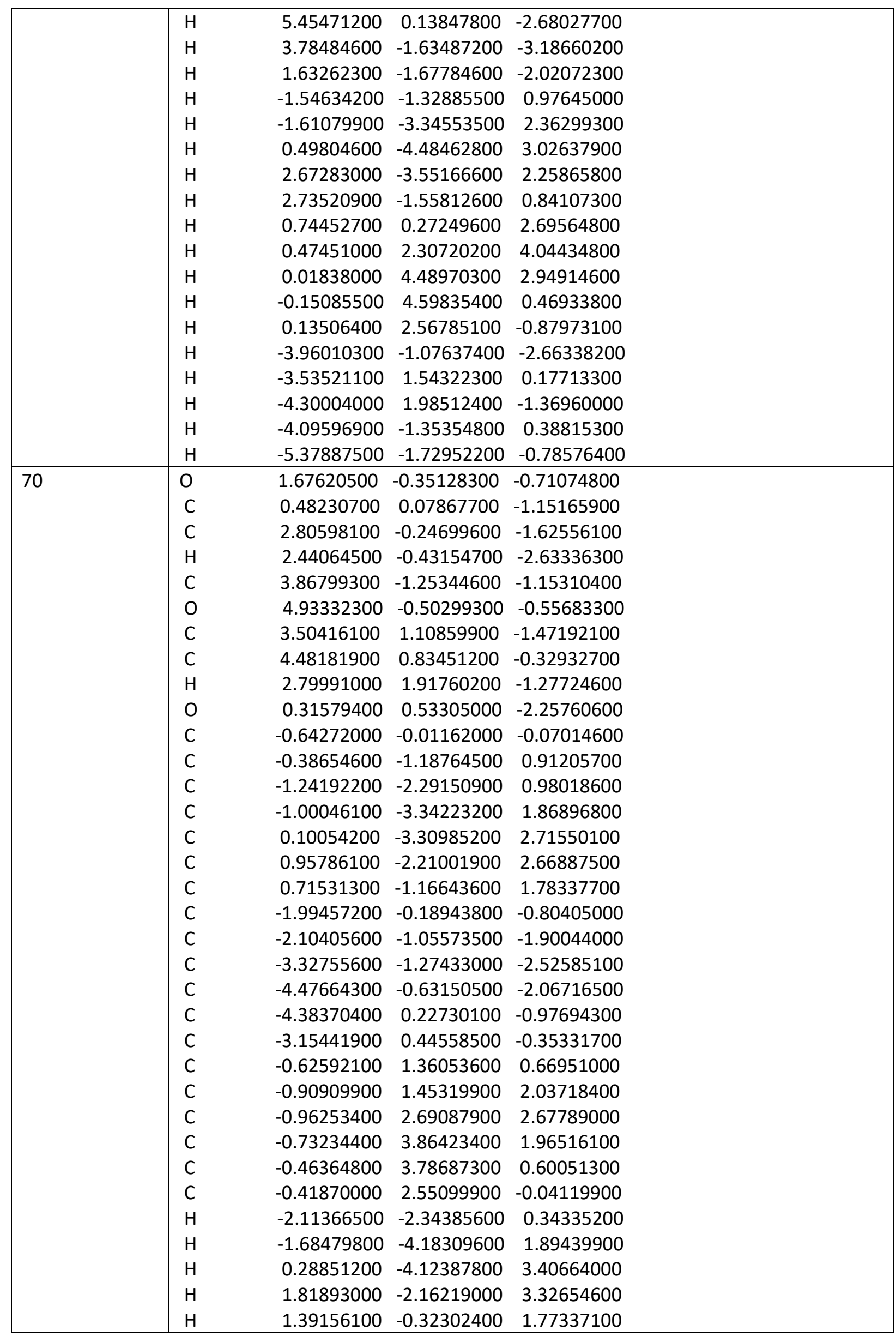




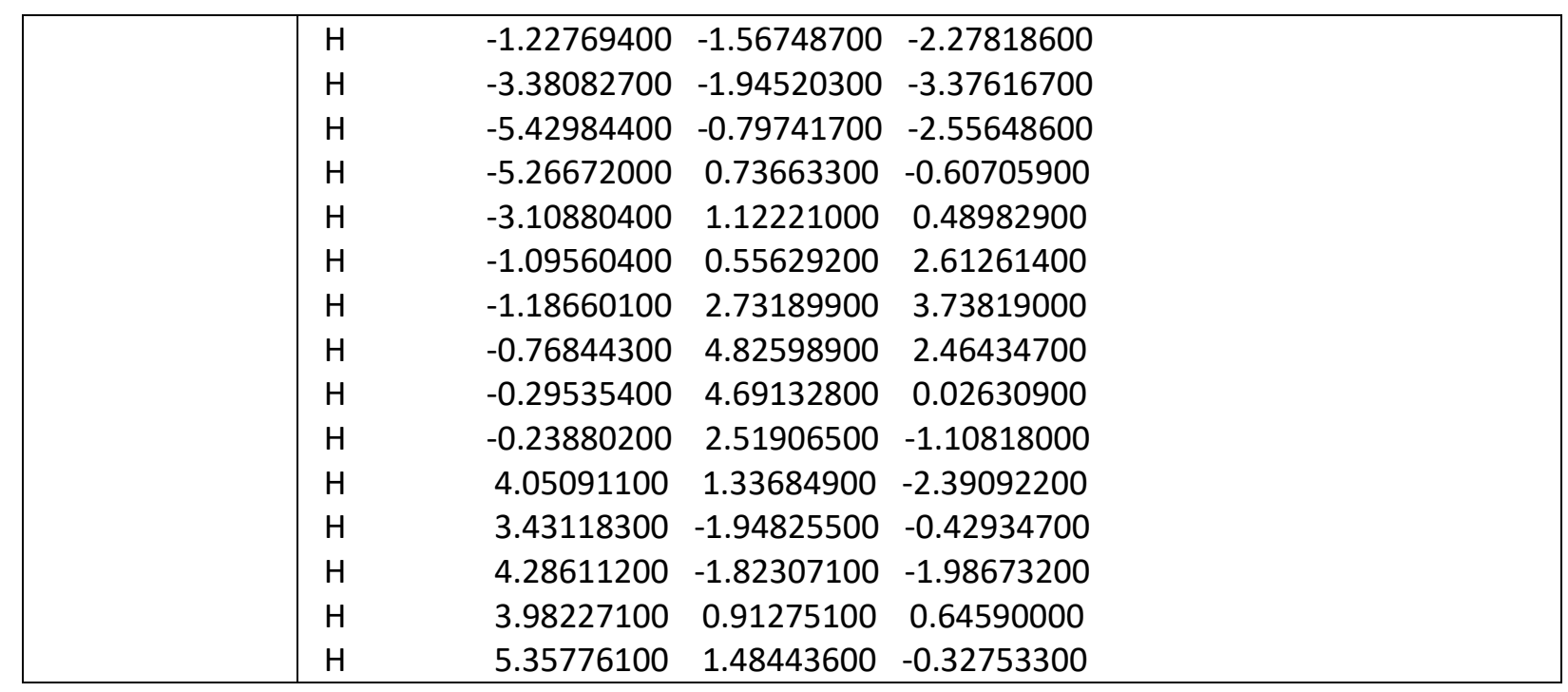

15 (optimized at the M06-2X/6-311++G(d,p) level)

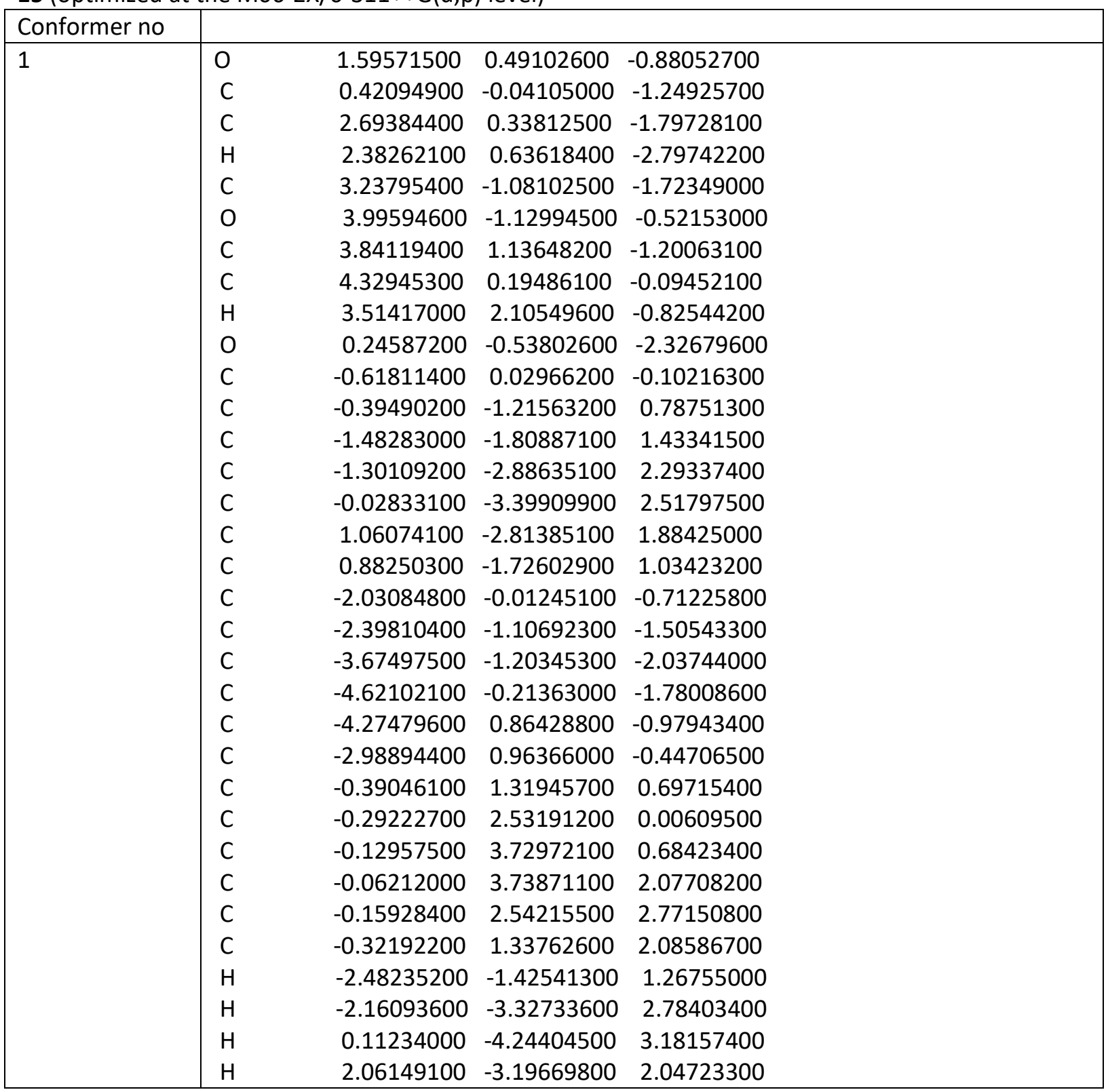




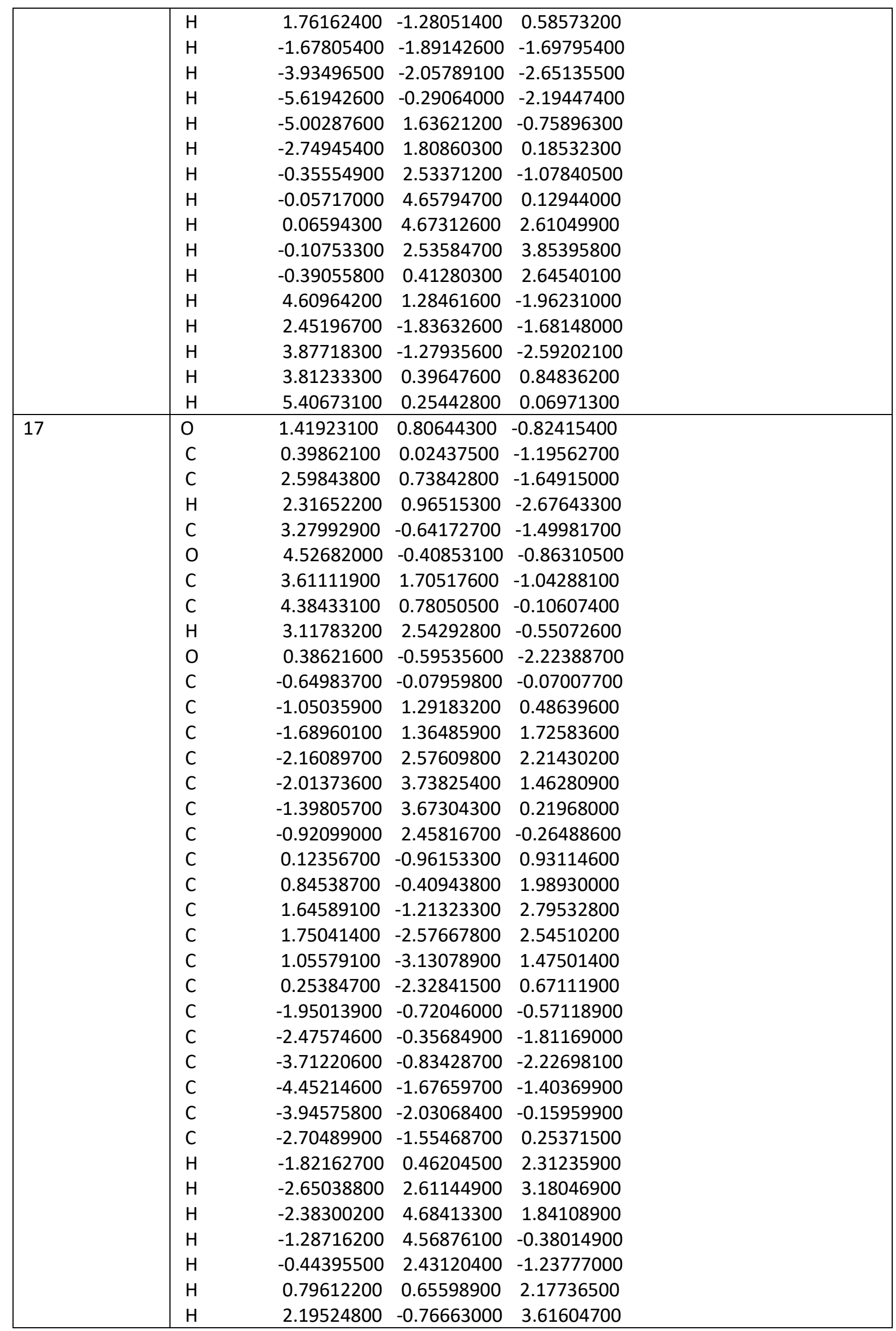




\begin{tabular}{|c|c|c|c|c|}
\hline & $\mathrm{H}$ & 2.37686900 & -3.20063300 & 3.17160800 \\
\hline & $\mathrm{H}$ & 1.14227900 & -4.18902600 & 1.25877000 \\
\hline & $\mathrm{H}$ & -0.26714200 & -2.76372900 & -0.17465900 \\
\hline & $\mathrm{H}$ & -1.91570400 & 0.30133000 & -2.46385400 \\
\hline & $\mathrm{H}$ & -4.09769400 & -0.54544700 & -3.19761200 \\
\hline & $\mathrm{H}$ & -5.41639100 & -2.04923700 & -1.72842500 \\
\hline & $\mathrm{H}$ & -4.51534100 & -2.67922400 & 0.49581400 \\
\hline & $\mathrm{H}$ & -2.32500500 & -1.84181400 & 1.22742700 \\
\hline & $\mathrm{H}$ & 4.27788100 & 2.07931900 & -1.82183600 \\
\hline & $\mathrm{H}$ & 2.65061400 & -1.29330600 & -0.88031700 \\
\hline & $\mathrm{H}$ & 3.46640200 & -1.12927300 & -2.45589600 \\
\hline & $\mathrm{H}$ & 3.80847700 & 0.58516700 & 0.80903900 \\
\hline & $\mathrm{H}$ & 5.37925200 & 1.13583800 & 0.15839900 \\
\hline \multirow{39}{*}{18} & 0 & 1.57599300 & 0.57737000 & -0.87419300 \\
\hline & $\mathrm{C}$ & 0.42137600 & 0.00473900 & -1.24658600 \\
\hline & $\mathrm{C}$ & 2.67416100 & 0.51035400 & -1.80094800 \\
\hline & $\mathrm{H}$ & 2.36814300 & 0.90393900 & -2.76979600 \\
\hline & $\mathrm{C}$ & 3.21942000 & -0.91048900 & -1.86331600 \\
\hline & 0 & 3.99320200 & -1.07683700 & -0.68303300 \\
\hline & $\mathrm{C}$ & 3.82136600 & 1.24039000 & -1.11909700 \\
\hline & $\mathrm{C}$ & 4.27060200 & 0.19638200 & -0.09327200 \\
\hline & $\mathrm{H}$ & 3.50505300 & 2.17980200 & -0.66701300 \\
\hline & 0 & 0.23763600 & -0.42094000 & -2.35225700 \\
\hline & $\mathrm{C}$ & -0.60738500 & -0.00897100 & -0.08486200 \\
\hline & $\mathrm{C}$ & 0.14733900 & -0.18266200 & 1.24195400 \\
\hline & $\mathrm{C}$ & 1.14627300 & -1.15824900 & 1.33559400 \\
\hline & $\mathrm{C}$ & 1.82107400 & -1.37768200 & 2.52668400 \\
\hline & $\mathrm{C}$ & 1.50246600 & -0.62651700 & 3.65753000 \\
\hline & $\mathrm{C}$ & 0.51035600 & 0.33975700 & 3.57657000 \\
\hline & $\mathrm{C}$ & -0.16225700 & 0.56302000 & 2.37470800 \\
\hline & $\mathrm{C}$ & -1.58747200 & -1.17161100 & -0.32257000 \\
\hline & $\mathrm{C}$ & -1.79074500 & -2.17556900 & 0.62188600 \\
\hline & $\mathrm{C}$ & -2.71711500 & -3.19308800 & 0.39271700 \\
\hline & $\mathrm{C}$ & -3.45463000 & -3.21844400 & -0.78106400 \\
\hline & $\mathrm{C}$ & -3.26848200 & -2.21127100 & -1.72588700 \\
\hline & $\mathrm{C}$ & -2.34923700 & -1.19827700 & -1.49743300 \\
\hline & $\mathrm{C}$ & -1.36265400 & 1.33766600 & -0.11118300 \\
\hline & $\mathrm{C}$ & -0.69967300 & 2.53277600 & -0.40022700 \\
\hline & $\mathrm{C}$ & -1.37164800 & 3.74970200 & -0.35694300 \\
\hline & $\mathrm{C}$ & -2.71630500 & 3.79811500 & -0.00903900 \\
\hline & $\mathrm{C}$ & -3.38118700 & 2.61645700 & 0.29702800 \\
\hline & $\mathrm{C}$ & -2.71220100 & 1.39789100 & 0.24344000 \\
\hline & $\mathrm{H}$ & 1.40977800 & -1.75151100 & 0.46455000 \\
\hline & $\mathrm{H}$ & 2.59914100 & -2.13099100 & 2.56948700 \\
\hline & $\mathrm{H}$ & 2.02703800 & -0.79535600 & 4.59054700 \\
\hline & $\mathrm{H}$ & 0.25384900 & 0.93093000 & 4.44806600 \\
\hline & $\mathrm{H}$ & -0.92738700 & 1.32833800 & 2.32901100 \\
\hline & $\mathrm{H}$ & -1.23612200 & -2.17291500 & 1.55104100 \\
\hline & $\mathrm{H}$ & -2.85765400 & -3.96276500 & 1.14267300 \\
\hline & $\mathrm{H}$ & -4.17402200 & -4.00911400 & -0.95918600 \\
\hline & $\mathrm{H}$ & -3.84433400 & -2.21358300 & -2.64393400 \\
\hline & $\mathrm{H}$ & -2.22313000 & -0.41533600 & -2.23388200 \\
\hline
\end{tabular}




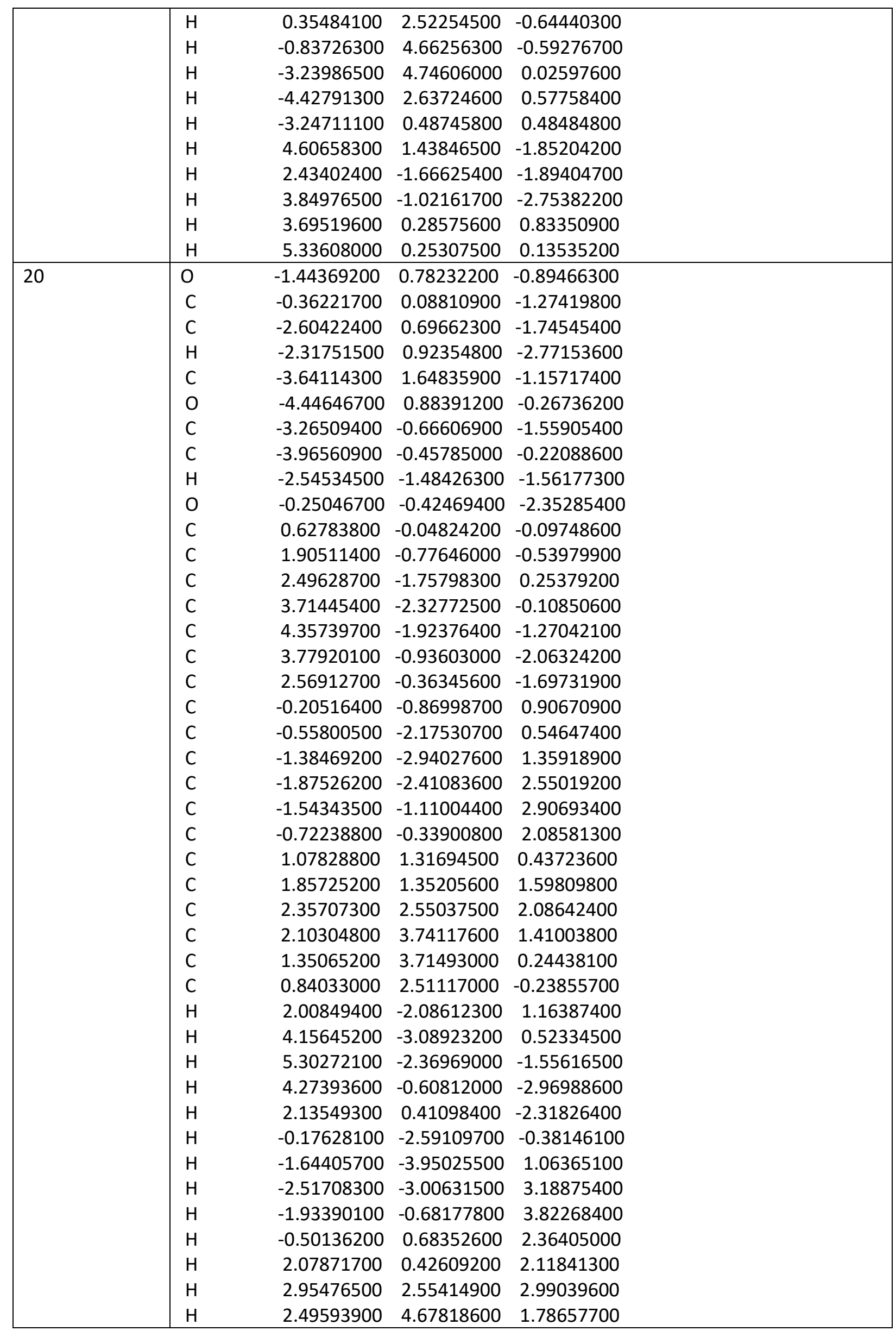




\begin{tabular}{|c|c|c|c|c|}
\hline & $\mathrm{H}$ & 1.15385900 & 4.63275200 & -0.29710900 \\
\hline & $\mathrm{H}$ & 0.25130200 & 2.51980500 & -1.14754000 \\
\hline & $\mathrm{H}$ & -3.99379100 & -0.82543900 & -2.35763900 \\
\hline & $\mathrm{H}$ & -3.15329600 & 2.46565900 & -0.62045100 \\
\hline & $\mathrm{H}$ & -4.27914500 & 2.06127400 & -1.94385400 \\
\hline & $\mathrm{H}$ & -3.25814500 & -0.57954500 & 0.60782700 \\
\hline & $\mathrm{H}$ & -4.81973000 & -1.11667300 & -0.06398000 \\
\hline \multirow[t]{45}{*}{34} & 0 & 1.45204100 & 0.67902400 & -0.93178700 \\
\hline & $\mathrm{C}$ & 0.38528700 & -0.06513600 & -1.25218200 \\
\hline & $\mathrm{C}$ & 2.63623200 & 0.47813200 & -1.72388700 \\
\hline & $\mathrm{H}$ & 2.36825900 & 0.48556900 & -2.77900600 \\
\hline & $\mathrm{C}$ & 3.32400900 & -0.80969800 & -1.27943600 \\
\hline & 0 & 4.00894800 & -0.44635400 & -0.09746300 \\
\hline & $\mathrm{C}$ & 3.63535600 & 1.56113300 & -1.30721300 \\
\hline & $\mathrm{C}$ & 4.59959000 & 0.81896300 & -0.35302700 \\
\hline & $\mathrm{H}$ & 3.10482600 & 2.37542000 & -0.81460700 \\
\hline & 0 & 0.32728700 & -0.73715900 & -2.24628300 \\
\hline & $\mathrm{C}$ & -0.65588600 & -0.06988200 & -0.11675900 \\
\hline & $\mathrm{C}$ & -2.10082500 & -0.19867400 & -0.61770400 \\
\hline & $\mathrm{C}$ & -3.10068900 & -0.35780600 & 0.34913000 \\
\hline & $\mathrm{C}$ & -4.43892500 & -0.42249000 & -0.00636900 \\
\hline & $\mathrm{C}$ & -4.81053700 & -0.31442200 & -1.34467200 \\
\hline & $\mathrm{C}$ & -3.82920100 & -0.14274300 & -2.30951100 \\
\hline & $\mathrm{C}$ & -2.48162400 & -0.08707600 & -1.95276800 \\
\hline & $\mathrm{C}$ & -0.60266100 & 1.22794500 & 0.70252700 \\
\hline & $\mathrm{C}$ & -0.61621900 & 1.23930600 & 2.09413200 \\
\hline & $\mathrm{C}$ & -0.66026000 & 2.44481200 & 2.79292000 \\
\hline & $\mathrm{C}$ & -0.69275100 & 3.65197300 & 2.10937900 \\
\hline & $\mathrm{C}$ & -0.69416400 & 3.64891000 & 0.71598000 \\
\hline & $\mathrm{C}$ & -0.65687200 & 2.44803200 & 0.02392000 \\
\hline & $\mathrm{C}$ & -0.18204600 & -1.31849800 & 0.66156200 \\
\hline & $\mathrm{C}$ & 1.03214100 & -1.27339800 & 1.35615300 \\
\hline & $\mathrm{C}$ & 1.53784700 & -2.40999700 & 1.97442200 \\
\hline & $\mathrm{C}$ & 0.84155400 & -3.61306700 & 1.90466800 \\
\hline & $\mathrm{C}$ & -0.35375800 & -3.67094100 & 1.19917000 \\
\hline & $\mathrm{C}$ & -0.85872100 & -2.53461700 & 0.57322100 \\
\hline & $\mathrm{H}$ & -2.82086400 & -0.42971300 & 1.39496800 \\
\hline & $\mathrm{H}$ & -5.19334800 & -0.55036500 & 0.76112000 \\
\hline & $\mathrm{H}$ & -5.85553300 & -0.36043800 & -1.62776600 \\
\hline & $\mathrm{H}$ & -4.10399600 & -0.05499600 & -3.35419500 \\
\hline & $\mathrm{H}$ & -1.73760500 & 0.02547500 & -2.72828000 \\
\hline & $\mathrm{H}$ & -0.59161200 & 0.30689600 & 2.64527600 \\
\hline & $\mathrm{H}$ & -0.66903500 & 2.43296100 & 3.87653100 \\
\hline & $\mathrm{H}$ & -0.72351100 & 4.58833000 & 2.65369800 \\
\hline & $\mathrm{H}$ & -0.73073400 & 4.58395200 & 0.16929600 \\
\hline & $\mathrm{H}$ & -0.67543200 & 2.45499200 & -1.06162400 \\
\hline & $\mathrm{H}$ & 1.59802700 & -0.34956500 & 1.39482500 \\
\hline & $\mathrm{H}$ & 2.48425900 & -2.35264300 & 2.49893200 \\
\hline & $\mathrm{H}$ & 1.23503900 & -4.50053900 & 2.38612900 \\
\hline & $\mathrm{H}$ & -0.89618200 & -4.60608100 & 1.12353600 \\
\hline & $\mathrm{H}$ & -1.77895200 & -2.60259200 & 0.00732200 \\
\hline & $\mathrm{H}$ & 4.15960000 & 1.96242300 & -2.17450000 \\
\hline
\end{tabular}




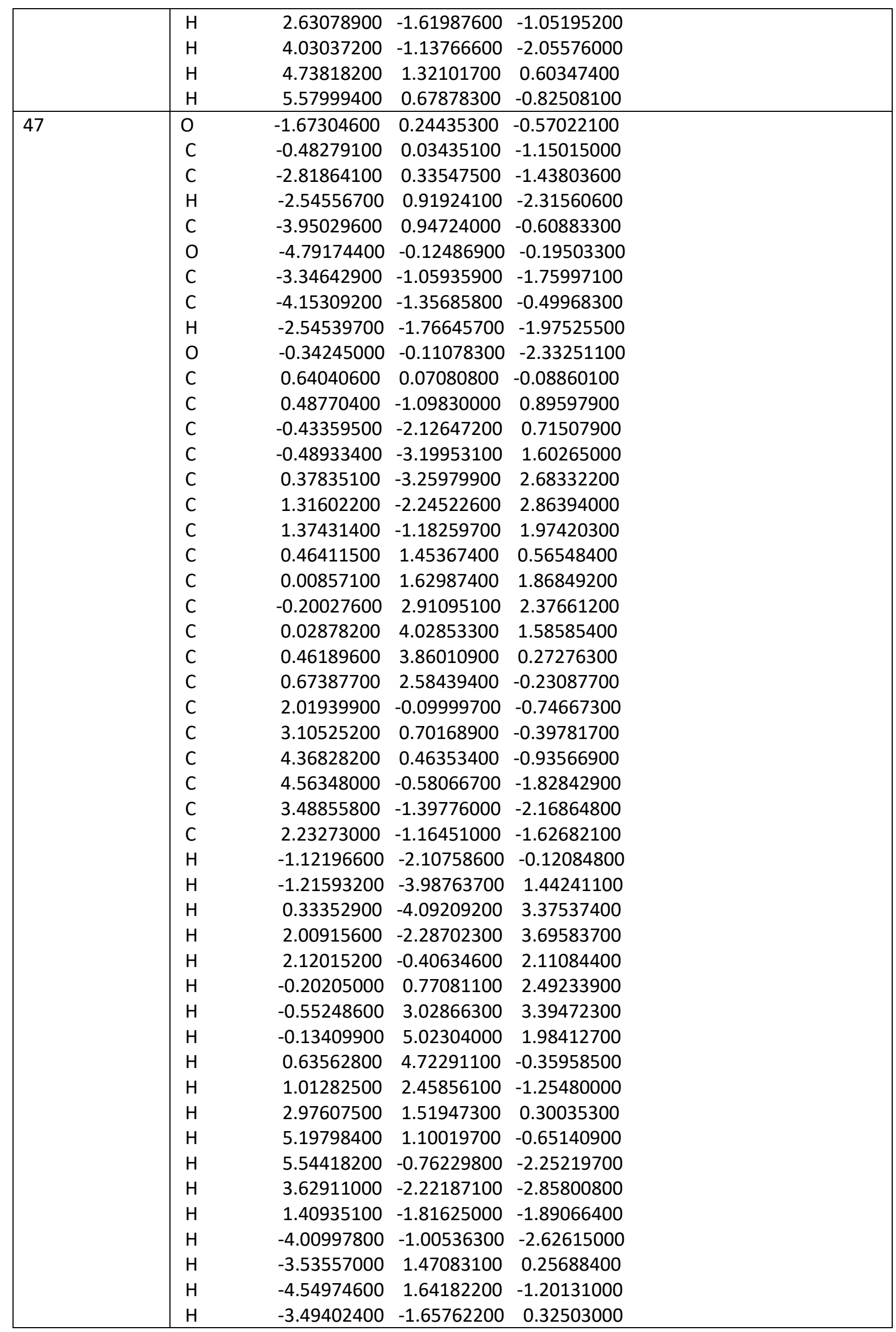




\begin{tabular}{|c|c|c|c|c|}
\hline & $\mathrm{H}$ & -4.92696000 & -2.11194000 & -0.63497500 \\
\hline \multirow[t]{49}{*}{58} & 0 & -1.50527700 & 0.59506400 & -0.97346500 \\
\hline & $\mathrm{C}$ & -0.37215700 & -0.04256500 & -1.29336600 \\
\hline & $\mathrm{C}$ & -2.63915100 & 0.37563200 & -1.82831300 \\
\hline & $\mathrm{H}$ & -2.37236400 & 0.62477400 & -2.85480200 \\
\hline & $\mathrm{C}$ & -3.75793100 & 1.21545900 & -1.23859500 \\
\hline & 0 & -4.21435600 & 0.46082200 & -0.13401700 \\
\hline & $\mathrm{C}$ & -3.17704800 & -1.05070300 & -1.65601100 \\
\hline & $\mathrm{C}$ & -4.23823600 & -0.90322000 & -0.54190600 \\
\hline & $\mathrm{H}$ & -2.37697300 & -1.74098000 & -1.38932100 \\
\hline & 0 & -0.21886000 & -0.60126300 & -2.34386500 \\
\hline & C & 0.63609100 & -0.00581100 & -0.11647800 \\
\hline & $\mathrm{C}$ & 2.04482200 & -0.34270100 & -0.63203900 \\
\hline & $\mathrm{C}$ & 2.26322100 & -1.49875800 & -1.39218600 \\
\hline & $\mathrm{C}$ & 3.53737400 & -1.83757800 & -1.82533100 \\
\hline & C & 4.62821400 & -1.03396300 & -1.50283000 \\
\hline & $\mathrm{C}$ & 4.42739900 & 0.10551000 & -0.73871100 \\
\hline & $\mathrm{C}$ & 3.14699900 & 0.44803600 & -0.30653000 \\
\hline & $\mathrm{C}$ & 0.13380000 & -1.06577200 & 0.90024500 \\
\hline & $\mathrm{C}$ & 0.97543700 & -2.06410200 & 1.39203500 \\
\hline & $\mathrm{C}$ & 0.52310500 & -2.97760600 & 2.34258400 \\
\hline & $\mathrm{C}$ & -0.77698700 & -2.90919700 & 2.82226500 \\
\hline & $\mathrm{C}$ & -1.61979600 & -1.90560800 & 2.35376600 \\
\hline & $\mathrm{C}$ & -1.17158400 & -0.99106700 & 1.40897900 \\
\hline & $\mathrm{C}$ & 0.62006600 & 1.39412900 & 0.51061200 \\
\hline & $\mathrm{C}$ & 0.63842500 & 2.51542400 & -0.32287300 \\
\hline & $\mathrm{C}$ & 0.71741100 & 3.79536100 & 0.20694600 \\
\hline & $\mathrm{C}$ & 0.78624800 & 3.97643200 & 1.58664600 \\
\hline & $\mathrm{C}$ & 0.77684400 & 2.86785500 & 2.42208700 \\
\hline & $\mathrm{C}$ & 0.69377300 & 1.58329700 & 1.88696800 \\
\hline & $\mathrm{H}$ & 1.43074100 & -2.14249300 & -1.64104000 \\
\hline & $\mathrm{H}$ & 3.67850600 & -2.73567300 & -2.41524400 \\
\hline & $\mathrm{H}$ & 5.62263900 & -1.29942800 & -1.84184700 \\
\hline & $\mathrm{H}$ & 5.26569800 & 0.73831000 & -0.47168900 \\
\hline & $\mathrm{H}$ & 3.02144900 & 1.34130400 & 0.29143500 \\
\hline & $\mathrm{H}$ & 1.99853600 & -2.13269400 & 1.04735600 \\
\hline & $\mathrm{H}$ & 1.20010700 & -3.74078000 & 2.70836900 \\
\hline & $\mathrm{H}$ & -1.12852200 & -3.61988200 & 3.56098400 \\
\hline & $\mathrm{H}$ & -2.63077400 & -1.81998000 & 2.73615800 \\
\hline & $\mathrm{H}$ & -1.83734900 & -0.19812600 & 1.09038100 \\
\hline & $\mathrm{H}$ & 0.60405500 & 2.38319300 & -1.40036200 \\
\hline & $\mathrm{H}$ & 0.72839900 & 4.65278700 & -0.45576400 \\
\hline & $\mathrm{H}$ & 0.84793000 & 4.97484400 & 2.00315400 \\
\hline & $\mathrm{H}$ & 0.83351200 & 2.99626700 & 3.49668000 \\
\hline & $\mathrm{H}$ & 0.69282100 & 0.72743200 & 2.55115900 \\
\hline & $\mathrm{H}$ & -3.61878800 & -1.39761600 & -2.59084100 \\
\hline & $\mathrm{H}$ & -3.41834500 & 2.18704900 & -0.88235100 \\
\hline & $\mathrm{H}$ & -4.55555200 & 1.34679900 & -1.98339900 \\
\hline & $\mathrm{H}$ & -4.01837100 & -1.51254700 & 0.33512900 \\
\hline & $\mathrm{H}$ & -5.23742900 & -1.16044900 & -0.91115100 \\
\hline 65 & 0 & -1.60725900 & 0.47330500 & -0.79099000 \\
\hline
\end{tabular}




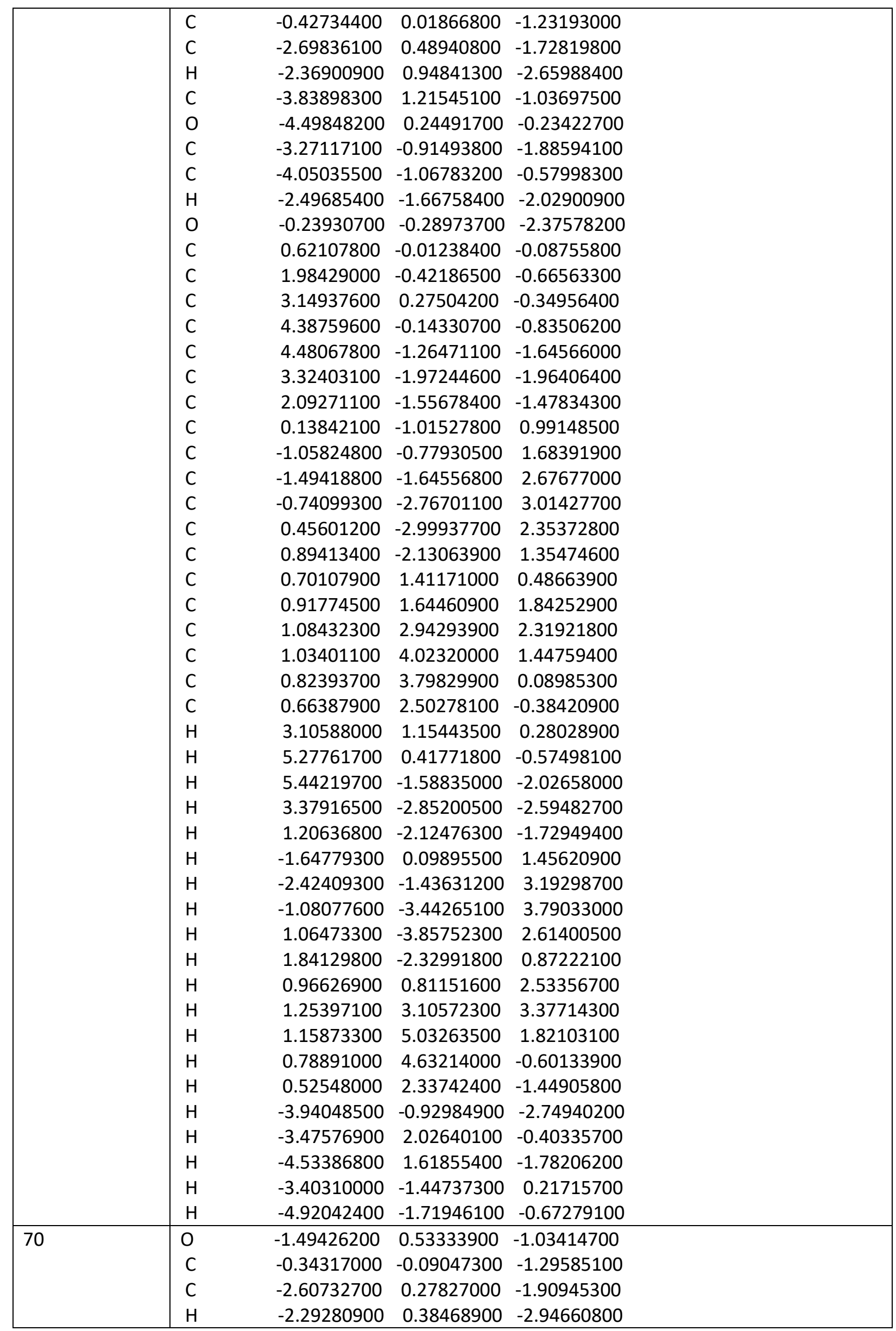




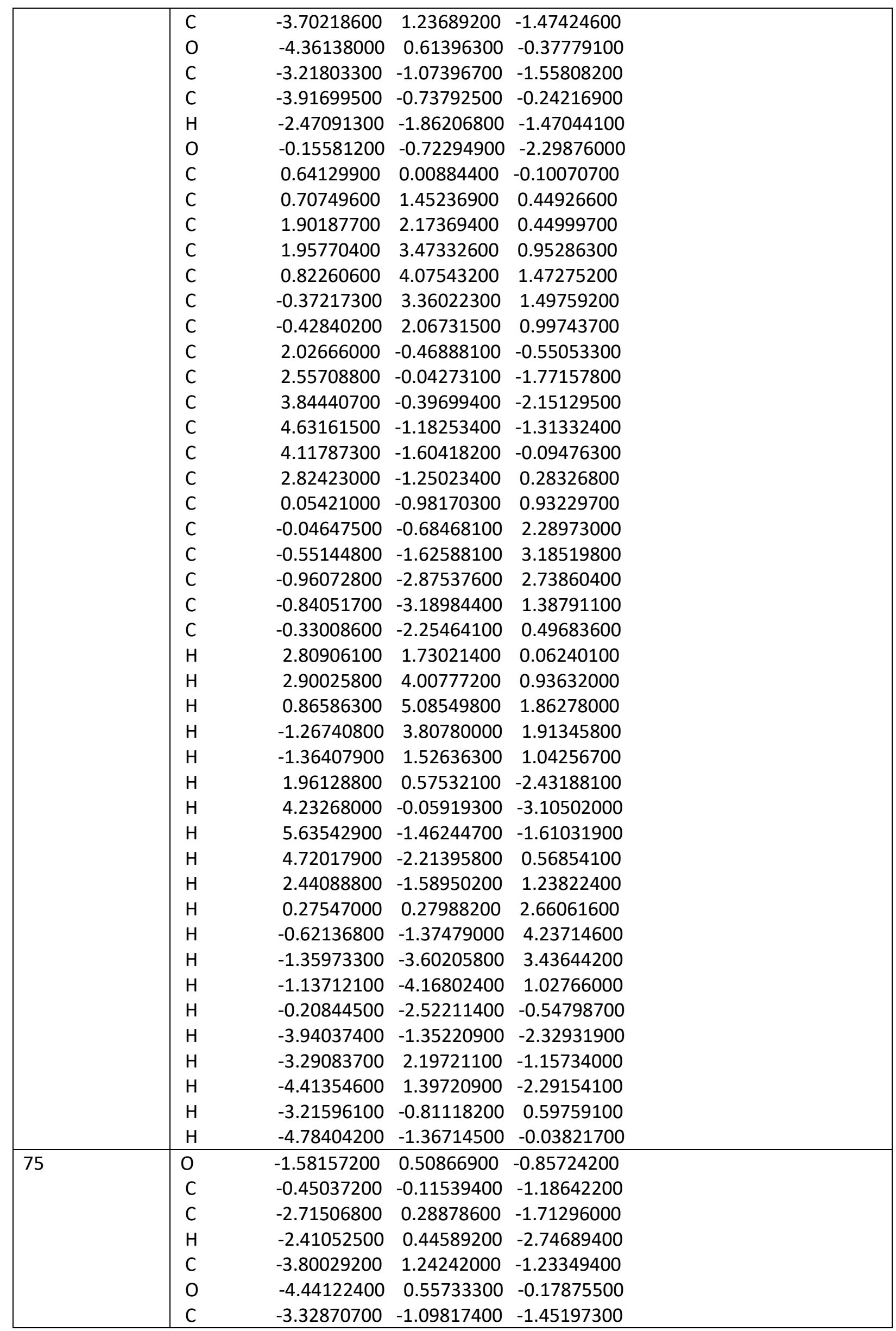




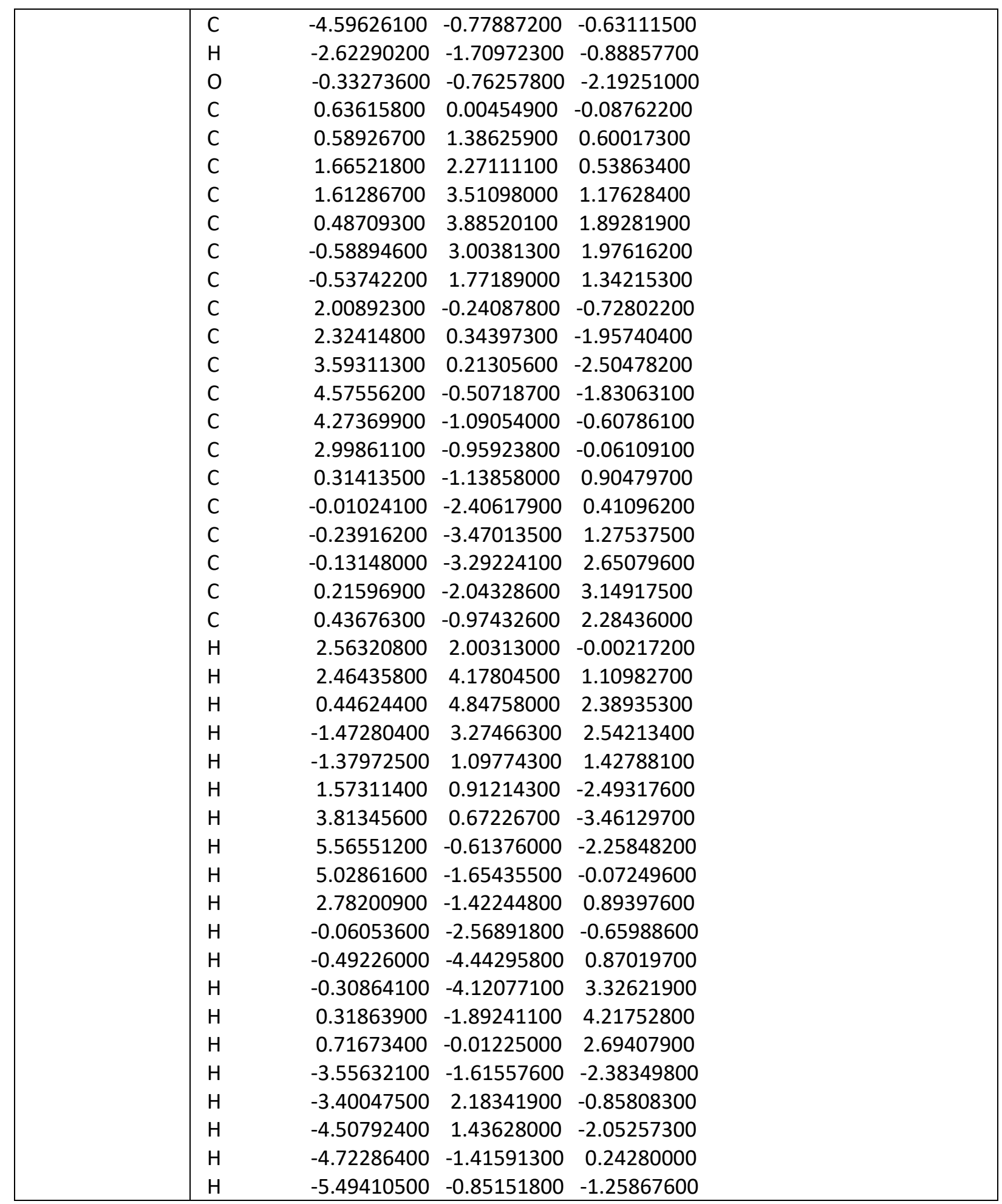

18 (optimized at the B3LYP/6-311G(d,p) level)

\begin{tabular}{|l|llll|}
\hline Conformer no & & & & \\
\hline 1 & O & -1.42887100 & -1.00169500 & -0.90951200 \\
& C & -2.45972000 & -0.25217300 & -1.34135800 \\
& C & -0.56676000 & -1.60231500 & -1.92016500 \\
& H & -1.19503400 & -1.84907100 & -2.78023100 \\
& C & 0.46529300 & -0.60063200 & -2.42060600 \\
& H & 1.04094600 & -1.04667100 & -3.23198500 \\
\hline
\end{tabular}




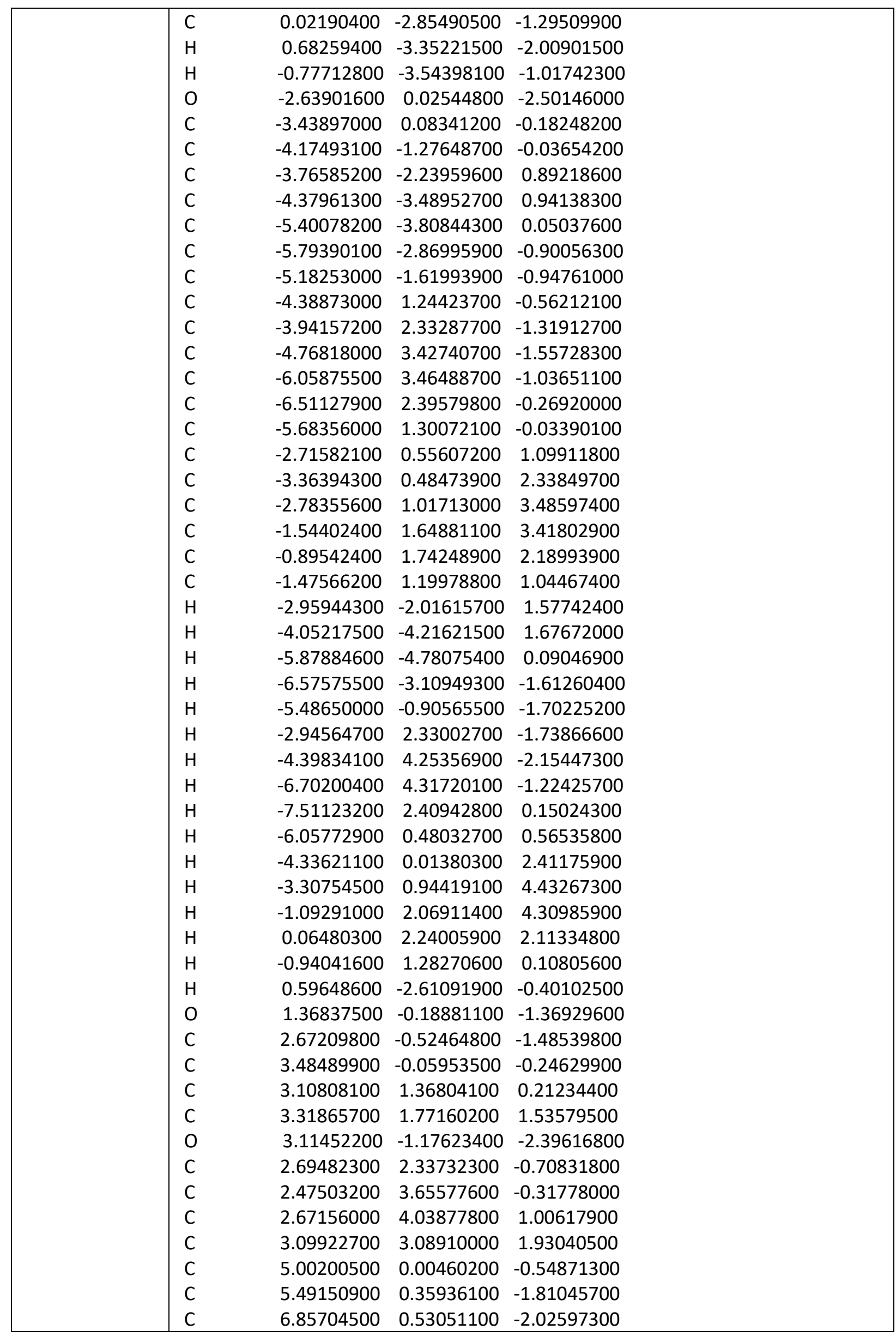




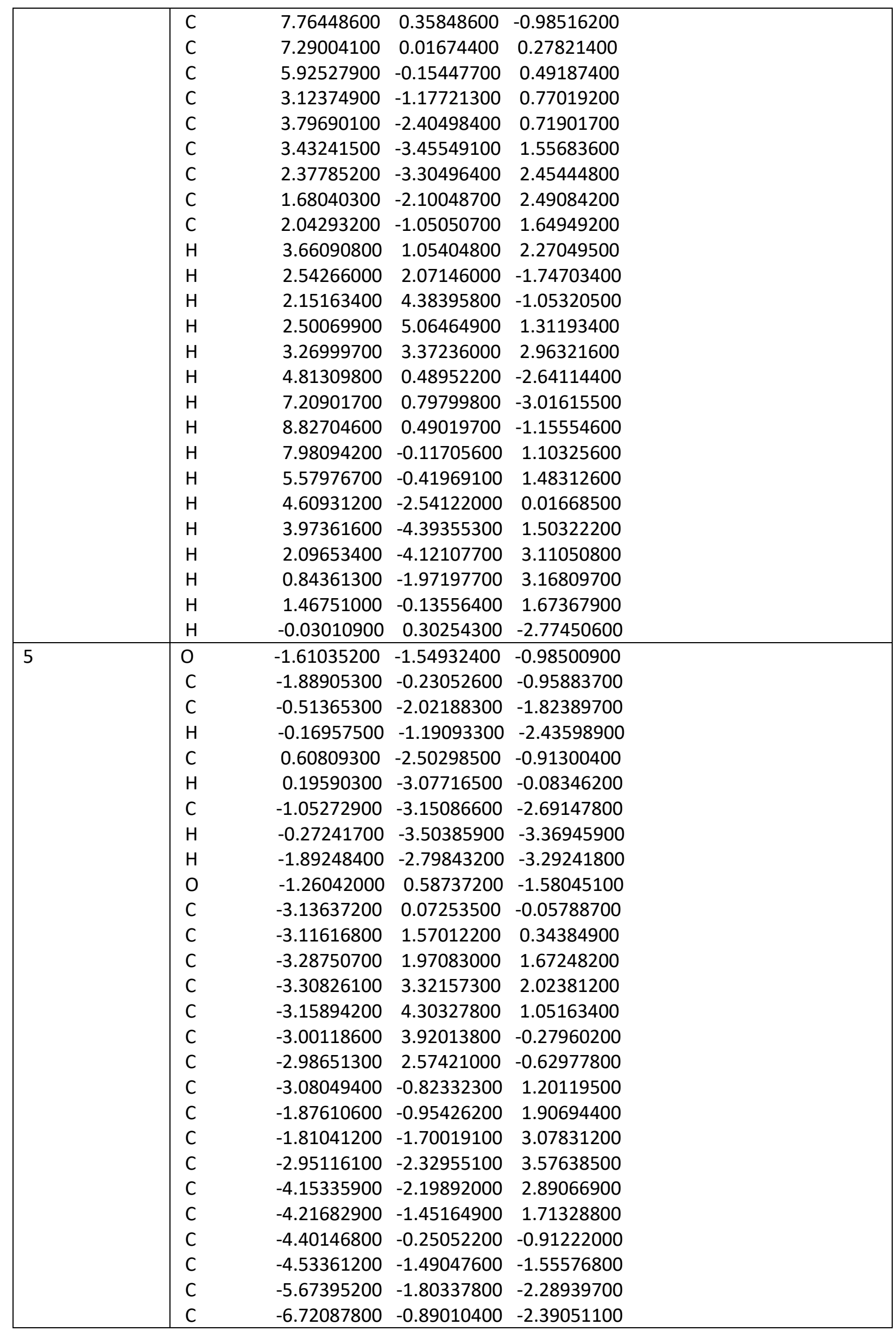




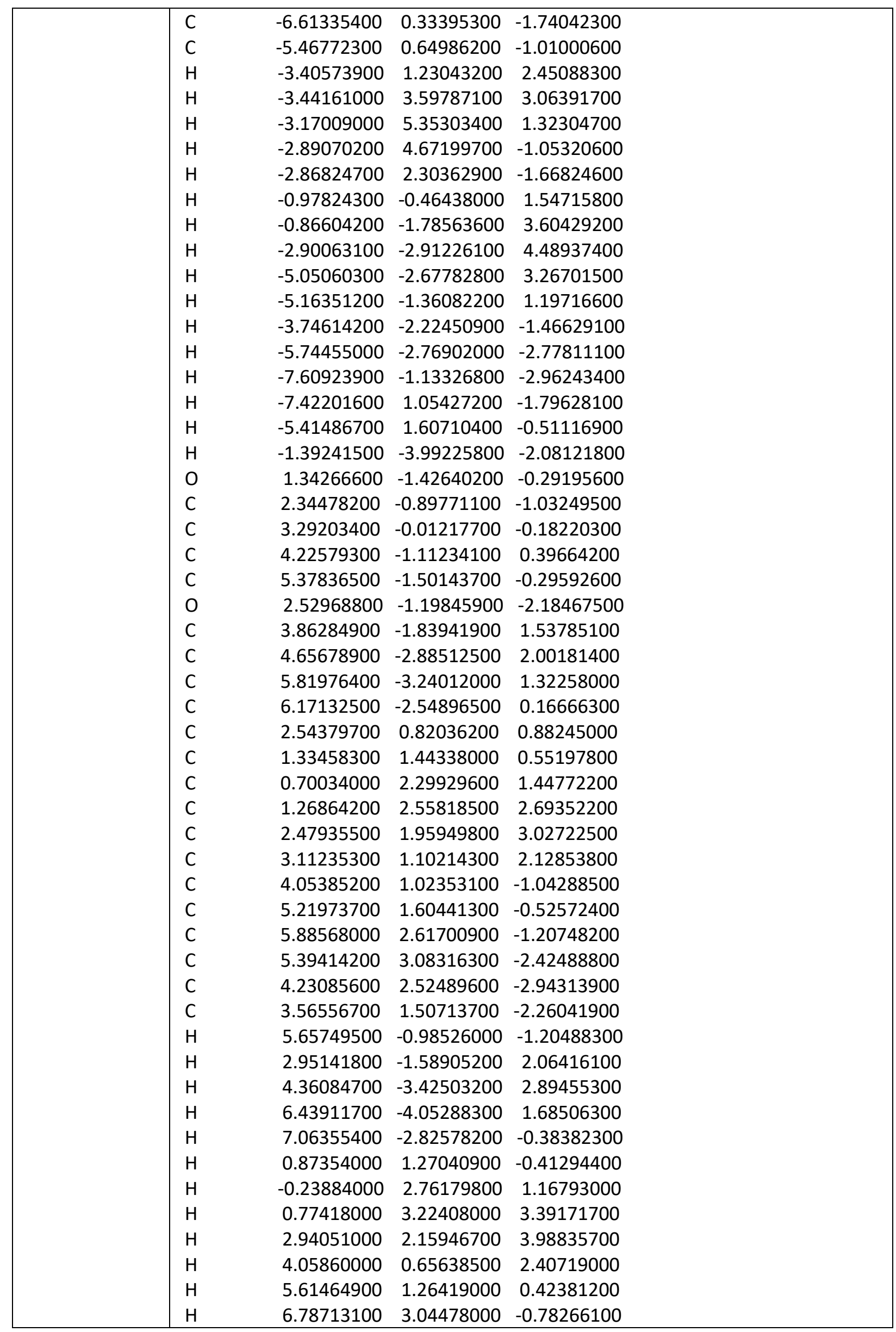




\begin{tabular}{|c|c|c|c|c|}
\hline & $\mathrm{H}$ & 5.91008500 & 3.87318600 & -2.95892200 \\
\hline & $\mathrm{H}$ & 3.83117700 & 2.87797300 & -3.88736400 \\
\hline & $\mathrm{H}$ & 2.67140000 & 1.08752600 & -2.69695700 \\
\hline & $\mathrm{H}$ & 1.30191300 & -3.11675500 & -1.48858600 \\
\hline 9 & 0 & 1.44896600 & 0.63132400 & -1.27106200 \\
\hline & $\mathrm{C}$ & 2.77666700 & 0.85691700 & -1.30940100 \\
\hline & C & 0.61492600 & 1.43866000 & -2.15333800 \\
\hline & $\mathrm{H}$ & 1.17516500 & 1.59646400 & -3.07836500 \\
\hline & $\mathrm{C}$ & -0.60410500 & 0.60512600 & -2.49148300 \\
\hline & $\mathrm{H}$ & -1.17992500 & 1.09215500 & -3.28226600 \\
\hline & $\mathrm{C}$ & 0.30380300 & 2.77738300 & -1.49879400 \\
\hline & $\mathrm{H}$ & -0.33957400 & 3.37108100 & -2.15385300 \\
\hline & $\mathrm{H}$ & 1.22662200 & 3.33259900 & -1.32897500 \\
\hline & $\mathrm{O}$ & 3.29418500 & 1.64487100 & -2.06043600 \\
\hline & $\mathrm{C}$ & 3.51489900 & 0.06723500 & -0.19313900 \\
\hline & $\mathrm{C}$ & 3.02973300 & -1.39627700 & -0.08356800 \\
\hline & $\mathrm{C}$ & 3.16890400 & -2.10767500 & 1.11356900 \\
\hline & C & 2.84697700 & -3.46057700 & 1.18873500 \\
\hline & $\mathrm{C}$ & 2.38384300 & -4.13776300 & 0.06336100 \\
\hline & $\mathrm{C}$ & 2.25683300 & -3.44708600 & -1.13869600 \\
\hline & $\mathrm{C}$ & 2.58034900 & -2.09436400 & -1.20958100 \\
\hline & $\mathrm{C}$ & 3.18335100 & 0.94696700 & 1.04294700 \\
\hline & $\mathrm{C}$ & 3.93413000 & 2.10192100 & 1.29776900 \\
\hline & $\mathrm{C}$ & 3.59920100 & 2.95563000 & 2.34569500 \\
\hline & $\mathrm{C}$ & 2.49829000 & 2.67884800 & 3.15253900 \\
\hline & $\mathrm{C}$ & 1.72522500 & 1.55143100 & 2.88789300 \\
\hline & $\mathrm{C}$ & 2.05780000 & 0.69985500 & 1.83676300 \\
\hline & $\mathrm{C}$ & 5.03471000 & -0.03368800 & -0.46786000 \\
\hline & $\mathrm{C}$ & 5.92714100 & -0.17208900 & 0.60231600 \\
\hline & $\mathrm{C}$ & 7.28557700 & -0.38578900 & 0.38646000 \\
\hline & $\mathrm{C}$ & 7.78430400 & -0.47233700 & -0.91038100 \\
\hline & $\mathrm{C}$ & 6.90694800 & -0.34765200 & -1.98330500 \\
\hline & $\mathrm{C}$ & 5.54763000 & -0.13483400 & -1.76578700 \\
\hline & $\mathrm{H}$ & 3.53543600 & -1.60535800 & 1.99972300 \\
\hline & $\mathrm{H}$ & 2.96593800 & -3.98613400 & 2.12992200 \\
\hline & $\mathrm{H}$ & 2.13320100 & -5.19087400 & 0.12034200 \\
\hline & $\mathrm{H}$ & 1.90849000 & -3.96082700 & -2.02782400 \\
\hline & $\mathrm{H}$ & 2.48281700 & -1.58573100 & -2.16060600 \\
\hline & $\mathrm{H}$ & 4.78459500 & 2.33678800 & 0.67050100 \\
\hline & $\mathrm{H}$ & 4.20013100 & 3.83985100 & 2.52672600 \\
\hline & $\mathrm{H}$ & 2.23980400 & 3.34066000 & 3.97161400 \\
\hline & $\mathrm{H}$ & 0.85396400 & 1.33049200 & 3.49430200 \\
\hline & $\mathrm{H}$ & 1.42534700 & -0.15271400 & 1.63056100 \\
\hline & $\mathrm{H}$ & 5.56217000 & -0.10821400 & 1.61966900 \\
\hline & $\mathrm{H}$ & 7.95243300 & -0.48553600 & 1.23577900 \\
\hline & $\mathrm{H}$ & 8.84216200 & -0.63630800 & -1.08213000 \\
\hline & $\mathrm{H}$ & 7.27811300 & -0.41379100 & -3.00008700 \\
\hline & $\mathrm{H}$ & 4.89332800 & -0.03173300 & -2.61913800 \\
\hline & $\mathrm{H}$ & -0.21185600 & 2.62992200 & -0.55047200 \\
\hline & 0 & -1.43267100 & 0.47187900 & -1.31934500 \\
\hline & $\mathrm{C}$ & -2.61751100 & -0.14592200 & -1.50280800 \\
\hline & $\mathrm{C}$ & -3.48315600 & -0.09444800 & -0.21336400 \\
\hline
\end{tabular}




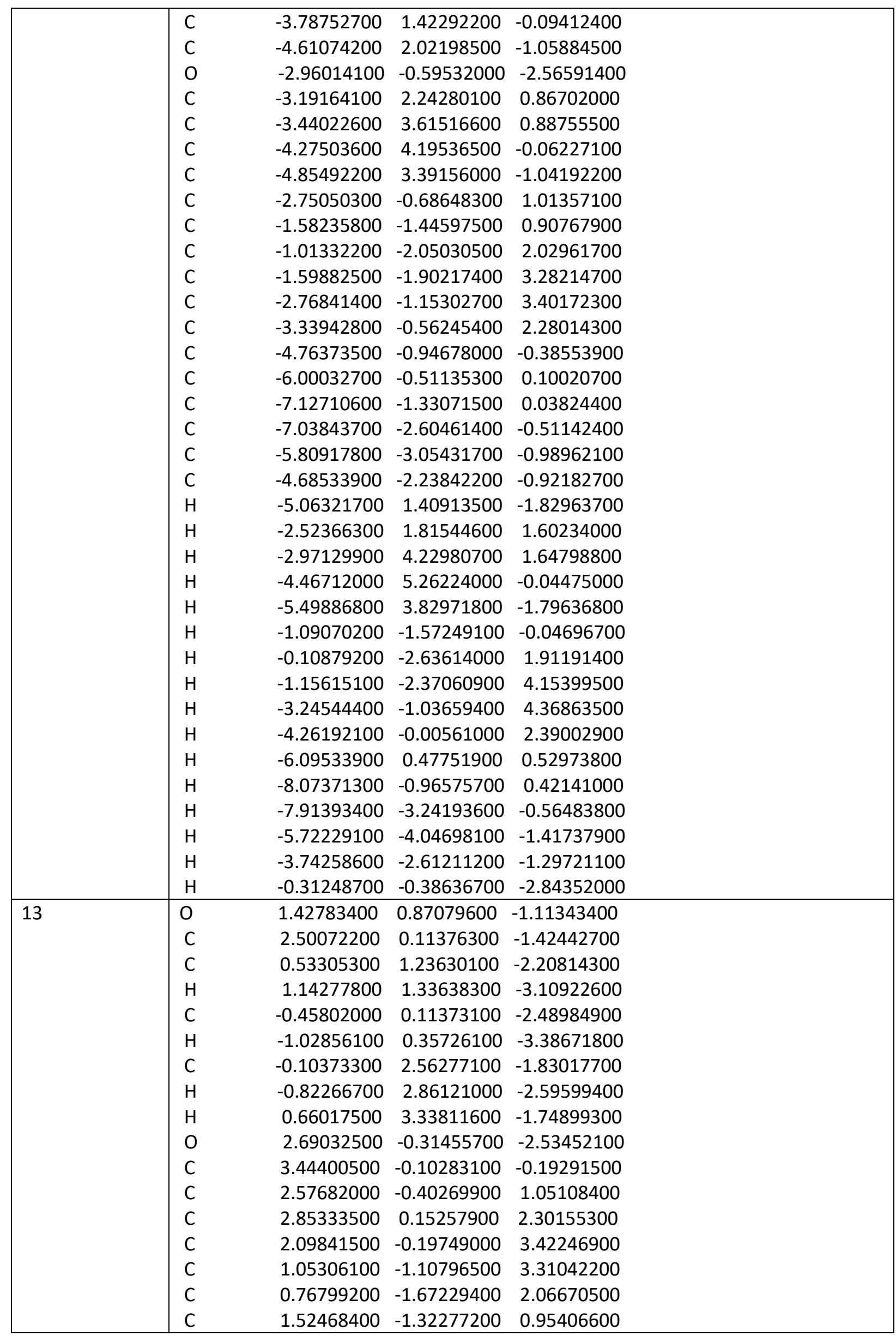




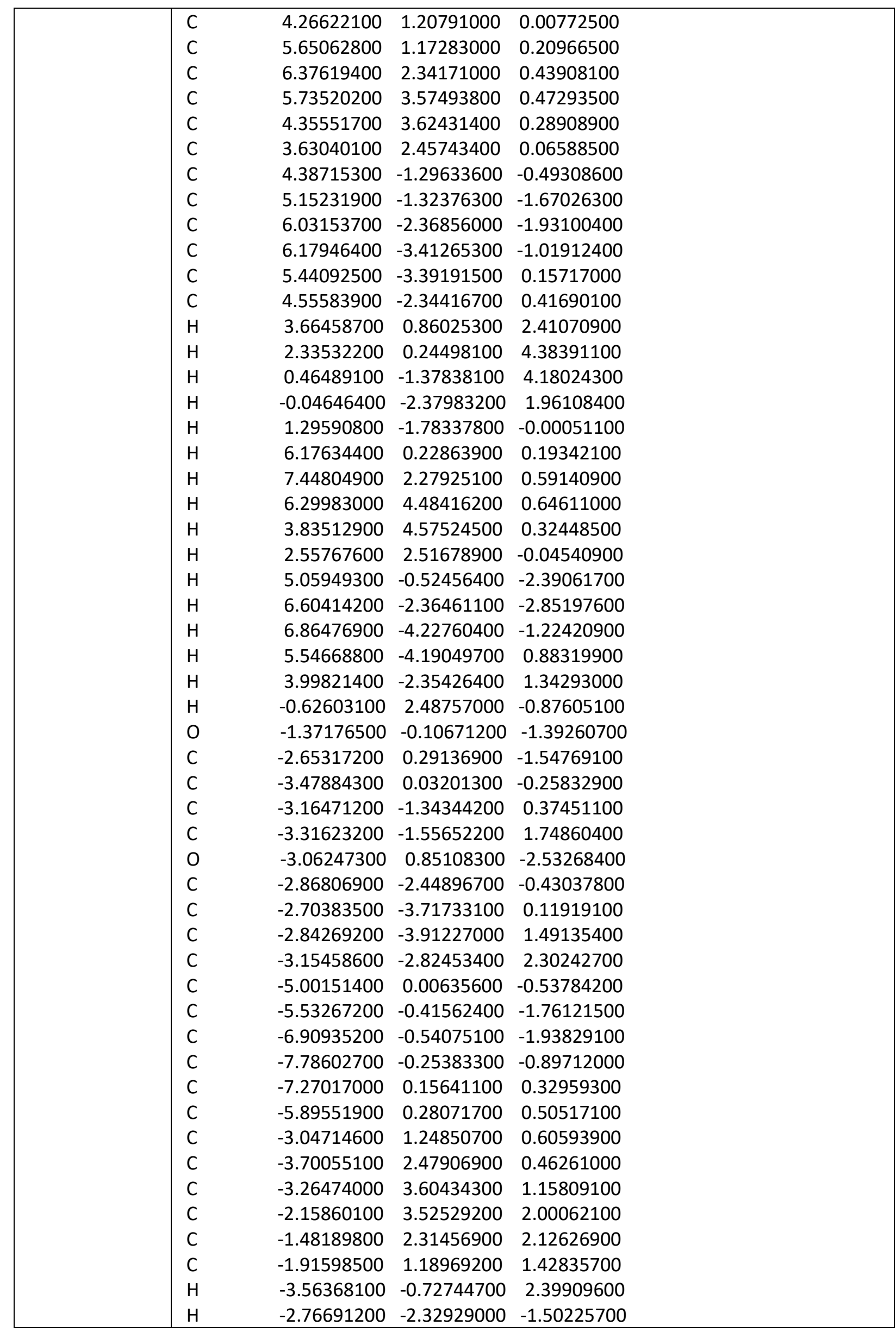




\begin{tabular}{|c|c|c|c|c|}
\hline & $\mathrm{H}$ & -2.47097900 & -4.55474100 & -0.52914400 \\
\hline & $\mathrm{H}$ & -2.71731600 & -4.89989100 & 1.92047900 \\
\hline & $\mathrm{H}$ & -3.27866500 & -2.95967700 & 3.37128000 \\
\hline & $\mathrm{H}$ & -4.87917000 & -0.63284700 & -2.59314800 \\
\hline & $\mathrm{H}$ & -7.29333100 & -0.86275600 & -2.89998100 \\
\hline & $\mathrm{H}$ & -8.85671600 & -0.34972400 & -1.03808800 \\
\hline & $\mathrm{H}$ & -7.93689000 & 0.37957600 & 1.15521100 \\
\hline & $\mathrm{H}$ & -5.51712700 & 0.59897000 & 1.46849900 \\
\hline & $\mathrm{H}$ & -4.55276000 & 2.56044100 & -0.19960500 \\
\hline & $\mathrm{H}$ & -3.79127000 & 4.54432800 & 1.03554600 \\
\hline & $\mathrm{H}$ & -1.82136000 & 4.40041800 & 2.54483800 \\
\hline & $\mathrm{H}$ & -0.60543500 & 2.23840100 & 2.75972200 \\
\hline & $\mathrm{H}$ & -1.35555700 & 0.26901500 & 1.51464600 \\
\hline & $\mathrm{H}$ & 0.07301100 & -0.82577500 & -2.63472100 \\
\hline \multirow{38}{*}{15} & 0 & -1.58670800 & -1.41332100 & -1.30963900 \\
\hline & $\mathrm{C}$ & -1.76658700 & -0.11586000 & -0.98669000 \\
\hline & $\mathrm{C}$ & -0.53597700 & -1.76056100 & -2.25924300 \\
\hline & $\mathrm{H}$ & -0.15579400 & -0.84272300 & -2.70261000 \\
\hline & $\mathrm{C}$ & 0.57403500 & -2.47235100 & -1.49841100 \\
\hline & $\mathrm{H}$ & 0.15086500 & -3.23070600 & -0.83992500 \\
\hline & $\mathrm{C}$ & -1.15311200 & -2.66189400 & -3.31972900 \\
\hline & $\mathrm{H}$ & -1.97854900 & -2.14958500 & -3.81640000 \\
\hline & $\mathrm{H}$ & -0.40639300 & -2.91488200 & -4.07593500 \\
\hline & 0 & -1.08823200 & 0.77257400 & -1.43467100 \\
\hline & $\mathrm{C}$ & -2.98239300 & 0.07212600 & -0.01641000 \\
\hline & $\mathrm{C}$ & -4.26241800 & 0.08438800 & -0.90883400 \\
\hline & $\mathrm{C}$ & -5.24181100 & 1.07367100 & -0.77183600 \\
\hline & $\mathrm{C}$ & -6.40348900 & 1.04929600 & -1.54367400 \\
\hline & $\mathrm{C}$ & -6.61367800 & 0.03564900 & -2.47144100 \\
\hline & $\mathrm{C}$ & -5.65412400 & -0.96476000 & -2.60874700 \\
\hline & $\mathrm{C}$ & -4.49824800 & -0.94369100 & -1.83417000 \\
\hline & $\mathrm{C}$ & -2.82793600 & 1.41858200 & 0.73852700 \\
\hline & $\mathrm{C}$ & -2.60473900 & 2.61561800 & 0.03859500 \\
\hline & $\mathrm{C}$ & -2.50926500 & 3.83128400 & 0.70706400 \\
\hline & $\mathrm{C}$ & -2.64560700 & 3.88956100 & 2.09301700 \\
\hline & $\mathrm{C}$ & -2.88312000 & 2.71598400 & 2.79774400 \\
\hline & $\mathrm{C}$ & -2.97339200 & 1.49526200 & 2.12715100 \\
\hline & $\mathrm{C}$ & -3.02526300 & -1.10394700 & 0.98616300 \\
\hline & $\mathrm{C}$ & -1.84857500 & -1.50993600 & 1.63123300 \\
\hline & $\mathrm{C}$ & -1.86885100 & -2.52323100 & 2.58297600 \\
\hline & C & -3.06844500 & -3.15048500 & 2.91894100 \\
\hline & $\mathrm{C}$ & -4.24361000 & -2.74732400 & 2.29543500 \\
\hline & $\mathrm{C}$ & -4.22117300 & -1.73271200 & 1.33700000 \\
\hline & $\mathrm{H}$ & -5.10775900 & 1.87242200 & -0.05629200 \\
\hline & $\mathrm{H}$ & -7.14367200 & 1.83091900 & -1.41261300 \\
\hline & $\mathrm{H}$ & -7.51442000 & 0.01993400 & -3.07464200 \\
\hline & $\mathrm{H}$ & -5.80621400 & -1.77176800 & -3.31719700 \\
\hline & $\mathrm{H}$ & -3.78117500 & -1.74567300 & -1.93524200 \\
\hline & $\mathrm{H}$ & -2.50329100 & 2.59833200 & -1.03602900 \\
\hline & $\mathrm{H}$ & -2.33047800 & 4.73772200 & 0.13917800 \\
\hline & $\mathrm{H}$ & -2.57235700 & 4.83856300 & 2.61260000 \\
\hline & $\mathrm{H}$ & -3.00042400 & 2.73955700 & 3.87558100 \\
\hline
\end{tabular}




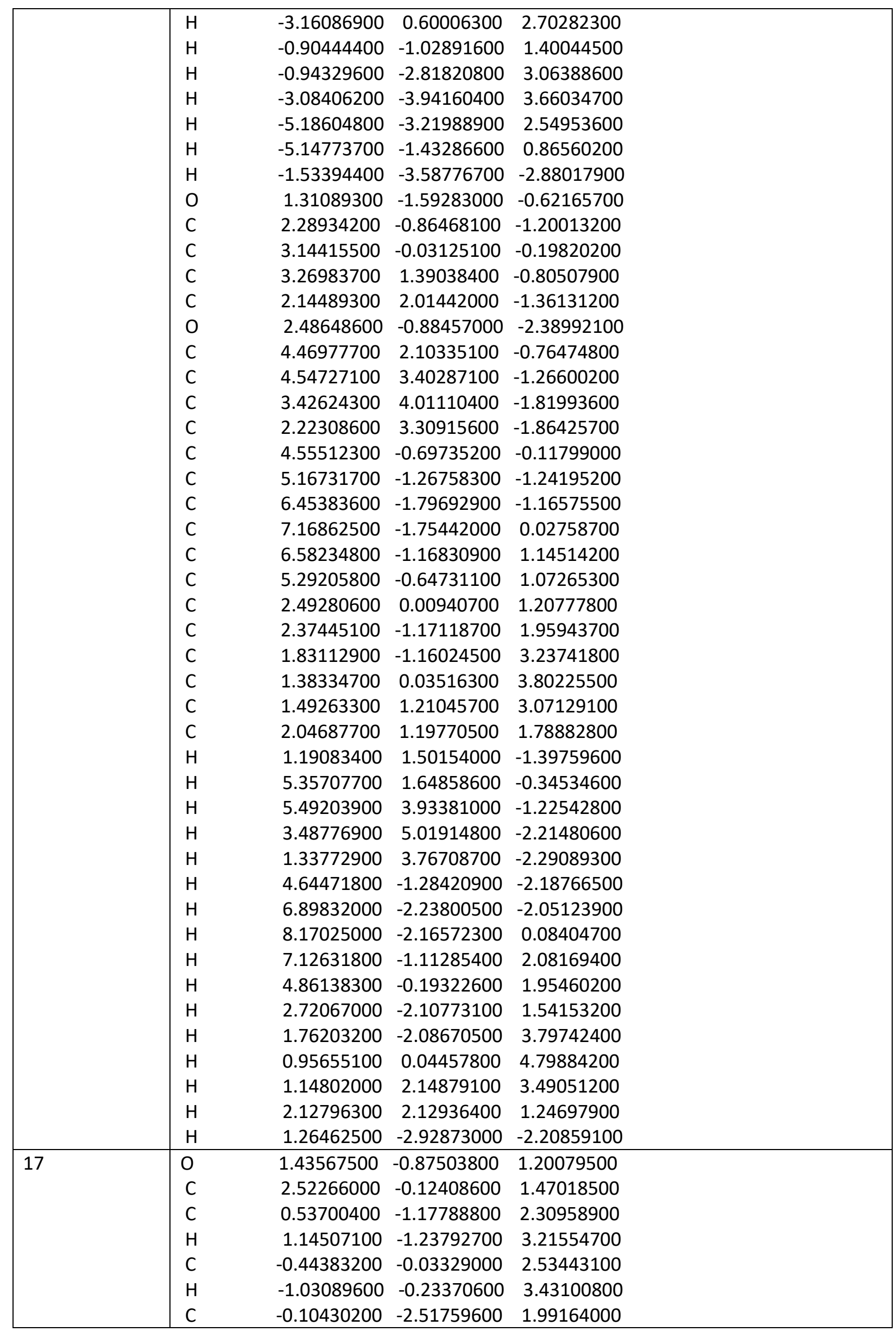




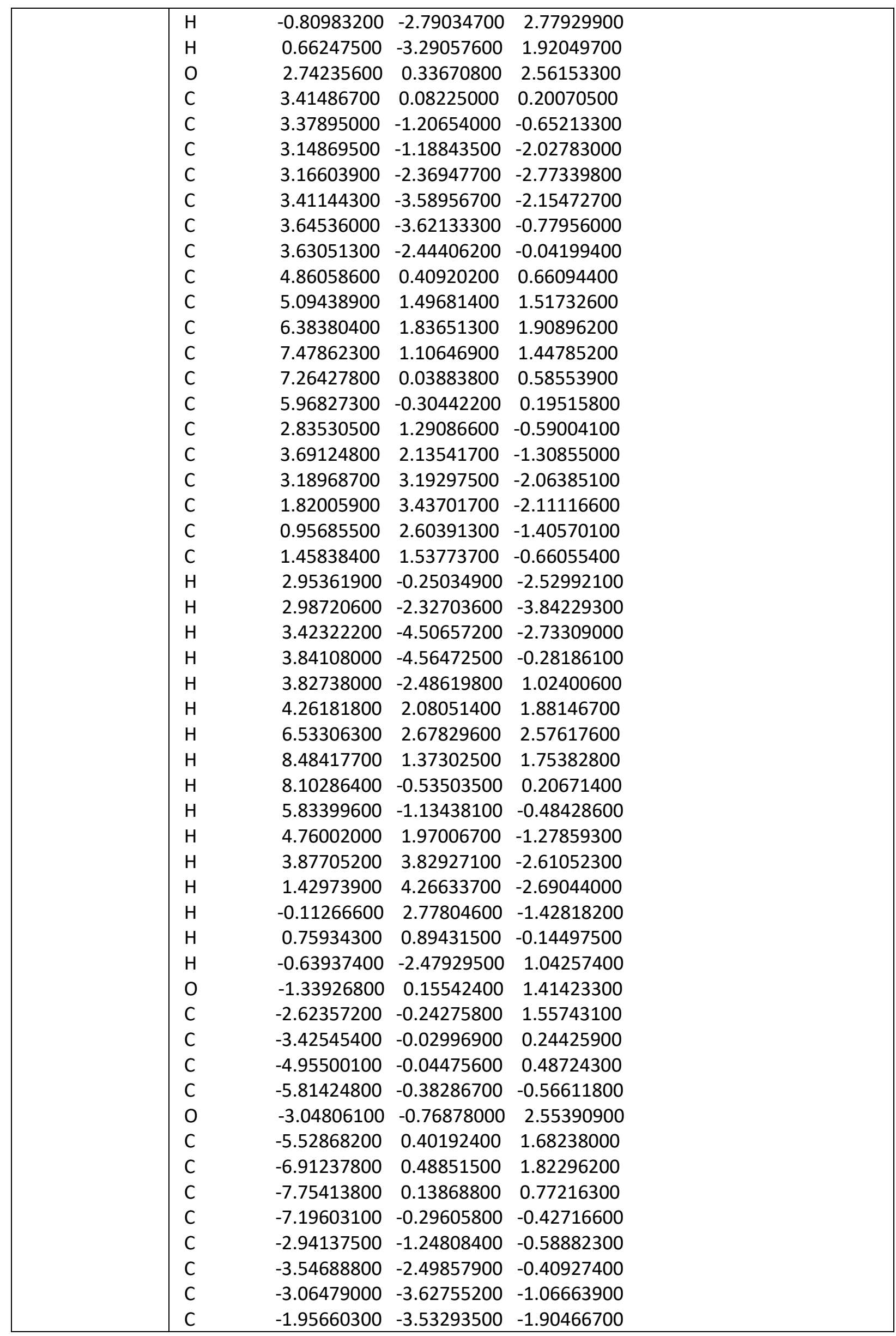




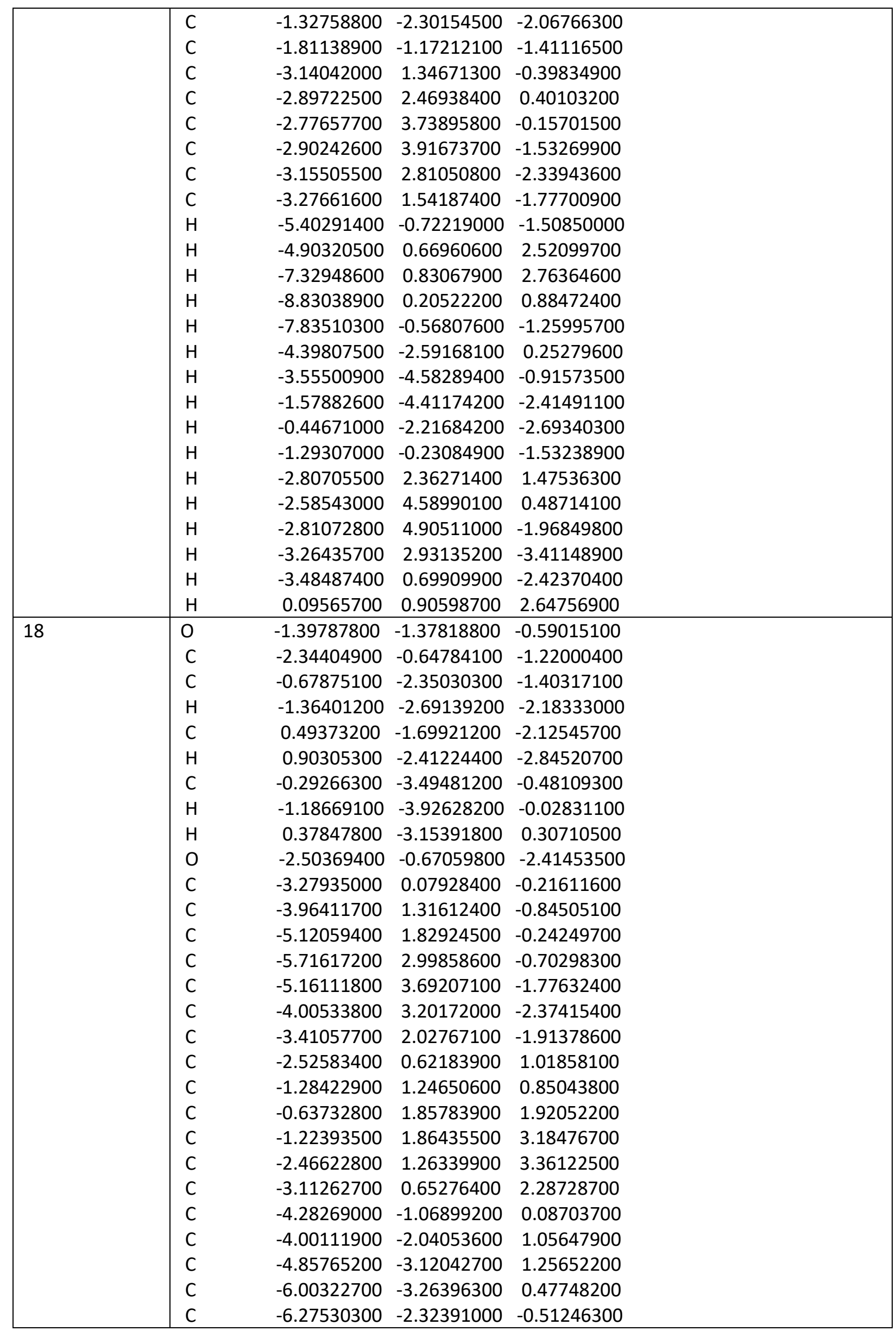




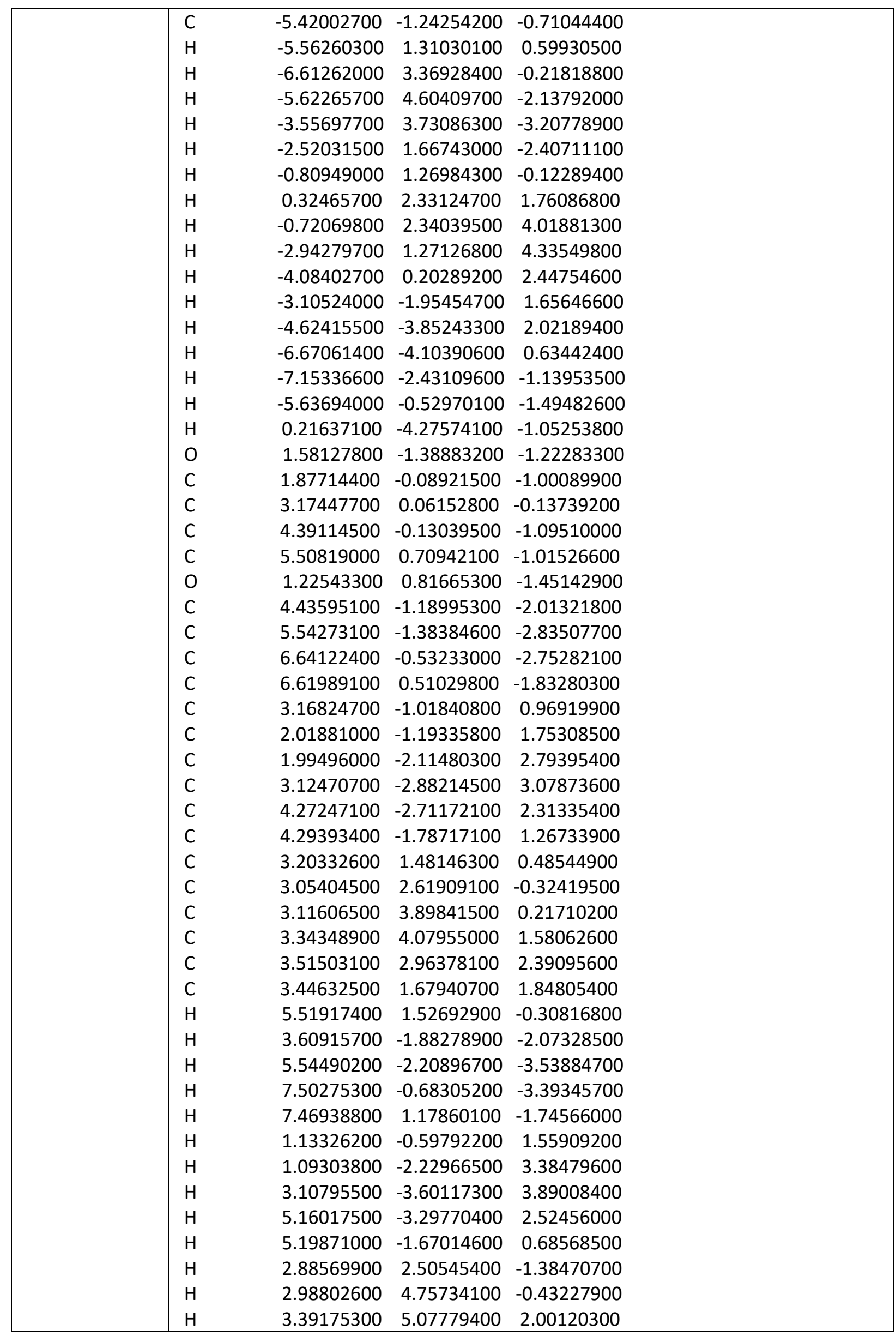




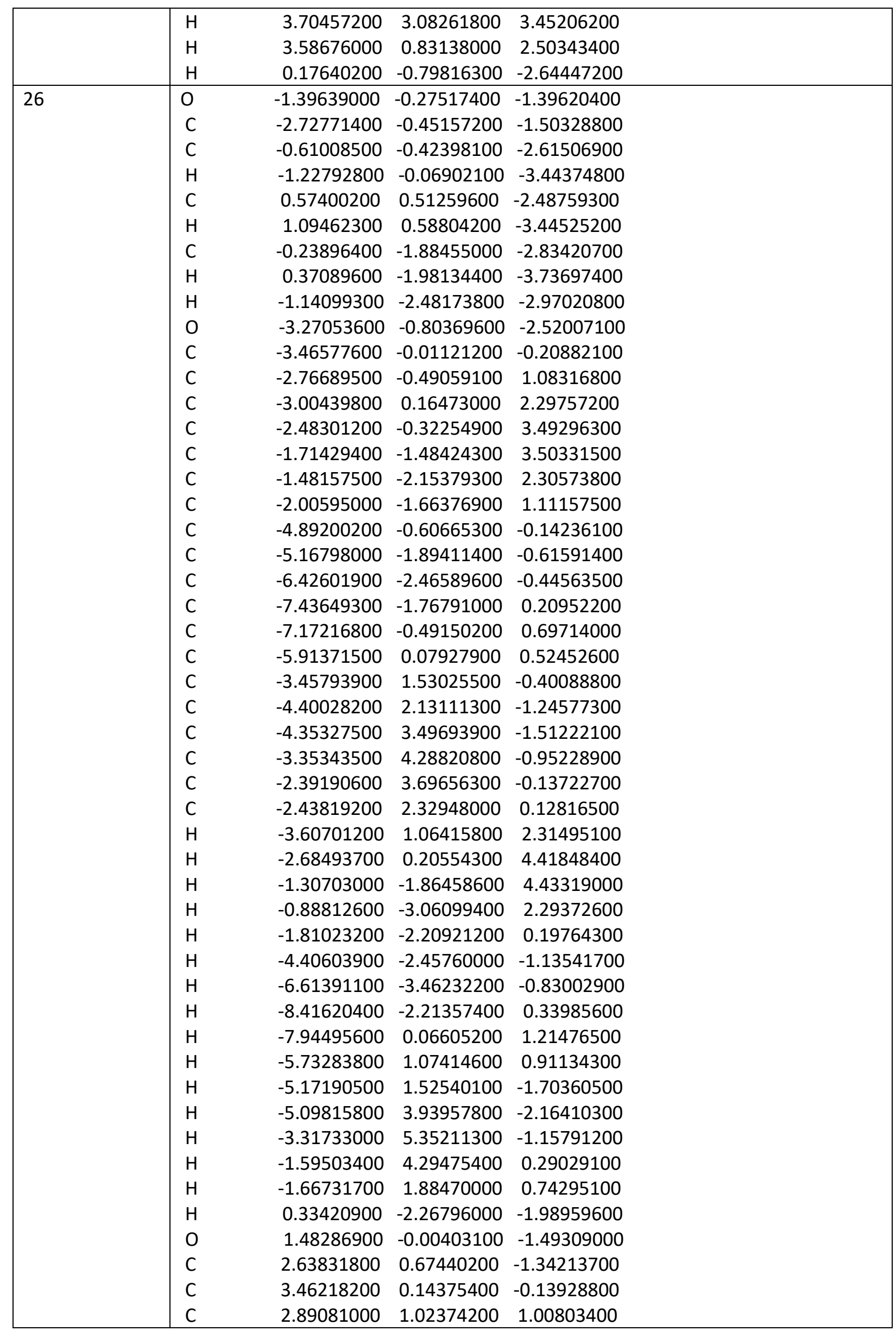




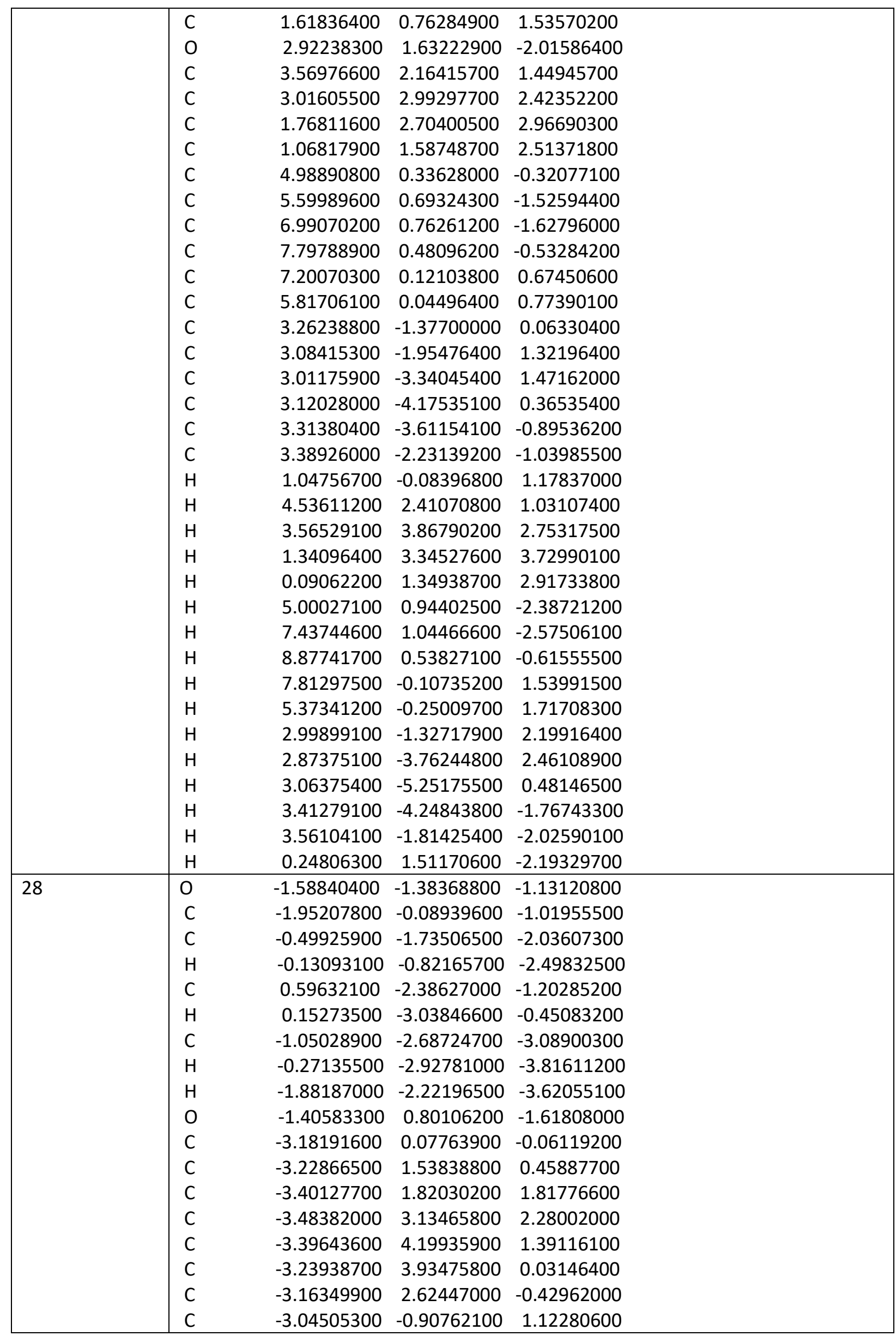




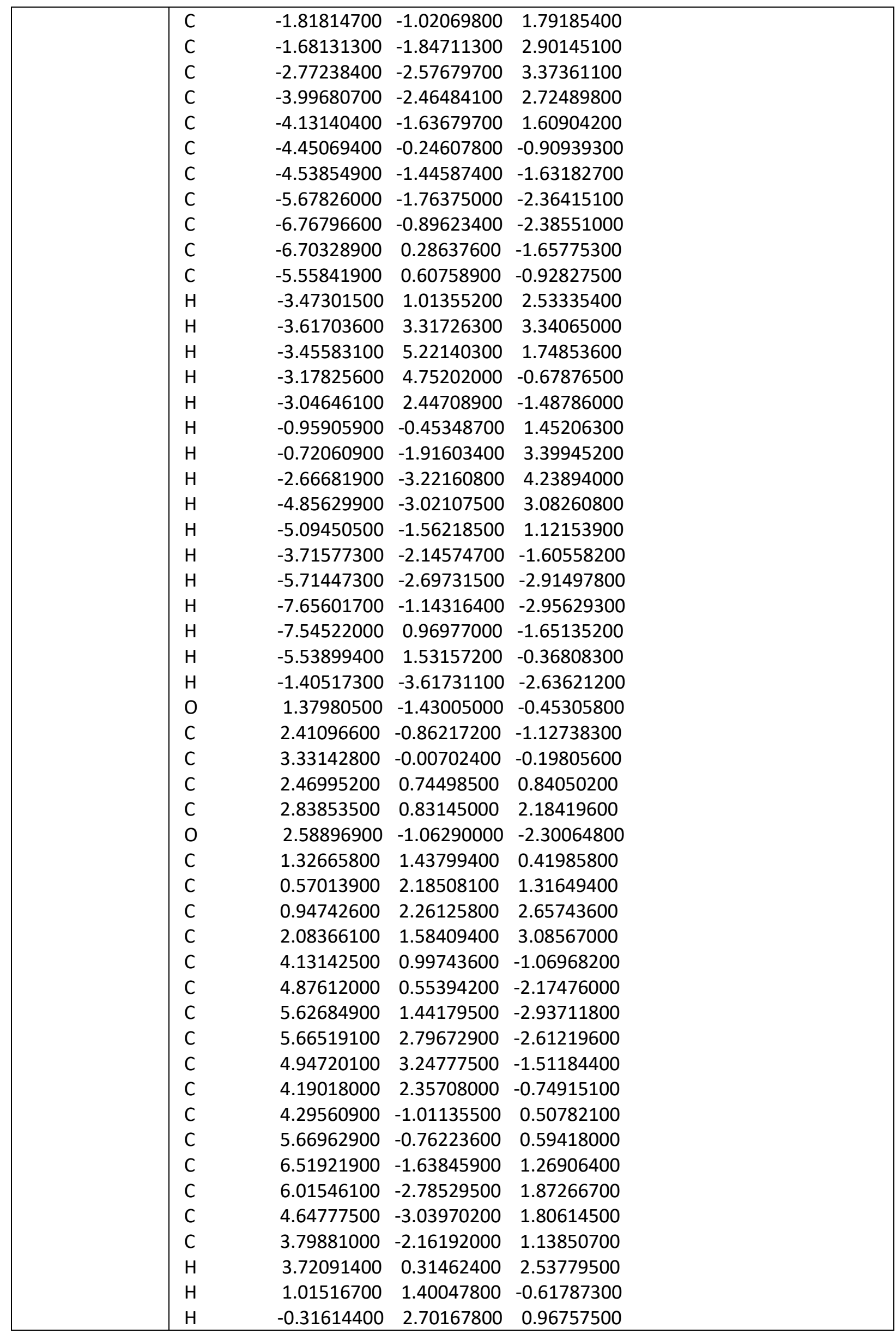




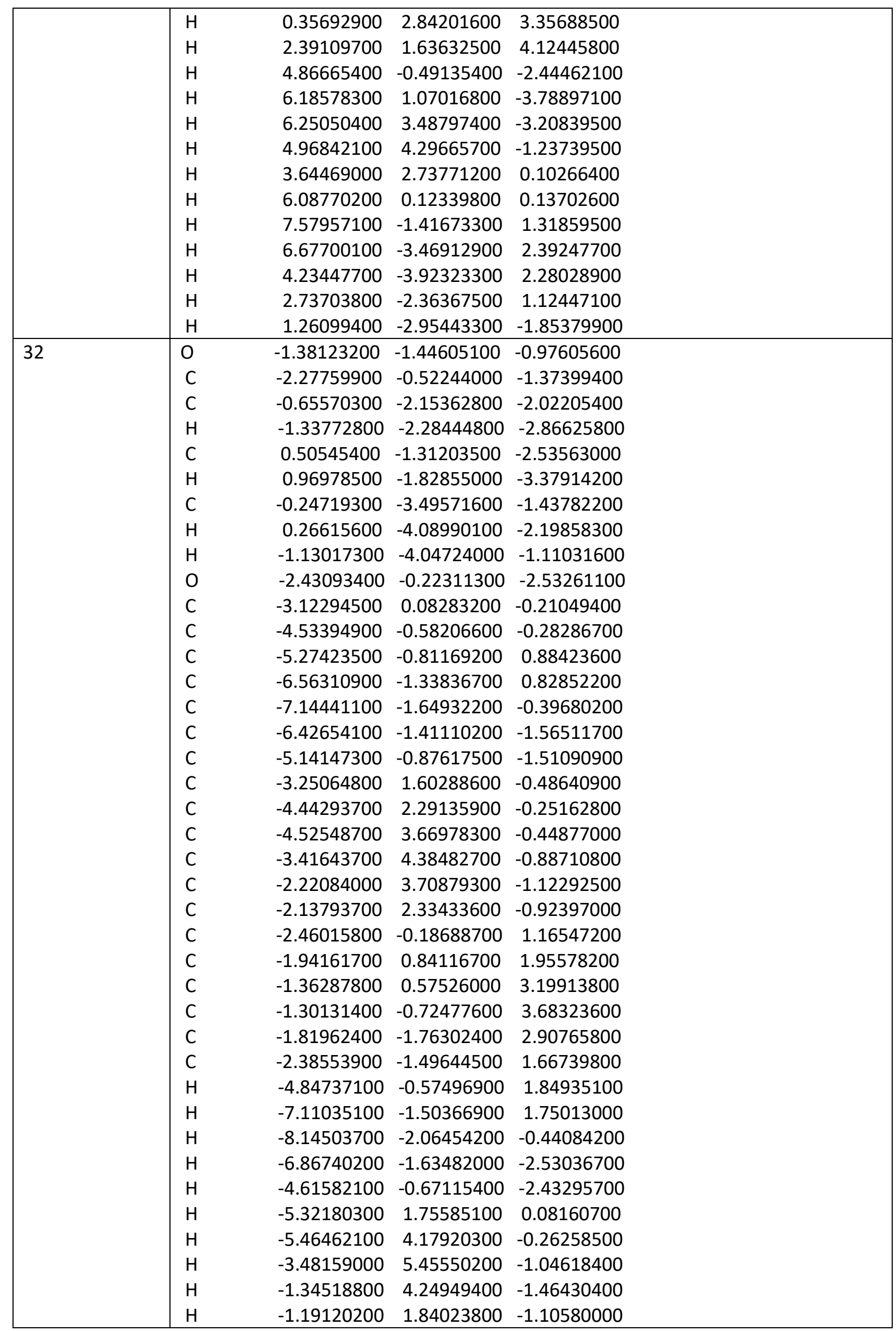




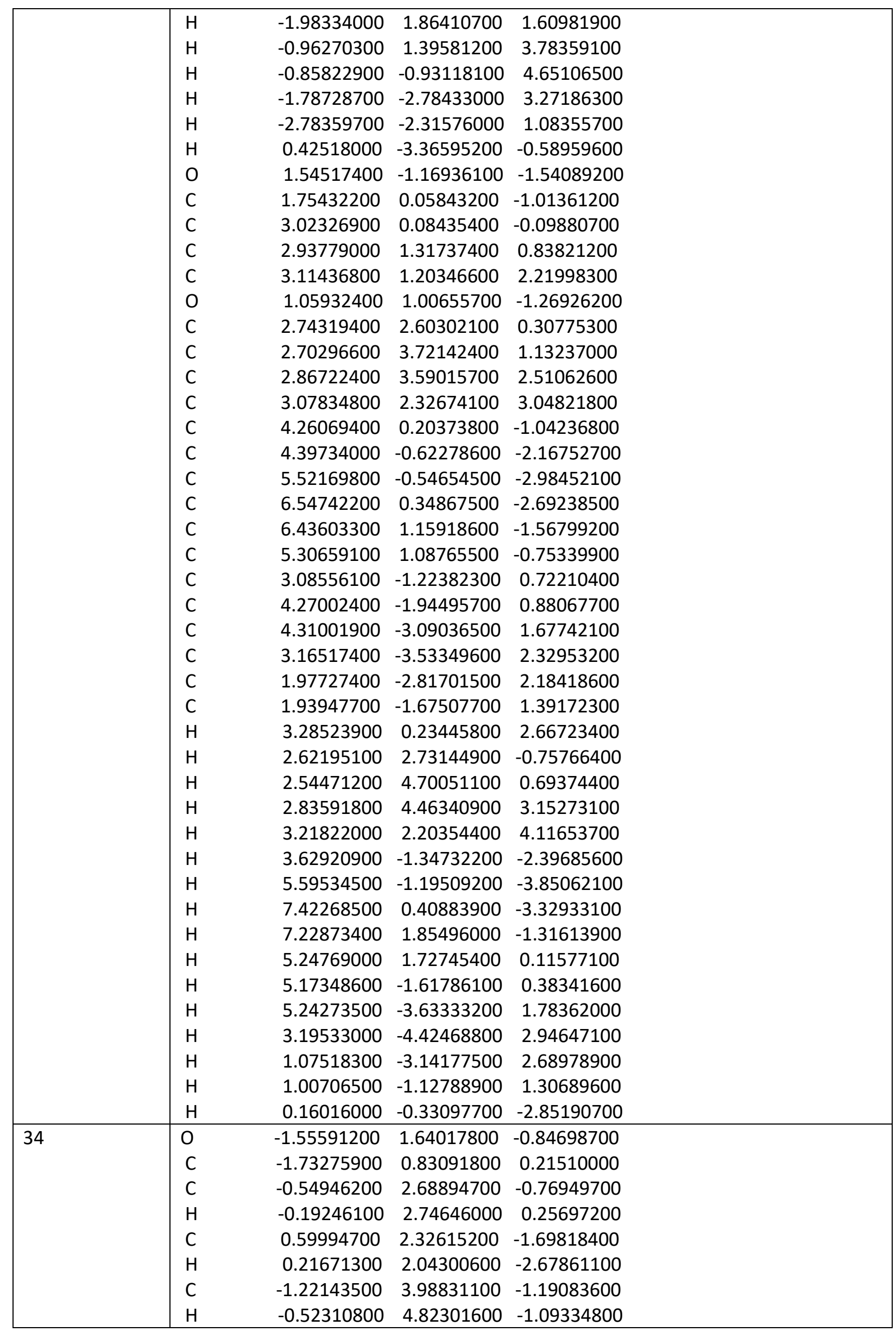




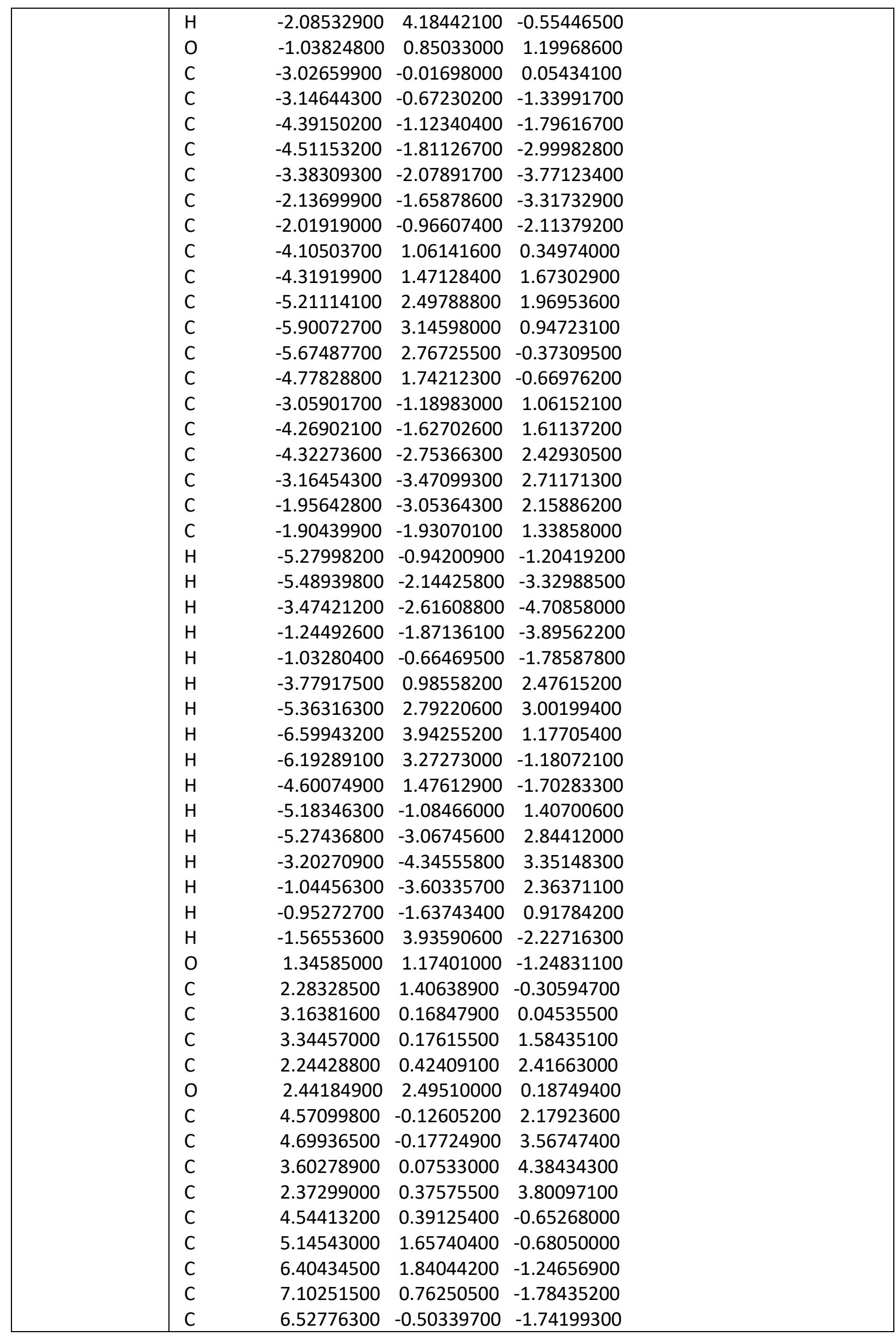




\begin{tabular}{|c|c|c|c|c|}
\hline & $C$ & 5.26358500 & -0.68651800 & -1.18333900 \\
\hline & C & 2.52367700 & -1.15997200 & -0.43326400 \\
\hline & $\mathrm{C}$ & 2.20731300 & -2.18983800 & 0.45636800 \\
\hline & C & 1.67175300 & -3.39808300 & 0.00309100 \\
\hline & $\mathrm{C}$ & 1.44501600 & -3.60414400 & -1.35125600 \\
\hline & $\mathrm{C}$ & 1.76598200 & -2.58961300 & -2.25349800 \\
\hline & $\mathrm{C}$ & 2.29866600 & -1.38895100 & -1.80084100 \\
\hline & $\mathrm{H}$ & 1.27265400 & 0.64974900 & 1.99247900 \\
\hline & $\mathrm{H}$ & 5.43856500 & -0.32140400 & 1.56304100 \\
\hline & $\mathrm{H}$ & 5.66359500 & -0.41093700 & 4.00537800 \\
\hline & $\mathrm{H}$ & 3.70338600 & 0.04123500 & 5.46349300 \\
\hline & $\mathrm{H}$ & 1.50676700 & 0.57384100 & 4.42227700 \\
\hline & $\mathrm{H}$ & 4.63372600 & 2.50397000 & -0.24440700 \\
\hline & $\mathrm{H}$ & 6.84120300 & 2.83297000 & -1.25904100 \\
\hline & $\mathrm{H}$ & 8.08328500 & 0.90653300 & -2.22361900 \\
\hline & $\mathrm{H}$ & 7.06010600 & -1.35866400 & -2.14349700 \\
\hline & $\mathrm{H}$ & 4.84306800 & -1.68239900 & -1.15876800 \\
\hline & $\mathrm{H}$ & 2.38161300 & -2.06159900 & 1.51534600 \\
\hline & $\mathrm{H}$ & 1.43892200 & -4.17800500 & 0.71953500 \\
\hline & $\mathrm{H}$ & 1.02770600 & -4.54050100 & -1.70362200 \\
\hline & $\mathrm{H}$ & 1.60756100 & -2.73699100 & -3.31628900 \\
\hline & $\mathrm{H}$ & 2.55123200 & -0.62131900 & -2.51978200 \\
\hline & $\mathrm{H}$ & 1.27578200 & 3.17739700 & -1.78819800 \\
\hline \multirow[t]{29}{*}{35} & 0 & -1.54594500 & -0.49632600 & -1.48893600 \\
\hline & $\mathrm{C}$ & -2.61083300 & 0.32877700 & -1.46147500 \\
\hline & $\mathrm{C}$ & -0.59741200 & -0.32668000 & -2.58247600 \\
\hline & $\mathrm{H}$ & -1.15384400 & 0.04885000 & -3.44439900 \\
\hline & $\mathrm{C}$ & 0.42297100 & 0.75063400 & -2.23725400 \\
\hline & $\mathrm{H}$ & 1.03048600 & 0.96394300 & -3.11747900 \\
\hline & $\mathrm{C}$ & -0.01761300 & -1.70046100 & -2.87088000 \\
\hline & $\mathrm{H}$ & 0.73369400 & -1.63286700 & -3.65965400 \\
\hline & $\mathrm{H}$ & -0.80769400 & -2.38277800 & -3.18862100 \\
\hline & 0 & -2.80034800 & 1.19023300 & -2.28476200 \\
\hline & $\mathrm{C}$ & -3.47961000 & 0.11288800 & -0.19288300 \\
\hline & $\mathrm{C}$ & -2.83214200 & 1.14790100 & 0.76915700 \\
\hline & $\mathrm{C}$ & -1.59607500 & 0.87783700 & 1.37211600 \\
\hline & $\mathrm{C}$ & -0.96968900 & 1.82898000 & 2.17383600 \\
\hline & $\mathrm{C}$ & -1.55543600 & 3.07767800 & 2.37084000 \\
\hline & $\mathrm{C}$ & -2.76543800 & 3.36927000 & 1.74779200 \\
\hline & $\mathrm{C}$ & -3.39442200 & 2.41632800 & 0.94930500 \\
\hline & $\mathrm{C}$ & -3.42350300 & -1.34812500 & 0.31206700 \\
\hline & $\mathrm{C}$ & -3.60504000 & -2.39477000 & -0.60120900 \\
\hline & $\mathrm{C}$ & -3.66232100 & -3.71754700 & -0.17985100 \\
\hline & $\mathrm{C}$ & -3.55145900 & -4.02805800 & 1.17497400 \\
\hline & $\mathrm{C}$ & -3.39067100 & -2.99838900 & 2.09517400 \\
\hline & $\mathrm{C}$ & -3.32878700 & -1.67144100 & 1.66757200 \\
\hline & $\mathrm{C}$ & -4.98112000 & 0.40209600 & -0.44332400 \\
\hline & $\mathrm{C}$ & -5.55249300 & 0.53906900 & -1.71142900 \\
\hline & $\mathrm{C}$ & -6.93014100 & 0.71001100 & -1.85911000 \\
\hline & $\mathrm{C}$ & -7.76343900 & 0.74937900 & -0.74800000 \\
\hline & $\mathrm{C}$ & -7.20639900 & 0.61039900 & 0.52236100 \\
\hline & $\mathrm{C}$ & -5.83605200 & 0.43346600 & 0.66860400 \\
\hline
\end{tabular}




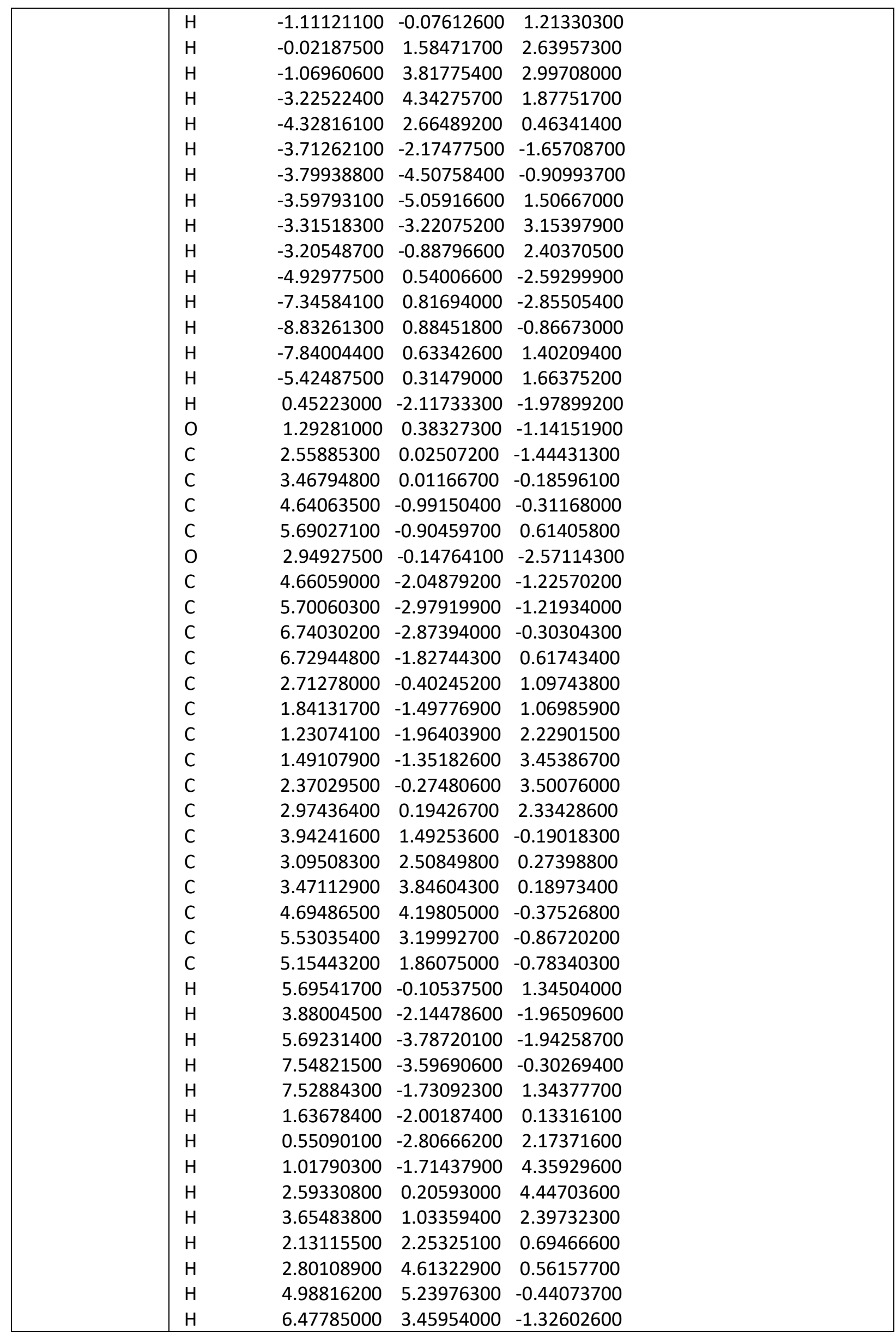




\begin{tabular}{|c|c|c|c|c|}
\hline & $\begin{array}{l}\mathrm{H} \\
\mathrm{H}\end{array}$ & $\begin{array}{r}5.80908900 \\
-0.08957900\end{array}$ & $\begin{array}{l}1.10016100 \\
1.65490000\end{array}$ & $\begin{array}{l}-1.18689900 \\
-1.91233200\end{array}$ \\
\hline \multirow[t]{50}{*}{36} & 0 & -1.47866400 & -1.44413700 & -0.43927300 \\
\hline & C & -2.29728200 & -0.56505800 & -1.05260200 \\
\hline & C & -0.61007300 & -2.24584300 & -1.28799100 \\
\hline & $\mathrm{H}$ & -0.32210000 & -1.63570500 & -2.14467300 \\
\hline & C & 0.61327300 & -2.59445200 & -0.46627500 \\
\hline & $\mathrm{H}$ & 0.32338300 & -2.96997400 & 0.51817100 \\
\hline & C & -1.36148500 & -3.49099300 & -1.74550800 \\
\hline & $\mathrm{H}$ & -1.65665800 & -4.10581200 & -0.89166200 \\
\hline & $\mathrm{H}$ & -0.73361500 & -4.09003000 & -2.41003800 \\
\hline & $\mathrm{O}$ & -2.28167300 & -0.36077900 & -2.23955300 \\
\hline & C & -3.33614900 & 0.03047300 & -0.06082200 \\
\hline & C & -2.71160500 & 0.53446800 & 1.25979900 \\
\hline & C & -3.54870000 & 0.81472100 & 2.34857600 \\
\hline & C & -3.04653400 & 1.37119300 & 3.51984300 \\
\hline & C & -1.69116600 & 1.67690300 & 3.62563100 \\
\hline & C & -0.85211800 & 1.42110000 & 2.54639400 \\
\hline & C & -1.35604300 & 0.85548100 & 1.37496300 \\
\hline & C & -4.28903900 & -1.18308300 & 0.11668100 \\
\hline & C & -5.15188000 & -1.53601600 & -0.93045500 \\
\hline & C & -5.95421000 & -2.67027100 & -0.84596700 \\
\hline & C & -5.90272000 & -3.48385100 & 0.28389100 \\
\hline & C & -5.03100800 & -3.15761400 & 1.31872000 \\
\hline & C & -4.22526900 & -2.02348400 & 1.23219200 \\
\hline & C & -4.03473700 & 1.26365200 & -0.68038400 \\
\hline & C & -5.39095400 & 1.51846000 & -0.45401900 \\
\hline & C & -5.99076700 & 2.68850700 & -0.91708100 \\
\hline & C & -5.24320500 & 3.63099900 & -1.61490000 \\
\hline & C & -3.88842100 & 3.39397600 & -1.83685100 \\
\hline & C & -3.29027600 & 2.22776700 & -1.37096800 \\
\hline & $\mathrm{H}$ & -4.60862400 & 0.60321900 & 2.27908500 \\
\hline & $\mathrm{H}$ & -3.71830700 & 1.57392100 & 4.34669900 \\
\hline & $\mathrm{H}$ & -1.29701400 & 2.11572900 & 4.53540700 \\
\hline & $\mathrm{H}$ & 0.20335900 & 1.65941300 & 2.60654200 \\
\hline & $\mathrm{H}$ & -0.67200500 & 0.66571000 & 0.55888200 \\
\hline & $\mathrm{H}$ & -5.19153100 & -0.91942300 & -1.82000800 \\
\hline & $\mathrm{H}$ & -6.61816600 & -2.91800500 & -1.66669100 \\
\hline & $\mathrm{H}$ & -6.52980300 & -4.36560900 & 0.35310900 \\
\hline & $\mathrm{H}$ & -4.97036100 & -3.78860200 & 2.19864900 \\
\hline & $\mathrm{H}$ & -3.54111100 & -1.79647300 & 2.03851500 \\
\hline & $\mathrm{H}$ & -5.99398200 & 0.79880900 & 0.08483000 \\
\hline & $\mathrm{H}$ & -7.04527900 & 2.85819600 & -0.72917400 \\
\hline & $\mathrm{H}$ & -5.70816400 & 4.54011300 & -1.97941800 \\
\hline & $\mathrm{H}$ & -3.29086200 & 4.12082900 & -2.37599200 \\
\hline & $\mathrm{H}$ & -2.23592000 & 2.07042100 & -1.55364200 \\
\hline & $\mathrm{H}$ & -2.26010900 & -3.20443800 & -2.29236600 \\
\hline & $\mathrm{O}$ & 1.41990200 & -1.41056200 & -0.27290300 \\
\hline & C & 2.76231300 & -1.54834100 & -0.39523400 \\
\hline & C & 3.50793600 & -0.19795100 & -0.13832600 \\
\hline & C & 3.67848700 & -0.04867700 & 1.40301400 \\
\hline & C & 4.84204300 & 0.51879300 & 1.93660500 \\
\hline
\end{tabular}




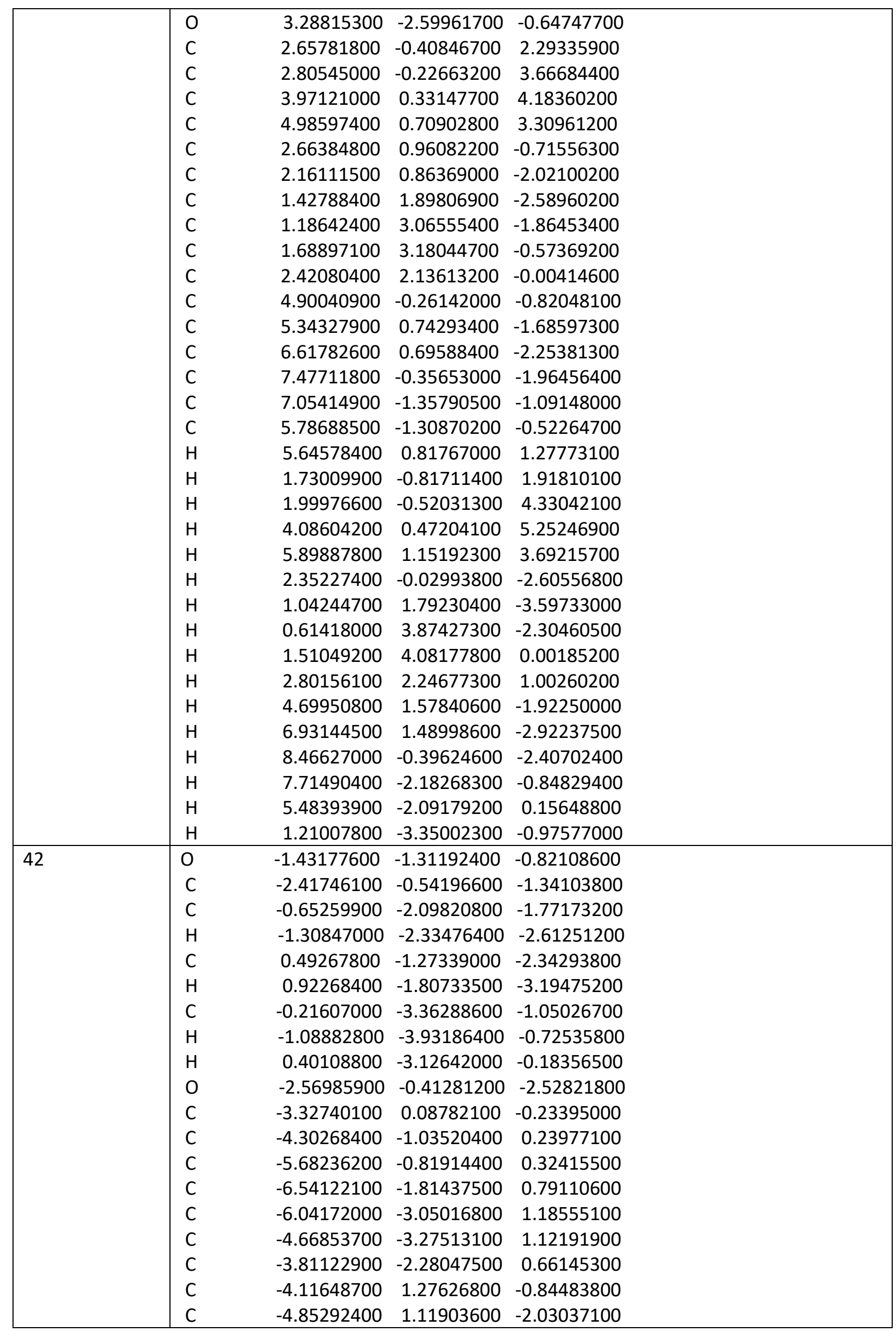




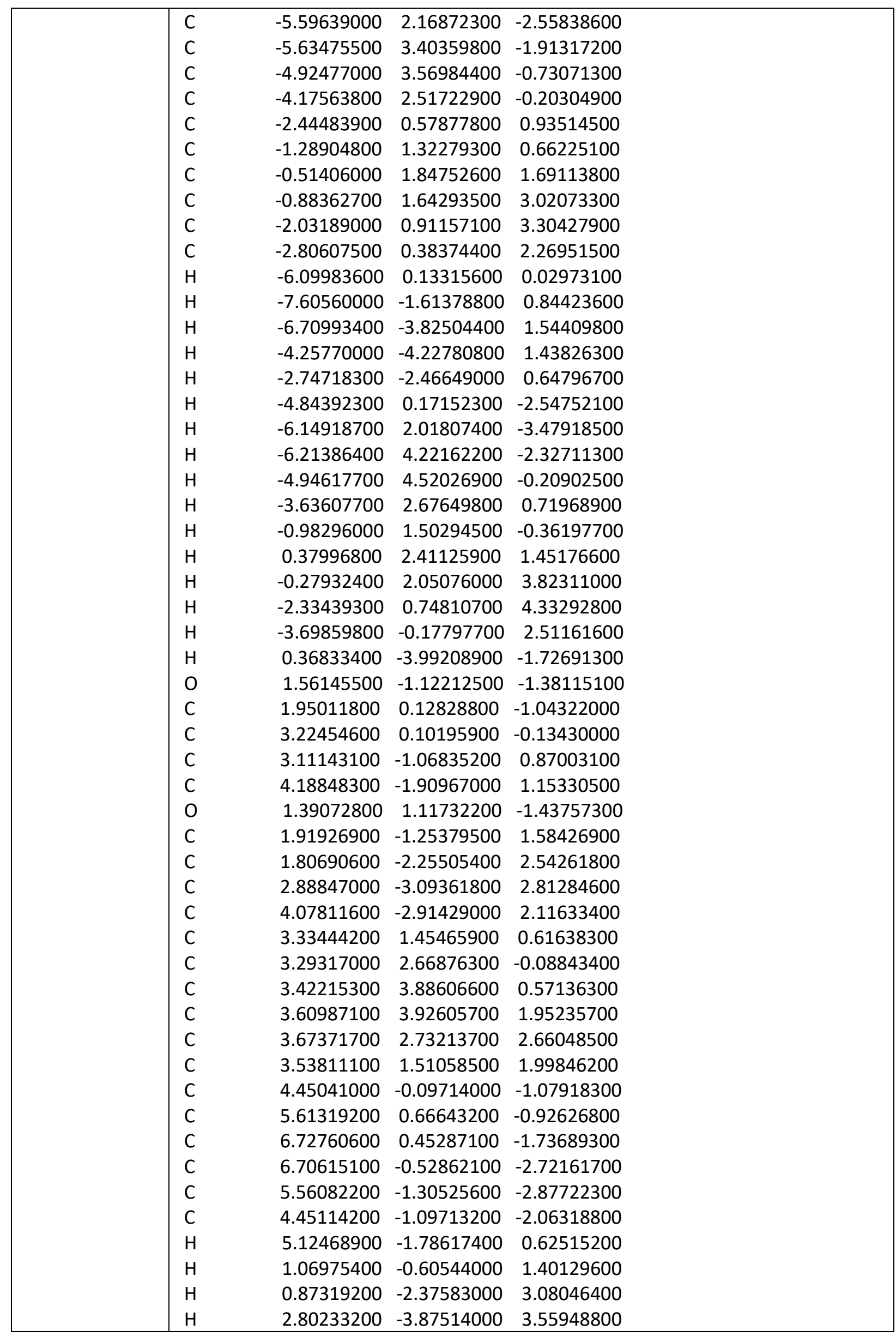




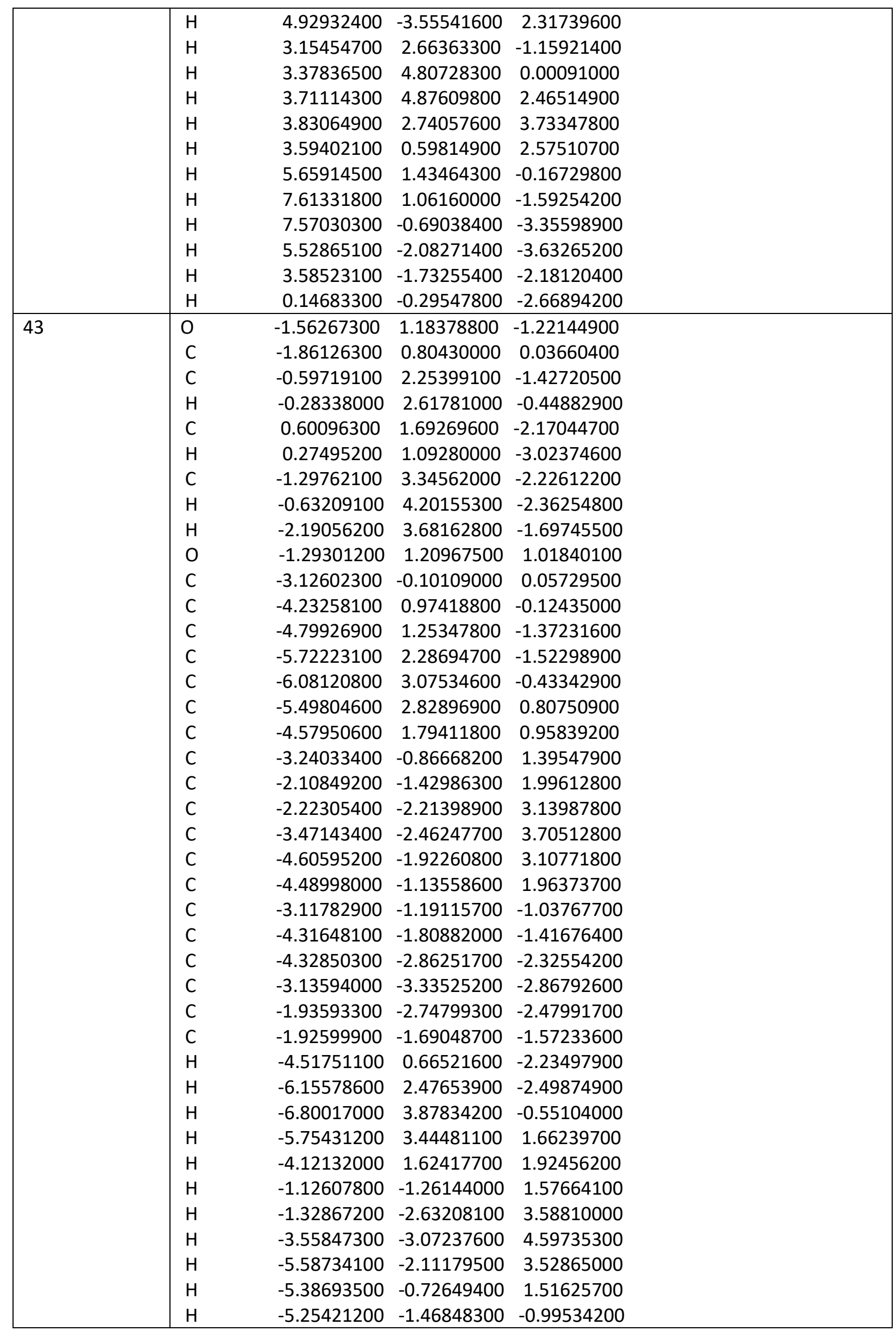




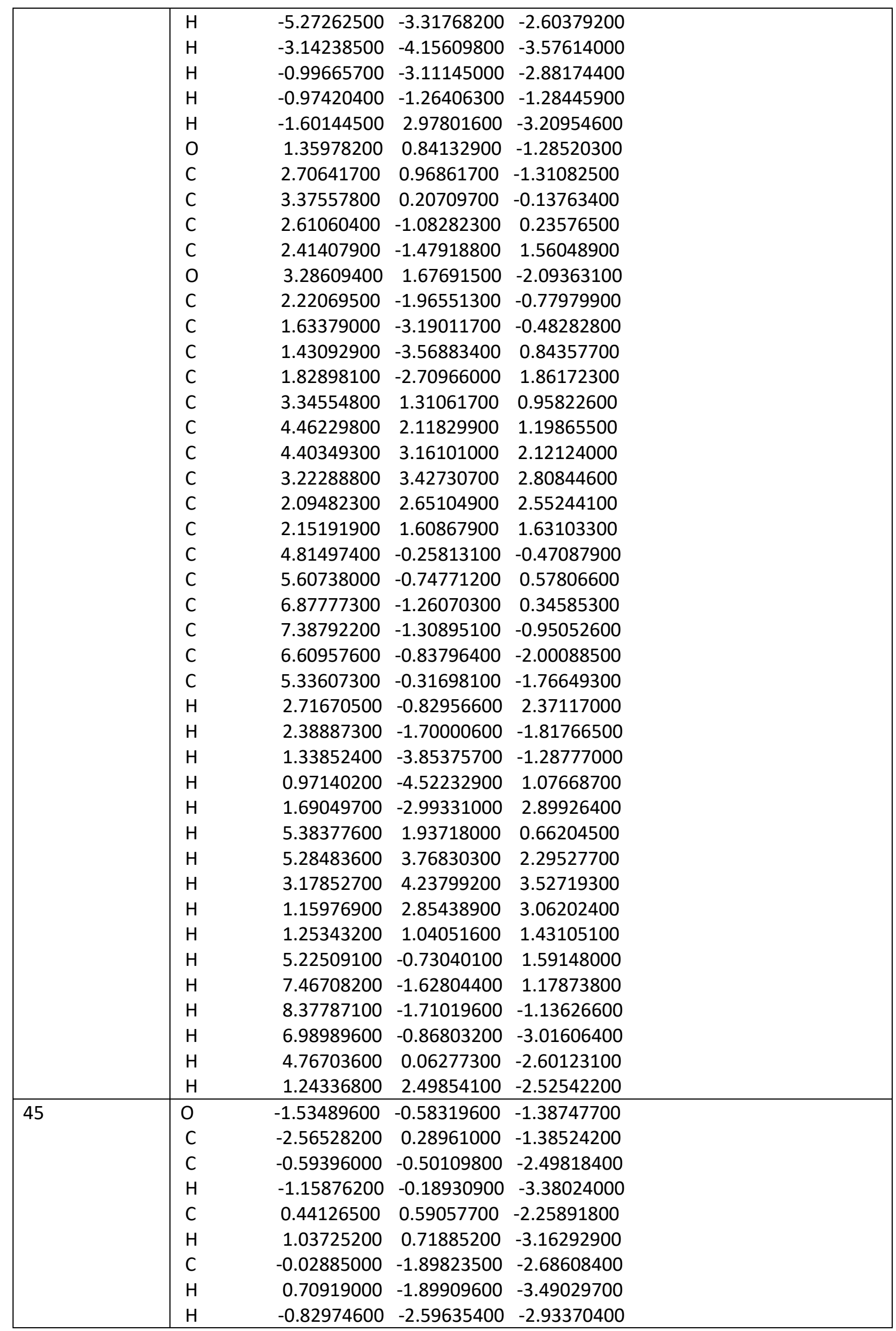




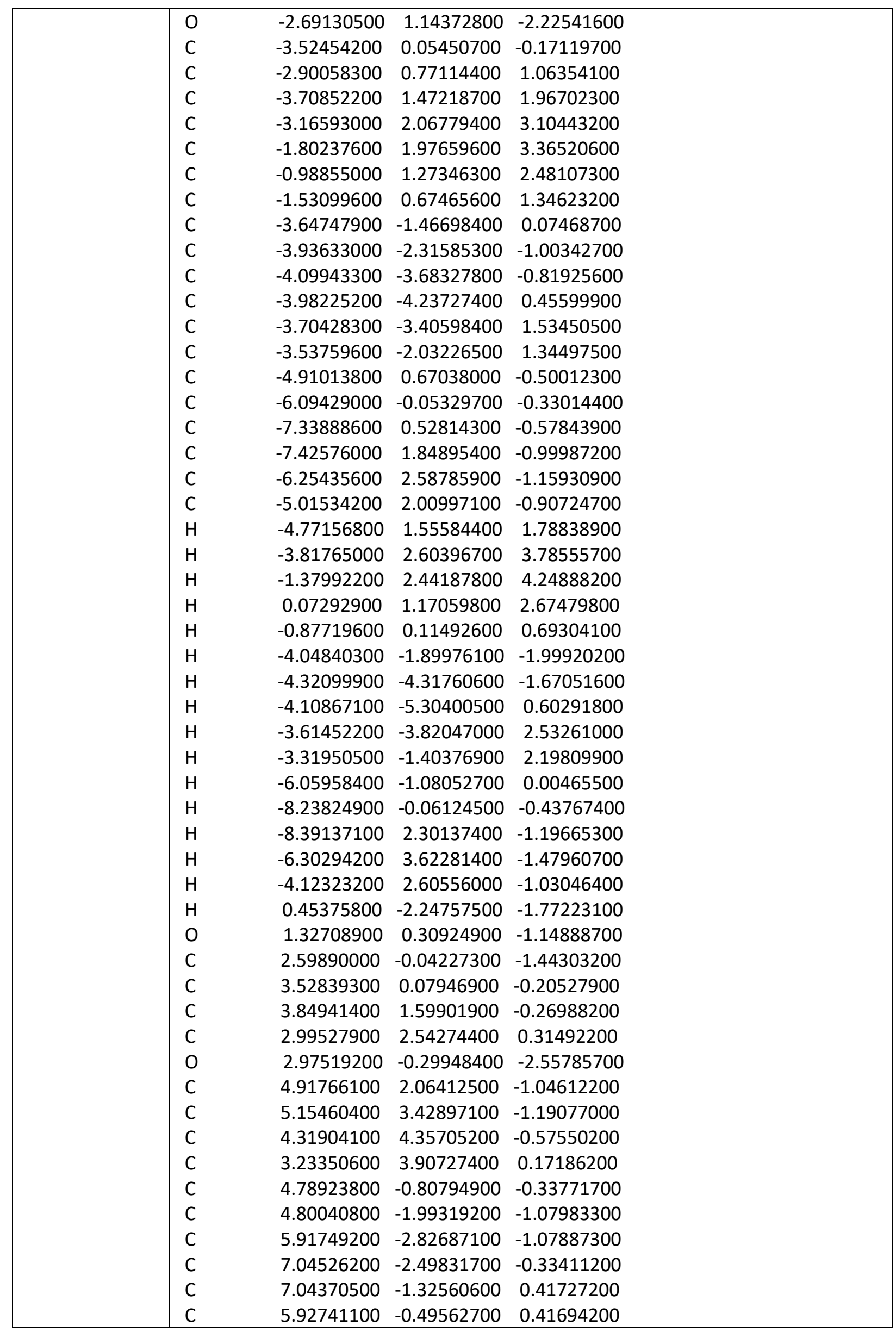




\begin{tabular}{|c|c|c|c|c|}
\hline & $C$ & 2.85674300 & -0.37197600 & 1.11041800 \\
\hline & C & 3.20873300 & 0.19937400 & 2.33817000 \\
\hline & $\mathrm{C}$ & 2.70251100 & -0.30218300 & 3.53581300 \\
\hline & C & 1.83328600 & -1.38927600 & 3.53236500 \\
\hline & $\mathrm{C}$ & 1.48329100 & -1.97493200 & 2.31838200 \\
\hline & $\mathrm{C}$ & 1.99546000 & -1.47449600 & 1.12522000 \\
\hline & $\mathrm{H}$ & 2.13267100 & 2.21213800 & 0.87781100 \\
\hline & $\mathrm{H}$ & 5.56613400 & 1.35607100 & -1.54513500 \\
\hline & $\mathrm{H}$ & 5.99256600 & 3.76396400 & -1.79178200 \\
\hline & $\mathrm{H}$ & 4.50480800 & 5.41943800 & -0.68627400 \\
\hline & $\mathrm{H}$ & 2.56270400 & 4.61804600 & 0.64150000 \\
\hline & $\mathrm{H}$ & 3.94627100 & -2.26937100 & -1.68058000 \\
\hline & $\mathrm{H}$ & 5.90116500 & -3.73661400 & -1.66885700 \\
\hline & $\mathrm{H}$ & 7.91371100 & -3.14730200 & -0.33703000 \\
\hline & $\mathrm{H}$ & 7.91126800 & -1.05487500 & 1.00884400 \\
\hline & $\mathrm{H}$ & 5.94590900 & 0.40864100 & 1.01244600 \\
\hline & $\mathrm{H}$ & 3.88673600 & 1.04266600 & 2.36745400 \\
\hline & $\mathrm{H}$ & 2.99352600 & 0.15980800 & 4.47283900 \\
\hline & $\mathrm{H}$ & 1.43515900 & -1.77695800 & 4.46304700 \\
\hline & $\mathrm{H}$ & 0.80778400 & -2.82248900 & 2.29579300 \\
\hline & $\mathrm{H}$ & 1.72079100 & -1.95907900 & 0.19663800 \\
\hline & $\mathrm{H}$ & -0.05586000 & 1.52605200 & -2.00789100 \\
\hline \multirow[t]{30}{*}{46} & 0 & -1.44827700 & -0.98385000 & -0.93722100 \\
\hline & $\mathrm{C}$ & -2.48598000 & -0.22107400 & -1.33568600 \\
\hline & $\mathrm{C}$ & -0.56901700 & -1.50604000 & -1.97749700 \\
\hline & $\mathrm{H}$ & -1.18160700 & -1.66947600 & -2.86791700 \\
\hline & $\mathrm{C}$ & 0.47725300 & -0.47221200 & -2.37274700 \\
\hline & $\mathrm{H}$ & 1.05579100 & -0.85290600 & -3.21473100 \\
\hline & $\mathrm{C}$ & -0.01132900 & -2.81992200 & -1.45782400 \\
\hline & $\mathrm{H}$ & -0.82672300 & -3.51613000 & -1.25582600 \\
\hline & $\mathrm{H}$ & 0.55319900 & -2.67294100 & -0.53611900 \\
\hline & 0 & -2.64327900 & 0.13751800 & -2.47608400 \\
\hline & $\mathrm{C}$ & -3.50096900 & 0.00975700 & -0.18240000 \\
\hline & $\mathrm{C}$ & -2.82956100 & 0.43342700 & 1.14297900 \\
\hline & $\mathrm{C}$ & -3.53773400 & 0.32279000 & 2.34672500 \\
\hline & $\mathrm{C}$ & -3.00901500 & 0.80353500 & 3.54031500 \\
\hline & $\mathrm{C}$ & -1.76056900 & 1.42169700 & 3.55724600 \\
\hline & $\mathrm{C}$ & -1.05297200 & 1.55424800 & 2.36680600 \\
\hline & $\mathrm{C}$ & -1.58272300 & 1.06547600 & 1.17338100 \\
\hline & $\mathrm{C}$ & -4.19166900 & -1.38065700 & -0.13069600 \\
\hline & $\mathrm{C}$ & -5.12246900 & -1.72304100 & -1.12129300 \\
\hline & $\mathrm{C}$ & -5.69388800 & -2.99184100 & -1.15866000 \\
\hline & $\mathrm{C}$ & -5.33711400 & -3.95055300 & -0.21329100 \\
\hline & $\mathrm{C}$ & -4.39235500 & -3.63130600 & 0.75795200 \\
\hline & $\mathrm{C}$ & -3.81789600 & -2.36199800 & 0.79303000 \\
\hline & $\mathrm{C}$ & -4.48236100 & 1.15576000 & -0.52498100 \\
\hline & $\mathrm{C}$ & -5.80872100 & 1.11740400 & -0.08168300 \\
\hline & $\mathrm{C}$ & -6.66767200 & 2.19536400 & -0.28513000 \\
\hline & $\mathrm{C}$ & -6.21571100 & 3.33968500 & -0.93456700 \\
\hline & $\mathrm{C}$ & -4.89415600 & 3.39560800 & -1.37000800 \\
\hline & $\mathrm{C}$ & -4.03694500 & 2.31908400 & -1.16317500 \\
\hline & $\mathrm{H}$ & -4.51774200 & -0.13778100 & 2.35419500 \\
\hline
\end{tabular}




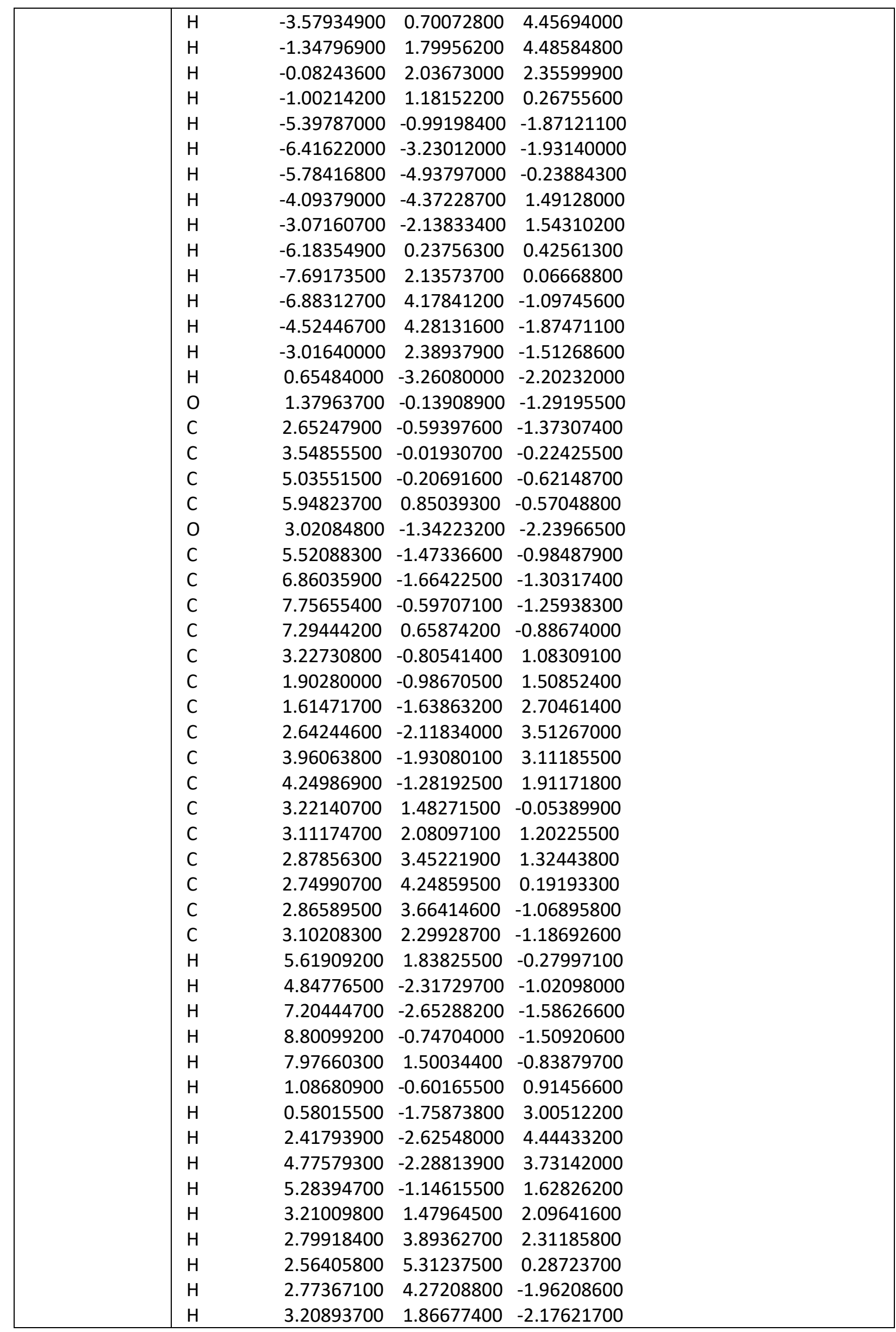




\begin{tabular}{|c|c|c|c|c|}
\hline & $\mathrm{H}$ & -0.00885000 & 0.46179700 & -2.65008400 \\
\hline \multirow[t]{51}{*}{48} & 0 & -1.40291800 & -0.43221900 & -1.37723000 \\
\hline & $\mathrm{C}$ & -2.74280200 & -0.56881300 & -1.47722400 \\
\hline & $\mathrm{C}$ & -0.61501200 & -0.84986100 & -2.53258800 \\
\hline & $\mathrm{H}$ & -1.21704100 & -0.65763100 & -3.42351500 \\
\hline & $\mathrm{C}$ & 0.60134200 & 0.04976300 & -2.60296100 \\
\hline & $\mathrm{H}$ & 1.12551200 & -0.11309300 & -3.54758600 \\
\hline & $\mathrm{C}$ & -0.29621400 & -2.33558800 & -2.43262700 \\
\hline & $\mathrm{H}$ & -1.21841800 & -2.91695900 & -2.46316800 \\
\hline & $\mathrm{H}$ & 0.24433400 & -2.55225000 & -1.51096200 \\
\hline & 0 & -3.27458700 & -1.02574100 & -2.45588600 \\
\hline & $\mathrm{C}$ & -3.48575100 & -0.05125400 & -0.19774000 \\
\hline & $\mathrm{C}$ & -4.94102900 & -0.58613400 & -0.21831900 \\
\hline & $\mathrm{C}$ & -5.50327800 & -1.21763400 & 0.89511700 \\
\hline & $\mathrm{C}$ & -6.82654100 & -1.66164300 & 0.88390300 \\
\hline & $\mathrm{C}$ & -7.61837700 & -1.48203700 & -0.24320100 \\
\hline & $\mathrm{C}$ & -7.07554400 & -0.84544400 & -1.35823900 \\
\hline & $\mathrm{C}$ & -5.75840200 & -0.40079400 & -1.34526000 \\
\hline & $\mathrm{C}$ & -3.46015500 & 1.50832000 & -0.24431500 \\
\hline & $\mathrm{C}$ & -2.24145800 & 2.19680600 & -0.35178800 \\
\hline & $\mathrm{C}$ & -2.19807800 & 3.58746100 & -0.35878000 \\
\hline & $\mathrm{C}$ & -3.37159900 & 4.33055900 & -0.24906200 \\
\hline & $\mathrm{C}$ & -4.58384900 & 3.66288800 & -0.12056700 \\
\hline & $\mathrm{C}$ & -4.62759200 & 2.26818900 & -0.11599200 \\
\hline & $\mathrm{C}$ & -2.74554700 & -0.57308900 & 1.05512300 \\
\hline & $\mathrm{C}$ & -2.58841200 & 0.21186600 & 2.19932500 \\
\hline & $\mathrm{C}$ & -1.98235000 & -0.30556900 & 3.34520700 \\
\hline & $\mathrm{C}$ & -1.52028700 & -1.61735800 & 3.36539600 \\
\hline & $\mathrm{C}$ & -1.68030600 & -2.41419100 & 2.23261800 \\
\hline & $\mathrm{C}$ & -2.29069300 & -1.89711400 & 1.09531200 \\
\hline & $\mathrm{H}$ & -4.91332900 & -1.37023800 & 1.78794500 \\
\hline & $\mathrm{H}$ & -7.23072900 & -2.14951100 & 1.76400700 \\
\hline & $\mathrm{H}$ & -8.64545100 & -1.82955700 & -0.25530400 \\
\hline & $\mathrm{H}$ & -7.67947700 & -0.69292400 & -2.24596500 \\
\hline & $\mathrm{H}$ & -5.36340000 & 0.09171000 & -2.22129800 \\
\hline & $\mathrm{H}$ & -1.31685700 & 1.64149600 & -0.41172400 \\
\hline & $\mathrm{H}$ & -1.23976100 & 4.08780400 & -0.44283400 \\
\hline & $\mathrm{H}$ & -3.33838700 & 5.41425600 & -0.25609100 \\
\hline & $\mathrm{H}$ & -5.50736700 & 4.22241000 & -0.02032100 \\
\hline & $\mathrm{H}$ & -5.58398900 & 1.77752800 & -0.00553000 \\
\hline & $\mathrm{H}$ & -2.94420000 & 1.23391800 & 2.20406500 \\
\hline & $\mathrm{H}$ & -1.87452600 & 0.32312600 & 4.22227900 \\
\hline & $\mathrm{H}$ & -1.04331600 & -2.01743800 & 4.25289000 \\
\hline & $\mathrm{H}$ & -1.32768800 & -3.43929700 & 2.23304900 \\
\hline & $\mathrm{H}$ & -2.42233600 & -2.53873600 & 0.23121300 \\
\hline & $\mathrm{H}$ & 0.32436100 & -2.64095800 & -3.27985600 \\
\hline & 0 & 1.49055800 & -0.25271100 & -1.50828800 \\
\hline & $\mathrm{C}$ & 2.67787900 & 0.38809400 & -1.52284600 \\
\hline & $\mathrm{C}$ & 3.48192400 & 0.13401100 & -0.22080400 \\
\hline & $\mathrm{C}$ & 5.01376600 & 0.22836600 & -0.42783900 \\
\hline & $\mathrm{C}$ & 5.82847000 & 0.20295300 & 0.71426900 \\
\hline & 0 & 3.00086800 & 1.13647200 & -2.41021300 \\
\hline
\end{tabular}




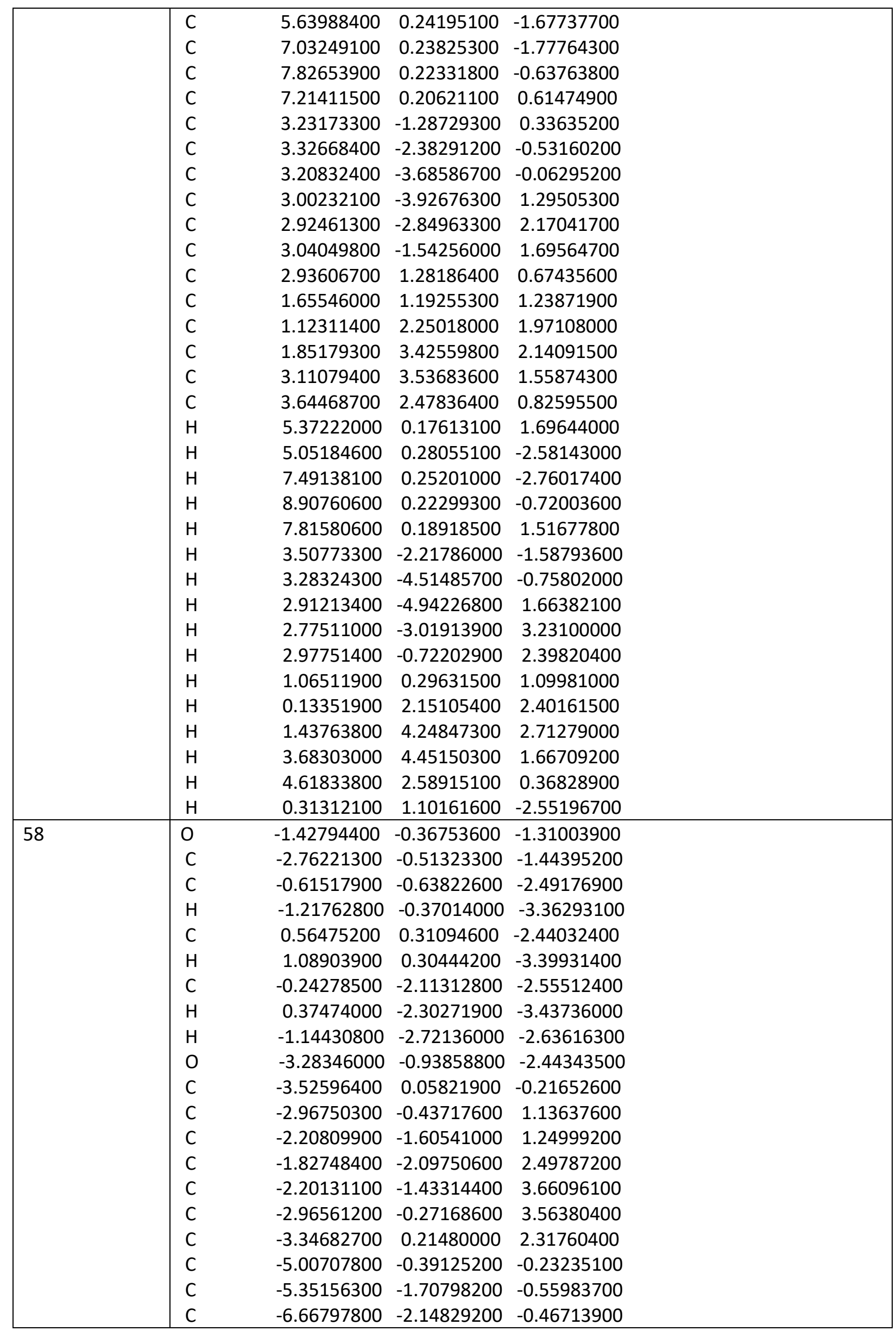




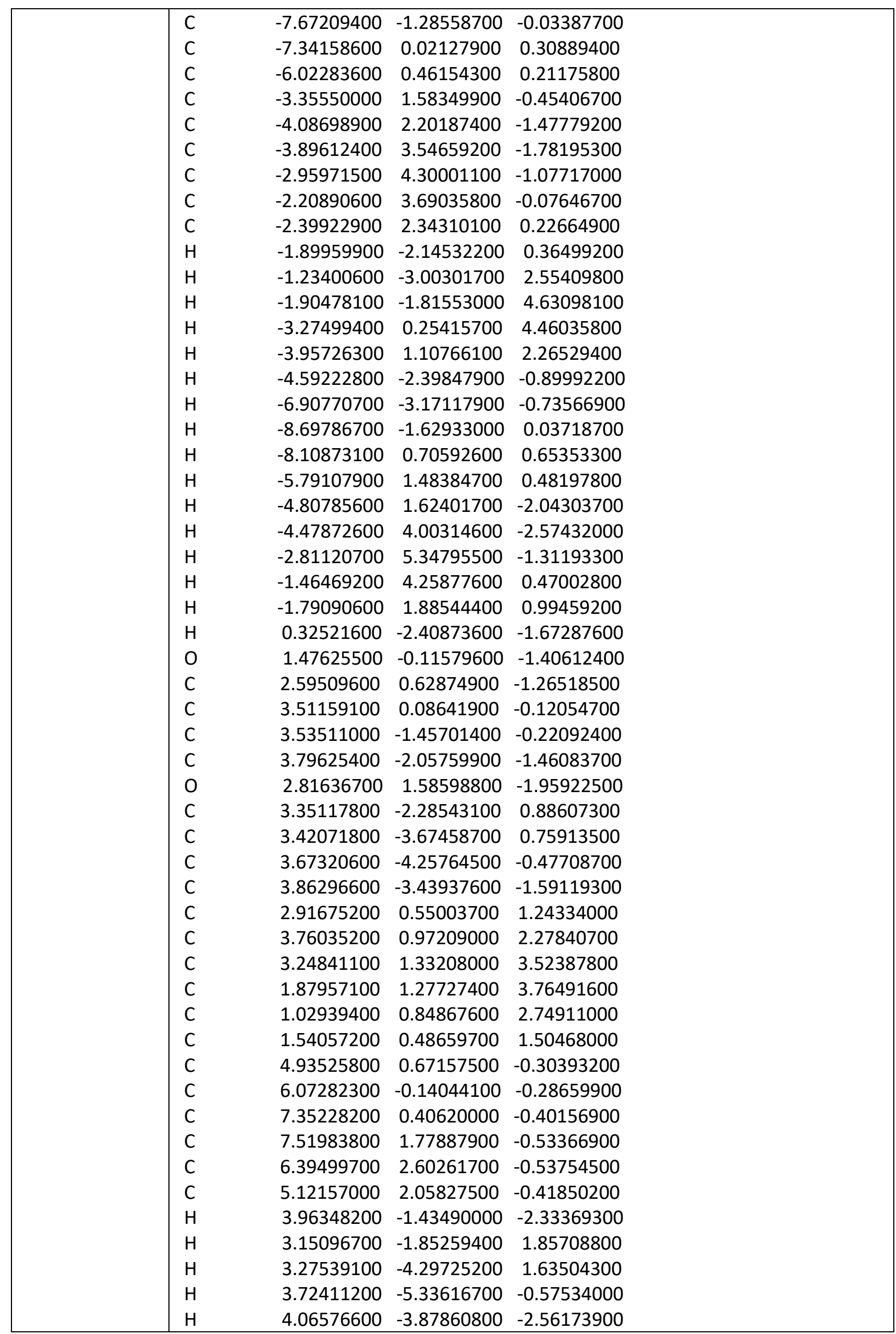




\begin{tabular}{|c|c|c|c|c|}
\hline & $\mathrm{H}$ & 4.82842900 & 1.02037100 & 2.11809700 \\
\hline & $\mathrm{H}$ & 3.92840100 & 1.65521300 & 4.30446800 \\
\hline & $\mathrm{H}$ & 1.48017000 & 1.55869400 & 4.73293000 \\
\hline & $\mathrm{H}$ & -0.03809400 & 0.77768800 & 2.92306300 \\
\hline & $\mathrm{H}$ & 0.85796500 & 0.13576200 & 0.74413900 \\
\hline & $\mathrm{H}$ & 5.97317500 & -1.21152400 & -0.17803500 \\
\hline & $\mathrm{H}$ & 8.21473200 & -0.25103300 & -0.38598800 \\
\hline & $\mathrm{H}$ & 8.51242700 & 2.20531400 & -0.62719400 \\
\hline & $\mathrm{H}$ & 6.50775200 & 3.67705900 & -0.63190700 \\
\hline & $\mathrm{H}$ & 4.26601800 & 2.71748800 & -0.41700000 \\
\hline & $\mathrm{H}$ & 0.23344700 & 1.33053700 & -2.23794600 \\
\hline \multirow[t]{41}{*}{59} & 0 & -1.60880500 & -1.65782700 & 0.84821100 \\
\hline & $\mathrm{C}$ & -1.74427800 & -0.76922300 & -0.15395800 \\
\hline & $\mathrm{C}$ & -0.66644700 & -2.75559000 & 0.69422500 \\
\hline & $\mathrm{H}$ & -0.42552800 & -2.85374900 & -0.36485100 \\
\hline & $\mathrm{C}$ & 0.61720000 & -2.47695100 & 1.47591600 \\
\hline & $\mathrm{H}$ & 0.40371100 & -1.87049200 & 2.35647000 \\
\hline & $\mathrm{C}$ & -1.38325800 & -3.99465400 & 1.21701300 \\
\hline & $\mathrm{H}$ & -0.77026200 & -4.88466200 & 1.05530100 \\
\hline & $\mathrm{H}$ & -2.33108900 & -4.12449200 & 0.69341200 \\
\hline & 0 & -1.03252000 & -0.74510300 & -1.12653900 \\
\hline & $\mathrm{C}$ & -3.01846900 & 0.10260600 & 0.02456700 \\
\hline & $\mathrm{C}$ & -2.91512100 & 1.40840700 & -0.79591000 \\
\hline & $\mathrm{C}$ & -4.06136200 & 2.00180300 & -1.33592400 \\
\hline & $\mathrm{C}$ & -3.99691500 & 3.23479900 & -1.98070500 \\
\hline & $\mathrm{C}$ & -2.78188500 & 3.90422400 & -2.09326500 \\
\hline & $\mathrm{C}$ & -1.63685200 & 3.33201800 & -1.54505400 \\
\hline & $\mathrm{C}$ & -1.70315300 & 2.10053400 & -0.89905100 \\
\hline & $\mathrm{C}$ & -3.26603900 & 0.55345600 & 1.48171600 \\
\hline & $\mathrm{C}$ & -2.20441500 & 0.77396000 & 2.36585400 \\
\hline & $\mathrm{C}$ & -2.43273900 & 1.29273000 & 3.63880700 \\
\hline & $\mathrm{C}$ & -3.72283000 & 1.60999000 & 4.05344700 \\
\hline & $\mathrm{C}$ & -4.78500800 & 1.41417200 & 3.17463400 \\
\hline & $\mathrm{C}$ & -4.55653800 & 0.89767200 & 1.90223500 \\
\hline & $\mathrm{C}$ & -4.08470000 & -0.88585300 & -0.52518600 \\
\hline & $\mathrm{C}$ & -4.78949400 & -1.74940100 & 0.32142500 \\
\hline & $\mathrm{C}$ & -5.66563100 & -2.70152600 & -0.19624400 \\
\hline & $\mathrm{C}$ & -5.84032500 & -2.82321100 & -1.57217300 \\
\hline & $\mathrm{C}$ & -5.12009600 & -1.99160800 & -2.42621500 \\
\hline & $\mathrm{C}$ & -4.24669000 & -1.03891100 & -1.90862700 \\
\hline & $\mathrm{H}$ & -5.01759600 & 1.49967100 & -1.25875900 \\
\hline & $\mathrm{H}$ & -4.90071200 & 3.66983000 & -2.39322200 \\
\hline & $\mathrm{H}$ & -2.72867600 & 4.86250100 & -2.59795600 \\
\hline & $\mathrm{H}$ & -0.68161400 & 3.83968400 & -1.61558700 \\
\hline & $\mathrm{H}$ & -0.79795000 & 1.68702700 & -0.47599400 \\
\hline & $\mathrm{H}$ & -1.18741600 & 0.55222000 & 2.07079600 \\
\hline & $\mathrm{H}$ & -1.59160700 & 1.45190200 & 4.30463400 \\
\hline & $\mathrm{H}$ & -3.89822200 & 2.01261600 & 5.04481600 \\
\hline & $\mathrm{H}$ & -5.79567900 & 1.66875300 & 3.47455400 \\
\hline & $\mathrm{H}$ & -5.39619500 & 0.76742900 & 1.23086200 \\
\hline & $\mathrm{H}$ & -4.65291800 & -1.68065800 & 1.39218200 \\
\hline & $\mathrm{H}$ & -6.20915700 & -3.35068900 & 0.48149900 \\
\hline
\end{tabular}




\begin{tabular}{|c|c|c|c|c|}
\hline & $\mathrm{H}$ & -6.52363400 & -3.56256800 & -1.97472300 \\
\hline & $\mathrm{H}$ & -5.23233000 & -2.08489000 & -3.50069900 \\
\hline & $\mathrm{H}$ & -3.67900600 & -0.41318800 & -2.58515800 \\
\hline & $\mathrm{H}$ & -1.59306600 & -3.90307900 & 2.28608700 \\
\hline & 0 & 1.64340300 & -1.84503100 & 0.67712400 \\
\hline & $\mathrm{C}$ & 1.79851500 & -0.50848700 & 0.76637400 \\
\hline & $\mathrm{C}$ & 3.06063900 & -0.01628900 & -0.01606500 \\
\hline & $\mathrm{C}$ & 4.26150700 & -0.17204800 & 0.96794300 \\
\hline & $\mathrm{C}$ & 5.22160500 & 0.83539400 & 1.11029800 \\
\hline & 0 & 1.06923600 & 0.18817000 & 1.42532700 \\
\hline & $\mathrm{C}$ & 4.45090600 & -1.36254000 & 1.68463400 \\
\hline & $\mathrm{C}$ & 5.54358600 & -1.52683600 & 2.53075100 \\
\hline & $\mathrm{C}$ & 6.48488600 & -0.51020700 & 2.67236400 \\
\hline & $\mathrm{C}$ & 6.32099100 & 0.66783300 & 1.95169000 \\
\hline & $\mathrm{C}$ & 2.85961500 & 1.47338100 & -0.40152000 \\
\hline & $\mathrm{C}$ & 2.55290600 & 2.43099300 & 0.57951800 \\
\hline & $\mathrm{C}$ & 2.40509500 & 3.77404000 & 0.25086100 \\
\hline & $\mathrm{C}$ & 2.57040000 & 4.20196100 & -1.06581600 \\
\hline & $\mathrm{C}$ & 2.88796800 & 3.26837400 & -2.04479000 \\
\hline & $\mathrm{C}$ & 3.03161900 & 1.91964500 & -1.71518900 \\
\hline & $\mathrm{C}$ & 3.27913600 & -0.88400000 & -1.27724400 \\
\hline & $\mathrm{C}$ & 2.19762400 & -1.17176100 & -2.12220900 \\
\hline & $\mathrm{C}$ & 2.38288100 & -1.89650100 & -3.29456700 \\
\hline & $\mathrm{C}$ & 3.65376100 & -2.34438200 & -3.65436900 \\
\hline & $\mathrm{C}$ & 4.73420600 & -2.05668400 & -2.82834300 \\
\hline & $\mathrm{C}$ & 4.54767900 & -1.33304100 & -1.64943800 \\
\hline & $\mathrm{H}$ & 5.11910800 & 1.75976800 & 0.55927500 \\
\hline & $\mathrm{H}$ & 3.75104100 & -2.17788500 & 1.56472300 \\
\hline & $\mathrm{H}$ & 5.66047300 & -2.45695500 & 3.07608000 \\
\hline & $\mathrm{H}$ & 7.33645500 & -0.63785600 & 3.33108300 \\
\hline & $\mathrm{H}$ & 7.04892200 & 1.46678100 & 2.03957000 \\
\hline & $\mathrm{H}$ & 2.42699900 & 2.12505600 & 1.60736400 \\
\hline & $\mathrm{H}$ & 2.16195600 & 4.48849600 & 1.02954900 \\
\hline & $\mathrm{H}$ & 2.45877300 & 5.25015900 & -1.32045000 \\
\hline & $\mathrm{H}$ & 3.02670400 & 3.58093500 & -3.07380700 \\
\hline & $\mathrm{H}$ & 3.28116700 & 1.21813200 & -2.49836900 \\
\hline & $\mathrm{H}$ & 1.20008400 & -0.83192700 & -1.87005800 \\
\hline & $\mathrm{H}$ & 1.52913400 & -2.10902500 & -3.92856600 \\
\hline & $\mathrm{H}$ & 3.79726700 & -2.90981300 & -4.56845800 \\
\hline & $\mathrm{H}$ & 5.72978800 & -2.39455600 & -3.09445100 \\
\hline & $\mathrm{H}$ & 5.40341600 & -1.12127200 & -1.02205500 \\
\hline & $\mathrm{H}$ & 1.06425600 & -3.42462700 & 1.77746700 \\
\hline 60 & 0 & -1.41836600 & -1.57421300 & -0.72142900 \\
\hline & $\mathrm{C}$ & -2.40666700 & -0.78561500 & -1.17641800 \\
\hline & $\mathrm{C}$ & -0.62994300 & -2.30818800 & -1.70070700 \\
\hline & $\mathrm{H}$ & -0.46760800 & -1.65996000 & -2.56305700 \\
\hline & $\mathrm{C}$ & 0.69362400 & -2.63432800 & -1.03028400 \\
\hline & $\mathrm{H}$ & 0.53360300 & -2.97801500 & -0.00840500 \\
\hline & $\mathrm{C}$ & -1.37664900 & -3.57181300 & -2.11548000 \\
\hline & $\mathrm{H}$ & -0.79352800 & -4.13134600 & -2.85134200 \\
\hline & $\mathrm{H}$ & -2.33121600 & -3.30910100 & -2.57105600 \\
\hline & 0 & -2.62048800 & -0.61794200 & -2.35191200 \\
\hline
\end{tabular}




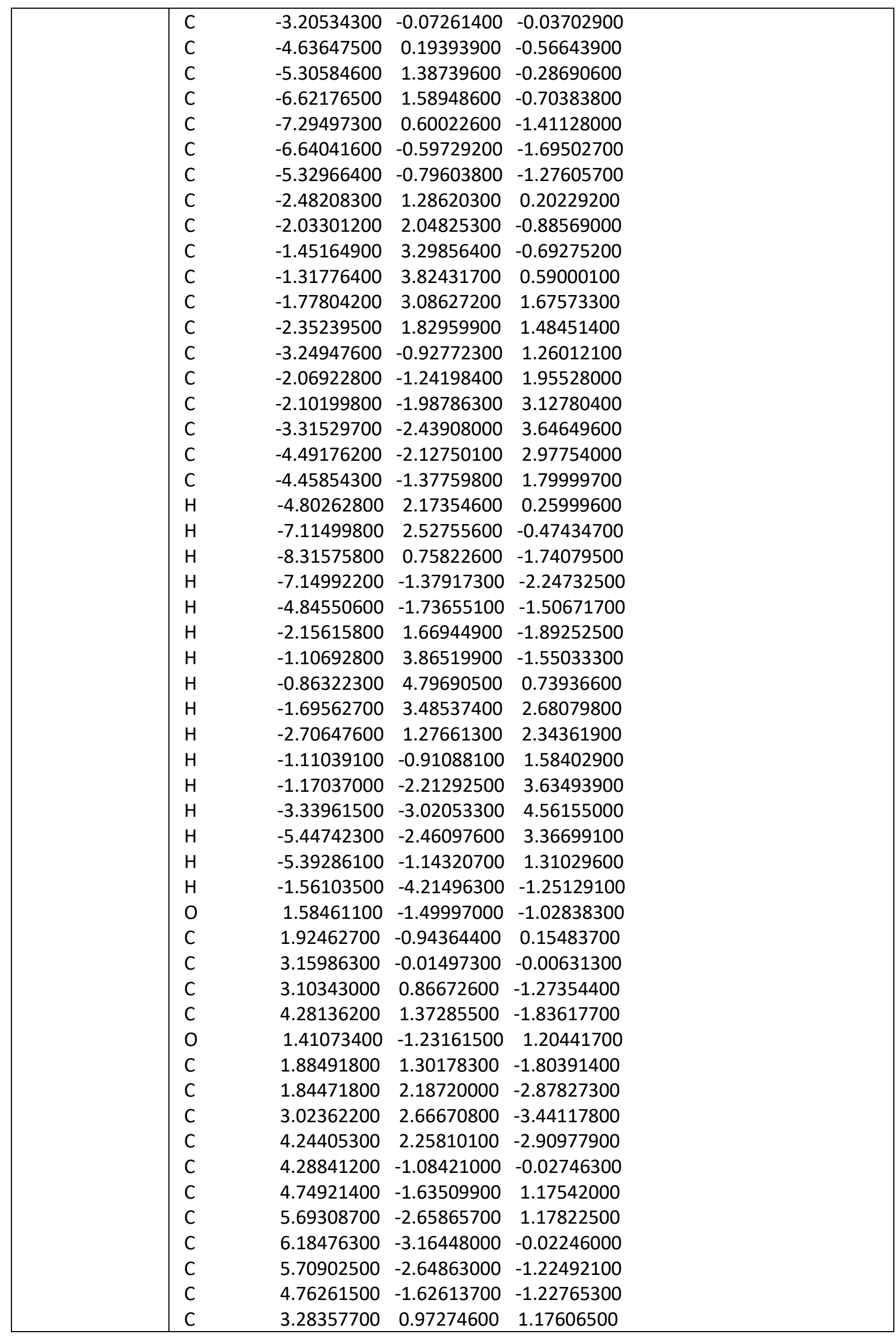




\begin{tabular}{|c|c|c|c|c|}
\hline & $C$ & 4.54122600 & 1.42942900 & 1.58733400 \\
\hline & C & 4.66641500 & 2.40456400 & 2.57346800 \\
\hline & $\mathrm{C}$ & 3.53217500 & 2.95097000 & 3.16697700 \\
\hline & C & 2.27529800 & 2.51686900 & 2.75534700 \\
\hline & $\mathrm{C}$ & 2.15142200 & 1.54259000 & 1.76823000 \\
\hline & $\mathrm{H}$ & 5.24177300 & 1.07686300 & -1.43325500 \\
\hline & $\mathrm{H}$ & 0.95021500 & 0.95809600 & -1.38046100 \\
\hline & $\mathrm{H}$ & 0.88482600 & 2.49990400 & -3.27349700 \\
\hline & $\mathrm{H}$ & 2.99223000 & 3.35388800 & -4.27911000 \\
\hline & $\mathrm{H}$ & 5.17286300 & 2.63070300 & -3.32748800 \\
\hline & $\mathrm{H}$ & 4.36304100 & -1.26393600 & 2.11606300 \\
\hline & $\mathrm{H}$ & 6.03958100 & -3.06279800 & 2.12284300 \\
\hline & $\mathrm{H}$ & 6.92281400 & -3.95871200 & -0.02096600 \\
\hline & $\mathrm{H}$ & 6.07009800 & -3.04389900 & -2.16801100 \\
\hline & $\mathrm{H}$ & 4.39012300 & -1.25018900 & -2.17108400 \\
\hline & $\mathrm{H}$ & 5.43747200 & 1.01998500 & 1.13857400 \\
\hline & $\mathrm{H}$ & 5.65400300 & 2.73627600 & 2.87464100 \\
\hline & $\mathrm{H}$ & 3.62666700 & 3.70799100 & 3.93744400 \\
\hline & $\mathrm{H}$ & 1.37997500 & 2.93578200 & 3.20056300 \\
\hline & $\mathrm{H}$ & 1.16072000 & 1.23109700 & 1.46848500 \\
\hline & $\mathrm{H}$ & 1.20861600 & -3.40434800 & -1.60727600 \\
\hline \multirow[t]{31}{*}{61} & 0 & 1.66995600 & -1.85309500 & 0.54664700 \\
\hline & $\mathrm{C}$ & 1.76876200 & -0.51972900 & 0.71157900 \\
\hline & $\mathrm{C}$ & 0.65753800 & -2.58659400 & 1.29953400 \\
\hline & $\mathrm{H}$ & 0.41412100 & -2.01304100 & 2.19452900 \\
\hline & $\mathrm{C}$ & -0.60829200 & -2.77350000 & 0.46114900 \\
\hline & $\mathrm{H}$ & -0.35946100 & -2.80328900 & -0.59989000 \\
\hline & $\mathrm{C}$ & 1.29286100 & -3.92554100 & 1.65298000 \\
\hline & $\mathrm{H}$ & 0.60580800 & -4.51974200 & 2.25987800 \\
\hline & $\mathrm{H}$ & 2.20878200 & -3.77365600 & 2.22551000 \\
\hline & $\mathrm{O}$ & 1.02069800 & 0.10838800 & 1.41775500 \\
\hline & $\mathrm{C}$ & 2.99472400 & 0.07555600 & -0.06181200 \\
\hline & $\mathrm{C}$ & 2.71089800 & 1.56771500 & -0.38330500 \\
\hline & $\mathrm{C}$ & 2.86554200 & 2.07986700 & -1.67486400 \\
\hline & $\mathrm{C}$ & 2.65876500 & 3.43342400 & -1.94565500 \\
\hline & $\mathrm{C}$ & 2.29406400 & 4.30642100 & -0.92792300 \\
\hline & $\mathrm{C}$ & 2.14567800 & 3.81269300 & 0.36764500 \\
\hline & $\mathrm{C}$ & 2.35664600 & 2.46490900 & 0.63782800 \\
\hline & $\mathrm{C}$ & 3.24146200 & -0.72765100 & -1.36010700 \\
\hline & $\mathrm{C}$ & 4.52060700 & -1.13020500 & -1.74788300 \\
\hline & $\mathrm{C}$ & 4.72565500 & -1.80145800 & -2.95476100 \\
\hline & $\mathrm{C}$ & 3.65369500 & -2.08124400 & -3.79402800 \\
\hline & $\mathrm{C}$ & 2.37188100 & -1.67812700 & -3.41933600 \\
\hline & $\mathrm{C}$ & 2.16806700 & -1.00689500 & -2.21881800 \\
\hline & $\mathrm{C}$ & 4.21650200 & -0.04615700 & 0.89976600 \\
\hline & $\mathrm{C}$ & 5.13067800 & 1.00207700 & 1.05040900 \\
\hline & $\mathrm{C}$ & 6.24950500 & 0.86886800 & 1.87192300 \\
\hline & $\mathrm{C}$ & 6.47964300 & -0.31506300 & 2.56446100 \\
\hline & $\mathrm{C}$ & 5.58558700 & -1.37198700 & 2.41323600 \\
\hline & $\mathrm{C}$ & 4.47341800 & -1.24092700 & 1.58665500 \\
\hline & $\mathrm{H}$ & 3.15316800 & 1.42646800 & -2.48594900 \\
\hline & $\mathrm{H}$ & 2.78592200 & 3.79741900 & -2.95913400 \\
\hline
\end{tabular}




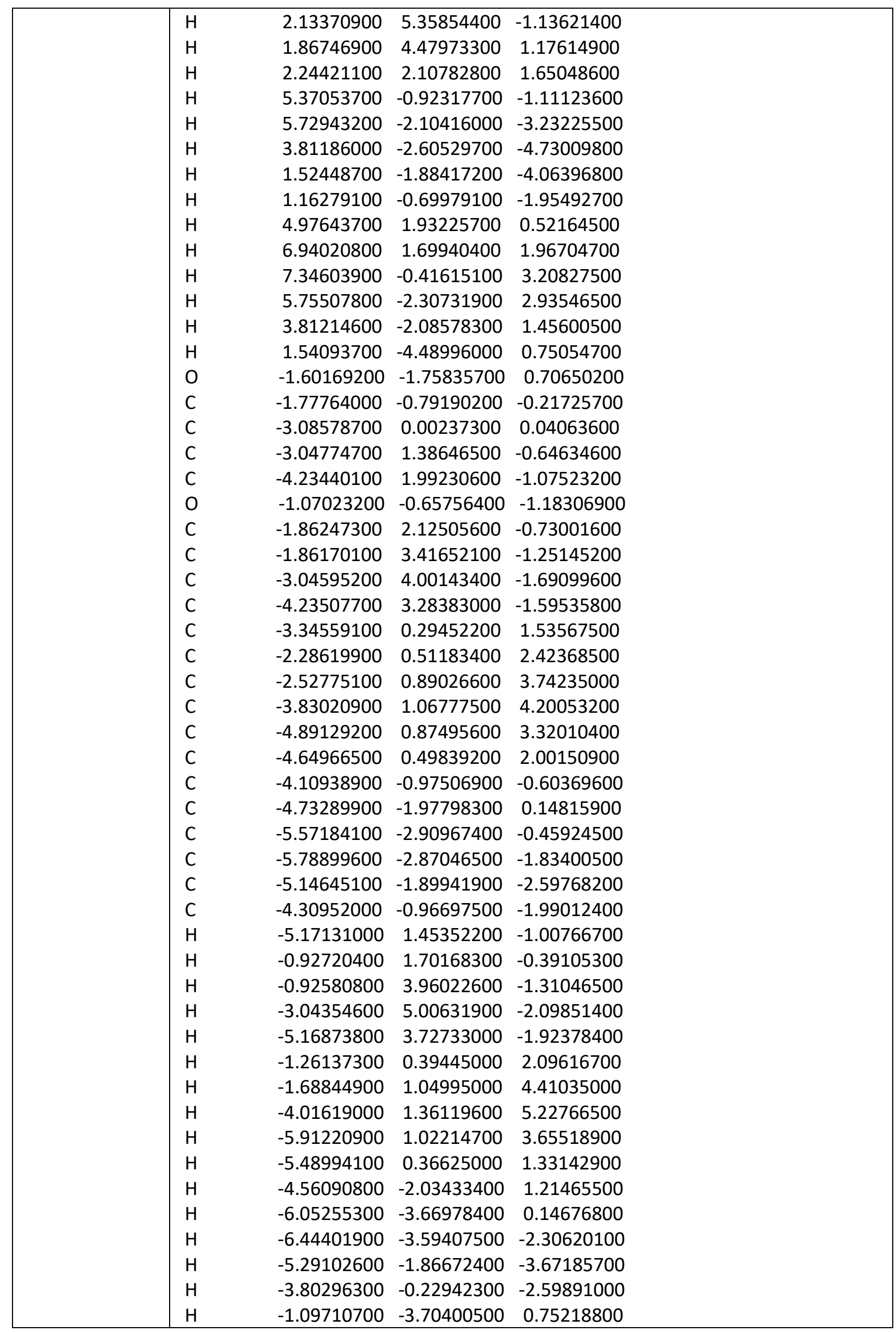




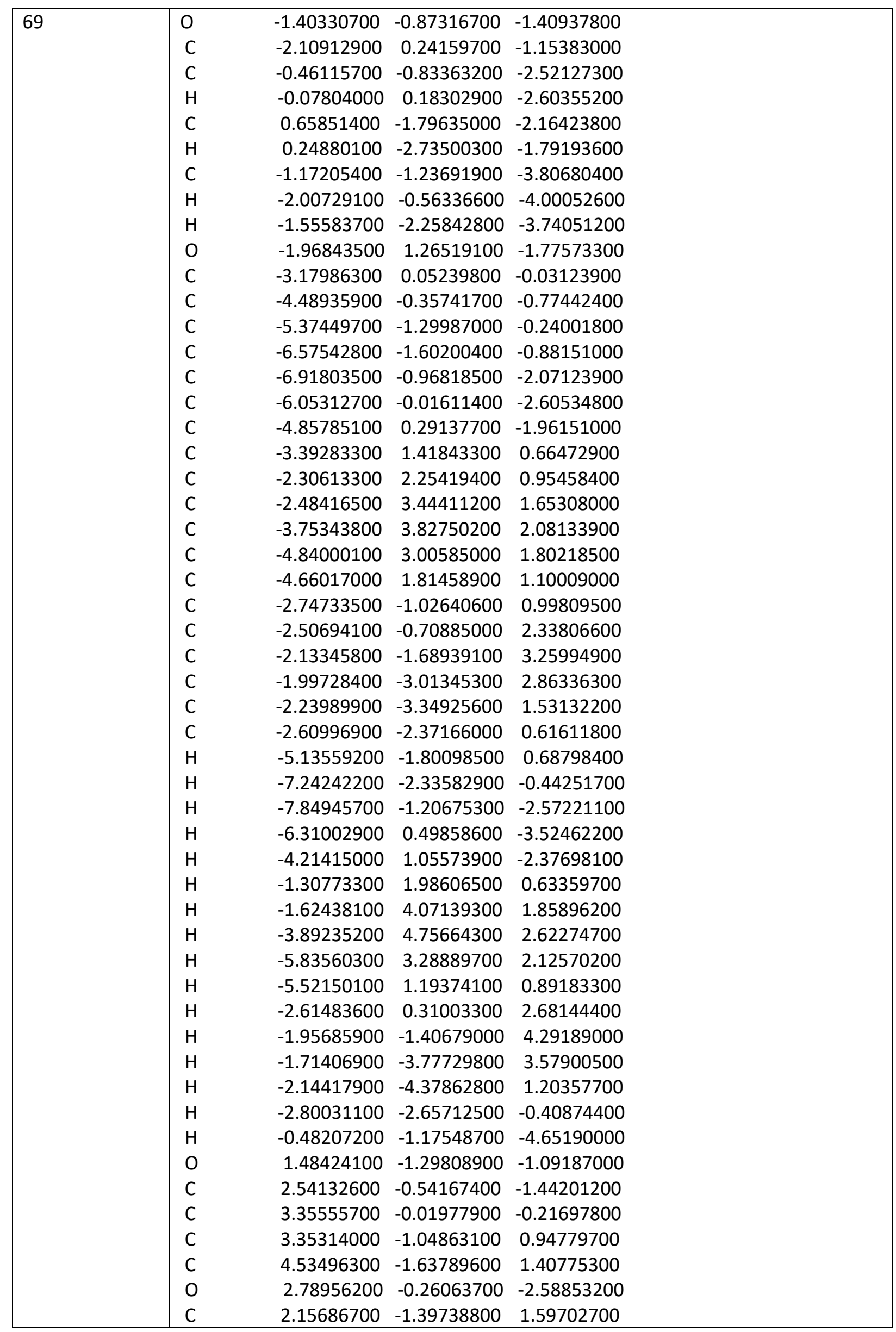




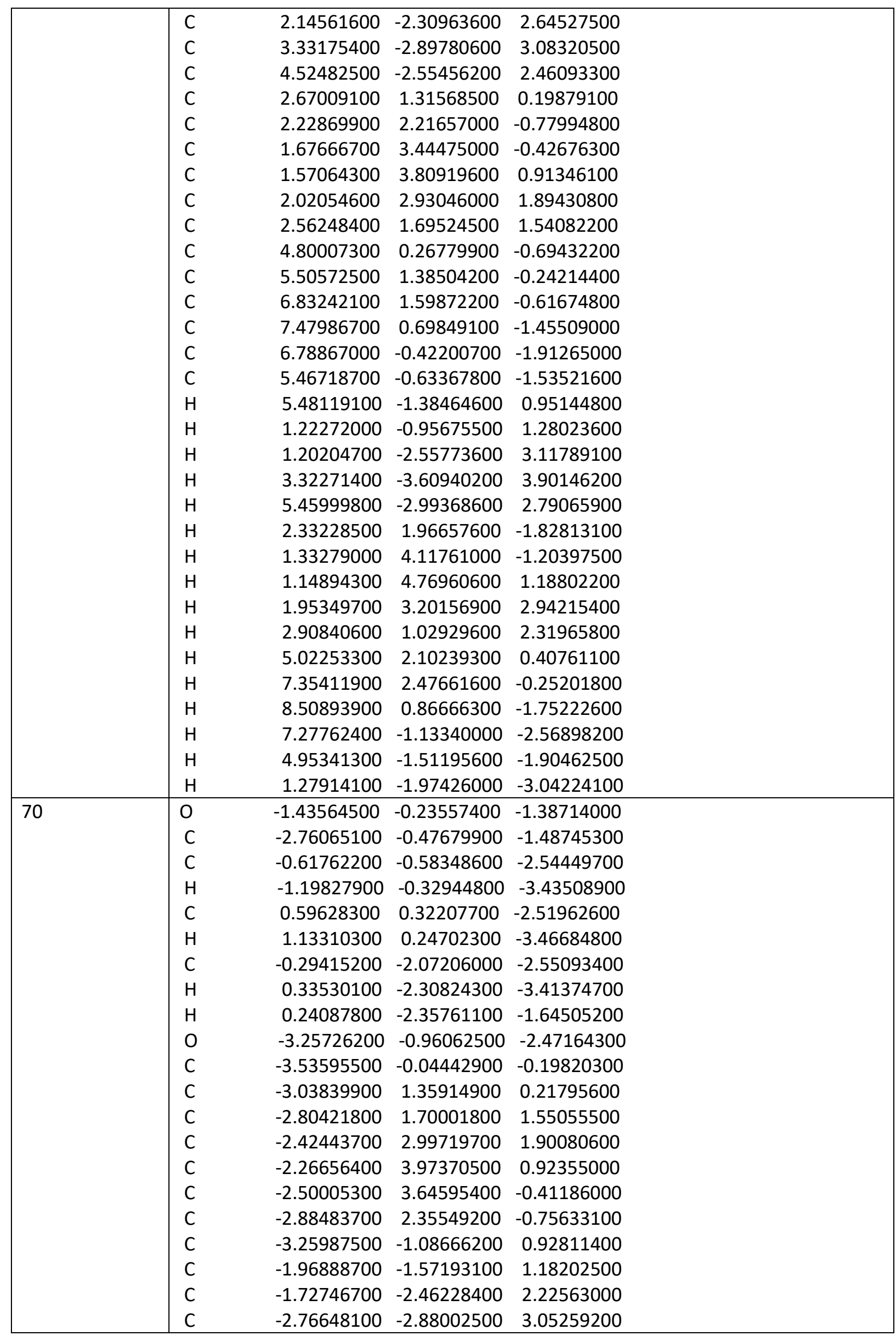




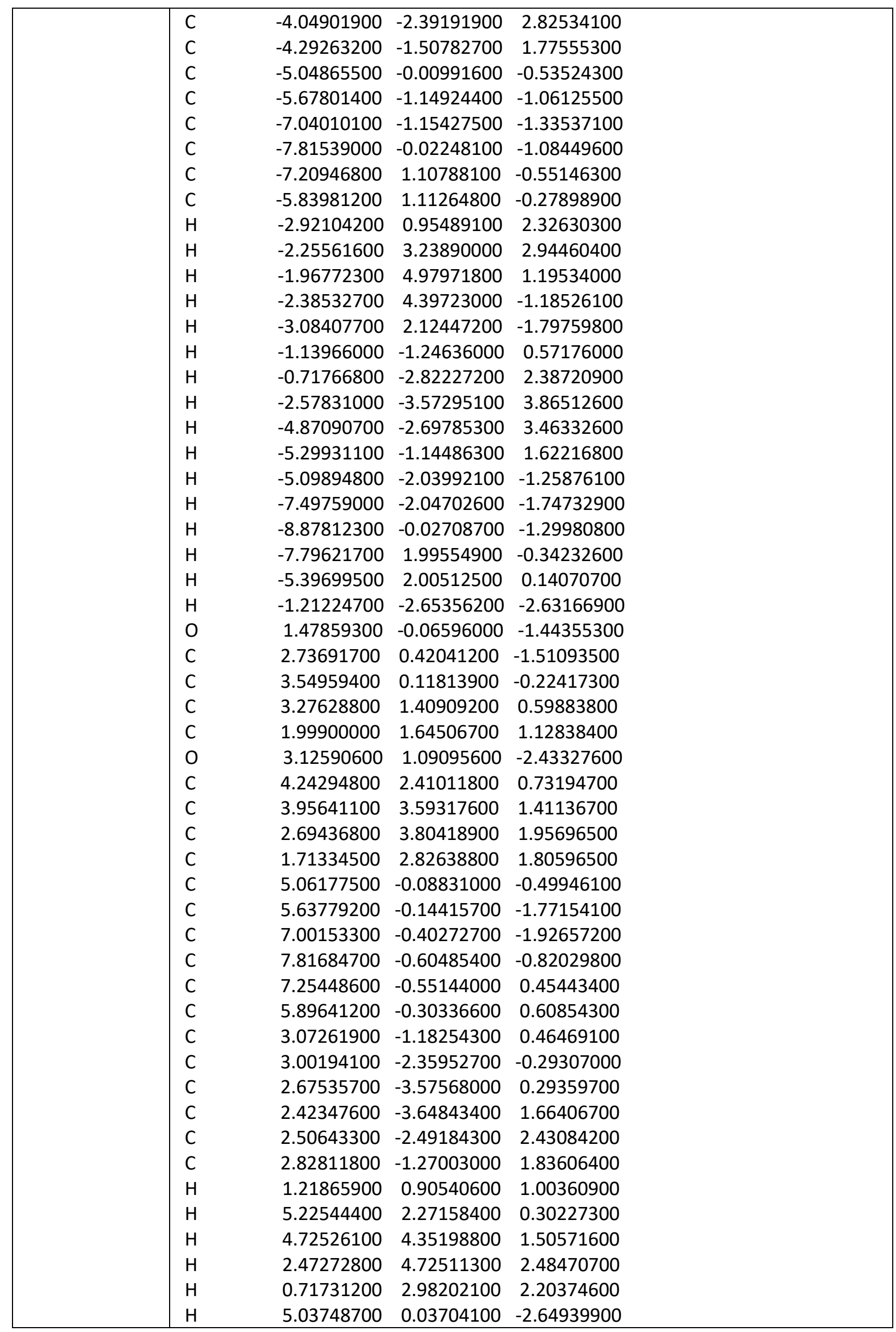




\begin{tabular}{|c|c|c|c|c|}
\hline & $\mathrm{H}$ & 7.42131200 & -0.43965900 & -2.92586100 \\
\hline & $\mathrm{H}$ & 8.87539600 & -0.80311800 & -0.94550300 \\
\hline & $\mathrm{H}$ & 7.87233300 & -0.71069100 & 1.33126000 \\
\hline & $\mathrm{H}$ & 5.47656900 & -0.28228400 & 1.60689400 \\
\hline & $\mathrm{H}$ & 3.22094100 & -2.32761200 & -1.35461700 \\
\hline & $\mathrm{H}$ & 2.62608900 & -4.47044700 & -0.31699900 \\
\hline & $\mathrm{H}$ & 2.17513100 & -4.59753800 & 2.12544200 \\
\hline & $\mathrm{H}$ & 2.32567700 & -2.53188200 & 3.49943600 \\
\hline & $\mathrm{H}$ & 2.89105500 & -0.38488900 & 2.45505100 \\
\hline & $\mathrm{H}$ & 0.29911500 & 1.36215600 & -2.37024900 \\
\hline \multirow[t]{42}{*}{71} & 0 & 1.72166100 & 0.36506500 & 0.35623100 \\
\hline & $\mathrm{C}$ & 2.62547600 & 0.19644400 & -0.62978800 \\
\hline & $\mathrm{C}$ & 0.36924600 & 0.74592300 & -0.02458000 \\
\hline & $\mathrm{H}$ & 0.12003700 & 0.22853800 & -0.95171800 \\
\hline & $\mathrm{C}$ & -0.48409500 & 0.20007600 & 1.11971100 \\
\hline & $\mathrm{H}$ & -0.29570800 & -0.86509200 & 1.24861800 \\
\hline & $\mathrm{C}$ & 0.26846100 & 2.25145500 & -0.20511900 \\
\hline & $\mathrm{H}$ & -0.76896700 & 2.53021800 & -0.40135200 \\
\hline & $\mathrm{H}$ & 0.87902600 & 2.57335900 & -1.04930700 \\
\hline & 0 & 2.37094000 & 0.34719200 & -1.79669200 \\
\hline & $\mathrm{C}$ & 4.03301500 & -0.10286500 & -0.04074900 \\
\hline & $\mathrm{C}$ & 5.00372500 & -0.64726500 & -1.11574900 \\
\hline & $\mathrm{C}$ & 6.37958800 & -0.41531300 & -0.99872000 \\
\hline & $\mathrm{C}$ & 7.28652300 & -0.99323100 & -1.88316000 \\
\hline & $\mathrm{C}$ & 6.83657500 & -1.82321500 & -2.90579400 \\
\hline & $\mathrm{C}$ & 5.47236800 & -2.07133600 & -3.02662600 \\
\hline & $\mathrm{C}$ & 4.56640300 & -1.49485100 & -2.13994500 \\
\hline & $\mathrm{C}$ & 3.98377000 & -1.19770300 & 1.04995300 \\
\hline & $\mathrm{C}$ & 3.01464300 & -2.20631500 & 1.01758200 \\
\hline & $\mathrm{C}$ & 3.03906100 & -3.25811700 & 1.93004600 \\
\hline & $\mathrm{C}$ & 4.03892600 & -3.32757800 & 2.89586100 \\
\hline & $\mathrm{C}$ & 5.02029200 & -2.34034500 & 2.92897800 \\
\hline & $\mathrm{C}$ & 4.99531200 & -1.29246700 & 2.01240500 \\
\hline & $\mathrm{C}$ & 4.43784900 & 1.30456700 & 0.47426200 \\
\hline & $\mathrm{C}$ & 4.21390500 & 1.70378800 & 1.79634000 \\
\hline & $\mathrm{C}$ & 4.49725000 & 3.00468400 & 2.20713800 \\
\hline & $\mathrm{C}$ & 4.99066200 & 3.93851700 & 1.30014300 \\
\hline & $\mathrm{C}$ & 5.18729600 & 3.56227000 & -0.02620000 \\
\hline & $\mathrm{C}$ & 4.90849100 & 2.26098300 & -0.43518300 \\
\hline & $\mathrm{H}$ & 6.75317700 & 0.22753500 & -0.21160400 \\
\hline & $\mathrm{H}$ & 8.34608400 & -0.79240300 & -1.76892000 \\
\hline & $\mathrm{H}$ & 7.53981700 & -2.27241200 & -3.59803200 \\
\hline & $\mathrm{H}$ & 5.10468200 & -2.71724900 & -3.81626700 \\
\hline & $\mathrm{H}$ & 3.51308900 & -1.69991300 & -2.26421600 \\
\hline & $\mathrm{H}$ & 2.22993100 & -2.18490700 & 0.27150900 \\
\hline & $\mathrm{H}$ & 2.27365600 & -4.02464600 & 1.88104400 \\
\hline & $\mathrm{H}$ & 4.05808300 & -4.14454600 & 3.60818600 \\
\hline & $\mathrm{H}$ & 5.81412400 & -2.38569200 & 3.66637400 \\
\hline & $\mathrm{H}$ & 5.77660300 & -0.54385500 & 2.04784600 \\
\hline & $\mathrm{H}$ & 3.80950800 & 0.99890000 & 2.51011300 \\
\hline & $\mathrm{H}$ & 4.32509600 & 3.28741200 & 3.23987100 \\
\hline & $\mathrm{H}$ & 5.21148100 & 4.95028800 & 1.62122000 \\
\hline
\end{tabular}




\begin{tabular}{|c|c|c|c|c|}
\hline & $\mathrm{H}$ & 5.55566900 & 4.28199900 & -0.74867800 \\
\hline & $\mathrm{H}$ & 5.05324500 & 1.98620200 & -1.47241000 \\
\hline & $\mathrm{H}$ & 0.60845400 & 2.76850300 & 0.69609600 \\
\hline & $\mathrm{O}$ & -1.88624800 & 0.42341700 & 0.86602000 \\
\hline & $\mathrm{C}$ & -2.53536800 & -0.51250200 & 0.13627300 \\
\hline & $\mathrm{C}$ & -4.01378700 & -0.09453500 & -0.10102400 \\
\hline & $\mathrm{C}$ & -3.85803200 & 1.18812900 & -0.96032900 \\
\hline & $\mathrm{C}$ & -3.36861400 & 1.06911900 & -2.26931100 \\
\hline & 0 & -1.99600000 & -1.49328300 & -0.30530600 \\
\hline & $\mathrm{C}$ & -4.07375500 & 2.47007900 & -0.44936200 \\
\hline & $\mathrm{C}$ & -3.84691500 & 3.59924000 & -1.23585400 \\
\hline & $\mathrm{C}$ & -3.38353900 & 3.46812500 & -2.54138900 \\
\hline & $\mathrm{C}$ & -3.13780600 & 2.19541500 & -3.05324600 \\
\hline & $\mathrm{C}$ & -4.76957100 & 0.13396000 & 1.22974000 \\
\hline & $\mathrm{C}$ & -4.26975100 & -0.27684800 & 2.46834400 \\
\hline & $\mathrm{C}$ & -5.02625900 & -0.13648000 & 3.63196800 \\
\hline & $\mathrm{C}$ & -6.29890200 & 0.41998300 & 3.58145900 \\
\hline & $\mathrm{C}$ & -6.81471100 & 0.82481700 & 2.35143100 \\
\hline & $\mathrm{C}$ & -6.06227600 & 0.67521900 & 1.19212400 \\
\hline & $\mathrm{C}$ & -4.78881100 & -1.21606600 & -0.83461300 \\
\hline & $\mathrm{C}$ & -5.70750400 & -0.92520700 & -1.84763900 \\
\hline & $\mathrm{C}$ & -6.47975900 & -1.93012700 & -2.42948600 \\
\hline & $\mathrm{C}$ & -6.34968800 & -3.24815200 & -2.00694700 \\
\hline & $\mathrm{C}$ & -5.44709600 & -3.54926900 & -0.98890200 \\
\hline & $\mathrm{C}$ & -4.68205300 & -2.54534800 & -0.40557600 \\
\hline & $\mathrm{H}$ & -3.16745600 & 0.08587700 & -2.67829000 \\
\hline & $\mathrm{H}$ & -4.41324800 & 2.59440200 & 0.56976700 \\
\hline & $\mathrm{H}$ & -4.02901800 & 4.58376900 & -0.81931400 \\
\hline & $\mathrm{H}$ & -3.20723100 & 4.34676700 & -3.15149000 \\
\hline & $\mathrm{H}$ & -2.76231000 & 2.07659900 & -4.06336600 \\
\hline & $\mathrm{H}$ & -3.28111500 & -0.70904300 & 2.54579800 \\
\hline & $\mathrm{H}$ & -4.61196200 & -0.46498100 & 4.57867600 \\
\hline & $\mathrm{H}$ & -6.88593600 & 0.53155200 & 4.48594100 \\
\hline & $\mathrm{H}$ & -7.81029000 & 1.25034000 & 2.29147000 \\
\hline & $\mathrm{H}$ & -6.49008900 & 0.97552300 & 0.24350700 \\
\hline & $\mathrm{H}$ & -5.82560200 & 0.09271500 & -2.19541000 \\
\hline & $\mathrm{H}$ & -7.18214500 & -1.67524500 & -3.21541800 \\
\hline & $\mathrm{H}$ & -6.94540100 & -4.03190000 & -2.46120200 \\
\hline & $\mathrm{H}$ & -5.33726200 & -4.57145000 & -0.64403900 \\
\hline & $\mathrm{H}$ & -3.99176700 & -2.80466400 & 0.38506500 \\
\hline & $\mathrm{H}$ & -0.27233500 & 0.73240300 & 2.04710900 \\
\hline 73 & 0 & 1.52243800 & 1.54488400 & 0.23271100 \\
\hline & $\mathrm{C}$ & 2.33453700 & 0.98625500 & -0.68739900 \\
\hline & $\mathrm{C}$ & 0.69072700 & 2.66019000 & -0.19198100 \\
\hline & $\mathrm{H}$ & 0.48298800 & 2.53942000 & -1.25532400 \\
\hline & $\mathrm{C}$ & -0.59333100 & 2.57156600 & 0.60516800 \\
\hline & $\mathrm{H}$ & -0.39157900 & 2.39229900 & 1.66229400 \\
\hline & $\mathrm{C}$ & 1.43738200 & 3.96425600 & 0.06225500 \\
\hline & $\mathrm{H}$ & 1.64986900 & 4.09325600 & 1.12623900 \\
\hline & $\mathrm{H}$ & 0.84485800 & 4.81516400 & -0.28377700 \\
\hline & 0 & 2.34689700 & 1.30822600 & -1.84803100 \\
\hline & $\mathrm{C}$ & 3.33350600 & -0.00576900 & -0.02938900 \\
\hline
\end{tabular}




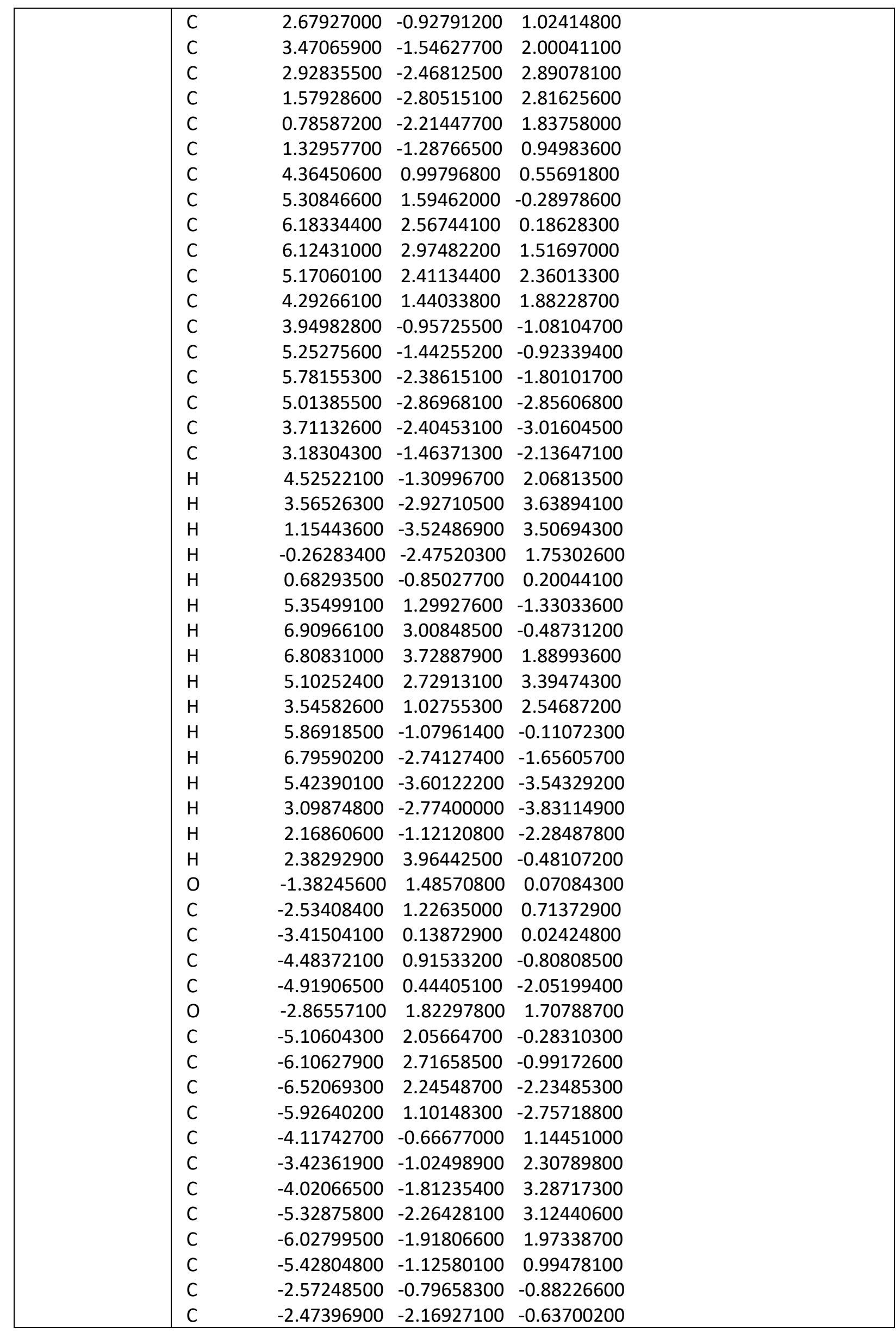




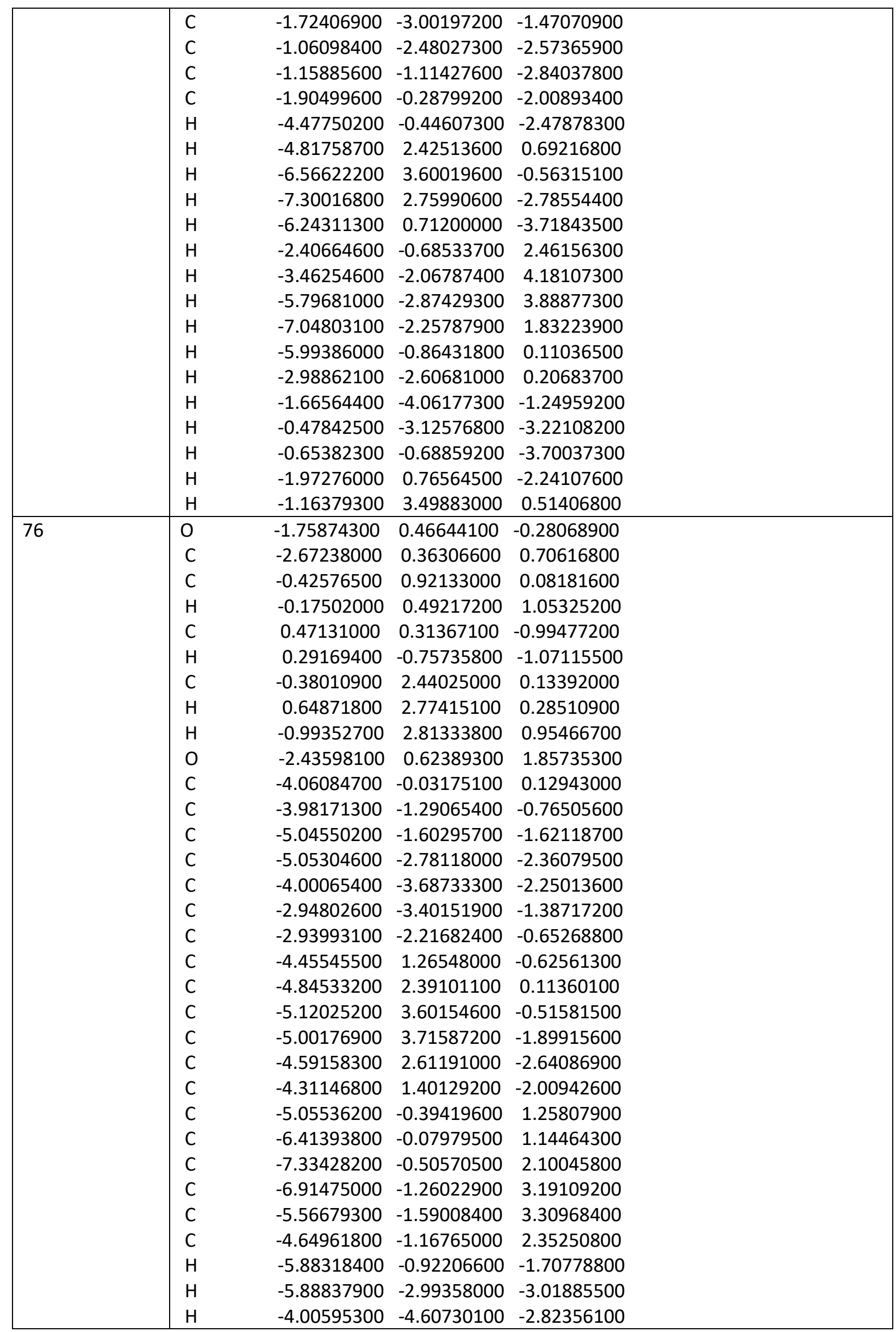




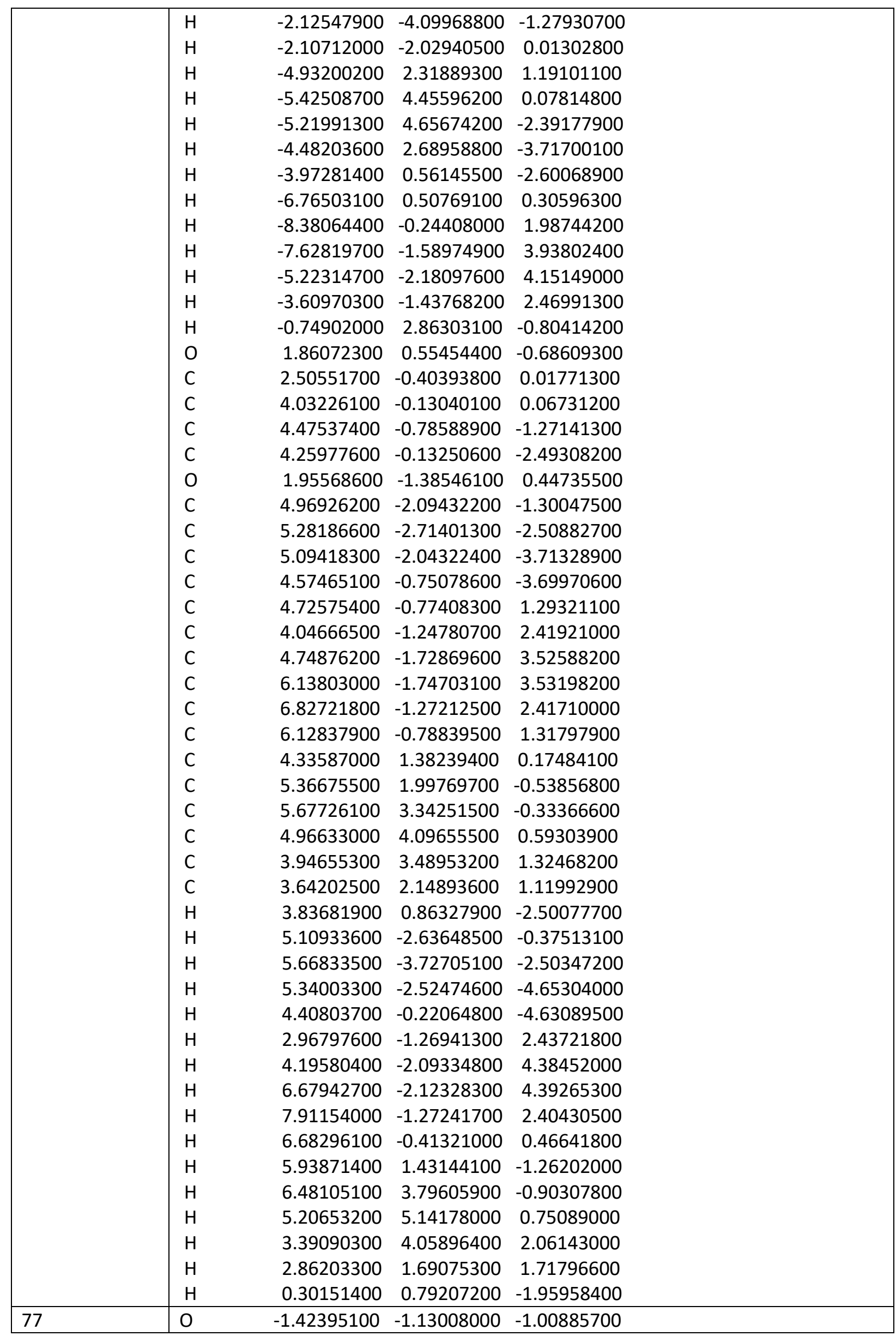




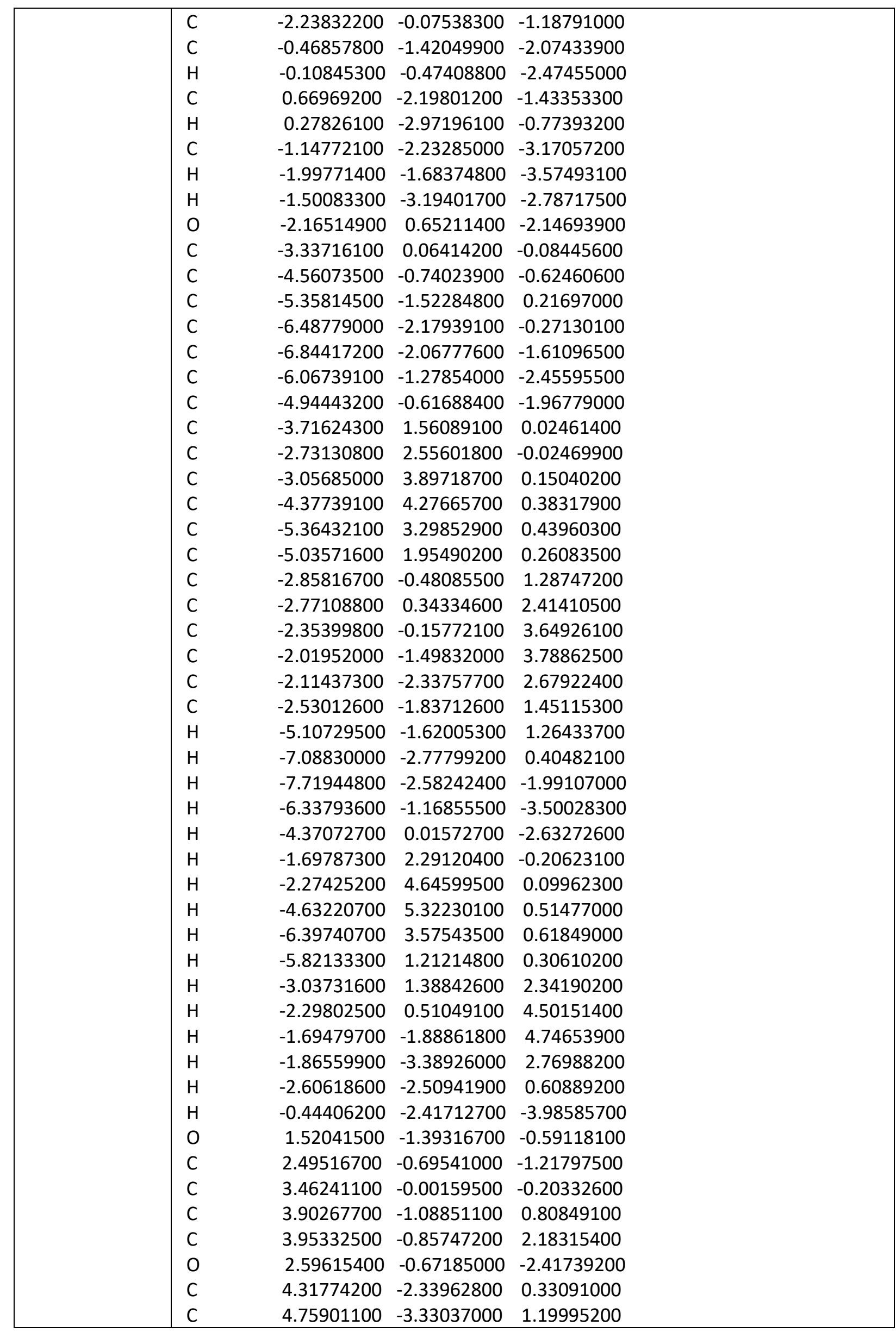




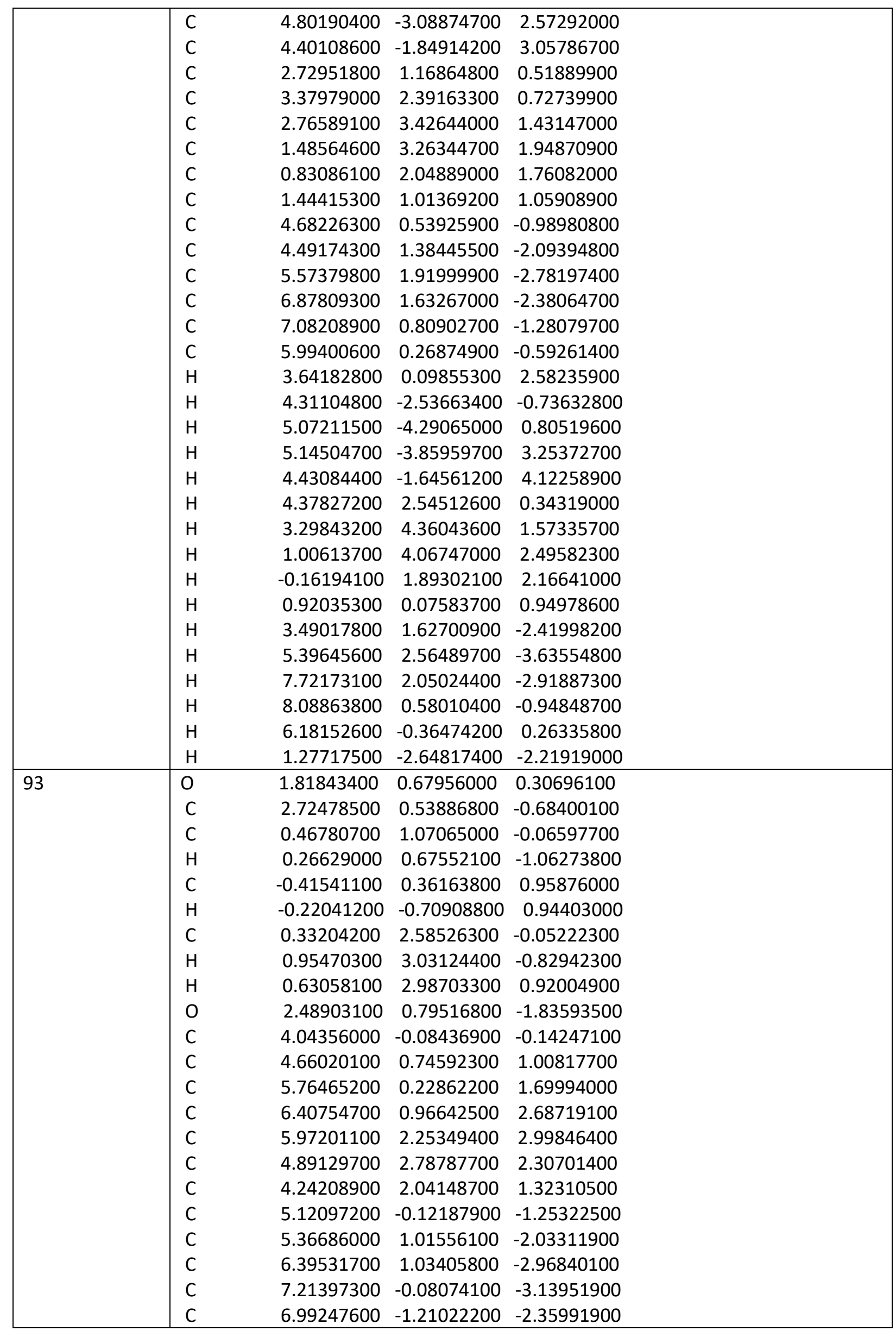




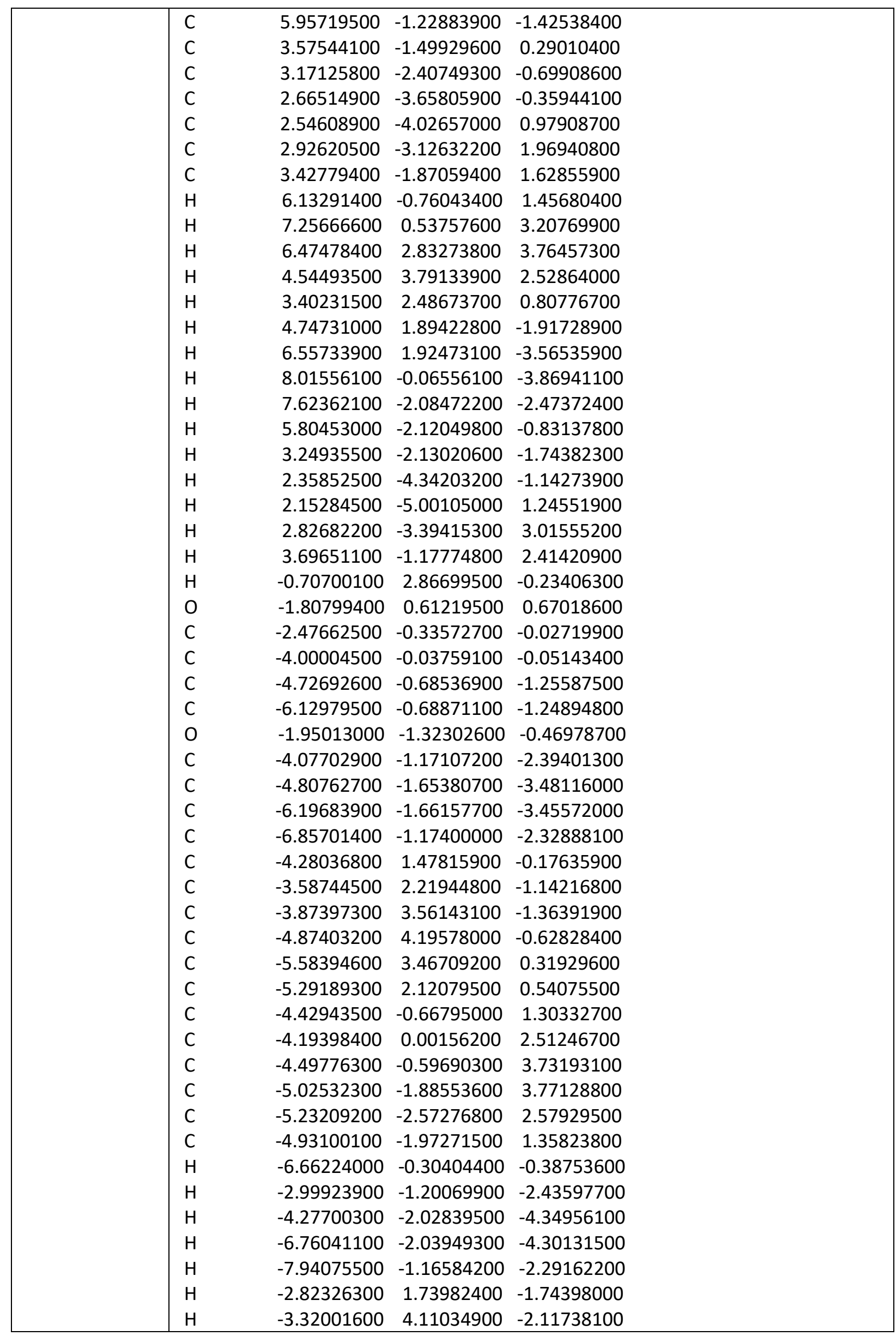




\begin{tabular}{|l|llll|}
\hline & H & -5.10040800 & 5.24199900 & -0.79940900 \\
H & -6.37306700 & 3.94173600 & 0.89203800 \\
H & -5.86364700 & 1.57505700 & 1.28000900 \\
H & -3.76379300 & 0.99416300 & 2.50073000 \\
H & -4.31589100 & -0.05422700 & 4.65301200 \\
H & -5.26240400 & -2.35148400 & 4.72109700 \\
H & -5.62457700 & -3.58340300 & 2.59358700 \\
H & -5.08612300 & -2.52749500 & 0.44278600 \\
H & -0.24701500 & 0.76198900 & 1.95915300 \\
\hline
\end{tabular}

18 (optimized at the B3LYP-GD3BJ/6-311G(d,p) level)

\begin{tabular}{|c|c|c|c|c|}
\hline Conformer no & & & & \\
\hline 13 & 0 & 1.29751500 & 0.86228200 & -1.34559100 \\
\hline & C & 2.41771100 & 0.15187300 & -1.55496800 \\
\hline & C & 0.47984700 & 1.19589300 & -2.49850800 \\
\hline & $\mathrm{H}$ & 1.14091200 & 1.30313300 & -3.36133800 \\
\hline & $\mathrm{C}$ & -0.45512100 & 0.03329000 & -2.79878200 \\
\hline & $\mathrm{H}$ & -1.07736400 & 0.26922700 & -3.66231700 \\
\hline & $\mathrm{C}$ & -0.20730000 & 2.50692400 & -2.16413300 \\
\hline & $\mathrm{H}$ & -0.89384300 & 2.78729100 & -2.96434100 \\
\hline & $\mathrm{H}$ & 0.53843300 & 3.29397100 & -2.04126100 \\
\hline & $\mathrm{O}$ & 2.74559300 & -0.24961100 & -2.64236400 \\
\hline & C & 3.18272600 & -0.09016200 & -0.22057000 \\
\hline & C & 2.19168000 & -0.73930800 & 0.76334600 \\
\hline & C & 2.20971200 & -0.43684600 & 2.12412600 \\
\hline & C & 1.36539400 & -1.10406400 & 3.01100800 \\
\hline & C & 0.49059900 & -2.08044300 & 2.54699900 \\
\hline & C & 0.46581900 & -2.39043600 & 1.18847600 \\
\hline & C & 1.31296300 & -1.72785200 & 0.30942100 \\
\hline & $\mathrm{C}$ & 3.68754000 & 1.26836600 & 0.33581000 \\
\hline & C & 5.02316700 & 1.44958700 & 0.70298600 \\
\hline & C & 5.45742800 & 2.64949500 & 1.26612400 \\
\hline & $\mathrm{C}$ & 4.56409600 & 3.69201700 & 1.47928900 \\
\hline & C & 3.22603000 & 3.51784800 & 1.13204100 \\
\hline & $\mathrm{C}$ & 2.79222900 & 2.32183900 & 0.57284400 \\
\hline & C & 4.36004700 & -1.04429800 & -0.49011300 \\
\hline & C & 5.27112100 & -0.76589000 & -1.51800600 \\
\hline & C & 6.37108400 & -1.58460700 & -1.73808000 \\
\hline & $\mathrm{C}$ & 6.59025900 & -2.70138100 & -0.93269200 \\
\hline & C & 5.69797100 & -2.98372900 & 0.09376000 \\
\hline & C & 4.59259000 & -2.16126500 & 0.31259100 \\
\hline & $\mathrm{H}$ & 2.88895800 & 0.31792800 & 2.49715200 \\
\hline & $\mathrm{H}$ & 1.39347800 & -0.85422900 & 4.06551000 \\
\hline & $\mathrm{H}$ & -0.17800600 & -2.59012400 & 3.22919600 \\
\hline & $\mathrm{H}$ & -0.22592200 & -3.13366000 & 0.81476100 \\
\hline & $\mathrm{H}$ & 1.29467000 & -1.99118900 & -0.74158200 \\
\hline & $\mathrm{H}$ & 5.73586000 & 0.65081700 & 0.56024900 \\
\hline & $\mathrm{H}$ & 6.50039000 & 2.75978100 & 1.54018700 \\
\hline & $\mathrm{H}$ & 4.90200200 & 4.62489800 & 1.91557300 \\
\hline & $\mathrm{H}$ & 2.50932900 & 4.31381800 & 1.30106200 \\
\hline & $\mathrm{H}$ & 1.74841900 & 2.19973300 & 0.33353300 \\
\hline
\end{tabular}




\begin{tabular}{|c|c|c|c|c|}
\hline & $\mathrm{H}$ & 5.11950000 & 0.09794000 & -2.14876200 \\
\hline & $\mathrm{H}$ & 7.05916400 & -1.34988300 & -2.54213100 \\
\hline & $\mathrm{H}$ & 7.44815900 & -3.34091900 & -1.10563600 \\
\hline & $\mathrm{H}$ & 5.85452400 & -3.84617000 & 0.73148800 \\
\hline & $\mathrm{H}$ & 3.91280100 & -2.39823300 & 1.11868700 \\
\hline & $\mathrm{H}$ & -0.77307400 & 2.42470500 & -1.23728600 \\
\hline & 0 & -1.29153500 & -0.26704700 & -1.66587100 \\
\hline & $\mathrm{C}$ & -2.53876000 & 0.24341900 & -1.65235000 \\
\hline & $\mathrm{C}$ & -3.18920000 & 0.04009000 & -0.26573200 \\
\hline & $\mathrm{C}$ & -2.96592700 & -1.37434900 & 0.29336600 \\
\hline & $\mathrm{C}$ & -3.03468100 & -1.60439900 & 1.66896000 \\
\hline & 0 & -3.02176600 & 0.85777500 & -2.56820000 \\
\hline & $\mathrm{C}$ & -2.85485400 & -2.47661400 & -0.55691600 \\
\hline & $\mathrm{C}$ & -2.79940400 & -3.77063900 & -0.04771700 \\
\hline & $\mathrm{C}$ & -2.86027200 & -3.98757400 & 1.32635000 \\
\hline & $\mathrm{C}$ & -2.98114600 & -2.89604200 & 2.18200500 \\
\hline & $\mathrm{C}$ & -4.71753300 & 0.20770200 & -0.31526200 \\
\hline & $\mathrm{C}$ & -5.46159700 & -0.20329400 & -1.42333800 \\
\hline & $\mathrm{C}$ & -6.85261200 & -0.16042700 & -1.40128600 \\
\hline & $\mathrm{C}$ & -7.52688200 & 0.28481600 & -0.26834100 \\
\hline & $\mathrm{C}$ & -6.79492900 & 0.68282900 & 0.84678200 \\
\hline & $\mathrm{C}$ & -5.40483800 & 0.64082100 & 0.82230900 \\
\hline & $\mathrm{C}$ & -2.48403100 & 1.15721300 & 0.53684800 \\
\hline & $\mathrm{C}$ & -2.95685000 & 2.47161700 & 0.45360900 \\
\hline & $\mathrm{C}$ & -2.27226400 & 3.51301900 & 1.07268600 \\
\hline & $\mathrm{C}$ & -1.09678700 & 3.25754200 & 1.77558400 \\
\hline & $\mathrm{C}$ & -0.60571400 & 1.95664900 & 1.83951700 \\
\hline & $\mathrm{C}$ & -1.28615700 & 0.91585100 & 1.21364000 \\
\hline & $\mathrm{H}$ & -3.13217000 & -0.76766100 & 2.34786600 \\
\hline & $\mathrm{H}$ & -2.81470200 & -2.33294300 & -1.62896500 \\
\hline & $\mathrm{H}$ & -2.71096400 & -4.60985600 & -0.72802500 \\
\hline & $\mathrm{H}$ & -2.81849700 & -4.99466500 & 1.72417100 \\
\hline & $\mathrm{H}$ & -3.03766200 & -3.04797900 & 3.25378900 \\
\hline & $\mathrm{H}$ & -4.95938400 & -0.54670100 & -2.31574000 \\
\hline & $\mathrm{H}$ & -7.40907000 & -0.47667500 & -2.27610400 \\
\hline & $\mathrm{H}$ & -8.61002200 & 0.31872700 & -0.25336200 \\
\hline & $\mathrm{H}$ & -7.30442600 & 1.02599400 & 1.73990000 \\
\hline & $\mathrm{H}$ & -4.85022600 & 0.95155000 & 1.69805800 \\
\hline & $\mathrm{H}$ & -3.85832400 & 2.67974000 & -0.10749000 \\
\hline & $\mathrm{H}$ & -2.65613200 & 4.52393200 & 0.99925100 \\
\hline & $\mathrm{H}$ & -0.56377900 & 4.06640700 & 2.26217200 \\
\hline & $\mathrm{H}$ & 0.31647300 & 1.74228200 & 2.36361500 \\
\hline & $\mathrm{H}$ & -0.86906900 & -0.07917000 & 1.24054000 \\
\hline & $\mathrm{H}$ & 0.12218600 & -0.86947800 & -2.98832000 \\
\hline 15 & 0 & -1.54044500 & -1.58042300 & -1.34097600 \\
\hline & $\mathrm{C}$ & -1.65211500 & -0.25278400 & -1.16215000 \\
\hline & $\mathrm{C}$ & -0.60803600 & -2.06275100 & -2.34394200 \\
\hline & $\mathrm{H}$ & -0.24294600 & -1.21165100 & -2.91384000 \\
\hline & $\mathrm{C}$ & 0.54041700 & -2.71901700 & -1.59591200 \\
\hline & $\mathrm{H}$ & 0.16419400 & -3.49789000 & -0.93351200 \\
\hline & $\mathrm{C}$ & -1.34994500 & -3.04565400 & -3.23171300 \\
\hline & $\mathrm{H}$ & -2.17893600 & -2.54381800 & -3.73309400 \\
\hline
\end{tabular}




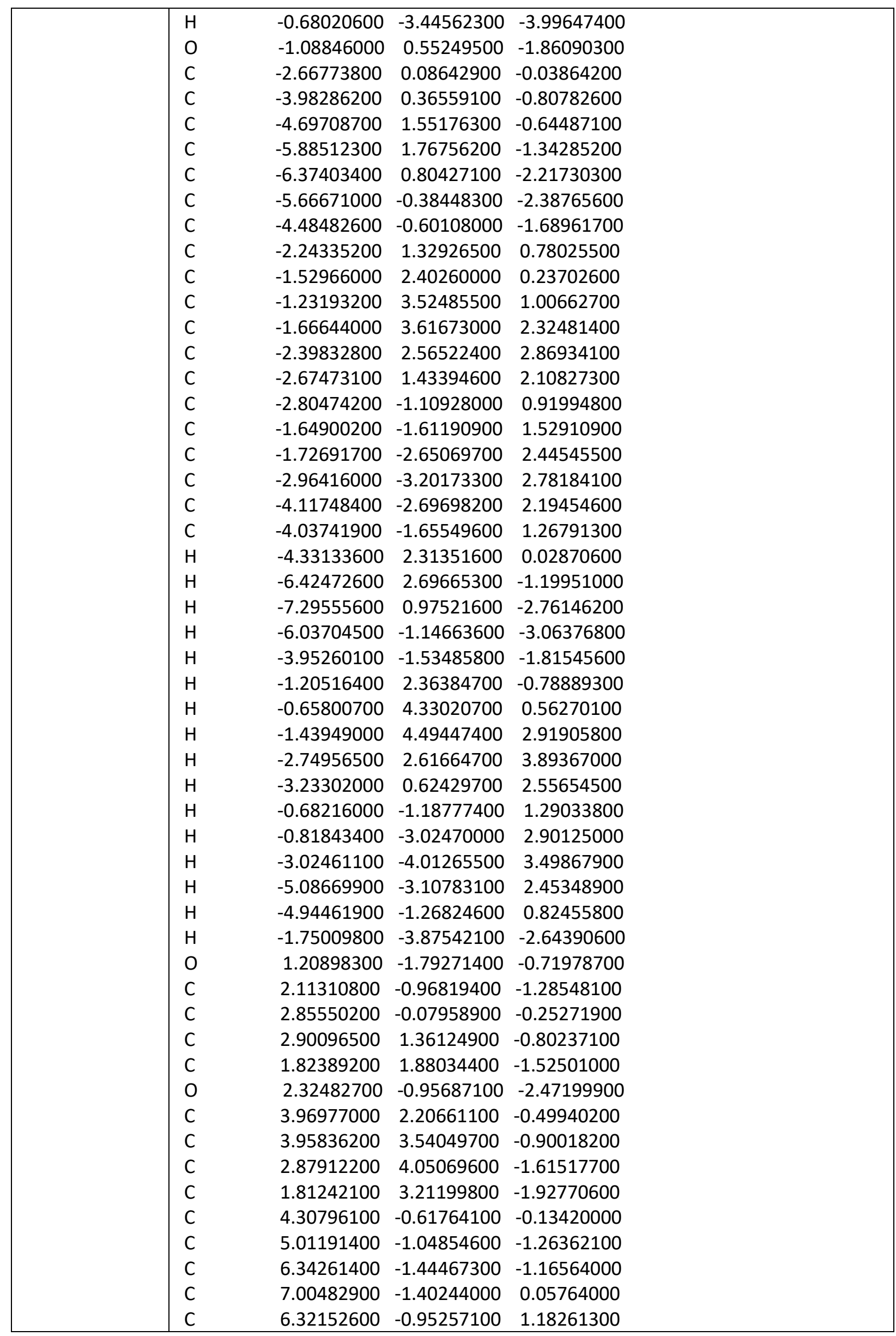




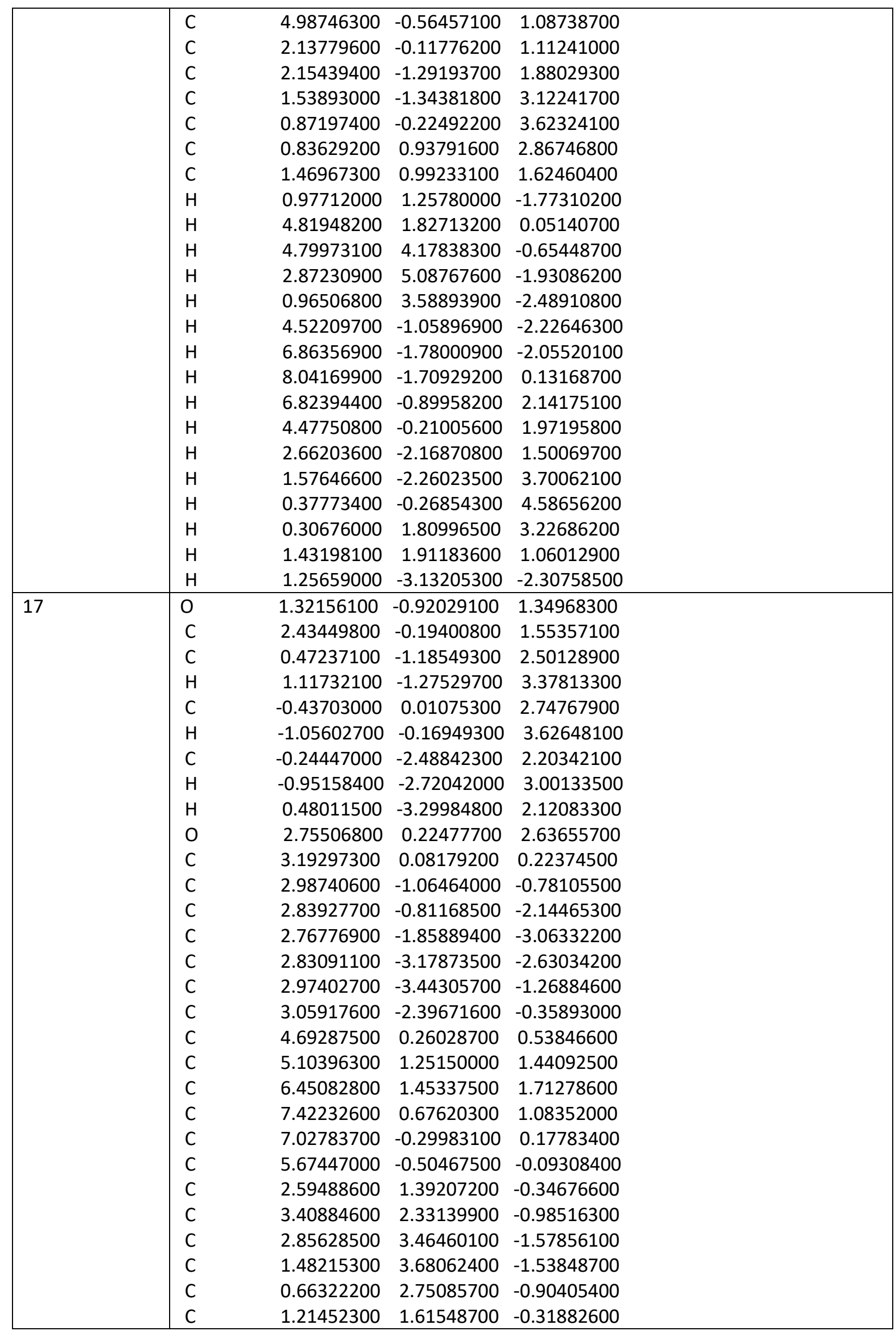




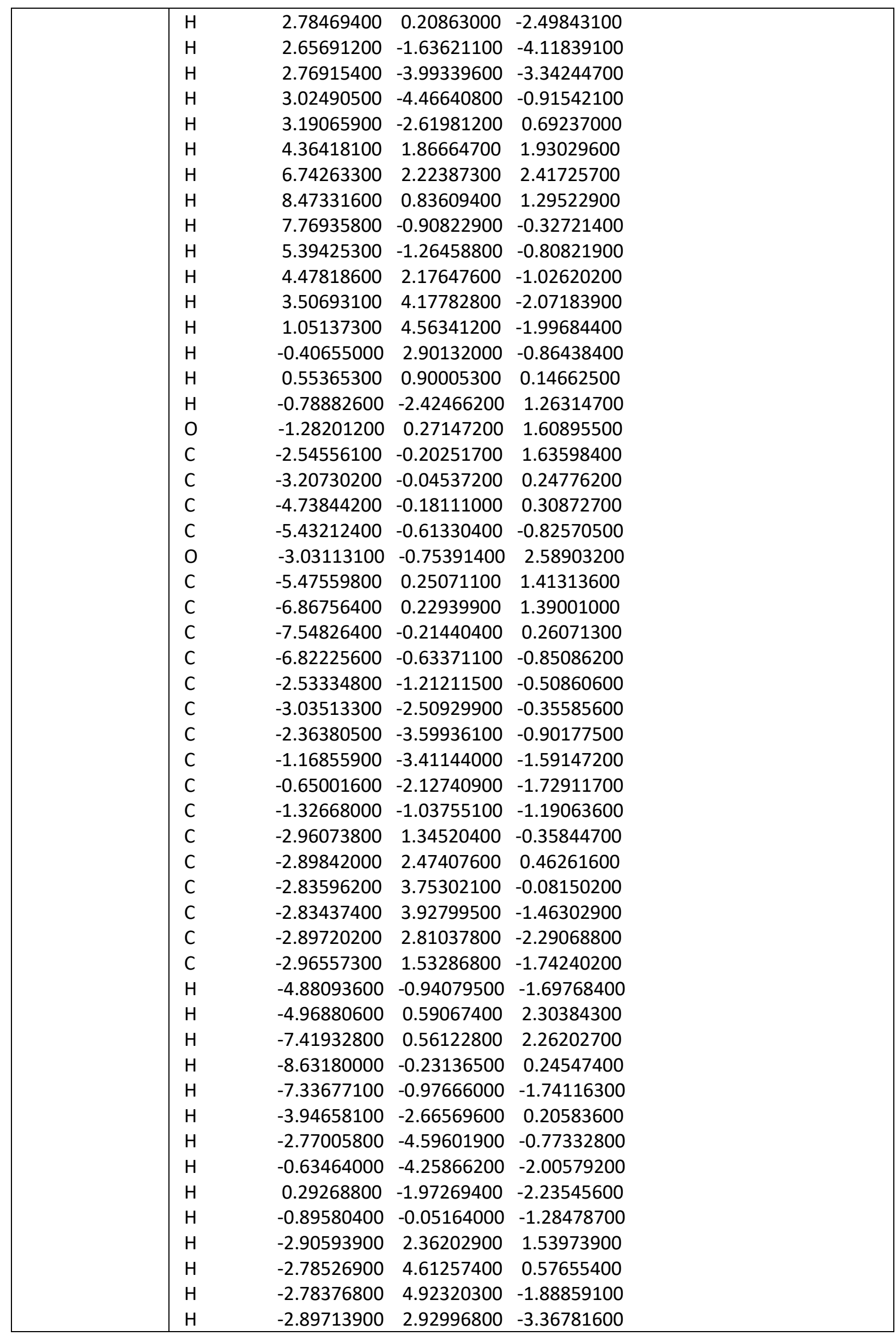




\begin{tabular}{|c|c|c|c|c|}
\hline \multirow{2}{*}{26} & $\begin{array}{l}\mathrm{H} \\
\mathrm{H}\end{array}$ & $\begin{array}{r}-3.02880700 \\
0.15719400\end{array}$ & $\begin{array}{l}0.67644800 \\
0.91195900\end{array}$ & $\begin{array}{c}-2.40044000 \\
2.88547500\end{array}$ \\
\hline & 0 & -1.36439800 & 0.02227600 & -1.69369000 \\
\hline & C & -2.68015500 & -0.23500200 & -1.65305700 \\
\hline & C & -0.68024200 & -0.04102900 & -2.96519900 \\
\hline & $\mathrm{H}$ & -1.31329400 & 0.43057300 & -3.72130900 \\
\hline & C & 0.56596500 & 0.79548000 & -2.77393300 \\
\hline & $\mathrm{H}$ & 1.17563100 & 0.80668300 & -3.67860100 \\
\hline & C & -0.39934100 & -1.48969300 & -3.33928200 \\
\hline & $\mathrm{H}$ & 0.17522700 & -1.53195200 & -4.26827900 \\
\hline & $\mathrm{H}$ & -1.33650900 & -2.02427700 & -3.49211900 \\
\hline & $\mathrm{O}$ & -3.34373700 & -0.49888200 & -2.62285300 \\
\hline & C & -3.19963300 & -0.02844900 & -0.21148400 \\
\hline & C & -2.28007400 & -0.68892600 & 0.83382900 \\
\hline & C & -2.33740300 & -0.27147400 & 2.16629500 \\
\hline & C & -1.58378000 & -0.90581400 & 3.14852000 \\
\hline & C & -0.75381100 & -1.97383200 & 2.81566700 \\
\hline & C & -0.70300600 & -2.41060200 & 1.49592500 \\
\hline & C & -1.46574100 & -1.77840500 & 0.51883600 \\
\hline & C & -4.58307000 & -0.66079700 & 0.00178100 \\
\hline & C & -4.92718200 & -1.86929700 & -0.60893100 \\
\hline & C & -6.13196400 & -2.49921600 & -0.31244100 \\
\hline & C & -7.01290600 & -1.93942400 & 0.60821900 \\
\hline & C & -6.67344700 & -0.74360500 & 1.23390500 \\
\hline & C & -5.46931500 & -0.11404400 & 0.93431600 \\
\hline & C & -3.20544600 & 1.51481400 & -0.13040500 \\
\hline & C & -4.28020100 & 2.24290600 & -0.64854400 \\
\hline & C & -4.24486000 & 3.63472500 & -0.67651100 \\
\hline & C & -3.12782100 & 4.31864900 & -0.20382900 \\
\hline & C & -2.03838100 & 3.59855200 & 0.28110400 \\
\hline & C & -2.07450000 & 2.20845200 & 0.30782900 \\
\hline & $\mathrm{H}$ & -2.97275200 & 0.56142300 & 2.43874100 \\
\hline & $\mathrm{H}$ & -1.64364700 & -0.56152800 & 4.17484900 \\
\hline & $\mathrm{H}$ & -0.15221800 & -2.45942100 & 3.57485400 \\
\hline & $\mathrm{H}$ & -0.05383500 & -3.23061800 & 1.21781900 \\
\hline & $\mathrm{H}$ & -1.40218300 & -2.13789900 & -0.49832900 \\
\hline & $\mathrm{H}$ & -4.26016800 & -2.32078300 & -1.32861500 \\
\hline & $\mathrm{H}$ & -6.38079300 & -3.43219000 & -0.80505600 \\
\hline & $\mathrm{H}$ & -7.95147100 & -2.43057000 & 0.83727000 \\
\hline & $\mathrm{H}$ & -7.34484000 & -0.29693500 & 1.95834000 \\
\hline & $\mathrm{H}$ & -5.22110600 & 0.81511500 & 1.42997700 \\
\hline & $\mathrm{H}$ & -5.14467100 & 1.71910600 & -1.03512700 \\
\hline & $\mathrm{H}$ & -5.09099500 & 4.18279100 & -1.07469200 \\
\hline & $\mathrm{H}$ & -3.10215100 & 5.40208700 & -0.22481100 \\
\hline & $\mathrm{H}$ & -1.14999700 & 4.11068800 & 0.63241900 \\
\hline & $\mathrm{H}$ & -1.20996000 & 1.66328400 & 0.65520600 \\
\hline & $\mathrm{H}$ & 0.17275900 & -1.97726700 & -2.54945000 \\
\hline & $\mathrm{O}$ & 1.30994800 & 0.21765200 & -1.68527900 \\
\hline & C & 2.58889400 & 0.60696200 & -1.54559300 \\
\hline & C & 3.15374500 & 0.14178300 & -0.18425400 \\
\hline & C & 2.47532000 & 1.15092600 & 0.77096400 \\
\hline & C & 1.36844700 & 0.81486300 & 1.55137200 \\
\hline
\end{tabular}




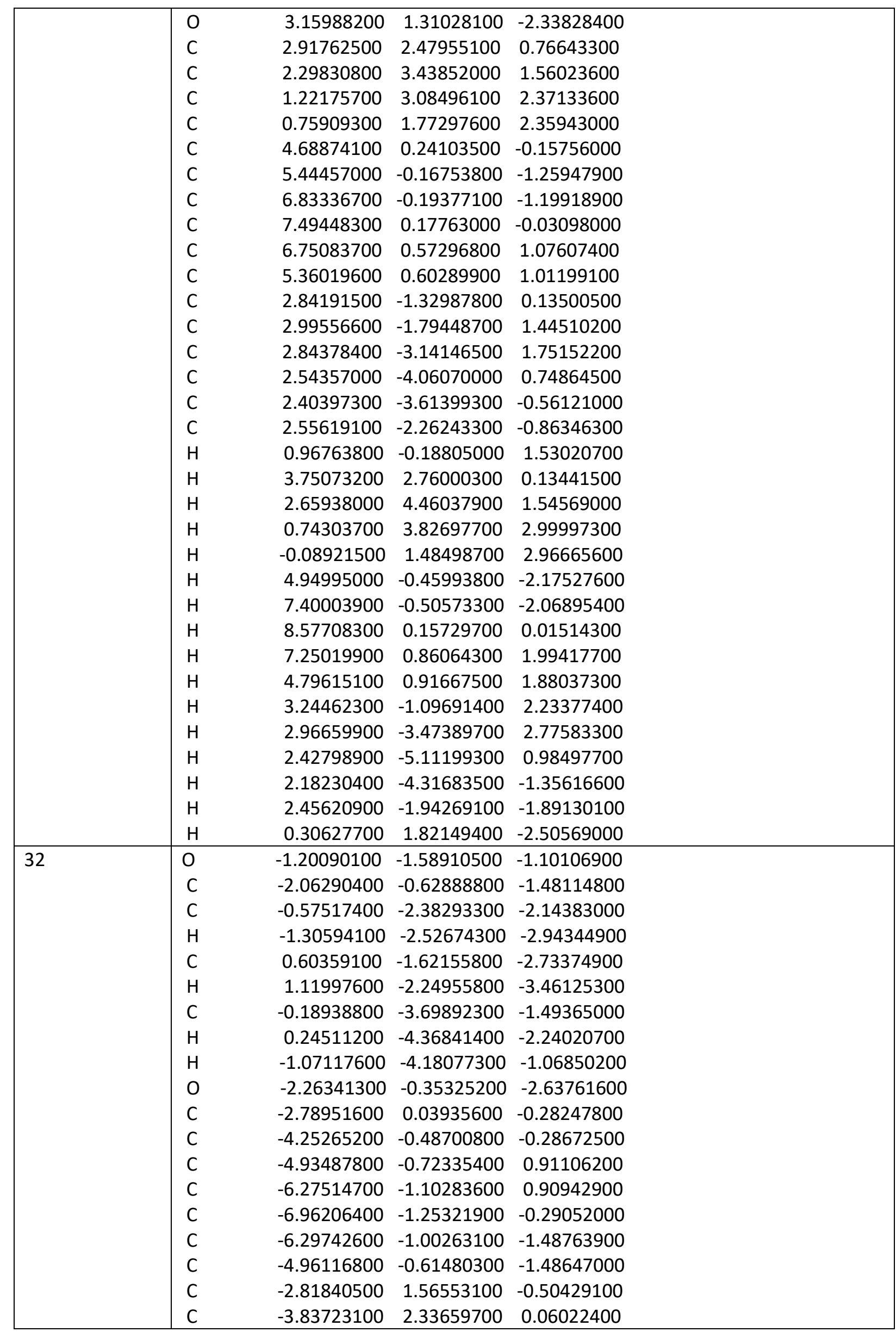




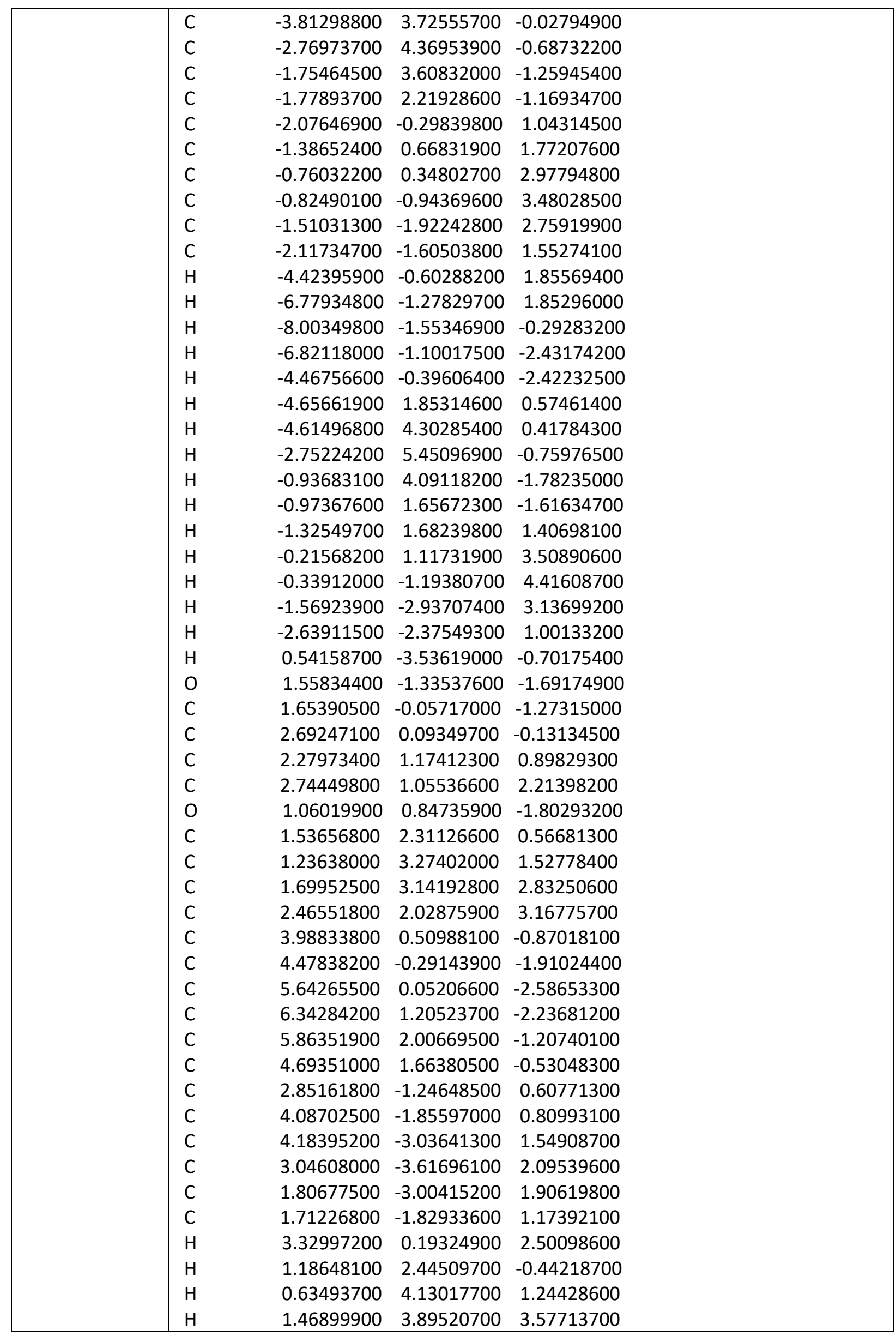




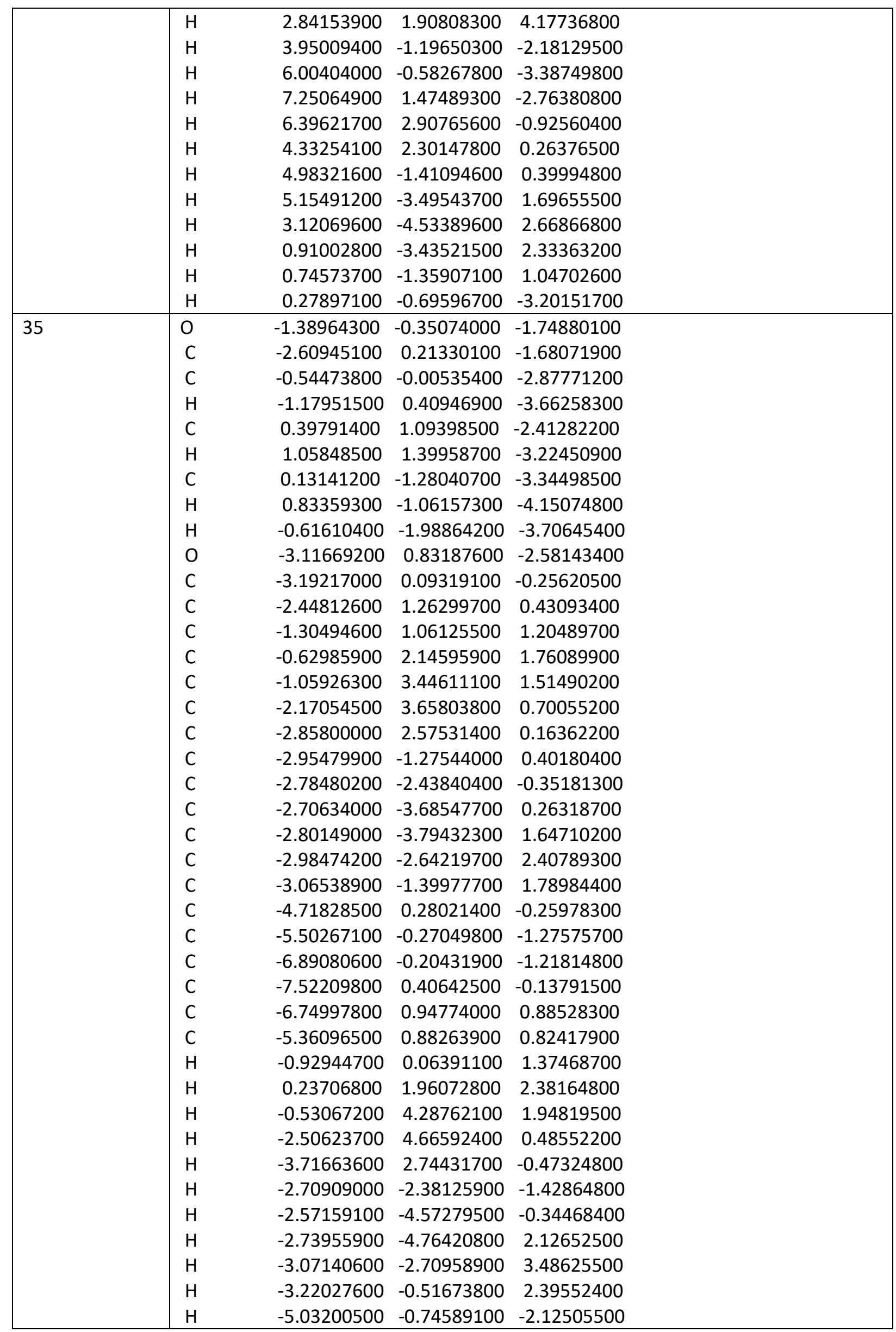




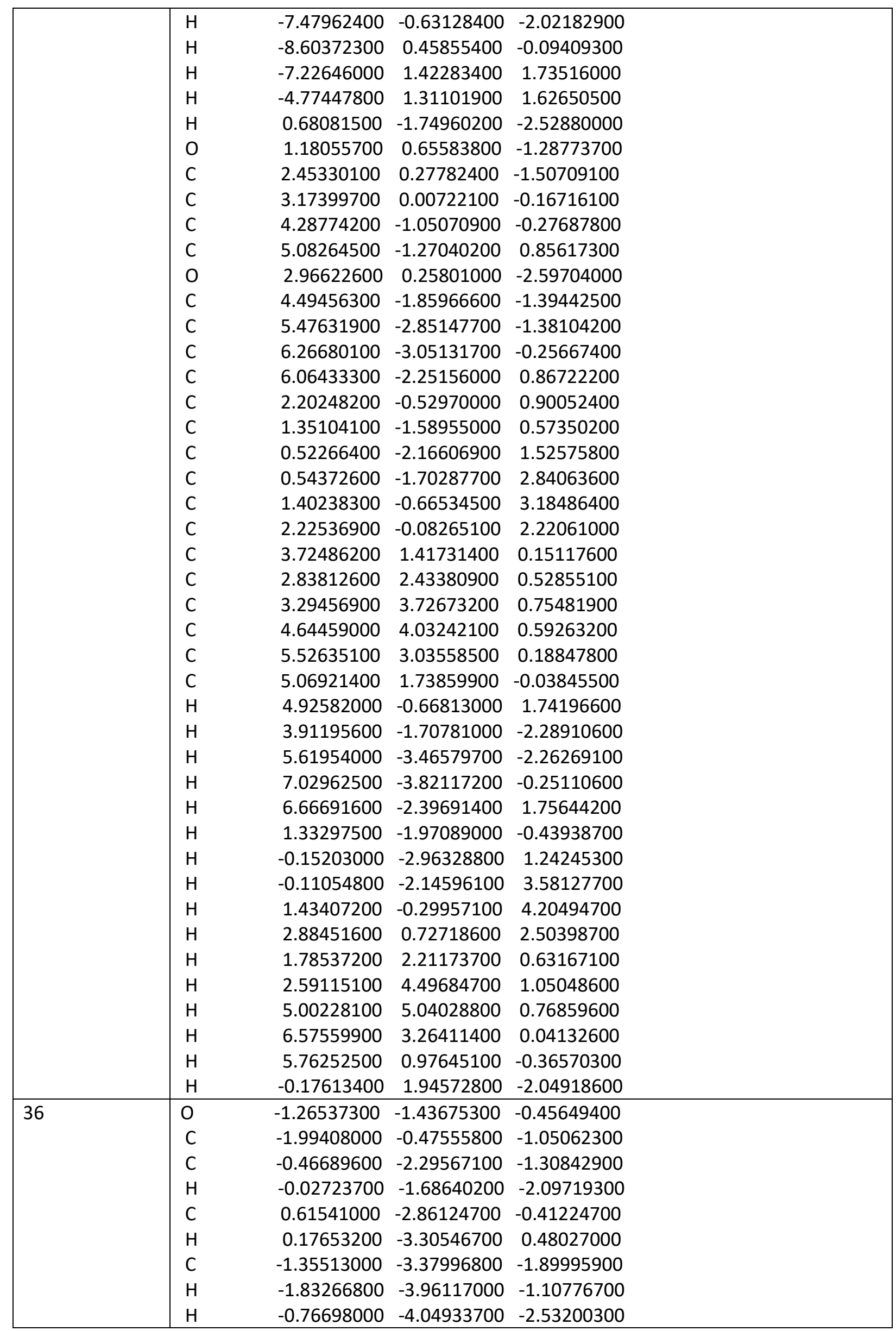




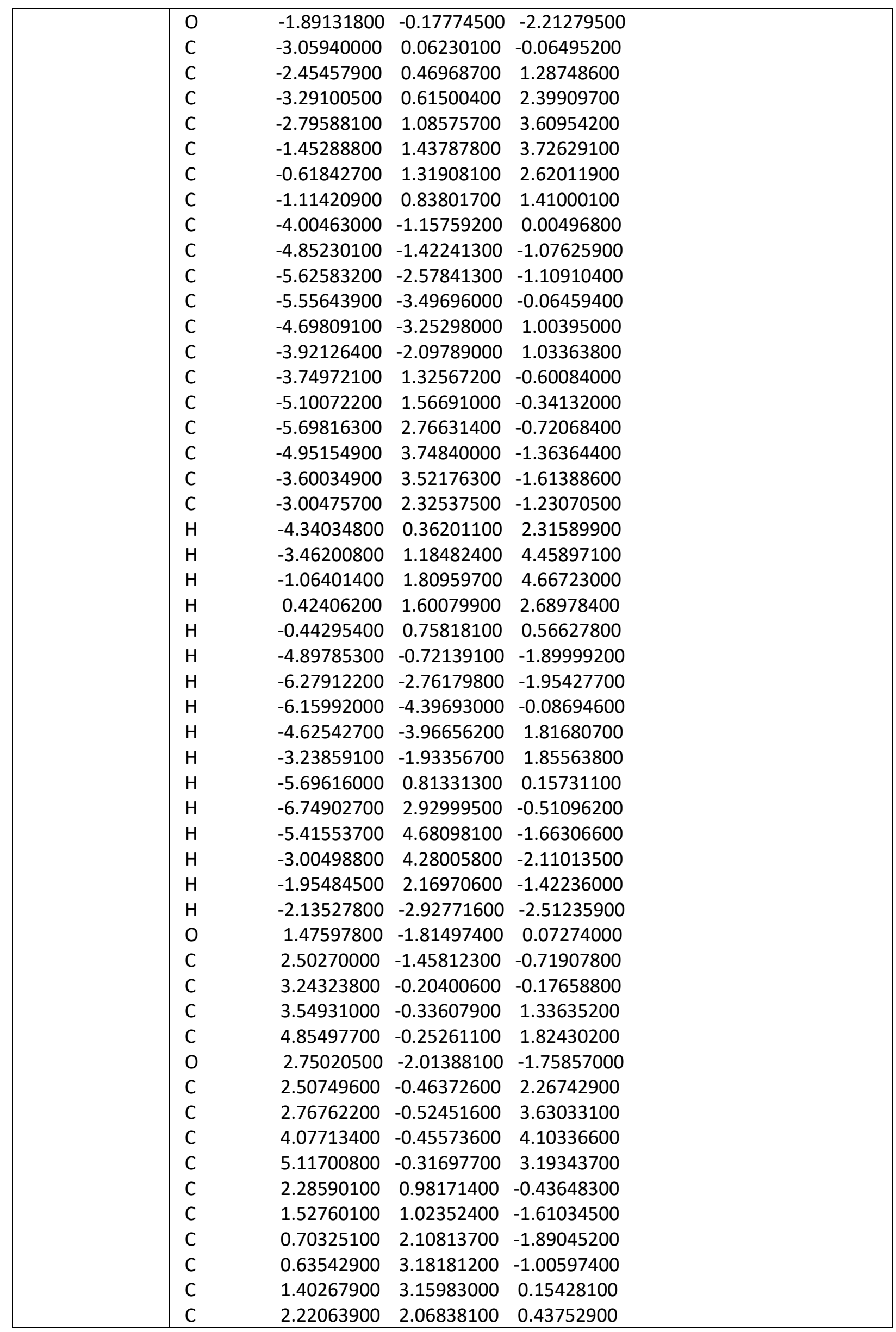




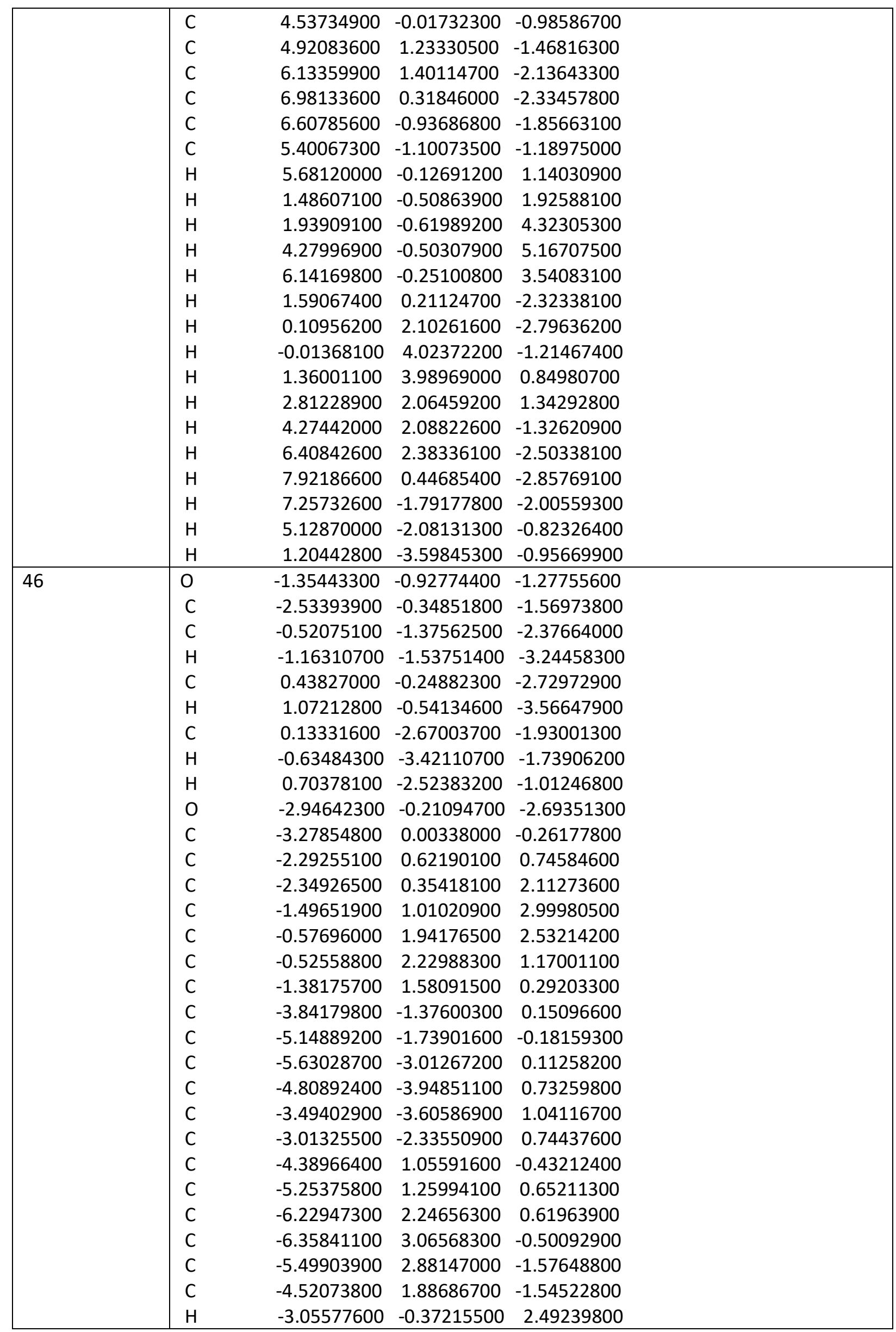




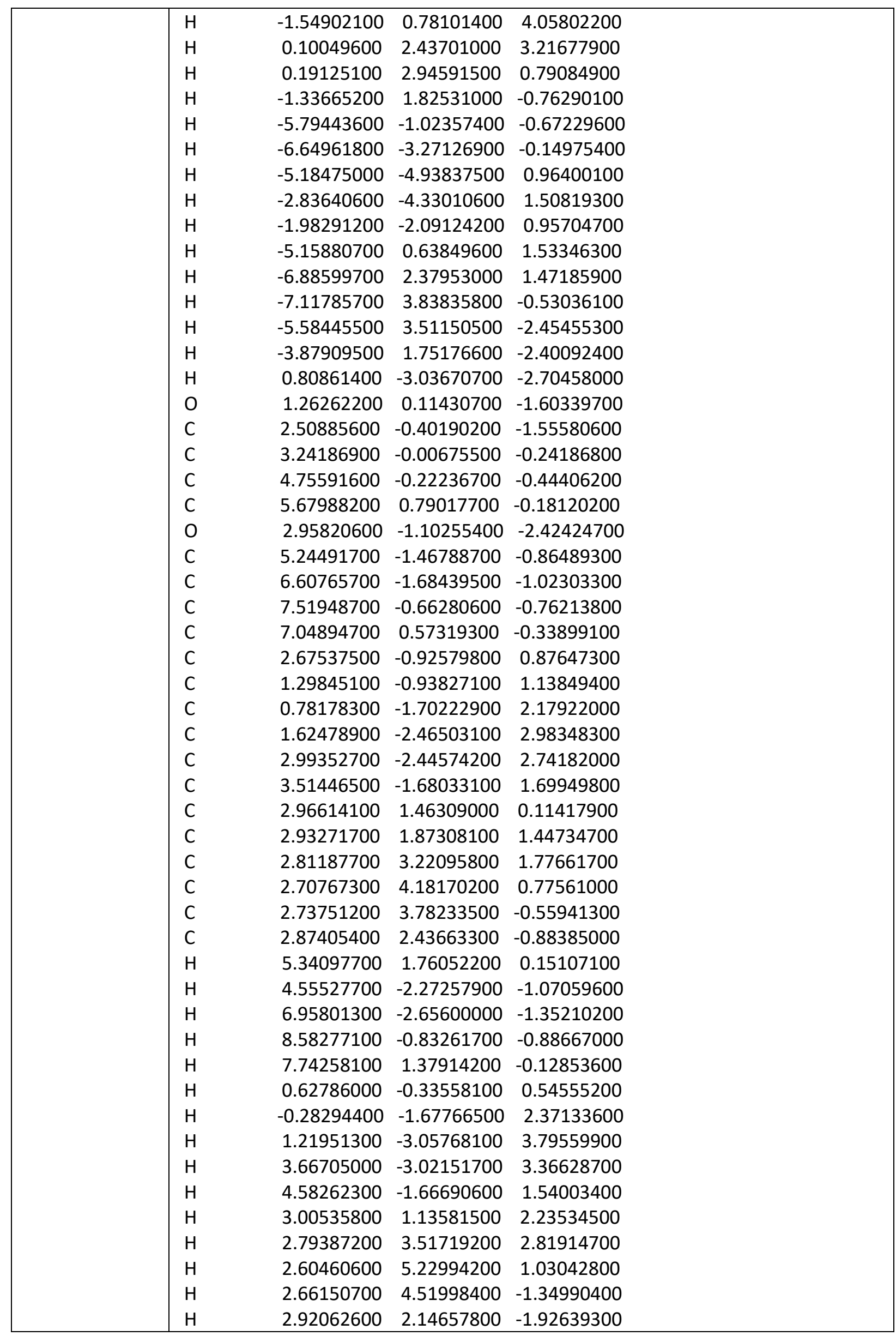




\begin{tabular}{|c|c|c|c|c|}
\hline & $\mathrm{H}$ & -0.12201200 & 0.65069300 & -2.98095500 \\
\hline \multirow{51}{*}{60} & 0 & -1.45669600 & -2.07700700 & -0.22247600 \\
\hline & $\mathrm{C}$ & -1.85413700 & -0.97764200 & -0.88200800 \\
\hline & $\mathrm{C}$ & -0.68184400 & -3.07831600 & -0.92771100 \\
\hline & $\mathrm{H}$ & -0.56287400 & -2.75167700 & -1.96055400 \\
\hline & $\mathrm{C}$ & 0.67512100 & -3.17943600 & -0.25392200 \\
\hline & $\mathrm{H}$ & 0.55888800 & -3.26749700 & 0.82598300 \\
\hline & $\mathrm{C}$ & -1.45570100 & -4.38499900 & -0.84549900 \\
\hline & $\mathrm{H}$ & -0.91513600 & -5.18435200 & -1.35796300 \\
\hline & $\mathrm{H}$ & -2.43129800 & -4.27171100 & -1.32031000 \\
\hline & 0 & -1.58291300 & -0.76481800 & -2.03818900 \\
\hline & $\mathrm{C}$ & -2.72678900 & -0.02659700 & -0.01270600 \\
\hline & $\mathrm{C}$ & -3.98739000 & 0.26847500 & -0.85079700 \\
\hline & $\mathrm{C}$ & -4.55955700 & 1.53853300 & -0.89001500 \\
\hline & $\mathrm{C}$ & -5.74325000 & 1.76362200 & -1.59168000 \\
\hline & $\mathrm{C}$ & -6.37000500 & 0.72225400 & -2.26681300 \\
\hline & $\mathrm{C}$ & -5.80597100 & -0.55185600 & -2.23244000 \\
\hline & $\mathrm{C}$ & -4.62856100 & -0.77325300 & -1.52887900 \\
\hline & $\mathrm{C}$ & -1.93646400 & 1.28888200 & 0.21530900 \\
\hline & $\mathrm{C}$ & -1.23387000 & 1.89250700 & -0.83307300 \\
\hline & $\mathrm{C}$ & -0.60025100 & 3.11809100 & -0.65573200 \\
\hline & $\mathrm{C}$ & -0.67554300 & 3.77969700 & 0.56614900 \\
\hline & $\mathrm{C}$ & -1.39165100 & 3.20066600 & 1.60857900 \\
\hline & $\mathrm{C}$ & -2.01233600 & 1.96502300 & 1.43711500 \\
\hline & $\mathrm{C}$ & -3.10960800 & -0.66738600 & 1.33603100 \\
\hline & $\mathrm{C}$ & -2.10482600 & -1.02474200 & 2.24707300 \\
\hline & $\mathrm{C}$ & -2.42623600 & -1.56514200 & 3.48436800 \\
\hline & $\mathrm{C}$ & -3.75998800 & -1.75625200 & 3.84734500 \\
\hline & $\mathrm{C}$ & -4.76292400 & -1.39484900 & 2.95798100 \\
\hline & $\mathrm{C}$ & -4.43935700 & -0.85186900 & 1.71287900 \\
\hline & $\mathrm{H}$ & -4.08268400 & 2.35921000 & -0.37192800 \\
\hline & $\mathrm{H}$ & -6.17053500 & 2.75963700 & -1.61077800 \\
\hline & $\mathrm{H}$ & -7.28700700 & 0.89903400 & -2.81678100 \\
\hline & $\mathrm{H}$ & -6.28318600 & -1.37350800 & -2.75411500 \\
\hline & $\mathrm{H}$ & -4.21025900 & -1.77277400 & -1.49869300 \\
\hline & $\mathrm{H}$ & -1.18818000 & 1.40626100 & -1.79453800 \\
\hline & $\mathrm{H}$ & -0.04128700 & 3.54599100 & -1.47775800 \\
\hline & $\mathrm{H}$ & -0.17668200 & 4.73149500 & 0.70557800 \\
\hline & $\mathrm{H}$ & -1.46996000 & 3.70579300 & 2.56466400 \\
\hline & $\mathrm{H}$ & -2.56531300 & 1.53498200 & 2.25950500 \\
\hline & $\mathrm{H}$ & -1.06466200 & -0.88185600 & 1.99144600 \\
\hline & $\mathrm{H}$ & -1.62971500 & -1.83360600 & 4.16906900 \\
\hline & $\mathrm{H}$ & -4.00981500 & -2.17624500 & 4.81506500 \\
\hline & $\mathrm{H}$ & -5.80497400 & -1.52720600 & 3.22608400 \\
\hline & $\mathrm{H}$ & -5.23761200 & -0.56836700 & 1.04197800 \\
\hline & $\mathrm{H}$ & -1.61367700 & -4.67428400 & 0.19583500 \\
\hline & 0 & 1.50013900 & -2.03990000 & -0.55922800 \\
\hline & $\mathrm{C}$ & 1.66845100 & -1.09945100 & 0.39646600 \\
\hline & $\mathrm{C}$ & 2.74623300 & -0.05905700 & -0.01611300 \\
\hline & $\mathrm{C}$ & 2.32375900 & 0.79068300 & -1.23752600 \\
\hline & $\mathrm{C}$ & 2.86892800 & 2.07371100 & -1.37759700 \\
\hline & 0 & 1.10582700 & -1.13498500 & 1.45864200 \\
\hline
\end{tabular}




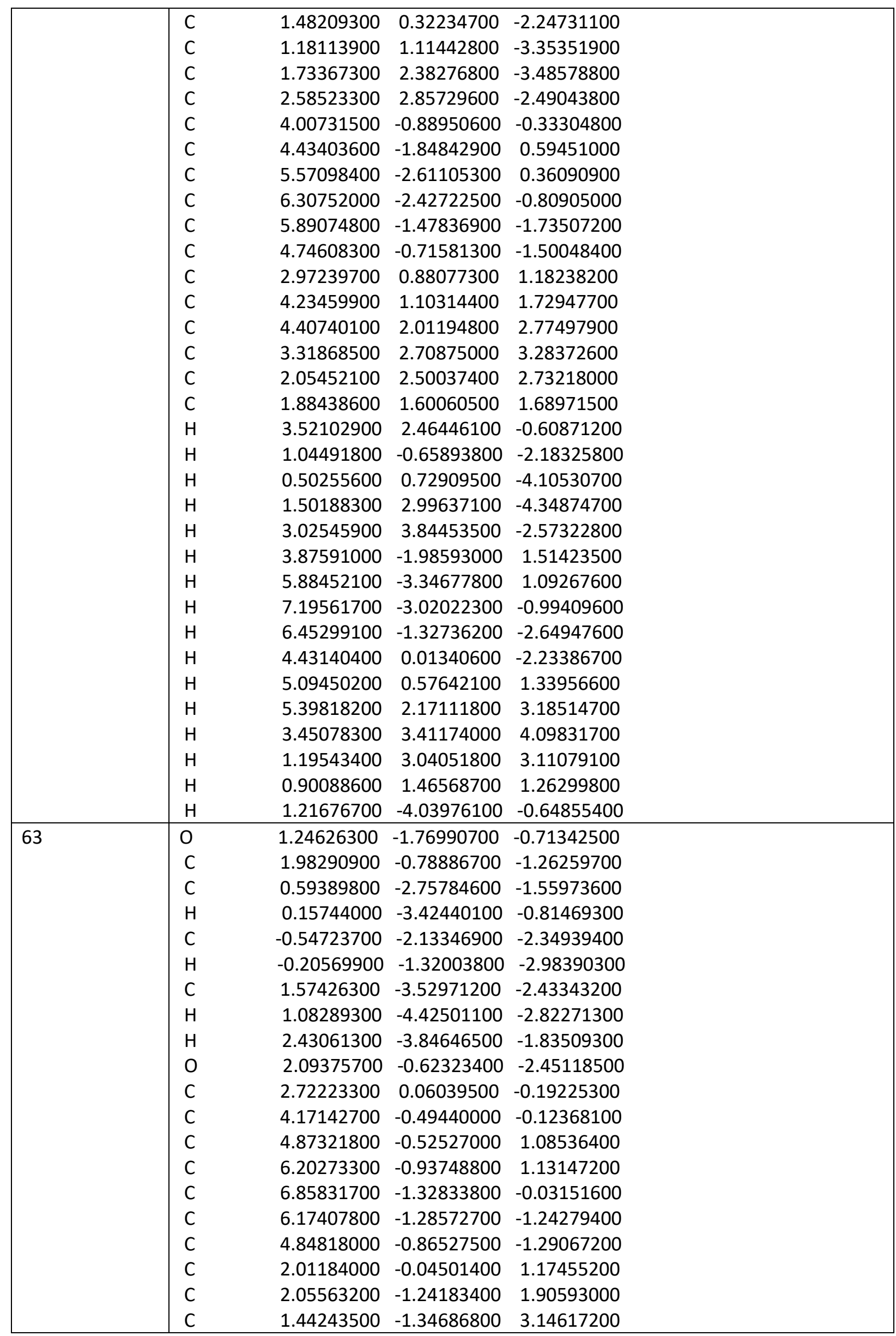




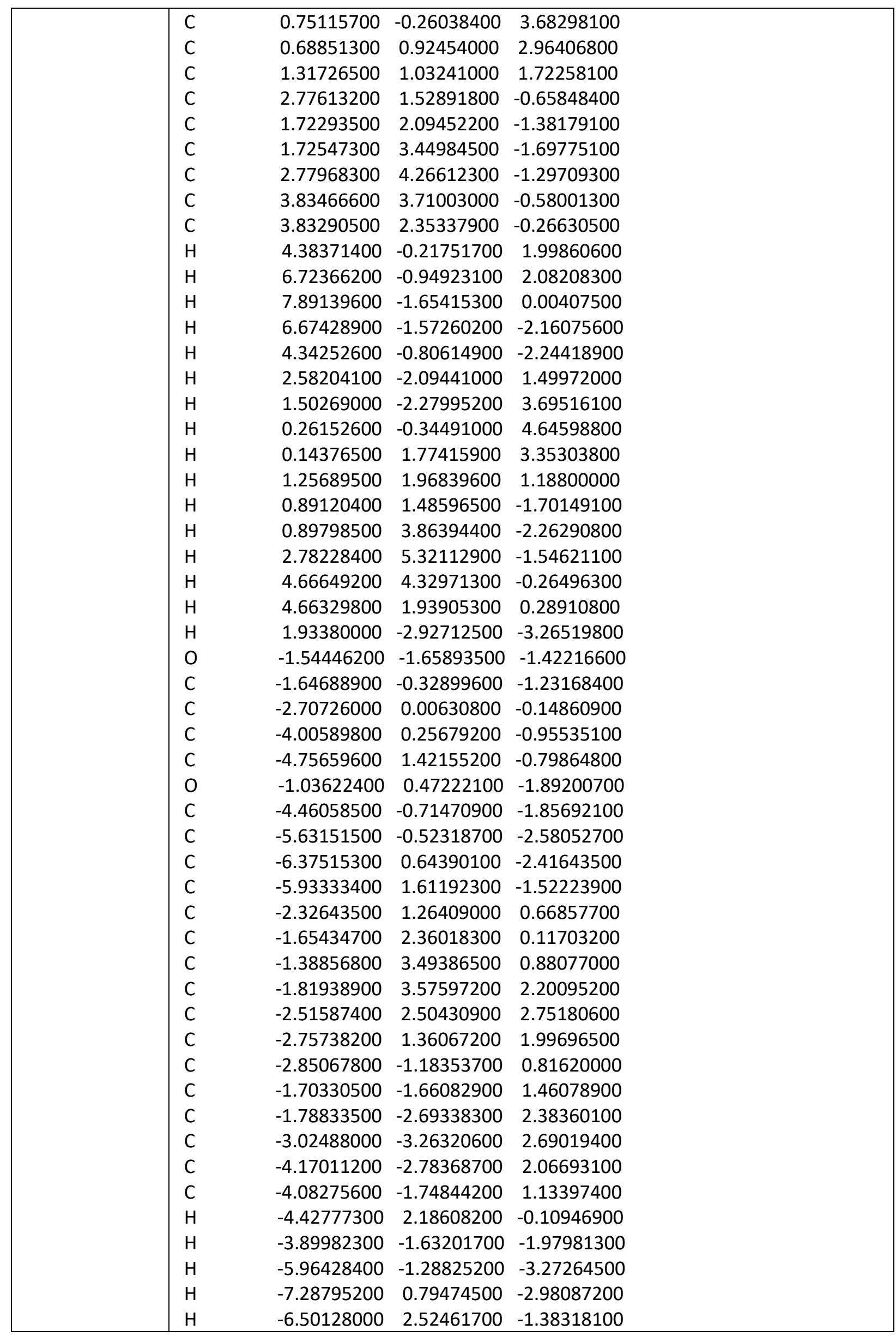




\begin{tabular}{|c|c|c|c|c|}
\hline & $\mathrm{H}$ & -1.33348900 & 2.32762800 & -0.90996100 \\
\hline & $\mathrm{H}$ & -0.84146800 & 4.31484000 & 0.43181600 \\
\hline & $\mathrm{H}$ & -1.61757600 & 4.46248600 & 2.79122900 \\
\hline & $\mathrm{H}$ & -2.86537400 & 2.54868600 & 3.77708600 \\
\hline & $\mathrm{H}$ & -3.28655000 & 0.53537700 & 2.45164600 \\
\hline & $\mathrm{H}$ & -0.73765600 & -1.22032700 & 1.24577400 \\
\hline & $\mathrm{H}$ & -0.88668800 & -3.04705800 & 2.86821300 \\
\hline & $\mathrm{H}$ & -3.09121400 & -4.06903700 & 3.41222300 \\
\hline & $\mathrm{H}$ & -5.13871200 & -3.20971900 & 2.30256400 \\
\hline & $\mathrm{H}$ & -4.98347000 & -1.38068200 & 0.66170100 \\
\hline & $\mathrm{H}$ & -1.03437300 & -2.90448500 & -2.94763000 \\
\hline \multirow[t]{41}{*}{77} & 0 & -1.54479000 & -1.77605400 & -1.05627600 \\
\hline & $\mathrm{C}$ & -1.47578700 & -0.44089800 & -0.89052800 \\
\hline & $\mathrm{C}$ & -0.59588400 & -2.39934400 & -1.96332200 \\
\hline & $\mathrm{H}$ & -0.23433600 & -1.63761200 & -2.65069600 \\
\hline & $\mathrm{C}$ & 0.57048100 & -2.94700500 & -1.15522500 \\
\hline & $\mathrm{H}$ & 0.21841900 & -3.66269400 & -0.41214400 \\
\hline & $\mathrm{C}$ & -1.34767900 & -3.49756500 & -2.69342600 \\
\hline & $\mathrm{H}$ & -2.20528000 & -3.07440500 & -3.21768500 \\
\hline & $\mathrm{H}$ & -1.71426100 & -4.25343600 & -1.99442600 \\
\hline & 0 & -0.63562200 & 0.24467500 & -1.41135700 \\
\hline & $\mathrm{C}$ & -2.66100600 & 0.08289200 & -0.02946100 \\
\hline & $\mathrm{C}$ & -3.94171400 & -0.45899200 & -0.69415800 \\
\hline & $\mathrm{C}$ & -4.97960600 & -1.03141400 & 0.03641900 \\
\hline & $\mathrm{C}$ & -6.13806600 & -1.47309900 & -0.60412700 \\
\hline & $\mathrm{C}$ & -6.27057100 & -1.35174300 & -1.98222600 \\
\hline & $\mathrm{C}$ & -5.23613800 & -0.77813100 & -2.72164700 \\
\hline & $\mathrm{C}$ & -4.08640300 & -0.33470500 & -2.08130400 \\
\hline & $\mathrm{C}$ & -2.62940200 & 1.62068600 & -0.06352900 \\
\hline & $\mathrm{C}$ & -1.46280100 & 2.28633700 & 0.33011300 \\
\hline & $\mathrm{C}$ & -1.41374500 & 3.67261600 & 0.36429200 \\
\hline & $\mathrm{C}$ & -2.53454800 & 4.42531100 & 0.01414700 \\
\hline & $\mathrm{C}$ & -3.70214800 & 3.77361200 & -0.36358900 \\
\hline & $\mathrm{C}$ & -3.74924000 & 2.37900200 & -0.40143600 \\
\hline & $\mathrm{C}$ & -2.55670900 & -0.40592100 & 1.43692800 \\
\hline & $\mathrm{C}$ & -2.96004300 & 0.44080400 & 2.47452500 \\
\hline & $\mathrm{C}$ & -2.96134600 & 0.00796800 & 3.79785800 \\
\hline & $\mathrm{C}$ & -2.55343200 & -1.28324100 & 4.11654000 \\
\hline & $\mathrm{C}$ & -2.15239300 & -2.13669700 & 3.09260000 \\
\hline & $\mathrm{C}$ & -2.16092400 & -1.70607700 & 1.76899900 \\
\hline & $\mathrm{H}$ & -4.88979900 & -1.13601300 & 1.10892100 \\
\hline & $\mathrm{H}$ & -6.93564500 & -1.91517800 & -0.01806800 \\
\hline & $\mathrm{H}$ & -7.16941100 & -1.69838500 & -2.47866000 \\
\hline & $\mathrm{H}$ & -5.32780700 & -0.67264600 & -3.79658300 \\
\hline & $\mathrm{H}$ & -3.29533300 & 0.12818600 & -2.66136600 \\
\hline & $\mathrm{H}$ & -0.59147800 & 1.71954200 & 0.61737900 \\
\hline & $\mathrm{H}$ & -0.49545700 & 4.16167000 & 0.66656100 \\
\hline & $\mathrm{H}$ & -2.49621500 & 5.50840400 & 0.03989300 \\
\hline & $\mathrm{H}$ & -4.58440900 & 4.34436900 & -0.63011300 \\
\hline & $\mathrm{H}$ & -4.66866000 & 1.88927600 & -0.69081000 \\
\hline & $\mathrm{H}$ & -3.27685300 & 1.44921800 & 2.25045700 \\
\hline & $\mathrm{H}$ & -3.28117900 & 0.68774100 & 4.57926600 \\
\hline
\end{tabular}




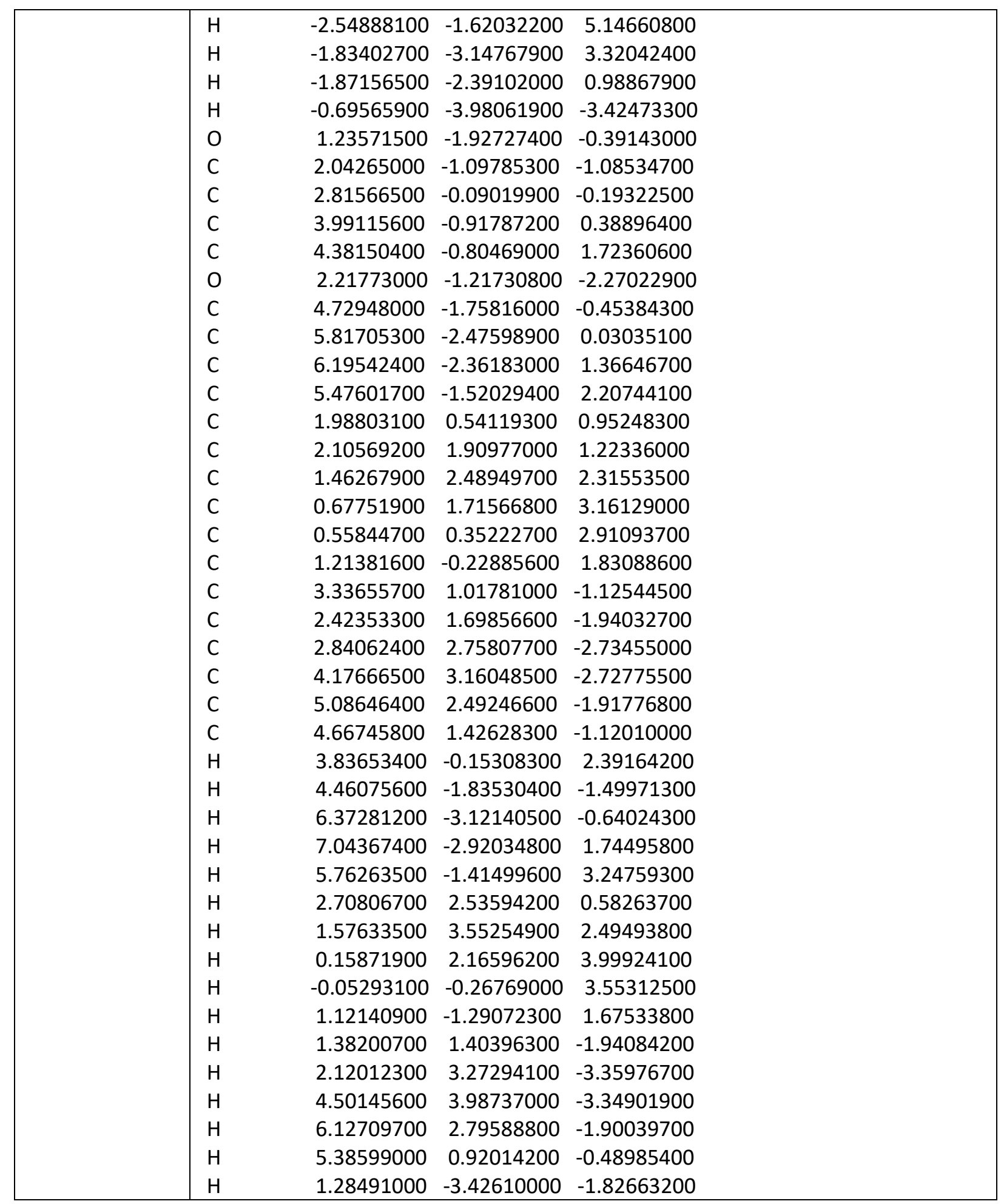

18 (optimized at the M06-2X/6-311G(d,p) level)

\begin{tabular}{|l|llll|}
\hline Conformer no & & & & \\
\hline 1 & O & -1.30405700 & -0.93445900 & -1.20937400 \\
& C & -2.44325200 & -0.30756600 & -1.52265700 \\
& C & -0.49802800 & -1.44801300 & -2.28355500 \\
& H & -1.15210300 & -1.69563500 & -3.12303300 \\
& C & 0.43519900 & -0.33980300 & -2.74180700 \\
& H & 1.05621100 & -0.68131200 & -3.57061100 \\
\hline
\end{tabular}




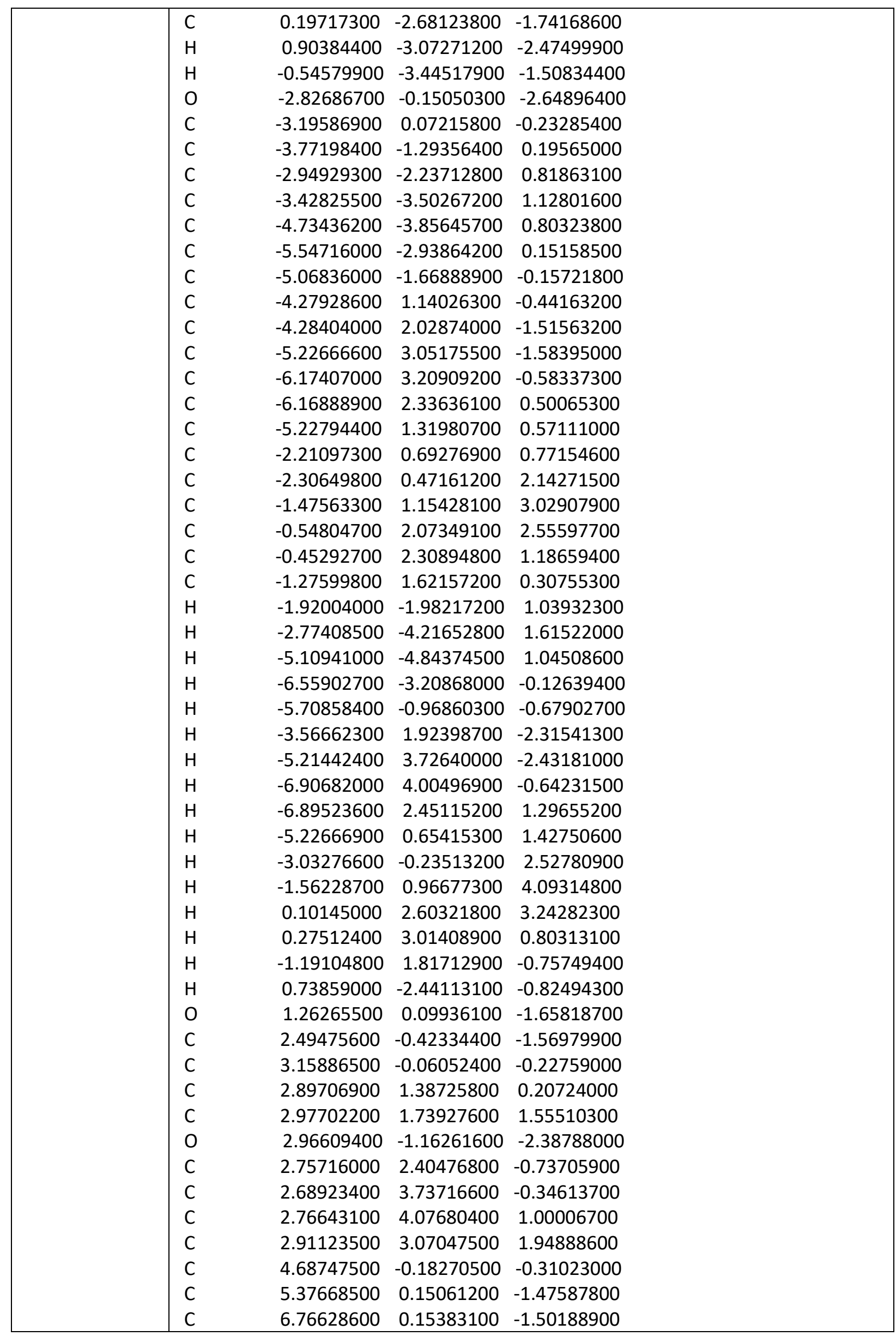




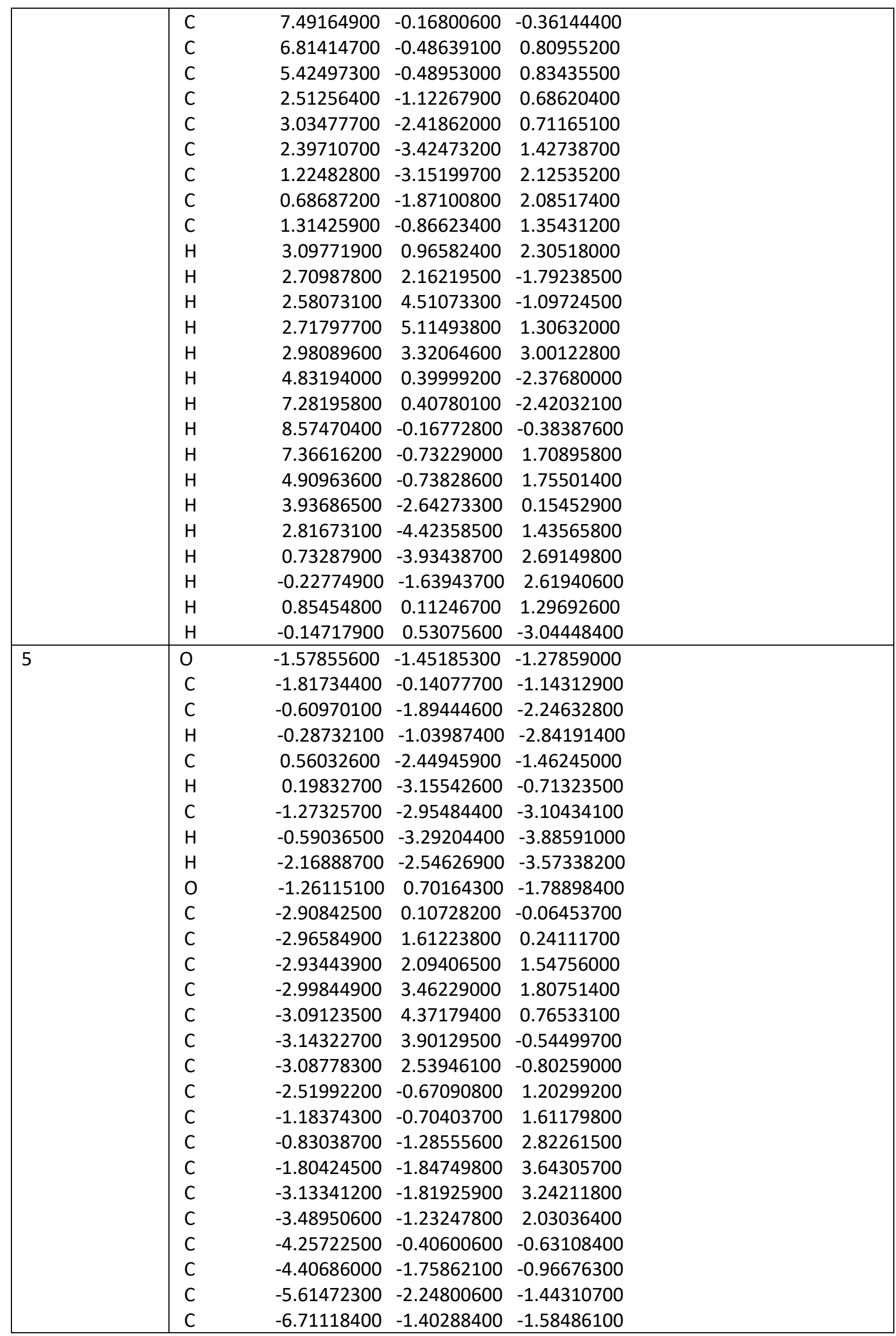




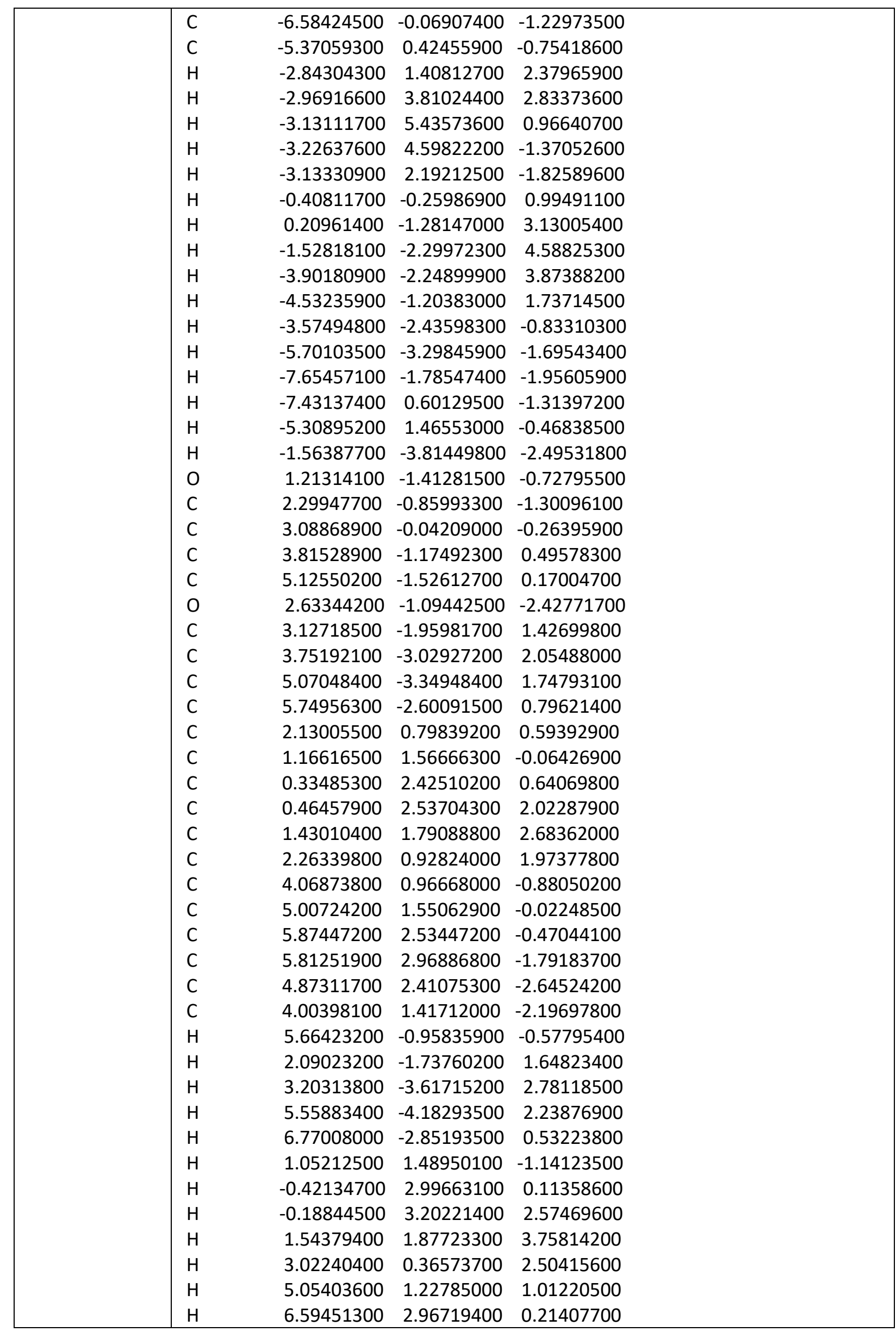




\begin{tabular}{|c|c|c|c|c|}
\hline & $\mathrm{H}$ & 6.48692000 & 3.73869400 & -2.14731800 \\
\hline & $\mathrm{H}$ & 4.80799800 & 2.74487200 & -3.67403100 \\
\hline & $\mathrm{H}$ & 3.29013500 & 0.99256800 & -2.88709600 \\
\hline & $\mathrm{H}$ & 1.27178200 & -2.93279500 & -2.13321700 \\
\hline \multirow[t]{48}{*}{13} & 0 & 1.29128400 & 0.88407700 & -1.34892000 \\
\hline & C & 2.38428200 & 0.13959700 & -1.54883400 \\
\hline & C & 0.47248400 & 1.19306400 & -2.49243500 \\
\hline & $\mathrm{H}$ & 1.12505200 & 1.31911600 & -3.36034000 \\
\hline & C & -0.43778100 & 0.01056100 & -2.78336300 \\
\hline & $\mathrm{H}$ & -1.05888400 & 0.22492200 & -3.65402400 \\
\hline & C & -0.24688700 & 2.48353700 & -2.15199400 \\
\hline & $\mathrm{H}$ & -0.95482700 & 2.74419200 & -2.93958900 \\
\hline & $\mathrm{H}$ & 0.48111200 & 3.28801500 & -2.03717300 \\
\hline & $\mathrm{O}$ & 2.68215900 & -0.30512600 & -2.62230700 \\
\hline & C & 3.15305800 & -0.08992300 & -0.22164500 \\
\hline & C & 2.15164200 & -0.71110100 & 0.76584100 \\
\hline & C & 2.16797700 & -0.39751100 & 2.12208400 \\
\hline & C & 1.32425400 & -1.06119800 & 3.01132400 \\
\hline & C & 0.45638500 & -2.04366800 & 2.55413400 \\
\hline & C & 0.42873500 & -2.36004400 & 1.19764400 \\
\hline & C & 1.27170100 & -1.69980000 & 0.31528400 \\
\hline & C & 3.68328000 & 1.26185700 & 0.31900900 \\
\hline & C & 5.01860300 & 1.41908400 & 0.69207000 \\
\hline & C & 5.47196400 & 2.61342400 & 1.24893200 \\
\hline & C & 4.60028000 & 3.67297100 & 1.44935200 \\
\hline & $\mathrm{C}$ & 3.26253900 & 3.52264000 & 1.09775100 \\
\hline & $\mathrm{C}$ & 2.80790800 & 2.33295600 & 0.54565600 \\
\hline & C & 4.30928600 & -1.06520400 & -0.48750100 \\
\hline & $\mathrm{C}$ & 5.21619300 & -0.79952700 & -1.52038300 \\
\hline & C & 6.30641800 & -1.62670600 & -1.74058800 \\
\hline & C & 6.51866900 & -2.73955700 & -0.93045800 \\
\hline & $\mathrm{C}$ & 5.63057100 & -3.00906200 & 0.09917500 \\
\hline & C & 4.53337200 & -2.17765500 & 0.31961100 \\
\hline & $\mathrm{H}$ & 2.84934700 & 0.35872000 & 2.49415300 \\
\hline & $\mathrm{H}$ & 1.35230000 & -0.80656400 & 4.06456100 \\
\hline & $\mathrm{H}$ & -0.20539900 & -2.55673200 & 3.24134700 \\
\hline & $\mathrm{H}$ & -0.26077700 & -3.11065300 & 0.82912300 \\
\hline & $\mathrm{H}$ & 1.25582300 & -1.96853700 & -0.73721500 \\
\hline & $\mathrm{H}$ & 5.72077600 & 0.60686400 & 0.56175100 \\
\hline & $\mathrm{H}$ & 6.51496800 & 2.70500700 & 1.52803100 \\
\hline & $\mathrm{H}$ & 4.95478500 & 4.60199600 & 1.87990200 \\
\hline & $\mathrm{H}$ & 2.56071700 & 4.33359800 & 1.25684800 \\
\hline & $\mathrm{H}$ & 1.75919600 & 2.23049600 & 0.30495400 \\
\hline & $\mathrm{H}$ & 5.06768700 & 0.06609000 & -2.15292600 \\
\hline & $\mathrm{H}$ & 6.99294500 & -1.40271800 & -2.54844000 \\
\hline & $\mathrm{H}$ & 7.36978700 & -3.38731800 & -1.10396100 \\
\hline & $\mathrm{H}$ & 5.78356300 & -3.86894600 & 0.74046900 \\
\hline & $\mathrm{H}$ & 3.85571100 & -2.40738800 & 1.13192800 \\
\hline & $\mathrm{H}$ & -0.79165700 & 2.38024600 & -1.21160200 \\
\hline & $\mathrm{O}$ & -1.26454600 & -0.28766800 & -1.65402600 \\
\hline & C & -2.49446000 & 0.24810400 & -1.62871800 \\
\hline & $\mathrm{C}$ & -3.15308400 & 0.04390700 & -0.25160800 \\
\hline
\end{tabular}




\begin{tabular}{|c|c|c|c|c|}
\hline & $\mathrm{C}$ & -2.92012600 & -1.36332100 & 0.31304500 \\
\hline & $\mathrm{C}$ & -2.95320300 & -1.58475400 & 1.68908400 \\
\hline & 0 & -2.96138200 & 0.88883300 & -2.52826300 \\
\hline & $\mathrm{C}$ & -2.85400300 & -2.46829900 & -0.53671900 \\
\hline & $\mathrm{C}$ & -2.81612300 & -3.75999400 & -0.02526300 \\
\hline & $\mathrm{C}$ & -2.84823500 & -3.97020000 & 1.34950800 \\
\hline & $\mathrm{C}$ & -2.91867500 & -2.87580800 & 2.20363700 \\
\hline & $\mathrm{C}$ & -4.67940500 & 0.19917900 & -0.31748200 \\
\hline & $\mathrm{C}$ & -5.40707300 & -0.18688900 & -1.44255000 \\
\hline & $\mathrm{C}$ & -6.79721500 & -0.15447100 & -1.43240400 \\
\hline & $\mathrm{C}$ & -7.48379900 & 0.25546000 & -0.29688200 \\
\hline & $\mathrm{C}$ & -6.76751900 & 0.62751400 & 0.83513400 \\
\hline & $\mathrm{C}$ & -5.37922800 & 0.59576100 & 0.82398100 \\
\hline & $\mathrm{C}$ & -2.46281300 & 1.17218400 & 0.54331800 \\
\hline & $\mathrm{C}$ & -2.96780900 & 2.47339400 & 0.47571900 \\
\hline & $\mathrm{C}$ & -2.28803600 & 3.52873700 & 1.07272700 \\
\hline & $\mathrm{C}$ & -1.08859500 & 3.30018700 & 1.73919500 \\
\hline & $\mathrm{C}$ & -0.56702100 & 2.01232400 & 1.78917300 \\
\hline & $\mathrm{C}$ & -1.24044400 & 0.95621500 & 1.18270300 \\
\hline & $\mathrm{H}$ & -3.01226200 & -0.74113900 & 2.36738000 \\
\hline & $\mathrm{H}$ & -2.84351800 & -2.32528000 & -1.61150400 \\
\hline & $\mathrm{H}$ & -2.76764800 & -4.60356200 & -0.70373100 \\
\hline & $\mathrm{H}$ & -2.82451200 & -4.97689600 & 1.74915300 \\
\hline & $\mathrm{H}$ & -2.95364800 & -3.02478000 & 3.27674500 \\
\hline & $\mathrm{H}$ & -4.89343100 & -0.50318300 & -2.34011200 \\
\hline & $\mathrm{H}$ & -7.34336100 & -0.45028100 & -2.32033400 \\
\hline & $\mathrm{H}$ & -8.56678100 & 0.28152300 & -0.29196800 \\
\hline & $\mathrm{H}$ & -7.28926600 & 0.94120200 & 1.73152800 \\
\hline & $\mathrm{H}$ & -4.83279900 & 0.88551200 & 1.71442300 \\
\hline & $\mathrm{H}$ & -3.89068100 & 2.66271400 & -0.05960700 \\
\hline & $\mathrm{H}$ & -2.69641600 & 4.53046500 & 1.01104700 \\
\hline & $\mathrm{H}$ & -0.56206400 & 4.12083800 & 2.21284700 \\
\hline & $\mathrm{H}$ & 0.37402900 & 1.81898100 & 2.29113300 \\
\hline & $\mathrm{H}$ & -0.79905200 & -0.03303500 & 1.19626600 \\
\hline & $\mathrm{H}$ & 0.16064500 & -0.88220100 & -2.96053400 \\
\hline 15 & 0 & -1.54637100 & -1.59234100 & -1.28986800 \\
\hline & $\mathrm{C}$ & -1.45279000 & -0.30708600 & -0.92401400 \\
\hline & $\mathrm{C}$ & -0.57346700 & -2.11372500 & -2.21503400 \\
\hline & $\mathrm{H}$ & -0.17377300 & -1.29293500 & -2.80943700 \\
\hline & $\mathrm{C}$ & 0.54362000 & -2.76740900 & -1.41902800 \\
\hline & $\mathrm{H}$ & 0.12530100 & -3.50660800 & -0.73516200 \\
\hline & $\mathrm{C}$ & -1.29660000 & -3.12578000 & -3.08206800 \\
\hline & $\mathrm{H}$ & -2.12438100 & -2.64259800 & -3.60225100 \\
\hline & $\mathrm{H}$ & -0.61472900 & -3.54252300 & -3.82517900 \\
\hline & 0 & -0.57379800 & 0.41605300 & -1.30256300 \\
\hline & $\mathrm{C}$ & -2.64083800 & 0.08865600 & -0.00938700 \\
\hline & $\mathrm{C}$ & -3.86788700 & 0.22065800 & -0.94615100 \\
\hline & $\mathrm{C}$ & -4.54396300 & 1.42821600 & -1.11355600 \\
\hline & $\mathrm{C}$ & -5.64438000 & 1.51818300 & -1.96407400 \\
\hline & $\mathrm{C}$ & -6.09144900 & 0.40418600 & -2.65744300 \\
\hline & $\mathrm{C}$ & -5.43428000 & -0.81091800 & -2.48599400 \\
\hline & $\mathrm{C}$ & -4.33897000 & -0.90274200 & -1.63954200 \\
\hline
\end{tabular}




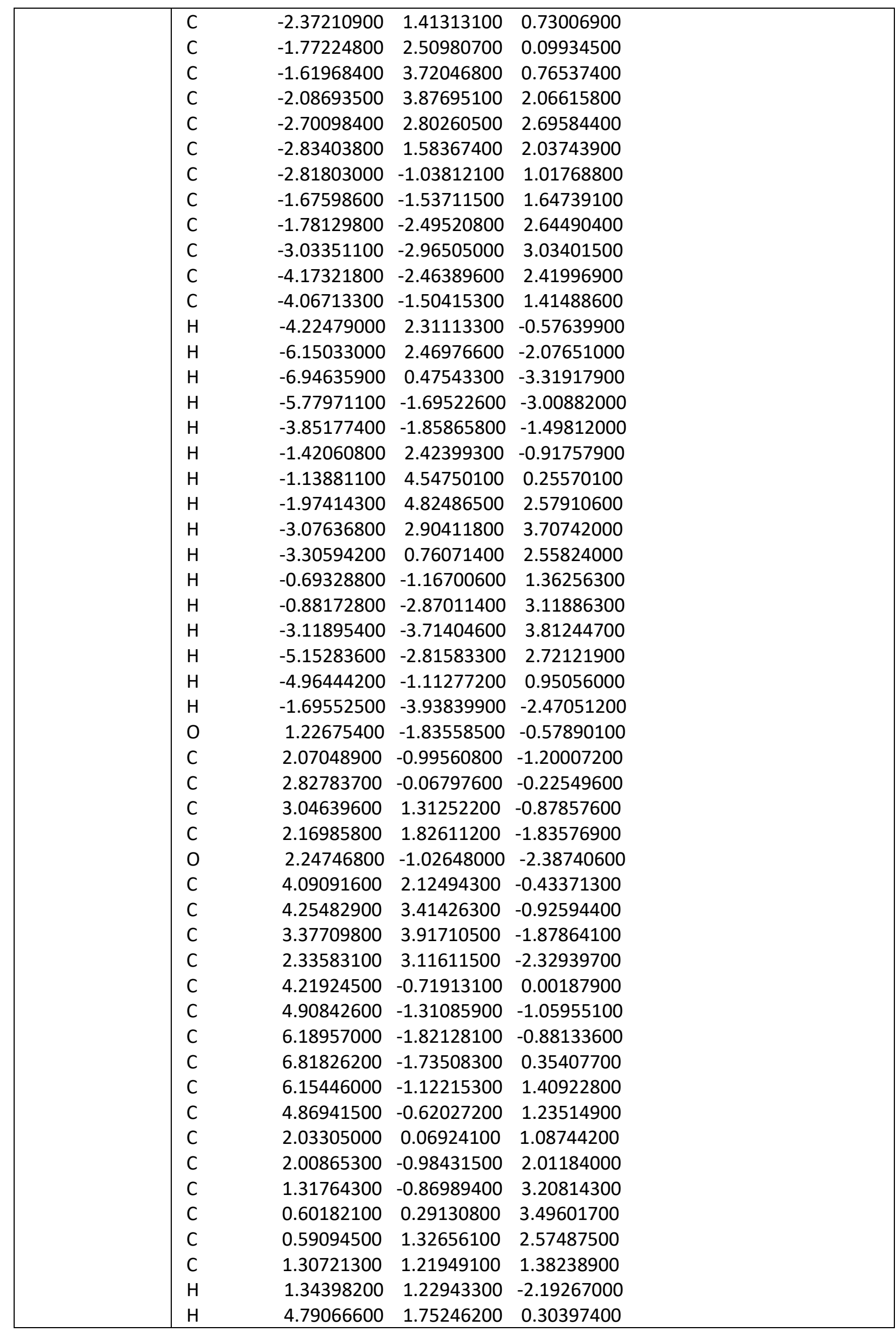




\begin{tabular}{|c|c|c|c|c|}
\hline & $\mathrm{H}$ & 5.07542700 & 4.02315400 & -0.56528100 \\
\hline & $\mathrm{H}$ & 3.50583700 & 4.92020300 & -2.26782000 \\
\hline & $\mathrm{H}$ & 1.64321100 & 3.49155600 & -3.07384900 \\
\hline & $\mathrm{H}$ & 4.45162000 & -1.35792800 & -2.03937100 \\
\hline & $\mathrm{H}$ & 6.69861400 & -2.28154200 & -1.72001200 \\
\hline & $\mathrm{H}$ & 7.81692400 & -2.13251900 & 0.49057900 \\
\hline & $\mathrm{H}$ & 6.63457900 & -1.02898800 & 2.37638800 \\
\hline & $\mathrm{H}$ & 4.37703600 & -0.13729900 & 2.06963100 \\
\hline & $\mathrm{H}$ & 2.54689300 & -1.89823500 & 1.79104100 \\
\hline & $\mathrm{H}$ & 1.33335100 & -1.69094800 & 3.91601000 \\
\hline & $\mathrm{H}$ & 0.05023700 & 0.37817300 & 4.42488000 \\
\hline & $\mathrm{H}$ & 0.02747200 & 2.23048000 & 2.77220300 \\
\hline & $\mathrm{H}$ & 1.28717200 & 2.04705800 & 0.68459100 \\
\hline & $\mathrm{H}$ & 1.25308300 & -3.23756100 & -2.10337800 \\
\hline \multirow[t]{38}{*}{17} & 0 & -1.29613100 & 0.93948300 & 1.34732900 \\
\hline & $\mathrm{C}$ & -2.39694800 & 0.20086200 & 1.54056500 \\
\hline & $\mathrm{C}$ & -0.44948800 & 1.16962900 & 2.49415000 \\
\hline & $\mathrm{H}$ & -1.08854500 & 1.27247500 & 3.37472300 \\
\hline & $\mathrm{C}$ & 0.42799700 & -0.05256000 & 2.72497800 \\
\hline & $\mathrm{H}$ & 1.03638700 & 0.09106500 & 3.61883700 \\
\hline & $\mathrm{C}$ & 0.30889400 & 2.44963000 & 2.20782700 \\
\hline & $\mathrm{H}$ & 1.03178800 & 2.64628600 & 3.00081900 \\
\hline & $\mathrm{H}$ & -0.38953100 & 3.28456900 & 2.13792800 \\
\hline & 0 & -2.70781100 & -0.23576600 & 2.61357300 \\
\hline & $\mathrm{C}$ & -3.15715600 & -0.08241100 & 0.21976000 \\
\hline & $\mathrm{C}$ & -2.94667000 & 1.03514300 & -0.81239300 \\
\hline & $\mathrm{C}$ & -2.87759700 & 0.74607900 & -2.17446400 \\
\hline & $\mathrm{C}$ & -2.83339800 & 1.76794800 & -3.12073200 \\
\hline & $\mathrm{C}$ & -2.84232700 & 3.09692600 & -2.71923000 \\
\hline & $\mathrm{C}$ & -2.90118400 & 3.39711200 & -1.36045800 \\
\hline & $\mathrm{C}$ & -2.96059400 & 2.37708100 & -0.42080200 \\
\hline & $\mathrm{C}$ & -4.65493200 & -0.23764400 & 0.54555000 \\
\hline & $\mathrm{C}$ & -5.06719400 & -1.24028400 & 1.43279800 \\
\hline & $\mathrm{C}$ & -6.41000400 & -1.42188000 & 1.72566500 \\
\hline & $\mathrm{C}$ & -7.37587000 & -0.61183700 & 1.13360900 \\
\hline & $\mathrm{C}$ & -6.98070500 & 0.37474300 & 0.24442100 \\
\hline & $\mathrm{C}$ & -5.63040500 & 0.55989500 & -0.04961100 \\
\hline & $\mathrm{C}$ & -2.57016200 & -1.40752900 & -0.31895400 \\
\hline & $\mathrm{C}$ & -3.38020000 & -2.34852400 & -0.95587300 \\
\hline & $\mathrm{C}$ & -2.82403600 & -3.48753700 & -1.53098100 \\
\hline & $\mathrm{C}$ & -1.45302600 & -3.70742700 & -1.47447900 \\
\hline & $\mathrm{C}$ & -0.63910900 & -2.77809000 & -0.83707500 \\
\hline & $\mathrm{C}$ & -1.19364200 & -1.63825900 & -0.26725100 \\
\hline & $\mathrm{H}$ & -2.86562400 & -0.28393700 & -2.50852600 \\
\hline & $\mathrm{H}$ & -2.78644900 & 1.51755900 & -4.17405300 \\
\hline & $\mathrm{H}$ & -2.80274800 & 3.89240100 & -3.45391900 \\
\hline & $\mathrm{H}$ & -2.90878300 & 4.42938900 & -1.03051100 \\
\hline & $\mathrm{H}$ & -3.02895400 & 2.62913800 & 0.63114900 \\
\hline & $\mathrm{H}$ & -4.32768300 & -1.88329400 & 1.89028300 \\
\hline & $\mathrm{H}$ & -6.70440000 & -2.20319600 & 2.41630500 \\
\hline & $\mathrm{H}$ & -8.42530100 & -0.75651300 & 1.36133600 \\
\hline & $\mathrm{H}$ & -7.71968200 & 1.00847000 & -0.23136900 \\
\hline
\end{tabular}




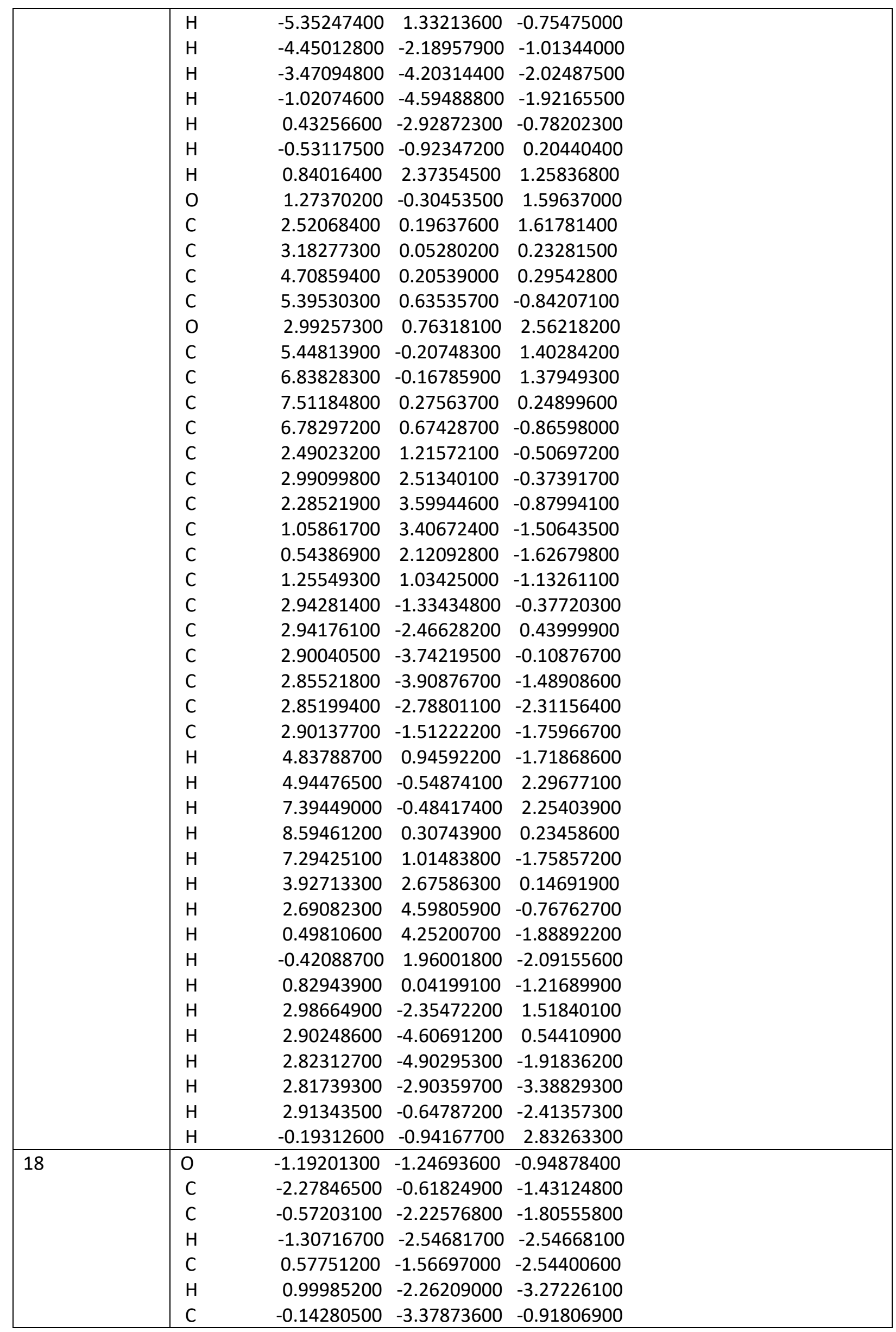




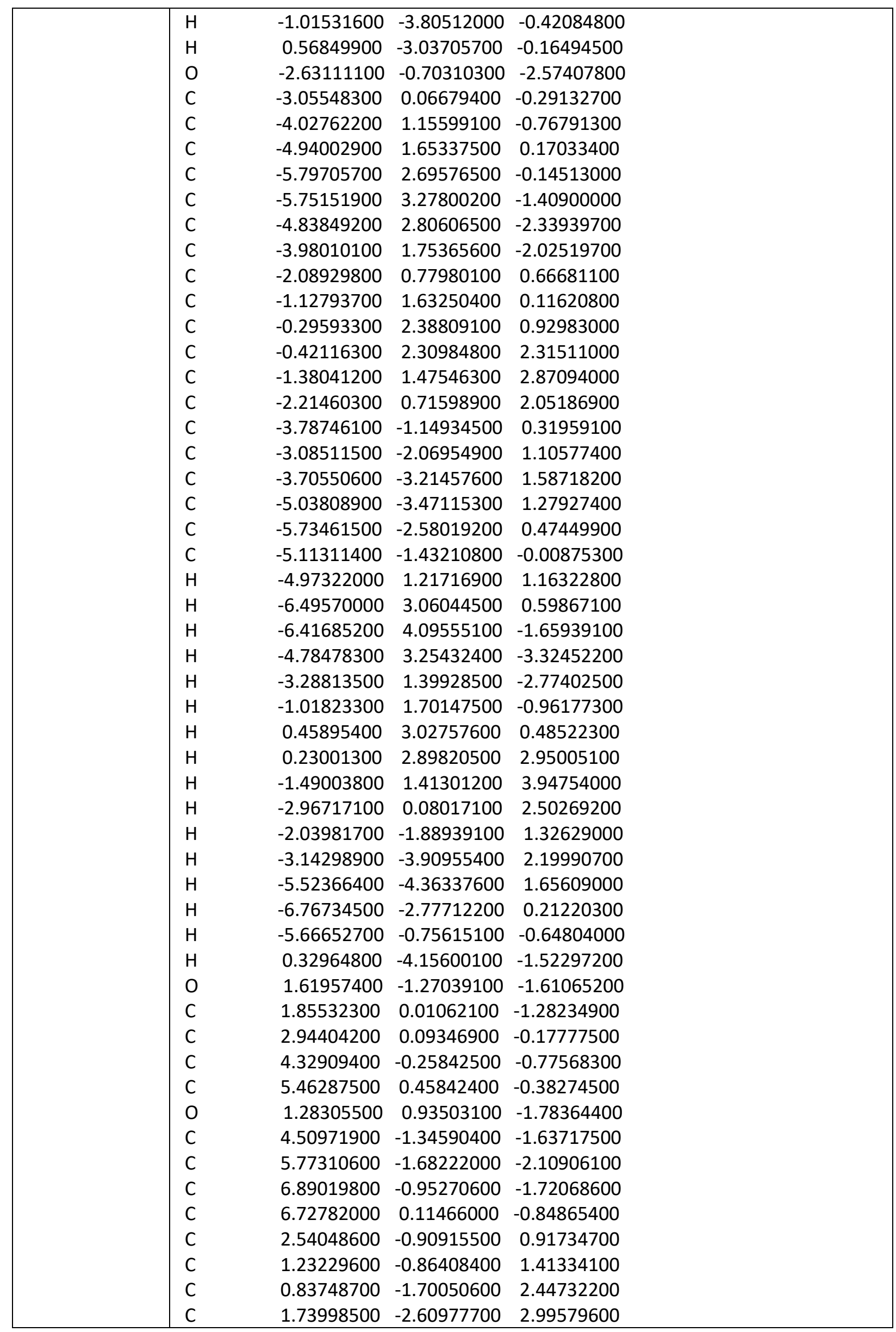




\begin{tabular}{|c|c|c|c|c|}
\hline & $C$ & 3.03575800 & -2.66518200 & 2.50300600 \\
\hline & C & 3.43747300 & -1.81615500 & 1.47190500 \\
\hline & $\mathrm{C}$ & 2.97236600 & 1.52574800 & 0.37592100 \\
\hline & C & 3.12709900 & 2.60158400 & -0.50620800 \\
\hline & $\mathrm{C}$ & 3.20124200 & 3.90353500 & -0.03494400 \\
\hline & $\mathrm{C}$ & 3.14419200 & 4.15938900 & 1.33396000 \\
\hline & $\mathrm{C}$ & 3.02525800 & 3.09734700 & 2.21775000 \\
\hline & $\mathrm{C}$ & 2.93749400 & 1.78941100 & 1.74241200 \\
\hline & $\mathrm{H}$ & 5.36767700 & 1.29311100 & 0.29890200 \\
\hline & $\mathrm{H}$ & 3.66272000 & -1.94989800 & -1.92784600 \\
\hline & $\mathrm{H}$ & 5.88083200 & -2.52515800 & -2.78154200 \\
\hline & $\mathrm{H}$ & 7.87415700 & -1.21701100 & -2.08892700 \\
\hline & $\mathrm{H}$ & 7.58703000 & 0.69078900 & -0.52584000 \\
\hline & $\mathrm{H}$ & 0.51469000 & -0.16379700 & 0.99579400 \\
\hline & $\mathrm{H}$ & -0.17514000 & -1.63030500 & 2.82916700 \\
\hline & $\mathrm{H}$ & 1.43387100 & -3.26466900 & 3.80291600 \\
\hline & $\mathrm{H}$ & 3.74729100 & -3.36742700 & 2.92157000 \\
\hline & $\mathrm{H}$ & 4.45579200 & -1.86757200 & 1.10676800 \\
\hline & $\mathrm{H}$ & 3.18849200 & 2.41628200 & -1.57020500 \\
\hline & $\mathrm{H}$ & 3.30869400 & 4.72120600 & -0.73772000 \\
\hline & $\mathrm{H}$ & 3.20379800 & 5.17626100 & 1.70336900 \\
\hline & $\mathrm{H}$ & 2.99712100 & 3.27790500 & 3.28621300 \\
\hline & $\mathrm{H}$ & 2.83539700 & 0.97793300 & 2.45162800 \\
\hline & $\mathrm{H}$ & 0.24767300 & -0.65763100 & -3.04523700 \\
\hline \multirow[t]{28}{*}{28} & 0 & -1.26821100 & -1.01212600 & -1.38260700 \\
\hline & $\mathrm{C}$ & -1.93147800 & 0.14158800 & -1.23413700 \\
\hline & $\mathrm{C}$ & -0.42701500 & -1.14522700 & -2.54416300 \\
\hline & $\mathrm{H}$ & 0.01350000 & -0.17253300 & -2.76800600 \\
\hline & $\mathrm{C}$ & 0.65419100 & -2.12556600 & -2.14172600 \\
\hline & $\mathrm{H}$ & 0.20817300 & -3.02189800 & -1.71071800 \\
\hline & $\mathrm{C}$ & -1.25187800 & -1.63759300 & -3.71894400 \\
\hline & $\mathrm{H}$ & -0.63351100 & -1.69074200 & -4.61671500 \\
\hline & $\mathrm{H}$ & -2.07476100 & -0.94658200 & -3.90618500 \\
\hline & 0 & -1.82138600 & 1.05545300 & -2.00449700 \\
\hline & $\mathrm{C}$ & -2.91160200 & 0.08626900 & -0.03113700 \\
\hline & $\mathrm{C}$ & -3.06214800 & 1.47644800 & 0.62075000 \\
\hline & $\mathrm{C}$ & -3.09958600 & 1.61027200 & 2.01051700 \\
\hline & $\mathrm{C}$ & -3.29524400 & 2.85275900 & 2.60853400 \\
\hline & $\mathrm{C}$ & -3.44894600 & 3.99085700 & 1.82994900 \\
\hline & $\mathrm{C}$ & -3.43039300 & 3.86868500 & 0.44390700 \\
\hline & $\mathrm{C}$ & -3.25575200 & 2.62667600 & -0.15440700 \\
\hline & $\mathrm{C}$ & -2.41864100 & -0.91729800 & 1.01932900 \\
\hline & $\mathrm{C}$ & -1.09586700 & -0.83307100 & 1.46373700 \\
\hline & $\mathrm{C}$ & -0.65242700 & -1.61347200 & 2.52122400 \\
\hline & $\mathrm{C}$ & -1.52166600 & -2.50752300 & 3.14425800 \\
\hline & $\mathrm{C}$ & -2.83208100 & -2.60676200 & 2.69999400 \\
\hline & $\mathrm{C}$ & -3.28069800 & -1.81091500 & 1.64617100 \\
\hline & $\mathrm{C}$ & -4.24749400 & -0.37930000 & -0.65758000 \\
\hline & $\mathrm{C}$ & -4.27393100 & -1.56598800 & -1.39979100 \\
\hline & $\mathrm{C}$ & -5.45704100 & -2.03386800 & -1.95348200 \\
\hline & $\mathrm{C}$ & -6.64315300 & -1.32969800 & -1.76872200 \\
\hline & $\mathrm{C}$ & -6.62972400 & -0.16086500 & -1.02158700 \\
\hline
\end{tabular}




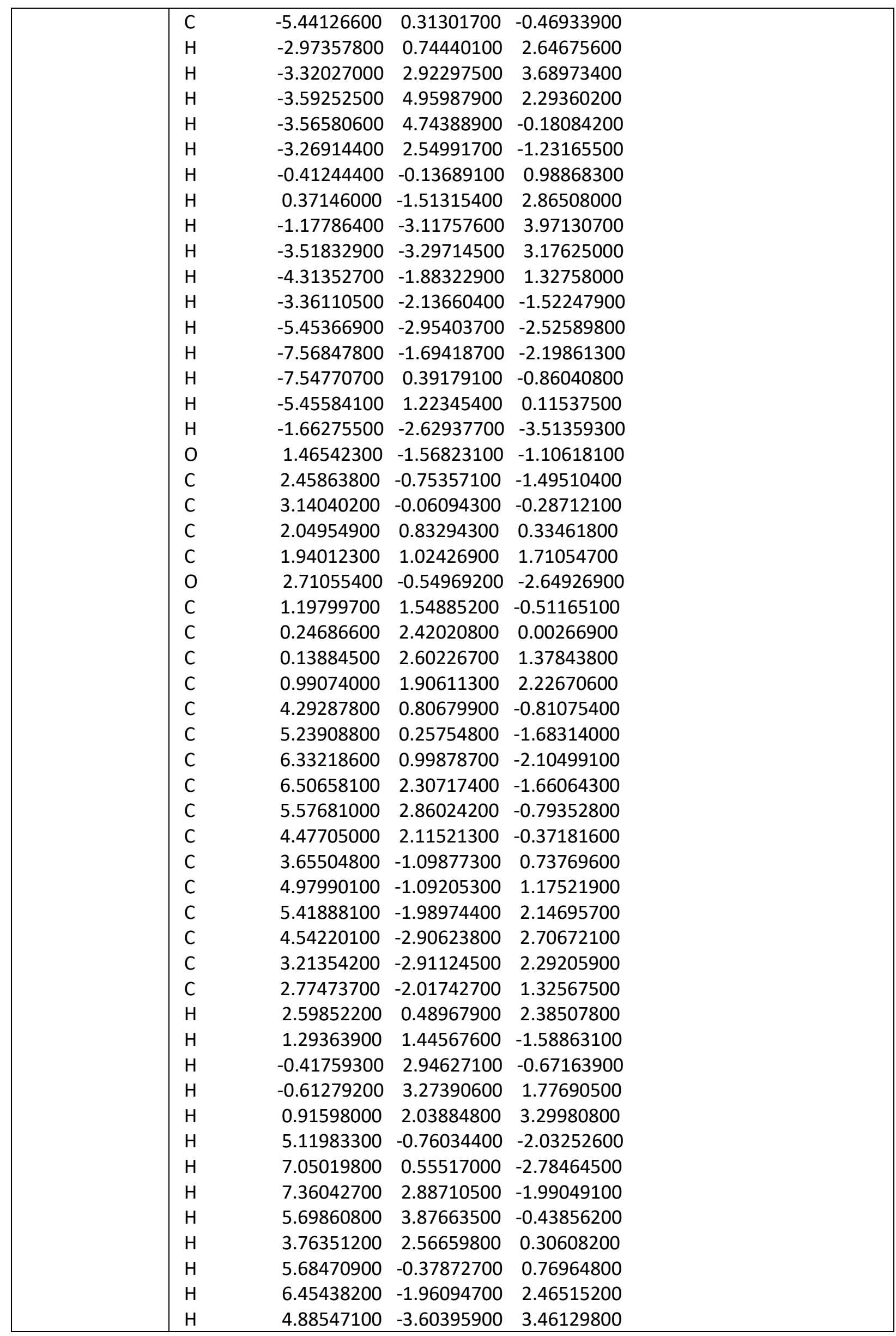




\begin{tabular}{|c|c|c|c|c|}
\hline & $\mathrm{H}$ & 2.50797100 & -3.61211000 & 2.72437700 \\
\hline & $\mathrm{H}$ & 1.73472200 & -2.02655600 & 1.03196100 \\
\hline & $\mathrm{H}$ & 1.27022800 & -2.38189400 & -3.00409300 \\
\hline \multirow[t]{49}{*}{32} & 0 & -1.26228200 & -1.61539400 & -1.01283900 \\
\hline & C & -2.05790500 & -0.61218000 & -1.41211500 \\
\hline & C & -0.61413100 & -2.37654200 & -2.04776100 \\
\hline & $\mathrm{H}$ & -1.34242400 & -2.56614500 & -2.84146200 \\
\hline & C & 0.52558500 & -1.57461700 & -2.65971100 \\
\hline & $\mathrm{H}$ & 1.01948900 & -2.17767100 & -3.42285500 \\
\hline & C & -0.15216600 & -3.66536000 & -1.39830700 \\
\hline & $\mathrm{H}$ & 0.28895300 & -4.32378700 & -2.14935300 \\
\hline & $\mathrm{H}$ & -1.00019300 & -4.17592300 & -0.94094300 \\
\hline & $\mathrm{O}$ & -2.21761800 & -0.33366200 & -2.56902500 \\
\hline & C & -2.80203800 & 0.06367500 & -0.23922300 \\
\hline & C & -4.17965200 & -0.64427500 & -0.14515700 \\
\hline & C & -4.81997800 & -0.82766400 & 1.08341500 \\
\hline & C & -6.09716800 & -1.37376900 & 1.14931100 \\
\hline & C & -6.76298100 & -1.74738600 & -0.01126800 \\
\hline & C & -6.14413300 & -1.55115300 & -1.23926600 \\
\hline & C & -4.87067100 & -0.99748500 & -1.30688700 \\
\hline & C & -3.04321300 & 1.55090000 & -0.56585300 \\
\hline & C & -4.14019600 & 2.21595900 & -0.01754900 \\
\hline & C & -4.32346700 & 3.57991700 & -0.21751500 \\
\hline & C & -3.41301600 & 4.30531800 & -0.97436200 \\
\hline & C & -2.31727900 & 3.65206300 & -1.52612900 \\
\hline & C & -2.13196300 & 2.28896300 & -1.32494200 \\
\hline & C & -1.98098000 & -0.08806700 & 1.05777700 \\
\hline & C & -1.24103100 & 0.97073100 & 1.57646400 \\
\hline & C & -0.50205600 & 0.82190100 & 2.74995400 \\
\hline & C & -0.50773200 & -0.38270800 & 3.43524400 \\
\hline & C & -1.23874100 & -1.45332800 & 2.92316400 \\
\hline & C & -1.94719000 & -1.31299200 & 1.73942200 \\
\hline & $\mathrm{H}$ & -4.32641700 & -0.52975300 & 1.99975100 \\
\hline & $\mathrm{H}$ & -6.57088600 & -1.50254400 & 2.11558800 \\
\hline & $\mathrm{H}$ & -7.75642800 & -2.17647800 & 0.04131100 \\
\hline & $\mathrm{H}$ & -6.65592900 & -1.82003700 & -2.15572400 \\
\hline & $\mathrm{H}$ & -4.42230500 & -0.82018200 & -2.27603700 \\
\hline & $\mathrm{H}$ & -4.86780100 & 1.67042200 & 0.56955200 \\
\hline & $\mathrm{H}$ & -5.18534900 & 4.07115200 & 0.21840200 \\
\hline & $\mathrm{H}$ & -3.55766200 & 5.36699400 & -1.13640200 \\
\hline & $\mathrm{H}$ & -1.59864700 & 4.20281800 & -2.12193300 \\
\hline & $\mathrm{H}$ & -1.26611100 & 1.80535200 & -1.75343500 \\
\hline & $\mathrm{H}$ & -1.22717700 & 1.92729400 & 1.07007300 \\
\hline & $\mathrm{H}$ & 0.07710000 & 1.66055800 & 3.11719700 \\
\hline & $\mathrm{H}$ & 0.05913000 & -0.49468200 & 4.35217400 \\
\hline & $\mathrm{H}$ & -1.25175400 & -2.40358100 & 3.44513500 \\
\hline & $\mathrm{H}$ & -2.49451300 & -2.15887700 & 1.34140300 \\
\hline & $\mathrm{H}$ & 0.59515500 & -3.45800300 & -0.63103300 \\
\hline & $\mathrm{O}$ & 1.53113800 & -1.29476300 & -1.67735200 \\
\hline & C & 1.48307000 & -0.10073800 & -1.06636300 \\
\hline & C & 2.68985600 & 0.08411500 & -0.11249100 \\
\hline & $\mathrm{C}$ & 2.45066500 & 1.23511000 & 0.88447300 \\
\hline
\end{tabular}




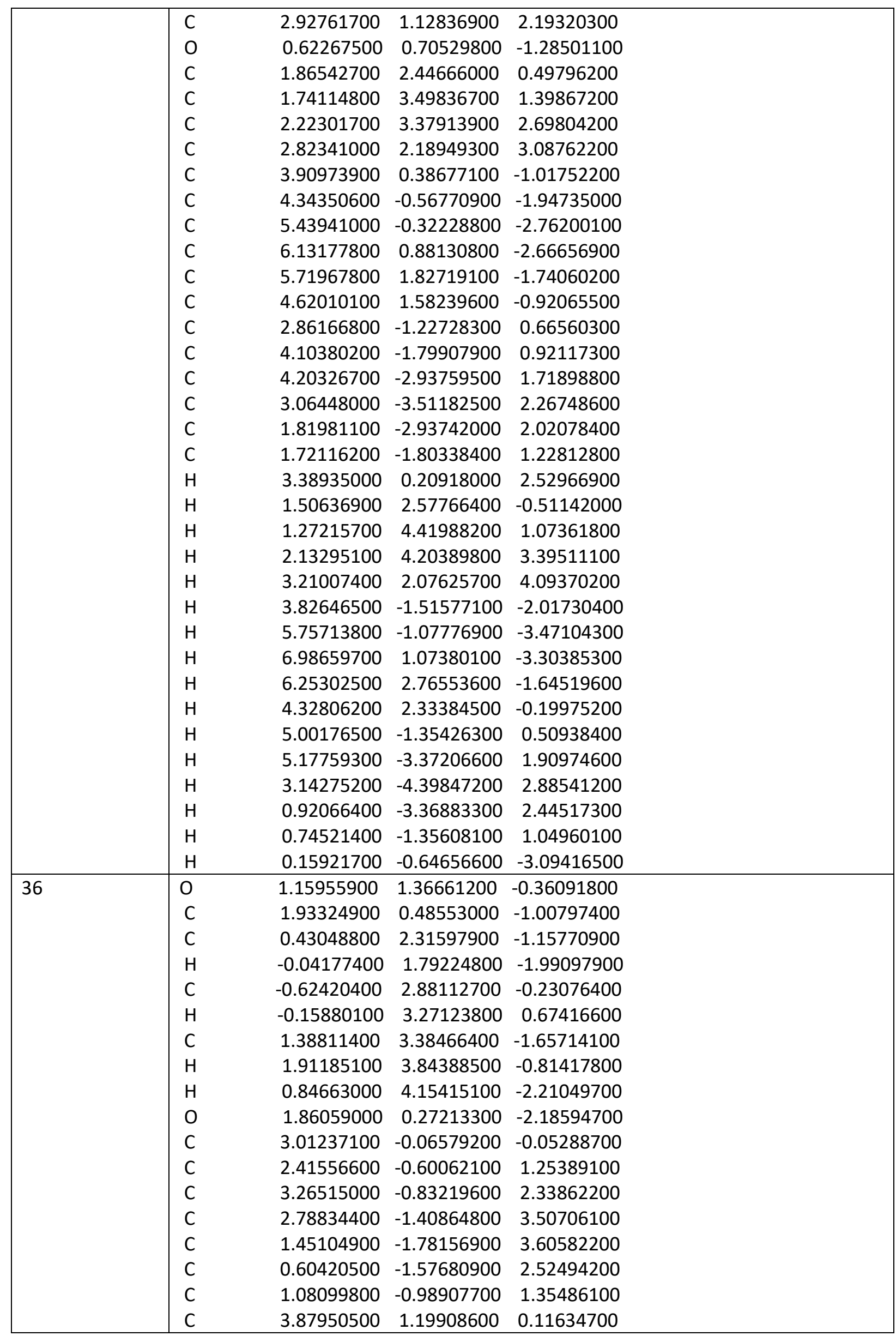




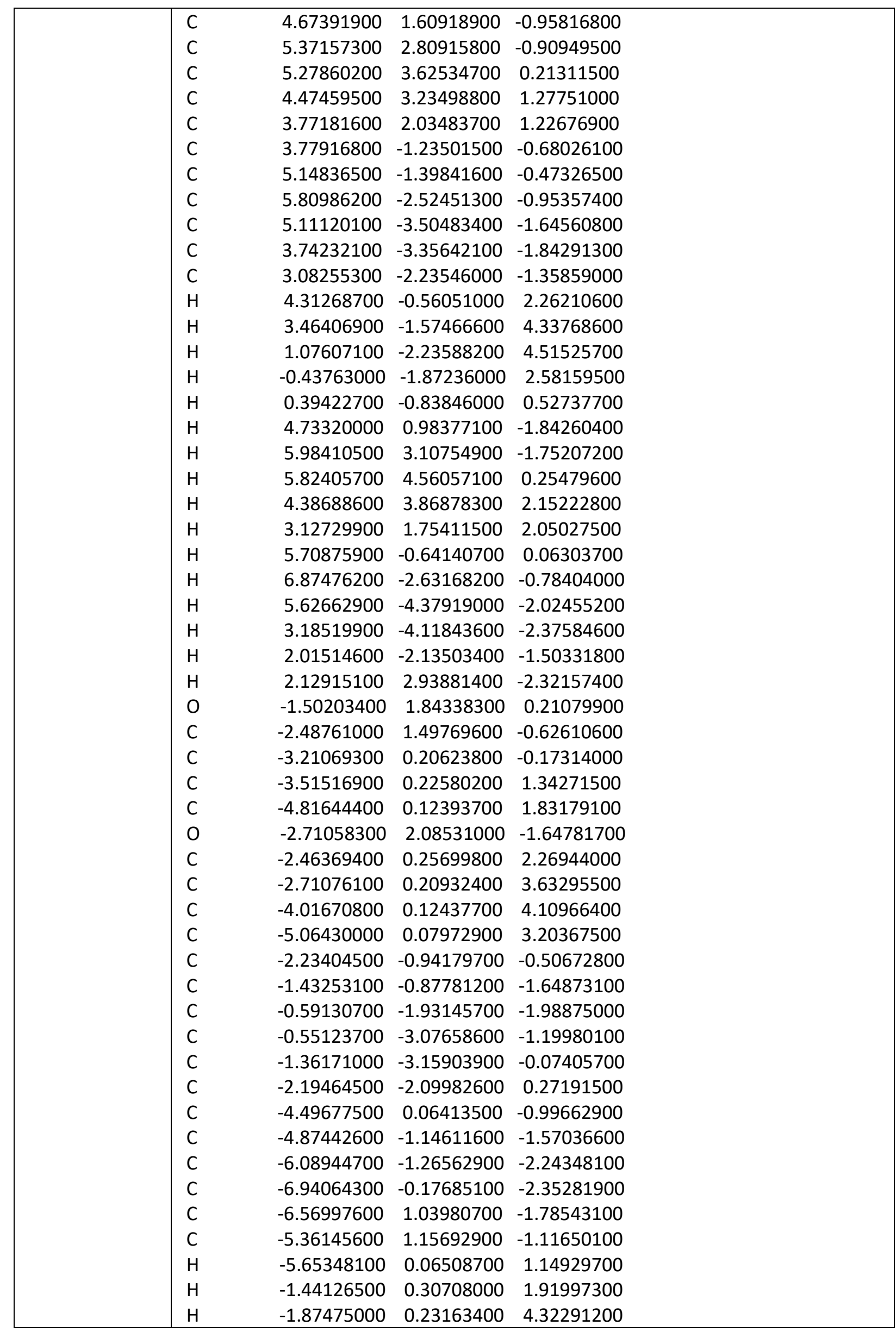




\begin{tabular}{|c|c|c|c|c|}
\hline & $\mathrm{H}$ & -4.21020300 & 0.08575800 & 5.17504100 \\
\hline & $\mathrm{H}$ & -6.08626000 & 0.00120900 & 3.55515100 \\
\hline & $\mathrm{H}$ & -1.47885400 & -0.01034000 & -2.29810400 \\
\hline & $\mathrm{H}$ & 0.03670200 & -1.84429800 & -2.86802200 \\
\hline & $\mathrm{H}$ & 0.10836900 & -3.89707900 & -1.45752900 \\
\hline & $\mathrm{H}$ & -1.34254000 & -4.04786400 & 0.54572900 \\
\hline & $\mathrm{H}$ & -2.82192000 & -2.17741900 & 1.15222600 \\
\hline & $\mathrm{H}$ & -4.22270500 & -2.00813600 & -1.50129300 \\
\hline & $\mathrm{H}$ & -6.36266800 & -2.21692400 & -2.68437300 \\
\hline & $\mathrm{H}$ & -7.88381300 & -0.26959500 & -2.87794300 \\
\hline & $\mathrm{H}$ & -7.22330400 & 1.90039700 & -1.86653600 \\
\hline & $\mathrm{H}$ & -5.08599500 & 2.10882600 & -0.67826800 \\
\hline & $\mathrm{H}$ & -1.19149200 & 3.66230700 & -0.73730400 \\
\hline \multirow[t]{39}{*}{42} & 0 & -1.42196000 & -1.44259300 & -1.15381200 \\
\hline & $\mathrm{C}$ & -2.43380800 & -0.64785900 & -1.52915700 \\
\hline & $\mathrm{C}$ & -0.62824000 & -2.04753300 & -2.19111300 \\
\hline & $\mathrm{H}$ & -1.27208800 & -2.24823100 & -3.04959500 \\
\hline & $\mathrm{C}$ & 0.43685300 & -1.05323600 & -2.61571100 \\
\hline & $\mathrm{H}$ & 0.98093100 & -1.42060800 & -3.49103900 \\
\hline & $\mathrm{C}$ & -0.06342000 & -3.32517800 & -1.59943000 \\
\hline & $\mathrm{H}$ & -0.87571300 & -4.00516100 & -1.34097600 \\
\hline & $\mathrm{H}$ & 0.51336500 & -3.09778400 & -0.70059000 \\
\hline & $\mathrm{O}$ & -2.70811600 & -0.44595800 & -2.67873100 \\
\hline & $\mathrm{C}$ & -3.11352100 & 0.02127300 & -0.30561800 \\
\hline & $\mathrm{C}$ & -3.64213000 & -1.03943100 & 0.68913900 \\
\hline & $\mathrm{C}$ & -4.95775900 & -1.00042600 & 1.15313200 \\
\hline & $\mathrm{C}$ & -5.41262300 & -1.91555300 & 2.10064200 \\
\hline & $\mathrm{C}$ & -4.56161100 & -2.88480400 & 2.60920300 \\
\hline & $\mathrm{C}$ & -3.24249400 & -2.92427900 & 2.16785200 \\
\hline & $\mathrm{C}$ & -2.78708600 & -2.01213800 & 1.22591200 \\
\hline & $\mathrm{C}$ & -4.25824600 & 0.91126900 & -0.80804200 \\
\hline & $\mathrm{C}$ & -5.21313000 & 0.38809500 & -1.68694400 \\
\hline & $\mathrm{C}$ & -6.30056600 & 1.14803800 & -2.08976400 \\
\hline & $\mathrm{C}$ & -6.46137500 & 2.44873700 & -1.61843800 \\
\hline & $\mathrm{C}$ & -5.52393200 & 2.97535300 & -0.74308300 \\
\hline & $\mathrm{C}$ & -4.42947300 & 2.21180700 & -0.34081900 \\
\hline & $\mathrm{C}$ & -2.01675800 & 0.88783300 & 0.34175300 \\
\hline & $\mathrm{C}$ & -1.17806600 & 1.63792300 & -0.48821400 \\
\hline & $\mathrm{C}$ & -0.21753000 & 2.48598600 & 0.04550800 \\
\hline & $\mathrm{C}$ & -0.08390900 & 2.60695500 & 1.42609500 \\
\hline & $\mathrm{C}$ & -0.92156900 & 1.87543600 & 2.25858500 \\
\hline & $\mathrm{C}$ & -1.88291500 & 1.01842200 & 1.72201000 \\
\hline & $\mathrm{H}$ & -5.64282200 & -0.24844200 & 0.78544400 \\
\hline & $\mathrm{H}$ & -6.44015000 & -1.85957800 & 2.44008900 \\
\hline & $\mathrm{H}$ & -4.91732300 & -3.59663200 & 3.34464300 \\
\hline & $\mathrm{H}$ & -2.55629900 & -3.66622500 & 2.56110300 \\
\hline & $\mathrm{H}$ & -1.75350600 & -2.04984100 & 0.91280700 \\
\hline & $\mathrm{H}$ & -5.10525300 & -0.62392300 & -2.05604400 \\
\hline & $\mathrm{H}$ & -7.02513300 & 0.72463600 & -2.77536300 \\
\hline & $\mathrm{H}$ & -7.31104000 & 3.04296100 & -1.93335100 \\
\hline & $\mathrm{H}$ & -5.63583100 & 3.98499100 & -0.36615600 \\
\hline & $\mathrm{H}$ & -3.71048100 & 2.64203900 & 0.34516100 \\
\hline
\end{tabular}




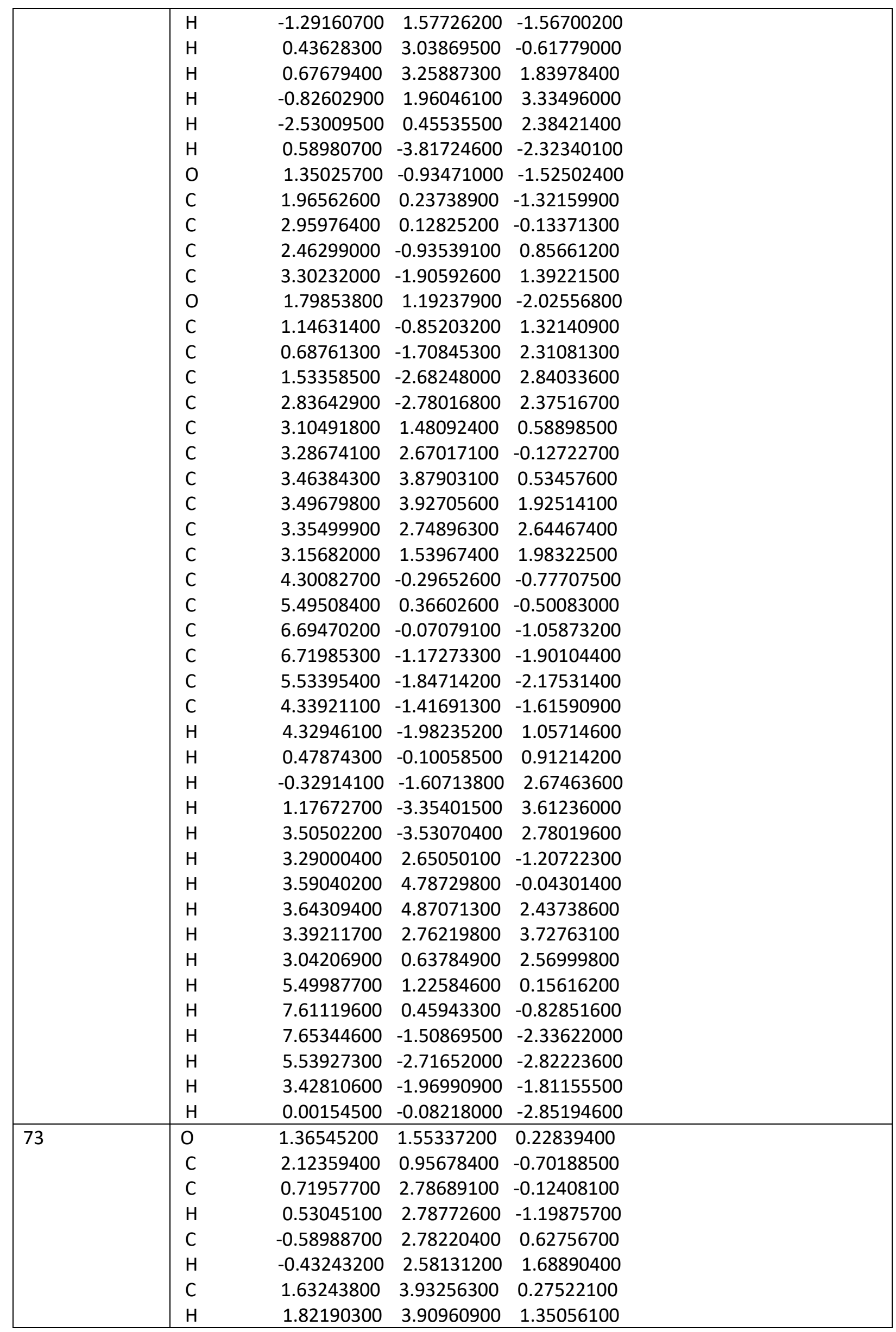




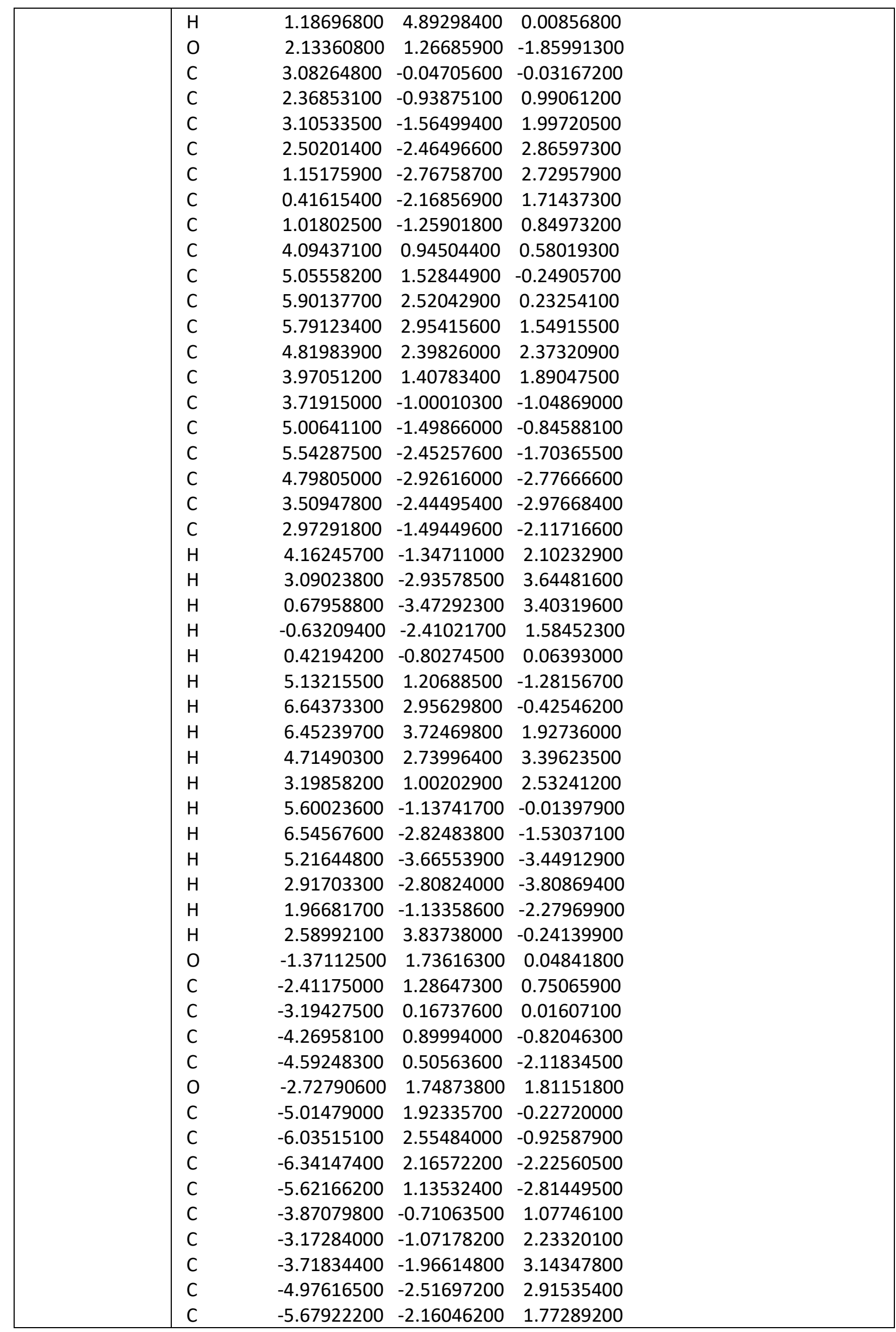




\begin{tabular}{|l|lrrr|}
\hline C & -5.13078000 & -1.26184500 & 0.86041600 \\
C & -2.26556700 & -0.68746600 & -0.87205100 \\
C & -2.12242800 & -2.05984500 & -0.67262800 \\
C & -1.26809900 & -2.81855200 & -1.47353600 \\
C & -0.55292400 & -2.22168800 & -2.49813900 \\
C & -0.70956700 & -0.85599600 & -2.73147600 \\
C & -1.55963700 & -0.10380700 & -1.93573900 \\
H & -4.04965900 & -0.30246900 & -2.59196900 \\
H & -4.80425500 & 2.21418900 & 0.79522500 \\
H & -6.59642900 & 3.34912700 & -0.44807800 \\
H & -7.13854300 & 2.65777900 & -2.77021600 \\
H & -5.85761800 & 0.81281100 & -3.82174000 \\
H & -2.19226000 & -0.65255600 & 2.42730100 \\
H & -3.16039700 & -2.22911600 & 4.03459400 \\
H & -5.40399800 & -3.21403700 & 3.62609900 \\
H & -6.66111300 & -2.57903100 & 1.58594300 \\
H & -5.69217200 & -0.99543100 & -0.02718800 \\
H & -2.67344500 & -2.55744800 & 0.11506600 \\
H & -1.16402000 & -3.87976900 & -1.28179500 \\
H & 0.11844300 & -2.81083100 & -3.11218600 \\
H & -0.16349100 & -0.36920200 & -3.53172800 \\
H & -1.68074600 & 0.95041100 & -2.14335900 \\
H & -1.11415500 & 3.73485800 & 0.51666600 \\
\hline
\end{tabular}

20 (optimized at the B3LYP/6-311G(d,p) level)

\begin{tabular}{|l|lrrr|}
\hline Conformer no & \multicolumn{4}{|c|}{} \\
\hline 1 & O & -1.38412400 & 0.01295600 & -1.37159700 \\
& C & -2.69773500 & -0.25877500 & -1.49227900 \\
& C & -0.59530100 & 0.03647500 & -2.59764200 \\
& H & -1.21864800 & 0.48297300 & -3.37643500 \\
C & 0.58845900 & 0.96954400 & -2.36101900 \\
& H & 1.15822000 & 1.02505000 & -3.29249800 \\
C & -0.20219300 & -1.37700000 & -3.00597300 \\
& H & -1.09376000 & -1.96378400 & -3.22751000 \\
H & 0.37143900 & -1.86231500 & -2.21611600 \\
C & 0.19037600 & 2.36998200 & -1.91727000 \\
& H & 1.07559900 & 2.99762800 & -1.81734500 \\
O & -3.22697200 & -0.51870000 & -2.54341600 \\
& C & -3.45405400 & -0.07016000 & -0.14561800 \\
C & -2.76045900 & -0.74424900 & 1.06104100 \\
& C & -3.21213400 & -0.44857300 & 2.35532200 \\
C & -2.68636100 & -1.09397000 & 3.46911800 \\
& C & -1.70041700 & -2.06744200 & 3.31652400 \\
& C & -1.25651600 & -2.38661100 & 2.03810900 \\
& C & -1.78227500 & -1.73300100 & 0.92319300 \\
& C & -4.85923900 & -0.71457100 & -0.22469000 \\
& C & -5.02075400 & -1.99200700 & -0.77482100 \\
& C & -6.25617500 & -2.62996900 & -0.75499700 \\
& C & -7.36067300 & -2.01028700 & -0.17385600 \\
C & -7.21153100 & -0.74737500 & 0.38860700 \\
& C & -5.97287700 & -0.10782600 & 0.36368700 \\
\hline
\end{tabular}




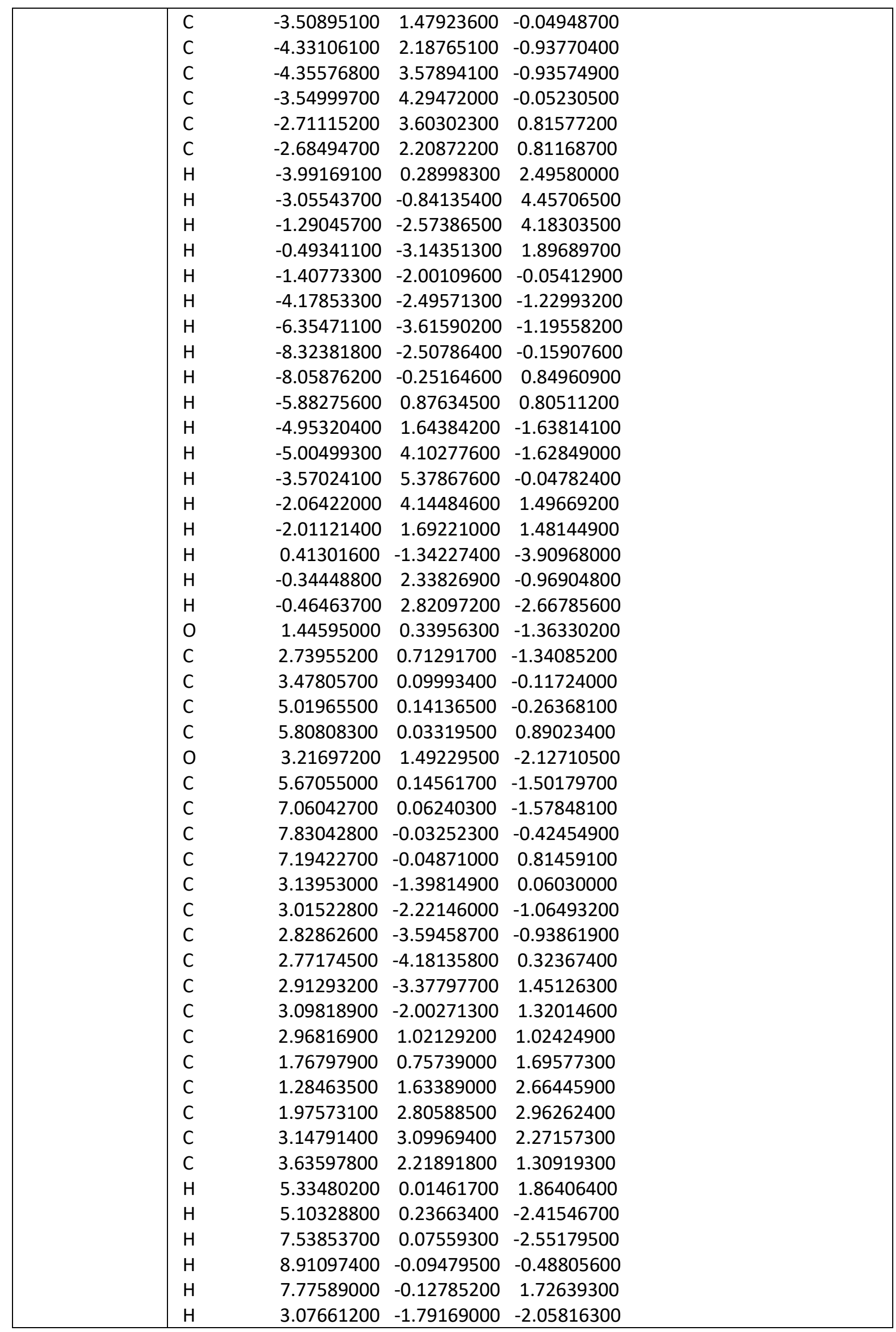




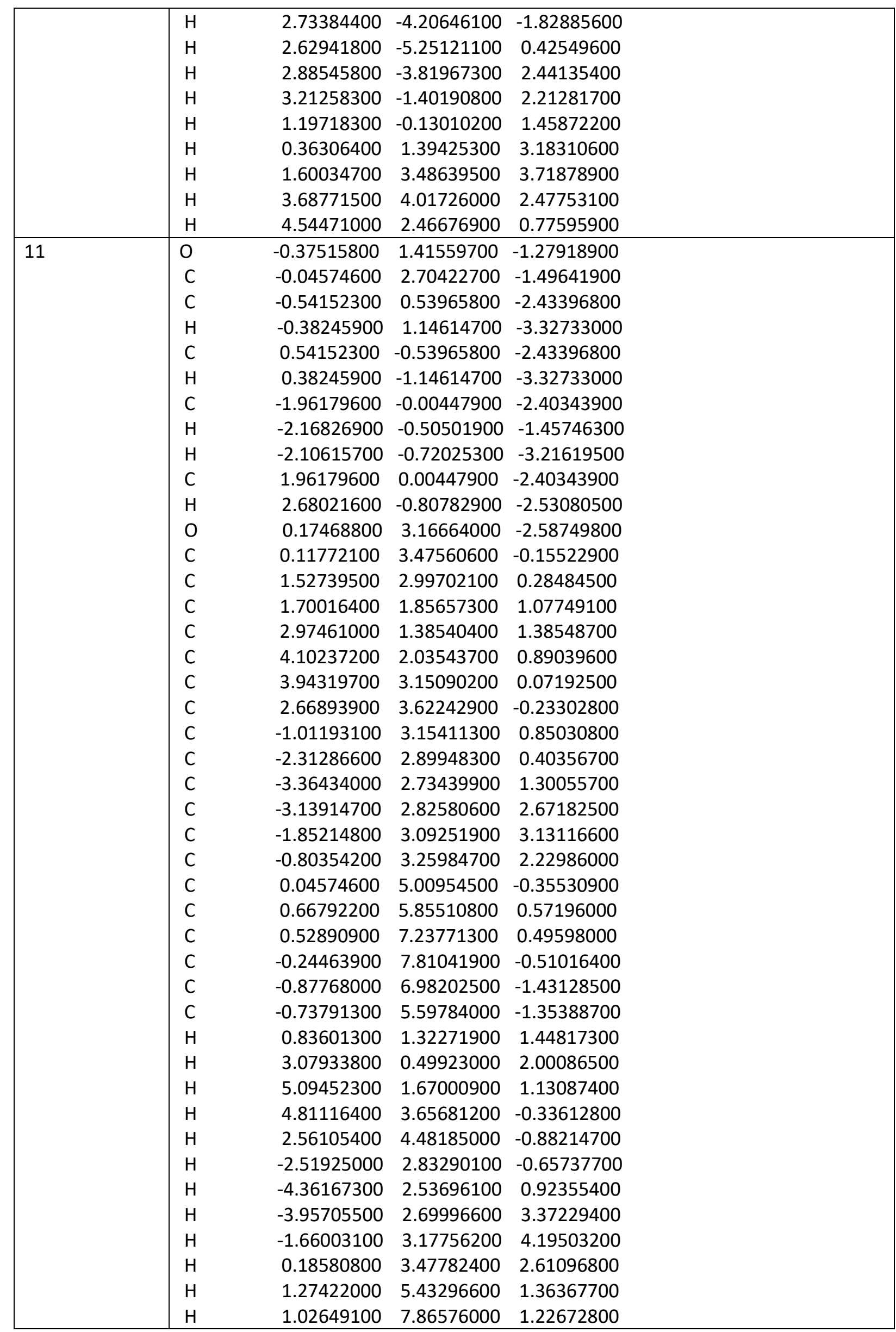




\begin{tabular}{|c|c|c|c|c|}
\hline & $\mathrm{H}$ & -0.35320100 & 8.88727100 & -0.57385400 \\
\hline & $\mathrm{H}$ & -1.48504300 & 7.41043300 & -2.22096400 \\
\hline & $\mathrm{H}$ & -1.23153900 & 4.98338400 & -2.09223100 \\
\hline & $\mathrm{H}$ & -2.68021600 & 0.80782900 & -2.53080500 \\
\hline & $\mathrm{H}$ & 2.16826900 & 0.50501900 & -1.45746300 \\
\hline & $\mathrm{H}$ & 2.10615700 & 0.72025300 & -3.21619500 \\
\hline & 0 & 0.37515800 & -1.41559700 & -1.27918900 \\
\hline & $\mathrm{C}$ & 0.04574600 & -2.70422700 & -1.49641900 \\
\hline & $\mathrm{C}$ & -0.11772100 & -3.47560600 & -0.15522900 \\
\hline & $\mathrm{C}$ & -1.52739500 & -2.99702100 & 0.28484500 \\
\hline & $\mathrm{C}$ & -1.70016400 & -1.85657300 & 1.07749100 \\
\hline & 0 & -0.17468800 & -3.16664000 & -2.58749800 \\
\hline & $\mathrm{C}$ & -2.66893900 & -3.62242900 & -0.23302800 \\
\hline & $\mathrm{C}$ & -3.94319700 & -3.15090200 & 0.07192500 \\
\hline & $\mathrm{C}$ & -4.10237200 & -2.03543700 & 0.89039600 \\
\hline & $C$ & -2.97461000 & -1.38540400 & 1.38548700 \\
\hline & $\mathrm{C}$ & 1.01193100 & -3.15411300 & 0.85030800 \\
\hline & $\mathrm{C}$ & 2.31286600 & -2.89948300 & 0.40356700 \\
\hline & $\mathrm{C}$ & 3.36434000 & -2.73439900 & 1.30055700 \\
\hline & $\mathrm{C}$ & 3.13914700 & -2.82580600 & 2.67182500 \\
\hline & $\mathrm{C}$ & 1.85214800 & -3.09251900 & 3.13116600 \\
\hline & $C$ & 0.80354200 & -3.25984700 & 2.22986000 \\
\hline & $\mathrm{C}$ & -0.04574600 & -5.00954500 & -0.35530900 \\
\hline & $\mathrm{C}$ & 0.73791300 & -5.59784000 & -1.35388700 \\
\hline & $\mathrm{C}$ & 0.87768000 & -6.98202500 & -1.43128500 \\
\hline & $\mathrm{C}$ & 0.24463900 & -7.81041900 & -0.51016400 \\
\hline & $\mathrm{C}$ & -0.52890900 & -7.23771300 & 0.49598000 \\
\hline & $\mathrm{C}$ & -0.66792200 & -5.85510800 & 0.57196000 \\
\hline & $\mathrm{H}$ & -0.83601300 & -1.32271900 & 1.44817300 \\
\hline & $\mathrm{H}$ & -2.56105400 & -4.48185000 & -0.88214700 \\
\hline & $\mathrm{H}$ & -4.81116400 & -3.65681200 & -0.33612800 \\
\hline & $\mathrm{H}$ & -5.09452300 & -1.67000900 & 1.13087400 \\
\hline & $\mathrm{H}$ & -3.07933800 & -0.49923000 & 2.00086500 \\
\hline & $\mathrm{H}$ & 2.51925000 & -2.83290100 & -0.65737700 \\
\hline & $\mathrm{H}$ & 4.36167300 & -2.53696100 & 0.92355400 \\
\hline & $\mathrm{H}$ & 3.95705500 & -2.69996600 & 3.37229400 \\
\hline & $\mathrm{H}$ & 1.66003100 & -3.17756200 & 4.19503200 \\
\hline & $\mathrm{H}$ & -0.18580800 & -3.47782400 & 2.61096800 \\
\hline & $\mathrm{H}$ & 1.23153900 & -4.98338400 & -2.09223100 \\
\hline & $\mathrm{H}$ & 1.48504300 & -7.41043300 & -2.22096400 \\
\hline & $\mathrm{H}$ & 0.35320100 & -8.88727100 & -0.57385400 \\
\hline & $\mathrm{H}$ & -1.02649100 & -7.86576000 & 1.22672800 \\
\hline & $\mathrm{H}$ & -1.27422000 & -5.43296600 & 1.36367700 \\
\hline 14 & 0 & 1.36749800 & 0.64157800 & -1.07900700 \\
\hline & $\mathrm{C}$ & 2.60265600 & 0.20676800 & -1.39317200 \\
\hline & $\mathrm{C}$ & 0.54885000 & 1.22454500 & -2.13699100 \\
\hline & $\mathrm{H}$ & 1.19043100 & 1.36161600 & -3.00965500 \\
\hline & $\mathrm{C}$ & -0.56508500 & 0.26177600 & -2.55231700 \\
\hline & $\mathrm{H}$ & -1.15660800 & 0.77079100 & -3.31557700 \\
\hline & $\mathrm{C}$ & 0.04439000 & 2.56389600 & -1.62212400 \\
\hline & $\mathrm{H}$ & 0.88628700 & 3.22882400 & -1.42249400 \\
\hline & $\mathrm{H}$ & -0.52607300 & 2.43822500 & -0.70158500 \\
\hline
\end{tabular}




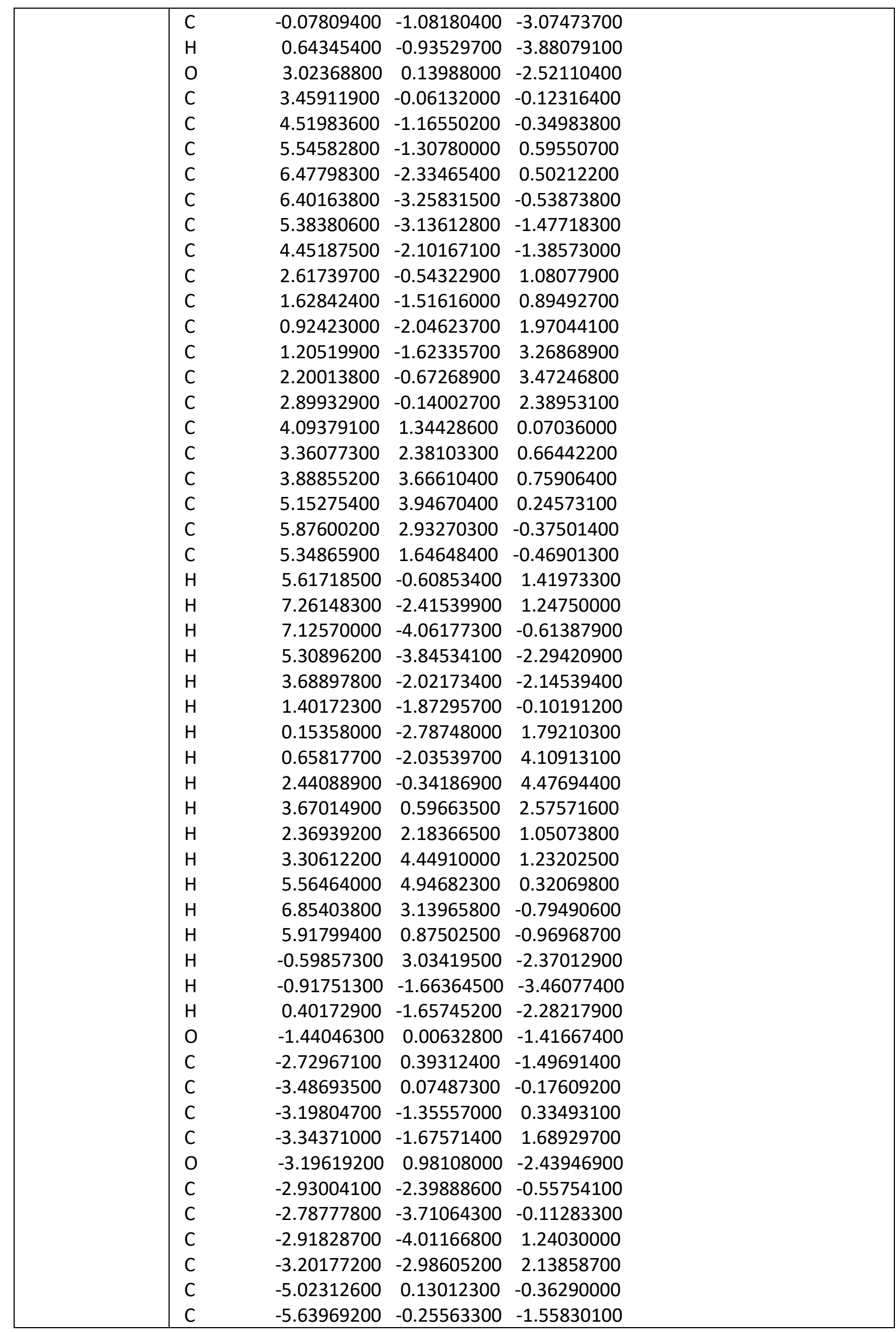




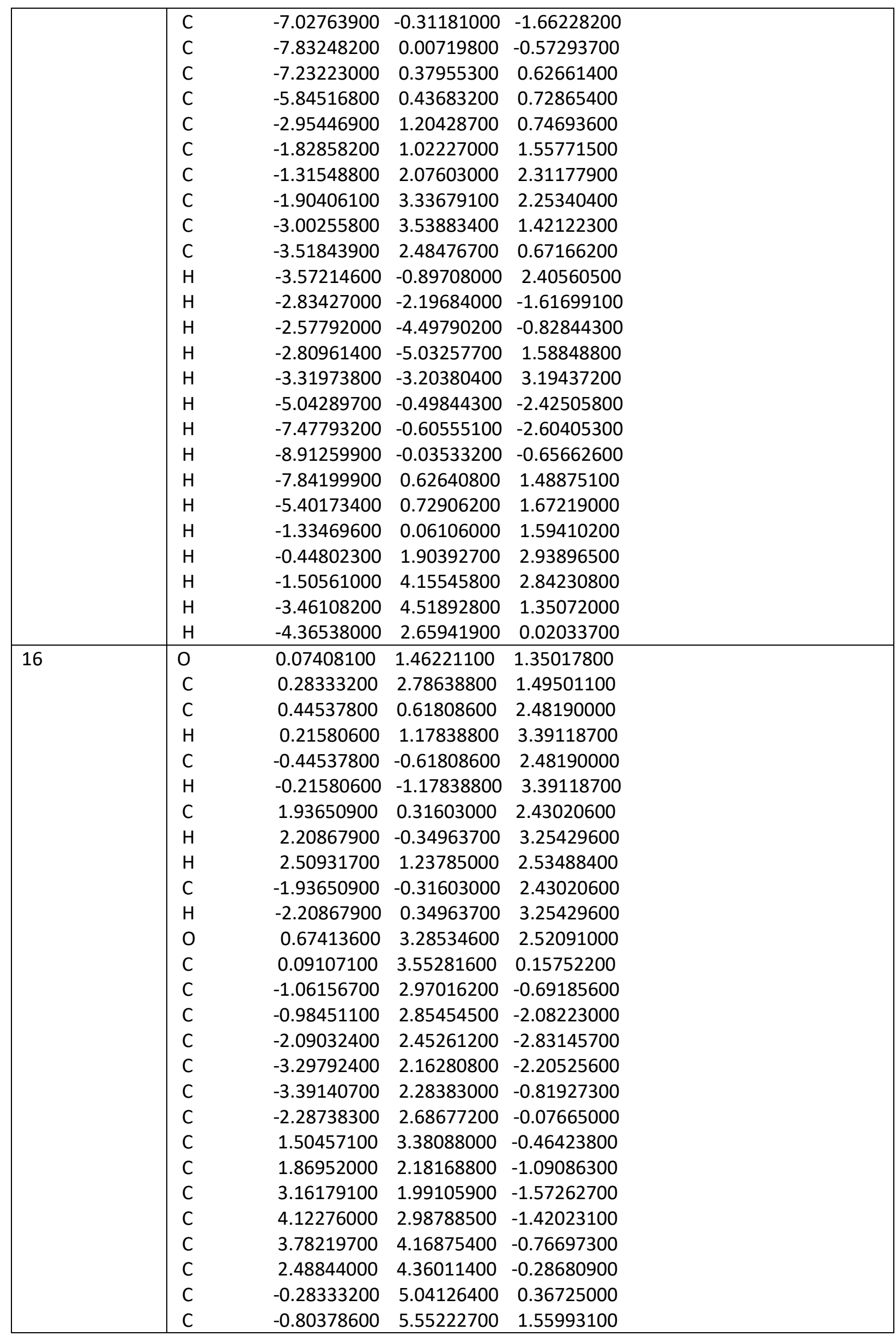




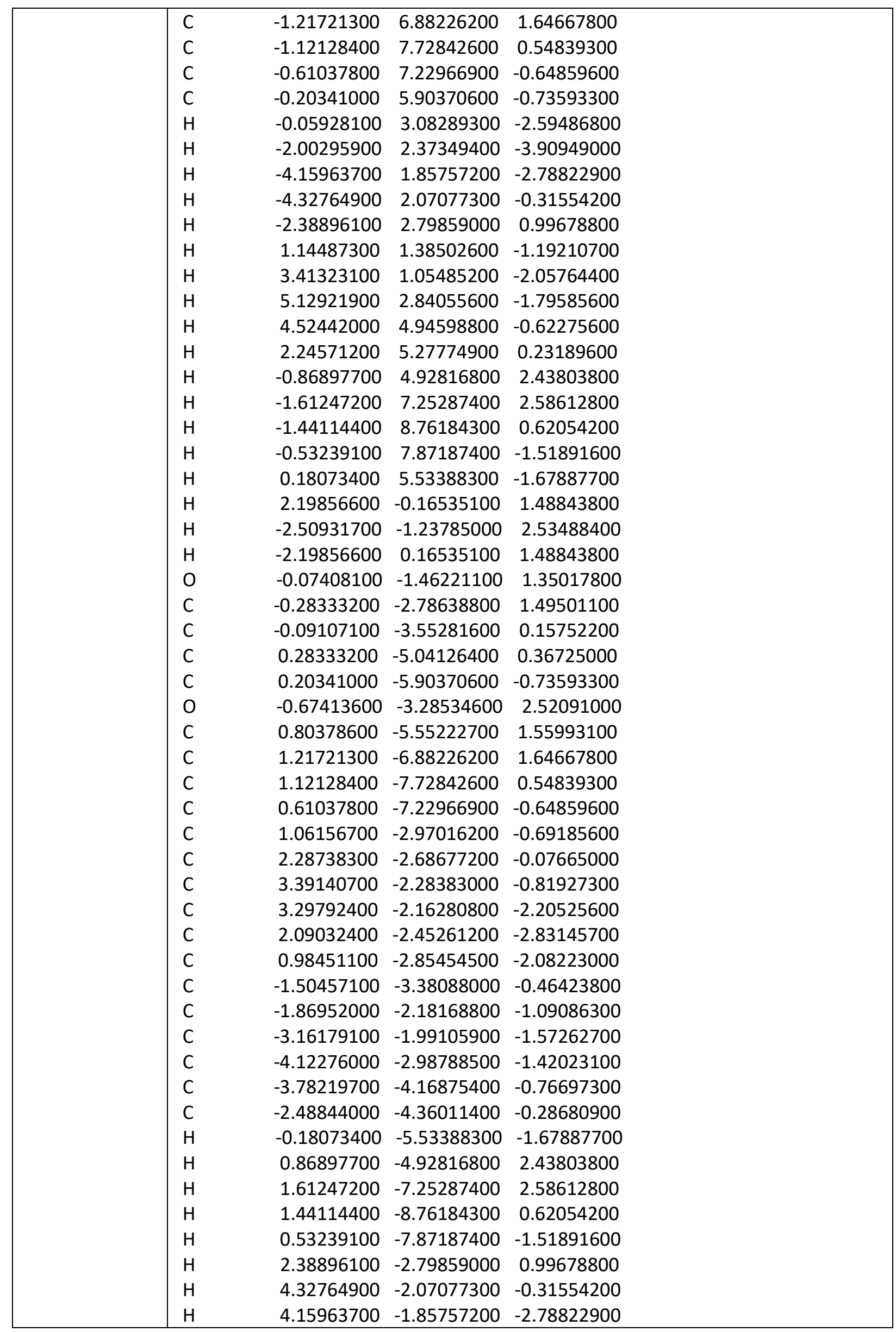




\begin{tabular}{|c|c|c|c|c|}
\hline & $\mathrm{H}$ & 2.00295900 & -2.37349400 & -3.90949000 \\
\hline & $\mathrm{H}$ & 0.05928100 & -3.08289300 & -2.59486800 \\
\hline & $\mathrm{H}$ & -1.14487300 & -1.38502600 & -1.19210700 \\
\hline & $\mathrm{H}$ & -3.41323100 & -1.05485200 & -2.05764400 \\
\hline & $\mathrm{H}$ & -5.12921900 & -2.84055600 & -1.79585600 \\
\hline & $\mathrm{H}$ & -4.52442000 & -4.94598800 & -0.62275600 \\
\hline & $\mathrm{H}$ & -2.24571200 & -5.27774900 & 0.23189600 \\
\hline \multirow[t]{45}{*}{17} & 0 & 1.41002400 & -0.27131900 & 1.42051500 \\
\hline & C & 2.69100800 & 0.13423100 & 1.54760900 \\
\hline & C & 0.54418800 & -0.20977800 & 2.59338300 \\
\hline & $\mathrm{H}$ & 1.15831500 & 0.11397200 & 3.43557300 \\
\hline & C & -0.53099700 & 0.86059200 & 2.39386700 \\
\hline & $\mathrm{H}$ & -1.16641100 & 0.83721400 & 3.28097300 \\
\hline & C & 0.00318700 & -1.61008800 & 2.84196600 \\
\hline & $\mathrm{H}$ & -0.52017800 & -1.99467300 & 1.96594700 \\
\hline & $\mathrm{H}$ & 0.82198500 & -2.29207000 & 3.07986300 \\
\hline & C & 0.01914400 & 2.26014400 & 2.16446300 \\
\hline & $\mathrm{H}$ & -0.79876200 & 2.98128500 & 2.12096200 \\
\hline & $\mathrm{O}$ & 3.15379600 & 0.57709900 & 2.56821100 \\
\hline & C & 3.44307400 & 0.05966200 & 0.18852500 \\
\hline & C & 3.12432800 & -1.23885800 & -0.58734600 \\
\hline & C & 3.14695400 & -1.27834000 & -1.98521200 \\
\hline & C & 2.99923000 & -2.48122100 & -2.67298500 \\
\hline & C & 2.83272100 & -3.67465200 & -1.97633800 \\
\hline & C & 2.81964600 & -3.65164500 & -0.58331500 \\
\hline & C & 2.96694500 & -2.44818000 & 0.09941100 \\
\hline & C & 4.98152200 & 0.04146200 & 0.36974200 \\
\hline & C & 5.60462300 & -0.47844600 & 1.50900600 \\
\hline & C & 6.99287300 & -0.58452400 & 1.57942700 \\
\hline & C & 7.78941700 & -0.18030700 & 0.51347400 \\
\hline & C & 7.18138400 & 0.32870800 & -0.63152500 \\
\hline & C & 5.79610800 & 0.43332400 & -0.70104000 \\
\hline & C & 2.94059600 & 1.36254000 & -0.49084500 \\
\hline & C & 3.59000900 & 2.57765300 & -0.23882100 \\
\hline & C & 3.09778900 & 3.77260700 & -0.75777500 \\
\hline & C & 1.93582700 & 3.77982300 & -1.52515200 \\
\hline & C & 1.26245800 & 2.58295000 & -1.75584100 \\
\hline & C & 1.75621000 & 1.38806100 & -1.23719600 \\
\hline & $\mathrm{H}$ & 3.28595700 & -0.36503000 & -2.54903800 \\
\hline & $\mathrm{H}$ & 3.01969200 & -2.48147300 & -3.75716600 \\
\hline & $\mathrm{H}$ & 2.72131100 & -4.61144400 & -2.51052900 \\
\hline & $\mathrm{H}$ & 2.69769300 & -4.57262300 & -0.02418700 \\
\hline & $\mathrm{H}$ & 2.96979000 & -2.45993700 & 1.18271700 \\
\hline & $\mathrm{H}$ & 5.01593100 & -0.78674800 & 2.35994200 \\
\hline & $\mathrm{H}$ & 7.44878600 & -0.98455400 & 2.47838500 \\
\hline & $\mathrm{H}$ & 8.86895400 & -0.26182200 & 0.57190600 \\
\hline & $\mathrm{H}$ & 7.78440700 & 0.64406000 & -1.47586800 \\
\hline & $\mathrm{H}$ & 5.34544300 & 0.82793900 & -1.60318600 \\
\hline & $\mathrm{H}$ & 4.48424300 & 2.59093600 & 0.37064700 \\
\hline & $\mathrm{H}$ & 3.62306800 & 4.69909900 & -0.55396600 \\
\hline & $\mathrm{H}$ & 1.55147900 & 4.70999300 & -1.92842600 \\
\hline & $\mathrm{H}$ & 0.33946100 & 2.57493200 & -2.32404800 \\
\hline
\end{tabular}




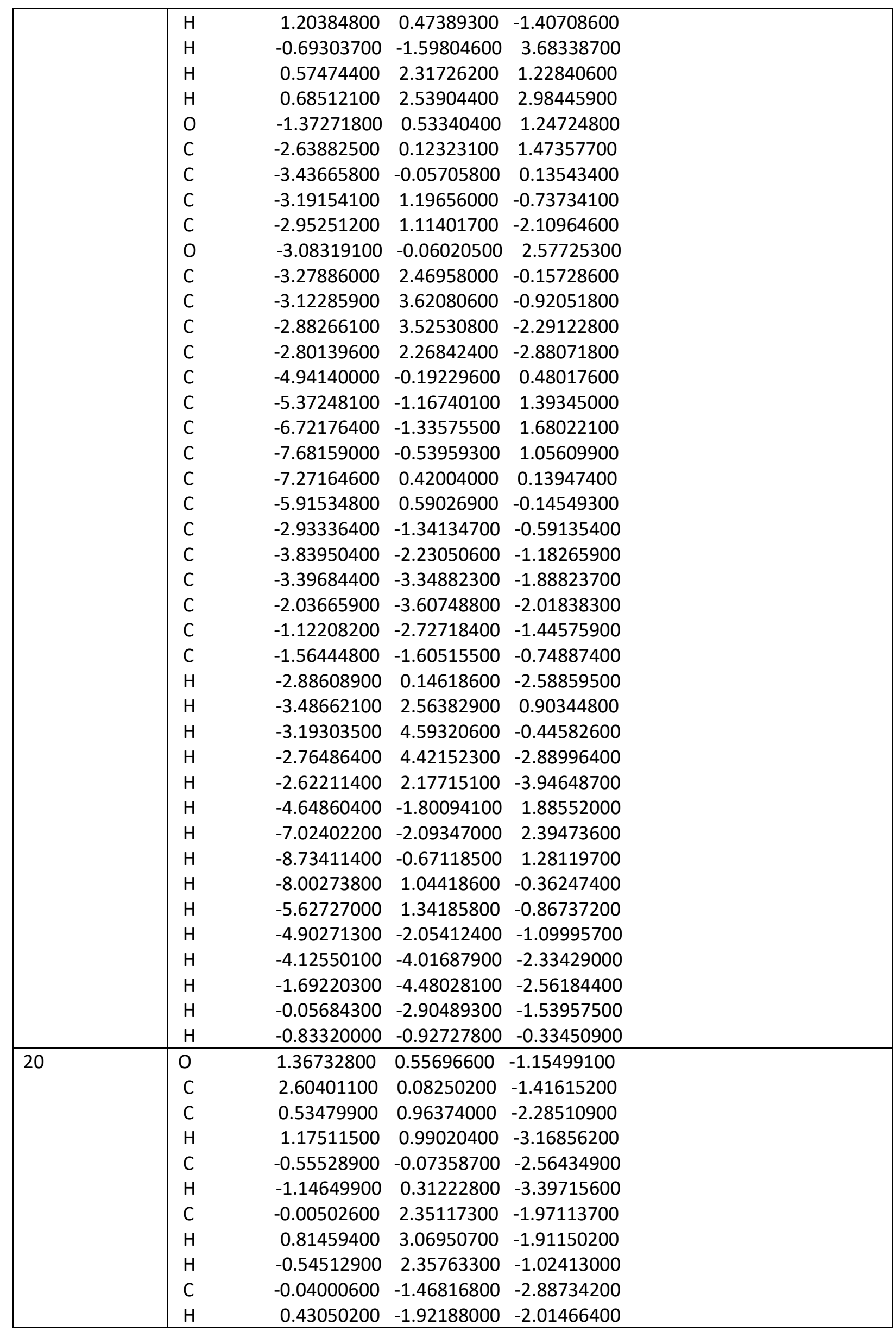




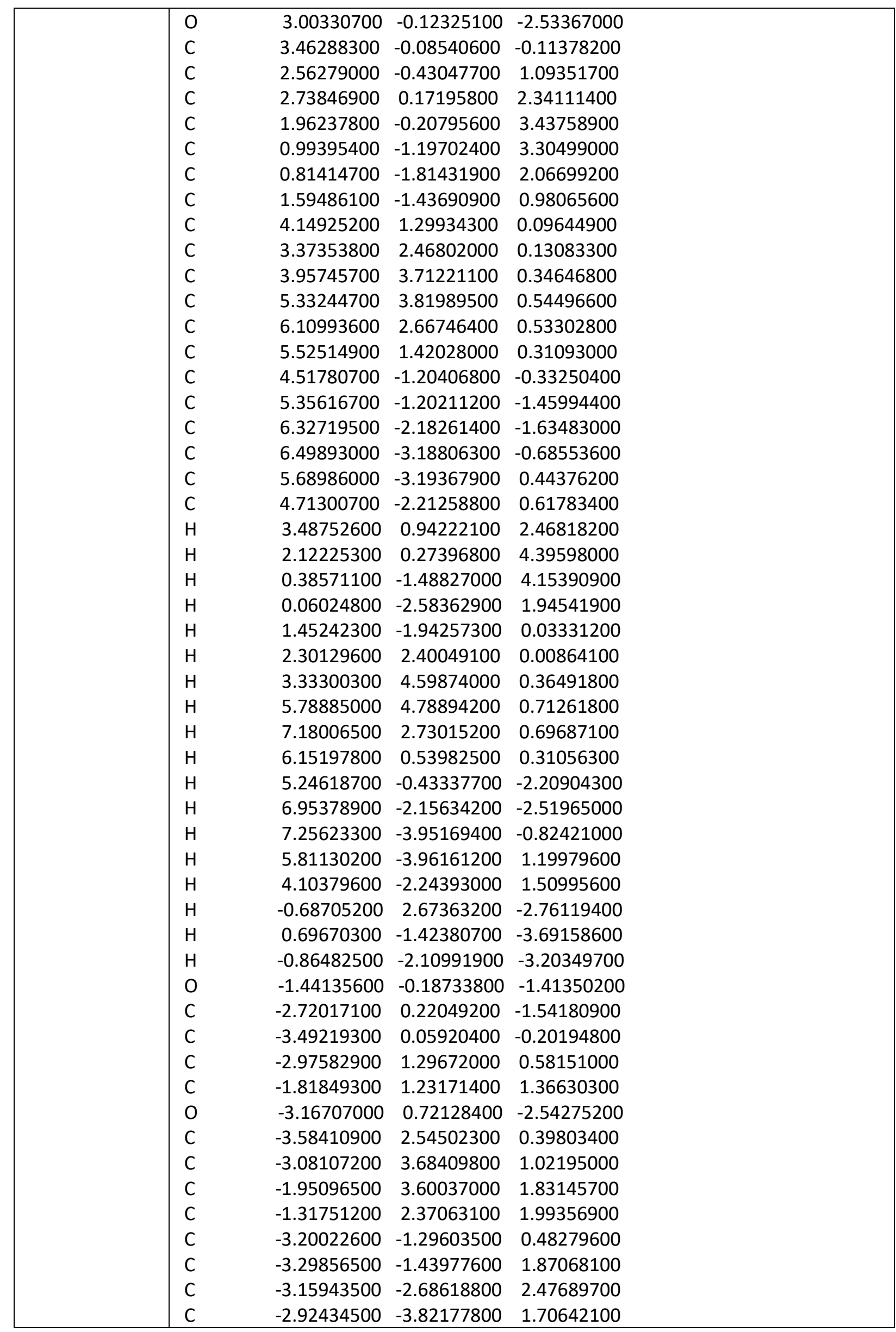




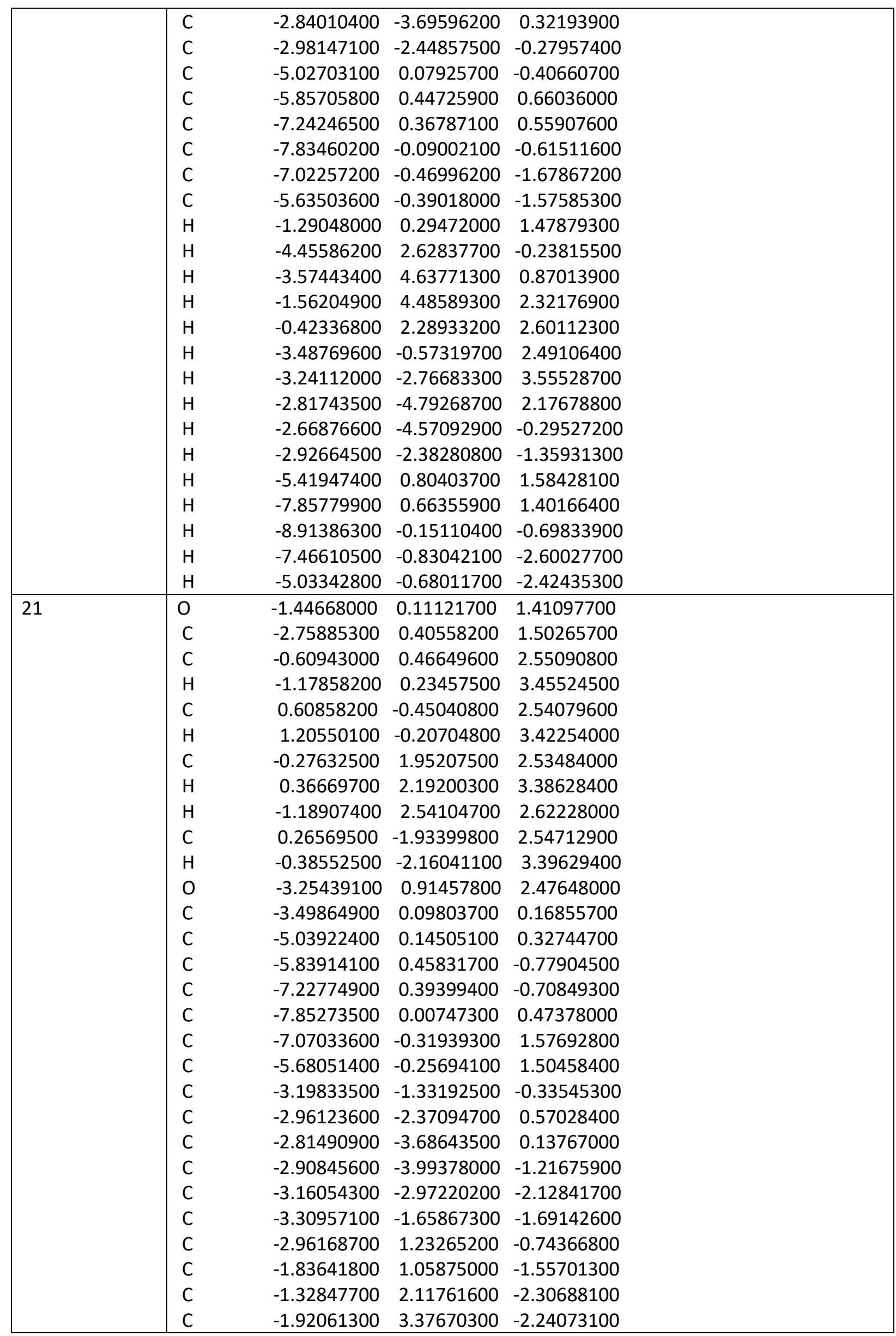




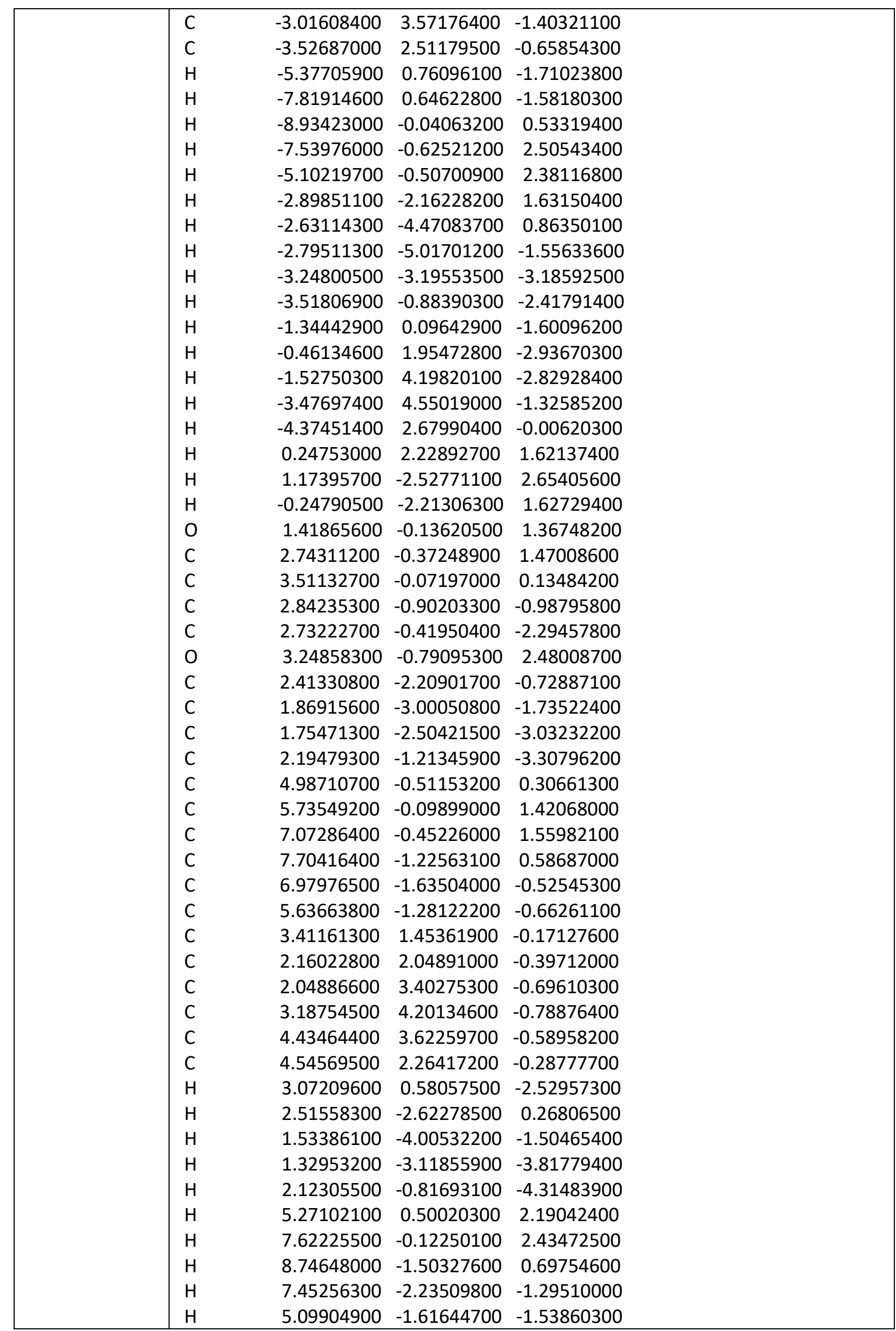




\begin{tabular}{|c|c|c|c|c|}
\hline & $\mathrm{H}$ & 1.26558800 & 1.44519900 & -0.34882200 \\
\hline & $\mathrm{H}$ & 1.06558400 & 3.82842900 & -0.86255500 \\
\hline & $\mathrm{H}$ & 3.10125600 & 5.25687300 & -1.02139900 \\
\hline & $\mathrm{H}$ & 5.33460800 & 4.22215300 & -0.67087100 \\
\hline & $\mathrm{H}$ & 5.53114700 & 1.84305000 & -0.15121700 \\
\hline \multirow[t]{47}{*}{22} & 0 & -1.42073500 & -0.35226700 & -1.26455900 \\
\hline & C & -2.72939400 & -0.66481500 & -1.29818500 \\
\hline & C & -0.58825900 & -0.83685200 & -2.35793300 \\
\hline & $\mathrm{H}$ & -1.18875600 & -0.78979100 & -3.26985700 \\
\hline & C & 0.57217900 & 0.13992600 & -2.51881100 \\
\hline & $\mathrm{H}$ & 1.17985700 & -0.20422200 & -3.35884300 \\
\hline & C & -0.15977500 & -2.27342600 & -2.09628000 \\
\hline & $\mathrm{H}$ & -1.03600400 & -2.92068300 & -2.04974900 \\
\hline & $\mathrm{H}$ & 0.39837200 & -2.34858900 & -1.16351800 \\
\hline & C & 0.14404300 & 1.58213100 & -2.75292700 \\
\hline & $\mathrm{H}$ & 1.02107000 & 2.21110000 & -2.91097300 \\
\hline & $\mathrm{O}$ & -3.22569400 & -1.29326000 & -2.19855700 \\
\hline & C & -3.50954800 & -0.05457400 & -0.08462800 \\
\hline & C & -2.87984400 & -0.52459500 & 1.26187600 \\
\hline & C & -1.53495500 & -0.25972400 & 1.57054100 \\
\hline & $\mathrm{C}$ & -0.98707900 & -0.63993800 & 2.79174200 \\
\hline & C & -1.76406900 & -1.29859200 & 3.74267300 \\
\hline & C & -3.09792800 & -1.56134000 & 3.45670500 \\
\hline & C & -3.64925400 & -1.17411800 & 2.23473600 \\
\hline & C & -4.99188600 & -0.49172700 & -0.17118500 \\
\hline & C & -5.33562400 & -1.82968900 & -0.41572800 \\
\hline & C & -6.66441600 & -2.23918000 & -0.42765500 \\
\hline & $\mathrm{C}$ & -7.68763200 & -1.32346400 & -0.18777600 \\
\hline & C & -7.36220200 & 0.00315400 & 0.06795200 \\
\hline & C & -6.02839100 & 0.41267700 & 0.07587900 \\
\hline & C & -3.40792100 & 1.48414500 & -0.26154100 \\
\hline & $\mathrm{C}$ & -3.15906300 & 2.35115400 & 0.80389600 \\
\hline & $\mathrm{C}$ & -3.13593700 & 3.73328600 & 0.60896800 \\
\hline & $\mathrm{C}$ & -3.35891400 & 4.27190800 & -0.65379200 \\
\hline & $\mathrm{C}$ & -3.61631500 & 3.41589600 & -1.72379400 \\
\hline & $\mathrm{C}$ & -3.64516400 & 2.03951100 & -1.52661500 \\
\hline & $\mathrm{H}$ & -0.90569700 & 0.24900300 & 0.85688600 \\
\hline & $\mathrm{H}$ & 0.05026000 & -0.40286500 & 2.99965900 \\
\hline & $\mathrm{H}$ & -1.33587900 & -1.59397700 & 4.69416600 \\
\hline & $\mathrm{H}$ & -3.72468300 & -2.06510400 & 4.18437700 \\
\hline & $\mathrm{H}$ & -4.69336700 & -1.38177500 & 2.05162900 \\
\hline & $\mathrm{H}$ & -4.56195700 & -2.56093500 & -0.60205100 \\
\hline & $\mathrm{H}$ & -6.89978500 & -3.27880700 & -0.62711000 \\
\hline & $\mathrm{H}$ & -8.72373700 & -1.64295800 & -0.20016900 \\
\hline & $\mathrm{H}$ & -8.14356900 & 0.72982600 & 0.26133100 \\
\hline & $\mathrm{H}$ & -5.80320400 & 1.45093000 & 0.27895200 \\
\hline & $\mathrm{H}$ & -2.98614200 & 1.95370100 & 1.79493500 \\
\hline & $\mathrm{H}$ & -2.94526100 & 4.38661900 & 1.45333500 \\
\hline & $\mathrm{H}$ & -3.33795500 & 5.34541400 & -0.80398900 \\
\hline & $\mathrm{H}$ & -3.80080100 & 3.81986200 & -2.71307200 \\
\hline & $\mathrm{H}$ & -3.87144700 & 1.38760700 & -2.36350000 \\
\hline & $\mathrm{H}$ & 0.47902000 & -2.62431400 & -2.91134400 \\
\hline
\end{tabular}




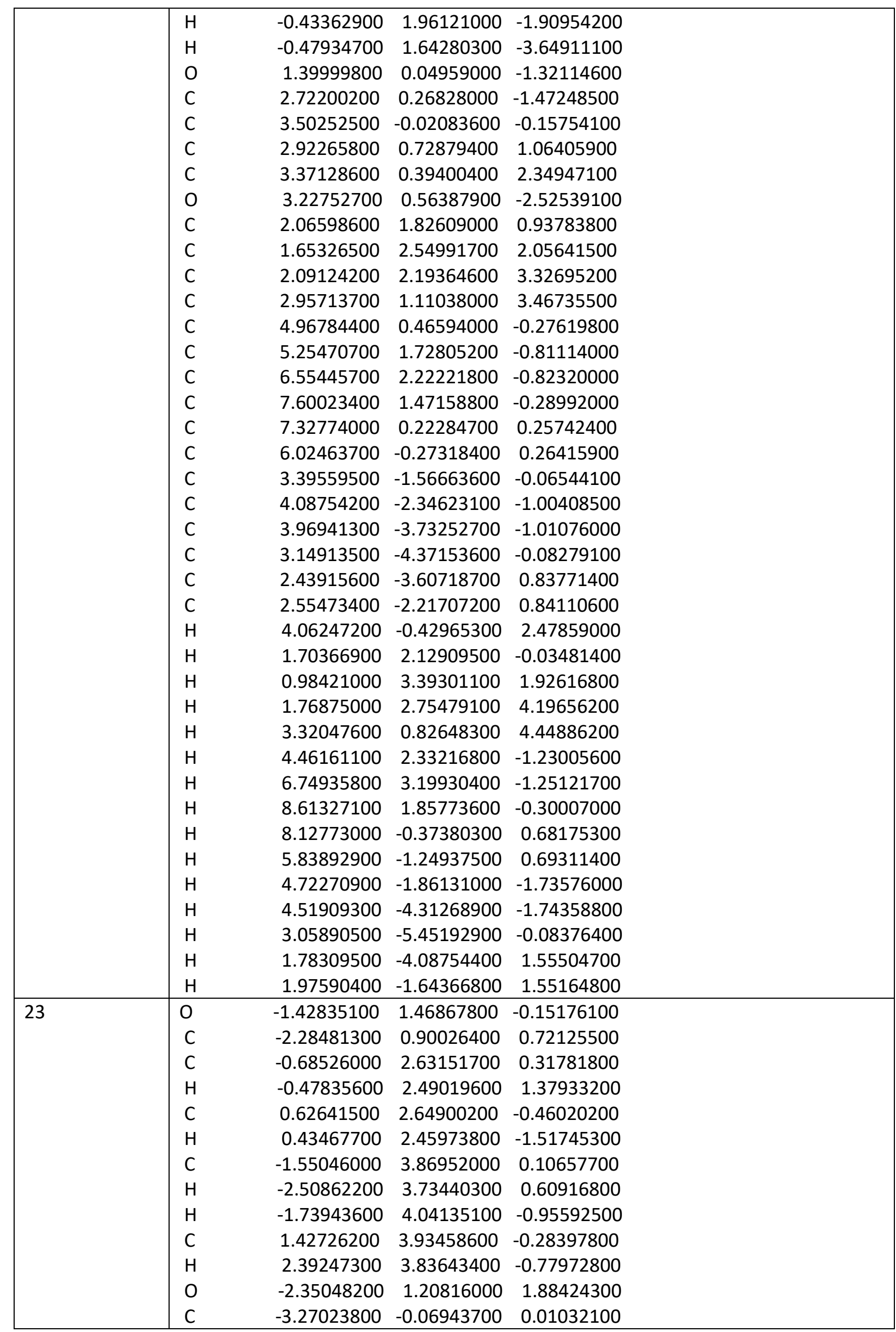




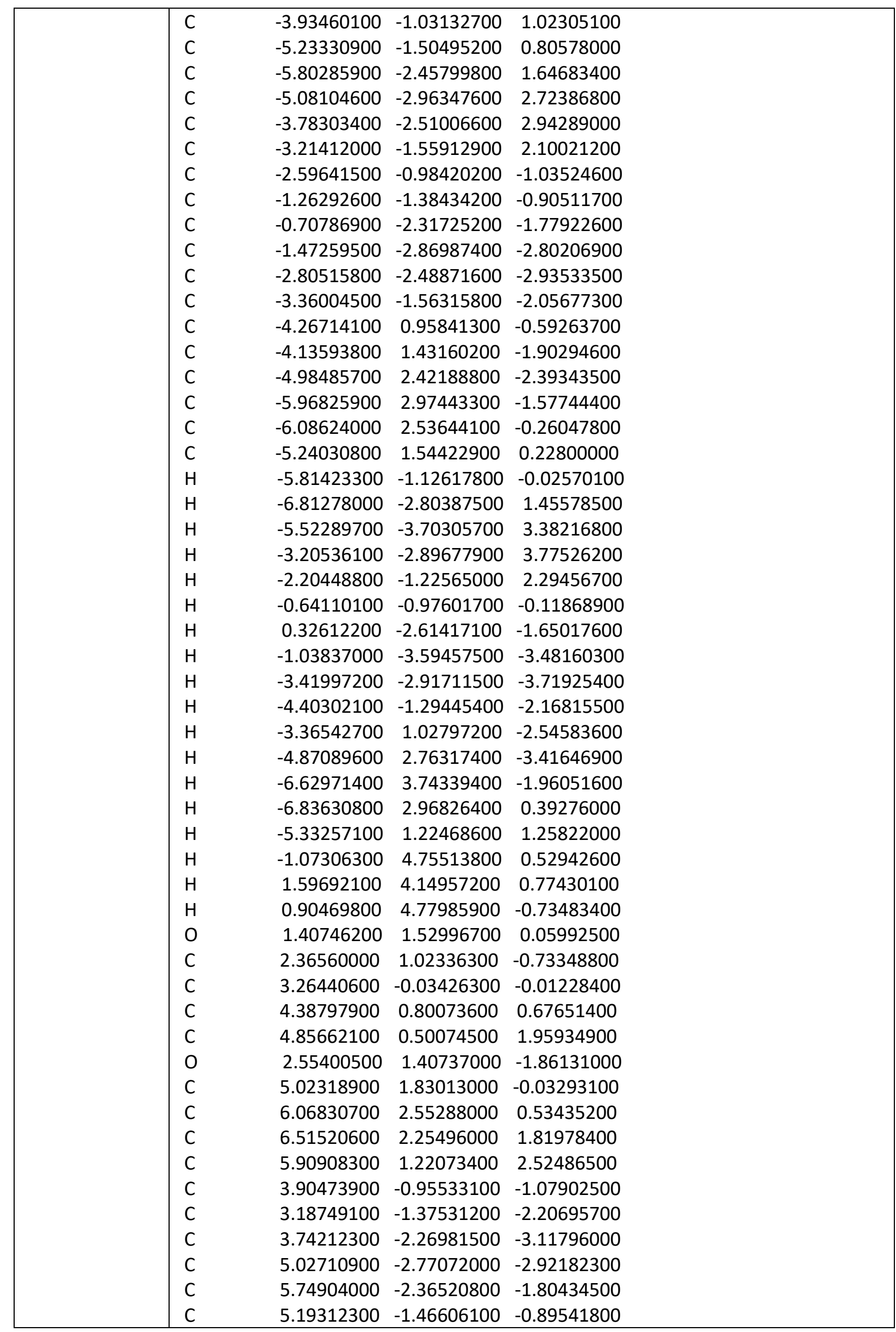




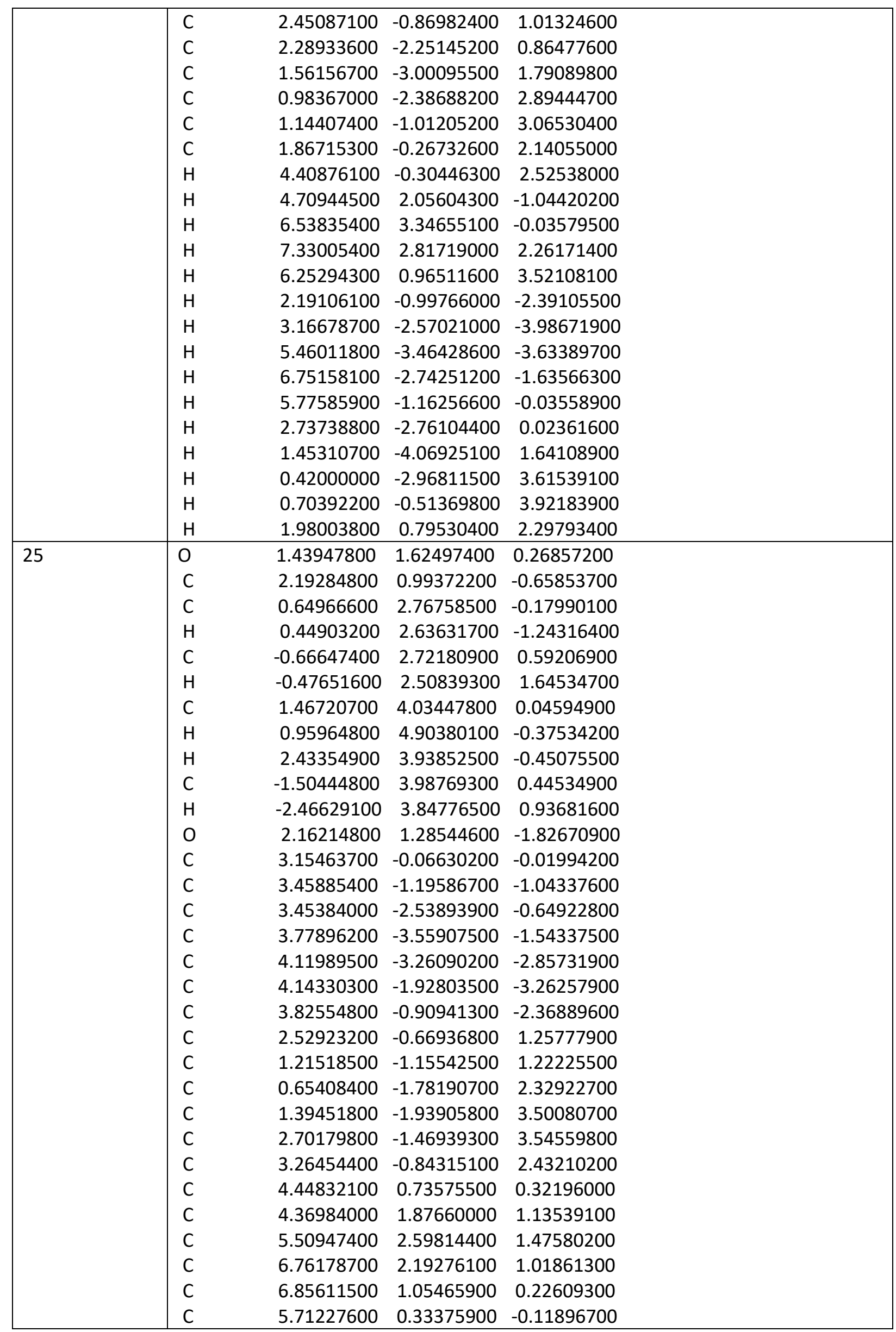




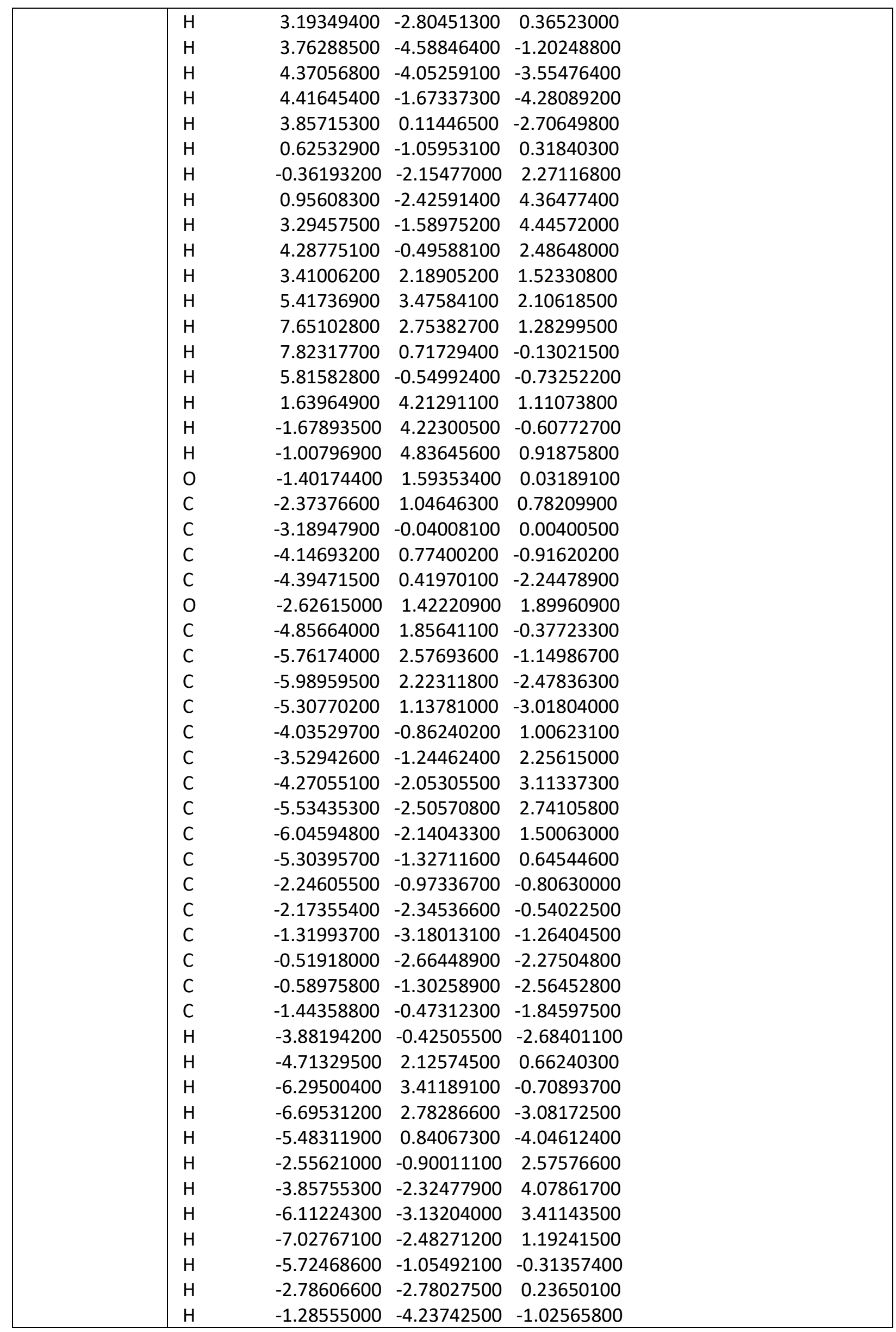




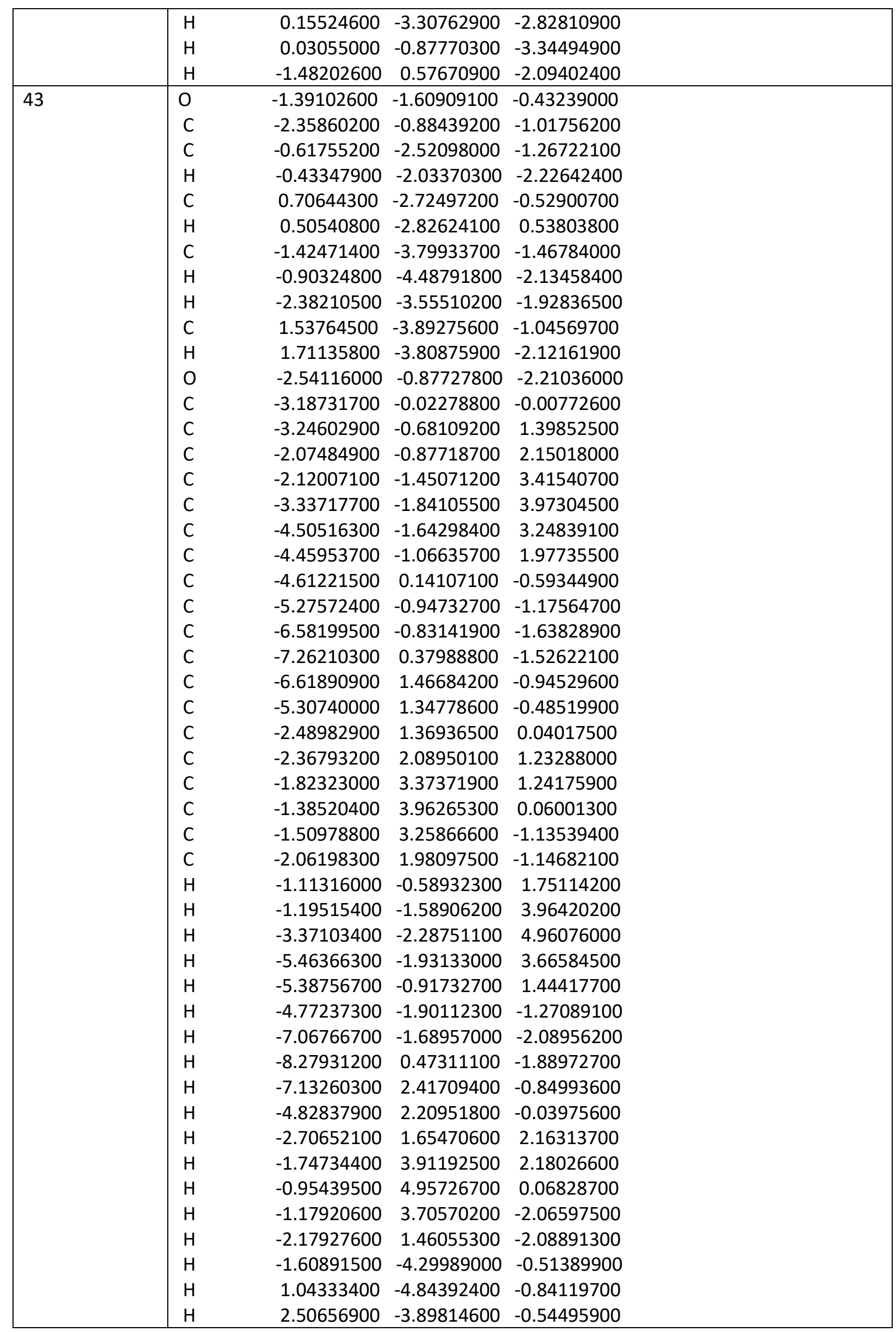




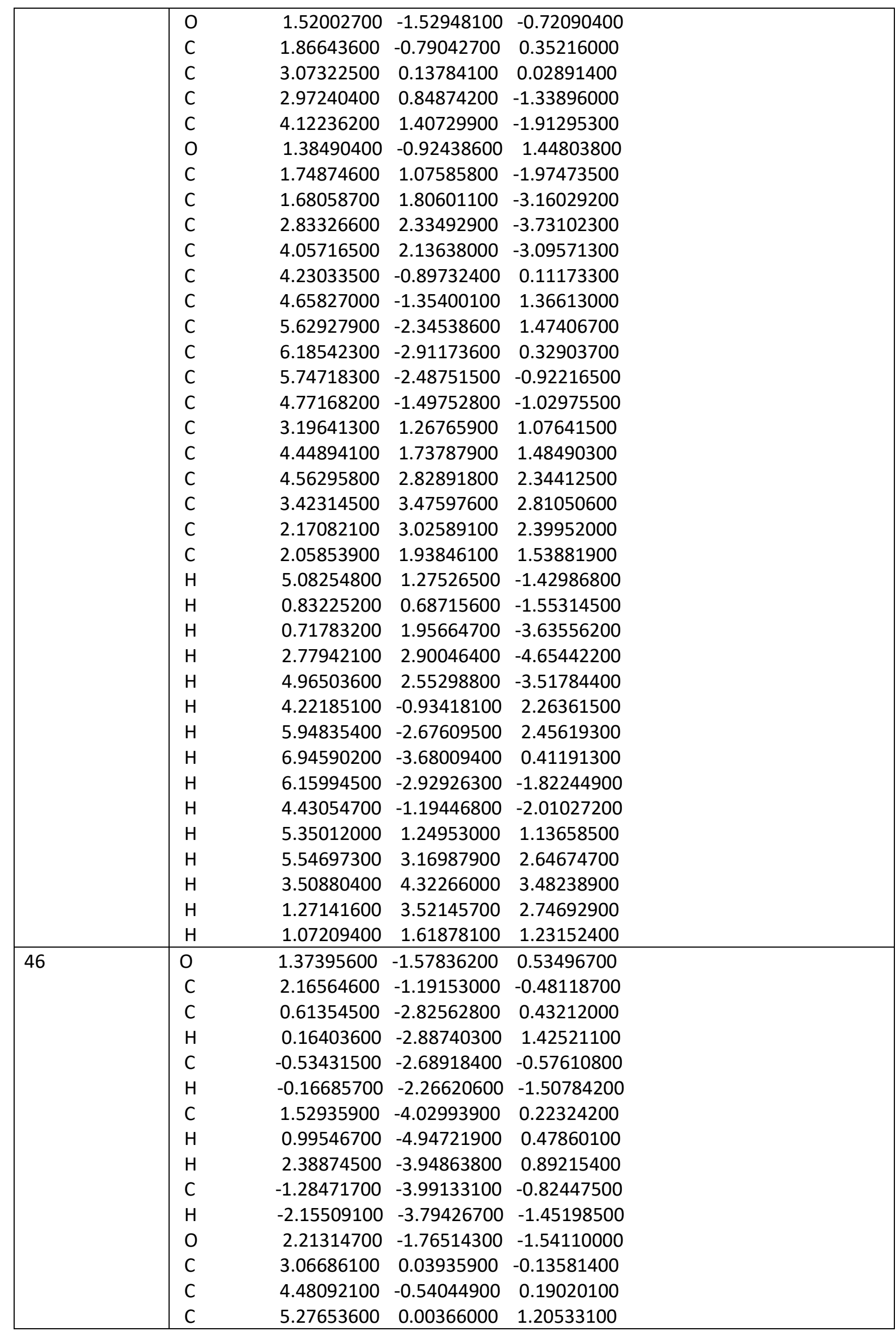




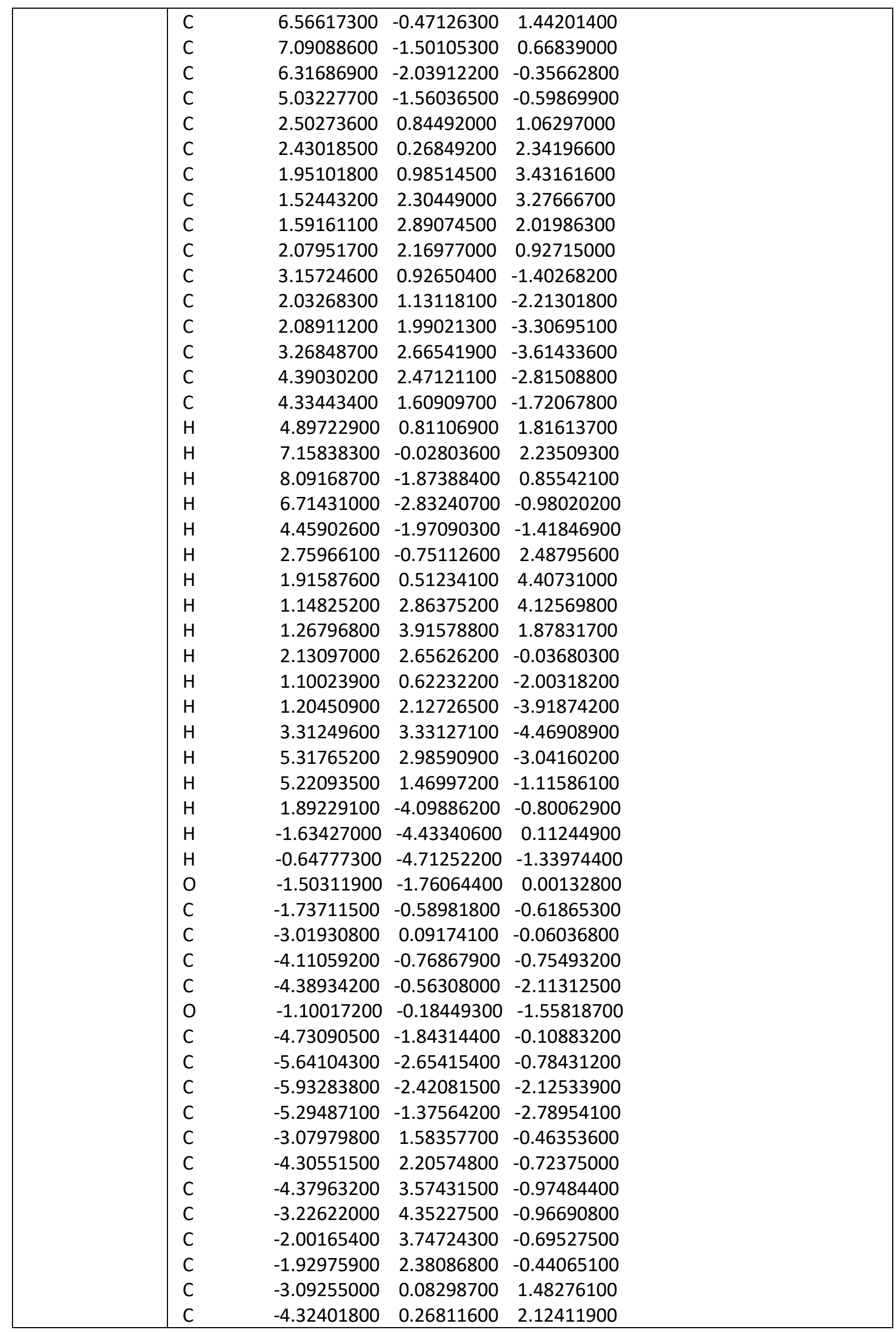




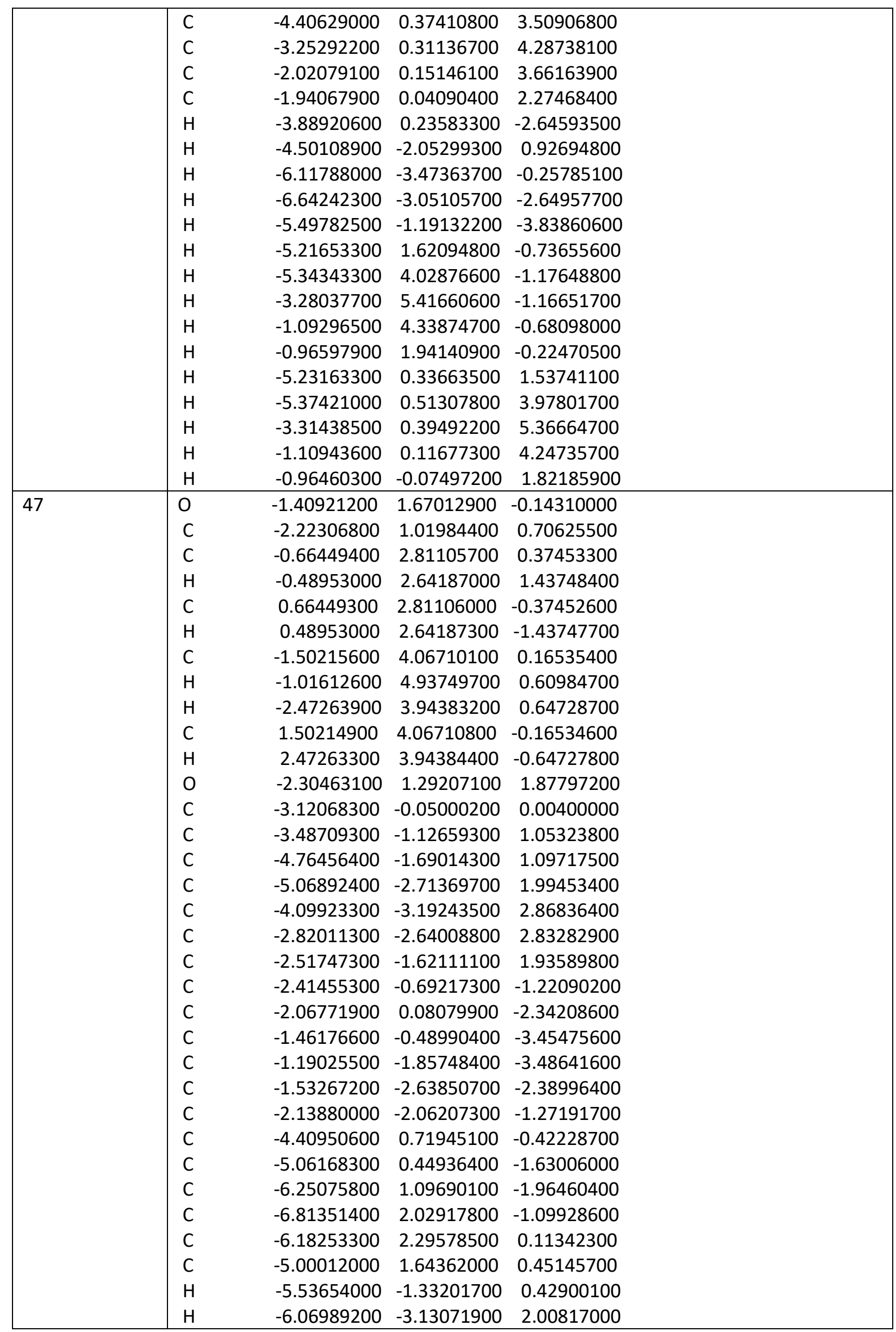




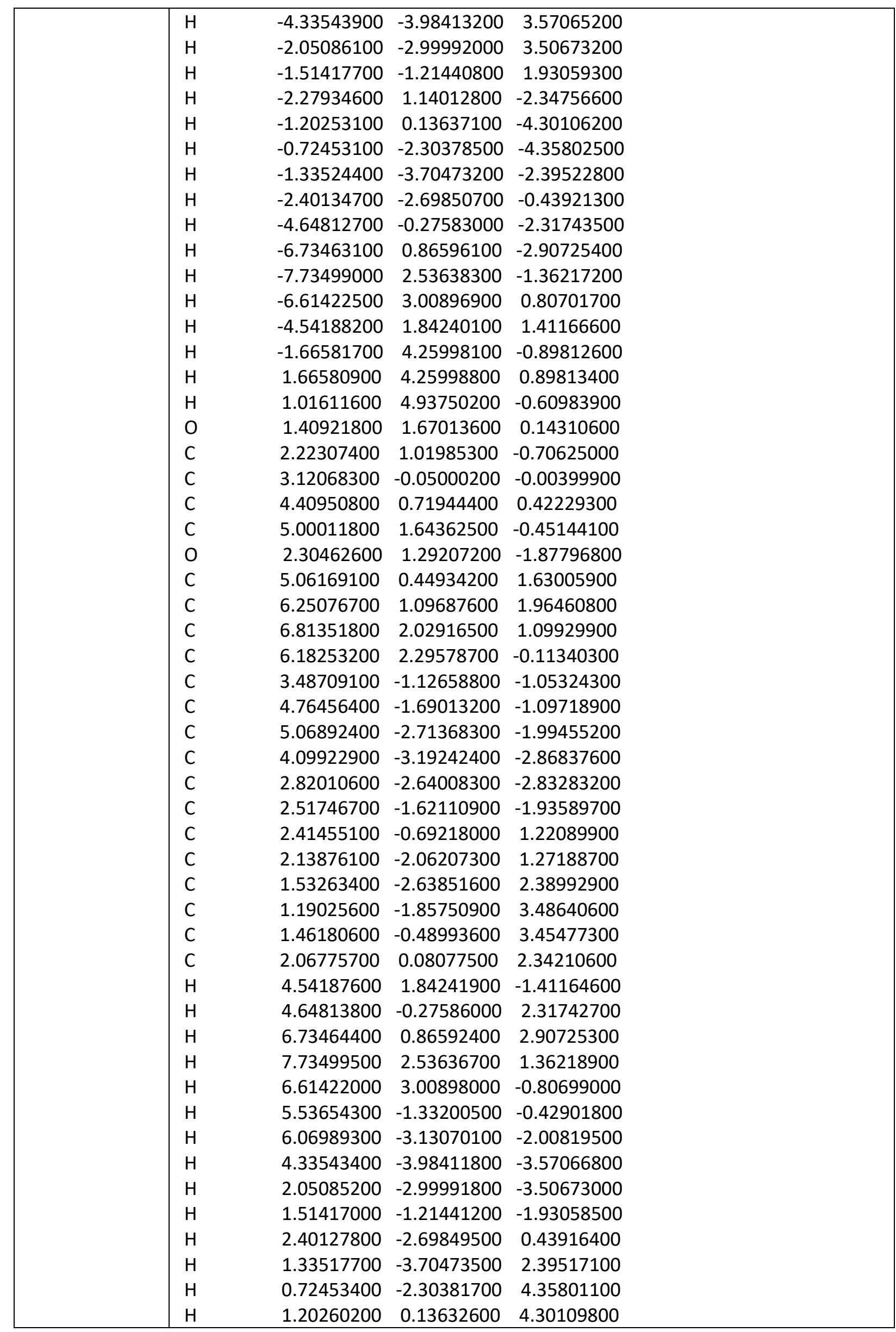


20 (optimized at the B3LYP-GD3BJ/6-311G(d,p) level)

\begin{tabular}{|c|c|c|c|c|}
\hline Conformer no & & & & \\
\hline 1 & 0 & -1.36096300 & 0.18834000 & -1.60886900 \\
\hline & $\mathrm{C}$ & -2.66826200 & -0.10858300 & -1.60610400 \\
\hline & C & -0.66812500 & 0.27630100 & -2.87536300 \\
\hline & $\mathrm{H}$ & -1.31178600 & 0.80697600 & -3.58123600 \\
\hline & C & 0.56507600 & 1.12447500 & -2.59847500 \\
\hline & $\mathrm{H}$ & 1.18442300 & 1.15688100 & -3.49767700 \\
\hline & C & -0.35325200 & -1.11798800 & -3.39592600 \\
\hline & $\mathrm{H}$ & -1.27693800 & -1.65279300 & -3.61595900 \\
\hline & $\mathrm{H}$ & 0.22061200 & -1.67363800 & -2.65451600 \\
\hline & C & 0.24268100 & 2.53151600 & -2.12261600 \\
\hline & $\mathrm{H}$ & 1.16353100 & 3.08485600 & -1.93860100 \\
\hline & $\mathrm{O}$ & -3.32118200 & -0.28541700 & -2.60262700 \\
\hline & C & -3.20153200 & -0.07609100 & -0.15447000 \\
\hline & C & -2.29218000 & -0.85646200 & 0.81351400 \\
\hline & C & -2.40481500 & -0.63668400 & 2.18956000 \\
\hline & $\mathrm{C}$ & -1.65364500 & -1.37571500 & 3.09710500 \\
\hline & C & -0.77678400 & -2.35977500 & 2.64509500 \\
\hline & C & -0.67566500 & -2.60585000 & 1.27993000 \\
\hline & $\mathrm{C}$ & -1.43140100 & -1.86465400 & 0.37608300 \\
\hline & C & -4.58600500 & -0.73092100 & -0.03826300 \\
\hline & C & -4.88648300 & -1.89796700 & -0.74565000 \\
\hline & C & -6.09382300 & -2.55923100 & -0.54741800 \\
\hline & C & -7.02122200 & -2.07346600 & 0.37056700 \\
\hline & C & -6.72584500 & -0.92066400 & 1.09112500 \\
\hline & C & -5.51846200 & -0.25816200 & 0.88850100 \\
\hline & C & -3.22386600 & 1.44530400 & 0.11183300 \\
\hline & C & -4.24779400 & 2.22629800 & -0.43422900 \\
\hline & C & -4.23247200 & 3.61119000 & -0.29788900 \\
\hline & C & -3.18617600 & 4.23935700 & 0.37395200 \\
\hline & C & -2.14813900 & 3.47094000 & 0.89327900 \\
\hline & C & -2.16339800 & 2.08623000 & 0.75388500 \\
\hline & $\mathrm{H}$ & -3.08357800 & 0.12287200 & 2.55546100 \\
\hline & $\mathrm{H}$ & -1.75558800 & -1.18249300 & 4.15904900 \\
\hline & $\mathrm{H}$ & -0.17973700 & -2.92862900 & 3.34807400 \\
\hline & $\mathrm{H}$ & 0.00670600 & -3.36040100 & 0.91079200 \\
\hline & $\mathrm{H}$ & -1.32548200 & -2.07420100 & -0.67839300 \\
\hline & $\mathrm{H}$ & -4.18024600 & -2.29175000 & -1.46249500 \\
\hline & $\mathrm{H}$ & -6.30892400 & -3.45829400 & -1.11348300 \\
\hline & $\mathrm{H}$ & -7.96192800 & -2.58945800 & 0.52369800 \\
\hline & $\mathrm{H}$ & -7.43425400 & -0.53248300 & 1.81402300 \\
\hline & $\mathrm{H}$ & -5.30529500 & 0.63785500 & 1.45645100 \\
\hline & $\mathrm{H}$ & -5.05570800 & 1.74695200 & -0.97225000 \\
\hline & $\mathrm{H}$ & -5.03776200 & 4.19923100 & -0.72282400 \\
\hline & $\mathrm{H}$ & -3.17552200 & 5.31794500 & 0.48047200 \\
\hline & $\mathrm{H}$ & -1.31088900 & 3.94187500 & 1.39516000 \\
\hline & $\mathrm{H}$ & -1.33256600 & 1.50849200 & 1.12925600 \\
\hline & $\mathrm{H}$ & 0.23205100 & -1.05045200 & -4.31652700 \\
\hline
\end{tabular}




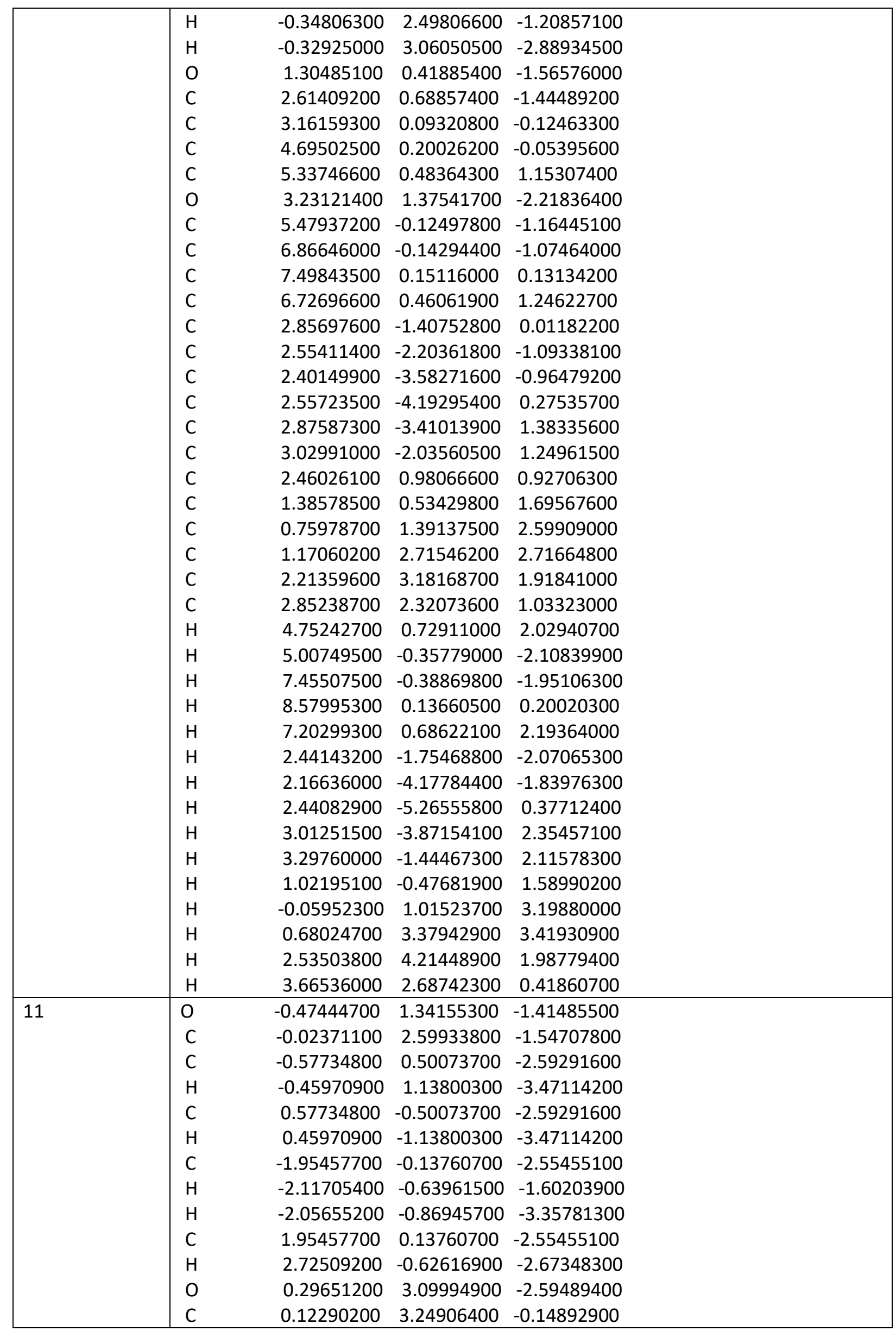




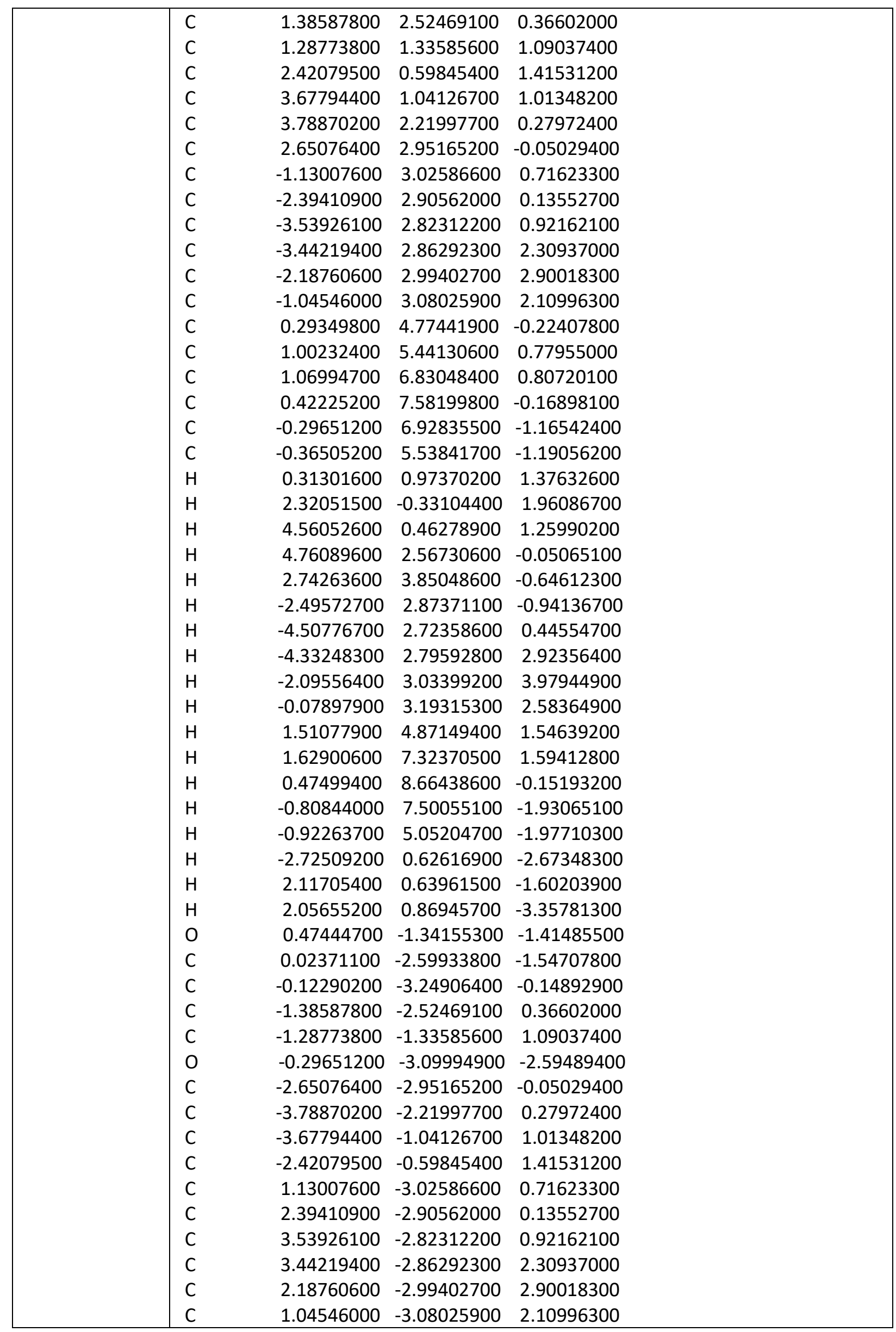




\begin{tabular}{|c|c|c|c|c|}
\hline & $\begin{array}{l}\text { C } \\
\mathrm{C} \\
\mathrm{C} \\
\mathrm{C} \\
\mathrm{C} \\
\mathrm{C} \\
\mathrm{H} \\
\mathrm{H} \\
\mathrm{H} \\
\mathrm{H} \\
\mathrm{H} \\
\mathrm{H} \\
\mathrm{H} \\
\mathrm{H} \\
\mathrm{H} \\
\mathrm{H} \\
\mathrm{H} \\
\mathrm{H} \\
\mathrm{H} \\
\mathrm{H} \\
\mathrm{H}\end{array}$ & $\begin{array}{r}-0.29349800 \\
0.36505200 \\
0.29651200 \\
-0.42225200 \\
-1.06994700 \\
-1.00232400 \\
-0.31301600 \\
-2.74263600 \\
-4.76089600 \\
-4.56052600 \\
-2.32051500 \\
2.49572700 \\
4.50776700 \\
4.33248300 \\
2.09556400 \\
0.07897900 \\
0.92263700 \\
0.80844000 \\
-0.47499400 \\
-1.62900600 \\
-1.51077900\end{array}$ & $\begin{array}{r}-4.77441900 \\
-5.53841700 \\
-6.92835500 \\
-7.58199800 \\
-6.83048400 \\
-5.44130600 \\
-0.97370200 \\
-3.85048600 \\
-2.56730600 \\
-0.46278900 \\
0.33104400 \\
-2.87371100 \\
-2.72358600 \\
-2.79592800 \\
-3.03399200 \\
-3.19315300 \\
-5.05204700 \\
-7.50055100 \\
-8.66438600 \\
-7.32370500 \\
-4.87149400\end{array}$ & $\begin{array}{r}-0.22407800 \\
-1.19056200 \\
-1.16542400 \\
-0.16898100 \\
0.80720100 \\
0.77955000 \\
1.37632600 \\
-0.64612300 \\
-0.05065100 \\
1.25990200 \\
1.96086700 \\
-0.94136700 \\
0.44554700 \\
2.92356400 \\
3.97944900 \\
2.58364900 \\
-1.97710300 \\
-1.93065100 \\
-0.15193200 \\
1.59412800 \\
1.54639200\end{array}$ \\
\hline 14 & $\begin{array}{l}\text { O } \\
C \\
C \\
H \\
C \\
H \\
C \\
H \\
H \\
C \\
H \\
O \\
C \\
C \\
C \\
C \\
C \\
C \\
C \\
C \\
C \\
C \\
C \\
C \\
C \\
C \\
C \\
C \\
C \\
C \\
C\end{array}$ & $\begin{array}{r}1.26615500 \\
2.53981700 \\
0.51760800 \\
1.20255500 \\
-0.55511600 \\
-1.19857300 \\
-0.03049100 \\
0.79237100 \\
-0.63039200 \\
-0.00474900 \\
0.66383500 \\
3.08736700 \\
3.22235300 \\
4.37778100 \\
5.20923800 \\
6.21840300 \\
6.41259500 \\
5.58561700 \\
4.57562100 \\
2.23855500 \\
1.38965000 \\
0.55086700 \\
0.55946200 \\
1.41731000 \\
2.24995700 \\
3.70177200 \\
2.77599800 \\
3.16746000 \\
4.48987100 \\
5.40915300 \\
5.01683400\end{array}$ & $\begin{array}{r}0.62739500 \\
0.25489400 \\
1.20902800 \\
1.35310500 \\
0.21223500 \\
0.70022300 \\
2.53615700 \\
3.21179800 \\
2.39687900 \\
-1.09322900 \\
-0.90072200 \\
0.27133600 \\
-0.05960600 \\
-1.06772800 \\
-1.24563600 \\
-2.19913800 \\
-3.01016000 \\
-2.85139000 \\
-1.88912200 \\
-0.68399700 \\
-1.71251000 \\
-2.36216900 \\
-2.00675000 \\
-1.00282400 \\
-0.34647400 \\
1.35551300 \\
2.29642000 \\
3.60071000 \\
3.99336300 \\
3.07294300 \\
1.76564800 \\
\end{array}$ & $\begin{array}{l}-1.28369900 \\
-1.48601500 \\
-2.38190300 \\
-3.21924200 \\
-2.81284600 \\
-3.54641300 \\
-1.88670500 \\
-1.64858000 \\
-0.98839700 \\
-3.35873900 \\
-4.19979500 \\
-2.55939800 \\
-0.13393800 \\
-0.25487400 \\
0.85823000 \\
0.85674000 \\
-0.26020700 \\
-1.36524500 \\
-1.36521300 \\
0.87169100 \\
0.45273600 \\
1.34760200 \\
2.69528600 \\
3.13057700 \\
2.22476700 \\
0.26371300 \\
0.73105300 \\
1.01039400 \\
0.81256700 \\
0.31955000 \\
0.03888400 \\
\end{array}$ \\
\hline
\end{tabular}




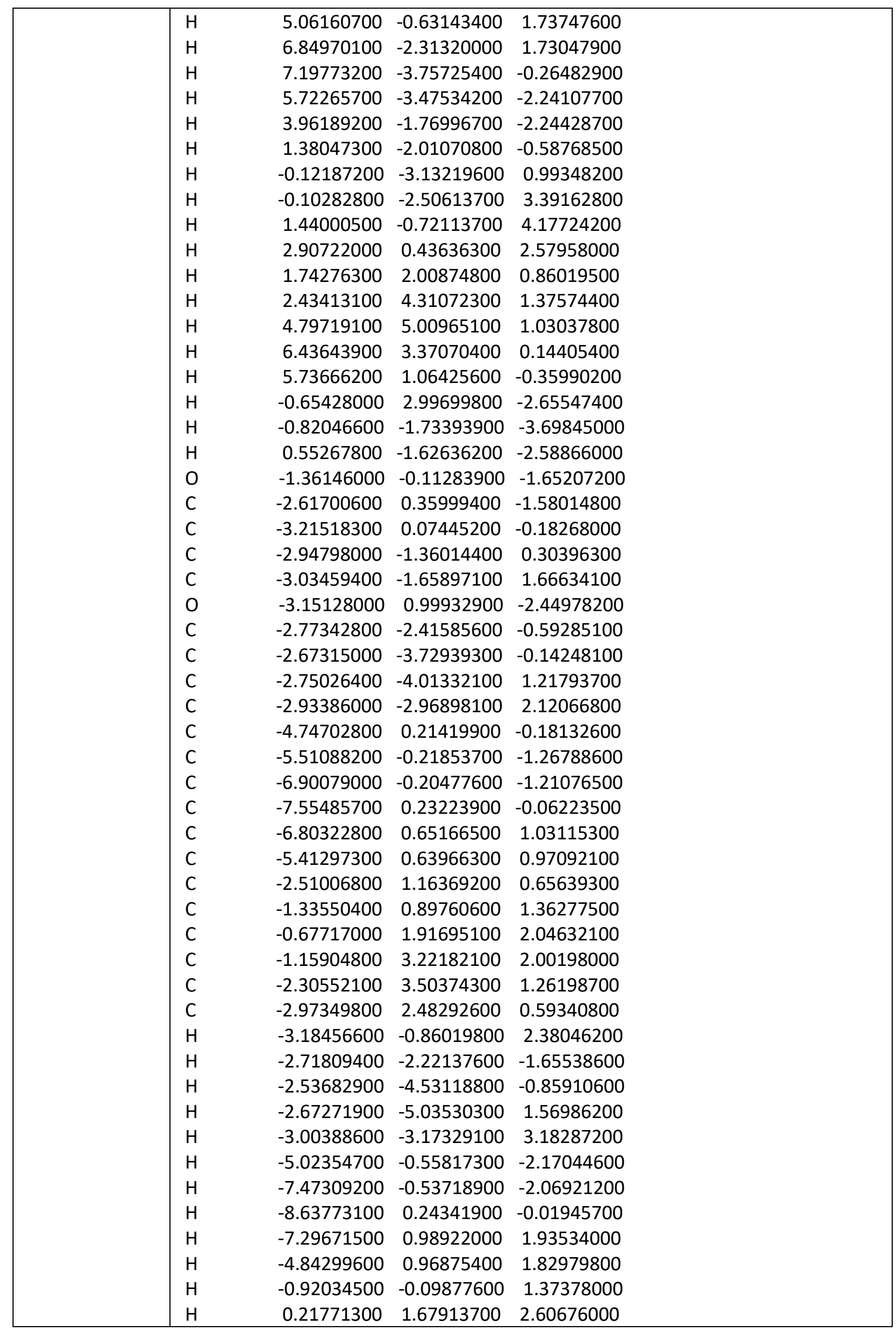




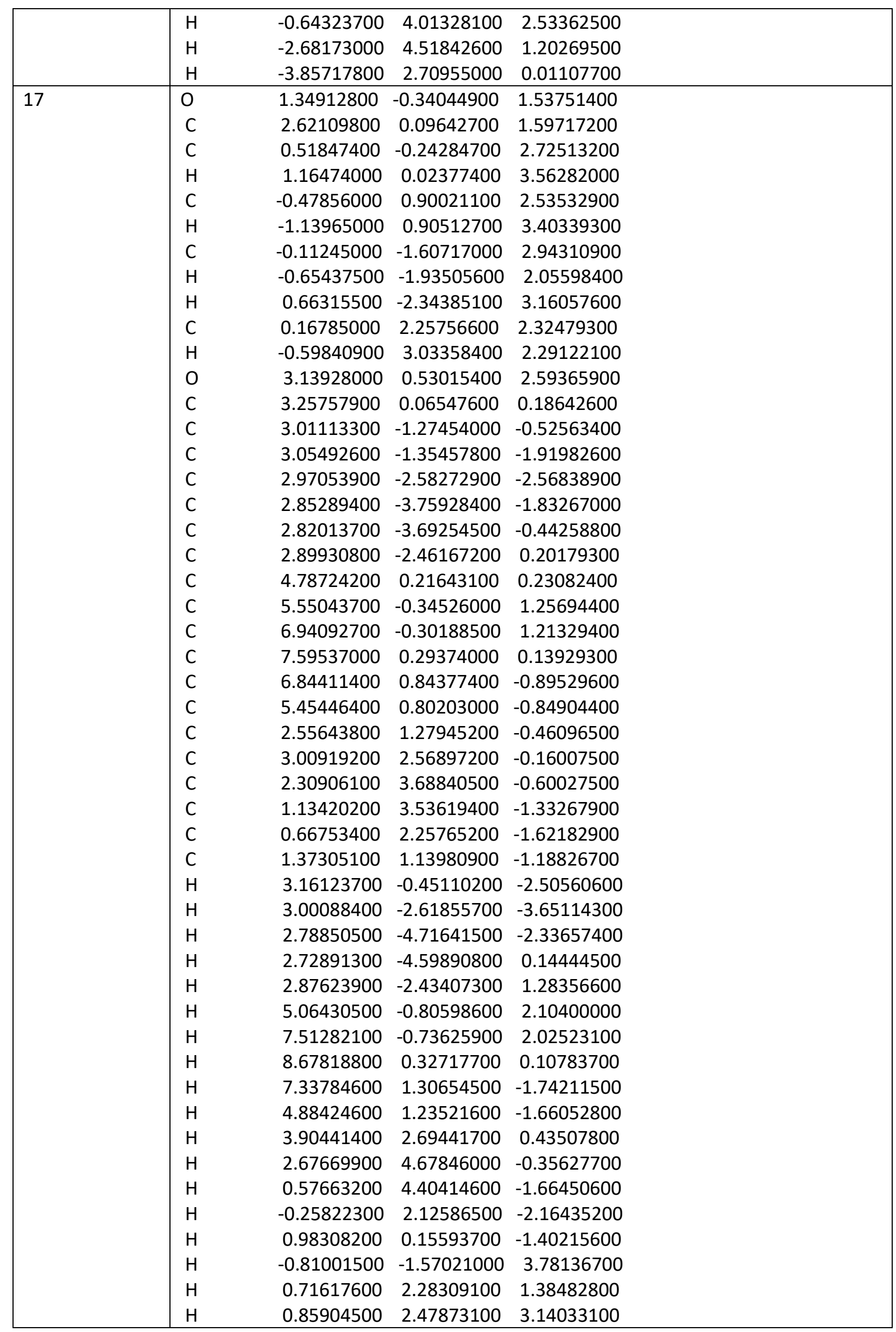




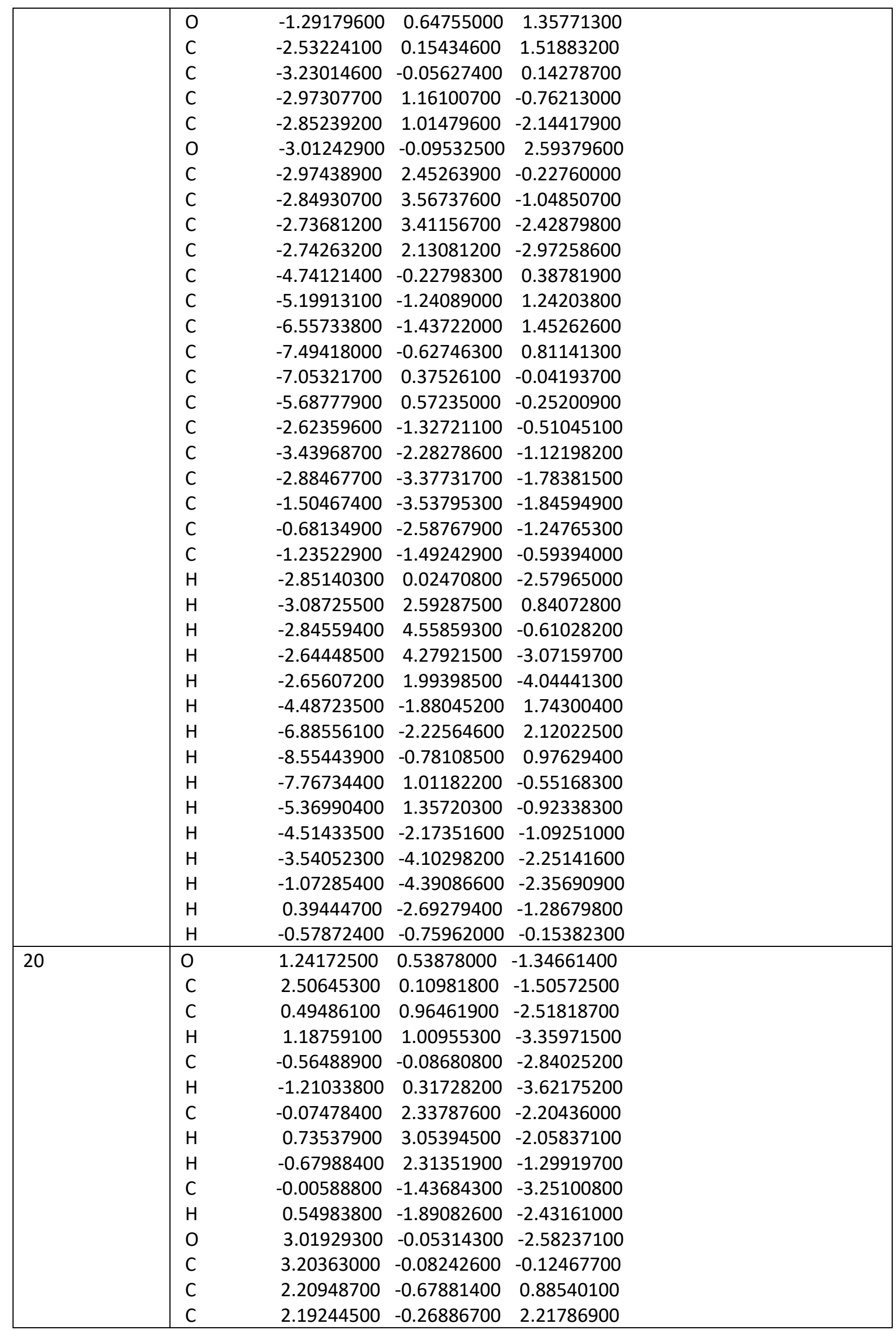




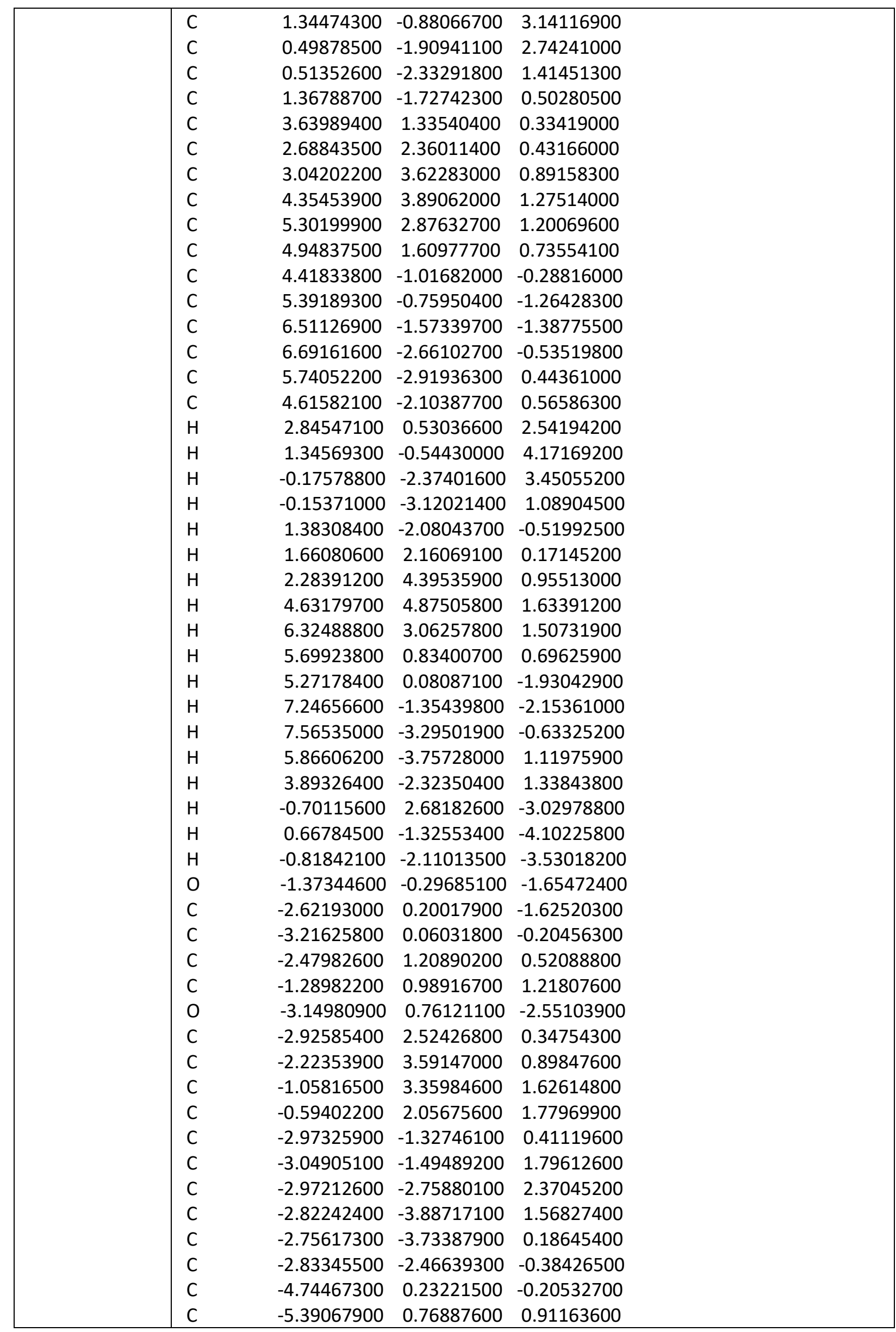




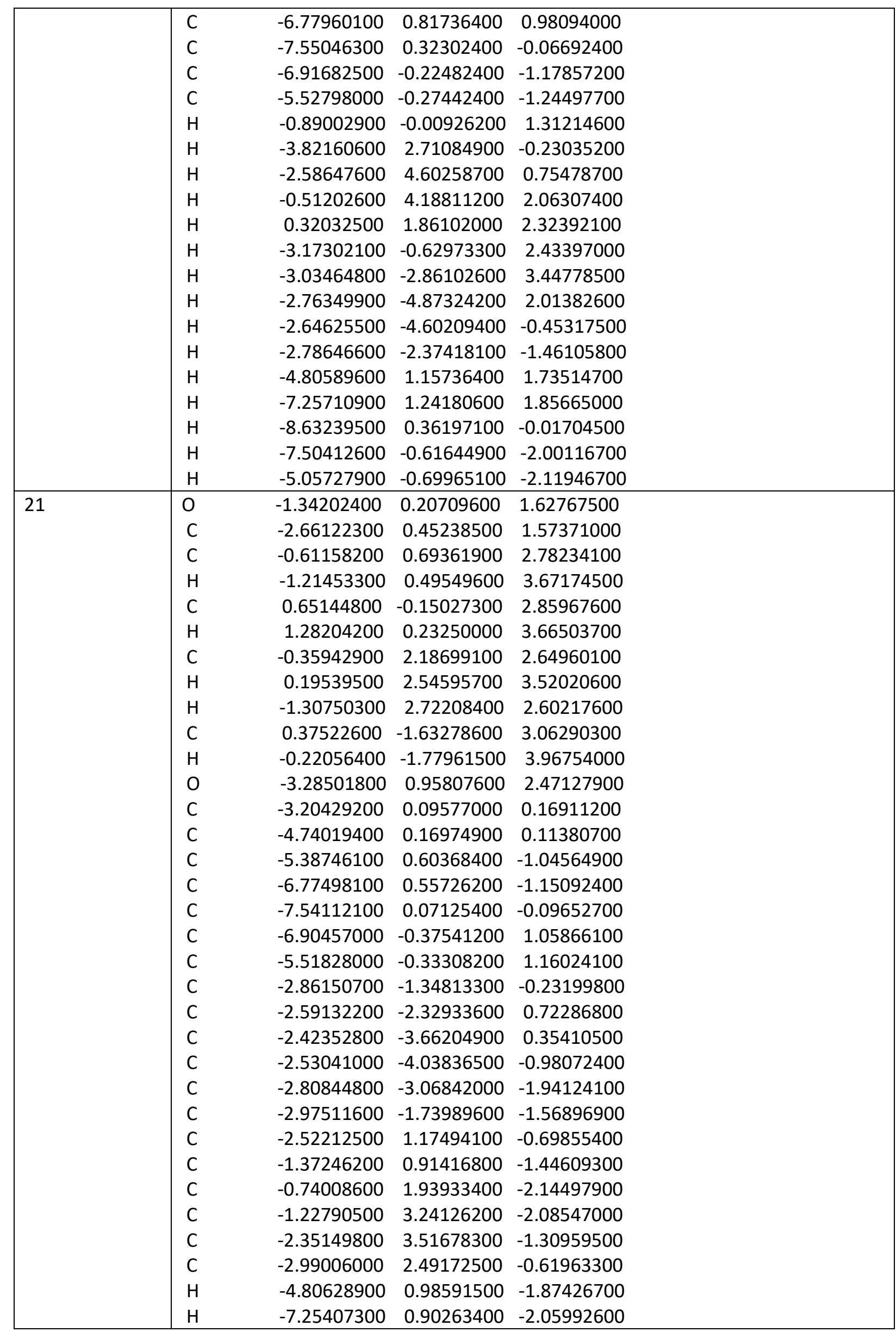




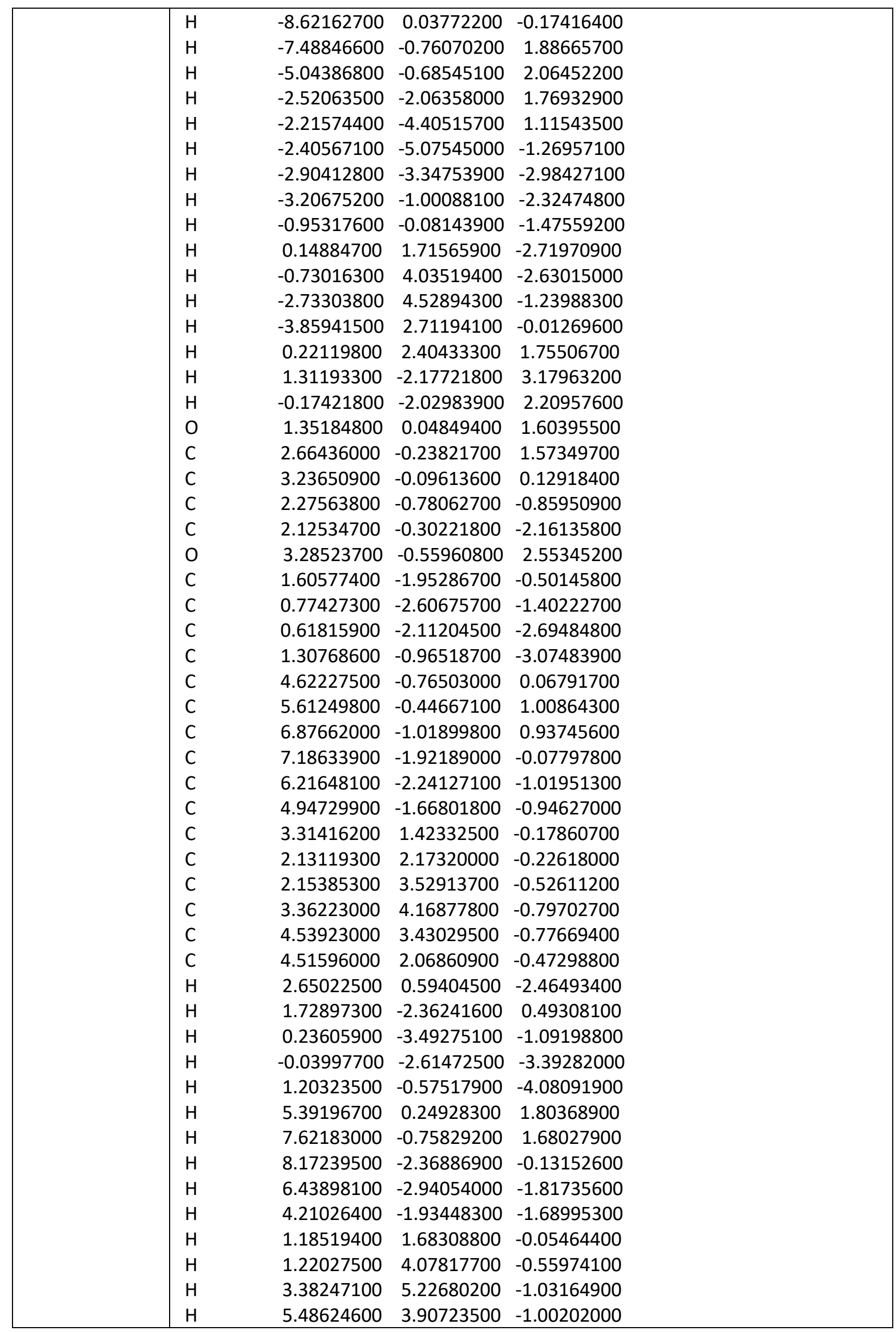




\begin{tabular}{|c|c|c|c|c|}
\hline & $\mathrm{H}$ & 5.44222600 & 1.51312300 & -0.47888900 \\
\hline \multirow[t]{51}{*}{22} & 0 & -1.35040500 & -0.50033100 & -1.39655300 \\
\hline & $\mathrm{C}$ & -2.62534000 & -0.88801900 & -1.24653500 \\
\hline & $\mathrm{C}$ & -0.56472600 & -1.08297700 & -2.46734800 \\
\hline & $\mathrm{H}$ & -1.19402700 & -1.12140900 & -3.36014700 \\
\hline & $\mathrm{C}$ & 0.56811400 & -0.09240300 & -2.70242200 \\
\hline & $\mathrm{H}$ & 1.22357000 & -0.48244300 & -3.48447800 \\
\hline & $\mathrm{C}$ & -0.09006200 & -2.47699100 & -2.09007200 \\
\hline & $\mathrm{H}$ & -0.94575000 & -3.13766000 & -1.95574400 \\
\hline & $\mathrm{H}$ & 0.49644000 & -2.44693900 & -1.17315900 \\
\hline & $\mathrm{C}$ & 0.10194900 & 1.31152100 & -3.05249000 \\
\hline & $\mathrm{H}$ & 0.96338100 & 1.95493800 & -3.23129500 \\
\hline & 0 & -3.15288900 & -1.70640600 & -1.95412500 \\
\hline & $\mathrm{C}$ & -3.29682000 & -0.08873200 & -0.08951000 \\
\hline & $\mathrm{C}$ & -2.66111100 & -0.48616700 & 1.26930600 \\
\hline & $\mathrm{C}$ & -1.27250500 & -0.44852700 & 1.46254000 \\
\hline & $\mathrm{C}$ & -0.72041100 & -0.69889100 & 2.71400000 \\
\hline & $\mathrm{C}$ & -1.53480700 & -1.00563300 & 3.80044000 \\
\hline & $\mathrm{C}$ & -2.91234300 & -1.04814200 & 3.62145900 \\
\hline & $\mathrm{C}$ & -3.46895800 & -0.78369100 & 2.37138800 \\
\hline & $\mathrm{C}$ & -4.80607300 & -0.38031300 & -0.08037900 \\
\hline & $\mathrm{C}$ & -5.26910300 & -1.70232500 & -0.08986300 \\
\hline & $\mathrm{C}$ & -6.62743000 & -1.98226200 & -0.01086700 \\
\hline & $\mathrm{C}$ & -7.55586000 & -0.94721500 & 0.08898400 \\
\hline & $\mathrm{C}$ & -7.10746400 & 0.36751500 & 0.11407600 \\
\hline & $\mathrm{C}$ & -5.74354000 & 0.64710700 & 0.03082700 \\
\hline & $\mathrm{C}$ & -3.03740400 & 1.39953000 & -0.40261900 \\
\hline & $\mathrm{C}$ & -2.56862600 & 2.30147300 & 0.54941300 \\
\hline & $\mathrm{C}$ & -2.37051100 & 3.64277500 & 0.21785600 \\
\hline & $\mathrm{C}$ & -2.63756300 & 4.09883300 & -1.06796600 \\
\hline & $\mathrm{C}$ & -3.11789300 & 3.20426700 & -2.02395300 \\
\hline & $\mathrm{C}$ & -3.31813100 & 1.87112300 & -1.69041200 \\
\hline & $\mathrm{H}$ & -0.61785500 & -0.19328400 & 0.64481300 \\
\hline & $\mathrm{H}$ & 0.35085400 & -0.61921000 & 2.84490100 \\
\hline & $\mathrm{H}$ & -1.10004900 & -1.19757200 & 4.77469100 \\
\hline & $\mathrm{H}$ & -3.56576500 & -1.27710500 & 4.45561100 \\
\hline & $\mathrm{H}$ & -4.54344700 & -0.80176100 & 2.26435800 \\
\hline & $\mathrm{H}$ & -4.56302000 & -2.51681400 & -0.15645900 \\
\hline & $\mathrm{H}$ & -6.96175700 & -3.01328800 & -0.02626600 \\
\hline & $\mathrm{H}$ & -8.61562700 & -1.16660200 & 0.14956500 \\
\hline & $\mathrm{H}$ & -7.81500000 & 1.18433800 & 0.19910600 \\
\hline & $\mathrm{H}$ & -5.41451300 & 1.67649000 & 0.05916300 \\
\hline & $\mathrm{H}$ & -2.35058300 & 1.96214400 & 1.55224200 \\
\hline & $\mathrm{H}$ & -2.00918500 & 4.33115600 & 0.97359100 \\
\hline & $\mathrm{H}$ & -2.47859900 & 5.13968700 & -1.32430300 \\
\hline & $\mathrm{H}$ & -3.33515500 & 3.54577900 & -3.02941200 \\
\hline & $\mathrm{H}$ & -3.70348200 & 1.18469200 & -2.43662900 \\
\hline & $\mathrm{H}$ & 0.53769700 & -2.88216700 & -2.88779400 \\
\hline & $\mathrm{H}$ & -0.50817500 & 1.72430300 & -2.24966300 \\
\hline & $\mathrm{H}$ & -0.50280600 & 1.28625300 & -3.96267800 \\
\hline & 0 & 1.32072900 & -0.07496500 & -1.46178800 \\
\hline & $\mathrm{C}$ & 2.62148400 & 0.25213400 & -1.51268000 \\
\hline
\end{tabular}




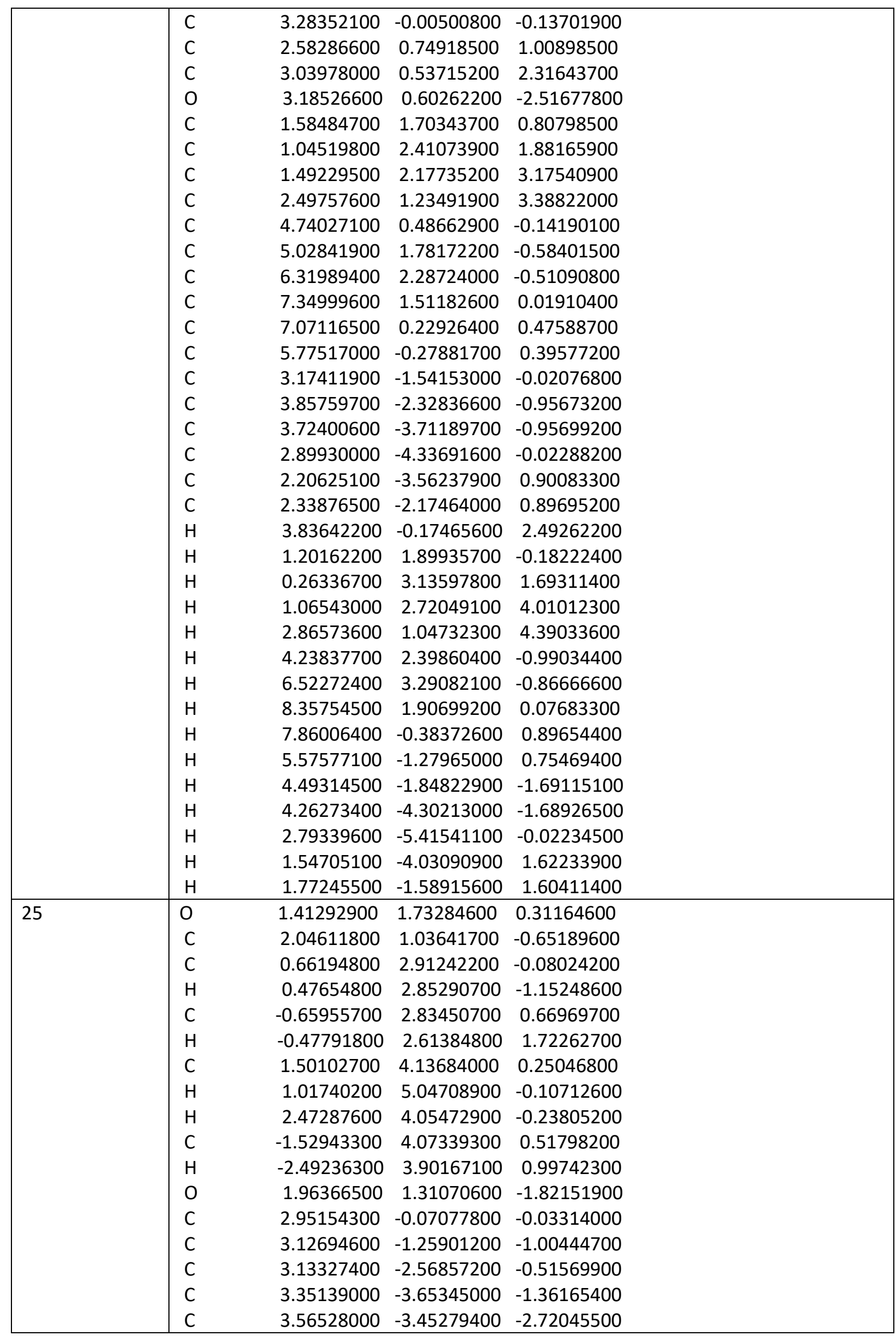




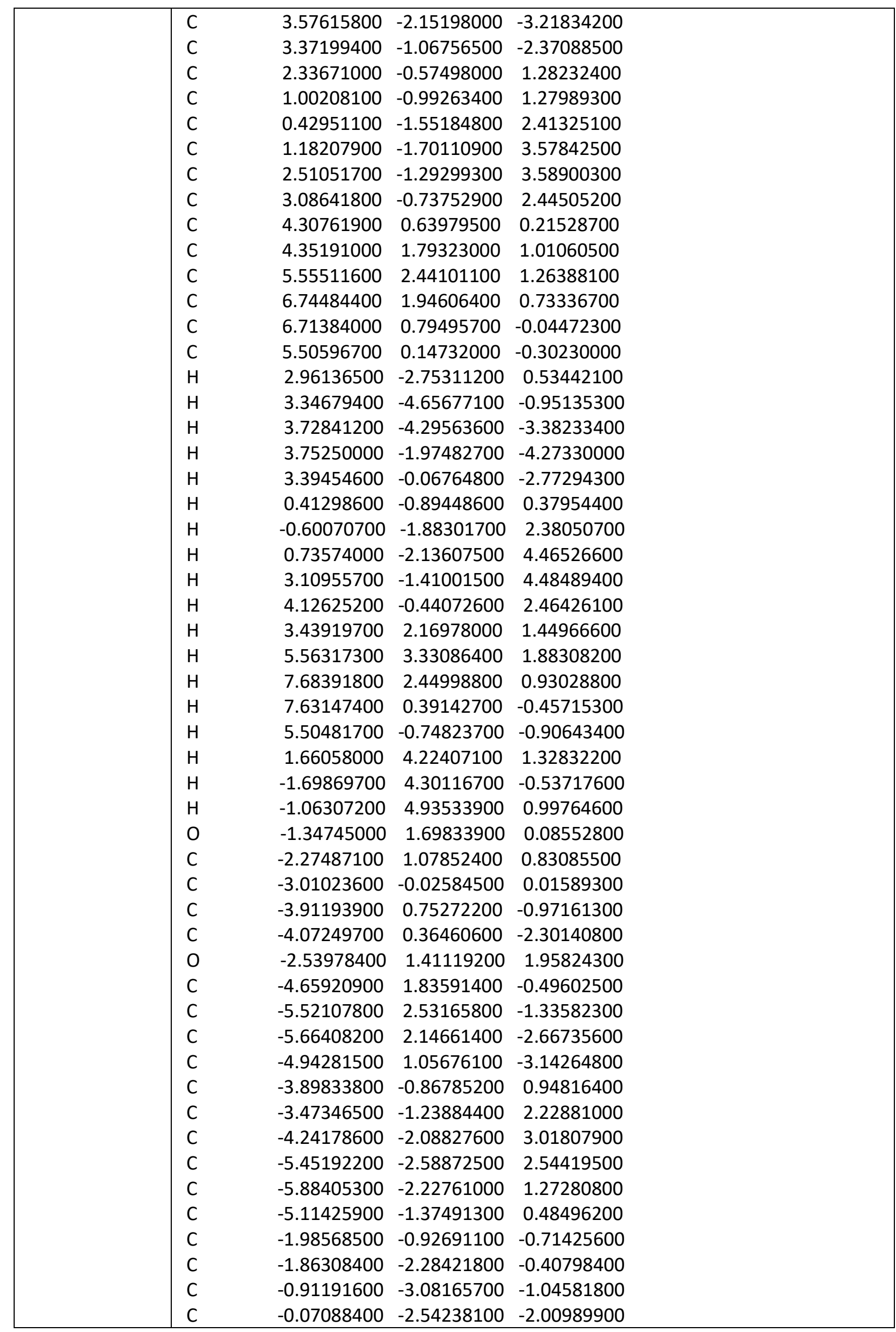




\begin{tabular}{|c|c|c|c|c|}
\hline & $C$ & -0.19890300 & -1.19556400 & -2.34299100 \\
\hline & C & -1.14460600 & -0.40158200 & -1.70802700 \\
\hline & $\mathrm{H}$ & -3.52305400 & -0.48384600 & -2.68484600 \\
\hline & $\mathrm{H}$ & -4.57844600 & 2.12330000 & 0.54535600 \\
\hline & $\mathrm{H}$ & -6.08650800 & 3.37085300 & -0.94726700 \\
\hline & $\mathrm{H}$ & -6.33594000 & 2.68692400 & -3.32400100 \\
\hline & $\mathrm{H}$ & -5.05423600 & 0.73768200 & -4.17260400 \\
\hline & $\mathrm{H}$ & -2.54457500 & -0.85427100 & 2.62093000 \\
\hline & $\mathrm{H}$ & -3.89241100 & -2.35550400 & 4.00889700 \\
\hline & $\mathrm{H}$ & -6.05121000 & -3.24865500 & 3.16073900 \\
\hline & $\mathrm{H}$ & -6.82427400 & -2.60583600 & 0.88815600 \\
\hline & $\mathrm{H}$ & -5.46771100 & -1.10583500 & -0.50117600 \\
\hline & $\mathrm{H}$ & -2.50387600 & -2.73099900 & 0.33795100 \\
\hline & $\mathrm{H}$ & -0.82839800 & -4.12728200 & -0.77291600 \\
\hline & $\mathrm{H}$ & 0.68589000 & -3.15104900 & -2.48723800 \\
\hline & $\mathrm{H}$ & 0.45848200 & -0.75146300 & -3.07864900 \\
\hline & $\mathrm{H}$ & -1.22003100 & 0.64018500 & -1.97633200 \\
\hline \multirow[t]{35}{*}{43} & 0 & -1.45794200 & -2.02887300 & -0.06199500 \\
\hline & $\mathrm{C}$ & -1.85789800 & -0.98273100 & -0.80027800 \\
\hline & $\mathrm{C}$ & -0.67410200 & -3.07716900 & -0.68687500 \\
\hline & $\mathrm{H}$ & -0.57528600 & -2.84447600 & -1.74762000 \\
\hline & $\mathrm{C}$ & 0.70196000 & -3.05996700 & -0.02913800 \\
\hline & $\mathrm{H}$ & 0.57578100 & -3.03089600 & 1.05349800 \\
\hline & $\mathrm{C}$ & -1.43634000 & -4.37519700 & -0.47254900 \\
\hline & $\mathrm{H}$ & -0.93965700 & -5.20717700 & -0.97405100 \\
\hline & $\mathrm{H}$ & -2.44227000 & -4.28065400 & -0.88412900 \\
\hline & $\mathrm{C}$ & 1.60946000 & -4.20169000 & -0.45968400 \\
\hline & $\mathrm{H}$ & 1.67287800 & -4.25945300 & -1.54909000 \\
\hline & 0 & -1.58131800 & -0.84992600 & -1.96716300 \\
\hline & $\mathrm{C}$ & -2.74872600 & 0.01736300 & -0.00582200 \\
\hline & $\mathrm{C}$ & -3.11524600 & -0.52540100 & 1.38962700 \\
\hline & $\mathrm{C}$ & -2.10071000 & -0.78446100 & 2.32259900 \\
\hline & $\mathrm{C}$ & -2.40598500 & -1.23794600 & 3.59814500 \\
\hline & $\mathrm{C}$ & -3.73387700 & -1.43732200 & 3.97800100 \\
\hline & $\mathrm{C}$ & -4.74682800 & -1.17218200 & 3.06632400 \\
\hline & $\mathrm{C}$ & -4.43920900 & -0.71708100 & 1.78251300 \\
\hline & $\mathrm{C}$ & -4.01620600 & 0.21881100 & -0.86131800 \\
\hline & $\mathrm{C}$ & -4.63406800 & -0.88517000 & -1.45768700 \\
\hline & $\mathrm{C}$ & -5.81443900 & -0.74328300 & -2.17663400 \\
\hline & $\mathrm{C}$ & -6.40553400 & 0.51203300 & -2.30842500 \\
\hline & $\mathrm{C}$ & -5.80246700 & 1.61508600 & -1.71456400 \\
\hline & $\mathrm{C}$ & -4.61546600 & 1.46962300 & -0.99783400 \\
\hline & $\mathrm{C}$ & -1.98868500 & 1.36373900 & 0.11828100 \\
\hline & $\mathrm{C}$ & -2.08833500 & 2.13555900 & 1.28060200 \\
\hline & $\mathrm{C}$ & -1.49408400 & 3.39363800 & 1.35492900 \\
\hline & $\mathrm{C}$ & -0.77763000 & 3.89905800 & 0.27475700 \\
\hline & $\mathrm{C}$ & -0.67795000 & 3.14148500 & -0.88816300 \\
\hline & $\mathrm{C}$ & -1.28981100 & 1.89483400 & -0.97060500 \\
\hline & $\mathrm{H}$ & -1.06468100 & -0.63381900 & 2.05459900 \\
\hline & $\mathrm{H}$ & -1.60157000 & -1.43208100 & 4.29849800 \\
\hline & $\mathrm{H}$ & -3.97162400 & -1.78935100 & 4.97543000 \\
\hline & $\mathrm{H}$ & -5.78466000 & -1.31243700 & 3.34657000 \\
\hline
\end{tabular}




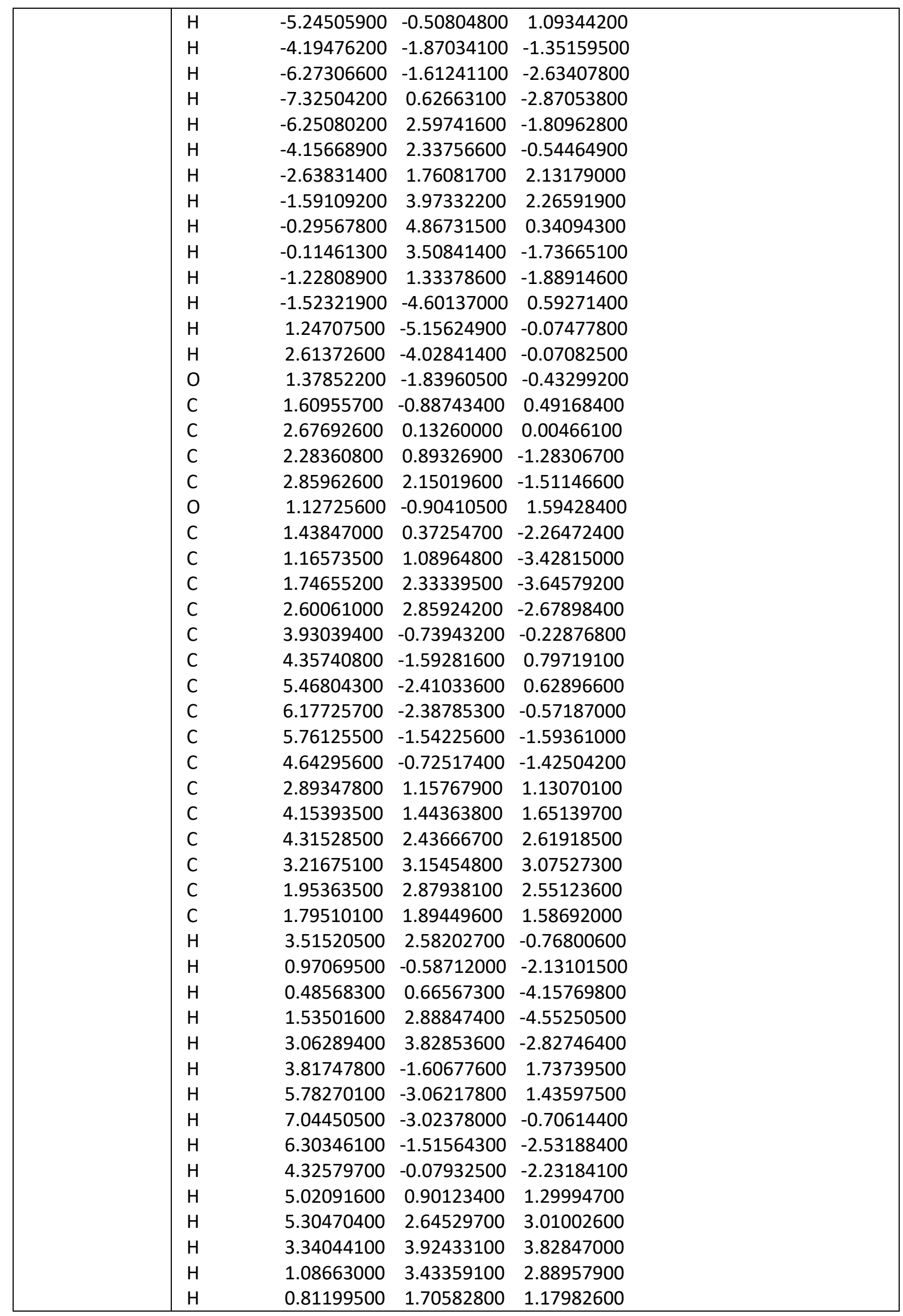


20 (optimized at the M06-2X/6-311G(d,p) level)

\begin{tabular}{|c|c|c|c|c|}
\hline Conformer no & & & & \\
\hline \multirow[t]{50}{*}{1} & 0 & -1.36986100 & 0.23909800 & -1.60990300 \\
\hline & C & -2.66410300 & -0.09487500 & -1.60723800 \\
\hline & $\mathrm{C}$ & -0.67612900 & 0.27552800 & -2.86553300 \\
\hline & $\mathrm{H}$ & -1.29759800 & 0.80614500 & -3.59283200 \\
\hline & C & 0.58321800 & 1.08482500 & -2.60463200 \\
\hline & $\mathrm{H}$ & 1.18284700 & 1.11062400 & -3.51879900 \\
\hline & $\mathrm{C}$ & -0.38562500 & -1.13910700 & -3.33961000 \\
\hline & $\mathrm{H}$ & -1.31756500 & -1.66498300 & -3.54681600 \\
\hline & $\mathrm{H}$ & 0.17535800 & -1.67362600 & -2.57078400 \\
\hline & C & 0.30937300 & 2.49017300 & -2.09901400 \\
\hline & $\mathrm{H}$ & 1.24681800 & 3.03505600 & -1.98423600 \\
\hline & 0 & -3.30336300 & -0.30043200 & -2.60091900 \\
\hline & C & -3.19801000 & -0.07262900 & -0.16163700 \\
\hline & $\mathrm{C}$ & -2.24421600 & -0.82130000 & 0.78422600 \\
\hline & C & -2.26232500 & -0.55636100 & 2.15372500 \\
\hline & C & -1.47896500 & -1.29543800 & 3.03364000 \\
\hline & C & -0.66429500 & -2.31726900 & 2.55742200 \\
\hline & $\mathrm{C}$ & -0.65352700 & -2.60245100 & 1.19672000 \\
\hline & $\mathrm{C}$ & -1.44121400 & -1.86434000 & 0.32205700 \\
\hline & C & -4.55457700 & -0.77481200 & -0.02338200 \\
\hline & C & -4.86803700 & -1.91154300 & -0.76912800 \\
\hline & $\mathrm{C}$ & -6.04937500 & -2.60700000 & -0.53726000 \\
\hline & $\mathrm{C}$ & -6.93280800 & -2.18732900 & 0.44937500 \\
\hline & C & -6.62156800 & -1.06624300 & 1.20928000 \\
\hline & $\mathrm{C}$ & -5.44249400 & -0.37027200 & 0.97549200 \\
\hline & $\mathrm{C}$ & -3.27417000 & 1.44445500 & 0.10215400 \\
\hline & $\mathrm{C}$ & -4.37438700 & 2.17793800 & -0.34615700 \\
\hline & C & -4.40725300 & 3.56037200 & -0.20097100 \\
\hline & C & -3.33578900 & 4.23135400 & 0.37851000 \\
\hline & $\mathrm{C}$ & -2.22161500 & 3.51106800 & 0.79393600 \\
\hline & $\mathrm{C}$ & -2.18915400 & 2.12925500 & 0.64671900 \\
\hline & $\mathrm{H}$ & -2.89570400 & 0.23630700 & 2.53699100 \\
\hline & $\mathrm{H}$ & -1.50824200 & -1.07140800 & 4.09394600 \\
\hline & $\mathrm{H}$ & -0.04294800 & -2.88811600 & 3.23763000 \\
\hline & $\mathrm{H}$ & -0.02016400 & -3.39134700 & 0.80980200 \\
\hline & $\mathrm{H}$ & -1.41840400 & -2.11051700 & -0.73344600 \\
\hline & $\mathrm{H}$ & -4.19487400 & -2.25605700 & -1.54245000 \\
\hline & $\mathrm{H}$ & -6.27788000 & -3.48280400 & -1.13311700 \\
\hline & $\mathrm{H}$ & -7.85335800 & -2.73014700 & 0.62716900 \\
\hline & $\mathrm{H}$ & -7.29603900 & -0.73015600 & 1.98787600 \\
\hline & $\mathrm{H}$ & -5.21441800 & 0.50214300 & 1.57690200 \\
\hline & $\mathrm{H}$ & -5.20658700 & 1.66366800 & -0.81303300 \\
\hline & $\mathrm{H}$ & -5.27236900 & 4.11346900 & -0.54712900 \\
\hline & $\mathrm{H}$ & -3.36470900 & 5.30842900 & 0.49301400 \\
\hline & $\mathrm{H}$ & -1.36331500 & 4.01755100 & 1.22169400 \\
\hline & $\mathrm{H}$ & -1.30154700 & 1.58095600 & 0.93515200 \\
\hline & $\mathrm{H}$ & 0.20690300 & -1.11270800 & -4.25690800 \\
\hline & $\mathrm{H}$ & -0.20301500 & 2.44346000 & -1.13715400 \\
\hline & $\mathrm{H}$ & -0.32355100 & 3.02883900 & -2.80769900 \\
\hline & 0 & 1.31300500 & 0.35233200 & -1.59993500 \\
\hline
\end{tabular}




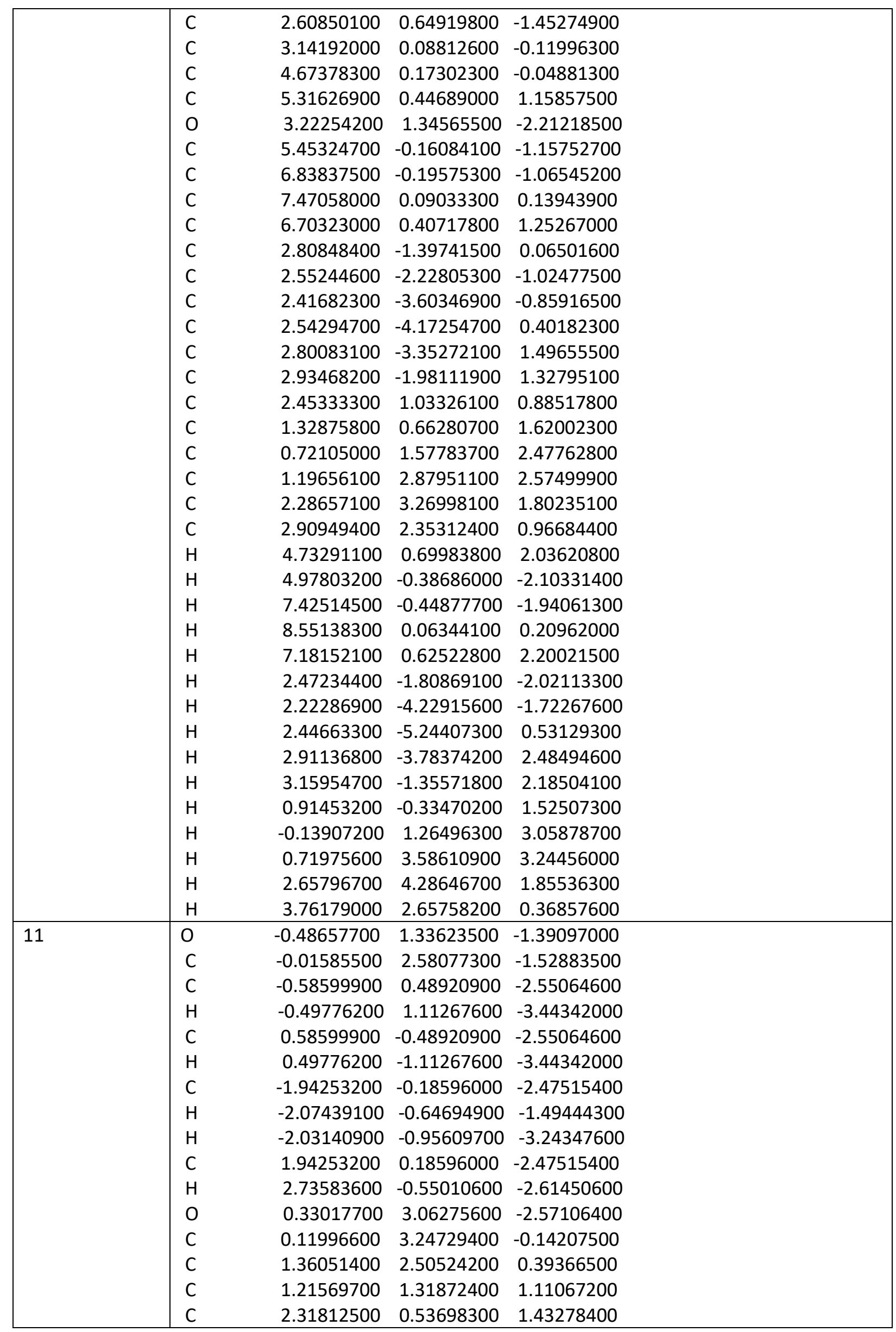




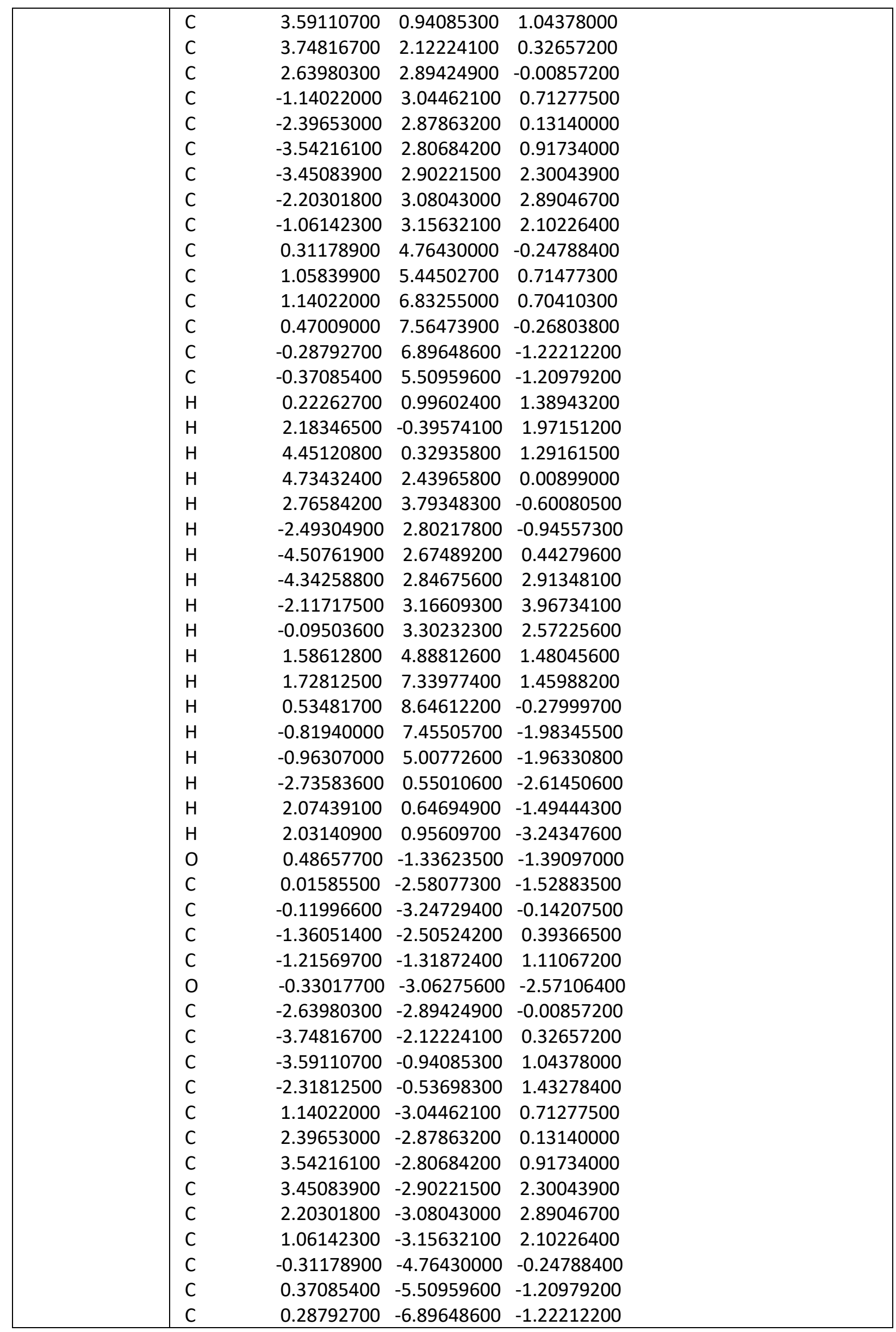




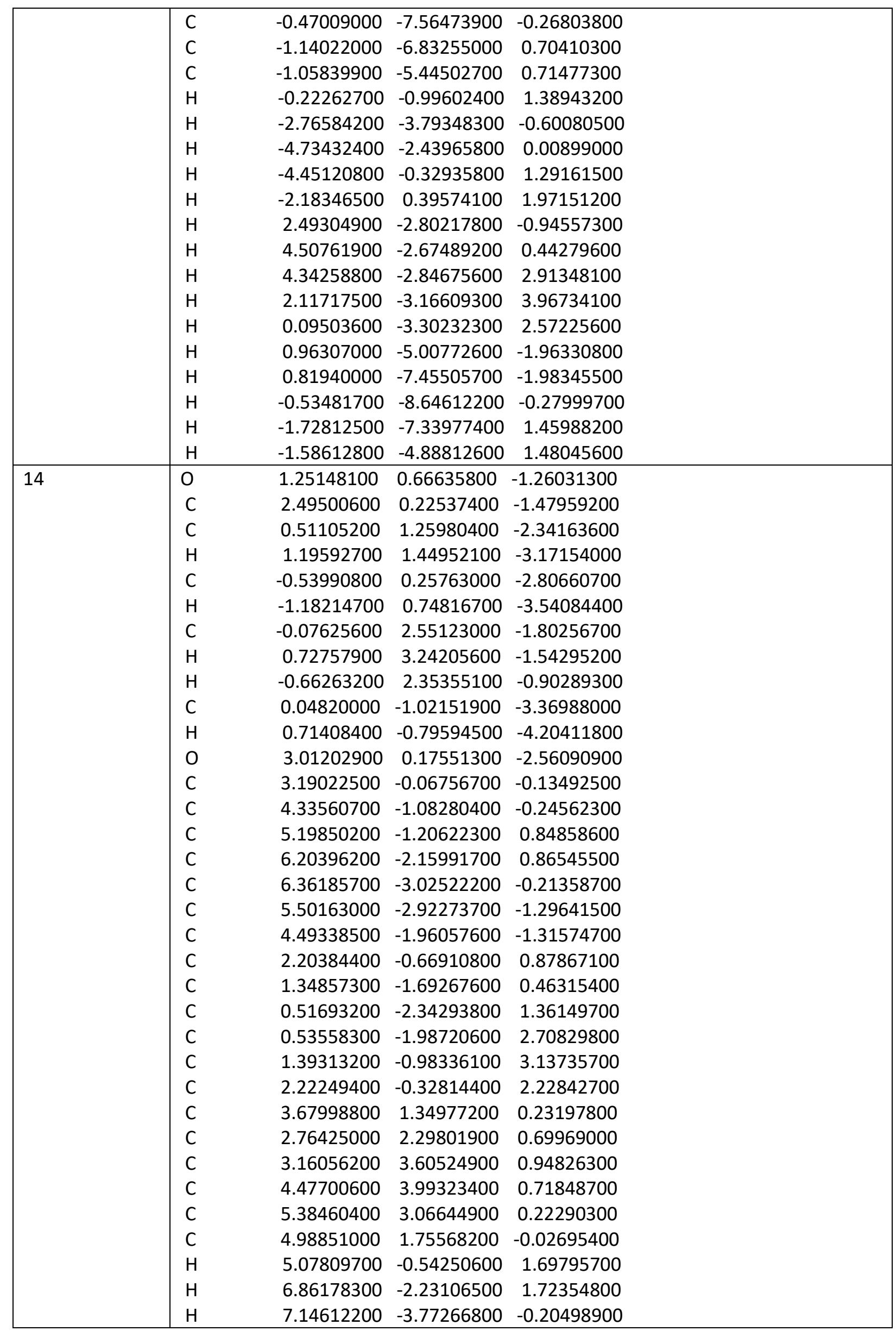




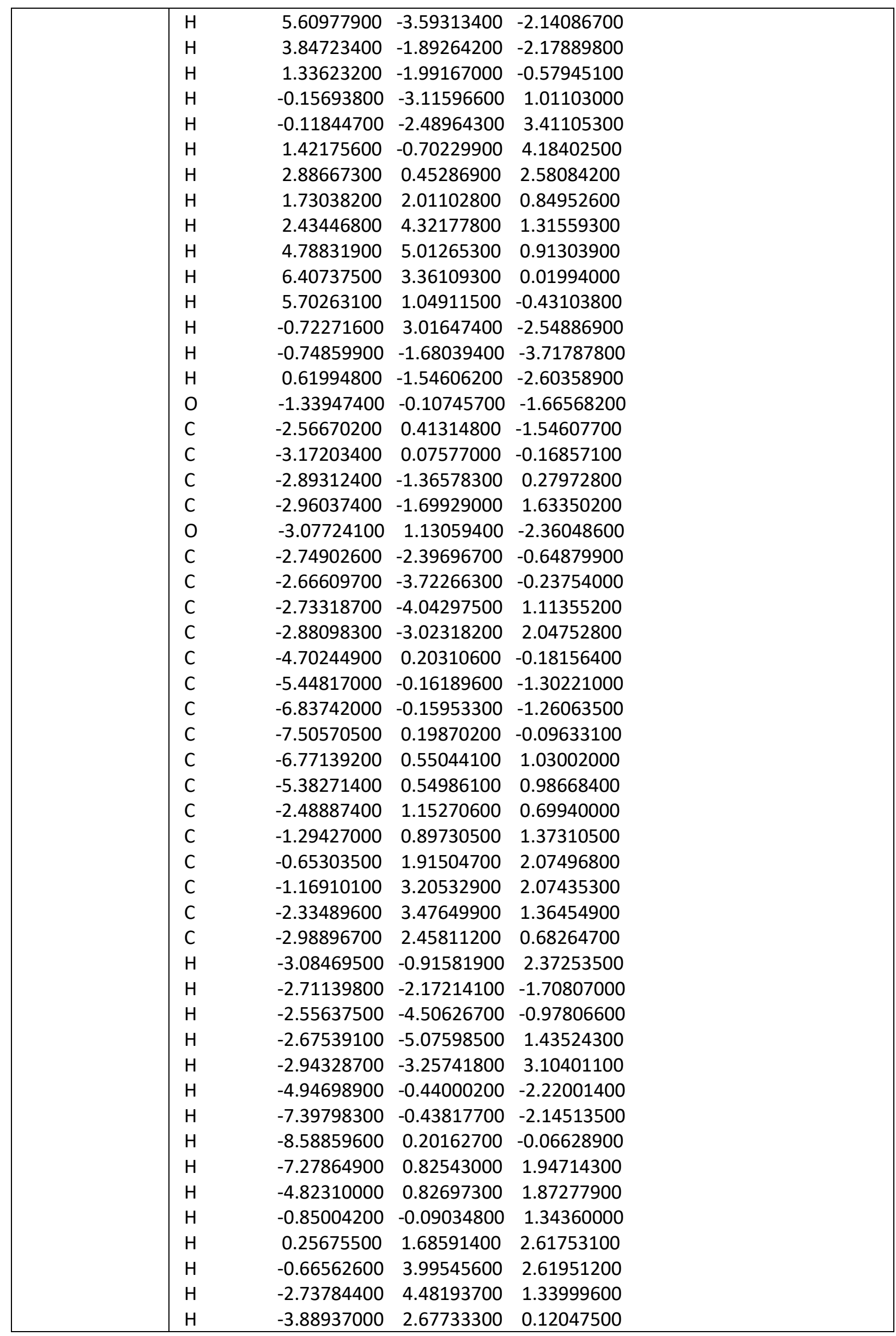




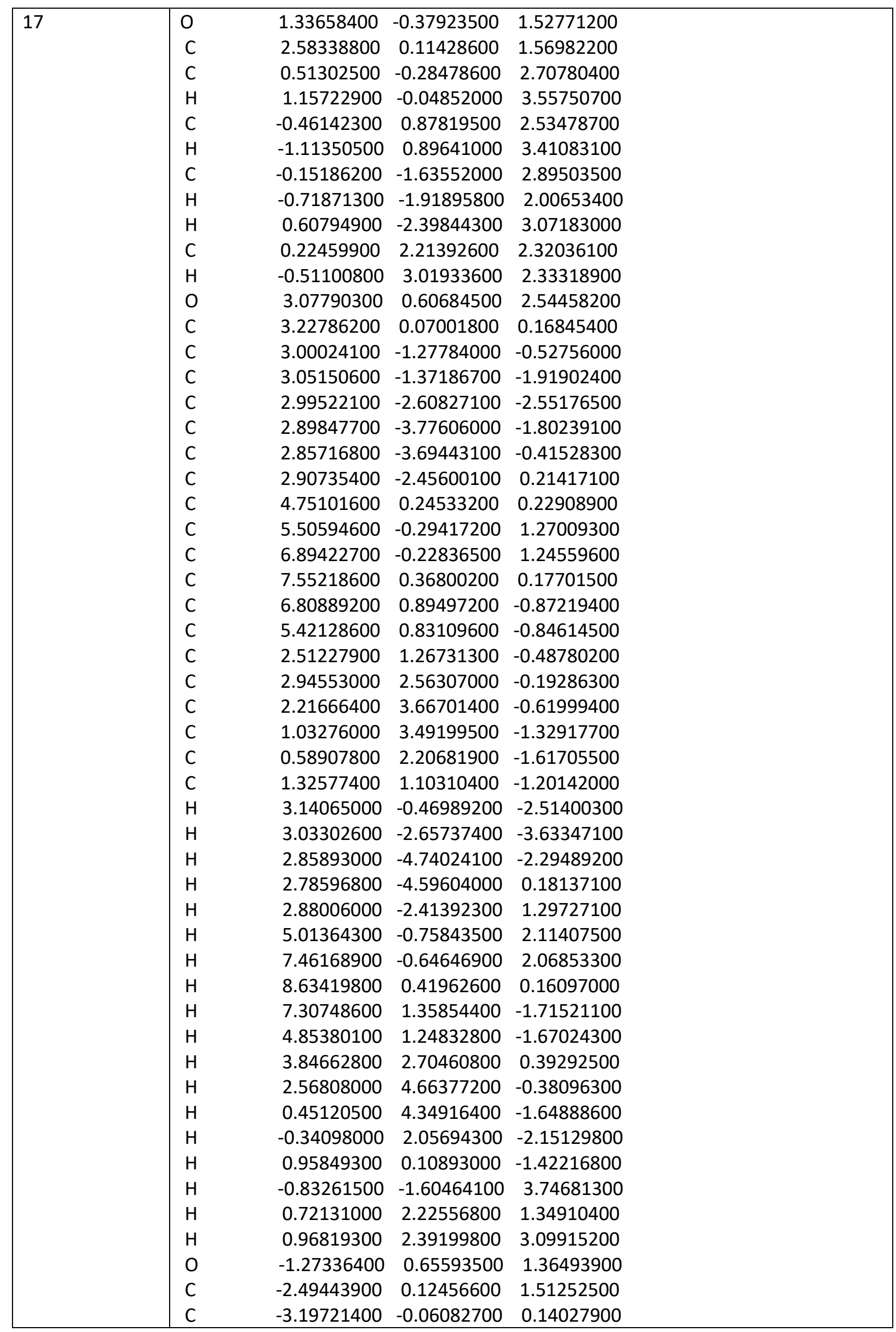




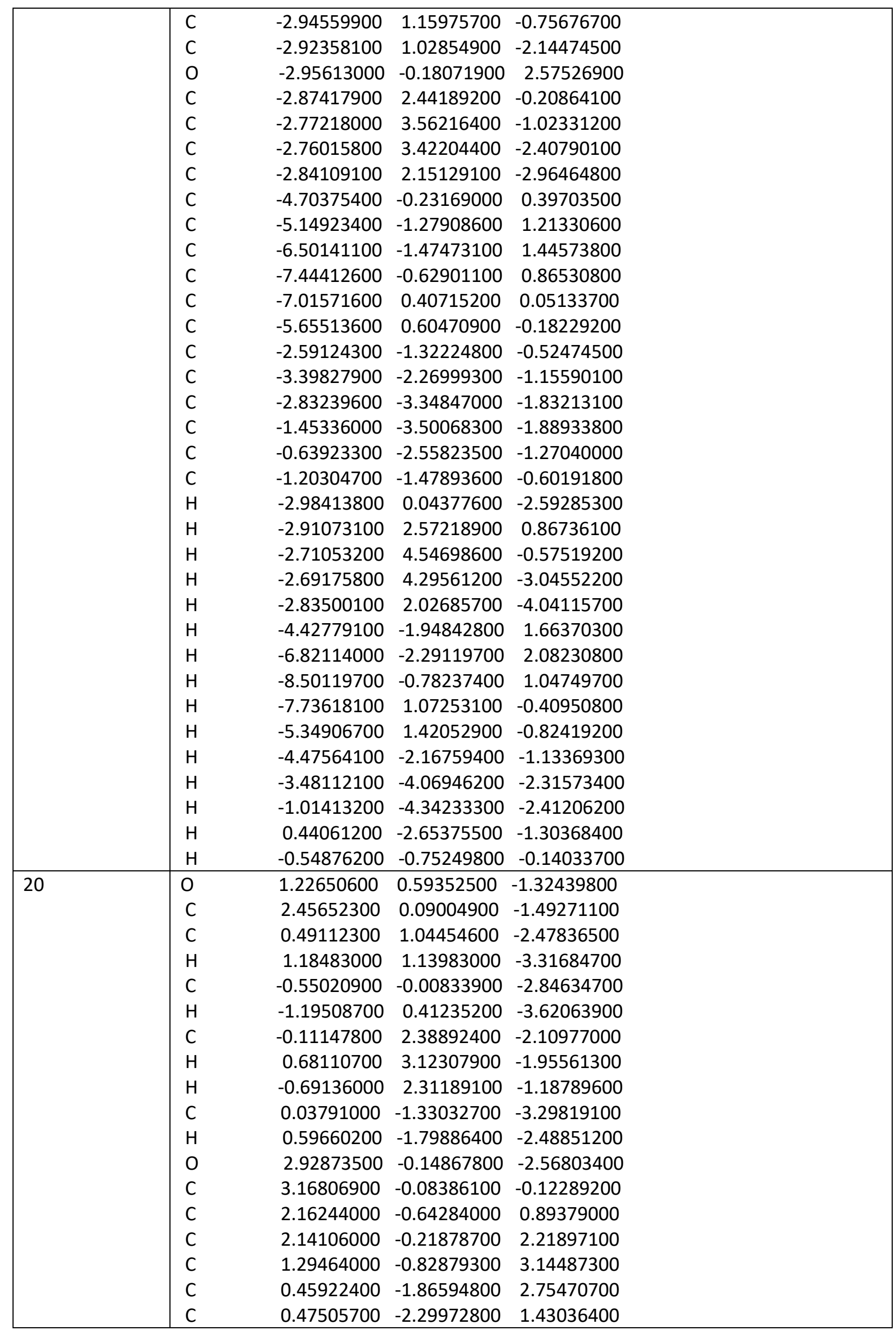




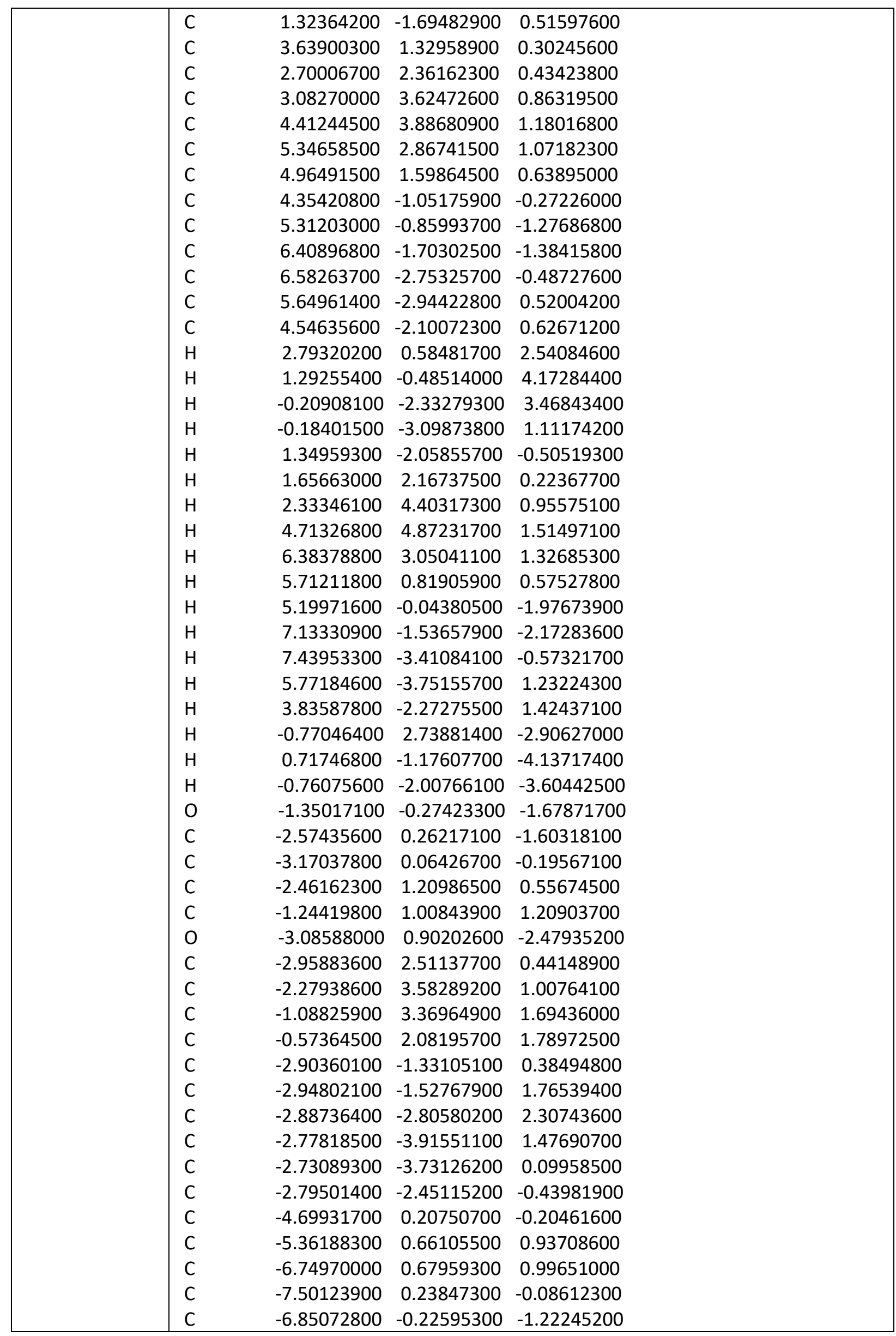




\begin{tabular}{|c|c|c|c|c|}
\hline & $C$ & -5.46203300 & -0.24547100 & -1.28028900 \\
\hline & $\mathrm{H}$ & -0.80353900 & 0.01924900 & 1.25373400 \\
\hline & $\mathrm{H}$ & -3.87778000 & 2.68485400 & -0.10617100 \\
\hline & $\mathrm{H}$ & -2.68250000 & 4.58388700 & 0.90958500 \\
\hline & $\mathrm{H}$ & -0.56302400 & 4.20168900 & 2.14918800 \\
\hline & $\mathrm{H}$ & 0.36144300 & 1.90235800 & 2.30701200 \\
\hline & $\mathrm{H}$ & -3.03969900 & -0.67301200 & 2.42613600 \\
\hline & $\mathrm{H}$ & -2.93369300 & -2.93355500 & 3.38290000 \\
\hline & $\mathrm{H}$ & -2.73486900 & -4.91323100 & 1.89712800 \\
\hline & $\mathrm{H}$ & -2.65158000 & -4.58621600 & -0.56162100 \\
\hline & $\mathrm{H}$ & -2.77115600 & -2.33193200 & -1.51673400 \\
\hline & $\mathrm{H}$ & -4.78829600 & 1.00673700 & 1.78950600 \\
\hline & $\mathrm{H}$ & -7.24226100 & 1.03876300 & 1.89232500 \\
\hline & $\mathrm{H}$ & -8.58363000 & 0.25425100 & -0.04355100 \\
\hline & $\mathrm{H}$ & -7.42463300 & -0.57439100 & -2.07295800 \\
\hline & $\mathrm{H}$ & -4.97567200 & -0.60616300 & -2.17694100 \\
\hline \multirow[t]{36}{*}{21} & 0 & -1.34710800 & 0.18730500 & 1.63837100 \\
\hline & $\mathrm{C}$ & -2.64997500 & 0.47800100 & 1.55696000 \\
\hline & $\mathrm{C}$ & -0.61571100 & 0.73227100 & 2.75119000 \\
\hline & $\mathrm{H}$ & -1.19863500 & 0.56640500 & 3.66208400 \\
\hline & $\mathrm{C}$ & 0.65654100 & -0.09240200 & 2.84120300 \\
\hline & $\mathrm{H}$ & 1.27025300 & 0.28883500 & 3.66201800 \\
\hline & $\mathrm{C}$ & -0.38206000 & 2.21760900 & 2.53839100 \\
\hline & $\mathrm{H}$ & 0.24775200 & 2.61413200 & 3.33763100 \\
\hline & $\mathrm{H}$ & -1.33394500 & 2.74827600 & 2.54837400 \\
\hline & $\mathrm{C}$ & 0.37564400 & -1.57567400 & 3.02411500 \\
\hline & $\mathrm{H}$ & -0.24421700 & -1.72826400 & 3.91086400 \\
\hline & 0 & -3.26036400 & 1.04439900 & 2.42037200 \\
\hline & $\mathrm{C}$ & -3.19055800 & 0.09759200 & 0.16529600 \\
\hline & $\mathrm{C}$ & -4.72399300 & 0.15723800 & 0.11230000 \\
\hline & $\mathrm{C}$ & -5.37469400 & 0.51969700 & -1.06777400 \\
\hline & $\mathrm{C}$ & -6.75983600 & 0.45730200 & -1.16676500 \\
\hline & $\mathrm{C}$ & -7.51894000 & 0.02644500 & -0.08615800 \\
\hline & $\mathrm{C}$ & -6.87900000 & -0.35016000 & 1.08869800 \\
\hline & $\mathrm{C}$ & -5.49422500 & -0.29238100 & 1.18529500 \\
\hline & $\mathrm{C}$ & -2.83164100 & -1.34317800 & -0.22048100 \\
\hline & $\mathrm{C}$ & -2.60373200 & -2.32010700 & 0.74790500 \\
\hline & $\mathrm{C}$ & -2.45576400 & -3.65736800 & 0.39202700 \\
\hline & $\mathrm{C}$ & -2.54215900 & -4.04026000 & -0.94068000 \\
\hline & $\mathrm{C}$ & -2.76552600 & -3.07223000 & -1.91496600 \\
\hline & $\mathrm{C}$ & -2.90707600 & -1.73800300 & -1.55710800 \\
\hline & $\mathrm{C}$ & -2.51266600 & 1.17670000 & -0.70246800 \\
\hline & $\mathrm{C}$ & -1.30821100 & 0.93946900 & -1.36633900 \\
\hline & $\mathrm{C}$ & -0.67474600 & 1.97006900 & -2.05534200 \\
\hline & $\mathrm{C}$ & -1.21526800 & 3.25055300 & -2.06492000 \\
\hline & $\mathrm{C}$ & -2.39382000 & 3.50220500 & -1.37073700 \\
\hline & $\mathrm{C}$ & -3.03430200 & 2.47322900 & -0.69142100 \\
\hline & $\mathrm{H}$ & -4.79694900 & 0.85914500 & -1.91953000 \\
\hline & $\mathrm{H}$ & -7.24372000 & 0.74626100 & -2.09222700 \\
\hline & $\mathrm{H}$ & -8.59897300 & -0.01869400 & -0.15945600 \\
\hline & $\mathrm{H}$ & -7.45885800 & -0.69191500 & 1.93787900 \\
\hline & $\mathrm{H}$ & -5.01472600 & -0.59115700 & 2.10788500 \\
\hline
\end{tabular}




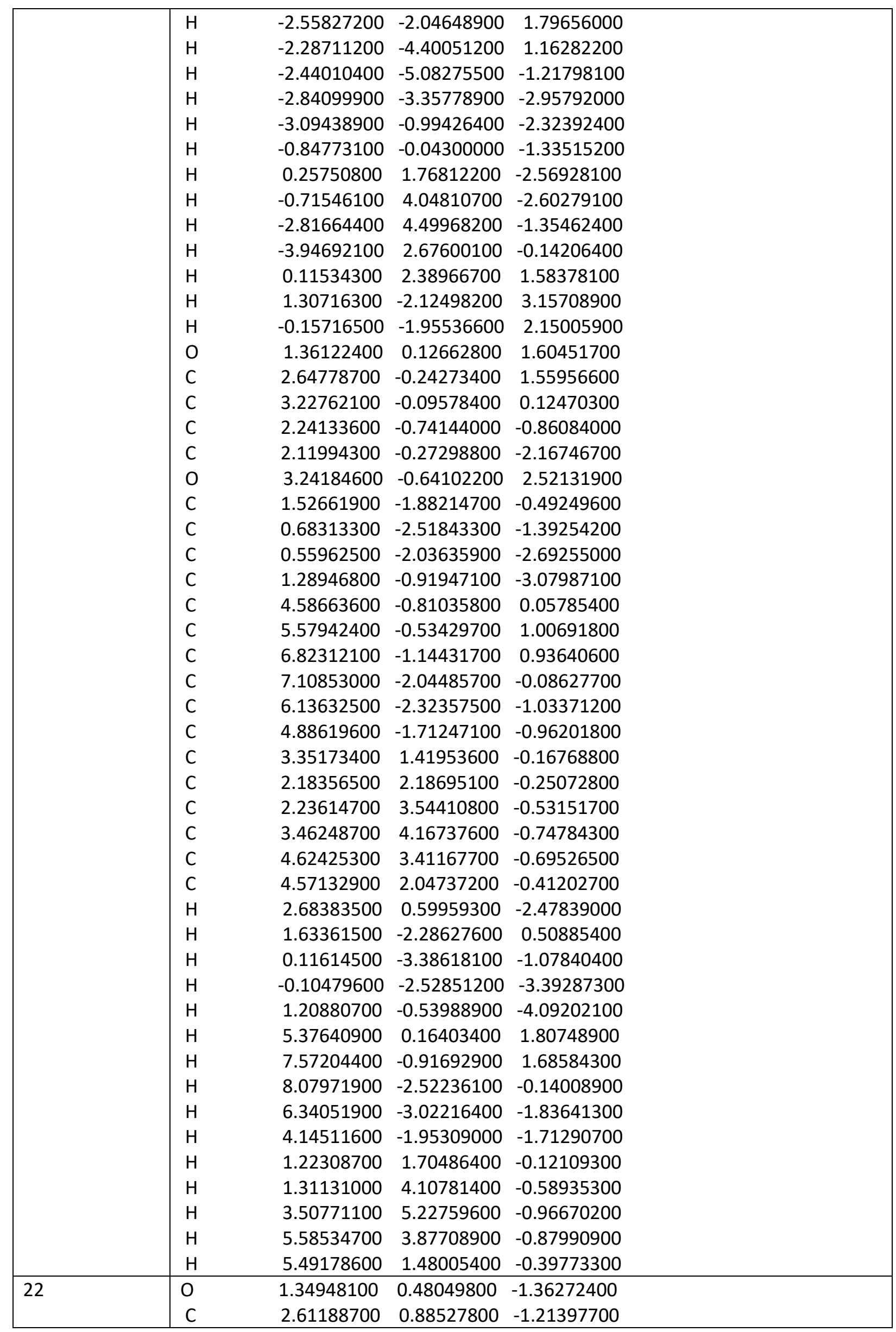




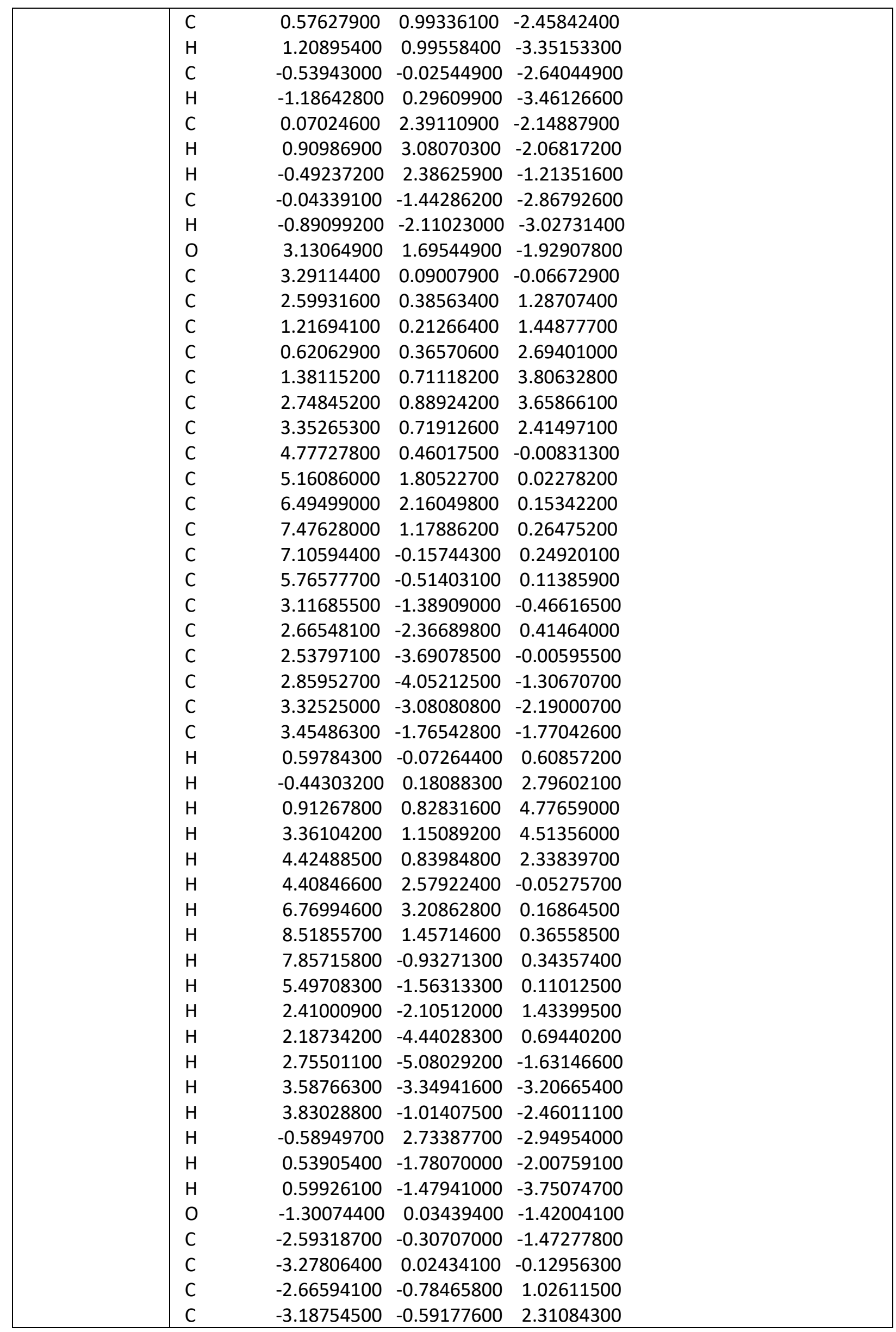




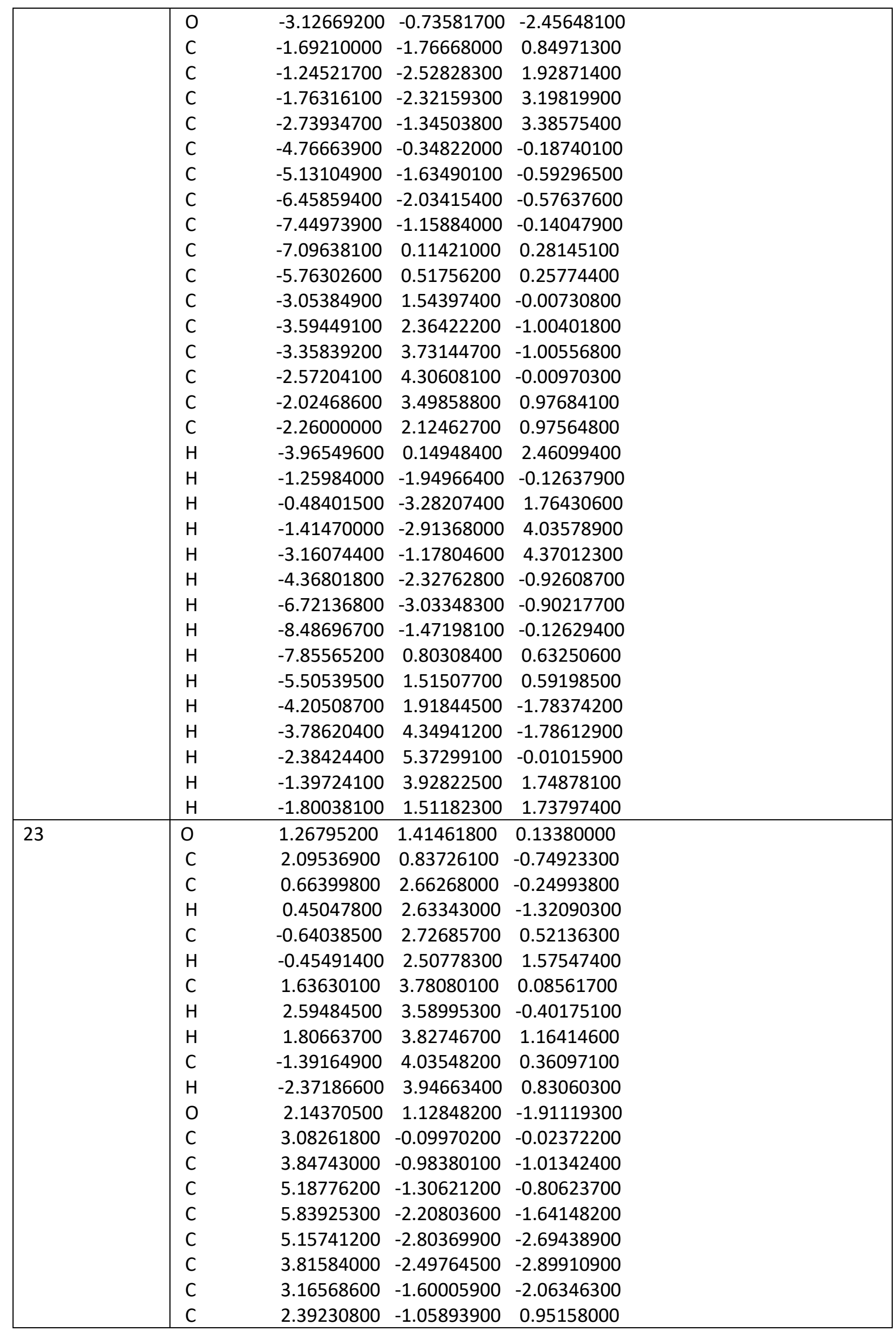




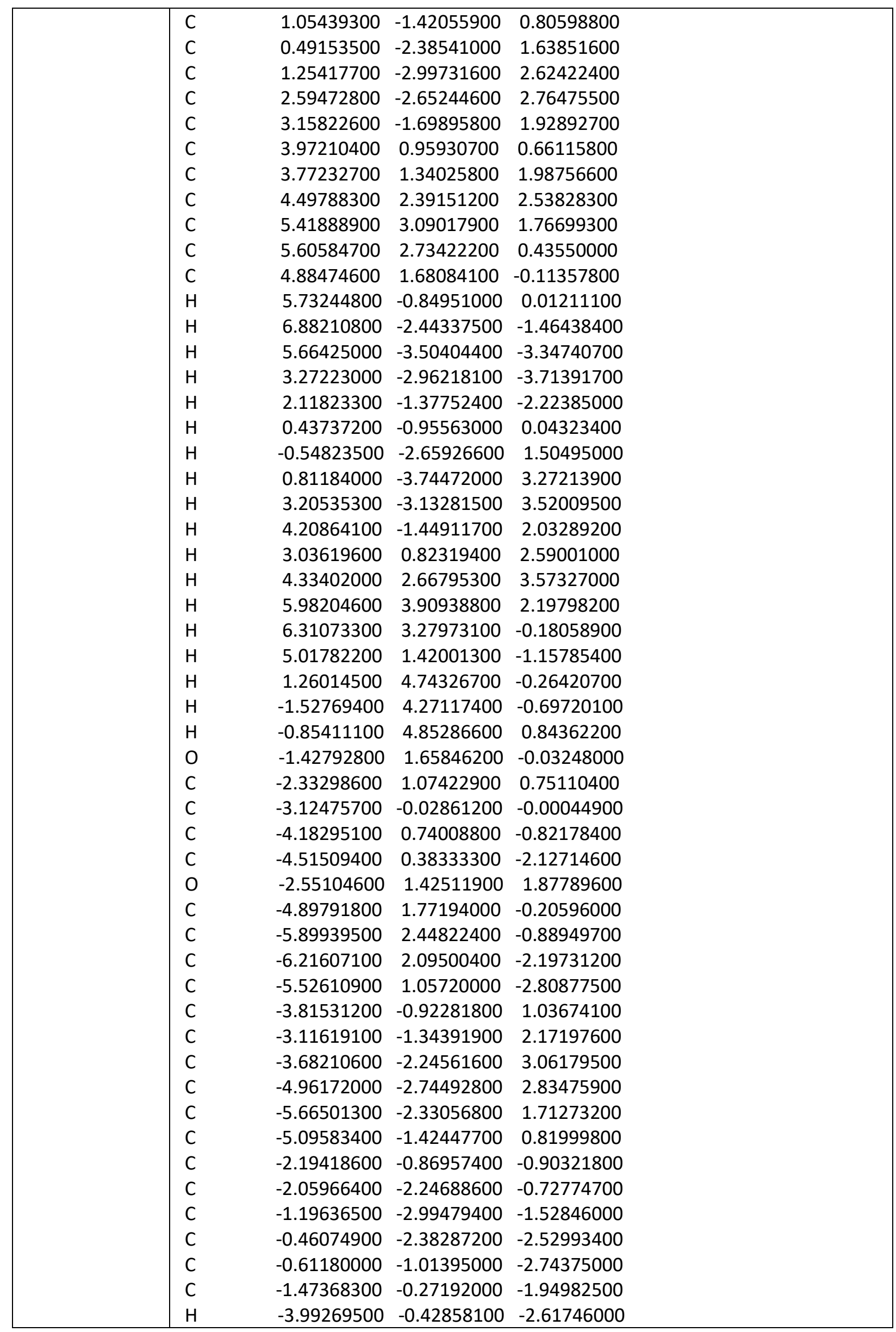




\begin{tabular}{|c|c|c|c|c|}
\hline & $\mathrm{H}$ & -4.68027600 & 2.03193800 & 0.82443800 \\
\hline & $\mathrm{H}$ & -6.43820100 & 3.24792400 & -0.39501400 \\
\hline & $\mathrm{H}$ & -6.99873300 & 2.62073900 & -2.73115900 \\
\hline & $\mathrm{H}$ & -5.77157800 & 0.76413300 & -3.82276500 \\
\hline & $\mathrm{H}$ & -2.12057000 & -0.96349500 & 2.36515800 \\
\hline & $\mathrm{H}$ & -3.12287900 & -2.55394200 & 3.93750500 \\
\hline & $\mathrm{H}$ & -5.40511900 & -3.44715200 & 3.53076600 \\
\hline & $\mathrm{H}$ & -6.66307500 & -2.70872000 & 1.52561200 \\
\hline & $\mathrm{H}$ & -5.65891700 & -1.11368400 & -0.05187700 \\
\hline & $\mathrm{H}$ & -2.62399000 & -2.75616200 & 0.04257800 \\
\hline & $\mathrm{H}$ & -1.10063200 & -4.05981700 & -1.35391100 \\
\hline & $\mathrm{H}$ & 0.21806900 & -2.96358700 & -3.14394500 \\
\hline & $\mathrm{H}$ & -0.05362200 & -0.51595300 & -3.52842900 \\
\hline & $\mathrm{H}$ & -1.59120100 & 0.78403500 & -2.14684700 \\
\hline \multirow[t]{38}{*}{25} & 0 & 1.37529200 & 1.69381400 & 0.34470500 \\
\hline & $\mathrm{C}$ & 2.03798800 & 1.04619200 & -0.62457700 \\
\hline & $\mathrm{C}$ & 0.63082800 & 2.87345700 & -0.01184400 \\
\hline & $\mathrm{H}$ & 0.41268000 & 2.83687100 & -1.08080200 \\
\hline & $\mathrm{C}$ & -0.67290700 & 2.78227300 & 0.76282900 \\
\hline & $\mathrm{H}$ & -0.46812800 & 2.53630100 & 1.80799700 \\
\hline & $\mathrm{C}$ & 1.47536200 & 4.08991300 & 0.32310600 \\
\hline & $\mathrm{H}$ & 0.99389800 & 5.00508800 & -0.02343500 \\
\hline & $\mathrm{H}$ & 2.44346800 & 4.00394500 & -0.17376600 \\
\hline & $\mathrm{C}$ & -1.53510000 & 4.02751700 & 0.65093600 \\
\hline & $\mathrm{H}$ & -2.49779800 & 3.84580100 & 1.12820700 \\
\hline & 0 & 1.98415200 & 1.36680100 & -1.77920300 \\
\hline & $\mathrm{C}$ & 2.94659500 & -0.06711200 & -0.03300700 \\
\hline & $\mathrm{C}$ & 3.12273100 & -1.23567500 & -1.02477700 \\
\hline & $\mathrm{C}$ & 3.14138300 & -2.55345800 & -0.56105400 \\
\hline & $\mathrm{C}$ & 3.37857900 & -3.61799200 & -1.42606200 \\
\hline & $\mathrm{C}$ & 3.59370000 & -3.38971900 & -2.77806900 \\
\hline & $\mathrm{C}$ & 3.58628300 & -2.08150000 & -3.25166200 \\
\hline & $\mathrm{C}$ & 3.36793700 & -1.01595000 & -2.38607200 \\
\hline & $\mathrm{C}$ & 2.34011700 & -0.59648100 & 1.27262900 \\
\hline & $\mathrm{C}$ & 1.00716300 & -1.01588900 & 1.25469900 \\
\hline & $\mathrm{C}$ & 0.43912900 & -1.62492200 & 2.36197900 \\
\hline & $\mathrm{C}$ & 1.19415100 & -1.81550800 & 3.51831000 \\
\hline & $\mathrm{C}$ & 2.51657400 & -1.39826200 & 3.54577300 \\
\hline & $\mathrm{C}$ & 3.09087300 & -0.79643700 & 2.42539200 \\
\hline & $\mathrm{C}$ & 4.29554700 & 0.64687400 & 0.22154200 \\
\hline & C & 4.32517300 & 1.78173600 & 1.04197000 \\
\hline & $\mathrm{C}$ & 5.51783800 & 2.44090200 & 1.30396600 \\
\hline & $\mathrm{C}$ & 6.71081200 & 1.97427700 & 0.75942100 \\
\hline & $\mathrm{C}$ & 6.69438600 & 0.84034100 & -0.03926500 \\
\hline & $\mathrm{C}$ & 5.49685400 & 0.18051100 & -0.30791600 \\
\hline & $\mathrm{H}$ & 2.96703600 & -2.76483100 & 0.48556100 \\
\hline & $\mathrm{H}$ & 3.38907700 & -4.62828300 & -1.03420400 \\
\hline & $\mathrm{H}$ & 3.77289700 & -4.21700200 & -3.45471400 \\
\hline & $\mathrm{H}$ & 3.76524900 & -1.88267500 & -4.30202800 \\
\hline & $\mathrm{H}$ & 3.39068800 & -0.00715400 & -2.77055200 \\
\hline & $\mathrm{H}$ & 0.41840700 & -0.87968800 & 0.35410000 \\
\hline & $\mathrm{H}$ & -0.58868000 & -1.96681700 & 2.31260400 \\
\hline
\end{tabular}




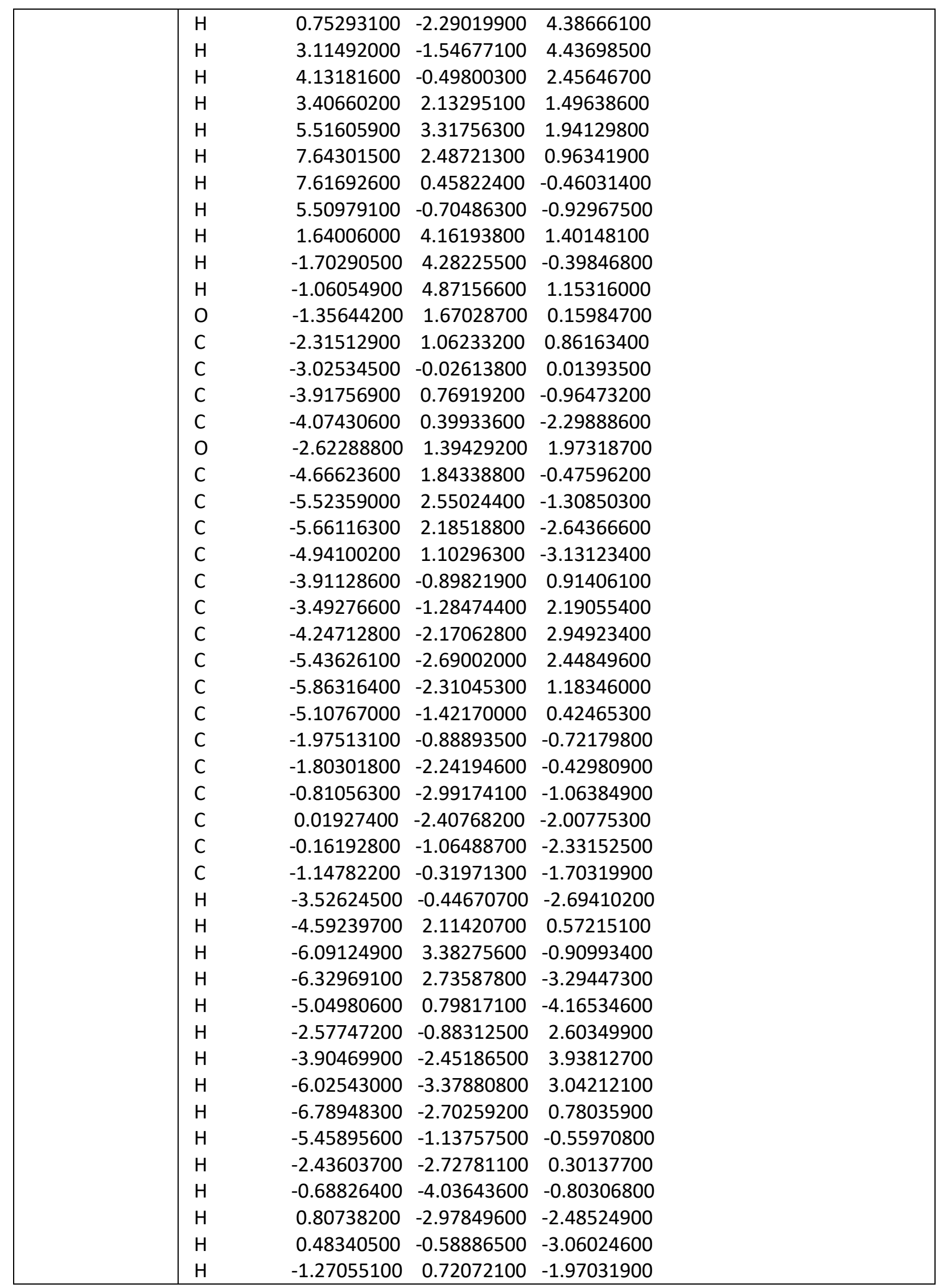


21 (optimized at the B3LYP/6-311G(d,p) level)

\begin{tabular}{|c|c|c|c|c|}
\hline \multicolumn{5}{|l|}{ Conformer no } \\
\hline \multirow[t]{50}{*}{1} & 0 & -1.75074800 & -1.68170200 & -0.23110800 \\
\hline & C & -2.08266200 & -0.46972200 & -0.72088900 \\
\hline & C & -0.70077500 & -2.43132300 & -0.93092500 \\
\hline & $\mathrm{H}$ & -0.02219700 & -1.70628600 & -1.37730100 \\
\hline & C & -1.33451300 & -3.28087100 & -2.02408100 \\
\hline & $\mathrm{H}$ & -0.55560100 & -3.81260100 & -2.57669200 \\
\hline & $\mathrm{H}$ & -1.87975900 & -2.65287900 & -2.73026300 \\
\hline & $\mathrm{O}$ & -1.50974200 & 0.03195700 & -1.65514100 \\
\hline & C & -3.28657900 & 0.17440800 & 0.05127300 \\
\hline & C & -3.07986100 & -0.04760800 & 1.56763800 \\
\hline & C & -1.83330500 & 0.23914800 & 2.14031000 \\
\hline & C & -1.62174000 & 0.10189200 & 3.50762500 \\
\hline & C & -2.66085000 & -0.31694100 & 4.33854700 \\
\hline & C & -3.90601600 & -0.59253400 & 3.78431800 \\
\hline & C & -4.11359300 & -0.45976700 & 2.41013400 \\
\hline & C & -4.59358300 & -0.52216700 & -0.43874200 \\
\hline & C & -4.69551100 & -1.92053200 & -0.48935700 \\
\hline & C & -5.87614600 & -2.54329200 & -0.88442600 \\
\hline & C & -6.99361500 & -1.78643800 & -1.22743800 \\
\hline & C & -6.91504800 & -0.39986500 & -1.16072200 \\
\hline & C & -5.73025600 & 0.22402900 & -0.77118100 \\
\hline & C & -3.32835600 & 1.68977800 & -0.27662200 \\
\hline & C & -3.41127300 & 2.65715200 & 0.72869400 \\
\hline & C & -3.48899400 & 4.01651100 & 0.42010600 \\
\hline & C & -3.48868700 & 4.43763300 & -0.90362600 \\
\hline & C & -3.42163300 & 3.48367500 & -1.91864500 \\
\hline & C & -3.34764000 & 2.13003400 & -1.61018100 \\
\hline & $\mathrm{H}$ & -1.01211500 & 0.58612500 & 1.52368200 \\
\hline & $\mathrm{H}$ & -0.64219500 & 0.32411900 & 3.91497700 \\
\hline & $\mathrm{H}$ & -2.49980400 & -0.42347900 & 5.40556200 \\
\hline & $\mathrm{H}$ & -4.72555600 & -0.91367100 & 4.41791800 \\
\hline & $\mathrm{H}$ & -5.09107100 & -0.67774200 & 2.00065300 \\
\hline & $\mathrm{H}$ & -3.85081600 & -2.52802200 & -0.20002800 \\
\hline & $\mathrm{H}$ & -5.92110900 & -3.62645400 & -0.91729300 \\
\hline & $\mathrm{H}$ & -7.91307700 & -2.27143100 & -1.53543500 \\
\hline & $\mathrm{H}$ & -7.77711400 & 0.20832200 & -1.41161900 \\
\hline & $\mathrm{H}$ & -5.70057500 & 1.30339700 & -0.72476100 \\
\hline & $\mathrm{H}$ & -3.41620100 & 2.36005700 & 1.76791700 \\
\hline & $\mathrm{H}$ & -3.54797200 & 4.74117900 & 1.22455300 \\
\hline & $\mathrm{H}$ & -3.54574300 & 5.49321400 & -1.14530600 \\
\hline & $\mathrm{H}$ & -3.42936900 & 3.79295900 & -2.95814700 \\
\hline & $\mathrm{H}$ & -3.30311600 & 1.41106400 & -2.41479900 \\
\hline & $\mathrm{H}$ & -2.02353500 & -4.01778600 & -1.60284700 \\
\hline & C & 0.02536100 & -3.25281800 & 0.12833900 \\
\hline & C & 0.79370300 & -2.41919000 & 1.14856000 \\
\hline & $\mathrm{H}$ & 0.14127000 & -1.67273400 & 1.59768600 \\
\hline & C & 1.45783800 & -3.25264200 & 2.23505700 \\
\hline & $\mathrm{H}$ & 2.02191900 & -2.61379800 & 2.91678600 \\
\hline & $\mathrm{H}$ & 2.13800700 & -3.99057800 & 1.80153100 \\
\hline & $\mathrm{H}$ & 0.69784500 & -3.78058900 & 2.81703000 \\
\hline
\end{tabular}




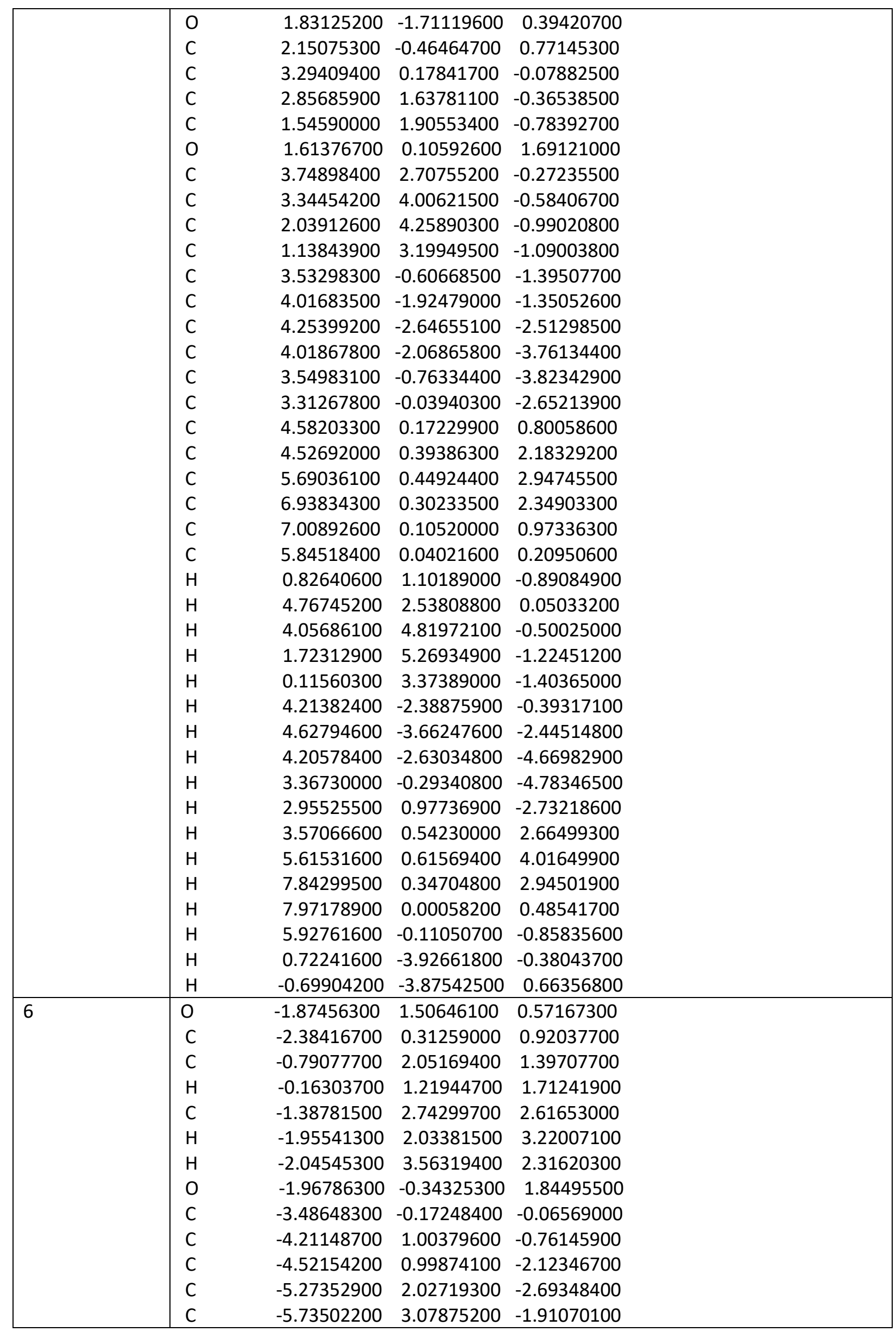




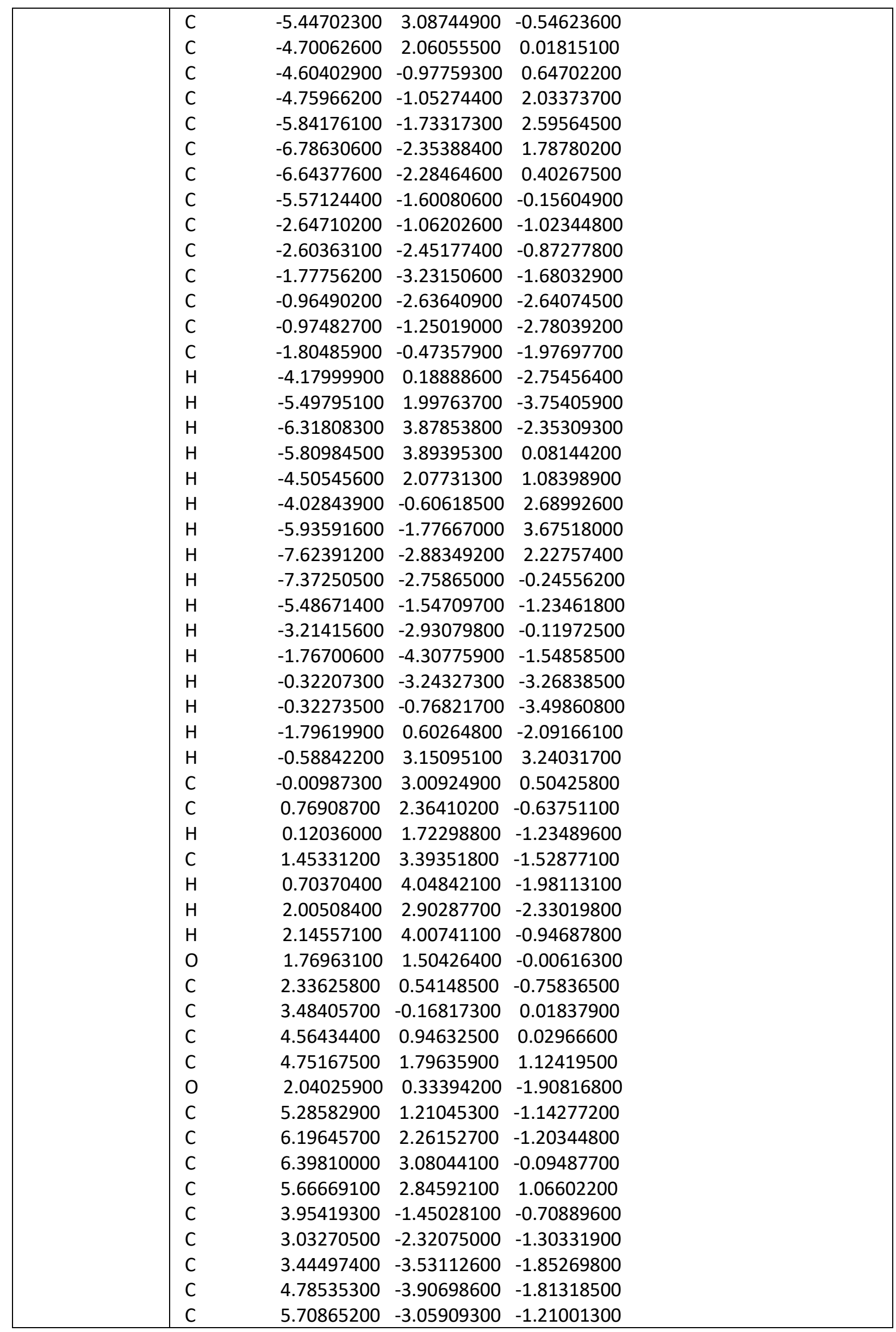




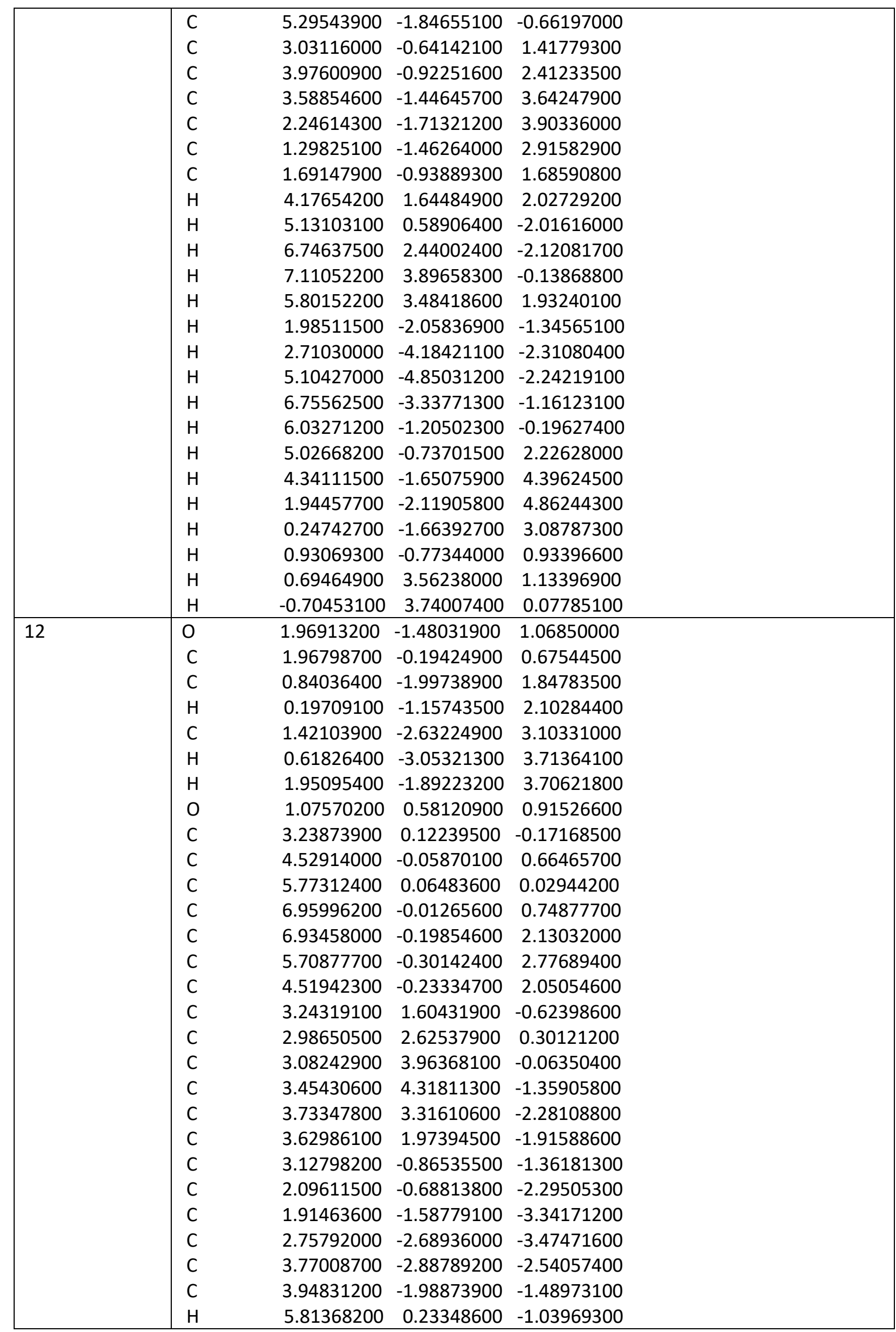




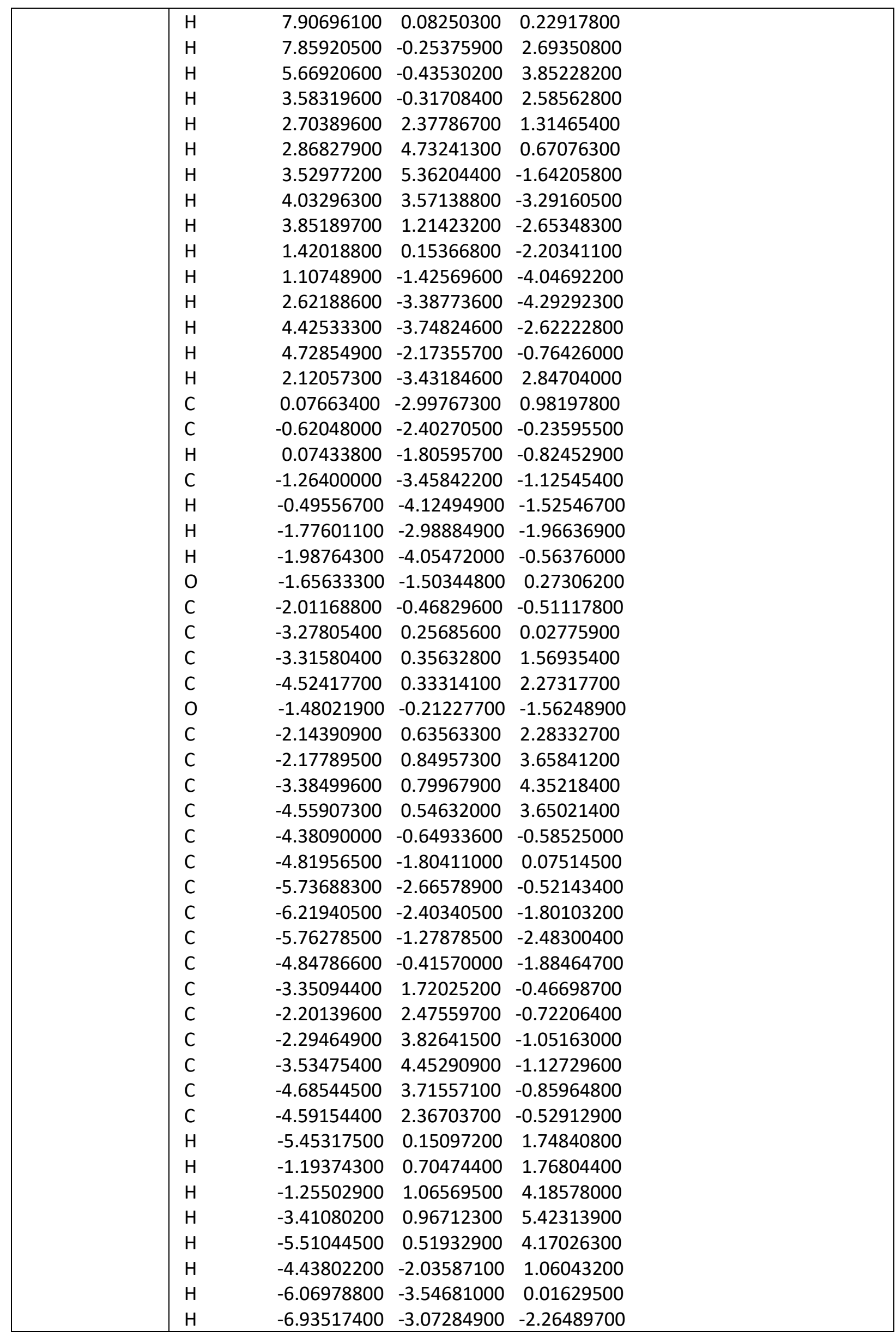




\begin{tabular}{|c|c|c|c|c|}
\hline & $\mathrm{H}$ & -6.11417600 & -1.07046000 & -3.48747900 \\
\hline & $\mathrm{H}$ & -4.48982400 & 0.44431300 & -2.43494400 \\
\hline & $\mathrm{H}$ & -1.22339700 & 2.01933300 & -0.66869400 \\
\hline & $\mathrm{H}$ & -1.38716800 & 4.38630900 & -1.24876700 \\
\hline & $\mathrm{H}$ & -3.60444000 & 5.50351200 & -1.38641900 \\
\hline & $\mathrm{H}$ & -5.66020800 & 4.18899000 & -0.90370300 \\
\hline & $\mathrm{H}$ & -5.49848900 & 1.81428800 & -0.31649500 \\
\hline & $\mathrm{H}$ & -0.67094400 & -3.49332900 & 1.61059800 \\
\hline & $\mathrm{H}$ & 0.77379300 & -3.76777900 & 0.63580700 \\
\hline \multirow[t]{43}{*}{15} & 0 & 1.93377900 & -1.69332700 & 0.60517900 \\
\hline & $\mathrm{C}$ & 1.90141500 & -0.36151900 & 0.43153100 \\
\hline & $\mathrm{C}$ & 0.84706100 & -2.33435500 & 1.35316300 \\
\hline & $\mathrm{H}$ & 0.21319600 & -1.54683900 & 1.75517500 \\
\hline & C & 1.49779500 & -3.12808600 & 2.47608600 \\
\hline & $\mathrm{H}$ & 0.72951000 & -3.60611400 & 3.08921300 \\
\hline & $\mathrm{H}$ & 2.08894300 & -2.47136000 & 3.11674800 \\
\hline & 0 & 0.97859200 & 0.34128500 & 0.76554300 \\
\hline & C & 3.24673100 & 0.19061200 & -0.11886700 \\
\hline & $\mathrm{C}$ & 3.03237300 & 1.50723300 & -0.90211600 \\
\hline & $\mathrm{C}$ & 4.07675100 & 2.43353200 & -1.00610700 \\
\hline & C & 3.95268700 & 3.57351200 & -1.79531100 \\
\hline & $\mathrm{C}$ & 2.77977100 & 3.80817700 & -2.50818100 \\
\hline & C & 1.74028200 & 2.88617500 & -2.42696300 \\
\hline & $\mathrm{C}$ & 1.86481300 & 1.74582200 & -1.63557800 \\
\hline & C & 3.95521500 & -0.75758100 & -1.11202100 \\
\hline & C & 3.22006400 & -1.52214200 & -2.02459600 \\
\hline & $\mathrm{C}$ & 3.85277100 & -2.28423400 & -3.00211400 \\
\hline & C & 5.24192000 & -2.29331900 & -3.09717800 \\
\hline & $\mathrm{C}$ & 5.98645700 & -1.52135300 & -2.21050100 \\
\hline & C & 5.34920700 & -0.75952500 & -1.23368000 \\
\hline & C & 4.00834200 & 0.38841300 & 1.22113400 \\
\hline & C & 4.75178300 & -0.65260900 & 1.79157700 \\
\hline & $\mathrm{C}$ & 5.34837600 & -0.50374900 & 3.04169400 \\
\hline & $\mathrm{C}$ & 5.19730500 & 0.68160800 & 3.75651300 \\
\hline & C & 4.43109300 & 1.71055600 & 3.21533400 \\
\hline & $\mathrm{C}$ & 3.83605300 & 1.56228700 & 1.96510800 \\
\hline & $\mathrm{H}$ & 4.99991100 & 2.26769200 & -0.46462000 \\
\hline & $\mathrm{H}$ & 4.77698800 & 4.27582100 & -1.85341700 \\
\hline & $\mathrm{H}$ & 2.67963000 & 4.69546000 & -3.12322200 \\
\hline & $\mathrm{H}$ & 0.82112200 & 3.04701200 & -2.97901000 \\
\hline & $\mathrm{H}$ & 1.03425300 & 1.05427500 & -1.59721800 \\
\hline & $\mathrm{H}$ & 2.13864000 & -1.51960500 & -1.98554600 \\
\hline & $\mathrm{H}$ & 3.25565100 & -2.86772500 & -3.69419500 \\
\hline & $\mathrm{H}$ & 5.73627300 & -2.88694600 & -3.85774700 \\
\hline & $\mathrm{H}$ & 7.06864800 & -1.50467300 & -2.27827600 \\
\hline & $\mathrm{H}$ & 5.95034100 & -0.15908300 & -0.56291900 \\
\hline & $\mathrm{H}$ & 4.86375900 & -1.58733900 & 1.25832200 \\
\hline & $\mathrm{H}$ & 5.92901400 & -1.32023600 & 3.45665200 \\
\hline & $\mathrm{H}$ & 5.66371700 & 0.79847300 & 4.72829000 \\
\hline & $\mathrm{H}$ & 4.28855500 & 2.63258500 & 3.76781000 \\
\hline & $\mathrm{H}$ & 3.22705700 & 2.36337000 & 1.56774900 \\
\hline & $\mathrm{H}$ & 2.15546700 & -3.90399600 & 2.07568800 \\
\hline
\end{tabular}




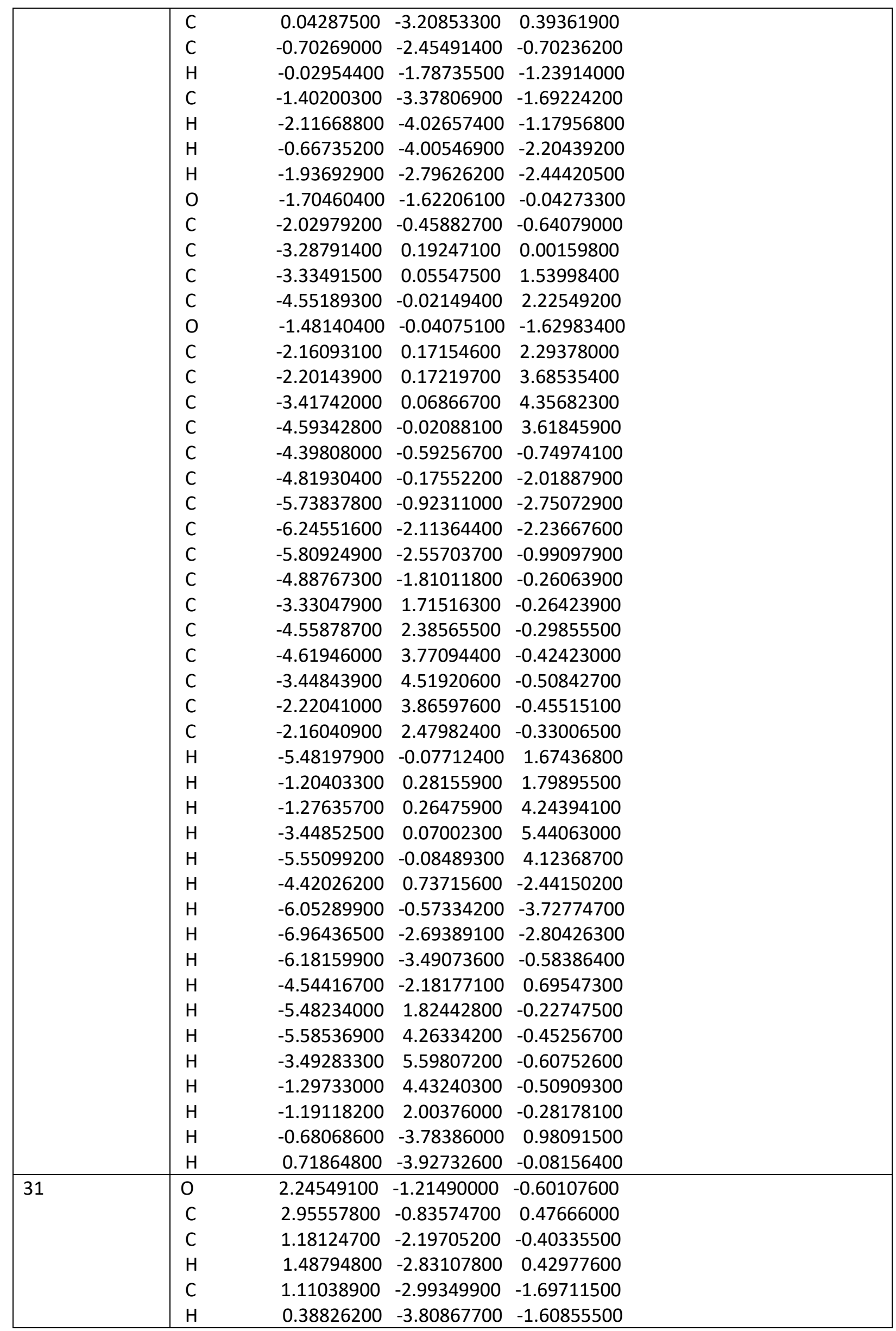




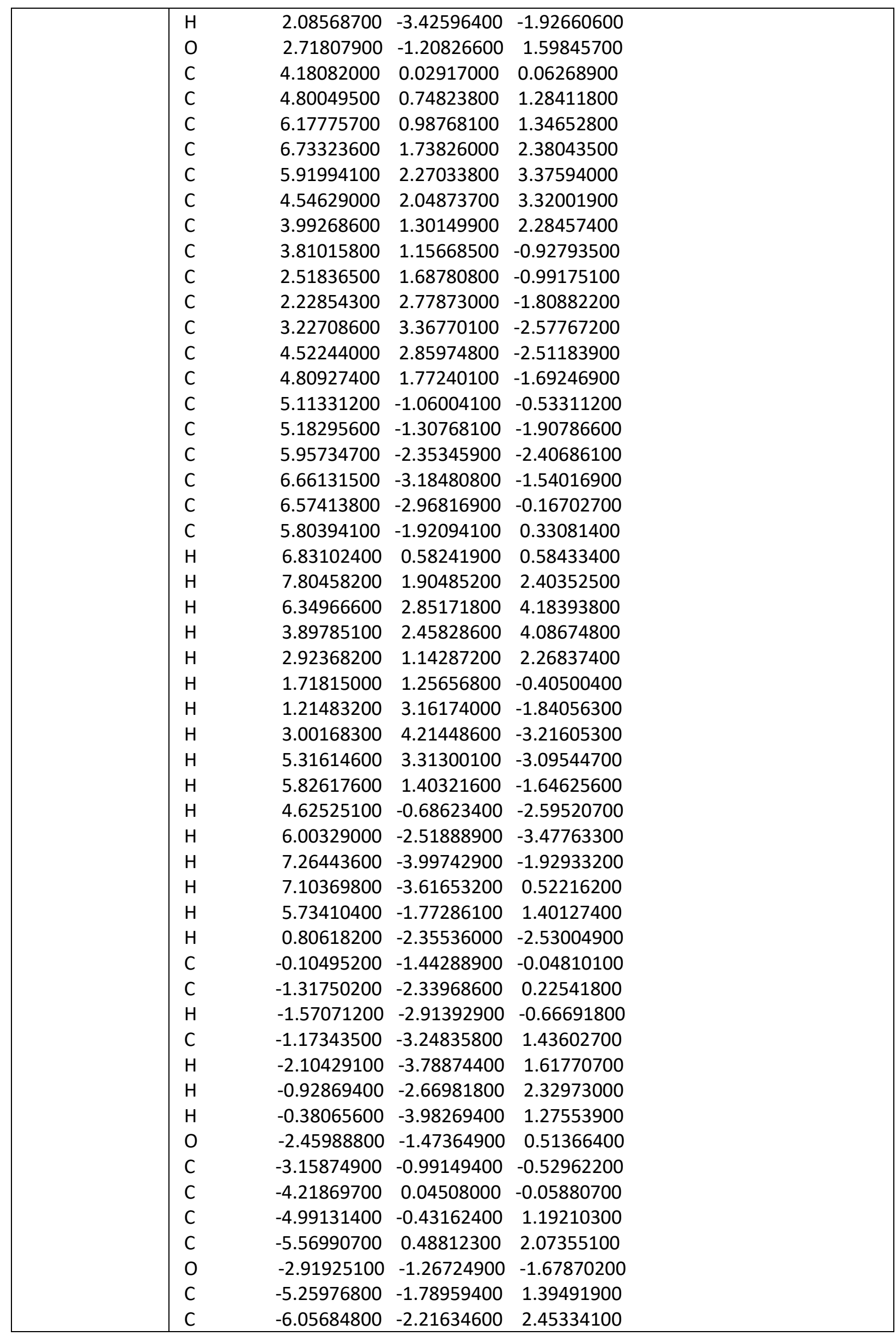




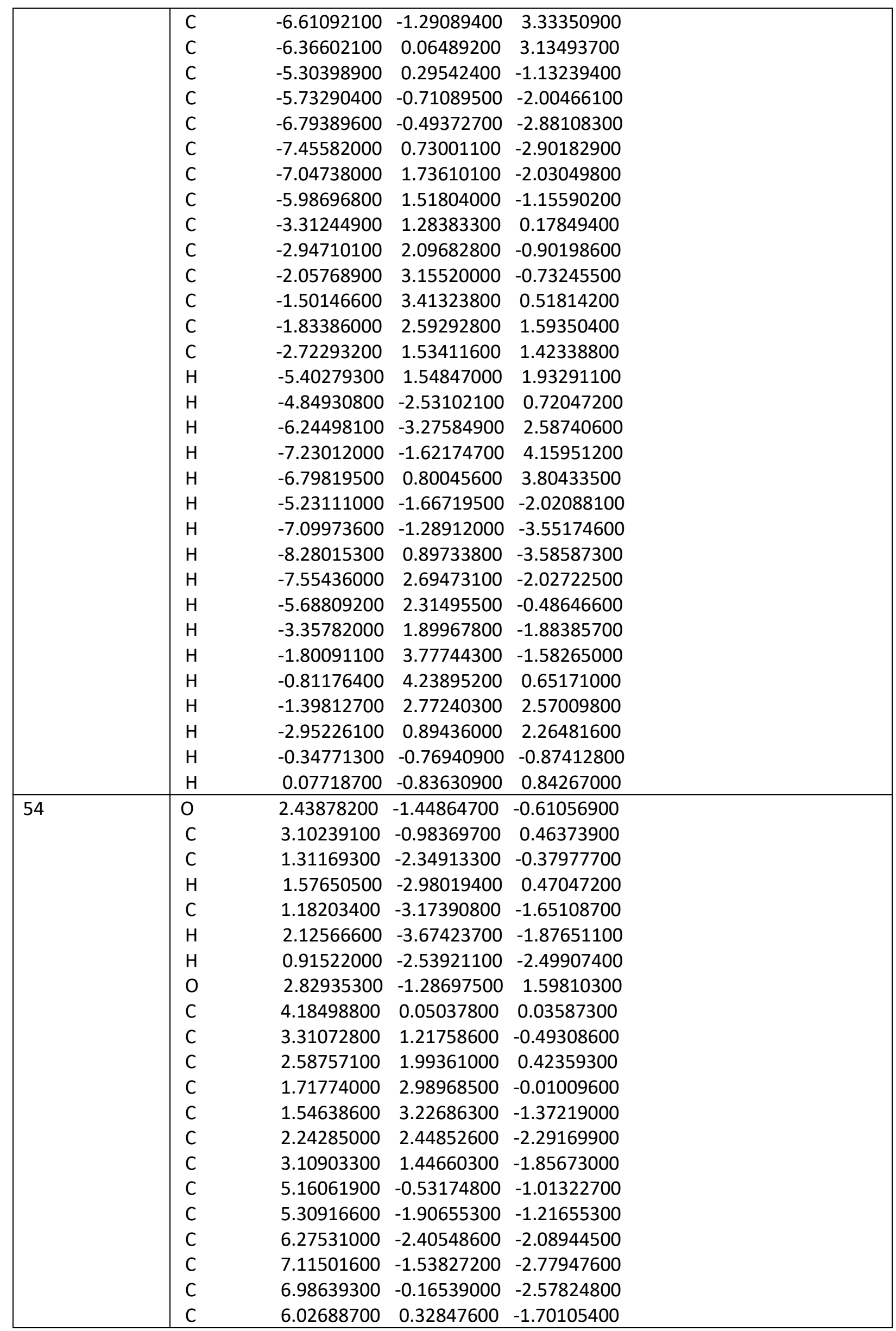




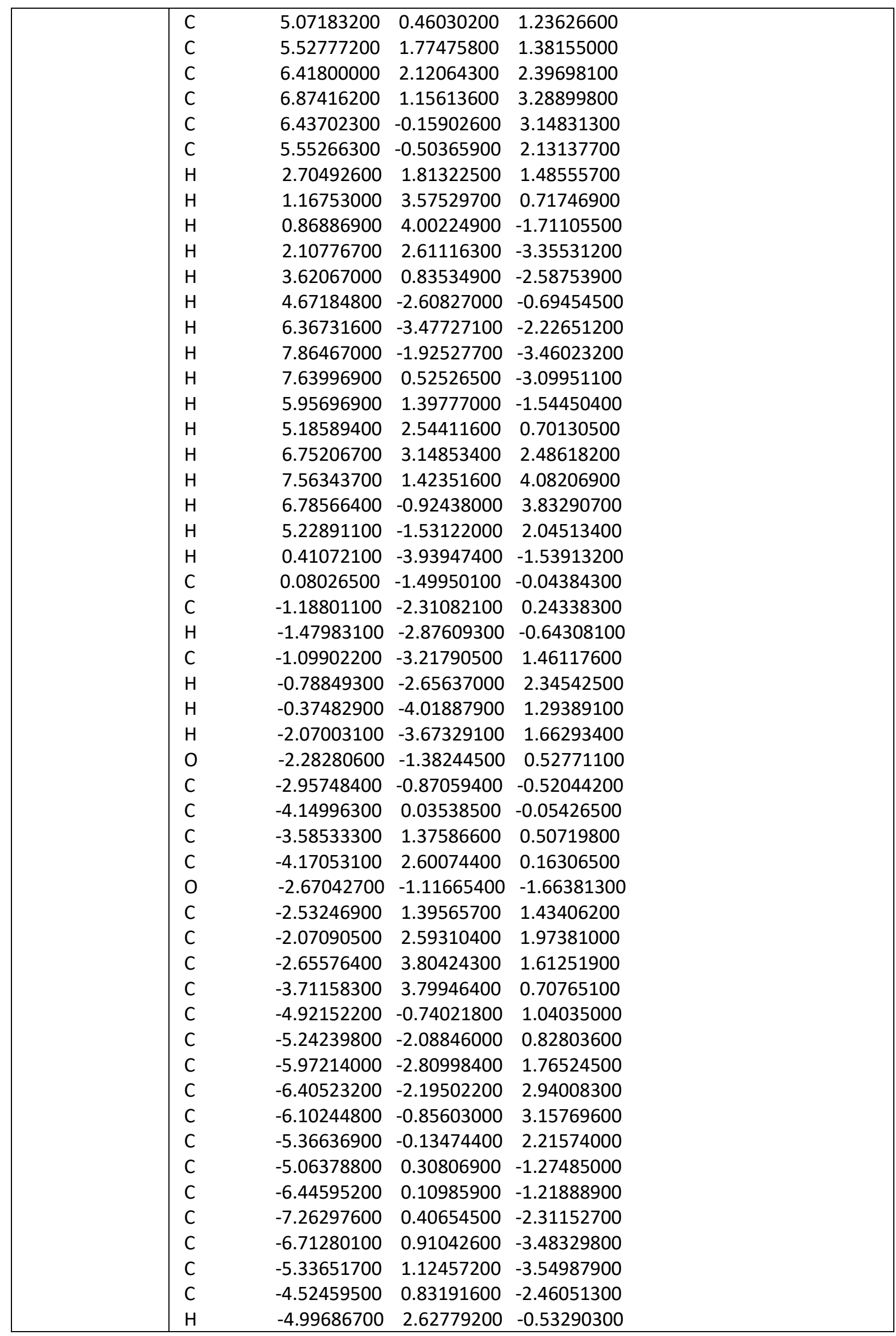




\begin{tabular}{|l|llll|}
\hline & $H$ & -2.07925200 & 0.46781500 & 1.74882000 \\
$H$ & -1.25366300 & 2.57322300 & 2.68639400 \\
$H$ & -2.29796000 & 4.73613600 & 2.03596600 \\
$H$ & -4.18864800 & 4.72994300 & 0.42016500 \\
$H$ & -4.93014200 & -2.57671700 & -0.08940700 \\
$H$ & -6.20539600 & -3.85235300 & 1.57782500 \\
$H$ & -6.97311500 & -2.75568900 & 3.67395600 \\
$H$ & -6.43536000 & -0.36300700 & 4.06436400 \\
$H$ & -5.14047800 & 0.90610900 & 2.40585900 \\
H & -6.90255400 & -0.27699800 & -0.31836200 \\
$H$ & -8.33197400 & 0.24014500 & -2.23695600 \\
H & -7.34514300 & 1.13778700 & -4.33435500 \\
$\mathrm{H}$ & -4.89065300 & 1.52173400 & -4.45516500 \\
$\mathrm{H}$ & -3.46171700 & 1.01131100 & -2.53466000 \\
$\mathrm{H}$ & -0.11209400 & -0.82687400 & -0.88283900 \\
$\mathrm{H}$ & 0.30807700 & -0.89049300 & 0.83431000 \\
\hline
\end{tabular}

21 (optimized at the B3LYP-GD3BJ/6-311G(d,p) level)

\begin{tabular}{|l|lrrr|}
\hline Conformer no & \multicolumn{4}{|c|}{} \\
\hline 1 & O & -1.60974500 & -1.72530100 & -0.20882400 \\
& C & -2.12580700 & -0.70336500 & -0.91331200 \\
& C & -0.69419200 & -2.62749900 & -0.89928000 \\
& H & 0.00431100 & -2.00838900 & -1.46087500 \\
& C & -1.45953500 & -3.55071300 & -1.83546700 \\
& H & -0.76375900 & -4.23905000 & -2.32186700 \\
& H & -1.97237700 & -2.97774700 & -2.60610900 \\
& O & -1.87887800 & -0.52563300 & -2.07907800 \\
& C & -3.09538100 & 0.14655400 & -0.03934600 \\
& C & -2.54324000 & 0.21577900 & 1.39444000 \\
& C & -1.21029400 & 0.59771800 & 1.58623200 \\
& C & -0.68526900 & 0.73948400 & 2.86388000 \\
& C & -1.49180100 & 0.50650800 & 3.97803800 \\
& C & -2.82016000 & 0.13873400 & 3.79810300 \\
& C & -3.34382400 & -0.00532200 & 2.51236600 \\
& C & -4.46242300 & -0.58567400 & -0.07924400 \\
& C & -4.54454200 & -1.95240200 & 0.21928800 \\
& C & -5.76931800 & -2.61027700 & 0.22706400 \\
& C & -6.94366000 & -1.91600500 & -0.05442700 \\
& C & -6.87595800 & -0.55663300 & -0.33626500 \\
& C & -5.64741400 & 0.10199000 & -0.34897800 \\
& C & -3.23289400 & 1.57167800 & -0.61378400 \\
& C & -3.21619700 & 2.68304400 & 0.23222100 \\
& C & -3.40257000 & 3.97112200 & -0.26615000 \\
& C & -3.60606000 & 4.17479200 & -1.62593500 \\
C & -3.64069300 & 3.07299400 & -2.47855200 \\
& C & -3.46901700 & 1.78687700 & -1.97818800 \\
H & -0.57993900 & 0.79951100 & 0.72919500 \\
& H & 0.35578300 & 1.00809300 & 2.98290800 \\
H & -1.08335400 & 0.61167500 & 4.97652600 \\
& H & -3.45789000 & -0.03907300 & 4.65668200 \\
H & -4.38060900 & -0.28674700 & 2.38760600 \\
\hline
\end{tabular}




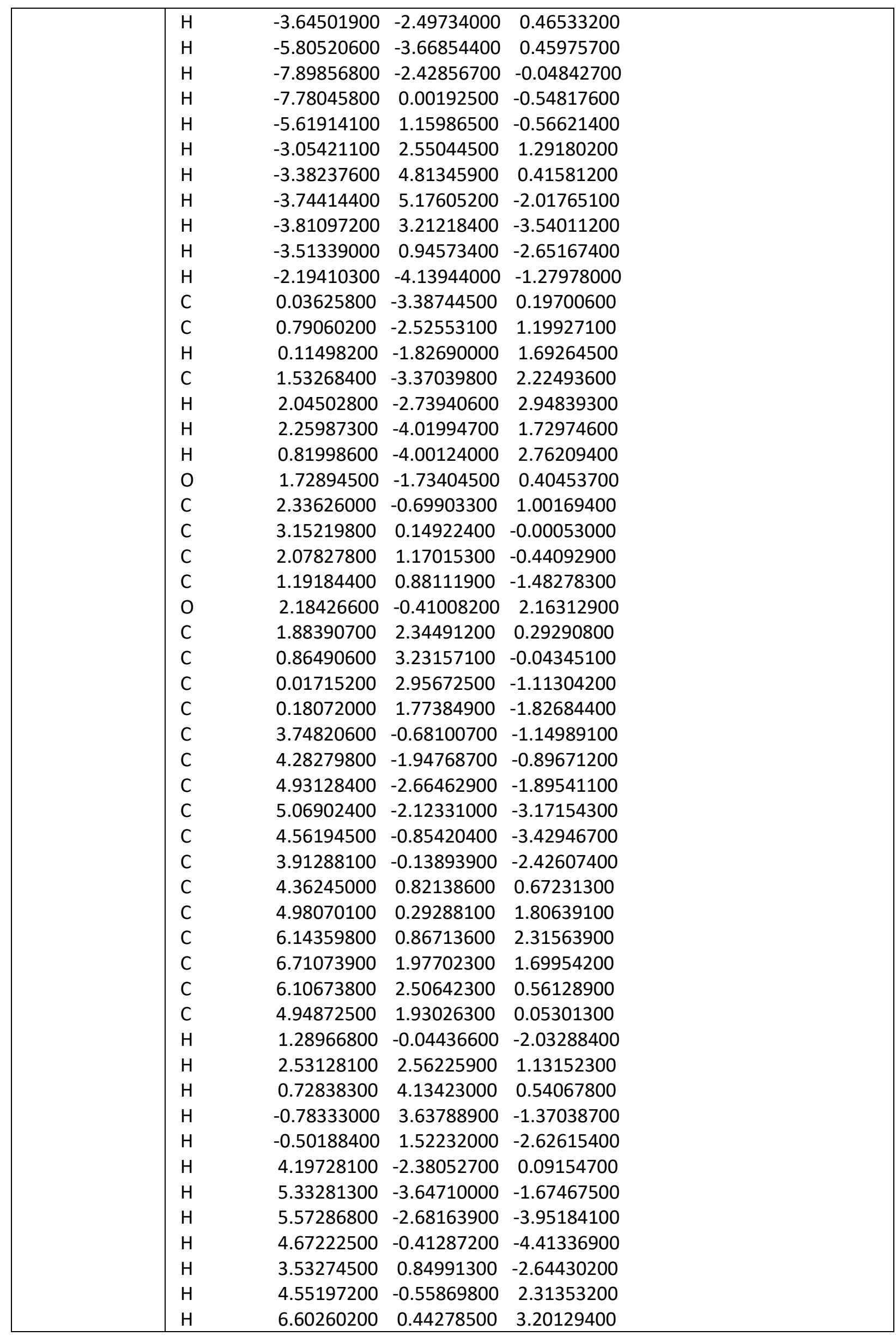




\begin{tabular}{|c|c|c|c|c|}
\hline & $\mathrm{H}$ & 7.61341900 & 2.42451000 & 2.09893600 \\
\hline & $\mathrm{H}$ & 6.53852300 & 3.36811400 & 0.06526200 \\
\hline & $\mathrm{H}$ & 4.49284500 & 2.34868400 & -0.83519000 \\
\hline & $\mathrm{H}$ & 0.74024000 & -4.07501500 & -0.28236200 \\
\hline & $\mathrm{H}$ & -0.68469600 & -3.99575300 & 0.75249200 \\
\hline 6 & 0 & -1.74761800 & 1.74055800 & 0.35475500 \\
\hline & $\mathrm{C}$ & -2.24157000 & 0.64187800 & 0.94285600 \\
\hline & $\mathrm{C}$ & -0.80086300 & 2.55123900 & 1.11493700 \\
\hline & $\mathrm{H}$ & -0.12688500 & 1.87321300 & 1.63703900 \\
\hline & C & -1.55215800 & 3.41316800 & 2.11726200 \\
\hline & $\mathrm{H}$ & -2.07611100 & 2.78939400 & 2.84083700 \\
\hline & $\mathrm{H}$ & -2.27412300 & 4.05346300 & 1.60400000 \\
\hline & 0 & -1.92546500 & 0.29015900 & 2.05476100 \\
\hline & $\mathrm{C}$ & -3.17214300 & -0.16307800 & 0.00360600 \\
\hline & $\mathrm{C}$ & -3.65022600 & 0.69083600 & -1.18650800 \\
\hline & C & -3.54863300 & 0.27306700 & -2.51167500 \\
\hline & $\mathrm{C}$ & -4.07097600 & 1.05020400 & -3.54726300 \\
\hline & C & -4.70764200 & 2.25311300 & -3.27111000 \\
\hline & $\mathrm{C}$ & -4.83252500 & 2.67034600 & -1.94541300 \\
\hline & C & -4.31620900 & 1.89317200 & -0.91899500 \\
\hline & $\mathrm{C}$ & -4.44725000 & -0.65272200 & 0.72392000 \\
\hline & $\mathrm{C}$ & -4.71225500 & -0.47140900 & 2.08131900 \\
\hline & $\mathrm{C}$ & -5.90689700 & -0.93040400 & 2.63927800 \\
\hline & $\mathrm{C}$ & -6.84886500 & -1.58548600 & 1.85696200 \\
\hline & $\mathrm{C}$ & -6.59070800 & -1.77633600 & 0.50012100 \\
\hline & C & -5.40839200 & -1.30937100 & -0.05715300 \\
\hline & C & -2.26616100 & -1.34640100 & -0.40754100 \\
\hline & $\mathrm{C}$ & -2.48081700 & -2.63796200 & 0.07505000 \\
\hline & C & -1.60802000 & -3.67180400 & -0.25626100 \\
\hline & $\mathrm{C}$ & -0.50412300 & -3.43049800 & -1.06663500 \\
\hline & $\mathrm{C}$ & -0.27262600 & -2.14270400 & -1.53855300 \\
\hline & C & -1.14132900 & -1.11041600 & -1.20488300 \\
\hline & $\mathrm{H}$ & -3.06529600 & -0.66473800 & -2.74737000 \\
\hline & $\mathrm{H}$ & -3.97798100 & 0.70495900 & -4.57058900 \\
\hline & $\mathrm{H}$ & -5.11173500 & 2.85733400 & -4.07500800 \\
\hline & $\mathrm{H}$ & -5.34058200 & 3.59938500 & -1.71295300 \\
\hline & $\mathrm{H}$ & -4.43810400 & 2.21755800 & 0.10757400 \\
\hline & $\mathrm{H}$ & -3.98337000 & 0.00558500 & 2.71740400 \\
\hline & $\mathrm{H}$ & -6.09098800 & -0.77451600 & 3.69622700 \\
\hline & $\mathrm{H}$ & -7.77385500 & -1.94280200 & 2.29446500 \\
\hline & $\mathrm{H}$ & -7.31471400 & -2.28365400 & -0.12712000 \\
\hline & $\mathrm{H}$ & -5.22692600 & -1.45070400 & -1.11493800 \\
\hline & $\mathrm{H}$ & -3.32670600 & -2.83899500 & 0.71719800 \\
\hline & $\mathrm{H}$ & -1.78890800 & -4.66658200 & 0.13438200 \\
\hline & $\mathrm{H}$ & 0.18377500 & -4.23141200 & -1.30871900 \\
\hline & $\mathrm{H}$ & 0.59123100 & -1.92138300 & -2.15002900 \\
\hline & $\mathrm{H}$ & -0.93380900 & -0.11431800 & -1.57067300 \\
\hline & $\mathrm{H}$ & -0.84835400 & 4.04931400 & 2.65953000 \\
\hline & $\mathrm{C}$ & -0.04195800 & 3.37234700 & 0.08378900 \\
\hline & C & 0.67753500 & 2.55469300 & -0.97764900 \\
\hline & $\mathrm{H}$ & -0.02363600 & 1.89560100 & -1.48745200 \\
\hline & C & 1.41282600 & 3.42321300 & -1.98686400 \\
\hline
\end{tabular}




\begin{tabular}{|c|c|c|c|c|}
\hline & $\mathrm{H}$ & 0.70152600 & 4.07009200 & -2.50614400 \\
\hline & $\mathrm{H}$ & 1.91614200 & 2.80434300 & -2.72893600 \\
\hline & $\mathrm{H}$ & 2.15007900 & 4.05256600 & -1.48195300 \\
\hline & 0 & 1.63412500 & 1.71921800 & -0.25816500 \\
\hline & C & 2.13139400 & 0.64893500 & -0.90151500 \\
\hline & C & 3.16400400 & -0.12586100 & -0.02794700 \\
\hline & C & 3.79528300 & 0.83485900 & 0.99374700 \\
\hline & C & 3.86717800 & 0.53476300 & 2.35121700 \\
\hline & 0 & 1.77278400 & 0.32917000 & -2.00737400 \\
\hline & C & 4.37234500 & 2.02943300 & 0.54375100 \\
\hline & C & 4.99381300 & 2.90157700 & 1.42593400 \\
\hline & C & 5.05431300 & 2.59486600 & 2.78625500 \\
\hline & C & 4.49152500 & 1.40981600 & 3.24233700 \\
\hline & C & 4.25267300 & -0.74156900 & -0.93558500 \\
\hline & C & 3.89984100 & -1.53837800 & -2.03367500 \\
\hline & C & 4.87200300 & -2.14952100 & -2.81601100 \\
\hline & C & 6.22329000 & -1.99450600 & -2.51338100 \\
\hline & C & 6.58577700 & -1.22664300 & -1.41402800 \\
\hline & C & 5.60981900 & -0.60879500 & -0.63240100 \\
\hline & C & 2.37504600 & -1.24807100 & 0.68878100 \\
\hline & C & 2.90601300 & -2.53124000 & 0.82893500 \\
\hline & C & 2.21228200 & -3.51241000 & 1.53521900 \\
\hline & C & 0.97841000 & -3.22735900 & 2.10972200 \\
\hline & C & 0.44118000 & -1.94996100 & 1.98030600 \\
\hline & C & 1.13781500 & -0.97409800 & 1.27884800 \\
\hline & $\mathrm{H}$ & 3.43585900 & -0.38465000 & 2.72191800 \\
\hline & $\mathrm{H}$ & 4.34196900 & 2.27158000 & -0.51225300 \\
\hline & $\mathrm{H}$ & 5.43377700 & 3.82019800 & 1.05460500 \\
\hline & $\mathrm{H}$ & 5.53802700 & 3.27432500 & 3.47844200 \\
\hline & $\mathrm{H}$ & 4.53421100 & 1.15666500 & 4.29549000 \\
\hline & $\mathrm{H}$ & 2.85914500 & -1.68110200 & -2.27511800 \\
\hline & $\mathrm{H}$ & 4.56996200 & -2.75525200 & -3.66288700 \\
\hline & $\mathrm{H}$ & 6.98074500 & -2.47340300 & -3.12317600 \\
\hline & $\mathrm{H}$ & 7.63097100 & -1.10431800 & -1.15375200 \\
\hline & $\mathrm{H}$ & 5.91846200 & -0.02677000 & 0.22372200 \\
\hline & $\mathrm{H}$ & 3.86515600 & -2.76998700 & 0.39122800 \\
\hline & $\mathrm{H}$ & 2.64323800 & -4.50262300 & 1.63163400 \\
\hline & $\mathrm{H}$ & 0.43448400 & -3.99515500 & 2.64745400 \\
\hline & $\mathrm{H}$ & -0.52466700 & -1.70348300 & 2.40046800 \\
\hline & $\mathrm{H}$ & 0.71071800 & 0.01340700 & 1.19649000 \\
\hline & $\mathrm{H}$ & 0.68678200 & 3.99426400 & 0.61245100 \\
\hline & $\mathrm{H}$ & -0.74223100 & 4.04264800 & -0.42393900 \\
\hline 16 & 0 & -1.26014200 & -1.31678500 & 0.32206900 \\
\hline & $\mathrm{C}$ & -2.40103900 & -0.96947400 & 0.92943600 \\
\hline & $C$ & -0.58991800 & -2.57637600 & 0.65903500 \\
\hline & $\mathrm{H}$ & 0.26873800 & -2.53714900 & -0.00705800 \\
\hline & $\mathrm{C}$ & -1.46527800 & -3.76577900 & 0.29789100 \\
\hline & $\mathrm{H}$ & -0.88372500 & -4.68465800 & 0.40825600 \\
\hline & $\mathrm{H}$ & -1.79308600 & -3.69269800 & -0.74008800 \\
\hline & 0 & -2.86355300 & -1.54080200 & 1.88724200 \\
\hline & $C$ & -3.10671800 & 0.16794200 & 0.14747300 \\
\hline & $\mathrm{C}$ & -2.08907000 & 1.10406400 & -0.53143300 \\
\hline
\end{tabular}




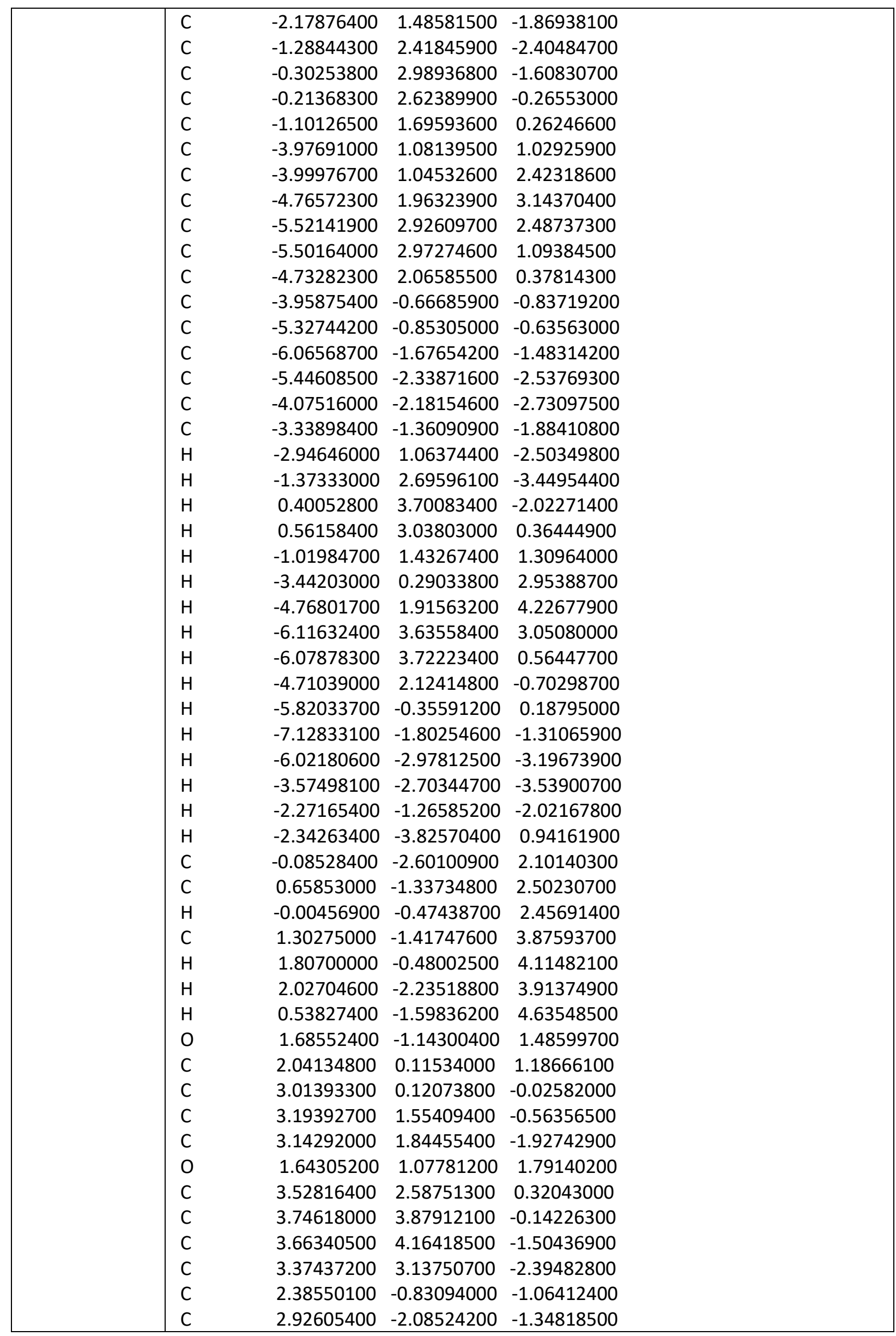




\begin{tabular}{|l|lrrr|}
\hline & C & 2.29596600 & -2.94441200 & -2.24745700 \\
C & 1.11813500 & -2.55917700 & -2.88072900 \\
C & 0.56658000 & -1.31237500 & -2.59376200 \\
C & 1.18584300 & -0.46328200 & -1.68445500 \\
C & 4.40864600 & -0.35813200 & 0.42858900 \\
C & 5.45926700 & -0.30482800 & -0.49566400 \\
C & 6.73965100 & -0.71301300 & -0.14680400 \\
C & 7.00329500 & -1.17027100 & 1.14395600 \\
C & 5.97144100 & -1.21513900 & 2.07292600 \\
C & 4.68280400 & -0.81623000 & 1.71665400 \\
H & 2.91617600 & 1.06361000 & -2.63884600 \\
H & 3.60846700 & 2.38292300 & 1.37762100 \\
H & 3.98871900 & 4.66489600 & 0.56392900 \\
H & 3.83517800 & 5.17191100 & -1.86462800 \\
H & 3.32393400 & 3.33547500 & -3.45946500 \\
$H$ & 3.83778500 & -2.40105600 & -0.86047200 \\
H & 2.73222400 & -3.91534300 & -2.45277000 \\
$H$ & 0.63366300 & -3.22326200 & -3.58727400 \\
H & -0.34957700 & -0.99236600 & -3.07464400 \\
$H$ & 0.72891600 & 0.48887100 & -1.45635400 \\
H & 5.26869600 & 0.06623900 & -1.49468500 \\
H & 7.53564500 & -0.66596400 & -0.88101500 \\
$H$ & 8.00364600 & -1.48219200 & 1.42036000 \\
H & 6.16126400 & -1.56236100 & 3.08209100 \\
$H$ & 3.89750000 & -0.87587600 & 2.45640500 \\
H & 0.58971500 & -3.45907700 & 2.19137300 \\
$H$ & -0.91577600 & -2.74973000 & 2.79193800 \\
\hline
\end{tabular}

21 (optimized at the M06-2X/6-311G(d,p) level)

\begin{tabular}{|l|llll|}
\hline Conformer no & & & & \\
\hline 1 & O & -1.64975900 & -1.72188000 & -0.13033200 \\
& C & -2.07249400 & -0.63366000 & -0.78654900 \\
& C & -0.73517800 & -2.60221100 & -0.83010500 \\
H & -0.02674400 & -1.98257300 & -1.38308800 \\
& C & -1.50898100 & -3.48946800 & -1.78896100 \\
H & -0.81646700 & -4.12679900 & -2.34210200 \\
& H & -2.06398400 & -2.88257800 & -2.50489900 \\
& O & -1.70806700 & -0.36232100 & -1.89798400 \\
& C & -3.09635600 & 0.19328900 & 0.03948400 \\
& C & -2.72614800 & 0.12324300 & 1.52705100 \\
& C & -1.40235000 & 0.36134400 & 1.91192900 \\
& C & -1.03054600 & 0.33670800 & 3.24822300 \\
& C & -1.98523200 & 0.08110600 & 4.23031400 \\
& C & -3.30230600 & -0.14772700 & 3.86055400 \\
& C & -3.67140800 & -0.13033800 & 2.51639500 \\
& C & -4.49263500 & -0.41949700 & -0.20375600 \\
& C & -4.67341800 & -1.79766700 & -0.33242200 \\
& C & -5.94584300 & -2.33816700 & -0.48673900 \\
& C & -7.06320200 & -1.51346500 & -0.49817900 \\
& C & -6.89637300 & -0.14164700 & -0.35066300 \\
& C & -5.62412100 & 0.40026400 & -0.20863300 \\
\hline
\end{tabular}




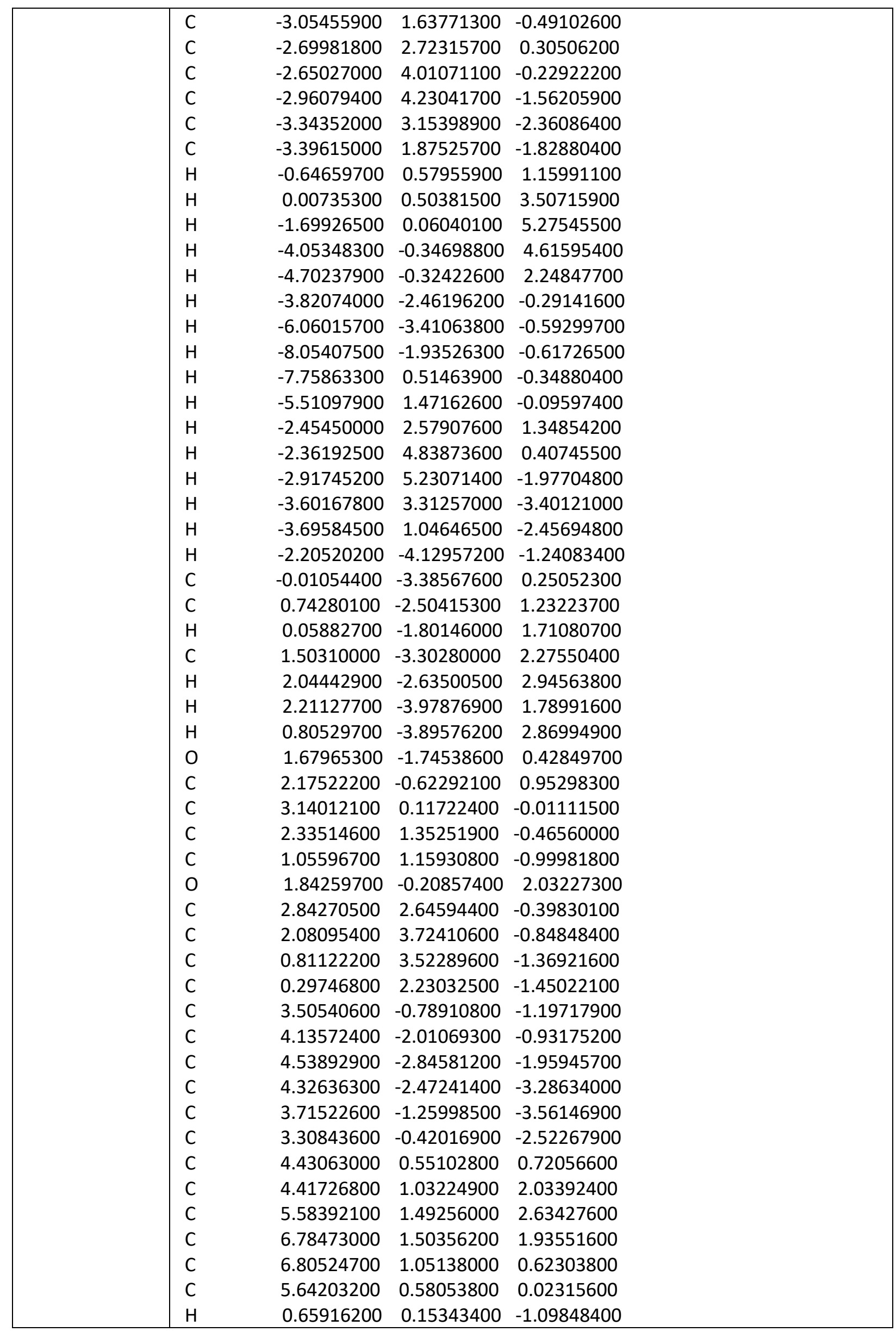




\begin{tabular}{|c|c|c|c|c|}
\hline & $\mathrm{H}$ & 3.83127400 & 2.82422000 & 0.00547100 \\
\hline & $\mathrm{H}$ & 2.49095800 & 4.72537100 & -0.78473900 \\
\hline & $\mathrm{H}$ & 0.21575600 & 4.36105300 & -1.71174100 \\
\hline & $\mathrm{H}$ & -0.68637900 & 2.05212400 & -1.86914700 \\
\hline & $\mathrm{H}$ & 4.31808600 & -2.29992200 & 0.09827400 \\
\hline & $\mathrm{H}$ & 5.02511000 & -3.78677500 & -1.73016900 \\
\hline & $\mathrm{H}$ & 4.64190000 & -3.12230100 & -4.09383700 \\
\hline & $\mathrm{H}$ & 3.55174600 & -0.95275700 & -4.58759900 \\
\hline & $\mathrm{H}$ & 2.84187200 & 0.52723100 & -2.76033900 \\
\hline & $\mathrm{H}$ & 3.49052500 & 1.05770100 & 2.58693500 \\
\hline & $\mathrm{H}$ & 5.54615300 & 1.85257000 & 3.65581300 \\
\hline & $\mathrm{H}$ & 7.69113800 & 1.86606900 & 2.40568500 \\
\hline & $\mathrm{H}$ & 7.72868700 & 1.06087400 & 0.05609300 \\
\hline & $\mathrm{H}$ & 5.68599100 & 0.23778100 & -1.00251800 \\
\hline & $\mathrm{H}$ & 0.69230000 & -4.07157800 & -0.23345800 \\
\hline & $\mathrm{H}$ & -0.73499700 & -3.98537000 & 0.81071100 \\
\hline \multirow[t]{36}{*}{12} & 0 & 1.98664500 & -1.45284800 & 1.23923100 \\
\hline & $\mathrm{C}$ & 1.81954000 & -0.23784400 & 0.71541600 \\
\hline & $\mathrm{C}$ & 0.87937200 & -2.08412500 & 1.92948900 \\
\hline & $\mathrm{H}$ & 0.17079900 & -1.31018400 & 2.22710100 \\
\hline & $\mathrm{C}$ & 1.47278400 & -2.77496300 & 3.14153200 \\
\hline & $\mathrm{H}$ & 0.68557000 & -3.27623300 & 3.70740700 \\
\hline & $\mathrm{H}$ & 1.96285300 & -2.05095000 & 3.79406300 \\
\hline & $\mathrm{O}$ & 0.82471000 & 0.42450500 & 0.85126900 \\
\hline & $\mathrm{C}$ & 3.00677900 & 0.14039000 & -0.19404800 \\
\hline & $\mathrm{C}$ & 4.36082200 & -0.18615800 & 0.44529000 \\
\hline & $\mathrm{C}$ & 5.48480900 & -0.36461400 & -0.36192400 \\
\hline & $\mathrm{C}$ & 6.74129600 & -0.55436000 & 0.19918800 \\
\hline & $\mathrm{C}$ & 6.89738800 & -0.55815600 & 1.58084400 \\
\hline & $\mathrm{C}$ & 5.78752300 & -0.36308800 & 2.39292000 \\
\hline & $\mathrm{C}$ & 4.53050400 & -0.17639600 & 1.82877600 \\
\hline & $\mathrm{C}$ & 3.03375200 & 1.64422100 & -0.49526800 \\
\hline & $\mathrm{C}$ & 2.69313200 & 2.58078600 & 0.48093100 \\
\hline & $\mathrm{C}$ & 2.82196400 & 3.94246800 & 0.23362200 \\
\hline & $\mathrm{C}$ & 3.30376200 & 4.39203600 & -0.99023700 \\
\hline & $\mathrm{C}$ & 3.66555300 & 3.46652600 & -1.96122100 \\
\hline & $\mathrm{C}$ & 3.53412700 & 2.10536000 & -1.71364500 \\
\hline & $\mathrm{C}$ & 2.66692600 & -0.72312000 & -1.42658300 \\
\hline & $\mathrm{C}$ & 1.69792900 & -0.27719100 & -2.32609100 \\
\hline & $\mathrm{C}$ & 1.23787000 & -1.10762800 & -3.34222100 \\
\hline & $\mathrm{C}$ & 1.73533800 & -2.39816700 & -3.46805000 \\
\hline & $\mathrm{C}$ & 2.68739300 & -2.85898700 & -2.56226300 \\
\hline & $\mathrm{C}$ & 3.14361100 & -2.03144900 & -1.54345200 \\
\hline & $\mathrm{H}$ & 5.37518600 & -0.35625400 & -1.44070700 \\
\hline & $\mathrm{H}$ & 7.60002800 & -0.69512400 & -0.44636900 \\
\hline & $\mathrm{H}$ & 7.87670200 & -0.70578300 & 2.02014800 \\
\hline & $\mathrm{H}$ & 5.89701600 & -0.35518800 & 3.47089800 \\
\hline & $\mathrm{H}$ & 3.67790900 & -0.02393500 & 2.48079500 \\
\hline & $\mathrm{H}$ & 2.31649100 & 2.25161600 & 1.44041100 \\
\hline & $\mathrm{H}$ & 2.54319400 & 4.65241100 & 1.00333100 \\
\hline & $\mathrm{H}$ & 3.40124600 & 5.45364100 & -1.18321100 \\
\hline & $\mathrm{H}$ & 4.05013800 & 3.80215100 & -2.91703000 \\
\hline
\end{tabular}




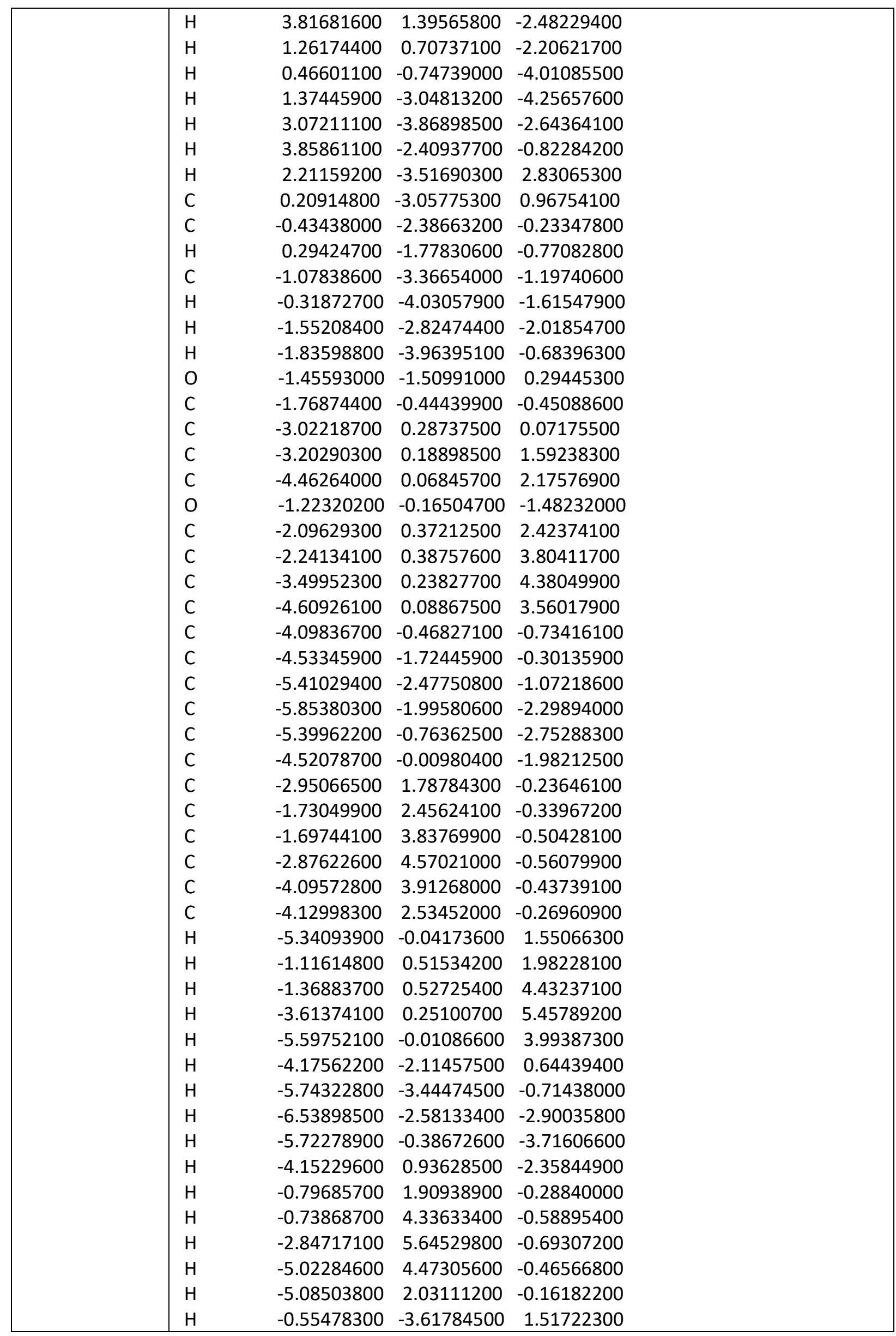




\begin{tabular}{|c|c|c|c|c|}
\hline & $\mathrm{H}$ & 0.95903100 & -3.77015000 & 0.60703500 \\
\hline \multirow[t]{51}{*}{15} & 0 & 1.64875600 & -1.71209600 & 0.14603700 \\
\hline & C & 1.70646800 & -0.41435300 & 0.46269200 \\
\hline & $\mathrm{C}$ & 0.82456400 & -2.58573900 & 0.95197800 \\
\hline & $\mathrm{H}$ & 0.15797200 & -1.96913900 & 1.55349900 \\
\hline & C & 1.75441400 & -3.38846500 & 1.84250900 \\
\hline & $\mathrm{H}$ & 1.17624200 & -4.06365300 & 2.47715500 \\
\hline & $\mathrm{H}$ & 2.33798800 & -2.72076300 & 2.48079800 \\
\hline & $\mathrm{O}$ & 0.94071800 & 0.13527600 & 1.20839000 \\
\hline & $\mathrm{C}$ & 2.98389800 & 0.23285800 & -0.11043600 \\
\hline & C & 2.86772400 & 1.76021400 & -0.14720800 \\
\hline & C & 3.97710900 & 2.57134200 & 0.08659100 \\
\hline & C & 3.89604200 & 3.95027500 & -0.07931300 \\
\hline & C & 2.70726200 & 4.53598000 & -0.49500400 \\
\hline & C & 1.60245200 & 3.73052700 & -0.75256300 \\
\hline & C & 1.68085100 & 2.35408000 & -0.58183500 \\
\hline & C & 3.26785500 & -0.18867000 & -1.55605800 \\
\hline & C & 2.21380400 & -0.42288300 & -2.43881000 \\
\hline & C & 2.46084200 & -0.69593800 & -3.77902600 \\
\hline & C & 3.76462800 & -0.72718000 & -4.26030700 \\
\hline & C & 4.81838200 & -0.46207200 & -3.39357500 \\
\hline & C & 4.57066900 & -0.18753600 & -2.05348900 \\
\hline & C & 3.99931000 & -0.30075700 & 0.92200000 \\
\hline & C & 4.68420900 & -1.49923200 & 0.71231700 \\
\hline & C & 5.49071500 & -2.03915300 & 1.70794300 \\
\hline & C & 5.60910400 & -1.40210400 & 2.93734200 \\
\hline & $\mathrm{C}$ & 4.90261200 & -0.22680400 & 3.16870700 \\
\hline & C & 4.09829200 & 0.31436400 & 2.17267700 \\
\hline & $\mathrm{H}$ & 4.91550300 & 2.12794600 & 0.39928100 \\
\hline & $\mathrm{H}$ & 4.76844800 & 4.56372100 & 0.11313000 \\
\hline & $\mathrm{H}$ & 2.64470600 & 5.60950300 & -0.62848300 \\
\hline & $\mathrm{H}$ & 0.67172700 & 4.16792300 & -1.09525300 \\
\hline & $\mathrm{H}$ & 0.81271700 & 1.74425200 & -0.80903700 \\
\hline & $\mathrm{H}$ & 1.18933500 & -0.36262100 & -2.08725000 \\
\hline & $\mathrm{H}$ & 1.62848100 & -0.87518200 & -4.44947700 \\
\hline & $\mathrm{H}$ & 3.95754400 & -0.94113600 & -5.30481400 \\
\hline & $\mathrm{H}$ & 5.83796000 & -0.46090000 & -3.76053600 \\
\hline & $\mathrm{H}$ & 5.39927100 & 0.03346000 & -1.39001900 \\
\hline & $\mathrm{H}$ & 4.57410500 & -2.02240100 & -0.22922000 \\
\hline & $\mathrm{H}$ & 6.01987400 & -2.96630300 & 1.52171800 \\
\hline & $\mathrm{H}$ & 6.23850100 & -1.82280300 & 3.71242600 \\
\hline & $\mathrm{H}$ & 4.97098800 & 0.26933600 & 4.12958600 \\
\hline & $\mathrm{H}$ & 3.52934900 & 1.21577000 & 2.36889400 \\
\hline & $\mathrm{H}$ & 2.44682600 & -3.97707700 & 1.23619600 \\
\hline & C & -0.00011000 & -3.43518500 & -0.00071700 \\
\hline & C & -0.82477900 & -2.58544800 & -0.95316600 \\
\hline & $\mathrm{H}$ & -0.15821600 & -1.96858000 & -1.55445800 \\
\hline & C & -1.75448800 & -3.38796900 & -1.84403800 \\
\hline & $\mathrm{H}$ & -2.44692900 & -3.97680400 & -1.23797900 \\
\hline & $\mathrm{H}$ & -1.17621200 & -4.06292100 & -2.47884300 \\
\hline & $\mathrm{H}$ & -2.33802900 & -2.72011100 & -2.48219300 \\
\hline & $\mathrm{O}$ & -1.64913400 & -1.71215700 & -0.14707600 \\
\hline
\end{tabular}




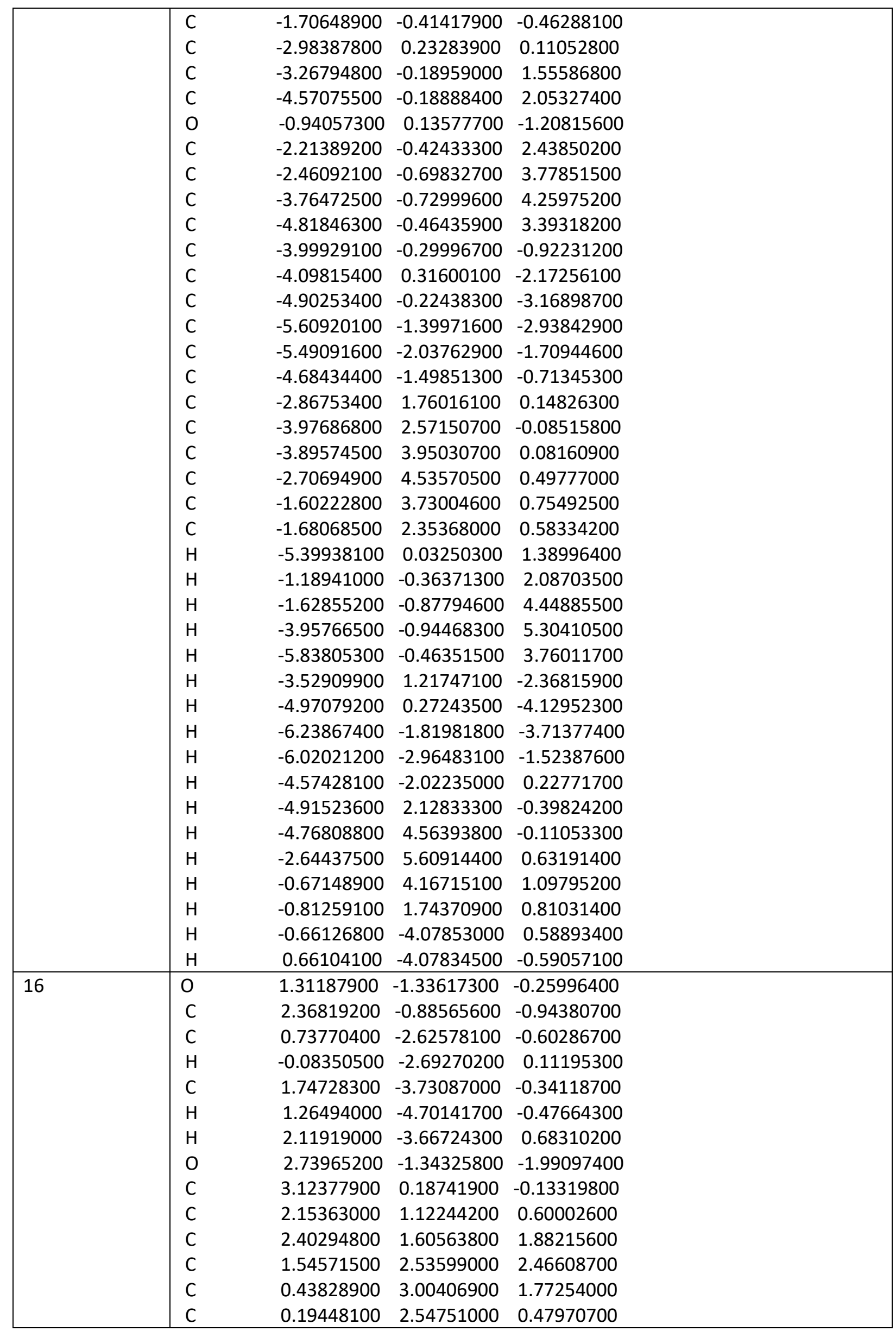




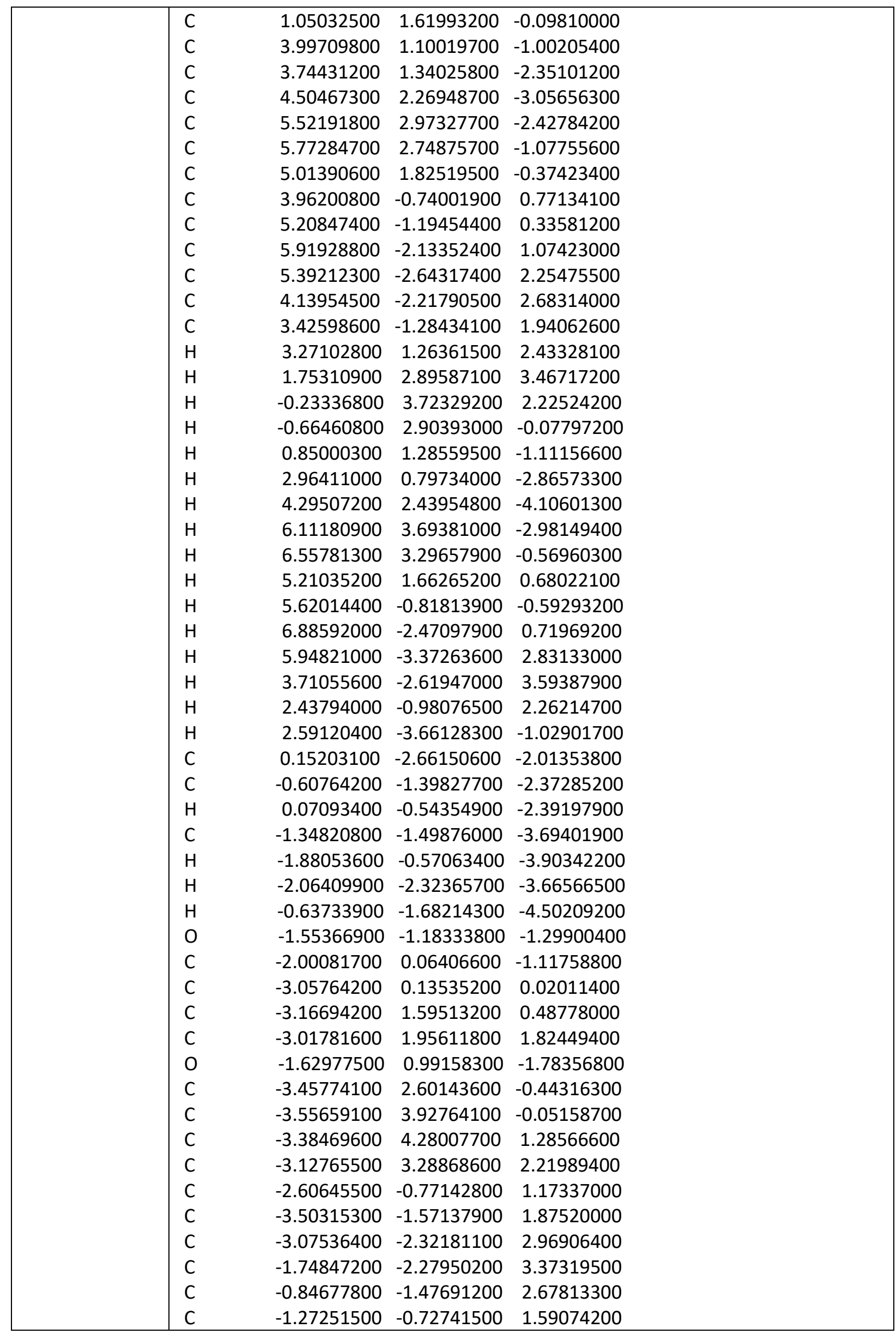




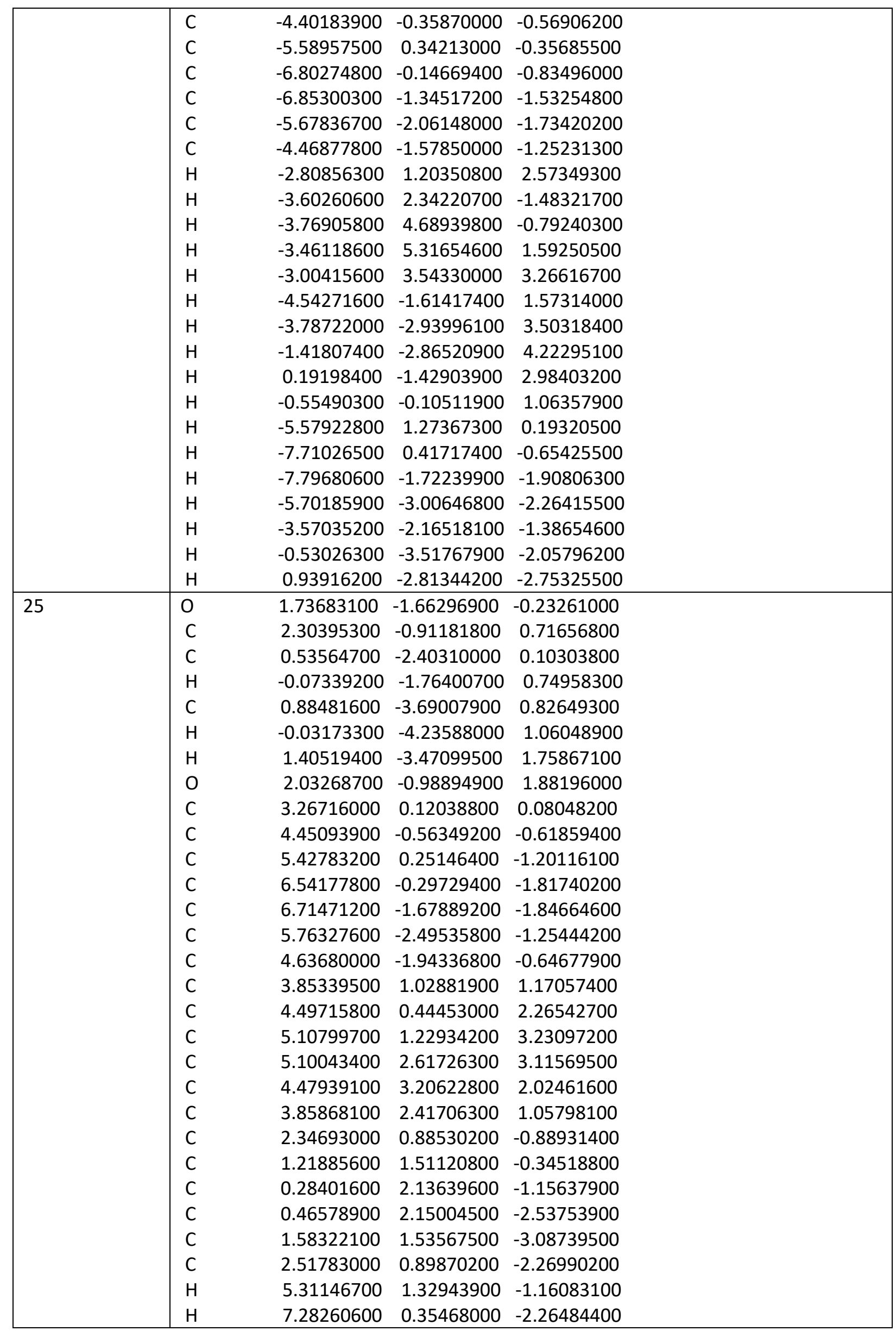




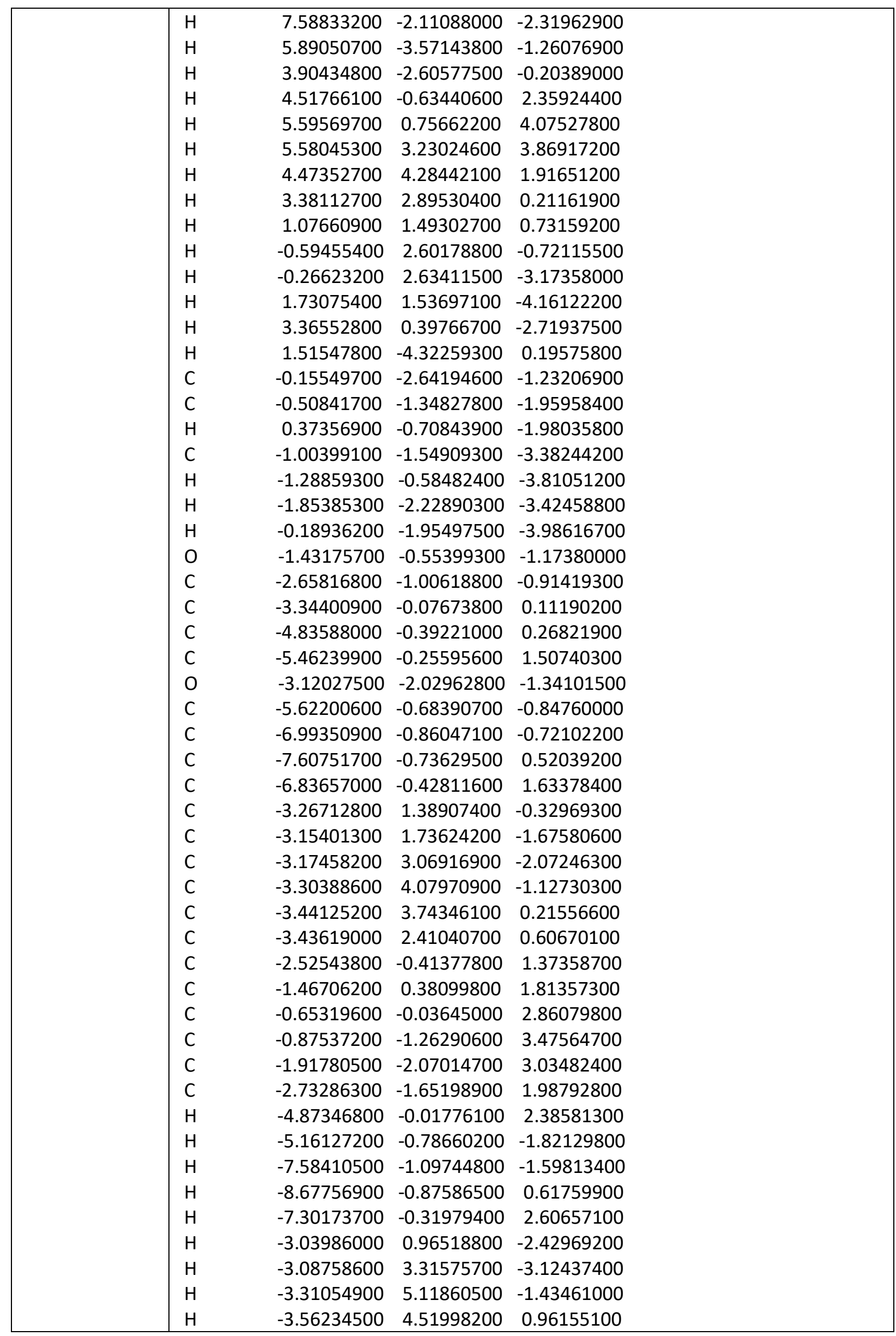




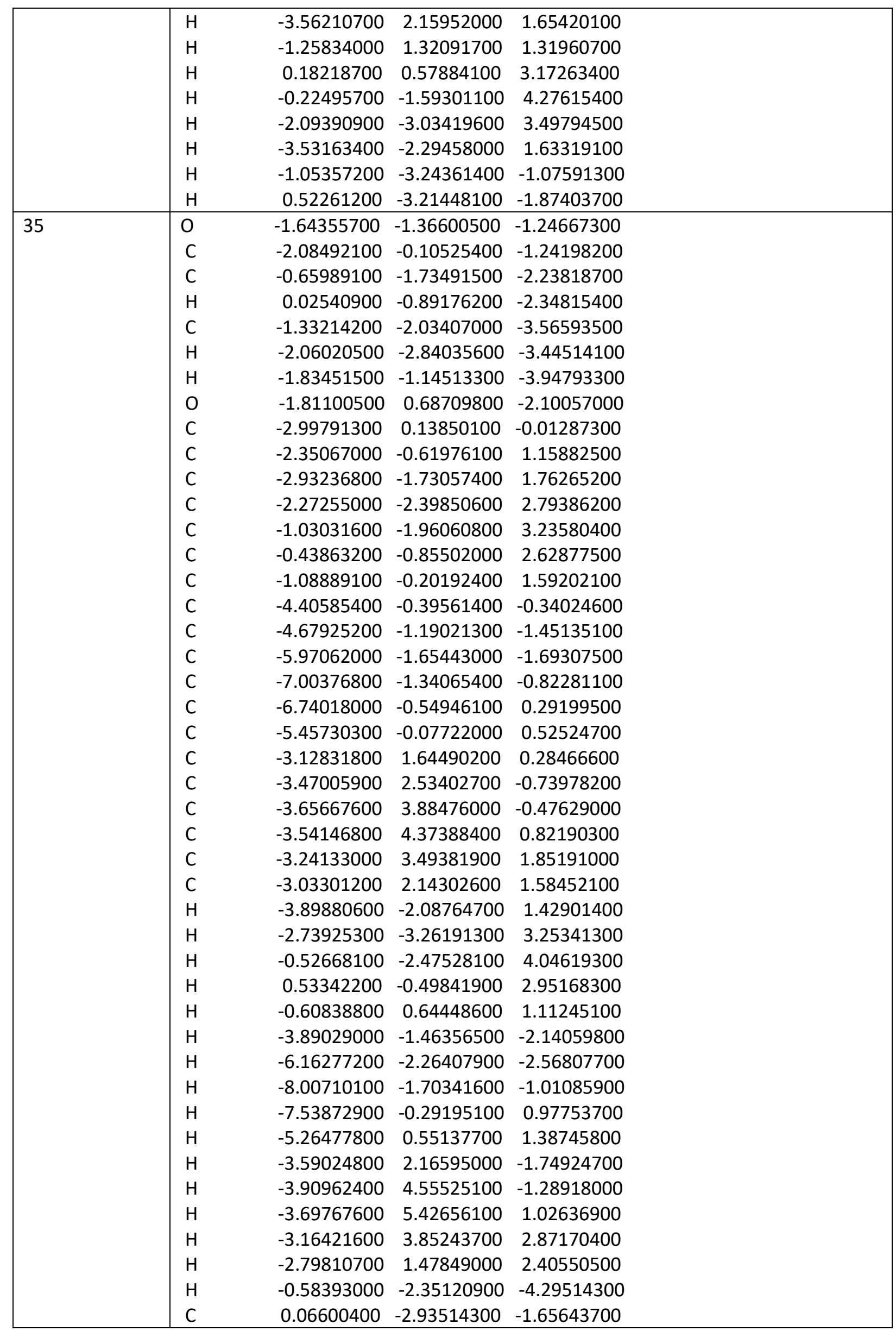




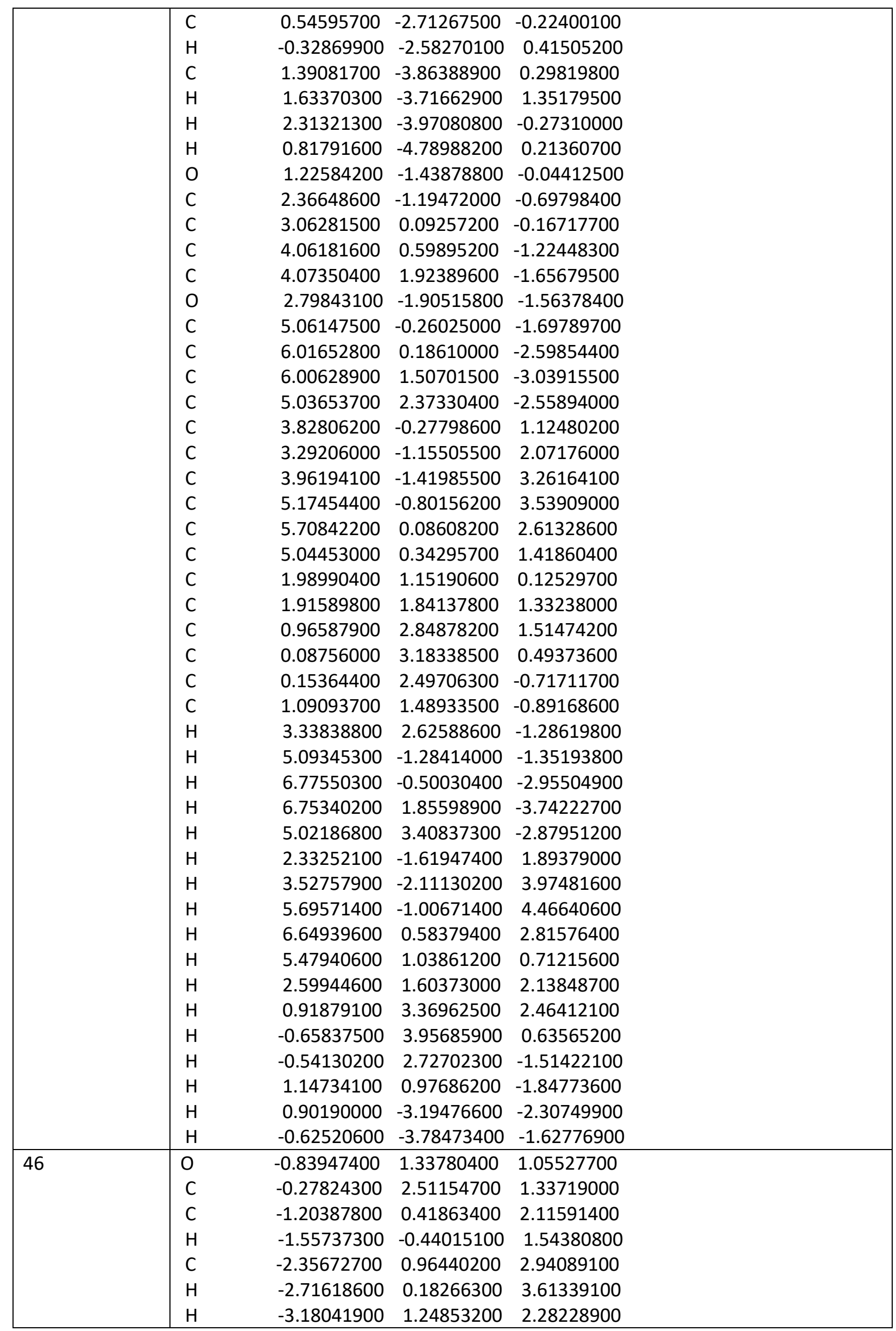




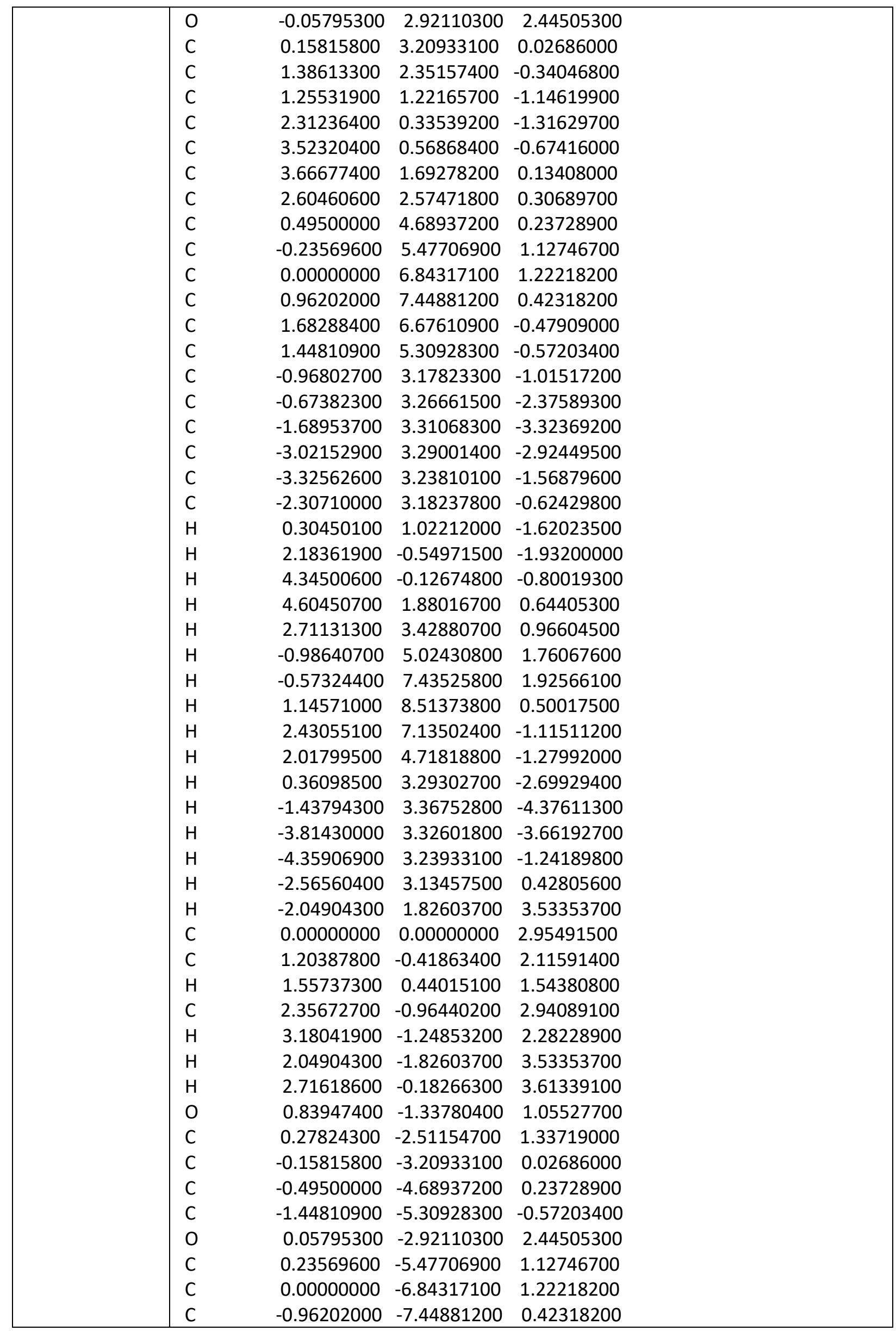




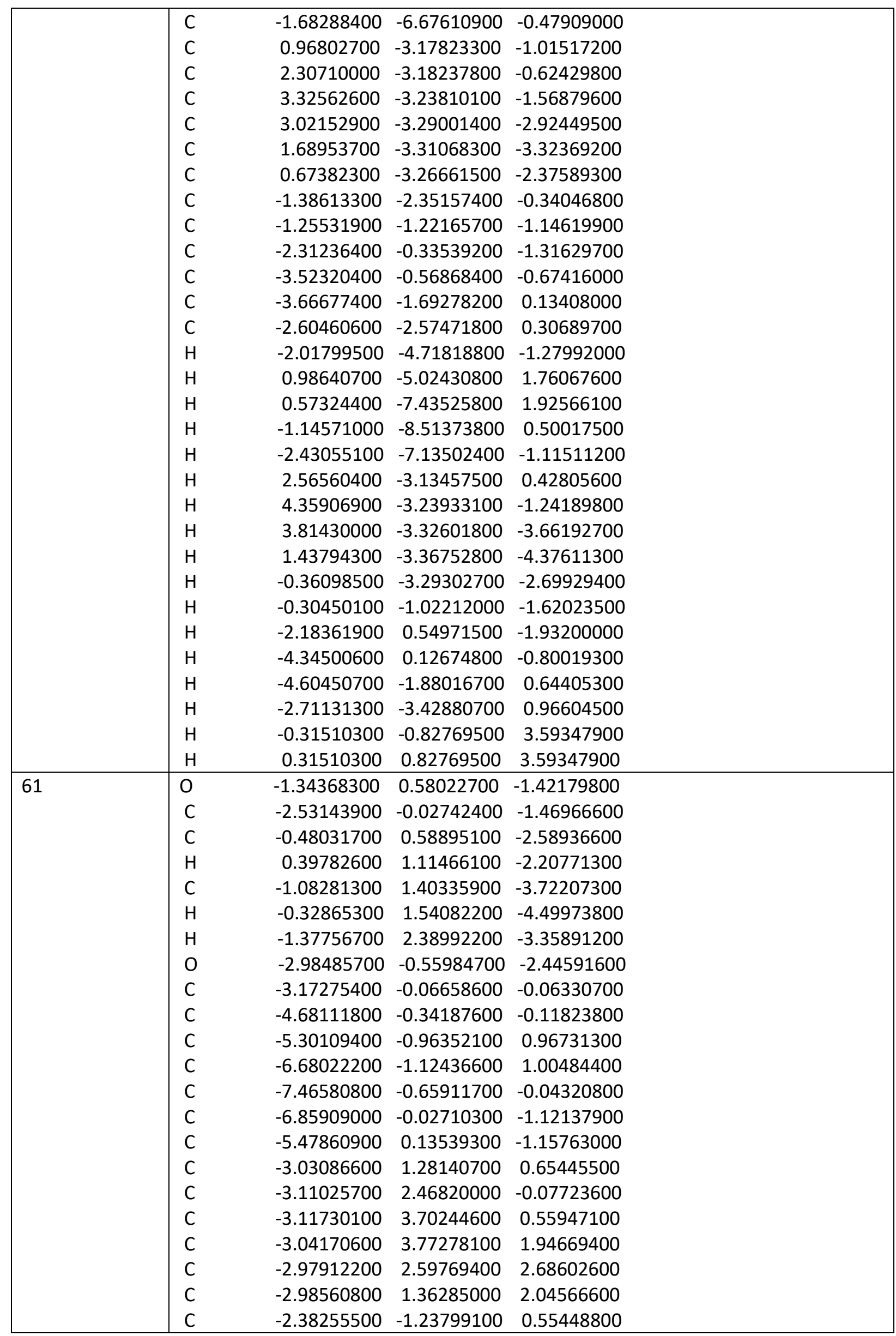




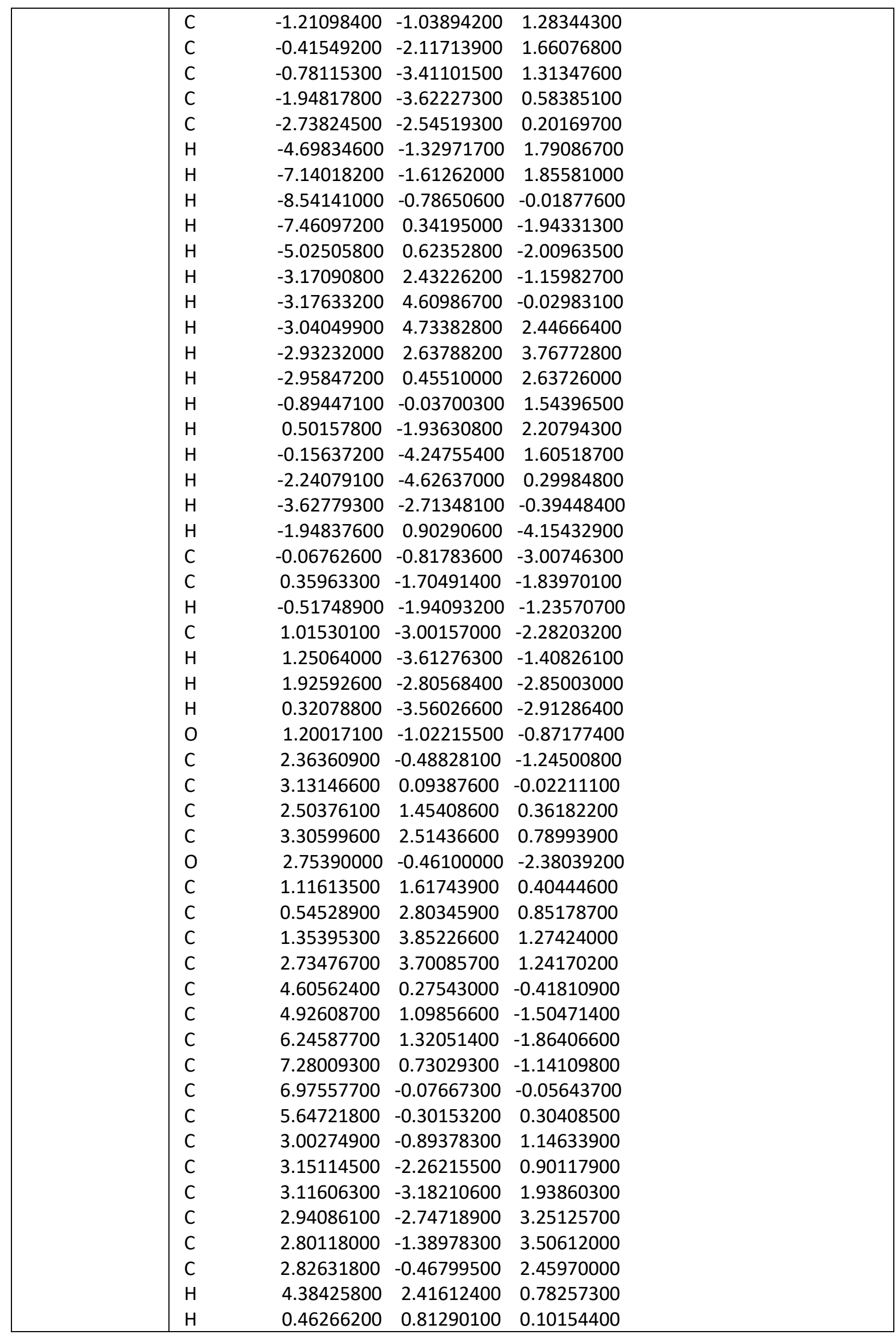




\begin{tabular}{|l|lrrr|}
\hline & $H$ & -0.53535900 & 2.89423600 & 0.87296300 \\
$H$ & 0.91176800 & 4.77624100 & 1.62840300 \\
$H$ & 3.37914900 & 4.50748300 & 1.57138400 \\
$H$ & 4.13186200 & 1.57213700 & -2.06674700 \\
$H$ & 6.47006100 & 1.95825000 & -2.71098800 \\
$H$ & 8.31206700 & 0.90470900 & -1.42169500 \\
$H$ & 7.76861000 & -0.53724200 & 0.52077900 \\
$H$ & 5.43589600 & -0.92805100 & 1.16096400 \\
$H$ & 3.30944300 & -2.60849300 & -0.11591200 \\
$H$ & 3.23171400 & -4.23842500 & 1.72540700 \\
H & 2.91477800 & -3.46207900 & 4.06491300 \\
$\mathrm{H}$ & 2.66596400 & -1.03849300 & 4.52223100 \\
$\mathrm{H}$ & 2.70870200 & 0.58712200 & 2.67506900 \\
$\mathrm{H}$ & 0.74899000 & -0.71794100 & -3.72448600 \\
$\mathrm{H}$ & -0.90364500 & -1.31703300 & -3.50261900 \\
\hline
\end{tabular}

22 (optimized at the B3LYP/6-311G(d,p) level)

\begin{tabular}{|c|c|c|c|c|}
\hline \multicolumn{5}{|l|}{ Conformer no } \\
\hline \multirow[t]{33}{*}{1} & 0 & -0.79709600 & 2.13039800 & -1.41739200 \\
\hline & C & -0.50222100 & 3.44166700 & -1.38789600 \\
\hline & $\mathrm{C}$ & -1.20244700 & 1.55010900 & -2.70065900 \\
\hline & $\mathrm{H}$ & -0.67288600 & 2.10269900 & -3.48009600 \\
\hline & $\mathrm{C}$ & -2.70514000 & 1.72362400 & -2.88261100 \\
\hline & $\mathrm{H}$ & -3.01287900 & 1.30969700 & -3.84655300 \\
\hline & $\mathrm{H}$ & -2.97482700 & 2.78094900 & -2.86950300 \\
\hline & $\mathrm{O}$ & -0.58411600 & 4.16744900 & -2.34679400 \\
\hline & $\mathrm{C}$ & 0.05194000 & 3.85809900 & 0.00785800 \\
\hline & C & -0.90364200 & 3.47754900 & 1.16330000 \\
\hline & $\mathrm{C}$ & -0.45467400 & 3.59047100 & 2.48662900 \\
\hline & C & -1.30415400 & 3.34882500 & 3.56041000 \\
\hline & C & -2.63612600 & 3.00344800 & 3.33911700 \\
\hline & $\mathrm{C}$ & -3.10161800 & 2.90721400 & 2.03231600 \\
\hline & $\mathrm{C}$ & -2.24377000 & 3.13988900 & 0.95683700 \\
\hline & $\mathrm{C}$ & 0.21250700 & 5.39501000 & 0.09876500 \\
\hline & $\mathrm{C}$ & -0.80605800 & 6.24249500 & -0.35546700 \\
\hline & C & -0.72246900 & 7.61986200 & -0.18296400 \\
\hline & C & 0.37634600 & 8.18587600 & 0.46003500 \\
\hline & $\mathrm{C}$ & 1.38711700 & 7.35550600 & 0.93124700 \\
\hline & C & 1.30415400 & 5.97508400 & 0.75265900 \\
\hline & $\mathrm{C}$ & 1.41064600 & 3.11050800 & 0.04743800 \\
\hline & $\mathrm{C}$ & 2.44470400 & 3.53961900 & -0.79721800 \\
\hline & C & 3.65598700 & 2.85664600 & -0.84974600 \\
\hline & $\mathrm{C}$ & 3.85679200 & 1.72218900 & -0.06512100 \\
\hline & $\mathrm{C}$ & 2.82811100 & 1.27071600 & 0.75660500 \\
\hline & C & 1.61354000 & 1.95444200 & 0.80507700 \\
\hline & $\mathrm{H}$ & 0.57080500 & 3.87899300 & 2.68190700 \\
\hline & $\mathrm{H}$ & -0.92568000 & 3.43984300 & 4.57248600 \\
\hline & $\mathrm{H}$ & -3.30210700 & 2.82077100 & 4.17477300 \\
\hline & $\mathrm{H}$ & -4.13835500 & 2.65273900 & 1.84063000 \\
\hline & $\mathrm{H}$ & -2.63711300 & 3.05065200 & -0.04632900 \\
\hline & $\mathrm{H}$ & -1.67067900 & 5.82938000 & -0.85591700 \\
\hline
\end{tabular}




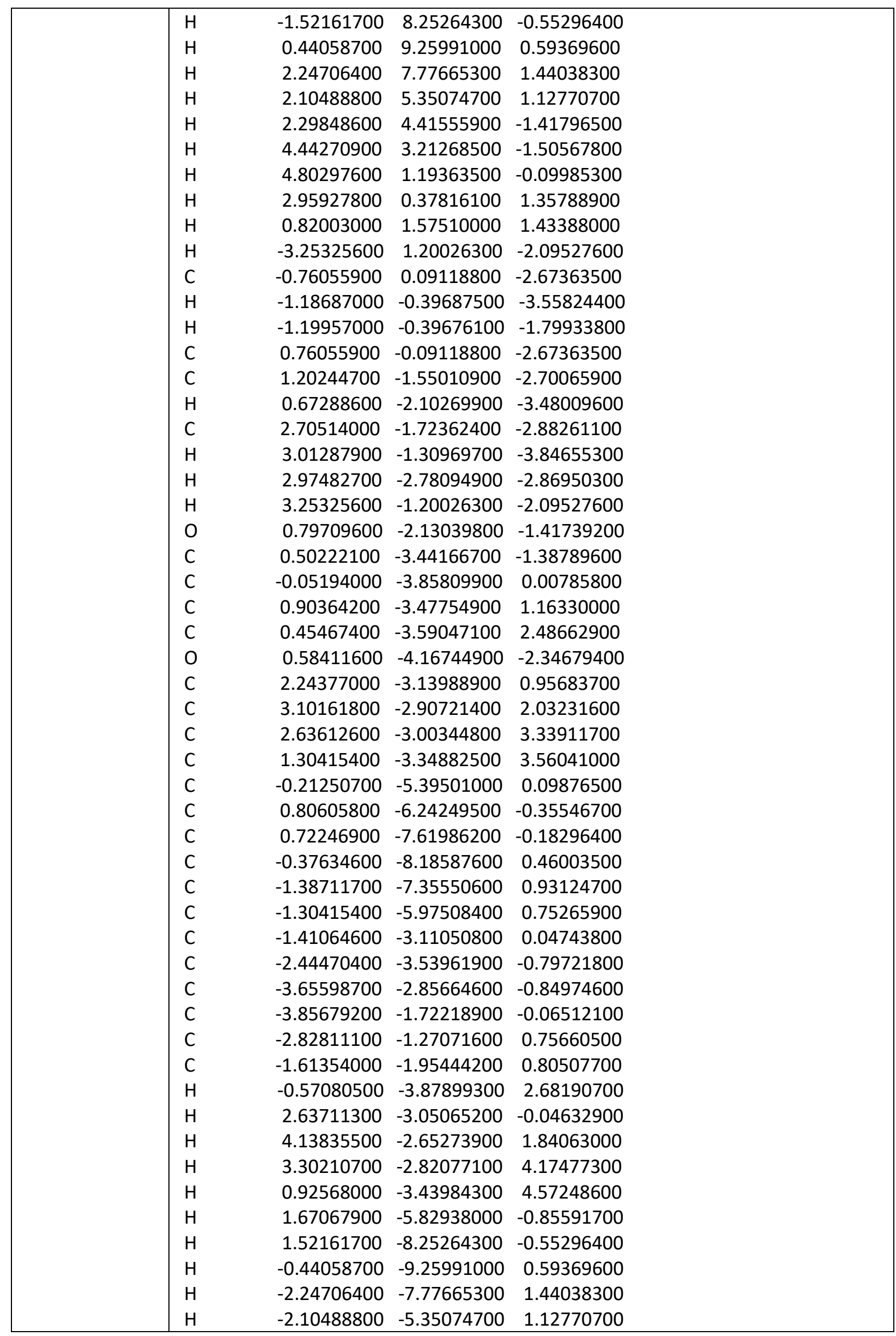




\begin{tabular}{|c|c|c|c|c|}
\hline & $\mathrm{H}$ & -2.29848600 & -4.41555900 & -1.41796500 \\
\hline & $\mathrm{H}$ & -4.44270900 & -3.21268500 & -1.50567800 \\
\hline & $\mathrm{H}$ & -4.80297600 & -1.19363500 & -0.09985300 \\
\hline & $\mathrm{H}$ & -2.95927800 & -0.37816100 & 1.35788900 \\
\hline & $\mathrm{H}$ & -0.82003000 & -1.57510000 & 1.43388000 \\
\hline & $\mathrm{H}$ & 1.19957000 & 0.39676100 & -1.79933800 \\
\hline & $\mathrm{H}$ & 1.18687000 & 0.39687500 & -3.55824400 \\
\hline 3 & 0 & -2.12706600 & -0.80610600 & -1.41612900 \\
\hline & $\mathrm{C}$ & -3.43910200 & -0.51451100 & -1.38781900 \\
\hline & $\mathrm{C}$ & -1.54445200 & -1.20992700 & -2.69883500 \\
\hline & $\mathrm{H}$ & -2.09922700 & -0.68364600 & -3.47893600 \\
\hline & $\mathrm{C}$ & -1.71108600 & -2.71354900 & -2.87933600 \\
\hline & $\mathrm{H}$ & -1.29523100 & -3.02041300 & -3.84273100 \\
\hline & $\mathrm{H}$ & -2.76722800 & -2.98791900 & -2.86655900 \\
\hline & 0 & -4.16391200 & -0.59856500 & -2.34725700 \\
\hline & $\mathrm{C}$ & -3.85796400 & 0.03891800 & 0.00754000 \\
\hline & $\mathrm{C}$ & -3.47652700 & -0.91605600 & 1.16319500 \\
\hline & $\mathrm{C}$ & -3.59121600 & -0.46752700 & 2.48651600 \\
\hline & $\mathrm{C}$ & -3.34880700 & -1.31676800 & 3.56031800 \\
\hline & $\mathrm{C}$ & -3.00083800 & -2.64806800 & 3.33904000 \\
\hline & $\mathrm{C}$ & -2.90279500 & -3.11315200 & 2.03222800 \\
\hline & $\mathrm{C}$ & -3.13626900 & -2.25553300 & 0.95674500 \\
\hline & $\mathrm{C}$ & -5.39528300 & 0.19646600 & 0.09715000 \\
\hline & $\mathrm{C}$ & -6.24050500 & -0.82354000 & -0.35810500 \\
\hline & $\mathrm{C}$ & -7.61816900 & -0.74252500 & -0.18675900 \\
\hline & $\mathrm{C}$ & -8.18677000 & 0.35505500 & 0.45607700 \\
\hline & $\mathrm{C}$ & -7.35867400 & 1.36721000 & 0.92830100 \\
\hline & $\mathrm{C}$ & -5.97794800 & 1.28684200 & 0.75086500 \\
\hline & $\mathrm{C}$ & -3.11322700 & 1.39914400 & 0.04793500 \\
\hline & $\mathrm{C}$ & -3.54378900 & 2.43242400 & -0.79693700 \\
\hline & $\mathrm{C}$ & -2.86340100 & 3.64520300 & -0.84858700 \\
\hline & $\mathrm{C}$ & -1.73013900 & 3.84831900 & -0.06282800 \\
\hline & $\mathrm{C}$ & -1.27716700 & 2.82044800 & 0.75909200 \\
\hline & $\mathrm{C}$ & -1.95828000 & 1.60437700 & 0.80665600 \\
\hline & $\mathrm{H}$ & -3.88175100 & 0.55739100 & 2.68176800 \\
\hline & $\mathrm{H}$ & -3.44125400 & -0.93865000 & 4.57239900 \\
\hline & $\mathrm{H}$ & -2.81755300 & -3.31385600 & 4.17471900 \\
\hline & $\mathrm{H}$ & -2.64625300 & -4.14937900 & 1.84053900 \\
\hline & $\mathrm{H}$ & -3.04562600 & -2.64854600 & -0.04643200 \\
\hline & $\mathrm{H}$ & -5.82537300 & -1.68723100 & -0.85847900 \\
\hline & $\mathrm{H}$ & -8.24916800 & -1.54271900 & -0.55754400 \\
\hline & $\mathrm{H}$ & -9.26103800 & 0.41727100 & 0.58882400 \\
\hline & $\mathrm{H}$ & -7.78182600 & 2.22624000 & 1.43732500 \\
\hline & $\mathrm{H}$ & -5.35543000 & 2.08863800 & 1.12667200 \\
\hline & $\mathrm{H}$ & -4.41885000 & 2.28443700 & -1.41850900 \\
\hline & $\mathrm{H}$ & -3.22052500 & 4.43128400 & -1.50470000 \\
\hline & $\mathrm{H}$ & -1.20362400 & 4.79566700 & -0.09683000 \\
\hline & $\mathrm{H}$ & -0.38543100 & 2.95349000 & 1.36118200 \\
\hline & $\mathrm{H}$ & -1.57780600 & 0.81151200 & 1.43558600 \\
\hline & $\mathrm{H}$ & -1.18575500 & -3.25850300 & -2.09112700 \\
\hline & $\mathrm{C}$ & -0.08757200 & -0.76124600 & -2.67174800 \\
\hline & $\mathrm{H}$ & 0.40260600 & -1.18543000 & -3.55621100 \\
\hline
\end{tabular}




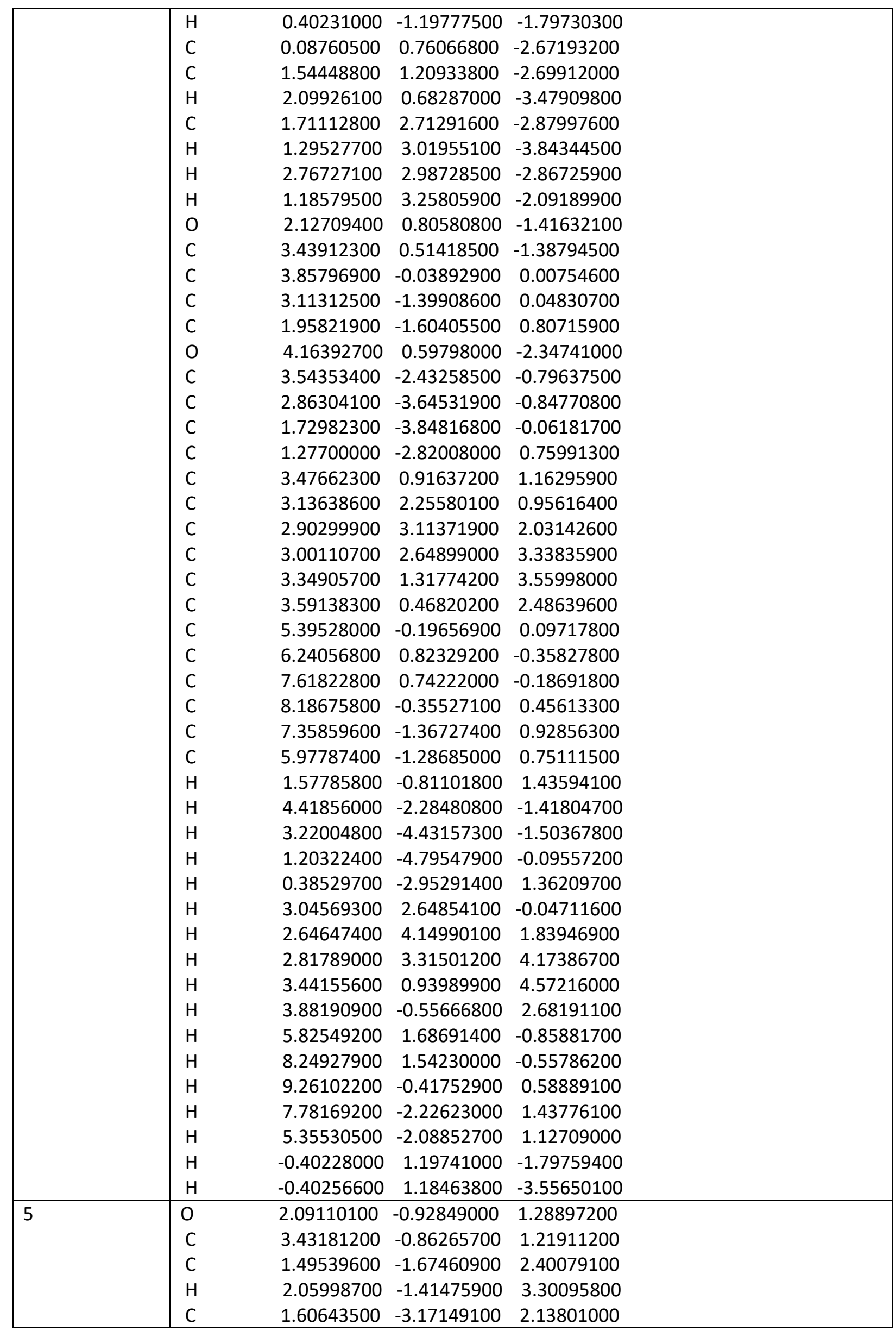




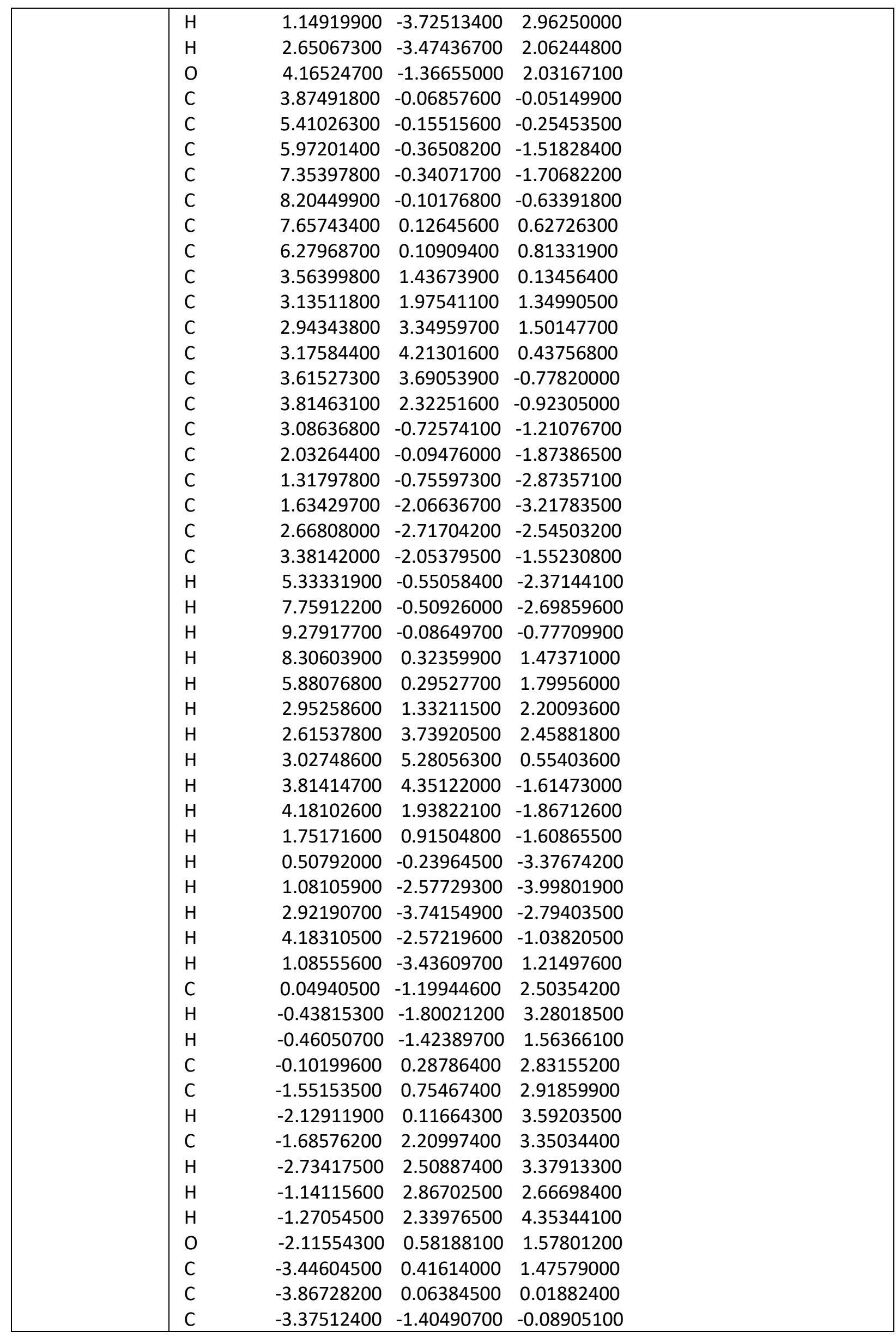




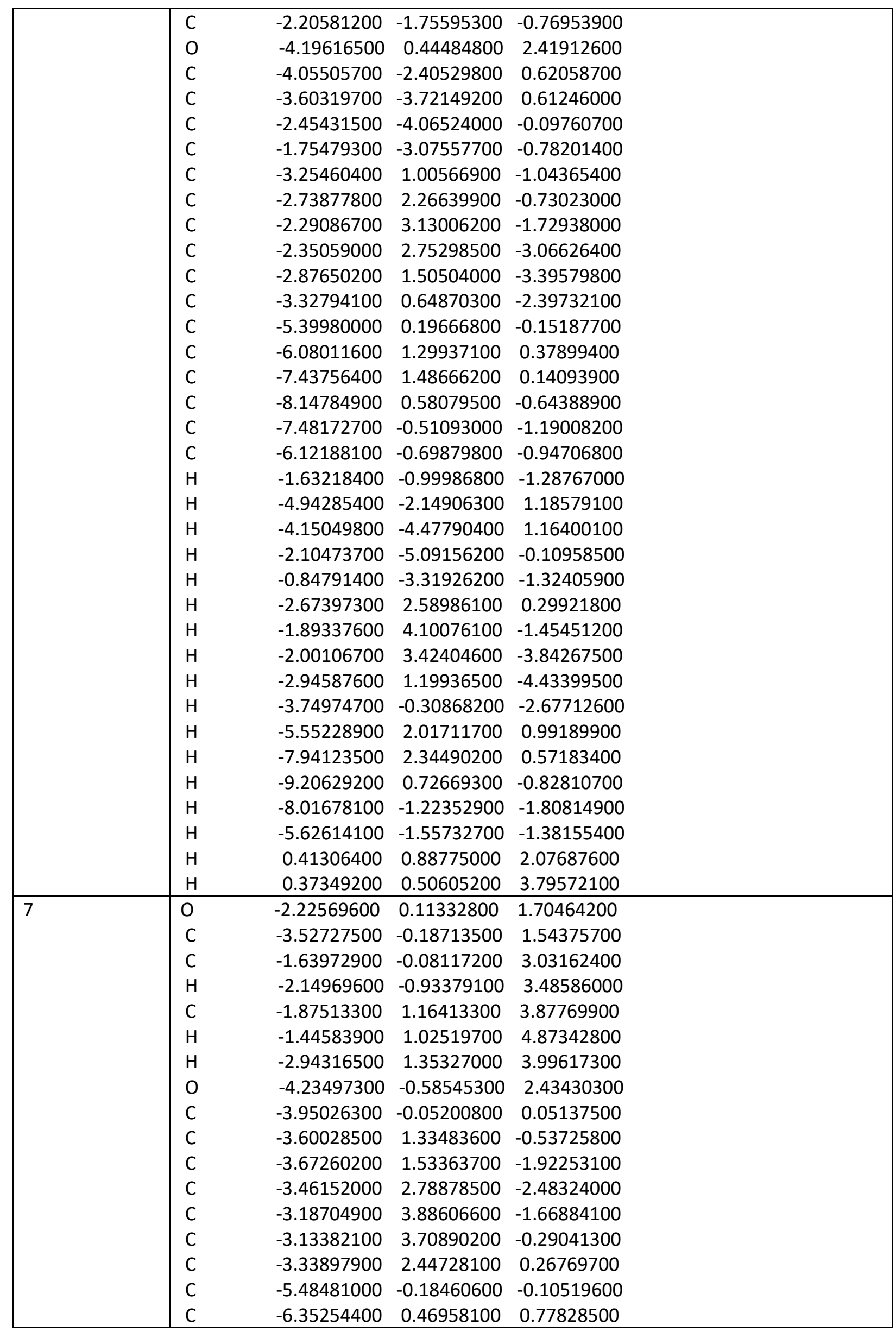




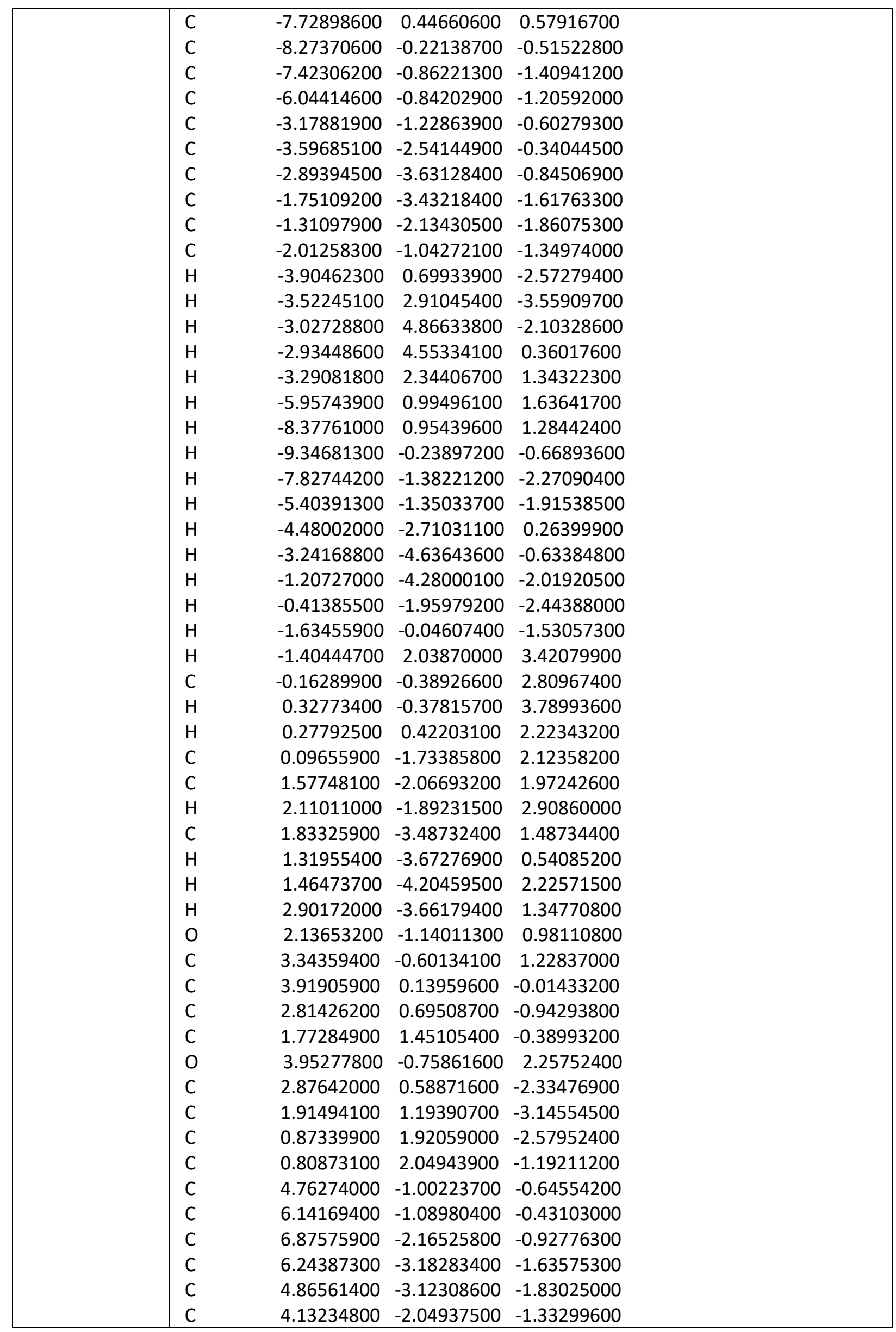




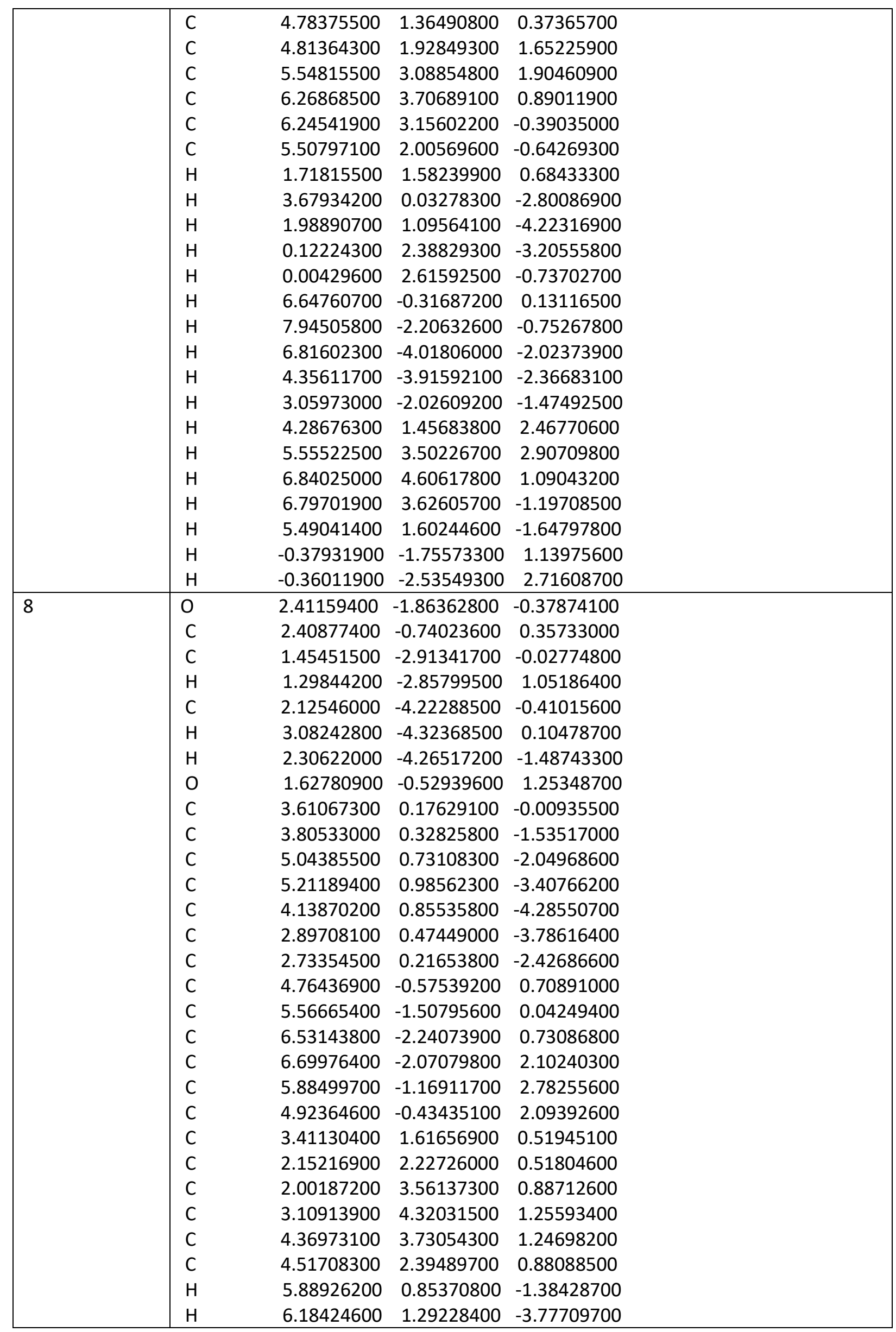




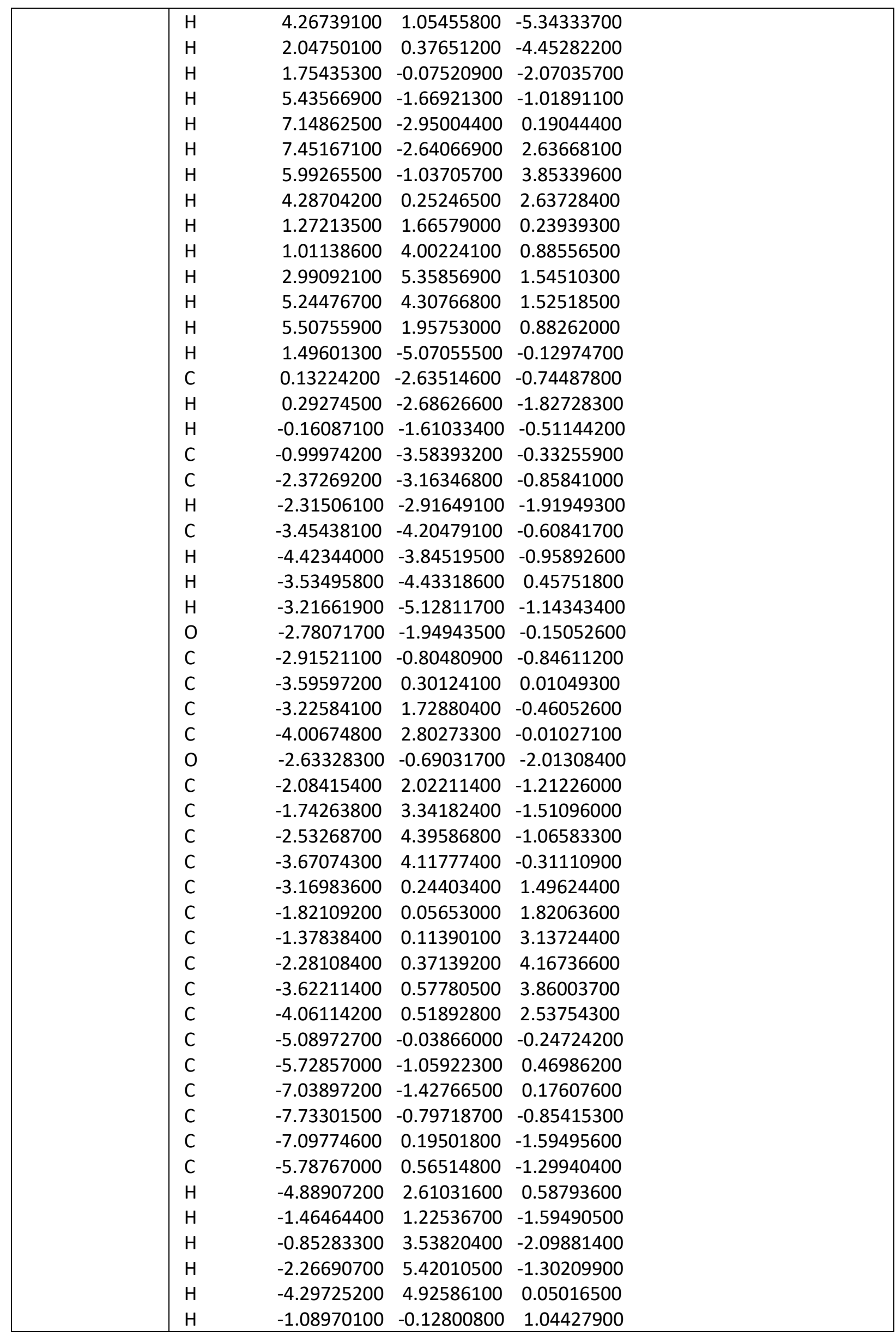




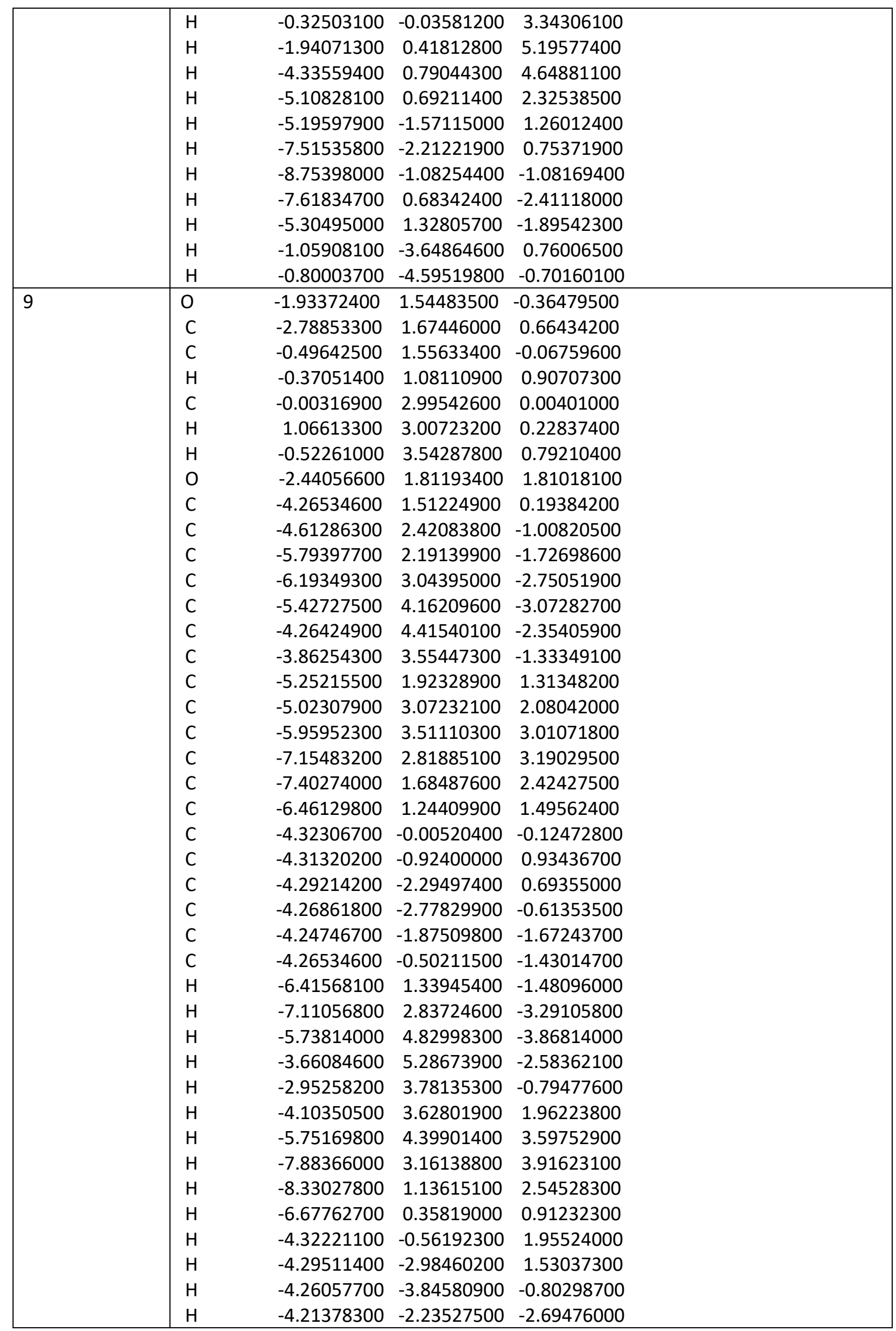




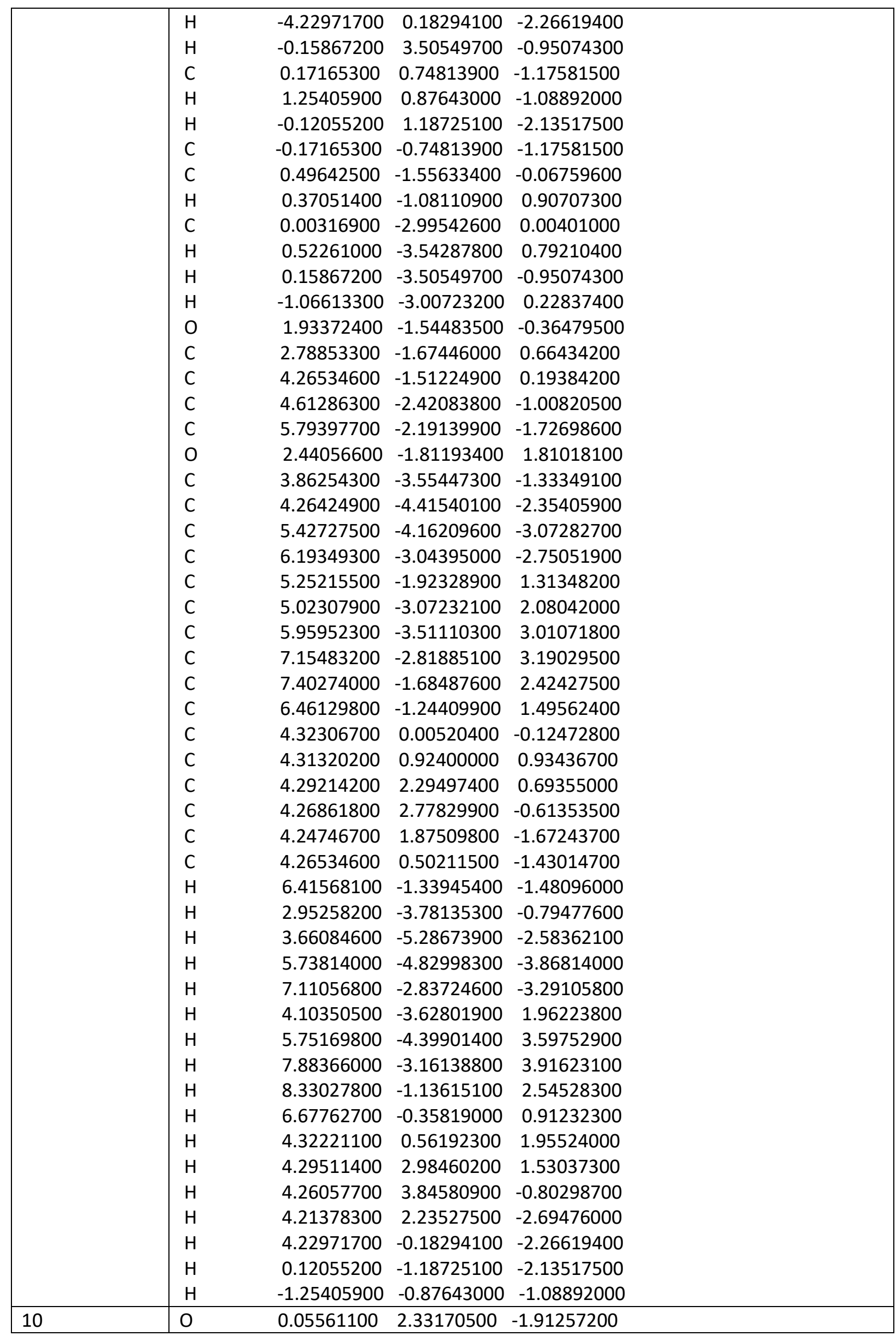




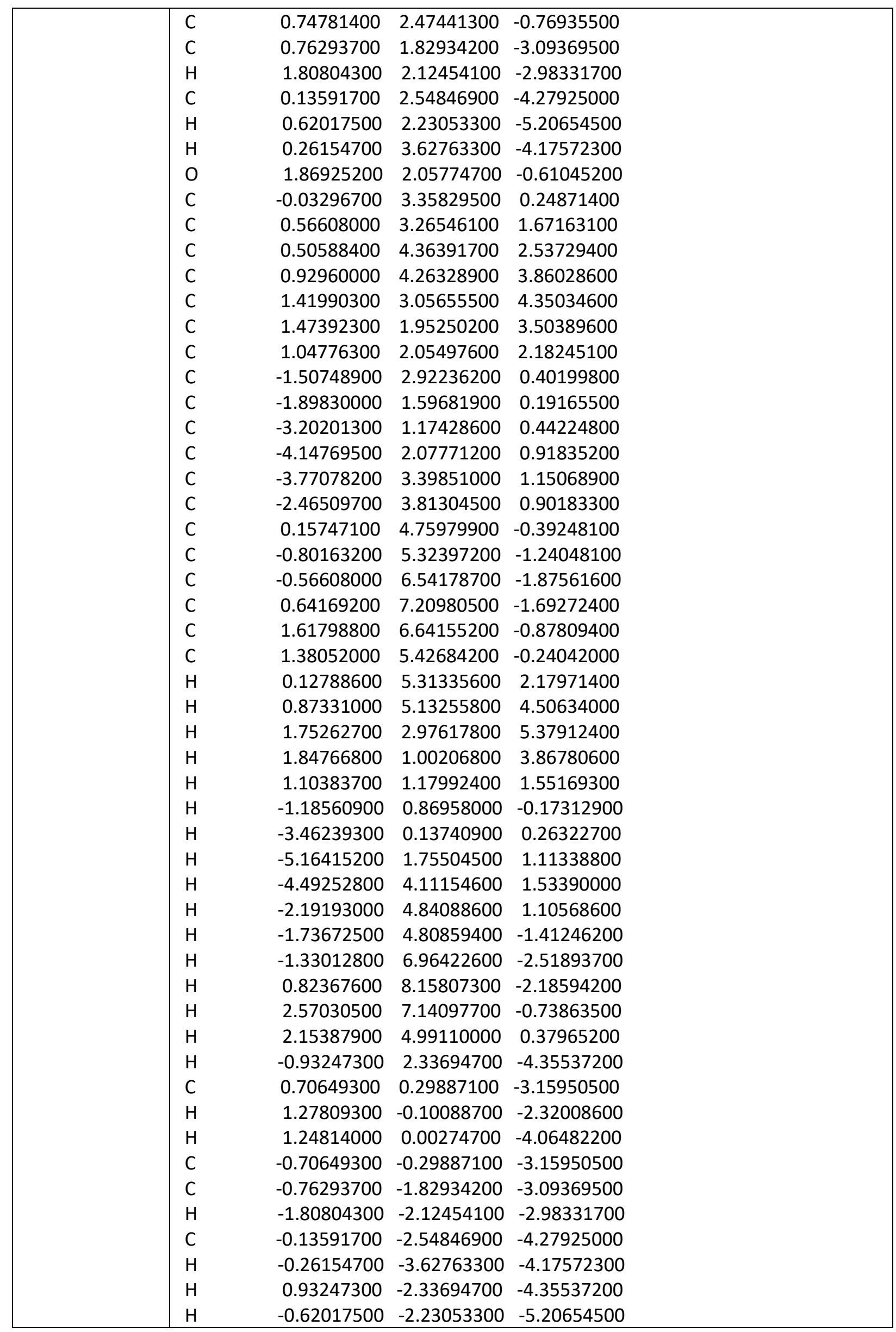




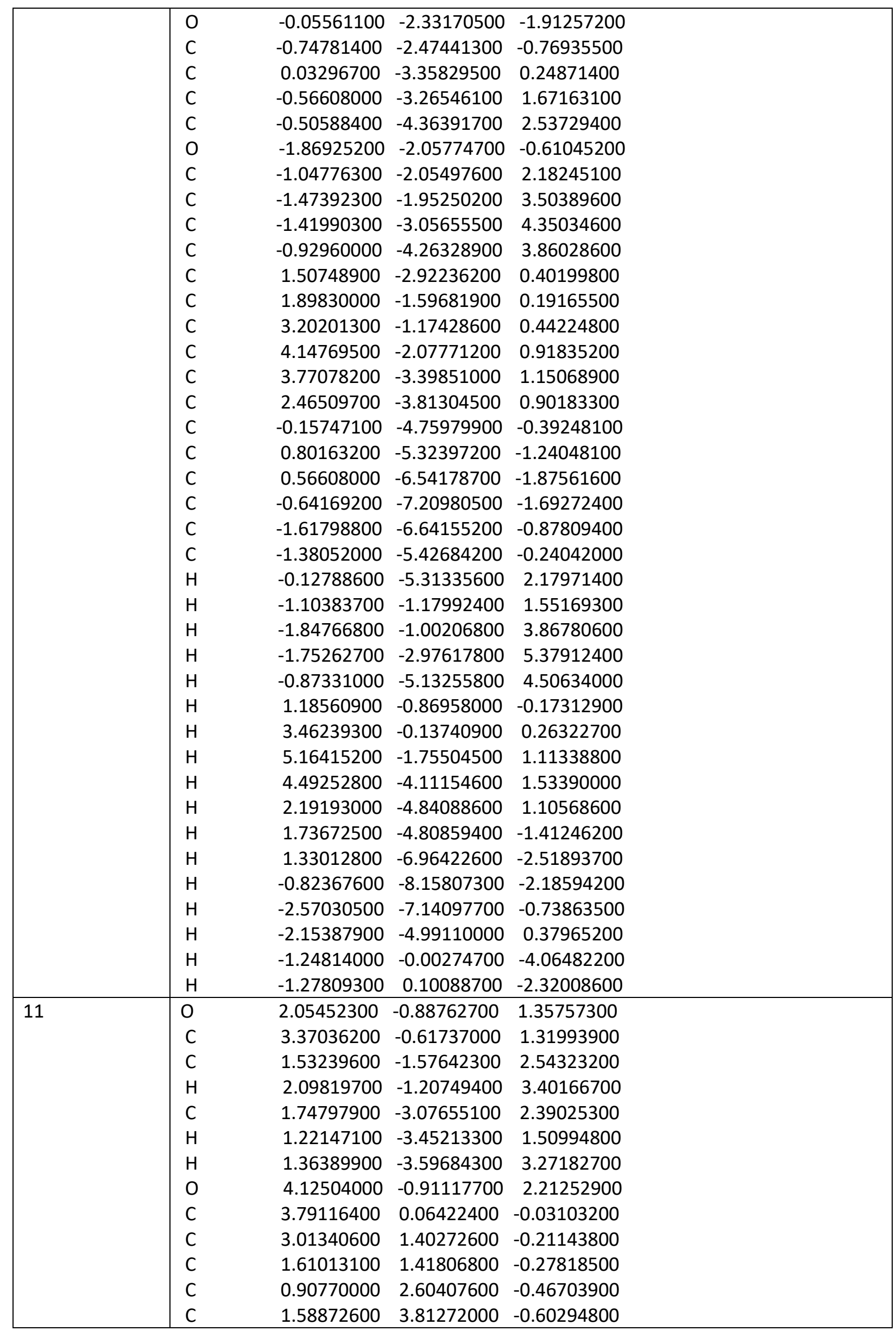




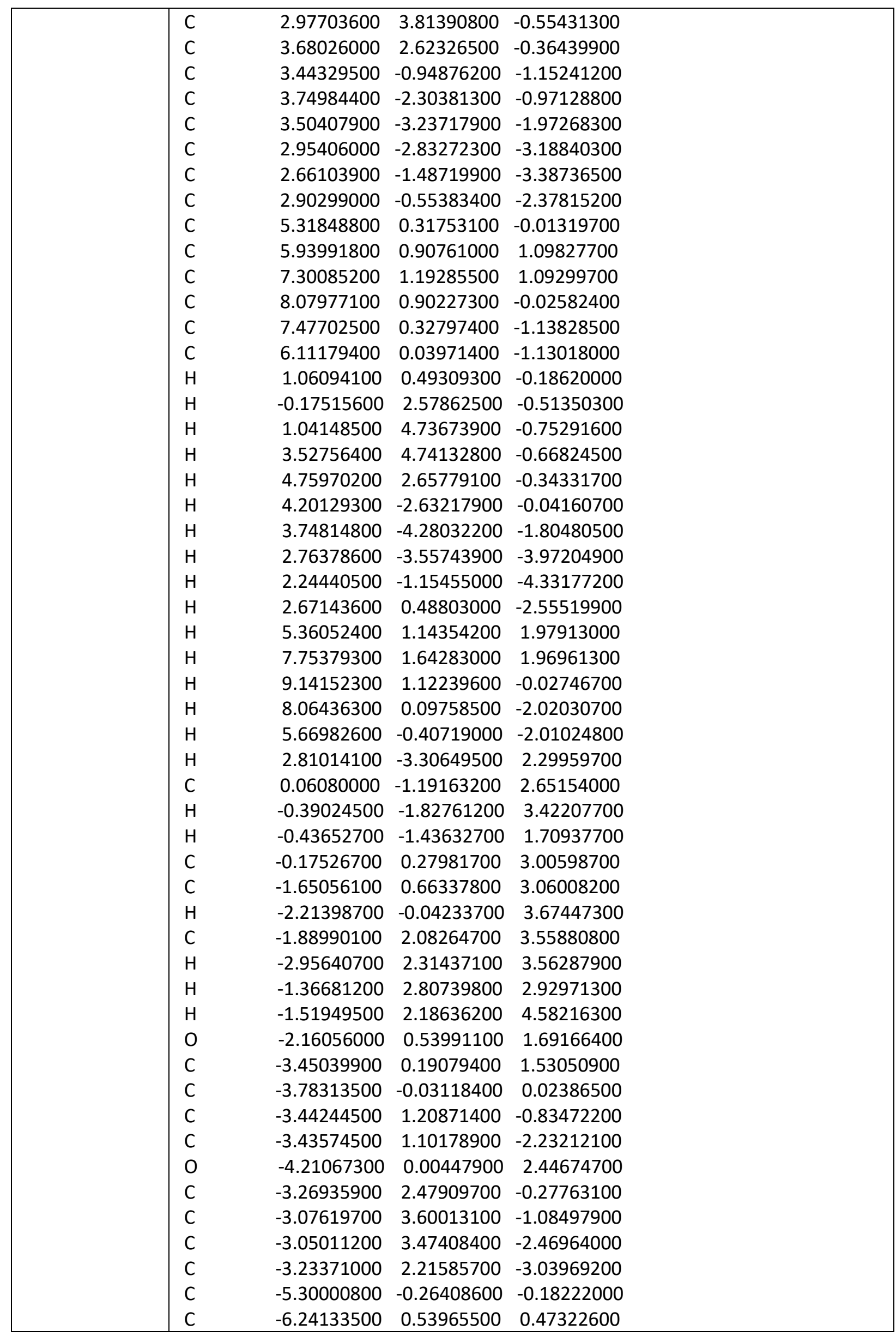




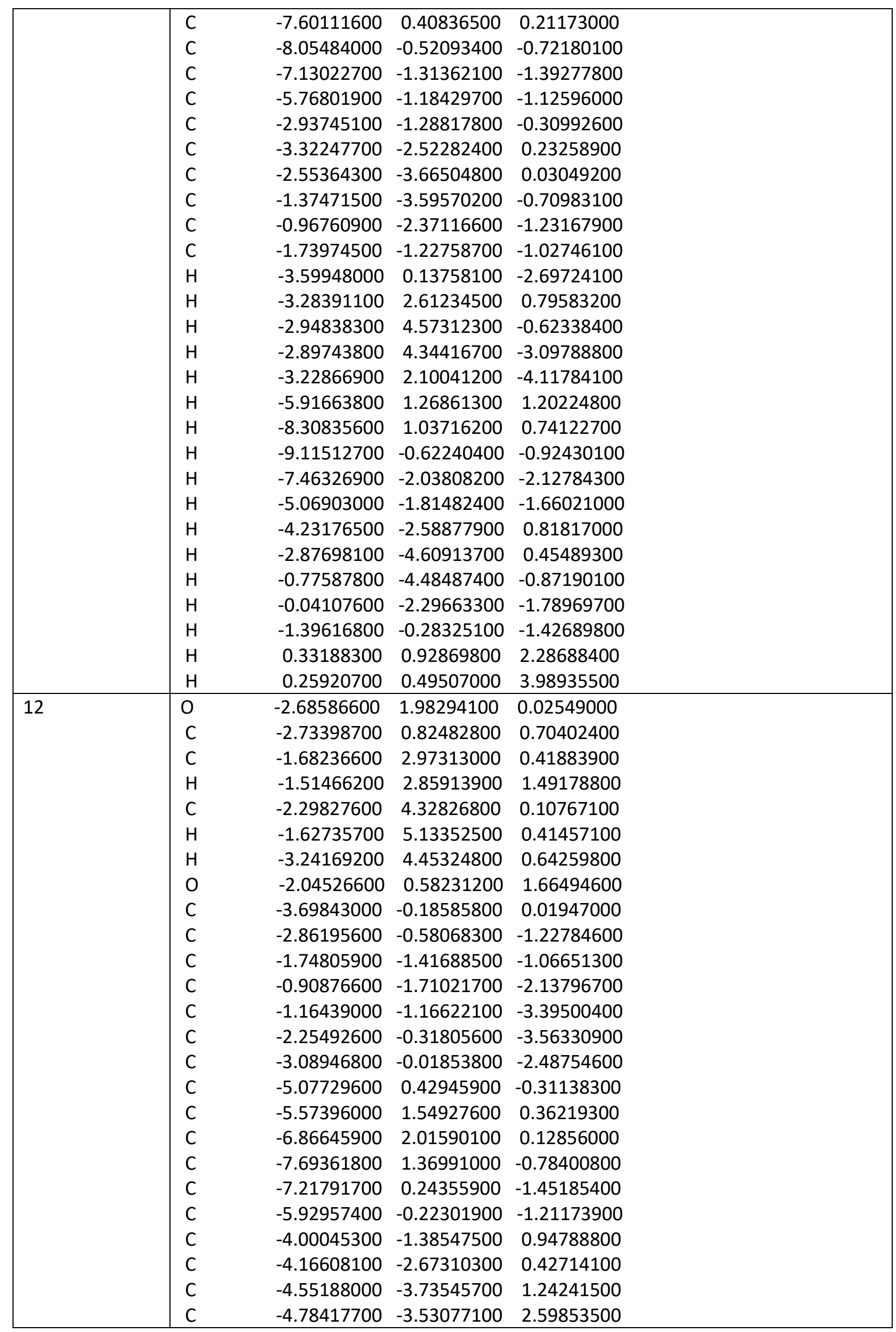




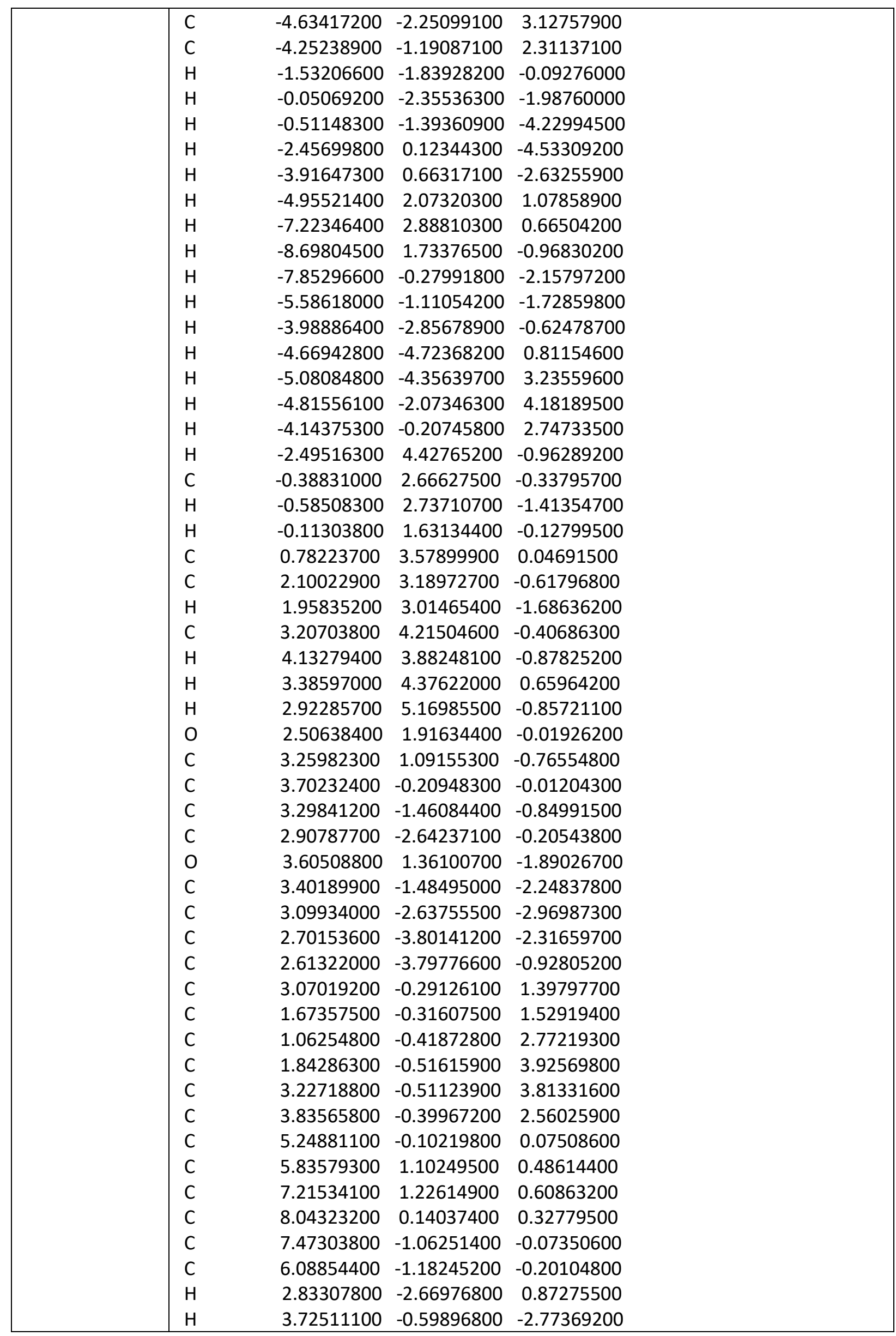




\begin{tabular}{|c|c|c|c|c|}
\hline & $\mathrm{H}$ & 3.18514700 & -2.62259700 & -4.05104500 \\
\hline & $\mathrm{H}$ & 2.47099200 & -4.69856500 & -2.88043400 \\
\hline & $\mathrm{H}$ & 2.31514500 & -4.69502000 & -0.39676900 \\
\hline & $\mathrm{H}$ & 1.05152700 & -0.26024700 & 0.64457500 \\
\hline & $\mathrm{H}$ & -0.01930500 & -0.41453700 & 2.83109400 \\
\hline & $\mathrm{H}$ & 1.37114700 & -0.59848900 & 4.89867800 \\
\hline & $\mathrm{H}$ & 3.84748000 & -0.59492800 & 4.69912300 \\
\hline & $\mathrm{H}$ & 4.91545800 & -0.40282400 & 2.50164700 \\
\hline & $\mathrm{H}$ & 5.20732400 & 1.95217400 & 0.72787900 \\
\hline & $\mathrm{H}$ & 7.64330000 & 2.17120000 & 0.92436200 \\
\hline & $\mathrm{H}$ & 9.11940700 & 0.23379900 & 0.42029900 \\
\hline & $\mathrm{H}$ & 8.10358800 & -1.91643100 & -0.29536000 \\
\hline & $\mathrm{H}$ & 5.66829700 & -2.12622400 & -0.52158700 \\
\hline & $\mathrm{H}$ & 0.92782700 & 3.57179200 & 1.13311500 \\
\hline & $\mathrm{H}$ & 0.56795800 & 4.61364000 & -0.23985500 \\
\hline \multirow[t]{37}{*}{13} & 0 & -2.48677300 & 1.86591400 & 0.02652400 \\
\hline & $\mathrm{C}$ & -2.79711300 & 0.80698400 & 0.79183500 \\
\hline & $\mathrm{C}$ & -1.49090100 & 2.82528000 & 0.51024600 \\
\hline & $\mathrm{H}$ & -1.31409600 & 2.60919600 & 1.56484400 \\
\hline & $\mathrm{C}$ & -2.11169700 & 4.20394500 & 0.33888600 \\
\hline & $\mathrm{H}$ & -3.05142600 & 4.26917100 & 0.89085600 \\
\hline & $\mathrm{H}$ & -2.31705100 & 4.40959100 & -0.71491600 \\
\hline & 0 & -2.34419400 & 0.61657500 & 1.89495500 \\
\hline & $\mathrm{C}$ & -3.70619700 & -0.20017200 & 0.03003300 \\
\hline & $\mathrm{C}$ & -4.60154900 & 0.49710600 & -1.02064300 \\
\hline & $\mathrm{C}$ & -4.80104600 & -0.02460100 & -2.30078000 \\
\hline & $\mathrm{C}$ & -5.69710800 & 0.57281000 & -3.18827500 \\
\hline & $\mathrm{C}$ & -6.41385000 & 1.70231100 & -2.81081100 \\
\hline & $\mathrm{C}$ & -6.23451100 & 2.22468300 & -1.53037300 \\
\hline & $\mathrm{C}$ & -5.34467900 & 1.62461700 & -0.64795700 \\
\hline & $\mathrm{C}$ & -4.68195700 & -0.95335500 & 0.96707300 \\
\hline & $\mathrm{C}$ & -4.94573800 & -0.57855300 & 2.28750700 \\
\hline & $\mathrm{C}$ & -5.90642100 & -1.25323200 & 3.04315500 \\
\hline & $\mathrm{C}$ & -6.61990900 & -2.31440500 & 2.49950500 \\
\hline & $\mathrm{C}$ & -6.36899800 & -2.69574700 & 1.18224400 \\
\hline & $\mathrm{C}$ & -5.41800800 & -2.01914600 & 0.42822500 \\
\hline & $\mathrm{C}$ & -2.62347900 & -1.13417900 & -0.57838100 \\
\hline & $\mathrm{C}$ & -2.30560600 & -2.36184200 & 0.01098400 \\
\hline & $\mathrm{C}$ & -1.25387800 & -3.13594300 & -0.47697400 \\
\hline & $\mathrm{C}$ & -0.48665500 & -2.69033400 & -1.54926000 \\
\hline & C & -0.77986800 & -1.45860600 & -2.13137500 \\
\hline & $\mathrm{C}$ & -1.83445400 & -0.68907400 & -1.64920400 \\
\hline & $\mathrm{H}$ & -4.25661200 & -0.90451000 & -2.61797500 \\
\hline & $\mathrm{H}$ & -5.83105700 & 0.14745100 & -4.17685500 \\
\hline & $\mathrm{H}$ & -7.10827000 & 2.16846200 & -3.50065700 \\
\hline & $\mathrm{H}$ & -6.79287800 & 3.09975300 & -1.21636200 \\
\hline & $\mathrm{H}$ & -5.23360600 & 2.03620300 & 0.34867200 \\
\hline & $\mathrm{H}$ & -4.39039500 & 0.22521700 & 2.74644200 \\
\hline & $\mathrm{H}$ & -6.08877200 & -0.94222800 & 4.06604200 \\
\hline & $\mathrm{H}$ & -7.36333400 & -2.83760400 & 3.09038800 \\
\hline & $\mathrm{H}$ & -6.91908800 & -3.51769400 & 0.73749500 \\
\hline & $\mathrm{H}$ & -5.24743900 & -2.32195700 & -0.59772900 \\
\hline
\end{tabular}




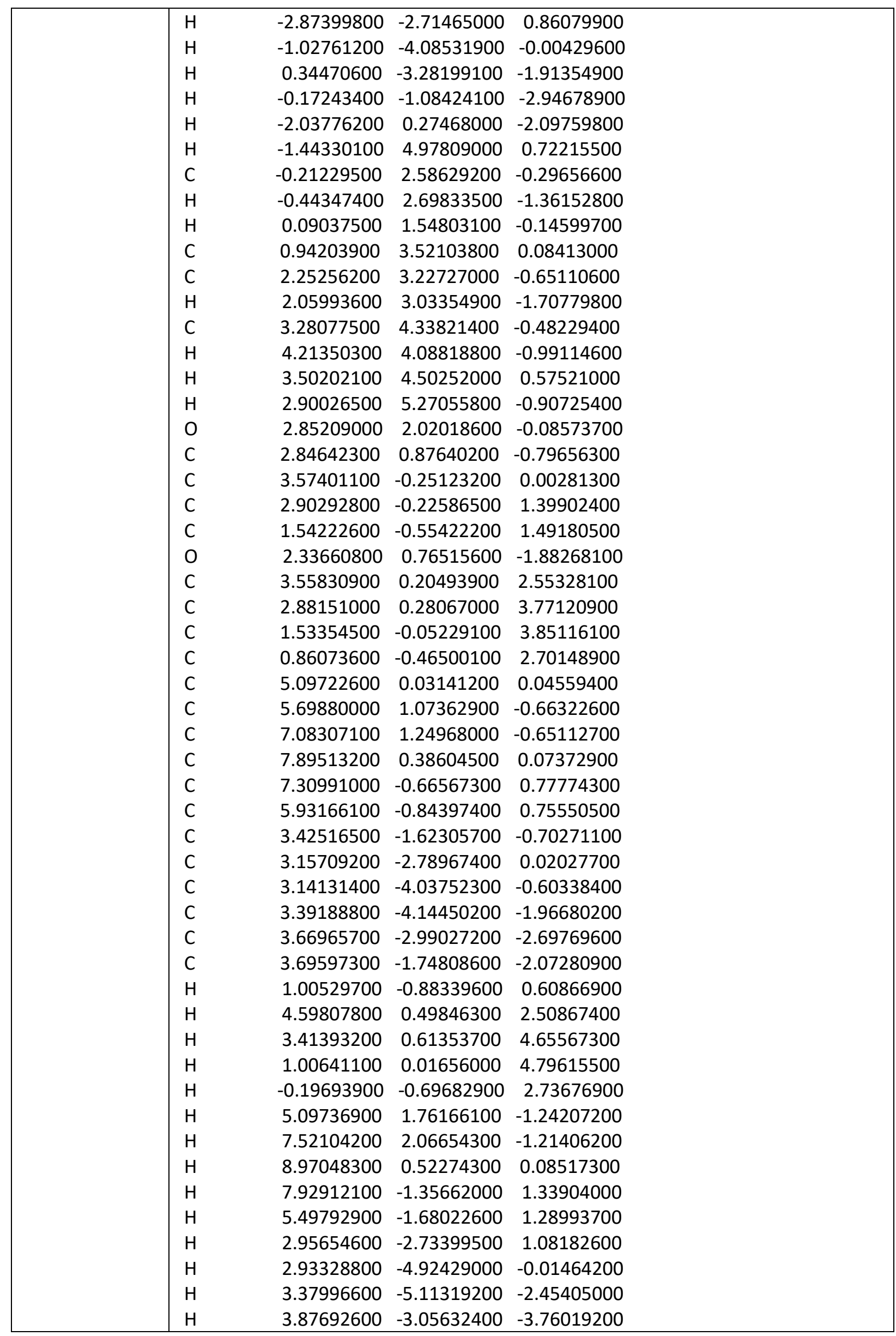




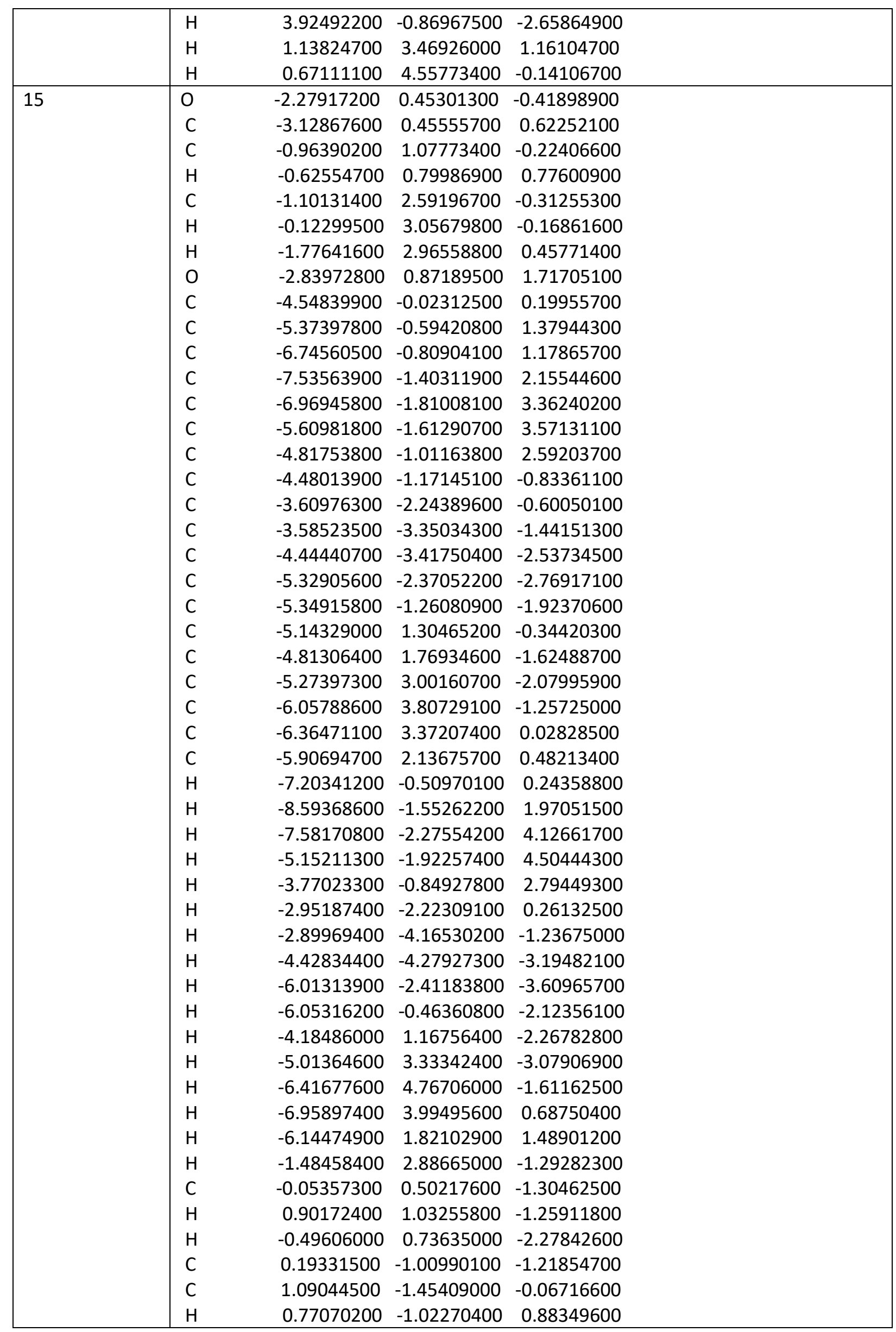




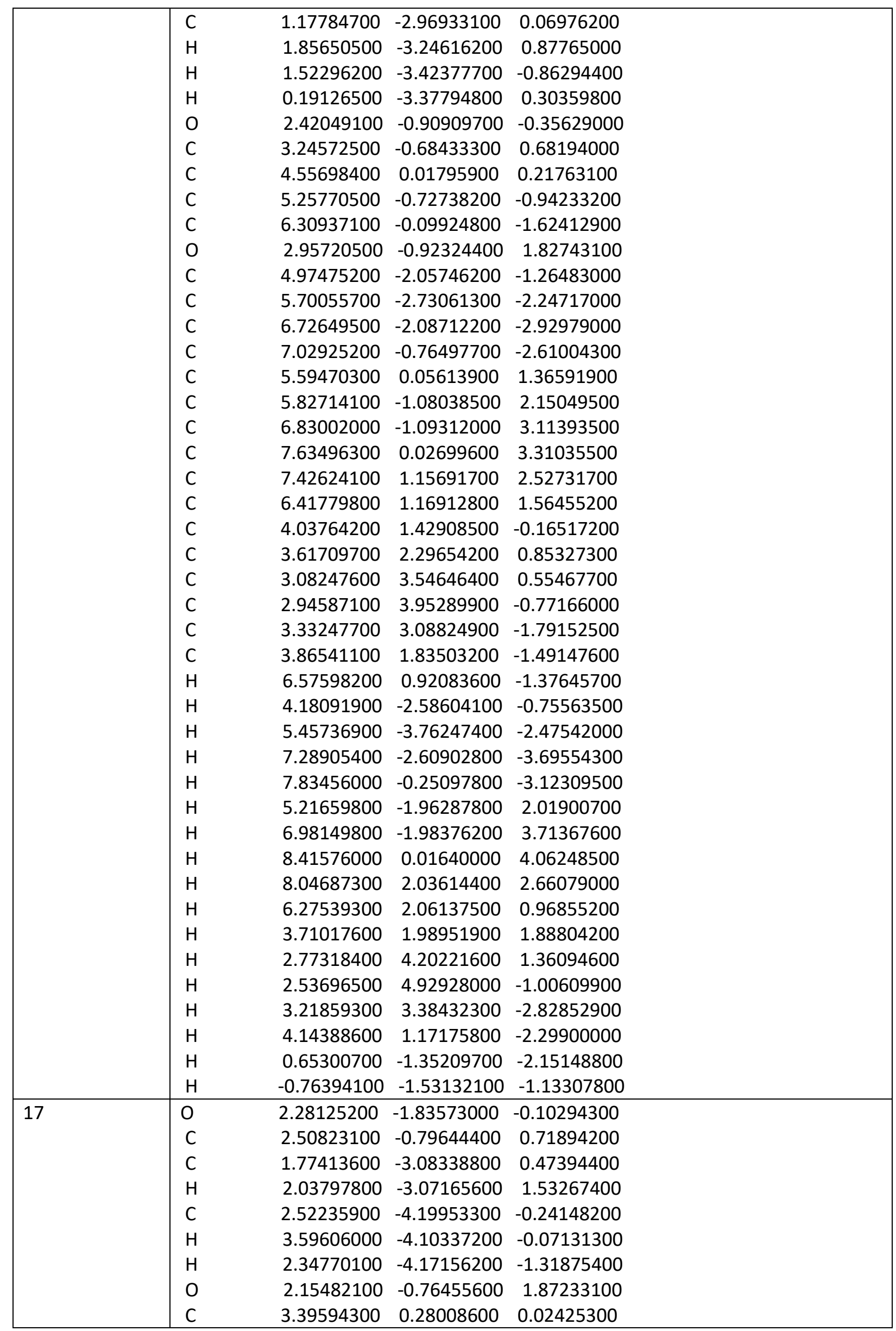




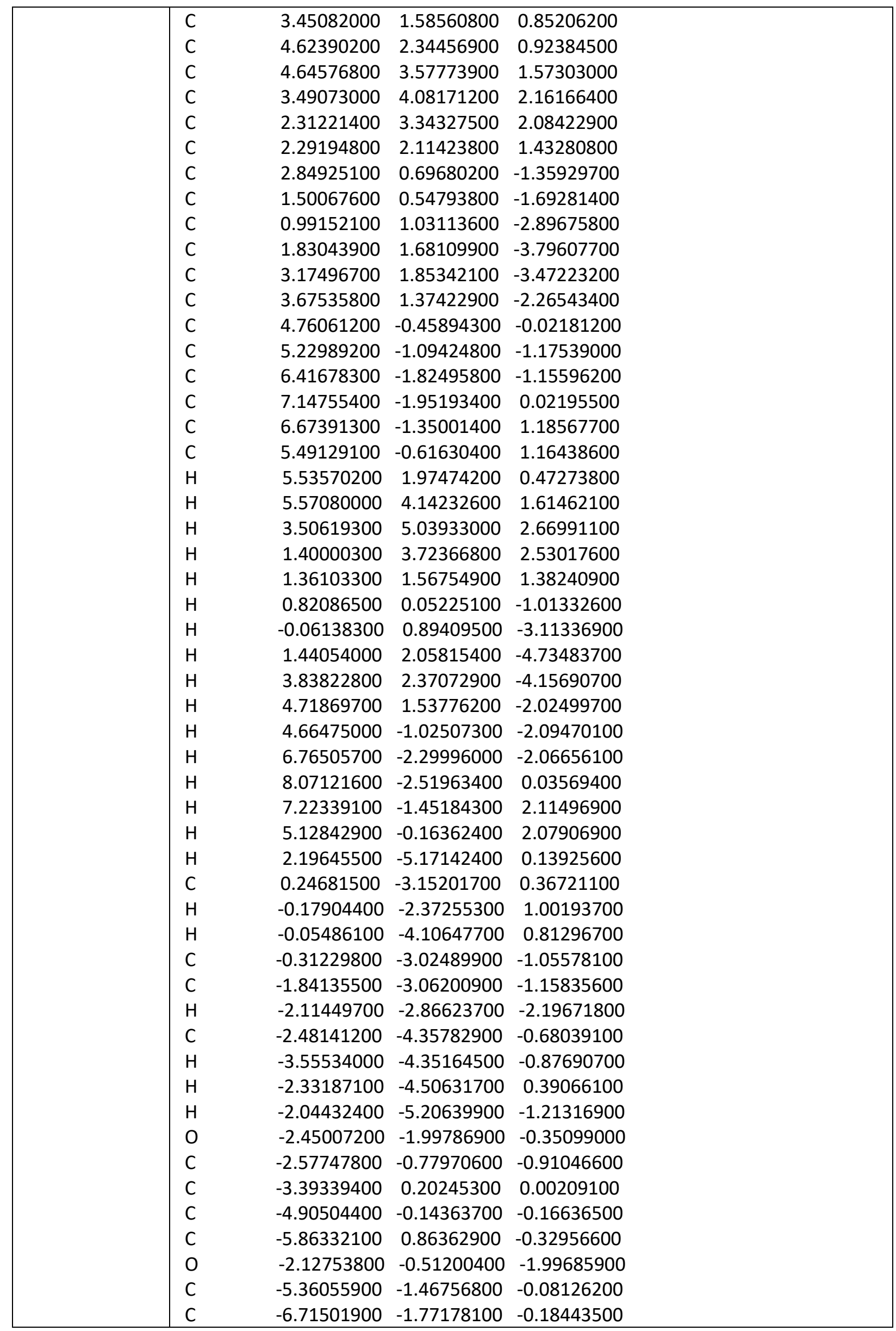




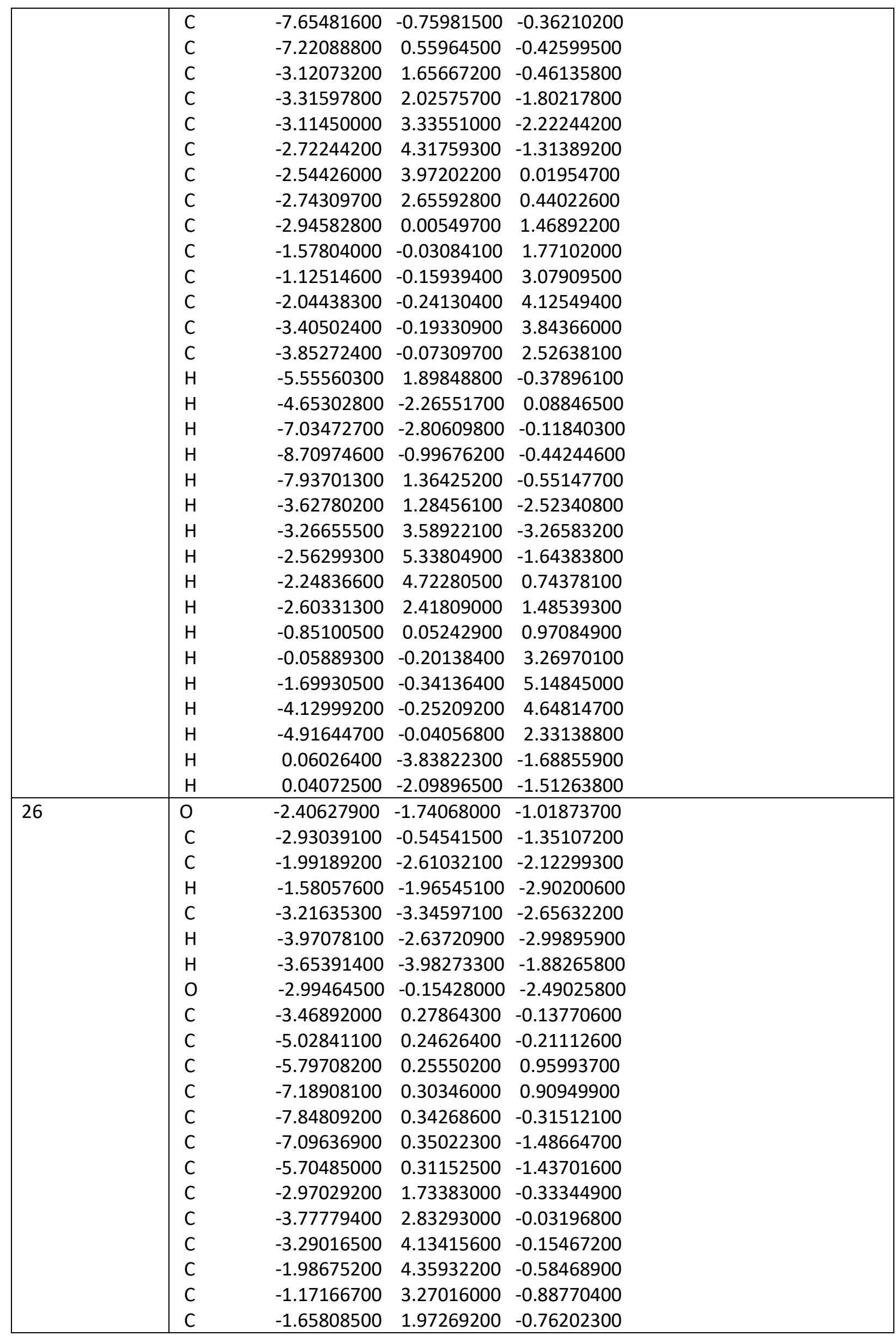




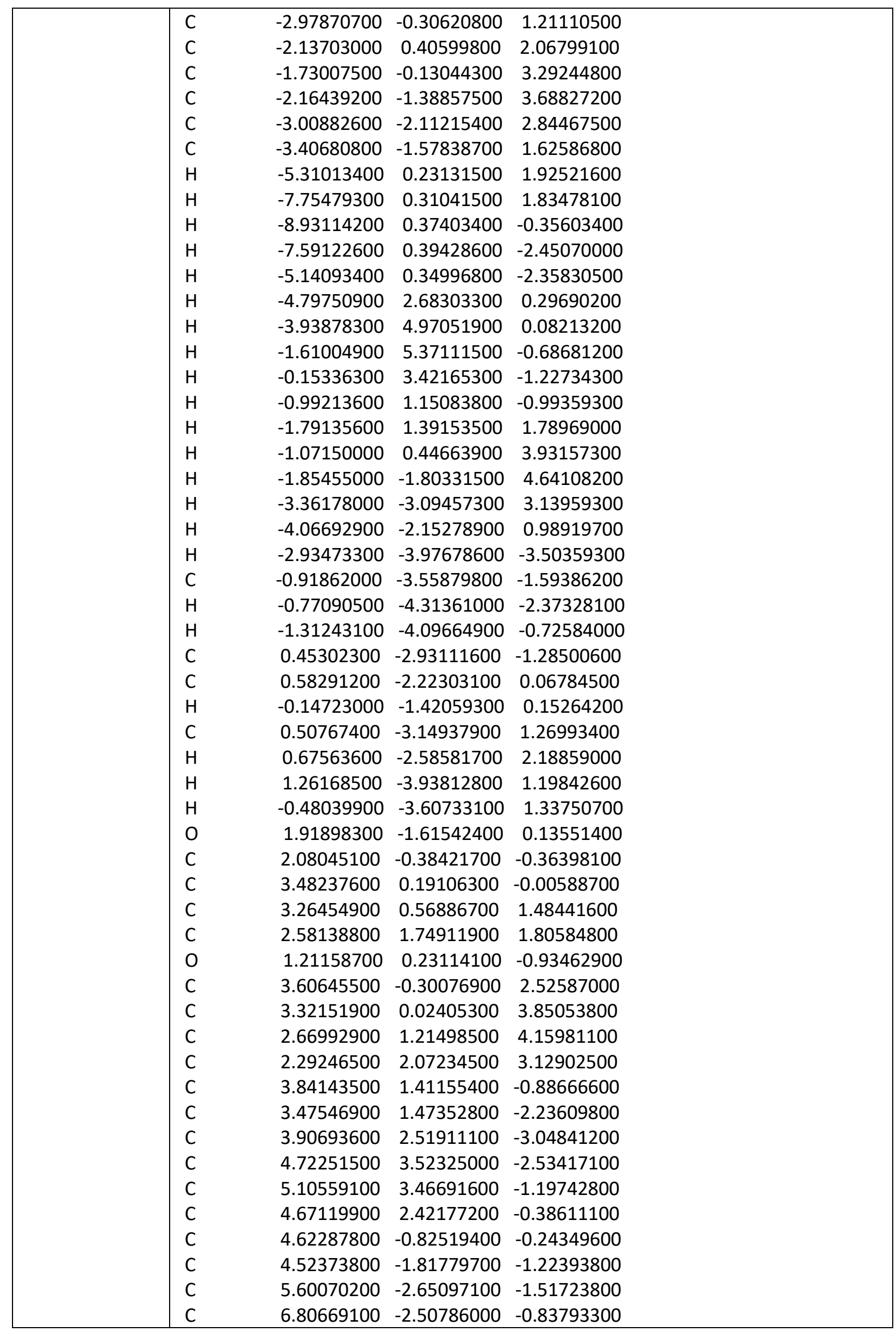




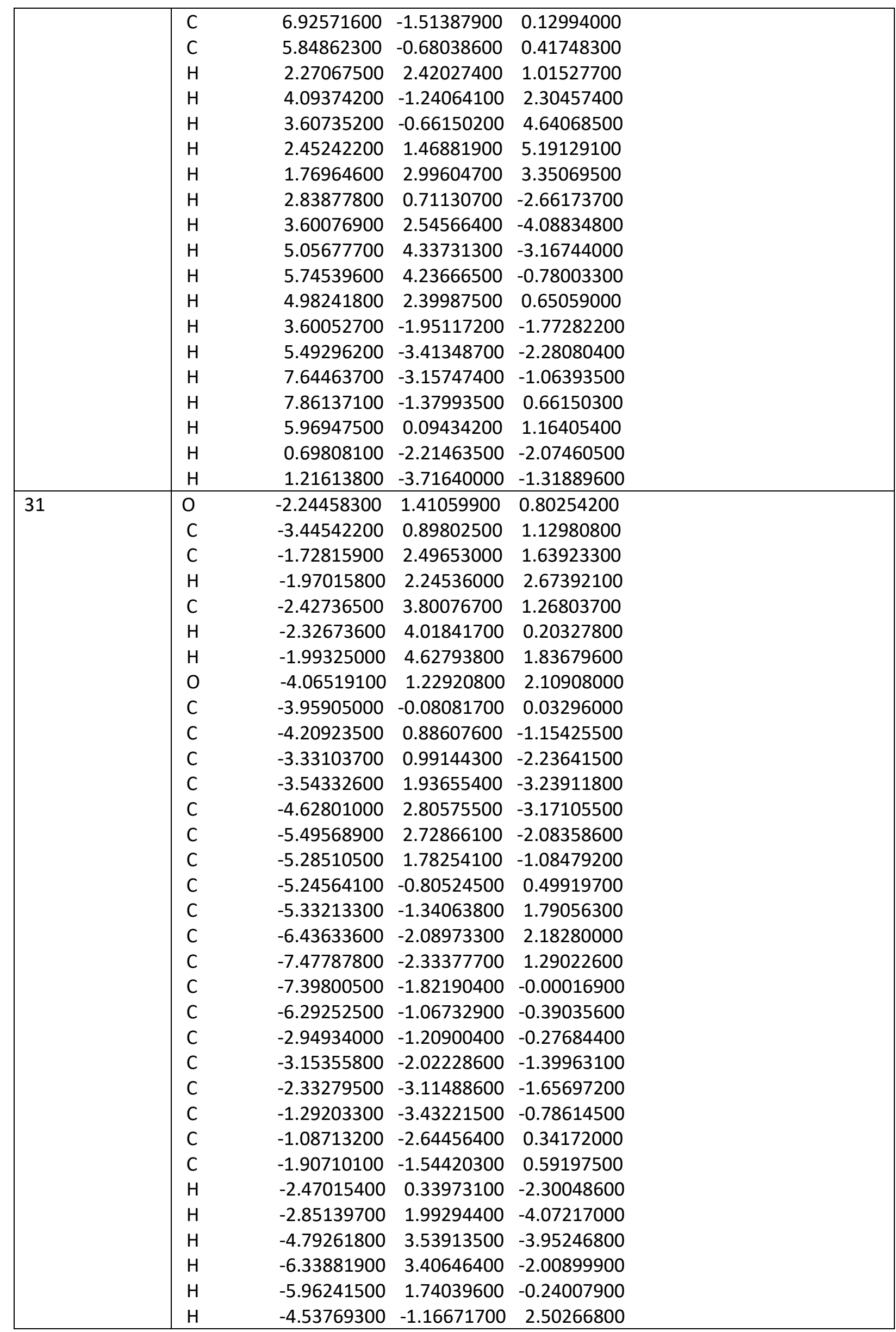




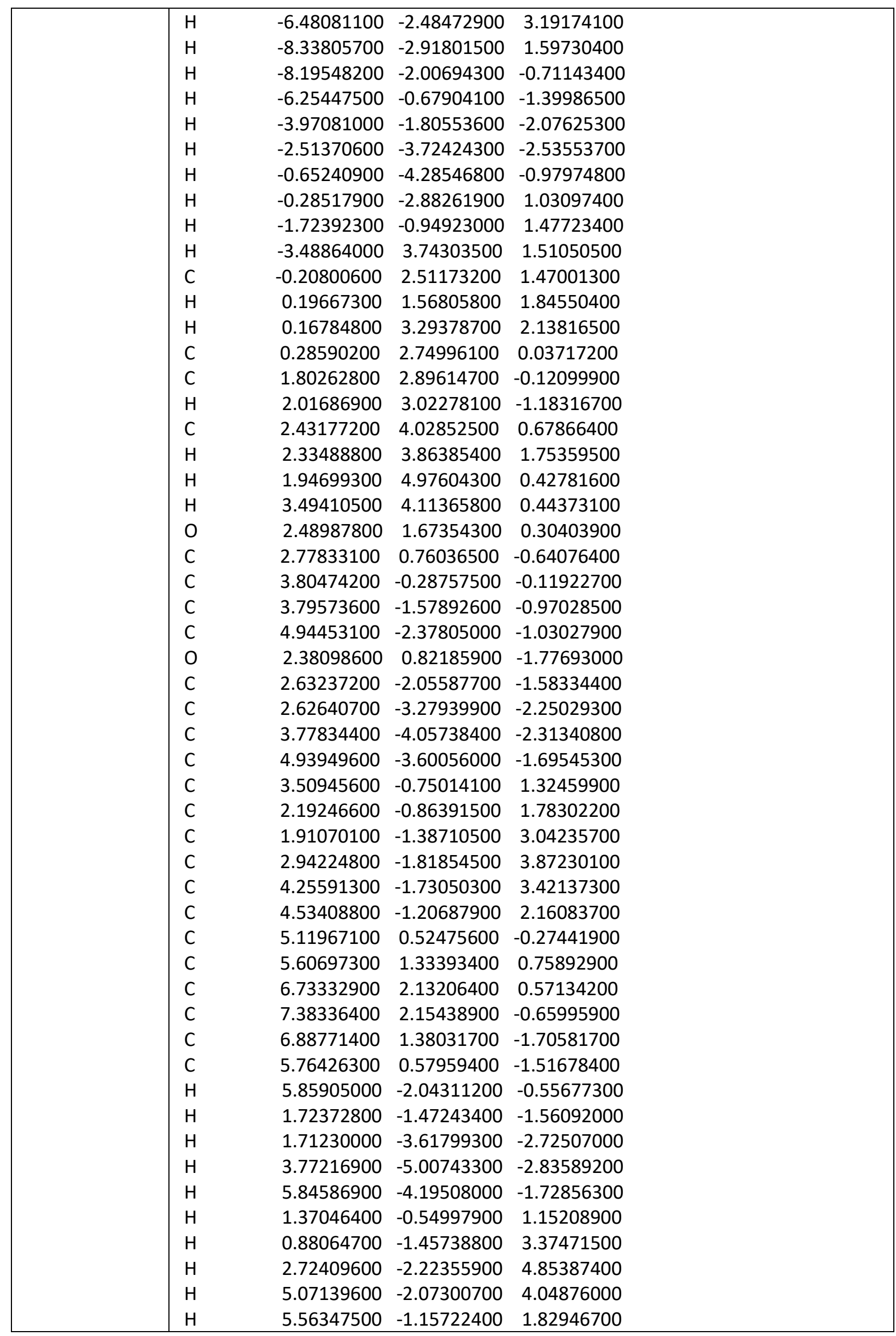




\begin{tabular}{|c|c|c|c|c|}
\hline & $\mathrm{H}$ & 5.10132500 & 1.34714000 & 1.71507900 \\
\hline & $\mathrm{H}$ & 7.09820700 & 2.74118100 & 1.39104700 \\
\hline & $\mathrm{H}$ & 8.26064300 & 2.77473000 & -0.80515800 \\
\hline & $\mathrm{H}$ & 7.37166100 & 1.40024600 & -2.67591700 \\
\hline & $\mathrm{H}$ & 5.38087300 & -0.00332600 & -2.34417600 \\
\hline & $\mathrm{H}$ & -0.15491300 & 3.66803000 & -0.36613500 \\
\hline & $\mathrm{H}$ & -0.05374300 & 1.93841600 & -0.60984100 \\
\hline \multirow[t]{45}{*}{36} & 0 & -2.17474000 & 0.72281000 & 1.31719800 \\
\hline & $\mathrm{C}$ & -3.51515200 & 0.62590800 & 1.35232200 \\
\hline & $\mathrm{C}$ & -1.51921700 & 1.44027300 & 2.41462500 \\
\hline & $\mathrm{H}$ & -2.01684400 & 1.13307900 & 3.33814000 \\
\hline & $\mathrm{C}$ & -1.68185800 & 2.94226200 & 2.21471000 \\
\hline & $\mathrm{H}$ & -1.19487200 & 3.47787200 & 3.03421300 \\
\hline & $\mathrm{H}$ & -2.73568000 & 3.22060800 & 2.20664400 \\
\hline & 0 & -4.18912600 & 1.03435000 & 2.26522100 \\
\hline & $\mathrm{C}$ & -4.06883700 & 0.03674700 & 0.02211000 \\
\hline & $\mathrm{C}$ & -3.25538000 & -1.19357900 & -0.44047700 \\
\hline & $\mathrm{C}$ & -3.09734600 & -1.50760500 & -1.79363900 \\
\hline & $\mathrm{C}$ & -2.47607500 & -2.69125900 & -2.18880900 \\
\hline & $\mathrm{C}$ & -2.00468300 & -3.59130700 & -1.23761100 \\
\hline & $\mathrm{C}$ & -2.16853900 & -3.29776700 & 0.11460500 \\
\hline & $\mathrm{C}$ & -2.78978200 & -2.11550600 & 0.50439900 \\
\hline & $\mathrm{C}$ & -3.96199700 & 1.27691300 & -0.90534000 \\
\hline & $\mathrm{C}$ & -5.00187000 & 2.21396700 & -0.94229700 \\
\hline & $\mathrm{C}$ & -4.88499600 & 3.38494100 & -1.68749600 \\
\hline & $\mathrm{C}$ & -3.71997100 & 3.64958800 & -2.40211400 \\
\hline & $\mathrm{C}$ & -2.66675400 & 2.73994400 & -2.34870400 \\
\hline & $\mathrm{C}$ & -2.78227400 & 1.57144700 & -1.59983400 \\
\hline & $\mathrm{C}$ & -5.52271700 & -0.47908100 & 0.15442000 \\
\hline & $\mathrm{C}$ & -6.29962100 & -0.61637600 & -1.00418700 \\
\hline & $\mathrm{C}$ & -7.57008500 & -1.18073300 & -0.96016200 \\
\hline & $\mathrm{C}$ & -8.09517400 & -1.63116000 & 0.24876200 \\
\hline & $\mathrm{C}$ & -7.33139300 & -1.51109200 & 1.40465600 \\
\hline & $\mathrm{C}$ & -6.05823000 & -0.94447900 & 1.35990800 \\
\hline & $\mathrm{H}$ & -3.46509500 & -0.82957500 & -2.55284900 \\
\hline & $\mathrm{H}$ & -2.36842600 & -2.90945300 & -3.24561600 \\
\hline & $\mathrm{H}$ & -1.52585500 & -4.51441300 & -1.54388700 \\
\hline & $\mathrm{H}$ & -1.81883500 & -3.99359000 & 0.86916000 \\
\hline & $\mathrm{H}$ & -2.92449100 & -1.91952400 & 1.56186300 \\
\hline & $\mathrm{H}$ & -5.90901200 & 2.02953900 & -0.38161300 \\
\hline & $\mathrm{H}$ & -5.70736400 & 4.09143600 & -1.70377900 \\
\hline & $\mathrm{H}$ & -3.62969600 & 4.55872800 & -2.98598000 \\
\hline & $\mathrm{H}$ & -1.74500400 & 2.94010200 & -2.88353700 \\
\hline & $\mathrm{H}$ & -1.94207400 & 0.89258000 & -1.54635400 \\
\hline & $\mathrm{H}$ & -5.91004400 & -0.27640100 & -1.95583900 \\
\hline & $\mathrm{H}$ & -8.14780400 & -1.26980100 & -1.87356800 \\
\hline & $\mathrm{H}$ & -9.08568100 & -2.07038600 & 0.28736300 \\
\hline & $\mathrm{H}$ & -7.72427800 & -1.85663700 & 2.35456000 \\
\hline & $\mathrm{H}$ & -5.49728200 & -0.84945200 & 2.27714400 \\
\hline & $\mathrm{H}$ & -1.22228500 & 3.25609300 & 1.27464000 \\
\hline & $\mathrm{C}$ & -0.06041300 & 0.99706000 & 2.41113700 \\
\hline & $\mathrm{H}$ & 0.45320100 & 1.57340300 & 3.18994400 \\
\hline
\end{tabular}




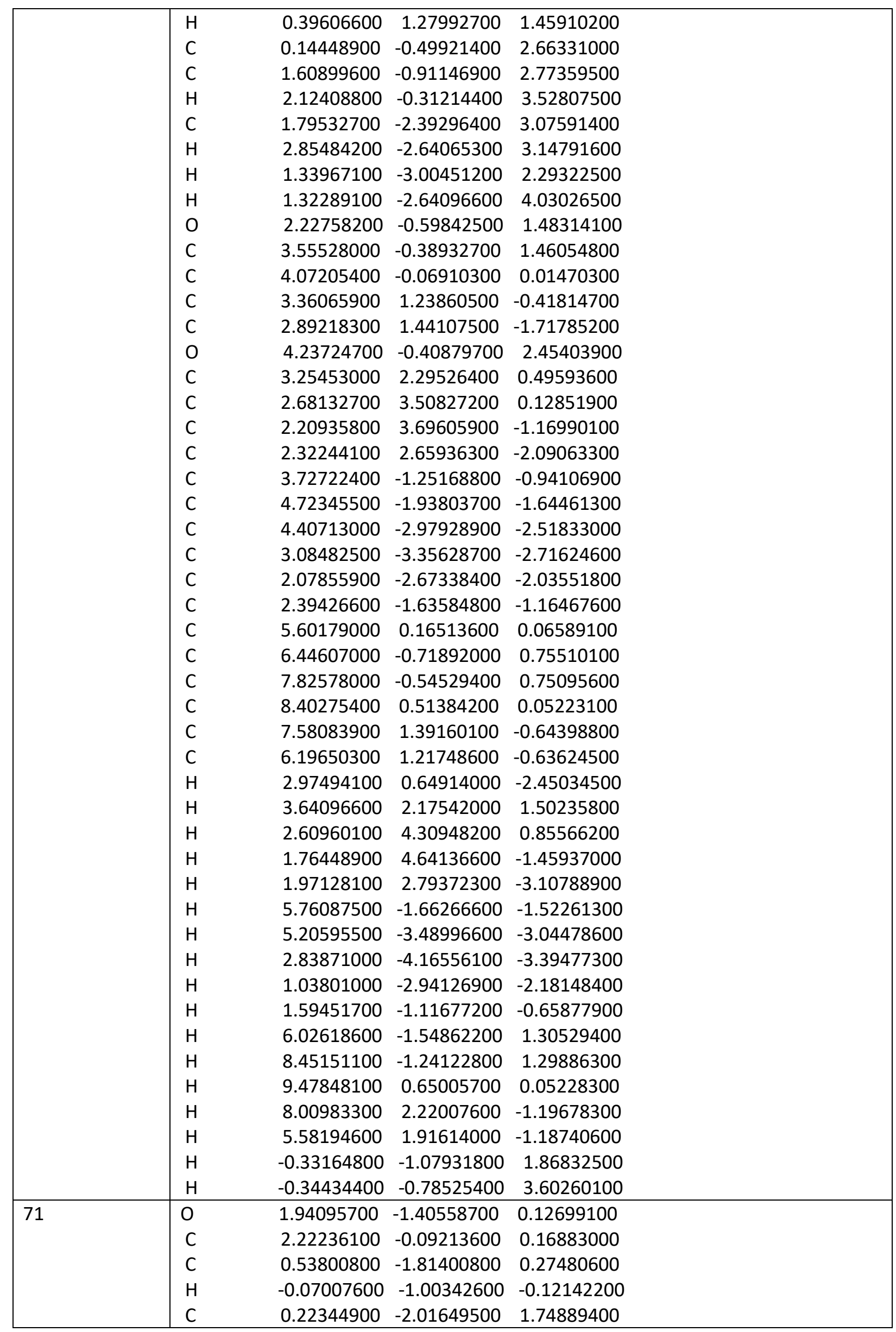




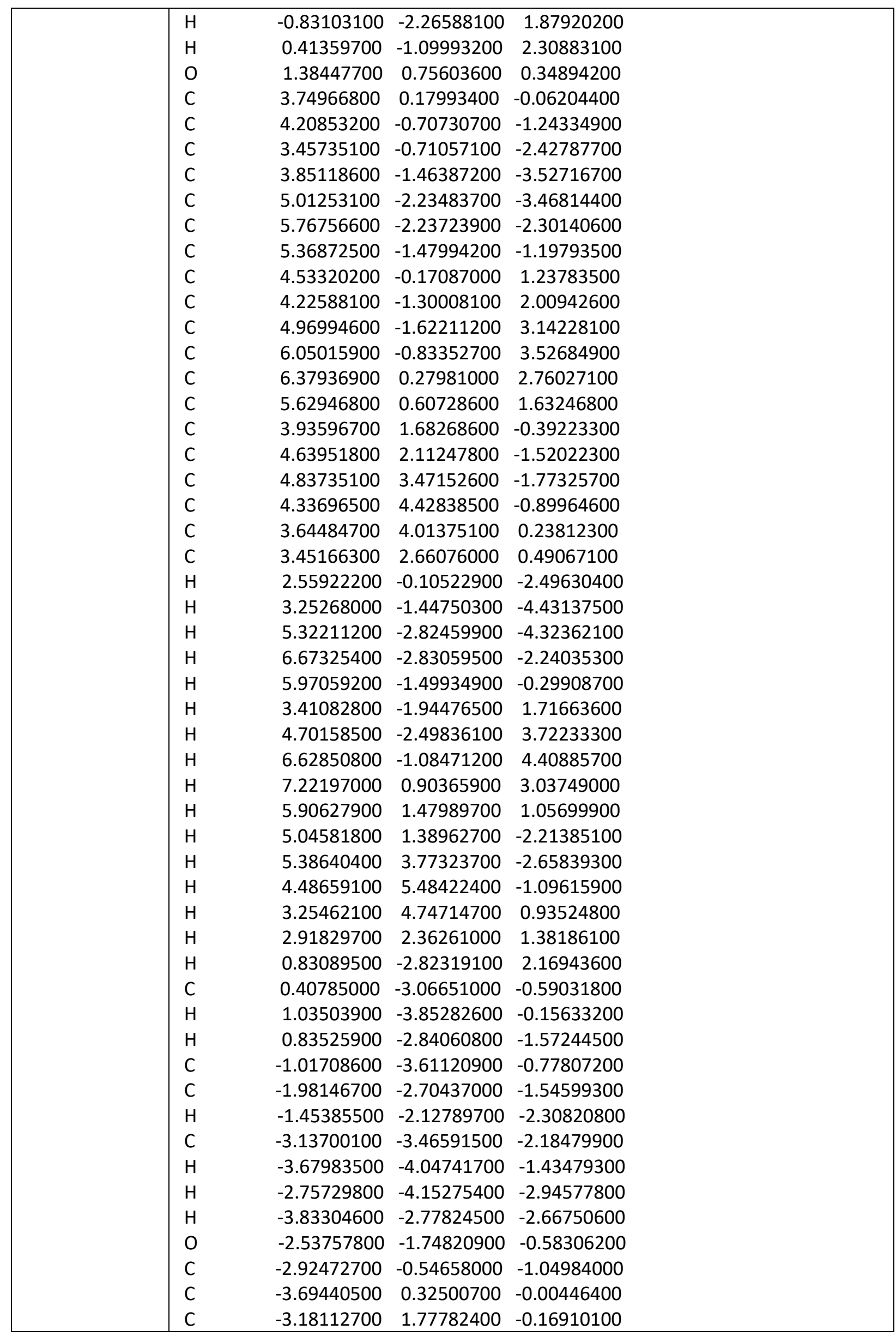




\begin{tabular}{|l|lrrr|}
\hline & C & -4.04942400 & 2.87152600 & -0.16145300 \\
O & -2.72037100 & -0.18684000 & -2.18296700 \\
C & -1.80613700 & 2.02702300 & -0.27127600 \\
C & -1.31544400 & 3.32462300 & -0.36767900 \\
C & -2.19319700 & 4.40744600 & -0.36330000 \\
C & -3.56003600 & 4.17463500 & -0.25734800 \\
C & -3.47958700 & -0.18849600 & 1.44301400 \\
C & -3.94859600 & -1.45815300 & 1.81968700 \\
C & -3.79205600 & -1.93179300 & 3.11616500 \\
C & -3.16303200 & -1.14525900 & 4.08227500 \\
C & -2.70217200 & 0.11614500 & 3.72928000 \\
C & -2.86119800 & 0.58963500 & 2.42440600 \\
C & -5.20599300 & 0.25232300 & -0.38959900 \\
C & -6.19784500 & 0.28390700 & 0.59870000 \\
C & -7.55064800 & 0.29193800 & 0.26282700 \\
C & -7.94496900 & 0.26790700 & -1.07106200 \\
C & -6.96954700 & 0.25309800 & -2.06414600 \\
C & -5.61750200 & 0.25413200 & -1.72961100 \\
H & -5.11727500 & 2.71585500 & -0.08569400 \\
H & -1.09263400 & 1.21249100 & -0.26242400 \\
H & -0.24543500 & 3.47989000 & -0.44370500 \\
H & -1.81395700 & 5.42024400 & -0.44299200 \\
H & -4.25636900 & 5.00612200 & -0.25353000 \\
H & -4.45041800 & -2.07906900 & 1.08998600 \\
H & -4.16788300 & -2.91580100 & 3.37511700 \\
H & -3.04276600 & -1.51202300 & 5.09558200 \\
H & -2.21788800 & 0.74679100 & 4.46660600 \\
H & -2.49933400 & 1.57865300 & 2.18206500 \\
H & -5.91852700 & 0.30798700 & 1.64318100 \\
H & -8.29420800 & 0.31745600 & 1.05186400 \\
H & -8.99700900 & 0.26796100 & -1.33341500 \\
H & -7.25716700 & 0.24803300 & -3.10981300 \\
H & -4.87780400 & 0.27364800 & -2.51784500 \\
H & -1.47481300 & -3.87228200 & 0.18125500 \\
H & -0.93502200 & -4.54725300 & -1.33951100 \\
\hline & & & \\
& & & \\
& &
\end{tabular}

22 (optimized at the B3LYP-GD3BJ/6-311G(d,p) level)

\begin{tabular}{|l|llll|}
\hline Conformer no & & & \\
\hline 1 & O & -0.89732700 & 1.97856600 & -1.52191500 \\
& C & -0.40953000 & 3.22365300 & -1.43556000 \\
& C & -1.28514800 & 1.46287200 & -2.83020700 \\
& H & -0.79643900 & 2.08686300 & -3.58050300 \\
& C & -2.79532800 & 1.57285600 & -2.95855400 \\
& H & -3.11886800 & 1.18116500 & -3.92613500 \\
& H & -3.11027800 & 2.61645400 & -2.89275000 \\
& O & -0.30265400 & 3.97972200 & -2.36726200 \\
& C & 0.07321400 & 3.49071800 & 0.01306500 \\
& C & -1.05232000 & 3.23560500 & 1.03030100 \\
& C & -0.74316800 & 3.13046100 & 2.39028500 \\
& C & -1.74317500 & 2.96920100 & 3.34266200 \\
& C & -3.08147600 & 2.93433900 & 2.95653200 \\
\hline
\end{tabular}




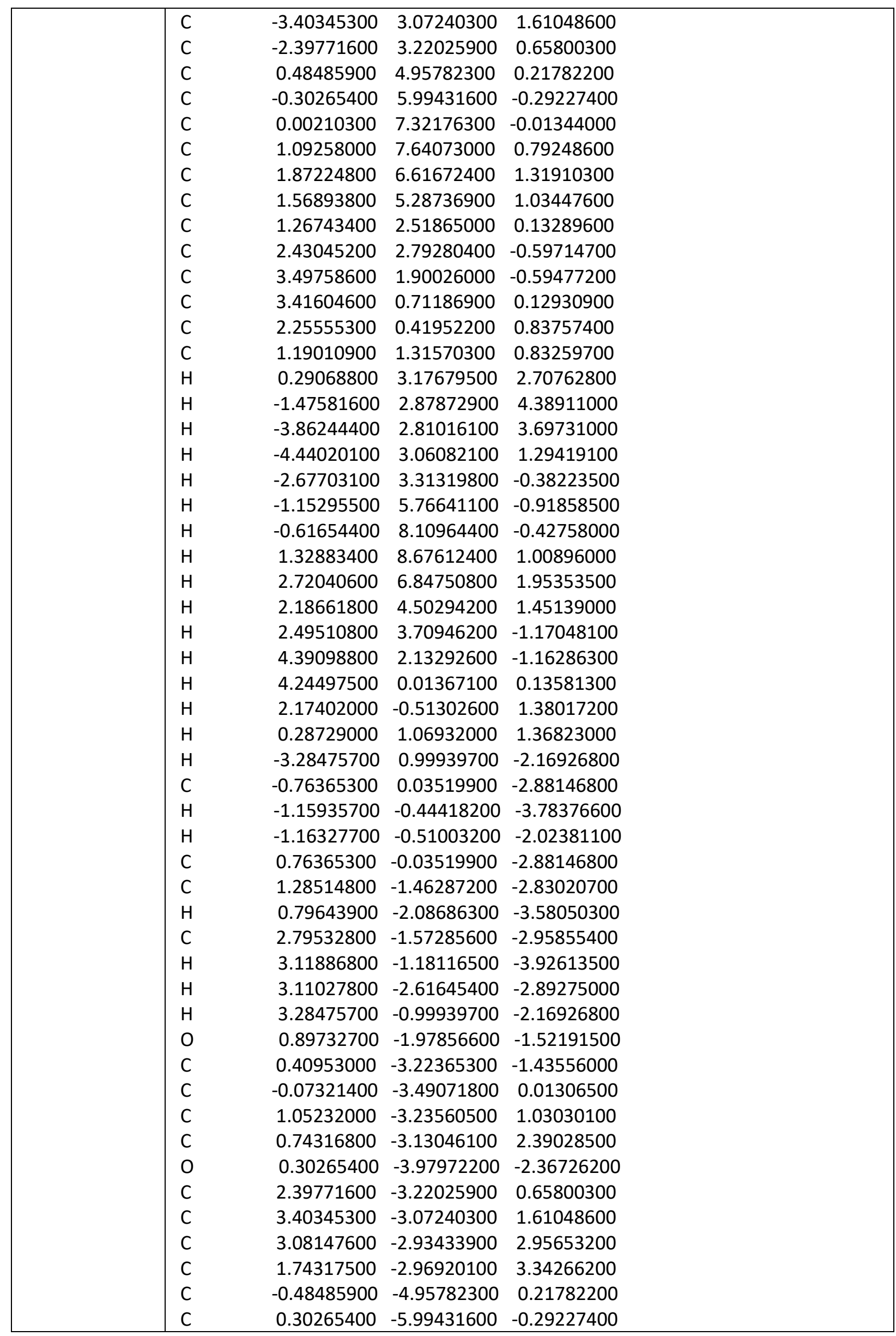




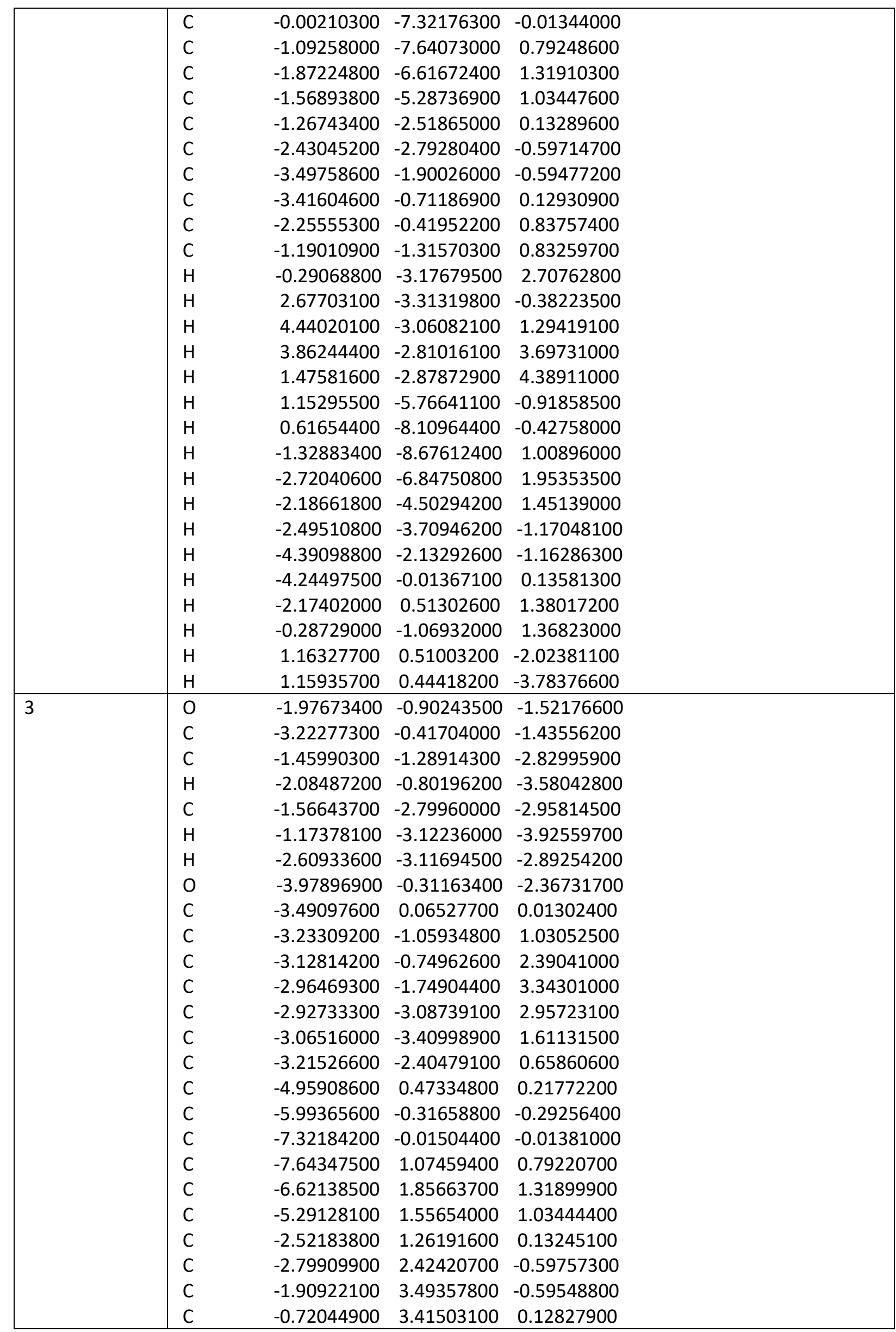




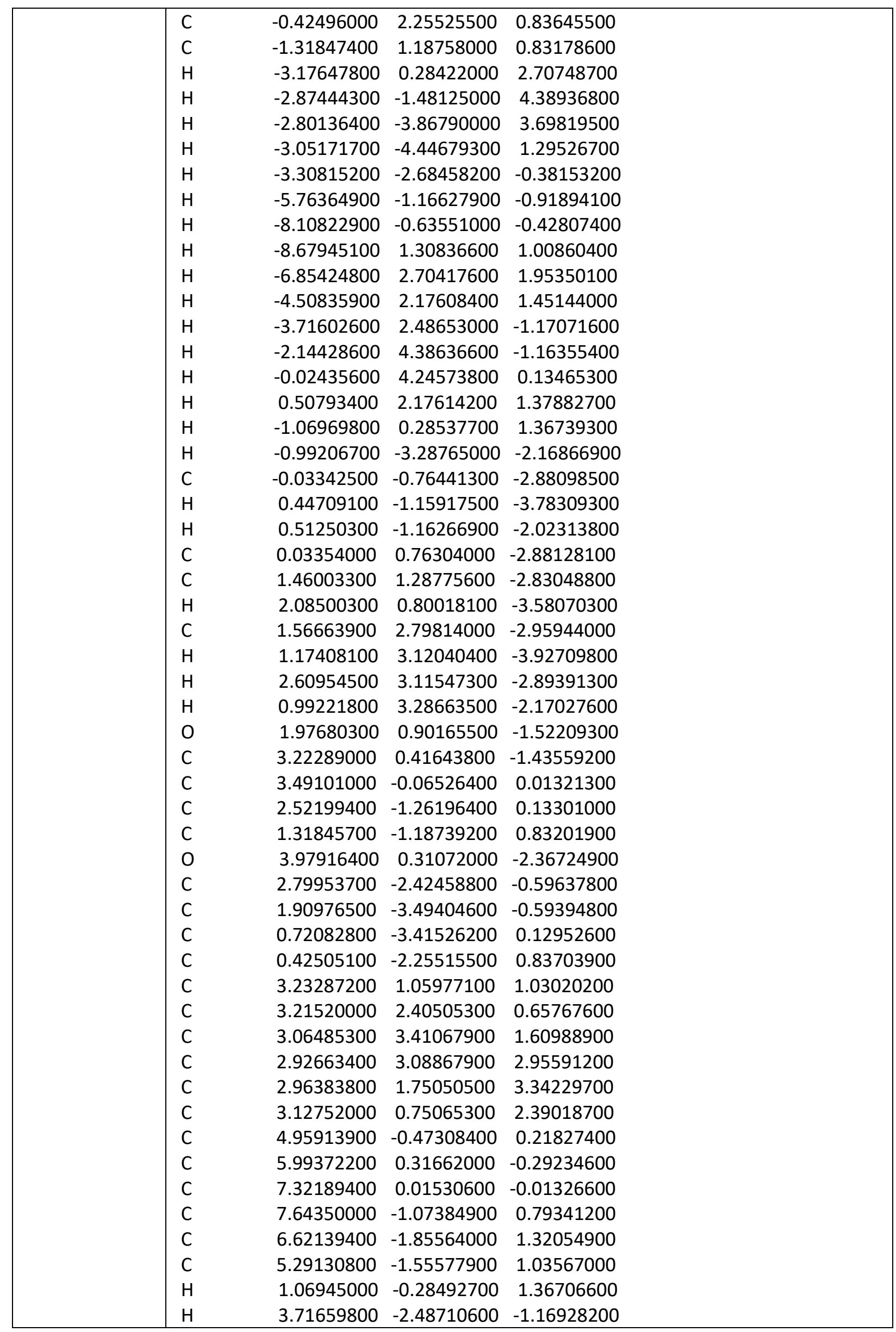




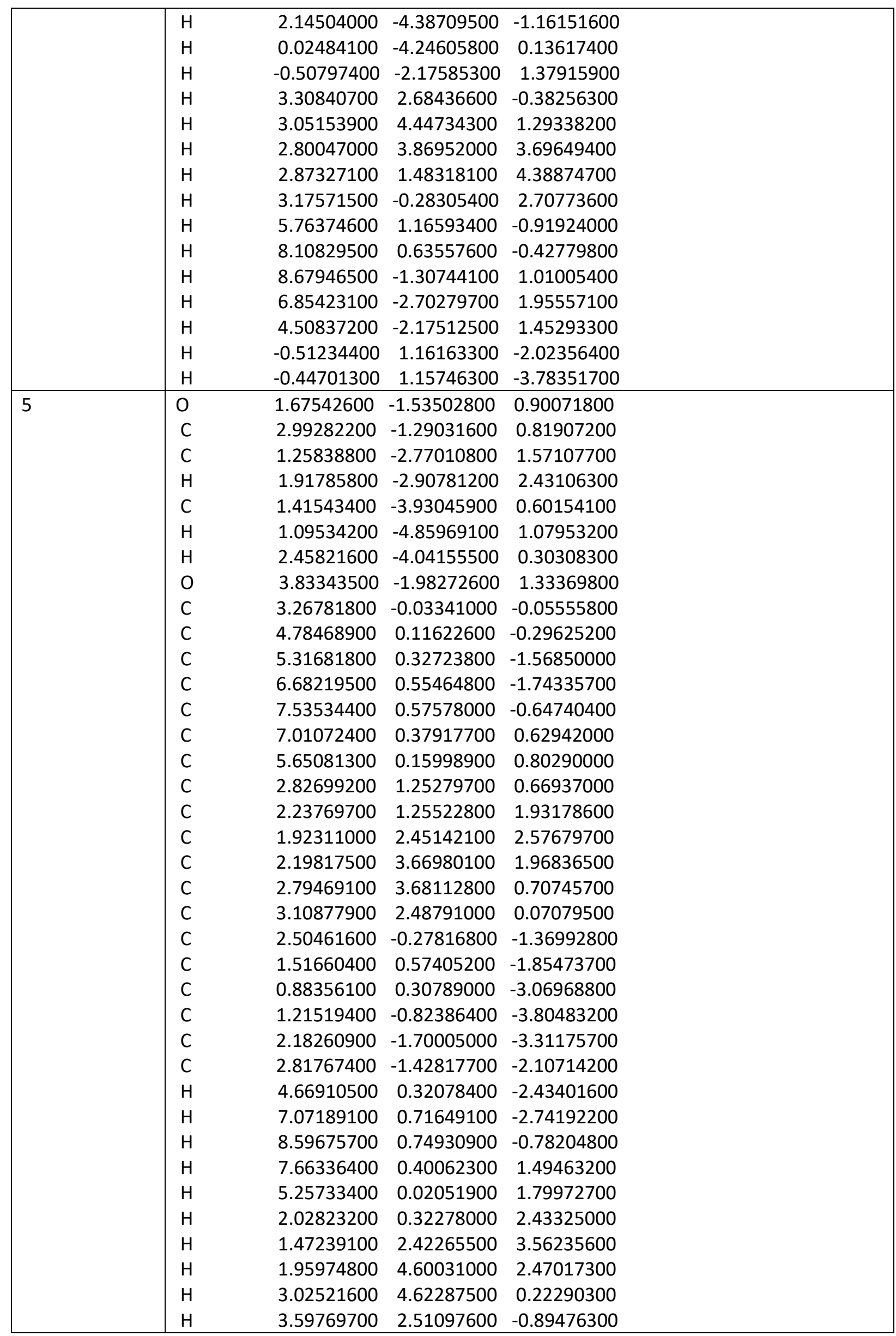




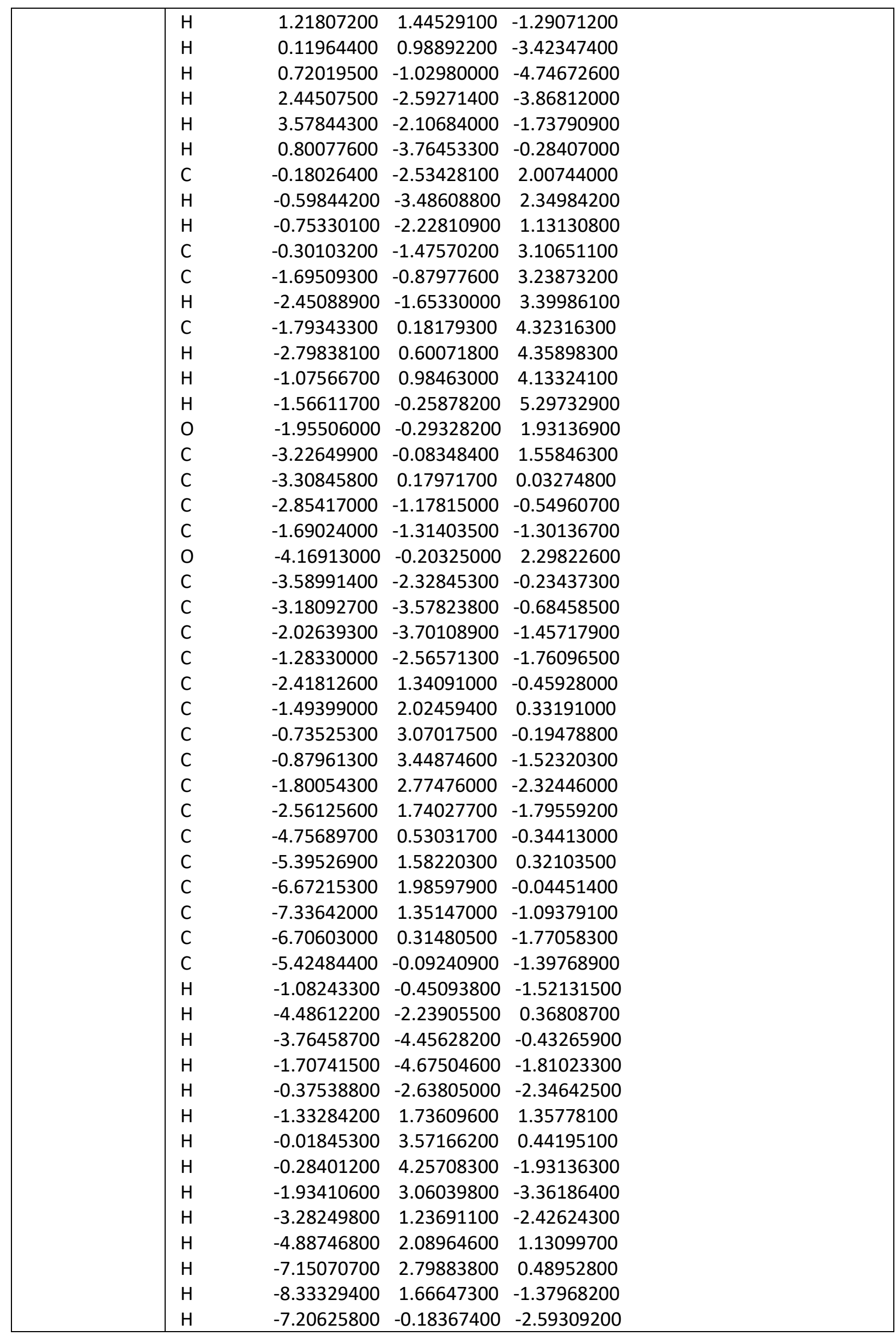




\begin{tabular}{|c|c|c|c|c|}
\hline & $\begin{array}{l}\mathrm{H} \\
\mathrm{H} \\
\mathrm{H}\end{array}$ & $\begin{array}{r}-4.94789100 \\
0.37546800 \\
0.00028300\end{array}$ & $\begin{array}{l}-0.89900400 \\
-0.64936000 \\
-1.88796000\end{array}$ & $\begin{array}{r}-1.93813900 \\
2.88205200 \\
4.07507400\end{array}$ \\
\hline \multirow[t]{49}{*}{7} & 0 & -2.12198800 & 0.74389200 & 1.74631800 \\
\hline & C & -3.33219400 & 0.19965800 & 1.54627200 \\
\hline & C & -1.64628000 & 0.90303200 & 3.11487500 \\
\hline & $\mathrm{H}$ & -2.26176300 & 0.26328400 & 3.74946200 \\
\hline & C & -1.82264800 & 2.35965700 & 3.51136000 \\
\hline & $\mathrm{H}$ & -1.45405200 & 2.52269000 & 4.52708100 \\
\hline & $\mathrm{H}$ & -2.87758600 & 2.63980800 & 3.48327700 \\
\hline & $\mathrm{O}$ & -4.08939600 & -0.11207500 & 2.42969000 \\
\hline & C & -3.54822800 & -0.06073900 & 0.03548600 \\
\hline & C & -3.32450300 & 1.20717200 & -0.80781300 \\
\hline & C & -3.19669200 & 1.09324200 & -2.19593100 \\
\hline & C & -3.12131800 & 2.22151400 & -3.00371300 \\
\hline & C & -3.18686400 & 3.49455800 & -2.44002200 \\
\hline & C & -3.32298500 & 3.62047400 & -1.06156300 \\
\hline & C & -3.38927400 & 2.48656100 & -0.25446500 \\
\hline & C & -4.99554300 & -0.48806500 & -0.26464700 \\
\hline & C & -6.07014300 & 0.16694600 & 0.34379700 \\
\hline & C & -7.38017900 & -0.13477100 & -0.01082000 \\
\hline & C & -7.64346800 & -1.08849700 & -0.99110800 \\
\hline & C & -6.58122600 & -1.73349600 & -1.61552300 \\
\hline & C & -5.26969600 & -1.43331200 & -1.25581200 \\
\hline & C & -2.52494600 & -1.18284600 & -0.23766100 \\
\hline & C & -2.78738000 & -2.47369800 & 0.23683000 \\
\hline & C & -1.85395000 & -3.49274200 & 0.07467300 \\
\hline & C & -0.63876100 & -3.23428100 & -0.55745400 \\
\hline & C & -0.35681500 & -1.94524300 & -0.99762800 \\
\hline & C & -1.28744500 & -0.92482700 & -0.82609900 \\
\hline & $\mathrm{H}$ & -3.16535500 & 0.11146800 & -2.65010000 \\
\hline & $\mathrm{H}$ & -3.02172000 & 2.10576300 & -4.07682900 \\
\hline & $\mathrm{H}$ & -3.13597000 & 4.37566600 & -3.06893100 \\
\hline & $\mathrm{H}$ & -3.37973300 & 4.60309100 & -0.60735100 \\
\hline & $\mathrm{H}$ & -3.49628500 & 2.61244900 & 0.81425600 \\
\hline & $\mathrm{H}$ & -5.88627900 & 0.91117000 & 1.10509800 \\
\hline & $\mathrm{H}$ & -8.19802400 & 0.37927800 & 0.48100300 \\
\hline & $\mathrm{H}$ & -8.66519300 & -1.32279900 & -1.26658900 \\
\hline & $\mathrm{H}$ & -6.76850800 & -2.47304400 & -2.38567300 \\
\hline & $\mathrm{H}$ & -4.45503800 & -1.94492600 & -1.75077100 \\
\hline & $\mathrm{H}$ & -3.72769900 & -2.67889600 & 0.73318100 \\
\hline & $\mathrm{H}$ & -2.07803000 & -4.48763800 & 0.44202700 \\
\hline & $\mathrm{H}$ & 0.08543400 & -4.02897700 & -0.69703800 \\
\hline & $\mathrm{H}$ & 0.59463200 & -1.72065400 & -1.45969000 \\
\hline & $\mathrm{H}$ & -1.03256000 & 0.07628400 & -1.13772000 \\
\hline & $\mathrm{H}$ & -1.26703500 & 3.00984400 & 2.83120800 \\
\hline & C & -0.19786400 & 0.43872200 & 3.10878100 \\
\hline & $\mathrm{H}$ & 0.24425100 & 0.67471700 & 4.08330000 \\
\hline & $\mathrm{H}$ & 0.34202400 & 1.01579900 & 2.35612800 \\
\hline & C & -0.04251600 & -1.05337900 & 2.81432400 \\
\hline & C & 1.41154900 & -1.47692600 & 2.67328900 \\
\hline & $\mathrm{H}$ & 2.01888000 & -1.07726500 & 3.48752800 \\
\hline
\end{tabular}




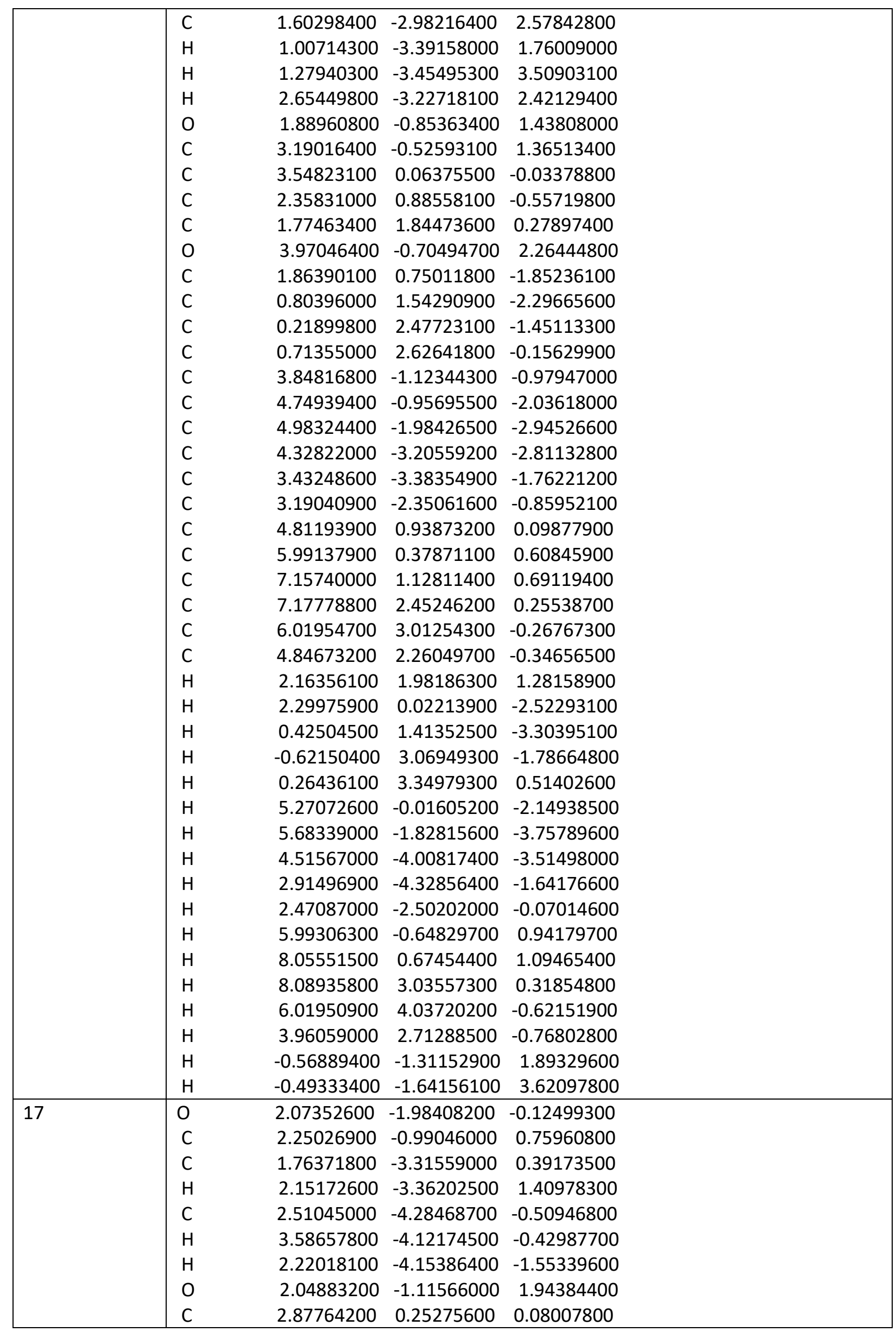




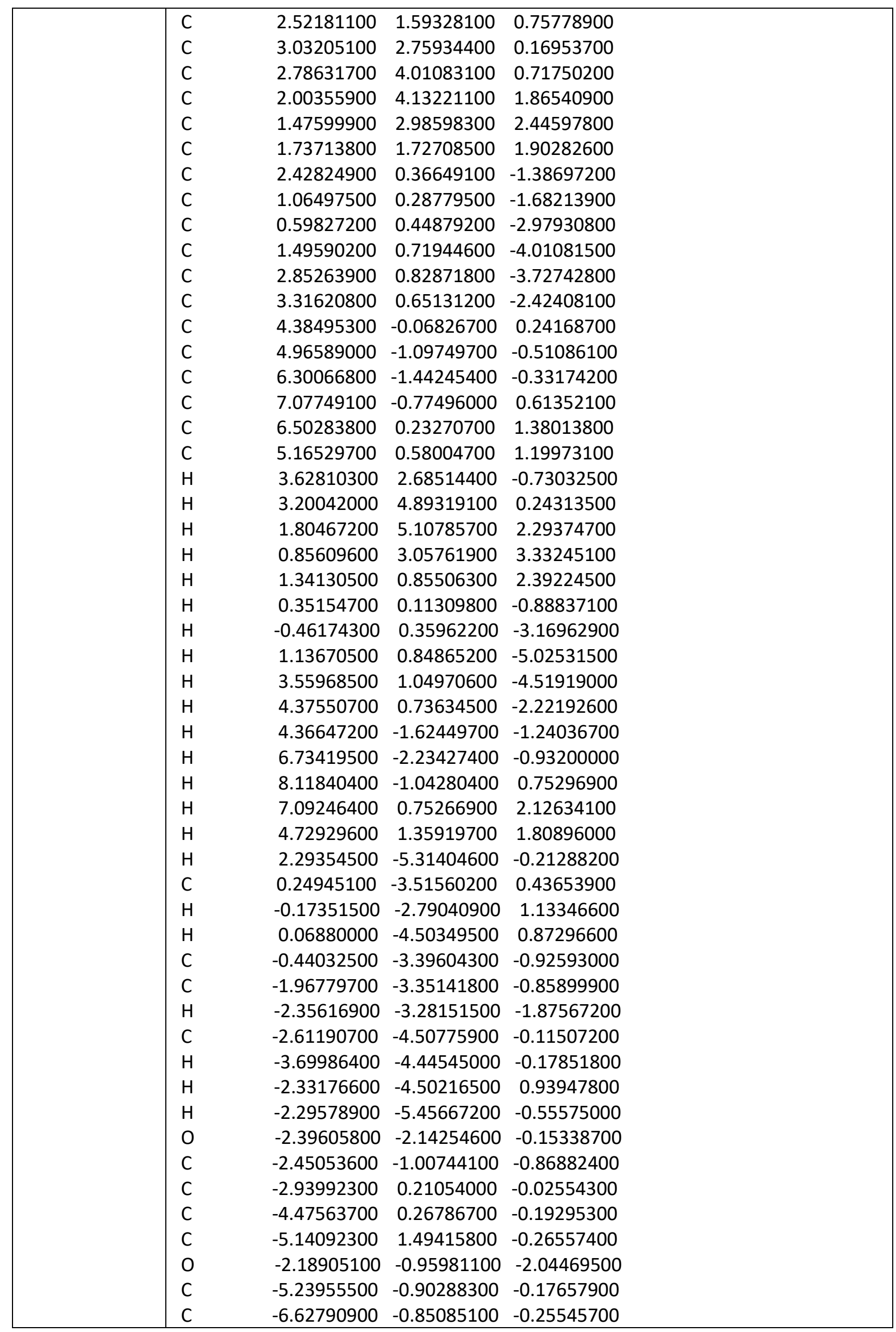




\begin{tabular}{|c|c|c|c|c|}
\hline & $\mathrm{C}$ & -7.28098000 & 0.37568500 & -0.33716900 \\
\hline & C & -6.53074100 & 1.54771400 & -0.33553300 \\
\hline & $\mathrm{C}$ & -2.26906500 & 1.46864900 & -0.61746800 \\
\hline & $\mathrm{C}$ & -2.55133800 & 1.84339700 & -1.93918900 \\
\hline & $\mathrm{C}$ & -1.92750800 & 2.94041900 & -2.51777800 \\
\hline & $\mathrm{C}$ & -1.00421300 & 3.68944000 & -1.78990900 \\
\hline & $\mathrm{C}$ & -0.73171500 & 3.34088700 & -0.47471600 \\
\hline & $\mathrm{C}$ & -1.36657500 & 2.24448100 & 0.10887100 \\
\hline & $\mathrm{C}$ & -2.58450400 & 0.02951000 & 1.45905200 \\
\hline & $\mathrm{C}$ & -1.30130000 & -0.38870800 & 1.82482400 \\
\hline & $\mathrm{C}$ & -0.93563800 & -0.52757500 & 3.15704700 \\
\hline & $\mathrm{C}$ & -1.85381000 & -0.22946900 & 4.16310200 \\
\hline & $\mathrm{C}$ & -3.12792200 & 0.20425700 & 3.81608100 \\
\hline & $\mathrm{C}$ & -3.49131700 & 0.32934200 & 2.47506100 \\
\hline & $\mathrm{H}$ & -4.57049100 & 2.41288600 & -0.26393500 \\
\hline & $\mathrm{H}$ & -4.74890200 & -1.86151000 & -0.07736100 \\
\hline & $\mathrm{H}$ & -7.19892500 & -1.77226800 & -0.24709300 \\
\hline & $\mathrm{H}$ & -8.36226000 & 0.41754800 & -0.39752000 \\
\hline & $\mathrm{H}$ & -7.02519000 & 2.51085500 & -0.38952500 \\
\hline & $\mathrm{H}$ & -3.25613500 & 1.26419600 & -2.51786900 \\
\hline & $\mathrm{H}$ & -2.15480600 & 3.20355600 & -3.54460200 \\
\hline & $\mathrm{H}$ & -0.50233300 & 4.53412300 & -2.24741200 \\
\hline & $\mathrm{H}$ & -0.01559000 & 3.90547200 & 0.10710200 \\
\hline & $\mathrm{H}$ & -1.13500400 & 1.99874900 & 1.13408000 \\
\hline & $\mathrm{H}$ & -0.57207600 & -0.60791400 & 1.05767200 \\
\hline & $\mathrm{H}$ & 0.06443500 & -0.86690800 & 3.39647300 \\
\hline & $\mathrm{H}$ & -1.57506000 & -0.33541000 & 5.20514300 \\
\hline & $\mathrm{H}$ & -3.85116000 & 0.44290900 & 4.58756000 \\
\hline & $\mathrm{H}$ & -4.49060000 & 0.65876500 & 2.22649000 \\
\hline & $\mathrm{H}$ & -0.17161400 & -4.24355500 & -1.56512900 \\
\hline & $\mathrm{H}$ & -0.09301900 & -2.49880300 & -1.44220500 \\
\hline 26 & 0 & -2.13032300 & -1.84993400 & -1.09310800 \\
\hline & $\mathrm{C}$ & -2.35069200 & -0.55702100 & -1.37174400 \\
\hline & $\mathrm{C}$ & -1.79856400 & -2.73532500 & -2.19721700 \\
\hline & $\mathrm{H}$ & -1.23347800 & -2.14843000 & -2.92353200 \\
\hline & $\mathrm{C}$ & -3.09284000 & -3.23400700 & -2.82333200 \\
\hline & $\mathrm{H}$ & -3.68681300 & -2.39315800 & -3.18299100 \\
\hline & $\mathrm{H}$ & -3.68092400 & -3.79431100 & -2.09192100 \\
\hline & 0 & -2.30097500 & -0.10776300 & -2.49086200 \\
\hline & $\mathrm{C}$ & -2.75029100 & 0.25859400 & -0.11227700 \\
\hline & $\mathrm{C}$ & -4.28760100 & 0.48430500 & -0.19383400 \\
\hline & $\mathrm{C}$ & -5.08972500 & 0.44606400 & 0.95045600 \\
\hline & $\mathrm{C}$ & -6.44958900 & 0.74041400 & 0.88265500 \\
\hline & $\mathrm{C}$ & -7.03573000 & 1.08041700 & -0.33166900 \\
\hline & $\mathrm{C}$ & -6.24265900 & 1.13706100 & -1.47455600 \\
\hline & $\mathrm{C}$ & -4.88302500 & 0.85074800 & -1.40608900 \\
\hline & $\mathrm{C}$ & -2.09538600 & 1.66267000 & -0.11463900 \\
\hline & $\mathrm{C}$ & -2.49358800 & 2.56419700 & 0.88123800 \\
\hline & C & -1.89527700 & 3.81195800 & 1.00429300 \\
\hline & $\mathrm{C}$ & -0.88711500 & 4.19520400 & 0.12023600 \\
\hline & $\mathrm{C}$ & -0.49686000 & 3.31675400 & -0.88343500 \\
\hline & $\mathrm{C}$ & -1.09629000 & 2.06248500 & -1.00226300 \\
\hline
\end{tabular}




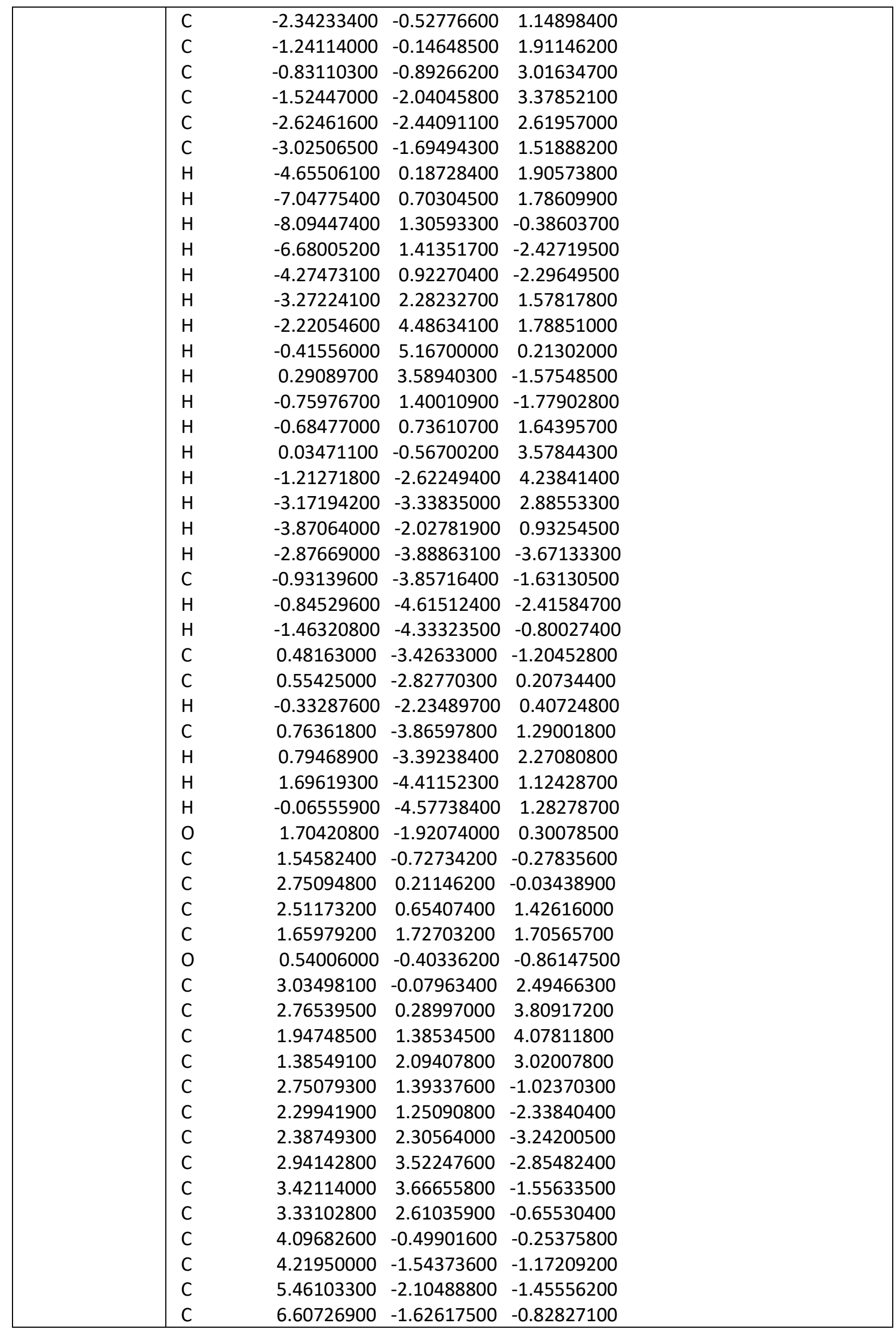




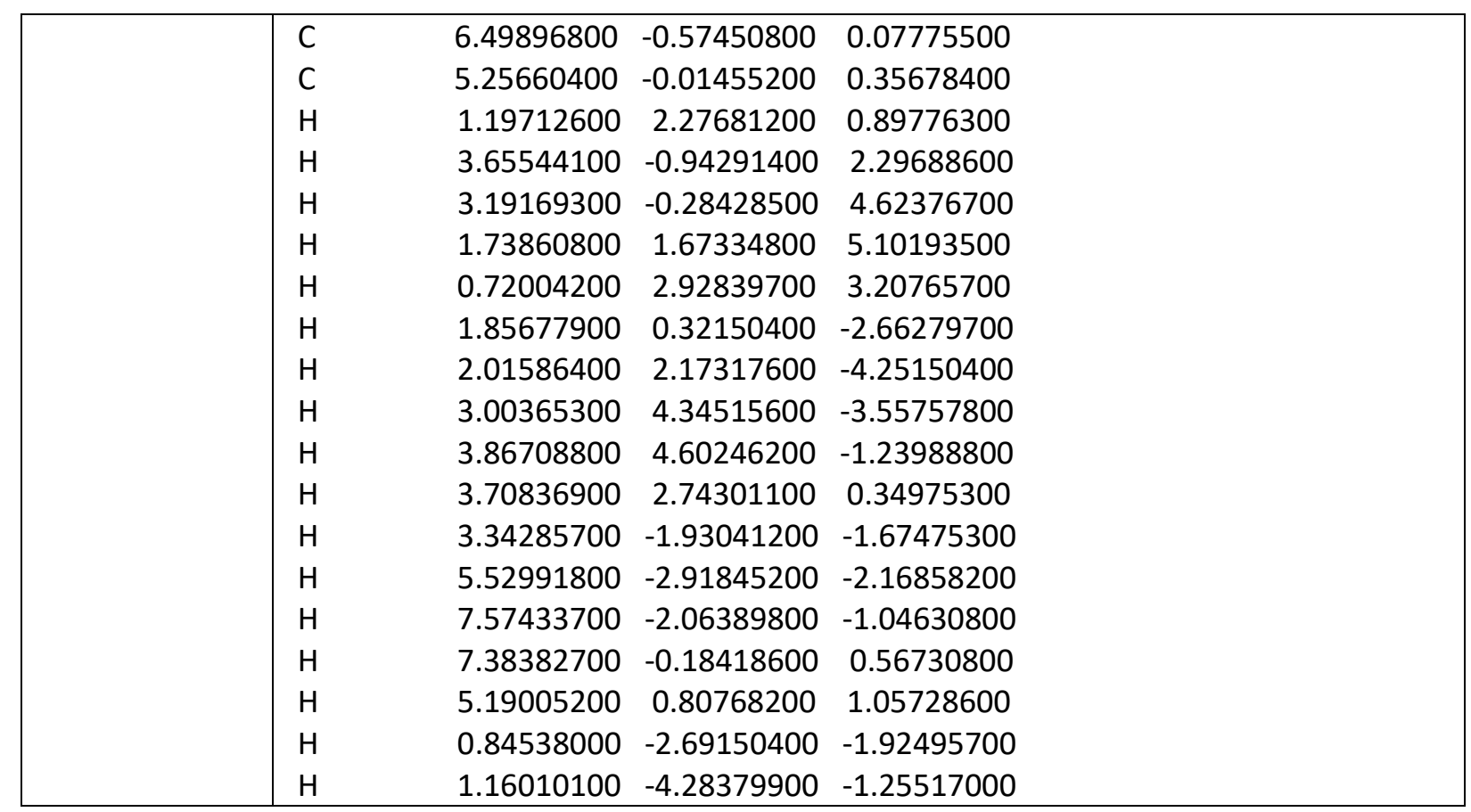

22 (optimized at the M06-2X/6-311G(d,p) level)

\begin{tabular}{|l|llll|}
\hline Conformer no & & & & \\
\hline 1 & O & -0.83205600 & 1.99166700 & -1.52716800 \\
& C & -0.42839400 & 3.25875200 & -1.41545400 \\
& C & -1.22702700 & 1.50140100 & -2.82693400 \\
& H & -0.70581900 & 2.09928900 & -3.57926100 \\
C & -2.72836500 & 1.66857000 & -2.97205000 \\
& H & -3.06010300 & 1.28569500 & -3.93921100 \\
& H & -2.99950800 & 2.72474900 & -2.91141300 \\
& O & -0.42137800 & 4.04776000 & -2.31855800 \\
& C & 0.07637200 & 3.50463300 & 0.02395700 \\
& C & -1.06064100 & 3.25761600 & 1.02624400 \\
& C & -0.76130000 & 3.17538300 & 2.38826600 \\
& C & -1.76606500 & 3.00700300 & 3.33110300 \\
& C & -3.09811400 & 2.94486500 & 2.93162000 \\
& C & -3.41041300 & 3.06233500 & 1.58384000 \\
& C & -2.39897900 & 3.21396100 & 0.63840800 \\
& C & 0.51841000 & 4.95893800 & 0.23402100 \\
& C & -0.28637400 & 6.00632900 & -0.22062700 \\
& C & 0.04642400 & 7.32578400 & 0.05178600 \\
& C & 1.18230900 & 7.62551200 & 0.79715900 \\
& C & 1.97677600 & 6.59167200 & 1.27219900 \\
& C & 1.64626700 & 5.26841300 & 0.99415200 \\
& C & 1.24212300 & 2.50238300 & 0.13859500 \\
& C & 2.39858000 & 2.74123500 & -0.61070400 \\
& C & 3.43842200 & 1.81982300 & -0.62429600 \\
C & 3.33507400 & 0.63674500 & 0.10354700 \\
& C & 2.17998000 & 0.37924600 & 0.83070900 \\
& C & 1.14148400 & 1.30602600 & 0.84294400 \\
H & 0.27252200 & 3.24238300 & 2.71018900 \\
H & -1.50917700 & 2.93378000 & 4.38114400 \\
\hline
\end{tabular}




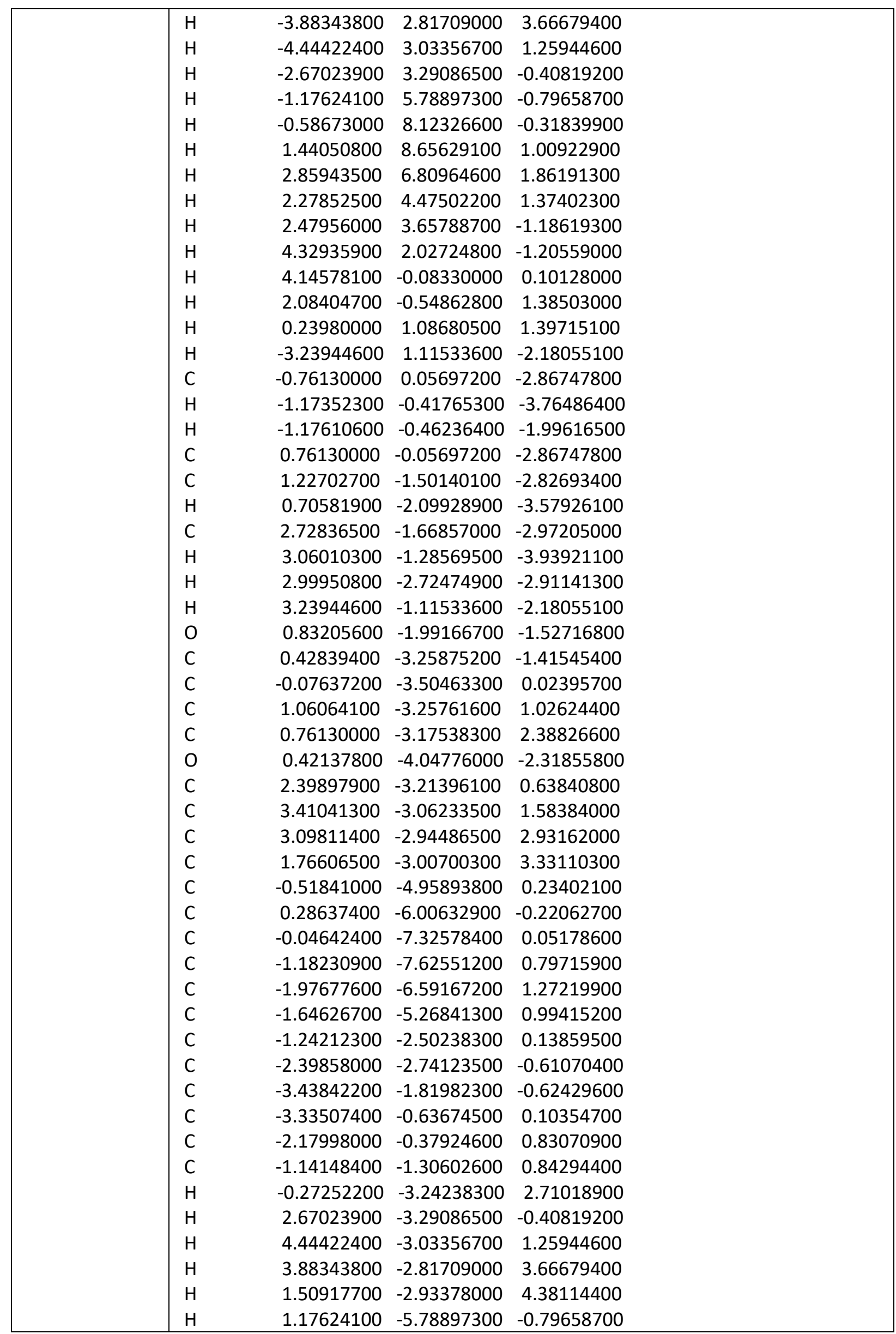




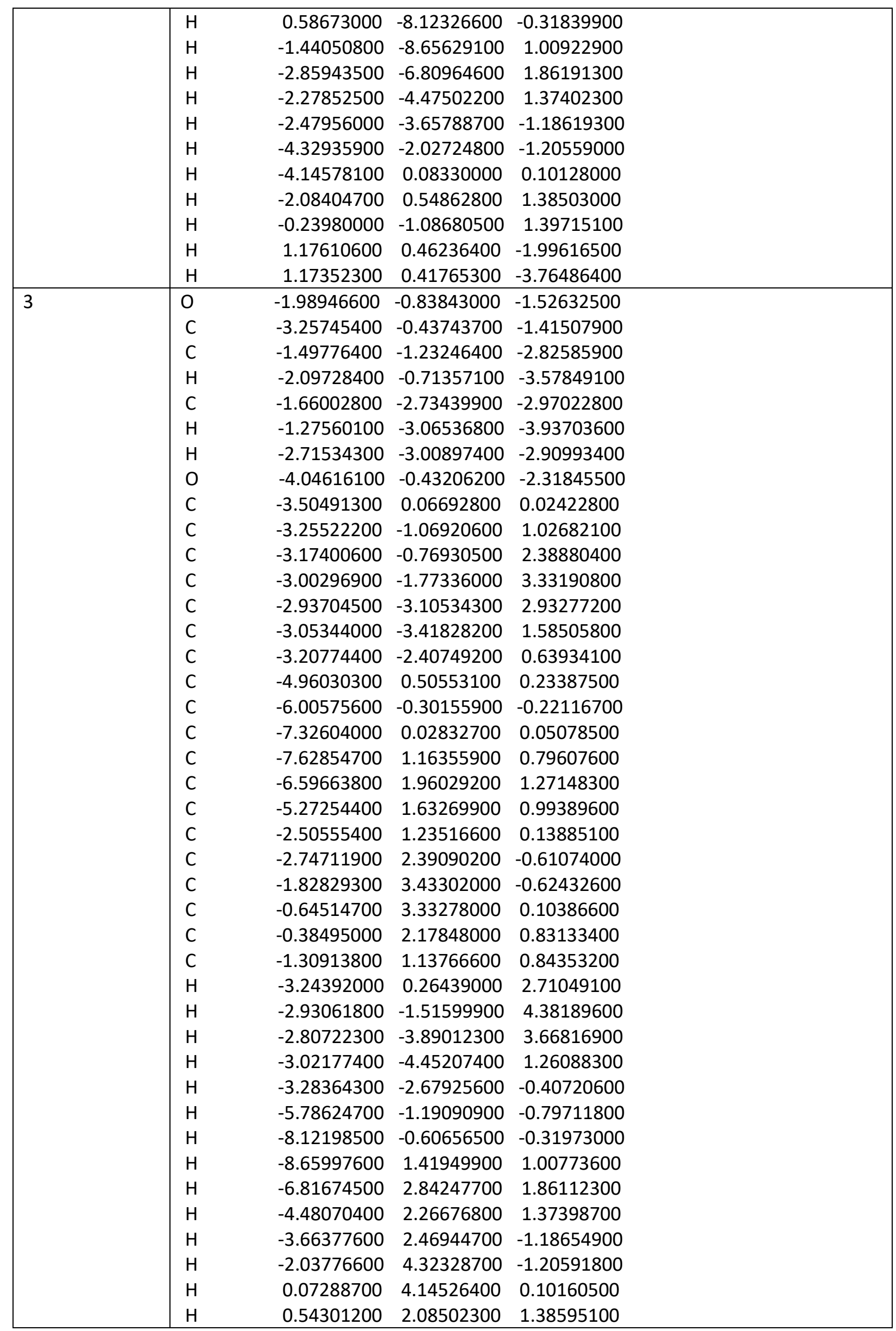




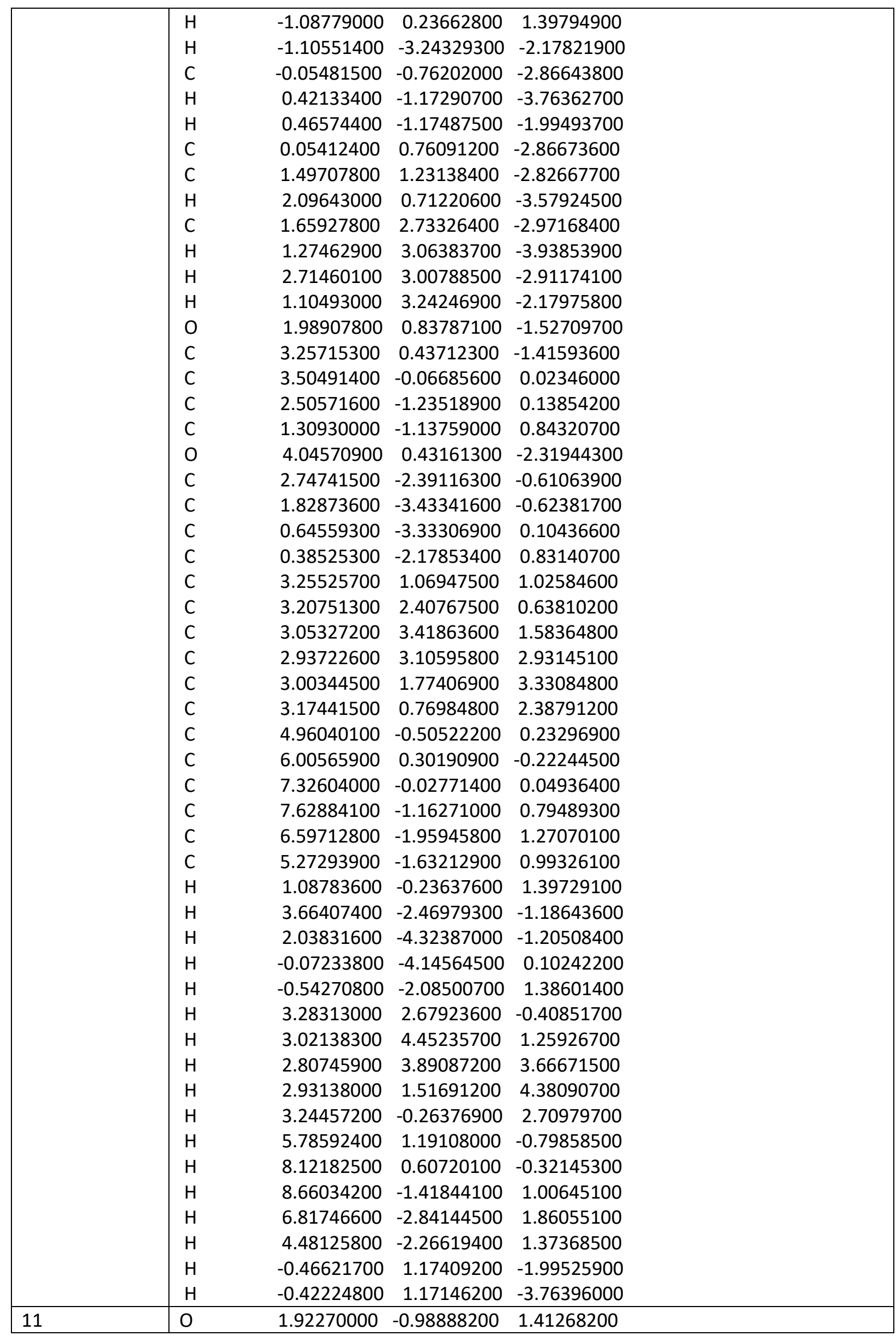




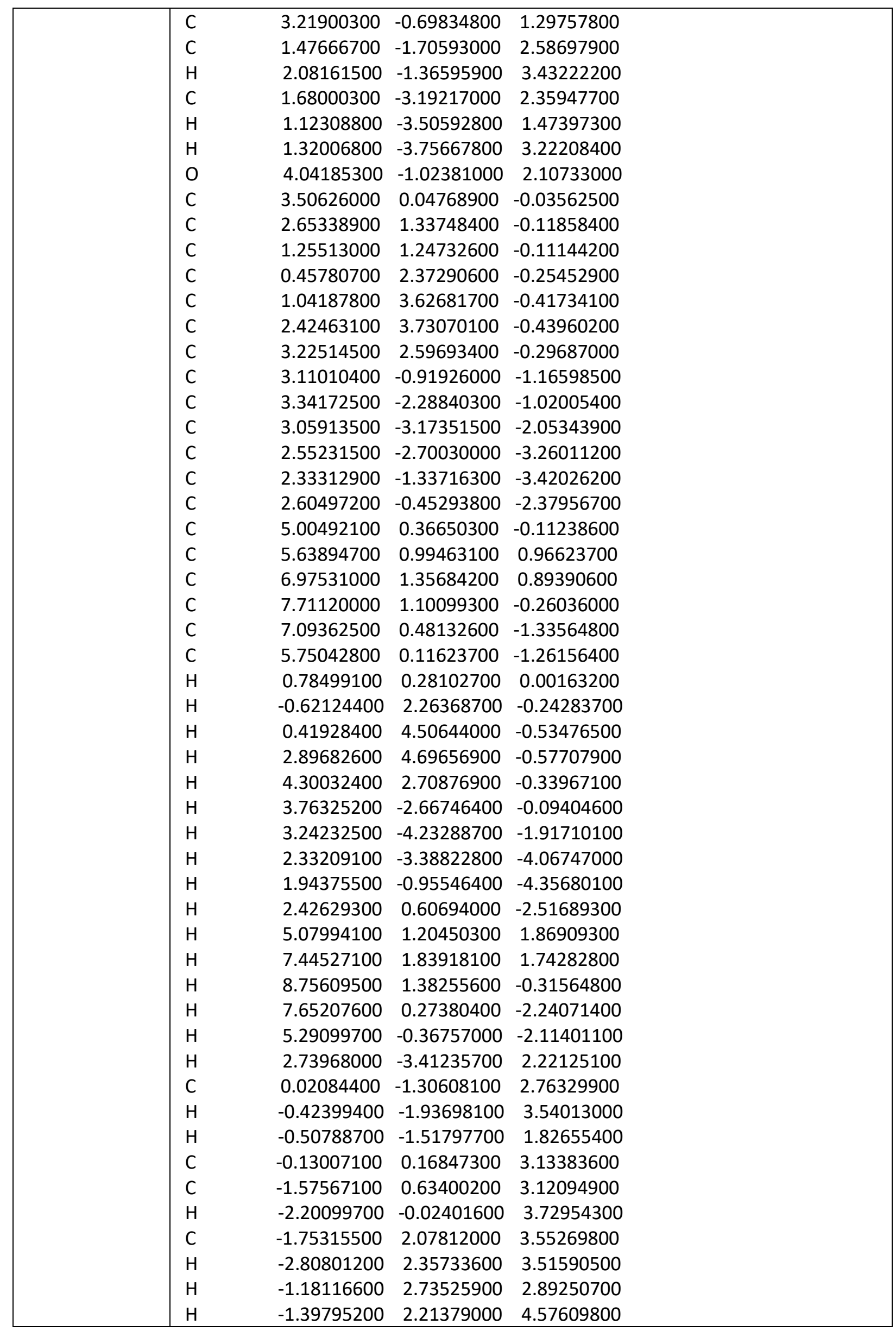




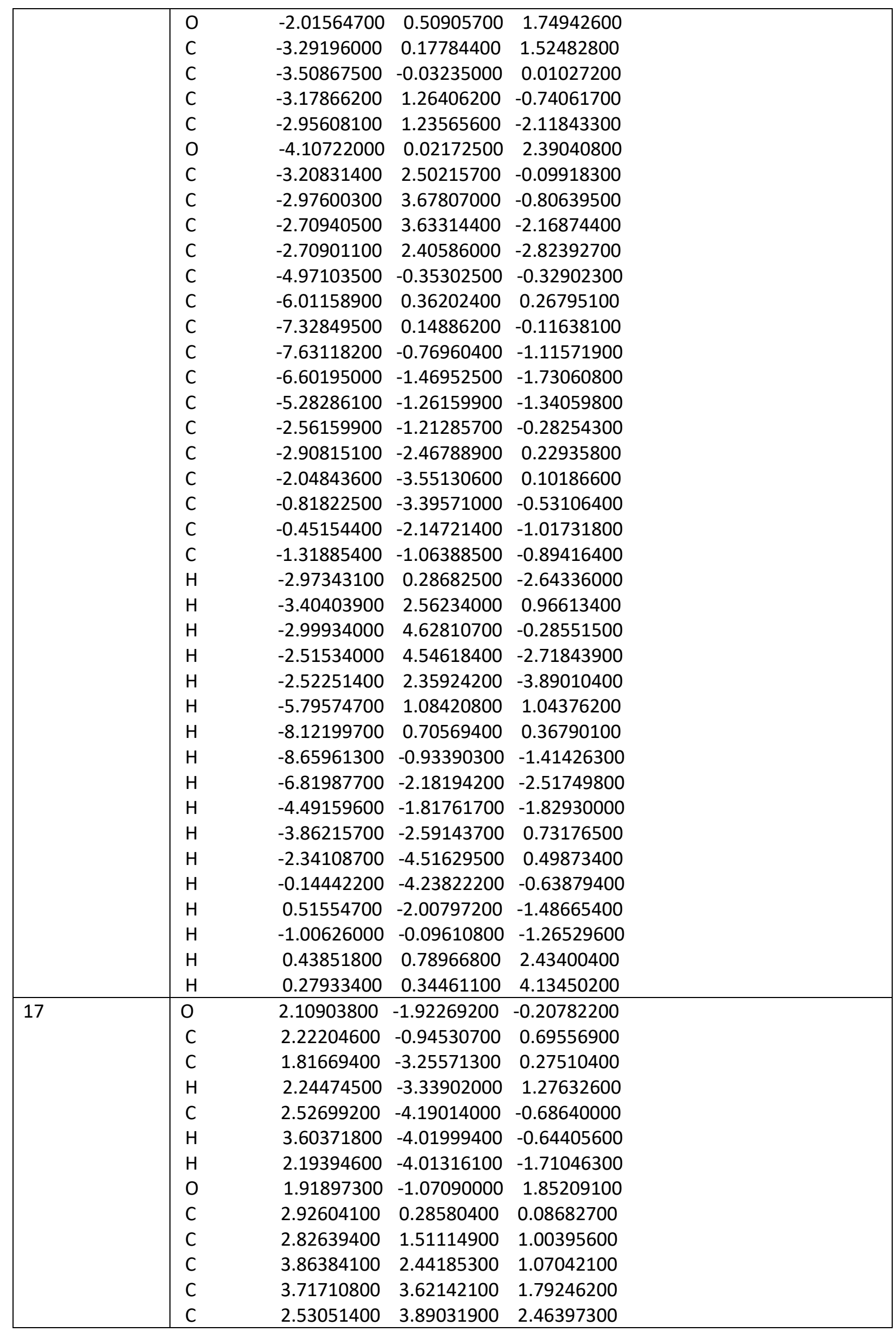




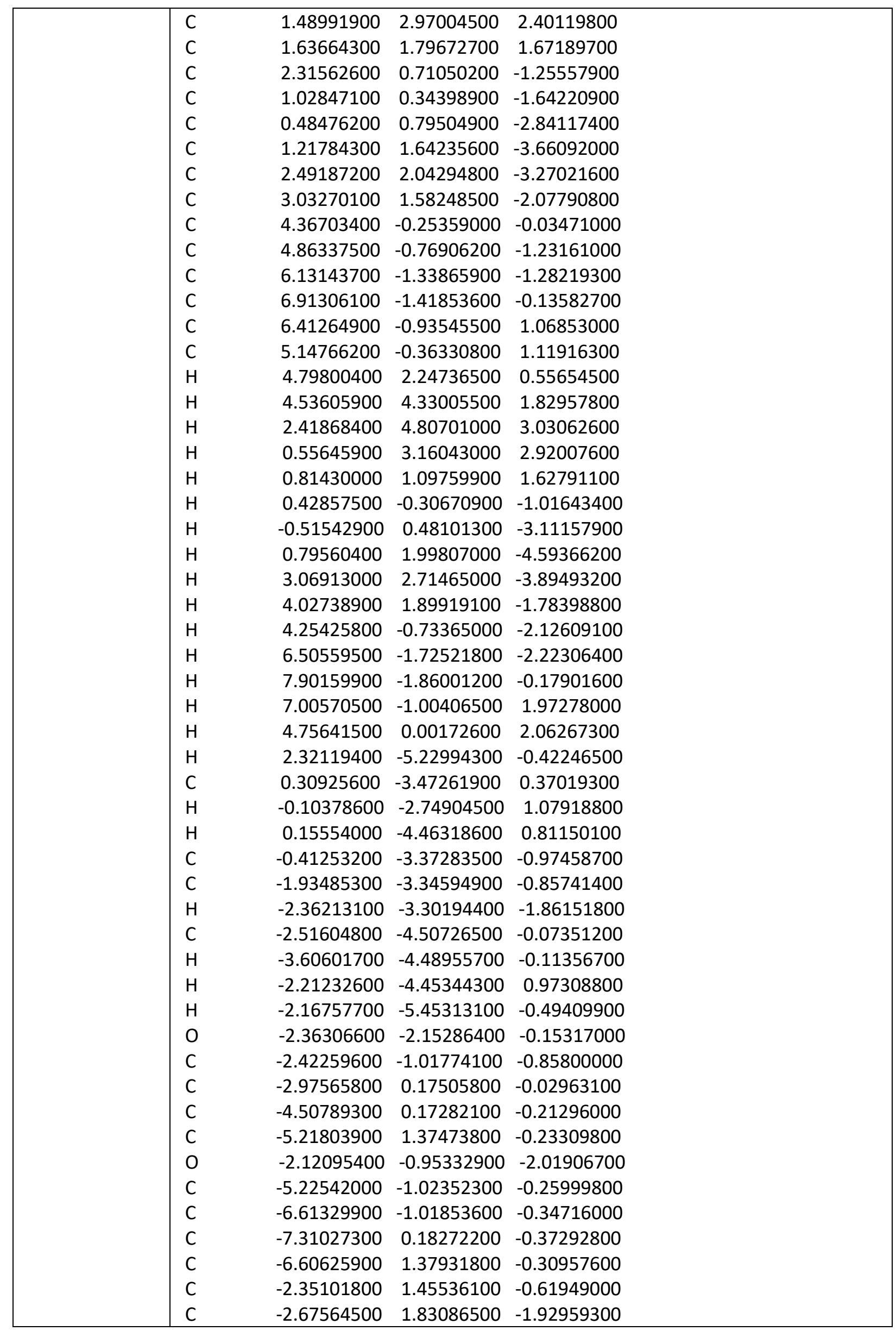




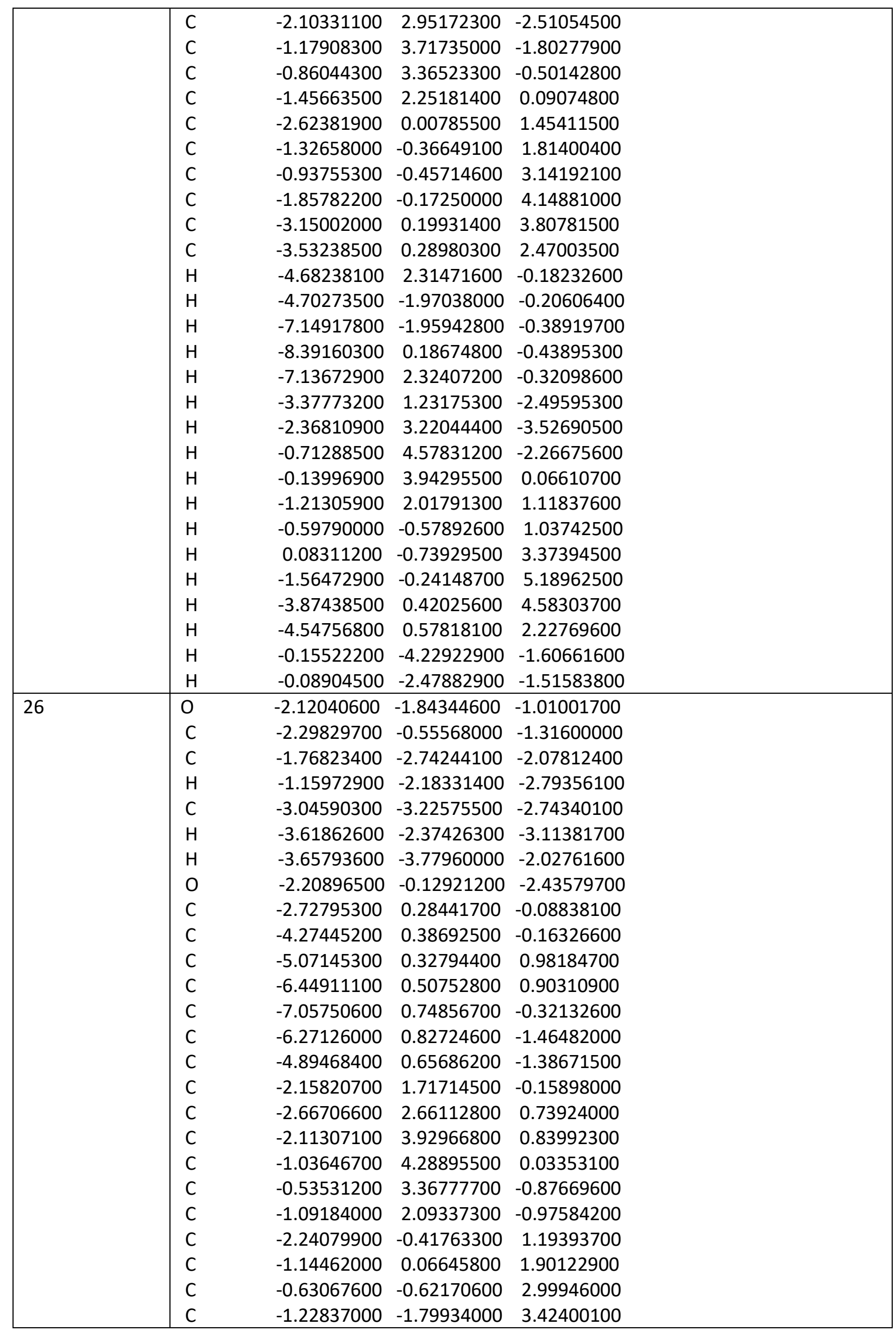




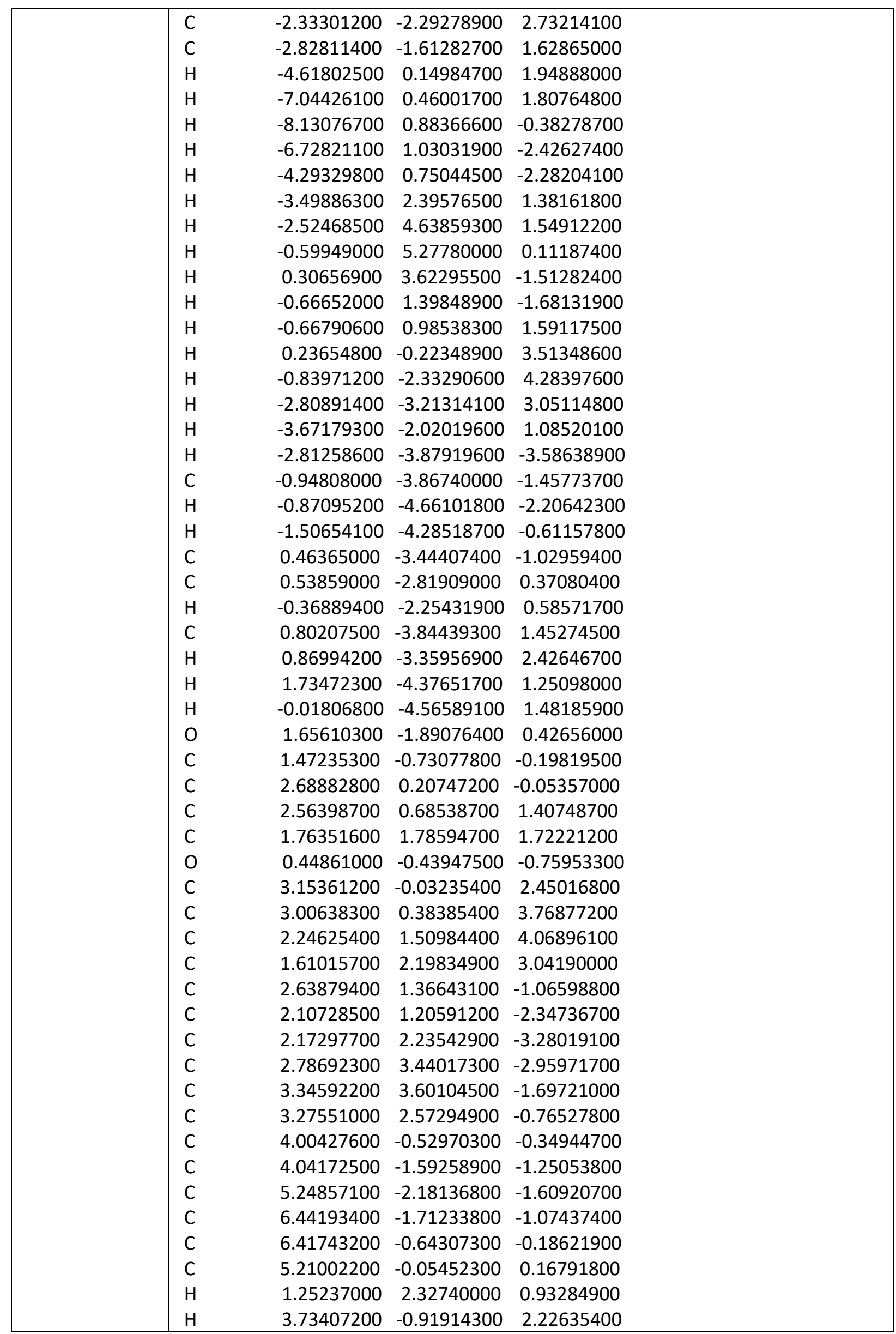




\begin{tabular}{|l|lrrr|}
\hline & H & 3.48726800 & -0.17556300 & 4.56267400 \\
$H$ & 2.13677600 & 1.83750800 & 5.09597600 \\
H & 0.98617600 & 3.05726200 & 3.26053700 \\
$\mathrm{H}$ & 1.62351800 & 0.27988400 & -2.62599700 \\
$\mathrm{H}$ & 1.73924300 & 2.08971200 & -4.26253800 \\
$\mathrm{H}$ & 2.83637200 & 4.24134800 & -3.68742300 \\
$\mathrm{H}$ & 3.84228000 & 4.52751000 & -1.43388600 \\
$\mathrm{H}$ & 3.71997600 & 2.71765600 & 0.21245700 \\
$\mathrm{H}$ & 3.12202400 & -1.97212300 & -1.68133800 \\
$\mathrm{H}$ & 5.25341400 & -3.00994800 & -2.30734100 \\
$\mathrm{H}$ & 7.38286300 & -2.17255300 & -1.35065800 \\
$\mathrm{H}$ & 7.34176700 & -0.26060300 & 0.23049300 \\
$\mathrm{H}$ & 5.20547900 & 0.78313100 & 0.85589400 \\
$\mathrm{H}$ & 0.83789100 & -2.72725400 & -1.76474900 \\
$\mathrm{H}$ & 1.13604700 & -4.30728800 & -1.05665000 \\
\hline
\end{tabular}




\section{References}

[1] SCIGRESS 2.5, Fujitsu Ltd

[2] M. J. Frisch, G. W. Trucks, H. B. Schlegel, G. E. Scuseria, M. A. Robb, J. R. Cheeseman, G. Scalmani, V. Barone, B. Mennucci, G. A. Petersson, H. Nakatsuji, M. Caricato, X. Li, H. P. Hratchian, A. F. Izmaylov, J. Bloino, G. Zheng, J. L. Sonnenberg, M. Hada, M. Ehara, K. Toyota, R. Fukuda, J. Hasegawa, M. Ishida, T. Nakajima, Y. Honda, O. Kitao, H. Nakai, T. Vreven, J. A. Montgomery Jr., J. E. Peralta, F. Ogliaro, M. Bearpark, J. J. Heyd, E. Brothers, K. N. Kudin, V. N. Staroverov, R. Kobayashi, J. Normand, K. Raghavachari, A. Rendell, J. C. Burant, S. S. Iyengar, J. Tomasi, M. Cossi, N. Rega, N. J. Millam, M. Klene, J. E. Knox, J. B. Cross, V. Bakken, C. Adamo, J. Jaramillo, R. Gomperts, R. E. Stratmann, O. Yazyev, A. J. Austin, R. Cammi, C. Pomelli, J. W. Ochterski, R. L. Martin, K. Morokuma, V. G. Zakrzewski, G. A. Voth, P. Salvador, J. J. Dannenberg, S. Dapprich, A. D. Daniels, Ö. Farkas, J. B. Foresman, J. V. Ortiz, J. Cioslowski, D. J. Fox, Gaussian 09, revision A.02, Gaussian, Inc., Wallingford CT, 2009.

[3] a) Becke, A. D. Density-functional thermochemistry. III. The role of exact exchange. J. Chem. Phys. 1993, 98, 5648-5652; b) Lee, C.; Yang, W.; Parr, R. G. Development of the Colle-Salvetti correlationenergy formula into a functional of the electron density. Phys. Rev. B 1988, 37, 785-789; c) Becke, A. D. Density-functional exchange-energy approximation with correct asymptotic behavior. Phys. Rev. A, 1988, 38, 3098-3100; d) Perdew, J. P. Density-functional approximation for the correlation energy of the inhomogeneous electron gas. Phys. Rev. B 1986, 33, 8822-8824.

[4] a) Zhao, Y.; Truhlar, D. G. The M06 suite of density functionals for main group thermochemistry, thermochemical kinetics, noncovalent interactions, excited states, and transition elements: two new functionals and systematic testing of four M06-class functionals and 12 other functionals. Theor. Chem. Acc. 2008, 120, 215-241; b) Jacquemin, D.; Perpète, E. A.; Ciofini, I.; Adamo, C.; Valero, R.; Zhao, Y.; Truhlar, D. G. On the Performances of the M06 Family of Density Functionals for Electronic Excitation Energies. J. Chem. Theor. 2010, 6, 2071-2085.

[5] a) Grimme, S.; Ehrlich, S.; Goerigk, L. Effect of the damping function in dispersion corrected density functional theory J. Comp. Chem. 2011, 32, 1456-1465; b) Antony, J.; Sure, R.; Grimme, S. Using dispersion-corrected density functional theory to understand supramolecular binding thermodynamics. Chem. Commun. 2015, 51, 1764-1774.

[6] Kwit, M.; Rozwadowska, M. D.; Gawroński, J.; Grajewska A. Density Functional Theory Calculations of the Optical Rotation and Electronic Circular Dichroism: The Absolute Configuration of 
the Highly Flexible trans-Isocytoxazone Revised. J. Org. Chem. 2009, 74, 8051-8063 and references therein.

[7] Yanai, T.; Tew, D.; Handy, N. A new hybrid exchange-correlation functional using the Coulombattenuating method (CAM-B3LYP). Chem. Phys. Lett. 2004, 393, 51-57.

[8] Harada, N.; Stephens, P. ECD cotton effect approximated by the Gaussian curve and other methods. Chirality 2010, 22, 229-233.

[9] CrysAlisPro 1.171.40.57a, Rigaku Oxford Diffraction, 2019.

[10] Sheldrick, G. M. SHELXT - Integrated space-group and crystal-structure determination. Acta Crystallogr. 2015, A71, 3-8.

[11] Sheldrick, G. M. Crystal structure refinement with SHELXL. Acta Crystallogr. 2015, C71, 3-8.

[12] Parsons, S.; Flack, H. D.; Wagner, T. Use of intensity quotients and differences in absolute structure refinement. Acta Crystallogr. 2013, B69, 249-259.

[13] Macrae, C. F.; Sovago, I.; Cottrell, S. J.; Galek, P. T. A.; McCabe, P.; Pidcock, E.; Platings, M.; Shields, G. P.; Stevens, J. S.; Towler, M.; Wood, P. A., Mercury 4.0: from visualization to analysis, design and prediction. J. Appl. Cryst. 2020, 45, 226-235.

[14] Dolomanov, O. V.; Bourhis, L. J.; Gildea, R. J.; Howard, J. A. K.; Puschmann, H. OLEX2: A Complete Structure Solution, Refinement and Analysis Program. J. Appl. Cryst. 2009, 42, 339-341.

[15] Spackman, M. A.; Jayatilaka, D. Hirshfeld surface analysis. CrystEngComm 2009, 11 ,19-32. 